The National Center on

Addiction and Substance Abuse

at Columbia University

DCOLUMBIA

633 Third Avenue

New York, NY 10017-6706

phone 2128415200

fax 2129568020

www.casacolumbia.org

Board of Directors

Jeffrey B. Lane

Chairman

Joseph A. Califano, Jr.

Founder and Chair Emeritus

William H. Foster, Ph.D.

President and $\mathrm{CEO}$

Lee C. Bollinger

Ursula M. Burns

Columba Bush

Kenneth I. Chenault

Peter R. Dolan

Victor F. Ganzi

Melinda B. Hildebrand

Ralph Izzo, Ph.D.

Gene F. Jankowski

David A. Kessler, M.D.

Jeffrey B. Kindler

Alan I. Leshner, Ph.D.

Rev. Edward A. Malloy, CSC

Doug Morris

Bruce E. Mosler

Manuel T. Pacheco, Ph.D.

Joseph J. Plumeri

Jim Ramstad

Shari E. Redstone

E. John Rosenwald, Jr.

Michael I. Roth

Mara Burros Sandler

Michael P. Schulhof

Louis W. Sullivan, M.D.

John J. Sweeney

Clyde C. Tuggle

\section{Directors Emeritus}

James E. Burke (1992-1997)

Jamie Lee Curtis (2001-2009)

Jamie Dimon (1995-2009)

Mary Fisher (1996-2005)

Betty Ford (1992-1998)

Douglas A. Fraser (1992-2003)

Barbara C. Jordan (1992-1996)

Leo Kelmenson (1998-2006)

Donald R. Keough (1992-2010)

LaSalle Leffall (1992-2001)

Nancy Reagan (1995-2000)

Linda J. Rice (1992-1996)

George Rupp (1993-2002)

Michael I. Sovern (1992-1993)

Frank G. Wells (1992-1994)

Michael A. Wiener (1997-2009)

\section{Addiction Medicine: Closing the Gap between Science and Practice}

June 2012

Funded by:

The Annenberg Foundation

The Diana, Princess of Wales Memorial Fund and The Franklin Mint The New York Community Trust Adrian and Jessie Archbold Charitable Trust 


\section{Board of Directors}

Lee C. Bollinger

President, Columbia University

Ursula M. Burns

Chairman and CEO, Xerox Corporation

Columba Bush

Former First Lady of Florida

Joseph A. Califano, Jr.

Founder and Chairman Emeritus, CASA Columbia

Kenneth I. Chenault

Chairman and CEO,

American Express Company

Peter R. Dolan

Chairman, ChildObesity180

William H. Foster, Ph.D.

President and CEO, CASA Columbia

Victor F. Ganzi

Chairman of the Board PGA Tour

Melinda B. Hildebrand

Ralph Izzo, Ph.D.

Chairman of the Board, CEO and President,

Public Service Enterprise Group, Inc. (PSEG)

Gene F. Jankowski

President, CBS Broadcasting, Retired

David A. Kessler, M.D.

Jeffrey B. Kindler

Jeffrey B. Lane

Chairman, CASA Columbia

Alan I. Leshner, Ph.D.

CEO, Executive Publisher, Science, American

Association for the Advancement of Science

\section{Directors Emeritus}

James E. Burke (1992-1997)

Jamie Lee Curtis (2001-2009)

Jamie Dimon (1995-2009)

Mary Fisher (1996-2005)

Betty Ford (1992-1998)

Douglas A. Fraser (1992-2003)

Barbara C. Jordan (1992-1996)

Leo-Arthur Kelmenson (1998-2006)
Rev. Edward A. Malloy, CSC

President Emeritus, University of Notre Dame

Doug Morris

CEO, Sony Music Entertainment

Bruce E. Mosler

Chairman, Global Brokerage,

Cushman \& Wakefield, Inc.

Manuel T. Pacheco, Ph.D.

President Emeritus, University of Arizona and

University of Missouri System

Joseph J. Plumeri

Chairman and CEO,

Willis Group Holdings PLC

Jim Ramstad

Former Member of Congress (MN-3)

Shari E. Redstone

President, National Amusements, Inc.

E. John Rosenwald, Jr.

Vice Chairman Emeritus, J.P.Morgan

Michael I. Roth

Chairman and CEO, The Interpublic Group

of Companies, Inc.

Mara Burros Sandler

Michael P. Schulhof

Chairman, GTI Holdings LLC

Louis W. Sullivan, M.D.

President Emeritus, Morehouse School of Medicine

John J. Sweeney

Clyde C. Tuggle

Senior Vice President, Chief Public Affairs and

Communications Officer, The Coca-Cola Company

Donald R. Keough (1992-2010)

LaSalle D. Leffall, Jr., M.D., F.A.C.S. (1992-2001)

Nancy Reagan (1995-2000)

Linda Johnson Rice (1992-1996)

George Rupp, Ph.D. (1993-2002)

Michael I. Sovern (1992-1993)

Frank G. Wells (1992-1994)

Michael A. Wiener (1997-2009)

Copyright (C2012. All rights reserved. May not be used or reproduced without the express written permission of The National Center on Addiction and Substance Abuse at Columbia University. 


\section{The CASA Columbia National Advisory Commission on Addiction Treatment}

Drew E. Altman, PhD (Chair)

President and Chief Executive Officer

The Henry J. Kaiser Family Foundation

Harvey V. Fineberg, MD, PhD

President

Institute of Medicine

Mark S. Gold, MD

University of Florida College of Medicine

and McKnight Brain Institute

Departments of Psychiatry, Neuroscience, Anesthesiology, Community Health \& Family

Medicine

Chairman, Department of Psychiatry

Shelly F. Greenfield, MD, MPH

Chief Academic Officer, McLean Hospital

Professor of Psychiatry, Harvard Medical School

Director, Clinical and Health Services Research

and Education

Division on Alcohol and Drug Abuse,

McLean Hospital

Elizabeth R. Kabler

President

Rosenstiel Foundation

Myles V. Lynk, JD

Peter Kiewit Foundation

Professor of Law and the Legal Profession

Faculty Fellow, Center for Law, Science \&

Innovation's Program in Public Health Law

and Policy

Sandra Day O'Connor College of Law

Arizona State University
June E. Osborn, MD

President Emerita

Josiah Macy, Jr. Foundation

Manuel T. Pacheco, PhD

President Emeritus, University of Arizona

and University of Missouri System

The Honorable Jose R. Rodriguez

Circuit Judge

Ninth Judicial Circuit of Florida

Reverend Msgr. Stephen J. Rossetti, PhD, DMin Clinical Associate Professor

Associate Dean for Seminary

and Ministerial Programs

The Catholic University of America,

The School of Theology and Religious Studies

Former President and Chief Executive Officer

Saint Luke Institute, Inc.

Leonard D. Schaeffer

Judge Robert Maclay Widney

Chair \& Professor

University of Southern California

Steven A. Schroeder, MD

Distinguished Professor of Health and Health

Care, Department of Medicine

Director, Smoking Cessation Leadership Center

University of California, San Francisco

Louis W. Sullivan, MD

President Emeritus

Morehouse School of Medicine 


\section{Table of Contents}

Foreword and Accompanying Statement by Drew Altman, PhD ...................................... i

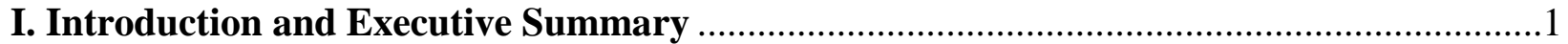

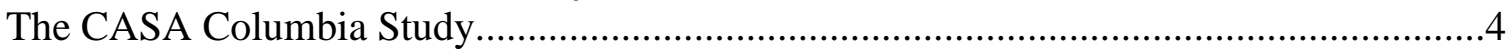

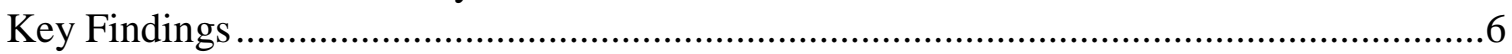

Addiction Is a Brain Disease.....................................................................................6

As with Other Health Conditions, There Are Clear Risk Factors for the Development of Addiction..............................................................................

Addiction Frequently Co-Occurs with Other Health Conditions ...................................

Addiction Can Be a Chronic Disease..........................................................................

A Lack of Standardized Terminology Compromises Effective Interventions................7

Multiple Addictive Substances and Behaviors Frequently Are Involved in Risky Use and Addiction ..................................................................................

Public Attitudes about the Causes of Addiction Are Out of Sync with the Science .....8

Physicians and Other Health Care Providers Should Be on the Front Line for Addressing this Disease ................................................................................

Screening and Intervention Are Effective at Addressing

Risky Substance Use and Forestalling Addiction .................................................

Effective Therapies to Treat and Manage Addiction Exist............................................9

The Importance of Tailored Interventions and Treatment............................................10

Public Attitudes about Addiction Treatment Reflect the Prevailing

Non-Medical Approach to Addiction Care ........................................................10

Most People in Need of Treatment Do Not Receive It ...............................................10

Most Referrals to Publicly Funded Treatment Come from the

Criminal Justice System.....................................................................................11

Less than Half of Treatment Admissions Result in Treatment Completion.................12

Patients Face Formidable Barriers to Receiving Addiction Treatment ........................12

The Spending Gap.............................................................................................12

Most Funding for Addiction Treatment Comes from Public Sources ..........................13

The Education, Training and Accountability Gap ………………………...................13

The Profound Disconnect between Evidence and Practice..........................................13

Recommendations and Next Steps................................................................................14

Reform Health Care Practice ..................................................................................14

Use the Leverage of Public Policy to Speed Reform in Health Care Practice ............15

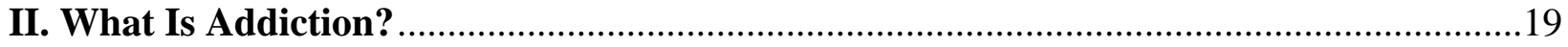



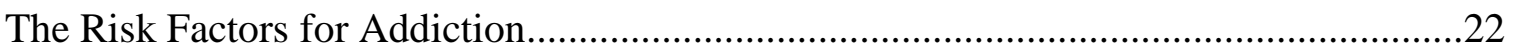

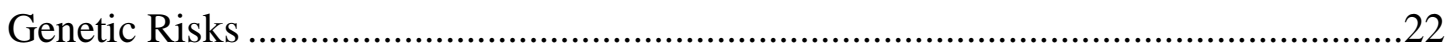



Psychological Risks .......................................................................................23

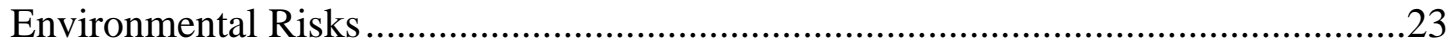

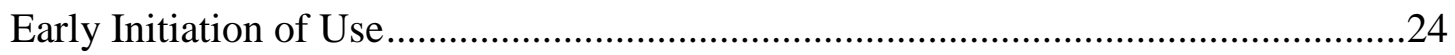

Risky Use and Addiction Frequently Co-Occur with Other Health Conditions................24

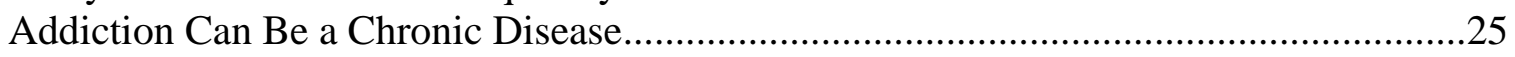




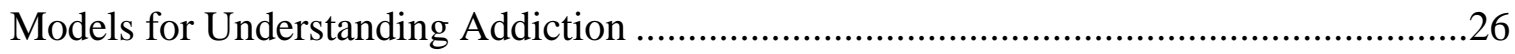

Evolving Approaches to Addressing Addiction …………….....................................26



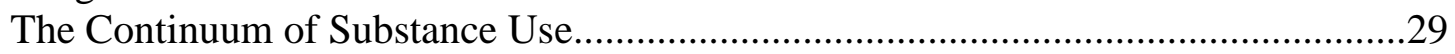

Public Attitudes about Addiction.................................................................................34

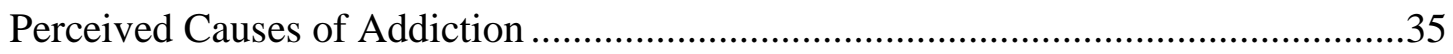

III. Prevalence and Consequences ...................................................................................

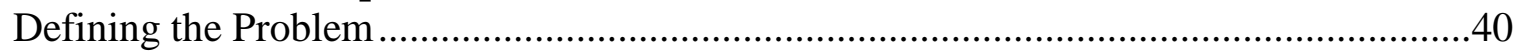

Risky Substance Users .................................................................................................

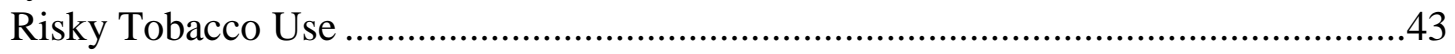

Risky Alcohol Use …………………………………….................................44

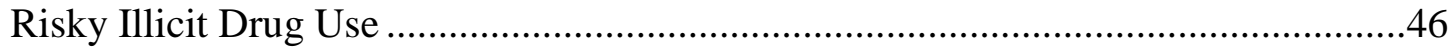

Risky Use of Controlled Prescription Drugs ...........................................................47

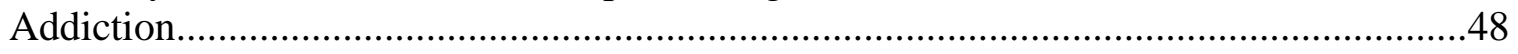

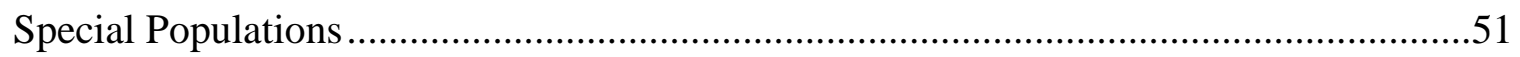



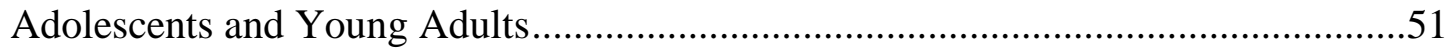

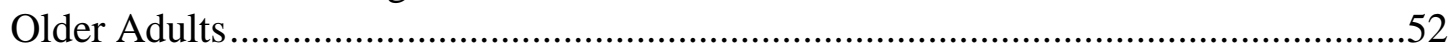

Co-Occurring Disorders ........................................................................................52

Members of the Military Exposed to Combat..............................................................53

Involvement in the Justice System.......................................................................55

Consequences of Risky Substance Use and Untreated Addiction ....................................55

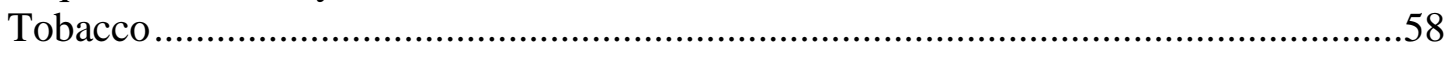

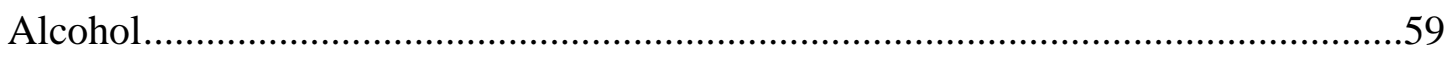

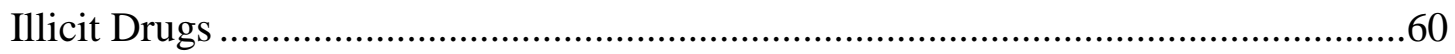

Controlled Prescription Drugs …………………………......................................61

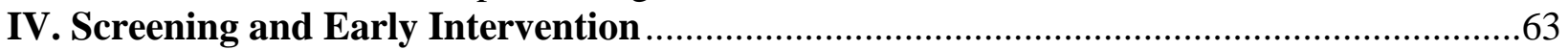

The Need for Patient Education, Screening and Intervention throughout the Lifespan ....64

Childhood and Adolescence ………………………………………………….....64

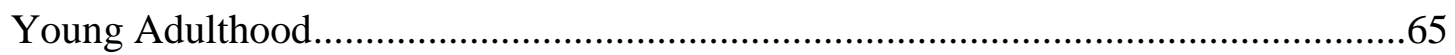

Middle and Later Adulthood................................................................................65

Attending to Co-Occurring Conditions ........................................................................66

Patient Education and Motivation............................................................................66



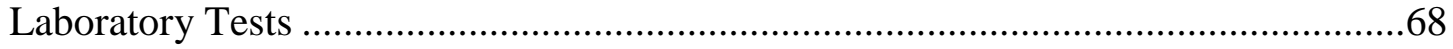

Brief Interventions and Treatment Referrals ...........................................................69

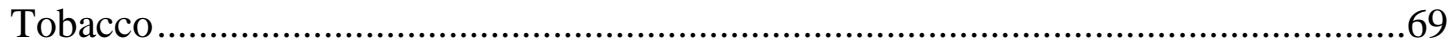

Alcohol and Other Drugs ...................................................................................

Effectiveness of Screening and Brief Interventions.......................................................71

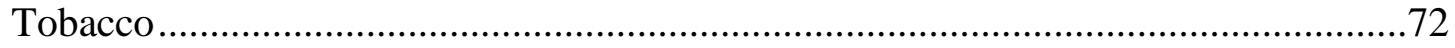

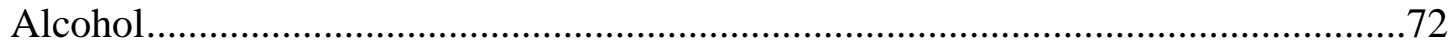

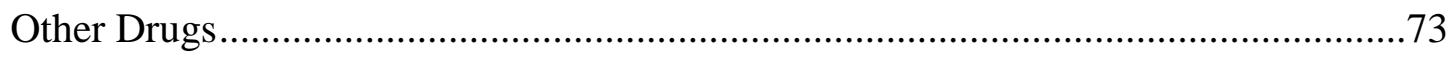

Implementing Screening and Brief Interventions in Health Care and Other Settings.......74

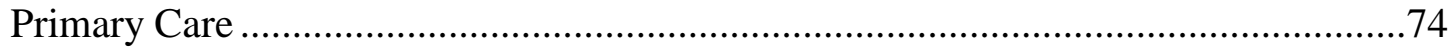



Health Care for Pregnant Women ............................................................................77 






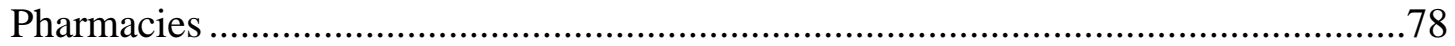

High School, College and University Settings …………….....................................78

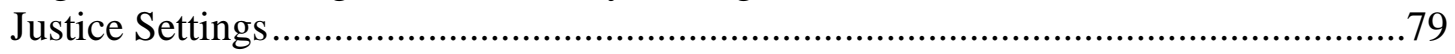

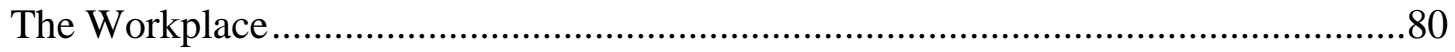

Government-Funded Social Service Systems ...........................................................

Barriers to Effective Implementation of Screening and Brief Interventions ......................81

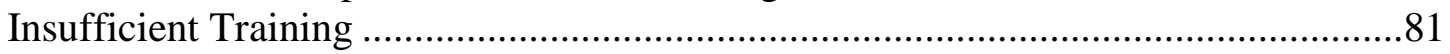

Competing Priorities/Insufficient Resources .........................................................82

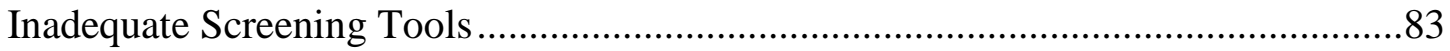

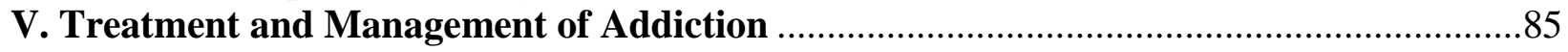

A Comprehensive Approach to Treatment ....................................................................86

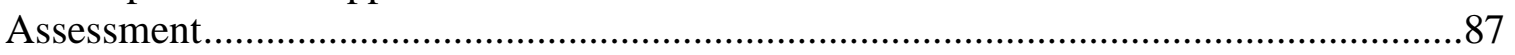

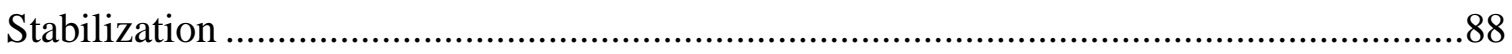

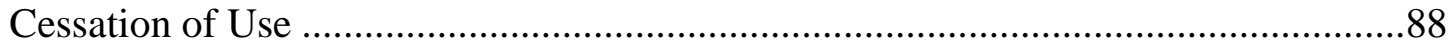

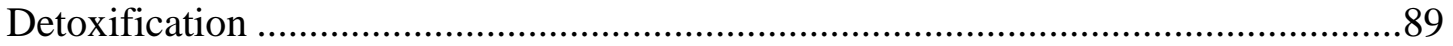

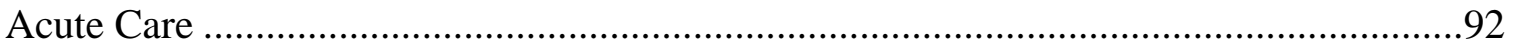

Pharmaceutical Therapies ................................................................................92

Psychosocial Therapies ....................................................................................102

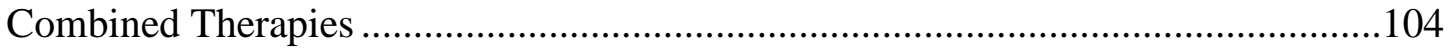

Nutrition and Exercise …………………............................................................106

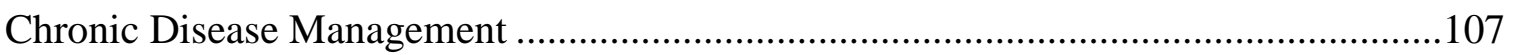

Medically Supervised Disease Management ……………........................................107

Case Management ......................................................................................108





Auxiliary Support Services ....................................................................................113

The Use of Technology in Addiction Treatment and Disease Management ....................114

Public Attitudes about Addictive Substances and the Need for Addiction Treatment ....114

Perceptions of the Relative Need for Treatment Based on Substance of Addiction .114

Perceptions of the Goals of Treatment...................................................................115

Perceptions of the Types of Interventions that Constitute Treatment........................116

Perceptions of the Effectiveness of Treatment .......................................................116



Co-occurring Medical Disorders............................................................................119

Co-occurring Mental Health Disorders ..........................................................................120

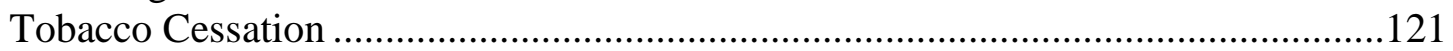

Treatment for Addiction Involving Alcohol and Other Drugs ..................................121

Adolescents .........................................................................................................122

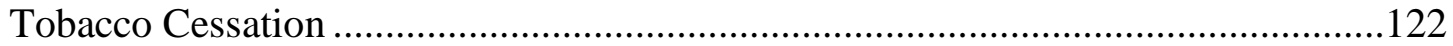

Treatment for Addiction Involving Alcohol and Other Drugs ..................................123

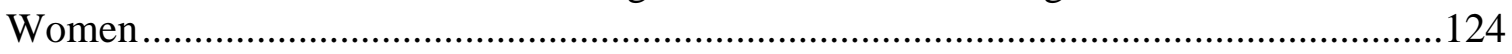

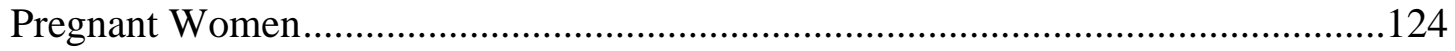

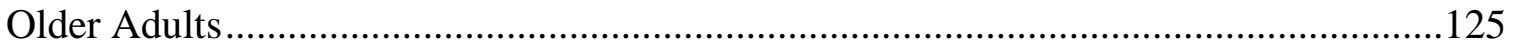

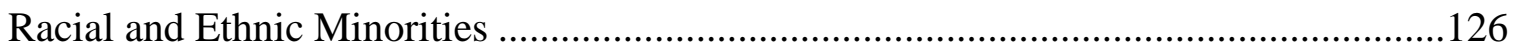


Individuals of Minority Sexual Orientation.............................................................126

Veterans and Active Duty Military .........................................................................127

Individuals Involved in the Justice System..............................................................128

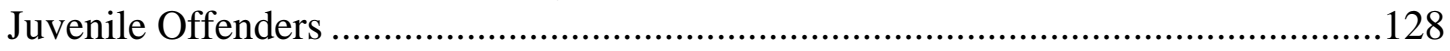

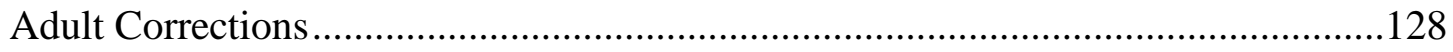

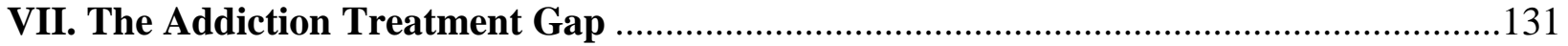

Most People in Need of Treatment Do Not Receive It ...................................................133

Variations in the Treatment Gap by Primary Substance Involved .............................134

Variations in the Treatment Gap by Key Patient Characteristics ...............................135

Regional Variations in the Treatment Gap ...........................................................137

Sources of Funding for Addiction Treatment ………….............................................137

Privately-Funded Treatment ……………………….....................................138

Publically-Funded Treatment..............................................................................138

Trends in Spending on Addiction Treatment......................................................139

Expenditures by Providers and Types of Services...................................................139

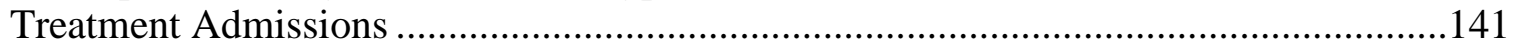

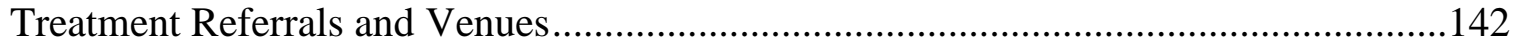

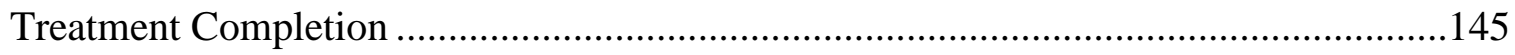

Variations in Treatment Completion by Source of Referral ......................................146

Variations in Treatment Completion by Primary Substance Involved .......................146

Variations in Treatment Completion by Key Patient Characteristics .........................146

Link between Funding Source, Type of Service Provided and Treatment Completion ..146

Barriers Patients Face in Accessing and Completing Addiction Treatment....................147

Misunderstanding of the Disease ..........................................................................147

Negative Public Attitudes and Behaviors Toward People with Addiction.................148

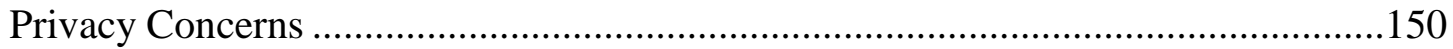

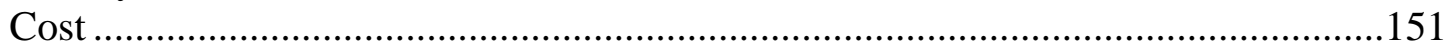

Lack of Information about How To Get Help …………........................................152

Limited Availability of Services ......................................................................152

Insufficient Social Support ……………………………….............................153

Conflicting Time Commitments .........................................................................153

Negative Perceptions of the Treatment Process........................................................153

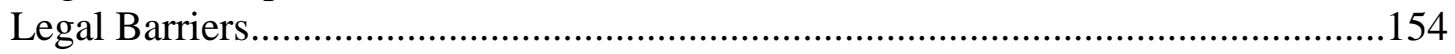

Barriers to Treatment Access and Completion in Special Populations ............................154



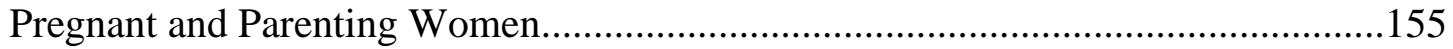



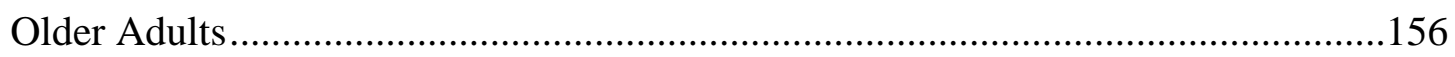

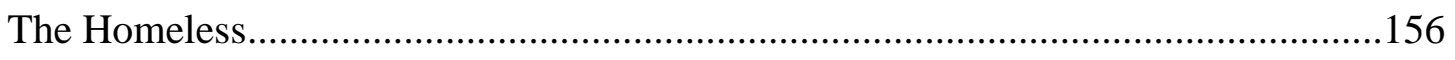

Veterans and Active Duty Military …………………........................................157



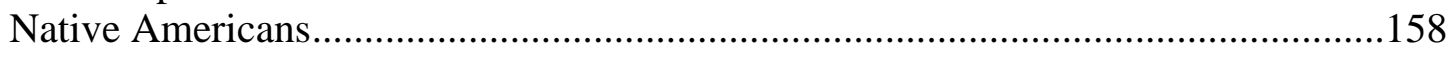




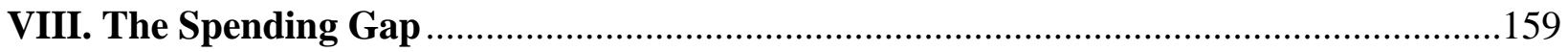

The Rational Approach to Risky Substance Use and Addiction .....................................159

Costs of Our Failure to Prevent and Treat Addiction as a Medical Condition .................160

The Largest Share of Costs Falls to the Health Care System .....................................160

Cost Savings of Addiction Screening, Intervention and Treatment ................................161

Screening and Early Intervention..........................................................................162

Addiction Treatment and Disease Management .......................................................164

Insurance Coverage of Addiction Treatment is Limited....................................................166



The Patient Protection and Affordable Care Act of 2010 ...........................................168

Gaps in Coverage within Public and Private Insurance Plans Continue to Impede Comprehensive Addiction Care ............................................................169

IX. The Education, Training and Accountability Gap ......................................................175

The Size and Shape of the Addiction Treatment Workforce..........................................176

Licensing and Credentialing Requirements for Individuals who Provide

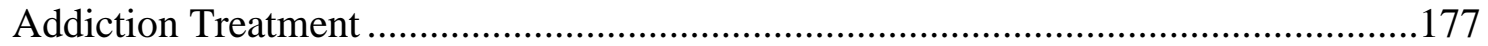

Medical Professionals .................................................................................178

Mental Health Professionals ...............................................................................183

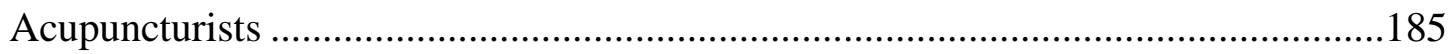

Addiction Counselors..........................................................................................186

Licensure, Certification and Accreditation Requirements for Addiction Treatment

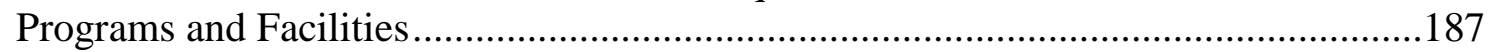

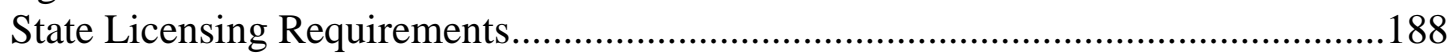

Federal Regulatory Requirements.......................................................................189

Accreditation Requirements...............................................................................190

Professional Staffing Requirements...................................................................191

Treatment Service Requirements ...................................................................193

Quality Assurance Requirements........................................................................195

X. The Evidence-Practice Gap ........................................................................................... 199

Current Approaches to Risky Substance Use and Addiction Are Inconsistent

with the Science and Evidence-Based Care...............................................................199

Patient Education, Screening, Brief Interventions and Treatment Referrals ..............200

Assessment, Stabilization and Acute Treatment....................................................204

Tailored Treatment Services ...............................................................................208

Chronic Disease Management ……………………………………………….....210

Barriers to Closing the Evidence-Practice Gap ……………......................................212

The Addiction Treatment Workforce is Not Qualified to Implement

Evidence-Based Practices ..............................................................................212

Health Professionals do not Implement Evidence-Based Addiction Care Practices .216

Inadequate Use and Development of Pharmaceutical Treatments for Addiction......219

Inadequate Quality Assurance ………………………....................................220

Inadequate Insurance Coverage .........................................................................222

No Overarching Organizing Body for Addiction Science and Treatment.................223

Efforts to Integrate Substance Use Prevention and Treatment into

Mainstream Medicine 


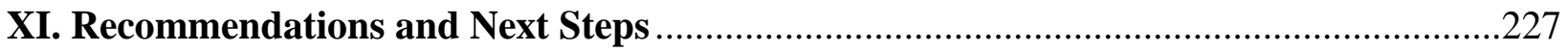



Incorporate Screening and Intervention for Risky Substance Use, and Diagnosis, Treatment and Disease Management for Addiction into Routine Medical Practice .........................................................................228

All Medical Schools and Residency Training Programs Should Educate and Train Physicians to Address Risky Substance Use and Addiction ......................229

Require Non-Physician Health Professionals to Be Educated and Trained to Address Risky Substance Use and Addiction ...................................................229

Develop Improved Screening and Assessment Instruments .....................................230

Establish National Accreditation Standards for All Addiction Treatment

Facilities and Programs that Reflect Evidence-Based Care................................230

Standardize Language Used to Describe the Full Spectrum of Substance Use and



Use the Leverage of Public Policy to Speed Reform in Health Care Practice ................231

Condition Grants and Contracts for Addiction Services on the Provision of

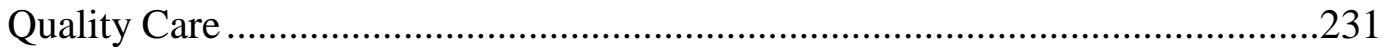

Educate Non-Health Professionals about Risky Substance Use and Addiction ........231 Identify Patients at Risk in Government Programs and Services where Costs of

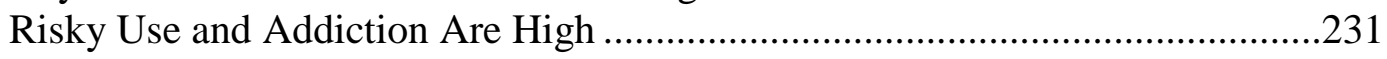

Develop Tools to Improve Service Quality ………………….................................231

License Addiction Treatment Facilities as Health Care Providers ............................232

Require Adherence to National Accreditation Standards that

Reflect Evidence-Based Care .........................................................................232

Require that All Insurers Provide Coverage for Comprehensive Addiction Care.....232

Expand the Addiction Medicine Workforce ..............................................................232

Implement a National Public Health Campaign .......................................................233

Invest in Research and Data Collection to Improve and Track Progress in Addiction

Prevention, Treatment and Disease Management...............................................233

Implement the National Institutes of Health's (NIH) Recommendation to Create a

Single Institute Addressing Substance Use and Addiction.................................234

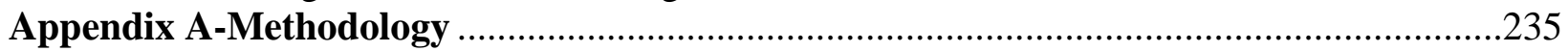

Appendix B-Key Informant Interview Guide and List of Key Informants........................245

Appendix C-National Addiction Belief and Attitude Survey (NABAS) ..............................253

Appendix D-Survey of New York State Addiction Treatment Directors .............................269

Appendix E-Survey of New York State Addiction Treatment Staff ..................................28

Appendix F- National Panel and National Online Survey of Members of Professional

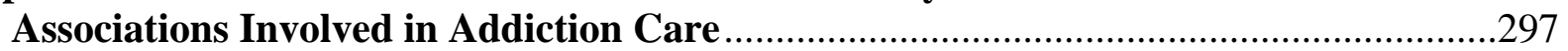





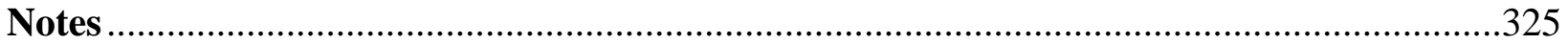






\section{Accompanying Statement by \\ Drew E. Altman, PhD, Chair, The CASA Columbia National Advisory Commission on Addiction Treatment}

In homes, doctors' offices, hospitals, schools, prisons, jails and communities across America, misperceptions about addiction are undermining medical care. Although advances in neuroscience, brain imaging and behavioral research clearly show that addiction is a complex brain disease, today the disease of addiction is still often misunderstood as a moral failing, a lack of willpower, a subject of shame and disgust. Addiction affects 16 percent of Americans ages 12 and older--40 million people. That is more than the number of people with heart disease ( 27 million), diabetes (26 million) or cancer (19 million). Another 32 percent of the population ( 80 million) uses tobacco, alcohol and other drugs in risky ways that threaten health and safety.

Like other public health and medical problems, we understand the risk factors for addiction. We have effective ways of screening for risky use and intervening. While as of now there is no cure for addiction, there are effective psychosocial and pharmaceutical treatments and methods of managing the disease. But as this landmark report by CASA Columbia shows in sharp detail, this is where the comparison with other health conditions ends. Unlike other diseases, we do little to effectively prevent and reduce risky use and the vast majority of people in need of addiction treatment do not receive anything that approximates evidence-based care.

The medical system, which is dedicated to alleviating suffering and treating disease, largely has been disengaged from these serious health care problems. The consequences of this inattention are profound. America's failure to prevent risky use and effectively treat addiction results in an enormous array of health and social problems such as accidents, homicides and suicides, child neglect and abuse, family dysfunction and unplanned pregnancies. CASA Columbia estimates that risky substance use and 
addiction are this nation's largest preventable and most costly health problems, accounting for one third of hospital inpatient costs, driving crime and lost productivity and resulting in total costs to government alone of at least $\$ 468$ billion each year.

In many ways, America's approach to addiction treatment today is similar to the state of medicine in the early 1900s. In 1908, the Council on Medical Education of the American Medical Association turned to the Carnegie Foundation for the Advancement of Teaching to conduct a survey of Medical Education in the U.S. That survey, which became known as the Flexner Report, was led by Abraham Flexner who famously observed of the discrepancy among physicians' qualifications, "there is probably no other country in the world in which there is so great a distance and so fatal a difference between the best, the average and the worst." This CASA Columbia report identifies a similar gulf in the knowledge and practice skills of addiction treatment providers today. The education and training of persons providing addiction treatment vary considerably by state. In many cases, entry requirements for the profession are minimal in terms of education and are based on apprenticeship models rather than on science-based instruction.

Flexner noted that the turn of the $19^{\text {th }}$ to $20^{\text {th }}$ century was a time of scientific progress in the understanding of disease and its treatment; however, due to the lack of a standardized and rigorous education for physicians, society reaped "but a small fraction of the advantage which current knowledge has the power to confer." Similarly, 100 years later, advances in science and medicine have drawn a much clearer picture of addiction--including its causes, correlates and how to treat it--yet we are woefully unprepared to apply this evidence to practice. Our medical professionals are not trained to look for risky use and addiction or to intervene or treat the disease. Without medical attention, the disease progresses, forcing doctors to expend valuable resources treating the more than 70 other conditions requiring medical attention that result from substance use and addiction, while taxpayers shoulder the costs of these health and other social consequences. This neglect by the medical system has led to the creation of a separate and unrelated system of addiction care that struggles to treat the disease without the resources or the knowledge base to keep pace with science and medicine.

Because addiction affects cognition and is associated primarily with the difficult social consequences that result from our failure to prevent and treat it, those who suffer from the disease are poor advocates for their own health. And due in large part to the shame, stigma and discrimination attached to the disease, individuals with addiction and their family members too often are isolated in their struggle to understand the disease and find help. Only recently have we begun to see those affected by the disease working to raise awareness in ways, for example, that families of breast cancer victims have done. But these efforts are small, challenged by public misunderstanding and have failed to raise sufficient funding for needed research.

Even individuals who can transcend the stigma face significant barriers to receiving effective care, and this report paints a dismal picture of a treatment 'non-system.' While almost half of Americans say they would go to their health care providers for help, most doctors are uninformed about this disease and rarely are equipped to offer a diagnosis, provide treatment or connect patients with appropriate specialty care. Insurance coverage varies widely. Services rarely are tailored to individual needs and are based primarily on an acute care model rather than recognizing the chronic nature of the disease. There are no national standards of care. Patients face a patchwork of treatment programs with vastly different approaches; many offer unproven therapies and little medical supervision. Some promise "one time" fixes; others offer posh residential treatment at astronomical prices with little evidence justifying the cost. Even for those who do have insurance coverage or can pay out-of-pocket, there are no outcome data reflecting the quality of treatment providers so that patients can make informed decisions. 
This report focuses long overdue attention on the disease of addiction. It clarifies the important difference between this disease and risky use of addictive substances; identifies the human and economic costs of our current approach to these health problems; and documents the breadth of available knowledge on how to prevent risky use and treat addiction.

As our nation struggles to reduce skyrocketing health care costs, there are few targets for cost savings that are as straightforward as preventing and treating risky substance use and addiction. This report shows that modest public health interventions and relatively inexpensive addiction therapies, compared with other medical treatments, would reduce this burden significantly.

The report calls for modernizing addiction treatment--to harness the scientific knowledge we have acquired to prevent risky use and treat this disease. This report is a call to action. Like the Flexner Report a century ago, it shines a bright light on the problem and offers a roadmap for action. Addiction Medicine: Closing the Gap between Science and Practice represents more than five years of intensive research, and draws on policy and treatment research conducted by CASA Columbia over two decades and on a wide body of scientific, clinical and policy research conducted by others. This major undertaking was the result of the work of a large team of dedicated individuals and institutions and was conducted with the able advice and counsel of The CASA Columbia National Advisory Commission on Addiction Treatment which I had the privilege to chair. The Commission includes an impressive group of individuals knowledgeable about the many aspects of substance use and addiction in America today. We are grateful for their expert assistance.

The project was made possible by the generous financial support of The Annenberg Foundation; The Diana, Princess of Wales Memorial Fund and The Franklin Mint; The New York Community Trust; and the Adrian and Jessie Archbold Charitable Trust.
Peter D. Hart Research Associates conducted the National Addiction Belief and Attitude Survey for this report; Survey Research Laboratory (SRL) of the University of Illinois at Chicago administered the survey of New York State addiction treatment providers. We are grateful to Karen Carpenter-Palumbo, former director of the New York State Office of Alcoholism and Substance Abuse Services (OASAS) for helping to make the New York State survey possible.

We thank the following organizations which generously helped connect CASA Columbia with treatment providers who participated in a national online survey of members of professional associations involved in addiction care: The American Academy of Addiction Psychiatry (AAAP); the American Association for the Treatment of Opioid Dependence (AATOD); the American Psychological Association (APA); the American Society of Addiction Medicine (ASAM); the Association for the Treatment of Tobacco Use and Dependence (ATTUD); NAADAC, the Association for Addiction Professionals; the National Association of Addiction Treatment Providers (NAATP); the National Association of County Behavioral Health and Developmental Disability Directors (NACBHDD); the National Council for Community Behavioral Healthcare (National Council); the State Associations of Addiction Services (SAAS); and Treatment Communities of America (TCA). Also, we thank the following organizations for connecting us with individuals in long-term recovery for CASA Columbia's online survey of this population: Hazelden, Freedom Institute, Faces and Voices of Recovery, Betty Ford Center, National Council on Alcoholism and Drug Dependence, Inc. (NCADD), Treatment Communities of America (TCA), Alcoholism and Substance Abuse Providers of New York State, Inc. (ASAP) and an anonymous treatment program alumni group. Finally, we are grateful to the 174 key informants who shared their insight and recommendations.

Susan E. Foster, MSW, CASA Columbia's Vice President and Director of Policy Research and Analysis, was the principal investigator and staff director for this effort. The senior research 
manager was Linda Richter, $\mathrm{PhD}$, Associate Director of the Division and CASA Columbia Scholar. The data collection and analysis was conducted by CASA Columbia's Data Analysis Center (SADAC ${ }^{\mathrm{SM}}$ ), headed by Roger Vaughan, DrPH, CASA Columbia Fellow and Professor of Clinical Biostatistics, Department of Biostatistics, Mailman School of Public Health at Columbia University, and associate editor for statistics and evaluation for the American Journal of Public Health. He was assisted by Elizabeth Peters and Sarah Tsai, MA. Emily Feinstein, JD, senior policy analyst, assisted with the research and writing. Other research staff members who worked on the project are: Nina Lei, Mark Stovell, Akiyo Kodera, Dina Feivelson, $\mathrm{PhD}$, Gina Hijjawi, PhD, Harold Wenglinsky, PhD, Swapna Reddy, JD, Kristen Keneipp, MHS, Nabil Ansari and Sarah Blachman. David Man, PhD, MLS, is CASA Columbia's librarian; he was assisted by Barbara Kurzweil. Jennie Hauser managed the bibliographic database and Jane Carlson handled administrative details.

While many individuals and institutions contributed to this effort, the findings and opinions expressed herein are the sole responsibility of CASA Columbia. 


\section{Chapter I \\ Introduction and Executive Summary}

A large and growing body of scientific research has demonstrated clearly that addiction involving nicotine, alcohol, illicit drugs and controlled prescription drugs ${ }^{*}$ is a complex brain disease. ${ }^{1}$ It affects 15.9 percent of the United States population ages 12 and older (40.3 million) ${ }^{\dagger 2}$--more than the share of the population with heart disease, ${ }^{\ddagger}$ diabetes or cancer. $^{3}$ Another 31.7 percent of the population (80.4 million), while not addicted, engages in risky use $\mathrm{e}^{\S}$ of addictive substances in ways that threaten health and safety. ${ }^{4}$

\footnotetext{
* In this report, we have used the general term addiction to apply to those who meet criteria for pastmonth nicotine dependence based on the Nicotine Dependence Syndrome Scale (NDSS) and those who meet diagnostic criteria for past year alcohol and/or other drug abuse or dependence (excluding nicotine) in accordance with the Diagnostic and Statistical Manual of Mental Disorders (DSM-IV). (The DSM, the most widely accepted diagnostic system in the U.S. for such conditions, refers to substance abuse and substance dependence collectively as substance use disorders. The diagnostic criteria for nicotine dependence in the NDSS parallel those of the DSMIV). This definition is consistent with the current move to combine abuse and dependence into an overarching diagnosis of addiction in the upcoming DSM-V. The term addiction also has been used in reference to compulsive behaviors involving eating, gambling and other activities that affect the brain's reward system and which may develop independent of or in combination with other manifestations of addiction. This report, however, focuses only on addiction involving nicotine, alcohol and other drugs. ${ }^{\dagger}$ This estimate excludes the institutionalized population, for which rates of addiction are higher. ${ }^{\ddagger}$ Includes coronary heart disease, angina pectoris, heart attack or any other heart condition or disease, excluding hypertension and stroke.

$\S$ Risky users of addictive substances are defined in this report as those who currently use tobacco products, exceed the U.S. Department of Agriculture (USDA) Dietary Guidelines for safe alcohol use, misuse controlled prescription drugs, use illicit drugs, or engage in some combination of these forms of substance use, but do not meet clinical diagnostic criteria for addiction. (See page 5.)
} 
Addiction and risky use constitute the largest preventable and most costly health problems facing the U.S. today. ${ }^{5}$ It is estimated that more than 20 percent of deaths in the U.S. are attributable to tobacco, alcohol and other drug use. ${ }^{6}$ Addiction and risky use cause or contribute to more than 70 other conditions requiring medical care, including cancer, respiratory disease, cardiovascular disease, HIV/AIDS, pregnancy complications, cirrhosis, ulcers and trauma. ${ }^{7}$ They also drive and contribute to a wide range of costly social consequences, including crime, accidents, suicide, child neglect and abuse, family dysfunction, unplanned pregnancies and lost productivity. ${ }^{8}$ Costs of addiction and risky substance use to government alone total at least $\$ 468$ billion each year. ${ }^{9}$

While both addiction and risky use of addictive substances contribute to these consequences, they are very different conditions. Addiction is a disease and, like other diseases, it can and should be diagnosed and treated in the context of the medical system, ${ }^{*}$ using available evidencebased practices. Risky use of addictive substances is a public health problem and tools are available for a wide range of professionals in the health, social services, education, criminal justice and other sectors to screen for it and intervene to reduce it and its consequences, including the potential development of addiction.

Despite the prevalence of these conditions, the enormity of the consequences that result from them and the availability of effective solutions, screening and early intervention for risky substance use is rare and the vast majority of people in need of addiction treatment do not receive anything that approximates evidencebased care. Nine out of 10 people (89.1 percent) who meet diagnostic criteria for addiction

\footnotetext{
* Involving interdisciplinary teams of physicians (of multiple medical specialties and subspecialties), nurses, physician assistants, nurses and nurse practitioners and graduate-level clinical mental health professionals (psychologists, social workers, counselors), working collaboratively with auxiliary and support personnel.
}

involving alcohol and drugs other than nicotine report receiving no treatment ${ }^{\dagger}$ at all; ${ }^{10}$ as a society, we do not even collect information on the number of people receiving treatment for addiction involving nicotine. There is no other disease that affects so many people, has such far-reaching consequences and for which there is such a broad range of effective interventions and treatments that is as neglected as the disease of addiction.

This report documents the significant body of evidence defining and describing the disease of addiction and the risky use of addictive substances. It reveals the size and shape of the populations in need of screening, intervention and treatment. It reviews the evidence of effective screening, intervention, diagnostic, treatment and disease management tools and therapies. It outlines the consequences and costs of our failure to prevent risky substance use and treat addiction effectively. Finally, it examines the profound gaps between those who need treatment and those who receive it, and between the services they receive and what constitutes quality care.

Key factors driving these gaps include:

\section{- Inadequate Integration of Addiction Care into Mainstream Medical Practice:}

Addiction prevention and treatment are for the most part removed from routine medical practice. ${ }^{11}$ In spite of the fact that about 80 percent of Americans ${ }^{\ddagger}$ visited at least one physician or other health care professional in

\footnotetext{
† Treatment is defined in this report as psychosocial and pharmaceutical therapies. Detoxification, mutual support programs, peer counseling, other support services (including religious-based counseling) and services received in an emergency department are excluded from the definition. Services provided in prison or jail settings also are excluded since they cannot be identified in the data sources used for this analysis; furthermore, in CASA Columbia's 2010 report (Behind Bars Update: Substance Abuse and America's Prison Population), more than 70 percent of those receiving addiction-related services in prisons and jails received support services rather than any form of professional treatment.

‡ Ages 18 and older.
} 
the past year, ${ }^{12}$ and more than two-thirds of people with addiction are estimated to be in contact with a primary or emergency care physician about twice a year, ${ }^{13}$ most physicians and other health professionals ${ }^{*}$ do not identify or diagnose the disease or know what to do with patients who present with identifiable and treatable signs and symptoms. And, unlike other diseases, physicians too often lack access to available, trained and certified addiction physician specialists for consultation or referral. Instead of addressing addiction, the medical field primarily has focused its efforts on treating the secondary and tertiary complications of addiction, allowing the public health epidemic to advance unchecked. Furthermore, whereas the main criterion for determining whether health care services should be provided to patients in mainstream medicine is the principle of medical necessity, ${ }^{14}$ patients needing addiction treatment may face stringent eligibility criteria for treatment entry, including insurance benefit restrictions, limited availability of treatment slots, long waiting lists, lack of child care and the requirement to comply with all rules and treatment protocols. ${ }^{15}$ There simply is no other disease where appropriate medical treatment is not provided by the health care system and where patients instead must turn to a broad range of practitioners largely exempt from medical standards.

\section{- Inadequate Education and Training of} Addiction Treatment Providers: The majority of those who currently make up the addiction treatment provider workforce are not equipped with the knowledge, skills or credentials necessary to provide the full range of evidence-based services to treat the disease.

\footnotetext{
* The term "health professional" as used in this report includes medical professionals (physicians, physician assistants, nurses and nurse practitioners, dentists, pharmacists) and graduate-level clinical mental health professionals (psychologists, social workers, counselors).
}

$>$ Addiction counselors, who make up the largest share of providers of addiction treatment services, provide care for patients with a medical disease yet they are not required to have any medical training and most states do not require them to have advanced education of any sort. $^{\dagger}$

$>$ Physicians and other medical professionals, who make up the smallest share of providers of addiction treatment services, receive little education or training in addiction science, prevention and treatment.

\section{Published in the American Journal of Public Health July, 1919}

There is urgent need for widespread and early education of the medical profession, legislators, administrative authorities and laity into the facts of addiction disease...

As a definite clinical entity of physical disease, addiction is practically untaught in the school and unappreciated by the average medical man...

In the light of available clinical information and study and in the light of competent laboratory research we are forced as a profession to admit that we have not treated our addiction sufferers with sympathetic understanding and clinical competency and that the blame for the past failure to control the [narcotic] drug problem rests largely upon the educational inadequacy of our medical profession, and institutions of scientific and public health education. ${ }^{16}$

\section{--Ernest S. Bishop, MD, FACP}

- Inadequate Accountability for Addiction Treatment Providers: Addiction treatment providers, facilities and programs are not adequately regulated or held accountable for providing treatment consistent with medical

\footnotetext{
${ }^{\dagger}$ Only six states require a bachelor's degree and only one state requires a master's degree.
} 
standards and proven treatment practices. The credentials of treatment providers vary dramatically from state to state and from program to program. Compounding the problem, quality assurance standards that do exist focus more on administrative processes than on measureable patient outcomes.

\section{- Inadequate Allocation of Financial}

Resources: Financial investments in addressing addiction and risky substance use are aimed disproportionately at coping with their costly health and social consequences rather than at the effective implementation of available prevention, intervention and treatment approaches. In 2010, only 1.0 percent ( $\$ 28.0$ billion) of total health care costs went to treating the disease of addiction. ${ }^{17}$ Spending on addiction treatment disproportionately falls to the public sector. In contrast to the role of private insurance in general health care spending--where it covers 54.4 percent of costs--private insurers cover only 20.8 percent of the costs of addiction treatment, and the private share has been decreasing. ${ }^{18}$

This profound gap between the science of addiction and current practice related to prevention and treatment is a result of decades of marginalizing addiction as a social problem rather than treating it as a medical condition. Much of what passes for "treatment" of addiction bears little resemblance to the treatment of other health conditions. Much of what is offered in addiction "rehabilitation" programs has not been subject to rigorous scientific study and the existing body of evidence demonstrating principles of effective treatment has not been taken to scale or integrated effectively into many of the treatment programs operating nationwide. This is inexcusable given decades of accumulated scientific evidence attesting to the fact that addiction is a brain disease with significant behavioral components for which there are effective interventions and treatments. It also is unfair to the thousands of addiction counselors who struggle, in the face of extreme resource limitations and no medical training, to provide help to patients with the disease of addiction and numerous co-occurring medical conditions.

America's tendency to frame risky use of addictive substances and addiction as the same issue and as moral or social problems has resulted in an unmitigated failure on the part of policymakers and the health care community to educate the public about these health problems in ways that can help prevent them and to offer effective interventions and treatments that match those offered for other health conditions; instead the focus has weighed heavily toward law enforcement. The end result is that we have declared war on drugs rather than mounting a rational approach to prevention, treatment and finding a cure for the disease of addiction. We largely have punished rather than treated those in need of help even though treatment for a disease and accountability for behavior are not antithetic concepts.

It is long past time for health care practice to catch up with the science. Failure to do so is a violation of medical ethics, a cause of untold human suffering and a profligate misuse of taxpayer dollars.

\section{The CASA Columbia Study}

Substance use can be understood as a continuum ranging from having never used tobacco, alcohol or another drug ${ }^{*}$ at one end to having an unmanaged chronic, relapsing disease ${ }^{\dagger}$ at the other. (Figure 1.A)

\footnotetext{
* Including any use of illicit drugs or the misuse of controlled prescription drugs.

${ }^{\dagger}$ This continuum focuses on substance use; the category labeled addiction includes those individuals who meet current clinical criteria for this disease but does not include all individuals with addiction.
} 
Figure 1.A

\section{Continuum of Substance Use}

\section{Percent of Population Age 12+ by Level of Substance Use*}

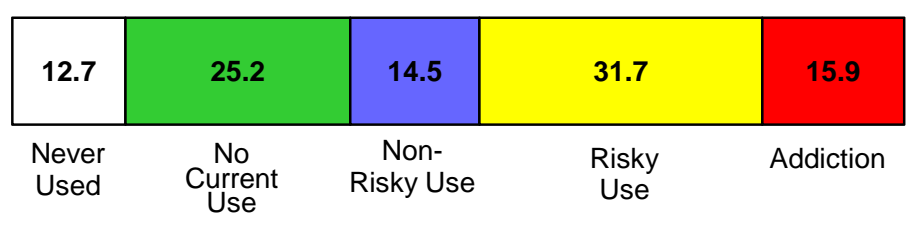

* Includes tobacco, alcohol, illicit drugs and misuse of controlled prescription drugs.

Source: CASA Columbia analysis of The National Survey on

Drug Use and Health (NSDUH), 2010.
Guidelines for safe alcohol use, ${ }^{\ddagger 20}$ misuse controlled prescription drugs, ${ }^{\S}$ use illicit drugs $^{* * 21}$ or engage in some combination of these forms of substance use but do not meet clinical diagnostic criteria for addiction. Risky use can result in devastating and costly health and social consequences including the disease of addiction. Risky users are targets for public health efforts aimed at reducing risky use and for health professionals' efforts to prevent risky use from progressing to the disease of addiction. Approximately onethird (31.7 percent) of the U.S. population ages 12 and older (80.4 million people) are risky substance users. ${ }^{22}$
While this report focuses primarily on those with the disease of addiction, it makes an important distinction between addiction and risky use of addictive substances:

- Those with the active disease of addiction ${ }^{*}$ are defined in this report as meeting the clinical diagnostic criteria for past month nicotine dependence or past year alcohol and/or other drug abuse or dependence. Individuals who meet diagnostic criteria for addiction are targets for appropriate, evidence-based clinical interventions by physicians and other health professionals. Addiction afflicts 15.9 percent of the U.S. population ages 12 and older (40.3 million people). ${ }^{19}$

- Risky users of addictive substances are defined in this report as those who currently ${ }^{\dagger}$ use tobacco products, exceed the U.S. Department of Agriculture (USDA) Dietary

\footnotetext{
* Available data allow us to include only those who meet behavioral criteria in accordance with the diagnostic standards, meaning in most cases that their disease is not currently being managed. Individuals who have the disease of addiction but do not meet diagnostic criteria for past month (nicotine) or past year (alcohol and other drug) addiction are not included.

${ }^{\dagger}$ In the past 30 days.
}

${ }^{\ddagger}$ The U.S. Department of Agriculture Dietary Guidelines for safe alcohol use are no more than one drink a day for women, no more than two drinks a day for men and no alcohol consumption for: (1) persons under the age of 21; (2) pregnant women; (3) individuals who cannot restrict their drinking to moderate levels; (4) individuals taking prescription or over-the-counter medications that can interact with alcohol; (5) individuals with certain specific medical conditions (e.g., liver disease, hypertriglyceridemia, pancreatitis); and (6) individuals who plan to drive, operate machinery, or take part in other activities that require attention, skill or coordination or in situations where impaired judgment could cause injury or death (e.g., swimming). Due to data limitations, we were unable to include categories 4-6 in our calculation of risky drinkers.

${ }^{\S}$ For data analysis purposes, the national survey examined for this report defines misuse of controlled prescription medications more generally as "taking a controlled prescription drug not prescribed for you or taking it in a manner not prescribed for the experience or feeling it causes." The misuse of overthe-counter medications also constitutes risky use; however, rates of risky use in this report do not include the misuse of these medications since they are not directly measured in the national surveys that were analyzed for this study.

${ }^{* *}$ Substances controlled (either through prohibited or restricted use) through the federal Controlled Substances Act of 1970, which created a system for classifying illicit and prescription drugs according to their medical value and their potential for misuse. In this analysis, illicit drugs include marijuana/hashish, cocaine/crack, heroin, hallucinogens, Ecstasy, methamphetamine and inhalants. 
To document the research on the causes, consequences and extent of risky substance use and addiction; the available tools for effective interventions and for treatment; the gap between the need for such interventions and treatments and the actual standard of care; the driving forces behind this substantial gap; and to develop concrete recommendations for minimizing it, CASA Columbia conducted: ${ }^{*}$

- A thorough review of more than 7,000 scientific articles, reports, books and other reference materials related to the science of addiction, the consequences of risky use and addiction, the prevention of risky use and treatment of addiction and barriers to improved care (see Appendix A);

- Secondary analysis of five national data sets (see Appendix A);

- Interviews with and suggestions from 174 leading experts in a broad range of fields relevant to the report, including researchers, physicians and other health professionals, other treatment providers, policymakers and members of professional associations, advocacy organizations, health insurers, pharmaceutical companies and organizations of people with the disease of addiction. Whereas the majority of these experts provided their thoughts in the context of an open-ended interview guide designed by CASA Columbia to explore key themes related to this project, some provided advice, suggestions and feedback about specific content to be included in this report (see Appendices A and B);

- Focus groups and a national general population survey assessing the attitudes and beliefs of 1,303 adults with regard to addiction and its treatment: the National Addiction Belief and Attitude Survey (NABAS) (see Appendices A and C);

- Two statewide surveys of addiction treatment providers in New York: one of 83

\footnotetext{
${ }^{*}$ See Appendix A for a more detailed description of the key methodological components of the study.
}

program directors and one of 141 staff treatment providers (see Appendices A, D and $\mathrm{E})$;

- A national panel of treatment providers and an online survey of 1,142 members of professional associations involved in addiction care (see Appendices A and F);

- An online survey of 360 individuals with a history of addiction who are managing the disease (see Appendices A and G);

- An in-depth analysis of state and federal governments' and professional associations' licensing and certification requirements for individual treatment providers and addiction treatment facilities and programs, as well as accreditation requirements for facilities and programs (see Appendix A); and

- A case study of addiction treatment in New York State and New York City that drew from the research described above and the findings of which are incorporated into the report where relevant (see Appendix A).

\section{Key Findings}

\section{Addiction Is a Brain Disease}

Addiction is a complex brain disease with significant behavioral characteristics. ${ }^{23}$ Nicotine, alcohol, illicit drugs and controlled prescription drugs all affect the pleasure and reward circuitry of the brain in similar ways. ${ }^{24}$ Over time, continued use of these substances can physically alter the structure and function of the brain, dramatically affect judgment and behavior $^{25}$ and drive a compulsion to obtain and use them, even in the face of mounting negative consequences. ${ }^{26}$ Growing evidence also points to structural and functional differences in the brain and to genetic factors that may predispose certain individuals to addiction. ${ }^{27}$ 


\section{As with Other Health Conditions, There Are Clear Risk Factors for the Development of Addiction}

Risk factors for developing addiction include a genetic predisposition, structural and functional brain vulnerabilities, psychological factors and environmental influences. Whereas biological, psychological and environmental factors--such as impairments in the brain's reward circuitry, compensation for trauma and mental health problems, easy access to addictive substances, substance use in the family or media and peer influences--play a large role in whether an individual starts to smoke, drink, or use other drugs, ${ }^{28}$ genetic factors are more influential in determining who develops the disease of addiction. ${ }^{29}$ A factor that is particularly predictive of risk, however, is the age of first use; in 96.5 percent of cases, addiction originates with substance use before the age of $21^{30}$ when the brain is still developing and is more vulnerable to the effects of addictive substances. $^{*} 31$

\section{Addiction Frequently Co-Occurs with Other Health Conditions}

Addiction frequently co-occurs with, contributes to or causes a wide range of medical conditions. Both risky substance use and addiction cause or contribute to more than 70 other conditions requiring medical care, such as heart disease and cancer, ${ }^{32}$ as well as mental health and behavioral disorders--including depression, anxiety, posttraumatic stress disorder, bipolar disorder, schizophrenia and other neuropsychiatric disorders. ${ }^{33}$

\section{Addiction Can Be a Chronic Disease}

There is tremendous variation in the severity and course of the disease of addiction and of its symptoms. Some individuals may experience one episode in which their symptoms meet clinical diagnostic criteria for addiction and be non-symptomatic thereafter. ${ }^{34}$ In many cases,

\footnotetext{
* These individuals also might have a predisposition to develop addiction, irrespective of their actual use of addictive substances.
}

however, addiction manifests as a chronic disease--a persistent or long-lasting illness-which requires ongoing professional treatment and management. ${ }^{35}$ However, very few people with addiction actually receive adequate, effective, evidence-based treatment, ${ }^{36}$ and the usual approach to treatment involves brief, episodic interventions rather than a model based on long-term chronic disease management. As a result, high rates of relapse, while comparable to other chronic diseases, may be due at least in part to inadequate or ineffective interventions and treatments. ${ }^{37}$

\section{A Lack of Standardized Terminology Compromises Effective Interventions}

Terms used to describe different levels of involvement with addictive substances--such as experimentation, use, misuse, excessive use, abuse, dependence and addiction--lack precision, obscuring important differences in the nature and severity of the illness and complicating our ability to intervene and treat it effectively. Even the word "treatment" lacks precision with regard to addiction, since historically it has been used to refer to a host of interventions, many of which are not based in the clinical and scientific evidence as are treatments for other diseases.

\section{Multiple Addictive Substances and Behaviors Frequently Are Involved in Risky Use and Addiction}

Traditionally, risky substance use and addiction have been addressed largely on a substancespecific basis. Growing understanding of the nature of risky use and the disease of addiction-including the risk factors, symptoms and the neuropsychological effects of addictive substances--helps to explain the significant proportion of risky users and those who are addicted who are involved with more than one addictive substance. Among risky substance users who do not meet diagnostic criteria for addiction, 30.6 percent are risky users of more than one substance. Among those who are addicted, 55.7 percent are risky users of one or 
more other substances and 17.3 percent have addiction involving multiple substances. ${ }^{38}$

Emerging research also suggests that other behavioral manifestations of addiction (e.g., obesity, gambling, sexual addiction) share common neuropsychological and genetic pathways with addiction involving substances, ${ }^{39}$ underscoring the importance of treating the antecedents, manifestations and consequences of addiction more generally. When treatments are too highly focused on a specific addictive substance or behavior, they may not be addressing the actual underlying disease of addiction or the possibility of addiction substitution, where a patient may replace one form of addiction with another. ${ }^{40}$

\section{Public Attitudes about the Causes of Addiction Are Out of Sync with the Science}

CASA Columbia's national survey of the attitudes and beliefs of adults in the U.S. with regard to addiction and its treatment (the $N A B A S$ ) found that while there is public recognition of the role of genetics and biological factors in the development of addiction, approximately one-third of Americans continue to view addiction as a sign of lack of will power or self-control. ${ }^{41}$

\section{Physicians and Other Health Professionals Should Be on the Front Line Addressing this Disease}

As with other diseases, addiction should be addressed within the medical system by physicians (including multiple medical specialties and sub-specialties) and a multidisciplinary team of health professionals including physician assistants, nurses and nurse practitioners, and graduate level clinical psychologists, social workers and counselors.

In order to treat addiction and reduce risky substance use and the related consequences, physicians and other health professionals must:

- Understand the risk factors, how these risks vary across the lifespan, how risky substance use that does not result in addiction has far-reaching adverse consequences and that addiction frequently co-occurs with other health conditions;

- Educate patients, and their families if relevant, about these risks, the nature of the disease of addiction and the adverse consequences of risky substance use;

- Screen for risky substance use and symptoms of addiction and co-occurring health conditions using tools that have been proven to be effective;

- Provide brief interventions when appropriate; and

- Treat and manage the disease or provide referrals to specialty care if needed.

Non-laboratory-based screening for risky substance use can be conducted by a wide range of trained health professionals and brief interventions can be conducted by physicians and by appropriately trained clinicians, supervised as necessary. All aspects of stabilization and treatment--including laboratory-based screening, assessment, acute care and disease management--should be managed by a physician, as is the case with other medical illnesses. Highly-trained clinical mental health professionals can provide psychosocial therapies as part of a treatment plan established and managed by the patient's physician. Case management can be provided by nurses and nurse practitioners, physician assistants and clinical mental health professionals if appropriately trained in addiction and if the services are performed under the supervision of a physician. Paraprofessionals and non-clinically trained and credentialed counselors can provide auxiliary services as part of a comprehensive treatment and disease management plan. 
Referrals to specialty addiction care should be made to trained and credentialed addiction physician specialists. $^{*} 42$

\section{Screening and Intervention Are Effective at Addressing Risky Substance Use and Forestalling Addiction}

Screening and brief interventions have been found to be effective tools for addressing the risky use of tobacco, ${ }^{43}$ alcohol, ${ }^{44}$ illicit drugs and controlled prescription drugs ${ }^{45}$ in multiple settings and in many population groups. ${ }^{46}$

A range of screening tools exist and typically include written or oral questionnaires and, less frequently, clinical and laboratory tests. However, most screening tools are substance specific; an instrument that screens for risky use or addiction involving all substances as a unified dimension--and that makes appropriate distinctions for age, culture and gender--has yet to be developed.

For those who screen positive for risky substance use that does not meet the threshold of clinical addiction, a brief intervention--typically involving motivational interviewing techniques and substance-related education--is an effective, low-cost approach to reducing risky substance use. $^{47}$

\section{Effective Therapies to Treat and Manage Addiction Exist}

For individuals showing signs of addiction, a comprehensive assessment of the stage and severity of the disease and the provision of treatment and disease management are critical to improving health and preventing further health and social consequences. ${ }^{48}$ As is true of other chronic diseases, while all patients with

\footnotetext{
* There are two major categories of addiction physician specialists: physician experts in addiction medicine--Diplomates of the American Board of Addiction Medicine (ABAM)--and physician experts in addiction psychiatry (psychiatrists with subspecialty certification in addiction psychiatry)-Diplomates of the American Board of Psychiatry and Neurology (ABPN).
}

addiction will not respond equally well to treatment, the provision of evidence-based treatment does increase the odds of success.

Addiction is a disease that can be treated and managed effectively within the medical profession using an array of evidence-based pharmaceutical and psychosocial approaches. In accordance with standard medical practice for the treatment of other chronic diseases, best practices for the effective treatment and management of addiction must be consistent with the scientific evidence of the causes and course of the disease. Best practices require: ${ }^{49}$

- Comprehensive assessment of the extent and severity of the disease, determination of a clinical diagnosis, evaluation of cooccurring health conditions and the development of a tailored treatment plan;

- Stabilization of the patient's condition via cessation of substance use and medically supervised detoxification, when necessary, as a precursor to treatment;

- Acute Care delivered by qualified health care professionals via evidence-based pharmaceutical and/or psychosocial addiction treatments, accompanied by treatment for co-occurring health conditions;

- Chronic Disease Management to help the patient maintain the progress achieved during acute treatment and prevent relapse. The process should be medically supervised and should involve pharmaceutical and/or psychosocial therapies and continued management of co-occurring health conditions as indicated; and

- Support Services including the provision of auxiliary services such as legal, educational, employment, housing and family supports, as well as nutrition and exercise counseling and connection to mutual support programs. 


\section{The Importance of Tailored Interventions and Treatment}

Each life phase presents unique vulnerabilities for risky substance use and the onset of the disease of addiction. Recognizing these differences as well as the basic risk factors for each is critical to reducing risky substance use and addiction.

Certain populations--such as pregnant women, ${ }^{50}$ the young ${ }^{51}$ and the elderly ${ }^{52}$--are more vulnerable to the damaging and addictive effects of tobacco, alcohol and other drugs. Among members of the military exposed to combat, ${ }^{53}$ persons with co-occurring health conditions ${ }^{54}$ and individuals involved in the justice system ${ }^{55}$ the likelihood of addiction is significantly higher than in the general population.

Treatment must be tailored to the particular stage and severity of the disease, a patient's overall health status, past treatments and any other personal characteristics and life circumstances that might affect patient outcomes. ${ }^{56}$ These include patients with cooccurring health conditions, adolescents, women, older adults, racial and ethnic minorities, individuals of minority sexual orientation, veterans and individuals involved in the justice system. The research evidence clearly demonstrates that a one-size-fits-all approach to addiction treatment typically is a recipe for failure. ${ }^{57}$

\section{Public Attitudes about Addiction Treatment Reflect the Prevailing Non-Medical Approach to Addiction Care}

CASA Columbia's NABAS found that although the American public appears to be supportive of assuring that individuals with addiction receive effective addiction treatment, public views about what constitutes addiction treatment do not comport with the science: more than half (60.1 percent) of respondents to the $N A B A S$ spontaneously offered mutual support programs such as AA or NA as a "treatment" intervention when asked what kinds of treatment come to mind when they think about treatment for addiction, ${ }^{58}$ despite the fact that such programs, while very helpful sources of support to many individuals with addiction, are not evidencebased treatments for the disease. ${ }^{59}$ The public also does not seem to distinguish between risky substance use and the disease of addiction.

\section{Most People in Need of Treatment Do Not Receive It}

As an indicator of the lack of attention afforded the disease of addiction, no single national data source exists to compare the proportion of the population in need of addiction treatment involving any addictive substance to the proportion that receives such treatment. While about seven out of 10 people with hypertension, major depression or diabetes get treatment for their medical conditions, only about one in 10 people with addiction involving alcohol or drugs other than nicotine do, ${ }^{*} 60$ (Figure 1.B), leaving a treatment gap of 20.7 million individuals. ${ }^{61}$

No data exist on the treatment gap for those with addiction involving nicotine. The proportion of individuals in need of addiction treatment involving alcohol and drugs other than nicotine who actually receive it has changed little since 2002, when 9.8 percent of those in need received treatment. $^{62}$

\footnotetext{
* For this comparison, CASA Columbia examined the referenced national survey data to determine the proportion of the population with each disease--those with diagnosed or undiagnosed hypertension (59.3 million); those with diagnosed or undiagnosed diabetes (25.8 million); those who met clinical criteria for a major depressive episode in the past year and/or received professional treatment (saw a doctor, received medication, a combination thereof) (22.4 million); and those who met clinical criteria for addiction involving alcohol or other drugs excluding nicotine in the past year and/or received professional treatment for alcohol and/or other drugs in the past year (23.2 million)--who received treatment.
} 


\section{Most Referrals to Publicly Funded Treatment Come from the Criminal Justice System}

CASA Columbia's national survey conducted for this study found that 46.8 percent of respondents would turn to a health professional-such as their physician (27.8 percent), a health professional other than their primary care physician (19.7 percent) or a mental health professional (9.2 percent) ${ }^{*}$--if someone close to them needed help for addiction. ${ }^{63}$ However, only 5.7 percent of referrals to publicly funded treatment came from a health care provider. In contrast, a full 44.3 percent of the referrals to treatment were from the criminal justice system, ${ }^{64}$ highlighting the fact that this disease typically is addressed only at the point at which it results in profound social consequences.

One-quarter (25.3 percent) of referrals came from individuals, including concerned family members, friends and the self-referred; 12.1 percent were referred by community sources such as social welfare organizations, religious organizations and mutual support programs; and 10.6 percent were referred by addiction treatment providers for additional treatment. Very few treatment referrals came from schools (1.4 percent) or from employers (0.6 percent). ${ }^{65}$ (Figure 1.C)


\footnotetext{
* Some respondents chose more than one response, so the 46.8 percent reflects those who chose either one of these health professionals.
} 


\section{Less than Half of Treatment Admissions Result in Treatment Completion}

In 2008, ${ }^{*}$ less than half (42.1 percent) of discharges from formal addiction treatment services were of admissions in which treatment was completed. ${ }^{66}$ The highest completion rates were from venues to which there were the fewest admissions:

- 14.8 percent of admissions were to shortterm residential services which had the highest completion rate of 54.8 percent;

- 11.4 percent of admissions were to longerterm residential treatment which had a completion rate of 45.5 percent; and

- 73.8 percent of admissions were to nonresidential services which had the lowest completion rate of 39.1 percent. $^{67}$

No data are available on the extent to which referrals were based on matching providers with individual treatment needs.

\section{Patients Face Formidable Barriers to Receiving Addiction Treatment}

In addition to the lack of treatment referrals from the health care system, many other barriers stand in the way of individuals accessing and completing addiction treatment. These include: a misunderstanding of the disease, negative public attitudes and behavior toward those with the disease, privacy concerns, insufficient insurance coverage of the costs of treatment, lack of information on how to get help, limited availability of services including a lack of addiction physician specialists, insufficient social support, conflicting time commitments, negative perceptions of the treatment process and legal barriers. Rarely is there only one obstacle to a person receiving needed treatment. ${ }^{68}$ Although comparable national data for barriers to accessing smoking cessation treatment are not available, research indicates that barriers similar to those facing individuals

\footnotetext{
* Most recent available data on discharges.
}

seeking treatment for addiction involving alcohol or other drugs stand in the way of smokers accessing tobacco cessation services. ${ }^{69}$

\section{The Spending Gap}

In 2010, the United States spent $\$ 43.8$ billion to treat diabetes ${ }^{70}$ which affects 25.8 million people, ${ }^{71} \$ 86.6$ billion to treat cancer ${ }^{72}$ which affects 19.4 million people ${ }^{73}$ and an estimated $\$ 107.0$ billion to treat heart conditions ${ }^{74}$ which affect 27.0 million people, ${ }^{75}$ but only $\$ 28.0$ billion to treat addiction ${ }^{\dagger}$ which affects 40.3 million people. ${ }^{\ddagger} 76$ Looking just at government spending, CASA Columbia calculated that in 2005, risky substance use- and addiction-related spending accounted for 10.7 percent of federal, state and local spending, and that for every dollar federal and state governments spent, 95.6 cents went to pay for the consequences of substance use; only 1.9 cents was spent on any type of prevention or treatment. ${ }^{\S}$ The taxpayer tab for government spending on the consequences of risky substance use and addiction alone totals almost $\$ 1,500$ a year for

\footnotetext{
${ }^{\dagger}$ There are no national data that document spending on treatment for addiction involving nicotine; although the cost estimate of $\$ 28.0$ billion applies to the treatment of addiction involving alcohol or other drugs excluding nicotine, the prevalence estimate of those with addiction (40.3 million) includes those with addiction involving nicotine.

${ }^{\ddagger}$ Due to data limitations, the prevalence estimates for cancer and heart conditions include individuals ages 18 and older who have ever been told by a doctor or other health professional that they have the condition (cancer/malignancy or a heart condition). The prevalence estimate for diabetes includes all ages and the estimate for addiction includes individuals ages 12 and older; for diabetes and addiction, the prevalence estimates include both diagnosed and undiagnosed cases. In each case, total costs of treatment are included without regard to age. The cost estimates for treating diabetes, cancer and heart conditions were inflated to 2010 dollars using the medical inflation factor (7.9 percent) found in SAMHSA's National Expenditures for Mental Health Services and Substance Abuse Treatment, 1986-2005 publication.

$\S^{\S}$ In addition, 0.4 cents was spent on research, 1.4 cents on taxation or regulation and 0.7 cents on interdiction.
} 
every person in America. ${ }^{77}$ Nearly one-third (32.3 percent) of all hospital inpatient costs are attributable to substance use and addiction. ${ }^{78}$

\section{Most Funding for Addiction Treatment Comes from Public Sources}

Spending on addiction treatment totaled an estimated \$28.0 billion in 2010. Whereas private payers (including private insurers and self-payers) are responsible for 55.6 percent (\$1.4 trillion) of medical expenditures in the U.S., they are responsible for only 20.8 percent ( $\$ 5.8$ billion) of addiction treatment spending. ${ }^{79}$

The concentration of spending for addiction treatment in public programs suggests that insurance across the board does not adequately cover costs of intervention and treatment, resulting in costly health and social consequences that stem from untreated addiction and that fall disproportionately to government programs. National data indicate that individuals with private insurance are three to six times less likely than those with public insurance to receive specialty addiction treatment. ${ }^{80}$

\section{The Education, Training and Accountability Gap}

Compounding the profound gap between the need for addiction treatment and the receipt of such care is the enormous gulf between the knowledge available about addiction and its prevention and treatment and the education and training received by those who provide or should provide care. In spite of the evidence that addiction is a disease:

- Most medical professionals who should be providing addiction treatment are not sufficiently trained to diagnose or treat it;

- Most of those who are providing addiction treatment are not medical professionals and are not equipped with the knowledge, skills or credentials necessary to provide the full range of evidence-based services to address addiction effectively; ${ }^{81}$ and
- Addiction treatment facilities and programs are not adequately regulated or held accountable for providing treatment consistent with medical standards and proven treatment practices. ${ }^{82}$

Further complicating this education, training and accountability gap is the fact that there are no national standards for the provision of addiction treatment and instead considerable inconsistency among states in the regulation of individual treatment providers and of the programs and facilities that provide addiction treatment services.

\section{The Profound Disconnect between Evidence and Practice}

The prevention and reduction of risky substance use and the treatment of addiction, in practice, bear little resemblance to the significant body of evidence-based practices that have been developed and tested; indeed only a small fraction of individuals receive interventions or treatment consistent with scientific knowledge about what works. ${ }^{83}$

Providing quality care to identify and reduce risky substance use and diagnose, treat and manage addiction requires a critical shift to science-based interventions and treatment by health care professionals--both primary care providers and specialists.

Significant barriers stand in the way of making this critical shift, including: an addiction treatment workforce starved of resources, operating outside the medical profession and lacking capacity to provide the full range of evidence-based practices including necessary medical care; a health professional that should be responsible for providing addiction screening, interventions, treatment and management but does not implement evidence-based addiction care practices; inadequate oversight and quality assurance of treatment providers and intervention practices; limited advances in the

\footnotetext{
* With the notable exception of the regulation of medication-assisted therapy for addiction involving opioids.
} 
development of pharmaceutical treatments and the adoption of existing pharmaceutical therapies; and a lack of adequate insurance coverage.

Recent efforts by government agencies and professional associations have begun to tackle these challenges to closing the evidence-practice gap, but are simply insufficient.

Nothing short of a significant overhaul in current approaches is required to bring practice in line with the evidence and with the standard of care for other public health and medical conditions. Given the prevalence of risky substance use and addiction in America and the extensive evidence on how to identify and address them, continued failure to do so raises the question of whether the insufficient care that patients with addiction usually do receive constitutes a form of medical malpractice. It also signals widespread system failure in health care service delivery, financing, professional education and quality assurance.

\section{Recommendations and Next Steps}

It is time for health care practice to catch up with the science. There is no silver bullet to making this happen; instead, a broad set of comprehensive reforms must be put in place. Toward this end, CASA Columbia makes the following recommendations:

\section{Reform Health Care Practice}

- Incorporate screening and intervention for risky substance use, and diagnosis, treatment and disease management for addiction into routine medical practice. As essential components of routine medical care, all physicians and other medical professionals should provide their patients with addiction-related screening and, as needed: brief interventions; comprehensive assessment to determine disease stage, severity and the presence of co-occurring health conditions; stabilization; acute treatment; chronic disease management; and connection to support and auxiliary services. Patients with severe cases of addiction should be referred to an addiction physician specialist.

- All medical schools and residency training programs should educate and train physicians to address risky substance use and addiction. All physicians should be educated and trained in the origins of risky substance use and addiction; prevention, intervention, treatment and management options; cooccurring conditions; and special population and specialty-care needs. These core clinical competencies should be required components of all medical school curricula, medical residency training programs, medical licensing exams, board certification exams and continuing medical education (CME) requirements, including maintenance of certification programs.

- Require non-physician health professionals to be educated and trained to address risky substance use and addiction. Develop core clinical competencies in addressing risky use and preventing and treating addiction for each type of non-physician health professional including, physician assistants, nurses and nurse practitioners, dentists, pharmacists and graduate-level clinical mental health professionals (psychologists, social workers, counselors). Assure that these core clinical competencies and specialized training are required components of all professional health care program curricula, graduate fellowship training programs, professional licensing exams and continuing education (CE) requirements. Require all nonphysician health professionals providing psychosocial addiction treatment services to have graduate-level clinical training in delivering these services. Require that all pharmaceutical treatments for addiction be provided only by a physician or in accordance with a treatment plan managed by a physician.

- Develop improved screening and assessment instruments. Screening instruments should be adjusted or developed 
to coincide with appropriate definitions of risky substance use, and assessment instruments should be adjusted or developed to mirror diagnostic criteria for addiction. Both screening and assessment instruments should address all types of addictive substances.

- Establish national accreditation standards for all addiction treatment facilities and programs that reflect evidence-based care. As a condition of accreditation, accrediting organizations should stipulate requirements for all facilities and programs providing addiction treatment with regard to professional staffing (e.g., requiring them to have a fulltime certified addiction physician specialist on staff to serve as medical director, oversee patient care and be responsible for all treatment services), intervention and treatment services (e.g., requiring them to provide comprehensive assessment and evidence-based treatment for addiction involving all substances that is tailored to the stage and severity of the disease, cooccurring conditions and patient characteristics), and quality assurance (e.g., requiring them to collect and report comprehensive quality assessment data, including process and outcome measurements).

- Standardize language used to describe the full spectrum of substance use and addiction. Recognize addiction as a primary medical disease and standardize the language related to the spectrum of substance use severity in current and forthcoming diagnostic instruments. Develop a classification system based both on observable behavior and neurobiological measures that underlie different manifestations of addiction and related conditions which currently are classified and addressed as distinct conditions.

\section{Use the Leverage of Public Policy to Speed Reform in Health Care Practice}

- Condition grants and contracts for addiction services on the provision of quality care. Federal, state and local governments should require--as a condition of receipt of public funds--that grants, contracts and non-insurance reimbursement for addiction treatment services utilize evidence-based prevention and treatment approaches, including pharmaceutical therapies (provided or managed by a physician demonstrating the core competencies of addiction medicine or addiction psychiatry) and psychosocial therapies (provided by medical professionals or graduate-level clinical mental health professionals trained and licensed in the core competencies of addiction treatment), as indicated; involve other health professionals, individuals providing auxiliary services and those providing peer support, working in a multidisciplinary team; and generate positive and measurable long-term patient outcomes.

- Educate non-health professionals about risky substance use and addiction.

Require that the topic of risky substance use and addiction be included in the education and training of government-funded professionals who do not provide direct addiction-related services but who come into contact with significant numbers of individuals who engage in risky substance use or who may have addiction. These include, but are not limited to law enforcement and other criminal justice personnel, legal staff, child welfare and other social service workers and educators.

- Identify patients at risk in government programs and services where costs of risky use and addiction are high. Federal, state and local governments should require that routine screening and brief interventions be provided by trained professionals in all educational, mental health, developmental disabilities, child welfare, housing, juvenile 
justice and adult corrections services that receive public funding; and that patients who screen positive for risky use or a potential diagnosis of addiction be connected with a trained health professional for intervention, diagnosis, treatment and disease management.

- Develop tools to improve service quality. Federal and state governments in collaboration with professional associations, accrediting organizations and other nonprofit organizations focusing on health care quality should develop and disseminate evidence-based tools, practice guidelines and performance measures oriented toward patient outcomes to improve the quality of addiction care (involving all substances) and require their implementation as a condition of continued licensure and/or accreditation.

- License addiction treatment facilities as health care providers. Federal, state and local governments should subject all addiction treatment facilities and programs to the same mandatory licensing processes as other health care facilities.

- Require adherence to national accreditation standards that reflect evidence-based care. As a condition of licensure, federal, state and local governments should stipulate that all facilities and programs providing addiction treatment adhere to established national minimum standards for accreditation.

- Require that all insurers provide coverage for comprehensive addiction care. Require that all health insurers-public and private--provide coverage for all insured individuals for patient education, screening and intervention for risky substance use and treatment and management of addiction (involving all substances associated with addiction) consistent with standards of medical practice, eliminating exemptions. As a condition of reimbursement, public payers and private insurance companies should be mandated to require that all addiction interventions and treatment be directly provided, supervised or managed by trained medical professionals. Public payers and private health insurance companies should encourage participating providers and facilities to adopt evidence-based practices, institute quality-improvement measures and assess patient outcomes. To help ensure comprehensive coverage and appropriate medical care, the Uniform Accident and Sickness Policy Provision Law (UPPL), which bars insurance coverage for injuries sustained by a person who was under the influence of alcohol or other drugs at the time of the injury, should be eliminated.

- Expand the addiction medicine workforce. Accelerate the work begun by the American Board of Addiction Medicine Foundation to develop residency training programs in addiction medicine and secure residency accreditation from the Accreditation Council for Graduate Medical Education (ACGME). Pursue and gain recognition of addiction medicine by the American Board of Medical Specialties (ABMS). Support the efforts of ACGMEaccredited addiction psychiatry residencies to increase the number of enrolled residents. Through these actions, assure that addiction medicine training programs are available to physicians, that training opportunities within addiction psychiatry are expanded, and that such specialty care is formally recognized and available in every hospital throughout the country and through every health care system.

- Implement a national public health campaign. Implement a nationwide public health campaign through federal agencies charged with protecting the public health to educate the public about all forms of risky substance use and addiction.

- Invest in research and data collection. Invest in research designed to improve and track progress in addiction prevention, treatment and disease management and to find a cure for addiction. 
- Implement the National Institutes of Health's (NIH) recommendation to create a single institute addressing substance use and addiction. Create a unified national institute focused on substance use and addiction, recognizing the overarching disease of addiction rather than continuing the focus on different manifestations of the disease--tobacco, alcohol, other drug use-and including the risky use of all addictive substances. Include in the research portfolio addiction involving behaviors other than substance use, and focus on the causes, correlates, consequences, interventions, policies and possible cures for all manifestations of the disease. The portfolio of the institute also should include health conditions resulting from risky use and addiction and other conditions which increase the risk of developing addiction. 


\section{Chapter II \\ What Is Addiction?}

Addiction ${ }^{*}$ is a complex brain disease with significant behavioral characteristics. ${ }^{1}$ In many but not all cases, it involves the use of nicotine, alcohol and other drugs. ${ }^{\dagger}$ Addiction involving these substances typically originates with use in adolescence when the brain is still developing and is more vulnerable to their effects. ${ }^{2}$ If untreated, it can become a chronic and relapsing condition, requiring ongoing professional treatment and management. ${ }^{3}$

Although there has been an evolution in scientific understanding of the disease, public attitudes and health care practice have not kept pace with the science. Terms used to describe different levels of substance use and addiction's many forms lack precision, obscuring important differences in the use of addictive substances and the nature and severity of the illness and complicating our ability to treat it effectively.

\footnotetext{
${ }^{*}$ In this report, we have used the general term addiction to apply to those who meet criteria for pastmonth nicotine dependence based on the Nicotine Dependence Syndrome Scale (NDSS) and those who meet diagnostic criteria for past year alcohol and/or other drug abuse or dependence (excluding nicotine) in accordance with the Diagnostic and Statistical Manual of Mental Disorders (DSM-IV). (The DSM refers to substance abuse and substance dependence as substance use disorders. The criteria for nicotine dependence in the NDSS parallel those of the DSMIV.) This definition is consistent with the current move to combine abuse and dependence into an overarching diagnosis of addiction in the upcoming DSM-V.

${ }^{\dagger}$ Other drugs include federally controlled illicit drugs and the misuse of controlled prescription drugs. The term addiction also has been used in reference to compulsive behaviors involving eating, gambling and other activities that affect the brain's reward system and which may develop independent of or in combination with other manifestations of addiction. This report, however, focuses only on addiction involving nicotine, alcohol and other drugs.
} 


\section{Addiction Is a Brain Disease}

Advances in neuroscientific research, including animal studies and brain imaging, demonstrate clearly that addiction is a primary and often chronic disease of the brain. ${ }^{*}$ The risk factors for developing the disease include a genetic predisposition and a range of biological, psychological and environmental influences. ${ }^{5}$ There is a growing body of evidence showing the brain circuits that are implicated in substance addiction in general also are involved in other compulsive or addictive behaviors such as those related to gambling, certain forms of disordered eating (e.g., bulimia, obesity) and compulsive sexual activity. ${ }^{6}$ For these reasons, researchers are beginning to explore whether substance addiction might be part of a syndrome characterized by:

- Shared neurobiological and psychosocial antecedents (risk factors);

- Production of desirable effects upon involvement with the rewarding object or activity;

- Shared manifestations and outcomes (e.g., biological ones such as tolerance or withdrawal or behavioral or psychological ones such as deceit, shame, guilt or depression); and

- A shared course of the disease (e.g., improvement, relapse, remission and the potential for progression to disability or death). ${ }^{7}$

...addiction is not about drugs, it's about brains. It is not the substances a person uses that make them an addict; it is not even the quantity or frequency of use. Addiction is about what happens in a person's brain when they are exposed to rewarding substances or rewarding behaviors, and it is more about reward circuitry in the brain and related brain structures than it is about the external chemicals or behavior that "turn on" that reward circuitry. ${ }^{14}$

\footnotetext{
${ }^{*}$ A primary disease indicates that it is not simply a
} symptom or effect of another disease or condition.
The focus of this report is on addiction involving nicotine, alcohol and other drugs. Use of these substances can result from an existing brain dysfunction; use also can alter the structure and function of the brain, dramatically affecting judgment and behavior. ${ }^{8}$ The amount and duration of substance use that results in brain changes and addiction depends on the individual and the particular substances used. ${ }^{\dagger 9}$

As yet, there is no conclusive biological marker of addiction; therefore the diagnosis of addiction is based on its symptoms including the compulsive use of addictive substances, significantly impaired function and persistent use despite negative consequences. ${ }^{\ddagger 10}$ These symptoms that characterize addiction are cognitive and behavioral manifestations of the underlying disease and its effects on the brain. ${ }^{11}$ The foundations of the disease may exist in certain individuals even before they ever use an addictive substance and, in some cases, once the disease develops it persists even when an individual is not actively engaged in substance use. $^{12}$

Unfortunately, keeping the emphasis on just the behavioral manifestations presumes that the problem is present when the behavior is present and the problem is resolved if the behavior is not present for whatever duration of time, even though the underlying disease process may be present and even progressing, or contributing to other manifestations, signs and symptoms that may be overlooked. ${ }^{13}$

\footnotetext{
${ }^{\dagger}$ The addictive potential of a substance is determined not only by its intrinsic ability to stimulate the reward circuits of the brain, but also by the speed with which it crosses the blood-brain barrier (i.e., how soon after initial ingestion/injection/inhalation it reaches receptors in reward circuits of the brain).

${ }^{\ddagger}$ Other physical signs such as intoxication, withdrawal, needle-related findings, co-infections, and laboratory findings--such as abnormalities in liver function tests or positive breath or urine tests-can aid in the diagnosis.
} 
The areas of the brain affected by this complex disease are among those that are responsible for survival--including the areas associated with motivation, decision making, risk and reward assessment, pleasure seeking, impulse control/ inhibition, emotion, learning, memory and stress control. ${ }^{15}$

In order to reinforce activities necessary for survival, the brain produces feelings of pleasure in response to the satisfaction of fundamental drives such as hunger, thirst and sex. ${ }^{16}$

Behaviors that lead to these rewards tend to be reinforced and thus perpetuated over time. ${ }^{17}$ On a neurological level, this reinforcement is a process carried out by chemical messengers that flood the reward circuits of the brain. ${ }^{18}$ Virtually all addictive substances affect the pleasure and reward circuitry deep in the brain ${ }^{*}$ which is activated by the neurotransmitter dopamine. $^{\dagger} 19$

\section{Definition of Addiction American Society of Addiction Medicine}

Addiction is a primary, chronic disease of brain reward, motivation, memory and related circuitry. Dysfunction in these circuits leads to characteristic biological, psychological, social and spiritual manifestations. This is reflected in an individual pathologically pursuing reward and/or relief by substance use and other behaviors.

Addiction is characterized by inability to consistently abstain, impairment in behavioral control, craving, diminished recognition of significant problems with one's behaviors and interpersonal relationships and a dysfunctional emotional response. Like other chronic diseases, addiction often involves cycles of relapse and remission. Without treatment or engagement in recovery activities, addiction is progressive and can result in disability or premature death. ${ }^{24}$

\footnotetext{
* The mesolimbic reward system.

${ }^{\dagger}$ The neurotransmitter serotonin also is involved in some forms of substance addiction; it plays a role in motivated or directed actions such as attaining addictive substances and also influences dopamine levels in the brain. Hallucinogenic drugs like LSD and Ecstasy target serotonin systems in the brain.
}

Addictive substances drive behavior by causing the release of more dopamine within brain reward circuits than almost all natural rewards including those tied to food and sex. ${ }^{20}$ With repeated use of addictive substances, the brain begins to expect this stimulation and an addicted individual may experience intense desire or cravings whenever the addictive substances are not readily available and especially when the individual is exposed to cues associated with substance use. ${ }^{21}$ Recent research points to evidence that use of one addictive substance can increase the risk of use of and addiction involving another substance; for example, nicotine use can prime the brain, making it more susceptible to developing addiction involving cocaine. $^{\ddagger 22}$ Signals in the environment such as seeing a drug-using friend or passing a bar, or emotional signals such as feeling stressed or sad also become associated with the addictive substance and spur the drive to obtain it. ${ }^{23}$

As use continues, the pleasure associated with the dopamine release that results from the ingestion of an addictive substance--or from its anticipation--can become consuming to the point where fundamental natural drives and associated behaviors lose their value in comparison. ${ }^{25}$

At the same time, the brains of substance-using individuals may adapt to the unnaturally high levels of dopamine that result from continued substance use and may respond by reducing the normal release of dopamine as well as the number of dopamine receptors in the brain. ${ }^{26}$ When this happens, the addictive substance may become necessary just for the person to feel normal. ${ }^{27}$ Compared to non-substance users, the brains of chronic substance users appear to have lower baseline levels of dopamine, making it difficult for them to achieve feelings of pleasure from substance use and other behaviors that once were pleasurable. ${ }^{28}$

Changes in the function and structure of the brain result in specific, compulsive behaviors aimed at obtaining and using addictive substances. ${ }^{29}$ The cognitive control of an

\footnotetext{
‡ This study provides a biological mechanism for the "gateway effect."
} 
addicted individual is so weakened that even when he or she wants to cut down or stop using an addictive substance, it becomes extremely difficult to do so. ${ }^{30}$

People may choose to take drugs, but no one chooses to be an addict.

--Participant

CASA Columbia Focus Group May 2008, Philadelphia, PA

\section{The Risk Factors for Addiction}

Genetic factors play a major role in the development of addiction as do individual biological and psychological characteristics and environmental conditions. ${ }^{31}$ These factors affect both the initial use of an addictive substance and the progression from initiation of substance use to regular use to addictive use. ${ }^{32}$

Whereas biological, psychological and environmental factors--such as impairments in the brain's reward circuitry, compensation for trauma and mental health problems, easy access to addictive substances, substance use in the family or media and peer influences--play a large role in whether an individual starts to smoke, drink or use other drugs, ${ }^{33}$ genetic factors are more influential in determining who progresses to risky use or addiction. ${ }^{34}$ A factor that is particularly predictive of risk, however, is the age of first use; almost all cases of addiction begin with substance use before the age of 21 , when the brain is still developing. ${ }^{35}$

\section{Genetic Risks}

Twin and adoption studies* confirm a genetic role in the likelihood of substance use and the

\footnotetext{
* These studies help distinguish the roles of genetics and environment in the development of addiction. Studies of adopted children allow researchers to compare the adopted child both to her biological parents with whom she shares genetic features but no environmental experiences and to her adopted parents with whom she shares environmental experiences but no genetic features. Studies of identical and fraternal twins allow researchers to isolate genetic similarities
}

development of addiction. ${ }^{36}$ Although certain specific genetic factors predispose an individual to addiction involving a particular substance, ${ }^{37}$ genetic factors also appear to contribute generally to the risk of use and addiction. ${ }^{38}$ Advances in genetic research have enabled scientists to identify individual genes, including genetic variations in components of the dopamine transmission system, ${ }^{39}$ implicated both in the likelihood of substance use and of addiction involving a variety of substances. ${ }^{40}$

Genetic variations may affect a person's ability to metabolize an addictive substance ${ }^{41}$ or to tolerate it. ${ }^{42}$ Studies have found that genetics account for between half and three quarters of the risk for addiction. ${ }^{\dagger} 43$ Genetic factors appear to be stronger drivers than environmental factors of initiation of substance use at an early age. ${ }^{44}$

Nicotine. Adolescents who do not have a certain variant of the gene that is responsible for the enzyme that metabolizes nicotine ${ }^{\ddagger}$ progress from smoking to addiction involving nicotine faster than adolescents with that type of gene. ${ }^{45}$ Other genetic variations in genes that determine how nicotine receptors in the brain function ${ }^{\S}$ also are linked to increased risk of addiction involving nicotine and difficulty quitting smoking. ${ }^{46}$

Alcohol. Individuals whose genetic makeup influences them to have a higher tolerance for alcohol are at increased risk of developing

from environmental similarities. Identical twins are genetically identical and fraternal twins share an average of 50 percent of their genes, but both types of twins typically experience a shared environment if reared together.

${ }^{\dagger}$ The majority of the genetics literature focuses on addiction involving alcohol; the estimated extent of genetic influence on addiction involving other drugs varies by the type of drug.

${ }^{\ddagger}$ CYP2A6.

$\S$ e.g., CHRNA5. 
addiction. $^{*} 47$ Adopted children with biological parents who have addiction involving alcohol are at least twice as likely as are adopted children without such parents to develop addiction involving alcohol. ${ }^{48}$ Individuals whose genetic makeup produces involuntary skin flushing and other unpleasant reactions to alcohol are at reduced risk of developing addiction involving alcohol. ${ }^{49}$

It's theoretically possible to take kids before they first drink, find out whether they have any gene variations, and say to them, 'If you choose to be a drinker, then be careful because it's very likely that you'll need to drink more to have the same effect. ${ }^{50}$

--Marc A. Schuckit, MD Distinguished Professor of Psychiatry Department of Psychiatry University of California, San Diego

Other Drugs. Genetic influences have been implicated in marijuana use ${ }^{51}$ and particular genes have been associated with marijuana cravings and withdrawal symptoms. ${ }^{52}$ Twin studies have found genetic risks for hallucinogen, opioid, sedative and stimulant use and addiction. ${ }^{53}$

\section{Biological Risks}

In addition to genetic variations, certain individuals have neurological, structural or functional differences that make them more susceptible to addictive substances. ${ }^{54}$ This is in part due to individual differences in how the brain produces and reacts to dopamine. ${ }^{55}$ Some research indicates that individuals with a naturally low level of dopamine response to addictive substances are at increased risk of engaging in substance use in order to achieve a greater experience of reward. Other research suggests that individuals with a biological

\footnotetext{
* There is some indication that these individuals have less cognitive impairment following the ingestion of alcohol and, therefore, may not perceive the negative experiential aspects of alcohol use in the same manner as individuals whose brains are more strongly affected by alcohol ingestion.
}

tendency toward heightened dopamine response also are at increased risk because of their enhanced or above average experience of reward or pleasure from engaging in substance use. ${ }^{56}$ Other biological risks may involve damage or deficits in the regions of the brain ${ }^{\dagger}$ responsible for decision making and impulse control. ${ }^{57}$

\section{Psychological Risks}

Clinical mental health disorders such as depression and anxiety and psychotic disorders such as schizophrenia, as well as behavioral disorders such as conduct disorder and attentiondeficit/hyperactivity disorder ${ }^{58}$--and sub-clinical symptoms of these conditions ${ }^{59}$--are strongly linked to substance use and addiction and to the risk of transitioning from substance use to addiction. ${ }^{60}$ Individuals whose brain development has been altered by stress are more sensitive to the effects of addictive substances and more vulnerable to the development of addiction. ${ }^{61}$ Likewise, individuals with posttraumatic stress disorder (PTSD), common among veterans and individuals in active military duty, are at increased risk of developing addiction. ${ }^{62}$ People who have risk-taking or impulsive personality traits ${ }^{63}$ or who have low self-esteem ${ }^{64}$ also are likelier to engage in substance use ${ }^{65}$ as are victims of trauma or abuse. ${ }^{66}$ Expectations play a role in substance use as well, since people who expect that using addictive substances will be a positive and rewarding experience--in terms of physical effects, mood or behavior--are likelier to smoke, drink alcohol or use other drugs than are those with more balanced or negative expectations. ${ }^{67}$

\section{Environmental Risks}

Many factors within an individual's family, social circle and community, as well as the larger cultural climate, increase the likelihood that an individual will use addictive substances and develop addiction.

\footnotetext{
${ }^{\dagger}$ e.g., in the orbitofrontal cortex and anterior cingulate cortex.
} 
People who grow up in homes in which parents routinely expose their children to smoking, excessive drinking or other drug use are at increased risk of substance use, as are those whose parents do not convey strong antisubstance use messages and expectations. ${ }^{68}$ The nature of the parent-child relationship is key; people who come from families with high levels of parent-child conflict, poor communication, weak family bonds and other indicators of an unhealthy parent-child relationship are at increased risk of substance use and addiction. ${ }^{69}$ Individuals whose peers engage in substance use or convey approval of such use are at increased risk as well. ${ }^{70}$

The simple fact of availability of addictive substances makes it likelier that an individual will use them. ${ }^{72}$ Homes where liquor and medicine cabinets are open to teens increase the chances that teens will use these substances. ${ }^{73}$ People who live in communities where addictive substances are readily available, where using such substances is considered normal or expected or where tobacco and alcohol retail outlets are prevalent are at increased risk. ${ }^{74}$ Widespread access to controlled prescription drugs contributes to the misuse of these substances $^{75}$ and increased access to marijuana marketed as medicine is linked to increased use. ${ }^{76}$ Community tolerance of high levels of substance use or of experimenting with and using addictive substances as a normal rite of passage for adolescents also increases the risk of use, as does lax enforcement of governmental policies and regulations restricting use. ${ }^{77}$

Exposure to advertising and marketing messages that promote or glamorize smoking and drinking increases the chances that these substances will be used and misused. ${ }^{78}$ Direct-to-consumer marketing of controlled prescription drugs may encourage substance use by conveying the message that there is a pill for every ill. ${ }^{79}$

Environmental influences can exacerbate existing genetic, biological and psychological risks for substance use, further increasing the chances that an individual will engage in risky substance use, sometimes to the point of addiction. $^{80}$

\section{Early Initiation of Use}

Adolescence is the critical period of vulnerability for the onset of substance use and the development of addiction. ${ }^{81}$ In 96.5 percent of cases, addiction originates with substance use before the age of $21 .^{82}$ Because the parts of the brain responsible for judgment, decisionmaking, emotion and impulse control are not fully developed until early adulthood, adolescents are more likely than adults to take risks, including experimenting with addictive substances. ${ }^{83}$ At the same time, because these regions of the brain are still developing, they are more vulnerable to the negative impact of addictive substances, further impairing judgment, interfering with brain development and increasing the risk of addiction. ${ }^{*} 84$ The combination of early initiation of use and genetic, biological, psychological or environmental risk factors dramatically hike the chances that addiction will develop. ${ }^{85}$

Adolescents with a genetic predisposition to addiction and/or co-occurring mental health problems are at the greatest risk of progressing from substance use to addiction. ${ }^{86}$

... [addiction] is not simply a disease of the brain, but it is a developmental disorder, and it begins early in life--during adolescence. ${ }^{71}$

--Nora D. Volkow, MD

Director

National Institute on Drug Abuse (NIDA)

\section{Risky Use and Addiction Frequently Co-occur with Other Health Conditions}

Individuals with addiction are likely to have cooccurring health conditions. ${ }^{87}$ Smoking causes bladder, esophageal, laryngeal, lung and oral cancer. ${ }^{88}$ From 2000-2004, the top three causes of smoking-attributable death were lung cancer,

\footnotetext{
* As is true of much of health research, the research on the neurological effects of addictive substances on the adolescent brain primarily has been conducted on animals.
} 
ischemic heart disease and chronic obstructive pulmonary disease. ${ }^{89}$ Alcohol consumption contributes to diseases that are among the top causes of death, including heart disease, cancer and stroke. ${ }^{90}$ Addiction involving alcohol is linked to cirrhosis, alcoholic hepatitis, chronic pancreatitis, cardiomyopathy, heart arrhythmias, stroke and neoplasms of the liver, pancreas and esophagus. ${ }^{91}$ Heavy alcohol use and addiction involving alcohol are associated with the incidence and re-infection of tuberculosis. ${ }^{92}$ Injection drug use is a risk factor for infectious diseases, including HIV, hepatitis $\mathrm{C}$ and hepatitis B. ${ }^{93}$ The incidence of various forms of cancer, ${ }^{94}$ heart disease ${ }^{95}$ and sexually-transmitted diseases ${ }^{96}$ are higher among those with addiction than among those without addiction.

Risky use and addiction also have high rates of co-occurrence with many mental health problems including depression, anxiety, posttraumatic stress disorder, bipolar disorder, schizophrenia and other neuropsychiatric disorders such as attention deficit/hyperactivity disorder, conduct disorder and eating disorders. $^{98}$ The association between addiction and co-occurring health conditions can result from several factors. Substance use may precipitate the onset of other conditions such as depression or anxiety disorders. ${ }^{99}$ Other times, the health conditions may precede the onset of addiction, as often occurs with mood disorders and attention deficit/hyperactivity disorder, ${ }^{100}$ as individuals attempt to self-medicate the pain or distress associated with their illness. ${ }^{101}$ The two types of conditions also may co-occur as a function of an underlying psychological or biological propensity, or substance use can exacerbate or complicate symptoms of existing health conditions. ${ }^{102}$

\section{Addiction Can Be a Chronic Disease}

Once an individual develops addiction, changes in the brain's reward circuitry may remain even after cessation of substance use. ${ }^{103}$ These changes leave addicted individuals vulnerable to physiological and environmental cues that they have associated with substance use, increasing the risk of relapse. In these cases, addiction is a chronic disease--like heart disease, hypertension, diabetes and asthma--defined as having a clear biological basis, a behavioral component, environmental influences, unique and identifiable signs and symptoms, a predictable course and outcome and the need for continued management following treatment. ${ }^{104}$

Like any other chronic condition, addiction rarely abates after a single course of medication or other treatment or after a single attempt to alter one's lifestyle or behavior. As is true of other chronic conditions, individuals with addiction can have symptom-free periods and periods of relapse. ${ }^{105}$ Many patients relapse multiple times and still others never achieve effective disease management. ${ }^{106}$ In fact, addiction frequently is characterized as a disease where relapse is virtually inevitable. Yet, this conception of addiction might be due to the focus of research studies on those with the most severe manifestations of addiction, who experience multiple episodes of symptom relapse and co-existing health and social problems over the course of many years or even a lifetime. ${ }^{107}$ Furthermore, very few people with addiction actually receive adequate, effective, evidence-based treatment. ${ }^{108}$ The seemingly high rates of relapse ${ }^{*}$ may be due, at least in part, to inadequate or ineffective interventions and treatments. ${ }^{109}$

It's not surprising to us now that when you stop the treatment, people relapse. It doesn't mean that the treatment doesn't work, it just means that you need to continue treatment. ${ }^{97}$

--Daniel Alford, MD, MPH

Associate Professor of Medicine Boston University School of Medicine

\footnotetext{
* Relapse rates for those with addiction are comparable to relapse rates for those with other chronic diseases.
} 


\section{Models for Understanding Addiction}

The model for how we understand addiction is critical because it influences how individuals with addiction are treated in society and guides the nature of the services provided to address the disease. ${ }^{110}$ The current model of addiction recognizes that it is a complex brain disease ${ }^{111}$ and that multiple determinants and systems influence substance use and its progression to addiction. ${ }^{112}$ Although this model is based on a large and growing body of scientific evidence, treatment practice and public attitudes still reflect earlier models of understanding the condition.

Since the 1700s, with few exceptions, ${ }^{113}$ two different models have dominated society's views on addiction, driven in large part by sociopolitical influences and also by developing knowledge about the science of addiction. ${ }^{114}$

The moral model of addiction framed addiction primarily as a failure of personal responsibility or morality. It asserted that addiction could be addressed simply by requiring personal responsibility and accountability on the part of the person who is addicted. This approach has contributed to:

- The stigma associated with addiction, attaching blame to the individual, creating shame and embarrassment, increasing the likelihood of discrimination and decreasing the chances that the addicted individual will seek or receive effective treatment. ${ }^{115}$

- Restrictions in benefits for addicted individuals. In 1995, a Congressional vote discontinued Supplemental Security Income (SSI) disability benefits to individuals whose primary diagnosis was addiction involving alcohol or other drugs. ${ }^{116}$ And, the majority of states in the U.S. currently are proposing or adopting legislation that condition the receipt of public services including welfare, unemployment assistance, job training, food stamps and public housing on passing a drug test. ${ }^{117}$
The disease model of addiction, in contrast to the moral model, acknowledged biology and genetics as significant contributors to addiction, drawing on advances in genetics and brain research. ${ }^{118}$ This approach has contributed to the concern that viewing addiction as a disease might:

- Release the individual from personal responsibility and the need for selfcontrol, ${ }^{119}$ and

- Engender feelings of hopelessness with regard to effective treatment and the possibility of recovery. ${ }^{120}$

These concerns, however, rarely are raised in relation to other health problems and appear, at least in part, to be reflective of the moral model of addiction.

\section{Evolving Approaches to Addressing Addiction}

America's approach to addressing substance use and addiction has been filled with contradiction. For example, at the turn of the $20^{\text {th }}$ century, cigarette smoking was frowned upon. ${ }^{121}$ Thanks to effective marketing by the tobacco industry, that view was replaced by one of tobacco use as glamorous and even healthful, ${ }^{122}$ only to be supplanted in the mid-1960s by a growing understanding that cigarette smoking is a significant contributor to poor health and disease. $^{123}$

Physicians prescribed marijuana and cocaine for a variety of ailments in the late part of the $19^{\text {th }}$ century only to scale back in the first decades of the $20^{\text {th }}$ century in response to increasing recognition of the adverse effects of these drugs and increasing regulatory restrictions on their use; today, there is a return to attempting to frame marijuana as medicine. ${ }^{124}$ Similarly, opium was used in the early part of the last century to treat diarrhea, dysentery and coughs. ${ }^{125}$ In response to the proliferation of marijuana, hallucinogen, cocaine and heroin use in the late 1960s and early 1970s--and their association with political protest, crime and 
addiction--and to the emerging "crack epidemic" in the late 1980s, substance use and addiction increasingly were criminalized. ${ }^{126}$ This trend toward criminalization was reflected in federal and state laws such as New York's Rockefeller Drug Laws which created mandatory minimum sentences of 15 years to life for possession of four ounces of narcotics (about the same as a sentence for second-degree murder). ${ }^{127}$ Later, prescription opioid medications were heavily marketed for pain which led to increased negative consequences associated with their use and renewed calls for increased legal restrictions. ${ }^{128}$ (See Text Box on page 28.)

The latter half of the $20^{\text {th }}$ century has seen more systematic and consistent progress in how addiction is perceived in the medical field. In 1956, the American Medical Association (AMA) declared that alcoholism is an illness and that it can and should be treated within the medical profession; ${ }^{130}$ in 1967, the AMA elaborated on this position in a manual for physicians declaring that alcoholism is characterized by a distinct pattern of symptoms, chronicity, progression, and by a tendency toward relapse and that it should be treated by physicians. ${ }^{131}$ The U.S. Comprehensive Alcohol Abuse and Alcoholism Prevention, Treatment, and Rehabilitation Act of 1970 recognized alcoholism as "an illness requiring treatment and rehabilitation.”

Alcoholism must be regarded as within the purview of medical practice. The Council on Mental Health, its Committee on Alcoholism, and the profession in general recognizes this syndrome of alcoholism as illness which justifiably should have the attention of physicians. ${ }^{133}$

--American Medical Association Reports of Officers Report of the Board of Trustees, 1956

In 1979, the AMA Council on Scientific Affairs published Guidelines for Physician Involvement in the Care of Substance-Abusing Patients which put the weight of policy behind the declaration that physicians are responsible for addressing alcohol and other drug use in their patients by engaging in diagnosis and referral (at a minimum) and preferably interventions that would ready the patient for treatment or actually providing treatment and follow-up care; the guidelines also specify the actions and knowledge required for each level of physician involvement. ${ }^{134}$ In 1989, a third of a century after declaring that alcoholism is a disease, the AMA adopted a policy declaring addiction involving other drugs--including nicotine--to be a disease. $^{135}$

Addiction involving alcohol and other drugs first was viewed by the field of psychiatry ${ }^{*}$ as a symptom of an underlying personality disorder in $1952 ;{ }^{136}$ in 1980 , addiction involving nicotine, alcohol and other drugs was described by the American Psychiatric Association (APA) ${ }^{\dagger}$ as an independent disorder--a substance use disorder for which the clinician was instructed to specify the substance involved in the addiction. ${ }^{137}$

From the mid-1990s through the present day there has been a growing backlash against a punitive approach to individuals with addiction, ${ }^{138}$ concomitant with the growth in scientific understanding of the brain processes underlying addiction and the development of evidence-based pharmaceutical and psychosocial therapies to treat it. ${ }^{139}$ Yet it was not until the late 1990s that addiction began to gain broader recognition as a brain disease. ${ }^{140}$

The history of addiction as a brain disease looks a lot like the history of atoms or germs, insofar as these were all older and controversial ideas for which scientific confirmation later became available. $^{129}$

--David T. Courtwright, PhD Professor, Department of History University of North Florida

\footnotetext{
* By the American Psychiatric Association's Diagnostic and Statistical Manual of Mental Disorder (DSM), first edition. (See page 31 for a description of the DSM.)

${ }^{\dagger}$ In the DSM-III.
} 


\section{Select Examples of Shifting Perceptions of Harms and Benefits of Addictive Substances in the U.S.}

Tobacco. Cigarette smoking generally was frowned upon at the turn of the $20^{\text {th }}$ century. ${ }^{141}$ However, highly effective marketing campaigns conducted during the early and mid- $20^{\text {th }}$ century by the major tobacco companies tremendously enhanced the appeal of smoking for men and women alike. The image of the cigarette smoker projected by such campaigns--and by the entertainment media--was that of glamour, sophistication and even fitness. Some medical professionals even signed on to the health message; tobacco advertisements featured physicians and some appeared in medical journals. ${ }^{142}$ In 1950, a landmark study linking smoking to lung cancer was published in the prestigious Journal of the American Medical Association. ${ }^{143}$ Over the next decade, more than 7,000 articles were published linking smoking with lung cancer and other life-threatening diseases. ${ }^{144}$ In 1964, the first U.S. Surgeon General's Report on Smoking and Health was published, concluding that smoking was hazardous to health and that immediate action was warranted. ${ }^{145}$ It spurred a major, highly successful public health effort to reduce smoking and other tobacco use. The truth about the dangers of smoking and the health consequences of second-hand smoke, finally reached the public through unmistakable health evidence and powerful anti-tobacco health and legal campaigns. Perceptions of smoking (and smokers) largely turned negative, public policy shifted toward widespread bans and restrictions on smoking, and smoking rates have declined significantly.

Alcohol. The duality of alcohol--as a staple for celebrations, religious rituals or relaxing with family and friends, and on the other hand as an underlying driver of crime, poverty, family dysfunction and illness--has a very long history. Alcohol was considered in early America to be helpful for curing ailments, natural and healthy when used in moderation, as an important source of nutrients and as a healthier alternative to water which often was contaminated. ${ }^{146}$ The $19^{\text {th }}$ and early $20^{\text {th }}$ centuries saw a change in attitudes wherein excessive alcohol use was seen as an impediment to worker productivity and as a contributing factor to problems such as domestic violence, poverty and crime. ${ }^{147}$ The initial push for moderation in alcohol use (temperance) soon became a push for prohibition. ${ }^{148}$ Once the futility of prohibition became apparent and the law ultimately repealed, alcohol has been both extolled through ubiquitous marketing and condemned for its tremendous societal and health-related costs. Conflicting views on the dangers versus benefits of alcohol use persist with a growing list of scientific findings that the health and social risks of excessive alcohol use and dangers of underage drinking outweigh any ostensible health benefits of non-excessive drinking.

Marijuana. In colonial America, marijuana was a major commercial crop alongside tobacco and was grown for its fiber much like cotton. In the 1920s, the recreational use of marijuana began to catch on, particularly among entertainers. Marijuana use at this time was not illegal and was not considered a social threat. Between the mid-19th and 20th centuries, marijuana was even prescribed for various medical conditions including labor pains, nausea and rheumatism. In the 1950s, marijuana use became increasingly popular and, in the 1960s, it became a symbol of rebellion against authority. In the Controlled Substances Act of 1970, the federal government classified marijuana along with heroin and LSD as a Schedule I drug--having the highest abuse potential and no accepted medical use-formalizing its illegality and highlighting its potential for addiction. The 1980s and early 1990s saw the passage of strict laws and mandatory sentences for possession of marijuana as well as the development of stronger strains of the drug. ${ }^{149}$ In recent years, a growing number of states have enacted laws bypassing the established process of bringing drugs to market in the U.S. which assures safety and efficacy and permitting the use of marijuana as "medicine."150

Opioids. The prescribing and dispensing of opioids by physicians and pharmacists were the major causes of the increase in narcotic use (and addiction) that occurred in the $19^{\text {th }}$ century. ${ }^{151}$ Opium and morphine were believed to cure a variety of health conditions. ${ }^{152}$ At the turn of the $20^{\text {th }}$ century, the Bayer Pharmaceutical Company promoted heroin as "the sedative for coughs." 153 At this time, there was some concern about the "moral degradation" caused by opioid use, but drug users were not subject to legal sanctions. ${ }^{154}$ Increased concern about the spread of opioid addiction prompted the passage of several Acts restricting the use and distribution of narcotics and making their nonmedical use illegal. ${ }^{155}$ Since that time, perceptions of narcotic/opioid use diverged dramatically, with prescription opioids marketed as beneficial and illegal opioids seen as a scourge. In the late 1990s, the growth in the use of prescribed pain relievers, tranquilizers and stimulants resulted in a widespread epidemic of prescription drug misuse and addiction among people of all ages, which now pose an even larger addiction problem than their illegal counterparts. ${ }^{156}$ In response, efforts to control this misuse have led, in some cases, to the under-treatment of pain. ${ }^{157}$ 


\section{Defining the Terms}

Part of the problem in understanding the nature of addiction is the lack of precision and uniformity in the terms used to describe it and distinguish it from substance use that threatens health and safety but is not addiction. ${ }^{158}$ Various terms such as experimentation, use, misuse, risky use, hazardous use, excessive use, abuse, dependence and addiction can be found in the research literature and in popular parlance. There are no standard connotations in use with regard to the severity of the behavior, the consequences of the behavior or the ability of the individual to stop the behavior. Nor is there recognition that the severity and consequences of different levels of use vary considerably by substance and with the age, gender and health status of the user.

Further confusion can be found in the misuse of the word "treatment" which historically has been used to refer to a host of interventions including detoxification, clinical treatment and professionally-administered disease management, social and mutual support and other auxiliary services. ${ }^{*} 159$ Rarely has the term treatment been reserved for those interventions that are based in the clinical and scientific evidence, as it is for the treatment of other diseases.

As with other diseases, precise definitions are essential to be able to stage the disease and develop effective interventions and treatments.

\section{The Continuum of Substance Use}

Substance use includes smoking or otherwise ingesting tobacco products, drinking alcohol or

\footnotetext{
* Detoxification services are not considered components of addiction treatment; rather, they are precursors to treatment designed to manage acute states of intoxication or withdrawal. Social and mutual support and other auxiliary services can be critical in supporting a patient through treatment and helping over the long term to sustain advances achieved through clinical treatment and to avoid relapse.
}

using other addictive substances. ${ }^{\dagger}$ The health consequences of use of these substances increase with frequency and quantity of use. ${ }^{160}$ The continuum of use ranges from no use on one end, to use that does not involve negative consequences, to risky use--a public health problem, to addiction--a medical problem.

\section{Risky Substance Use: A Public Health}

Problem. Risky substance use is defined, for the purposes of this report, as the use of tobacco, alcohol or other drugs in a way that can increase the probability of harm to the user or to others but does not meet clinical criteria for addiction. ${ }^{\ddagger}$ More specifically, it includes the following (sometimes-overlapping) categories:

- Use of any addictive substance by people under the age of 21 , for whom there is no known safe level of use. ${ }^{\S}$

The notion of critical periods, so integral to the development of language or musical skills, is relevant to the development of addiction as well. It is clear from my work in addiction medicine that use of addictive substances is like a traumatic brain injury to a young person. Any use by young people before brain development is complete equals risky use. ${ }^{161}$

--Mark S. Gold, MD

University of Florida College of Medicine and McKnight Brain Institute Departments of Psychiatry, Neuroscience, Anesthesiology, Community Health \& Family Medicine Chairman, Department of Psychiatry

\footnotetext{
${ }^{\dagger}$ Regardless of the legal status of the substance.

₹ The term risky use was chosen to reflect a range of health and social risks inherent in the use of these substances and to underscore the fact that significant hazards can result from such use even in the absence of clinical addiction. See Chapter III for a complete account of the health and social consequences of each of the following types of substance use that accounts for their designation as risky substance use behaviors. $\S$ Age 21 was selected because of the cultural marker of adulthood in the U.S., including the minimum legal drinking age, although research documents the continued development of the brain (and consequent vulnerability to the effects of addictive substances) into the mid-twenties.
} 
- Use of any addictive substance by pregnant women, for whom there is no known safe level of use.

- Use of any tobacco product; there is no known safe level of use of tobacco products.

- Alcohol use in excess of the established dietary guidelines for safe alcohol use of no more than one drink per day for women and two drinks per day for men, as described by the U.S. Departments of Agriculture (USDA) Dietary Guidelines." ${ }^{* 2}$ This includes the non-mutually exclusive categories of binge drinking, ${ }^{\dagger}$ heavy drinking $^{\ddagger}$ and heavy binge drinking. ${ }^{\S}$

- Misuse (non-medical use) of controlled prescription drugs and over-the-counter medications $^{* *}$--i.e., using them for purposes

* The guidelines also stipulate no alcohol consumption for: (1) persons under the age of 21; (2) pregnant women; (3) individuals who cannot restrict their drinking to moderate levels; (4) individuals taking prescription or over-the-counter medications that can interact with alcohol; (5) individuals with certain specific medical conditions (e.g., liver disease, hypertriglyceridemia, pancreatitis); and (6) individuals who plan to drive, operate machinery or take part in other activities that require attention, skill or coordination, or in situations where impaired judgment could cause injury or death (e.g., swimming). Although drinking alcohol, as described in (4) through (6) is considered risky substance use, estimates of rates of risky substance use in this report do not include these criteria since they are not directly measured in the national surveys that were analyzed for this study.

${ }^{\dagger}$ Consuming five or more drinks on the same occasion (within a few hours) on at least one day in the past 30 days.

${ }^{\ddagger}$ When a woman consumes more than three drinks on a given day or more than seven drinks during a given week or when a man consumes more than four drinks on a given day or more than 14 drinks during a given week.

${ }^{\S}$ Binge drinking on at least five occasions in the past 30 days.

${ }^{* *}$ In accordance with the federal Controlled Substances Act of 1970 which created a system for classifying illicit and prescription drugs according to their medical value and their potential for misuse. not prescribed or intended such as to get high, feel stimulated or sedated; taking more of the substance than prescribed or recommended; or taking the substance too often or for a longer period of time than was prescribed or recommended. ${ }^{\dagger \dagger} 163$

- Use of non-prescribed federally controlled

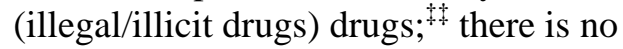
known safe level of use of these drugs.

The public, and treatment sources alike, often are confused as to the distinction among use, heavy use and addictive disease. ${ }^{164}$

--Stuart Gitlow, MD, MPH, MBA Executive Director Annenberg Physician Training Program in Addictive Disease Associate Clinical Professor Mount Sinai School of Medicine Acting President \& AMA Delegate American Society of Addiction Medicine

Addiction: A Medical Disease. At the end of the continuum of substance use are those who meet clinical criteria for addiction. Yet even among these individuals, there is tremendous variation in the severity and course of the disease. At the extreme end of the continuum is

\footnotetext{
${ }^{\dagger \dagger}$ For data analysis purposes, the national survey examined for this report defines misuse of controlled prescription medications more generally as "taking a controlled prescription drug not prescribed for you or taking it in a manner not prescribed for the experience or feeling it causes.” The misuse of overthe-counter medications also constitutes risky use; however, rates of risky substance use in this report do not include the misuse of these medications since they are not directly measured in the national surveys that were analyzed for this study.

¥ Substances controlled (either through prohibited or restricted use) by the federal Controlled Substances Act of 1970 which created a system for classifying illicit and prescription drugs according to their medical value and their potential for misuse. In the analyses presented in this report, illicit drugs include marijuana/hashish, cocaine/crack, heroin, hallucinogens, Ecstasy, methamphetamine and inhalants. (See Chapter III.)
} 
the most severe form of the illness: chronic relapsing disease.

There are two leading sources of diagnostic classifications for addiction (called substance use disorders): The American Psychiatric Association's Diagnostic and Statistical Manual of Mental Disorders (DSM-IV) and the World Health Organization's International Statistical Classification of Disease (ICD). Both reflect the historical classification of addiction as a mental disorder based on measures of its behavioral symptoms rather than the current science documenting the medical nature of the problem as an underlying brain disease. ${ }^{166}$

\section{Diagnostic and Statistical Manual of Mental} Disorders (DSM-IV). The DSM-IV is the main diagnostic reference of mental health professionals in the United States and presents three types of disorders that are drug specific: (1) alcohol use disorders, (2) drug use disorders and (3) nicotine dependence. Both alcohol and drug use disorders involve two levels of severity--abuse and dependence--while nicotine only involves dependence. The criteria for abuse are similar for alcohol and other drugs, and the criteria for dependence are similar for all three drug types. ${ }^{167}$

The DSM-IV defines substance abuse as "a maladaptive pattern of substance use leading to clinically significant impairment or distress.”168 The term substance abuse, though widely used-and often used beyond its clinical definition to describe any kind of risky substance use--has become controversial due to its negative, valueladen and pejorative connotation. ${ }^{169}$

The term substance dependence is defined in the DSM-IV as “.... cluster of cognitive, behavioral and physiological symptoms indicating that the individual continues use of the substance despite significant substance-related problems."170

\section{DSM-IV Criteria $^{165}$}

\section{Substance Abuse}

To be diagnosed with substance abuse, an individual must exhibit one or more of the following four symptoms within a 12-month period: ${ }^{*}$

- Recurrent use resulting in failure to fulfill major role obligations at work, school or home;

- Recurrent use in physically hazardous situations;

- Recurrent use resulting in legal problems; and

- Continued use despite persistent or recurrent social or interpersonal problems.

\section{Substance Dependence}

To be diagnosed with substance dependence, an individual must exhibit three or more of seven symptoms within a 12-month period:

- Tolerance--the need for markedly increased amounts of the substance to achieve intoxication or the desired effect or a markedly diminished effect with the continued use of the same amount of the substance;

- Withdrawal--maladaptive behavioral change with co-occurring physiological and cognitive changes that occurs when use of a substance is reduced or discontinued, or usage of a substance to relieve or avoid withdrawal symptoms;

- Taking increasing amounts or using the substance over a longer period than intended;

- A persistent desire or unsuccessful efforts to reduce or control substance use;

- Spending a significant amount of time in activities to obtain the drug, use it or recover from use;

- Neglecting or giving up important social, occupational or recreational activities because of use; and

- Continuing use of the substance despite knowledge of having a persistent or recurring physical or psychological problem that is caused or worsened by use.

* The symptoms must never have met criteria for substance dependence for the class of substances used. 
Definitional Problems. The first two symptoms of the DSM-IV's substance dependence diagnosis--tolerance and withdrawal-- reflect physiological dependence on a substance as a result of its regular use. ${ }^{171}$ There has been considerable confusion about the difference between physiological dependence on a substance and addiction involving that substance. This is further confused by the use of the same term "dependence" to apply to both conditions.

According to the DSM-IV diagnostic criteria, tolerance and withdrawal symptoms neither are necessary nor sufficient for a diagnosis of substance dependence ${ }^{172}$ (e.g., an individual may meet diagnostic criteria for addiction involving cocaine or methamphetamine in the absence of symptoms of tolerance and withdrawal). ${ }^{173}$ There also are cases where an individual is physically dependent on a substance but does not meet clinical criteria for addiction. ${ }^{174}$ This occurs relatively frequently in relation to the use of controlled prescription pain relievers. Prolonged use of these medications may result in physical symptoms, including tolerance and withdrawal as a result of the body's adaptation to taking the drug, ${ }^{175}$ but may not include the loss of control and other behavioral symptoms associated with addiction. ${ }^{176}$

Other symptoms of substance dependence also can be observed in patients legitimately using certain prescription medications. ${ }^{177}$ For example, if a patient's pain is under-treated, he or she may become preoccupied with finding a pain medication or may take a pain medication for a longer time than originally was prescribed. ${ }^{178}$ Mischaracterizing this behavior as addiction may result in further withholding of legitimate pain treatment. ${ }^{\dagger 179}$

Issues have been raised with regard to the reliability and validity of the nicotine

\footnotetext{
* Opioids.

${ }^{\dagger}$ While the under-treatment of pain has been acknowledged, there are emerging indications that many physicians may now be overprescribing addictive opioid medications for the treatment of patients with non-malignant chronic pain.
}

dependence criteria as well: they have been described as ambiguous, failing to address important aspects of addiction involving nicotine such as craving, too subjective and intertwined with an individual's social context (e.g., with regard to assessing the "costs" of continued use) and limited in their ability to predict key outcomes such as extent of tobacco use, withdrawal severity and likelihood of future cessation. ${ }^{180}$

Proposed Modifications to the DSM Diagnostic Criteria. Partially because of these definitional problems, work is under way to redefine and reorganize the DSM's diagnostic criteria for substance use disorders. These revisions are proposed to be included in the upcoming DSM$\mathrm{V}$ version, which is estimated to be released in May 2013. ${ }^{181}$ Among the proposed revisions is the suggestion to replace the two categories of substance abuse and substance dependence with a single diagnostic category of substance use and addictive disorders. ${ }^{\ddagger 182}$ This modification recognizes a broad category of addiction including substance addiction and other compulsive behaviors that reflect a common neuropathology. ${ }^{183}$ Separate disorders would be identified for each type of addictive substance or behavior and for multiple addictive disorders. The addictive disorder diagnosis would contain 11 criteria; meeting two or three criteria would result in a diagnosis of "moderate" severity, while meeting four or more criteria would result in a diagnosis of "severe." The presence of physical dependence (symptoms of tolerance or withdrawal) also would be identified. ${ }^{\S} 184$

\footnotetext{
‡ Initially, the Substance-Related Disorders Work Group for the revisions to the DSM considering renaming this category "Addiction and Related Disorders."

$\S$ The proposed revised criteria clearly state that tolerance and withdrawal are "not counted for those taking medications under medical supervision such as analgesics, antidepressants, anti-anxiety medications or beta-blockers." This stipulation is designed to prevent a patient who becomes physically dependent on a prescription drug received during the course of medical care from being diagnosed with addiction (i.e., a substance use disorder).
} 
One issue being raised in the proposed revisions to the DSM is that of patients who are at heightened risk of developing addiction in the near future but do not now meet the diagnostic criteria. ${ }^{185}$ One suggestion ${ }^{*}$ is to add a severity category of "mild" to the proposed "moderate" and "severe" diagnosis categories. This modification would help to identify risky substance users and increase the chances that they receive needed services to prevent their risky use from progressing to addiction, while reducing the risk of their receiving unnecessary treatment which might accompany a more severe diagnosis. ${ }^{186}$

\section{International Statistical Classification of} Diseases (ICD). Like the DSM, the ICD does not present a disease category of addiction; rather, it presents mental health disorders that are linked to psychoactive substance use including nicotine, alcohol and other drugs. ${ }^{187}$

Like the DSM, the ICD offers two categories related to addiction: in this case, harmful use and dependence syndrome. Harmful use is defined as "a pattern of psychoactive substance use that is causing damage to health. The damage may be physical (e.g., hepatitis following injection of drugs) or mental (e.g., depressive episodes secondary to heavy alcohol intake)." The ICD defines dependence syndrome as "a cluster of behavioral, cognitive and physiological phenomena that may develop after repeated substance use. Typically, these phenomena include a strong desire to take the drug, impaired control over its use, persistent use despite harmful consequences, a higher priority given to drug use than to other activities and obligations, increased tolerance and a physical withdrawal reaction when drug use is discontinued."188

The DSM vs. the ICD. While there is considerable overlap between the diagnostic definitions provided in the DSM and the ICD, the ICD definitions connote more of a disease state while the DSM definitions imply more of a behavioral disorder. In addition, the ICD is used

\footnotetext{
* Made by NAADAC, the Association for Addiction Professionals.
}

more frequently internationally while the DSM is used more frequently in the United States and Canada. ${ }^{189}$ Access to the ICD codes is free to the public via the Internet, whereas providers must pay the American Psychiatric Association for access to the DSM manual and codes, either by purchasing the text version of the manual or by paying to access the information online. ${ }^{190}$ The DSM is used more commonly than the ICD in research studies, whereas the ICD has become the primary tool used by health care facilities to index health care data. ${ }^{191}$ The DSM criteria for addiction involving nicotine are used less frequently by researchers and clinicians than criteria for addiction involving alcohol and other drugs. $^{\dagger} 192$

Like the DSM, the ICD currently is undergoing revisions and there is hope that the parties involved in the revisions to these classification systems will take the opportunity to standardize the language used to describe the full spectrum of clinical addiction, ${ }^{193}$ with an increased focus on the disease itself rather than different disorders linked to specific substances.

It also is important to clarify that addiction is a medical condition with significant behavioral components. ${ }^{194}$ Recognizing that current diagnostic classification systems like the DSM and ICD do not sufficiently take into account the shared underlying genetic and neurobiological dimensions of addiction and various health conditions--instead relying primarily on subjective reports of symptoms of seemingly unique and unitary conditions--the National Institute of Mental Health (NIMH) has begun to classify these conditions in a new way. The Research Domain Criteria project (RDoC) aims to develop a classification system based on observable behavior and neurobiological measures that underlie different manifestations of addiction and related conditions which currently are classified and addressed as distinct conditions. ${ }^{195}$ Should this new system take hold,

\footnotetext{
${ }^{\dagger}$ Measures of addiction involving nicotine that are considered to have greater predictive validity in terms of outcomes include the Fagerstrom Test for Nicotine Dependence (FTND) and Nicotine Dependence Symptom Scale (NDSS).
} 
it eventually may help practitioners diagnose and address the disease of addiction and its multiple manifestations in a more unified and coherent manner in clinical practice.

\section{Public Attitudes about Addiction}

Public attitudes about a particular disease or health condition and the people who suffer from it historically have been linked to the public's understanding of its causes and amenability to treatment. This nation has a long history of isolating and stigmatizing individuals with health problems that were not well understood, from tuberculosis to cancer, depression and HIV-AIDS. Once scientific understanding of the condition is solidified and the information permeates public understanding, public attitudes towards the condition and those who have it often change. The availability of effective treatments also can have a profound impact on driving this change. Addiction is a prime example of a disease where public attitudes have yet to catch up with the science, although attitudes are shifting. ${ }^{196}$

A 2005 online survey of 1,000 adults ages 20 and older about addiction involving alcohol found that 63 percent of the general public see it primarily as a personal or moral weakness (19 percent) or equally as a personal or moral weakness and as a disease or health problem (44 percent); only 34 percent see it primarily as a disease or health problem. In contrast, only 11 percent of individuals who are managing the disease (i.e., in recovery) see it either primarily as a personal or moral weakness (two percent) or as both a personal or moral weakness and a disease or health problem (nine percent); 81 percent see it primarily as a disease or health problem. This survey also found that 43 percent of physicians consider addiction involving alcohol to be a personal or moral weakness (nine percent see it primarily as a personal or moral weakness and 34 percent see it equally as a personal or moral weakness and as a disease or health problem); 56 percent see it primarily as a disease or health problem. ${ }^{197}$
A more recent survey of treatment providers in the U.S. and the United Kingdom, published in 2011, found that the belief that addiction is a disease is stronger among those who provide for-profit treatment while the belief that addiction is a choice is stronger among providers of public or not-for-profit treatment ${ }^{*}{ }^{198}$--the more common form of treatment in the United States. ${ }^{199}$

Results from a nationally representative survey conducted in 2008 found that 44 percent of the public believes that people with addiction involving alcohol could stop drinking if they had enough willpower (73 percent of young adults, age 18 to 24, hold this view). Fewer Americans think the same is true for addiction involving other drugs (38 percent of the public; 66 percent of young adults). ${ }^{200}$

The average person in the U.S. views addiction with a sense of hopelessness. They realize that not all users of alcohol or other addictive drugs will become addicted. They know that some users will become addicted but that others will not. This creates a belief that the addict can-and should--use willpower to stop using. ${ }^{201}$

-- J. Paul Molloy, JD Chief Executive Officer Oxford House

A 2009 nationally representative survey of 1,000 adults ages 18 and older underscores the tremendous stigma still associated with addiction: individuals with addiction involving alcohol or other drugs commonly were described by respondents with words such as "sinner," "irresponsible," "selfish,” "stupid," "loser," "undisciplined,” "pathetic” and “weak.,"202

\footnotetext{
${ }^{*}$ Differences also were found between providers who were members of a group of addiction professionals, had been treating addiction for longer, had stronger spiritual beliefs, had a past addiction problem and were older (tending to endorse the belief that addiction is a disease) versus those who were not members of a group of addiction professionals, had less strong spiritual beliefs and were younger (tending to endorse the belief that addiction is a choice).
} 
However, as a sign of increasing acceptance of addiction as a disease, this same survey found that the majority (78 percent) regard addiction involving drugs other than nicotine or alcohol as a chronic disease. $^{203}$ Another 2009 nationally representative survey of adults ages 18 and older also found that the majority of the respondents believe that addiction is a health condition that requires ongoing attention and support (83 percent). ${ }^{204}$

\section{Perceived Causes of Addiction}

For decades, public misconceptions about the origins of addiction have led to negative attitudes and discrimination against those afflicted with the disease and hindered progress not only in understanding it, but also in developing and providing effective treatments for it. ${ }^{205}$ In 2008, CASA Columbia probed these perspectives with a nationally representative survey of American attitudes toward substance use and addiction--the National Addiction Belief and Attitude Survey (NABAS). Although public attitudes increasingly appear to be reflective of the science of addiction, people still often hold conflicting views about the causes of addiction, many of which are inconsistent with the growing body of evidence.

CASA Columbia's NABAS found, for example, that while many people understand that factors such as genetics, family history, other health problems and availability of addictive substances play a role in the development of addiction and that loss of control is a defining characteristic of the disease, a significant proportion of Americans cite "lack of will power or self-control” as a primary causal factor. Surprisingly, respondents who had a personal experience with addiction--either that of a family member or friend or their own--did not differ much from the general public in these perceptions of the key contributors to addiction. Respondents also had different views on the causes of addiction depending on the substance involved. $^{206}$
Tobacco/Nicotine. Despite the fact that genetics account for up to 75 percent of the risk for addiction involving nicotine, ${ }^{207}$ the $N A B A S$ found that only one in four respondents (25.4 percent) cited "a predisposition to addiction, due to genetics or family history” as a primary causal factor when given a list of potential causes of addiction involving tobacco/nicotine.

Respondents were most likely to cite an "inability to resist peer pressure" (43.5 percent); "easy availability of tobacco among youth" (38.7 percent); "stress or anxiety about work, family or other problems” (37.7 percent); and "lack of willpower or self-control” (33.0 percent) as primary causal factors. ${ }^{208}$

(Figure 2.A)



Alcohol. Public attitudes are more aligned with the science regarding the role of genetics in addiction involving alcohol. Genetics account for 48 to 66 percent of the risk that someone who drinks alcohol will become addicted ${ }^{209}$ and nearly half (47.6 percent) of respondents to the NABAS cite "a predisposition to addiction, due to genetics or family history" as a primary causal factor. Other perceived primary causes of addiction involving alcohol include "stress or anxiety about work, family or other problems" (44.9 percent); “emotional disorders or mental illness, such as depression or anxiety” (35.3 percent); "lack of willpower or self-control" 
(29.7 percent); "easy availability of alcohol among youth" (29.2 percent); and "inability to resist peer pressure” (28.2 percent). ${ }^{210}$ (Figure 2.B)

Other Drugs. Although genetic factors account for up to 78 percent of the risk for developing addiction involving illegal or controlled prescription drugs (depending on the type of drug studied and the severity of the addiction), ${ }^{211}$ only 27.5 percent of NABAS respondents cited "a predisposition to addiction, due to genetics or family history" as a primary cause of addiction involving prescription drugs. The most commonly cited factor that may cause people to become addicted to prescription drugs was "emotional disorders or mental illness, such as depression or anxiety” (40.8 percent). ${ }^{212}$ Co-occurring mental health disorders do appear to play a significant role in the risk that drug use will progress to addiction. ${ }^{213}$ Other primary causes that were mentioned by respondents to the NABAS include "stress or anxiety about work, family or other problems” (36.9 percent); “easy availability of prescription medications among youth" (36.7 percent); and a "lack of willpower or self-control” (26.9 percent). ${ }^{214}$ (Figure 2.C)

Thirty percent cited "a predisposition to addiction, due to genetics or family history" as a primary cause of addiction involving illegal drugs. The most commonly cited factor that may cause people to become addicted to illegal drugs was an "inability to resist peer pressure” (41.9 percent). Other factors that were mentioned as a primary cause of addiction involving illicit drugs include "easy availability of illegal drugs among youth" (35.1 percent); "emotional disorders or mental illness, such as depression or anxiety" (34.8 percent); "stress or anxiety about work, family or other problems" (29.9 percent); and a "lack of willpower or self-control" (28.7 percent). ${ }^{215}$ (Figure 2.D)
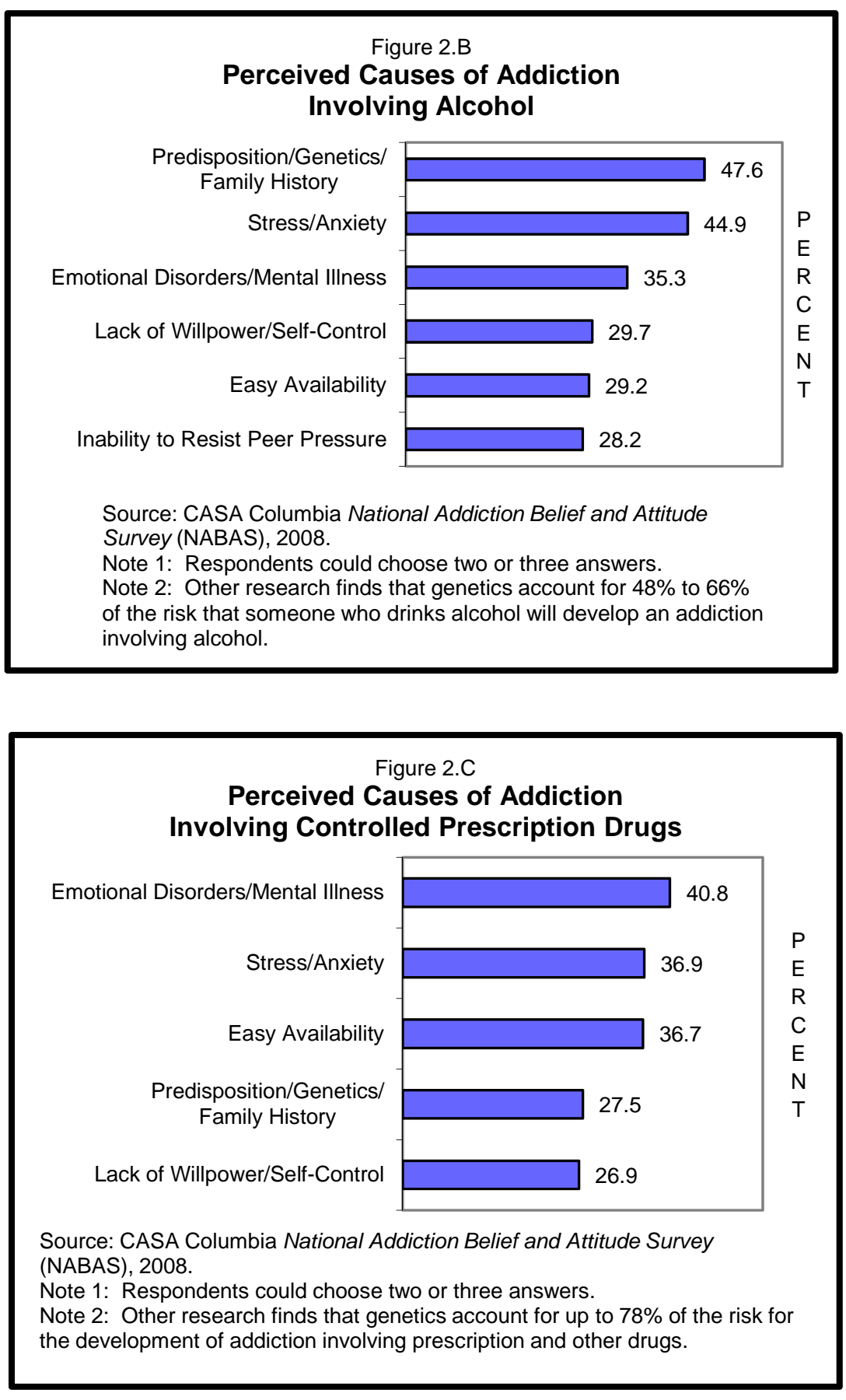


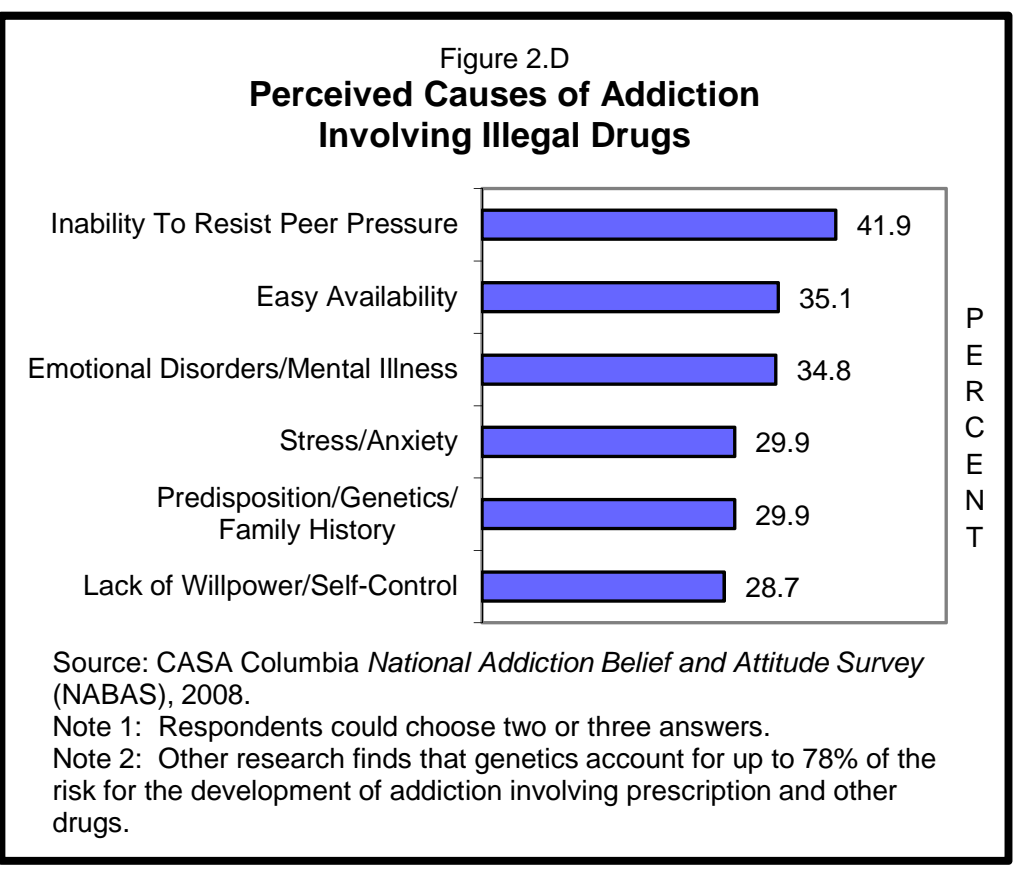

The NABAS found little indication that respondents attribute addiction primarily to an "absence of religious faith or spiritual grounding” or to “distorted moral values."216 
$-38-$ 


\section{Chapter III \\ Prevalence and Consequences}

More than one in seven (15.9 percent, 40.3 million) people in the United States ages 12 and older currently meet clinical criteria for addiction $^{* 1}$--more than the share of the population with cancer, diabetes or heart disease. $^{\dagger 2}$ An additional but unknown number of people have the disease but are managing it effectively and so do not meet these behavioral criteria. $^{\ddagger 3}$ Another third of the population (31.7 percent, 80.4 million), while not addicted, currently ${ }^{\S}$ use one or more addictive substances in ways that threaten their own health and safety or the health and safety of others. ${ }^{4}$

Risky substance use and addiction are the largest preventable and most costly public health and medical problems in the U.S. ${ }^{5}$ Together they are the leading causes of preventable death and cause or contribute to more than 70 other conditions requiring medical care. ${ }^{6}$ The damaging effects of risky substance use and addiction extend to a wide range of costly social

* Defined as meeting criteria for past-month nicotine dependence based on the Nicotine Dependence Syndrome Scale (NDSS) and meeting diagnostic criteria for past year alcohol and/or other drug abuse or dependence (excluding nicotine) in accordance with the Diagnostic and Statistical Manual of Mental Disorders (DSM-IV). (The DSM refers to substance abuse and substance dependence collectively as substance use disorders. The criteria for nicotine dependence in the NDSS parallel those of the DSMIV.) This estimate excludes the institutionalized population, for which rates of addiction are higher. ${ }^{\dagger}$ Includes coronary heart disease, angina pectoris, heart attack or any other heart condition or disease, excluding hypertension and stroke.

₹ There are no national data on the proportion of the population that has been diagnosed with addiction and is effectively managing the disease. Existing national survey data indicate that 10 percent of adults ages 18 and older report that they "once had a problem with drugs or alcohol but no longer do" (sometimes referred to as "sustained recovery"), but it is not clear what proportion of this group ever was diagnosed with addiction as distinguished from "having a problem."

$\S$ In the past 30 days. 
consequences including crime, lost productivity, child neglect and abuse, family dysfunction and developmental disabilities.

Certain populations--including pregnant women, adolescents and young adults and the elderly-are more vulnerable to the damaging effects of addictive substances. Other populations-including those with co-occurring disorders, members of the military exposed to combat and those in the justice system--are more likely to engage in risky use or have addiction.

\section{Defining the Problem}

Substance use can be understood as a continuum ranging from having never smoked or used alcohol or another drug ${ }^{*}$ at one end to having an unmanaged chronic, relapsing disease ${ }^{\dagger}$ at the other. (Figure 3.A)

\section{Figure 3.A \\ Continuum of Substance Use \\ Percent of Population Age 12+ by Level of Sustance Use*}

\begin{tabular}{|c|c|c|c|c|}
\hline 12.7 & 25.2 & 14.5 & 31.7 & 15.9 \\
\hline $\begin{array}{c}\text { Never } \\
\text { Used }\end{array}$ & $\begin{array}{c}\text { No } \\
\text { Current } \\
\text { Use }\end{array}$ & $\begin{array}{c}\text { Non- } \\
\text { Risky Use }\end{array}$ & $\begin{array}{c}\text { Risky } \\
\text { Use }\end{array}$ & Addiction \\
\end{tabular}

* Includes tobacco, alcohol, illicit drugs and misuse of controlled prescription drugs.

Source: CASA Columbia analysis of The National Survey on Drug Use and Health (NSDUH), 2010.

Most people (87.3 percent) ages 12 and older in the U.S. have used one or more addictive

\footnotetext{
* Including any use of illicit drugs or the misuse of controlled prescription drugs.

${ }^{\dagger}$ This continuum focuses on substance use; the category labeled addiction includes those individuals whose substance use meets current clinical criteria for this disease but does not include all individuals with addiction. There are some individuals with the disease of addiction who do not currently use any addictive substances and others who currently may fit into other use categories along the continuum.
}

substances at some point in their lives, and 62.1 percent are current users. $^{\ddagger 7}$ Those who have never used an addictive substance, are not current users $^{\S}$ or do not engage in risky substance use are appropriate targets for public health efforts aimed at preventing risky use and addiction; however, these individuals are not the focus of this report.

While this report focuses primarily on those with the disease of addiction, it makes an important distinction between addiction and risky use of addictive substances:

- Those with the disease of addiction are defined in this report as meeting the clinical diagnostic criteria for past month nicotine dependence or past year alcohol and/or other drug abuse or dependence. Individuals who meet diagnostic criteria for addiction are targets for appropriate, evidence-based clinical interventions by physicians and other health professionals.

- Risky users of addictive substances are defined in this report as those who currently use tobacco products, exceed the U.S. Department of Agriculture (USDA) Dietary Guidelines for safe alcohol use, ${ }^{* *} 8$

${ }^{\ddagger}$ Current substance use among the entire U.S. population ages 12 and older: 51.7 percent drink alcohol; 27.6 percent use tobacco products; 7.5 percent use illicit drugs; and 2.8 percent misuse controlled prescription drugs.

${ }^{\S}$ Having used the substance in the past 30 days.

*** The U.S. Department of Agriculture Dietary Guidelines for safe alcohol use are no more than one drink a day for women, no more than two drinks a day for men and no alcohol consumption for: (1) persons under the age of 21; (2) pregnant women; (3) individuals who cannot restrict their drinking to moderate levels; (4) individuals taking prescription or over-the-counter medications that can interact with alcohol; (5) individuals with certain specific medical conditions (e.g., liver disease, hypertriglyceridemia, pancreatitis); and (6) individuals who plan to drive, operate machinery or take part in other activities that require attention, skill or coordination or in situations where impaired judgment could cause injury or death (e.g., swimming). Due to data limitations, we were unable to include categories 4-6 in our calculation of risky drinkers. 
misuse controlled prescription drugs, ${ }^{*}$ use illicit drugs ${ }^{\dagger}{ }^{9}$ or engage in some combination of these forms of substance use but do not meet clinical diagnostic criteria for addiction. Risky use can result in devastating and costly health and social consequences, including the disease of addiction. Risky users are targets for public health efforts aimed at reducing risky use and of medical and other health professional efforts to prevent risky use from progressing to the disease of addiction.

To determine the scope of risky substance use and addiction in the United States, CASA Columbia examined national prevalence data ${ }^{\ddagger}$ for the total U.S. non-institutionalized population ages 12 and older. For these analyses, we examined current (past 30 day) use of tobacco, alcohol and other drugs and the rate of addiction involving these substances. Our definition of addiction in this report is consistent with the parameters used in the national survey upon which our analyses are based--the National Survey on Drug Use and Health (NSDUH)-which categorizes respondents on the basis of diagnostic criteria used in the Nicotine Dependence Syndrome Scale (NDSS) for nicotine dependence and in the DSM-IV for

\footnotetext{
* For data analysis purposes, the national survey examined for this report defines misuse of controlled prescription medications more generally as "taking a controlled prescription drug not prescribed for you or taking it in a manner not prescribed for the experience or feeling it causes." The misuse of overthe-counter medications also constitutes risky use; however, rates of risky use in this report do not include the misuse of these medications since they are not measured directly in the national surveys that were analyzed for this study.

† Substances controlled (either through prohibited or restricted use) through the federal Controlled Substances Act of 1970 which created a system for classifying illicit and prescription drugs according to their medical value and their potential for misuse. In this analysis, illicit drugs include marijuana/hashish, cocaine/crack, heroin, hallucinogens, Ecstasy, methamphetamine and inhalants.

₹ The most recent data available (2010) from the Substance Abuse and Mental Health Association's (SAMHSA) National Survey on Drug Use and Health (NSDUH).
}

alcohol and other drug abuse or dependence. We also examined variations in the rates of risky use and addiction by age, gender and race/ ethnicity. Because of the heightened vulnerability to addictive substances while the brain is still developing, ${ }^{\S 10}$ we present data for the following age groups: 12- to 17-year olds; 18- to 25-year olds; and those ages 26 and older. While the NSDUH was used for these analyses to present consistent data across age groups, it is important to note that these data appear to underestimate rates of current substance use among adolescents by at least half.* 11

\section{Risky Substance Users}

Approximately one-third (31.7 percent) of the U.S. population ages 12 and older (80.4 million people) currently ${ }^{\dagger \dagger}$ use one or more addictive substances in ways that threaten their own health and safety--including increasing the risk of addiction--or the health and safety of others, but do not meet clinical criteria for addiction. (Table 3.1) Nearly one-third (30.6 percent) of risky users engage in risky use of two or more substances. Alcohol is the substance most frequently implicated in risky substance use, followed by tobacco. ${ }^{12}$

\footnotetext{
$\S$ See Chapter II.

** Because the NSDUH is conducted in the home where an adult must be present, reported prevalence rates for teens are significantly lower than reported rates in school-based surveys where greater anonymity is assured. For example, the Youth Risk Behavior Survey's (YRBS) prevalence estimates for 12- to 17-year olds are 2.15 to 2.75 times as high as those presented in the NSDUH.

${ }^{+\dagger}$ In the past 30 days.
} 


\begin{tabular}{|c|c|c|}
\hline \multicolumn{3}{|c|}{$\begin{array}{c}\text { Table } 3.1 \\
\text { Prevalence of Current Risky Use }{ }^{*} \text { of } \\
\text { Addictive Substances in } \\
\text { U.S. Population, Ages 12+, } 2010\end{array}$} \\
\hline & $\begin{array}{l}\text { Percent of } \\
\text { Total } \\
\text { Population }\end{array}$ & $\begin{array}{l}\text { Number } \\
\text { (in } \\
\text { Millions) }\end{array}$ \\
\hline Total Risky Use & 31.7 & 80.4 \\
\hline $\begin{array}{l}\text { Tobacco With/Without } \\
\text { Other Substances }\end{array}$ & 18.5 & 47.0 \\
\hline Tobacco Only & 6.7 & 17.0 \\
\hline $\begin{array}{l}\text { Alcohol With/Without } \\
\text { Other Substances }\end{array}$ & 27.2 & 69.0 \\
\hline Alcohol Only & 14.3 & 36.2 \\
\hline $\begin{array}{l}\text { Illicit Drugs } \\
\text { With/Without } \\
\text { Other Substances }\end{array}$ & 5.8 & 14.6 \\
\hline Illicit Drugs Only & 0.6 & 1.6 \\
\hline $\begin{array}{l}\text { Controlled Prescription } \\
\text { Drugs With/Without } \\
\text { Other Substances }\end{array}$ & 2.1 & 5.4 \\
\hline $\begin{array}{l}\text { Controlled Prescription } \\
\text { Drugs Only }\end{array}$ & 0.5 & 1.1 \\
\hline Multiple Substances & 9.7 & 24.6 \\
\hline \multicolumn{3}{|c|}{$\begin{array}{l}{ }^{*} \text { Individuals included in the risky use category used } \\
\text { the substance in the past } 30 \text { days but do not meet } \\
\text { diagnostic criteria for addiction. } \\
\text { Source: CASA Columbia analysis of The National } \\
\text { Survey on Drug Use and Health (NSDUH), } 2010 .\end{array}$} \\
\hline
\end{tabular}

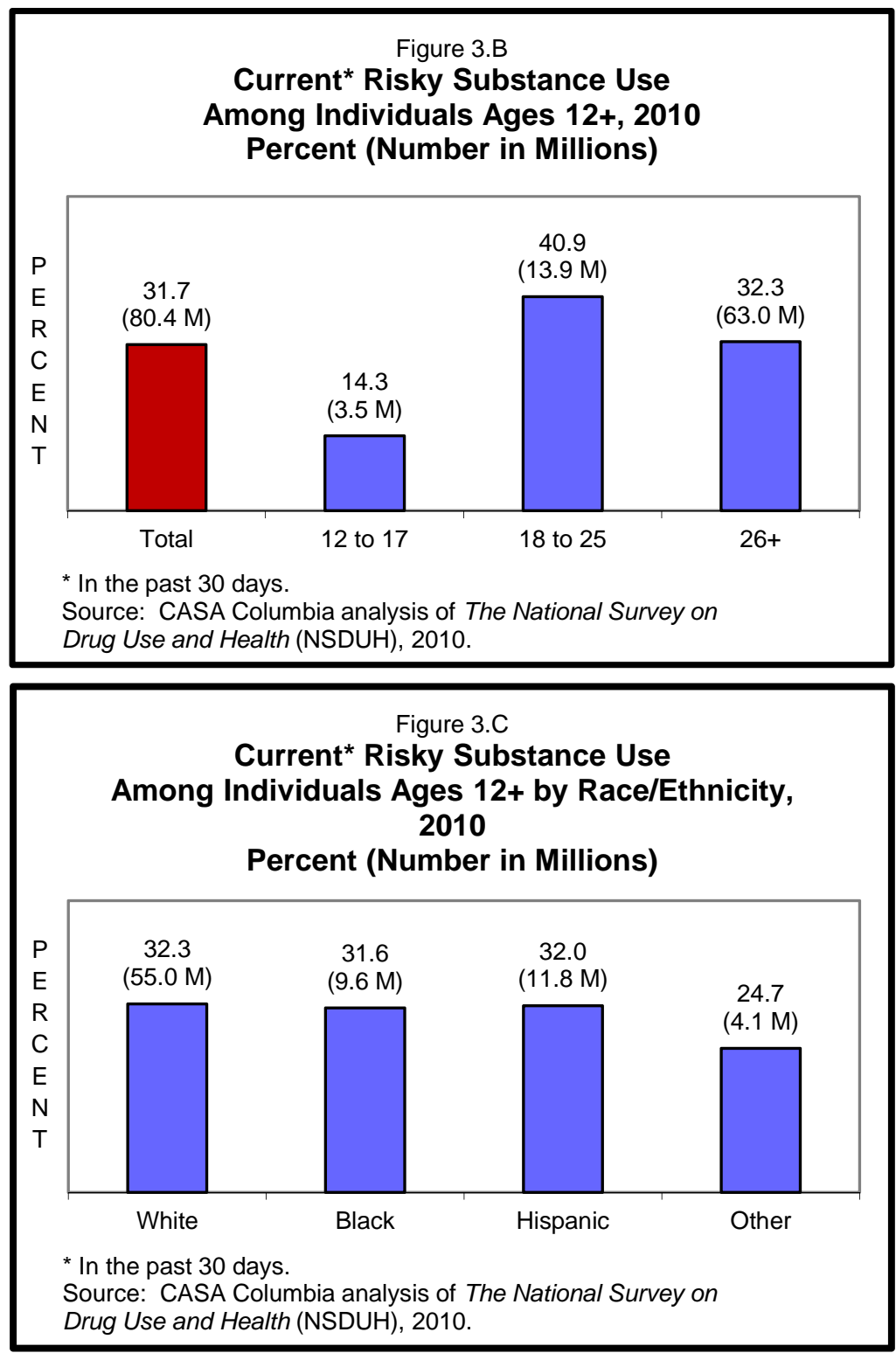

Young adults, ages 18- to 25- years old, are more likely to engage in risky substance use than any other age group. (Figure 3.B) Men are somewhat likelier to be risky substance users than women: 33.9 percent (41.8 million) vs. 29.6 percent (38.6 million). ${ }^{13}$

Whites, Hispanics and blacks are likelier to engage in risky substance use than persons of other races/ethnicities. ${ }^{*} 14$ (Figure 3.C)

\footnotetext{
* "Other" races/ethnicities include American Indian/Alaska Native, Asian, Native Hawaiian/Other Pacific Islander and multiracial non-Hispanic. These races/ethnicities were combined for purposes of analysis because there are too few respondents in each category to calculate meaningful prevalence data for each category separately. The "other races/ethnicities" category is reported as a group despite the fact that substance use prevalence rates
}

The proportion of the population engaging in risky substance use has remained stable over the past decade; in 2002, 31.1 percent (73.1 million) were risky substance users, as were 31.7 percent (80.4 million) in 2010. ${ }^{15}$

vary between each racial/ethnic group in this category. 


\section{Risky Tobacco Use}

In 2010, 18.5 percent of the U.S. population ages 12 and older (47.0 million) reported current risky use ${ }^{*}$ of a tobacco product:

- $\quad$ Cigarettes: 14.0 percent (35.5 million);

- $\quad$ Cigars: 4.2 percent (10.6 million);

- Smokeless tobacco products: 3.0 percent (7.7 million); and

- $\quad$ Pipe tobacco: 0.7 percent (1.7 million). ${ }^{\dagger} 16$

The majority (59.1 percent) of risky tobacco users ${ }^{\ddagger}$ also are risky users of alcohol or other drugs. While not having addiction involving nicotine, 18.2 percent of risky tobacco users have addiction involving alcohol and/or other drugs. ${ }^{17}$

Among all age groups, 18- to 25- year olds have the highest rate of risky tobacco use. ${ }^{18}$ (Figure 3.D)

Men are nearly twice as likely as women to be risky tobacco users (24.1 percent, 29.7 million vs. 13.3 percent, 17.3 million). ${ }^{19}$
Risky tobacco use is slightly higher among blacks than among whites and Hispanics; risky use in these groups is higher than among persons of other races/ethnicities. ${ }^{20}$ (Figure 3.E)
Figure 3.D

Current* Risky Tobacco Use

Among Individuals Ages 12+, 2010

Percent (Number in Millions)
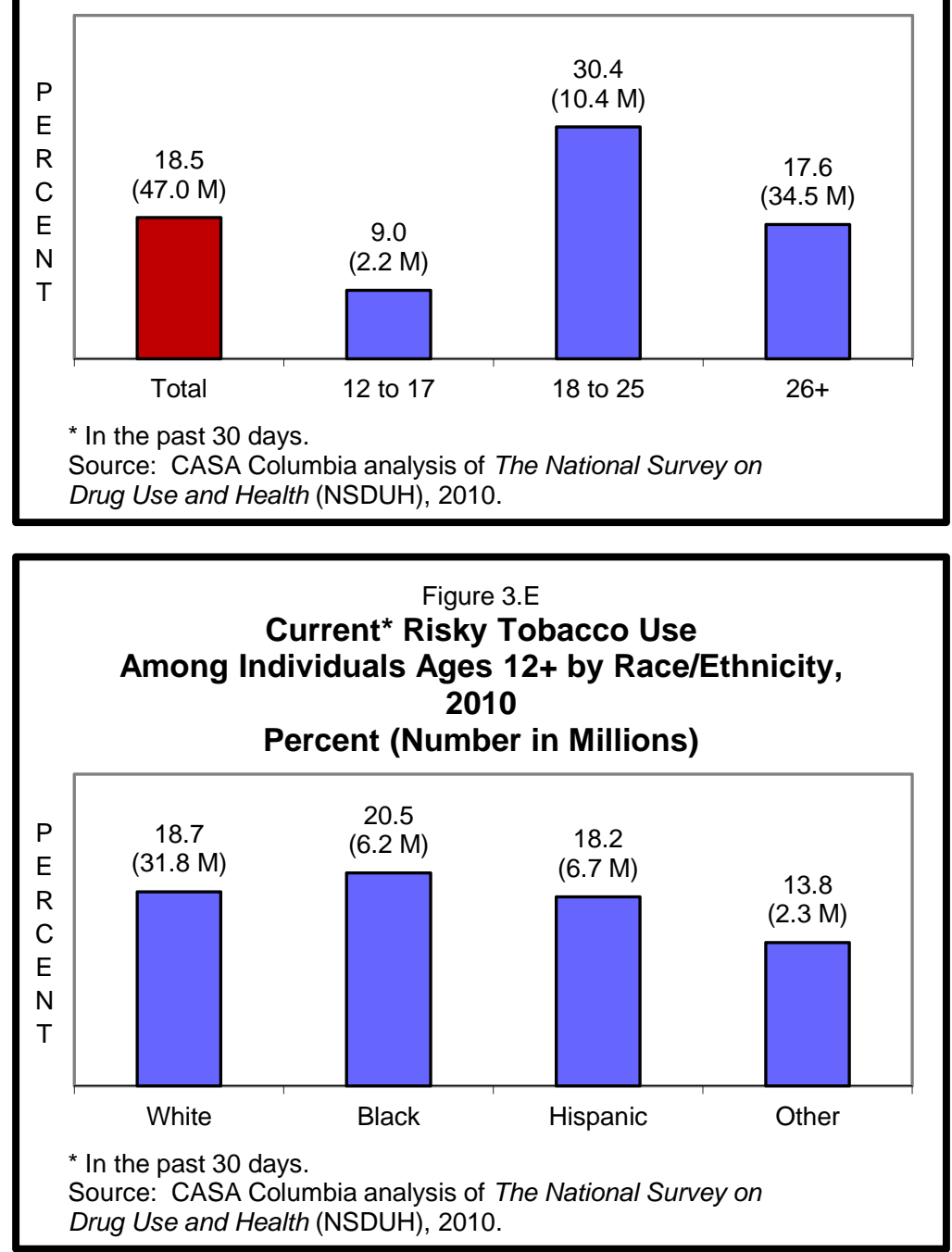

\footnotetext{
${ }^{*}$ Used a tobacco product in the past 30 days but do not meet criteria for addiction involving nicotine (i.e., nicotine dependence, as defined by the NDSS).

${ }^{\dagger}$ These percentages represent the proportion of the U.S. population ages 12 and older reporting risky use of each type of tobacco product. These percentages do not add up to the total of 18.5 percent because of multiple substance use. Nationally representative data on the use of water/ hookah pipes to smoke tobacco are not available.

${ }^{\ddagger}$ Who do not have addiction involving nicotine.
} 
Between 2002 and 2010, risky tobacco use in the U.S. population ages 12 and older decreased slightly, from 19.9 percent (46.8 million) in 2002 to 18.5 percent (47.0 million) in 2010. Among adolescents (ages 12 to 17), the decrease in risky tobacco use was more pronounced, from 12.0 percent in 2002 to 9.0 percent in $2010 .^{21}$

\section{Risky Alcohol Use}

In 2010, 27.2 percent of the U.S. population ages 12 and older (69.0 million) were current risky drinkers: ${ }^{22}$

- 24.2 percent were age 21 and older who, on average, exceeded the USDA guidelines for safe alcohol use;

- $\quad 2.9$ percent were under age 21 and drank alcohol; and

- 0.1 percent were adult pregnant women who drank alcohol. ${ }^{\dagger 23}$

Within this population of risky drinkers:

- $\quad 69.6$ percent $^{\ddagger}$ were heavy drinkers-consuming more than three drinks on any day or more than seven drinks in any week for women, and more than four drinks on

\footnotetext{
* Based on the USDA Guidelines for safe alcohol use described on page 40. Excluded from the category of risky drinkers are those who meet diagnostic criteria for addiction involving alcohol in the past year.

Because the analyses were restricted to the data available in the NSDUH, the component of risky alcohol use that reflects the USDA guidelines of no more than one drink a day for women and two for men was measured by the item: "On the days that you drank during the past 30 days, how many drinks did you usually have?” As such, it represents the average amount respondents drink on days that they drink. If a woman drinks, on average, more than one drink a day on days that she drinks or if a man drinks, on average, more than two drinks a day on days that he drinks, she or he would be considered a risky drinker in this analysis.

${ }^{\dagger}$ A portion of this category is included in the adult risky drinker category above.

₹ 18.9 percent of the population (ages 12 and older), 48.0 million people.
}

any day or more than 14 drinks in any week for men; ${ }^{\S}$

- 65.1 percent $^{* *}$ were current binge drinkers-consuming five or more drinks on the same occasion on at least one day in the past 30 days; and

- 14.7 percent $^{\dagger \dagger}$ were current heavy binge drinkers--binge drinking on at least five days in the past 30 days.

More than half (52.6 percent) of all current drinkers are risky drinkers. Nearly half (46.4 percent) of risky drinkers ${ }^{\S \S}$ also are risky users of tobacco or other drugs. While not having addiction involving alcohol, 14.2 percent of risky drinkers have addiction involving nicotine and/or other drugs. ${ }^{25}$

As with tobacco, 18- to 25-year olds have the highest rates of risky alcohol use. ${ }^{26}$ One in 10 adolescents ages 12 to 17 are risky drinkers. ${ }^{* * *} 27$ (Figure 3.F)

\footnotetext{
${ }^{\S}$ Due to limitations of the NSDUH data set, weekly consumption was computed as an average of monthly drinking divided by 4.33 , or 52 weeks / 12 months. ${ }^{* *} 17.7$ percent of the population, 44.9 million people. ${ }^{+\dagger} 4.0$ percent of the population, 10.1 million people.

㧊 Rates of heavy, binge, and heavy binge drinkers include only risky alcohol users who do not have addiction involving alcohol.

$\S \S$ Who do not have addiction involving alcohol.

${ }^{* * *}$ For individuals under age 21 , any alcohol use (in the past 30 days) that does not meet criteria for addiction is considered risky drinking.
} 


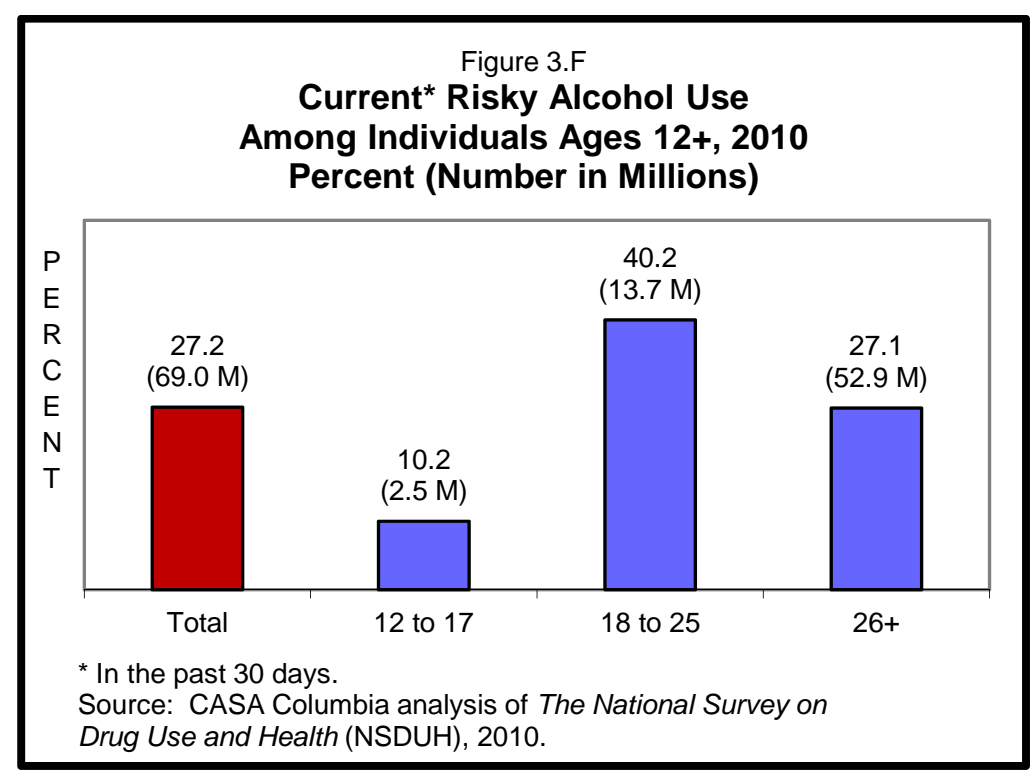

Those ages 18 to 25 engage in binge and heavy binge drinking at significantly higher rates than those ages 26 and older. ${ }^{* 28}$ (Table 3.2)

More men than women engage in risky drinking (28.3 percent, 34.9 million vs. 26.2 percent, 34.1 million). This difference becomes more pronounced at higher levels of drinking: men are almost twice as likely as women to be heavy drinkers (23.8 percent vs. 14.3 percent) and binge drinkers (23.6 percent vs. 12.1 percent), and three times as likely to be heavy binge drinkers (6.2 percent vs. 1.9 percent). ${ }^{29}$

Overall, whites are more likely to engage in risky drinking compared to persons of other races/ethnicities; however, Hispanics are slightly

\footnotetext{
* Other national data indicate that while the prevalence and intensity of binge drinking is highest among individuals ages 18 to 34, adults ages 65 and older who binge drink do so more frequently than any other age group. Data regarding involvement in each type of substance in this table and in subsequent tables demonstrating demographic differences are presented as any involvement with the substance (with or without involvement with other substances) because presenting risky use for each substance on its own (without other substance involvement) results in cell sizes that are too small to present reliable data. As such, data on specific substances and multiple substances shown in the tables are not mutually exclusive.
}

more likely to engage in heavy and binge drinking. $^{30}$ (Table 3.3)

$$
\text { Table } 3.2
$$

Prevalence of Current Heavy, Binge and Heavy Binge Drinking," by Age, 2010 Percent (Number in Millions)

\begin{tabular}{l|c|c|c}
\hline & $\begin{array}{c}\text { Heavy } \\
\text { Drinking }\end{array}$ & $\begin{array}{c}\text { Binge } \\
\text { Drinking }\end{array}$ & $\begin{array}{c}\text { Heavy } \\
\text { Binge } \\
\text { Drinking }\end{array}$ \\
\hline $\begin{array}{l}\text { Total Heavy, Binge, } \\
\text { and Heavy Binge } \\
\text { Drinking, Ages 12+ }\end{array}$ & $\begin{array}{c}\mathbf{1 8 . 9} \\
\mathbf{( 4 8 . 0} \mathbf{~ M )}\end{array}$ & $\begin{array}{c}\mathbf{1 7 . 7} \\
\mathbf{( 4 4 . 9 M )}\end{array}$ & $\begin{array}{c}\mathbf{4 . 0} \\
\mathbf{( 1 0 . 1 M )}\end{array}$ \\
\hline Age: & & & \\
\hline 12- to 17-years old & 5.2 & 5.0 & 0.8 \\
\hline 18 - to 25-years old & 28.8 & 28.0 & 7.3 \\
\hline 26+ years old & 18.9 & 17.5 & 3.8 \\
\hline${ }^{*}$ Were risky users of alcohol in the past 30 days but do not \\
meet diagnostic criteria for addiction involving alcohol. \\
$\begin{array}{l}\text { Source: CASA Columbia analysis of The National Survey on } \\
\text { Drug Use and Health (NSDUH), 2010. }\end{array}$ \\
\hline
\end{tabular}

Table 3.3

Prevalence of Current Risky, Heavy, Binge and Heavy Binge Drinking," by Race/Ethnicity, 2010 Percent (Number in Millions)

\begin{tabular}{l|c|l|l|l}
\hline & White & Black & Hispanic & Other \\
\hline Total Risky & $\begin{array}{c}\mathbf{2 8 . 5} \\
\text { Alcohol Use }\end{array}$ & $\begin{array}{l}\mathbf{2 4 . 9} \\
\mathbf{( 4 8 . 4 M )}\end{array}$ & $\begin{array}{l}\mathbf{2 7 . 0} \\
\mathbf{( 9 . 9 M} \mathbf{M})\end{array}$ & $\begin{array}{l}\mathbf{1 9 . 2} \\
\mathbf{( 3 . 2 M )}\end{array}$ \\
\hline Heavy Drinking & 19.7 & 16.0 & 20.7 & 12.6 \\
\hline Binge Drinking & 18.1 & 15.6 & 20.1 & 11.9 \\
\hline Heavy Binge & & & & \\
Drinking & 4.7 & 2.2 & 2.9 & 1.9 \\
\hline \\
"Were risky users of alcohol in the past 30 days but do not \\
meet diagnostic criteria for addiction involving alcohol. \\
Source: CASA Columbia analysis of The National Survey on \\
Drug Use and Health (NSDUH), 2010.
\end{tabular}

Between 2002 and 2010, risky alcohol use in the U.S. population ages 12 and older increased slightly, from 26.4 percent (62.0 million) in 2002 to 27.2 percent (69.0 million) in $2010 .^{31}$ This increase was consistent across all forms of risky drinking:

- Heavy drinking, from 18.2 percent to 18.9 percent; 
- $\quad$ Binge drinking, from 16.9 percent to 17.7 percent; and

- Heavy binge drinking from 3.8 percent to 4.0 percent. $^{32}$

\section{Risky Illicit Drug Use}

In 2010, 5.8 percent of the U.S. population ages 12 and older (14.6 million) reported current risky use of illicit drugs, ${ }^{*}$ primarily marijuana: ${ }^{33}$

- Marijuana/hashish: 5.6 percent (14.2 million);

- $\quad$ Cocaine/crack: 0.3 percent (0.8 million);

- Hallucinogens: 0.3 percent (0.8 million);

- Ecstasy: 0.2 percent (0.4 million); and

- Inhalants: 0.2 percent (0.5 million)..$^{\dagger 34}$

Nearly all risky illicit drug users (81.6 percent) also are risky users of tobacco, alcohol or controlled prescription drugs. While not having addiction involving illicit drugs, 40.3 percent has addiction involving nicotine, alcohol and/or controlled prescription drugs. ${ }^{35}$

Risky use of illicit drugs is highest among 18- to 25-year olds; adolescents ages 12 to 17 are more likely to be risky users of illicit drugs than adults ages 26 and older. (Figure 3.G) Risky marijuana use follows the same pattern, with 13.6 percent (4.7 million) of 18 - to 25 -year olds, 4.9 percent (1.2 million) of 12 to 17 -year-olds and 4.0 percent of those ages 26 and older (7.9 million) engaging in risky use of marijuana. ${ }^{\ddagger} 36$

\footnotetext{
${ }^{*}$ Used an illicit drug in the past 30 days but do not have addiction involving illicit drugs. Data on the risky use of methamphetamine and heroin cannot be reported separately due to small sample size.

${ }^{\dagger}$ These percentages do not add up to the total of 5.8 percent because of multiple substance use.

${ }^{*}$ Data on the risky use of other types of illicit drugs cannot be reported separately by age, gender or race/ethnicity due to small sample sizes.
}

Men are nearly twice as likely as women to be risky users of illicit drugs (7.5 percent, 9.3 million vs. 4.1 percent, 5.3 million). Men also are nearly twice as likely as women to be risky marijuana users (7.1 percent, 8.8 million vs. 3.8 percent, 4.9 million). ${ }^{37}$

Blacks and whites are more likely to engage in risky use of illicit drugs than Hispanics or persons of other races/ethnicities. ${ }^{38}$ (Figure 3.H)
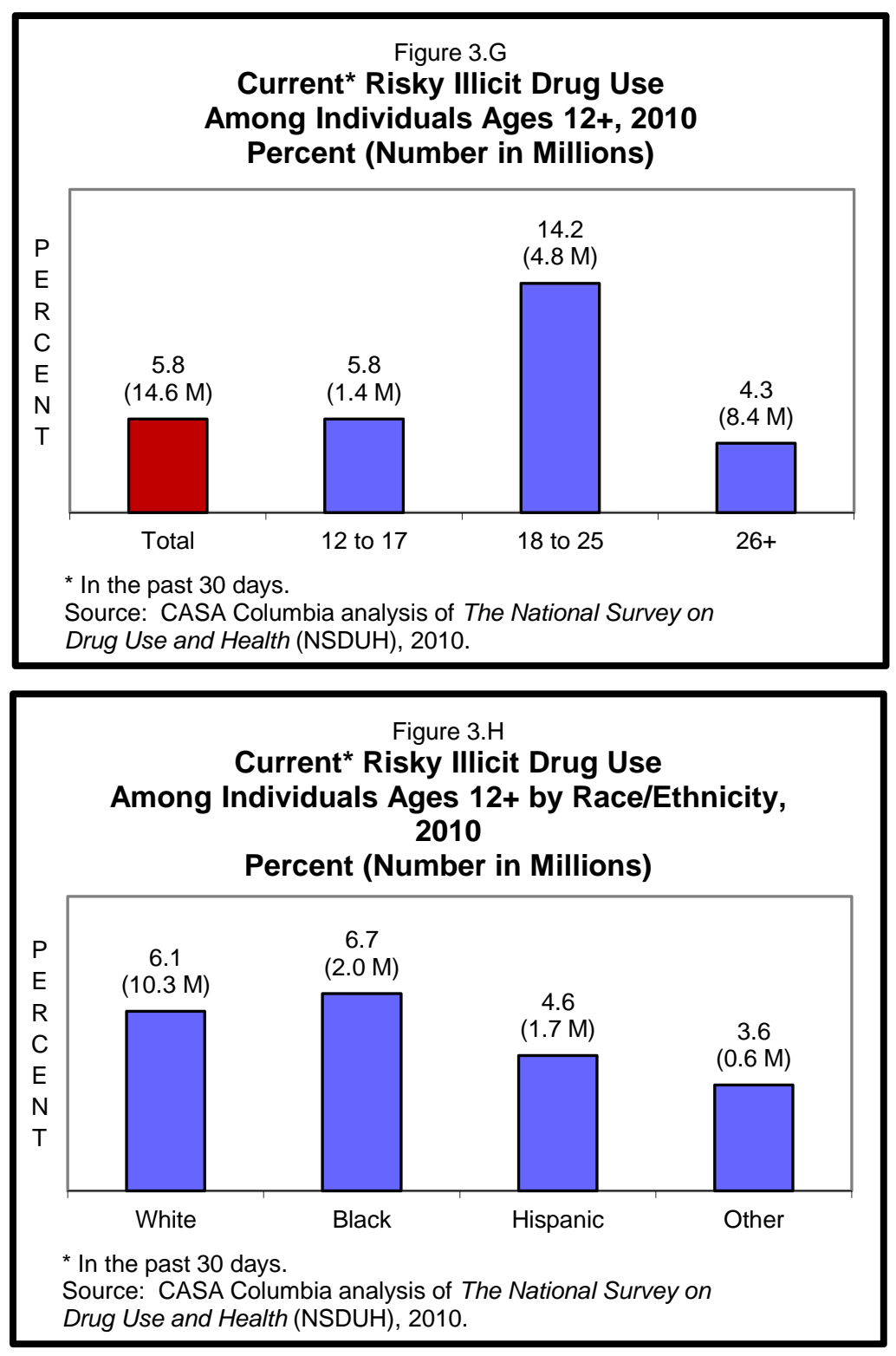
With regard to the risky use of marijuana specifically, blacks are more likely to be risky users (6.3 percent, 1.9 million), than whites (5.7 percent, 9.8 million), Hispanics (4.1 percent, 1.5 million) or persons of other races/ethnicities (3.3 percent, 0.6 million). ${ }^{39}$

The rate of risky use of illicit drugs has increased slightly between 2002 (5.0 percent, 11.9 million) and 2010 (5.8 percent, 14.6 million) driven primarily by an increase in the rate of risky use of marijuana (4.6 percent, 10.7 million in 2002 and 5.6 percent, 14.2 million in 2010). ${ }^{40}$

\section{Risky Use of Controlled Prescription Drugs}

In 2010, 2.1 percent of the U.S. population ages 12 and older (5.4 million) reported risky use of controlled $^{\dagger}$ prescription drugs, with opioids (pain relievers) the most frequently misused: ${ }^{41}$

- $\quad$ Opioids: 1.6 percent (3.9 million);

- Tranquilizers: 0.6 percent (1.5 million); and

- $\quad$ Stimulants: 0.3 percent (0.7 million). ${ }^{\ddagger 42}$

Of those who are risky users ${ }^{\S}$ of controlled prescription drugs, 65.8 percent (3.6 million) also are risky users of tobacco, alcohol or illicit drugs. While not having addiction involving controlled prescription drugs, 43.6 percent of risky users of these drugs have addiction involving nicotine, alcohol and/or an illicit drug. ${ }^{43}$

\footnotetext{
* Misused a controlled prescription drug in the past 30 days but do not have addiction involving prescription drugs.

${ }^{\dagger}$ Controlled by the U.S. Drug Enforcement Administration because of their potential for misuse.

${ }^{\ddagger}$ Data on the risky use of sedatives cannot be reported separately due to small sample size. These percentages do not add up to the total of 2.1 percent because of multiple substance use.

${ }^{\S}$ Who do not have addiction involving prescription drugs.
}

Risky use of controlled prescription drugs is highest among 18- to 25-year olds; more 12- to 17 -year olds report risky use of these drugs than those ages 26 and older. ${ }^{44}$ (Figure 3.I)



This pattern is the same for the risky use of opioids specifically: 2.0 percent of 12 - to 17 year olds, 3.2 percent of 18- to 25-year olds and 1.2 percent of individuals ages 26 and older are risky users of opioids. ${ }^{* * 45}$

Men are slightly likelier to be risky users of controlled prescription drugs (2.3 percent, 2.8 million) than women (2.0 percent, 2.6 million). With regard to opioids, men also are more likely to be risky users (1.8 percent, 2.2 million) than women (1.3 percent, 1.7 million). ${ }^{46}$

\footnotetext{
** Data on the risky use of other classes of controlled prescription drugs cannot be reported separately by age, gender or race/ethnicity due to small sample size.
} 
Whites are more likely to be risky users of controlled prescription drugs than persons of other races/ethnicities. ${ }^{47}$ (Figure 3.J)

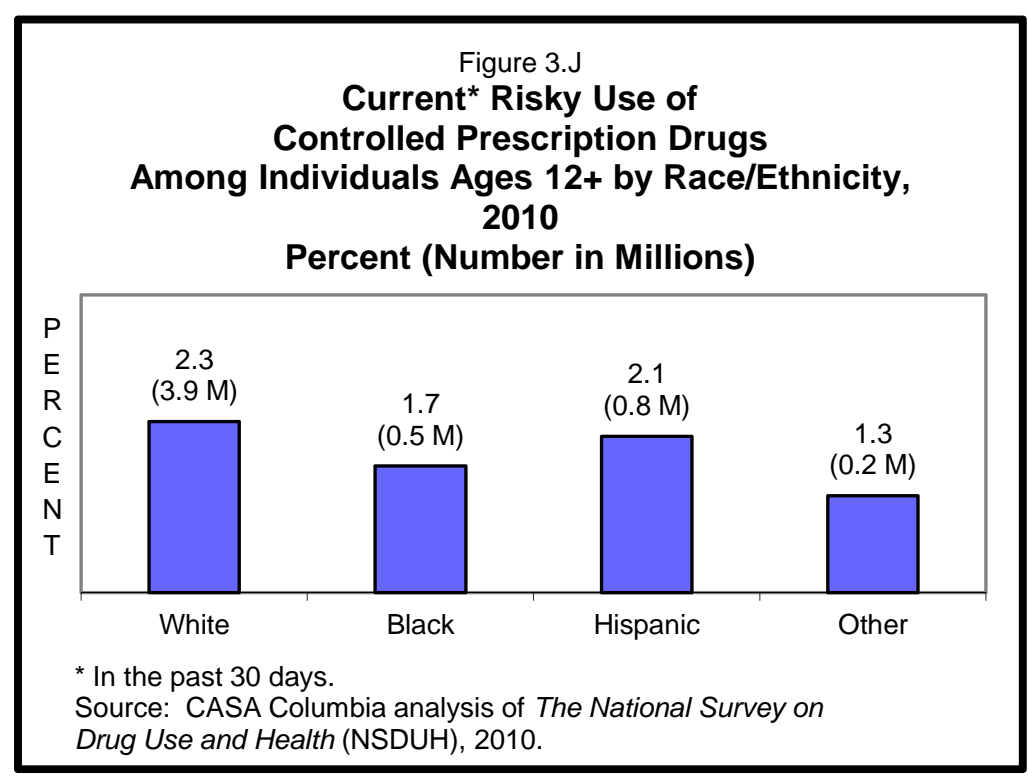

However, with regard to the specific case of opioids, Hispanics are slightly likelier than whites (1.7 percent, 0.6 million vs. 1.6 percent, 2.8 million$)^{*}$ to misuse opioids. ${ }^{48}$

The rate of risky use of controlled prescription drugs has remained relatively stable between 2002 (2.2 percent, 5.2 million) and 2010 (2.1 percent, 5.4 million); the rate of risky use of opioids also has remained stable between 2002 (1.5 percent, 3.6 million) and 2010 (1.6 percent, 3.9 million) ${ }^{49}$

\footnotetext{
${ }^{*}$ Data on risky opioid use among blacks (1.3 percent) and persons of other races/ethnicities (1.0 percent) cannot be reported as statistically significant due to small sample sizes.
}

\section{Addiction $^{\dagger}$}

In 2010, a total of 15.9 percent of the U.S. population ages 12 and older (40.3 million people) met clinical diagnostic criteria for addiction. ${ }^{\ddagger 50}$

Addiction involving nicotine and alcohol are the most prevalent manifestations of addiction, followed by addiction involving illicit drugs and controlled prescription drugs. ${ }^{51}$ (Table 3.4)

\footnotetext{
† The term "addiction" is used synonymously in this report with the NDSS criteria for past 30 day nicotine dependence, and the DSM-IV clinical diagnostic criteria for past year alcohol and/or other drug abuse and dependence (excluding nicotine) in accordance with the Diagnostic and Statistical Manual of Mental Disorders (DSM-IV). (The DSM refers to substance abuse and substance dependence collectively as substance use disorders. The diagnostic criteria for nicotine dependence in the NDSS parallel those of the DSM-IV). These are the criteria used by the NSDUH to classify respondents as having a substance use disorder. This definition is consistent with the current move to combine abuse and dependence into an overarching diagnosis of addiction in the upcoming DSM-V. Available data allow us to include in our prevalence estimates only those who meet behavioral criteria in accordance with the current diagnostic standards, meaning that their disease is not effectively managed or has not reached the point of behavioral symptoms. Individuals who have the disease of addiction but do not meet diagnostic criteria for past month (nicotine) or past year (alcohol or other drug) addiction are not included.

${ }^{\ddagger}$ This estimate excludes the institutionalized population, for which rates of addiction are higher.
} 


\begin{tabular}{|c|c|c|}
\hline \multicolumn{3}{|c|}{$\begin{array}{c}\text { Table } 3.4 \\
\text { Prevalence of Addiction in } \\
\text { U.S. Population, Ages 12+, } \mathbf{2 0 1 0}\end{array}$} \\
\hline & $\begin{array}{l}\text { Percent of } \\
\text { Total } \\
\text { Population }\end{array}$ & $\begin{array}{l}\text { Number } \\
\text { (in } \\
\text { Millions) }\end{array}$ \\
\hline Total Addiction $^{*}$ & 15.9 & 40.3 \\
\hline $\begin{array}{l}\text { Nicotine With/Without } \\
\text { Other Substances }\end{array}$ & 9.0 & 22.9 \\
\hline Nicotine Only & 7.1 & 18.0 \\
\hline $\begin{array}{l}\text { Alcohol With/Without } \\
\text { Other Substances }\end{array}$ & 7.1 & 18.0 \\
\hline Alcohol Only & 5.0 & 12.6 \\
\hline $\begin{array}{l}\text { Illicit Drugs } \\
\text { With/Without Other } \\
\text { Substances }\end{array}$ & 2.2 & 5.6 \\
\hline Illicit Drugs Only & 0.8 & 2.1 \\
\hline $\begin{array}{l}\text { Controlled Prescription } \\
\text { Drugs With/Without } \\
\text { Other Substances }\end{array}$ & 0.9 & 2.4 \\
\hline $\begin{array}{l}\text { Controlled Prescription } \\
\text { Drugs Only }\end{array}$ & 0.2 & 0.6 \\
\hline Multiple Substances & 2.7 & 7.0 \\
\hline
\end{tabular}

* Based on past 30-day nicotine dependence, as defined in the NDSS, and past-year alcohol and/or other drug abuse or dependence, as defined in the DSM-IV.

Source: CASA Columbia analysis of The National Survey on Drug Use and Health (NSDUH), 2010.

Among those with addiction, 55.7 percent are risky users of one or more other substances and 17.3 percent have addiction involving multiple substances, ${ }^{*}$ including:

- 21.3 percent of those with addiction involving nicotine;

- $\quad 30.1$ percent of those with addiction involving alcohol;

- $\quad 75.2$ percent of those with addiction involving controlled prescription drugs; and

- 62.1 percent of those with addiction involving illicit drugs. ${ }^{52}$

\footnotetext{
* Met diagnostic criteria for two or more of the following: past 30-day nicotine dependence and/or past year abuse/dependence involving alcohol, illicit drugs and/or controlled prescription drugs.
}

Those ages 18 to 25 have the highest rate of addiction, including the highest rates involving each type of substance, than any other age group. $^{53}$ (Figure 3.K; Table $3.5^{\dagger}$ )

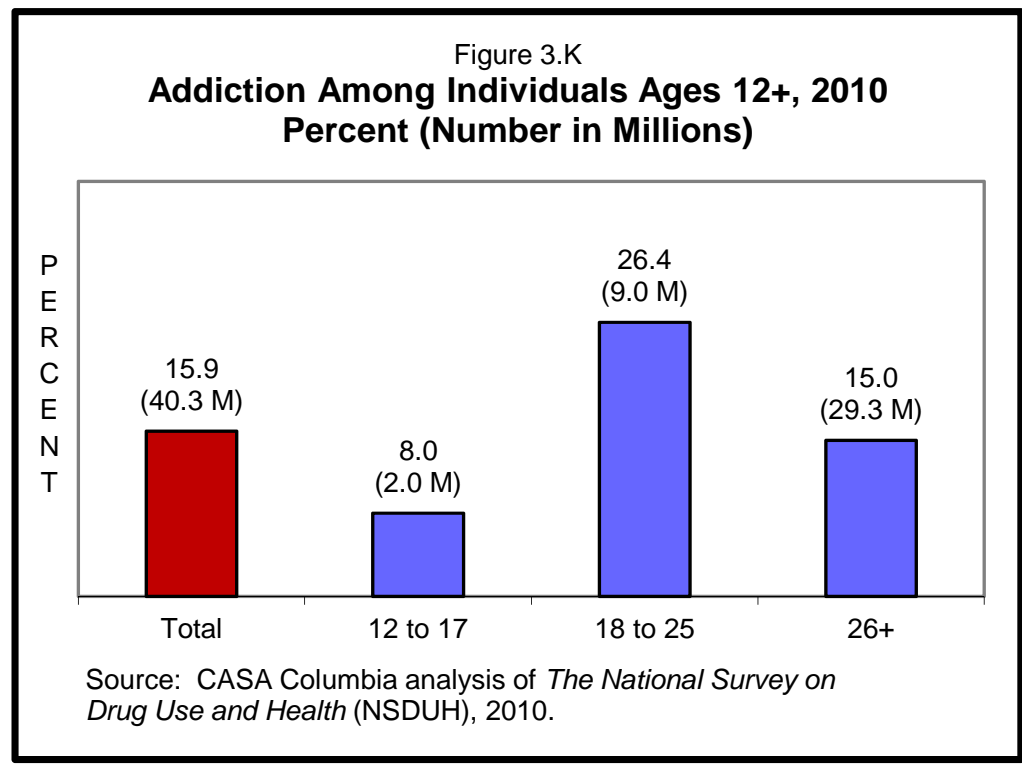

\footnotetext{
${ }^{\dagger}$ Data regarding addiction involving each type of substance in this table and in subsequent tables demonstrating demographic differences are presented as addiction involving the substance, with or without addiction involving other substances, because presenting addiction related to each substance on its own (without other substance addiction) results in cell sizes that are too small to present reliable data. As such, data on specific substances and multiple substances shown in the tables are not mutually exclusive.
} 
Table 3.5

Prevalence of Addiction, by Age Group, 2010 Percent (Number in Millions)

\begin{tabular}{|c|c|c|c|}
\hline & $\begin{array}{l}12 \text { - to } \\
17 \text { - years } \\
\text { old }\end{array}$ & $\begin{array}{l}\text { 18- to } \\
\text { 25- years } \\
\text { old }\end{array}$ & $\begin{array}{c}26+ \\
\text { years } \\
\text { old }\end{array}$ \\
\hline $\begin{array}{l}\text { Total Addiction, } \\
\text { Ages 12+ }\end{array}$ & $\begin{array}{c}8.0 \\
(2.0 \mathrm{M})\end{array}$ & $\begin{array}{l}26.4 \\
(9.0 \mathrm{M})\end{array}$ & $\begin{array}{c}15.0 \\
(29.3 \mathrm{M})\end{array}$ \\
\hline $\begin{array}{l}\text { Nicotine With/Without } \\
\text { Other Substance } \\
\text { Addiction }\end{array}$ & 1.8 & 10.4 & 9.7 \\
\hline $\begin{array}{l}\text { Alcohol With/Without } \\
\text { Other Substance } \\
\text { Addiction }\end{array}$ & 4.6 & 15.8 & 5.9 \\
\hline $\begin{array}{l}\text { Illicit Drugs } \\
\text { With/Without Other } \\
\text { Substance Addiction }\end{array}$ & 4.1 & 6.5 & 1.2 \\
\hline $\begin{array}{l}\text { Controlled Prescription } \\
\text { Drugs With/Without } \\
\text { Other Substance } \\
\text { Addiction }^{* *}\end{array}$ & $(1.2)$ & $(2.2)$ & $(0.7)$ \\
\hline Multiple Substances & 2.7 & 6.7 & 2.1 \\
\hline \multicolumn{4}{|c|}{$\begin{array}{l}{ }^{*} \text { Based on past 30-day nicotine dependence, as defined in } \\
\text { the NDSS, and past-year alcohol and/or other drug abuse } \\
\text { or dependence, as defined in the DSM-IV. } \\
\text { ** The rate of addiction involving controlled prescription } \\
\text { drugs is too low to assure statistical reliability of data by } \\
\text { age. } \\
\text { Source: CASA Columbia analysis of The National Survey } \\
\text { on Drug Use and Health (NSDUH), } 2010 \text {. }\end{array}$} \\
\hline
\end{tabular}

It is important to note that 8.0 percent of adolescents ages 12 to 17 have addiction. ${ }^{54}$ The percent of adults who meet clinical criteria for addiction declines with age. ${ }^{*}$ The explanation for this is not well understood and reliable national data are not available on the proportion of those with addiction for whom the disease is chronic. It may be that some young people receive treatment or otherwise successfully manage the disease to the point where they no longer meet diagnostic criteria for addiction as they get older, while for some other young people the disease may be fatal.

* 23.7 percent (8.6 million) of adults ages 26-34; 16.8 percent (10.5 million) of adults ages $35-49$; 13.8 percent ( 7.9 million) of adults ages $50-64$; and 5.8 percent ( 2.3 million) of adults ages 65 and older.
Men are more likely to have addiction than women (19.1 percent, 23.6 million vs. 12.8 percent, 16.6 million). While rates of addiction involving nicotine and controlled prescription drugs are similar for both genders, rates of addiction involving all other substances are twice as high among men as women. ${ }^{55}$

(Table 3.6)

\section{Table 3.6 \\ Prevalence of Addiction, ${ }^{*}$ by Gender, 2010 Percent (Number in Millions)}

\begin{tabular}{|c|c|c|}
\hline & Males & Females \\
\hline $\begin{array}{l}\text { Total Addiction, } \\
\text { Ages 12+ }\end{array}$ & $\begin{array}{c}19.1 \\
(23.6 \mathrm{M})\end{array}$ & $\begin{array}{c}12.8 \\
(16.6 \mathrm{M})\end{array}$ \\
\hline $\begin{array}{l}\text { Nicotine With/Without Other } \\
\text { Substance Addiction }\end{array}$ & 9.9 & 8.2 \\
\hline $\begin{array}{l}\text { Alcohol With/Without Other } \\
\text { Substance Addiction }\end{array}$ & 9.5 & 4.8 \\
\hline $\begin{array}{l}\text { Illicit Drugs With/Without } \\
\text { Other Substance Addiction }\end{array}$ & 2.9 & 1.6 \\
\hline $\begin{array}{l}\text { Controlled Prescription Drugs } \\
\text { With/Without Other } \\
\text { Substance Addiction }^{* *}\end{array}$ & (1.1) & $(0.8)$ \\
\hline Multiple Substances & 3.5 & 2.0 \\
\hline \multicolumn{3}{|c|}{$\begin{array}{l}{ }^{*} \text { Based on past 30-day nicotine dependence, as } \\
\text { defined in the NDSS, and past-year alcohol and/or } \\
\text { other drug abuse or dependence, as defined in the } \\
\text { DSM-IV. } \\
\text { ** The rate of addiction involving controlled } \\
\text { prescription drugs is too low to assure statistical } \\
\text { reliability of data by gender. } \\
\text { Source: CASA Columbia analysis of The National } \\
\text { Survey on Drug Use and Health (NSDUH), } 2010 .\end{array}$} \\
\hline
\end{tabular}


Whites are more likely to have addiction than individuals of other races/ethnicities. Rates of addiction involving nicotine are highest among whites, while rates of addiction involving alcohol are highest among whites and Hispanics. Rates of addiction involving illicit drugs are highest among blacks. ${ }^{56}$ (Table 3.7)

Between 2002 and 2010, the rate of addiction declined from 17.6 percent (41.4 million) to 15.9 percent ( 40.3 million), driven primarily by the decline in rates of addiction involving nicotine (10.5 percent in 2002 to 9.0 percent in 2010). ${ }^{57}$

\section{Special Populations}

Certain populations--such as pregnant women, the young and the elderly--are more vulnerable to the damaging and addictive effects of tobacco, alcohol and other drugs. Among individuals with co-occurring disorders, members of the military exposed to combat and persons involved in the justice system, the likelihood of risky use and addiction is significantly higher than in the general population.

\section{Pregnant Women}

In 2010, 16.2 percent of pregnant women were risky users of tobacco, alcohol or other drugs* and 14.7 percent of pregnant women met clinical criteria for addiction. ${ }^{\dagger}$ Although pregnant women are less likely to engage in risky substance use or have addiction than nonpregnant women, ${ }^{58}$ any substance use by pregnant women is concerning because of the risk of pregnancy complications, adverse health consequences for the fetus and health and behavioral consequences for children prenatally exposed to addictive substances. ${ }^{59}$

\section{Adolescents and Young Adults}

Use of tobacco, alcohol and other drugs while the brain is still developing increases the risk of addiction. Because the brain continues to

\footnotetext{
* Compared to 34.2 percent of non-pregnant women.

${ }^{\dagger}$ Compared to 16.2 percent of non-pregnant women.
}

\section{Table 3.7}

\section{Prevalence of Addiction," by Race/Ethnicity, 2010 Percent (Number in Millions)}

\begin{tabular}{|c|c|c|c|c|}
\hline & White & Black & Hispanic & Other \\
\hline $\begin{array}{l}\text { Total Addiction, } \\
\text { Ages 12+ }\end{array}$ & $\begin{array}{c}17.6 \\
(29.9 \mathrm{M})\end{array}$ & $\begin{array}{c}13.4 \\
(4.0 \mathrm{M})\end{array}$ & $\begin{array}{l}12.9 \\
(4.7 \mathrm{M})\end{array}$ & $\begin{array}{c}9.9 \\
(1.6 \mathrm{M})\end{array}$ \\
\hline $\begin{array}{l}\text { Nicotine With/ } \\
\text { Without Other } \\
\text { Substance Addiction }\end{array}$ & 10.9 & 6.9 & 4.2 & 4.9 \\
\hline $\begin{array}{l}\text { Alcohol With/ Without } \\
\text { Other Substance } \\
\text { Addiction }\end{array}$ & 7.4 & 5.8 & 7.6 & 5.0 \\
\hline $\begin{array}{l}\text { Illicit Drugs With/ } \\
\text { Without Other } \\
\text { Substance Addiction }\end{array}$ & 1.9 & 3.5 & 2.9 & 1.6 \\
\hline $\begin{array}{l}\text { Controlled Prescription } \\
\text { Drugs With/Without } \\
\text { Other Substance } \\
\text { Addiction }^{* *}\end{array}$ & (1.0) & $(0.6)$ & (1.0) & $(0.7)$ \\
\hline Multiple Substances & 2.9 & 2.9 & 2.4 & 2.0 \\
\hline \multicolumn{5}{|c|}{$\begin{array}{l}{ }^{*} \text { Based on past 30-day nicotine dependence, as defined in the } \\
\text { NDSS, and past-year alcohol and/or other drug abuse or dependence, } \\
\text { as defined in the DSM-IV. } \\
{ }^{* *} \text { The rate of addiction involving controlled prescription drugs is too } \\
\text { low to assure statistical reliability of data by race/ethnicity. } \\
\text { Source: CASA Columbia analysis of The National Survey on Drug } \\
\text { Use and Health (NSDUH), } 2010 \text {. }\end{array}$} \\
\hline
\end{tabular}

develop into the mid-20s, the use of tobacco, alcohol, controlled prescription drugs and illicit drugs among 12- to 25-year olds is a significant public health concern. ${ }^{60}$ Despite this, risky substance use is high in this age group:

- About half (50.6 percent, 29.6 million) of 12- to 25-year olds have used a tobacco product $^{\ddagger}$ in their lifetime;

- Two-thirds (64.9 percent, 37.9 million) of 12- to 25-year olds have used alcohol in their lifetime;

- Four in 10 (40.6 percent, 23.7 million) 12to 25-year olds have used an illicit drug in their lifetime; and

- $\quad$ Approximately one-fifth (21.0 percent, 12.3 million) of 12- to 25-year olds have misused

\footnotetext{
‡ Includes cigarettes, cigars, pipes and smokeless tobacco.
} 
a controlled prescription drug in their lifetime. ${ }^{61}$

\section{Older Adults}

The body's tolerance to addictive substances declines with age, ${ }^{62}$ while the quantity and frequency of prescription drug use typically increases. ${ }^{63}$ These factors contribute to an increased chance of risky substance use and addiction. Also, as the "Boomer" generation ages, seniors are reporting increasingly higher rates of substance use and addiction, due to the higher rates of substance use in this age cohort compared with prior generations. ${ }^{64}$ Currently, 25.2 percent of the population ages 50 and older engages in risky substance use and 10.6 percent has addiction. ${ }^{*} 65$

\section{Co-occurring Disorders}

Addiction frequently co-occurs with other health conditions. ${ }^{66}$ CASA Columbia's analysis of national data indicates that in the past year, 57.5 percent of non-institutionalized individuals ages 18 and older ${ }^{\dagger}$ with addiction also have another health condition:

- 31.9 percent have been told by a doctor that they have a medical condition ${ }^{\ddagger}$ (not including mental health disorders); and

- $\quad 39.4$ percent meet clinical criteria for a mental health disorder. ${ }^{\S 67}$ (Figure 3.L)

\footnotetext{
* The sample size is too low to provide any further statistically reliable data on older adults ages 65 and older.

${ }^{\dagger}$ Data on mental health disorders among 12- to 17year olds are not available in the NSDUH.

${ }^{\ddagger}$ In the past year. Includes asthma, bronchitis, cirrhosis of the liver, diabetes, heart disease, hepatitis, high blood pressure, HIV/AIDs, lung cancer, pancreatitis, pneumonia, STDs, sinusitis, sleep apnea, stroke, tinnitus, tuberculosis and ulcer. ${ }^{\S}$ Includes those with a current or past year mental, behavioral or emotional disorder (e.g., depression and anxiety; excluding developmental disorders and addiction) that meets DSM-IV criteria, or those with a major depressive episode in the past year.
}

Figure 3.L

Rates of Mental Health Disorders Among Individuals Ages 18+ with Addiction Involving Specific Substances, 2010 Percent (Number in Millions)

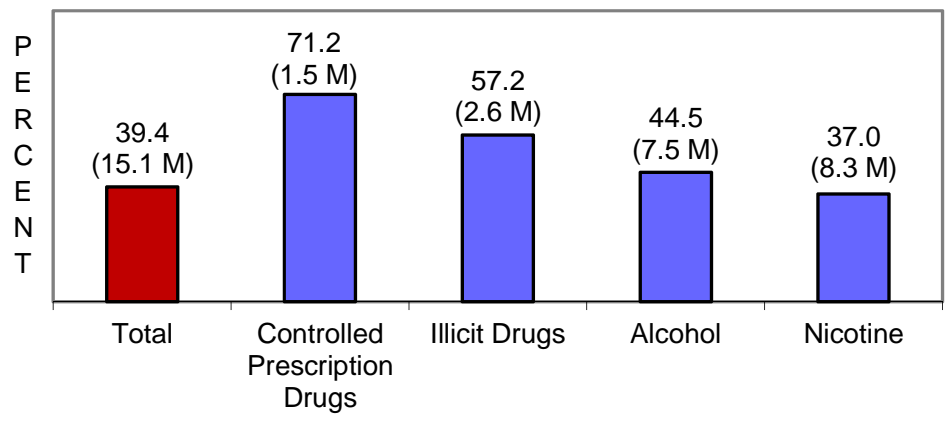

Source: CASA Columbia analysis of The National Survey on Drug Use and Health (NSDUH), 2010.

People with mental health disorders also are more likely to be risky substance users and to have addiction than those without a mental health disorder. Among those ages 18 and older who have a mental health disorder, ${ }^{* *} 30.6$ percent are risky substance users and 31.4 percent have addiction. ${ }^{68}$

In total, 6.6 percent of the non-institutionalized U.S. population ages 18 and older (15.1 million) meet clinical criteria for both addiction and a mental health disorder. ${ }^{\dagger \dagger} 69$ Those with cooccurring addiction and mental health disorders also are likelier to have other co-occurring chronic illnesses such as hypertension, asthma and arthritis. ${ }^{70}$

The rates of co-occurring mental health disorders appear to be even higher among people seeking treatment for addiction. One large-scale study of adolescents and adults in addiction treatment found that two-thirds of the patients had co-occurring mental health disorders in the year prior to treatment admission, with 18- to 25-year olds most likely to have co-occurring

\footnotetext{
** 20.9 percent of adults (18 and older) in the U.S. population meet clinical criteria for a mental health disorder.

†† These rates are higher among institutionalized persons; for example, CASA Columbia's 2010 study, Behind Bars Update: Substance Abuse and America's Prison Population, found that 24.4 percent of prison and jail inmates have both addiction and a cooccurring mental health disorder.
} 
disorders. Attention deficit/hyperactivity disorder and conduct disorder are the most common co-occurring mental health disorders in young patients ${ }^{*}$ being treated for addiction, and anxiety and depression are the most common cooccurring mental health disorders in older patients; trauma-related disorders are common across age groups. ${ }^{71}$

The link between mental illness and smoking is particularly striking: ${ }^{72}$ individuals age 18 and older with a mental illness involving serious functional impairment ${ }^{\dagger}$ are nearly twice as likely as those without such illnesses to have smoked cigarettes in the past year (49.8 percent vs. 27.4 percent). ${ }^{73}$ Up to 60 percent of those with depression, up to 70 percent of those with bipolar disorder and up to 88 percent of those with schizophrenia either are current or former smokers. ${ }^{\ddagger 74}$ Those with clinical anxiety ${ }^{\S}$ are approximately twice as likely to be current smokers (39.2 percent vs. 22.2 percent), have smoked twice as many cigarettes in the past month (139.5 cigarettes vs. 63.4 cigarettes) and are more than twice as likely to have addiction involving nicotine (21.2 percent vs. 8.4 percent) as smokers without anxiety. ${ }^{* *}$ Slightly more than one-quarter of the population (26.9 percent) has addiction, a mental health disorder or a serious mental illness, ${ }^{\dagger \dagger}$ yet this group smokes 72.9 percent of all cigarettes. ${ }^{75}$

\footnotetext{
* Ages 17 and younger.

† National data from 2010. A diagnosable mental, behavioral or emotional disorder (excluding developmental disorders and addiction involving alcohol or drugs other than nicotine) of sufficient duration to meet diagnostic criteria specified in the DSM-IV that has resulted in serious functional impairment, substantially interfering with or limiting one or more major life activities. Comparable data are not available for 12- to 17-year olds.

$\ddagger$ These data are from a review of studies of clinical and population-based samples.

$\S$ Ages 12 and older.

** Among smokers, those with anxiety smoked an average of 355.5 cigarettes in the past month compared to 286.2 cigarettes among smokers without anxiety.

${ }^{\dagger \dagger}$ A mental, behavioral or emotional disorder that has resulted in serious functional impairment which substantially interferes with or limits one or more major life activities.
}

\section{Members of the Military Exposed to Combat}

Exposure to combat increases the risk of addiction and co-occurring mental health problems such as post-traumatic stress disorder (PTSD), anxiety and depression. Addiction involving alcohol is one of the most commonlyreported disorders among Vietnam War veterans, ${ }^{76}$ and co-occurring addiction and mental health disorders are most prevalent among veterans of the Vietnam era. ${ }^{77}$ Military personnel and veterans of the more recent conflicts in Afghanistan and Iraq also are at increased risk of risky use and addiction as well as co-occurring mental health disorders. ${ }^{78}$

A study of smoking among individuals who were deployed or not deployed to Iraq and Afghanistan found that those who were deployed were more likely than those who were not deployed to initiate smoking if they never smoked before (2.3 percent vs. 1.3 percent), to resume smoking if they had smoked in the past (39.4 percent vs. 28.7 percent) and to have a greater increase in their smoking rate if they were smokers (57 percent vs. 44 percent). The same study found that those who were deployed and reported combat exposure were 1.6 times more likely to initiate smoking (among neversmokers) and 1.3 times more likely to resume smoking (among past smokers) compared to those who were not exposed to combat. The likelihood of resumption of smoking postdeployment was associated with length of deployment. $^{\ddagger} 79$

One study using data from 2005 found that 40 percent of veterans from military operations in Iraq and Afghanistan who sought Veterans Administration (VA) health care screened positive for risky alcohol use and 22 percent screened positive for addiction involving

\footnotetext{
㧊 More than nine months and deploying multiple times.
} 
alcohol. $^{*} 80$ Another study ${ }^{\dagger}$ found that the prevalence of risky alcohol use ${ }^{\ddagger}$ was higher after deployment to Iraq or Afghanistan compared to pre-deployment. ${ }^{81}$ A study of soldiers who were interviewed three to four months after returning from deployment to Iraq found that 25 percent engaged in risky alcohol use. ${ }^{\S}$ Soldiers who had higher rates of exposure to threats of injury or death were more likely to engage in risky alcohol use. ${ }^{82}$ A study of reserve/National Guard and active duty personnel found that reserve/National Guard personnel who were deployed with combat exposure were 1.6 times more likely than those deployed without combat exposure to experience new-onset heavy weekly drinking $^{* *}$ (8.8 percent vs. 5.6 percent) and 1.5 times more likely to report new-onset binge drinking $^{\dagger \dagger}$ (25.6 percent vs. 19.3 percent); active duty personnel who were deployed to Iraq or Afghanistan and reported combat exposure were 1.3 times more likely than those who were deployed without combat exposure to report new-onset binge drinking (26.6 percent vs. 22.0 percent). ${ }^{83}$ A study of National Guard Brigade Combat Team soldiers deployed to Iraq from March 2006 to July 2007 found that 13 percent

\footnotetext{
${ }^{*}$ Based on scores on a version of the AUDIT instrument (see Appendix H). Risky drinking was defined as an AUDIT-C score of three or higher for women and four or higher for men. Addiction was defined as an AUDIT-C score of four or higher for women and six or higher for men.

${ }^{\dagger}$ Of four U.S. combat infantry units (three Army units and one Marine Corps unit).

${ }^{\ddagger}$ Measured with a two-question instrument asking, "In the last year, have you ever drunk or used more drugs than you meant to?" and "Have you felt you wanted or needed to cut down on your drinking or drug use in the last year?"

${ }^{\S}$ Answering yes either to: "In the past four weeks, have you felt you wanted or needed to cut down on your drinking?" or "In the past four weeks, have you used alcohol more than you meant to?”

${ }^{* *}$ Men who consumed more than 14 drinks per week and women who consumed more than seven drinks per week.

${ }^{+\dagger}$ Those who reported drinking five or more drinks (for men) or four or more drinks (for women) on at least one day of the week or those who reported "drinking five or more alcoholic beverages" on at least one day or occasion during the past year.
}

met criteria for addiction involving alcohol when they returned from deployment. 84

The risky use of prescription drugs also is common among active duty personnel. One study found that in 2008, 9.9 percent of service members misused prescription drugs ${ }^{\S \S}(14.5$ percent of Army, 9.1 percent of Navy, 10.2 percent of Marine Corps, 7.5 percent of Air Force and 8.0 percent of Coast Guard service members), ${ }^{85}$ a rate far higher than the 2.1 percent who have misused controlled prescription drugs in the general population. ${ }^{86}$

Soldiers exposed to combat who experience a traumatic brain injury (TBI) are at particularly high risk for the risky use of alcohol or other drugs and for addiction. One study of service members who were discharged from military service found that those with mild TBI were 2.6 times more likely and those with moderate TBI were 5.4 times more likely to be discharged for addiction involving alcohol or for drug use compared to the total discharge population. ${ }^{87}$ Another study of service members with blastinduced mild-to-moderate injuries between 2004 and 2007 found that more than six percent of service members with a mild TBI had postdeployment addiction involving alcohol. ${ }^{88}$

Post-traumatic stress disorder (PTSD) also is prevalent in the military population and cooccurs at high rates with addiction. One study of Iraq and Afghanistan veterans who were firsttime users of VA health care, found that among those who met criteria for addiction involving alcohol and/or other drugs, 63 to 76 percent also met diagnostic criteria for PTSD. ${ }^{89}$

\footnotetext{
\# Sixty-two percent of these soldiers met criteria prior to deployment while 38 percent had a new onset--that is, they met criteria post-deployment. $\$ \S$ In the past 30 days, misused prescription-type amphetamines/stimulants (including methamphetamine), tranquilizers/muscle relaxers, barbiturates/sedatives or opioids/pain relievers.
} 


\section{Involvement in the Justice System}

Adolescents (ages 12 to 17) who have a lifetime history of arrest are three times more likely to engage in risky substance use or have addiction compared with adolescents who have no arrest record (60.1 percent vs. 19.8 percent). ${ }^{90}$ They are:

- Twice as likely to be risky substance users (24.5 percent vs. 13.7 percent); and

- Five-and-a-half times as likely to have addiction (35.6 percent vs. 6.2 percent). ${ }^{91}$

Previous research by CASA Columbia has found that 78.4 percent of 10 - to 17 -year olds who are in juvenile justice systems are substanceinvolved; $^{*} 9252.4$ percent of juvenile or youthful offenders incarcerated in state prisons and local jails meet clinical criteria for addiction involving alcohol or other drugs. ${ }^{93}$

Those ages 18 and older who have ever been arrested are almost twice as likely to engage in risky substance use or have addiction compared to those with no arrest record (74.5 percent vs. 45.0 percent). More specifically, while they are slightly more likely to be risky substance users without having addiction (38.1 percent vs. 32.5 percent), they are three times as likely to meet diagnostic criteria for addiction (36.4 percent vs. 12.5 percent). ${ }^{94}$

\footnotetext{
* They were under the influence of alcohol or other drugs while committing their crime, test positive for drugs, are arrested for committing an alcohol or other drug offense, admit having substance-related problems or addiction or share some combination of these characteristics.
}

Previous research by CASA Columbia found that the majority (84.8 percent) of all inmates are substance involved; ${ }^{\dagger} 64.5$ percent of the inmate population (nearly 1.5 million people) has addiction involving alcohol or drugs other than nicotine. $^{\ddagger 95}$

\section{Consequences of Risky Substance Use and Untreated Addiction}

A broad range of health and social consequences result from risky substance use and addiction, including those discussed above for special populations. ${ }^{96}$ Risky substance use and addiction constitute the largest preventable public health problems and the leading causes of preventable death in the U.S. ${ }^{97}$ Of the nearly 2.5 million deaths in 2009, an estimated minimum of 578,819 were attributable to tobacco, alcohol or other drugs. ${ }^{\S} 98$ (Table 3.8)

\footnotetext{
† They had a history of using illicit drugs regularly, met clinical criteria for addiction, were under the influence of alcohol or other drugs when they committed their crime, had a history of alcohol treatment, were incarcerated for an alcohol or other drug law violation, committed their offense to get money to buy drugs or had some combination of these characteristics.

${ }^{\ddagger}$ Due to data limitations, the estimated rate of addiction in the adult inmate population does not include nicotine dependence. However, other research suggests that the rate of tobacco use in the justice population is higher than in the general population. For example, in 2005, 37.8 percent of state inmates and 38.6 percent of federal inmates smoked in the month of their arrest. In contrast, the current smoking rate in the general population at that time was approximately 10 percentage points lower (24.9 percent).

$\S$ These numbers do not reflect the share of deaths from a wide range of other health conditions attributable to risky substance use and addiction.
} 


\begin{tabular}{|c|c|}
\hline \multicolumn{2}{|c|}{$\begin{array}{c}\text { Table } 3.8 \\
\text { Deaths Attributable to Substance Use }\end{array}$} \\
\hline & Deaths/Year \\
\hline $\begin{array}{l}\text { Total Deaths Attributable to } \\
\text { Substance Use }\end{array}$ & 578,819 \\
\hline Tobacco & 443,000 \\
\hline Alcohol & 98,334 \\
\hline Other drugs & $37,485^{*}$ \\
\hline \multicolumn{2}{|c|}{$\begin{array}{l}{ }^{*} \text { Based on data from } 2009 . \\
\text { Sources: Tobacco--Centers for Disease Control and } \\
\text { Prevention (2008); Alcohol--CASA Columbia } \\
\text { analysis of the Alcohol and public health: Alcohol- } \\
\text { Related Disease Impact (ARDI) (2012); Other } \\
\text { Drugs--Centers for Disease Control and Prevention } \\
\text { (2010); Kochanek, K., et al. (2011). }\end{array}$} \\
\hline
\end{tabular}

Four out of every 10 (39.6 percent) fatal traffic crashes involve a driver who is under the influence of alcohol ${ }^{*}$ or who tested positive for other drugs. ${ }^{99}$

Overdose deaths caused by controlled prescription drugs and illicit drugs have increased five-fold since $1990^{100}$ and now surpass the total number of deaths caused by traffic accidents. ${ }^{101}$

Individuals with addiction are at increased risk of potentially fatal diseases including cancer, ${ }^{102}$ heart disease $\mathrm{e}^{103}$ and sexually-transmitted diseases. ${ }^{104}$ More specifically, smoking contributes to multiple types of cancer as well as heart and respiratory disease. ${ }^{105}$ Alcohol contributes to some of the leading causes of death, including heart disease, cancer and stroke, as well as to other serious illnesses such as cirrhosis, hepatitis and pancreatitis. ${ }^{106}$ Injection drug use contributes to HIV, hepatitis C and hepatitis B. ${ }^{107}$ CASA Columbia's research found that risky substance use and addiction cause or contribute to more than 70 other conditions requiring medical care, including cancer, respiratory disease, cardiovascular disease, pregnancy complications, HIV/AIDS, cirrhosis, ulcers and trauma. ${ }^{108}$ (See Table 3.9)

\footnotetext{
${ }^{*}$ Has a blood alcohol concentration of 0.08 or higher.
}

The many negative consequences of our failure to prevent risky substance use and treat addiction extend beyond the individual to family, friends, community and society. ${ }^{109}$ Risky substance use and untreated addiction contribute to family dysfunction and financial troubles, disrupted social relationships, unsafe sexual practices, unplanned pregnancies, lost work productivity, legal problems, poor academic and career performance, homelessness, property and violent crimes, domestic violence, child abuse and neglect, rapes and other sexual assaults and motor vehicle crashes and fatalities. ${ }^{110}$

Risky substance use and addiction adversely affect the mental health of other family members as well. Family members ages 19 and older are at approximately twice the risk of having addiction or clinical depression as those ages 19 and older in families without a member with addiction, and they have higher health care costs. ${ }^{111}$

Children and adolescents are particularly vulnerable to the health consequences of substance use. ${ }^{112}$ Approximately 70 percent of child welfare cases are caused or exacerbated by parental risky use and addiction. ${ }^{113}$ Children exposed to parental substance use are at increased risk of emotional and behavioral problems, conduct disorder, poor developmental outcomes and risky substance use and addiction in adolescence and adulthood. ${ }^{114}$ Children and adolescents with family members who have addiction are more likely to be diagnosed with a number of medical conditions, including asthma, depression, headaches, attention deficit/ hyperactivity disorder, trauma and addiction, than children in families of similar demographic characteristics who do not have a member with addiction. ${ }^{115}$ 
Table 3.9

\section{Conditions Requiring Medical Care Attributable to Risky Use and Addiction}

\begin{tabular}{l}
\hline Tobacco-Related Conditions \\
\hline Malignant neoplasm (Cancer) \\
\hline Lip, oral cavity, pharynx \\
\hline Esophagus \\
\hline Stomach \\
\hline Pancreas \\
\hline Larynx \\
\hline Trachea/lung/bronchus \\
\hline Cervix uteri \\
\hline Kidney and renal pelvis \\
\hline Urinary bladder \\
\hline Acute myeloid leukemia
\end{tabular}

\section{Alcohol-Related Conditions}

\begin{tabular}{|l|l}
\hline Cardiovascular disease & Perinatal conditions \\
\hline Ischemic heart disease & Short gestation, low birth weight \\
\hline Other heart disease & Respiratory distress syndrome \\
\hline Cerebrovascular disease & Other respiratory \\
\hline Aortic aneurysm & \\
\hline Other circulatory disease & \\
\hline Respiratory diseases & \\
\hline Pneumonia, influenza & \\
\hline Bronchitis, emphysema & \\
\hline Chronic airway obstruction & \\
\hline & \\
\hline
\end{tabular}

Acute pancreatitis

Alcohol abuse/Alcohol dependence syndrome

Alcohol cardiomyopathy

Alcohol polyneuropathy

Alcohol-induced chronic pancreatitis

Alcoholic gastritis

Alcoholic liver disease

Alcoholic myopathy

Alcoholic psychosis

Breast cancer (females only)

Cholelithiases

Chronic hepatitis

Chronic pancreatitis

Degeneration of nervous system due to alcohol

Epilepsy

Esophageal cancer

Laryngeal cancer

Liver cancer

Liver cirrhosis unspecified

Oropharyngeal cancer

Portal hypertension

Prostate cancer (males only)

Psoriasis

Spontaneous abortion (females only)

Stroke hemorrhagic

Stroke ischemic

Supraventricular cardiac dysrhythmia

Prenatal, infant

Birth trauma

Digestive

Hemorrhage/hemolysis/endocrine/

jaundice/hematologic

Esophageal varices

Fetal alcohol syndrome

Gastro esophageal hemorrhage

Hypertension

Ischemic heart disease
Hypoxia/asphyxia/respiratory

Infections

Integument/temperature regulation

Length of gestation and fetal growth

Low birth weight/prematurity/

intrauterine growth restriction death

\section{Other Drug-Related Conditions}

Drug-induced psychosis

Hepatitis

\section{HIV/AIDS}

Inflammatory and toxic neuropathy

\section{Acute Conditions}

Air-space transport

Alcohol poisoning

Aspiration

Child maltreatment

Drowning

Excessive blood alcohol level

Fall injuries

Fire injuries

Firearm injuries

Homicide

Hypothermia

Motor-vehicle non-traffic crashes

Motor-vehicle traffic crashes

Occupational and machine injuries

Other road vehicle crashes

Poisoning (not alcohol)

Suicide

Water transport accident

* Other-drug related conditions also may include kidney, liver and respiratory diseases as well as the accident, suicide and poisoning categories listed above in the Alcohol-Related Conditions section.

Sources: Centers for Disease Control and Prevention. (2004b); Centers for Disease Control and Prevention. (2008a); Centers for Disease Control and Prevention. (2008b); Merrill, J. \& Fox, K. (1998); The National Center on Addiction and Substance Abuse (CASA) at Columbia University. (1993); The National Center on Addiction and Substance Abuse (CASA) at Columbia University. (1994). 


\section{Tobacco}

Tobacco use is the leading preventable cause of death and disability in the United States. An estimated one in five, or 443,000 , deaths each year are attributable to cigarette smoking and exposure to tobacco smoke; ${ }^{116}$ nearly 400,000 deaths per year are attributable to smokingrelated diseases. ${ }^{117}$ (Table 3.10)

\begin{tabular}{|c|c|}
\hline \multicolumn{2}{|c|}{$\begin{array}{c}\text { Table } 3.10 \\
\text { Average Tobacco-Attributable Deaths Due to } \\
\text { Smoking-Related Disease in the } \\
\text { United States, 2000-2004 } \\
\text { Select Examples }\end{array}$} \\
\hline & $\begin{array}{c}\text { Average Deaths } \\
\text { Per Year }\end{array}$ \\
\hline Total & 392,683 \\
\hline Cancers: & 160,848 \\
\hline Lung, trachea, bronchus & 125,522 \\
\hline Esophagus & 8,592 \\
\hline Pancreas & 6,683 \\
\hline Urinary bladder & 4,983 \\
\hline Lip, oral cavity, pharynx & 4,893 \\
\hline Kidney, renal pelvis & 3,043 \\
\hline Larynx & 3,009 \\
\hline Stomach & 2,484 \\
\hline Acute myeloid leukemia & 1,192 \\
\hline Cervix, uterus (females only) & 447 \\
\hline Cardiovascular Diseases: & 128,497 \\
\hline Ischemic heart disease & 80,005 \\
\hline Other heart disease & 21,004 \\
\hline Cerebrovascular disease & 15,922 \\
\hline Aortic Aneurysm & 8,419 \\
\hline Atherosclerosis & 1,893 \\
\hline Other arterial disease & 1,254 \\
\hline Respiratory Diseases: & 392,683 \\
\hline Chronic airway obstruction & 78,988 \\
\hline Bronchitis, emphysema & 13,927 \\
\hline Pneumonia, influenza & 10,423 \\
\hline \multicolumn{2}{|c|}{$\begin{array}{l}{ }^{*} \text { These data do not reflect all tobacco-attributable deaths } \\
\text { For example, deaths due to secondhand smoke and fire } \\
\text { burn are not included. } \\
\text { Source: Centers for Disease Control and Prevention } \\
\text { (2011). }\end{array}$} \\
\hline
\end{tabular}

Tobacco use contributes to approximately 30 percent of cancer and heart disease-related deaths ${ }^{118}$ and numerous other health conditions including respiratory illness and chronic kidney disease. ${ }^{119}$ An estimated 8.6 million Americans suffer from a serious smoking-related illness. ${ }^{120}$
Although the prevalence of tobacco use has declined over the past two decades, ${ }^{121}$ mortality rates have remained constant due to an increase in population size, the identification of new diseases linked to smoking and the fact that cohorts that smoked heavily during their lifetime are now reaching an age with the highest incidence of smoking-attributable diseases. ${ }^{122}$

Over approximately the past four decades, an estimated 94,000 infant deaths have been linked to prenatal exposure to smoking. ${ }^{123}$ Pregnant women who smoke put their babies at increased risk for a host of health problems including placenta previa, ${ }^{\dagger}$ stillbirth and sudden infant death syndrome (SIDS). ${ }^{124}$ Smoking during pregnancy increases the risk for preterm birth ${ }^{125}$ and pregnant smokers are 1.6 times more likely to have a low birth weight baby than pregnant nonsmokers (11.9 percent vs. 7.5 percent). ${ }^{126}$ Merely reducing the number of cigarettes women smoke during pregnancy results in birth weight gain; but even light smokers ${ }^{\ddagger}$ are twice as likely as nonsmokers to have low birth weight infants. $^{\S 127}$ Low birth weight is a leading risk factor for neonatal and infant mortality, can result in restricted childhood development and increases the risk of chronic disease, developmental delays and cognitive impairment. ${ }^{128}$

The negative long-term health consequences for children exposed to prenatal smoking include increased risk for substance-related problems, depression, ${ }^{129}$ attention deficit/hyperactivity disorder, conduct disorders and childhood obesity. ${ }^{130}$ The nicotine in tobacco products can produce structural and chemical changes in the developing adolescent brain and make young people who smoke vulnerable to future addiction and to certain forms of mental illness, including panic attacks, panic disorder and other anxiety disorders. $^{131}$

\footnotetext{
* 1964 to 2004.

$\dagger$ The complete or partial obstruction of the cervical opening by the placenta.

¥ Smoke less than half a pack a day.

$\S$ In a study of low-income black women.
} 
There also are serious health consequences for nonsmokers exposed to environmental tobacco smoke (ETS). ${ }^{132}$ Children exposed to ETS are at increased risk of developing acute lower respiratory infections, ear infections, asthma and chronic respiratory symptoms, ${ }^{133}$ and of becoming smokers and developing asthma in adulthood. ${ }^{134}$ Exposure to ETS increases the risk of lung, breast and other cancers, heart disease, stroke and respiratory illnesses. ${ }^{135}$

Recently, the term "third-hand smoke" has been developed to describe the invisible but toxic gases and particles--including heavy metals, carcinogens and radioactive materials--that form a residue on smokers' hair, clothing and household items and remain for weeks or months after the second-hand smoke has cleared. ${ }^{136}$ Like second-hand smoke, third-hand smoke is a cancer risk. ${ }^{137}$

\section{Alcohol}

Alcohol use is the third leading cause of death in the United States (after tobacco use and poor diet/physical inactivity) and is responsible for approximately 3.5 percent of all deaths. ${ }^{138} \mathrm{An}$ estimated 98,334 people die from alcoholrelated causes in the U.S. each year, including chronic diseases (e.g., liver disease, cancer) and acute causes (e.g., accidents, homicides). ${ }^{139}$ (Table 3.11)

Of the 13,555 substance-related traffic fatalities in 2009, 10,185 involved drivers who were alcohol impaired (BAC of .08 or higher). ${ }^{140}$ Young people are at greater risk of becoming a victim of an alcohol-related traffic fatality compared to older people. ${ }^{141}$ In 2009, 35 percent of 21- to 24-year old drivers involved in fatal motor vehicle traffic crashes were under the influence of alcohol, ${ }^{*}$ more than any other age group. $^{\dagger 142}$

\footnotetext{
${ }^{*}$ Had a BAC of .08 or higher.

${ }^{\dagger}$ Compared to 19 percent of 15- to 20-year olds; 33 percent of 25- to 29-year olds; 29 percent of 30- to 34-year olds; 26 percent of 35- to 44-year olds; 22 percent of 45- to 54-year olds; 13 percent of 55- to 64-year olds; and 7 percent of 65- to 74-year olds.
}

\section{Table 3.11 \\ Average Alcohol-Attributable Deaths in the United States 2001-2005, Select Examples}

\begin{tabular}{l|c}
\hline Total & $\begin{array}{c}\text { Average Deaths } \\
\text { Per Year }\end{array}$ \\
\hline Chronic Causes: & $\mathbf{9 8 , 3 3 4}$ \\
\hline Alcoholic liver disease & 12,219 \\
\hline Stroke, hemorrhagic & 8,725 \\
\hline Liver cirrhosis, unspecified & 7,055 \\
\hline Esophageal cancer & 4,225 \\
\hline Alcohol dependence syndrome & 3,857 \\
\hline Liver cancer & 3,431 \\
\hline Breast cancer (females only) & 1,835 \\
\hline Oropharyngeal cancer & 1,528 \\
\hline Laryngeal cancer & 1,460 \\
\hline Hypertension & 1,480 \\
\hline Prostate cancer (males only) & 1,025 \\
\hline Acute Causes: & \\
\hline Motor-vehicle traffic crashes & 13,819 \\
\hline Homicide & 7,787 \\
\hline Suicide & 7,235 \\
\hline Fall injuries & 5,532 \\
\hline Poisoning (not alcohol) & 5,416 \\
\hline Fire injuries & 1,158 \\
\hline Drowning & 868 \\
\hline Alcohol poisoning & 370 \\
\hline Hypothermia & 269 \\
\hline Aspiration & 204 \\
\hline Child maltreatment & 168 \\
\hline Source: CASA Columbia analysis of the Alcohol and \\
public health: Alcohol-Related Disease Impact (ARDI). \\
\hline
\end{tabular}

In 2009, alcohol was reported in at least onequarter (24.3 percent) of substance-related emergency department (ED) visits. ${ }^{\ddagger}$ These reports, however, significantly underestimate the prevalence of alcohol-related emergency department visits for two reasons: the data set does not include visits linked to alcohol use in combination with other drugs for persons under the age of 21, and many EDs do not screen for

\footnotetext{
${ }^{\ddagger}$ Measured in terms of patient visits, not individual drug reports. The analyses of the Substance Abuse and Mental Health Services Administration, Drug Abuse Warning Network (DAWN) data assess the number of drug mentions associated with a drugrelated emergency department visit; up to four drugs plus alcohol may be recorded for each drug-related visit.
} 
substance use because of state laws that exclude alcohol and other drug-related injuries from medical insurance coverage. ${ }^{*} 143$

Risky alcohol use and addiction involving alcohol are associated with neurological problems including dementia, stroke and neuropathy; ${ }^{144}$ cardiovascular problems including myocardial infarction, cardiomyopathy, atrial fibrillation and hypertension; ${ }^{145}$ psychiatric problems including depression, anxiety and suicide; ${ }^{146}$ liver diseases including hepatitis and cirrhosis; ${ }^{147}$ and gastrointestinal problems including pancreatitis and gastritis. ${ }^{148}$ Other associated conditions include increased risk of cancer of the liver, breast, mouth, throat, esophagus and colon, ${ }^{149}$ and recent research suggests that risky alcohol use may contribute to the physiological process that causes cancer cells to metastasize. ${ }^{150}$

Heavy alcohol use during pregnancy is associated with miscarriage and stillbirth and is one of the primary causes of severe mental and developmental delays in infants. ${ }^{151}$ Fetal alcohol syndrome (FAS) is the leading known cause of preventable mental retardation. ${ }^{152}$

\section{Illicit Drugs}

In 2008, ${ }^{\dagger}$ there were an estimated 16,044 deaths attributed to the use of illicit drugs. ${ }^{153}$ Of the 13,555 substance-related traffic fatalities in 2009, 5,938 involved drivers impaired either by illicit drugs alone (3,146 deaths) or illicit drugs in combination with alcohol (2,792 deaths). ${ }^{154}$

In 2009, illicit drugs were involved in an estimated 973,591 emergency department visits; ${ }^{\ddagger}$ accounting for 35.9 percent of substancerelated ED visits; ${ }^{155}$ cocaine, marijuana and

\footnotetext{
* In 25 states, Uniform Accident and Sickness Policy Provision Laws (UPPL) exclude alcohol and other drug-related injuries from medical insurance coverage, creating a barrier to conducting screening for risky substance use (see Chapters IV and X).

${ }^{\dagger}$ Most recent available data that distinguishes between illicit and controlled prescription drugs.

${ }^{\ddagger}$ Measured in terms of patient visits, not individual drug reports.
}

heroin were the most frequently mentioned illicit drugs. ${ }^{156}$

Injection drug use behavior--including sharing needles and other injection paraphernalia--is associated with the spread of HIV/AIDS. ${ }^{157}$ It is estimated that more than one-third of all AIDSrelated deaths in the U.S. have occurred among illicit drug users and their sexual partners. ${ }^{158}$

Morbidity (secondary illness) and mortality (death) data related to illicit drug use may represent the direct pathological effects or medical toxicities from the drug (e.g., cocaine producing seizures or strokes or inhalants producing cardiac arrhythmias that can lead to sudden cardiac deaths), but also the infections transmitted via drug self-administration (e.g., intravenous or intramuscular selfadministration). ${ }^{159}$

Marijuana use is associated with sexually transmitted disease due to unsafe sexual behaviors engaged in while under the influence of the drug, bronchitis and lung cancer; cocaine use is associated with pancreatitis; heroin use is associated with hepatitis and tuberculosis; hallucinogen use is associated with tinnitus and sexually transmitted disease; and inhalant use is associated with HIV/AIDS, sexually transmitted disease, tuberculosis, bronchitis, asthma, sinusitis and tinnitus. ${ }^{\S} 160$

Illicit drug use also is linked to mental health problems. Marijuana use is associated with the onset of psychotic disorders, particularly in individuals with an underlying vulnerability to the illness; ${ }^{161}$ several longitudinal studies have linked marijuana use with the subsequent onset of schizophrenia, and case studies have linked synthetic cannabinoids with psychosis. ${ }^{162}$ Marijuana and inhalant use are associated with

\footnotetext{
$\S$ These health consequences are associated primarily with long-term use of illicit drugs. The analyses adjusted for potentially confounding factors such as the duration of controlled prescription drug misuse, alcohol use, tobacco use, daily cigarette smoking history and demographic variables (age, gender, race/ethnicity, educational attainment, health insurance status and family income).
} 
anxiety and depression, and cocaine and heroin use are associated with anxiety. ${ }^{*} 163$

Methamphetamine, cocaine and other stimulant use (including the use of amphetamine-related and other "designer drugs") are associated with violent behavior, hallucinations, paranoia and delusions which can be acute time-limited symptoms or which can persist long after the cessation of use. ${ }^{\dagger 164}$ There is a range of substance-induced disorders included in the DSM-IV that reflect secondary mental health effects of addictive substance use. ${ }^{165}$

Approximately 160,000 pregnancies in 2004 were associated with illicit drug use. ${ }^{166}$ Illicit drug use during pregnancy increases the risk of neurological and cognitive deficits in the fetus and future behavior problems. ${ }^{167}$ Heavy marijuana use has been associated with low birth weight, premature delivery and complications in delivery. ${ }^{168}$ Marijuana and cocaine exposure have been linked to impaired attention, language and learning skills, as well as to behavioral problems. ${ }^{169}$

Infants exposed to prenatal illicit drug use are at increased risk of low birth weight, ${ }^{170}$ developmental and educational problems and future substance use and addiction. ${ }^{171}$

\section{Controlled Prescription Drugs}

In $2008,{ }^{\ddagger}$ there were an estimated 20,044 overdose deaths ${ }^{\S}$ attributable to risky use of controlled prescription drugs. The majority of these deaths (73.8 percent or 14,800$)$ were attributable to the risky use of prescription opioids. ${ }^{172}$ Overdose deaths from controlled prescription drugs have increased significantly

\footnotetext{
* Adjusting for potentially confounding factors such as those listed above.

${ }^{\dagger}$ Producing what is described in the DSM-IV as a Substance-Induced Mental Disorder.

${ }^{\ddagger}$ Most recent available data that distinguishes between illicit and controlled prescription drugs.

$\S$ Data on other causes of death (e.g., accidents) that are attributable to the misuse of controlled prescription drugs are not available.
}

over recent years and now surpass the number of overdose deaths caused by illicit drugs. ${ }^{* *} 173$

\section{Enough prescription painkillers were prescribed in 2010 to medicate every American adult around-the-clock for a month. ${ }^{174}$ \\ --Centers for Disease Control and Prevention}

In 2009, there were 224 deaths that involved drivers impaired by controlled prescription drugs (or an unknown combination of prescription drugs, alcohol and other drugs). ${ }^{175}$ The risky use of controlled prescription drugs was involved in an estimated 1,079,683 emergency department visits, ${ }^{\dagger \dagger}$ accounting for 39.8 percent of all substance-related emergency department visits in the U.S. Among prescription drug-related ED visits, 73.3 percent involved opioids, stimulants, sedatives and barbiturates. ${ }^{\text {邦 } 176}$

The risky use of prescription opioids can result in a range of consequences from drowsiness and constipation to depressed breathing, at high doses. Even a large single dose of opioids can lead to severe respiratory depression or death. ${ }^{177}$ One study found that individuals with addiction involving opioids had significantly higher rates of comorbid health conditions, including hepatitis, pancreatitis and psychiatric illness than those without addiction involving opioids. ${ }^{178}$

At high doses, risky use of prescription stimulants can produce anxiety, paranoia, seizures ${ }^{179}$ and serious cardiovascular complications including stroke. ${ }^{180}$ Other possible adverse effects include slowed growth in children, allergic reactions, potentially fatal interactions with other drugs ${ }^{\S \S}$ and sudden death. ${ }^{181}$

\footnotetext{
** Specifically, heroin and cocaine.

${ }^{+\dagger}$ Measured in terms of patient visits, not individual drug reports.

㧊 The remaining prescription drug-related ED visits involved antidepressants and antipsychotics (12.3 percent) or other types of prescription drugs (14.4 percent).

$\S \S$ Such as MAO inhibitors.
} 
Long-term risky use of benzodiazepines is associated with cognitive impairment, other adverse effects and a high risk of drug interactions, addiction and withdrawal syndrome that can be life threatening. ${ }^{182}$ Likewise, risky use of barbiturates, such as butalbital and phenobarbital, can lead to changes in alertness, irritability and memory loss. ${ }^{183}$ If combined with certain medications or alcohol, tranquilizers and sedatives can slow both heart rate and respiration, which can be fatal. ${ }^{184}$

Taking certain controlled prescription drugs during pregnancy, such as alprazolam (Xanax) or phenobarbital, may harm the developing fetus. ${ }^{185}$ 


\section{Chapter IV \\ Screening and Early Intervention}

Nearly one-third (31.7 percent) of the U.S. population ( 80.4 million people ages 12 and older) engages in substance use that threatens their own health or safety or the health and safety of others, but does not meet clinical diagnostic criteria for addiction. ${ }^{1}$ Few of these individuals, however, are routinely screened for risky use of addictive substances or receive any services designed to reduce such use such as brief interventions. ${ }^{2}$ Of those who do receive some form of screening, in most cases it involves only one type of substance use-tobacco or alcohol--which fails to identify risky use of other substances or recognize that 30.6 percent of risky users who are not addicted engage in risky use of more than one substance. ${ }^{3}$

In order to reduce risky use and its far-reaching health and social consequences, which may include the development of addiction, health care practitioners must: ${ }^{4}$

- Understand the risk factors, ${ }^{*}$ how these risks vary across the lifespan and how risky use-whether or not it progresses to addiction-can have devastating outcomes for individuals, families and communities;

- Educate patients, and their families if relevant, about these risks and the adverse consequences of risky use;

- Screen for risky use of addictive substances and related problems using tools that have been proven to be effective; and

- Provide brief intervention when appropriate.

Health care practitioners also should conduct further assessment if the patient presents with signs or symptoms of addiction and treat the patient or provide referrals to specialty care if needed, as discussed in Chapters V and VI.

\footnotetext{
${ }^{*}$ See Chapter II.
} 
Screening and early intervention services should be provided in regular health care settings, but also can be highly successful when offered in other venues to individuals at high risk, including students, those in justice programs and those in social service settings. To assure that these health care services are provided, a range of barriers must be addressed, including insufficient training of health care and other professionals and a lack of trained specialty providers to which patients with addiction can be referred for treatment; time constraints and insufficient financial and workforce resources; and screening tools that may fail to detect cases of risky use of addictive substances.

\section{The Need for Patient Education, Screening and Intervention throughout the Lifespan}

For many health conditions, certain developmental periods are associated with increased risk of acquiring a disease. ${ }^{5}$

Addiction, in most cases, has its roots in adolescence with the initiation of risky use of addictive substances, ${ }^{6}$ but the onset of risky use and addiction can occur at any point in the lifespan. Each life phase presents unique vulnerabilities that must be recognized, as well as the basic risk factors (discussed in Chapter II) that may be present at any time in life.

\section{Childhood and Adolescence}

Adolescence is the critical period for the onset of substance use and its consequences, ${ }^{*}$ but signs of risk sometimes can be observed much earlier. In addition to the overall risks associated with substance use, children and adolescents with heightened risk of engaging in substance use, of experiencing the adverse consequences of risky use and of developing addiction include:

- Those with certain genetic predispositions or structural or functional brain characteristics that make them more susceptible to addictive substances; ${ }^{8}$

\footnotetext{
${ }^{*}$ See Chapter II.
}

- Young children whose temperaments' are more active, impatient, aggressive and nonconforming than their peers, ${ }^{9}$

- Those with behavioral disorders including oppositional defiant disorder ${ }^{\dagger}$ and conduct disorder, ${ }^{\sharp 10}$ those who engage in bullying ${ }^{\S 11}$ and those who have sleep problems; ; 12 and

- Children who are maltreated, abused or have suffered other trauma. ${ }^{13}$

As children age, moving through elementary and middle school--a period that coincides with firsttime exposure to cigarettes and other drugs--they are presented with increasing academic and social challenges and responsibilities that increase their risk of trying addictive substances and engaging in substance use. ${ }^{14}$

During adolescence and into early adulthood the brain undergoes considerable developmental changes, explaining why adolescence is such a risky period for the onset of substance use and addiction. ${ }^{15}$ Hormonal changes that occur during adolescence also pose a biological risk for substance use in this age group. The surge in the female hormone estrogen and the male hormone testosterone during puberty is associated with risk taking and sensation

\footnotetext{
${ }^{\dagger}$ According to the American Academy of Child and Adolescent Psychiatry, oppositional defiant disorder refers to a pattern of disobedient, hostile and defiant behavior directed toward authority figures. Common behavioral symptoms include defiance, spitefulness, negativity, hostility and verbal aggression.

* Conduct disorder is diagnosed in youth who exhibit enormous difficulty following rules and behaving in a socially-acceptable manner. These children may bully others, start fights, show aggression toward animals, steal or engage in sexually inappropriate behavior.

$\S$ Past-year participation in the following acts: (a) hit and pushed or threatened another student, (b) called another student mean names, (c) told another student you will not like her/him unless she/he did what you wanted, (d) made people not like another student, (e) told lies or spread rumors about another student, (f) not let another student be in your group of friends. ${ }^{* *}$ Mothers' reports of children and teens having trouble sleeping and overtiredness.
} 
seeking. The lack of fully developed decisionmaking and impulse-control skills combined with the hormonal changes of puberty compromise an adolescent's ability to assess risks and make them uniquely vulnerable to substance use. ${ }^{16}$

Other psychological and social challenges faced by adolescents--such as the struggle to develop a sense of identity, feeling less satisfied with one's appearance and experiencing peer pressure to conform--contribute to the risk. ${ }^{17}$

\section{Young Adulthood}

In recent years, researchers have begun to recognize the developmental stage of young adulthood--often referred to as emerging adulthood--as a period of life that is strongly associated with risky use. ${ }^{18}$ Young adults facing heightened risk include:

- College students--* --while approximately two-thirds of college students who engage in substance use began to smoke, drink or use other drugs in high school or earlier, the culture on many college campuses permits and promotes risky use rather than curtailing it. $^{19}$

- Young adults facing work-related stress or instability in living arrangements, social relations or academic or career choices. ${ }^{20}$ As marriage and parenthood have become delayed, the phase of life devoted to academics and career development has stretched well into the twenties. Young adults may turn to addictive substances to relieve these forms of stress and selfmedicate their anxiety and emotional troubles. $^{21}$

\section{Middle and Later Adulthood}

Major life events and transitions increase the chances that an individual will engage in risky use of addictive substances. ${ }^{22}$ Adults may turn to risky use when:

\footnotetext{
* Much of the research conducted on young adults is based on college student samples.
}

- Coping with the stresses of child rearing, balancing a career with family and managing a household; ${ }^{23}$

- Facing divorce, caring for an adult family member or grandchildren or coping with the death of a loved one, ${ }^{24}$

- Struggling with retirement, the loss of independent living or financial problems; ${ }^{25}$ or,

- Coping with an illness, including increasing physical ailments such as arthritis or other forms of chronic pain. ${ }^{26}$

Middle aged and older adults who engage in risky use may be even more vulnerable to the health consequences of such use since physical tolerance for alcohol and other drugs declines with age: the ways in which addictive substances are absorbed, distributed, metabolized and eliminated in the body change as people get older. ${ }^{27}$ With regard to alcohol, several biological factors account for reduced tolerance. The amount of lean body mass (muscle and bone) and water in older adults' bodies decreases as the amount of fat increases, with less water to dilute the alcohol. Reduced liver and kidney function slows down the metabolism and the elimination of alcohol from the body, including the brain. These factors allow the effects of alcohol to take hold more quickly and depress brain function to a greater extent than in younger people, impairing physical coordination and cognitive function. ${ }^{28}$

The increasing susceptibility to substanceinduced neurotoxicity with age is a growing concern as the "Boomer" generation, a population with higher rates of risky use, ages. ${ }^{29}$ The interaction of prescribed and other drugs with alcohol also is of great concern for the physical and mental health of middle and older adults who are likelier than younger people to use prescription and over-the-counter medications. $^{30}$ 


\section{Attending to Co-occurring Conditions}

Individuals who engage in risky use or who have addiction frequently suffer from other cooccurring health conditions. ${ }^{*} 31$ Therefore, any attempt to identify risky use of addictive substances, evaluate an individual's risk for developing addiction or assess a substance user's need for intervention or treatment must involve identification of co-occurring conditions and plans to address them. Being informed of a patient's health conditions that might be caused or exacerbated by substance use or that might cause or exacerbate the patient's addiction will help medical professionals determine appropriate interventions and provide effective care. $^{32}$ Similarly, medical professionals treating patients with medical conditions that frequently co-occur with risky use and addiction--such as hypertension, gastritis and injuries--should be prompted to screen for risky use of addictive substances that may cause or aggravate these conditions.

\section{Patient Education and Motivation}

Educating patients and motivating them to reduce their risky use of addictive substances is a critical component of preventive care. ${ }^{33}$ As part of routine medical practice, medical and other health professionals should educate their patients (and parents of young patients) about:

- The adverse consequences of risky use and the nature of addiction--that it is a disease that can be prevented and treated effectively;

- The risk factors for substance use, tailoring the information to the patient's age, gender, mental health history and other relevant medical, social and demographic characteristics;

- Times of increased risk for substance use, such as adolescence, key life transitions and stressful life experiences; and

\footnotetext{
${ }^{*}$ See Chapter II.
}

- Steps patients can take to prevent risky use of addictive substances and the onset of addiction, e.g., by delaying initiation of substance use, following guidelines for the safe use of alcohol and of controlled prescription medications, being vigilant for signs and symptoms of risk and seeking professional help at the first sign of trouble.

\section{Screening ${ }^{\dagger}$}

Screening, a staple of public health practice that dates back to the $1930 \mathrm{~s},{ }^{34}$ serves to identify early signs of risk for or evidence of a disease or other health condition and distinguish between individuals who require minimal intervention and those who may need more extended treatment. ${ }^{35}$ It is an effective method of preventive care in many medical specialties, and risky use of addictive substances is no exception. Screening for risky use of addictive substances is comparable to offering regularly scheduled pap smears or colonoscopies to identify cancer indicators. ${ }^{36}$

\footnotetext{
${ }^{\dagger}$ Despite the distinction between screening and assessment tools, the term screening often is used to subsume the concept of assessment or interchangeably with the term in the clinical and research literatures. Nevertheless, Chapter V addresses assessments specifically. In addition, while there is some overlap between screening or assessment procedures used to identify risky use of addictive substances and methods used to diagnose a clinical addiction, a formal diagnosis of addiction is based on the demonstration of specific symptoms included in the most recent versions of the Diagnostic and Statistical Manual of Mental Disorders (DSM) or the International Statistical Classification of Diseases (ICD). (See Chapter II.)
} 
It's like taking a blood pressure. You don't just take the pressure of patients you suspect of having high blood pressure. You take everyone's blood pressure. And, with public health screening, you're typically not shooting for a diagnosis, but just an initial measure of a patient's level of risk. ${ }^{38}$

--Dan Hungerford, DrPH Epidemiologist National Center for Injury Prevention and Control Centers for Disease Control and Prevention

Screening tools typically are brief and easy to administer and are to be implemented with a relatively broad population to identify indications of risk involving smoking, drinking or using other drugs. Screening tools typically include written or oral questionnaires and, less frequently, clinical and laboratory tests. (See Appendix $\mathrm{H}$ for a description of commonly-used screening instruments.)

An instrument that can be used to screen for risky use of all addictive substances--rather than separately for each substance--and that makes appropriate distinctions for young people and by gender has yet to be developed.

In recent years, attempts have been made to develop and validate more simple screening tools that can be used in primary care settings. For example:

- A single-item measure of current tobacco use $^{*}$ has been validated on adult populations for use in research protocols ${ }^{37}$ but also can be used clinically to determine if a patient is a current smoker.

- The National Institute on Alcohol Abuse and Alcoholism (NIAAA) recommends a singlequestion screening test for unhealthy alcohol

\footnotetext{
${ }^{*}$ Have you smoked one or more cigarettes in the past month?
}

use $^{\dagger}$ to be asked of patients of all ages who admit to sometimes drinking alcohol. ${ }^{39}$

- The NIAAA, in collaboration with the American Academy of Pediatrics (AAP) recently introduced a simple screening tool for identifying early signs of risky alcohol use in young people ages 9-18. The screener begins with two simple questions assessing the child's own alcohol use and that of his or her friends. Depending on the patient's age, positive responses to these items would be followed by more in-depth questions assessing the level of the patient's risk and the provision of appropriate brief interventions. $^{40}$

- A recent study found that a single-question screening test to identify other drug use in a diverse sample of adult primary care patients was effective in accurately identifying other drug use and may be beneficial in helping physicians identify potential medication interactions and associated risks of prescribing specific medications. ${ }^{41}$

Single-item screening tools can help narrow the patient population that requires further assessment for the identification of addiction. ${ }^{42}$ However, most instruments focus on specific substances rather than the range of addictive substances that pose a risk for addiction. The National Institute on Drug Abuse (NIDA) has begun to move in the direction of a more unified look at risky use and addiction with the development of a "quick screen" for use in general medical settings. The instrument actually contains four separate screens and asks patients about the frequency of their past-year use of each of the following types of substances: (1) tobacco, (2) alcohol, ${ }^{\S}$ (3) prescription drugs

\footnotetext{
${ }^{\dagger}$ How many times in the past year have you had 5 or more drinks in a day (for men)/4 or more drinks in a day (for women)?

${ }^{*}$ How many times in the past year have you used an illegal drug or used a prescription medication for nonmedical reasons?

${ }^{\S}$ With separate measures of risk for males and females--the frequency of having five or more drinks in a day for men and four or more drinks in a day for women.
} 
(for nonmedical reasons) and (4) illicit drugs. Response options for each, on a five-point scale, range from never to daily or almost daily. Used online, the screening tool tallies the responses to generate a score indicating the patient's level of risk for each type of substance and the recommended level of intervention. It also provides additional resources to help practitioners intervene appropriately. ${ }^{43}$

Implementing screening and brief intervention would be a revolution in 21st century medical practice. It would help reduce billions of dollars annually in lost productivity, injury and social costs associated with risky behaviors. It would also encourage those with chronic conditions to get the treatment they need. But medical practices are unlikely to take action without pressure from others who would benefit. The question is, when will society demand this change? ${ }^{44}$

--John C. Higgins-Biddle, PhD Assistant Professor (Retired) Community Medicine and Health Care University of Connecticut Health Center

\section{Laboratory Tests}

One approach to screening is to examine laboratory values of urine, hair, blood, sweat, saliva or carbon monoxide ${ }^{*}$ to determine the presence of nicotine, ${ }^{45}$ alcohol ${ }^{46}$ or other drugs. ${ }^{47}$ Laboratory tests also can be used to look for biological symptoms of chronic substance use. ${ }^{48}$ For example, to assess heavy alcohol use, doctors can look for elevated levels of the blood proteins gamma-glutamyl transferase (GGT) or carbohydrate-deficient transferrin (CDT). ${ }^{\dagger}$ The size of red blood cells also increases with prolonged heavy alcohol use. ${ }^{49}$ The validity of testing for these markers as a means of identifying risky alcohol use is limited by the fact that they are not necessarily unique to risky drinkers. ${ }^{50}$ For instance, increased GGT also

\footnotetext{
${ }^{*}$ Carbon monoxide breathalyzer tests used to detect smoking.

${ }^{\dagger}$ Heavy drinkers are defined in this context as individuals who consume four or more drinks per day. CDT is less accurate at determining heavy drinking in women and adolescent populations.
}

can be caused by nonalcoholic liver disease. At the same time, looking for biological markers is more objective than using a patient's selfreports, ${ }^{51}$ as it is not subject to patients' or examiners' biases.

Unlike tests for other diseases such as diabetes and hypertension which can be diagnosed using blood sugar or blood pressure measurements, there is not a conclusive test that physicians can conduct to determine with certainty the presence of the disease of addiction. ${ }^{52}$ With few exceptions, ${ }^{\ddagger}$ laboratory tests for nicotine, alcohol and other drugs generally inform health care providers of whether patients recently ${ }^{\S}$ have been using these substances rather than being indicators of chronic use or addiction. ${ }^{53}$

Individuals, groups and organizations may be hesitant to agree to laboratory tests for substance use for legal, financial or personal reasons. ${ }^{54}$ Widespread use of these tests is costly ${ }^{55}$ and, as with any other biological testing, the possibility

\footnotetext{
* A liver function test that indicates an elevated level of GGT and a complete blood count that indicates that the red blood cells have a greater than normal mean corpuscular volume (MCV) are evidence of chronic heavy alcohol use.

${ }^{\S}$ With regard to smoking, high levels of nicotine or cotinine indicate active tobacco use or use of nicotine replacement therapy (NRT); moderate concentrations indicate a smoker who has not had tobacco or nicotine for two to three weeks; lower levels may indicate a non-smoker who has been exposed to environmental tobacco smoke; and very low to nondetectible concentrations are found in non-smokers who have not been exposed to environmental tobacco smoke or a smoker who has not used tobacco or nicotine for several weeks. An alcohol test called the EtG can detect alcohol up to 80 hours after very extensive drinking episodes; however, in 2006, the Substance Abuse and Mental Health Services Administration (SAMHSA) released an advisory saying that the EtG test was not appropriate for assessing alcohol use because it is highly sensitive and unable to distinguish between alcohol absorbed into the body from actual consumption and from exposure to many common commercial and household products that contain alcohol. Laboratory tests can capture instances of other drug use for days or weeks after use, depending on the drug.
} 
of errors such as false positives, contamination or mislabeling exists. ${ }^{56}$

Given these concerns, biological tests may be most useful in verifying conclusions drawn from other forms of screening and assessment instruments ${ }^{57}$ or in specific circumstances where any substance use is sanctioned (e.g., use by those who are underage, pregnant, have health problems, in jobs that require sustained attention and vigilance* or by juvenile or criminal offenders). ${ }^{58}$

\section{Brief Interventions and Treatment Referrals}

For those who screen positive for risky use of addictive substances that does not meet the threshold of clinical addiction, providing brief interventions is an effective, low-cost approach to reducing risky use. ${ }^{59}$

Brief interventions generally include feedback about the extent and effects of patients' substance use and recommendations for how they might change their behavior. ${ }^{60}$ Brief interventions often involve motivational interviewing techniques ${ }^{\dagger}$ and substance-related education; the exact approach may differ depending on the target population. ${ }^{61}$ Brief interventions can be conducted face-to-face, over the phone or via computerized feedback to patients. ${ }^{62}$ They can be performed by health professionals after relatively limited training. ${ }^{63}$ Providing brief interventions can save lives and reduce a broad range of negative health and social consequences including addiction.

\footnotetext{
* e.g., truck drivers, air traffic controllers, physicians.

${ }^{\dagger}$ Motivational interviewing is a patient-centered approach to counseling. Counselors attempt to initiate behavior change through reflective listening. They help patients resolve any ambivalence toward reducing their substance use through an empathetic discussion of the discrepancies between their values and self-image and their current substance use behavior. Counselors stress ideas of self-efficacy and optimism to their patients. (For a more detailed discussion, see Chapter V.)
}

For individuals showing signs of addiction, providing treatment or referral to specialty care is critical to managing the condition and preventing further health and social consequences. $^{64}$

\section{Tobacco}

Brief interventions for smoking and other tobacco use can be provided by trained healthcare practitioners and generally occur in clinical and primary-care settings. ${ }^{65}$ According to clinical guidelines, practitioners should provide brief interventions based on the "Five A's":

- Ask. The process begins with inquiries about tobacco use, which should be made during every visit.

- Advise. Individuals who smoke should be advised in a clear, strong and personalized manner to quit.

- Assess. Practitioners should determine whether or not a patient is willing to attempt to quit.

- Assist. If the patient is willing to attempt to quit, the practitioner should provide assistance by helping patients create a quit plan, providing counseling and pharmaceutical treatment recommendations, offering problem solving and skills training and distributing supplementary educational materials. One intervention approach is known as the "Five R's" where a technique is implemented to help motivate patients to quit smoking. ${ }^{66}$

- Arrange. Schedule follow-up contact, either in person or by phone. ${ }^{67}$

\footnotetext{
\$ See Chapters V and VI.
} 


\section{The “Five R's”}

Employing an empathetic counseling style, practitioners should:

- Relevance: Encourage patients to indicate why quitting is personally relevant.

- Risks: Help patients identify the acute, long-term and environmental risks they take by continuing to smoke.

- Rewards: Help point out the rewards that will come with cessation.

- Roadblocks: Ask patients to identify any roadblocks they may face during their attempt to quit and suggest potential solutions for each.

- Repetition: Repeat this process every time they see the patient.

Brief interventions for smoking cessation should include a follow-up visit scheduled shortly after a patient's quit date. ${ }^{68}$

A more simple approach that is gaining traction is to restrict the brief intervention to the first two "A's"--Ask and Advise--and then refer the patient, usually to a telephone quitline or a smoking cessation service, where the other three "A's" are performed. ${ }^{69}$ A recent review of research found that compared to just providing advice, physicians who offered assistance in quitting to all patients who smoke regardless of their stated willingness to quit, could prompt an additional 40 percent to 60 percent of smokers to try quitting. ${ }^{70}$

There is some evidence to suggest that shorter interventions for smoking cessation may be more successful than longer ones, perhaps because of the direct, instructional nature of the brief intervention. A study of smokers with addiction involving alcohol enrolled in an addiction treatment program found that 35 percent of those who received a 10-minute brief intervention for tobacco use were abstinent a month later compared to only 13 percent of those who received a more extensive, 50-minute

\footnotetext{
* In the form of counseling or nicotine replacement therapy (see Chapter V).
}

motivational interview session. ${ }^{\dagger}$ The brief advice session directly told patients to quit smoking and assisted participants in accessing additional information or help to reach that goal. In contrast, the more extensive motivational interview focused on the advantages and disadvantages of smoking, imagining life without smoking, providing personalized feedback and setting stage-specific goals. ${ }^{71}$

\section{Alcohol and Other Drugs}

Based on screening results, brief interventions for alcohol and other drug use begin with feedback about the quantity and frequency of a patient's substance use, and the potential consequences the patient may face as a result. ${ }^{72}$ Brief interventions typically involve the counseling technique of motivational interviewing. ${ }^{\$ 73}$

Health care practitioners trained in providing brief intervention services try to help patients decide to change their substance use behavior in light of the adverse medical and social consequences of risky use of addictive substances and the many ways in which it may conflict with their values and goals, and then offer advice on how patients may do so. ${ }^{74}$

The advice for adults ${ }^{\S}$ may include:

- Setting a specific limit on consumption;

- Learning to recognize the antecedents of substance use and developing skills to avoid use in those situations;

\footnotetext{
$\dagger$ After six months, the abstinence rates of both groups had fallen to 13 percent and two percent, respectively.

* See Chapter V.

$\S$ Given the dangers of substance use during the vulnerable period of brain development that continues into young adulthood, the advice for adolescents and young adults who have not reached the legal ages for smoking or drinking alcohol, should focus less on limiting risky use and more on abstaining from use of all addictive substances.
} 
- Planning ahead to limit drinking and other drug use;

- Pacing one's use; and

- Learning to cope with the everyday problems that may lead to substance use. ${ }^{75}$

"FRAMES":
Key Elements of a Brief Intervention for
Reducing the Risky Use of
Alcohol and Other Drugs
76
A frequently-used brief intervention for the risky
use of alcohol and other drugs includes six core
elements identified and verified through
empirical research that can be summed up by the
acronym "FRAMES":
- Feedback regarding personal risk or
- $\quad$ Responsibility for change;
- Advice to change;
- Menu of options for reducing substance use;
- $\quad$ Empathetic counseling style; and
Self-efficacy in terms of ability and
responsibility to change.

Helping patients understand how they can change their behavior and encouraging them to be optimistic about their ability to do so are important components of most brief interventions. ${ }^{77}$ Interventions delivered in an empathetic counseling style are more effective than those that rely on confrontation or coercion. ${ }^{78}$ While brief interventions can avert the development of a more serious substance use problem, ${ }^{79}$ individuals with the disease of addiction require more intensive care and should be treated or referred to specialty care.

\section{Effectiveness of Screening and Brief Interventions}

The combination of screening and brief interventions ${ }^{*}$ has shown positive results for tobacco, ${ }^{80}$ alcohol, ${ }^{81}$ illicit drugs and the misuse

\footnotetext{
${ }^{*}$ Sometimes referred to as SBI.
}

of controlled prescription drugs, ${ }^{82}$ across many settings and population groups. ${ }^{\dagger} 83$

Several large-scale studies have explored the effectiveness of screening and brief interventions in reducing the consumption of addictive substances as well as the serious problems and costs that accompany such behavior, including visits to emergency departments, hospitalization, high-risk injection drug use, criminal activity, psychiatric stress and depression. ${ }^{84}$ One study found that adult patients ${ }^{\ddagger}$ receiving a brief intervention after a positive screen by their primary care physicians for risky alcohol use experienced 20 percent fewer emergency department visits and 37 percent fewer days of hospitalization than patients who did not receive the intervention. ${ }^{85}$ Participants who received screening and brief interventions also had significantly fewer arrests for alcohol or controlled drug violations (two vs. 11 arrests). ${ }^{86}$

A large-scale study conducted in a broad range of medical settings across six states found that 22.7 percent of the patients in the study screened positive for risky alcohol or other drug use or addiction. Sixteen percent of the patients who were screened received a recommendation for a brief intervention, 3.2 percent received a recommendation for brief treatment and 3.7 percent received a referral to specialty

\footnotetext{
${ }^{\dagger}$ It is difficult to compare the effectiveness rates of different research trials and programs as many of them use restricted populations and vary in the length and intensity of the intervention. Despite these methodological discrepancies, there are strong data showing the effectiveness of screening and brief interventions in addressing risky use of addictive substances. Studies reporting successful outcomes tend to reflect situations where participation was voluntary and may not reflect outcomes in a population with mandatory participation. Furthermore, most of these studies examine the use of screening and brief interventions in primary care settings as it pertains to alcohol use and not to other drug use.

* Between the ages of 18 to 65 who visited a physician's office for routine care.
} 
treatment. ${ }^{*}$ The study found that brief interventions, brief treatments or referrals to specialty treatment for those who screened positive for risky alcohol and/or illicit drug use at baseline were associated with a 38.6 percent reduction in rates of heavy alcohol use and a 67.7 percent reduction in rates of illicit drug use; however, it is important to note that this study was not a randomized controlled trial. ${ }^{87}$

Screening and brief intervention services also have proven effective in increasing entry rates to specialized addiction treatment programs for patients with addiction ${ }^{88}$ and for patients who are required to wait to enter standard treatment programs. ${ }^{89}$

\section{Tobacco}

When clinics and medical offices require screening for tobacco use, tobacco-using patients are likelier to achieve smoking cessation. This may be because clinics and medical offices that have tobacco screening systems in place are approximately 1.7 times as likely to provide smokers with interventions as offices that do not require patients to undergo tobacco screening. ${ }^{90}$

A systematic review of 31 studies examining the effects of smoking interventions provided by nurses in hospital settings found that the smoking cessation rate of patients who received brief interventions was 1.3 times the cessation

\footnotetext{
* All patients in the study were screened for alcohol and other drug use; however, different instruments were used in each study site and the thresholds for being classified as being a risky substance user varied considerably among the sites. Those with moderate risk substance use patterns received brief interventions such as the FRAMES intervention or motivational interviews; those with heavy use patterns received brief treatment which consisted of brief but more intense interventions such as enhanced motivational interviews (MI), motivational enhancement therapy (MET) or cognitive behavioral therapy (CBT) (see Chapter $\mathrm{V}$ for an explanation of these therapies); and those who met clinical criteria for addiction were referred to specialty treatment. There was no control or comparison group in this study.
}

rate of control group patients..$^{\dagger 91}$ Another largescale study found that smokers who reported that they had received a brief smoking cessation intervention from their primary care provider were more than three times likelier to quit smoking than those who did not receive such counseling (34.9 percent vs. 10.5 percent among patients without co-occurring addiction involving alcohol or other drugs or mental health disorders and 31.3 percent vs. 6.0 percent among those with such co-occurring conditions). ${ }^{92}$

\section{Alcohol}

Screening and brief interventions for risky alcohol use have demonstrated efficacy in primary care and emergency/trauma settings. ${ }^{93}$ One study, conducted in a primary care setting with patients who screened positive for risky alcohol use, found a greater decline in the number of people who reported binge drinking ${ }^{\S}$ among those receiving a brief intervention (from 85.0 percent at baseline to 61.5 percent three years after the first intervention) compared to those in the control group (from 86.9 percent at baseline to 70.7 percent three years after the first intervention). ${ }^{* *}$ Likewise, there was a greater decline in the number of people who reported heavy drinking ${ }^{\dagger \dagger}$ among those receiving the brief intervention (from 46.7 percent at baseline to 23.2 percent three years after the first intervention) compared to those in the control

\footnotetext{
${ }^{\dagger}$ The effects of the interventions appear to be strongest among cardiac rehabilitation patients. * As indicated by a positive response to the question, In the past 12 months, did any of the general medical providers talk to you about quitting or avoiding smoking?

$\S$ Defined in this study as consuming more than five drinks on one occasion during the previous 30 days.

${ }^{* *}$ The brief intervention included a health information booklet, two face-to-face, 15-minute intervention sessions with a physician spaced one month apart and two follow-up calls from nurses during the weeks following their interventions; the control group only received the information booklet. Patients were randomly assigned to one of these two conditions.

${ }^{1 \dagger}$ Consuming more than 20 drinks during the past week for men and more than 13 drinks during the past week for women.
} 
group (from 49.2 percent at baseline to 34.6 percent three years after the first intervention). The intervention participants also experienced 55 percent fewer nonfatal motor vehicle crashes and 46 percent fewer arrests. ${ }^{94}$ Another study found that a 30-minute brief intervention was associated with significantly fewer at-risk patients ${ }^{*}$ being arrested for driving under the influence of alcohol during the next three years (11.3 percent of brief intervention patients had a DUI arrest vs. 21.9 percent of control group patients). ${ }^{95}$

A systematic review of emergency departmentbased screening and brief intervention programs found that 82 percent of the studies included in the review demonstrated a positive effect. ${ }^{\dagger 96}$ Even in non-emergency cases and cases seemingly unrelated to substance use (e.g., general surgery and anesthesia consults), routinely screening all pre-surgical patients for risky use and addiction can prevent complications from surgery, and brief interventions or referral to treatment can prevent the presenting condition from worsening. ${ }^{97}$ Brief interventions conducted in trauma centers for patients who engage in risky alcohol use have been associated with a 47 percent reduction in re-injuries requiring emergency department or trauma center admission and a 48 percent reduction in re-injuries requiring hospital admission. ${ }^{98}$ Brief interventions with follow-up are more effective than single-contact interventions: ${ }^{99}$ a review comparing multisession and single-session brief alcohol interventions found that those who received multi-session brief interventions reported a 13 to 34 percent greater reduction in the average number of drinks per week after six to 12

\footnotetext{
* Specifically, those who have a BAC $\geq 80 \mathrm{mg} / \mathrm{dL}$ or a score $\geq 8$ on the AUDIT screening instrument.

${ }^{\dagger}$ Ninety percent showed a decrease in alcohol consumption, 13 percent showed a decrease in emergency department visits and hospitalizations, 13 percent showed a decrease in negative social consequences and 13 percent showed an increase in referrals for follow-up and/or treatment.
}

months than those receiving single-session brief interventions. ${ }^{\ddagger} 100$

\section{Other Drugs}

Although the research on screening and brief interventions for other drug use is quite limited and therefore data supporting these services is scarcer than in relation to tobacco and risky alcohol use, ${ }^{101}$ the available research suggests that screening and brief interventions can reduce other drug use among patients in primary care facilities, emergency departments, trauma centers and other hospital units. ${ }^{102}$ In one study conducted at six health care sites across the country, patients who screened positive for drug use (other than tobacco or alcohol) received screening and brief interventions, brief treatment or referrals to treatment based on the severity of their drug use. Six months after receiving these interventions, the percentage of patients reporting past month marijuana, cocaine, methamphetamine, heroin or other drug use-including the misuse of prescription sedatives and opioids as well as hallucinogens and inhalants--decreased significantly. ${ }^{103}$ However, this study did not contain a control condition so the extent to which substance use would have decreased without these interventions cannot be determined. (Figure 4.A)

Patients in the study reported significant increases in health status (from fair to good) and employment (from 31.3 percent to 36.1 percent) at the six-month follow-up as well. There also were significant decreases in the percentage of patients reporting past-month emotional problems (from 25.6 percent to 17.6 percent), arrests (from 12.2 percent to 4.4 percent) and homelessness (from 11.8 percent to 6.4 percent). ${ }^{104}$

\footnotetext{
* Ranges rather than single values are cited because the figures come from a meta-analysis comparing results from multiple studies.
} 


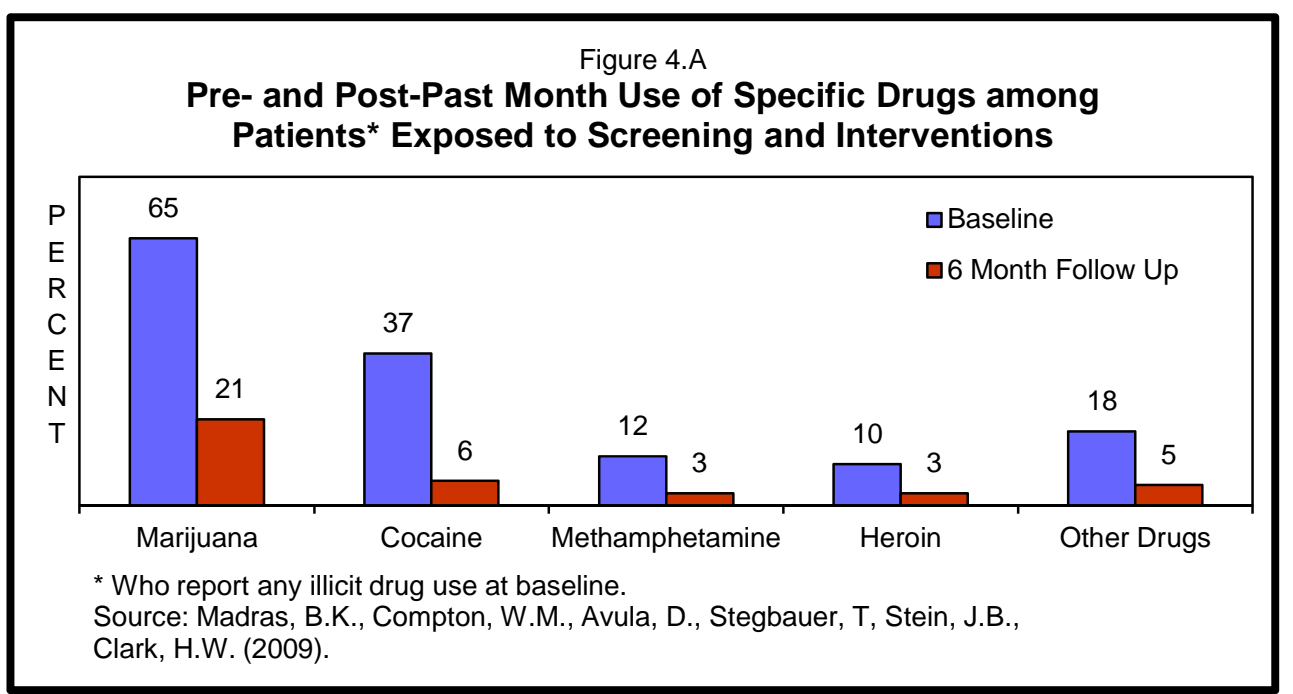

Implementing Screening and Brief Interventions in Health Care and Other Settings

While screening and brief interventions can be provided in a broad range of venues, ${ }^{107}$ health care settings may be the most effective. Physicians and other Another study found that a screening and brief intervention program ${ }^{*}$ for heroin and cocaine users implemented during a routine medical visit was related to greater abstinence among intervention versus control participants from cocaine use ( 22.3 percent vs. 16.9 percent) and heroin use ( 40.2 percent vs. 30.6 percent) six months following the intervention. ${ }^{105}$

Brief interventions also can reduce regular amphetamine use. Six months after screening positive for amphetamine use, individuals who received brief interventions ${ }^{\dagger}$ were significantly likelier to be abstinent than users who received only self-help booklets. Amphetamine users who received brief interventions also showed decreased psychiatric distress scores and depression levels. ${ }^{106}$

\footnotetext{
${ }^{*}$ Consisting of toxicological and questionnaire screening during routine care at an inner-city teaching hospital, as well as brief motivational interviews, active referrals, a list of treatment providers and a follow-up booster phone call. ${ }^{\dagger}$ In this study, each participant had four 45 - to 60 minute individual therapy sessions guided by a therapist manual and a self-help booklet. The sessions included role-play and take-home exercises, concentrating on coping strategies and relapse prevention. The first session focused on increasing motivation to reduce drug use, the second on reducing cravings through muscle relaxation and self talk, the third on controlling thoughts about drug use and the fourth on coping with lapses and developing skills to use in high-risk situations.
}

health care providers, including dental professionals, nurses and pharmacists, typically are a consistent, trusted and influential presence in the lives of children and adults and their professional position grants them the authority and credibility to deliver effective, evidencebased interventions to patients at risk for complications related to their substance use, including addiction. Part of the success of incorporating these services for risky use of addictive substances into standard medical practice is that people tend to be more receptive to health messages once they are in a health care setting. Patients view additional screening, information, brief intervention or referral to treatment as part of the health care they sought initially. ${ }^{108}$ The use of technology to assist in the completion of screening and brief interventions holds promise for helping to integrate these practices into routine health care delivery. ${ }^{109}$

\section{Primary Care}

About 80 percent of Americans ${ }^{\ddagger}$ visited at least one physician or other health care professional in the past year, ${ }^{110}$ and the American Society of Addiction Medicine (ASAM) estimates that more than two-thirds of people with addiction are in contact with a primary or emergency care physician about twice a year. ${ }^{111}$ Integrating screening and brief interventions into routine medical check-ups can be an effective way of

\footnotetext{
Ages 18 and older.
} 
identifying smoking, risky drinking and other drug use before negative consequences occur, including the development of addiction. ${ }^{112}$

\section{The 2010 National Drug Control Strategy Recommends:}

- Increasing health care providers'

knowledge and use of screening and brief intervention techniques through enhanced medical and nursing school educational programs;

- Increasing screening and early intervention for substance use in all health care settings; and

- Increased reimbursement for screening and brief interventions in primary care. ${ }^{113}$

Although the most promising research findings regarding the benefits of implementing these services in health care and other settings have been found in primary care settings, clinical trials are lacking in this area. ${ }^{114}$ Given the strong scientific evidence to date supporting the use of screening and brief interventions, particularly for smoking and risky alcohol use, several national medical societies already have endorsed this approach, including:

- The American Society of Addiction Medicine (ASAM) has identified screening and brief interventions as an effective method for catching substance-related problems early and preventing the development of addiction; ASAM encourages medical and insurance professionals to redesign their primary care and funding practices to accommodate these programs. $^{115}$

- The American Academy of Family Physicians recommends that adults, including pregnant women, be screened for tobacco use and provided with cessation interventions or brief counseling and that screening and brief interventions for alcohol use be used in health care settings as well. ${ }^{116}$
- The American College of Obstetricians and Gynecologists' Committee on Ethics declared that obstetrician-gynecologists have an ethical obligation to conduct universal screening, brief intervention and referral to treatment for their patients. ${ }^{117}$

- The American Dental Association advises dentists to address the issue of risky use and addiction with patients and refer them to appropriate addiction treatment if needed. ${ }^{118}$

Even the United States Preventive Services Task Force (USPSTF), which is constrained by available medical evidence, has recommended screening and brief intervention for risky alcohol use for all adults in primary care settings, ${ }^{2} 119$ and that all adults, including pregnant women, be screened for tobacco use and receive appropriate cessation counseling. ${ }^{\dagger 120}$ And, the National Quality Forum has endorsed screening and brief interventions for tobacco and alcohol use in general health and mental health-care settings. ${ }^{121}$

Adolescent Health Care. Screening the adolescent population for substance use may be the single most effective preventive step that can be taken to address the problem of risky use and

\footnotetext{
* The USPSTF recommends this service with a B rating, indicating that the net benefits of the intervention outweigh any potential harms. ${ }^{\dagger}$ The USPSTF recommends this service with an A rating, indicating that the net benefits of the intervention substantially outweigh the harms; for pregnant women, the Task Force recommends that the counseling services be tailored to pregnancy. As a result of limited research on the topic, the USPSTF concluded that there currently is insufficient clinical evidence to determine the balance of benefits and harms of routine (asymptomatic) screening or interventions for tobacco or alcohol use among adolescents or for screening individuals for illicit drug use in primary care practice settings. A recent report by the USPSTF, however, identified 11 highpriority areas in preventive medicine that have critical evidence gaps that, if addressed through targeted research, are likely to result in recommendations for practice. Among these 11 high-priority areas are screening and providing counseling for adolescent alcohol use and screening all individuals for illicit drug use.
} 
addiction in this country since the consequences of risky use of addictive substances among adolescents are so profound and individuals with addiction typically began engaging in risky use in adolescence. ${ }^{122}$

The primary care office is a key setting for adolescent screening and brief intervention related to substance use; using computer technology to achieve these ends meets the needs of both patients and physicians by making the process more efficient and easier for physicians, producing more honest responses from patients, and increasing both providers' and patients' satisfaction with the encounter. ${ }^{126}$

--John R. Knight, MD

Director,

Center for Adolescent Substance Abuse Research Associate Professor of Pediatrics, Harvard Medical School Senior Associate in Medicine, Associate in Psychiatry, Children's Hospital Boston

The U.S. Public Health Service's clinical practice guideline for tobacco use and dependence recommends that clinicians ask adolescent patients about their tobacco use and provide them with brief interventions to aid in quitting. ${ }^{123}$

Professional medical associations such as the American Medical Association (AMA) and the American Academy of Pediatrics (AAP) support screening adolescent patients for substance use, and promote the use of screening and brief intervention techniques among their constituents: ${ }^{124}$

- The AMA's Guidelines for Adolescent Preventive Services recommend that physicians ask all adolescents annually about their use of tobacco, alcohol and other drugs, including over-the-counter drugs, controlled prescription drugs and anabolic steroids. ${ }^{125}$

- The American Academy of Pediatrics (AAP) encourages pediatricians to:
$>$ Be knowledgeable about addiction and skilled in recognizing the risk factors for substance use among young people;

$>$ Screen all patients for substance use at annual medical examinations and, if possible, at other medical visits as well using screening methods that are validated, nonjudgmental and that protect confidentiality;

$>$ Provide brief interventions and refer patients, when necessary, to treatment or specialty care;

$>$ Consider and address co-occurring disorders; and

$>$ Promote substance use prevention programs and media responsibility with regard to depictions of substance use. ${ }^{127}$

\section{Emergency and Trauma Care}

Emergency Departments (EDs) and trauma centers are particularly critical venues for screening and brief intervention since so many emergency and trauma cases involve risky substance use. One study found that up to 31 percent of all patients treated in hospital EDs ${ }^{*}$ who were screened for risky alcohol use ${ }^{\dagger}$ had a positive result. ${ }^{\$ 128}$ Many trauma patients meet criteria for addiction; a study of seriously injured trauma patients shows that 24.1 percent of them met diagnostic criteria for alcohol dependence and 17.7 percent for other drug dependence. ${ }^{129}$ Further, substance-using individuals frequently rely on EDs for much of their health-care needs. $^{130}$

\footnotetext{
* Fifty percent of severely injured trauma patients and 22 percent of minor trauma patients receive their injuries under the influence of alcohol.

${ }^{\dagger}$ Based on the CAGE questionnaire.

* This most likely is a conservative estimate since many EDs do not screen for alcohol or other drug use because of insurance laws (i.e., the Uniform Accident and Sickness Policy Provision Law-UPPL) restricting payment if the reason for admission was substance related.
} 
EDs and trauma centers are ideal settings for the "teachable moment" that is thought to be one of the key components of the positive impact of a brief intervention. ${ }^{131}$ Most individuals who experience substance-related accidents and injuries do not meet clinical criteria for addiction ${ }^{132}$ but are excellent candidates for brief interventions. One study of young adults admitted to a hospital emergency department found that those who were alcohol-involved ${ }^{*}$ and who received a brief motivational intervention followed by two telephone booster sessions showed greater reductions in alcohol use than those who just received one to three minutes of feedback, in which they were provided information about how much they drink, what happens when they drink and how their alcohol intake compares to their peers (a reduction of up to 53 percent vs. 18 percent). ${ }^{133}$

Research [related to screening and brief intervention] began in the ED. The earliest study--conducted in 1957--was a controlled trial with 200 dependent drinkers at Massachusetts General Hospital. Patients who had a nonjudgmental, respectful conversation inviting them to attend an outpatient program were more likely than other patients to complete one appointment (65.0 percent vs. 5.4 percent) and five appointments (42.0 percent vs. 1.1 percent). ${ }^{140}$

ED and trauma physicians were some of the first to recommend the adoption of screening and brief interventions, ${ }^{134}$ their focus to date has been on excessive alcohol use which is the leading risk factor for injury: ${ }^{135}$

- The American College of Emergency Physicians recommends screening and brief interventions for alcohol use. ${ }^{136}$

- The American College of Surgeons Committee on Trauma requires that Level I and Level II trauma centers have a mechanism in place to identify patients who

\footnotetext{
* Screened positive for alcohol use, reported drinking in the six hours before their accident or had a history of risky drinking (as determined by their AUDIT score).
}

engage in risky alcohol use and Level I Centers must have a mechanism in place to intervene with these patients. ${ }^{\dagger 137}$

An important point of access to the health care system for adolescents is through the ED; approximately 12.7 percent of substance-related ED visits are made by individuals ages 12 to 20 years old ( 5.7 percent by those ages 12 to 17 and 7.0 percent by those ages 18 to 20$).^{138}$ Interventions conducted in the ED may reach adolescents who do not attend school regularly or who do not have a primary care physician. ${ }^{139}$

\section{Health Care for Pregnant Women ${ }^{\ddagger}$}

Given the considerable impact of substance use on reproductive health and pregnancy, women (especially those who are pregnant or of reproductive age) are an ideal target for screening and brief intervention services. ${ }^{141}$ Because there is no universally safe level of substance use during pregnancy, any use should be screened for and addressed. The American College of Obstetricians and Gynecologists recommends that because of these risks, all women--regardless of present pregnancy status-should be screened for alcohol use at least yearly and provided with intervention and referral services if necessary. ${ }^{142}$

One study found that pregnant smokers who received brief counseling and behavioral interventions in a public maternity hospital had a higher rate of smoking abstinence (33.3 percent) than pregnant smokers who received usual care (8.3 percent). ${ }^{143}$ Another study found that pregnant smokers in community health centers who received brief interventions were more likely to be abstinent by the end of their pregnancy than women receiving usual prenatal care (past-month abstinence rate of 26 percent vs. 12 percent). However, in this study, the higher rates of smoking abstinence following a

\footnotetext{
${ }^{\dagger}$ The focus in this area primarily has been on alcohol rather than tobacco or other drugs.

${ }^{\star}$ Research on screening and brief interventions for pregnant women focuses primarily on tobacco and alcohol use. No studies of the use of such services in pregnant women who use other drugs were found.
} 
brief intervention disappeared at three and sixmonth post-partum follow-ups, ${ }^{144}$ indicating a need for more intensive treatment services.

Screening and brief interventions in prenatal care settings have been found to reduce alcohol use significantly, ${ }^{145}$ as well as the chances of low birth-weight deliveries. ${ }^{146}$ Brief interventions for alcohol use among pregnant women are effective even when provided in a community setting by non-medical professionals. ${ }^{147}$ Programs that include spouses and partners are particularly effective. ${ }^{148}$

\section{Mental Health Care}

Despite the high rate of co-occurring addiction and mental health disorders, ${ }^{149}$ screening and brief interventions for risky substance use are not common practice in mental health settings and are not standard practice in the field of psychiatry. ${ }^{150}$

There are effective tools for screening patients with mental health conditions in mental health settings for risky use of addictive substances ${ }^{151}$ and there is evidence that interventions can be effective in addressing such use among those with psychiatric conditions. ${ }^{152}$ For example, a study evaluating the effectiveness of a screening and brief intervention program in a primary health and mental health care setting at a university found that six weeks after receiving the intervention, participants decreased their alcohol use. ${ }^{*} 153$

\section{Dental Care}

Dental professionals can play a unique role in detecting substance use among their patients, providing brief interventions and referring patients to treatment. ${ }^{154}$ Risky use and addiction have a significant impact on multiple components of dentistry including patients' oral

\footnotetext{
* Including the average number of drinks consumed per week over the past 30 days, the highest number of drinks consumed on one occasion in the past 30 days and the number of times in the preceding two weeks participants had consumed five or more drinks on one occasion.
}

health ${ }^{155}$ and the safety of common treatments and interventions including the prescription of controlled medications such as opioid pain relievers. ${ }^{156}$ The fact that dental health maintenance and treatment require routine and often repeated visits makes dental professionals a consistent and potentially influential presence in the lives of people who engage in risky use of addictive substances. ${ }^{157}$ Dental patients are receptive to their dentists' involvement in the prevention and treatment of risky use and addiction. A 2005 survey of patients visiting an emergency dental clinic found that 80 percent believed dentists should ask their patients how much alcohol they drink; 90 percent believed that dentists should warn patients to drink less or quit if it is affecting their oral health. ${ }^{158}$

\section{Pharmacies}

The responsibilities of pharmacists with regard to the prevention and early intervention of risky use and addiction extend to administering prescription medications; pharmacists are the best source of information regarding the safe and effective use of medications and the adverse effects that arise from their misuse. They also can be instrumental in controlling the diversion of prescription medications for misuse by monitoring the number of prescriptions filled by a patient, looking for false or altered prescription forms ${ }^{159}$ and recognizing when a patient is "doctor shopping" or in need of treatment. ${ }^{160}$

\section{High School, College and University Settings}

Screening and brief intervention programs reduce risky use of addictive substances among students by changing their attitudes, beliefs and expectations regarding tobacco, alcohol and other drug use. ${ }^{161}$

School health programs, in collaboration with primary care providers, are important opportunities for screening adolescents and young adults for substance use, primarily

\footnotetext{
${ }^{\dagger}$ The practice of patients visiting various health care providers to obtain multiple prescriptions for the drugs they misuse.
} 
because young people spend a majority of their time in school. Few schools, however, take advantage of this opportunity. ${ }^{162}$ A CASA Columbia survey of school personnel, conducted for its 2011 report, Adolescent Substance Use: America's \#1 Public Health Problem, found that only 7.4 percent reported that their schools screen all students for signs of risky alcohol or other drug use; 9.0 percent of high school teachers reported that their schools screen particular groups of high-risk students for signs of risky alcohol or other drug use. ${ }^{163}$

The college setting also is ideal for intervening with young people at risk via screening and brief interventions because of the high rates of substance use in the college population; an estimated 67.2 percent $^{*}$ are risky users or have addiction. ${ }^{\dagger 169}$ To date, the majority of the screening- and intervention-related research among college students has focused on alcohol, most likely because alcohol typically is the substance most likely to be used by college students. ${ }^{170}$ Screening and brief interventions have proven successful in reducing risky alcohol use and its consequences in this population. ${ }^{171}$ The Department of Education recommends the implementation of screening and brief intervention programs in all college health centers. $^{172}$

\footnotetext{
${ }^{*}$ Of enrolled college students, ages 18-22.

$\uparrow 43.9$ percent are risky users but do not have addiction and 23.3 percent have addiction, i.e., meet clinical diagnostic criteria for past month nicotine dependence and/or past year alcohol and/or other drug abuse or dependence.
}

\section{Brief Alcohol Screening and Intervention of College Students (BASICS) Program}

The BASICS program targets risky drinkers (defined as those who drink heavily and are at risk for or already have experienced problems related to alcohol use) between the ages of 18 and $25 .{ }^{164}$ Students are identified for participation in the programs through screening or through referral from medical, housing or disciplinary services. ${ }^{165}$ The program consists of two one-hour interviews and a brief online assessment survey about drinking habits and history, as well as beliefs and attitudes, while giving instructions for monitoring one's own drinking between interviews. In the second interview, students receive personalized face-toface feedback about their alcohol use compared with peer norms, consequences of and risk factors for drinking and strategies for reducing alcohol use and related problems. ${ }^{166}$ The BASICS program has proven to be effective and cost-effective. ${ }^{167}$ In one study, students who received the BASICS intervention as college freshmen were more likely than risky drinkers who did not participate in the intervention to have reduced their alcohol consumption four years later (67 percent vs. 55 percent). ${ }^{168}$

\section{Justice Settings}

Juvenile justice programs and facilities are ideal venues for screening and brief interventions; CASA Columbia's 2004 report, Criminal Neglect Substance Abuse, Juvenile Justice and The Children Left Behind, found that four of every five children and adolescents in the juvenile justice system are substance involved. ${ }^{\$ 13}$ Unfortunately, jurisdictions typically do not provide adequate screening or brief intervention services ${ }^{174}$ even though there are several screening tools that have been validated for use with juvenile offenders. ${ }^{175}$

\footnotetext{
*Under the influence of alcohol or other drugs while committing their crime, test positive for drugs, are arrested for committing an alcohol or other drug offense, admit to having a substance use problem or share some combination of these characteristics.
} 
One national study of juvenile residential facilities found that 15 percent of the facilities that reported information about evaluating residents for substance-related issues ${ }^{*}$ indicated that they did not screen at all, 20 percent reported that they screened some youth and 64 percent reported that they screened all youth; 41 percent reported using a standardized screening instrument. ${ }^{176}$ Even those facilities that screen youth and use a standardized screening instrument do not necessarily provide appropriate interventions or treatment based on screening findings. ${ }^{177}$

The criminal justice system includes even higher concentrations of individuals whose crimes are linked to their use of alcohol and/or other drugs. CASA Columbia's 2010 report on substance use in this population, Behind Bars II: Substance Abuse and America's Prison Population, found that 84.8 percent of inmates in America are substance involved. ${ }^{\dagger 178}$ Screening can be used to identify those in need of intervention and to make appropriate referrals to treatment, which ultimately can help to reduce crime and prison overcrowding and save taxpayer money. ${ }^{179}$

Despite abundant evidence of the efficacy of screening and brief interventions, ${ }^{180}$ standardized screening and interventions are not implemented regularly in justice settings. ${ }^{181}$ Although inmates are guaranteed the right to medical care, ${ }^{182}$ they routinely are denied access to appropriate screening, intervention and treatment services for the disease of addiction. ${ }^{183}$

\section{The Workplace}

The majority of people ages 18 and older who meet clinical criteria for addiction (63.8 percent)

\footnotetext{
* Of the 2,658 facilities in the final sample, 2,128 reported information about screening.

† Substance-involved inmates are those who either had a history of using illicit drugs regularly; met clinical criteria for addiction; were under the influence of alcohol or other drugs when they committed their crime; had a history of alcohol treatment; were incarcerated for a drug law violation; committed their offense to get money to buy drugs; were incarcerated for an alcohol law violation; or had some combination of these characteristics.
}

or who engage in risky use but do not have addiction (73.0 percent) are employed full or part time; ${ }^{184}$ individuals who engage in risky substance use or are addicted have higher rates of absenteeism, decreased work productivity and higher health care costs. ${ }^{185}$

If approached as a health issue, the workplace is an ideal venue for offering confidential screening, brief interventions and treatment referrals. Workplaces increasingly rely on Employee Assistance Programs (EAPs) ${ }^{186}$ confidential counseling programs for employees that offer assistance with health or other problems that can adversely affect job performance. ${ }^{187}$ One recent survey of human resource professionals found that 60 percent reported that their organizations offered employee wellness programs and 42 percent had health screening programs. ${ }^{188}$ Only recently, however, have there been attempts to utilize EAPs and similar workplace programs to provide screening and brief intervention services to employees. ${ }^{189}$

Researchers have demonstrated that providing these services to employees who contact an EAP program for assistance can be effective at identifying risky use and addiction. ${ }^{190}$ Several pilot studies" have demonstrated the effectiveness of identifying risky drinking ${ }^{\S}$ among employees via EAP services and of having those who were identified agree to follow-up counseling. ${ }^{191}$ According to a national employer survey, however, only 29 percent of employers offer screening to their employees $^{* *}$ for risky alcohol use and 60 percent of that group provide brief interventions. ${ }^{192}$ Comparable data on the proportion of employers that screen or provide interventions for employees who smoke or use other drugs are not available.

\footnotetext{
¥ Conducted in partnership with Aetna and OptumHealth.

${ }^{\S}$ Using the AUDIT.

** Of the employers that provide screening, most reported using the EAP, human resources, occupational health and safety and educational outreach programs to conduct the screening.
} 
Unfortunately, much workplace screening takes the form of drug testing and is used for compliance purposes. In this light, it frequently is viewed as infringing on workers' privacy; ${ }^{193}$ workers may worry about the confidentiality of their test results and whether they will be used to deny employment or to impose other forms of discrimination. ${ }^{194}$ The drug-testing process can be costly as well. ${ }^{195}$ In conducting workplace drug testing, the American Society of Addiction Medicine (ASAM) recommends that a positive drug test be used only as evidence that substance use occurred, not as evidence of functional impairment or addiction; the interpretation of drug test results should include the use of a credentialed Medical Review Officer; and controlled prescription medications should be included in the screening. ${ }^{196}$

\section{Government-Funded Social Service Systems}

Government agencies can play an important role in providing screening and brief interventions to a range of clients including those receiving housing, welfare and child protection, services for the elderly, and in HIV and STD clinics. ${ }^{197}$ Government-funded social service systems can identify substance use risk in individuals participating in their programs and provide interventions, treatments or referrals to specialty care when addiction is identified.

A significant proportion of individuals who participate in government programs have many risk factors for substance use and addiction and can benefit from screening and brief intervention services. Identifying individuals at risk and providing effective interventions for those in need may help to reduce their risk of further substance use, job loss, domestic violence and other crime and, ultimately, can lead to costsavings through decreased demand for government services. ${ }^{198}$ Despite the logic of this approach, there is little research on the effectiveness of screening and brief interventions in these populations and, instead of implementing these services, some states are now imposing or considering drug testing as a precondition for cash assistance and other services and a basis for denying both program eligibility and needed medical care. ${ }^{* 199}$

\section{Barriers to Effective Implementation of Screening and Brief Interventions}

The failure of our health care providers, schools, employers, justice programs and social service programs to implement effective screening, brief interventions and treatment referrals for those who engage in risky use of addictive substances represents a tremendous missed opportunity to help countless Americans avoid the far-reaching consequences of risky use and the disease of addiction. A significant barrier to change is the fact that services aimed at preventing and addressing risky use and addiction traditionally have not been paid for by health insurance plans; as a result, there are few incentives for health professionals to make them a priority in the care of their patients. To close the gap in needed services, specific barriers in addition to insufficient funding must be addressed, including insufficient training of health care and other professionals and a lack of specialty care providers; competing priorities and insufficient resources; and inadequate screening tools. ${ }^{200}$

\section{Insufficient Training}

Many physicians and other health professionals do not screen their patients for risky use of addictive substances, provide early interventions or treat or refer for specialty care, or they do so inadequately because they simply have not been properly trained. ${ }^{\dagger}$ Education about risky use and the disease of addiction, their impact on a patient's health and other medical conditions, and how to implement screening, interventions and treatment is not sufficiently integrated into medical education or residency training programs. $^{201}$ Among those programs that do address substance use and addiction, many have shortcomings in the curriculum such as insufficient instruction, limited number of

\footnotetext{
* The Constitutionality of these policies is being tested in the courts.

${ }^{\dagger}$ See Chapters IX and X.
} 
courses and limited time spent in courses on the topic of addiction. ${ }^{202}$

Inadequate training in risky use and addiction means that many physicians do not recognize these conditions in their patients, do not believe that substance-related interventions are effective, ${ }^{203}$ are unaware of what do with a patient who screens positive for risky use or addiction or are uninformed about effective resources to which they could refer patients in need of more in-depth assessment or of specialty treatment. $^{204}$

Most schools lack employees or consulting personnel with the necessary training and resources for identifying students who engage in risky use of addictive substances and attaining appropriate intervention services for those students who need them. ${ }^{205}$ CASA Columbia's survey of school personnel conducted for its 2011 report, Adolescent Substance Use: America's \#1 Public Health Problem, found that three-fourths of teachers are unable to identify a professional in their schools who would be able to help students with a substance use problem. Only 26.9 percent of teachers report that their schools train educators and other school staff to identify and respond to student substance use. ${ }^{206}$ Other national surveys likewise find that high school counselors and school psychologists generally report low competence in providing direct substance-related intervention services to students and a lack of relevant opportunities to become trained in doing so. ${ }^{207}$ Most schools have not set up partnerships with health care providers trained in conducting screening or early interventions to refer students who engage in risky use nor do they have links to appropriate treatment programs to which they refer students with addiction. ${ }^{208}$

CASA Columbia's 1999 report, No Safe Haven: Children of Substance-Abusing Parents, found that insufficient training among most child welfare workers and family court judges greatly contributes to the lack of effective screening practices in the child welfare system. ${ }^{209}$ CASA Columbia's research, published in its 2010 report Behind Bars II: Substance Abuse and America's Prison Population, found that probation and parole officers in the justice system need to be better trained as well. ${ }^{210}$

A related barrier to screening for risky use of addictive substances and providing brief interventions is the lack of effective and appropriate specialty treatment services available for referral when addiction is identified. ${ }^{211}$ Although having more trained addiction physician specialists is critical to providing care for those with severe forms of the disease, the lack of such specialty providers is not a legitimate barrier to providing screening and brief interventions. Neither is it a legitimate reason for general health care professionals to be unprepared to provide addiction treatment that does not require specialty care. These services are designed to be provided in non-specialty care settings, along with some forms of assessment and treatment (see Chapter V). The real barrier in this case remains the lack of knowledge about risky use and addiction and insufficient training in addressing these issues among health professionals.

\section{Competing Priorities/Insufficient Resources}

Lack of time and resources in the face of competing priorities is one of the most prominent barriers to implementation of screening and brief interventions among health professionals, ${ }^{212}$ school personnel ${ }^{213}$ and government agencies. ${ }^{214}$

Because the general model in medicine today (which is reflected or driven by insurance reimbursement structures) is procedure-oriented and reactive more than preventive, and because insurance coverage for screening and brief interventions for substance use has been rare, these services end up falling low on most physicians' lists of competing priorities for their time and attention. ${ }^{215}$

Schools and government agencies that administer justice programs or provide social services also face competing priorities and financial constraints that serve as barriers to

\footnotetext{
* See Chapter VIII.
} 
their implementation of screening and brief intervention services. ${ }^{216}$ Too often, state policymakers or administrators of these programs fail to understand how risky use and addiction impede progress in achieving their organizational goals.

The priorities of protecting patient confidentiality and maintaining an amicable and trusting doctor-patient relationship also may impede health professionals' implementation of these practices. ${ }^{217}$ While existing federal regulations ${ }^{*}$ protect the privacy of patients receiving addiction-related services in settings that are federally assisted and that are primary providers of these services, the regulations do not apply to other service venues. ${ }^{218}$ These ambiguous rules serve as a disincentive to health professionals to offer screening and brief intervention services and an incentive to keep substance-related services divorced from mainstream medicine. ${ }^{219}$

\section{Inadequate Screening Tools}

Another barrier to the effective implementation of screening and brief intervention has been that widely-used screening tools do not adequately identify the full range of incidences of risky use. $^{\dagger}$ These tools also do not follow consistent standards nor are they designed to be tailored to the unique patterns, symptoms and consequences of substance use of different age groups, genders, races/ethnicities and cultures or of individuals with co-occurring conditions, for whom a lower level of use may constitute risk relative to an average respondent. ${ }^{\ddagger 220}$ Further, most screening instruments focus on specific substances independently rather than identifying risky use of all addictive substances or risk for addiction as a unified disease. Reliance on

\footnotetext{
* Known as 42 CFR, Part 2 (Confidentiality of Alcohol and Drug Abuse Patient Records).

† See Appendix H.

* For example, any use of addictive substances by children, adolescents or pregnant women constitutes risky use; risky alcohol use is defined differently for women vs. men; and substance use by some individuals with co-occurring health conditions poses extreme risks even at levels that may be considered relatively safe among those without such conditions.
}

instruments that screen only for one type of substance increases the likelihood that risky use will not be adequately detected or that interventions will fail to reduce risky use across the board.

Only a few screening instruments have undergone rigorous scientific examination to determine their reliability, validity, sensitivity and specificity--key elements determining the effectiveness of such instruments. ${ }^{\S 221}$ Rather than using objective and standardized measures of risky use and risk for addiction, many of the more commonly-used screening instruments determine risk by relying on respondents' subjective reports of their own reactions to their use of addictive substances and the reactions of those around them, or their experiences of adverse social and health consequences associated with such use. For example, while risky alcohol use commonly is defined simply as drinking in excess of the established dietary guidelines of no more than one drink per day for women and two drinks per day for men, the CAGE Questionnaire simply asks four items related to the respondent's alcohol use--(1) Have you ever felt you should Cut down on your drinking? (2) Have people Annoyed you by criticizing your drinking? (3) Have you ever felt bad or Guilty about your drinking? (4) Have you ever had a drink first thing in the morning to steady your nerves or to get rid of a hangover (Eye-opener) $?^{222}$--none of which assesses directly the quantity and/or frequency of use.

Likewise, the CRAFFT, a six-item questionnaire for screening adolescents for risky alcohol and other drug use (excluding nicotine) asks: (1) Have you ever ridden in a Car driven by

\footnotetext{
${ }^{\S}$ See Appendix H. Reliability is whether the instrument produces the same results under the same conditions when taken on multiple occasions. Validity is how accurately the instrument measures what it is intended to measure. Sensitivity refers to an instrument's ability to identify correctly the presence of a condition; the higher the sensitivity the less likely the instrument is to produce false positives. Specificity is an instrument's ability to identify correctly those without the condition; the higher the specificity, the less likely the instrument is to produce false negatives.
} 
someone (including yourself) who was high or had been using alcohol or drugs? (2) Do you ever use alcohol or drugs to Relax, feel better about yourself or fit in? (3) Do you ever use alcohol or drugs while you are by yourself Alone? (4) Do you ever Forget things you did while using alcohol or drugs? (5) Do your Family or Friends ever tell you that you should cut down on your drinking or drug use? (6) Have you ever gotten into Trouble while you were using alcohol or drugs? An affirmative answer to each question is worth one point and a cut-off score of two is recommended for identifying risky alcohol and other drug use, ${ }^{223}$ even though any use of addictive substances by adolescents is considered risky. ${ }^{224}$

The typical screening process also may fail to distinguish those individuals with a higher level of substance involvement and the associated health and social consequences (including the risk for addiction) from those with lower levels of involvement--a distinction necessary for providing appropriate interventions. ${ }^{225}$ 


\section{Chapter V \\ Treatment and Management of Addiction}

Addiction $^{*}$ is a disease that can be treated and managed effectively at venues where regular medical care is delivered by physicians, including addiction physician specialists, and including a multi-disciplinary team of other health professionals using an array of evidencebased pharmaceutical and psychosocial ${ }^{\dagger}$ approaches. In accordance with standard medical practice for the treatment of other chronic diseases, best practices for the effective treatment and management of addiction must be consistent with the scientific evidence of the causes and course of the disease. Best practices require: ${ }^{1}$

- Comprehensive assessment of the extent and severity of the disease, determination of a clinical diagnosis, evaluation of cooccurring health conditions and the development of a tailored treatment plan;

\footnotetext{
* In this report, we have used the general term addiction to apply to those who meet criteria for pastmonth nicotine dependence based on the Nicotine Dependence Syndrome Scale (NDSS) and those who meet diagnostic criteria for past year alcohol and/or other drug abuse or dependence (excluding nicotine) in accordance with the Diagnostic and Statistical Manual of Mental Disorders (DSM-IV). (The DSM refers to substance abuse and substance dependence collectively as substance use disorders. The criteria for nicotine dependence in the NDSS parallel those of the DSM-IV.) This definition is consistent with the current move to combine abuse and dependence into an overarching diagnosis of addiction in the upcoming DSM-V.

${ }^{+}$Psychosocial therapy is a general term for nonpharmaceutical-based interventions and includes various forms of individual and group psychotherapy that address psychological, behavioral and social issues that contribute to risky substance use and addiction. Behavioral therapies are those psychosocial interventions that focus more directly on addressing the patient's substance-related behaviors, typically through behavioral reinforcement approaches, with less of an emphasis on the psychological or social determinants of their substance use.
} 
- Stabilization of the patient's condition via cessation of substance use and medically supervised detoxification, when necessary, as a precursor to treatment;

- Acute Care via evidence-based pharmaceutical and/or psychosocial addiction treatments, accompanied by treatment for co-occurring health conditions, delivered by qualified health professionals;

- Chronic Disease Management to help the patient maintain the progress achieved during acute treatment and prevent relapse. The process should be medically supervised and should involve pharmaceutical and/or psychosocial therapies and continued management of co-occurring health conditions as indicated; and

- $\quad$ Support Services including encouragement to participate in mutual support programs and the provision of auxiliary support services such as legal, educational, employment, housing and family supports.

\section{A Comprehensive Approach to Treatment}

A comprehensive approach to addiction requires recognition of addiction as a primary disease and that all substances and behaviors associated with addiction are addressed in treatment, rather than a focus only on an individual addictive substance. It is all too common, for example, for addiction involving nicotine to be ignored in the course of treating addiction involving alcohol or other drugs. Accordingly, when treating addiction, it is critical to recognize the high rates of co-occurrence of different manifestations of addiction and the possibility of the existence of an addiction syndrome, in which common pathways underlie related addictive behaviors (e.g., obesity or bulimia and addiction involving alcohol) and in which individuals may switch from one object of addiction ${ }^{*}$ to another (e.g., from prescription opioids to heroin, from addictive substances to pathological gambling). ${ }^{5}$

\footnotetext{
* Including the source of reward or relief.
}

Similarly, treatments for one manifestation of addiction tend to have spillover effects, either ameliorating the symptoms of other manifestations of addiction or proving effective in the treatment of multiple expressions of addiction (e.g., naltrexone for the treatment of addiction involving opioids, alcohol as well as gambling) ${ }^{6}$

Numerous studies have shown that addiction treatments are just as effective as those for other illnesses. ${ }^{2}$

--Alan I. Leshner, PhD Chief Executive Officer American Association for the

Advancement of Science Executive Publisher, Science Former Director National Institute on Drug Abuse (NIDA)

[I would define treatment for addiction] the way I would define treatment for any medical problem-good thorough evaluation, intervention tailored for that specific person based on a good assessment, use of a range of tools--behavioral, pharmacological, family and other social support, housing, jobs. ${ }^{3}$

--John Rotrosen, MD

Professor, Department of Psychiatry New York University School of Medicine VA NY Harbor Healthcare System

The goal of the 'good and modern' system is to provide a full range of high-quality services that meet the range of age, gender, cultural and other circumstances that the individual brings to treatment. It is grounded in a public health model that addresses system and service coordination; health promotion and prevention, screening and early intervention; treatment and recovery; and resiliency supports to promote social integration and optimal health and productivity. ${ }^{4}$

--H. Westley Clark, MD, JD, MPH

Director

Center for Substance Abuse Treatment, SAMHSA

When treatment is too highly focused on a specific substance or behavior, it may not be addressing the actual underlying causes of the addiction or the possibility of "addiction 
hopping," where a patient replaces one addiction object with another. Treating the disease of addiction involves addressing not only the specific object of the addiction, but the antecedents, manifestations and consequences of addiction more generally. ${ }^{7}$

The bottom line is that addiction is an illness that we are able to treat and manage, if not cure, provided that we focus on the person with the addiction, the family and the community-- $a$ holistic approach to a sprawling problem. ${ }^{8}$

--Harold S. Koplewicz, MD Child and Adolescent Psychiatrist President Child Mind Institute

\section{Assessment}

Once a patient has been screened for risky use and identified as requiring professional services beyond a brief intervention, a physician-working with other health professionals--should perform a comprehensive assessment of the patient's medical, psychological and substance use history and current health status, present symptoms of addiction, potential withdrawal syndrome and related addictive behaviors. This thorough assessment is a necessary precursor to treatment initiation and must involve a trained physician. ${ }^{9}$ The assessment should utilize reliable and valid interview-based instruments and biological tests as needed. The goals of the assessment are to help the provider determine the nature, stage and extent of the disease and whether the patient meets clinical criteria for an

\footnotetext{
* Despite the distinction between screening and assessment tools, the term screening often is used to subsume the concept of assessment or interchangeably with the term in the clinical and research literatures. Furthermore, while there is some overlap between screening or assessment procedures used to identify risky substance use and methods used to diagnose a clinical addiction, a formal diagnosis of addiction should be based on the demonstration of specific symptoms included in the most recent versions of the Diagnostic and Statistical Manual of Mental Disorders (DSM) or the International Statistical Classification of Diseases (see Chapter II).
}

addiction diagnosis, ${ }^{\dagger}$ establish whether cooccurring medical, including mental health, problems exist and allow for the development of an appropriate and specific treatment plan. ${ }^{10}$

Assessment tools, as distinguished from screening tools, are meant to determine the presence and severity of a clinical condition and should parallel, at least in part, established diagnostic criteria for the disease. ${ }^{\ddagger}$ Assessments tools also might examine social, family and personal factors that might relate to or co-occur with substance use. ${ }^{11}$ This information can help health care practitioners determine the most appropriate intervention for their patients.

A comprehensive assessment helps to create the foundation for effective treatment that is individualized and tailored to the patient. ${ }^{\S 12}$ The assessment should gather information about many aspects of the individual including the physiological, behavioral, psychological and social factors that contribute to the patient's substance use and that might influence the treatment process. ${ }^{13}$ For example, in addition to determining the patient's health status, the stage and severity of the disease ${ }^{14}$ and the family history of addiction, the assessment should determine personality traits such as temperament; family and social dynamics; the extent and quality of the patient's family and social support networks; prior treatment attendance and response to previous treatment experiences; ${ }^{15}$ and the patient's motivation and commitment to disease management.

Assessments also should cover the situations and behaviors that may increase risk for relapse and those that protect against relapse. ${ }^{16}$ It is important that assessment instruments also offer some degree of cultural sensitivity and that they are age and gender appropriate. ${ }^{17}$

\footnotetext{
† See Appendix H for some examples of assessment instruments used by practitioners and researchers to help make these diagnoses.

‡ This, however, is not always the case in commonlyused assessment instruments (see Appendix H).

$\S$ Much of the research on comprehensive assessments relates to addiction involving alcohol.
} 
The comprehensive assessment should result in a treatment plan that is developed in concert with the patient by a physician with input from other health professionals. The treatment plan should articulate clearly the treatment goals and particular interventions aimed at meeting each of those goals. The plan should be monitored and revised as needed should the patient's status or needs change. ${ }^{18}$

The comprehensive assessment also should result in a detailed and thorough written report, which should be incorporated into the patient's health record, that:

- Provides a clinical diagnosis and identifies the particular manifestations and severity of the disease;

- Identifies factors that contribute to or are related to the disease;

- Identifies a treatment plan to address these risk factors and ensure that the treatment plan is implemented and monitored effectively; and

- Provides connections to specialty care--i.e., an addiction physician specialist or other specialty providers--as needed and to auxiliary and support services. ${ }^{19}$

\section{Stabilization*}

The first step in addressing addiction involving nicotine, alcohol or other drugs is cessation of use and, if necessary, medically managing the clearance of toxic substances from the patient's system via a clinical process often referred to as detoxification. Detoxification itself addresses intoxication or withdrawal but is not treatment of addiction. ${ }^{20}$ In most cases, cessation of use is the necessary first step to formal treatment protocols. While cessation of use can in some

\footnotetext{
* Note that some of the medications described for use in the stabilization (tobacco cessation and alcohol/other drug detoxification) process will be described in greater detail later in this chapter in the discussions of acute treatments for addiction and chronic disease management.
}

cases be a self-managed process, patients typically need professional assistance.

\section{Cessation of Use}

Tobacco. Smoking cessation, while unpleasant for most persons going through it, is not unsafe and does not require medical monitoring. Patients undergoing smoking cessation may experience certain withdrawal symptoms including cravings, irritability, impatience, hostility, anxiety, depressed mood, difficulty concentrating, decreased heart rate, increased appetite and sleep disturbances. ${ }^{21}$ The calming effect many smokers feel when smoking usually is associated more with the relief of nicotine withdrawal symptoms than with the effects of the nicotine itself. Withdrawal symptoms can commence in as little as a few hours after the last dose of nicotine, peak within a few days, and either subside within several weeks or, in some cases, persist for months. ${ }^{22}$

Nicotine replacement therapy (NRT)--through the use of nicotine patches, gum, nasal spray, inhalers, lozenges and sub-lingual tablets--is a common pharmaceutical aid for persons attempting smoking cessation. NRT lessens withdrawal symptoms, increasing the chance that a smoker will quit successfully. ${ }^{23}$ NRT replaces some of the nicotine formerly obtained by smoking. ${ }^{24}$ However, the nicotine in NRT is delivered more slowly and at lower dose levels than through smoking, so NRT is more likely to reduce cravings than wholly eliminate them. ${ }^{25}$ Some NRTs mimic the sensations of smoking (the inhaler) or otherwise occupy the mouth (gum, lozenges and sub-lingual tablets). ${ }^{26}$ Research indicates that the most effective use of NRT involves replicating the experience of smokers: using nicotine patches to maintain a baseline serum nicotine level along with the gum or lozenges to produce a boost of serum nicotine levels periodically. ${ }^{27}$

Other pharmaceutical therapies such as antidepressants (bupropion SR) and nicotine agonists (varenicline) can help people quit 
smoking and maintain their abstinence. ${ }^{*} 28$

Alcohol and Other Drugs. Some patients with addiction involving alcohol and other drugs can reduce and ultimately cease substance use without medical supervision, particularly if they are not physically dependent ${ }^{\dagger}$ on the substances involved, the disease is not advanced and they have sufficient personal supports to help them through the cessation process.

\section{Detoxification}

For patients who demonstrate physical dependence on a substance, cessation of use on their own may be unsafe and medically supervised detoxification may be required to manage withdrawal symptoms and complications. $^{29}$

Detoxification occurs when toxic substances that come from the ingestion of alcohol or other drugs are removed from the body via metabolism through the liver and excretion through the kidneys. ${ }^{30}$ Medically-assisted detoxification aims to reduce the risk of discomfort and potential physical harm for patients who are experiencing withdrawal. ${ }^{31}$

The detoxification process often requires the assistance of medical professionals and may involve the use of pharmaceutical therapies to guide people safely through withdrawal. Medical professionals may collaborate with supportive, non-medical personnel or with medical personnel in other health facilities to facilitate the withdrawal process. ${ }^{32}$ Detoxification is an important and often necessary prerequisite to effective acute addiction treatment. ${ }^{33}$ It should serve as the catalyst for entry into the treatment system but does not itself constitute treatment. ${ }^{34}$

There are three main components to effective detoxification:

\footnotetext{
${ }^{*}$ These medications are described in more detail later in the chapter.

${ }^{\dagger}$ Physical dependence is characterized by symptoms of tolerance and/or withdrawal (see Chapter II).
}

1. Evaluation. Examine the patient and determine if symptoms are acutely present-ideally using standardized instruments to measure the severity of withdrawal ${ }^{\ddagger 35}$--and documenting vital signs and other physical manifestations of withdrawal. Assess for the presence of co-occurring medical and mental health conditions and determine, through the use of drug testing, which substances are present in the person's body or were used recently. ${ }^{36}$

2. Stabilization. Assist patients through withdrawal to re-establish a state of physiological stability with or without the use of medications. ${ }^{37}$

3. Facilitation of Treatment Entry. Provide or connect patients to addiction treatment and a continuing care plan. ${ }^{38}$

Alcohol Detoxification. In alcohol detoxification services, the cessation of alcohol ingestion in an alcohol-tolerant individual is coupled with certain medications to help prevent the dangerous effects that may accompany alcohol withdrawal. Withdrawal from alcohol typically takes up to seven to 10 days, but with medical management, stabilization can be achieved sooner. ${ }^{\S 39}$ During the first six to 48 hours of withdrawal from alcohol, symptoms may include anxiety, nausea, agitation and difficulty concentrating. ${ }^{40}$ More severe symptoms can include hallucinations and seizures. ${ }^{41}$ Alcohol withdrawal delirium, also known as delirium tremens (DTs), is the most severe and dangerous withdrawal symptom and usually appears two to four days after the last drink. $^{42}$ Some symptoms of alcohol withdrawal, including DTs and seizures, can be life-

\footnotetext{
‡ These include the Clinical Institute Withdrawal Assessment-Alcohol Revised (CIWA-Ar), the Clinical Opiate Withdrawal Scale (COWS) and the Finnegan Neonatal Abstinence Score.

$\S$ The duration of detoxification varies with the severity of addiction and some withdrawal symptoms, such as sleep disturbances, may persist for several weeks.
} 
threatening. ${ }^{*} 43$ These withdrawal symptoms can be more severe in persons who have undergone prior multiple episodes of alcohol withdrawal, a process known as the kindling effect. ${ }^{\dagger 44}$

Benzodiazepines, which have calming, sedating effects, have been shown to prevent the onset of certain alcohol withdrawal symptoms and acutely relieve such symptoms including alcohol-induced seizures and DTs. ${ }^{45}$ A large review study found that whereas benzodiazepines are more effective than placebos at treating seizures in patients going through alcohol withdrawal, there is no evidence that they are more effective than other medications used to treat alcohol withdrawal syndrome or that particular benzodiazepines are more effective than others. ${ }^{46}$ Benzodiazepines commonly used to treat the anxiety and agitation symptoms associated with alcohol withdrawal include diazepam, ${ }^{\ddagger} 47$ chlordiazepoxide, ${ }^{48}$ lorazepam and oxazepam. ${ }^{49}$

Because the combined effects of benzodiazepines and alcohol can be life threatening or even fatal, ${ }^{50}$ patients must be advised not to drink while on benzodiazepine medications. This is particularly important since benzodiazepines commonly are prescribed for alcohol withdrawal on an outpatient basis where patients' drinking may not be monitored adequately. ${ }^{51}$ Another cautionary note is that benzodiazepines have addictive potential in their own right; therefore, their use must be monitored carefully. ${ }^{52}$

Carbamazepine, an anti-seizure medication, may be an effective alternative to benzodiazepine medications for treating alcohol withdrawal. ${ }^{53}$ Carbamazepine also may be effective at

\footnotetext{
* Although DTs occur only in about five percent of patients undergoing detoxification, mortality from DTs historically has been as high as 18.5 percent. Swift detection and proper treatment can lower this to about five percent.

${ }^{\dagger}$ Kindling is the increase in neuronal responses produced by repeated stimulation. Kindling leads to a worsening of withdrawal symptoms with each attempt at alcohol detoxification.

${ }^{\ddagger}$ Diazepam also may relieve muscle spasms and seizures associated with alcohol withdrawal.
}

addressing alcohol cravings. ${ }^{54}$ The medication's ability to treat seizures, the minimal potential for misuse, the significant potential to treat mood disorder and the lack of sedating effects are some of its advantages. ${ }^{55}$

Opioid Detoxification. For patients with addiction involving illicit or prescription opioids, including heroin, hydrocodone or oxycodone, withdrawal symptoms are not life threatening, but they can be extremely uncomfortable ${ }^{56}$ and must be managed effectively to prevent relapse. ${ }^{57}$ Opioid withdrawal symptoms can include abdominal pain, muscle aches, agitation, diarrhea, dilated pupils, insomnia, nausea, runny nose, sweating and vomiting. ${ }^{58}$ Withdrawal symptoms generally last from seven days to several weeks. ${ }^{59}$ Because medical complications can develop, patients must undergo regular monitoring including physical examinations, a complete review of the functioning of the body's organs and psychological status and, when necessary, laboratory evaluations. ${ }^{60}$

The goal of medical detoxification is a safe, comfortable and complete withdrawal from opioids. Abrupt discontinuation of opioids, especially for a patient who has developed physical dependence on the drug, typically is not recommended; instead, in the case of such dependence involving prescription opioids, the patient is tapered or weaned off the opioid medication. ${ }^{\S 61}$ However, for addiction involving illicit opioids, it is not legal to prescribe a tapering dose of the illicit drug so another method must be used.

An alternative to tapering is management of withdrawal symptoms using non-opioid medications--such as clonidine ${ }^{62}$--to decrease the agitation and discomfort associated with opioid withdrawal, or other medications that can relieve the symptoms of acute withdrawal such as nonsteroidal anti-inflammatory drugs (NSAIDs) to treat muscle pain, antiemetics for nausea and non-addicting sleeping medications such as

\footnotetext{
$\S$ The use of a tapering dose calculator can help in this process and can be accessed online at: www.agencymeddirectors.wa.gov/guidelines.asp
} 
trazadone to treat insomnia. ${ }^{63}$ Detoxification also can be achieved using specific medicallyprescribed opioids that have less potential for misuse (methadone or buprenorphine) and then tapering the patient off these medications when possible. $^{64}$

The prescription of methadone for addiction treatment is restricted by federal regulations; ${ }^{*}$ it only can be prescribed for detoxification from opioids in licensed facilities. ${ }^{\dagger 65}$ Buprenorphine can be dispensed or prescribed for illicit or prescription opioid withdrawal in any outpatient setting by qualified physicians who have the required waiver from the Drug Enforcement Administration (DEA). ${ }^{\ddagger 6}$ While use of these medically-prescribed opioids can result in physical dependence, they are considered less dangerous because they have less potential for misuse and addiction than other opioids; ${ }^{\S 67}$ they also are prescribed to patients by a licensed physician in a medical care setting. They work by occupying the opioid receptors in the brain, blocking or minimizing the effects of more addicting opioid drugs; therefore, a patient on methadone or buprenorphine maintenance largely is protected from inadvertent overdose. ${ }^{68}$

CNS $^{* *}$ Stimulant Detoxification. Cessation of CNS stimulant (cocaine, amphetamine) use may result in withdrawal symptoms if the user

\footnotetext{
* It is not restricted when prescribed for pain management.

${ }^{\dagger}$ Unless a patient has been hospitalized for another medical condition.

${ }^{\ddagger}$ Becoming qualified to prescribe and distribute buprenorphine involves an eight-hour approved program in treating addiction involving opioids, an agreement that the physician/medical practice will not treat more than 30 patients for addiction involving opioids with buprenorphine at any one time within the first year and up to 100 thereafter, and assurance that the trained physician will refer patients to necessary supplemental psychosocial services. Physicians who meet the qualifications are issued a waiver by the Substance Abuse and Mental Health Services Administration (SAMHSA) and a special identification number by the DEA.

${ }^{\S}$ Methadone and buprenorphine also are used for stabilization and maintenance of addiction involving opioids.

Central Nervous System.
}

develops addiction involving these drugs; these symptoms are not life-threatening and generally are less severe than those associated with withdrawal from alcohol or opioids. ${ }^{\dagger \dagger} 69$ Symptoms of stimulant withdrawal commonly include decreased energy, insomnia, agitation, increased appetite, depressed mood, anxiety and drug craving. ${ }^{70}$

Evidence for the effectiveness of pharmaceutical detoxification to assist in stimulant withdrawal is limited. ${ }^{71}$ A vaccine to treat addiction involving cocaine and ease withdrawal symptoms currently is being tested. ${ }^{\ddagger} 72$ But more research is needed to determine the vaccine's place in the cocaine detoxification process and how it can be implemented safely. ${ }^{73}$ Although there are no available medications proven to be effective in mitigating the symptoms of amphetamine withdrawal, ${ }^{74}$ several medications currently being researched may prove useful in alleviating the symptoms. ${ }^{\S \S 75}$

CNS Depressant Detoxification. Withdrawal from CNS depressants may produce complications and, in some circumstances, can be life-threatening. ${ }^{76}$ Symptoms of withdrawal from certain prescription CNS depressants, such as benzodiazepines, are similar to those for alcohol withdrawal, with seizures and delirium being the most serious. In the elderly, there is a risk of falls and myocardial infarctions during benzodiazepine withdrawal. ${ }^{77}$ Benzodiazepine withdrawal symptoms more specifically include seizure, hypersensitivity, impaired perception of movement, nausea and tension. ${ }^{* * *} 78$ It is common for people detoxifying from

\footnotetext{
${ }^{\dagger \dagger}$ During withdrawal from stimulants, there is a risk of depression or negative thoughts and feelings that may lead to suicidal thoughts or attempts.

㧊 See page 98 for a discussion of vaccines under development for addiction treatment.

$\S \S$ Drugs under investigation for this purpose include modafinil, propranolol and bupropion; these investigations are of off-label uses of approved drugs. *** Symptoms of withdrawal from benzodiazepines often mimic the conditions for which those drugs initially were prescribed--mood and anxiety disorders. As such, it sometimes is unclear if the patient is presenting with withdrawal symptoms or with symptoms of the underlying condition.
} 
benzodiazepines to experience significant withdrawal symptoms lasting between 10 and 14 days, ${ }^{79}$ and symptoms can persist for four to six months. ${ }^{80}$ Therefore, it is recommended that benzodiazepine detoxification extend over a period of weeks or months--tapering the patient off the drugs over time. Another option for detoxification from benzodiazepines is to prescribe a different drug from the class, one with a longer half-life, such as chlorodiazepoxide or clonazepam. ${ }^{81}$

Detoxification Venues. Detoxification can take place in a variety of settings including the patient's home (monitored and managed by trained clinicians), physicians' offices, nonhospital addiction or mental health treatment facilities, urgent care centers and emergency departments, intensive outpatient and partial hospitalization programs and acute care inpatient settings. ${ }^{*} 2$

For planned, monitored or medically-assisted detoxification, health-care providers, considering the specific needs of the patient, typically determine the venue for detoxification. Patients should be placed in the least restrictive setting possible. ${ }^{83}$ Beginning in the 1970 s, there was a movement toward medical ambulatory detoxification, primarily for alcohol, that maintained high safety and efficacy profiles while being more cost effective than inpatient detoxification. Ambulatory care was intended to supplement rather than replace inpatient medical detoxification with the understanding that there were some people for whom inpatient care still was necessary. The ability to continue to meet life responsibilities as well as relatively lower costs are advantages of outpatient detoxification. ${ }^{84}$

The primary substance involved in the addiction, the severity of the symptoms and particular patient characteristics (e.g., age, co-occurring substance use and other health conditions) all play important roles in determining the appropriate venue for detoxification. For

\footnotetext{
* Such as acute care general hospitals, acute care addiction treatment units within those hospitals, acute care psychiatric hospitals and other specialty hospitals licensed to provide addiction treatment.
}

example, patients with a history of severe withdrawals or multiple withdrawals should not be placed in nonmedical settings for detoxification. ${ }^{85}$ For patients deemed a danger to themselves or others, medically-managed intensive inpatient treatment or emergency hospitalization in a psychiatric facility is recommended. ${ }^{86}$ For patients with mild or moderate withdrawal symptoms, outpatient detoxification can be just as effective as inpatient, provided the patients have a positive and helpful social support network. ${ }^{87}$

\section{Acute Care}

Effective, clinical treatments for addiction include a significant and growing range of pharmaceutical and/or psychosocial therapies delivered by qualified health professionals. Due to the complex nature of addiction and its physiological, psychological and environmental risk factors, a multi-pronged approach to its treatment that includes a combination of pharmaceutical and psychosocial therapies typically yields the best results. ${ }^{88}$ Because of the extent to which addiction co-occurs with a broad range of other health problems, effective medically-managed acute treatment protocols also should address both co-occurring disorders and patients' nutrition and exercise requirements. ${ }^{89}$

Guidelines set forth by the American Society of Addiction Medicine's (ASAM) patient placement criteria increasingly are being used by treatment programs, government programs, managed care companies and other organizations to appropriately match patient needs to specific treatment services and to determine the appropriate level of care. ${ }^{90}$

\section{Pharmaceutical Therapies}

As with most medical conditions, pharmaceutical therapies can be an important component of addiction treatment, ${ }^{91}$ particularly for patients who are highly motivated to adhere to the medication regimen. ${ }^{\dagger} 92$ (Table 5.1)

\footnotetext{
${ }^{\dagger}$ For less motivated patients, supervised administration of the medication may be necessary.
} 
Table 5.1

Food and Drug Administration (FDA) Approved and Promising Pharmaceutical Therapies and the Types of Substances They Address

\begin{tabular}{|c|c|c|c|c|c|}
\hline Type of Medication $^{\text {a } 93}$ & $\begin{array}{c}\text { Addiction } \\
\text { Involving } \\
\text { Alcohol } \\
\end{array}$ & $\begin{array}{c}\text { Addiction } \\
\text { Involving } \\
\text { Nicotine }\end{array}$ & $\begin{array}{c}\text { Addiction } \\
\text { Involving } \\
\text { Cocaine }\end{array}$ & $\begin{array}{c}\text { Addiction } \\
\text { Involving } \\
\text { Opioids }\end{array}$ & $\begin{array}{c}\text { Addiction } \\
\text { Involving } \\
\text { Marijuana }\end{array}$ \\
\hline \multicolumn{6}{|l|}{$\begin{array}{l}\text { Reduce Craving/ } \\
\text { Withdrawal Symptoms }\end{array}$} \\
\hline Acamprosate (Campral) & $\mathrm{X}$ & & & & \\
\hline $\begin{array}{l}\text { Bupropion (Zyban, } \\
\text { Wellbutrin) }\end{array}$ & & $\mathrm{X}$ & & & \\
\hline $\begin{array}{l}\text { Nortriptyline (Pamelor, } \\
\text { Aventyl) }\end{array}$ & & $\mathrm{X}^{*}$ & & & \\
\hline Clonidine (Catapres) & & $\mathrm{X}^{*}$ & & & \\
\hline $\begin{array}{l}\text { Baclofen (Kemstro, } \\
\text { Lioresal, Gablofen) }\end{array}$ & $\mathrm{X}^{*}$ & & & & \\
\hline Ondansetron (Zofran) & $\mathrm{X}^{*}$ & & & $\mathrm{X}^{*}$ & \\
\hline $\begin{array}{l}\text { Gabapentin (Fanatrex, } \\
\text { Gabarone, Gralise, } \\
\text { Neurontin) }\end{array}$ & & & & & $\mathrm{X}^{*}$ \\
\hline \multicolumn{6}{|l|}{ Reward Reduction } \\
\hline Disulfiram (Antabuse) & $\mathrm{X}$ & & & & \\
\hline $\begin{array}{l}\text { Naltrexone (ReVia, } \\
\text { Depade, Vivitrol) }\end{array}$ & $\mathrm{X}$ & & & $\mathrm{X}$ & \\
\hline Varenicline (Chantix) & & $\mathrm{X}$ & & & \\
\hline $\begin{array}{l}\text { Modafinil (Provigil, } \\
\text { Alertec, Modavigil) }\end{array}$ & & & $\mathrm{X}^{*}$ & & \\
\hline Topiramate (Topamax) & $\mathrm{X}^{*}$ & & $\mathrm{X}^{*}$ & & \\
\hline Vaccines & & $\mathrm{X}^{*}$ & $\mathrm{X}^{*}$ & & \\
\hline \multicolumn{6}{|l|}{$\begin{array}{l}\text { Maintenance/Medication- } \\
\text { Assisted Therapies }\end{array}$} \\
\hline $\begin{array}{l}\text { Nicotine Replacement } \\
\text { Therapy (NRT) }\end{array}$ & & $\mathrm{X}$ & & & \\
\hline Methadone & & & & $\mathrm{X}$ & \\
\hline $\begin{array}{l}\text { Buprenorphine/Naloxone } \\
\text { (Subutex, Suboxone) }\end{array}$ & & & & $\mathrm{X}$ & \\
\hline $\begin{array}{l}\text { Oral Tetrahydrocannabinol } \\
\text { (THC) }\end{array}$ & & & & & $\mathrm{X}^{*}$ \\
\hline
\end{tabular}

${ }^{\text {a }}$ Some of these medications are FDA-approved for treating addiction involving the particular substance, while others are not. Specifically, the $\mathrm{X}$ indicates the type of substance for which the medication has demonstrated efficacy and has received FDA-approval for use in the treatment of addiction involving the noted substance. The $\mathrm{X}^{*}$ notation indicates that ongoing research is demonstrating promising clinical utility for the medication or research already has demonstrated clinical utility for the medication in treating addiction involving the noted substance, but these medications are not (yet) approved by the FDA for use as a pharmaceutical treatment for addiction involving that particular substance. Medications that have been approved by the FDA for other purposes can be prescribed off- label (for uses other than that for which it received FDA approval) based on clinical evidence. However, in spite of clinical evidence of efficacy, these medications may never receive FDA approval since adding new indications to a medication's FDA-approved label would necessitate significant investments in FDA-required studies. ${ }^{94}$ 
Pharmaceutical agents for addiction treatment may work via one of the following mechanisms of action or by a combination of these mechanisms:

- $\quad$ Reducing cravings for the addictive substance and/or reducing aversive withdrawal symptoms;

- Creating an aversion to the addictive substance or attenuating the rewarding effects of the addictive substance, eventually limiting its appeal; or

- Producing moderated effects resembling those of the addictive substance and serving as a less addicting replacement for the substance of addiction.

Differences in the factors that contribute to addiction and that determine how the disease will manifest itself ${ }^{\dagger}$ require different and tailored approaches to treatment, particularly with regard to pharmaceutical treatments. For example, individuals with addiction involving alcohol who drink primarily for the rewarding effects may be quite different biologically from those who drink primarily as a means of relieving stress or reducing negative feelings; as such, the efficacy of a specific pharmaceutical treatment may depend on whether it addresses the reward experienced from using the addictive substance or whether it serves as a safer medication for providing relief from negative feelings. ${ }^{96}$ Likewise, certain genetic or biological characteristics may determine how effective a certain type of pharmaceutical intervention will be for an individual with addiction; for example, naltrexone has been found to be a more effective medication for the treatment of addiction

\footnotetext{
* For ease of presentation, a medication is categorized in this discussion based on its primary mechanism of action.

${ }^{\dagger}$ In individuals with a significant genetic susceptibility, progression from use to addiction is relatively quick and severe, whereas in individuals with a low genetic susceptibility, progression from use to addiction will result from prolonged exposure to addictive substances and considerable environmental risk factors, such as stress. (See Chapter II.)
}

involving alcohol in patients with a family history of the disease than in those without a family history. ${ }^{97}$ A true understanding of these differences is in its infancy, but appears to be a critical factor in tailoring pharmaceutical treatments to achieve the maximum therapeutic benefit. $^{98}$

What we hope to do is to actually have a menu of treatments that clinicians could choose from. If one drug doesn't work or they can't tolerate it," patients would "try another one and so forth, and hopefully they'll find one that is effective." 95

--Raye Z. Litten, PhD Associate Director Division of Treatment and Recovery Research National Institute on Alcohol Abuse and Alcoholism (NIAAA)

\section{Medications that Reduce Craving and/or} Withdrawal Symptoms. A number of medications work on the brain chemicals and pathways of individuals with addiction to reduce cravings for the addictive substance and in some cases reduce symptoms of withdrawal from the substance.

Acamprosate (brand name Campral), approved by the Food and Drug Administration (FDA) in 2004 to treat addiction involving alcohol, helps to normalize brain activity and function that has been disrupted by heavy alcohol use ${ }^{99}$ and reduce withdrawal symptoms such as anxiety and insomnia. ${ }^{100}$ It is prescribed for treatment patients who have discontinued their use of alcohol. ${ }^{101}$

Studies regarding the effectiveness of acamprosate have been mixed in the U.S., but more consistently positive in Europe. ${ }^{\ddagger 102}$ Several large-scale, controlled studies have found that acamprosate can double the abstinence rate among treatment patients at one to two years following program completion compared to patients who receive placebos, and

\footnotetext{
₹ The differences in effectiveness findings appear to be due to methodological differences.
} 
is associated with better treatment retention rates. ${ }^{103}$ Acamprosate generally is safe to use, as it does not appear to have a potential for addiction, has virtually no overdose risk, has mostly mild side effects and does not interact significantly with other medications. ${ }^{104}$

Antidepressant medications also have proven to be effective in smoking cessation. The mechanism driving the efficacy of antidepressants as cessation agents is not yet fully understood. It may be that antidepressant medications compensate for nicotine's antidepressive effects during withdrawal, lessening this withdrawal symptom. Alternatively, antidepressant medications may work-independent of their antidepressant qualities--on the neural pathways or the nicotine receptors that are active in addiction involving nicotine.* 105

- Bupropion sustained release (SR) (brand names Zyban and Wellbutrin) is a prescription antidepressant medication that can be used alone or in combination with nicotine replacement therapy (NRT) for smoking cessation. ${ }^{106}$ It is believed to work by minimizing cravings and withdrawal symptoms during the early stages of tobacco cessation. ${ }^{107}$ Bupropion may be effective in relieving negative mood and feelings that smokers may experience when going through smoking cessation. ${ }^{108}$ The neurological effects of bupropion that aid in its efficacy as a cessation medication may include blocking the re-uptake of two neurotransmitters that are active in addiction involving nicotine--dopamine and norepinephrine--and blocking nicotine receptors. ${ }^{109}$ The medication reduces the severity of nicotine withdrawal and the depression that may accompany smoking cessation. ${ }^{110}$ Another advantage of bupropion for smoking cessation is that it tends to lessen the weight gain that often accompanies--and derails--smoking cessation attempts. ${ }^{111}$

\footnotetext{
${ }^{*}$ For example, by blocking nicotine receptors in the brain.
}

Patients generally are advised to begin daily bupropion treatment one to two weeks prior to quitting so that adequate blood levels of the medication can be reached. ${ }^{112}$ The standard course of treatment is seven to 12 weeks, although this period can be extended for up to six months if necessary. ${ }^{113}$

Possible side effects include insomnia, dry mouth, nausea and a small risk of seizures. ${ }^{114}$ Bupropion SR carries a black box warning $^{\dagger}$ of increased suicidal tendencies among children, adolescents and young adults. ${ }^{115}$

A meta-analysis of 24 bupropion studies found that the drug can nearly double smokers' chances of achieving abstinence lasting longer than five months compared to a placebo. ${ }^{116}$ Other analyses found similar results. ${ }^{117}$ The efficacy of the drug does not seem to be sensitive to longer follow-up periods (up to 12 months), treatment setting, dosage or the level of supplementary psychosocial therapy. ${ }^{118}$

Bupropion also may be a promising treatment for methamphetamine addiction; it appears to reduce cravings and the rewarding effects of methamphetamine. ${ }^{119}$ However, evidence supporting its ability to increase abstinence rates is mixed and further research is needed to establish bupropion as an effective treatment for methamphetamine addiction. ${ }^{120}$

- Preliminary research indicates that another antidepressant, nortriptyline (brand names Pamelor and Aventyl), may be an effective smoking cessation aid. ${ }^{121}$ Nortriptyline has been found to double patients' chances of cessation compared to placebos. ${ }^{122}$

However, the medication has not yet been approved by the FDA for use as a smoking cessation aid; therefore, it only is recommended for use by patients who have not responded well to NRT or bupropion. ${ }^{123}$

\footnotetext{
${ }^{\dagger}$ A black box warning from the FDA denotes the most serious warning of adverse effects for a medication.
} 
Clonidine (brand name Catapres), a medication normally used to lower blood pressure, may reduce withdrawal symptoms and assist in smoking cessation. ${ }^{124}$ However, the many side effects associated with clonidine suggests that it only be prescribed to patients seeking to quit smoking if they are unwilling or unable to use other first-line cessation medications. ${ }^{125}$

Baclofen (brand names Kemstro, Lioresal and Gablofen), a medication normally used to treat spasticity, has been found to reduce cravings and withdrawal symptoms in patients with addiction involving alcohol, helping them to reduce their drinking and to achieve and maintain abstinence; however, the existing evidence is too limited to recommend baclofen for addiction involving alcohol. ${ }^{126}$ A double-blind placebo-controlled study ${ }^{*}$ in humans found that more baclofen patients than placebo patients achieved and maintained abstinence (70.0 percent vs. 21.1 percent); baclofen patients also demonstrated a larger reduction in average number of drinks, alcohol craving, relapse rates and anxiety levels relative to placebo patients in this study. ${ }^{127}$ There appears to be a dose-response relationship for baclofen, with greater effectiveness at higher doses. ${ }^{128}$

Ondansetron (brand name Zofran), an antinausea drug, ${ }^{129}$ has been shown to be effective in reducing alcohol use cravings and alcohol use among patients with addiction involving alcohol, particularly among those with an early onset addiction. ${ }^{130}$ The drug may be more effective in patients with specific genotypes. ${ }^{\dagger} 131$ There also is emerging evidence that ondansetron might be effective in treating withdrawal symptoms in patients with addiction involving opioids. ${ }^{132}$

Gabapentin (brand names Fanatrex, Gabarone, Gralise, Neurontin), a medication used to treat neuropathic pain and epileptic seizures, has been found to reduce withdrawal symptoms and the

\footnotetext{
* Sample size of 39.

' Specifically, those with the L/L genotype of the 5HTTLPR polymorphism. "L” signifies long alleles and "S" signifies short alleles. Possible genotypes are $\mathrm{L} / \mathrm{L}, \mathrm{S} / \mathrm{S}$ and $\mathrm{S} / \mathrm{L}$.
}

use of marijuana in patients with addiction involving cannabis. ${ }^{133}$

Reward Reduction Medications. Certain medications work to reduce the rewarding or pleasurable effects of addictive substances, over time decreasing their appeal.

Disulfiram (brand name Antabuse), approved by the FDA in 1949 to treat addiction involving alcohol, works by producing unpleasant physiological effects in individuals who consume alcohol; these aversive reactions serve as a deterrent to alcohol consumption among those with addiction involving alcohol. ${ }^{\ddagger 134}$

For addiction involving alcohol, disulfiram blocks the production of the enzyme aldehyde dehydrogenase which enables the liver to metabolize alcohol. ${ }^{135}$ The obstruction of this process leads to a build up of acetaldehyde ${ }^{\S}$ in the system, causing hangover-like symptoms-nausea, vomiting, flushing, rapid heart rate-shortly after alcohol consumption. ${ }^{136}$ The medication has not been found to reduce cravings; its preventive qualities come from replacing the pleasurable effects of alcohol with unpleasant effects. ${ }^{137}$

Although disulfiram is one of the oldest pharmaceutical treatments for addiction involving alcohol, there are few scientifically valid studies of its efficacy. ${ }^{138}$ The medication appears to be most effective when its use is supervised to assure compliance. ${ }^{139}$ Researchers suggest that the relatively limited evidence of the efficacy of the drug may be linked to low adherence rates: in one study only 20 percent of participants who completed the trial complied with disulfiram treatment. Among patients who completed the trial, those who received a

\footnotetext{
₹ Disulfiram is the most commonly-used aversion medication for the treatment of addiction involving alcohol. Another example of a medication that produces a similar aversive effect in individuals who consume alcohol is calcium carbimide (brand name Temposil); other medications used for non-addiction clinical purposes, such as cephalosporins which are used to treat bacterial infections, have side effects similar to disulfiram when alcohol is consumed. $\S$ The major metabolite of ethanol.
} 
standard dose of disulfiram reported significantly fewer drinking days than their peers over the course of a year. ${ }^{140}$ One explanation for the variation in effectiveness may be related to addiction severity: those with more severe addiction may drink despite the adverse reactions caused by disulfiram, or avoid taking the medication altogether. ${ }^{141}$

Naltrexone, used in the treatment of addiction involving alcohol and opioids, blocks opioid receptors in the brain, leading to reductions in the reinforcing effects of these drugs. ${ }^{142}$ It does this by disrupting the transmission of dopamine-and thus the endorphin rush--caused by alcohol and opioid ingestion. ${ }^{143}$

Oral naltrexone (brand names ReVia and Depade), which is available as a daily tablet, was approved by the FDA to treat addiction involving alcohol in 1984. Injectable naltrexone (brand name Vivitrol), which is an extended release monthly deep intramuscular gluteal injection, ${ }^{*} 144$ was approved by the FDA in 2006 for addiction involving alcohol and in 2010 for the treatment of addiction involving opioids. ${ }^{145}$ Naltrexone is prescribed for patients who have not ingested opioids for seven to 10 days because it can cause serious withdrawal symptoms if used at the same time as an opioid. ${ }^{146}$

Because of naltrexone's mechanism of actionreducing the reward or "high" associated with substance use-- some patients may not take the medication regularly. ${ }^{147}$ Noncompliance also may be associated with experiencing uncomfortable side effects ${ }^{\dagger}$ at the start of a course of treatment. ${ }^{148}$ Unless participants adhere to their treatment regimen 70 to 90 percent of the time, ${ }^{\ddagger}$ naltrexone does not produce significant outcomes. ${ }^{149}$ Injectable

\footnotetext{
${ }^{*}$ When injected, the pharmacological agent releases its active compound in a consistent way over a long period of time.

${ }^{\dagger}$ Side effects can include gastrointestinal problems such as nausea, vomiting and abdominal pain and central nervous system-related symptoms such as headache and fatigue.

${ }^{\ddagger}$ The necessary adherence rate varies by the duration of treatment.
}

naltrexone provides two advantages which may improve retention and success rates: the need for a monthly injection rather than a daily pill and the need for regular contact with medical and other supporting staff in the course of a clinic visit which is required to obtain the medication. ${ }^{150}$ A disadvantage of the injection formulation, however, is that it has more side effects at higher doses. ${ }^{151}$

For addiction involving alcohol, the medication is more effective at reducing heavy drinking than increasing abstinence rates. ${ }^{152}$ Several randomized, placebo-controlled trials found that compared to patients taking placebos, patients taking naltrexone report significantly fewer drinking days, fewer drinks per drinking day, reduced cravings and reduced relapse. ${ }^{153}$

Because naltrexone blocks the euphoric effects of opioids and does not produce a high when taken, there is much less potential for misuse or diversion than there is for other pharmaceutical treatments, such as methadone, for addiction involving opioids. ${ }^{154}$ In one randomized, controlled study, patients with addiction involving heroin who received naltrexone injections were significantly likelier to have remained in treatment over the course of the study than patients receiving a placebo injection. $^{\S 155}$ Buprenorphine, ${ }^{* *}$ when added to naltrexone, has been found to improve retention in treatment. ${ }^{156}$ Relapsing to the use of opioids after beginning naltrexone treatment can increase patients' risk of overdosing, due to naltrexone's effect on increasing the sensitivity of opioid receptors in the brain to the effects of opioids. ${ }^{157}$

Varenicline (brand name Chantix) is an effective therapy for smoking cessation that works by reducing the rewarding effects of nicotine among patients who smoke while on the medication and by reducing the craving and withdrawal symptoms that occur among abstinent patients. ${ }^{158}$

\footnotetext{
$\S$ Naltrexone patients also were less likely than placebo patients to test positive for cocaine, benzodiazepine, marijuana and amphetamine use. *** See page 101 .
} 
Research indicates that varenicline use, compared with a placebo, can significantly increase a patient's chances of attaining continual abstinence from smoking over six months. ${ }^{159}$ A large-scale analysis of several randomized controlled trials found that the medication was significantly more effective than placebos or bupropion in relieving cravings and in increasing the likelihood of achieving continuous abstinence over a 12-month period. ${ }^{160}$

While nausea is the most commonly-reported side effect, insomnia, headaches and nightmares also are prevalent. ${ }^{161}$ In February 2008--two years after Chantix was approved--the FDA released a public health advisory warning patients that the medication has the potential to aggravate psychiatric illnesses and in some cases lead to the development of neuropsychiatric symptoms, such as anxiety, tension, depression or suicide attempts. ${ }^{162}$ This advisory led to modifications in the product labeling and the medication guide advising medical professionals to monitor all patients taking the medication for neuropsychiatric symptoms. ${ }^{163}$ More recently, medical professionals were advised to monitor use of the medication among patients with cardiovascular disease since Chantix has been linked to adverse cardiovascular effects in these patients. ${ }^{164}$

Modafinil, a stimulant medication (brand names Provigil, Alertec and Modavigil), used to treat narcolepsy and other sleep disorders, reduces the stimulating effects of cocaine. ${ }^{165}$ At the same time, it may reduce cocaine cravings and withdrawal symptoms. ${ }^{166}$ In one study, patients ${ }^{*}$ with addiction involving cocaine who received daily doses of modafinil for eight weeks submitted nearly twice as many clean urine samples than placebo patients during the course of the study and were more than twice as likely to achieve at least three weeks of prolonged abstinence. ${ }^{167}$ Another study found modafinil to be effective in reducing cocaine use and cocaine craving in patients with addiction involving

\footnotetext{
* Who met clinical diagnostic criteria for dependence and who used at least $\$ 200$ worth of cocaine during the prior month.
}

cocaine $^{\dagger}$ who took part in individual psychosocial therapy. ${ }^{168}$

Topiramate (brand name Topamax), an anticonvulsant, has been validated by randomized controlled trials to treat addiction involving alcohol. ${ }^{169}$ It is believed to work by reducing the release of dopamine and thus the rewarding effects of alcohol use and the urge to drink. ${ }^{170}$ It appears to reduce alcohol withdrawal symptoms and can be used in patients who are not yet abstinent from alcohol. ${ }^{171}$ Topiramate also is a promising pharmaceutical treatment for addiction involving cocaine, but additional research is needed to establish its efficacy. ${ }^{172}$ Preliminary research suggests that the anticonvulsant and mood-stabilizing medications carbamazepine and valproate also may be effective in treating addiction involving alcohol. $^{173}$

Vaccines. Recent research in pharmacotherapy for substance addiction has examined the use of vaccines in the treatment process. ${ }^{174}$ These vaccines work by producing a sufficient quantity of antibodies that bind to the substance and prevent or significantly impede it from entering the brain, ${ }^{\ddagger}$ reducing the accumulation of the substance in the brain and ultimately decreasing its rewarding effects. ${ }^{175}$ Much of the work on vaccines for addiction is still in the preclinical phase of development. ${ }^{176}$

Vaccines for addiction involving nicotine ${ }^{\S}$ are farthest along in the development phase. They are proving to be safe, with limited adverse side effects and have shown promise for helping smokers quit. ${ }^{177}$ However, these vaccines still are undergoing clinical trials to test for safety and efficacy. While they may be helpful in reducing the rewarding effects of nicotine in those who already are addicted, they do not

\footnotetext{
${ }^{\dagger}$ Who did not have co-occurring addiction involving alcohol.

${ }^{\ddagger}$ Antibodies typically are comprised of larger molecules than addictive substances, making them less able to cross into the brain.

$\S$ NicVax, Nic002 (also known as NicotineQB) and TA-Nic are the vaccines currently under investigation.
} 
prevent addiction and should be used in conjunction with other therapeutic approaches. $^{178}$

A cocaine vaccine also has been developed and was found in a preliminary study to reduce cocaine use. ${ }^{179}$ Researchers also are exploring a vaccine or antibody administrations for other addictive stimulants including methamphetamine ${ }^{180}$ and phencyclidine $(\mathrm{PCP})^{181}$ and have found promising results in animal samples.

\section{Maintenance Medications/Medication Assisted Therapies. Certain medications used to treat addiction function by delivering a less dangerous or less addicting version of the addictive substance during the acute care phase of addiction treatment. ${ }^{*}$ These medications may function by reducing cravings or withdrawal symptoms and/or by reducing the rewards associated with the addictive substance. Maintenance medications have proven to be highly effective in treating a variety of manifestations of addiction and in disease management.}

Nicotine Replacement Therapy (NRT). NRT, when used as directed, provides lower doses of nicotine at a slower rate than smoking, ${ }^{182}$ thereby easing nicotine withdrawal symptoms. ${ }^{183}$ For many smokers, it works best as an aid to managing nicotine-related cravings when used in conjunction with psychosocial therapies. In most cases of acute care treatment, a therapeutic level of nicotine is reached and then use is reduced in order to eliminate the medication entirely or reach a maintenance level. ${ }^{184}$ Because most patients who use NRT control their treatment regimen on their own, there is a risk that the nicotine intake from NRT products may be higher than intended for those who do not use them as directed or who use them while continuing to smoke. ${ }^{185}$

Nicotine gum, lozenges and inhalers and nasal sprays deposit nicotine in the bloodstream through the lining of the mouth or nose, whereas

\footnotetext{
* These medications also may be used for an extended period of time for disease management.
}

the nicotine patch delivers the nicotine through the skin. ${ }^{186}$ Nicotine gum and lozenges both are over-the-counter medications; inhalers and sprays require prescriptions. The nicotine patch is available both over-the-counter and by prescription. $^{187}$

A meta-analysis ${ }^{\dagger}$ of 13 studies found that use of nicotine gum can increase significantly smokers' chances of quitting for at least six months. ${ }^{188}$ The use of nicotine lozenges nearly doubles the chance of achieving continuous abstinence over at least a six-month period. ${ }^{189}$ Side effects of nicotine gum and lozenges include sore throat, heartburn, jaw pain and nausea. ${ }^{190}$

Nicotine inhalers come in cartridges which release nicotine vapor when puffed that is absorbed through the lining of the mouth and through the back of the throat. ${ }^{191}$ Two metaanalyses found that inhaler use can nearly double patients' abstinence rate over at least a six-month period, relative to those who received a placebo. ${ }^{192}$ The primary side effect is local irritation. ${ }^{193}$ Nicotine nasal spray is aerosolized nicotine that comes in a spray pump. The nicotine is sprayed into the nostrils and absorbed rapidly by the nasal membranes. ${ }^{194}$ Metaanalyses indicate that patients almost double their chances of achieving and maintaining abstinence at six months with the use of a nasal spray versus a placebo. ${ }^{195}$ The primary side effect of the medication is local irritation. ${ }^{196}$ This form of NRT has the highest potential for misuse: 15 to 20 percent of patients report using the spray for longer than the recommended period and five percent report using a higher dose than recommended. ${ }^{197}$

The nicotine patch is available in both single and step-down dosages. An eight-week course of

\footnotetext{
${ }^{\dagger}$ The review included only those studies that had been published in peer-reviewed journals; however, some studies were supported by the pharmaceutical industry. Most of the studies included in the analysis drew participants from self-selecting populations of smokers and, in general, the studies' participants received counseling regardless of whether they were randomly assigned to receive medication or placebos. (This is true of all meta-analyses of tobacco cessation interventions reported here.)
} 
treatment is most common; ${ }^{198}$ however, one study found that an extended course of treatment--24 weeks--improves outcomes. ${ }^{199}$ Unlike other forms of NRT, patches do not address the behavior of smoking by occupying the hands or the mouth or inhaling, but by passively and slowly delivering the nicotine. ${ }^{200}$ A meta-analysis of 24 studies found that the use of nicotine patches for six to 14 weeks can nearly double a smoker's chance of sustaining abstinence for at least six months compared to placebos. ${ }^{201}$ Unlike nicotine gum, there is little evidence supporting a connection between higher doses and higher efficacy rates, or between longer treatments and better outcomes. ${ }^{202}$ The patch also appears to be effective regardless of additional psychosocial interventions. ${ }^{203}$ The primary side effect of the patch is skin sensitivity and irritation. ${ }^{204}$

Contrary to the many controlled studies supporting the efficacy of NRT, a populationbased survey of adult smokers who recently had quit smoking casts doubt on its effectiveness in smoking cessation and relapse prevention. In this study, nearly one-third (30.6 percent) of smokers who had quit smoking within the previous two years reported having relapsed, regardless of whether they used NRT for the recommended amount of time and regardless of whether the use of NRT was accompanied by professional behavioral counseling. While this study appears to call into question the efficacy of NRT in achieving smoking cessation in the general population, ${ }^{205}$ it actually underscores the fact that NRT is not a comprehensive treatment approach to addiction involving nicotine, but rather an aid to smoking cessation to be used in conjunction with other evidence-based acute care and chronic disease management approaches.

Methadone. Methadone is a synthetic opioid used as replacement therapy for patients with addiction involving opioids. ${ }^{*}$ Methadone reduces cravings and withdrawal symptoms by

\footnotetext{
* Methadone can be used in the stabilization, acute treatment and disease management/maintenance phases of treatment for patients with addiction involving opioids.
}

stimulating opioid receptors in the brain ${ }^{206}$ and reduces the rewarding effects of opioids by blocking their euphoric effects. ${ }^{207}$ Although methadone is an opioid, it does not produce the same euphoric rush characteristic of heroin or oxycodone; its effects are slower and steadier. ${ }^{208}$

Methadone can be taken orally and has a long half-life with a slow onset of action. ${ }^{209}$

Methadone is safe when used as directed. It allows individuals with addiction involving opioids to function relatively normally. ${ }^{210}$ Potential side effects of methadone include drowsiness, weakness, headache and nausea, and side effects are more likely to occur when starting methadone, when switching from another narcotic/opioid medication to methadone and when the dosage is increased. ${ }^{211}$

Multiple studies have found that participation in methadone maintenance treatment (MMT) is related to significant reductions in patients' use of opioids, criminal behavior, injection drug use, needle sharing and risky sexual behavior which can increase the risk of HIV and sexuallytransmitted diseases; ${ }^{\dagger}$ MMT also is related to higher treatment retention rates. ${ }^{212}$

Methadone does, however, carry the potential for misuse ${ }^{213}$ and the risk of overdose. ${ }^{214}$ While methadone is effective in inhibiting the effects of opioids like heroin and morphine, it does not appear to inhibit the effects of alcohol or other drugs; rather methadone may magnify the effects of alcohol and other CNS depressants. ${ }^{215}$ Its use in concert with other addictive substances can result in adverse interactions. Methadone also may accumulate in the body to a toxic level if it is taken too often, or in larger than recommended quantities. ${ }^{216}$ Because of the risk of methadone misuse and its consequences, methadone distribution is regulated by strict federal and state guidelines. ${ }^{217}$ Methadone prescribed for addiction involving opioids can

\footnotetext{
${ }^{\dagger}$ Because methadone does not require intravenous injection, methadone users are less likely to engage in needle sharing and because they do not need the same amount of money to obtain heroin, they are less likely to engage in prostitution compared to their heroin-using counterparts.
} 
be obtained only through specially-licensed treatment programs. ${ }^{* 218}$

Buprenorphine. Buprenorphine is used in the treatment of addiction involving opioids and, when used as directed, functions both by reducing craving for addictive opioids and by easing withdrawal symptoms. ${ }^{219}$ At low doses, buprenorphine enables patients with addiction involving opioids to discontinue their use of the drugs without experiencing withdrawal symptoms. $^{220}$

There are two forms of the medication: buprenorphine alone (brand name Subutex) and a buprenorphine/naloxone combination therapy (brand name Suboxone). ${ }^{221}$ Approved in 2002 by the FDA for treating addiction involving opioids, Subutex generally is prescribed during acute treatment followed by Suboxone for maintenance therapy. ${ }^{222}$ The naloxone component of Suboxone serves to reduce the rewarding effects of opioids and helps to prevent the misuse of the medication which can occur if Suboxone is crushed and then injected or snorted to achieve a high. ${ }^{223}$

Buprenorphine must be administered under the supervision of a trained physician. ${ }^{224}$ It can be prescribed by physicians who are certified in addiction medicine $^{\dagger}$ or who complete at least eight hours of training ${ }^{\ddagger}$ in the treatment and management of addiction involving opioids. ${ }^{\S 225}$ Once such training is completed, physicians may submit an application to the Substance Abuse and Mental Health Services Administration (SAMHSA) and receive an identification

\footnotetext{
* Such restrictions apply only to the use of methadone in addiction treatment and not when physicians prescribe methadone to treat or manage pain. ${ }^{+}$Through the American Board of Addiction Medicine or the American Osteopathic Association. ${ }^{\ddagger}$ Approved training includes training provided by the American Society of Addiction Medicine, the American Academy of Addiction Psychiatry, the American Medical Association, the American Osteopathic Association, the American Psychiatric Association or any other organization that the Secretary of Health determines is appropriate. ${ }^{\S}$ Physicians also must have the ability to provide or refer patients to any necessary ancillary services.
}

number from the DEA allowing them to prescribe the medication. ${ }^{226}$

Buprenorphine provides moderate relief from opioid withdrawal and has less risk of misuse and overdose than methadone. ${ }^{227}$ Another advantage to buprenorphine is that it can be dosed less frequently than every day and still have a beneficial effect, which could help to enhance medication adherence. ${ }^{228}$ Promising results are emerging from preliminary research on low-frequency dosing with sustained-release formulations of the medication. ${ }^{229}$ Despite these advantages, buprenorphine has similar side effects to methadone and other opioids including nausea, vomiting and constipation. ${ }^{230}$

Buprenorphine is equally effective as methadone in treating addiction involving opioids. ${ }^{231}$ Research reviews of pharmaceutical treatments for addiction involving opioids have found that regardless of the dose, buprenorphine is better than placebos for ensuring patient retention, ${ }^{232}$ and that higher doses increase the likelihood of retention and abstinence relative to lower doses. ${ }^{233}$ A randomized, controlled trial found that patients receiving buprenorphine were significantly likelier to have negative urinalyses than placebo patients and to report decreased cravings for opioid drugs. ${ }^{234}$

Recent research has begun to explore, using animal models, the benefits of using buprenorphine to treat addiction involving methamphetamine. $^{235}$

Oral THC. Preliminary research suggests that oral tetrahydrocannabinol (THC), made from the psychoactive ingredient contained in cannabis, may serve to reduce withdrawal symptoms and cravings in patients with addiction involving marijuana without producing marijuana-like intoxication effects. ${ }^{236}$ However, research on oral THC and other pharmaceutical therapies for use in the treatment of patients with addiction involving marijuana, while promising, is in an early stage and clinical trials are needed to support their use in clinical interventions. ${ }^{237}$ 


\section{Psychosocial Therapies}

Psychosocial treatments for substance addiction aim to alter patients' attitudes and behaviors with regards to the use of tobacco, alcohol and other drugs. These therapies enhance patients' skills in coping with life challenges, navigating high-risk situations, avoiding substance use triggers, controlling cravings and coping with lapses. ${ }^{238}$ Some therapies focus on enhancing patients' motivations to change their substancerelated behaviors. Other therapies focus on helping patients alter their environments in order to reduce pressures and cues to use, or provide positive or negative reinforcements to help patients change their attitudes and behavior. ${ }^{239}$

Psychosocial therapies are critical components of almost every treatment regimen, regardless of a patient's primary substance of addiction; when combined with pharmaceutical treatments they enhance treatment efficacy. ${ }^{240}$

As is true of treatments for most other health conditions, successful treatment for patients with addiction takes into account patients' social and financial circumstances as well as their physical well-being. And, as is the case for other health conditions, not all approaches work equally well for all patients; the effectiveness of a particular approach depends on patient circumstances such as the severity of the addiction, the primary substance involved in the addiction, the extent of social support and the presence of co-occurring disorders; the venue in which the treatment is provided; and the nature and dynamics of the provider-patient relationship. ${ }^{241}$

The following are brief descriptions of the primary psychosocial therapies for addiction treatment:

\section{Motivational Interviewing (MI) and} Motivational-Enhancement Therapy (MET). Motivational techniques capitalize on patients' readiness to stop using addictive substances and enter treatment by bolstering their motivation to change their substance use behaviors. ${ }^{242}$ In acute care, motivational therapies are employed early in the treatment process. They also may be used in conjunction with other psychosocial and pharmaceutical approaches. ${ }^{243}$

Motivational Interviewing (MI), which can occur in inpatient or outpatient settings, is rooted in the idea that individuals with addiction often feel ambivalent about their substance use and the need to change their behaviors. ${ }^{244} \mathrm{MI}$ techniques help patients deal with this ambivalence and strengthen their commitment to engage in behavior change. ${ }^{245}$ Motivational Enhancement Therapy (MET) is an adaptation of $\mathrm{MI}^{*}$ that restricts the intervention to four sessions. $^{\dagger} 246$

Both MI and MET have proven efficacy in addressing adolescent and adult addiction and are cost-effective approaches to treating addiction involving nicotine, alcohol and other drugs. $^{247}$ Two main benefits of MI/MET are increased treatment retention and program completion, ${ }^{248}$ which are associated with improved treatment outcomes. ${ }^{249}$ A study of tobacco cessation among patients who had previously had a heart attack found that those receiving MI were more likely to achieve abstinence after a year than patients who only received brief advice about quitting (65.5 percent vs. 37.0 percent). ${ }^{\ddagger 250}$ An evaluation of MET across five treatment sites found that it was associated with greater reductions in alcohol and other drug use over a 12-week period than standard individual counseling. ${ }^{\S} 251$

\footnotetext{
* Both modalities are based on the Transtheoretical Model of Behavior Change.

${ }^{\dagger}$ MET was developed for Project MATCH (1997), a large study of treatment efficacy that compared the effectiveness of three treatment modalities: 12 sessions of cognitive behavioral therapy (CBT), 12 sessions of Twelve-Step Facilitation Therapy or four sessions of MET. All three groups showed significant and comparable declines in alcohol use up to three years later.

₹ These rates are based on at least one week of abstinence corroborated by a family member.

${ }^{\S}$ More than 450 individuals with addiction were randomly assigned to receive three sessions either of MET or standard individual counseling during a onemonth period.
} 


\section{Motivational Interviewing}

The MI therapist attempts to:

- Express empathy through reflective listening;

- Recognize discrepancies between patients' goals or values and their current substance use;

- Provide normative feedback on the discrepancy between patients' substance use and that of their peers;

- Adjust to patient resistance rather than oppose it directly;

- Avoid arguments and direct confrontations; and

- Support patients' sense of self-efficacy to change their behavior. ${ }^{252}$

\section{Cognitive Behavioral Therapy (CBT).}

Cognitive Behavioral Therapy (CBT) involves training in social skills, self-control and stress management through activities such as role playing, behavioral modeling and feedback. ${ }^{253}$ It is designed to help patients identify, recognize and avoid thought processes, behaviors and situations that are associated with substance use; manage cravings; refuse offers of tobacco, alcohol or other drugs; and develop better problem-solving and coping skills. ${ }^{254}$

CBT generally is used as a short-term intervention and can be tailored both to inpatient and outpatient programs via group or individual therapy. ${ }^{255}$ The therapy has been proven effective for adolescents and adults and for a variety of manifestations of substance addiction. ${ }^{256}$ CBT has demonstrated efficacy for specific populations such as women with addiction and individuals with co-occurring disorders. ${ }^{*} 257$

\section{Community Reinforcement Approach (CRA).} The Community Reinforcement Approach (CRA) is a multi-phase, intensive 24-week outpatient treatment for addiction involving

\footnotetext{
${ }^{*}$ CBT may work as well for other populations and other substances, but available data largely are focused on the ones described here.
}

alcohol and drugs other than nicotine. ${ }^{258}$ Counseling sessions focus on improving family relations, learning skills to reduce substance use, acquiring vocational skills and developing recreational activities and social networks that can help to minimize the drive to engage in substance use. ${ }^{259}$ CRA also assists patients in developing communication, problem-solving and drug refusal skills. ${ }^{260}$ CRA is based on the notion that patients must be taught life skills and shown that living substance free can be more rewarding than a life of addiction. ${ }^{261}$

There is evidence of the effectiveness of CRA for treating patients with addiction involving alcohol and drugs other than nicotine. ${ }^{262}$ Effectiveness is enhanced when coupled with pharmaceutical interventions and abstinencebased incentive programs, such as the provision of vouchers exchangeable for retail items contingent on negative urinalysis results. ${ }^{263}$ Participation in a CRA intervention also has shown positive supplementary effects, such as increased employment rates and decreased criminal involvement. ${ }^{264}$

\section{Contingency Management (CM).} Contingency Management (CM) is an intervention that uses positive and negative reinforcement to alter behavior, although rewarding positive behavior has been demonstrated to be more effective than punishing negative behavior. ${ }^{265}$ Most CM interventions provide patients with vouchers and incentives for meeting treatment-related goals such as producing a drug-free urine test. Incentives can include cash rewards, vouchers to purchase desired items or treatment-related privileges such as receiving multiple doses of medication at one time to avoid having to make numerous clinic visits. ${ }^{266}$

The effectiveness of CM has been demonstrated for addiction involving nicotine, alcohol, marijuana, cocaine, methamphetamine and opioids. ${ }^{267} \mathrm{CM}$ can improve program retention, ${ }^{\dagger}$ increase abstinence and help prevent relapse. It is most successful when used in conjunction

\footnotetext{
${ }^{\dagger}$ Improved program retention is associated with other positive treatment outcomes.
} 
with other interventions, such as the community reinforcement approach (CRA). ${ }^{* 268}$

\section{Behavioral Couples/Family Therapy.} Couples- and family-based treatments aim to improve communication and support and reduce conflict between couples and within families that have a member with addiction. ${ }^{269}$ Since lack of social and family support often is a barrier to treatment enrollment, the support of family members is important in helping individuals with addiction enter and complete treatment. Studies have found family and couples therapy to be effective for adolescents and adults, men and women and racial/ethnic minorities as well as for individuals for whom the primary substances of addiction are alcohol, marijuana, opioids or cocaine. ${ }^{270}$

A family approach to treatment generally is more effective than individual-based programs and tends to have higher retention rates than other evidence-based interventions. ${ }^{271}$

Combining couples/family therapy with other forms of individual-based treatments, such as cognitive behavioral therapy (CBT), tends to increase treatment effectiveness. ${ }^{272}$

[Addiction] is a family disease and you cannot treat an addict without bringing in the family and children. ${ }^{275}$

--John Schwarzlose Chief Executive Officer Betty Ford Center

\section{Combined Therapies}

Treatment programs that combine pharmaceutical and psychosocial treatments typically are more effective for individuals with addiction than the use of either form of intervention alone. ${ }^{273}$

\footnotetext{
* Stronger effects were found when the voucher was delivered immediately after the patient met the contingency requirement and when vouchers were of a higher value.
}

Our efforts to date have taught us some humbling lessons about addictive diseases, namely, that they are complex biopsychosocial entities which defy simple "either/or" solutions. ${ }^{274}$

--Norman S. Miller, MD

Professor of Medicine and Psychiatry Michigan State University

Combination therapy is successful for multiple reasons. First, the provision of one treatment modality tends to enhance compliance with the other. ${ }^{276}$ For example, medication may help patients better tolerate withdrawal symptoms that otherwise might have discouraged their participation in psychosocial therapy and psychosocial therapy might encourage patients to initiate and maintain a course of pharmaceutical therapy. ${ }^{277}$ Medications used in conjunction with psychosocial interventions have been found to increase patients' likelihood of remaining in treatment and maintaining abstinence. $^{278}$ Second, because there is no one treatment that works perfectly for every patient, patients who are provided with more than one treatment approach have an increased chance of success. ${ }^{279}$ Third, each modality may produce different outcomes, increasing overall success. For instance, in the case of smoking cessation, pharmaceutical therapy helps patients face withdrawal symptoms and maintain abstinence, while psychosocial treatments improve behavioral, cognitive and coping skills that are particularly useful for ensuring compliance with treatment and preventing relapse. ${ }^{280}$

Addiction Involving Nicotine. The combination of nicotine replacement therapy (NRT) and psychosocial approaches to smoking cessation increases patients' chances of quitting and their chances of achieving long-term abstinence. ${ }^{281}$ A review of combined therapy studies shows that the inclusion of NRT produced up to a 15-percentage point increase in efficacy rates over psychosocial treatment alone. ${ }^{282}$ One explanation for the improved results of combined therapies for tobacco cessation is that NRT is the primary mechanism behind patients' initial quitting success while the psychosocial therapies give patients the tools 
they need to avoid relapse over the longer term. ${ }^{283}$ Forms of pharmaceutical therapy other than NRT, such as antidepressants, also can enhance the benefits of psychosocial treatment for smoking cessation. ${ }^{284}$

\section{A Spectrum of Smoking Cessation Treatments}

Smokers of less than five cigarettes per day will have a good chance of success in quitting by choosing a quit date, getting rid of tobacco and using freely-available counseling/support services. Smokers of 6-14 cigarettes per day probably are moderately dependent and will benefit from an approved smoking cessation aid (nicotine patch, gum, lozenge, inhaler or nasal spray, bupropion, varenicline). Smokers of 15 or more cigarettes per day probably are highly dependent and will benefit from more intensive counseling and possibly combination pharmaceutical therapy. ${ }^{285}$

Addiction Involving Alcohol. In one study, patients with addiction involving alcohol who received daily doses of naltrexone were less likely than those taking placebos to relapse if they also participated in psychosocial therapies, including cognitive behavioral therapy (CBT) (38 percent vs. 60 percent) or motivationalenhancement therapy (MET) (44 percent vs. 56 percent) over the course of 12 weeks. ${ }^{286}$ Patients in another study ${ }^{\dagger}$ who received CBT for a three-month period were likelier to achieve abstinence by the end of the 12-week program if they also received daily doses of acamprosate (38 percent vs. 14 percent). ${ }^{287}$ Another study found that six months after treatment completion, disulfiram patients in a community reinforcement approach (CRA) program spent significantly less time drinking than patients who used only disulfiram (abstinent 28.3 days vs. 8.0 days that month). ${ }^{288}$

\footnotetext{
${ }^{*}$ CBT patients who took naltrexone spent significantly more of their time in treatment abstinent from alcohol than any of the other study groups.

${ }^{\dagger}$ The study groups were not randomized but matched based on gender, age, previous treatment episodes, detoxification history and average alcohol intake.
}

Addiction Involving Other Drugs. With regard to treatment for addiction involving opioids, incorporating family therapy into a treatment regimen that includes naltrexone therapy enhances treatment outcomes with regard to medication compliance; abstinence from opioids and other drugs during treatment and during a year of follow-up; and measures of drug-related, legal and family problems at oneyear follow-up. ${ }^{289}$ A meta-analysis of 30 studies conducted in outpatient methadone treatment settings found that the inclusion of contingency management (CM) is related to fewer positive urine tests submitted by patients with addiction involving opioids. ${ }^{290} \mathrm{CM}$ also has been found to augment naltrexone treatment for addiction involving opioids by increasing patients' compliance with their treatment regimen. Naltrexone patients who received contingency management in the form of vouchers in exchange for clean urinalyses, on average, stayed in treatment longer (7.4 weeks vs. 5.6 weeks), submitted more opioid-free urine samples (18.9 vs. 13.5) and were abstinent continuously over longer periods of time (49.1 days vs. 37.7 days) than patients who received naltrexone without a CM component. ${ }^{291}$ Another study found that patients on methadone maintenance treatment who received weekly community reinforcement approach (CRA) sessions demonstrated significantly greater reductions in drug problem severity ${ }^{\ddagger}$ than patients who received standard methadone maintenance services. ${ }^{292}$

Other research finds that cognitive behavioral therapy (CBT) patients who received daily doses $^{\S}$ of modafinil versus a placebo provided significantly more clean urine tests (42.3 percent vs. 24.0 percent) and were likelier to achieve abstinence from cocaine over at least a threeweek period (33 percent vs. 13 percent). ${ }^{293}$

\section{Addiction Involving Poly-Substances.}

Research on the best methods of treating individuals with addiction involving multiple substances is limited. One study found that methadone maintenance patients with addiction

\footnotetext{
‡ As measured by the Addiction Severity Index. $\S 400$ mgs.
} 
involving both opioids and cocaine fared better if they were randomly assigned to receive bupropion versus placebos and CM versus no psychosocial intervention. ${ }^{294}$ Other research points to the potential utility of combining an antidepressant ${ }^{*}$ with CM for patients with addiction involving opioids and cocaine who are maintained on buprenorphine. ${ }^{295}$ Patients who received this combined therapy provided more drug-free urine samples during treatment and achieved a period of continuous abstinence that was, on average, twice as long as patients with addiction involving opioids and cocaine who were in the control conditions. $^{\dagger 296}$ Preliminary evidence also suggests that daily doses of naltrexone $^{\ddagger}$ combined with CBT may be effective for treating addiction involving alcohol and cocaine, particularly among men. ${ }^{297}$

\section{Nutrition and Exercise}

A healthy nutrition and exercise regimen can mitigate the symptoms of withdrawal, enhance the effects of evidence-based treatment and help sustain successful treatment outcomes. ${ }^{298}$ Furthermore, because different addictive behaviors can share common causes, patients in treatment for addiction involving nicotine, alcohol or other drugs may substitute unhealthy foods in an attempt to satisfy addictive cravings. ${ }^{299}$ This is particularly evident in the common case of weight gain following smoking cessation. As such, a comprehensive approach to addiction treatment includes interventions aimed at ensuring good nutrition and exercise.

Nicotine, alcohol and other drug use also disrupt normal body functioning--resulting in nutritional deficiencies, dehydration or electrolyte imbalance--and often lead to unhealthy lifestyle changes such as poor diet and irregular eating habits. ${ }^{300}$ Providing patients in addiction treatment with nutritional programming may help them to reverse some of the damage that smoking, drinking and using other drugs can inflict on their bodies. The improvements in

\footnotetext{
* desipramine

${ }^{\dagger}$ Who took placebos combined with CM, desipramine without CM or placebos without CM. $\ddagger 150 \mathrm{mg}$.
}

general health and mood that may result from healthy eating habits also could help patients maintain their abstinence. ${ }^{301}$

Some individuals in treatment attempt to compensate for the lack of alcohol, for example, by consuming significant amounts of sugar and other carbohydrates which may increase serotonin $^{\S}$ levels. $^{302}$ A healthier approach, according to one theory, suggests that eating foods that are rich in the precursors of the neurotransmitters which are depleted when a substance user abstains will reduce cravings for those substances and facilitate the treatment process. ${ }^{303}$ These include protein-rich foods such as meat, fish, dairy products and nuts. ${ }^{304}$

Exercise also stimulates brain cells that reinforce dopamine-related reward pathways. ${ }^{305}$ This reinforcement may allow substance users to experience pleasurable effects from exercise which potentially could reduce their substancerelated cravings. ${ }^{306}$ Exercise generally is beneficial in reducing symptoms of depression and anxiety that often co-occur with and contribute to addiction. ${ }^{307}$ Another theory regarding the utility of exercise in a comprehensive treatment program is that as individuals develop a mastery of exercise techniques, they increase their self-efficacy--the belief that one can master new skills--which can be applied to disease management strategies. ${ }^{308}$ Patients who exercise in group settings also may benefit from social support networks and social interactions that do not involve tobacco, alcohol or other drug use. ${ }^{309}$

Exercise moderates the effects of nicotine withdrawal symptoms including reductions in cravings, negative mood, ${ }^{310}$ sleep disturbances ${ }^{311}$ and tension. ${ }^{312}$ One study found that exercisers in a smoking cessation program were twice as likely as those who did not exercise to demonstrate continual abstinence by the end of the three-month program, and three and 12 months following treatment completion. After a year, exercise participants were 36 percent less

\footnotetext{
$\S$ A neurotransmitter involved in mood, emotion, sleep, appetite and some aspects of addiction.
} 
likely than those who did not exercise to have relapsed. ${ }^{313}$

\section{Chronic Disease Management}

Although the reasons are not yet clear, some individuals may experience one episode in which their symptoms meet clinical diagnostic criteria for addiction and be non-symptomatic thereafter. ${ }^{314}$ In many cases, however, addiction manifests as a chronic disease--a persistent or long-lasting illness--which requires ongoing professional treatment and management. ${ }^{315}$ This may be due to a preexisting brain dysfunction or to changes that occur in the brain in response to repeated exposure to addictive substances which increase the vulnerability of the individual to relapse, even after cessation of substance use. ${ }^{316}$

All chronic diseases--regardless of whether they are genetically based, driven by biological or environmental influences or originate from some combination of these factors--require long term, evidence-based medical management of the disease by qualified health professionals and may include pharmaceutical and/or psychosocial therapies as indicated, to assure that patients remain symptom free and that co-occurring health conditions and the patient's nutrition and exercise requirements are addressed. ${ }^{317}$

Chronic disease management can improve patient functioning, suppress symptoms, prevent the development of additional diseases or cooccurring conditions and reduce relapse. ${ }^{318}$

Relapse can result from a wide range of factors including cravings and withdrawal symptoms; interpersonal conflict, peer pressure and other stressors; and the patient's emotional state, motivation level, self-efficacy and ability to cope with high-risk situations. ${ }^{319}$ Recent research also points to individual differences in brain structure as a risk factor for relapse: patients in treatment for addiction involving alcohol who had reduced volumes of gray matter ${ }^{*}$ in the regions of the brain associated

\footnotetext{
* Gray matter volumes were measured via magnetic resonance imaging (MRI). Gray matter is considered a reliable indicator of neural count and functionality.
}

with impulse control and cognitive function were more likely to relapse after a shorter period of time than those with higher volumes of gray matter in these brain regions. ${ }^{320}$ Yet another obvious but rarely considered factor that may contribute to relapse risk is that the treatment the patient received simply was inadequate, either in terms of the type of intervention provided or the length or intensity of the treatment. ${ }^{321}$

Chronic disease management, as it applies to addiction treatment and relapse prevention, seeks to address and prevent those factors that increase the likelihood of relapse by ensuring that treatment delivery is effective and that personal, psychological and environmental risk factors for relapse are addressed and mitigated. ${ }^{322}$ Specifically, physicians supervising addiction treatment should assess the need for chronic disease management and ensure continuing care. ${ }^{323}$

\section{Medically Supervised Disease Management}

Health care providers are optimally situated to provide clinical disease management. ${ }^{324}$ Following acute treatment, the disease management process is critical to help maintain health and prevent relapse. ${ }^{325}$

Patients who have received acute treatment for addiction may require maintenance medications such as methadone, buprenorphine, naltrexone, disulfiram or NRT to prevent relapse. ${ }^{326}$ Since many patients with addiction have co-occurring health conditions that may complicate their treatment or exacerbate the risk of relapse, medical professionals should carefully supervise and coordinate treatment for all conditions. ${ }^{327}$

Medical professionals supervising patient care also may perform toxicology screens to monitor patients' substance use following acute treatment and modify clinical interventions accordingly. ${ }^{328}$

Medical professionals also should supervise the psychosocial interventions that patients receive following acute addiction treatment. Psychosocial therapy often is a critical component of relapse prevention. ${ }^{329}$ Collaborating with other health professionals, as 
needed, physicians should work to educate patients about the precipitants of cravings and relapse and help them to cope adaptively with the associated psychological and environmental risk factors.

\section{The Physician Health Program}

An addiction treatment model that provides effective long-term disease management is the Physician Health Program (PHP) established by state medical societies and licensing boards to treat physicians who have addiction. ${ }^{330}$ Today, all 50 states and the District of Columbia have a PHP. ${ }^{331}$ The PHP assists the participating physician in finding clinical treatment services, ${ }^{332}$ provides case management of individuals undergoing treatment and maintains relationships with the authorized treatment facilities that evaluate and treat referred physicians. ${ }^{333}$ Contingency management in the form of negative consequences for violating its terms (e.g., losing one's license to practice medicine, malpractice suits, disciplinary actions from hospital boards) is a critical part of the program. ${ }^{334}$ PHPs must have the oversight of a medical director; some have an MD on staff who serves as a medical consultant. ${ }^{335}$

Treatment managed by a PHP is more intense and lasts longer than that for the general population, with three to six months spent in structured therapy followed by five years of contingency management, and it includes pharmaceutical therapy, psychosocial interventions and nutrition and exercise counseling. ${ }^{336}$ PHPs also typically have a mutual support program component. ${ }^{337}$ Long-term monitoring is employed to reduce relapse: when relapse or signs of possible relapse occur, the reaction is therapeutic, not punitive. ${ }^{338}$ Physicians who relapse tend to improve again after a treatment adjustment. $^{339}$

More than 80 percent of physicians who participate in PHPs return to work and remain substance free for a minimum of five years after receiving treatment. ${ }^{340}$ Seventy-one percent of participating physicians retain their medical license and are employed after five years. ${ }^{341}$

Key elements of the PHP program that appear to contribute to its success and that are replicable in the general population include high intensity care for an extended duration and the inclusion of longterm monitoring and disease management. ${ }^{342}$

\section{Case Management}

Many of the activities involved in chronic disease management and oversight can be performed by professionally-trained case managers working in a variety of settings such as physicians' offices, hospital or out-patient addiction treatment programs, justice facilities or social service agencies. They should, however, work under the supervision of a trained physician and other medical professionals. $^{343}$

\section{A Case Management Approach: Recovery Management Checkups}

Recovery Management Checkups (RMC)--in which patients are contacted, evaluated and linked to additional support services including those that address co-occurring conditions--have been shown to be an effective approach to chronic disease management by helping patients engage in and stay in treatment. ${ }^{344}$

Research finds that adolescents and adults in addiction treatment who receive RMCs--based on a method that involves locating individuals for checkup, assessing eligibility for intervention, linking individuals to treatment services, engaging participants in treatment and retaining participation for at least 14 days in residential treatment or seven days in intensive or regular outpatient treatment--demonstrate higher levels of treatment participation and abstinence rates and reduced time to readmission for treatment following relapse. ${ }^{345}$ Patients receiving such checkups were significantly likelier than patients receiving usual care to return to treatment (55 percent vs. 37 percent), do so sooner (384 days earlier) and to be abstinent ( 480 days vs. 430 days) over a two-year follow-up. ${ }^{346}$

Professional case managers can help to navigate and coordinate resources within the fragmented health care system and ensure that patients receive the services they need to keep addiction symptoms in check. ${ }^{347}$ The role of the case manager is to link patients to the health and social services appropriate to their specific needs, ensure that patients follow through with referrals, help patients identify and access a 
variety of additional support programs including family and peer support and auxiliary services, provide educational materials on relapse prevention and promptly intervene in the case of relapse. ${ }^{348}$ Monitoring the course of a patient's treatment and connecting patients with services when they are needed are common public health approaches to addressing chronic illnesses. ${ }^{349}$

\section{An Example of an Effective Case Management Program for Women}

CASASARD $^{\text {SM }}$ is a welfare demonstration program for mothers in Essex and Atlantic counties in New Jersey who have addiction. The program is designed to get women engaged in treatment and employment services, help them become sober and move successfully to stable employment. CASASARD ${ }^{\mathrm{SM}}$ uses an intensive case management approach to provide services for these women.

CASA Columbia's research has found that women with addiction involving alcohol or other drugs (excluding nicotine) who receive income assistance through CASASARD ${ }^{\mathrm{SM}}$ were more likely to initiate treatment (66.5 percent vs. 50.3 percent) and complete their programs (43.5 percent vs. 22.7 percent) if they were provided with case management services rather than standard care. Looking at abstinence as one outcome measure, over a 12-month post-referral period, women participating in the CASASARD $^{\text {SM }}$ program had a 64 percent higher monthly abstinence rate than their peers in the standard care program, and were likelier to have remained completely abstinent by the end of the 12-month period (41 percent vs. 25 percent). ${ }^{354}$ After another 12 months, the abstinence rate among CASASARD ${ }^{\mathrm{SM}}$ participants had increased to 47 percent while the abstinence rate among non-participants remained relatively unchanged (24 percent). ${ }^{355}$

Case management is associated with an increased chance that people with addiction will access treatment for relapse, remain in treatment for a longer period of time ${ }^{350}$ (which is associated with better treatment outcomes), utilize support services ${ }^{351}$ and demonstrate improved social outcomes. ${ }^{*} 352$ A meta-analysis of case management for patients in treatment for addiction involving alcohol or drugs other than nicotine found that case management can improve patients' family and social relationships, living situations and health. ${ }^{353}$

\section{Support Services}

Comprehensive care for patients with addiction requires not only proper assessment, stabilization, acute care and chronic disease management, but support services as well. These may include:

- Mutual support programs to bolster disease management efforts and avoid the recurrence of disease symptoms; and

- Auxiliary support services to address legal, educational, employment, housing, parenting and child-care issues that may impede disease management. ${ }^{356}$

Health care providers are optimally situated to facilitate links to these support and auxiliary services. ${ }^{357}$

\section{Mutual Support Services}

Mutual support programs, sometimes referred to as self-help groups, can be a significant part of a comprehensive approach to caring for a patient with addiction. In fact, for many people with addiction, these programs have been the main help available to them and have been both lifesaving and critical to helping them manage their disease. ${ }^{358}$ These programs allow individuals with addiction to seek and provide social, emotional and informational support within a group of their peers. Participation in these programs can increase the chances of achieving and maintaining abstinence as well as

\footnotetext{
* In one study, veterans with addiction involving illicit drugs who were assisted by case managers experienced a larger increase in the number of days they spent gainfully employed than their peers who went unassisted which, in turn, was associated with lower rates of substance use, incarceration and arrests resulting in convictions.
} 
help patients reduce their substance-related problems. ${ }^{359}$ They are incorporated into many formal treatment programs, can be an important part of chronic disease management and constitute the most frequently-accessed resource among people with addiction involving alcohol and other drugs and related problems. ${ }^{360}$

National data indicate that an average of five million people ages 12 and older attend an alcohol or other drug use mutual support group each year. Slightly less than half (45.3 percent) of the attendees participated in support groups for addiction involving alcohol; 21.8 percent participated in support groups for addiction involving other drugs and 33.0 percent participated in support groups for co-occurring addiction involving alcohol and other drugs. ${ }^{*} 361$

Although there are many types of mutual support programs, most have many characteristics in common: ${ }^{362}$

- Group members share the same problem or status;

- Groups are run using self-directed leadership; that is, members, rather than an outside governing figure, are in charge of the program;

- Group members share their experiences and lessons learned;

- Members share the goal of changing some aspect of their personal behavior;

- $\quad$ Reciprocal helping is the norm;

- Members participate on a voluntary basis; and

- Programs may accept donations but do not charge for membership. ${ }^{363}$

Some organizations focus more on providing fellowship, information or self-acceptance while others attempt to address the origin of

\footnotetext{
${ }^{*}$ Comparable data on rates of participation in Nicotine Anonymous are not available.
}

participants' addiction problems. ${ }^{364}$ Programs also vary in terms of the methods used to address addiction, the extent to which there is an emphasis on spirituality or religion, whether members are encouraged to participate in political advocacy, ${ }^{\dagger}$ whether friends and family members are included in group meetings, the extent to which the group facilitates connection with treatment professionals working in an advisory role ${ }^{\ddagger}$ and whether the program accepts external funds. ${ }^{365}$

The 12-step groups, such as Alcoholics Anonymous (AA) and Narcotics Anonymous (NA) are some of the best known and most widely available mutual support programs. ${ }^{\S 366}$ They provide participants with 12 steps to follow during the process of recovery. ${ }^{367}$ These programs generally begin with the acceptance of one's addiction and--through a process of selfexploration--participants take action to make changes toward recovery. ${ }^{368}$

Other core components of these programs are taking responsibility for the recovery process, sharing personal experiences, recognizing the existence of a higher power, helping others and lifetime commitment to the program. ${ }^{369}$ Outside of the group meetings, sponsorship ${ }^{* *}$ plays an important role in most 12-step programs. Each new group member finds a sponsor to serve as a role model, program guide and first line of support in the case of a potential or actual relapse. ${ }^{370}$ It is estimated that $\mathrm{AA}$, the oldest

\footnotetext{
† e.g., unlike organizations such as Alcoholics Anonymous (AA) and Narcotics Anonymous (NA) which require anonymity, the Faces and Voices of Recovery organization seeks to organize and mobilize individuals with histories of addiction, their families and friends in public policy advocacy efforts.

${ }^{\ddagger}$ Professionals may work in an advisory role or they may serve as guest lecturers.

$\S$ There also are 12-step programs for individuals dealing with gambling and other types of addiction, and with a family member's or friend's addiction, such as Al-Anon and Alateen.

** Sponsors typically have completed at least one year of abstinence with the program and are of the same sex as the new member (or a different sex for gay members).
} 
and best known of the 12-step programs, has more than 2,000,000 members in more than 114,000 groups in more than 180 different countries. ${ }^{371}$

Other examples of mutual support programs that do not follow the traditional 12-steps approach and that are secular in nature include SMART

Recovery (Self-Management and Recovery Training), ${ }^{372}$ Secular Organizations for Sobriety (SOS), Women for Sobriety and LifeRing Secular Recovery. ${ }^{373}$ Groups vary based on the population they serve. ${ }^{374}$

Participation in mutual support programs-including 12-step and secular-based programs-has been associated with improved psychological functioning, reduced substance use and reduced health-care costs and utilization. ${ }^{375}$ However, there are methodological limitations to the effectiveness studies that examine mutual support programs, restricting the extent to which their ability to curb substance use and help manage addiction can be determined. ${ }^{376}$ Most are not randomized controlled studies, research on the programs is difficult to conduct due to the anonymous nature of group membership ${ }^{377}$ and there may be an inherent bias in the research in that AA and other 12-step groups may attract patients who are more motivated to change. ${ }^{378}$ Despite the limited empirical evidence demonstrating the effectiveness of mutual support programs like AA, anecdotal evidence of their effectiveness abounds. ${ }^{379}$

One finding that emerges consistently from the available research is that patients who had been in addiction treatment and then followed up with involvement in mutual support programs fare better than those who do not. ${ }^{380}$ A study that followed treatment patients with addiction involving alcohol for three years found that those who were more involved with mutual support programs ${ }^{*}$ were likelier than other patients to be abstinent the next year. Mutual support program members who were not abstinent the year following group participation

\footnotetext{
* Including, but not limited to, AA, SMART

Recovery and Women for Sobriety.
}

still drank less on the days that they did consume alcohol. ${ }^{381}$ Other research finds that those who attend AA or another 12-step group following treatment have about twice the rate of abstinence as those who do not participate in these mutual support programs. ${ }^{382}$

Reviews of the research on the effectiveness of mutual support programs suggest that it may not be the specific content of the programs or their processes that are associated with positive outcomes, but rather the fact that they provide free, long-term and easily-accessible exposure to people and messages that support recovery, ${ }^{383}$ which is a key element of chronic disease management. ${ }^{384}$

Twelve Step Facilitation. Twelve Step Facilitation (TSF) is a formalization and professionalization of the 12-step mutual support model which involves a brief, structured and manual-driven approach implemented over the course of 12 to 15 sessions by a trained counselor or treatment provider. ${ }^{385}$ During these sessions, providers will advocate abstinence, explain the basic concepts of the 12-steps and actively support and facilitate the patient's involvement in 12-step programs. TSF can be implemented in an individual or group format or including the patient's significant other. It has been used in acute treatment and as a method of providing support services for chronic disease management. ${ }^{386}$

TSF has been used to address addiction involving alcohol, ${ }^{387}$ marijuana and stimulants. ${ }^{388}$ Evaluations of TSF are limited; however, several studies have found it to be comparable in effectiveness to psychosocial treatments such as CBT and MET. ${ }^{389}$ It is listed in SAMHSA's National Registry of Evidencebased Programs and Practices ${ }^{390}$ and as an evidence-based approach by the National Institute on Drug Abuse (NIDA). ${ }^{391}$

Residential Programs. Some mutual support approaches, such as the Therapeutic Community (TC) model, are residential and incorporate elements of treatment. Other residential programs such as recovery homes or sober living houses provide mutual support only. These 
programs are non-professional, generally lowcost communal homes that provide supportive, substance-free living environments to individuals attempting to establish or maintain sobriety. ${ }^{392}$ Homes may be democratically run or hierarchically structured with house managers in charge of other residents. Typically, participation in additional aftercare services ${ }^{*}$ is encouraged or required. ${ }^{393}$

Therapeutic Communities. The TC model, used primarily but not exclusively with the justice population, is a highly structured residential program that requires a long-term commitment (six to 24 months). ${ }^{394}$ It is based on mutual support principles and incorporates behavior modification techniques, education classes and residential job duties. ${ }^{395}$ This approach aims to re-socialize the patient to a substance-free, crime-free lifestyle through peer influence, personal responsibility and skill training. ${ }^{396}$ TC participants commonly include individuals with relatively long histories of addiction, involvement in serious criminal activities and significantly impaired social functioning. ${ }^{397}$ The mutual support aspect of TCs operates on a hierarchical basis; patients who have been involved in the program longer provide support and serve as role models for newer patients. ${ }^{398}$

A large, national study found that patients enrolled for at least 90 days in a $\mathrm{TC}^{\dagger}$ were significantly less likely to have used cocaine (28 percent vs. 55 percent), tested positive for drug use (19 percent vs. 53 percent), reported daily alcohol use (9 percent vs. 15 percent) or have spent time in jail ( 24 percent vs. 54 percent) a year after program participation than those who spent fewer than 90 days in the program. ${ }^{399}$ The year following successful TC completion showed lasting effects along several indicators compared to the year prior to TC entry: the rate of weekly cocaine use fell from 66.4 percent to 22.1 percent; weekly heroin use, from 17.2 percent to 5.8 percent; heavy alcohol use, from 40.2 percent to 18.8 percent; illegal activity, from 40.5 percent to 15.9 percent; less than full-

\footnotetext{
* Not defined.

${ }^{\dagger}$ Long-term residential programs, most of which were TCs.
}

time employment, from 87.6 percent to 77.0 percent; and reported suicidal thoughts, from 23.6 percent to 13.2 percent. $^{400}$

For patients with co-occurring mental health disorders, a Modified Therapeutic Communities (MTC) model takes into account patients' psychiatric symptoms, ${ }^{\ddagger}$ potential cognitive impairments and reduced levels of functioning due to substance use, including poor control over urges and short attention spans. MTCs are more flexible, less intense and more individualized than standard TCs. ${ }^{401}$

Sober Living Houses. Sober Living Houses provide a substance-free living environment for individuals with addiction involving alcohol, illicit drugs and controlled prescription drugs. No formal treatment services are provided but residents are mandated or strongly encouraged to participate in mutual support programs and must comply with house rules which include maintaining abstinence, paying rent, participating in house chores and attending house meetings. ${ }^{402}$ Failure to comply with these rules results in dismissal from the home. ${ }^{403}$

One study of the combination of participating in a sober living house and receiving outpatient treatment interviewed participants within their first week of entering the houses and again at six-, 12-, and 18-month follow-ups. ${ }^{\S}$ The study found significant improvements over time on measures of alcohol and other drug use, arrests and employment. ${ }^{404}$

The Oxford House Model. Oxford House, founded in 1975, is one of the most prevalent and well-studied examples of communal-living environments of this nature. ${ }^{405}$ Unlike sober living houses, they encourage but never mandate participation in mutual support programs. ${ }^{406}$ Typically, eight to 15 residents of the same sex live in each home. Most recently have received detoxification or some form of treatment and many have been homeless or spent time in jail at

\footnotetext{
‡ Although care is not medically-supervised.

$\S$ The average length of stay in the sober living houses was over five months but there was considerable variation.
} 
some point in their lives. ${ }^{407}$ Homes are run independently by the residents; there are no onsite professional treatment providers. The homes are supported financially by residents who are required to pay the rent on time and maintain their sobriety. ${ }^{*}$ Contrary to a chronic disease management approach, members who relapse are expelled immediately from the house and must demonstrate 30 days of sobriety before reapplying for residence. ${ }^{408}$ Residents are expected to attend weekly meetings where house issues are discussed and where they are encouraged to attend mutual support programs. $^{409}$

One study found that two years after completing treatment and entering an Oxford House, current and former residents were more successful at decreasing their use of alcohol and other drugs than those who completed treatment but simply received standard aftercare referrals to outpatient programs or self-help groups. ${ }^{410}$ Over that period, those who lived in an Oxford house were less than half as likely as those who received usual care to use any substance (31.3 percent vs. 64.8 percent), less likely to be currently facing criminal charges ( 0.0 percent vs. 5.6 percent) and more likely to be employed (76.1 percent vs. 48.6 percent) two years later. Only 15.6 percent of participants who had lived in an Oxford House for at least six months relapsed after two years and residents ages 36 and younger demonstrated a two-year relapse rate of only 6.7 percent. ${ }^{411}$

\section{Auxiliary Support Services}

Patients who complete treatment successfully may find themselves facing relapse due to the anxiety of coping with other health problems, unemployment, child care, homelessness, criminal justice and other social problems. ${ }^{412}$ Matching patients with the services necessary to address these problems decreases the risk of relapse. $^{413}$

\footnotetext{
* New houses often are started with federal or state loans stemming from the 1988 Anti-Drug Abuse Act; the loans are paid back by the residents.
}

According to data from a national multi-site study of a variety of treatment modalities, the majority of treatment patients' perceived a need for auxiliary support services including family counseling services (70.9 percent), supplementary medical care (63.5 percent), mental health care (63.4 percent), housing (63.0 percent) and vocational services (61.4 percent). ${ }^{414}$ (Figure 5.A)

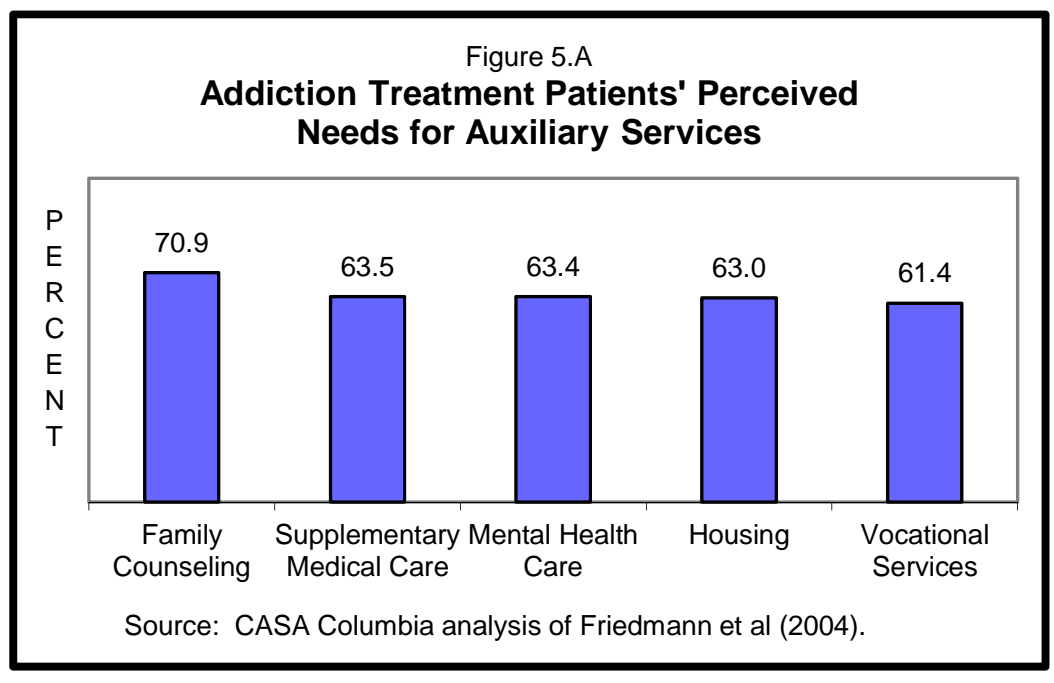

The perceived needs for supplementary medical, including mental health, care underscore the historic disconnect of addiction treatment from medical care. Individuals who had their perceived needs for vocational and housing services met showed significant reductions in illicit drug use. $^{\dagger 415}$

Evidence from a smaller study also demonstrated that matching treatment patients with services they need can decrease the severity of their substance use. ${ }^{\ddagger}$ Six-months after enrolling in the study, patients who reported a need for housing services and had their needs matched experienced a greater reduction in their substance use severity score compared to patients with housing needs who were not matched to services (50 percent vs. 23 percent

\footnotetext{
† Patients' needs were determined by their rating of a service as "somewhat or very important." Because patients were not randomly assigned to receive services, the reductions in illicit drug use cannot be linked directly to having their perceived service needs met.

${ }^{\ddagger}$ As measured by the Addiction Severity Index.
} 
reduction). ${ }^{*}$ Similarly, patients who reported a need for child care services and had their needs addressed experienced a greater reduction in their substance use severity score compared to patients with child care needs who were not matched to needed services (45 percent vs. 20 percent). ${ }^{\dagger} 416$

\section{The Use of Technology in Addiction Treatment and Disease Management}

An emerging approach to identifying, treating and managing addiction--the utilization of online computer tools and other technology-based interventions to enhance access and delivery--is showing some promising results. ${ }^{417}$ These include telephone contact with treatment providers and e-mail, text messaging, smartphone apps and online support groups. ${ }^{418}$

Online approaches that employ evidence-based practices via Web sites or tele- or videoconferencing offer key advantages. They can provide psychosocial therapies to patients at lower cost than traditional face-to-face approaches. ${ }^{419}$ They allow patients who live far from specialty treatment providers or who lack resources to access psychosocial therapies or supplemental services in a convenient manner. ${ }^{420}$

Researchers are just beginning to investigate the utility of technology-based services for patients with addiction. While most existing studies are methodologically weak or flawed, ${ }^{421}$ a randomized controlled trial of a digitallydelivered smoking cessation intervention found significantly higher long-term abstinence rates compared to a control group receiving only a self-help booklet (22.3 percent vs. 13.1 percent). ${ }^{422}$ Another randomized control study found that patients enrolled in a methadone maintenance program who participated in Internet-based group therapy sessions reported

\footnotetext{
* There was a 41 percent reduction among patients who never reported needing the services. Patients were not randomly assigned to receive services.

† There was a 31 percent reduction among those who never reported needing the service.
}

greater satisfaction with the program than patients who participated in traditional, face-toface group therapy sessions; reductions in positive drug urine tests during the six-week study were comparable between the two groups, suggesting that Internet-based therapy may be an effective treatment tool. ${ }^{423}$ A recent study found that a smartphone-based support system shows potential for preventing relapse in addiction involving alcohol; ${ }^{424}$ however, further research is needed on this particular program and, more generally, on the outcomes of technology-based services and how they compare to traditional service delivery methods. ${ }^{425}$

Concerns about technology-based services include the risks to privacy and confidentiality of information transmitted over the Internet, safety and efficacy, and issues of licensing posed by treatment delivered across state lines. ${ }^{426}$

\section{Public Attitudes about Addictive Substances and the Need for Addiction Treatment}

Although the American public appears to be supportive of assuring that individuals with addiction receive effective addiction treatment, the view of most individuals about the relative need for treatment for individuals who use addictive substances and what constitutes effective treatment does not match the science of best practices.

\section{Perceptions of the Relative Need for Treatment Based on Substance of Addiction}

CASA Columbia's NABAS found significant differences in respondents' views of the type and extent of substance use that indicates a substance use problem and the need to seek treatment. Public perceptions do not reflect the continuum of substance use or distinguish between risky use and the need for intervention versus addiction and the need for treatment:

- The majority of respondents reported that any use of the illegal drugs heroin (84.0 
percent), cocaine (76.0 percent) or methamphetamine (73.9 percent) indicates that the user has a serious problem and should seek treatment.

- $\quad 42.5$ percent thought that any misuse of a controlled prescription drug is a serious problem requiring treatment; 29.6 percent thought that daily or more than daily misuse of these drugs is serious enough to require treatment.

- $\quad 35.8$ percent felt that any use of marijuana indicates that the user has a serious problem and should seek treatment; the same amount (35.9 percent) thought that daily or more than daily use of marijuana is a serious problem that requires treatment. complete abstinence should be the main goal of treatment for individuals with addiction involving illicit drugs (65.7 percent), alcohol (60.0 percent), tobacco (50.1 percent) or controlled prescription drugs (47.1 percent). Whereas the next most common response for the goal of addiction treatment was that the goal should be set by the patient, a small but significant proportion indicated that reduced use should be the primary goal; this was particularly true for addiction involving prescription drugs, ${ }^{\dagger}$ tobacco and alcohol, but less so for addiction involving illicit drugs. A focus solely on reducing the negative consequences of substance use (the "harm reduction" approach) rather than reducing or eliminating use was endorsed by fewer than five percent of the respondents as a main goal of treatment. ${ }^{428}$ (Figure 5.B)
- $\quad 78.0$ percent reported that alcohol use is a serious problem requiring treatment if it occurs daily or more than daily; 6.8 percent reported that any drinking is a serious problem requiring treatment. $^{427}$

- $\quad 49.5$ percent felt that smoking was a serious problem requiring treatment if

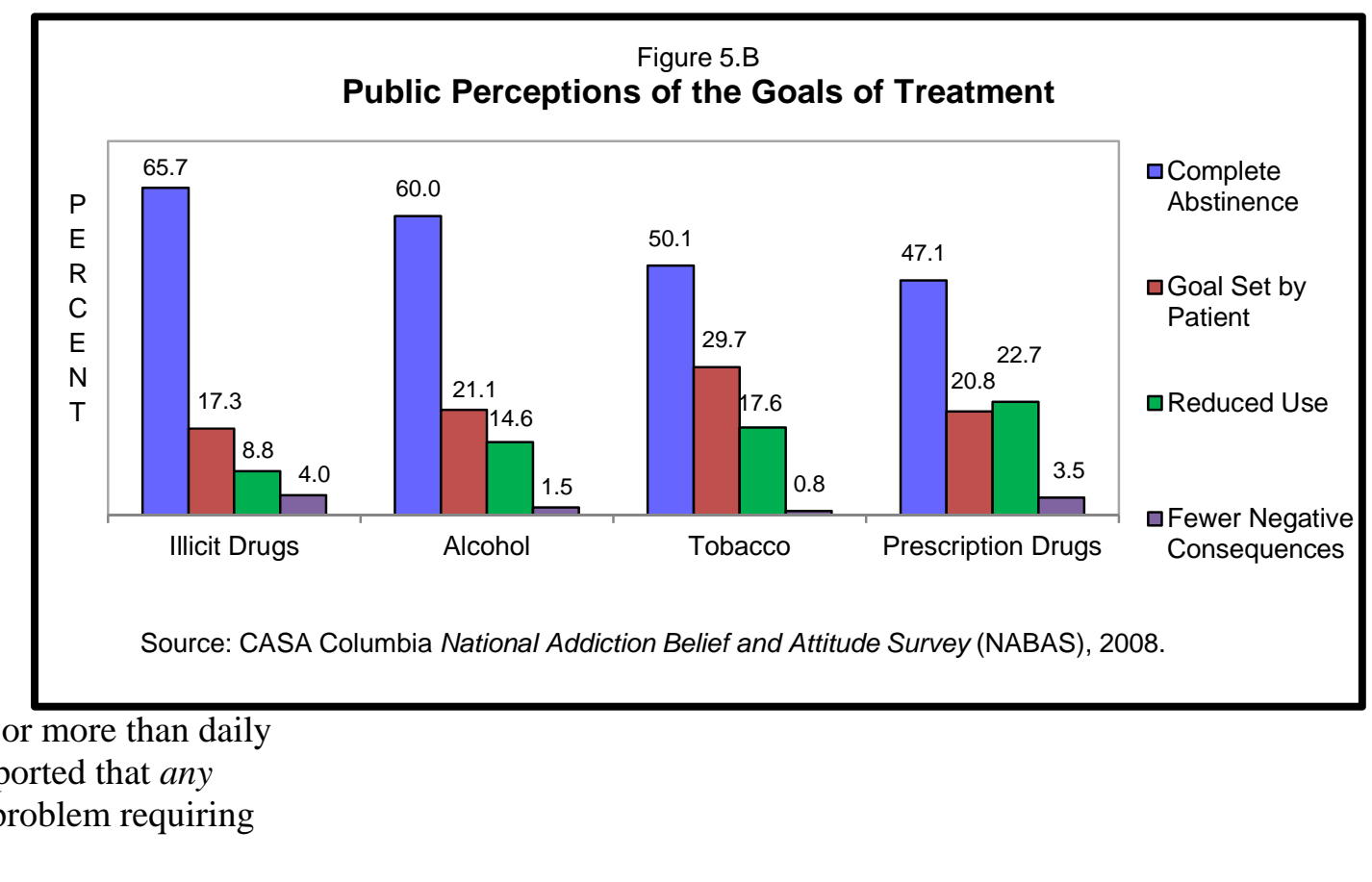

\section{Perceptions of the Goals of Treatment}

From a medical perspective, the goal of addiction treatment would be to restore and maintain health and eliminate or reduce risky behavior that threatens health and safety. Most respondents to the $N A B A S^{*}$ indicated that

\footnotetext{
* In the NABAS survey, respondents were asked which of the following they thought should be the main treatment goal for someone with addiction
}

involving tobacco, alcohol, illicit drugs and prescription drugs: complete abstinence, reduced use, fewer negative consequences from use, or that the goal should be set by the patient.

${ }^{\dagger}$ For prescription drugs, reduced use was a more common response (22.7 percent) than that the goal should be set by the patient (20.8 percent). 


\section{Perceptions of the Types of Interventions that Constitute Treatment}

Although a wide range of psychosocial and pharmaceutical therapies are available to treat addiction, 60.1 percent of respondents to the NABAS spontaneously offered mutual support programs such as AA or NA as a "treatment" intervention when asked what kinds of treatment come to mind when they think about treatment for addiction. This is despite the fact that a mutual support program, while a very helpful resource to many individuals with addiction, is not an evidence-based treatment for the disease. Forty percent (39.9 percent) mentioned a hospital visit, 11.7 percent mentioned residential rehabilitation clinics and 10.4 percent mentioned outpatient treatment as what comes to mind when they think of addiction treatment (although these are treatment venues, not treatments approaches). Although only 11.6 percent mentioned the utilization of prescription medications for treating addiction (Figure 5.C), a separate question found that 54.7 percent of respondents (46.8 percent of those with a history of addiction) stated that it is good that there are medicines to treat addiction because addiction is a medical condition that medicine can help. Perhaps reflective of a lack of knowledge about effective treatment for addiction, 38.3 percent of respondents (48.6 percent of those with a history of addiction) indicated it is not good news that there are medicines to treat addiction because treating addiction with medication only serves to replace one addiction with another. ${ }^{429}$

Some individuals with addiction may not access appropriate treatment because of the belief that the use of pharmaceutical treatments, such as methadone, to treat addiction is contrary to the abstinence-based perspectives of addiction treatment espoused by many mutual support/12-step programs. A recent study found that adherence to such beliefs leads many heroin users to underutilize methadone maintenance therapy despite their knowledge of its effectiveness. ${ }^{430}$

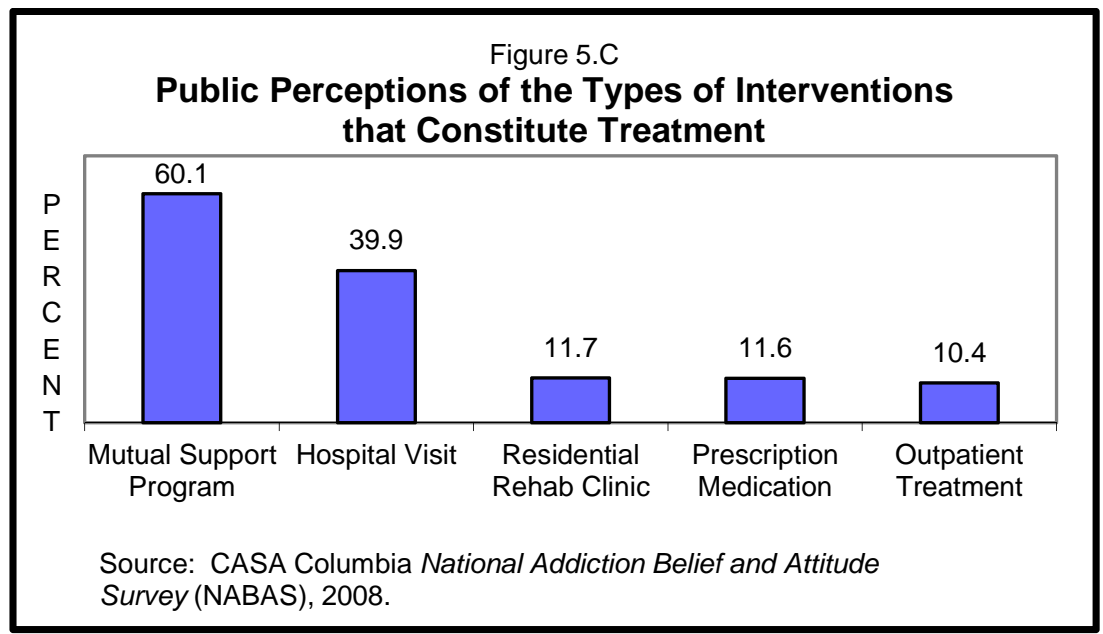

\section{Perceptions of the Effectiveness of Treatment}

Despite respondents' limited awareness of effective science-based treatments for addiction, the majority of respondents to the NABAS reported believing that if a person admits to having a problem and wants to get better, addiction treatment is somewhat or very effective:* approximately eight in 10 indicated that treatment for addiction involving alcohol (82.5 percent) or prescription drugs (79.9 percent) is effective, and about three quarters indicated that treatment for addiction involving illicit drugs (73.6 percent) and tobacco (73.1 percent) is effective. ${ }^{431}$ (Figure 5.D)



\footnotetext{
* It is important to note, however, that a significant proportion of the public defines treatment in a way that is not consistent with the science of best practices in treatment.
} 
These data are consistent with those of other recent surveys. One found that the majority of Americans believe that treatment programs can help people with addiction involving alcohol (81 percent), prescription drugs (79 percent), marijuana (78 percent) or other illicit drugs (69 percent). ${ }^{432}$ Another found that 88 percent of Americans believe that addiction treatment is extremely or very important in helping people get better and 77 percent believe that long-term recovery is unlikely to be successful without treatment and continued support. ${ }^{433}$ 
-118- 


\section{Chapter VI \\ Tailored Treatment for Special Populations}

Diseases manifest differently in different individuals and, as with other health conditions, a one-size-fits-all approach to addiction treatment typically is a recipe for failure. ${ }^{1}$ For addiction treatment to be effective, it must be tailored to the individual patient, including the particular stage and severity of the disease, overall health status including any co-occurring conditions, past treatments and any other life circumstances that might affect patient outcomes. $^{2}$ Treatment approaches also must be appropriate to the patient's age, gender, race/ethnicity and cultural background. ${ }^{3}$

Although research on the effectiveness of various treatment approaches for special populations is very limited, particular subgroups for whom there is some documented evidence of the benefits of specialized treatment include: those with co-occurring health conditions, adolescents, women, older adults, racial and ethnic minorities, individuals of minority sexual orientation, veterans and those in active duty military, and individuals involved in the justice system.

\section{Co-occurring Medical Disorders}

Because addiction causes, contributes to and cooccurs with multiple other diseases, including 70 other conditions requiring medical care such as heart disease and cancer, ${ }^{+4}$ physicians and other medical professionals must address these cooccurring health conditions in the course of caring for their patients and assure that medication interactions and the use of

\footnotetext{
* The following discussion is a brief overview of tailored treatment approaches for special populations and is not an exhaustive or definitive account of all possible treatments of this nature. More research is needed to identity the best treatment approaches for the special populations discussed in this chapter as well as for others not included here.

${ }^{\dagger}$ See Chapter III.
} 
potentially addictive medications are considered and monitored. ${ }^{5}$

For example, patients treated with opioids for pain may be at risk for developing addiction, particularly if they do not take them as prescribed. ${ }^{6}$ Likewise, the frequent use of addictive substances such as cocaine can result in a range of gastrointestinal and cardiac complications that can affect various organs in the body; medications used in detoxification and addiction treatment may further complicate these medical conditions. ${ }^{7}$

The efficacy of particular addiction treatment approaches has not been examined systematically in patient populations with cooccurring medical conditions. However, medical and other health professionals should plan carefully the treatment protocols for patients with co-occurring addiction and other medical conditions, be prepared to monitor and address emergent reactions that may arise in the course of treating these patients and consult with specialists in other medical sub-specialties when necessary. ${ }^{8}$

\section{Co-occurring Mental Health Disorders"}

In the late 1970s, treatment professionals began to recognize the effect that co-occurring mental health disorders had on patients' success in addiction treatment. Around the same time, treatment professionals and programs began to document the large number of addiction and mental health treatment seekers suffering from both sets of problems. ${ }^{\dagger 9}$

Traditionally, patients with co-occurring addiction and mental health disorders were sent to one treatment setting or another to address their problems sequentially. ${ }^{10}$ Patients have

\footnotetext{
* The treatment programs discussed in this section represent the main evidence-based treatment modalities for individuals with co-occurring addiction and mental health disorders. It is not a comprehensive list of available programs.

${ }^{\dagger}$ See Chapter III for data on the prevalence of cooccurring addiction and mental health disorders.
}

been advised either to "solve" their addiction problems before entering mental health treatment or stabilize their mental health problems before entering addiction treatment. ${ }^{11}$ This approach has not been effective since each condition tends to exacerbate the other. ${ }^{12}$

Current standards call for treatment programs serving patients with addiction and mental health disorders to provide integrated care that treats both conditions simultaneously. ${ }^{13}$ Treatment providers may coordinate addiction and mental health treatment services by combining therapies or by managing the care while patients receive both types of treatments concurrently. ${ }^{14}$

\section{Essential Program Components and Principles for Treating Patients with Co-occurring Disorders ${ }^{15}$}

- Coordinated treatment and recovery plan;

- Access to addiction and regular medical and mental health services within the same facility or through collaborating programs;

- Specialists to provide addiction treatment, psychiatric services and other health care services as needed;

- Patient information about the nature of the disorders, the importance of lifestyle changes and adherence to treatment regimens and strategies for relapse prevention;

- Comprehensive support services to address issues such as housing and unemployment;

- Access to mutual support programming; and

- Reintegration of patients with their families and communities.

Integrating addiction treatment and mental health care for patients with co-occurring disorders increases retention and yields positive outcomes, including higher abstinence rates and reduced hospitalization and arrest rates. ${ }^{16}$ Integrated treatment also helps providers prevent adverse drug interactions among their patients and ensure that proper medication dosage is used to treat both conditions. ${ }^{17}$ 


\section{Tobacco Cessation}

Smoking rates are high among individuals with mental health disorders, due to common neurobiological and psychosocial risk factors, the tendency to smoke as a means of selfmedication and a reduced ability to manage the difficult process of cessation. ${ }^{18}$

Practice guidelines for smoking cessation underscore the importance of providing smoking cessation services to patients with co-occurring mental health disorders, utilizing motivational and cognitive-behavioral approaches and combining psychosocial therapies with pharmaceutical interventions--practices that mirror those that are recommended for the general population. ${ }^{19}$ However, in implementing these approaches, care must be taken to ensure that interventions are tailored to the clinical needs of the patient and that such interventions do not contraindicate other treatments the patient might be receiving for his or her mental illness. ${ }^{20}$

Bupropion has been approved by the FDA as both a smoking cessation medication and an antidepressant, making it uniquely suited to treat individuals with co-occurring mood disorders and addiction involving nicotine. ${ }^{21}$ Preliminary findings indicate that use of bupropion, in conjunction with nicotine replacement therapy (NRT), can be particularly helpful in treating patients with co-occurring mental health disorders. ${ }^{22}$ However, research on smoking cessation interventions in populations with cooccurring mental health disorders is very limited, in part because patients with such disorders historically have been excluded from many smoking cessation studies. ${ }^{23}$

Monitoring a patient's smoking and cessation activities is extremely important for those with mental illnesses since tobacco use can affect the treatment of mental health disorders. ${ }^{24}$ For example, because smoking may influence the metabolism of certain commonly prescribed psychiatric medications, ${ }^{*}$ dosages of these medications may need to be adjusted when a

\footnotetext{
* Smokers typically need twice the dosage of these medications than nonsmokers.
}

patient is cutting back or quitting their ingestion of tobacco products. ${ }^{25}$

\section{Treatment for Addiction Involving Alcohol and Other Drugs}

Psychosocial interventions have proven effective for patients with co-occurring mental health disorders and addiction. ${ }^{26}$ Patients with cooccurring schizophrenia and addiction appear to respond positively to psychosocial approaches that include cognitive behavioral therapy (CBT), motivational interviewing (MI) and family therapy components. ${ }^{27}$ Patients with cooccurring addiction and mood disorders respond well to behavioral skills training. ${ }^{\dagger 28}$ Integrated group therapy (IGT), a CBT-based intervention for co-occurring addiction and bipolar disorders, was found to be significantly more beneficial than a standard group therapy program: in one study, IGT patients used alcohol and other drugs half as often as other patients did during the intervention and three months after treatment. ${ }^{29}$

There also is evidence of the efficacy of pharmaceutical interventions for patients with co-occurring disorders. ${ }^{30}$ Antidepressants, including selective serotonin reuptake inhibitors (SSRIs), are an effective pharmaceutical treatment for many individuals with cooccurring mood disorders and addiction involving alcohol ${ }^{31}$ and may be effective for patients with co-occurring mental health disorders and addiction involving opioids or sedatives as well. Stimulating antidepressants, such as desipramine or bupropion, may be more useful for treating patients with co-occurring depression and addiction involving cocaine. ${ }^{32}$ Preliminary research also suggests that certain anticonvulsant medications may be effective in treating patients with co-occurring mood or anxiety disorders and addiction involving alcohol. $^{33}$

\footnotetext{
${ }^{\dagger}$ The behavioral skills training model utilized a psycho-educational approach to teach patients selfmanagement skills and provide opportunities for practice.
} 
While evidence regarding effective treatments for addiction and co-occurring trauma disorders is limited, psychotherapeutic approaches, typically involving CBT and a psychoeducational component, appear to be quite effective. $^{34}$

\section{Integrated Treatment for Co-occurring Disorders}

Originally developed to treat patients with a serious mental illness, Assertive Community Treatment (ACT) was modified in the late 1990s to serve patients with co-occurring addiction and mental health disorders. ${ }^{35}$ The ACT model is an effective way of delivering integrated dual disorders treatment (IDDT), also known as integrated treatment for co-occurring disorders. This integrated approach to treatment for people with mental illness and addiction should include the following components: staged interventions, assertive outreach, motivational interventions, counseling, social support interventions, a longterm perspective to treatment, comprehensiveness and cultural sensitivity and competence. ${ }^{36}$ The approach relies on a multidisciplinary team of providers and intensive outreach activities-including providing services to patients in their homes and communities--to keep participants actively engaged in a high-intensity outpatient treatment model. ${ }^{37}$ The services provided combine treatment approaches such as motivational interviewing (MI) or cognitive behavior therapy (CBT) with support services such as psycho-educational instruction about addiction, 12-step mutual support programming and life skills training. ${ }^{38}$ Patients are monitored closely and have access to crisis intervention services 24 hours a day. ${ }^{39}$

Participation in this type of program is associated with reduced alcohol and other drug use (based on clinicians' reports), reduced hospital utilization, lower post-treatment relapse rates and improved quality of life. $^{40}$

\section{Adolescents}

Treatment approaches for adolescents must be tailored to the profound neurochemical, physical, cognitive, emotional and social changes that take place during adolescent development and to the heightened influence of family and peers relative to adult patients. ${ }^{41}$ Because early initiation of substance use is related so strongly to the risk of addiction, ${ }^{42}$ interventions for young people demonstrating early signs of risky substance use and treatment for addiction is imperative. ${ }^{43}$ Treatment approaches with a strong evidence base in adult populations are not necessarily applicable to the treatment needs of adolescents with addiction. ${ }^{44}$

The clinical presentation of addiction often differs in adolescents compared to adults: adolescents typically do not demonstrate the same extent of physical dependence (i.e., tolerance and withdrawal) symptoms; progression from use to addiction often is more rapid; and co-occurrence with mental health disorders is more common. ${ }^{45}$

Treatment programs for adolescents should be developmentally appropriate and family oriented. ${ }^{46}$ Special care should be taken when providing group-based therapy to adolescents who may be more vulnerable than adults to potential negative peer influences; this is especially true when the group contains members with significant behavioral problems. ${ }^{47}$ A significant proportion of adolescents with addiction have histories of trauma or adverse life experiences as well as co-occurring disorders that must be addressed in treatment. ${ }^{48}$

\section{Tobacco Cessation}

A range of effective options exists for teen smoking cessation, including NRT, ${ }^{49}$ educational programs that offer life-skills training and counseling interventions. ${ }^{50}$ A meta-analysis of 48 smoking cessation program studies from 1970 to 2003 for adolescents ages 12-19 found that the odds of quitting for smokers in these programs increased by 46 percent. Higher quit rates were found in programs that included 
motivational-enhancement therapy (MET), CBT and social influence approaches in which adolescents address the influences that promote or maintain smoking behavior. ${ }^{51}$

\section{Treatment for Addiction Involving Alcohol and Other Drugs}

Psychosocial therapies such as $\mathrm{CBT}^{52}$ and family-based therapies are effective treatments for adolescents with addiction. ${ }^{53}$ Interventions that integrate a family component into psychosocial interventions are particularly effective for adolescent patients. ${ }^{54}$ Adolescents generally seem to fare better in treatment programs that include family members in counseling sessions or that encourage families to take an active role in the treatment process. ${ }^{55}$

Particular types of family-based therapies that have proven effective for adolescents include:

- Multidimensional Family Therapy (MDFT)-an outpatient family-based treatment program that addresses adolescent alcohol and other drug use in relation to individual-, family-, peer- and community-level influences. ${ }^{56}$ One study found that adolescents who received MDFT were likelier than those who received other interventions, such as group therapy or educational interventions, to complete their treatment and to demonstrate reduced alcohol and other drug use directly following treatment and one year later. ${ }^{57}$

- Functional Family Therapy (FFT)--a comprehensive approach to treatment implemented in the home or in clinical or school settings based on the idea that behaviors influence and are influenced by multiple systems in the adolescent's life, including the family. The three-month program consists of engaging and motivating adolescents and families; the development and implementation of an individually tailored, long-term behavior change plan; and an attempt to generalize positive behavior change to other areas of family functioning. Research suggests that interventions that include FFT produce better treatment outcomes than those without an FFT component. ${ }^{58}$

You have an addicted family system. The family needs education and therapy, especially with adolescents in treatment. ${ }^{59}$

--John Coppola

Executive Director

New York Association of Alcoholism and Substance Abuse Providers, Inc. (ASAP)

...It is clear the family plays an important role in encouraging and supporting recovery, especially in adolescents. ${ }^{60}$

--Jose Szapocznik, PhD Professor and Chair, Department of Epidemiology and Public Health Director, Center for Family Studies Director, Miami Clinical Translational Science Institute

- Multi-Systemic Therapy (MST)--a familybased approach to addressing risk factors associated with serious antisocial behavior in children and adolescents who use alcohol or other drugs. The treatment generally takes place in familiar environments (homes, schools or other neighborhood settings) which contributes to a high retention rate. ${ }^{61}$ In addition to addressing substance use, MST also attempts to reduce criminal and other forms of problem behavior and decrease future involvement with juvenile justice and child welfare systems. ${ }^{62}$ MST is associated with reduced alcohol and other drug use during treatment and for at least six months following program completion, ${ }^{63}$ and is particularly effective for those involved with the juvenile justice system. ${ }^{64}$

A large study of adolescent treatment participants in different types of programs ${ }^{*}$ found that in the year following treatment, the percentage of adolescents using marijuana at least weekly was cut by approximately half.

\footnotetext{
* The study included more than 1,100 adolescent treatment participants from 23 different programs in four cities.
} 
Heavy drinking rates fell from 33.8 percent to 20.3 percent. The rate of criminal activity also declined and indicators of psychosocial adjustment, school attendance and academic performance improved significantly. ${ }^{65}$

As is true for adults, comprehensive and continuing care is critical for adolescents with addiction. Assertive continuing care (ACC) is a method of enhancing engagement in treatment by moving responsibility for service utilization from adolescents and their caregivers to treatment providers. ${ }^{66}$ ACC is characterized by at least weekly face-to-face sessions conducted in the home or community settings that are convenient for the adolescent and increase the likelihood of retention and low patient-toprovider ratios. Interventions used in ACC include an adolescent-oriented community reinforcement approach (A-CRA) and intensive case management. ${ }^{67}$ Research on this approach demonstrates that adolescents receiving ACC were significantly likelier to be abstinent from marijuana following discharge from residential treatment than adolescents who did not receive ACC; ${ }^{*}$ they also had higher rates of retention in aftercare services. $^{68}$

Very few studies have examined the use and effectiveness of pharmaceutical interventions for the treatment of adolescent patients with addiction involving alcohol or other drugs; ${ }^{69}$ best practice suggests that if they are employed they should be used as a supplement to psychosocial therapies. ${ }^{70}$

\section{Women}

A considerable body of evidence demonstrates the importance of addressing gender differences in the treatment process, particularly for women with histories of trauma and those who are pregnant or parenting. ${ }^{71}$ Women often smoke, drink and use other drugs for different reasons than men and addictive substances affect women

\footnotetext{
* All participants received referrals to adolescent outpatient treatment providers for continuing care in their communities following discharge from residential treatment.
}

differently than men; this may indicate different treatment needs. $^{72}$ Because the life roles and responsibilities of women typically differ from men, their support service needs may differ as well. ${ }^{73}$ Women with addiction have high rates of co-occurring mental health disorders, including mood, anxiety and eating disorders that should be addressed in the treatment process, and high rates of trauma histories that can influence treatment outcomes. ${ }^{74}$

The safety and security of 'all female' [programs] allow women to disclose things that are very difficult and that may be impossible within a co-ed arrangement. It enhances a woman's ability to feel understood and accepted without judgment, shame or guilt. ${ }^{75}$

Gender-specific treatment programs appear to be particularly beneficial for women with a history of sexual abuse and domestic violence. ${ }^{76}$

Women who were abused as children suffer from substance-related problems that are more severe than those of their peers, including lifetime use of a greater number of substances, higher alcohol and other drug severity scores ${ }^{\dagger}$ and greater financial and interpersonal problems. ${ }^{77}$ Women who report exposure to physical, sexual or emotional abuse during childhood are more prone to relapse than other treatment patients, and may be less likely to improve during and after treatment. ${ }^{78}$ Women with trauma histories require a more empowering and less confrontational approach; being told that they are powerless over their addiction--a common tactic in many treatment programs that are centered on the 12-step model-can bring back feelings of powerlessness from sexual and other abuse. ${ }^{79}$

\section{Pregnant Women}

Pregnant women require special consideration in stabilization, acute treatment and disease management protocols, particularly with regard

\footnotetext{
${ }^{\dagger}$ Based on the Modified Michigan Alcohol-Drug Screening Test.
} 
to pharmaceutical interventions. ${ }^{*} 80$ Withdrawal from addictive substances during detoxification can be highly risky to a fetus; for example, sudden withdrawal from certain opioids and sedatives can lead to fetal distress or death. ${ }^{81}$ As a result, detoxification protocols should include careful monitoring of the pregnant woman and her fetus and medical supervision of the detoxification process itself--for example, through the use of buprenorphine to treat or prevent opioid withdrawal during pregnancy ${ }^{\dagger}--$ preferably under the direction of a physician with experience in perinatal addiction. ${ }^{82}$ Medically supervised detoxification typically takes place during the second trimester because of the risk of miscarriage in the first trimester and the increased risk of premature delivery or fetal death in the third trimester. ${ }^{83}$

Research on the safety and efficacy of pharmaceutical therapies for addiction treatment among pregnant women is limited. ${ }^{84}$ Certain medications, such as disulfiram, are not considered safe for pregnant women, while others, such as methadone, are less risky and may be preferable to continued substance use. ${ }^{85}$

Case management is particularly critical for pregnant women with addiction. ${ }^{86}$ Case management services typically assure standardized assessments, access to prenatal and pediatric care, mental health services, vocational and parenting classes, childcare and transportation services. ${ }^{87}$

Pregnant women with co-occurring addiction and mental health disorders require additional medical monitoring because pregnancy can aggravate certain symptoms of mental illness, including depression and anxiety. ${ }^{88}$ Hormonal changes, increased stress and pregnancy-specific medications all can contribute to the potential exacerbation of mental illness symptoms. ${ }^{89}$

\footnotetext{
* Specific psychosocial treatment approaches that work for pregnant women with addiction do not appear to differ from those found to be effective in the general population of women.

${ }^{\dagger}$ Opioid withdrawal during pregnancy can lead to fetal death.
}

Although federal law requires that pregnant women receive priority admission into addiction treatment programs, allowing them to bypass waiting lists, ${ }^{90}$ numerous barriers prevent many pregnant women from accessing needed treatment. ${ }^{\ddagger}$ While pregnant women may be more motivated than other women to receive addiction treatment because of the known risks of substance use to pregnant women and their babies, they are less likely to stay in treatment once admitted, and reductions in substance use often are transient and dissipate once their children are born. ${ }^{91}$

The Center for Substance Abuse Treatment's (CSAT) Pregnant and Postpartum Women demonstration program provided comprehensive clinical, medical and social services, over the course of six to 12 months, to pregnant women and mothers of children under the age of one. ${ }^{92}$ During the six months following discharge from this program, 61 percent of the women had achieved and maintained their abstinence from alcohol and other drugs. Program participation also was associated with increased employment rates and decreased rates of arrest, foster care involvement and premature deliveries, low birth weight and infant death. ${ }^{93}$

\section{Older Adults}

Treatment approaches for older adults must take into account their increased risk of developing addiction involving prescription drugs due to the use of medication to treat chronic pain, sleep disorders, depression and anxiety--problems that are common in this age group--as well as the fact that their bodies become even more vulnerable to the effects of alcohol and other drugs with age. ${ }^{\S 94}$ Co-occurring health conditions and medical complications due to age-related health problems can interfere with addiction treatment and make it harder for older adults to follow treatment instructions and plans. $^{95}$

\footnotetext{
${ }^{\ddagger}$ See Chapter VII.

$\S$ See Chapter IV for a discussion of the unique risks that older adults face with regard to substance use and addiction.
} 
Psychosocial interventions, particularly motivational interviewing (MI) and motivational enhancement therapy (MET), are regarded as effective approaches for older adults, especially those who may resist changing their substance use behavior. ${ }^{96}$

Before prescribing a potentially addictive medication to an adult patient, a full assessment of the patient's use of other addictive substances and deliberate counseling with regard to the risks of physical dependence and the dangers of combining addictive substances is necessary. When treating older patients with pharmaceutical therapy, long-acting benzodiazepines and disulfiram should be avoided because of their toxicity. Naltrexone has been tolerated well by older adults and there is some evidence of its effectiveness in this population. $^{97}$

To improve treatment compliance, older patients also may require specialized services to assure appropriate interventions for dietary deficiencies or auditory or visual impairments; to provide psychosocial interventions that help patients cope with loneliness, loss or depression which are more common in this age group; and to help bolster supportive social networks. ${ }^{98}$

\section{Racial and Ethnic Minorities}

Treatment providers should take into consideration the substance-related health disparities--including the likelihood of cooccurring disorders--that exist among racial/ethnic groups. ${ }^{* 9}$ Different racial/ethnic groups also may vary in their metabolism, response to dosages and side effects of pharmaceutical interventions for addiction. ${ }^{100}$

While data are not available on specific psychosocial therapies that work best for

\footnotetext{
* e.g., Whites and Hispanics have a higher severity of alcohol problems than other racial/ethnic groups (see Chapter III); Hispanic and black men have higher rates of cirrhosis mortality than white men; and Alaska Native and white men report higher rates of alcohol-related and non-alcohol-related major depressive disorder than Hispanic and black men.
}

particular racial/ethnic groups, treatment providers should ensure that programs are effective for individuals of every racial and ethnic background by making them sensitive to racial, ethnic and cultural conditions that may affect the treatment process. ${ }^{101}$ For example, providers should make sure that language barriers are addressed and require cultural competency training for staff. ${ }^{102}$ These measures improve communication and increase trust and understanding, which in turn result in greater recognition of patients' needs, increased patient engagement in treatment and better treatment compliance. ${ }^{103}$

\section{Individuals of Minority Sexual Orientation}

Treatment goals for lesbian, gay, bisexual and transgender (LGBT) individuals are the same as treatment goals for other individuals in terms of reducing use or achieving abstinence, but treatment should also focus on the unique characteristics of LGBT patients. For example, higher rates of discrimination against lesbian, gay and bisexual adults may be associated with higher rates of risky use and addiction in this population, compared with heterosexuals. ${ }^{104}$

Treatment providers should screen for other health problems and adverse experiences that may be more common in the LGBT population including co-occurring mental health disorders, suicidal thoughts or behaviors, sexuallytransmitted infections (in particular HIV/AIDS and hepatitis A and B) and sexual abuse. ${ }^{105}$ Gay and bisexual men make up nearly half of those living with HIV in the United States; ${ }^{106}$ the prevalence of mental health disorders is higher among lesbian, gay and bisexual adults ${ }^{\dagger}$ than among heterosexual adults; ${ }^{\ddagger 107}$ and a review study found that lifetime prevalence estimates of sexual abuse range from 15.6 percent to 85.0 percent for lesbian or bisexual women and from 11.8 percent to 54.0 percent for gay or bisexual men. ${ }^{108}$

\footnotetext{
${ }^{\dagger}$ Or those who report same-sex sexual partners.

‡ Or those who report opposite-sex sexual partners.
} 
When assessing available social support for LGBT patients, treatment providers should recognize that LGBT individuals may experience conflict with their family of origin, especially around issues of sexual orientation or gender identity, and it is important for treatment providers to understand the interpersonal relationships of their LGBT patients. In addition, providers should be particularly careful to protect the confidentiality of LGBT patients because in many states LGBT individuals lack legal protections against discrimination in housing, the job market or social services. ${ }^{109}$

Despite the need to tailor addiction treatment to the needs of LGBT individuals, national data show that only 6.2 percent of treatment facilities offered any type of tailored treatment specifically for LGBT patients in $2010 .{ }^{110} \mathrm{~A}$ 2007 national study found that services provided by 70.8 percent of LGBT-specialized programs did not differ from services provided to patients in other programs and only about seven percent of facilities offered LGBT-specific services, such as special groups for LGBT patients or having counselors who were trained specifically in LGBT issues. ${ }^{111}$

Research evaluating the impact of treatment specific to the needs of LGBT individuals is lacking. Among a small sample of individuals in addiction treatment, gay and bisexual men in LGBT-specialized treatment reported better outcomes, such as achieving abstinence and completing treatment, than gay and bisexual men in traditional treatment. ${ }^{112}$

\section{Veterans and Active Duty Military}

The U.S. Department of Veterans Affairs (VA) and the Department of Defense have developed practice guidelines for evidence-based addiction treatment, and endorsed the U.S. Public Health Services' Clinical Practice Guidelines for smoking cessation. ${ }^{113}$ Among the recommended practices for addressing risky use of addictive substances and addiction in veterans and active duty members of the military are the following: ${ }^{114}$
- Active duty members involved in an incident in which substance use is suspected to be a contributing factor are required to be referred to specialty addiction care for evaluation.

- A treatment team shall convene with the patient and command in order to review the treatment plan and goals.

- Rehabilitation and referral services for the patient require an individualized plan designed to identify the continued support of the patient with at least monthly monitoring during the first year after inpatient treatment.

- For patients who do not stabilize and refuse to engage in any type of ongoing care with any provider (e.g., medical, psychiatric or addiction specialty), consider involving supportive family members or significant others if the patient agrees. This may include a first line supervisor when appropriate and the unit commander.

Of particular importance in the veteran and active duty military populations is the need to attend to co-occurring mental health conditions, particularly post-traumatic stress disorder (PTSD), which are common in this population. ${ }^{115}$

The VA has outlined certain treatment guidelines for veterans and military personnel with addiction and co-occurring PTSD, most of which mirror the treatment approaches outlined in Chapter $\mathrm{V}$ for the general population.

However, the VA notes that treatment for the two conditions can be delivered simultaneously, that the clinician should use first-stage treatment approaches such as motivational interviewing to initiate treatment and specifically states that tobacco cessation services should be integrated into the treatment protocol. ${ }^{116}$ The most commonly used treatment approach for veteran patients with co-occurring addiction and PTSD involves cognitive behavioral therapy (CBT). ${ }^{117}$ 
The importance of attending to co-occurring conditions in this population is highlighted by a study of veterans with co-occurring PTSD and addiction who participated in addiction treatment. ${ }^{*} 118$ This study found that receiving treatment for PTSD during the initial year of addiction treatment almost quadrupled participants' odds of being in remission from addiction $^{\dagger}$ five years later. ${ }^{119}$

\section{Individuals Involved in the Justice System}

In 2006, the National Institute on Drug Abuse (NIDA) developed evidence-based principles for the effective treatment of individuals in the justice system who have addiction. These principles include providing comprehensive assessments of the extent and severity of offenders' substance involvement, addressing the presence of co-occurring conditions that might call for specialized treatment services, assessing treatment progress and adjusting interventions accordingly. ${ }^{120}$

For both juveniles and adult offenders with addiction, the use of treatment-based alternatives to incarceration represents an important step in treating the disease. Drug courts, prosecutorialbased diversion programs and intensive treatment-based probation are some of the approaches that have proved effective in the justice field. ${ }^{121}$ Services also can be provided by professionals, using evidence based practices, during incarceration and after release. These initiatives provide addiction treatment, assure collaboration between justice authorities and treatment providers and hold the offender legally accountable for treatment compliance. ${ }^{122}$

\footnotetext{
* The sample was drawn from male inpatients participating in a multisite VA program evaluation of addiction treatment.

${ }^{\dagger}$ Defined as having (1) abstained from all 13 substances investigated, (2) had no problems related to alcohol or other drugs and (3) consumed $3 \mathrm{oz}$. $(88.79 \mathrm{ml})$ or less of alcohol per day on maximum drinking days in the past month. Freedom from problems related to substance use was reflected by a response of "never" to each of 15 problems in the areas of health, work, legal situation and finances.
}

\section{Juvenile Offenders}

Comprehensive treatment and management of addiction in the juvenile justice population result in decreased substance use, crime (recidivism), homelessness and high-risk sexual behavior; improved school performance, productivity, employment and future earning power; and better health and psychological adjustment. ${ }^{123}$

For adolescents in the juvenile justice system, screening and comprehensive assessments are critical for identifying an adolescent's needs and for connecting the juvenile with effective interventions and treatments. Comprehensive assessments can take place at various points, including at the first interview after referral to juvenile court (often conducted by an intake officer) where results may be used to refer the adolescent to more appropriate community health services rather than incarceration. Assessments also may be conducted upon admission to a pre-trial detention center to await adjudication or upon admission to a postadjudication community program or correctional facility. ${ }^{124}$

Addiction treatment for juvenile offenders should reflect the standards of evidence-based care for adolescents in the general population, with a special focus on family-based treatment models. Effective treatment approaches include multidimensional family therapy (MDFT), functional family therapy (FFT) and multisystemic therapy (MST). ${ }^{125}$

\section{Adult Corrections}

Treatment tailored to criminal justice populations should ensure that treatment plans correspond with correctional supervision requirements as well as the medical and social support services that the patient may need. ${ }^{126}$

Cognitive behavioral therapy (CBT), motivational enhancement therapy (MET) and contingency management (CM) have been shown to be effective treatment approaches for inmates, ${ }^{127}$ particularly in that they help train patients to recognize errors in judgment that lead to substance use and to criminal behavior, ${ }^{128}$ and 
help to increase treatment engagement and retention. ${ }^{129}$ As is true in the general population, evidence-based pharmaceutical interventions should be provided to patients in the criminal justice population along with psychosocial therapies. $^{130}$

Individuals with addiction facing release and reentry should be assured appropriate postrelease community-based treatment, disease management and support services. ${ }^{131}$ 
-130- 


\section{Chapter VII \\ The Addiction Treatment Gap}

In spite of the facts that 15.9 percent of the U.S. population ages 12 and older report meeting clinical criteria for addiction and that evidencebased interventions and treatments are widely available, research clearly indicates that only a small fraction of those in need of addiction treatment receives it and, of those who do, few receive evidence-based care. Determining the exact size and shape of the addiction treatment gap in this country is impossible due to significant data limitations; ' however, 89.1 percent of those who meet criteria for addiction involving alcohol and drugs other than nicotine report receiving no treatment ${ }^{\dagger}$ at all. $^{\ddagger 1}$

\footnotetext{
${ }^{*}$ There are no national data on the total number of people who receive treatment for the disease. Rather, existing data on addiction treatment exclude addiction involving nicotine, and data on the types of services offered and venues in which they are provided are available only for providers that receive public funds. Because some addiction treatment providers accept both public and private funding, the available data on providers serving publicly-funded patients also include an unidentifiable number of patients who receive private funding.

${ }^{\dagger}$ For the purposes of the present analyses, treatment includes: (1) services received at non-intensive or intensive non-residential settings (including alcohol or other drug rehabilitation facilities, mental health centers or facilities or doctors' or mental health professionals' private offices); and (2) short- or longterm residential settings (including alcohol or other drug rehabilitation facilities, hospital or mental health centers or facilities) in the past 12 months. Detoxification, services received in an emergency department or in prison or jail settings, mutual support programs, peer counseling and other support services (including religious-based counseling) are excluded from these analyses.

${ }^{\ddagger}$ While 31.7 percent of the U.S. population ages 12 and older engage in risky use but do not meet criteria for addiction, there has been no attempt to document nationally the proportion of this population that receives evidence-based screening and brief interventions, leaving the size of the intervention gap for risky users unknown. Therefore, this chapter focuses exclusively on the treatment gap for individuals with addiction.
} 
Of those whose addiction involves nicotine, the only data available are for persons ages 18 and older who have tried to quit smoking; less than one-third (31.7 percent) of these individuals ${ }^{*}$ receive smoking cessation services. ${ }^{\dagger 2}$

A key factor contributing to the treatment gap is the way treatment costs are covered. In contrast to the role of private insurance in medical care spending--where it covers 55.6 percent of costs, private insurers cover only 20.8 percent of the costs of addiction treatment, and the private share has been decreasing. Instead, public spending accounts for 79.2 percent of the costs of addiction treatment in the United States. ${ }^{3}$ This skewing of services to populations dependent on public resources is in part a result of our failure to prevent and treat this disease. Public spending also has concentrated available resources for treatment in non-residential services operated outside of the mainstream health care system. In both public and private plans, insurance coverage for addiction care has been limited in the range of covered services. ${ }^{4}$

National data indicate that people in need of help for addiction largely choose to turn to a health professional; however, only 5.7 percent of referrals to addiction treatment come from health professionals. The largest share of referrals--44.3 percent--comes from the criminal justice system, ${ }^{5}$ demonstrating our nation's attention to the social consequences of addiction rather than to prevention and treatment of the disease. Even those who are referred to treatment may face long waits for admission and the longer the wait the less likely patients are to enter or complete treatment. ${ }^{6}$

Only 42.1 percent of those receiving treatment for addiction involving alcohol or drugs other than nicotine complete their course of care. ${ }^{7}$ The highest treatment completion rates are from venues to which there are the least referrals-residential treatment; the lowest treatment

\footnotetext{
* Among current smokers who tried to quit in the past year and former smokers who successfully quit in the past two years.

${ }^{\dagger}$ In the form of counseling or smoking cessation medications.
}

completion rates are from venues to which there are the most referrals--non-residential treatment. $^{8}$

A range of factors contribute to these spending, referral and service delivery patterns that account for the treatment gap, including a misunderstanding of the disease of addiction, a lack of appropriate disease staging and treatment services, ${ }^{\ddagger}$ negative public attitudes and behavior toward those with the disease, privacy concerns, cost, lack of information on how to get help, limited availability of services including a lack of trained addiction physician specialists, insufficient social support, conflicting time commitments, negative perceptions of the treatment process and legal barriers. Certain populations with addiction, including those with co-occurring health conditions, pregnant and parenting women, adolescents, older adults, the homeless, veterans and those in active duty military, individuals living in rural areas and Native Americans, face additional barriers.

This chapter examines the disconnect between those in need of treatment and those who receive it. The fact that those who do receive some form of treatment rarely receive quality, evidencebased care is discussed in Chapter X.

\footnotetext{
‡ See Chapter X.
} 


\section{Most People in Need of Treatment Do Not Receive It}

In 2010, 15.9 percent of the U.S. population ages 12 and older--40.3 million people--met diagnostic criteria for addiction. ${ }^{*}$ Although some treatment providers are beginning to address the disease of addiction comprehensively, including all involved substances, no single national data source exists to compare the proportion of the population in need of addiction treatment ${ }^{\dagger}$ involving any addictive substance (including nicotine) to the proportion that receives such treatment. CASA Columbia's analysis of the treatment gap in the following discussion, therefore, is based on available data and limited to the 9.1 percent (23.2 million) whose addiction involves alcohol or other drugs excluding nicotine. ${ }^{\ddagger 9}$

CASA Columbia's analysis indicates that only one in 10 (10.9 percent, 2.5 million) of those in need of addiction treatment (excluding nicotine) receive it, leaving a treatment gap of 20.7 million individuals. The proportion of individuals in need of addiction treatment who actually receive it has changed little since 2002, when 9.8 percent of those in need received it. ${ }^{10}$ This is in stark contrast to the much smaller

\footnotetext{
* Those meeting criteria for addiction include individuals who met the Nicotine Dependence Syndrome Scale (NDSS) criteria for past month nicotine dependence, or the DSM-IV clinical diagnostic criteria for past year alcohol and/or other drug abuse or dependence. This estimate excludes the institutionalized population, for which rates of addiction are higher.

${ }^{\dagger}$ Those in need of treatment are defined not only as those who met DSM-IV diagnostic criteria for past year alcohol and/or other drug abuse or dependence, but also those who have received formal treatment for addiction involving alcohol and/or other drugs in the past year. Due to data limitations, individuals in need of treatment for addiction involving nicotine are not included in this analysis.

* Another nine percent (22.9 million) of the population has addiction involving nicotine; 2.7 percent of the population (7.0 million) has addiction involving multiple substances, including alcohol, illicit drugs, controlled prescription drugs and/or nicotine.
}

treatment gaps that exist for other major health conditions including hypertension, diabetes and major depression. ${ }^{\S 11}$

(Figure 7.A)



\footnotetext{
$\S$ For this comparison, CASA Columbia examined the referenced national survey data to determine the proportion of the population with each disease--those with diagnosed or undiagnosed hypertension (59.3 million); those with diagnosed or undiagnosed diabetes (25.8 million); those who met clinical criteria for a major depressive episode in the past year and/or received professional treatment (saw a doctor, received medication, some combination thereof) (22.4 million); and those who met clinical criteria for addiction involving alcohol or other drugs excluding nicotine in the past year and/or received professional treatment for alcohol and/or other drugs in the past year (23.2 million)--who received treatment.
} 
Other national data indicate that, in 2010 among smokers age 18 and older, 68.8 percent tried to stop smoking ${ }^{*}$ but only 31.7 percent of those who tried to stop received some type of smoking cessation service (psychosocial or pharmaceutical treatment). ${ }^{\dagger 12}$

\section{Variations in the Treatment Gap by Primary Substance Involved}

In 2010, 7.4 percent of the population needed treatment for addiction involving alcohol ${ }^{\ddagger}$ and 3.1 percent needed treatment for addiction involving another drug (excluding nicotine); ${ }^{\S} 1.3$ percent of the total population needed treatment for addiction involving multiple substances, excluding nicotine. ${ }^{* *}$ While considerably more people needed treatment for addiction involving alcohol (18.7 million) compared to those needing treatment for addiction involving an illicit or controlled prescription drug (7.9 million) or multiple substances (3.4 million), ${ }^{\dagger \dagger} 13$ they were less likely to receive it:

- $\quad 8.8$ percent (1.7 million) of those in need of treatment for addiction involving alcohol received it, leaving a treatment gap of 17.1 million people;

\footnotetext{
* The source of these data does not present the proportion of the sample that smoked, just the proportion of smokers that tried to quit.

Among current smokers who tried to quit in the past year and former smokers who successfully quit in the past two years. Trend data are not available for these measures.

$\ddagger 18.1$ percent of these individuals also needed treatment for addiction involving other drugs.

$\S 43.1$ percent of these individuals also needed treatment for addiction involving other drugs, excluding nicotine.

** The 7.4 percent of those needing treatment for addiction involving alcohol and the 3.1 percent needing treatment for addiction involving other drugs overlap with the category needing treatment for multiple substances. Looking at mutually exclusive categories, 6.0 percent needed treatment for addiction involving alcohol only and 1.8 percent needed treatment for addiction involving other drugs (excluding nicotine) only.

${ }^{+\dagger}$ Individuals in need of treatment for addiction involving multiple substances also are included in the other two categories.
}

- $\quad 19.9$ percent (1.6 million) of those in need of treatment for addiction involving another drug (excluding nicotine) received it, leaving a treatment gap of 6.3 million people; and

- 28.5 percent (1.0 million) of those in need of treatment for addiction involving multiple substances (excluding nicotine) received it, leaving a treatment gap of 2.4 million people. $^{14}$ (Figure 7.B)

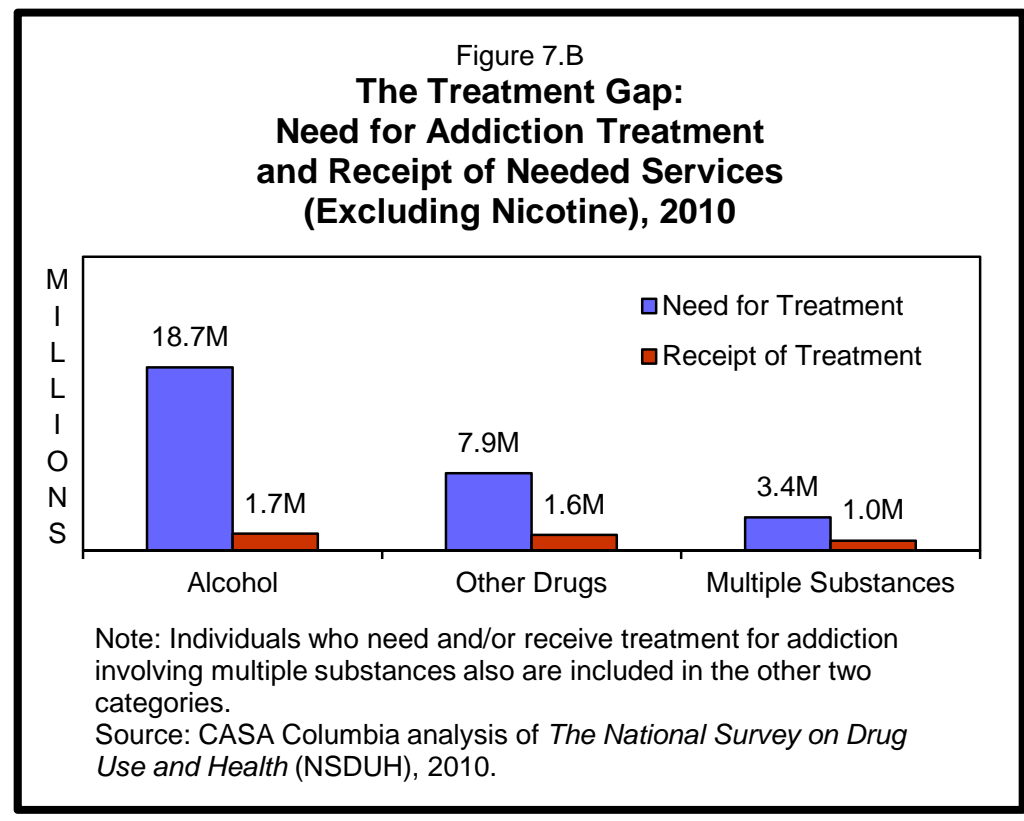

Of the 31.7 percent of those ages 18 and older who wanted to stop smoking in 2010, 5.9 percent received cessation counseling ${ }^{\ddagger \ddagger}$ and 30.0 percent received medications; ${ }^{\S \S} 4.3$ percent received both. ${ }^{15}$

\footnotetext{
\# 3.1 percent used a telephone quitline, 2.6 percent received one-on-one counseling and 2.4 percent were involved in a stop smoking clinic, class or support group.

$\$ \$ 14.6$ percent used the nicotine patch, 11.2 percent used the medication varenicline, 8.9 percent used the nicotine gum or lozenge, 3.2 percent used the medication bupropion and 1.0 percent used the nicotine spray/inhaler.
} 


\section{Variations in the Treatment Gap by Key Patient Characteristics}

The addiction treatment gap is substantial across all demographic groups, with the greatest need among adolescents, young adults and Hispanics. ${ }^{16}$

Gender. In 2010, twice as many males as females were in need of addiction treatment (12.2 percent vs. 6.2 percent). ${ }^{17}$ That year:

- 10.5 percent of males in need of treatment received it, leaving a treatment gap of 13.5 million; and

- $\quad 11.7$ percent of females in need of treatment received it, leaving a treatment gap of 7.2 million. ${ }^{18}$

Although more males than females had addiction involving nicotine in 2010 (9.9 percent vs. 8.2 percent), ${ }^{19}$ more female than male smokers were interested in quitting (70.7 percent vs. 67.3 percent) and treatment was more prevalent among females than among males (35.1 percent vs. 28.8 percent). ${ }^{20}$

Age. In 2010, young people ages 18 to 25 were the most likely of all age groups to need addiction treatment:

- $\quad 7.5$ percent of teens ages 12 to 17 and 20.5 percent of young adults ages 18 to 25 were in need of treatment (15.1 percent of all 12 to 25 year olds) but only 1.1 percent of all 12 to 25 year olds received it, ${ }^{*}$ leaving a treatment gap of 8.2 million; and

- $\quad 7.4$ percent of adults ages 26 and older were in need of treatment but only 1.0 percent received it, leaving a treatment gap of 12.5 million. ${ }^{21}$

\footnotetext{
* The sample sizes for those who received needed treatment is too small to report statistically reliable data for 12- to 17-year olds and 18- to 25-year olds separately.
}

Although more 18- to 25-year olds than adults ages 26 and older had addiction involving nicotine (10.4 percent vs. 9.7 percent) in $2010{ }^{22}$ those ages 26 and older ${ }^{\dagger}$ were more likely to be interested in quitting, and smoking cessation treatment was higher among those ages 25 and older (29.4 percent of 25- to 44-year olds; 42.3 percent of 45-to 64-year olds; and 35.9 percent of those ages 65 and older) than among 18- to 24-year olds (15.8 percent). ${ }^{23}$

Race/Ethnicity. Similar to racial/ethnic disparities that have been found for other health conditions, ${ }^{24}$ Hispanics were slightly likelier than white or black people to need addiction treatment (10.1 percent vs. 9.3 percent and 8.8 percent, respectively) but less likely to receive it in $2010:^{25}$

- 7.0 percent of Hispanics in need of treatment received it, leaving a treatment gap of 3.5 million;

- $\quad 11.7$ percent of whites in need of treatment received it, leaving a treatment gap of 13.9 million; and

- $\quad 11.9$ percent of blacks in need of treatment received it, leaving a treatment gap of 2.4 million. $^{\ddagger 26}$

Whites were likelier than blacks or Hispanics to have addiction involving nicotine (10.9 percent vs. 6.9 percent and 4.2 percent, respectively), ${ }^{27}$ but adult black smokers were likelier than adult white and Hispanic smokers to be interested in quitting in 2010 (75.6 percent vs. 69.1 percent and 61.0 percent, respectively). White smokers, however, were likelier to use smoking cessation treatments than black or Hispanic smokers (36.1 percent vs. 21.6 percent and 15.9 percent, respectively). ${ }^{28}$ Other research supports the

\footnotetext{
† Interest in quitting was higher among adults ages 25 to 44 (72.5 percent) and ages 45 to 64 (69.0 percent) relative to those ages 18 to 24 (66.7 percent); older adults ages 65 and older were the least interested in quitting (53.8 percent).

₹ A recent study found that black and Hispanic adolescents are significantly less likely to receive addiction treatment relative to white adolescents.
} 
finding that black and Hispanic smokers are less likely than white smokers to use smoking cessation interventions such as nicotine replacement therapy (NRT) and other pharmaceutical therapies. $^{29}$

Special Populations. The need for addiction treatment is disproportionately higher for individuals with co-occurring mental health disorders, for individuals involved in the justice system and for members of the military, including veterans, returning from active combat in Iraq and Afghanistan:

- In 2010, one in five (20.7 percent) noninstitutionalized individuals ages 18 and older ${ }^{*}$ with a diagnosed mental health disorder $^{\dagger}$ were in need of addiction treatment (relative to 6.3 percent of those without a diagnosed mental health disorder); 14.2 percent of those in need of addiction treatment received it, leaving a treatment gap of 8.5 million adults with co-occurring addiction and mental health disorders. ${ }^{\ddagger 30}$ In 2010 , only 37.6 percent of facilities nationwide that provided addiction treatment services offered services specific to patients with co-occurring addiction and mental health disorders. ${ }^{31}$

- In 2010, adults with a mental illness were more than 1.5 times as likely as those without a mental illness to have smoked cigarettes in the past year (41.3 percent vs.

\footnotetext{
${ }^{*}$ Comparable data on those with mental health disorders are not available for those ages 12-17. ${ }^{\dagger}$ And/or a reported major depressive episode.

‡ Research indicates that young people with cooccurring conditions are more easily identified as needing treatment than those with addiction only. One study found that unmet treatment need (i.e., the treatment gap) was greatest for teens with addiction (37 percent) compared to a relatively lower treatment gap of 23 percent among those with a psychiatric disorder only and 19 percent among those with cooccurring disorders. Other research confirms that rates of addiction treatment are approximately three times higher for adolescent illicit drug users who report having received mental health treatment in the past 12 months compared to those who have not received mental health treatment.
}

25.1 percent). ${ }^{32}$ Even though patients with mental health disorders are likelier to smoke $^{33}$ and about as likely to want to quit smoking as smokers in the general population, ${ }^{34}$ research suggests that smoking cessation services for these patients are rare. ${ }^{\S 35}$

- In 2010, nearly half (46.8 percent) of individuals who were arrested or booked in the past year, but not incarcerated at the time of the survey, were in need of addiction treatment (relative to 8.0 percent of those who had not been arrested or booked in the past year); 27.9 percent of those in need of treatment received it, leaving a treatment gap of 2.5 million individuals. ${ }^{* *}$ In 2010 , only 26.8 percent of facilities nationwide that provided addiction treatment services ${ }^{\dagger \dagger}$ offered services specific to patients involved in the criminal justice system. ${ }^{37}$ Among inmates, only 11.2 percent receive treatment. $^{38}$

- Individuals involved in the justice system also are likelier than the general population to smoke ${ }^{39}$ but are less likely to receive tobacco cessation services. ${ }^{40}$ One survey of 500 correctional facilities ${ }^{\ddagger \ddagger}$--including jails, prisons and juvenile facilities--found that 80 percent reported that their facilities had no tobacco cessation programs at all. ${ }^{41}$

- In 2005, of veterans from the military operations in Iraq and Afghanistan who sought health care from the Department of Veterans Affairs (VA), 40 percent screened positive for risky alcohol use and 22 percent

\footnotetext{
$\S$ Based on research documenting health care practice; national data on the use of smoking cessation treatments in this population are not available.

** Comparable treatment admission data from the Treatment Episode Data Set (TEDS), described on page 141 and in Appendix A, are not available for this population.

${ }^{+\dagger}$ Excluding facilities such as jails, prisons or other organizations that treat incarcerated individuals exclusively.

¥ Accredited by the National Commission on Correctional Health Care.
} 
screened positive for a possible addiction involving alcohol. ${ }^{*}$ However, of those who screened positive for risky alcohol use, only 31 percent were advised in the past year to drink less or stop drinking; 41 percent of those with a possible addiction involving alcohol were given such advice. ${ }^{42}$ Another study found that 11.8 percent of soldiers who returned from Iraq reported risky alcohol use but only 0.2 percent were referred to treatment and only 21.6 percent of those who were referred were seen within 90 days. ${ }^{43}$ And although the Handbook on Uniform Mental Health Services requires access to opioid maintenance therapy, fewer than one in five veterans diagnosed with addiction involving opioids receive this kind of therapy on an ongoing basis. ${ }^{44}$

\section{Regional Variations in the Treatment Gap}

Current national data on regional differences in the proportion of individuals in need of addiction treatment are not available. ${ }^{\dagger}$ However, other research indicates that the disparity between the number of people who need addiction treatment and the number who receive it varies substantially among states and regions of the country, ${ }^{45}$ with southern and southwestern states having the largest estimated treatment gaps in the nation and the northeast having the smallest estimated treatment gap. ${ }^{46}$

Although reasons for these geographical differences in the treatment gap are not well understood, ${ }^{47}$ possible explanations include variations among states in funding of treatment services, including differences in coverage of the costs of these services in state managed care systems. ${ }^{48}$ One study found that only 21 percent of the variation among states in the percent who receive treatment was associated with variations

\footnotetext{
* Based on scores on a version of the AUDIT instrument (see Appendix H). Risky drinking was defined as an AUDIT-C score of four or higher for men and three or higher for women. Addiction was defined as an AUDIT-C score of four or higher for women and six or higher for men.

${ }^{\dagger}$ Public access NSDUH files have not contained geographic variables since 1998.
}

in the prevalence of addiction; the rest of the variation is attributable to these other factors. ${ }^{49}$

\section{Sources of Funding for Addiction Treatment}

Spending on addiction treatment totaled an estimated $\$ 28.0$ billion in 2010. Whereas private payers (including private insurers and self-payers) are responsible for 55.6 percent (\$1.4 trillion) of medical expenditures in the U.S., they are responsible for only 20.8 percent ( $\$ 5.8$ billion) of addiction treatment spending. ${ }^{50}$

Public payers picked up the tab for 79.2 percent (\$22.2 billion) of expenses for addiction treatment, with state and local governments paying for 44.8 percent ( $\$ 12.6$ billion) and the federal government paying for 34.4 percent (\$9.6 billion). ${ }^{51}$ (Figures 7.C)

The concentration of spending for addiction treatment in public programs suggests that insurance across the board does not adequately cover costs of intervention and treatment, with costly health and social consequences falling to government programs. National data ${ }^{\ddagger}$ indicate that those with private insurance are three to six times less likely than those with public insurance to receive specialty addiction treatment. ${ }^{\S 52}$

\footnotetext{
‡ From a study examining data from the 2002-2007 editions of the NSDUH. It is not possible to determine from these data why treatment access differed based on insurance type since the study could not take into account important factors such as individual characteristics and circumstances that may relate both to type of insurance and likelihood of treatment access (e.g., symptom severity, SES).

$\S$ Defined in the study as treatment received in hospital inpatient units, outpatient and residential addiction treatment facilities, mental health facilities and methadone maintenance facilities. Services provided by private physicians (including psychiatrists), independent practice mental health practitioners, clergy, in prisons/jails and by self-help groups are not included in the definition of treatment. Those with no insurance are almost twice as likely as those with private insurance to receive specialty treatment.
} 


\section{Public vs Private Spending}



Medical Care

Source: CASA Columbia (2012) analysis of the National Expenditures for Mental Health Services \& Substance Abuse Treatment 1986-2005 (Table A.1) SAMHSA Spending Estimates Project, 2010; CMS Office of the Actuary, National Health Statistics Group with our estimated dollar updates.
Addiction Treatment

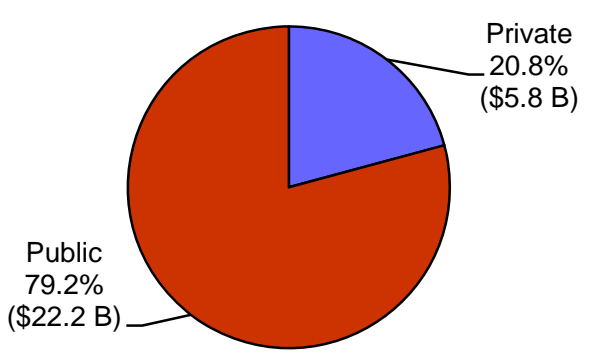

\section{Privately-Funded Treatment}

In 2010, 20.8 percent ( $\$ 5.8$ billion) of addiction treatment expenditures came from private sources. Private spending includes:

- $\quad 56.6$ percent (\$3.3 billion) from private health insurance;

- 30.5 percent ( $\$ 1.8$ billion) from self-pay by patients; and

- 12.9 percent (\$0.8 billion) from other private sources, such as charitable donations and charity care/fee waivers. ${ }^{53}$ (Figure 7.D)

\section{Publicly-Funded Treatment}

In 201079.2 percent ( $\$ 22.2$ billion) in addiction treatment expenditures came from public sources. Public spending includes:

- 45.3 percent ( $\$ 10.1$ billion) from state and local government non-Medicaid sources;

- 26.3 percent (\$5.9 billion) from Medicaid;

\section{Figure 7.D \\ Components of Private Addiction \\ Treatment Spending (Total \$5.8B)}

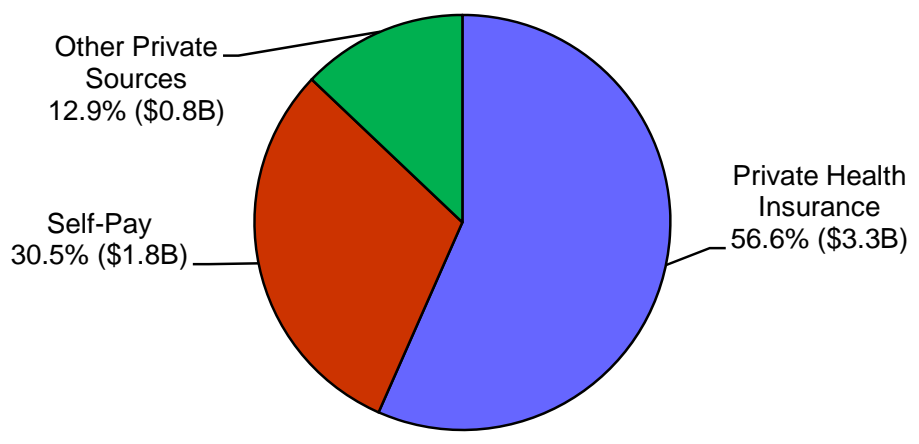

Source: CASA Columbia analysis of the National Expenditures for Mental Health Services \& Substance Abuse Treatment 1986-2005. (2012).

- 19.9 percent ( $\$ 4.4$ billion) from other federal funds such as block grants to states; and

- $\quad 8.5$ percent (\$1.9 billion) from Medicare. ${ }^{*} 54$ (Figure 7.E)

\footnotetext{
* Medicaid and Medicare are government programs with state and federal contributions. Therefore, the estimates reported in the federal, state and local categories are exclusive of the estimates of public funds spent through Medicare and Medicaid programs.
} 


\section{Figure 7.E \\ Components of Public Addiction \\ Treatment Spending (Total \$22.2B)}

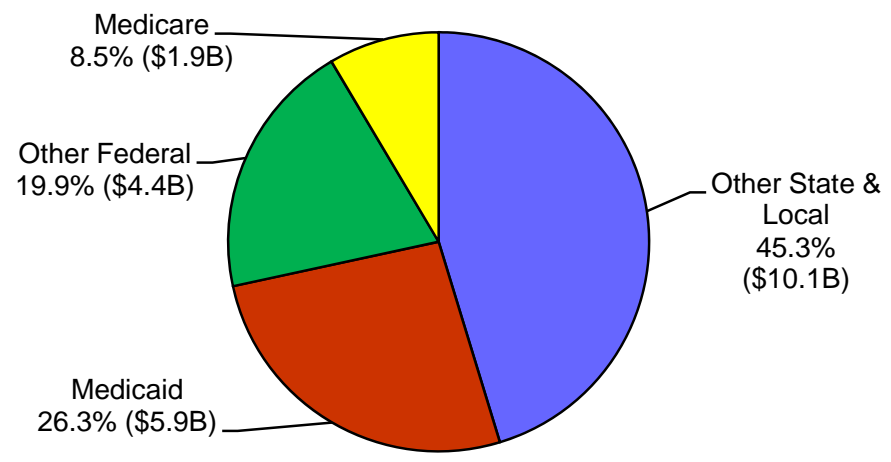

Source: CASA Columbia analysis of the National Expenditures for Mental Health Services \& Substance Abuse Treatment 19862005. (2012).

\section{Expenditures by Providers and Types of Services}

Most expenditures for addiction treatment are to non-hospital based providers offering outpatient services.

Providers. Treatment providers whose primary role is addiction treatment account for 61.7 percent ( $\$ 17.3$ billion) of the total spending on addiction treatment (\$28.0 billion). ${ }^{57}$ These expenditures include:

- 43.4 percent ( $\$ 12.2$ billion) by specialty addiction treatment centers;

\section{Trends in Spending on Addiction Treatment}

Between 1986 and 2010, direct spending on addiction treatment increased from $\$ 9.1$ billion to an estimated $\$ 28.0$ billion. In constant dollars, direct spending on addiction treatment nearly doubled during this period, while the portion of direct addiction treatment paid by private sources fell from 39.8 percent to 20.8 percent and the portion paid by public sources rose from 60.2 percent to 79.2 percent. While there also was a shift from private to public payment of medical care expenditures during this period, the shift was far less pronounced; private source payment of medical expenditures dropped from 61.2 percent in 1986 to 55.7 percent in 2005 while public source payment rose from 38.8 percent to 44.3 percent. $^{55}$

I was not able to get help until I got myself into the criminal justice system and was then placed into treatment as a condition of parole. I tried many times to get help for my addiction but due to the lack of insurance and money, was denied. $^{56}$
- 12.8 percent ( $\$ 3.6$ billion) by specialty units in general hospitals; and

- 5.5 percent ( $\$ 1.5$ billion) by specialty hospitals. ${ }^{58}$

The remaining expenditures (38.3 percent, $\$ 10.7$ billion) are accounted for by facilities and individuals that have another primary mission but also provide addiction-related services, including:

- $\quad 8.8$ percent ( $\$ 2.5$ billion) by specialty mental health centers;

- $\quad 7.9$ percent ( $\$ 2.2$ billion) by health professionals other than physicians such as counselors, social workers and psychologists;

- 6.8 percent ( $\$ 1.9$ billion) by general hospital non-specialty units such as detoxification," when a patient with addiction is admitted via the emergency room;

\footnotetext{
* Not considered to be addiction treatment.
} 
- $\quad 6.7$ percent (\$1.9 billion) in insurance administration;

- $\quad 6.3$ percent ( $\$ 1.8$ billion) by physicians;

- 1.2 percent ( $\$ 0.4$ billion) by nursing homes and home health care providers; and

- $\quad 0.6$ percent ( $\$ 0.2$ billion) in retail prescription drugs. $^{59}$ (Figure 7.F)

Services. Total addiction treatment spending in 2010 ( $\$ 28.0$ billion) included:

- $\quad 92.7$ percent (\$25.9 billion) for services, including:

48.3 percent ( $\$ 13.5$ billion) for outpatient services (nonhospital, 38.4 percent, $\$ 10.8$ billion and hospital, 9.8 percent, \$2.8 billion);

$>\quad 27.9$ percent ( $\$ 7.8$ billion) for residential (non-hospital inpatient) services; and

16.5 percent ( $\$ 4.6$ billion) for inpatient hospital services;

- $\quad 6.7$ percent ( $\$ 1.9$ billion) in insurance administration fees; and

- $\quad 0.6$ percent ( $\$ 0.2$ billion) for retail prescription drugs. ${ }^{60}$ (Figure 7.G)



\section{Spending by Service (Total \$28.0B)}



Source: CASA Columbia analysis of the National Expenditures for Mental Health Services \& Substance Abuse Treatment 1986-2005. (2012). 


\section{Treatment Admissions}

There are no national data on individuals who receive addiction treatment in the U.S.; the national data that are available represent admissions to treatment facilities for addiction involving alcohol or other drugs (excluding nicotine) ${ }^{*}$ for the 79.2 percent of addiction treatment funding that comes from public sources, although some of these facilities also may receive private funding. ${ }^{\dagger}$

${ }^{*}$ While some addiction treatment programs may address nicotine, they do not report these services in their treatment admission data.

${ }^{\dagger}$ These analyses are based on data from the Treatment Episode Data Set (TEDS), a national database of information on the demographic and addiction characteristics of admissions to and discharges from addiction treatment facilities, routinely collected by each state substance abuse agency and submitted to the Substance Abuse and Mental Health Services Administration (SAMHSA). TEDS collects information about the beginning and termination of treatment episodes; multiple episodes of treatment for the same client are counted separately. The 2009 TEDS data are based on information on the demographic and substance use characteristics of the approximately 1.5 million annual admissions to alcohol and other drug addiction treatment facilities (excluding detoxification), among those ages 12 and older, that report to individual state substance abuse agency data systems. The TEDS attempts to include all admissions to providers receiving public funding; however, because each state or jurisdiction decides the TEDS eligibility of a provider, there is no independent check on the actual sources of funding. Although SAMHSA requests submission of TEDS data on all admissions to any publicly funded treatment facility, reporting in some state agencies is structured so that only patients treated with public funds are included in the TEDS. The number of patients in these facilities whose treatment is not publicly funded is unknown. Because the scope of facilities included in the TEDS is affected by differences in state licensure, certification, accreditation and disbursement of public funds, the TEDS, while comprising a significant proportion of all admissions to addiction treatment, does not include all admissions. (See Appendix A for more information about TEDS.) It is important to note that CASA Columbia's analyses of TEDS data exclude detoxification services in calculating rates of
In 2009, ${ }^{\ddagger}$ there were 1.5 million admissions to publicly-funded addiction treatment facilities. ${ }^{\S}$ More than half (55.6 percent) of the admissions were for addiction involving multiple substances. For 38.6 percent of the admissions, alcohol was the primary substance of addiction, compared with 29.0 percent for illicit drugs other than marijuana, 22.4 percent for marijuana and 7.9 percent for controlled prescription drugs. ${ }^{61}$ (Figure 7.H)

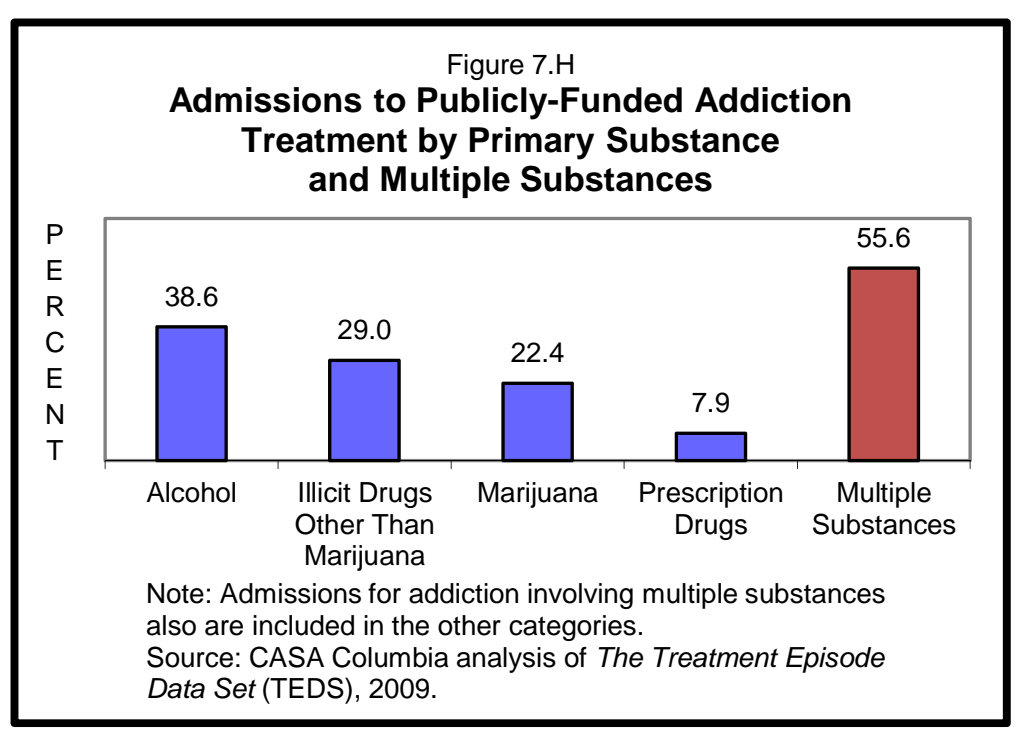

Admissions to publicly-funded addiction treatment reflect greater attention to addiction involving illicit drugs other than marijuana and multiple substances relative to the prevalence of addiction involving these substances in the population. $^{* *} 62$

admissions to addiction treatment; therefore, data reported in this report may differ from those published in TEDS reports.

₹ The most recent available data.

${ }^{\S}$ Included in the TEDS database.

** Of those needing addiction treatment, 80.7 percent involve alcohol, 18.9 percent involve marijuana, 10.3 percent involve controlled prescription drugs and 7.0 percent involve illicit drugs other than marijuana; 14.6 percent involve multiple substances. (Categories are not mutually exclusive.) 
Between 2002 and 2009, treatment admissions to publicly-funded addiction treatment increased by 7.0 percent (from 1.4 million to 1.5 million). During that time, admissions where prescription drugs were the primary substances of addiction increased the most--by 92.7 percent; admissions where marijuana was the primary substance of addiction increased by 13.1 percent. At the same time, admissions where illicit drugs other than marijuana were the primary substances of addiction declined by 11.0 percent and admissions where alcohol was the primary substance of addiction declined by 6.5 percent. Between 2002 and 2009, admissions for addiction involving multiple substances declined by 1.2 percent. $^{63}$ (Figure 7.I)

\section{Treatment Referrals and Venues}

CASA Columbia's NABAS found that 46.8 percent of respondents would turn to a health professional--such as their physician (27.8 percent), a health professional other than their primary care physician* $^{*}$ (19.7 percent) or a mental health professional (9.2 percent) $)^{\dagger}$--if someone close to them needed help for addiction. ${ }^{64}$ (Figure 7.J)

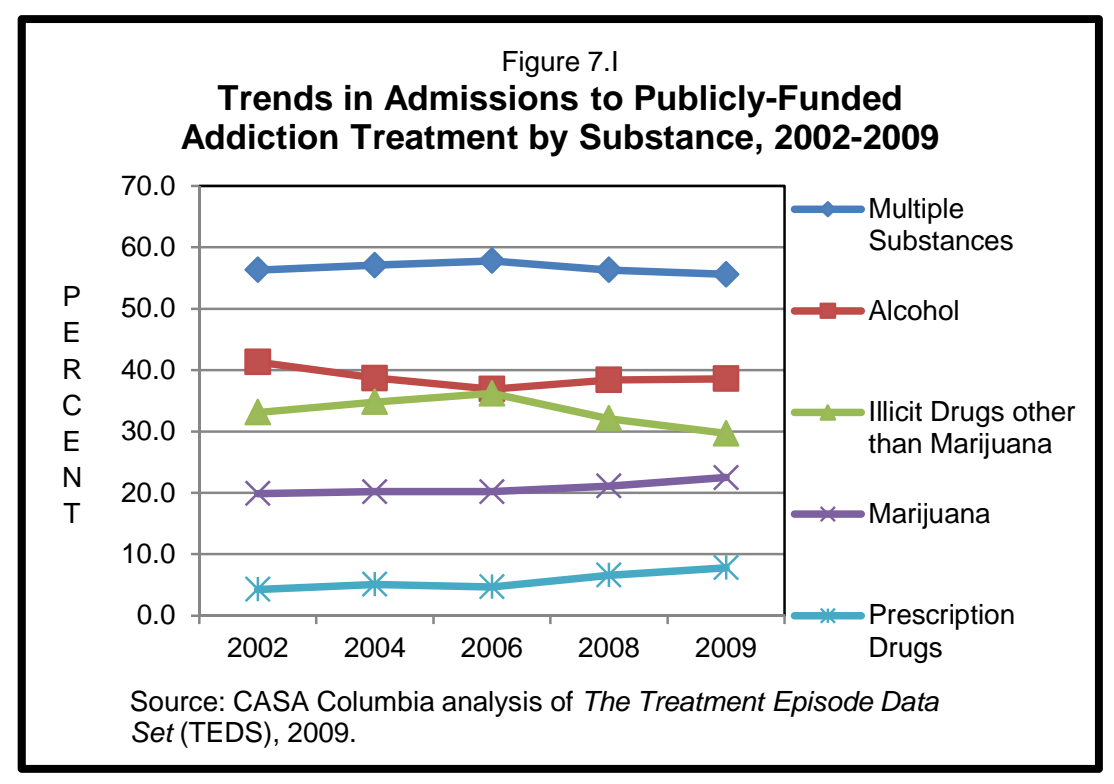

Figure 7.J

Where People Would Turn for Information/Help with Addiction for Someone Close to Them

\begin{tabular}{|c|c|}
\hline Primary Care Physician & $\square 27.8$ \\
\hline Mutual Support Program & 21.0 \\
\hline Other Health Professional & 19.7 \\
\hline "Do Research/Look It Up" & 18.8 \\
\hline Addiction Treatment Center & 11.0 \\
\hline Friend/Family Member & 10.7 \\
\hline Church/Clergy or Relgious/Spiritual Leader & 9.8 \\
\hline Mental Health Professional & 9.2 \\
\hline Addiction Hotline/Helpline & 7.2 \\
\hline
\end{tabular}

\footnotetext{
* Not including mental health professionals.

${ }^{\dagger}$ Some respondents chose more than one response so the 46.8 percent reflects those who chose either one of these health professionals.
} 
Another national survey found that 65 percent of adults would turn to a health care provider for a problem involving alcohol. ${ }^{65}$ Despite these findings, the smallest proportion of referrals to publicly-funded addiction treatment comes from health professionals. ${ }^{66}$

Of all the admissions to publicly-funded addiction treatment in 2009, 44.3 percent were referred by the criminal justice system. ${ }^{*}$ Onequarter (25.3 percent) of referrals came from individuals, including concerned family members, friends and the self-referred; $;^{\dagger} 12.1$ percent were referred by community sources such as social welfare organizations, religious organizations and mutual support programs; 10.6 percent were referred by addiction treatment providers ${ }^{\S}$ for additional treatment and

${ }^{*}$ Referrals from the criminal justice system include referrals from any police official, judge, prosecutor, probation officer or other person affiliated with a federal, state or county judicial system. This category also includes referrals by a court for DWI/DUI, patients referred in lieu of or for deferred prosecution, during pretrial release, before or after official adjudication, as well as referrals of those on pre-parole, pre-release, work or home furlough or Treatment Alternatives for Safe Communities (TASC).

${ }^{\dagger}$ Separate data on each of these categories are not available in the TEDS dataset.

${ }^{\ddagger}$ Community sources of referral also include government agencies that provide aid in the areas of poverty relief, unemployment, shelter or social welfare and referrals from defense attorneys. According to the TEDS data, defense attorneys are not included in the criminal justice system category; prosecutors are included in that category. These community referral categories cannot be examined separately in the TEDS dataset.

$\S$ Addiction service providers are those programs, clinics or health care providers whose principal objective is treating patients with addiction, or where a program's services are related to substance use prevention or education. TEDS distinguishes between transfers within a single, continuous treatment episode and the initiation of a new treatment episode but, because TEDS relies on state administrative systems that appear to differ greatly in their ability to distinguish transfers within a continuous treatment episode from the initiation of a new treatment episode, some transfers may be reported by TEDS as new treatment episodes.
5.7 percent were referred by a health care provider. $^{* *}$ Very few treatment referrals came from schools (1.4 percent) ${ }^{\dagger \dagger}$ or from employers or Employee Assistance Programs (0.6 percent). $^{67}$ (Figure $7 . \mathrm{K}^{\ddagger \ddagger}$ )

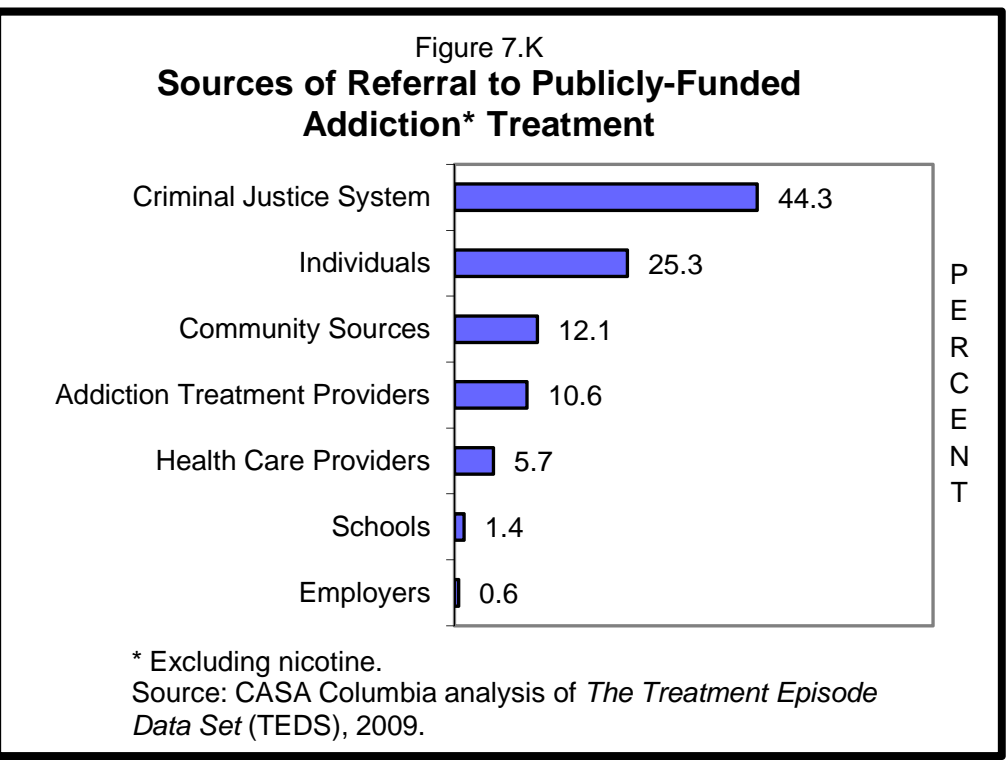

Given that addiction is a medical disease requiring the intervention of trained medical professionals and the high prevalence of this disease in the general population, ${ }^{\S \S}$ the fact that only 5.7 percent of treatment admissions are referred by health professionals highlights the extent to which health professionals fail to address this disease in their practice. The fact that the largest proportion of referrals to addiction treatment comes from the criminal justice system further underscores how extensively we neglect to address addiction until the consequences are too dire to ignore. ${ }^{68}$ The

\footnotetext{
** Referrals to treatment programs from health care providers include those from physicians (including psychiatrists) or other licensed health professionals, or from a general hospital, psychiatric hospital, mental health program or nursing home.

${ }^{+\dagger}$ Including a school principal, counselor, teacher, student assistance program (SAP), the school system or an educational agency.

㧊 These data are from the TEDS dataset.

Comparable data on referral to treatment for nicotine addiction (smoking cessation) are not available. The data reported here do not include referrals to detoxification programs.

$\S \S$ See Chapters II and III.
} 
separation of addiction treatment from mainstream health care is demonstrated further by the fact that most treatment venues are not licensed health care institutions. ${ }^{69}$ (See Chapter IX.)

Available data on treatment venues to which referrals are made distinguish between intensive and non-intensive services provided in nonresidential settings and between short- and longer-term services provided in residential settings: ${ }^{70}$

- Non-residential services include individual, family, group and/or pharmaceutical therapies provided on an ambulatory or outpatient basis. Intensive services are those that last at least two or more hours per day for three or more days per week.

- Residential services include addiction treatment provided in a non-hospital setting, and 24-hour per day medical care in a hospital facility that includes addiction treatment. Short-term services include those that last for 30 days or less and longer-terms services are those that last for more than 30 days.

There are no data available to match the need for specific services with referrals to specific treatment venues. Although referrals to treatment primarily come when addiction has advanced to the point of serious social consequences (e.g., the criminal justice system) and often involve co-occurring health conditions, in 2009, the majority of the 1.5 million treatment admissions were to nonintensive and non-residential venues: ${ }^{71}$

- 63.3 percent were for non-intensive, nonresidential services;

- $\quad 14.6$ percent were for intensive, nonresidential services;
- $\quad 11.9$ percent were for short-term residential services; and

- 10.2 percent were for longer-term residential services. $^{72}$ (Figure 7.L)



While most admissions regardless of referral source are to non-residential venues (77.9 percent), certain referral sources are even likelier than average to result in admissions to nonresidential services, including:

- $\quad$ schools (97.8 percent);

- $\quad$ the criminal justice system (85.5 percent);

- $\quad$ employers (84.0 percent); and

- $\quad$ community sources (79.3 percent). ${ }^{73}$

In contrast, while only 22.0 percent of treatment admissions overall are referred to residential treatment venues, 47.8 percent of those referred by addiction treatment providers are to residential treatment venues. ${ }^{74}$ (Table 7.1) 
Table 7.1

Admissions to Different Types of Treatment Service Venues by Source of Referral, 2009 (Percent)

\begin{tabular}{l|c|c|c|c}
\hline $\begin{array}{l}\text { Source of } \\
\text { Referral }\end{array}$ & $\begin{array}{c}\text { Non- } \\
\text { Intensive } \\
\text { Non- } \\
\text { Residential }\end{array}$ & $\begin{array}{c}\text { Intensive } \\
\text { Non- } \\
\text { Residential }\end{array}$ & $\begin{array}{c}\text { Short-Term } \\
\text { Residential }\end{array}$ & $\begin{array}{c}\text { Longer- } \\
\text { Term } \\
\text { Residential }\end{array}$ \\
\hline Total & $\mathbf{6 3 . 3}$ & $\mathbf{1 4 . 6}$ & $\mathbf{1 1 . 9}$ & $\mathbf{1 0 . 2}$ \\
\hline $\begin{array}{l}\text { Criminal } \\
\text { Justice } \\
\text { System }\end{array}$ & 69.7 & 15.8 & 6.3 & 8.1 \\
\hline $\begin{array}{l}\text { Individual } \\
\text { Referrals }\end{array}$ & 61.0 & 13.7 & 14.2 & 11.1 \\
\hline $\begin{array}{l}\text { Community } \\
\text { Sources }\end{array}$ & 62.3 & 17.0 & 11.2 & 9.6 \\
\hline $\begin{array}{l}\text { Addiction } \\
\text { Treatment } \\
\text { Providers }\end{array}$ & 41.3 & 10.9 & 29.0 & 18.8 \\
\hline $\begin{array}{l}\text { Health Care } \\
\text { Providers }\end{array}$ & 60.2 & 12.7 & 17.5 & 9.6 \\
\hline Schools & 88.2 & 9.6 & 1.3 & 0.9 \\
\hline Employers & 67.3 & 16.7 & 13.6 & 2.5 \\
\hline Soure: CASA & & & & \\
\hline
\end{tabular}

Source: CASA analysis of The Treatment Episode Data Set (TEDS), 2009.

\section{Treatment Completion}

In 2008, ${ }^{*}$ less than half (42.1 percent) of discharges from addiction treatment services ${ }^{\dagger}$ were of admissions in which treatment was completed. $^{\ddagger 75}$ The highest completion rates were from venues to which there were the least referrals:

- 14.8 percent of admissions were to shortterm residential services which had the highest completion rate of 54.8 percent;

- $\quad 11.4$ percent of admissions were to longerterm residential treatment which had a completion rate of 45.5 percent; and

- $\quad 73.8$ percent of admissions were to nonresidential services which had the lowest completion rate of 39.1 percent. $^{76}$

(Figure 7.M)

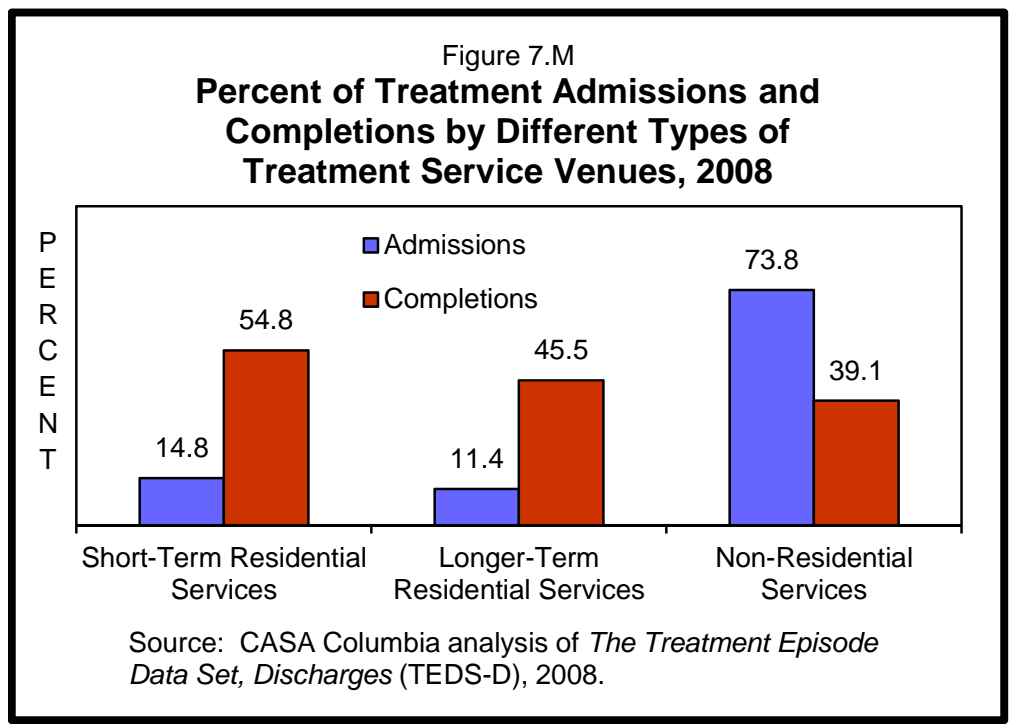

\footnotetext{
* Most recent available data on discharges.

${ }^{\dagger}$ Specifically, those that received state funds and reported data to TEDS. Data include only those discharges that could be linked to admission data in the 2008 TEDS dataset. The general completion rate among all discharges (regardless of whether they were linked to admission data) was 42.1 percent. ${ }^{\ddagger}$ All parts of the treatment plan or program were completed.
} 
Of those discharges that did not represent a completed treatment episode, 46.6 percent dropped out of treatment, 25.9 percent were transferred to another treatment service (whether or not the patient attended that program is unknown), 12.8 percent were terminated by the program and 4.5 percent were incarcerated. The remainder failed to complete treatment for some other reason. ${ }^{*} 77$

\section{Variations in Treatment Completion by Source of Referral}

Admissions to addiction treatment for which the source of referral was an employer were the most likely to complete treatment (57.2 percent of admissions), followed by referrals from the criminal justice system (48.1 percent).

Admissions referred by health care providers and individual sources--including concerned family members, friends and the self-referred-were the least likely to complete treatment (34.6 percent and 33.9 percent of admissions, respectively). ${ }^{78}$ Concern about potential loss of a job or criminal sanctions might help account for higher rates of treatment completion among those referred by employers or the criminal justice system. (Table 7.2)

Table 7.2

Treatment Completion by Source of Referral

\begin{tabular}{|c|c|}
\hline Source of Referral & Percent \\
\hline Total & 42.1 \\
\hline Employers & 57.2 \\
\hline Criminal Justice System & 48.1 \\
\hline Addiction Treatment Providers & 44.5 \\
\hline Schools & 41.1 \\
\hline Community Sources & 36.6 \\
\hline Health Care Providers & 34.6 \\
\hline Individual Referrals & 33.9 \\
\hline \multicolumn{2}{|c|}{$\begin{array}{l}\text { Source: The Treatment Episode Data Set (TEDS) } \\
\text { discharge data, } 2008 .\end{array}$} \\
\hline
\end{tabular}

* i.e., moving, illness or hospitalization, death, other reason out of patient's control or the reason for discharge is unknown or not recorded.

\section{Variations in Treatment Completion by Primary Substance Involved}

Patients admitted to treatment with addiction involving alcohol as the primary substance had the highest rate of treatment completion (50.7 percent) compared with 39.3 percent involving marijuana, 35.4 percent involving other illicit drugs and 35.3 percent involving prescription drugs. The treatment completion rate for admissions involving multiple substances was 38.9 percent. $^{79}$

\section{Variations in Treatment Completion by Key Patient Characteristics}

Male patients admitted to treatment were likelier than females to complete treatment (48.5 percent vs. 42.6 percent of admissions). No significant age-related differences in treatment completion were found. ${ }^{80}$ With regard to racial/ethnic differences in treatment completion, Hispanics admitted to treatment were more likely to complete treatment than were whites or blacks (46.8 percent vs. 37.9 percent and 35.4 percent of admissions, respectively). ${ }^{\dagger} 81$

\section{Link between Funding Source, Type of Service Provided and Treatment Completion}

Individuals who are privately insured are substantially less likely to enter addiction treatment than those with public insurance. ${ }^{\ddagger} 82$ Publicly-funded admissions to addiction treatment are likelier than privately-funded admissions to be for more intensive services:

\footnotetext{
${ }^{\dagger}$ Similarly, a study of patients receiving treatment for addiction involving alcohol found that black patients were less likely than white or Hispanic patients to complete their treatment program, regardless of whether they were enrolled in non-residential treatment (17.5 percent vs. 26.7 percent and 29.7 percent, respectively) or residential treatment (30.7 percent vs. 46.1 percent and 42.9 percent, respectively).

${ }^{\ddagger}$ Controlling for type of substance, severity of the addiction, demographic characteristics, current health status and whether the individual is a daily cigarette smoker.
} 
- $\quad$ intensive outpatient (25.9 percent vs. 15.1 percent),

- $\quad$ short-term residential (21.6 percent vs. 14.1 percent), and

- $\quad$ longer-term residential (7.6 percent vs. 3.0 percent). ${ }^{83}$

Privately-funded admissions are likelier than publicly-funded admissions to be to nonintensive outpatient services (67.6 percent vs. 44.7 percent), have a higher rate of treatment completion (53.7 percent vs. 42.9 percent) and have a lower rate of transfer to another facility (12.2 percent vs. 17.9 percent). ${ }^{84}$

Existing data do not provide an explanation for these differences and no data are available on treatment needs and outcomes by funding source and type of service provided. Possible contributing factors, however, might include that privately-funded admissions are likelier to involve less severe cases of addiction, those with private resources may have greater access to effective support services or quality care, or those with private insurance may be less likely to seek treatment perhaps due to the perceived stigma. ${ }^{85}$

\section{Barriers Patients Face in Accessing and Completing Addiction Treatment}

In addition to the limited private sector coverage of addiction treatment and the lack of treatment referrals from the health care system, many other barriers stand in the way of individuals accessing and completing addiction treatment. These include: a misunderstanding of the disease, negative public attitudes and behavior toward those with the disease, privacy concerns, cost, lack of information on how to get help, limited availability of services including a lack of physicians trained in addiction care, insufficient social support, conflicting time commitments, negative perceptions of the treatment process and legal barriers. Other factors having to do with treatment quality are discussed in Chapter X. Rarely is there only one obstacle to a person receiving needed treatment. ${ }^{86}$

Although comparable national data for barriers to accessing smoking cessation treatment are not available, research indicates that barriers similar to those facing individuals seeking addiction treatment involving alcohol or other drugs stand in the way of smokers accessing tobacco cessation services. ${ }^{87}$

\section{Misunderstanding of the Disease}

One of the most frequently reported barriers to accessing addiction treatment has been described as patient denial. ${ }^{88}$ However, what is commonly viewed as denial might also be characterized as a misunderstanding of the disease. As is the case for seeking treatment for other health conditions such as diabetes, hypertension or heart disease, ${ }^{89}$ most cases of denial that serve as barriers to treatment access actually involve cases in which a person with symptoms of addiction does not recognize that he or she has a treatable disease, ${ }^{90}$ underestimates the severity of the disease ${ }^{91}$ or does not believe that the symptoms can be allayed through treatment. ${ }^{92}$ Such feelings stem not only from a lack of public awareness about the true nature of addiction--that it is a brain disease that can be treated effectively--but from the disease itself-one effect of addictive substances on the brain is that judgment, self-awareness and insight become impaired. ${ }^{93}$ Continuing to misuse substances despite the associated harms is a defining symptom of the disease of addiction ${ }^{94}$ and in many cases results from the changes that addictive substances produce in the structure and function of the areas of the brain that control judgment, decision making and behavioral inhibition and control. ${ }^{95}$

In one survey of people with a history of addiction in their families, 60 percent cited denial as the biggest obstacle to getting help for addiction. ${ }^{96}$ The majority (71.7 percent) of respondents to CASA Columbia's NABAS think that a main reason why people with addiction do not get the help they need is that they refuse to admit to having a problem or that they do not 
want to quit. ${ }^{*} 97$ Another study found that between eight and 16 percent of people who had been assessed but had not entered treatment cited some form of denial as the barrier. $^{\dagger 98}$

The most frequently-mentioned barrier to accessing treatment for addiction involving alcohol and drugs other than nicotine is not being ready to stop using these substances. ${ }^{\ddagger 99}$ A study of current smokers in Wisconsin found that the main barriers to quitting that participants reported were not being ready to stop smoking because they enjoy it (79 percent), would crave it (75 percent) or are afraid of losing the stress relief associated with it (63 percent). ${ }^{100}$

One national survey found that among respondents who needed ${ }^{\S}$ but did not receive treatment, only 3.3 percent of those with addiction involving alcohol, 8.3 percent of those with addiction involving another drug (excluding nicotine) and 12.4 percent of those with addiction involving multiple substances (excluding nicotine) perceived a need for treatment. ${ }^{101}$

Treatment providers also cite denial as the main barrier to treatment access: CASA Columbia's survey of treatment providers in New York State found that 85.5 percent of program directors and 84.9 percent of staff providers said that denial of an addiction problem "very much" stands in the way of people looking for needed addiction treatment. ${ }^{102}$

Misunderstanding of the disease of addiction also is reflected in public policies and health care practices that fail to integrate treatment for

\footnotetext{
${ }^{*}$ Respondents were asked to select two or three reasons from a list read to them by the interviewer. ${ }^{\dagger}$ Eight percent of respondents believed their "drug use is not causing any problems" while 16.1 percent believed they "could handle their drug use on their own.”

‡ As reported in a national survey of individuals ages 12 and older who recognized they needed treatment, made an effort to get treatment, but did not receive treatment. These estimates are from combined national data from 2006-2009.

${ }^{\S}$ Met clinical diagnostic criteria for addiction involving alcohol or drugs other than nicotine.
}

all addictive substances including nicotine into standard treatment protocols. (See Chapter X.)

\section{Negative Public Attitudes and Behaviors Toward People with Addiction}

Related to widespread misunderstanding of the disease of addiction is the stigma attached to it-the well documented, strong disapproval of or discrimination against those with the disease-and the fear of repercussions which prevent people with addiction from getting help. ${ }^{103}$ Although stigma is a subjective experience-perceived disapproval by others and subsequent embarrassment may or may not reflect a more objective reality--there is a long history of blaming and looking down on people with addiction ${ }^{104}$ rather than sympathizing with them as we do for those with other health conditions. The fear of disapproval or rejection can derive from an individual's own low self-esteem or sense of shame about having addiction or it can derive from a fear of abandonment by friends or family because of the substance use itself, the consequences that result or because of the decision to pursue treatment. ${ }^{105}$

Twenty-nine percent of the respondents to CASA Columbia's NABAS reported that the main reason why people with substance-related problems do not get the help they need is a fear of social embarrassment or shame. ${ }^{106}$ Another national survey found that two-thirds (67 percent) of the public believe that a stigma exists toward people who have been treated for addiction involving alcohol or other drugs. ${ }^{* *} 107$ A related study found that 80 percent of the public believes that there is a stigma against people with addiction involving alcohol and 51 percent believe this stigma is maintained even after treatment or cessation of alcohol use. ${ }^{108}$

Smokers also face a stigma, particularly in light of increasing anti-smoking policies and awareness of the health risks of smoking and exposure to environmental tobacco smoke. ${ }^{109}$

\footnotetext{
** This survey excluded addiction involving nicotine. Stigma was defined for respondents as "something that detracts from the character or reputation of a person; a mark of disgrace.”
} 
One survey of New York City residents found that 39 percent said that "Most people think less of a person who smokes" and 21 percent said that "Most people believe smoking is a sign of personal failure."110

Over 40 percent (41.4) of respondents to the $N A B A S$ reported that discrimination against people in recovery ${ }^{*}$ from addiction involving nicotine, alcohol or prescription or illegal drugs is a very (15.8 percent) or somewhat (25.6 percent) serious problem in their community; 51.0 percent of those who had their own history of addiction thought it was a very (24.5 percent) or somewhat (26.5 percent) serious problem. ${ }^{111}$

Thirty-eight percent of the respondents to the $N A B A S$ reported that the main reason why people with addiction do not get the help they need is a fear of adverse consequences like losing a job, getting expelled from school or losing child custody. Treatment providers also see this as a barrier to treatment access: CASA Columbia's survey of treatment providers in New York State found that a significant proportion of the respondents said that the fear that one will be discriminated against “somewhat" (48.2 percent of program directors and 51.4 percent of staff providers) or "very much" (39.8 percent of program directors and 30.4 percent of staff providers) stands in the way of people looking for needed addiction treatment, as does fear of losing one's job (96.3 percent of directors and 94.2 percent of staff providers said this "somewhat" or "very much" stands in the way of people looking for needed treatment). ${ }^{112}$

Another recent study found that while there are no significant differences in employment rates between people with a history of addiction and those without, those with a history of addiction report significantly higher rates of involuntary job loss. The analyses conducted for the study, which controlled for other factors that predict employment outcomes, suggest that employer discrimination may be an important contributing factor to job instability in this population. The authors speculate that while the Americans with

${ }^{*}$ Defined as effectively managing their disease.
Disabilities Act of 1990 is successful in protecting those with a history of addiction from experiencing discrimination in the hiring process, it might be less successful in protecting those people from job termination. ${ }^{113}$

Individuals with a lifetime diagnosis of addiction involving alcohol are less likely to access treatment or support services ${ }^{\dagger}$ if they perceive that addiction carries a stigma. ${ }^{114}$

Respondents to the NABAS admit that they would discriminate against people who have a history of addiction, being less likely to hire former smokers or those in recovery from addiction involving alcohol or other drugs. ${ }^{115}$

- $\quad 53.6$ percent of respondents said that they would be less likely to hire a qualified applicant upon learning that he or she is in recovery from addiction involving illicit drugs;

- $\quad 41.1$ percent would be less likely to hire a qualified applicant in recovery from addiction involving controlled prescription drugs;

- $\quad 27.9$ percent would be less likely to hire a qualified applicant in recovery from addiction involving alcohol; and

- 6.6 percent would be less likely to hire a qualified applicant who is a former smoker. ${ }^{116}$

In comparison, 18.0 percent said they would be less likely to hire a qualified applicant who had been treated for depression, about nine percent said that they would be less likely to hire a qualified applicant who had been treated either

\footnotetext{
${ }^{\dagger}$ Defined in this study as including services delivered in an inpatient ward, outpatient clinic, rehabilitation program, halfway house, emergency room or crisis center or by a private physician, psychiatrist, psychologist, social worker or other professional; alcohol or other drug detoxification; and selfhelp/mutual support programs (e.g. AA) or social services (family services or services delivered by an employee assistance program or by clergy).
} 
for obesity or heart disease and about five percent said the same for diabetes, asthma or cancer. ${ }^{* 117}$ (Figure 7.N)
Perhaps because of the lingering view that addiction results from lack of will power or self control that can be remedied with a simple

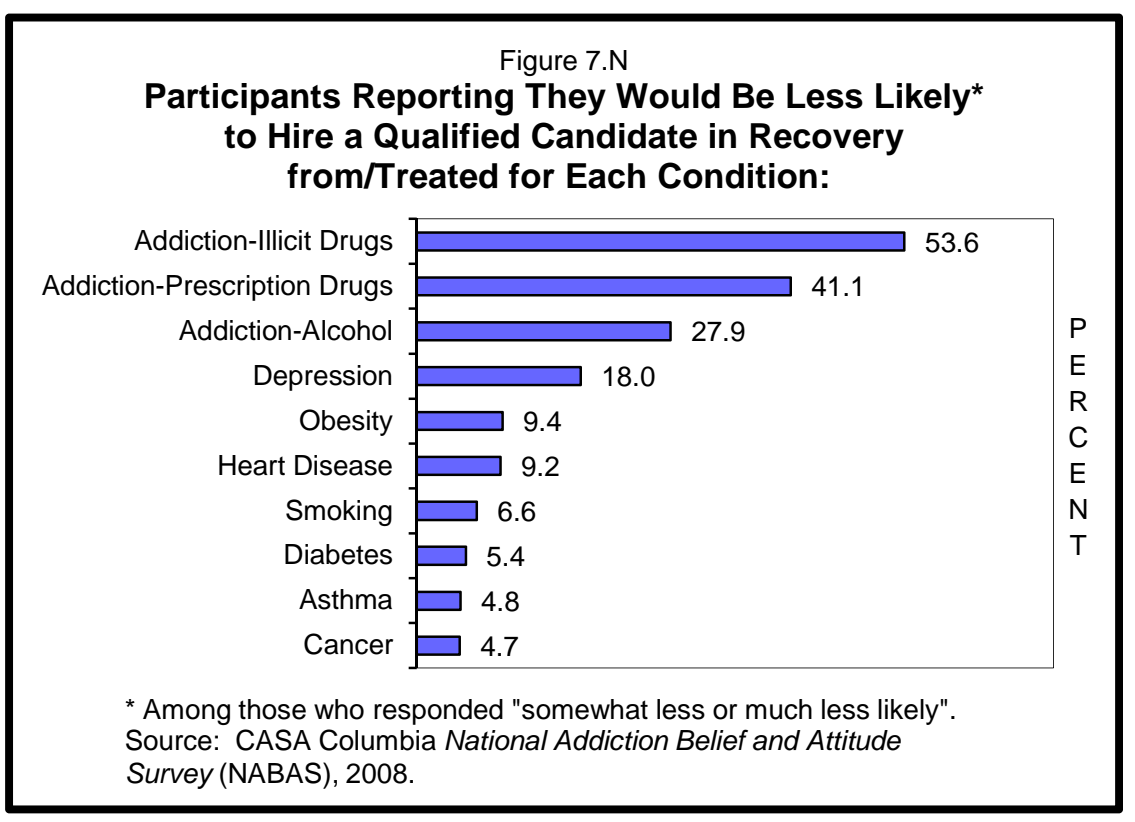

change of mind, stigma and discrimination against addicted individuals are all too common. Recent research finds that people with addiction are seen as more blameworthy and dangerous compared to individuals with a "mental" illness, and those with a "mental" illness are viewed more negatively than those with a "physical" illness.

Consequently, those with addiction are avoided more and helped less. ${ }^{121}$ Research also indicates that enhancing the public's understanding of addiction or mental health disorders as having a neurobiological basis relates to

Another study found that 43 percent of the public would be less likely to vote for a gubernatorial candidate who was in recovery from addiction involving alcohol or other drugs (excluding nicotine). ${ }^{118}$

Discrimination against those with addiction is manifested on the governmental and institutional levels as well. Insurance companies generally provide less coverage for addiction treatment services than for other medical services. ${ }^{119}$ Although the passage of the Paul Wellstone and Pete Domenici Mental Health Parity and Addiction Equity Act (MHPAEA) of 2008, and the subsequent 2010 Patient Protection and Affordable Care Act, were significant breakthroughs, the laws contain exemptions and loopholes that may continue to limit access to addiction treatment. $^{\dagger 120}$

\footnotetext{
* The stigma associated with addiction is compounded by its high rate of co-occurrence with mental illness.

${ }^{\dagger}$ For example, MHPAEA does not require health insurance plans to offer coverage for mental health or addiction treatment services, employers with fewer than 50 employees are not covered by the law, and if the projected cost increase is too high for health
}

increased public support for providing treatment services to individuals with these conditions, but has not yet translated into less of a stigma or discrimination associated with these conditions. $^{122}$

\section{Privacy Concerns}

Because of negative public attitudes toward addiction and the consequent potential for stigma and discrimination, prospective patients for addiction treatment may have concerns about the extent to which their identity and the details of their treatment will stay private and confidential. $^{123}$

Prospective patients sometimes believe that providers violate patients' confidentiality. ${ }^{124}$

insurance plans (more than two percent in the first year and more than one percent in subsequent years), then insurance companies may request an exemption from the law. Under the ACA, mental health and addiction treatment services must be offered in individual and small group health plans as part of essential health benefits, but the scope of these benefits will likely vary by state. See Chapter VIII for a more complete discussion of health insurance coverage for addiction prevention and treatment services. 
Privacy concerns are particularly acute in rural populations where patients may fear a lack of anonymity due to relatively smaller and more close-knit communities. ${ }^{125}$

Another element of privacy concerns involves the aversion of some individuals to key elements of the therapeutic process--participating in individual or group therapy where patients discuss personal or intimate details with therapists or with other patients. ${ }^{126}$ In one study of individuals with addiction, 36.5 percent of respondents said they do not like talking in groups, 35.6 percent said they do not like to talk about their personal lives with other people and 32.1 percent said they do not like being asked personal questions. ${ }^{127}$ In a study of risky alcohol users, 50 percent cited a combination of concerns about privacy, labeling, asking for help and sharing problems as a reason for not seeking treatment. $^{128}$

\section{Cost $^{*}$}

Cost is one of the most frequently-reported and long-standing barriers to receiving addiction treatment. ${ }^{129}$ Approximately 50 million Americans, or 16.3 percent of the United States population, had no health insurance in 2010. ${ }^{130}$ Twenty-nine million insured people are underinsured $^{\dagger 131}$ perhaps prompting them to postpone needed treatment. People who are uninsured and underinsured not only have higher rates of chronic, relapsing addiction, ${ }^{132}$ but also generally receive less preventive care, are at more advanced disease stages at the time of diagnosis, receive less treatment for health conditions and have higher mortality rates than those with comprehensive coverage. ${ }^{133}$

A 2009 national survey found that nearly half (49 percent) of U.S. adults feel that they would not be able to afford treatment for addiction involving alcohol or other drugs ${ }^{\ddagger}$ if they or

\footnotetext{
* See Chapter VIII for a more complete discussion of cost-related issues in addiction treatment.

${ }^{\dagger}$ Those with health insurance, but with very high medical expenses relative to their incomes.

‡ This survey did not address addiction involving nicotine.
}

someone in their family needed it. This perception was true across income levels: 67 percent of adults with annual incomes under $\$ 50,000$ said they would not be able to afford treatment, as did 30 percent of those with incomes between $\$ 75,000$ and $\$ 100,000$ and 25 percent of those with incomes above $\$ 100,000$. In addition to those with the lowest income level, other groups most concerned about affording addiction treatment include those with a high school degree or less (65 percent), those living outside of metropolitan areas (56 percent) and adults ages 18-34 (56 percent). The survey also found that 75 percent believe that people with addiction may not get treatment because they lack insurance coverage or cannot afford it. ${ }^{134}$

Cost is a barrier to seeking treatment even for people with adequate health insurance. ${ }^{135}$ Some addiction treatment programs do not accept any insurance payments--private or public--for their services, ${ }^{136}$ and insurance coverage for addiction treatment, when it exists, often has higher copays and limited service coverage. ${ }^{137}$

With the current funding stream, you must be very rich or very poor to get treatment. ${ }^{138}$

--Johnny Allem

Founder and President Aquila Recovery, Chartered Former President and CEO Johnson Institute

People with private insurance tend to have greater concerns about cost and are likelier to cite cost as a barrier to treating addiction than people with Medicaid or Medicare. Those with public insurance focus more on accessibility issues (waiting times, eligibility) as barriers to treatment. ${ }^{139}$ This disparity may be due to the fact that some private insurance companies do not cover addiction treatment and some employers do not extend their benefit plans to include addiction treatment coverage. ${ }^{140}$

Twenty-eight percent of respondents to the NABAS think that one of the main reasons why people do not get the help they need for 
addiction is because of insufficient available and affordable treatment services. ${ }^{141}$ Treatment providers see this as a barrier to treatment access as well: CASA Columbia's survey of treatment providers in New York State found that approximately 90 percent of the respondents said that inability to pay "somewhat" (44.6 percent of program directors and 42.8 percent of staff providers) or "very much" (45.8 percent of program directors and 50.7 percent of staff providers) stands in the way of people receiving needed addiction treatment. ${ }^{142}$

Cost not only is a barrier to entering treatment, but to staying in treatment as well. One study found that people randomly assigned to receive free methadone maintenance therapy stayed in their treatment programs longer than those randomly assigned to fee-for-service treatment, even though the fee was only $\$ 2.50$ per day. ${ }^{143}$

\section{Lack of Information about How to Get Help}

A significant barrier to obtaining addiction treatment is the lack of knowledge about where to go for help and the limited ability of physicians, parents and other family members, teachers, coaches, employers, clergy and law enforcement to identify the signs of addiction in others and know how to help patients access effective treatment. ${ }^{144}$ The NABAS found that 16 percent of respondents believe that a main reason why people with addiction do not get the help they need is because of insufficient information about how and where to get help. ${ }^{145}$

\section{Limited Availability of Services}

A significant barrier to treatment is the lack of appropriate and accessible treatment services. ${ }^{146}$ This barrier is due to insufficient training of medical professionals and treatment options and the lack of treatment options tailored to individual needs; excessively restrictive eligibility criteria in some treatment facilities; long waiting times for treatment entry and a lack of trained addiction physician specialists and a lack of inclusion of addiction medicine as a recognized field of practice by the American
Board of Medical Specialties, as discussed in Chapters IX and X.

Approximately half of respondents to CASA Columbia's NABAS reported that insufficient treatment programs and services for people with addiction is a somewhat or very serious problem in their communities. ${ }^{147}$ Another national poll found that nearly half (47 percent) of the respondents said that treatment services are lacking in their community; only one quarter indicated that there are enough affordable, accessible quality treatment centers and services. ${ }^{*} 148$ CASA Columbia's survey of treatment providers in New York State found that a significant proportion of the respondents said that a lack of conveniently located treatment programs "somewhat" (62.2 percent of program directors and 56.8 percent of staff providers) or "very much" (17.1 percent of program directors and 21.6 percent of staff providers) stands in the way of people seeking needed addiction treatment. ${ }^{149}$

Some individuals who need addiction treatment face eligibility criteria for program entry that are too stringent--including a patient's ability to pay and a required agreement to comply with all rules and treatment protocols regardless of individual goals. ${ }^{150}$ In contrast, the main criterion for treatment access in mainstream medicine is the principle of medical necessity, determined by a physician; ${ }^{151}$ patients seeking medical treatment rarely have to meet a threshold level of problem severity or agree to comply in advance with particular rules, unless those rules are designed to protect the patient's health and safety (e.g., refusing to give a patient a certain medication that is contraindicated for medical reasons, requiring cessation of certain medications or behaviors prior to a surgical procedure).

\footnotetext{
* Those reporting the greatest concern about the limited treatment options in their communities included respondents with incomes below $\$ 50,000$ (52 percent), blacks (67 percent), those who knew someone with addiction (58 percent) and those who did not have health insurance (56 percent).
} 
Patients who do meet eligibility criteria and are ready to enter treatment often face a long wait for services--according to some estimates, up to 70 days $^{152}$--due to limited treatment availability. ${ }^{153}$ This barrier can undermine an individual's fragile resolve to enter treatment. ${ }^{154}$ Long waiting times may bias treatment entry in favor of those most likely to succeed with treatment, ${ }^{155}$ as a longer wait time to enter a program is associated with pretreatment attrition. ${ }^{156}$ One study found that the longer patients have to wait between clinical assessment and the first treatment session, the less likely they are to complete subsequent treatment sessions. ${ }^{157}$ CASA Columbia's survey of treatment providers in New York State found that a significant proportion of the respondents said that long waiting lists "somewhat" (40.2 percent of program directors and 49.3 percent of staff providers) or "very much" (22.0 percent of program directors and 21.0 percent of staff providers) stand in the way of people accessing needed addiction treatment. ${ }^{158}$

\section{Insufficient Social Support}

A lack of social support can serve as a barrier to treatment access. Individuals with addiction may be discouraged openly by family or friends from entering treatment or may have more subtle fears that loved ones will think badly of them or will be embarrassed or ashamed if they enter treatment. ${ }^{159}$ Even among those who may otherwise seek treatment, continued substance use in an addicted individual's family or social network can increase the risk of continued use, reduce the likelihood of treatment entry and derail treatment efforts. ${ }^{160}$

\section{Conflicting Time Commitments}

Competing responsibilities and time commitments related to one's family and career are common reasons for not seeking treatment. ${ }^{161}$ One study found that the opportunity cost of a person's time--including both time spent traveling to non-residential treatment programs and time spent in treatment that otherwise would be spent on work or leisure activities--is one of the main barriers to seeking treatment. $^{162}$

\section{Negative Perceptions of the Treatment Process}

Among those with addiction who accept that they need to treat their disease are those who believe they can manage it themselves, without professional assistance. ${ }^{163}$ Such a belief may derive from a misperception of what symptoms and what level of symptom severity constitute the disease of addiction and require professional assistance, or it may derive from the belief that treatment simply is not effective and will not help. ${ }^{164}$ Some of this concern may be warranted given the nature of the services offered. (See Chapter X.)

Twenty percent of respondents to the NABAS think that a main reason why people with substance-related problems do not get the help they need is that they do not believe that treatment would help. ${ }^{165}$ Treatment providers see this as a barrier to treatment access as well: CASA Columbia's survey of treatment providers in New York State found that a significant proportion of the respondents said that the belief that treatment does not work "somewhat" (63.9 percent of program directors and 59.0 percent of staff providers) or "very much" (32.5 percent of program directors and 31.7 percent of staff providers) stands in the way of people looking for needed addiction treatment. ${ }^{166}$

Some individuals with addiction have negative perceptions or a fear of treatment providers and programs that may keep them from seeking and accessing treatment. ${ }^{167}$ These perceptions can be based on an individual's prior negative experience in treatment, anticipation that the treatment process will be unpleasant, assumptions about the limited efficacy of treatment or a general fear of what might happen in treatment. ${ }^{168}$

With regard to smoking cessation, there is a prevalent belief among smokers that certain interventions, particularly nicotine replacement therapy (NRT), may not be safe. ${ }^{169}$ One study found that 66.0 percent of current and former smokers expressed concerns about the safety of NRT; those expressing concern were less likely 
to use NRT in a cessation attempt (30 percent vs. 49 percent). ${ }^{170}$

\section{Legal Barriers}

Unlike other chronic health conditions, addiction involving illicit drugs, by definition, marks a person as having engaged in illegal activity. Treatment providers see this as a barrier to treatment access: CASA Columbia's survey of treatment providers in New York State found that a significant proportion of the respondents said that fear of being sent to prison or jail "somewhat" (41.0 percent of program directors and 42.8 percent of staff providers) or "very much" (42.2 percent of program directors and 37.0 percent of staff providers) stands in the way of people looking for needed addiction treatment. ${ }^{171}$

\section{Barriers to Treatment Access and Completion in Special Populations}

The barriers to treatment outlined above apply to most individuals with addiction; however, certain populations face additional barriers that exacerbate the difficulty of accessing needed treatment. $^{*}$

\section{Individuals with Co-occurring Conditions}

An estimated 20 percent of the U.S. population has a disability that limits their functioning. ${ }^{\dagger} 172$

\footnotetext{
* The special populations discussed in this section do not necessarily mirror those discussed in Chapter VI since not all populations that require specialized screening or treatment protocols have barriers to treatment access that surpass or differ from those of the general population (e.g., individuals involved in the justice system). Likewise, not all special populations that have additional or unique barriers to treatment access necessarily require specialized screening or treatment protocols (e.g., rural populations).

${ }^{\dagger}$ Including individuals with sensory disabilities involving sight or hearing; physical disabilities that limit basic physical activities such as walking, lifting, carrying; mental disabilities that involve difficulty in learning, remembering or concentrating; or disabilities that impede self-care such as dressing, bathing or getting around.
}

People with disabilities use addictive substances at nearly twice the rate of the general population. ${ }^{173}$ While individuals with cooccurring addiction and mental health disorders such as anxiety and depression access treatment at higher rates than individuals in the general population (although most treatment facilities do not provide services tailored to this population), ${ }^{174}$ those with other disabilities that impede functioning access treatment services at substantially lower rates than the general population. ${ }^{175}$

Many health and social service professionals fail to identify the presence of risky substance use or addiction in people with disabilities, ${ }^{176}$ despite their high rate of substance use. ${ }^{177}$ Many barriers stand in the way of treatment for people with disabilities, such as erroneous attitudes or beliefs of medical providers, ${ }^{\ddagger}$ lack of staff training in how to work with disabled people and treatment methods and materials that are not tailored to the needs of people with disabilities. ${ }^{178}$ People with disabilities who have addiction also may be deterred by accommodation barriers to treatment, such as lack of personal or public transportation to a treatment center ${ }^{179}$ and facilities that do not have adequate accommodations, such as restrooms, parking facilities, hallways and ramps that are accessible to patients with mobility impairments. ${ }^{180}$

Treatment personnel often do not have the proper training to meet the physical and other health care needs of patients with disabilities, such as knowledge of sign language or Braille. ${ }^{181}$ In 2009, 27.7 percent of facilities offered services in sign language for the hearing impaired. ${ }^{182}$

\footnotetext{
${ }^{\ddagger}$ e.g., believing that people with disabilities deserve pity so they should be allowed more latitude to engage in substance use.
} 


\section{Pregnant and Parenting Women}

Barriers to treatment access and completion can be particularly acute for women who are pregnant or post-partum, despite the fact that pregnancy may provide substance-using women with the motivation they need to reduce their substance use or seek treatment. ${ }^{183}$ In 2010, only 15.9 percent of facilities nationwide that provided addiction treatment services offered services specific to pregnant or post-partum women. ${ }^{184}$

Limited availability of child care for parenting women in addiction treatment is a significant barrier to treatment entry and retention. National data indicate that only 6.5 percent of outpatient addiction treatment facilities that serve women offer child care, although facilities that served women only were significantly likelier than facilities serving both men and women to offer child care services. ${ }^{185}$

Some pregnant smokers report reluctance to quit smoking due to fear of weight gain, ${ }^{186}$ not believing in the harmful effects of smoking to themselves or their fetus due to prior pregnancies with no observable harm and a social environment where smoking is prevalent. ${ }^{187}$

Some women also fear being branded as "bad mothers" by treatment personnel. ${ }^{188}$ One study found that more than one-third of pregnant smokers reported being apprehensive about attending smoking cessation counseling because they believed the counselor would judge them harshly. ${ }^{189}$

Pregnant and parenting women might shy away from accessing treatment for addiction involving alcohol or other drugs (excluding nicotine) for fear that entering treatment may result in losing custody of their children; ${ }^{190}$ they may be apprehensive of the involvement of child protective services if they were to be identified as having addiction. ${ }^{191}$ CASA Columbia's survey of treatment providers in New York State found that a significant proportion of the respondents said that fear of losing child custody "somewhat" (25.3 percent of program directors and 37.0 percent of staff providers) or "very much" (73.5 percent of program directors and 58.7 percent of staff providers) stands in the way of people looking for needed addiction treatment. $^{192}$

Substance use during pregnancy is considered a form of child abuse under civil child-welfare statutes in 15 states and is considered grounds for civil commitment (i.e., forced admission into a treatment program) in three states. ${ }^{193}$ Pregnant substance users have been charged with such crimes as fetal abuse, child abuse and neglect, delivering drugs to a minor, corruption of a minor, assault with a deadly weapon and manslaughter. ${ }^{194}$ Some states have used prenatal substance use as grounds to terminate parental rights. ${ }^{195}$

\section{Adolescents}

Few diseases affecting adolescents are as extensively under-treated as addiction, even though addiction is a disease with firm roots in adolescence. ${ }^{196}$ The significant treatment gap in the adolescent population--which is particularly acute among black and Hispanic youth ${ }^{197}$--is due in large part to the failure to understand the developmental nature of addiction and the failure of health care professionals to look for and prevent risky substance use or to identify and address addiction in their adolescent patient populations. ${ }^{198}$

The Society for Adolescent Health and Medicine has called for greater access for adolescents and young adults to health care through nontraditional health care providers such as school health centers, community health centers and other public health agencies that rely primarily on public funding, and expanded insurance coverage. ${ }^{199}$ Unfortunately, systems responsible for the welfare of young people-schools, juvenile justice, child welfare-- too often miss opportunities to intervene with young people in need of treatment and continue to allow them to fall through the cracks undetected and unaided. ${ }^{200}$

Treatment models with a strong evidence base in adult populations are not necessarily applicable 
to the treatment needs of adolescents with addiction. ${ }^{201}$ Yet, effective evidence-based treatment approaches for adolescents do exist. ${ }^{202}$ Despite this, in 2010, only 28.8 percent of facilities nationwide that provided addiction treatment services offered adolescent-specific services. ${ }^{203}$

Barriers to treatment for adolescents include lack of support among parents and school personnel and lack of interest on the part of adolescents in participating in treatment. ${ }^{204}$ These barriers may result in difficulty recruiting adolescents to participate in treatment. ${ }^{205}$ One national survey found that adolescents frame their reasons for not wanting to participate in treatment as they "are not ready to stop substance use," "didn't want others to find out" and because they "could handle the problem without treatment"--barriers that are similar to those offered by the general population and are at least in part reflections of the disease itself and of the stigma attached to it. ${ }^{206}$ Other barriers include insufficient research on the safety and efficacy of evidence-based addiction treatments for use in adolescent populations, particularly pharmaceutical therapies. ${ }^{207}$

\section{Older Adults}

Older adults are less likely than younger people to be identified as having addiction or to be referred to treatment; ${ }^{208}$ those who do try to access treatment often have difficulty finding age-appropriate treatment services. ${ }^{209}$ In 2010, only 6.9 percent of facilities nationwide that provided addiction treatment services offered services specific to older adults. ${ }^{210}$

Older smokers, for example, may be less aware of the harmful consequences of tobacco use and may focus more on the perceived benefits, such as its use as an aid in coping with stress or controlling weight. ${ }^{211}$ Some may feel that it is "too late" to reverse the effects of smoking and therefore may not be motivated to seek out smoking cessation services; this belief is reflected in the failure of many health care providers to counsel older adult patients to stop smoking and support them through a cessation attempt. $^{212}$ Some physicians may have concerns about the safety of prescribing pharmaceutical interventions for smoking cessation to older patients. ${ }^{213}$

Adults who develop late onset addiction involving alcohol are less likely than those with early onset addiction to have a family history of addiction (21 percent vs. 72 percent) ${ }^{*}$ and are more likely to try to hide their illness; further, the symptoms associated with addiction involving alcohol--such as disorientation or confusion--may be misinterpreted as cognitive or physical deficits such as dementia, depression or other medical problems common in the elderly. ${ }^{214}$

CASA Columbia's 1998 report, Under the Rug: Substance Abuse and the Mature Woman, found that when physicians were asked for five possible diagnoses of a hypothetical 68-year-old female patient with an array of complaints typical of risky use of alcohol or prescription drugs, only one percent identified a substance use problem as a likely diagnosis. Contrary to the evidence, only 62 percent of physicians reported believing that addiction treatment is somewhat or very effective for older women. ${ }^{215}$

\section{The Homeless}

Mental illness and co-occurring addiction are highly prevalent in the chronically homeless population. ${ }^{216}$ Addiction, like other health problems in the homeless population, too often goes unaddressed until it is severe enough to require costly urgent care and hospitalization, resulting in a great deal of unnecessary human suffering and a serious, yet avoidable burden on health care systems. ${ }^{217}$

Seeking addiction treatment may be a low priority for homeless individuals who must contend with the competing needs of securing food, clothing and shelter and who rarely have a network of social support to help them access and succeed in treatment. ${ }^{218}$ Given homeless individuals' lack of resources, their ability to find appropriate treatment programs and pay for

\footnotetext{
* In a study of men ages 60 and older admitted to a VA geriatric outpatient treatment program.
} 
services can be extremely limited. ${ }^{219}$ However, even with greater motivation and better access to treatment, the environment in which many homeless people spend their time--with high rates of ambient substance use, crime and violence--may present additional barriers to seeking and receiving treatment. ${ }^{220}$

Lack of collaboration between social service providers, public health systems and addiction treatment programs is another significant obstacle to addressing addiction in the homeless population, resulting in a missed opportunity to increase access to treatment. ${ }^{221}$

Homeless individuals, who frequently have cooccurring addiction and mental health disorders, often are rejected by community-based residential programs since housing programs for the mentally ill frequently exclude substance users, and those for substance users often exclude those with serious mental illnesses. ${ }^{222}$ One study found that 60 percent of homeless people who admitted to having addiction reported that they were not eligible for addiction treatment or subsidized housing. Forty-two percent of those who did receive treatment reported that their treatment was ineffective because of a lack of aftercare and housing services. ${ }^{223}$

Another study found that receipt of public insurance was the strongest predictor of access to treatment among homeless people relative to other predictors. Jail stays, emergency department visits and non-residential medical care visits were not associated with increased access to treatment, suggesting that referral links between these services and addiction treatment are not adequate. ${ }^{224}$

\section{Veterans and Active Duty Military}

According to the U.S. Department of Defense's Task Force on Mental Health, service members may be concerned that their substance-related problems might impede their career advancement, which can lead them to avoid seeking timely care. ${ }^{225}$ Soldiers may be reluctant to seek treatment for addiction because self-referrals can be reported to their superiors; the military has an established policy of reporting mental health and substance-related problems to superiors. ${ }^{226}$ The use of illicit drugs or the misuse of controlled prescription drugs can be grounds for dishonorable discharge. ${ }^{227}$

Any referral for additional mental health care in a military treatment facility must be documented in an individual's personal record which can deter people from seeking such treatment. ${ }^{228}$ Data from 2007 show that since military operations began in Iraq, army commanders have dismissed more than twice as many soldiers for drug use ${ }^{*}$ than they did in the same period before the war. ${ }^{229}$ Drug use is categorized as a form of "misconduct," which discontinues some or all military benefits. ${ }^{230}$

Another barrier to accessing treatment for veterans is the long wait time for initial appointments or between appointments. ${ }^{231}$ Veterans with co-occurring health problems also face barriers to treatment, including the practice of requiring individuals to be substance free prior to entering treatment for other co-occurring conditions that are common among military personnel, such as post-traumatic stress disorder (PTSD). ${ }^{232}$

More generally, there is a significant shortage of medical and mental health professionals to address the complex medical and psychological treatment needs of individuals returning from military combat, as well as those of their family members. ${ }^{233}$

\section{Rural Populations}

Rural populations face a considerable gap in the receipt of needed addiction treatment. ${ }^{234}$

Limited accessibility to treatment services as a function of geographic location presents a significant obstacle to treatment access for people living in rural areas ${ }^{235}$ since general medical and specialty treatment services typically are located in urban centers. ${ }^{236}$ Only 8.9 percent of all addiction treatment facilities are located in a rural county that is not adjacent

\footnotetext{
* The use of illicit drugs or the misuse of controlled prescription drugs.
} 
to an urban county. ${ }^{237}$ In 2010, 8.9 million rural residents had no access to inter-city public transportation, ${ }^{238}$ posing a significant barrier to treatment access.

Rural residents tend to have lower incomes and are less likely than non-rural residents to have health insurance, which limits their ability to afford and pay for treatment. ${ }^{239}$ And since rural residents are more likely than urban residents to be self-employed, they have fewer encounters with employee assistance programs. ${ }^{240}$ For these reasons, rural residents who engage in risky substance use or have other health problems tend to delay seeking preventive care, resulting in the need for more costly care in the future. ${ }^{241}$

\section{Native Americans}

National data on racial/ethnic differences in the addiction treatment gap are limited with regard to Native Americans due to small sample sizes for this population. ${ }^{242}$ However, existing data suggest that Native Americans are the likeliest of all racial/ethnic groups to smoke and to meet clinical criteria for addiction involving alcohol and other drugs. ${ }^{243}$ National data also suggest that the group with the largest treatment gap is Native Americans. ${ }^{244}$ One estimate indicates that less than one-fifth of addiction treatment programs nationally offer specialty services for Native Americans. ${ }^{245}$ 


\section{Chapter VIII The Spending Gap}

Despite the evidence that risky substance use can be identified and reduced through screening and early intervention, and that addiction can be treated and managed effectively with psychosocial and pharmaceutical interventions, ${ }^{*}$ financial investments in addressing risky substance use and addiction are aimed disproportionately at coping with the costly health and social consequences of the disease rather than at prevention and treatment. This spending gap impairs health and imposes extraordinary and unnecessary costs to taxpayers. The continued inadequacy of insurance coverage for these services further flies in the face of a fiscally-sound approach to disease prevention, treatment and management.

\section{The Rational Approach to Risky Substance Use and Addiction}

The goals of medicine are the prevention of disease, the diagnosis and treatment of illness or injury and the relief of pain and suffering. ${ }^{1}$ The general standard for determining what health care services should be provided to patients is the "reasonable and necessary" 2 or the "medically necessary" standard. ${ }^{3}$ The definition of what is considered necessary generally is made by health care payers based on the strength of the clinical evidence supporting the effectiveness of interventions in improving health outcomes. ${ }^{4}$ In the Medicare and Medicaid programs, medical necessity is defined in various ways but generally as the prevention, diagnosis or treatment of illness or injury that endangers life, causes suffering or pain, causes physical deformity or malfunction or results in illness or infirmity. ${ }^{5}$ Some states also require that Medicaid services not be more costly than reasonable available alternatives. ${ }^{6}$

\footnotetext{
${ }^{*}$ See Chapters IV and V.
} 
The American Medical Association (AMA) supports the ideal that all patients have "a basic right to available, adequate health care” regardless of the ability to pay. ${ }^{7}$ This ideal is based on several arguments which assert a moral obligation to treat injuries or diseases that impede normal functioning. ${ }^{*} 8$

Addiction is not unique as a health condition for which a lack of understanding of the nature of the disease and its causes has resulted in assigning blame to the patient and to inadequate or misguided interventions; other historical examples include tuberculosis, cancer, depression and HIV-AIDS. ${ }^{9}$ However, once a body of evidence exists about the nature of an illness and how to address it, that information is incorporated into medical practice and reimbursement policies based on the obligation of the profession to treat disease. Addiction is a glaring example of practice lagging behind the science. ${ }^{10}$ The science is unambiguous-addiction is a complex brain disease with significant behavioral characteristics ${ }^{11}$ that results in unhealthy compulsive behaviors, ${ }^{12}$ diminished cognitive control, ${ }^{13}$ clinicallysignificant impairment or distress ${ }^{14}$ and that can lead to long-term disability and death. ${ }^{15}$ Our continued failure to prevent and treat the disease is inconsistent with ethical standards and the goals of medical practice.

\footnotetext{
${ }^{*}$ The collective social protection argument posits that certain essential services, including safety and health care, are a "collective responsibility" of society and should be provided to all. The principle of fair equality of opportunity calls upon institutions to provide individuals with basic services needed to pursue the normal range of opportunities that are essential to a good life--to cultivate one's talents, develop skills and formulate one's own life goals.
}

\section{Costs of Our Failure to Prevent and Treat Addiction as a Medical Condition}

Risky substance use and addiction constitute the leading cause of death and disability in the United States. ${ }^{16}$ The result of not providing effective prevention and treatment services for addiction is that the cost of addiction accrues, driving many other diseases, later manifesting as more expensive care and spilling out to costly social consequences. ${ }^{\dagger}$

Looking just at government spending, CASA Columbia calculated that in 2005, ${ }^{\ddagger}$ risky substance use- and addiction-related spending accounted for 10.7 percent of federal, state and local spending, and that for every dollar federal and state governments spent, 95.6 cents went to pay for the consequences of substance use; only 1.9 cents was spent on any type of prevention or treatment. ${ }^{\S}$ The taxpayer tab for government spending on the consequences of risky substance use and addiction alone totals $\$ 467.7$ billion a year, almost $\$ 1,500$ a year for every person in America. ${ }^{17}$

\section{The Largest Share of Costs Falls to the Health Care System}

The largest share of spending on the consequences of risky substance use and addiction is in health care. ${ }^{18}$ Persons with addictive diseases are among the highest-cost health care users in America: ${ }^{19}$ they have higher utilization rates, more frequent hospital admissions, longer hospital stays and require more expensive health care services. ${ }^{20}$ Nearly a third (32.3 percent) of all hospital inpatient costs is attributable to tobacco, alcohol and other drug use and addiction. ${ }^{21}$

Even family members of individuals with addiction have approximately 30 percent greater

\footnotetext{
${ }^{\dagger}$ See Chapter III.

${ }^{\ddagger}$ Most recent data available.

$\S$ In addition, 0.4 cents was spent on research, 1.4 cents on taxation or regulation and 0.7 cents on interdiction.
} 
health care costs compared to families with similar demographic characteristics who do not have a member with an addiction diagnosis. ${ }^{22}$

The health care costs associated with addiction also stem from the impact that addiction has on the ability to treat other diseases. Addiction affects the body in ways that complicate health care, for example, by weakening the immune system. ${ }^{23}$ These costs, however, rarely are recognized. One study found that from 1994 to 2002, admissions of patients with addiction increased 46 percent but hospital costs increased 134 percent; because only one percent of these patients had a primary diagnosis of addiction, the rise in costs was attributed to treating the cooccurring medical illness (i.e., the primary diagnosis). ${ }^{24}$

In 2010 , only $\$ 28.0$ billion (1.0 percent) of total health care costs ${ }^{*}$ was spent on addiction treatment-related services involving alcohol or drugs other than nicotine. Approximately $\$ 13.0$ billion was spent on treatment-related services involving alcohol and $\$ 15.0$ billion on treatment-related services involving controlled prescription or illicit drugs. $^{\dagger 25}$ Total costs of treatment for addiction involving nicotine are unknown.

In 2010, the U.S. spent $\$ 43.8$ billion to treat diabetes ${ }^{26}$ which affects 25.8 million people, ${ }^{27}$ $\$ 86.6$ billion to treat cancer ${ }^{28}$ which affects 19.4 million people $\mathrm{e}^{29}$ and an estimated $\$ 107.0$ billion to treat heart conditions ${ }^{30}$ which affect 27.0 million people, ${ }^{31}$ but only $\$ 28.0$ billion to treat addiction $^{\ddagger 32}$ which affects 40.3 million people. $^{\S 33}$

\footnotetext{
*Including medical, mental health and direct treatment costs.

${ }^{\dagger}$ Treatment-related services include: detoxification (which is not considered treatment) and diagnostic and treatment services provided in inpatient settings (usually a hospital), outpatient/ambulatory settings (such as in a hospital outpatient department or emergency department and in physicians' and other medical professionals' offices and clinics) and residential settings (24-hour care).

₹ There are no national data that document spending on treatment for addiction involving nicotine; although the cost estimate of $\$ 28.0$ billion applies to
}

\section{Cost Savings of Addiction Screening, Intervention and Treatment}

There are no national data available on total health care spending for screening or intervention services; ${ }^{* *} 34$ therefore, data on cost savings from these services and from addiction treatment come from individual studies rather than national data sets.

In an effort to increase resources directed to screening, intervention and treatment, much attention has been focused on highlighting the cost effectiveness of these services. ${ }^{35}$ While cost effectiveness certainly is an important component of resource allocation and targeting, this standard as applied to addiction is a stark reminder of the stigma attached to the disease. ${ }^{36}$ The United States does not use cost effectiveness as a measure to determine which health care treatments should be covered; in fact,

the treatment of addiction involving alcohol and other drugs excluding nicotine, the prevalence estimate of those with addiction (40.3 million) includes those with addiction involving nicotine.

${ }^{\S}$ Due to data limitations, the prevalence estimates for cancer and heart conditions include individuals ages 18 and older who have ever been told by a doctor or other health professional that they have the condition (cancer/malignancy or a heart condition). The prevalence estimate for diabetes includes all ages and the estimate for addiction includes individuals ages 12 and older; for diabetes and addiction, the prevalence estimates include both diagnosed and undiagnosed cases. In each case, total costs of treatment are included without regard to age. The cost estimates for treating diabetes, cancer and heart conditions were inflated to 2010 dollars using the medical inflation factor (7.9 percent) found in the Substance Abuse and Mental Health Services Administration's (SAMHSA) National Expenditures for Mental Health Services and Substance Abuse Treatment, 1986-2005 publication.

${ }^{* *}$ The 2010 National Drug Control Strategy reports on spending in one grant program through SAMHSA, which spent \$29.1 million in grants to eight states to provide screening, brief intervention and referral to treatment services in general medical settings, and to 11 grant recipients to include training in these services in medical residency training programs. 
almost no other disease is held to this standard. ${ }^{37}$ However, if cost-effectiveness were an essential factor in determining which medical services to fund, then screening, interventions and treatment for addiction certainly would be considered a good investment.

One study found that the annual benefits in avoided health care spending (i.e., cost-saving) per person achieved through substance-related interventions in 2001 dollars ranged from \$634 for brief physician interventions for risky drinkers to $\$ 3,951$ for standard residential addiction treatment; the average annual savings per person was $\$ 1,939$ across all interventions. ${ }^{38}$

The projected cost to insurers of offering comprehensive addiction treatment benefits also is modest. According to a 1999 study, the cost of providing managed, ${ }^{*}$ comprehensive ${ }^{\dagger}$ addiction treatment benefits with low copayments $^{\ddagger}$ and no annual limits was $\$ 5.11$ per member in 1996-1997. ${ }^{39}$ Adding managed, unlimited addiction treatment benefits to a plan that previously did not offer addiction treatment benefits would increase costs ${ }^{\S}$ only by an estimated 0.3 percent. $^{* *} 40$ In 2001, the Congressional Budget Office estimated that mandating parity for mental health and addiction treatment benefits would increase group health insurance premiums by 0.9 percent initially and by 0.4 percent in total after accounting for the market-driven responses of health plans, employers and workers to the higher premiums. $^{\dagger+41}$ Studies of the effect of mandated parity in Federal Employee Health Benefit Plans have concluded that total plan spending per

\footnotetext{
* Benefits carved out and provided by a large managed behavioral health care organization.

${ }^{\dagger}$ Including outpatient, intensive outpatient, inpatient and residential treatment.

\$10 or less.

$\S$ Costs include payments to providers; administrative fees and profits are not included.

** Assuming annual Health Maintenance Organization (HMO) insurance premiums of $\$ 1,500$ per member.

${ }^{\dagger \dagger}$ Market-driven responses include: reductions in employers offering and employees enrolling in employer-sponsored insurance, changes in the types of plan offerings and reductions in scope of benefits.
}

member did not increase significantly while out of pocket expenses for those who received treatment benefits declined. ${ }^{42}$

Because cost-effectiveness research to date has for the most part focused on the cost savings of providing a particular service within a particular population, it is not yet possible to generate an overall estimate of the potential cost savings of screening all patients for all forms of risky substance use and providing appropriate interventions, or for assessing the need for treatment and providing these services.

However, as the following examples reveal, the opportunity for cost savings is substantial.

\section{Screening and Early Intervention}

Cost-benefit studies of screening and brief interventions for tobacco and alcohol use among adults and pregnant women have demonstrated a range of potential costs savings. ${ }^{43}$ Numerous studies have demonstrated that medical costs for patients with addiction increase significantly as these patients age, ${ }^{44}$ implying that the greatest cost savings can be achieved by early intervention and treatment. ${ }^{\S \S 45}$ In the health care field, treatment costs of up to $\$ 50,000$ for each year of life saved are considered to be a worthwhile investment in health (i.e., cost effective); in specialty care, such as cancer, treatment costs of up to $\$ 200,000$ may be considered cost effective. ${ }^{46}$

Smoking. Smoking cessation programs yield positive health outcomes at the low cost of $\$ 5,000$ per healthy year gained ${ }^{* * *} 47$ compared to $\$ 56,200$ per year for Aspirin and statin therapy

\footnotetext{
㧊 Research is presented related to screening and interventions for smoking and risky alcohol use. Comparable research related to other drug use is not available.

$\S \S$ Cost-benefit studies calculate the total cost savings that result from providing treatment (sometimes called return on investment); whereas costeffectiveness studies determine the treatment costs of extending a patient's life by one year, or per qualityadjusted life year (QALY), a year of perfect health. ${ }^{* * *}$ Cost effectiveness as measured by costs minus savings for each year of healthy life attributable to the intervention.
} 
to prevent coronary heart disease, ${ }^{*} 48$ \$191,635 per year for diet/exercise to prevent diabetes in high-risk adults ${ }^{\dagger}$ and $\$ 30,619$ per year for biennial mammograms to screen for cancer among women ages 50 to $79 .{ }^{49}$

Screening pregnant women who smoke can be especially cost effective, given that the smokingattributable medical care needed by infants whose mothers smoked while pregnant is an estimated \$367 million per year. ${ }^{\ddagger 50}$ A onepercent reduction in the prevalence of smoking in the U.S. population, including among pregnant women, would reduce the number of low-birth weight births by $2,000,{ }^{\S}$ resulting in $\$ 21$ million in avoided direct medical costs. Continuing at that rate for another six years, more than 57,000 low-birth weight births could be prevented, saving $\$ 572$ million. ${ }^{51}$

The American Legacy Foundation projected that a reduction in Medicaid costs of nearly one billion dollars ${ }^{* *}$ could be achieved by preventing the current cohort of 24-year-olds ${ }^{\dagger \dagger}$ from smoking. If prevention and cessation efforts were successful in motivating all Medicaid recipients who smoke to quit, states' Medicaid expenditures would be, on average, 5.6 percent lower, resulting in a total of $\$ 9.7$ billion in savings after five years. ${ }^{52}$

Risky Alcohol Use. Screening and brief intervention for risky alcohol use rank among the top most cost-effective prevention services available, ${ }^{53}$ along with colorectal cancer

\footnotetext{
* For 45-year old men with a 10 -year risk for coronary heart disease of 7.5 percent. The calculation includes the cost of medication plus medical care including care for adverse events (e.g., Aspirin-induced gastrointestinal bleeding and resulting morbidity and mortality).

${ }^{\dagger}$ Costs include individually-tailored diet and exercise plans, visits to a nutritionist and physical training sessions.

‡ In 1996 dollars.

$\S$ Based on 1995 birth rates.

** Over the cohort's lifetime.

${ }^{\dagger+}$ The researchers chose this age because nearly all smokers begin smoking before age 24, whereas younger smokers may still be experimenting with tobacco.
}

screening, hypertension screening and influenza immunization. $^{54}$

Research findings suggest that early interventions ${ }^{\ddagger \ddagger}$ for risky alcohol use may result in health care cost savings of up to $\$ 43,000$ for every $\$ 10,000$ invested. ${ }^{55}$ A study of primary care screening and brief physician intervention for adult risky drinkers yielded a net benefit of $\$ 947$ per person. $^{56}$

The use of screening and brief interventions in hospitals has demonstrated promising returns on investment. ${ }^{57}$ A study of screening and brief interventions for risky alcohol use among adults in trauma centers estimated that over a threeyear period, the cost savings associated with screening were $\$ 89$ per patient ${ }^{\S \S}$ and the cost savings associated with brief interventions lasting 30 minutes were $\$ 330$ per patient. ${ }^{* * *}$ In total, the implementation of a hospital-based alcohol screening and brief intervention program for risky alcohol use was estimated to reduce health care costs by $\$ 3.81$ for every dollar spent. $^{58}$ Brief interventions ${ }^{\dagger \dagger \dagger}$ with adolescents ages 18 and 19 who were admitted to a trauma center for alcohol-related injuries also have been found to be more cost-effective than standard care. $^{59}$

The return on investment in preventing Fetal Alcohol Syndrome (FAS) further underscores the importance of screening and early interventions. The added medical costs for a child with FAS are estimated to be more than $\$ 2,300$ per year for the first 21 years of a child's life. An alcohol intervention program costing $\$ 50,000$ that could successfully prevent at least one case of FAS annually would pay for itself in just six years. ${ }^{60}$

\footnotetext{
㧊 Consisting of two doctor visits and two nurse follow-up calls.

$\S \S$ Cost per screening was $\$ 16$.

${ }^{* * *}$ Cost per intervention was $\$ 38$. Savings were calculated based on average hospitalization and emergency department costs; hospital recidivism rates for trauma patients with and without addiction involving alcohol; and the efficacy rate of screening, brief interventions and referrals to treatment at reducing injury, recidivism and hospital readmission. ${ }^{\dagger+\dagger}$ Using motivational interviewing.
} 


\section{Addiction Treatment and Disease Management}

Persons with untreated addiction have higher health care utilization rates and more frequent hospital stays, generating billions of dollars in largely avoidable health care charges. ${ }^{61}$ Addiction treatment, particularly evidence-based treatment, not only reduces health care costs in both the short and long term, it saves lives and reduces other social consequences and costs as well. ${ }^{*} 62$ Some research suggests that treatment "pays for itself," often on the day it is delivered and the total cost savings from addiction treatment continue to accrue over time. ${ }^{63}$

While the total economic benefits of treatment are greater than the cost of treatment, ${ }^{64}$ administrators and policymakers too often disregard benefits of treatment that accrue beyond the narrow silo of each individual government program. For example, health care payers may refuse to acknowledge the significant cost savings that may accrue outside the health care system (increased productivity, reduced crime, etc.). An example of this can be seen in an analysis of data from the VA health care system from 1998 to 2006 showing that providing addiction treatment resulted in an overall increase in health care costs, presumably because expanding access to treatment brought more sick patients into the health care system and more of their co-occurring medical conditions subsequently were identified and treated. The one exception was opioid maintenance therapy which paid for itself in health care savings. This study did not account for potential longer-term reductions in health care costs or for potential cost savings in other sectors because it was taking the perspective of an insurance payer--via the VA health care system $^{65}$--and this perspective tends to look at short-term costs rather than long-term savings.

Most studies--even those that look only within a particular system for costs and benefits--find immediate and longer-term savings associated with addiction treatment:

\footnotetext{
* The referenced studies do not include detailed examples of the nature of the treatment provided.
}

- A longitudinal study of patients treated for addiction in Kaiser Permanente's Medical Care Program found an average reduction of 30 percent in medical costs three years posttreatment. Significant declines were seen in areas such as the number of inpatient hospital days and emergency department visits, which are high-cost services. ${ }^{66}$

- A comparison of adult patients who met clinical criteria for addiction involving alcohol or drugs other than nicotine who were enrolled in an outpatient treatment program with a control group $^{\dagger}$ found that those enrolled in the treatment program were less likely to be hospitalized 18 months after treatment than before treatment. The study also found that treatment can cut health care costs associated with addiction by about one quarter, primarily by reducing the number of annual hospital stays and the likelihood of emergency room visits. ${ }^{67}$

- An analysis of data from patients in treatment for addiction involving alcohol or drugs other than nicotine in California found a benefit-cost ratio of more than seven to one: ${ }^{\ddagger}$ the average cost of treatment was $\$ 1,583$ and the benefits ${ }^{\S}$ were $\$ 11,487$. Most of the savings were attributed to reduced crime and increased employment. ${ }^{68}$

- An analysis of statewide data from Washington State found that treatment was associated with an annual \$2,500 reduction in medical expenses ${ }^{* *}$ among adult patients

\footnotetext{
${ }^{\dagger}$ Adults who met criteria for addiction involving alcohol or other drugs but did not receive treatment. ${ }^{\ddagger}$ Nine months after treatment.

$\S$ Including earnings from employment and reductions in the costs related to emergency department visits, incarceration and crime.

${ }^{* *}$ Including inpatient and outpatient hospital care, physicians' services, prescription drugs and nursing home care. Most of the reductions in medical spending were within Medicaid expenditures.
} 
with addiction ${ }^{*}$ receiving general assistance welfare. $^{69}$ A more recent analysis in Washington State of the return on investment from an increase in addiction treatment for disabled adults enrolled in Medicaid or medical coverage through a general assistance welfare program between 2006 and 2009 found a savings of \$2.07 in medical and skilled nursing facility expenses for every dollar spent on treatment ${ }^{\dagger}$ over the four-year period. ${ }^{70}$

- A performance audit of the costs and savings to the Colorado Medicaid Program-which in 2006, implemented a benefit to provide outpatient addiction treatment for services related to tobacco, alcohol and other drugs for all Medicaid beneficiaries-found that the program cost \$2.4 million over the course of three years while medical costs for patients receiving services under this program declined by approximately $\$ 3.5$ million. ${ }^{\text {71 }}$

- A simulation of potential cost savings in the justice system demonstrated that treating all arrestees who are at risk of addiction would cost $\$ 13.7$ billion and save more than $\$ 46$ billion (for every dollar spent on treatment, more than three dollars in benefits accrue). Treating all arrestees who are "probably guilty"§ and who are at risk of addiction would result in a reduction in recidivism rates in the range of 16 to 34 percent, depending on the modality of treatment (with long-term residential treatment yielding the greatest reduction in recidivism, roughly 27 to 34 percent). ${ }^{72}$

\footnotetext{
* Measured as receiving a clinical diagnosis of alcohol or other drug dependence or psychosis, receiving detoxification services or having been referred for alcohol or other drug assessment by the state division of alcohol and substance abuse.

${ }^{\dagger}$ In this study, treatment included outpatient, residential and opioid maintenance therapy and case management.

‡ Analysis based on available Medicaid claims data, not a controlled longitudinal study.

$\S$ As phrased by the authors of the study who state that an admission of guilt generally is required for enrollment in court-monitored treatment.
}

Addiction Involving Nicotine. One study found that 24.6 percent of adult depressed smokers who received six sessions of mental health counseling and up to 10 weeks of nicotine replacement therapy (NRT) with the patch were abstinent from smoking after 18 months; the total cost of treatment (smoking cessation services plus mental health care) was $\$ 9,580$ per life year*** gained. $^{73}$

Following the implementation of Medicaidcovered pharmaceutical therapy for addiction involving nicotine, Massachusetts had a 46 percent annual decrease in hospitalizations for heart attacks and a 49 percent annual decrease in cases of coronary atherosclerosis. ${ }^{\dagger \dagger 74}$

Addiction Involving Alcohol. For individuals with addiction involving alcohol, a number of pharmaceutical interventions have been found to be cost effective, including medical management with naltrexone therapy and combined naltrexone and acamprosate therapy. ${ }^{75}$

Even among patients who already have developed an alcohol-related illness (such as alcohol-related liver damage or psychosis), treatment may reduce future health care costs. Naltrexone therapy is related to less of an increase in health care expenditures for individuals with alcohol-related illnesses compared to a control group ${ }^{\ddagger \ddagger}$ (\$63 increase among naltrexone recipients vs. \$814 increase among controls). Those in the control group were more likely to have an alcohol-related visit to the emergency department during the study compared to patients taking naltrexone (15 percent vs. nine percent). ${ }^{76}$

Addiction Involving Other Drugs. One study examined the cost effectiveness of providing

\footnotetext{
** The cost of extending a patient's life by one year is a common metric used in cost-effectiveness studies. ${ }^{+\dagger}$ There were, however, no significant changes in rates of hospital admissions for respiratory conditions including pneumonia, asthma, chronic obstructive pulmonary disease and respiratory failure.

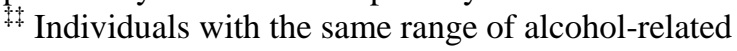
illnesses who were not prescribed naltrexone.
} 
treatment ${ }^{*}$ to pregnant illicit drug users and calculated an average net savings of \$4,644 per mother/infant pair. ${ }^{77}$

An examination of health care and pharmacy costs for patients with addiction involving opioids in a large U.S. health plan revealed that total health care costs (including inpatient, outpatient and pharmacy costs) six-months posttreatment were 29 percent lower for patients who received medication than for patients who received medication-free treatment ( $\$ 10,192$ vs. $\$ 14,353)^{78}$

A study of the cost effectiveness of short-term opioid replacement therapy ${ }^{\dagger}$ compared to detoxification only calculated a costeffectiveness ratio of $\$ 1,376$ in opioid replacement therapy costs per quality-adjusted life year (QALY). ${ }^{79}$ Another study projected that methadone maintenance therapy costs $\$ 5,915$ for every year of life gained. ${ }^{\ddagger} 80$ In HIV populations, expanding methadone maintenance capacity for heroin users is cost effective, at $\$ 8,200$ per QALY gained in communities with 40 percent HIV prevalence among injection drug users and \$10,900 per QALY gained in communities with five percent HIV prevalence among injection drug users. ${ }^{81}$

The use of evidence-based approaches in treatment will be driven by policy. We need to use the payment system to drive changes in practice. ${ }^{82}$

--Jeffrey Samet, MD Professor of Medicine and Social Behavior, Clinical Addiction Research and Education (CARE) Program Boston University School of Medicine

\footnotetext{
* One week of residential care followed by intensive outpatient (day treatment) services through labor/ delivery.

${ }^{\dagger}$ Patients, ages 15-21, received 12 weeks of buprenorphine-naloxone therapy and also were offered twice-weekly counseling.

$\ddagger$ Assuming annual treatment costs of \$5,250.
}

\section{Insurance Coverage of Addiction Treatment is Limited}

Recently-enacted federal and state parity laws have expanded coverage for addiction treatment where offered, and the Patient Protection and Affordable Care Act (ACA) holds potential for further expansion of access and benefits. However, insurance coverage of addiction treatment remains severely limited in both the populations and services that are covered. The absence of mandated coverage in all health plans means that some health plans may continue to choose not to provide coverage for addiction treatment, persisting to deny access to patients who need it. ${ }^{83}$

\section{Parity Laws}

Federal and state parity laws require private insurers that provide mental health and addiction treatment services to provide them on par with medical services. In general, restrictions placed on addiction services (e.g., co-pays, deductibles) cannot be more restrictive than restrictions placed on other medical services. ${ }^{84}$

Specifically, while the Mental Health Parity Act (MHPA), passed in 1996, did not apply to addiction treatment, ${ }^{85}$ the 2008 Paul Wellstone and Pete Domenici Mental Health Parity and Addiction Equity Act (MHPAEA) was enacted, in part, to address this omission. ${ }^{86}$ The MHPAEA provisions apply to:

- Plans sponsored by private and public sector employers with more than 50 employees and that include medical/surgical and mental health/addiction benefits; ${ }^{\S}$

\footnotetext{
$\S$ Applies to plan years beginning on or after July 1, 2010.
} 
- $\quad$ Medicaid managed care plans ${ }^{*} 87$ and benchmark/benchmark-equivalent plans ${ }^{\dagger}$ that provide mental health/addiction benefits; and

- State plans within the Children's Health Insurance Program (CHIP). ${ }^{88}$

Under the law, plans that offer addiction treatment benefits cannot impose limitations on these benefits that are more restrictive than limitations placed on medical and surgical benefits. $^{\ddagger 89}$

In addition to the federal parity laws, 49 states and the District of Columbia have some type of parity law for mental health services; at least 38 states include coverage addiction involving alcohol and/or other drugs. While the scope of these laws varies, some are stronger than MHPAEA. ${ }^{\S}$

\footnotetext{
* This includes traditional and benchmark/benchmark equivalent managed care plans. In Medicaid benchmark/equivalent plans' benefits are determined by comparison to BlueCross/BlueShield, state employee benefit plans, certain Health Maintenance Organization (HMO) plans or benefits that include the basic services defined in Section 1937(b)(2) of the Social Security Act.

${ }^{\dagger}$ The ACA extends certain MHPAEA parity requirements to Medicaid benchmark and benchmark equivalent plans that are not managed care plans that provide mental health or addiction treatment benefits. F The interim final regulations, which went into effect on April 5, 2010, address how health plans must comply with MHPAEA and how health plans may define covered services that are consistent with current medical standards. The regulations define classification of benefits (such as inpatient, innetwork or prescription drug benefits); financial requirements (such as deductibles and out-of-pocket maximums); treatment limitations defined both quantitatively (limits on day visits or frequency of treatment limits) and qualitatively (standards for provider admission into a network or prescription drug formulary design); and how to determine whether these restrictions comply with the law. ${ }^{\S}$ State parity laws generally fall into three categories: (1) mental health parity/equal coverage laws in which insurers must provide the same level of benefits for addiction treatment as they do for other health conditions; (2) minimum mandated mental health benefit laws in which some coverage must be offered
}

A recent government evaluation found that postMHPAEA, 96 percent of employers' plans continued to offer both mental health and addiction treatment services, two percent continued to cover only mental health services and another two percent discontinued their coverage of addiction treatment services. Plans also indicated that they had reduced service limitations on addiction-related services after MHPAEA. In general, patients' average costsharing burden declined after the MHPAEA, with the exception of co-payments for office visits which increased slightly. ${ }^{91}$ (Table 8.1)

\section{Table 8.1 \\ Employers' Insurance Coverage of Addiction Benefits Since Enactment of the MHPAEA}

\begin{tabular}{l|c|c}
\hline & $\begin{array}{c}2008 \text { Plan } \\
\text { Year }\end{array}$ & $\begin{array}{c}2010 / 2011 \\
\text { Plan Year }\end{array}$ \\
\hline $\begin{array}{l}\text { Employers including } \\
\text { addiction benefits in } \\
\text { most popular plan }\end{array}$ & $97 \%$ & $97 \%$ \\
\hline $\begin{array}{l}\text { Employers placing } \\
\text { limits on office visits } \\
\text { for addiction care }\end{array}$ & $33 \%$ & $8 \%$ \\
\hline $\begin{array}{l}\text { Employers placing } \\
\text { limits on inpatient } \\
\text { days for addiction care }\end{array}$ & $27 \%$ & $8 \%$ \\
\hline $\begin{array}{l}\text { Average office visit } \\
\text { copayment }\end{array}$ & $\$ 25$ & $\$ 27$ \\
\hline $\begin{array}{l}\text { Average office visit } \\
\text { coinsurance }\end{array}$ & $22 \%$ & $19 \%$ \\
\hline $\begin{array}{l}\text { Average outpatient } \\
\text { services copayment }\end{array}$ & $\$ 39$ & $\$ 33$ \\
\hline $\begin{array}{l}\text { Average outpatient } \\
\text { services coinsurance }\end{array}$ & $26 \%$ & $19 \%$ \\
\hline $\begin{array}{l}\text { Source: United States Government Accountability } \\
\text { Office. (2011). }\end{array}$
\end{tabular}

Analysis of national data found that in states with broad parity laws (where benefits for the treatment of addiction and mental illness are mandated in at least some health plans and must be offered at parity with medical and surgical benefits), there was a 12.8 percent increase

but disparities in level of benefits provided are permitted; and (3) "mandated offering laws" in which an option of coverage for addiction treatment is offered and, if coverage is accepted, benefits must be equal with other health benefits. 
between 1992 and 2007 in total treatment admissions compared to states with weak parity laws (in which parity laws were absent or did not include addiction treatment services). In comparison, in states with limited parity laws (in which parity was mandated but with limitations such as applying to certain groups or a limited number of health plans), there was only a 4.7 percent increase (compared to states with weak parity laws) in addiction treatment admissions during this time. ${ }^{92}$

Among adolescents who received both an addiction and psychiatric diagnosis, those living in a state with a parity law expanding coverage for addiction treatment are 4.5 times as likely to be diagnosed with addiction and 3.2 times as likely to receive treatment as adolescents in states without such parity laws. ${ }^{93}$

\section{The Patient Protection and Affordable Care Act of 2010}

The Patient Protection and Affordable Care Act (ACA) of 2010 was enacted to expand access to insurance for the uninsured, to make health care more affordable and to reform health care delivery systems to improve quality. ${ }^{94}$ The federal government estimates that the ACA could expand coverage for addiction treatment to an additional 4.8 million Americans if coverage is offered at parity with other health benefits. ${ }^{*} 95$

The ACA has the potential for increasing access to addiction-related services by (1) increasing the number of people who are covered by health insurance ${ }^{96}$ and (2) requiring that addiction treatment benefits be offered by certain ${ }^{\dagger}$ health

\footnotetext{
* This estimate was for those who buy coverage in the individual market, and while these plans must provide some form of addiction treatment as part of Essential Health Benefits, states have not yet defined their Essential Health Benefits. It is unclear how many states will include the full range of necessary services for addiction treatment in their definition. ${ }^{\dagger}$ Including new small fully-insured or self-insured plans, new individual market health plans, qualified health plans (as defined by the ACA), Basic Health Programs and Medicaid benchmark/equivalent plans.
}

plans as part of an "essential health benefit" package. ${ }^{97}$ The ACA also extends requirements of MHPAEA to some health plans to which the law did not previously apply.

One main goal of the ACA is to increase the number of people who have health insurance by making more people eligible for Medicaid, ${ }^{99}$ allowing individuals who do not have insurance through their job to obtain insurance in state exchanges (transparent and competitive marketplaces), offering them income-based tax credits and subsidies ${ }^{100}$ and allowing young adults under age 26 to remain covered by their parents’ insurance plan. ${ }^{\S} 101$

The ACA also attempts to change the way health care is delivered to improve quality and integrate addiction treatment into medical care, for example through demonstration projects like Medicaid health homes, where teams of health professionals care for individuals with chronic conditions including addiction; ${ }^{102}$ accountable care organizations (ACOs), in which groups of health professionals coordinate services for Medicare fee-for-service patients; ${ }^{103}$ and through temporary funding to expand the role of community health centers. ${ }^{104}$ If these initiatives are successful and become common practice, they will help to integrate the treatment of addiction into mainstream medical practice and expand the use of pharmaceutical therapies. ${ }^{105}$

\footnotetext{
‡ The ACA requires that qualified health plans offered through the exchanges, individual (nongroup) market plans and Medicaid non-managed care benchmark and benchmark-equivalent plans comply with MHPAEA.

$\S$ Even if they are married, in school or eligible to enroll in their employer's plan. Plans that existed on March 23, 2010 do not have to offer dependent coverage until 2014 if the dependent is eligible for employer-sponsored insurance.
} 


\section{Gaps in Coverage within Public and Private Insurance Plans Continue to Impede Comprehensive Addiction Care}

\author{
Screening and Interventions for Risky \\ Substance Use. Recent developments in \\ Medicare and Medicaid reimbursement have \\ begun to remove some of the cost barriers that \\ health professionals faced in routinely screening \\ their patients for risky use of addictive \\ substances and conducting early interventions \\ when necessary. ${ }^{106}$
}

For example, the U.S. Department of Health and Human Services' Centers for Medicare and Medicaid Services (CMS) adopted Medicaid billing codes for screening and brief intervention services; in January 2007, these codes became effective. ${ }^{107}$ The Medicaid codes cover these services related to alcohol and other drugs (excluding nicotine). ${ }^{108}$ These codes are available for health care providers in individual states to use but there is no requirement for providers to use the codes. Individual states determine which services are reimbursed and, to be operational, states have to enable the billing codes; however, many have not done so. ${ }^{*} 109$ With regard to smoking, the only screening services that states explicitly are required to provide are those that fall under the Early and Periodic Screening, Detection and Treatment (EPSDT) Program--the child health component of Medicaid which is required for each state to finance appropriate and necessary pediatric services, including tobacco cessation services for youth; as of $2000,{ }^{\dagger} 15$ states explicitly require providers to screen youth for tobacco use. ${ }^{110}$ (See next section on treatment for information about coverage for smoking cessation services.)

In 2008, CMS adopted Medicare billing codes for structured assessments ${ }^{\ddagger}$ and brief intervention services related to the risky use of alcohol and other drugs (excluding nicotine) for

\footnotetext{
* And these codes do not cover screening and intervention services for all addictive substances.

${ }^{\dagger}$ Most recent available data.

‡ Structured assessments involve the use of validated tools such as AUDIT or DAST (see Appendix H).
}

patients who show signs/symptoms of substance-related problems. ${ }^{111}$ These services were reimbursed only when reasonable and necessary to diagnose or treat illness or injury. ${ }^{112}$ In October 2011, CMS determined that Medicare would provide coverage in primary care settings $s^{\S}$ for preventive annual alcohol screening ${ }^{* *}$ of all patients and up to four brief, face-to-face interventions for Medicare beneficiaries who screen positive for risky alcohol use but who do not meet clinical criteria for addiction involving alcohol. ${ }^{113}$ Medicare does not reimburse for population-wide screening and brief interventions to address the risky use of illicit and prescription drugs because it is not yet recommended by the U.S. Preventive Services Task Force (see Chapter IV). ${ }^{\dagger \dagger} 114$ Although there are no specific Medicare codes for general tobacco use screening, questions about tobacco use are considered part of the medical history to be collected, for example, during the Initial Preventive Physical Examination for those new to Medicare. ${ }^{115}$ As of August 2010, Medicare does cover preventive tobacco cessation counseling for smokers who do not present with signs or symptoms of tobacco-related disease. The benefit includes two individual tobacco cessation counseling attempts per year, with each attempt consisting of up to four sessions. ${ }^{116}$

Despite the facts that the American Medical Association (AMA) has published Current Procedural Terminology (CPT) codes for screening for tobacco use $\mathrm{e}^{\ddagger 117}$ and risky use of alcohol and other drugs ${ }^{118}$ and that most private insurance plans cover these services, ${ }^{119}$ patients are not routinely screened for risky use or provided brief interventions if indicated. A 2009 survey found that very few claims have been paid by commercial insurance plans for

\footnotetext{
$\S^{\S}$ Including outpatient hospital settings.

${ }^{* *}$ But not for other substances. Medicare allows providers to choose any screening tool that is appropriate for their clinical population and setting.

${ }^{+1}$ Medicare does cover structured assessments to evaluate and provide interventions for patients who exhibit symptoms of addiction involving drugs.

\# CPT codes for tobacco screening include the codes for "health and behavior assessment" and other preventive medicine services.
} 
alcohol and other drug screening and brief intervention services. ${ }^{120}$ Although 76 percent of smokers in commercial health plans reported being advised by their physician to stop smoking in a 2008 survey; only half recalled being advised about smoking cessation strategies or medications. ${ }^{121}$

The ACA was designed to increase the number of individuals covered under Medicaid and commercial insurance, ${ }^{* 122}$ but also allows states facing budget deficits to scale back eligibility under certain circumstances. ${ }^{\dagger 123}$ Because of economic constraints, states appear to be cutting back on health services rather than assuring access to these services. ${ }^{\ddagger 124}$

An additional resource problem that stands in the way of screening and early interventions is a legal statute dating back to 1947, the Uniform Accident and Sickness Policy Provision Law $(U P P L) .{ }^{125}$ Under the UPPL, insurers can deny payment for treatment of injuries sustained by a person under the influence of alcohol or other drugs. $^{\S 126}$ In states that adopted UPPL, EDs and trauma centers might not screen for risky substance use because reimbursement for the emergency or trauma services they provide could be denied; as a result, critical opportunities for intervention are missed. ${ }^{127}$ Because of the barriers the UPPL imposes to

\footnotetext{
* The ACA mandates no co-pay in private insurance and self-insured plans for alcohol screening and counseling; alcohol and other drug use assessments for adolescents; tobacco use screening for all adults; and cessation interventions for tobacco users.

${ }^{\dagger}$ States are permitted to scale back eligibility if they have expanded their Medicaid programs to nonpregnant, non-disabled adults with incomes over 133 percent of the poverty level.

${ }^{\ddagger}$ At least 31 states have implemented cuts that will restrict eligibility for health insurance programs and/or access to health care services.

$\S$ The law originally was adopted to control insurance costs at a time when little knowledge was available about addiction and when access to treatment was limited. The statute states: "The insurer shall not be liable for any loss sustained or contracted in consequence of the insured's being intoxicated or under the influence of any narcotic unless administered on the advice of a physician.”
}

needed screening and interventions for risky substance use, the AMA has been working to overturn the law and prohibit other state laws modeled on it. ${ }^{* *} 128$ As of January 2011, 25 states still have the UPPL or a history of court decisions that permit insurers to use an alcohol exclusion to deny payment for treatment; 16 states and the District of Columbia prohibit denial of benefits. ${ }^{\dagger \dagger} 129$ A similar legal provision allows many states to deny disability payments or workers' compensation to individuals harmed while under the influence of alcohol or while participating in an illegal act, such as driving under the influence. ${ }^{130}$

Treatment for Addiction. Current coverage of addiction treatment is not designed to prevent and treat the disease effectively. There is considerable variability in coverage within and among public and private insurance plans which poses significant barriers to treatment accessibility.

Medicaid. The Medicaid program is the major public health coverage program for low-income Americans. ${ }^{131}$ With the exception of the ACA's “essential health benefits” requirement, addiction treatment services are not federally mandated in Medicaid, ${ }^{132}$ nor are any addiction treatment services that are eligible for reimbursement defined at the federal level. ${ }^{133}$ However, states may provide addiction-related services under several of the mandatory benefit categories:

- Physician services; ${ }^{134}$

- Inpatient services provided in a general hospital, such as inpatient detoxification, ${ }^{135}$ but not including room and board charges in

\footnotetext{
** In 2001, the National Association of Insurance Commissioners, which originally adopted the UPPL as a model law in 1947 and encouraged states to implement it, reversed their position and recommended the repeal of the UPPL. Since that time, several organizations along with the AMA have pledged support for repealing the UPPL.

${ }^{\dagger+}$ Other states do not explicitly permit or prohibit alcohol exclusion provisions.

㧊 Applies to Medicaid benchmark/equivalent plans.
} 
residential specialty treatment settings or inpatient care for patients ages 22- to 64years old in institutions for mental disease; ${ }^{136}$ and

- Outpatient hospital care, ${ }^{*}$ including detoxification, individual, group or family counseling and/or psychotherapy, and diagnosis, treatment, assessment and medication management. ${ }^{137}$

Optional benefit categories under which addiction-related treatment services also may be provided include:

- Outpatient rehabilitation services, including diagnostic and treatment services. States providing optional benefits under Medicaid often choose this option since it does not require services to be provided under the direction of a physician and instead permits the delivery of services including mutual support by community paraprofessionals and peers; ${ }^{138}$

- Clinic services; ${ }^{139}$ and

- Case management services. ${ }^{140}$

Last, states may provide addiction treatment services as part of a Medicaid managed care waiver program. ${ }^{\dagger 141}$

Because state Medicaid plans vary widely in their eligibility requirements and benefits, individuals have substantially different access to care depending on the state in which they live. Some states cover a broad range of addictionrelated services; others provide only a few and two states cover no such services. ${ }^{142}$

State Medicaid programs also distinguish between services offered for addiction treatment

\footnotetext{
* Several states specifically exclude addiction treatment from the outpatient services benefits and some place limits on the services such as number of visits per year.

${ }^{\dagger}$ A 2006 survey found that 31 states offer addiction treatment through a managed care waiver program.
}

involving nicotine and treatment involving alcohol and other drugs.

With regard to smoking, Medicaid plans are required to provide tobacco cessation counseling and pharmaceutical treatments to pregnant women, children and adolescents. States are free to choose whether or not to include tobacco cessation benefits for other enrollees. ${ }^{143}$ In 2009, 18 states covered individual counseling for all Medicaid enrollees, seven states covered counseling only for enrollees in some programs (fee-for-service or managed care) and six states covered it only for pregnant women. Eight states covered group counseling for all Medicaid enrollees, five covered group counseling only for enrollees in some programs (fee for service or managed care) and five states covered group counseling for pregnant women only. As of 2009, 34 states covered the nicotine patch for all Medicaid enrollees, 33 covered bupropion, 32 covered nicotine gum, 32 covered varenicline, 28 covered nicotine nasal spray, 27 covered nicotine inhalers and 25 covered nicotine lozenges. ${ }^{144}$ As of 2011, six state Medicaid programs provide comprehensive coverage for smoking cessation treatments for all Medicaid enrollees, while five state Medicaid programs provide no coverage for cessation treatment for any enrollees. ${ }^{145}$

Medicare. Medicare is a federally-funded system for financing health care for U.S. citizens ages 65 and older and people under age 65 with certain disabilities. ${ }^{146}$ Medicare covers the following services, when medically necessary:

- Inpatient hospital services for detoxification for addiction involving alcohol and outpatient services for detoxification for addiction involving drugs other than nicotine; ${ }^{147}$

- Inpatient rehabilitation treatment for addiction involving alcohol, controlled prescription drugs and illicit drugs in an acute care or psychiatric hospital; ${ }^{148}$

- Outpatient hospital-based diagnostic and therapeutic services for treatment of 
addiction involving alcohol, and outpatient hospital services involving detoxification, rehabilitation and treatment for addiction involving drugs other than nicotine; ${ }^{149}$

- Clinic-based treatment services for addiction involving alcohol and detoxification services for addiction involving opioids, under physician supervision; ${ }^{150}$ and

- Tobacco cessation counseling from a qualified physician or practitioner for all smokers ${ }^{*}$ and tobacco cessation medications prescribed by a physician. ${ }^{151}$

Methadone maintenance therapy also is covered on an outpatient basis, but only when indicated for pain, and in hospitals for treatment of addiction involving opioids. ${ }^{152}$

Differences in copayments for outpatient addiction treatment and other outpatient services will be phased out by $2014 .^{153}$

Private Health Insurers. Historically, private health insurers have provided less coverage for the treatment of addiction than for other health conditions by setting lower annual or lifetime limits on benefits, ${ }^{\dagger}$ covering fewer inpatient days or outpatient visits and increasing cost sharing through higher deductibles and copayments. ${ }^{154}$ A survey of private health plans found that, while only 16 percent of private insurance offerings imposed lifetime limits on addiction treatment, 94 percent had annual limits for outpatient services and 89 percent had annual limits for inpatient services. Private insurance offerings were more likely to limit visits (outpatient) or days (inpatient) than to limit spending. ${ }^{155}$

\footnotetext{
${ }^{*}$ Medicare covers two individual cessation counseling attempts per year, and each attempt may include up to four sessions.

${ }^{\dagger}$ Annual limits are caps that insurers place on the benefits an enrollee is entitled to each year. Limits can apply to particular services (e.g., hospitalizations), number of visits or dollar amount of covered services. Lifetime limits are caps on expenditures, on specific services or both during an individual's lifetime.
}

Children's Health Insurance Program (CHIP). Under CHIP, formerly the State Children's Health Insurance Program (SCHIP), states are entitled to federal matching funds up to specified limits to finance health care for low-income children $^{\ddagger}$ who do not qualify for Medicaid. ${ }^{156}$ States can provide benefits related to substance use and addiction under CHIP by expanding children's eligibility under Medicaid, by creating a separate insurance program or through some combination of these approaches. States that opt simply to expand their Medicaid programs are required to follow the rules and requirements of Medicaid. ${ }^{157}$ States that provide benefits by creating unique CHIP programs (outside of Medicaid) must provide a benefits package equivalent to one of several "benchmark" insurance plans. ${ }^{\S} 158$ States have latitude in designing their CHIP program. ${ }^{159} \mathrm{~A}$ 2000 study found that almost all states provided at least one of detoxification, inpatient/ residential or outpatient services, though many states imposed annual limits (e.g., 20 or 60 visits per year) or lifetime benefit limits (e.g., $\$ 16,000$ or $\$ 20,000){ }^{160}$

\section{Gaps in Addiction Care Coverage Within Parity and Health Reform Initiatives. With regard to federal parity laws, MHPAEA includes an exemption if the financial burden of implementing the law is too great, ${ }^{* *} 161$ and small employers (with less than 50 employees) are exempt from the law completely. ${ }^{162}$ Under MHPAEA, insurance plans may cap services}

\footnotetext{
‡ Through waivers, states may expand CHIP eligibility to pregnant women, low-income parents and adults without children.

$\S$ Such as the Blue Cross/Blue Shield Standard Option Service Benefit Plan offered under the Federal Employees Health Benefits Program (FEHBP), a plan that is available to the state's employees or a plan offered by the HMO with the largest enrollment in the state outside of Medicaid. States also may use a benefits package that is actuarially equivalent to one of the benchmark plans, an already existing state-funded plan or any other plan approved by the federal government.

${ }^{* *}$ Health plans are exempt if complying with the law results in a cost increase of greater than two percent in the first plan year and greater than one percent in subsequent years.
} 
(e.g., number of visits per year) as long as the caps are equivalent to those placed on medical services. ${ }^{163}$ Placing blanket limitations on allowed visits or length of stay, however, does not accord with best practices for treating cases of addiction that are chronic and relapsing. ${ }^{164}$

Challenges to implementing MHPAEA in practice include a lack of education among medical professionals in how to screen, intervene and treat addiction and a lack of addiction physician specialists. ${ }^{165}$

With regard to state parity laws, self-insured employer-sponsored health plans are exempt from state regulation under the federal Employee Retirement Income Security Act (ERISA) of 1974. ${ }^{166}$ Furthermore, coverage for mental health and addiction services varies dramatically by state, depending on the strength of the state's parity law.

With regard to the ACA, despite the improvements in treatment coverage that will arise from its passage, many limitations remain both in policy and practice. Its impact on treatment access remains to be seen since many of the provisions of the law have not yet taken effect. For example, the expansion of Medicaid as a payer likely will result in reductions in federal and state grants for residential care (which is not covered by Medicaid). As a result, care may transition toward outpatient treatment ${ }^{167}$ which may be inadequate in some cases, such as for treating patients with more severe addiction.

Expanding access to insurance is necessary but alone is not sufficient to expand access to care. In 2006, Massachusetts enacted health care reform legislation similar to the ACA which, among other things, established universal health insurance through individual mandates to purchase insurance and government subsidies. ${ }^{168}$ In the years following, addiction treatment admission rates did not increase significantly. Despite the fact that the total uninsured population dropped to three percent, a large number (23-30 percent) of patients with addiction remained uninsured, either due to noncompliance with the mandate to obtain insurance, inability to pay even with subsidies or logistical barriers such as lack of documentation or a stable home address. For some of those who were successful in becoming insured, coinsurance and co-payments rendered treatment unaffordable. ${ }^{169}$ Furthermore, expanding insurance coverage does not automatically translate into expanded screening and diagnosis by health professionals or capacity to treat large numbers of newly-insured patients.

Similarly, requiring parity for addiction benefits in Federal Employee Health Benefit (FEHB) plans did not result in increased treatment rates. An analysis of nine large FEHB plans in the two years before and after the parity requirement found that the number of new diagnoses of addiction increased; however, utilization rates for addiction treatment benefits were unaffected. ${ }^{170}$

The ACA includes 10 categories of essential health benefits (EHB)--including addiction treatment--that must be provided by newlycreated individual and small group plans. ${ }^{171}$ Yet rather than defining what these services must include, the federal government has proposed that each state design its own EHB package, ${ }^{*}{ }^{172}$ meaning that benefits will vary across states. Furthermore, the EHB provisions do not apply to self-insured group health plans, large group market health plans or already existing small and individual market ("grandfathered") health plans. ${ }^{173}$ The provisions that would expand coverage and require EHBs do not go into effect until 2014. ${ }^{174}$

Efforts to control spending and legal challenges may limit some intended effects of the ACA. States may respond to fiscal challenges by attempting to control costs in their Medicaid programs by cutting services. ${ }^{175}$ Moreover, the ACA faces challenges in the U.S. Supreme

\footnotetext{
${ }^{*}$ Within limits--the plan must be comparable to a benchmark plan: (1) the largest plan by enrollment in any of the three largest small group insurance products in the state's small group market; (2) any of the largest three state employee health benefit plans by enrollment; (3) any of the largest three national FEHBP plan options by enrollment; or (4) the largest insured commercial non-Medicaid HMO operating in the state.
} 
Court over two provisions that would expand coverage: the Medicaid expansion and the requirement for individuals not covered under public programs or by employer-sponsored insurance to purchase insurance; the Court's decision would have implications not just for these provisions but for other parts of the law as well. ${ }^{* 176}$

\footnotetext{
${ }^{*}$ For example, if the Supreme Court decides that the individual mandate is unconstitutional and not severable from the rest of the legislation, the entire ACA would be struck down. If it is deemed unconstitutional and severable, then the prohibitions against excluding patients with pre-existing conditions and charging higher premiums based on a person's medical history also might be invalidated.
} 


\section{Chapter IX}

\section{The Education, Training and Accountability Gap}

Compounding the profound gap between the need for prevention, intervention, treatment and disease management for addiction and the receipt of such care is the enormous deficit of trained providers; there is a wide gulf between existing knowledge about addiction and its prevention and treatment and the education and training received by those who provide or should provide care. In spite of the evidence that risky use of addictive substances is a public health problem and addiction is a disease:

- Most health professionals ${ }^{*}$ are not sufficiently trained to educate patients about risky use and addiction, conduct screening and interventions for risky use or diagnose and treat addiction;

- Most of those who currently are providing addiction treatment are not medical professionals and are not equipped with the knowledge, skills or credentials necessary to prove the full range of evidence-based services to address addiction effectively; ${ }^{\dagger 1}$ and

\footnotetext{
* The term "health professional" as used in this report includes medical professionals (physicians, physician assistants, nurses and nurse practitioners, dentists, pharmacists) and graduate-level clinical mental health professionals (psychologists, social workers, counselors). All health professionals can be trained to educate patients about risky use and addiction and screen for these conditions; brief interventions also can be conducted by appropriately trained health professionals. Diagnosis and treatment requires a trained physician with the exception of psychosocial treatments which can be provided by trained graduate-level clinical mental health professionals working with a managing physician.

${ }^{\dagger}$ The National Quality Forum (2005) defines evidence-based addiction care to include: screening, brief interventions, treatment planning, psychosocial interventions, pharmaceutical therapy, retention strategies and chronic care management. Effective implementation requires particular skills and training.
} 
- Addiction treatment facilities and programs are not adequately regulated or held accountable for providing treatment consistent with medical standards and proven treatment practices. ${ }^{2}$

Further complicating the education, training and accountability gap in addiction treatment is the fact that there are no national standards; instead, there is considerable inconsistency among states in the regulation of individual treatment providers and of the programs and facilities that provide addiction treatment services.

For just about all known diseases other than addiction, treatment is provided within a highlyregulated health care system. In contrast, patients with the disease of addiction are referred to a broad range of providers largely exempt from medical training and standards (for many of whom the main qualification may be that they themselves have a history of addiction) who work within a fragmented system of care with inconsistent regulatory oversight.

\section{The Size and Shape of the Addiction Treatment Workforce}

Given the extensive prevalence of addiction in the U.S. and the frequently extensive treatment needs of individuals with addiction, there is a significant shortage of qualified addiction treatment providers. ${ }^{3}$ According to data collected from 1996 to 1997, there are 134,000 full-time staff and 201,000 total staff (including part-time and contract staff) working in addiction treatment. $^{\dagger}$ Only a small proportion of these workers, however, have medical training. ${ }^{4}$

\footnotetext{
* With the notable exception of the regulation of medication-assisted therapy for addiction involving opioids.

† This estimate includes physicians, registered nurses, other medical personnel, doctoral level counselors, master's level counselors, counselors with other degrees, non-degreed counselors and other staff. Data on the numbers of professionals who currently are providing some type of addiction treatment are not available.
}

Trained medical professionals and other graduate-level health professionals are less likely than other types of providers to work fulltime in addiction treatment; rather, staff members with higher levels of education are more likely to be hired on a contract/part-time basis. ${ }^{5}$ A nationally representative survey of addiction treatment facilities found that onequarter of the program directors were not fulltime employees; only two of the programs surveyed were directed by a physician; 54 percent employed a part-time physician; less than 15 percent employed a nurse; and psychologists and social workers rarely were on staff. ${ }^{6}$ An older study ${ }^{\ddagger}$ found that medical professionals and graduate-level counselors each made up only about 17 percent of the full-time staff of addiction treatment facilities and that only 12.8 percent of facilities had a physician on staff full time. ${ }^{\S 7}$ Another study found that more than a third of clinical supervisors lack any type of graduate degree. ${ }^{8}$

Unlike patient care in the mainstream medical system, which is delivered by highly educated and trained professionals, the staff primarily responsible for patient care in addiction treatment facilities is comprised largely of addiction counselors, many of whom while highly dedicated to addiction care have only a bachelor's degree or, in some cases, no posthigh school education. ${ }^{9}$ The Bureau of Labor Statistics reports that there were 76,600 addiction counselors in 2011 . $^{* *}$ One study found that 50 percent of facilities have full-time counselors on staff who have no degree; 58.5 percent have a bachelor's level counselor, 61.9 percent have a master's level counselor and 12.0 percent have a doctorate level counselor. ${ }^{11}$

\footnotetext{
‡ Data are from 1996/1997.

$\S 25.8$ percent had a full-time registered nurse and 17.5 percent had other full-time medical staff.

** Addiction counselors are those who "counsel and advise individuals with alcohol, tobacco, drug or other problems such as gambling and eating disorders. May counsel individuals, families or groups or engage in prevention programs.” This estimate excludes social workers, psychologists and mental health counselors who provide these services.
} 
Even among physicians, who constitute the group most qualified to treat patients with the medical disease of addiction, required training in addiction is minimal. ${ }^{12}$ And there is a severe shortage of physicians with expertise in addiction treatment via the medical specialty fields of addiction medicine and addiction psychiatry. ${ }^{13}$

The American Medical Association (AMA) estimates that of the 985,375 active physicians, there are only 582 addiction physician specialists: 227 addiction medicine physicians and 355 addiction psychiatrists ${ }^{*}$--the two medical sub-specialties specifically trained in addiction science and its treatment--totaling 6/100ths of one percent of all active physicians. ${ }^{14}$ However, according to the American Board of Addiction Medicine (ABAM), these estimates are low since they come from a voluntary, self-report survey in which physicians who choose to respond are asked to indicate their specialty and typically mark the field of their primary board certification rather than their subspecialty. ${ }^{15}$

Although there are no recent data identifying the actual number of practicing specialists in addiction medicine or addiction psychiatry, ABAM has certified 2,584 addiction medicine specialists and estimates that the number of fulltime practicing addiction medicine specialists may be about five times the amount of the AMA estimate--approximately $1,200 .^{\dagger 16}$ This estimate still falls far short of the estimated minimum of 6,000 full-time addiction medicine specialists currently needed to meet addiction treatment demands. ${ }^{17}$ Even this projection of workforce need in addiction medicine may underestimate the need in several ways: (1) it does not include adolescents; (2) it does not include addiction involving nicotine; ${ }^{\ddagger}(3)$ it does not include institutionalized individuals; (4) it assumes that only those who meet clinical criteria for

\footnotetext{
* Based on data from 2010.

${ }^{\dagger}$ Data on the number of practicing addiction medicine specialists who are involved directly in patient care are not available.

${ }^{*}$ Unless addressed in the context of addiction involving alcohol or other drugs.
}

substance dependence as distinguished from substance abuse require any form of specialty care; and (5) it is based on data that are six years old. ${ }^{18}$ Adjusting ABAM's estimate to address these gaps could increase substantially the number of addiction medicine specialists required to provide needed care.

Likewise, due to the limitations of the AMA survey and the absence of other data, it is impossible to know how many of the 1,137 physicians who are board certified in addiction psychiatry as of $2011^{19}$ currently are practicing in that subspecialty or how much overlap there is with the number of physicians certified in addiction medicine.

\section{Licensing and Credentialing Requirements for Individuals who Provide Addiction Treatment ${ }^{\S}$}

To help assure adherence to minimum standards in the delivery of medical care, the licensing and credentialing requirements of individuals who may provide such care are clearly delineated and regulated. For physicians, these include extensive graduate-level classroom-based and clinically-supervised training, a focus on

\footnotetext{
${ }^{\S}$ The information provided in this section is based on an extensive review of publicly available documents conducted by CASA Columbia in 2010. This entailed online reviews and updates (using the Internet and the Lexis/Nexis database) of publiclyavailable federal and state laws and regulations (in all 50 states and the District of Columbia) and of professional association Web sites. CASA Columbia's analysis examined the minimum licensing and certification requirements to practice in each profession in the 50 states and the District of Columbia, and optional certifications. The analysis sought to develop a summary overview of the regulatory landscape. However, because licensing and certification requirements are found in a wide variety of laws and regulations and can change on a state by state basis, and may have changed in certain states since the time of the review, findings from this review cannot be guaranteed to be complete and current. Unless cited to another source, the findings presented regarding licensing and certification requirements are derived from this review. See Appendix A for a description of the methodology.
} 
standards of medical practice ${ }^{20}$ and the adoption of evidence-based practices for those who wish to become board certified. ${ }^{21}$ In contrast, there are no clearly delineated, consistent and regulated national standards that stipulate who may provide addiction treatment in the United States; instead, standards vary by payer and by state.

In 2010, CASA Columbia examined the addiction-related licensing and certification requirements of individuals who most typically are the providers of addiction treatment and related services in the United States. These include medical professionals such as physicians, physician assistants, nurses and nurse practitioners; mental health professionals such as psychologists, social workers and counselors/therapists; acupuncturists; and addiction counselors. Licensure is a mandatory process required by state law; licensing standards are designed to ensure minimum competency required to practice one's profession and protect public health, safety and welfare. Certification is a voluntary process administered by non-governmental organizations, typically professional associations. $^{22}$ Certification demonstrates additional expertise within a specific area of one's profession (i.e., a specialty). ${ }^{23}$

Of all these groups, addiction counselors provide the majority of addiction treatment in the U.S. ${ }^{24}$ Indeed the only category of providers specifically required to be licensed to provide addiction treatment in most states is addiction counselors. Yet the requirements in some states for becoming an addiction counselor include only a high school diploma and some practical training--typically involving a focus on the 12step model. ${ }^{25}$ Training approximates an apprenticeship model which may fail to promote systematic adoption of evidence-based practices. ${ }^{26}$ Historically, personal experience with addiction (i.e., being "in recovery") was the primary qualification necessary to become an addiction counselor. ${ }^{27}$

Unlike providers of medical care who are trained in evidence-based medical practices, few among the broad range of providers who may treat patients with addiction are trained in or knowledgeable about evidence-based practices in addiction prevention and treatment. ${ }^{28}$ While medical professionals and some mental health professionals may have the training and skills needed to implement research-based treatments-and regularly come into contact with patients in the target population of risky substance users and those with addiction--most are unprepared to address these conditions. And while addiction counselors, who constitute the largest proportion of the workforce in specialty treatment facilities, ${ }^{29}$ specifically address addiction, most lack an education grounded in the science of addiction and are not equipped to deliver evidence-based treatments including appropriate medical care and treatment of cooccurring health conditions. ${ }^{30}$

Compounding this problem is that the diversity in education and training among the different types of individuals providing addiction treatment results in inconsistent treatment approaches and care for patients with addiction. $^{31}$

\section{Medical Professionals}

Medical professionals have been regulated at the state level since Colonial times. ${ }^{32}$ Rooted in their police powers, states have the authority to prohibit the performance of ineffective and dangerous treatments, to license professionals and to define their scope of practice. ${ }^{33}$ For specific licensing standards, states largely defer to professional boards and national organizations that accredit education programs. Medical professionals must complete an accredited professional education program and pass a national licensing exam to become licensed by the state in which they practice their profession. State licensing requirements may include minimum education, training or skills demonstrated by earning a specified degree; time spent in clinical training requirements; and passing a licensing exam.

Because risky use of addictive substances is a public health issue and addiction is a medical condition, medical professionals--particularly physicians--should be on the front lines in 
treating patients with these conditions, working with a team of other qualified health professionals. However, separate courses in addiction medicine rarely are taught in medical school $^{34}$ and there are no addiction medicine residencies among the 9,034 accredited U.S. residency programs currently training 116,404 residents. ${ }^{35}$ Physicians, therefore, lack the basic education and training in addiction medicine that is needed to understand the science of addiction, translate research evidence into practice, screen for risky use, diagnose and provide treatment for addiction and the broad range of co-occurring health problems, or refer patients to other specialists as needed. ${ }^{36}$

Physicians. To become a physician, an individual must earn a bachelor's degree, complete four years of medical school to earn an M.D. (allopathic physician) or a D.O. (osteopathic physician) and complete an additional three to seven years of post-graduate training in a medical residency training program (also called graduate medical education). ${ }^{37}$ To become licensed to practice medicine, physicians must pass a three-step licensing exam; allopathic candidates take the United States Medical Licensing Exam (USMLE). ${ }^{38}$ The USMLE includes addiction as a possible subtopic in each step. ${ }^{*} 39$ Those who choose to practice osteopathic medicine must take the Comprehensive Osteopathic Medical Licensing Examination (COMLEX) administered by the National Board of Osteopathic Medical Examiners (NBOME). ${ }^{40}$ Dimension 1 of the COMLEX exam is devoted to "Patient Presentation" where addiction is listed as a subtopic. $^{\dagger 41}$ These requirements are set by national accreditation organizations (that accredit schools and residency programs) and professional boards (that provide education and licensing standards) to which states defer when they require professional licensing. ${ }^{42}$

\footnotetext{
* Within the content areas "Central and Peripheral Nervous Systems: Abnormal Processes” in Step 1 of the exam, "Mental Disorders" in Step 2 and

"Behavioral/Emotional Disorders" in Step 3.

${ }^{\dagger}$ Within the content areas "Population Health Concepts: Disease Detection and Monitoring” and "Cognition, Behavior, Sensory and Central Nervous Systems, Substance Abuse and Pain.”
}

Although physicians in the United States have extensive competency requirements regarding most illnesses, their level of required competency in addiction medicine is minimal given the prevalence of risky substance use and addiction in most patient populations. ${ }^{43}$ No reliable national data exist on the proportion of medical school curricula devoted to the topic of addiction. A national survey of residency training program directors in seven medical specialties revealed that 56.3 percent of the programs report having required curriculum content in preventing and treating addiction, but that the median number of curricular hours of training ranges from three (emergency medicine and obstetrics/gynecology) to 12 (family medicine). ${ }^{44}$ While most allopathic medical schools do include some addiction content in required coursework, ${ }^{45}$ research suggests that the average school requires few hours of its fouryear curriculum to be devoted to the topic. ${ }^{46}$

Physicians may choose to become board certified in a medical specialty, which demonstrates that they have the knowledge, skills and experience to treat patients within that specialty. ${ }^{47}$ The American Board of Medical Specialties (ABMS) has adopted a Maintenance of Certification (MOC) program for all specialties in which physicians must stay abreast of the latest advances in their specialty and demonstrate use of best practices. ${ }^{48}$

CASA Columbia reviewed the board certification exam requirements of the six medical specialties that interact most often and regularly with patients who engage in risky substance use or have addiction to determine their addiction-related content: 
- Internal Medicine--two percent of the general board exam. ${ }^{49}$ In addition, substance use/addiction is listed as possible subtopics in the geriatric medicine ${ }^{*} 50$ and infectious disease ${ }^{51}$ subspecialty exams; however, the exact proportions are not specified.

- Pediatrics--1.5 percent of the general exam, ${ }^{52}$ five percent of the adolescent medicine exam ${ }^{53}$ and two percent of the developmental-behavioral pediatrics subspecialty exam. ${ }^{54}$ Substance-related topics also are listed in the pediatric emergency medicine, child abuse pediatrics, and neonatal-perinatal medicine subspecialty exams; however, the exact proportion is not specified. ${ }^{55}$

- Family Medicine--no specification in the general board exam, ${ }^{56}$ but the pharmacology of and testing for the use of addictive substances is included as a possible subtopic in the optional sports medicine subspecialty certification exam. ${ }^{57}$

- Psychiatry--included as subtopics in the general board exam, but the proportion of the total content is not specified. ${ }^{58}$ Also one-half percent of the forensic psychiatry and six percent of the psychosomatic medicine subspecialty exams are devoted to substance use/addiction. ${ }^{59}$ Substance use/addiction also is listed as a subtopic in

\footnotetext{
* Subspecialty certifications in the same area may be offered by more than one medical board. For example, the geriatric medicine subspecialty certification administered by the American Board of Internal Medicine also can be obtained by physicians specializing in family medicine; the adolescent medicine certification administered by the American Board of Pediatrics also can be obtained by physicians specializing in internal medicine and family medicine; the pediatric emergency medicine exam administered by the American Board of Pediatrics also can be obtained by physicians specializing in emergency medicine; and the sports medicine subspecialty certification administered by the American Board of Family Medicine also can be obtained by physicians specializing in internal medicine, pediatrics and emergency medicine.
}

the child and adolescent psychiatry, geriatric psychiatry and pain medicine subspecialty exams. $^{60}$

- Emergency Medicine--included as a subtopic in the qualifying examination, although the exact proportion and content are unspecified. ${ }^{61}$

- Obstetrics/Gynecology--included in a subtopic of the general written board certification exam, although the exact proportion is unspecified. Substance use/addiction assessment and counseling are listed as one of 40 patient cases that may be covered in the oral exam. ${ }^{62}$ The subspecialty of maternal-fetal medicine explicitly lists substance use/addiction as a competency for the certification exam, but the exact proportion and content are unspecified. $^{63}$

There are two areas of specialty medical practice in addiction: addiction medicine and addiction psychiatry.

Addiction Medicine. The American Board of Addiction Medicine (ABAM) offers a voluntary certification in addiction medicine to physicians across a range of medical specialties. ${ }^{64}$ The role of the addiction medicine physician, as a member of an interdisciplinary team of health professionals, includes examining patients to establish the presence or absence of a diagnosis of addiction; assessing associated health conditions that are brought on or exacerbated by the use of addictive substances; participating in the development and management of an integrated treatment plan; prescribing and monitoring patients' use of addiction treatment medications and therapies; providing direct treatment and disease management for individuals with severe cases of addiction and providing consultation to other primary and specialty care providers. ${ }^{65}$ To become certified in addiction medicine, applicants must meet specific educational and clinical requirements including: 
- Graduating from a medical school in the U.S. or Canada approved by the Liaison Committee on Medical Education (LCME) or the Committee on Accreditation of Canadian Medical Schools (CACMS) or from a school of osteopathic medicine approved by the American Osteopathic Association (AOA); ${ }^{* 6}$

- Being an ABMS board-certified physician or having completed a residency training program $^{\dagger}$ in any medical specialty, plus 50 hours of addiction medicine educational course work (continuing medical education/CME); ${ }^{67}$

- Completing at least 1,920 hours in teaching, research, administration and clinical care related to prevention and treatment for individuals who are at risk for or have addiction, or completing a one-year ABAM Foundation-accredited addiction medicine residency training program; ${ }^{68}$

- Passing a five and a half-hour computerbased examination; ${ }^{69}$ and

- Holding a valid and unrestricted license to practice medicine in the United States, its territories or Canada. ${ }^{70}$

Physicians must maintain their certification through ABAM's Maintenance of Certification (MOC) program of continuing education and periodic examinations. ${ }^{71}$

\footnotetext{
* If applicants are graduates of medical schools outside the U.S. or Canada, they must have a currently valid standard certificate from the Educational Commission for Foreign Medical Graduates (ECFMG) or have passed the Medical Council of Canada Evaluating Examination (MCCEE).

${ }^{\dagger}$ Residency programs must be accredited by one of the following: the Accreditation Council for Graduate Medical Education (ACGME), the Royal College of Physicians and Surgeons of Canada, the Professional Corporation of Physicians of Quebec or residency programs accepted by any member board of the American Board of Medical Specialties (ABMS) as qualifying to sit for that member board's certification examination.
}

Though not yet a member board of the ABMS, ABAM is working to gain recognition of addiction medicine as a medical specialty. ${ }^{72}$

Addiction Psychiatry. The American Board of Psychiatry and Neurology, Inc. offers optional certification in addiction psychiatry. Addiction psychiatrists are trained to identify and treat cooccurring addiction and psychiatric disorders in individuals seeking treatment for either condition, and in therapies tailored to specific subgroups of patients with addiction. ${ }^{73}$

Addiction psychiatry is recognized by the ABMS. ${ }^{74}$ Candidates for certification are boardcertified psychiatrists who have completed a one-year fellowship in addiction psychiatry. ${ }^{75}$

Physician Assistants. Physician assistants are licensed to assist physicians in the practice of medicine, enabling them to perform many of the same duties that physicians perform, including medical assessments and prescribing medication. ${ }^{76}$ The precise scope of their practice varies according to the regulations of each state.

All states license and regulate physician assistants and require graduation from an accredited physician assistant's program and passing of the Physician Assistant National Certifying Examination (PANCE) administered by the National Commission on Certification of Physician Assistants (NCCPA). ${ }^{77}$ States defer to the national accreditation agency and professional board for content requirements. All physician assistants must complete two years of college course work in basic and behavioral sciences and earn a degree from a program ${ }^{\ddagger}$ accredited by the Accreditation Review Commission on Education for the Physician Assistant (ARC-PA). ${ }^{78}$ Most programs (more than 80 percent) award a master's degree. ${ }^{79}$

\footnotetext{
‡ Physician assistant programs are approximately 27 months long.
} 
The ARC-PA requires physician assistant programs to provide some instruction in the "detection and treatment of substance abuse" although the nature and extent of this instruction is not specified. ${ }^{80}$ The PANCE exam may include addiction in its psychiatry/behavioral exam category. ${ }^{*} 81$

As of 2010, all states permit physicians to delegate prescription privileges to physician assistants ${ }^{82}$ and all states except Florida and Kentucky allow physician assistants to prescribe certain controlled substances under medical supervision. ${ }^{83}$ Yet physician assistants, like other medical professionals, receive little training in addiction in spite of the fact that they can prescribe controlled substances. ${ }^{\dagger}$

Nurses. States offer several categories of licensing in the nursing profession, each with different standards, practice limitations and supervision requirements. As they do for physicians, states defer to national accreditation agencies and professional boards for specific licensing standards for nurses. To be a licensed registered nurse (RN), one must graduate from an accredited nursing program which includes earning either a bachelor's of science degree in nursing (BSN), an associate's degree in nursing (ADN) or completing a diploma program (administered in hospitals). ${ }^{84}$ Graduates from each program are eligible to take the National Council Licensure Exam (NCLEX), administered by the National Council of State

\footnotetext{
${ }^{*}$ Each question addresses one organ system along with one of seven practice areas: history taking and physical examinations, using laboratory and diagnostic studies, formulating a most likely diagnosis, health maintenance, clinical interventions, pharmaceutical therapeutics and applying basic science concepts.

${ }^{\dagger}$ A few states require physician assistants to be trained in addiction. Physician assistants in CA who wish to prescribe controlled substances without advanced approval from a supervising physician are required to take a controlled substance education course which includes assessment of risky substance use and addiction. In OK, all physician assistants must complete one hour of continuing education per year on the topic of addiction.
}

Boards of Nursing, and they must pass this exam to become a licensed $\mathrm{RN}^{85}$

Even though most nurses interact regularly with individuals who are risky users or who have addiction, ${ }^{86}$ CASA Columbia's review found that in all but several states ${ }^{\ddagger}$ addiction-related education is not required explicitly in curriculum guidelines for state nursing programs. Other research found that many nursing education programs do not teach current information related to addiction. ${ }^{87}$ The National League for Nursing Accrediting Commission and the Commission on Collegiate Nursing Education, the two main accrediting agencies for nursing schools, do not require addiction to be part of nursing curricula. ${ }^{88}$ Addiction, including the topic of smoking cessation, may be included as topics on the licensing exams for registered and practical/vocational nurses. ${ }^{89}$ The American Academy of Nursing recently published new core clinical competencies in mental health that should be expected of all RNs. They include knowledge about the disease of addiction, addiction treatment, the pharmacology of commonly-misused illicit and prescription drugs, comprehensive screening, motivational interviewing, patient outcome evaluation, comprehension of research literature and the adoption of evidence-based practices. ${ }^{90}$

In most states, advanced practice nurses (APN) must earn a master's degree and are authorized to prescribe both non-controlled and controlled substances; ${ }^{91}$ however, few states explicitly include addiction-related content in their training requirements.

The International Nurses Society on Addictions (IntNSA) offers an optional certificate in addiction nursing; to qualify, candidates must

\footnotetext{
${ }^{\ddagger}$ In CA, RNs must complete studies in alcohol and other drug addiction; however, the exact amount is not specified. In IL, programs in practical nursing must include a course in pharmacology which must include topics on substance use and addiction. IN and $\mathrm{RI}$ require some addiction education and $\mathrm{NJ}$ requires six contact hours in pharmacology related to controlled substances including the prevention and management of addiction involving these substances.
} 
have 200 hours (one year) of addiction-specific experience as an $\mathrm{RN}$ and pass an examination. ${ }^{92}$

\section{Mental Health Professionals}

As is true of medical professionals, the licensing of mental health professionals is regulated by the states, which defer to national accreditation organizations and professional boards.

Psychologists. Licensed clinical and counseling psychologists must obtain a doctorate degree from a PhD or PsyD program at an accredited or government-chartered institution acceptable to the American Psychological Association (APA) licensing board. ${ }^{93}$ They also must pass a national licensing examination, the Examination for Professional Practice in Psychology, administered by the Association of State and Provincial Psychology Boards. ${ }^{94}$ Vermont and West Virginia offer a psychologist license at the master's level.

In the course of their practice--whether in the mental health care system, the general health care system, the correctional system, schools and universities or specialty addiction treatment programs--clinical and counseling psychologists encounter many patients who engage in risky substance use or have addiction. Psychologists often hold administrative and supervisory positions in specialty addiction treatment programs and other health and social welfare systems with large numbers of substanceinvolved individuals. ${ }^{95}$ Yet CASA Columbia's review found that in most states, addiction is not a required element of psychologists' training. ${ }^{\dagger}$

In the national licensing exam for psychologists, ${ }^{\ddagger}$ addiction-related content may appear as part of the required knowledge base in

\footnotetext{
* A number of states also license temporary or renewable psychological associate licenses for master's level supervised practitioners.

${ }^{\dagger}$ CA requires all licensed psychologists to have some level of addiction-related education; applicants must complete at least a semester course in addiction detection and treatment.

‡ The Examination for Professional Practice in Psychology, administered by the Association of State and Provincial Psychology Boards (ASPPB).
}

several content areas, including "biological bases of behavior" and "assessment and diagnosis.”§ 96

The APA had offered an optional certificate related to substance use and addiction which, although recognized by some state agencies, ${ }^{* *}$ was not required for a psychologist to treat patients with addiction; the only requirement for this certification was experience in treating addiction as a licensed psychologist for at least one year. ${ }^{97}$ As of January 1, 2011, the APA Practice Organization (APAPO) discontinued accepting new applications for the Certificate of Proficiency in the Treatment of Alcohol and Other Psychoactive Substance Use Disorders but continues to support the credential for previously-certified psychologists who maintain their certification by engaging in appropriate continuing education. The decision to discontinue the certification program for new applicants was based in part on insufficient interest in obtaining the credential by licensed psychologists. ${ }^{98}$

Mental Health Counselors/Therapists. All states license mental health counselors ${ }^{99}$ and, with the exception of the "professional counselor" license in Illinois, ${ }^{\dagger \dagger}$ all states require at least a master's degree in counseling or a related field.

The National Board for Certified Counselors (NBCC) offers certification to become a National Certified Counselor (NCC); in addition, three optional national specialty certifications are offered on the graduate level to become a school counselor, clinical mental

\footnotetext{
$\S$ Although these content areas constitute 40 percent of the test, the exact proportion of substance use/addiction content is not specified.

** There are a number of different ways that state agencies have recognized the certificate. Most states use it as one way for psychologists to be listed as registered addiction treatment providers or as one way to qualify them as clinical supervisors. The significance of these categories depends on the state's regulations.

†† IL requires only a bachelor's degree. IL also has a “clinical professional counselor” license which requires a master’s degree.
} 
health counselor and master addictions counselor. ${ }^{*} 100$ The National Counselor Examination for Licensure and Certification (NCE), administered by NBCC, is required for those seeking certification. The NCE exam does not appear to contain substance use/addictionrelated content; ${ }^{101}$ however, the specialty certification exam administered by the NBCC for the master addictions counselor exam focuses on addiction. ${ }^{102}$ The master addictions counselor certification requires (1) a minimum of 12 semester hours of graduate coursework in addiction content or 500 continuing education hours in addiction content; (2) three years of supervised experience as an addiction counselor at a minimum of 20 hours per week (two of the three years must have been completed after obtaining the master's degree in counseling); and (3) a passing score on the examination for master addictions counselor. ${ }^{103}$ National certification is voluntary; however, in some states, counselors are exempt from taking a state certification exam if they pass the national exam. $^{104}$

Professional counselors are trained to provide treatment for mental, emotional and behavioral disorders and tend to encounter in their practice individuals who engage in risky substance use or have addiction. ${ }^{105}$ Although risky substance use and addiction may be addressed under broad course content areas, CASA Columbia's review found few explicit state licensing requirements that mandate a specific number of addictionrelated education hours in their curriculum guidelines. ${ }^{\dagger}$

The Council for Accreditation of Counseling and Related Educational Programs (CACREP), which accredits master's programs in numerous counseling specialties, requires all programs to provide students with an understanding of "theories and etiology of addictions and addictive behaviors, including strategies for

\footnotetext{
${ }^{*}$ The NCC is a pre- or co-requisite for the specialty credentials.

${ }^{\dagger}$ More frequently, states mention substance use and addiction as topics that may be addressed under content areas such as human growth and development or that may be taken as electives or continuing education courses.
}

prevention, intervention and treatment.” The CACREP specifically includes additional substance-related content in the curricular requirements for clinical mental health counseling; marriage, couple and family counseling; school counseling; and student affairs and college counseling programs. ${ }^{106}$ Surveys of these programs reveal, however, that the majority of CACREP-accredited master's programs in counseling offer elective courses but do not require substance use/addictionrelated course work. When asked to identify which courses teach addiction-related content, CACREP programs typically cite supervised clinical settings (practicums and internships), where students encounter patients who engage in risky substance use or have addiction, rather than classroom-based courses. Most counseling degree programs do not provide any addiction education prior to the clinical experience. ${ }^{107}$

Marriage and Family Therapists. Marriage and family therapists either must have a master's degree, a doctoral degree or three to four years of post-graduate clinical training for licensure. ${ }^{108}$ According to CASA Columbia's review, few states have explicit addiction-related education requirements (such as minimum number of hours of addiction content); most states that mention addiction include it as an optional or suggested topic or as a topic eligible for continuing education credit. The national licensing exam in Marital and Family Therapy is administered by the Association of Marital and Family Therapy Regulatory Boards (AMFTRB). In drafting the Examination in Marital and Family Therapy, the Examination Advisory Committee draws from a list of the 56 knowledge areas required for entry-level practice in marital and family therapy, which includes two areas related to substance use and addiction. $^{\ddagger 109}$

Social Workers. Some social workers function as mental health professionals, providing counseling services. Social workers must earn,

\footnotetext{
‡ The substance-related areas in the AMFTRB are "effect of substance abuse and dependence on individual and family functioning" and "addiction treatment modalities.”
} 
at a minimum, a bachelor's degree in social work (BSW); however, a master's degree in social work (MSW) often is required for positions in health care settings or to provide clinical services. ${ }^{110}$

The Association of Social Work Boards (ASWB), which regulates the profession, administers four separate examinations: the bachelor's exam, master's exam, advanced generalist exam and clinical exam. ${ }^{*}$ Each state determines the examination required for licensure.

More than 25 percent of patients whom social workers come into contact with either have a primary or secondary diagnosis of addiction. ${ }^{111}$ While addiction-related content is mentioned explicitly as a topic area that may be included in the bachelor- and clinical-level licensing examinations, ${ }^{112}$ CASA Columbia's review identified just two states--California and Alaska-that require social workers to complete coursework in addiction. ${ }^{\dagger 113}$

The National Association of Social Workers offers an optional Certified Clinical Alcohol, Tobacco, and Other Drugs Social Worker (CCATODSW) certification for master's level social workers with 180 hours of addictionrelated education and two years of paid, supervised post-graduate work experience in both clinical social work and addiction counseling. ${ }^{\ddagger 114}$

\footnotetext{
* In general, the bachelor's exam is designed for those with a BSW and no post-degree experience; the master's exam is for those with an MSW and no postdegree experience; the advanced generalist exam is for those with an MSW and at least two years of postdegree experience in non-clinical settings; and the clinical exam is for those with an MSW and at least two years of post-degree experience in direct clinical practice settings.

${ }^{\dagger}$ In CA, the applicant must obtain at least 15 hours of addiction-related training. In AK, licensed social workers must complete 45 hours of continuing education, including six hours of addiction-related education during their bi-annual license renewal cycle.

$\ddagger$ Nationwide data on the number of practicing social workers with this certification are not available.
}

\section{Acupuncturists}

Acupuncture sometimes is used as an alternative/complementary treatment for addiction. Estimates are that several hundred addiction treatment clinics in the U.S. and Europe offer auricular acupuncture, a procedure that targets chronic diseases, including addiction through needle therapy of the ear (typically used to treat cocaine addiction). ${ }^{115}$ Despite its widespread use, there is very little research supporting the efficacy of auricular acupuncture for the treatment of addiction. ${ }^{116}$

CASA Columbia's review found that most states require individuals who practice acupuncture to be licensed. Licensing requirements include earning a degree from an accredited acupuncture school and, except in four states, professional certification or examination by the National Certification Commission for Acupuncture and Oriental Medicine (NCCAOM). In Alabama and North Dakota, which do not have licensure requirements for acupuncturists, only physicians, osteopaths or chiropractors may practice acupuncture as permitted by their scope of practice or via specialty certification. ${ }^{\S 117}$

CASA Columbia's review identified several states that require specialized training and certification to practice auricular acupuncture. In California, acupuncture training programs are required to teach both auricular acupuncture and principles of public health, including treatment of addiction. ${ }^{118}$ Acupuncturists certified through NCCAOM for Acupuncture and Oriental Medicine are not required explicitly to have addiction-related training, although substancerelated knowledge is in the exam content for the "diplomate in acupuncture" certification. ${ }^{119}$ The National Acupuncture Detoxification Association (NADA) ${ }^{120}$ certifies Acupuncture

\footnotetext{
$\S$ In KS, acupuncturists may practice under the supervision of a medical doctor, osteopath or chiropractor. In OK, medical doctors may practice acupuncture. In SD, chiropractors may practice acupuncture. WY has no laws or regulations permitting or prohibiting acupuncture practice.
} 
Detoxification Specialists (ADS) and has its own set of training guidelines. ${ }^{* 121}$

\section{Addiction Counselors}

Addiction counselors, also known as Substance Abuse Counselors (SACs), Credentialed Alcoholism and Substance Abuse Counselors (CASACs) or Alcohol and Drug Counselors (ADCs), make up the largest share of full-time addiction treatment provider staff in facilities providing addiction treatment in the United States. ${ }^{122}$

Addiction counselors can be licensed, certified or both. ${ }^{123}$ CASA Columbia's review found that 37 states require addiction counselors either to be licensed or certified, although even these states may provide important exemptions. ${ }^{\dagger}$

The review also found that to become a licensed addiction counselor in a given state, generally one must meet the state's minimum education and training requirements and pass a designated exam. There is no one national licensing exam used to measure competencies. ${ }^{124}$ The state education qualifications for licensure vary greatly. ${ }^{\ddagger 125}$ According to CASA Columbia’s review, more than half of states that offer licensing require a master's degree for the

\footnotetext{
* The NADA training guidelines include 30 hours of didactic classroom training (e.g., history of the profession; the NADA protocol which defines the five points where needles are to be applied; techniques; clean needle; integration with larger treatment program; ethics) and 40 hours of hands-on clinical work (content not specified), supervised by a licensed acupuncturist.

${ }^{\dagger}$ Such as supervised students engaged in practicum, licensed health care professionals (e.g., MDs, RNs, psychologists) working within their scope of profession, religious leaders working within the scope of their ministerial duties, government employees working within the scope of their employment, and short-term services provided by non-residents who are certified in another state.

₹ There are several tiers of addiction counselors--such as Certified Alcohol and Drug Abuse Counselor (CADAC) or Substance Abuse Counselor (SAC)-each with its own proficiency requirements (based on education and experience). The highest level usually requires a master's or doctorate degree.
}

highest level of licensure, although several states offer the highest level of licensure to individuals who only have a bachelor, associate or high school degree. A 1998 survey similarly found that 56 percent of licensed addiction counselors have at least a master's degree. ${ }^{126}$

A variety of state boards and non-governmental organizations are designated with responsibility for certifying addiction counselors. ${ }^{127}$ Similar to the licensure process, certification recognizes that addiction counselors have met what the organization deems to be minimum standards of education and clinical experience. ${ }^{128}$ In some states addiction counselors must be certified while in other states certification is voluntary. ${ }^{129}$ Some state certification boards defer to standards established by professional membership or credentialing organizations, such as the International Certification and Reciprocity Consortium (IC\&RC) or NAADAC, the Association for Addiction Professionals, ${ }^{\S} 130$ which do not stipulate a particular required degree for the entry level certification. ${ }^{131}$

The IC\&RC establishes minimum education and training standards for the credentialing of addiction counselors--each state certification board that is a member of the IC\&RC consortium either may follow the minimum standards or impose higher standards. ${ }^{132}$ The focus of the minimum requirements for the IC\&RC Alcohol and Drug Counselor (ADC) certification is more on clinical work experience than on classroom-based education: requirements include 270 hours of education in the eight domains of addiction counseling, 300 hours of supervised practical experience in the eight domains, 6,000 hours of supervised

\footnotetext{
$\S$ The National Association for Alcoholism and Drug Abuse Counselors (NAADAC) changed its name in 2001 to NAADAC, the Association for Addiction Professionals to reflect the increasing number of addiction professionals who address forms of addiction involving behaviors other than substance use, such as gambling.

${ }^{* *}$ The eight domains include: clinical evaluation; treatment planning; referral; service coordination; counseling; client, family and community education; documentation; and professional and ethical responsibilities.
} 
professional work experience specific to the eight domains and successful completion of a written examination. ${ }^{133}$

NAADAC, the Association for Addiction Professionals offers three levels of certification for addiction professionals based on experience and education. ${ }^{134}$ Like the IC\&RC requirements for the National Certified Addiction Counselor, Level I (NCAC I), the focus primarily is on clinical work experience rather than on classroom-based education. Requirements include: (1) a current state certificate or license as an addiction counselor; (2) 270 contact hours of addiction counseling training; (3) three years of full-time work experience or 6,000 hours of supervised experience as an addiction counselor; and (4) successful completion of a written examination. ${ }^{135}$

These certification requirements reflect a great reliance on apprenticeships as the training model for addiction counselors. ${ }^{136}$

CASA Columbia's state-by-state analysis of regulations and statutes found that 14 states do not require addiction counselors in all settings to be licensed or certified. ${ }^{*}$ Six states do not mandate any degree to become credentialed as an addiction counselor; ${ }^{\dagger}$ in 14 states, an individual may become licensed or certified as an addiction counselor with the minimum education requirement of a high school diploma or a GED; an associate's degree is required in 10

\footnotetext{
* In AR, counselors who are not licensed or certified must register with the state; CT, FL and NY do not require supervised staff and MA does not require staff in licensed facilities to be licensed or certified; ID, MS, OR, and SD require licensing or certification of counselors only in certain facilities (e.g., licensed facilities or those that receive public funds); HI insurance law requires clinical licensure and certification to qualify for reimbursement; in $\mathrm{MO}$, staff who are not licensed or certified have a limited scope of practice; in WV, only counselors in opioid treatment facilities must be certified or licensed; and $\mathrm{AK}$ and $\mathrm{NH}$ do not appear to require counselors to be licensed or certified.

${ }^{\dagger}$ In states where no degree is required, certification requirements typically include 270 hours of education in addiction counseling.
}

states, a bachelor's degree is required in six states and a master's degree is the minimum requirement in only one state. ${ }^{\ddagger}$

CASA Columbia's survey of New York State addiction treatment staff providers found that 35.0 percent had a graduate degree, 27.7 percent had a bachelor's degree, 29.9 percent had some college or an associate's degree and 6.6 percent had only a high school or GED degree. ${ }^{137}$

In spite of the limited education and training requirements and the apprenticeship model of training, the essential practice dimensions of addiction counseling are defined as including clinical evaluation; treatment planning; referral; service coordination; and individual, group, family and couples counseling ${ }^{138}$--practices that would seem to require far more extensive and structured clinical training than the field of addiction counseling requires.

\section{Licensure, Certification and Accreditation Requirements for Addiction Treatment Programs and Facilities}

Just as licensing and certification requirements are insufficient to assure that those providing addiction treatment have the knowledge and skills to do so, government and professional oversight of addiction treatment facilities and programs is insufficient to insure that patients receive clinically-indicated, quality care.

Regulatory oversight of health care facilities may include state licensure, certification ${ }^{\S}$ and/or accreditation by a national accrediting organization. Licensing and certification standards may include:

\footnotetext{
${ }^{\ddagger}$ CASA Columbia reviewed the minimum education requirements for certification/ licensure in each state. In many states, counselors who meet only the minimum education requirements must be supervised.

${ }^{\S}$ In the case of opioid maintenance therapy.
} 
- Professional staffing requirements to assure that clinical staff are adequately qualified to implement evidence-based practices;

- Requirements related to services to be delivered; or

- Requirements that treatment services actually help patients achieve desired outcomes of improved health and functioning. ${ }^{139}$

Like health care facilities, addiction treatment programs* are subject to state licensing requirements; however, for addiction treatment programs, these requirements typically are set by state agencies separately charged with authority to oversee addiction services rather than by the agencies responsible for regulating health care facilities. ${ }^{140}$ CASA Columbia's review of licensing and certification requirements for addiction treatment facilities and programs ${ }^{\dagger}$ found that the nature and extent of these regulations vary significantly among states and that certain addiction treatment programs (e.g., many state-run and religious programs) are exempt entirely from state regulation. In facilities that are subject to state regulation, the staffing requirements do not consistently mandate the involvement of professionals who are capable of providing a full range of effective interventions (including pharmaceutical and psychosocial therapies), services rarely are required to reflect best practices and quality

\footnotetext{
${ }^{*}$ Including (1) organizations or facilities that provide non-hospital based residential or outpatient addiction treatment, or opioid replacement therapy (e.g., methadone maintenance clinics) and (2) hospitalbased inpatient and outpatient addiction treatment programs.

${ }^{\dagger}$ This entailed online reviews and analyses of publicly available documents related to federal and state laws and regulations (in all 50 states and the District of Columbia), including the use of the Lexis/Nexis database to supplement information related to state laws and regulations available on the Internet. Because licensing and certification requirements are found in a wide variety of laws and regulations and can change on a state by state basis, findings from this review cannot be guaranteed to be complete and current.
}

assurance requirements seldom stipulate that patient outcome data be collected, analyzed or made available to the public. For no other health condition are such exemptions from routine governmental oversight considered acceptable practice.

\section{State Licensing Requirements}

Health care facilities such as hospitals and ambulatory care (outpatient) clinics are licensed by state departments of health, whereas the licensing authority for addiction treatment facilities and programs is distributed across a variety of governmental agencies and the content of statutes and regulations varies greatly by state. ${ }^{141}$

Most (81.9 percent) addiction treatment facilities and programs that voluntarily participate in a national survey of such providers ${ }^{\ddagger}$ are licensed by the state agency responsible for addiction services (which varies from state to state): 41.4 percent of facilities are licensed by the state department of health; 35.3 percent by the state mental health department; and 7.1 percent by the hospital licensing authority. ${ }^{\S 142}$

Despite the abundant evidence that addiction and mental health conditions co-occur at very high rates and are best addressed in an integrated manner, in some states, treatment facilities and programs cannot be dually licensed to provide both mental health and addiction treatment services. ${ }^{143}$

\footnotetext{
‡ The National Survey of Substance Abuse Treatment Services (N-SSATS) of the Substance Abuse and Mental Health Services Administration (SAMHSA) is a national survey of public and private addiction treatment programs and facilities in the United States, excluding treatment programs in jails and prisons. Participation is voluntary and the survey does not represent all treatment providers.

$\S$ These categories are not mutually exclusive. More than half (57.7 percent) of the addiction treatment facilities that participate in the survey are not licensed either by the state department of health or the hospital agency--the two departments responsible for licensing health care facilities.
} 
A significant number of states exempt state agency-run programs (e.g., in the justice and welfare systems) from licensure. A national study of state-run treatment programs found that, excluding programs provided or operated by the state addiction treatment agencies, only about one-half of state agencies that operate treatment programs require the programs they operate to meet state licensure standards: ${ }^{144}$

- 38 states report that the department of corrections operates treatment programs, but only 18 of these require treatment programs operated by the corrections departments to meet state licensing standards. ${ }^{*} 145$

- 13 states report that their juvenile justice system operates treatment programs, with only six states requiring these programs to adhere to state licensing standards. ${ }^{146}$

- Seven states identify their departments of child and family services as operating treatment programs, with only four requiring adherence to state licensing standards. ${ }^{147}$

- 18 states identified 21 other government agencies (e.g., departments of public welfare, mental health and criminal justicerelated agencies other than the department of corrections) that operate treatment programs, but in only 11 states are these programs required to adhere to state licensing standards. ${ }^{148}$

CASA Columbia also found substantial inconsistencies in the regulation of faith-based programs that offer addiction-related services. While most states do not appear explicitly to address faith-based programs in their laws or regulations, some states explicitly exempt such programs from regulation. $^{\dagger}$

\footnotetext{
* A few states qualified the licensing requirement, saying that it only applied to certain types of facilities (e.g., one state noted that the requirement was for residential treatment facilities only).

${ }^{\dagger}$ Exemptions appear to apply to programs that solely are spiritual or religious in nature.
}

\section{Federal Regulatory Requirements}

The federal government does not regulate addiction treatment facilities or programs, with the exception of those that provide opioid maintenance therapy. ${ }^{149}$ However, the federal government imposes certain conditions of participation on qualifying programs and facilities through federal health insurance programs, including Medicare, Medicaid, TRICARE for members of the active duty military and the veterans' health insurance program (CHAMP VA)--whether they are mainstream health care or addiction treatment providers. ${ }^{150}$ Like state licensing laws, these provisions include requirements regarding staffing, services and quality assurance mechanisms.

To be eligible to receive Medicare/Medicaid reimbursement, most treatments must be provided by or under the supervision of a physician. ${ }^{151}$ State Medicaid programs have the option of covering addiction treatment under the Medicaid rehabilitation option, Medicaid clinic services, targeted case management and Medicaid managed care waiver programs. The rehabilitation option is the most flexible for treatment coverage in that it does not require services to be medically provided or supervised. ${ }^{152}$ The Medicare/Medicaid Conditions of Participation impose extensive requirements on participating facilities including staffing, services and quality assurance provisions. $^{\ddagger 153}$

\footnotetext{
‡ In addition, under the Government Performance Results Act (GPRA) of 1993, federally-operated programs or those that receive federal funds for addiction treatment are required to meet certain reporting requirements.
} 
All opioid maintenance therapy facilities are required by federal law to obtain certification from the U.S. Department of Health and Human Services' Substance Abuse and Mental Health Services Administration (SAMHSA) demonstrating compliance with established standards for opioid maintenance therapy programs. ${ }^{154}$ It is a pre-requisite of certification that a program be accredited by an organization approved by SAMHSA. ${ }^{*} 155$

\section{Accreditation Requirements}

In lieu of or in addition to state licensure, addiction treatment facilities and programs may be accredited by a national accreditation organization. While adopting these standards largely is voluntary, ${ }^{156}$ some states grant licenses to programs that have been accredited, ${ }^{157}$ such that the program is deemed to have met the state licensure requirements because it has been approved by a national accrediting body. ${ }^{\dagger 158} \mathrm{By}$ granting “deemed status" licenses to accredited programs, the state essentially delegates to the accrediting body its responsibility for ensuring that the facility or program meets state licensure requirements. ${ }^{159}$ Accreditation standards are more detailed than state licensing requirements and while some require facilities and programs to use evidence-based practices or to analyze patient outcomes, ${ }^{160}$ not all do. Furthermore, accreditation standards do not require staff to be qualified to provide a full range of evidencebased treatment, and accreditation does not necessarily ensure that quality care is delivered. ${ }^{161}$

The accreditation process entails a review of the facility's or program's structure and operational practices and evaluation of the organization's clinical care processes and outcomes to confirm that they comply with standards set by the

\footnotetext{
${ }^{*}$ To be approved by SAMHSA to accredit opioid maintenance therapy programs, an accrediting organization must demonstrate how its accreditation process will ensure that programs meet the standards described in federal regulations.

${ }^{\dagger}$ Select examples of states that allow providers to have deemed status for state license or require accreditation for Medicaid include: AL, AK, AZ, AR, CT and DE.
}

accrediting body. ${ }^{162}$ Accreditation generally is considered a higher standard of oversight than state licensing, in that it incorporates "ideal or optimum” standards rather than minimum standards, and focuses on continuous quality improvement. ${ }^{163}$

A 2007 study of the association between accreditation and nine indicators of quality care (categorized as staff-to-patient ratio, treatment comprehensiveness and treatment sufficiency) in 566 outpatient addiction treatment programs found only several correlations between accreditation and quality of care measures. ${ }^{164}$ Another study that examined the adoption of evidence-based dosing practices in methadone maintenance programs found that voluntary accreditation was strongly related to adoption of these practices; however, after accreditation became mandatory in 2000, this relationship disappeared. The authors speculated that programs that voluntarily seek accreditation tend to be resource-rich (in funds, staff and training) and more motivated to improve their quality of care, and therefore more likely to adopt evidence-based practices. ${ }^{165}$

The five organizations that accredit addiction treatment programs and facilities in the United States are:

- The Commission on Accreditation of Rehabilitation Facilities (CARF). CARF is an independent, nonprofit organization that offers accreditation for addiction treatment programs and facilities and integrated addiction/mental health treatment programs and facilities; it is the largest accreditor of addiction treatment programs and facilities. ${ }^{166}$ CARF accreditation is widely accepted by state licensing agencies toward the fulfillment of licensing requirements for addiction treatment programs and facilities. ${ }^{167}$

- The Joint Commission on Accreditation of Healthcare Organizations (Joint Commission). The Joint Commission is the largest accreditor of all health care services in the U.S. ${ }^{168}$ and the second largest accreditor of addiction treatment programs 
and facilities. ${ }^{169}$ Joint Commission accreditation is widely accepted by state licensing agencies toward the fulfillment of licensing requirements for addiction treatment programs and facilities. ${ }^{170}$

- The Council on Accreditation (COA). COA is an international accrediting organization. Originally known for accrediting family and children services agencies, COA currently accredits numerous services, including addiction treatment. ${ }^{171} \mathrm{COA}$ accreditation is accepted by fewer states (approximately 10) relative to accreditation by CARF or the Joint Commission. ${ }^{172}$

- The National Committee for Quality Assurance (NCQA). NCQA is a nonprofit health care quality improvement organization that accredits health care organizations. ${ }^{173}$ As of 2005, Florida and Michigan were the only states that accepted NCQA accreditation as fulfilling licensing requirements for addiction treatment programs and facilities. ${ }^{174}$

- The National Commission on Correctional Health Care (NCCHC). The NCCHC is the only organization authorized by the federal government to accredit opioid maintenance therapy programs that specialize in correctional settings. ${ }^{175}$

Of the 13,339 addiction treatment programs and facilities voluntarily responding to a national survey: ${ }^{\dagger}$

- $\quad 56.9$ percent (7,595 facilities) are not accredited by CARF, the Joint Commission, COA or NCQA;

- 21.8 percent (2,909 facilities) are accredited by CARF;

\footnotetext{
* The most recent data available. More states may currently accept the accreditation.

${ }^{\dagger}$ The National Survey of Substance Abuse Treatment Services (N-SSATS). Accreditation by the NCCHC was not measured in the survey. The categories are not mutually exclusive, as programs and facilities may have multiple accreditations.
}

- $\quad 19.2$ percent (2,556 facilities) are accredited by the Joint Commission;

- 5.0 percent (664 facilities) are accredited by COA; and

- 2.8 percent (371 facilities) are accredited by NCQA. ${ }^{176}$

\section{Professional Staffing Requirements}

In licensing standards for medical facilities, physicians generally are responsible for patient care. $^{\ddagger 177}$ In contrast, addiction treatment may be provided not only by people who lack medical training, but by individuals with no license and no graduate education or clinical training at all. $^{178}$

State licensing laws, federal requirements and accreditation standards regarding which professionals may provide and supervise addiction treatment services in facilities and programs vary significantly. While some states require addiction treatment programs to have a medical director who is an M.D. on staff, others impose minimum education/training requirements on directors, supervisors and staff. Accreditation standards do not recognize addiction treatment generally as requiring medical care; rather, they only require physician oversight for certain services, deferring to states with regard to which professionals are qualified to provide addiction treatment. Similarly, federal requirements stipulate that some types of care must be provided under the supervision of a physician, while services such as "rehabilitation services" do not require supervision by a physician. ${ }^{179}$

State Staffing Requirements. In licensing regulations for addiction treatment facilities and programs, states typically specify:

\footnotetext{
${ }^{\ddagger}$ Dentists and other health professionals may be responsible for services they are qualified to perform or supervise.
} 
- A wide range of "qualified providers"* (e.g., addiction counselors, social workers, nurses) who may offer and/or supervise treatment ${ }^{\dagger}--$ most of whom are not qualified to provide medical care or are not trained in addiction;

- The total proportion of patient care that must be provided by “qualified providers"; and

- The ratio of staff to patients. ${ }^{180}$

Although state licensing requirements allow an array of practitioners to provide addiction treatment, the licensing requirements for addiction treatment facilities and programs typically state that "medical services" must be provided or supervised by a physician; however, medical services are defined as detoxification, opioid replacement therapy or the assessment, diagnosis and treatment of co-occurring medical or mental health conditions, not as addiction treatment itself. ${ }^{181}$

According to CASA Columbia's review, 43 states require non-hospital-based ${ }^{\ddagger}$ outpatient and residential addiction treatment programs to employ (at least part time) a physician to serve either as medical director or on staff; however, this requirement applies primarily to programs that provide opioid maintenance therapy which, by federal regulation, must be supervised by a physician. ${ }^{182}$ Few states require non-hospitalbased programs that do not provide opioid maintenance therapy to have a physician serving as medical director or on staff; 10 states require residential treatment programs to have a physician either as a medical director or on staff and eight states require the same of outpatient treatment programs. Without a physician as medical director or on staff, addiction treatment programs cannot provide a full range of evidence-based treatment services including

\footnotetext{
* Qualified providers are those who, under state law, may provide addiction treatment.

${ }^{\dagger}$ See section on Licensing and Credentialing Requirements for Individuals who Provide Addiction Treatment, above.

${ }^{\ddagger}$ Hospital licensing requirements require physician supervision of patient care; however, the majority of addiction services offered are not hospital based.
}

pharmaceutical therapy and treatment of cooccurring health conditions.

Consistent with these requirements, addiction treatment services typically are not required to be supervised by a physician, other than for the provision of narrowly-defined "medical services." While states may require that addiction services be overseen by a clinical or program director, that position is not required to be filled by a physician.

Approximately two-thirds of states in CASA Columbia's review specify that residential treatment programs (31 states) and outpatient treatment programs (29 states) must have a program or clinical director. Among the 21 states that specify the minimum educational/ training requirements for this position, few have particularly high standards:

- Eight states require a minimum of a master's degree;

- Six states require the director to be a licensed or certified addiction counselor;

- Four states require a minimum number of years of experience; ${ }^{\S}$

- One state requires a bachelor's degree;

- One state requires an associate’s degree; and

- One state simply requires the person to demonstrate competence to perform certain services.

A national survey of treatment professionals conducted in 1998 found that 60.6 percent of individuals who were responsible for supervising clinical services in addiction treatment facilities had graduate degrees and 77.5 percent of these supervisors were certified or licensed as addiction/mental health professionals. Among facility directors, 64.0 had a graduate degree and 68.9 percent were

\footnotetext{
$\S \mathrm{AZ}$ additionally specifies that the individual must have a high school diploma or GED.
} 
certified or licensed as addiction/mental health professionals. ${ }^{183}$

CASA Columbia's survey of directors* of addiction treatment programs in New York State found that 67.5 percent had a graduate degree, 16.9 percent had a bachelor's degree, 14.5 percent had some college or an associate's degree and 1.2 percent had only a high school or GED degree. ${ }^{184}$

Although state licensing laws sometimes dictate that clinical supervisors, such as program or medical directors, have special training (including certification) or experience in addiction treatment, ${ }^{185}$ they generally allow facilities more flexibility to determine the necessary qualifications, including level of experience, education or training, as well as the composition of other clinical staff. ${ }^{186}$

Federal Staffing Requirements. In states that provide addiction treatment using Medicaid funding, hospital and clinic services must be provided under the direction of a physician, ${ }^{187}$ but if states choose to provide services under the optional benefits category of rehabilitative care, they are exempt from this requirement. ${ }^{188}$ If services are required to be provided under the direction of a physician, the facility physician is not required to be on staff full time, but must be present for sufficient time to provide medical direction, care and consultation in accordance with accepted principles of medical practice. The facility and the staff providing care also are required to hold appropriate state licenses, certifications or registrations. ${ }^{189}$

The federal regulations for opioid maintenance therapy programs specify that each program must employ a medical director and that all providers, including addiction counselors, comply with the credentialing requirements of their respective profession. ${ }^{190}$

\footnotetext{
* Some of the program directors also may have served as clinical supervisors, but the survey did not distinguish between the two roles.
}

Accreditation Requirements Regarding Staffing. ${ }^{\dagger}$ CARF standards require that programs that offer detoxification, inpatient care, partial hospitalization, residential treatment serving "persons with medical needs" or opioid maintenance therapy have a medical director who is a physician. Organizations that seek elective accreditation for assertive community treatment $(\mathrm{ACT})^{\ddagger}$ services must have a physician on staff. Other services do not require physician supervision. ${ }^{191}$

While CARF requires staff to be licensed or certified by a credentialing body that uses a competency-based process, ${ }^{\S}$ it leaves the determination of which practitioners are qualified to provide addiction treatment to state laws and professional associations, thus allowing for great variation in education and training requirements. ${ }^{192}$

The Joint Commission allows programs to define the qualifications required for staff to perform their job and requires staff who provide care to be licensed, certified or registered "in accordance with the law." Only opioid maintenance therapy programs must have a physician on staff; this person must have experience in addiction medicine, including medication-assisted treatment. ${ }^{193}$

\section{Treatment Service Requirements}

Licensed health care facilities must deliver care that meets standards of medical practice; state regulations tend to defer to health care providers--for whom there are well-delineated standards of education and training in health care practice--to determine appropriate medical practice (e.g., which services to provide and how to provide them). ${ }^{194}$ In contrast, those who provide addiction treatment often have minimal

\footnotetext{
${ }^{\dagger}$ CASA Columbia reviewed the standards of the two largest accreditors of addiction treatment programs-CARF and the Joint Commission.

${ }^{\ddagger}$ As described in Chapter VI, ACT is a treatment approach for patients with co-occurring addiction and mental health conditions.

${ }^{\S}$ Focused on the ability to demonstrate adequate skills, knowledge and capacity to perform a specific set of job functions.
} 
education or training in addiction science, and there are no accepted standards of practice that apply across facilities and programs. ${ }^{195}$

While most states regulate addiction treatment services, they treat these services as completely separate and distinct from medical care, ${ }^{* 196}$ and state regulations regarding addiction treatment service requirements vary considerably and tend to be vague. Accreditors specify required services in greater detail than state licensing regulations, as do the federal requirements for facilities providing opioid maintenance therapy.

State Services Requirements. According to CASA Columbia's review, the majority of states regulate the content of addiction services by requiring adherence to specific guidelines, but the extent of the regulation varies considerably. ${ }^{\dagger}$ In addition to listing the types of required services, the frequency and timing of services are specified in some cases; for example, patients receiving detoxification must receive a psychosocial assessment within 72 hours of admission ${ }^{197}$ or patients in intensive outpatient programs must receive individual or group therapy for a minimum of six hours over at least two days a week. ${ }^{198}$

More than 30 states require addiction treatment programs and facilities to utilize the American Society of Addiction Medicine (ASAM) patient placement criteria, which guide providers in matching patient needs to specific treatment services and determining the appropriate level of care for patients. ${ }^{199}$

State regulations related to addiction treatment services tend to specify the categories of services that addiction facilities and programs must offer--such as individual, family and group

\footnotetext{
* Except for opioid maintenance therapy and some detoxification services, which are considered medical care and must be supervised by a physician.

${ }^{\dagger}$ Regulations were considered to provide detailed guidelines if they included, for example, specific pharmaceutical dosing schedules or specific assessment criteria (e.g., including onset/duration of problems, previous interventions/outcomes, health history/current medical care needs and daily living skills).
}

counseling; alcohol and other drug education; activity therapy and social services ${ }^{200}$--but are not particularly specific in requiring that the services follow evidence-based practices. A survey conducted in 2006 found that three states--Oregon, North Carolina and Alaska-have enacted legislation that mandate or encourage the use of evidence-based practices in addiction treatment programs; only Oregon mandates programs to implement evidencebased practices under penalty of fiscal sanctions. ${ }^{201}$ However, the reach of the Oregon law is limited to programs and facilities that are funded by the state. ${ }^{\ddagger 202}$

Federal Services Requirements. The federal regulations for opioid maintenance therapy programs include specific admission criteria, services and procedures for patient care. Admission is limited to patients who meet clinical diagnostic criteria for opioid dependence $e^{\S}$ and the person must currently have addiction and must have become addicted within one year before treatment admission. ${ }^{* *} \mathrm{~A}$ physician must perform a full medical examination before admission. Opioid maintenance therapy programs must provide medical care, addiction counseling, vocational and educational services and other assessment and treatment services. The regulations stipulate that random testing for addictive drugs must be performed on all patients periodically; ${ }^{\text {} \dagger}$ that treatment medication dosing must be calculated by a physician familiar with the "most up-todate” labeling; and that for patients receiving

\footnotetext{
‡ The Department of Corrections, the Oregon Youth Authority, the State Commission on Children and Families and the part of the Oregon Health Authority that deals with mental health and addiction issues. $\S$ Determined using accepted clinical criteria, such as those in the DSM-IV.

** The one-year requirement may be waived for persons released from penal institutions (within six months after release), pregnant women (a program physician must certify pregnancy) or previouslytreated patients (up to two years after discharge).

${ }^{+\dagger}$ All patients are to be drug tested initially and then eight times per year for patients in maintenance treatment and monthly for persons receiving longterm detoxification treatment.
} 
methadone, the initial dose cannot exceed 30 milligrams. ${ }^{203}$

\section{Accreditation Service Requirements.}

Accreditation organizations delineate detailed specifications related to addiction services provided by facilities and programs.

CARF requires that addiction services be evidence based, explicitly stating that care should incorporate "current research, evidencebased practice, peer-reviewed scientific and health publications, clinical practice guidelines and/or expert professional consensus.” The facility must have written policies regarding service delivery including screening, referrals, medication use and control, ${ }^{*}$ discharge and follow up. ${ }^{204}$ CARF sets forth procedural requirements for the above services, as well as orientation, assessment and the development of "person-centered plans" for treatment. ${ }^{\dagger}$ CARF offers accreditation standards for each of the "core program areas" that programs and facilities may elect to have accredited, such as assertive community treatment (ACT), community integration, ${ }^{\ddagger}$ detoxification, day treatment, drug court treatment, partial hospitalization and inpatient services. Standards for the core program areas are very detailed and cover which services must be provided, by whom and how often. ${ }^{\S 205}$

\footnotetext{
* The process of physically controlling, transporting, storing and disposing of medications, including those that are self-administered by patients.

${ }^{\dagger}$ Treatment plans developed with the input of patients regarding their goals, needs, strengths, abilities, preferences, desired outcomes and cultural background.

‡ A form of treatment that provides opportunities for community participation as a means of optimizing personal, social and vocational competency in order to live successfully in a community.

${ }^{\S}$ For example, day treatment must be provided at least three hours a day, four days a week; must include three interventions from a designated list of interventions (e.g., family therapy, group counseling, individual psychotherapy, occupational therapy, alcohol and other education) plus two additional activities (nutritional/fitness/leisure/social); the program must be directed by a qualified behavioral health practitioner (a person certified, licensed, registered or credentialed by a governmental entity or
}

The Joint Commission requires facilities to have written procedures for eligibility for admission and for most services. The standards for addiction treatment programs include procedural requirements governing essential services such as screening and assessments; medical history/physical examinations; screening for physical pain, nutritional status and signs of trauma/abuse/neglect/exploitation; special provisions for services provided to vulnerable populations (children, individuals with intellectual/developmental disabilities); care that reflects patients' needs, preferences and goals; coordination of care; necessary referrals; primary physical care; and discharge/transfer that assures continuity of care. ${ }^{206}$ The standards do not specify what kind of psychosocial or pharmaceutical services should be provided, leaving much room for variability in the quality of patient care.

Because opioid maintenance therapy is considered "medical care," Joint Commission standards for opioid maintenance therapy programs are more numerous and detailed. For example, such programs must address relapse prevention in discharge planning; use DSM-IV or other accepted clinical criteria to diagnose addiction; perform an initial toxicology test and a comprehensive screening of patients' medical conditions; document current chronic or acute medical conditions; provide hepatitis A and B immunizations; manage current misuse of other drugs that are not the primary focus of treatment; and include smoking cessation as an integral component of care. ${ }^{207}$

\section{Quality Assurance Requirements}

Quality assurance requirements can be imposed by state and federal governments, by accreditors or by payers. Such requirements focus on assuring specific organizational structures,

\footnotetext{
a professional association as meeting the requirements necessary to provide mental health or alcohol and other drug services); screening and assessments must be provided; and when psychiatric services are necessary, a psychiatrist must be available 24-hours a day.
} 
processes and outcomes as ways of assuring quality. $^{208}$

Accreditation of health care facilities tends to focus on structural measures (e.g., physical plant adequacy, nursing ratios, certification of providers, availability of certain services). While patient outcomes (e.g., survival, function, quality of life) are in many ways the most important variables, they are difficult to collect and analyze. Patient outcomes may be affected by factors independent of the quality of a specific health care service delivered, including co-occurring conditions, patient compliance and lifestyle. Outcome data also are subjective and vary according to the setting and the particular instruments used to measure them. Given these barriers, quality assurance efforts tend to focus on the process of care, which examines the frequency with which interventions known to correlate with positive outcomes are performed. ${ }^{209}$

State Quality Assurance Requirements. State quality assurance requirements for addiction treatment programs and facilities are highly variable and focus mainly on process. For example, a program that is run efficiently and maintains good records would be considered a quality program regardless of patient outcomes.

CASA Columbia's state-by-state analysis of regulations and statutes found that 45 states have some type of quality assurance requirements for addiction treatment facilities and programs, although this may take the form of simply requiring the establishment of a quality improvement plan. Fewer than half of these states (21 states) have explicit requirements that patient outcome data be collected but, even for those that do, the types of data required rarely are specified. Four of the states that require the collection of patient outcome data have explicitly-defined categories of such data, but these categories focus primarily on reductions in specific types of substance use or its consequences--such as reduction or elimination of the use of illicit drugs, reduction or elimination of associated criminal activities or improvement of quality of life. The absence of other indicators linked to improved health and function or effective disease management is noticeably absent.

CASA Columbia's review found little evidence that states impose sanctions on facilities that fail to meet the outcome reporting requirements and, for those that do, the penalties are minor (e.g., a $\$ 100$ fine).

\section{Federal Quality Assurance Requirements.}

Federal quality assurance requirements vary by program. Federal Medicare/Medicaid conditions of participation address quality assurance issues by requiring utilization review committees to assure compliance with written procedures for evaluating admissions, continued care and discharges; linking treatment plans to established goals; clinical record keeping; and requiring that the group of professionals within the program or facility that develops patient care policies review and act upon recommendations from the utilization review committee. ${ }^{210}$

The federal regulations for opioid maintenance therapy programs require continual quality assurance measures which must include ongoing assessment of patient outcomes (not specified) and annual review of program policies and procedures. ${ }^{211}$

All federal agencies that operate programs or provide funds related to addiction treatment are required to report on the performance of those programs in accordance with the Government Performance Results Act (GPRA) of 1993. In order to fulfill their requirements under the Act, each federal agency must develop a strategic plan (including annual performance targets) covering each of their programs, conduct evaluations to assess how well a program is working and why particular outcomes have (or have not) been achieved and produce program performance reports based on analyses of program effectiveness. ${ }^{212}$

SAMHSA is the leading federal agency that funds addiction treatment; it administers the Substance Abuse Prevention and Treatment Block Grant (SAPTBG), a federal grant that funds state prevention, treatment, recovery 
supports and other services. ${ }^{* 213}$ SAPTBG is a major source of funding for state-run or statefunded treatment programs. ${ }^{214}$ SAMHSA's performance targets and outcome measures for this program include required data collection and reporting on the number of admissions to treatment programs receiving public funding and on outcomes, including abstinence, ${ }^{\dagger}$ employment, school enrollment, justice system involvement and housing. ${ }^{215}$

\section{Accreditation Requirements for Quality}

Assurance. CARF requires addiction treatment facilities and programs to conduct a records review to assess the quality and appropriateness of services provided, as well as a review of the patterns of service utilization. Programs must analyze patient outcomes--including abstinence; reduction in relapse, criminal activity and hospitalizations; improved psychological function; housing situation; employment status-to determine the effectiveness of services. ${ }^{216}$

Joint Commission standards for addiction treatment facilities and programs focus more on performance improvement measures by requiring programs to conduct data collection and data analysis and to identify any opportunities for improving performance (e.g., in reducing errors, incorporating patient goals or comparison with external quality measures). Only opioid maintenance therapy programs are required specifically to collect outcome data, which include measures of the use of illicit opioids, criminal involvement, health status, retention in treatment and abstinence. ${ }^{217}$

\footnotetext{
* States that receive SAPTBG funds must, among other things: spend five percent of the funds on increasing treatment capacity for pregnant women and women with dependent children by establishing new programs or expanding the capacity of existing programs, improve the process for referring individuals to treatment facilities that can provide the most appropriate treatment modality, and make continuing education available to employees of funded facilities.

${ }^{\dagger}$ Measures of client abstinence reflect no past-month use of alcohol or other drugs at discharge. Discharge is defined as the date of last service.
} 
-198- 


\section{Chapter X \\ The Evidence-Practice Gap}

The prevention and reduction of the risky use of addictive substances and the treatment of addiction, in practice, bear little resemblance to the significant body of evidence-based practices that have been developed and tested; ${ }^{*}$ indeed only a small fraction of individuals receive interventions or treatment consistent with scientific knowledge about what works. ${ }^{1}$

Providing quality care to identify and reduce risky use and diagnose, treat and manage addiction requires a critical shift to sciencebased interventions and treatment by medical professionals--both primary care providers and specialists.

Significant barriers stand in the way of making this critical shift, including an addiction treatment workforce that is largely unqualified to implement evidence-based practices; a health professional that should be responsible for providing addiction screening, interventions, treatment and management but does not implement evidence-based addiction care practices; inadequate oversight and quality assurance of treatment providers and intervention practices; limited advances in the development of pharmaceutical treatments; and a lack of adequate insurance coverage.

Recent efforts by government agencies and professional associations have begun to tackle these challenges to closing the evidence-practice gap, but are insufficient.

\section{Current Approaches to Risky} Substance Use and Addiction Are Inconsistent with the Science and with Evidence-Based Care

Unlike other public health problems and diseases that are met with qualified medical care

\footnotetext{
${ }^{*}$ See Chapters IV-VI.
} 
and cutting-edge interventions, the reigning paradigm in the health community for addressing risky substance use and addiction is to fail to address the problem directly. Instead, risky users of addictive substances are in most cases sanctioned in terms of the consequences that result--such as accidents, crimes, domestic violence, child neglect or abuse--while effective interventions to reduce risky use rarely are provided. Those with addiction frequently are referred to support services, often provided by similarly-diagnosed peers who struggle with limited resources and no medical training, to assist them in abstaining from using addictive substances. While social support approaches are helpful and even lifesaving to many--and can be important supplements to medically-supervised, evidence-based interventions--they do not qualify as treatment for a medical disease.

In short, the gap between the evidence regarding what works in interventions for risky substance use and in the treatment and management of addiction versus on-the-ground practice is wide, and nothing short of a significant overhaul in current approaches is required to bring practice in line with the evidence and with the standard of care for other public health and medical conditions.

While a wide range of trained health professionals can screen for risky use of addictive substances and provide brief interventions, physicians should be essential providers of the full range of addiction treatment services. They should provide this care in collaboration with multiple medical specialties and sub-specialties and a multi-disciplinary team of health professionals, including physician assistants, nurses and nurse practitioners and graduate-level clinical mental health professionals (psychologists, social workers, counselors).

Given the prevalence of addiction in society and the extensive evidence regarding how to identify, intervene and treat it, continued failure to do so signals widespread system failure in health care service delivery, financing, professional education and quality assurance. It also raises the question of whether the low levels of care that addiction patients usually do receive constitutes a form of medical malpractice.

\section{Patient Education, Screening, Brief Interventions and Treatment Referrals}

Despite the documented benefits of screening and early intervention practices, ${ }^{*}$ medical and other health professionals' considerable potential to influence patients' substance use decisions, and the long list of professional health organizations that endorse the use of such activities, most health professionals do not educate their patients about the dangers of risky substance use or the disease of addiction, screen for risky substance use, conduct brief interventions when indicated, treat the condition or refer their patients to specialty care if needed. $^{2}$

Since the 1950s, screening has been considered an important element of general health care and as early as 1968, the World Health Organization (WHO) laid out the principles of early disease detection. ${ }^{3}$ Based on those principles, risky substance use and signs of addiction are highly conducive to screening by general health practitioners: they are significant health problems with well-understood natural histories, there are non-invasive tests and easily-detected symptoms and early interventions result in favorable outcomes. ${ }^{4}$ Unfortunately, there is a considerable gap between what current science suggests constitutes risky substance use and the thresholds set in some of the most common screening instruments for determining that an individual meets criteria for risky substance use and is in need of intervention (see Chapter IV).

Medical and other health professionals are in ideal positions to educate patients, conduct screening and brief interventions and refer patients to treatment. The vast majority of adults (82.2 percent) and children (92.1 percent) had regular contact with a health professional in $2010 .^{5}$ There are many venues where health professionals can conduct patient education, screening and brief interventions with relative ease and most patients would be receptive to

\footnotetext{
${ }^{*}$ See Chapter IV.
} 
these practices. ${ }^{6}$ These include primary care medical offices, dental offices, pharmacies, school-based health clinics, mental health centers and clinics, emergency departments and trauma centers, hospitals or encounters with the justice system due to substance-involved crimes. ${ }^{7}$

Yet, there is no evidence to suggest that medical and other health professionals routinely avail themselves of these opportunities. ${ }^{8}$ To the contrary, a 2000 CASA Columbia survey found that only 32.1 percent of physicians regularly do even one of the following: administer a health history form to patients at least annually with one or more substance use questions; administer a screening instrument to detect the risky use of one or more substances; discuss substance use with pregnant patients; or "almost always" ask patients about their substance use when they suspect a patient has a problem. ${ }^{9}$

...Primary care physicians do not routinely provide any comprehensive screening for substance use disorders... [When they do provide treatment referrals, however, some encounter] addiction treatment services as a "black hole"... They are not informed of patient progress, treatment completion or non-completion or recommendations for continuing care. This contrasts significantly with referrals to other specialists wherein the treatment is regularly communicated and a collaborative relationship is maintained. ${ }^{10}$

The consequences of failure to identify risky use or detect signs of addiction can be life threatening. Mistaking symptoms of risky substance use for signs of other conditions may lead to a misdiagnosis or to prescribing medications that are unnecessary, produce dangerous drug interactions, compound an existing addiction problem ${ }^{*}$ or create additional health risks. ${ }^{11}$

\footnotetext{
* Thirty percent of patient respondents in CASA Columbia's 2000 survey of physicians and patients said their physician knew about their addiction and still prescribed psychoactive drugs such as sedatives or Valium.
}

This gap between evidence and practice is particularly acute for adolescents because of the critical importance of prevention and early intervention in this population. Screening and intervention services by health professionals for adolescents rarely is part of routine practice despite the abundance of guidelines and recommendations for screening this population; the knowledge that nearly three out of four high school students have used tobacco, alcohol or other drugs; and the availability of effective interventions. ${ }^{12}$

Current Practices Related to Tobacco. The majority (68.8 percent) of adults who are current smokers report that they want to quit smoking, ${ }^{\dagger}$ yet less than half (48.3 percent) of smokers who saw a health professional in the past year reported receiving advice to quit. ${ }^{\ddagger 13}$ A survey of $6^{\text {th- }}$ through $12^{\text {th }}$-grade students found that 64.5 percent reported that in the past year no medical doctor, dentist or nurse asked them whether they smoked cigarettes, ${ }^{\S 14}$ even though screening and interventions by health professionals can have a substantial impact on young smokers. ${ }^{15}$

A large-scale analysis of national data ${ }^{* *}$ of clinical preventive services delivered to asymptomatic patients in clinical settings estimates that only 35 percent of the population receives tobacco screening and brief interventions in accordance with the

\footnotetext{
${ }^{\dagger} 52.4$ percent of current and former smokers (those who quit smoking in the past year for six months or longer) had made a quit attempt that lasted longer than one day in the past year; however, only 6.2 percent report that they have quit successfully. ${ }^{\ddagger}$ Medicare beneficiaries were the most likely to receive smoking cessation advice (59.0 percent) and those without health insurance were the least likely to receive smoking cessation advice (35.3 percent).

$\S$ CASA Columbia's analysis of data from that survey found that, of those who smoked, only 21.4 percent said that these health professionals told them to stop smoking.

** Including the National Health Interview Survey (NHIS), the Behavioral Risk Factor Surveillance System (BRFSS) and the Healthcare Effectiveness Data and Information Set (HEDIS) performance data.
} 
recommendations of the United States Preventive Services Task Force (USPSTF). ${ }^{16}$

Another study found that while physicians knew of their patients' smoking status at 68 percent of office visits, they provided smoking cessation counseling at only 20 percent of visits by smokers. ${ }^{17}$ Analysis of national data found that physicians provided counseling in 22.5 percent of visits by current adult tobacco users; only 2.4 percent of current tobacco users were prescribed medications for smoking cessation. ${ }^{*} 18$ A national survey of medical professionals-including primary care physicians, emergency medicine physicians, psychiatrists, registered nurses, dentists, dental hygienists and pharmacists--indicates that whereas most report asking patients if they smoke and advising those who smoke to quit, they are much less likely to follow through with assessments or referrals to a smoking cessation program. ${ }^{19}$

A study of physicians in family medicine, internal medicine, obstetrics/gynecology and psychiatry found similar results. Although most (86 percent) report asking patients about their smoking and advising them to quit, few do much more than that: only 17 percent said they usually arrange for a follow-up visit to address the patient's smoking and only 13 percent said they usually refer patients who smoke to appropriate treatment. ${ }^{20}$ One study found that pulmonologists, cardiologists and family physicians were the physician specialists most likely to be familiar with resources regarding treatment for addiction involving nicotine and most likely to refer patients to treatment, whereas psychiatrists, neurologists, ophthalmologists and surgeons were the least likely to have such familiarity or to refer patients. $^{21}$

A survey of clinical psychologists found that one-third (32.3 percent) had not thought about offering smoking cessation counseling to their patients. ${ }^{22}$ And although most registered nurses ask patients about smoking (87.3 percent), only 65.6 percent advise those who smoke to quit and

\footnotetext{
${ }^{*}$ Both female patients and patients ages 65 and older were less likely to be prescribed medication.
}

less than half offer interventions (49.4 percent provide materials with quitline information and 34.0 percent refer the patient to a smoking cessation program). ${ }^{23}$ Another national survey of nurses' interventions with patients who smoke found similar results. ${ }^{\dagger 24}$

While the U.S. Clinical Practice Guideline on Treating Tobacco Use and Dependence encourages dental clinicians to screen their patients for tobacco use $\mathrm{e}^{25}$ and has been promulgated widely by the United States Public Health Service and the Agency for Healthcare Research and Quality, approximately three in 10 dental professionals still do not advise patients who smoke to quit and approximately threequarters do not refer a patient who smokes to a smoking cessation program. ${ }^{26}$ This is despite the fact that many patients expect their dentists to inquire about their smoking status and to discuss smoking cessation with them; 30 percent of dental patients report that they would try to quit smoking if their dentist suggested they do so. ${ }^{27}$ Dentists who implement an effective smoking cessation intervention can expect that up to 10 to 15 percent of their patients who smoke will quit in a given year. ${ }^{28}$

Pharmacists, as a profession, rarely provide tobacco cessation counseling; only about seven percent of patients report being asked by a pharmacist about tobacco use. ${ }^{29}$ This is in spite of the facts that pharmacists are one of the most accessible groups of health professionals and they work in settings frequented by smokers and where tobacco cessation products are available. ${ }^{30}$ The majority of smokers (83 percent) believe that pharmacists should be involved in providing smoking cessation interventions, 73 percent would join a smoking cessation program offered at a conveniently-located pharmacy ${ }^{31}$ and 63 percent who already use nicotine replacement therapy (NRT) believe that smoking cessation counseling by pharmacists would increase a

\footnotetext{
${ }^{\dagger}$ Seventy-three percent frequently asked patients about tobacco use and 62 percent advised patients about quitting. Only 24 percent of nurses recommended medications to patients for cessation, 22 percent referred patients to cessation resources and 10 percent recommended use of a quitline.
} 
smoker's likelihood of being able to quit. ${ }^{32}$ Something as simple as keeping NRT products behind the pharmacy counter where customers would have to ask for them, or within view of the pharmacist but accessible to customers, ${ }^{*}$ is related to a greater likelihood of pharmacistinitiated smoking cessation counseling. In one study, pharmacists who stored NRT products behind the counter were 4.7 times likelier to provide counseling ${ }^{\dagger}$ than pharmacists who stored the products out of customers' sight; those who stored them within view but still accessible to customers were three times likelier to offer counseling than those who stored them out of customers' sight. ${ }^{33}$

\section{Current Practices Related to Alcohol and Other Drugs. A national survey of patients} who had visited a general medical provider in the past year found that only 29 percent were asked about alcohol or other drug use; 9.2 percent were given the suggestion to stop using, 13.6 percent were given a brief intervention and 5.3 percent were referred to counseling. Of those in the sample who reported having been asked by a general medical provider about their alcohol or other drug use and were identified as risky drinkers, less than half (48.6 percent) received any type of advice from their doctor concerning their substance use. ${ }^{34}$

Another study found that 19.6 percent of patients who were identified by primary care practitioners as misusing addictive substances but who were not diagnosed with addiction did not receive a recommendation for an active intervention. ${ }^{35}$

A national survey of current and former drinkers, ages 18-39 years, found that 67 percent saw a physician in the past year but only 49 percent of excessive drinkers ${ }^{\ddagger}$ were asked about

\footnotetext{
* This approach might be less likely to deter customers from purchasing the NRT products, since some smokers may be hesitant to ask for assistance.

${ }^{\dagger}$ To four or more customers per month.

${ }^{\ddagger}$ Those exceeding the National Institute on Alcohol Abuse and Alcoholism's (NIAAA) guidelines of no more than four drinks per day or 14 drinks per week for men and no more than three drinks per day or seven drinks per week for women.
}

their drinking and only 21 percent of them were counseled about risky drinking. ${ }^{\S}$ While respondents ages 18-25 years were most likely to engage in excessive drinking, they were least likely to be asked about their alcohol use (34 percent of excessive drinkers ages 18 to 25 years vs. 54 percent of excessive drinkers ages 26 to 39 years). ${ }^{36}$

Efforts to educate patients and connect them with needed services also are inadequate in emergency departments (EDs). ${ }^{37}$ The American College of Surgeons Committee on Trauma designated alcohol and other drug screening as an "essential diagnostic test" at Level I and Level II trauma centers, ${ }^{38}$ yet many trauma centers do not provide any screening or brief intervention services for those who may need them. ${ }^{39}$ A national survey of ED directors found that only 15 percent reported having formal screening and intervention policies in their EDs. While nearly two-thirds (64.5 percent) reported routinely screening for risky alcohol use via a serum alcohol level and 23.6 percent reported using standardized screening instruments, only nine percent reported offering brief interventions by trained personnel for risky alcohol use. ${ }^{40}$

Although the majority of surgeons (89 percent) say that alcohol is a major burden on their trauma center (an estimated 40 to 50 percent of trauma patients have positive blood alcohol levels) ${ }^{41}$ and 76 percent consider other drug use to be a significant burden, ${ }^{42}$ trauma center and ED physicians often fail to address the underlying alcohol and other drug problems that cause patients' injuries. ${ }^{43}$

Despite evidence of the effectiveness of EDbased screening and brief interventions for substance-using adolescents, ${ }^{44}$ a study of adolescents admitted to hospitals following trauma injuries in which 15.5 percent screened positive for alcohol in their blood found that only 59 percent of those who screened positive were referred for intervention services. ${ }^{45}$

\footnotetext{
$\S 14$ percent were advised about low-risk drinking guidelines and seven percent were advised to cut down.
} 


\section{Assessment, Stabilization and Acute Treatment}

Despite the existence of effective assessment, stabilization and treatment options, addiction treatment today for the most part is not based in the science of what works. ${ }^{46}$ Depending on disease stage and a range of other health and social factors, some people with addiction may be able to stop using addictive substances and manage the disease with support services only; however, most individuals with the disease require clinical treatment. ${ }^{47}$ The failure of many providers to properly assess the stage and severity of the disease and provide effective psychosocial and pharmaceutical therapies appears in large part to be due to a lack of appropriate education and training. ${ }^{*} 48$

While physicians are somewhat better at assessing tobacco use among their patients and discussing options for treatment, there is still much room for improvement. A recent national survey found that approximately two-thirds of primary care physicians (68.5 percent) and psychiatrists (63.8 percent) report discussing medication options for smoking cessation with their patients, as do 22.6 percent of dentists and 14.5 percent of emergency medicine physicians. $^{49}$

CASA Columbia's 2000 survey of physicians and patients found that 94 percent of primary care physicians (excluding pediatricians) failed to identify addiction as a possible diagnosis when asked to offer five possible diagnoses of a patient with symptoms of risky alcohol use. Most patients responding to that survey (53.7 percent) reported that their primary care physician did nothing about their addiction; 10.7 percent said their physician knew about it and still did nothing. The majority of patients (74.1 percent) said their primary care physician was not involved in their decision to seek treatment and 16.7 percent said their physician was involved only "a little."

CASA Columbia's research also found that 40.8 percent of pediatricians failed to diagnose

\footnotetext{
* See Chapter IX.
}

addiction when presented with a classic description of an adolescent patient with symptoms of addiction involving drugs (other than nicotine or alcohol). ${ }^{51}$ However, another study found that, among adolescent patients diagnosed with addiction, primary care physicians recommended some type of followup $^{\dagger}$ for 94.7 percent of the patients. ${ }^{52}$ A study of adolescents admitted to an inpatient psychiatric unit $^{\ddagger}$ found that one-third met clinical criteria for addiction, but outpatient clinicians had not identified addiction in any of these patients before admission to the inpatient unit. ${ }^{53}$

Other research found that only 13 percent of patients who received an addiction-related diagnosis while visiting an ED received followup addiction treatment services within two weeks of the visit. ${ }^{\S}$ More than 200 patients in the study had another ED visit within two months of their initial ED visit, ${ }^{54}$ suggesting that hospitals do not appropriately address patients' addiction or provide them with referrals to treatment. $^{* * 55}$ A study of ED patients admitted with cocaine-related chest pain found that threequarters (74.7 percent) had not received any treatment three months after discharge. ${ }^{56}$

\section{Detoxification Frequently is Considered Treatment Rather Than a Precursor to} Treatment. A minority of patients who participate in detoxification programs go on to receive treatment, despite evidence that

\footnotetext{
${ }^{\dagger}$ Defined in this study as any plan beyond periodic screening, including notification of parents, referral to counseling, return visit with the primary care physician or noting that the patient already was in counseling for substance use.

‡ Patients were admitted to the unit for psychiatric conditions other than addiction.

$\S$ The primary diagnosis for patients included in the study was addiction for 28 percent of the sample, mental health issues for 13 percent of the sample and medical (non-psychiatric) disorders for 59 percent of the sample.

** Another study found that patients with unmet addiction treatment needs are nearly twice as likely to be admitted to the hospital and nearly one-and-a-half times as likely to have made at least one ED visit in the past year compared to patients without unmet treatment needs.
} 
treatment beyond detoxification typically is a medical necessity. ${ }^{57}$ In 2008 , only 12.6 percent of discharges from detoxification programs transferred to a treatment facility. About one in 10 (10.8 percent) of alcohol detoxification discharges were transferred to a treatment facility, as were 20.7 percent of marijuana detoxification discharges, 13.6 percent of other illicit drug detoxification discharges, ${ }^{\dagger} 18.2$ percent of prescription drug detoxification discharges and 13.9 percent of multiple substance detoxification discharges. ${ }^{58}$ Another study found that only 32.8 percent of Medicaidenrolled adult patients discharged from detoxification received follow-up care within 30 days of discharge. ${ }^{\ddagger 59}$

\section{Addiction Treatment Rarely Addresses} Smoking. Although recent scientific evidence underscores the unitary nature of the disease of addiction and the consequent need to address addiction involving all substances, ${ }^{60}$ many addiction treatment providers continue to address addiction involving alcohol, illicit drugs and controlled prescription drugs while largely ignoring addiction involving nicotine. ${ }^{61}$

Smoking cessation services are not commonly implemented in addiction treatment settings ${ }^{62}$ or in psychiatric treatment settings. ${ }^{63}$

The reluctance to provide smoking cessation services to patients in treatment for addiction involving alcohol or other drugs stems in part from an unfounded concern that it might jeopardize patients' ability to abstain from alcohol or other drug use. ${ }^{64}$ There is no evidence that quitting smoking interferes with

\footnotetext{
${ }^{*}$ Most recent available data.

${ }^{\dagger} 19.4$ percent of opioid (other than heroin) detoxification discharges, 16.4 percent of cocaine/crack detoxification discharges and 12.4 percent of heroin detoxification discharges transferred to a treatment facility.

₹ This disparity stems in part from financial constraints. Some insurance plans pay only for medical detoxification but not for addiction treatment including psychosocial and pharmaceutical therapies. Some plans that do cover both detoxification and treatment manage them separately, making continuity of care difficult.
}

the effectiveness of treatment for addiction involving alcohol or other drugs. ${ }^{65}$ In fact, research shows improved addiction treatment outcomes among patients who receive smoking cessation services, including reduced risk of relapse following treatment and improved outcomes for co-occurring addiction involving alcohol. ${ }^{66}$ In light of this evidence, some states ${ }^{\S}$ are banning smoking in addiction treatment facilities and requiring that smoking cessation services be provided to patients. ${ }^{67}$ Making smoking cessation a key component of addiction treatment programs would go a long way toward improving treatment outcomes as well as the long-term health of patients with addiction. ${ }^{68}$

One study found that fewer than half (43 percent) of addiction treatment programs in the United States offer formal smoking cessation services; no data are available on the extent to which nicotine addiction is fully integrated into these treatment programs. ${ }^{* *}$ Among those that do offer cessation services, more offer pharmaceutical interventions than psychosocial interventions (37 percent vs. 18 percent). ${ }^{69}$

Although rates of smoking among adolescent addiction treatment patients are high and effective interventions are available, ${ }^{70}$ less than half (42.8 percent) of treatment programs designed specifically for adolescents offer smoking cessation services; 13 percent offer a comprehensive formal program with pharmaceutical therapy, 15.3 percent offer counseling only and 14.5 percent offer pharmaceutical therapy only. ${ }^{71}$

Less than 20 percent of addiction treatment providers received any training in smokingrelated issues in the past year. ${ }^{72}$ This is despite the fact that the majority (between 65 and 87 percent $)^{\dagger \dagger}$ of patients in addiction treatment

\footnotetext{
$\S_{\text {NY, NJ, WA. }}$

** CASA Columbia's survey of directors of addiction treatment programs in New York State found that the majority (89.2 percent) indicated that treatment for addiction involving nicotine is offered in their addiction treatment programs.

${ }^{\dagger \dagger}$ Data based on a review of several studies.
} 
programs smoke--a rate more than three times that in the general population. ${ }^{73}$

\section{Pharmaceutical Treatments are \\ Underutilized. A key factor in integrating} addiction treatment into mainstream medicine is broader implementation of pharmaceutical interventions, when indicated. ${ }^{74}$ Yet providers of addiction treatment vastly underutilize evidence-based pharmaceutical therapies. ${ }^{75}$

CASA Columbia's survey of directors of addiction treatment programs in New York State found that less than half (47.0 percent) indicated that pharmaceutical treatments are offered in their addiction treatment programs. ${ }^{76}$

A national longitudinal survey of programs that offer addiction services ${ }^{*}$ found that the percentage of programs offering nicotine replacement therapy (NRT) decreased significantly from 38.0 percent during 20022004 to 33.8 percent four years later. Programs were more likely to continue offering NRT if they were medically oriented (i.e., located in a hospital setting with access to physicians). ${ }^{77}$

Underutilization of pharmaceutical treatments is particularly common in treatment programs that are publicly funded, small, not located in a hospital, not accredited ${ }^{\dagger}$ and have few medical professionals--including physicians and nurses-on staff. ${ }^{78}$ National data indicate that among privately- and publicly-funded treatment programs, approximately half have adopted at least one pharmaceutical treatment for addiction. ${ }^{\ddagger 79}$

\footnotetext{
* Including privately-funded treatment organizations, publicly-funded treatment organizations and therapeutic communities.

${ }^{\dagger}$ By the Joint Commission or the Commission on Accreditation of Rehabilitation Facilities (CARF). (See Chapter IX for a description of these accrediting organizations.)

‡ Approximately 51 percent of privately-funded programs and 25 percent of publicly-funded programs adopted buprenorphine in their treatment of addiction, 40 percent of private programs and 19 percent of public programs adopted acamprosate or tablet naltrexone, 30 percent of private programs and 16 percent of public programs adopted disulfiram and
}

The limited adoption of pharmaceutical treatments for addiction, when indicated, is due in large part to a lack of qualified medical staff in addiction treatment programs to prescribe and monitor medication protocols. ${ }^{80}$ Thirty-eight percent of publicly-funded programs do not even have access to a prescribing physician, nor do 23 percent of privately-funded programs. ${ }^{81}$

Treatment providers seem to have more negative attitudes toward the use of pharmaceutical therapies relative to psychosocial therapies. ${ }^{82}$ Some treatment programs see pharmaceutical treatments for addiction, such as the use of methadone maintenance treatment for addiction involving opioids, as incompatible with abstinence-based treatment approaches; ${ }^{83}$ there is a stigma among some providers attached to the use of pharmaceuticals to achieve abstinence from a drug to which the patient is addicted. One of the key predictors of the underutilization of pharmaceutical treatments is adherence of treatment providers to a strong 12-step ideology for addiction treatment. ${ }^{84}$

CASA Columbia's survey of treatment providers in New York State found that respondents were more likely to say that recreational therapy/ leisure skills training is a "very important" intervention for a treatment facility to offer to patients $^{\S}$ than to say the same of pharmaceutical treatments. $^{* *} 85$

Addiction treatment medications also may be underutilized by physicians themselves due in part to insufficient evidence regarding optimal dosages of certain pharmaceutical therapies, durations of use, how to combine the use of medications with counseling and the

less than 20 percent of private programs and less than 10 percent of public programs adopted injectable naltrexone in their treatment protocols.

$\S 51.8$ percent of program directors, 54.7 percent of staff providers.

** 28.0 percent of program directors, 33.8 percent of staff providers for methadone maintenance treatment and 43.9 percent of program directors, 45.7 percent of staff providers for other medication treatments for addiction such as buprenorphine, disulfiram or naltrexone. 
generalizability of research-based efficacy findings to different patient populations. ${ }^{86}$

Although one study found that each additional physician on staff in a treatment program was associated with a doubling of the odds that the program would adopt the use of pharmaceutical treatments for addiction, ${ }^{87}$ having access to a staff physician does not guarantee access to or use of pharmaceutical treatments. ${ }^{88}$ One study found that 82 percent of publicly-funded addiction treatment programs with access to a physician did not prescribe any treatment medications for addiction involving alcohol; the same is true of 41 percent of privately-funded treatment programs with access to a prescribing physician. $^{89}$

The treatment of addiction involving opioids presents one of the most glaring examples of the underutilization of clinically-effective and costeffective pharmaceutical treatments for addiction. ${ }^{91}$ Buprenorphine is a pharmaceutical treatment for addiction involving opioids that, despite a rich body of evidence demonstrating its efficacy, safety ${ }^{92}$ and cost effectiveness, ${ }^{93}$ is significantly underutilized in practice. ${ }^{94}$ The majority (86 percent) of addiction counselors report not being aware of the effectiveness of buprenorphine. $^{95}$

We're seeing less interest [in prescribing buprenorphine] than we expected, especially among primary care physicians. ${ }^{96}$

--Robert Lubran, MPA Director

Division of Pharmacological Therapies Center for Substance Abuse Treatment (CSAT) SAMHSA

Specific additional obstacles to the widespread use of buprenorphine by physicians include cost, lack of insurance coverage and availability problems due to pharmacies not stocking the medication. ${ }^{97}$

Physicians' biases against patients with addiction may contribute to the limited adoption of pharmaceutical treatments as well. ${ }^{98}$ Survey results from a random sample of internal medicine, family medicine, psychiatry and pain management physicians in Maryland found that only 36 percent of respondents were willing to prescribe buprenorphine to an established patient and only 28 percent were willing to prescribe the medication to a new patient. Seventeen percent of physicians unwilling to prescribe the medication said that addiction involving opioids is best described as a habit rather than an illness; none of the physicians willing to prescribe the medication agreed with this statement. Half of the Maryland doctors who were not willing to prescribe buprenorphine reported that they believe that treatment for addiction involving opioids is beyond the scope of practice of officebased physicians and 46 percent reported not wanting patients with addiction involving opioids in their clinics. ${ }^{99}$

The reason I am not interested [in prescribing buprenorphine] is I see this as an opportunity for drug users who are by class the most lying, scheming, dishonest group of patients. They need hard-based, no-nonsense treatment programs. I can't stand their manipulative behavior. ${ }^{90}$

Anonymous Physician

The fact that buprenorphine can be prescribed in physicians' offices for at-home use was heralded as a step forward in the treatment of addiction involving opioids. Addiction professionals anticipated the medication's potential to help addiction treatment become a more central component of medical practice. ${ }^{101}$ However, these hopes have not come to fruition. ${ }^{102}$

There is no other comparable example in medicine where you have evidence-based treatments that are not available. ${ }^{100}$

--Shelly Greenfield, MD, MPH

Chief Academic Officer, McLean Hospital Professor of Psychiatry, Harvard Medical School

Director, Clinical and Health Services Research and Education Division of Alcohol and Drug Abuse, McLean Hospital 
Nutrition and Exercise Are Not Integrated into Addiction Treatment. Although good nutrition and exercise are important components of comprehensive addiction treatment ${ }^{*}{ }^{103}$-particularly in light of recent research underscoring common brain circuitry involved in substance-related addiction and obesity ${ }^{104}$--the extent to which nutrition and exercise are incorporated into addiction treatment has not been examined. One small study ${ }^{\dagger}$ found that 56 percent of dietitians and nutrition program managers working in addiction treatment facilities reported that their facilities offered nutrition-related addiction education in group settings to only about half of their patients. Fifty-six percent of respondents reported offering nutrition-related addiction education in individual settings to an average of 18 percent of their patients. $^{105}$

\section{Formal Treatment Is Conflated with Support}

Services. The overwhelming salience and considerable evidence--although largely anecdotal--of the benefits of mutual support programs like Alcoholics Anonymous (AA), for example, have led many people to conflate such support services with actual addiction treatment rather than to recognize them as highly useful systems of support that should accompany or follow evidence-based clinical treatment. ${ }^{\ddagger 106}$ Mutual support programs are facilitated by members whose main credential is that they themselves have experienced an addictive disorder and have learned to manage it. Such support, however, is quite different than treatment for a medical condition. ${ }^{\S 107}$ Few would argue that any other disease be treated

\footnotetext{
* See Chapter V.

${ }^{\dagger}$ Using a non-randomized sample.

$\ddagger$ There are some exceptions where, depending on the severity of disease symptoms and the patient's health status and degree of social support, certain patients are able to manage their addiction with support services only or no interventions at all. In addition, it should be noted that Twelve-Step Facilitation, discussed in Chapter V, is a formalization and professionalization of the 12-step mutual support model and has been deemed an evidence-based treatment for addiction.

$\S$ AA openly recognizes addiction as a medical condition.
}

solely via support groups composed of those who themselves have had the condition.

\section{Tailored Treatment Services}

Whereas research clearly indicates that to be effective interventions should be tailored not only to the stage and severity of a patient's illness but also to a patient's co-occurring conditions and other personal characteristics and life circumstances that might affect treatment outcome, most health professionals and addiction treatment programs follow a one-sizefits-all approach to treatment.

\section{Disease Severity Rarely is Assessed and Interventions Rarely are Tailored to Stage and Severity of Disease. It is standard recommended medical practice to assess the stage and severity of a patient's disease in order to develop an effective treatment plan and tailor treatment accordingly. ${ }^{108}$ Assessment of disease severity is an essential part of addiction treatment as well. ${ }^{109}$ One of the most widely- used tools, both in research and clinical practice, for assessing the severity of addiction is the Addiction Severity Index, although even this instrument fails to address addiction involving nicotine. ${ }^{110}$ Yet, the extent to which treatment providers tailor treatment protocols based on the findings of such assessments is limited. ${ }^{111}$ Instead, addiction treatment programs typically utilize a non-tailored approach to patient care. ${ }^{112}$}

Having patients pass through a rigid, timelimited treatment program that assumes uniformity in disease symptoms and severity simply burdens patients with unnecessarily extensive interventions or with interventions that are too brief or superficial to have a significant impact on their symptoms. Yet the standard treatment for addiction is non-intensive outpatient treatment, ${ }^{113}$ often without adequate professional follow-up care and disease management. Even residential treatment typically is limited to a 28-day stay in a program despite little evidence that the condition remits after such a brief period of time. ${ }^{114}$ Given this standard approach to treatment that focuses primarily on acute care only, it is little wonder that relapse is so common and that addiction 
continues to be seen as an intractable condition largely resistant to treatment.

We are treating these folks with severe and persistent addiction with a time limited-treatment of three or four weeks, maybe six or eight, maybe at the most three months, if you want to include what's called aftercare. Now, why would we think that treating a chronic disorder for a few weeks would lead to improvement 10 years later or five years later or even a year later? It doesn't make any sense. ${ }^{115}$

--Mark Willenbring, MD

Director, Division of Treatment and Recovery

National Institute on Alcohol

Abuse and Alcoholism

(currently, Associate Professor of Psychiatry, University of Minnesota)

Interventions Do Not Adequately Address Co-occurring Conditions. In standard medical practice, it is recommended that health professionals assess the presence of co-occurring conditions in order to develop an effective treatment plan and tailor treatment accordingly. ${ }^{116}$ Although such assessments are critical in addiction treatment given the very high rate of co-occurring conditions in people with addiction, treatment programs frequently do not address co-occurring health conditions or do so in a suboptimal way. ${ }^{117}$

Implementing a one-size-fits-all approach to treatment based solely on a clinical diagnosis without consideration of co-occurring health conditions often amounts to a waste of time and resources. CASA Columbia's survey of directors of addiction treatment programs in New York State found that less than half (48.2 percent) of the program directors indicated that treatment for co-occurring mental and physical health disorders is offered in their programs. ${ }^{118}$

A recent study of patients in residential treatment for addiction who had co-occurring mental health conditions underscores the importance of tailoring treatment to the needs of the patient population. In this study, those with co-occurring mental health conditions reported less satisfaction with treatment, saw fewer benefits to stopping their substance use, had less belief in their efforts to control their substance use to maintain abstinence and were less likely to employ positive coping skills than patients with addiction who did not have co-occurring mental health conditions. ${ }^{119}$

I lost my son to addiction and ultimately suicide. From the time I knew he had a problem until the day he died, I tried everything at my disposal to help him get quality care. He went to eight different programs and they all had a different approach; many offered conflicting advice. Only four of them looked at Brian as a whole person; the other four only looked at his addiction. Only five included a comprehensive medical assessment; in fact, only three even had a full-time doctor on the premises. And although they each viewed his condition as a chronic disease requiring effective aftercare and long term management, none of them had an effective recommendation for this, nor any follow up from that program. In a letter to me during one of his stays in a residential program, he offered the insight that much of his previous rounds of treatment had addressed his addiction in isolation--as if it were unrelated to any underlying emotional problems. In the last weeks of his life, Brian was suffering from severe depression. On the day before he died, his aftercare program made the decision, without consulting Brian's therapist, or his parents, to terminate their relationship with him. At the time Brian most needed help, he was left alone. And so was I. ${ }^{120}$

--Gary Mendell, father Lost his son Brian, age 25, to addiction and suicide

Although people with mental illness smoke at significantly higher rates than the general population, smoking cessation services rarely are provided by mental health treatment professionals. ${ }^{121}$ Generally psychiatrists are less likely than family physicians to inquire about smoking, offer advice on quitting or assess patients' willingness to quit. ${ }^{122}$ Yet, because individuals with severe mental illness interact with psychiatrists to a greater extent than with primary care physicians (who typically are the main referral source or provider of smoking cessation interventions), patients in mental 
health settings who smoke may be even less likely to receive the smoking cessation services they need. ${ }^{123}$ Psychiatrists may eschew smoking cessation interventions because they believe "patients have more immediate problems to address;" some medical and other health professionals also may be reluctant to encourage smoking cessation because they feel it "gives patients with psychiatric illnesses comfort while dealing with their mental illness symptoms."124 Contrary to these beliefs, research shows that smokers with mental health conditions are as motivated as those in the general population to quit smoking ${ }^{125}$ and one study showed that the majority (79 percent) of mentally ill smokers want to quit. ${ }^{126}$

\section{Interventions Rarely are Tailored to Patient} Characteristics that Might Affect Treatment Outcomes. Not taking into account a patient's age, gender, race/ethnicity, socioeconomic status or system of personal supports in designing a treatment intervention can compromise otherwise effective treatment plans. Chapter VI of this report outlines specific treatment needs of special populations and Chapter VII shows the gaps in needed treatment for some of these groups. While the baseline level of addiction-related services offered to the general population is inadequate, the deficiency in tailored services offered to populations with special treatment needs is even more glaring. ${ }^{127}$

\section{Chronic Disease Management}

For many individuals, addiction manifests as a chronic disease, requiring disease and symptom management over the long term. ${ }^{128}$ In recent years, there has been growing recognition of the importance of comprehensive disease management in the treatment of chronic health conditions for which there is no known cure, where relapse episodes are considered an expected part of the disease course and where long-term symptom management is considered routine care. While this approach increasingly has been adopted for diseases such as diabetes, ${ }^{129}$ hypertension ${ }^{130}$ and asthma, ${ }^{131}$ addiction treatment largely remains stuck in the acute-care model. ${ }^{132}$
Patients with addiction, regardless of the stage and severity of their disease, typically receive a diagnosis followed by a swift course of treatment administered by individuals without any medical training and then minimal to no follow-up care. ${ }^{133}$ In contrast to other chronic diseases, positive results from a short-term intervention or treatment for addiction are expected to endure indefinitely and relapse commonly is viewed as a sign of treatment failure, at best, and as evidence of a deficit in patients' willpower or dedication to managing their condition, at worst, rather than as a result of inadequate treatment or follow-up care. ${ }^{134}$

Evidence of the acute care approach to addiction treatment is that current Medicare and Medicaid regulations indicate that hospital readmissions for patients with addiction involving alcohol are not to be treated as extensions of the original treatment but rather as a new admission to treat the same condition. Readmissions can be seen as evidence that treatment is not working and typically are not covered unless a physician can document a change in the patient's physical, emotional or social condition that makes it reasonable to expect that additional treatment would improve the patient's condition, or documents why the initial treatment was insufficient. ${ }^{135}$ Given that addiction often is a chronic disease and that relapse is possible, limitations on hospital readmissions may reduce access to needed care and reflect a fundamental mischaracterization of the disease and its expected course of treatment. 


\section{Examples of the Evidence-Practice Gap in Addiction Treatment throughout History}

The use of addiction treatments that are not grounded in science has a long history. While most of these approaches have been discredited with time, some have proven to be prescient in their foreshadowing of current treatment approaches, both those that are evidence based and those that continue to profit from claims about being able to treat or even cure addiction in manners that largely are not based on scientific evidence.

For example, in the late 1700s, Dr. Benjamin Rush, the "father of American psychiatry," was the first American doctor to say that "habitual drunkenness should be regarded not as a bad habit but as a disease"136 that should be treated. ${ }^{137}$ In the late $19^{\text {th }}$ century, medically-based addiction treatment mostly involved trying to cure individuals of their addiction, often with the use of other addictive substances. ${ }^{138}$

By 1910, private sanitariums in the United States offered specialized treatment for addicted individuals--but only for those who could afford the expense. Similar to today, many of the "treatment experts" opening facilities were savvy businessmen or enterprising physicians, including Harvey Kellogg (later of cereal fame) and Dr. Leslie E. Keeley. Between 1892 and 1893, almost 15,000 people with addiction were treated at the famous, yet controversial Keeley Institutes. ${ }^{139}$ Keeley's treatment for addiction involved bichloride of gold remedies, a substance purportedly containing gold that would cure addiction involving alcohol and opioids. The use of bichloride of gold became highly controversial and was opposed by the American Medical Association (AMA). After the death of Dr. Keeley in 1900, the popularity and ultimately, the existence of his institutes waned. ${ }^{140}$

Although Keeley's treatments were later discredited, his position that addiction was decidedly a disease rather than a religious or moral failing was ahead of its time. His use of "shot treatments" or hypodermic treatments that induced vomiting was a precursor to later aversion therapies and his introduction of clubs for addicted individuals to receive social support to maintain sobriety was a precursor to the mutual support programs that remain prominent today. His focus on helping people quit smoking in the 1920s was prescient in its characterization of nicotine as a harmful and addicting drug. ${ }^{141}$

Addiction treatment tactics that are based more on the personal charisma of the founders, catchy phrases and simplistic approaches than on the science of what works in addiction continue to proliferate and show no sign of waning. A simple Google search produces an abundance of "rehabilitation" approaches and facilities with slogans such as: Learn how to heal the underlying causes of dependency--and be free of addiction forever $!^{142}$ A recent study examining treatments that a panel of experts believes qualifies as quackery in addiction treatment ${ }^{*}$ found such treatments as electrical stimulation of the head, past-life therapy, electric shock therapy, psychedelic medication and neuro-linguistic programming to be "certainly discredited." ${ }^{143}$ Nevertheless, unsubstantiated interventions continue to be used to this day, many of which prey upon the desperation of addicted individuals and their families.

In the late 1930s and early 1940s, many hospitals would not admit patients for the treatment of addiction involving alcohol, so lay approaches became an important option. ${ }^{144}$ Alcoholics Anonymous (AA), founded in 1935, was premised on laypersons addicted to alcohol helping one another overcome their addiction and related problems. ${ }^{145}$ While the mutual support/self-help approach maintained the perspective of addiction as a disease--formalized in the development of the principles underlying the Minnesota Model in the 1950s--the "rehabilitative model" of treatment was seen as distinct from "the medical model." Standard medical interventions that normally would be applied to diseases were not a significant part of the treatment, nor were medical or other health professionals called upon to play key roles in treating the disease. ${ }^{146}$ This model remains the dominant approach to addressing addiction in the United States. Yet, its limitations and failure to address addiction the way other diseases are addressed have led to a call to integrate addiction treatment into mainstream medical care.

* Based on the composite opinions of a panel of 75 experts regarding 65 addiction treatments which they rated on a continuum from "not at all discredited" to "certainly discredited." 


\section{Barriers to Closing the Evidence- Practice Gap}

America's approach to addressing risky substance use and providing addiction treatment has evolved outside of the mainstream public health and medical systems. ${ }^{*} 47$ As a result:

- Most primary providers of intervention and treatment for risky substance use and addiction do not have the requisite training or qualifications to implement the existing range of evidence-based practices and face many organizational and structural barriers to providing services;

- Most health professionals do not implement evidence-based addiction care practices;

- Performance and outcome measures that should be a routine part of quality assurance in mainstream medicine are limited and rarely implemented in addiction treatment;

- The pharmaceutical industry lacks the incentive to develop new and effective pharmaceutical interventions for addiction treatment; and

- Insurance coverage for evidence-based intervention, treatment and disease management is inadequate.

Because of the vast chasm between the health care system and approaches to preventing risky substance use or treating addiction, medical professionals fail to address risky substance use or addiction or take responsibility for intervention or treatment, risky substance use is addressed primarily in terms of its consequences and addiction treatment providers are not held to the same standards as providers of mainstream medical care.

\footnotetext{
* See Chapter IX for an in-depth discussion of the education and training requirements for those providing services for risky substance use and addiction.
}

The lack of integration of screening and intervention and addiction treatment into mainstream health care has led to many missed opportunities to help patients who engage in risky substance use or who have addiction and has contributed to the high rates of relapse and enormous health and social consequences associated with risky use and addiction.

One of the fundamental barriers to providing effective treatment is the fact that addiction is not integrated into medical practice. And a lot of medical people like and want it that way; they do not want to deal with addiction; they do not like to deal with the people and they do not feel effective addressing the problem. ${ }^{148}$

--Keith Humphreys, PhD Professor

Stanford Medical School

\section{The Addiction Treatment Workforce is Not Qualified to Implement Evidence-Based Practices}

For most illnesses, the preponderance of treatment interventions, including coordination of services, occurs within mainstream medicine and is provided and managed by trained medical professionals. Other highly-trained and credentialed health professionals may be part of a team of providers working with a physician. Motivated and experienced non-professionals may serve additional vital functions--such as providing social support to encourage adherence to a treatment plan and help patients maintain important lifestyle changes that can reduce the risk of relapse--but their roles and services do not supersede or replace those of the medical team.

In contrast, the majority of care for individuals with addiction is provided by people without any medical training at all and rarely is supervised by medical professionals. Physicians and other medical professionals typically are absent from or on the periphery of the treatment process, occasionally being called in to provide a prescription or medically monitor a detoxification protocol. 
Addiction treatment services primarily are provided through community-based treatment programs that were established in the 1970s and 1980s before the medical model gained support and changes in the financial structure led to increasing demands for accountability and efficiency. ${ }^{149}$ The treatment provider workforce, although frequently highly dedicated, is composed primarily of certified alcoholism and substance abuse counselors (CASACs)--a profession for which a college degree typically is not required ${ }^{*}$ and in which counselors receive limited on-the-job training in evidence-based practices. ${ }^{150}$

The result is that much of the current treatment provider workforce is not equipped to provide consistent evidence-based treatment, including administering and monitoring medication protocols, implementing complex psychosocial interventions, addressing co-occurring health conditions or responding to medical problems that may arise among individuals undergoing addiction treatment. ${ }^{151}$

CASA Columbia's survey of addiction treatment providers in New York State provides a case in point. It found that 37.2 percent of staff providers do not have a bachelor's degree ${ }^{\dagger}$ and only 67.5 percent of program directors and 35.0 percent of staff providers have a graduate degree of any type. ${ }^{152}$

Further, most treatment providers see the current state of affairs with regard to the staffing of treatment programs as reasonable. When asked which type of professional they think is best qualified to provide addiction treatment services, nearly three quarters of respondents to CASA Columbia's New York survey (71.3 percent of program directors and 72.6 percent of staff

\footnotetext{
* See Chapter IX for specific credentialing requirements.

† 29.9 percent had some college or an associate's degree, 6.6 percent had only a high school degree or GED and 0.7 percent did not complete high school or have a GED.
}

providers) said substance abuse counselors (CASACs); 16.3 percent of directors and 17.8 percent of staff providers said social workers, 7.5 percent of directors and 6.7 percent of staff providers said psychologists and only 1.3 percent of directors and 1.5 percent of staff providers said physicians. ${ }^{153}$

Only 9.9 percent of program directors and 13.7 percent of staff providers indicated that a medical degree is a "very important" qualification for treatment providers to have; 3.7 percent of directors and 6.5 percent of staff providers thought that way about a doctoral degree. A quarter (24.1 percent of directors and 26.1 percent of staff providers) felt that a master's degree was a very important qualification, and about a quarter (26.8 percent of directors and 27.3 percent of staff providers) felt that way about a college/bachelor's degree. $^{154}$ (Figure 10.A)

\section{New York State Addiction Treatment Providers' Beliefs about the Importance of the Following Provider Educational Qualifications*}

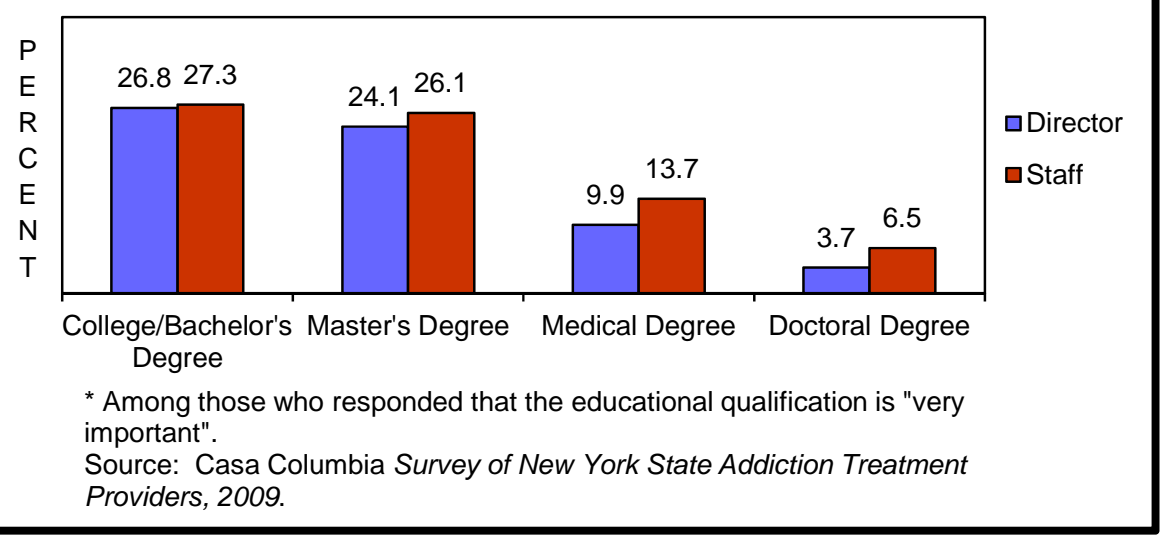

In contrast, the majority of treatment providers in CASA Columbia's survey (61.4 percent of program directors and 76.3 percent of staff providers) thought that personal experience with addiction is an important qualification for 
addiction treatment providers to have. ${ }^{*} 155$

CASA Columbia's survey also found that only 11.3 percent of program directors and 8.1 percent of staff providers believe that the addiction treatment system "should be integrated into the medical system such that addiction is treated as a health condition by health care professionals." Two-thirds of directors (66.3 percent) and 60.3 percent of staff providers, however, say that the addiction treatment system "should be two-tiered with health care professionals providing psychotherapeutic and pharmacological interventions and individuals with histories of addiction (in recovery) providing recovery support services.” Fewer directors (7.5 percent) but more staff providers (21.3 percent) indicated that "the system should revolve around a recovery support model with self-help/mutual support programs as the main intervention.” Fifteen percent of directors and 10.3 percent of staff providers think that nothing about the system should change. ${ }^{156}$

Despite this variability in perceptions regarding what the treatment system should look like, there is general agreement that the current system is riddled with organizational and structural problems that impede the delivery of quality care, including inadequate financial resources, high staff turnover and restricted professional advancement. ${ }^{157}$ While limited education and training of most providers and a treatment culture largely steeped in the self-help model stand in the way of adopting new, science-based practices, ${ }^{158}$ organizational and structural problems within the current treatment system compound these limitations and further prevent the delivery of evidence-based care. ${ }^{159}$

CASA Columbia's survey of treatment providers in New York State found that respondents were most likely to cite a high volume of paperwork

\footnotetext{
* Among program directors, 28.9 percent said personal experience is slightly important, 25.3 percent said it is moderately important and 7.2 percent said it is very important. Among staff providers, 36.0 percent said personal experience is slightly important, 21.6 percent said it is moderately important and 18.7 percent said it is very important.
}

(reporting requirements that take up too much time and resources), insufficient salaries to attract and retain high-quality staff, insufficient program funding and insufficient insurance coverage for patients as barriers that "very much" stand in the way of providers' ability to provide effective services to people in need of addiction treatment--reflecting both frustration with organizational barriers to quality care and a non-medical approach to treatment. An insufficient number of trained medical-level (MD/DO) professionals was least likely to be named by providers as a barrier that "very much" stands in the way of providing effective treatment services. $^{160}$ (Table 10.1)

\section{Table 10.1 \\ New York State Addiction Treatment Providers' Opinions of Barriers that "Very Much" Stand in the Way of Providing Effective Addiction Services}

\begin{tabular}{l|c|c}
\hline & $\begin{array}{c}\text { Program } \\
\text { Directors }\end{array}$ & $\begin{array}{c}\text { Staff } \\
\text { Providers }\end{array}$ \\
\hline $\begin{array}{l}\text { High volume of paperwork/reporting } \\
\text { requirements }\end{array}$ & 76.8 & 72.7 \\
\hline $\begin{array}{l}\text { Insufficient salaries to attract and retain } \\
\text { high-quality staff }\end{array}$ & 74.7 & 69.8 \\
\hline Insufficient program funding & 62.2 & 63.3 \\
\hline Insufficient insurance coverage & 60.2 & 61.6 \\
\hline $\begin{array}{l}\text { Insufficient number of appropriately trained } \\
\text { staff with the qualifications necessary to } \\
\text { provide treatment for co-morbid conditions }\end{array}$ & 56.1 & 37.4 \\
\hline $\begin{array}{l}\text { Insufficient number of trained master's- } \\
\text { level professionals }\end{array}$ & 28.0 & 20.9 \\
\hline $\begin{array}{l}\text { Insufficient number of appropriately trained } \\
\text { staff with the qualifications necessary to } \\
\text { provide pharmacological therapies }\end{array}$ & 26.5 & 19.7 \\
\hline $\begin{array}{l}\text { Limited motivation among staff members to } \\
\text { be knowledgeable about evidence-based } \\
\text { best practices }\end{array}$ & 24.1 & 19.7 \\
\hline $\begin{array}{l}\text { Insufficient professional standards related } \\
\text { to knowledge and clinical skills for } \\
\text { individuals providing addiction treatment }\end{array}$ & 20.5 & 21.7 \\
\hline $\begin{array}{l}\text { Insufficient federal- or state-level education } \\
\text { and training requirements for individuals } \\
\text { providing addiction treatment }\end{array}$ & 18.1 & 23.2 \\
\hline $\begin{array}{l}\text { Insufficient access to information about } \\
\text { best practices }\end{array}$ & 13.3 & 15.9 \\
\hline $\begin{array}{l}\text { Insufficient number of trained medical-level } \\
\text { (MD/DO) professionals }\end{array}$ & 12.3 & 13.0 \\
\hline $\begin{array}{l}\text { Source: CASA Columbia Survey of New York State Addiction } \\
\text { Treatment Providers, 2009. }\end{array}$ & \\
\hline
\end{tabular}


Most Addiction Treatment Providers Do Not Adopt Evidence-Based Practices. ${ }^{*}$ Many addiction treatment providers address addiction in ways that have not been evaluated or proven effective. While this is due in part to the inadequate education and training of treatment providers in evidence-based practices, ${ }^{161}$ the larger issue is the significant mismatch between the qualifications that are necessary for implementing many evidence-based practices and the qualifications that the majority of the treatment workforce currently possesses. ${ }^{162}$ Because most treatment providers are not adequately trained, they are not capable of performing health assessments, prescribing pharmaceutical medications, treating cooccurring health conditions or managing a chronic disease, each of which is an essential evidence-based addiction treatment practice. ${ }^{163}$ Most also are not trained in the scientific method or clinical research, further impeding their ability to integrate clinical research findings into treatment practice. ${ }^{164}$

Education and awareness about evidence-based practices, however, are not sufficient to ensure proper implementation of those practices; ${ }^{165}$ additional steps are necessary to translate evidence into practice. Although significant progress has been made in the past decade in disseminating knowledge about evidence-based practices and improving attitudes toward them, less is known about how well such practices are implemented in treatment facilities and programs. ${ }^{166}$

One study found that treatment programs that are most likely to adopt clinical and practice guidelines, including the use of standardized assessment tools, are those that offer a broad range of services--medical services, counseling, pharmaceutical therapy and support services--

\footnotetext{
* Evidence-based practice involves the use of current evidence in making decisions about patient care. Evidence-based practices aim to combine the best available research and clinical judgment while taking into account patient characteristics and needs.
}

and those that receive funding from managed care organizations. $^{\dagger} 167$

We have entered the world of showing providers what is evidence based. Now we must show providers how to implement it... We haven't effectively brought practical research results to individual providers... so they can use it. ${ }^{168}$

--William H. Janes Former Director Florida Office of Drug Control Office of the Governor

Evidence from research findings is not generally accessible and understandable to providers; we're failing miserably at that. ${ }^{169}$

--Joseph M. Amico, MDiv President

National Association of Lesbian and Gay Addiction Professionals

(now Vice President, and association now called

The Association of Lesbian, Gay, Bisexual, Transgender Addiction Professionals and Their Allies)

According to the American Psychological Association, evidence-based practice is the integration of the best available research and clinical expertise within the context of patient characteristics, culture, values and preferences. Today we have a few places trying to implement these practices but with major limitations; few ever implement the practice the way it was researched. ${ }^{170}$

$$
\begin{array}{r}
\text {--Stephanie Covington, PhD, LCSW } \\
\text { Co-Director } \\
\text { Center for Gender and Justice } \\
\text { Institute for Relational Development } \\
\text { La Jolla, CA }
\end{array}
$$

\footnotetext{
${ }^{\dagger}$ Programs with managed care involvement were less likely to be freestanding facilities and more likely to be associated with multi-service hospital or medicalbased clinics. They also were more likely to have staff with advanced degrees and less likely to have staff in recovery. The authors of this study speculate that programs with managed care contracts might be likelier than those without such contracts to be encouraged to have standardized practices.
} 
One study found that support staff ${ }^{*}-$-who make up 24 percent of the treatment provider workforce in the National Institute on Drug Abuse's (NIDA) Clinical Trial Network (CTN) ${ }^{\dagger}$ and have more direct patient contact than professional counselors and medical personnel-showed little enthusiasm for evidence-based practices; they also were more likely to support intervention techniques that employ confrontation and coercion--techniques that contradict evidence-based practice. ${ }^{171}$ In contrast, treatment providers affiliated with CTN who advocated for the use of new evidencebased practices tended to be more highly educated. ${ }^{172}$

A study of counselors' attitudes toward evidence-based psychosocial and pharmaceutical practices $^{\ddagger}$ found that those who had more specific training in the practices and those who worked in treatment centers where the particular practices were used routinely tended to perceive evidence-based practices as more acceptable for treating addiction. ${ }^{173}$ Providers with higher educational degrees are more likely to be supportive of evidence-based practices than those with lower-level degrees. ${ }^{174}$ In contrast, providers with a strong 12-step orientation to treatment tend to perceive evidence-based practices as less acceptable. ${ }^{175}$

For many recovering paraprofessional counselors, their counseling "trump card" is that their personal experience is exemplary of how recovery works. ${ }^{177}$

\footnotetext{
* Support staff is distinct from counselors, managers or supervisors and medical personnel.

${ }^{\dagger} \mathrm{CTN}$ is a partnership between NIDA researchers and community treatment providers to deliver new evidence-based treatments to a broader population of patients and to conduct multi-site clinical trials to determine the effectiveness of new therapies in diverse settings.

₹ Including the use of buprenorphine, methadone, naltrexone, disulfiram, motivational enhancement therapy and voucher-based motivational incentives.
}

\section{Health Professionals do not Implement Evidence-Based Addiction Care Practices ${ }^{\S}$}

Mainstream medical and other health professionals do not adequately address risky substance use or the disease of addiction in their professional practice, in part because they are not trained to do so. ${ }^{* *} 176$ and in part because they do not see it as a legitimate element of their role as health professionals.

Education and training alone, however, is insufficient to change practice. For example, while numerous guidelines have been produced and disseminated by government agencies, ${ }^{\dagger \dagger} 178$ professional associations ${ }^{179}$ and quality improvement organizations such as the National Quality Forum (NQF) ${ }^{180}$ and the Agency for Healthcare Research and Quality (AHRQ), ${ }^{181}$ to help health professionals conduct evidencebased practices related to risky substance use and addiction, physicians and other health care providers commonly fail to adhere to these clinical practice guidelines. ${ }^{182}$

A recognized cadre of addiction physician specialists is essential to help educate and train other physicians, serve as equal partners in regular medical practice and provide specialty care. $^{183}$

Efforts also must be made to translate physician training into practice. A lack of time and resources make it difficult for physicians to remain up to date with the latest guidelines and recommendations, and limited reimbursement may prevent some physicians from taking the time to implement practice recommendations. ${ }^{184}$

\footnotetext{
$\S$ See Chapter IX for a detailed discussion of the addiction-related credentialing requirements for health professionals.

** Most of the research related to the training of health professionals in addiction-related services focuses on tobacco cessation rather than interventions for addiction involving alcohol and other drugs. ${ }^{\dagger \dagger}$ e.g., The Substance Abuse and Mental Health Services Administration (SAMHSA) produces the National Registry of Evidence-Based Programs and Practices (NREPP), an online searchable database of evidence-based interventions for mental health and addiction prevention and treatment.
} 
These factors, however, are not sufficient to justify the lack of medical attention to a disease affecting 16 percent of the population.

Physicians. Poor training in the care of patients with addiction relates to low confidence among physicians in their ability or competence to treat such patients, negative attitudes toward patients with addiction, pessimism about the effectiveness of treatment and low rates of implementation of evidence-based practices related to screening, brief interventions and treatment. ${ }^{185}$

Physicians can be the first line of defense against risky substance use and addiction, but they need the right tools and resources. ${ }^{186}$

--Nora D. Volkow, MD Director

National Institute on Drug Abuse

Most doctors do not look at addiction as part of their job. They may assess, but they don't intervene. ${ }^{187}$

--Brian Hurley Chair

Physicians-in-training Committee American Society of Addiction Medicine

Only a small proportion of primary care physicians feel "very prepared" to detect particular types of risky use (alcohol--19.9 percent; illicit drugs--16.9 percent; prescription drugs--30.2 percent), which is in stark contrast to the much higher percentages of physicians who report feeling "very prepared" to identify hypertension (82.8 percent), diabetes (82.3 percent) and depression (44.1 percent). ${ }^{188} \mathrm{~A}$ state-based 2006 survey of primary care physicians found that the vast majority (88 percent) screen for diabetes in adults with risk factors such as obesity, hypertension and a family history of diabetes. ${ }^{189}$

A 2004 survey showed that less than one third of certain medical professionals--registered nurses, dentists, psychiatrists and emergency medicine physicians--had received training in smoking cessation. ${ }^{190}$ Another national study found that only half of psychiatry residency programs offer training in tobacco cessation, ${ }^{191}$ even though a state-based survey found that 94 percent of psychiatry residents would be interested in receiving available training. ${ }^{192}$ A study of fourth-year medical students in New York City found that the majority (85 percent) did not know of local smoking cessation programs to which to refer patients. ${ }^{193}$ And a national survey of directors and assistant directors of U.S. medical school obstetrics/gynecology training programs found that only nine percent reported offering students at least 15 minutes of time dedicated to improving students' tobacco cessation skills and only one-third (32.9 percent) reported that their programs taught students both how to intervene with patients who smoke and how to refer them for follow-up. ${ }^{194}$

Medical curricula, by providing insufficient information about recent advances in the neurological science of addiction, perpetuate misconceptions about the disease of addiction and inhibit the acceptance of biological models to explain the disease. ${ }^{195}$

Inadequate training with regard to tobacco, alcohol and other drug use also derives from limited exposure to role models in the field who have knowledge about these issues. ${ }^{196}$ Curriculum time and the number of faculty with expertise in addiction education pale in comparison to curriculum time and the number of faculty with expertise in education for health conditions with similar prevalence rates as addiction, such as cancer and heart disease. ${ }^{197}$

As medical students, many of us are perplexed by the lack of a formal standard of care regarding addiction. The sad thing is, many of my fellow students and I feel that too many of our attending physicians have not demonstrated to us that they believe that addiction can and should be addressed and that attitude affects patient care for the worse. ${ }^{198}$

--Kimberly Fitzgerald Fourth-year medical student

More than 20 years ago, the subspecialty of addiction psychiatry officially was established, ${ }^{199}$ yet there often are more addiction 
psychiatry residency positions available than there is demand for them. Although a survey of psychiatry residents found that most had positive attitudes towards addiction psychiatry, few residents believed that addiction psychiatrists were well paid and less than half (45 percent) believed a career in addiction psychiatry would be satisfying. ${ }^{200}$

The lack of physician training in addiction and its treatment has very real effects on patient care. For example, overwhelming evidence has proven that smoking cessation interventions are clinically effective and cost effective and that a patient's chances of quitting smoking are nearly doubled if a health professional advises him or her to quit. ${ }^{201}$ Yet many medical schools do not require clinical training in smoking cessation. ${ }^{202}$

There are trained clinicians who do not fully understand the nature of addiction.

I am amazed at how many if not most medical professionals have no understanding and little education on the subject.

My relapse was in part due to ignorance in the medical profession and lack of medical addiction understanding during a lifethreatening illness.

...I escaped the clutches of doctors and psychiatrists with their prescription pads and rotten advice due to lack of understanding, due to lack of education. ${ }^{203}$

--Respondents to CASA Columbia's Survey of Individuals in Long-Term Recovery

Other Health Professionals. Doctoral-level clinical psychologists are highly trained in psychosocial therapies, many of which can be applied effectively to addressing addiction in the significant proportion of their patient population that has co-occurring addiction and mental health disorders. ${ }^{204}$ Yet because few psychologists receive adequate training in screening and intervention for risky substance users and in diagnosing, treating or referring patients with addiction, some fail to identify risky use or addiction or lack confidence in their ability to provide psychosocial therapies. ${ }^{205}$ For example, one study found that 17.1 percent of clinical psychologists reported that a barrier to providing smoking cessation counseling was their "lack of training in tobacco cessation skills." Other reported barriers also were related to a lack of knowledge about the disease: that smoking was "not the client's presenting problem" (57.1 percent); that smoking cessation is not "a priority for my patients" (28.7 percent); "it may interfere with therapy goals" (21.2 percent); and "smoking patients are not interested in smoking cessation counseling" (19.8 percent). ${ }^{206}$

Dental professionals also receive inadequate training in caring for patients with risky substance use and addiction, ${ }^{207}$ despite significant evidence of the important role they can play in screening, intervention and referral to treatment. ${ }^{208}$ There are no national standards for tobacco cessation education in U.S. dental schools and the ability to provide tobacco cessation services is not considered a clinical competency. ${ }^{209}$ Only about half of dental schools and dental hygienist programs ${ }^{*}$ have tobacco cessation clinical activities integrated in their student clinics. ${ }^{210}$ This is despite the fact that dental professionals are highly receptive to receiving substance-related training ${ }^{211}$ and that appropriate training early in a clinician's career increases the likelihood that such interventions will be adopted and implemented in practice. ${ }^{212}$

Nurses constitute the largest group of health care professionals ${ }^{213}$ with extensive patient contact; therefore, they are ideally situated to perform patient education, screening and brief intervention services. ${ }^{214}$ Yet, nurses are not adequately prepared to perform these services, particularly tobacco cessation for which research indicates they can be particularly effective. ${ }^{215}$ Barriers to the implementation of smoking interventions include a reported lack of motivation, self- efficacy, institutional support, time and training. ${ }^{216}$ Nursing school curricula have little tobacco control content; there is a lack of tested curricula, nurse educators are not

\footnotetext{
* Forty-seven percent of dental schools and 55 percent of dental hygienist programs.
} 
trained in it and it is considered to be a low priority in already overloaded curricula. ${ }^{217}$

Although pharmacists who engage in tobacco cessation interventions are effective in providing those services, ${ }^{218}$ and despite the important role pharmacists can play in preventing the misuse of controlled prescription drugs, ${ }^{219}$ most are not well trained to perform these functions, have little confidence to do so and believe that most patients are not interested in having them intervene. $^{220}$

A study in California found that the majority of pharmacists (88 percent) indicated that they would be interested in receiving specialized training in tobacco cessation counseling but fewer than eight percent had received any formal training. ${ }^{221}$ A study of pharmacists in Florida found that 29.2 percent reported that they received no addiction-related education in pharmacy school and 53.7 percent reported that they had never referred a patient to addiction treatment. ${ }^{222}$

\section{Inadequate Use and Development of Pharmaceutical Treatments for Addiction}

The underutilization of pharmaceutical therapies in addiction treatment is another example of the disconnect between addiction treatment services and medical care. Many addiction treatment providers are unable to prescribe pharmaceutical therapies and medical professionals who could prescribe such therapies fail to address addiction. A related problem is that some medical professionals appear to have a disproportionate concern about the safety risks of addiction medications relative to medications aimed at treating other medical conditions. For example, although side effects for some addiction medications have been noted and safety concerns raised--particularly with regard to smoking cessation treatments, ${ }^{223}$ side effects exist for many medications aimed at treating other health conditions and typically are acknowledged as an acceptable risk of treatment.

Even when utilizing pharmaceutical treatments, medical professionals often fail to prescribe them at therapeutic doses. For example, methadone, which is used in treatment for addiction involving opioids, often is not prescribed as clinically recommended, undermining its effectiveness. ${ }^{224}$ Specifically, although it is well understood that dosages between 60-100 mg per day promote retention in treatment and reduction of opioid use, ${ }^{225} 34$ percent of patients are given doses of less than $60 \mathrm{mg}$ per day and 17 percent are given doses of less than 40 mg per day. ${ }^{* 226}$ Treatment programs more likely to give suboptimal doses of methadone include those with directors who take a 12-step approach to addiction treatment. $^{227}$

The Institute of Medicine and the Office of National Drug Control Policy (ONDCP) have recommended that treatment for addiction involving opioids be integrated into mainstream medical practice to improve availability and quality. ${ }^{228}$ New York City has been a leader in this area with its Methadone Medical Maintenance program established more than 25 years ago, ${ }^{229}$ yet only 56 percent of need for methadone treatment in New York City was met in $2009 ;{ }^{230}$ inadequate training of providers may have restricted its expansion and integration with mainstream medical care.

Furthermore, despite the potentially vast market for pharmaceutical treatments for addiction, the pharmaceutical industry has not made substantial investments in the development of new and effective addiction treatment medications. ${ }^{231}$ One of the most significant contributing factors to the increased medical treatment of mental health disorders, such as depression and anxiety, in the past two decades has been the development and marketing of pharmaceutical treatments for these conditions. ${ }^{232}$ However in recent years, the pharmaceutical industry has cut back dramatically on investments in the development of new pharmaceutical therapies for these and other mental health conditions. ${ }^{233}$ The large profits that pharmaceutical companies were able to accrue from medications that were modified

\footnotetext{
* Although initial methadone treatment begins at dosages under $40 \mathrm{mg} /$ day, the amount is increased gradually until cravings disappear.
} 
versions of drugs already approved by the Food and Drug Administration meant that investments in innovative new medications were limited. Coupled with the rising cost of research and development, the fact that pharmaceutical companies face dramatic losses once the patents on many of their largest money-making drugs expire makes the current climate for the development of new innovative medications quite unfavorable. ${ }^{234}$

This is particularly unfortunate since recent advances in addiction science have highlighted specific neurotransmitter receptors and transporters that underlie addiction and that are promising targets for the development of medications to prevent and treat addiction. ${ }^{235}$

Aside from economic concerns, other factors inhibiting investments in new pharmaceutical interventions for addiction include an underestimation of the market for addiction treatment medications (i.e., the size of the population with addiction), the belief that the majority of those with addiction lack health insurance and the ability to pay for medications, and the long-standing stigma associated with the use of illegal substances and the disease of addiction. ${ }^{236}$

For the most part, SUDs [substance use disorders] have not been high priority targets for the pharmaceutical industry. Even for smoking cessation, which offers a huge potential market, investments are negligible compared with the costs associated with developing medications to treat the consequences of smoking. ${ }^{237}$

\section{--Volkow \& Skolnick, 2012}

Translating the rapidly-evolving science of addiction into science-based treatments will require dramatic changes in incentives for the pharmaceutical industry to invest in innovative medications, increased public understanding that addiction is a treatable disease, and increased involvement of the health care system in its prevention and treatment. ${ }^{238}$

\section{Inadequate Quality Assurance}

Addiction treatment providers do not speak with clarity or consistency about what the goals of treatment are, what counts as quality treatment, how performance and outcomes should be measured and what practices should be implemented to improve treatment and achieve measurable outcomes. ${ }^{* 239}$ And because addiction treatment for the most part is not integrated into mainstream health care, quality assurance standards and efforts to improve such standards in mainstream medicine largely do not apply to addiction treatment. ${ }^{240}$ For example, organizations like the Council on Graduate Medical Education and the National Advisory Council on Nurse Education and Practice are public-private partnerships with Congressional mandates to provide sustained assessment of the needs of the medical and nursing fields. ${ }^{241}$ However, these organizations do not appear to address addiction treatment and comparable organizations for addiction treatment do not exist.

Efforts to assure quality treatment within the mainstream medical system for patients with addiction are not typically adhered to. For example, as far back as 1979, the American Medical Association (AMA) adopted as policy the AMA Guidelines for Physician Involvement in the Care of Substance-Abusing Patients, which states that every physician must assume clinical responsibility for the diagnosis and referral of patients with addiction and explicates the particular competencies needed to fulfill that responsibility. Other similar efforts followed, with a national conference sponsored by the Office of National Drug Control Policy, the Leadership Conference on Medical Education in Substance Abuse, in 2004. Despite these and other efforts by government and professional organizations to put forth guidelines and principles aimed at ensuring proper training in risky substance use and addiction, physicians continue to be insufficiently equipped to address the needs of their substance-involved patients. ${ }^{242}$

\footnotetext{
* See Chapter IX.
} 
Efforts to assure quality treatment for addiction have proven highly fallible, with many barriers standing in the way of adequate performance and outcome measurement, including limited consensus regarding core quality standards and measures, inadequately developed measures and improvement mechanisms, and inadequate infrastructure and technical capacity. ${ }^{243}$

Further, the way in which addiction treatment is evaluated differs considerably from the evaluation of the effectiveness of treatment for other health conditions like diabetes, hypertension and asthma. Specifically, evaluations of the effectiveness of treatment of chronic diseases typically are conducted while the patient continues to receive treatment, since the treatment is considered critical to managing the disease. In the case of chronic addiction, however, evaluations of treatment effectiveness usually are conducted after the treatment has been withdrawn. ${ }^{244}$ In other words, we use an acute care model to evaluate treatments for a chronic condition.

Furthermore, because treatment facilities in some states cannot be licensed to provide both mental health and addiction treatment services, the mental health and addiction treatment systems are divided further. Given the large number of patients who suffer from co-occurring addiction and psychiatric disorders, the inability to treat both conditions concurrently within the same program is a significant barrier to providing quality care; it reduces the diagnosis and treatment of co-occurring conditions, impedes coordination of care, and increases the number of transfers which disrupts treatment. ${ }^{245}$

\section{Lack of Consensus Regarding the Main Goals} of Treatment. The primary goals of medical care are the prevention, diagnosis and treatment of illness, injury and disease, and the consequent relief of pain and suffering. ${ }^{246}$ The picture is not as clear in addiction treatment: there is little agreement among addiction treatment providers about what the goals of treatment are or should be and whether successful treatment is defined by abstinence, a reduction in clinical symptoms or a reduction in negative health and social consequences. $^{247}$ Goals are not defined as improving health and function or disease stabilization as they are with other health conditions. Such inconsistency in goals makes measuring and assessing the effectiveness of treatment very difficult.

CASA Columbia's survey of members of key treatment provider associations ${ }^{*}$ found that the majority (78.5 percent) "strongly agree" that improvements in functioning (e.g., employment, education, parenting, family stability, crime and recidivism, health, happiness, citizenship) is an important goal of addiction treatment; about half (52.5 percent) "strongly agree" that complete abstinence is an important goal; 44.0 percent "strongly agree" that remission of clinical symptoms is an important goal; and 43.8 percent "strongly agree" that reduced substance use is an important goal. Nearly half (45.1 percent) of respondents "strongly agree" that the patient should be allowed to set the goal that is right for him or her. ${ }^{248}$

Respondents to CASA Columbia's survey of treatment program directors and staff providers in New York State were more uniform in their support of complete abstinence as the main treatment goal for someone with addiction involving nicotine, alcohol or other drugs. ${ }^{249}$ (Table 10.2)

\footnotetext{
${ }^{*}$ See Appendix F.
} 
Table 10.2

New York State Addiction Treatment

Providers' Opinions of the Main Goal of Treatment, by Substance

\begin{tabular}{l|r|r}
\hline & $\begin{array}{c}\text { Program } \\
\text { Directors }\end{array}$ & $\begin{array}{c}\text { Staff } \\
\text { Providers }\end{array}$ \\
\hline Tobacco & & \\
\hline Complete abstinence & 49.4 & 48.2 \\
\hline Reduced use & 10.8 & 21.3 \\
\hline Fewer negative consequences & 4.8 & 11.3 \\
\hline Goal set by patient & 41.0 & 41.1 \\
\hline Other goal & 3.6 & 6.4 \\
\hline Alcohol & & \\
\hline Complete abstinence & 61.4 & 70.2 \\
\hline Reduced use & 3.6 & 9.2 \\
\hline Fewer negative consequences & 10.8 & 9.2 \\
\hline Goal set by patient & 25.3 & 22.0 \\
\hline Other goal & 2.4 & 3.5 \\
\hline Other Drugs & & \\
\hline Complete abstinence & 66.3 & 65.2 \\
\hline Reduced use & 8.4 & 9.2 \\
\hline Fewer negative consequences & 13.3 & 9.9 \\
\hline Goal set by patient & 30.1 & 26.2 \\
\hline Other goal & 3.6 & 5.7 \\
\hline $\begin{array}{l}\text { Source: CASA Columbia Survey of New York State } \\
\text { Addiction Treatment Providers, 2009. }\end{array}$ \\
\hline
\end{tabular}

Inadequate Performance and Outcome

Measurement. Government and private funders and insurance companies increasingly are pressing addiction treatment programs to demonstrate the effectiveness of their programs and services. ${ }^{250}$ However, most programs do not measure performance or treatment outcomes or have reliable evaluation data to demonstrate the efficacy of their services. ${ }^{251}$ There is no national, unified body that sets standards for addiction treatment. ${ }^{252}$

CASA Columbia's survey of treatment program directors in New York State found that, when asked about the three main ways that their program evaluates how well it is doing, the most common response offered was "program completion rates" (68.7 percent); the next most common response was "random client feedback/testimonials" (54.2 percent). Neither of these measures adequately reflects treatment quality or effectiveness. Long-term follow up of patients is not typical, as only 34.9 percent of program directors reported following patients for more than six months after treatment exit to assess how well their patients were faring. ${ }^{253}$ Further complicating the measurement of outcomes is the failure to understand that addiction in many cases is a chronic disease that requires management over time.

The effectiveness of addiction treatment is evaluated based on perverted measures of success...you'd never judge the benefits of an antihypertensive medication AFTER the medication had been stopped. ${ }^{254}$

--Michael M. Miller, MD Past President American Society of Addiction Medicine

Most addiction treatment programs lack resources to develop and implement effective measurement systems. ${ }^{255}$ Integrating addiction treatment into mainstream health care, however, would permit the performance and outcome measures and standards that have been well established for the treatment of other health conditions to be applied to addiction treatment as well.

\section{Inadequate Insurance Coverage}

In spite of recent expansions in coverage for intervention and addiction treatment through health care parity laws and the Affordable Care Act, insurance coverage for addiction and related services remains severely limited compared with coverage for other health conditions. ${ }^{* 256}$ This lack of insurance coverage for effective--and cost-effective--intervention and addiction treatment services provides a further barrier to increasing the attention of health care professionals to this disease. The end result is that millions of Americans are denied treatment, health care costs continue to rise as do social consequences and costs, and taxpayers foot the bill. ${ }^{257}$

\footnotetext{
${ }^{*}$ See Chapter VIII.
} 


\section{No Overarching Organizing Body for Addiction Science and Treatment}

Unlike other major health conditions, there is no highly credible and unifying organization, within or outside of the National Institutes of Health (NIH), which sets standards and advocates for addiction prevention and treatment, including all manifestations of addiction.

A key mission of the National Cancer Institute is to conduct and support "research, training, health information dissemination and other programs with respect to the cause, diagnosis, prevention and treatment of cancer, rehabilitation from cancer, and the continuing care of cancer patients and the families of cancer patients." 258 Likewise, according to the American Heart Association's description of its organization, "we fund innovative research, fight for stronger public health policies and provide lifesaving tools and information to prevent and treat these diseases."259

Although there are federal institutes and organizations dedicated to substance use and addiction, including the National Institute on Drug Abuse (NIDA), the National Institute on Alcohol Abuse and Alcoholism (NIAAA) and the Substance Abuse and Mental Health Services Administration (SAMHSA) as well as other organizations and institutes for which substance use and addiction are part of their focus, ${ }^{*}$ there is no unified body that promotes research, public awareness and prevention and treatment quality for the disease of addiction. The result is a disjointed array of programs and efforts that often have quite different perspectives and approaches to addressing the issues surrounding substance use and addiction, which further contributes to the segmented view of addiction as multiple substance- and behavior-specific problems, rather than a single disease with different manifestations.

\footnotetext{
* Such as the National Cancer Institute (NCI), primarily in relation to smoking, the National Institute of Mental Health (NIMH) and the Centers for Disease Control and Prevention (CDC).
}

A recent plan by the NIH would combine public health initiatives related to substance use and all addiction-related research within the NIH into a new institute focused on substance use and addiction. ${ }^{260}$ This proposal is controversial with regard to how the merger will affect the funding and research portfolios not only of NIDA and NIAAA but also of other NIH institutes with interests that involve substance use and addiction (e.g., those that address cancer, HIV, prenatal care, birth defects, obesity, pain control). ${ }^{261}$ Its strength is a clear and longoverdue recognition of the nature of the disease of addiction and the importance of addressing it in a unified way.

\section{Efforts to Integrate Substance Use Prevention and Treatment into Mainstream Medicine}

Recent efforts aimed at improving the prevention of risky substance use and the treatment of addiction have concentrated on the application of quality improvement and quality assurance measures such as those that have gained traction in mainstream health care practice:

- Developing, disseminating and promoting evidence-based practices; ${ }^{262}$

- Improving the treatment provider workforce through enhanced education and training; ${ }^{263}$

- Improving performance measures; ${ }^{264}$

- Shifting evaluation efforts from a focus on performance-based measures that document the process of service delivery to a focus on patient outcomes, and rewarding those programs that demonstrate positive patient outcomes; ${ }^{265}$ and

- Using electronic health records and other forms of health information technology to track and monitor patients and help improve the coordination and delivery of care. ${ }^{266}$ 
Many of these efforts, however, have been aimed at shoring up and maintaining a separate system of addiction treatment outside of mainstream health care practice. Although these are steps in the right direction, the research documented in this report demonstrates clearly that the current treatment infrastructure is riddled with barriers to closing the vast gap between research evidence and practice. ${ }^{267}$ As such, modifications to the existing system simply will not suffice to make significant, meaningful and lasting improvements in how risky substance use and addiction are addressed in the United States.

What is required is an integration of screening and intervention for risky substance use and treatment and management of addiction into mainstream health care, assuring that addiction treatment is managed by trained medical professionals and supported by an array of highly-trained clinical mental health professionals and other providers of essential support and auxiliary services. ${ }^{268}$ Such integrated care leads to improved patient outcomes and significantly-reduced medical costs. $^{269}$

Several recent developments have begun to make significant strides in this direction:

- Efforts to expand insurance coverage for screening and brief interventions in primary care settings, to offer equitable coverage for addiction treatment services and to ensure accountability by establishing quality benchmarks in patient care as a condition for coverage. ${ }^{270}$

- Efforts by professional societies and associations to professionalize addiction treatment, have the medical field respond to risky substance use as a public health problem and addiction as a medical condition, and integrate care for risky substance use and addiction into medical practice. Notable examples include:

$>$ The American Society of Addiction Medicine (ASAM) which is seeking to improve the quality of care for risky substance use and addiction by: educating physicians and other health professionals about these issues; supporting research and prevention efforts; advocating for state and federal policies that would expand access to treatment; and working to establish addiction medicine as a recognized medical specialty. ${ }^{271}$

$>$ The American Board of Addiction Medicine (ABAM), which was founded in 2007 with the encouragement and assistance of ASAM, is working toward the goal of establishing addiction medicine as a medical specialty recognized by the American Board of Medical Specialties (ABMS). ABAM's sister organization, the ABAM Foundation, began accrediting one- or two-year fellowship training programs-"secondary residencies"--in addiction medicine open to physicians completing training in any field certified by the ABMS member boards.* Such programs have the ability to train the physician experts needed to meet the demand within health care teams at all locations where regular medical care is received--from hospitals to community clinics. The ABAM Foundation also is working toward having its fellowship training programs accredited by the Accreditation Counsel for Graduate Medical Education (ACGME). ${ }^{272}$ NIAAA and NIDA recently awarded grants to SUNY Buffalo and to Boston University Medical Center to help support this effort. ${ }^{273}$

The American Society of HealthSystems Pharmacists, a member of the National Pharmacy Partnership for Tobacco Cessation, which has created a resource center to provide pharmacists with tobacco cessation information and tools to help patients stop smoking. ${ }^{274}$

\footnotetext{
* The ABAM Foundation to date has accredited 10 addiction medicine training programs across the country, with more programs under development.
} 
- Efforts by government agencies to promote physician education and training in addiction science and addiction treatment and upgrade health care professionals' skills through the development and dissemination of screening tools and evidence-based clinical guidelines. ${ }^{275}$ Notable examples include:

$>$ The National Institute on Drug Abuse's NIDAMED, a physician outreach initiative aimed at encouraging doctors to screen patients for risky substance use, including tobacco, alcohol and other drugs. NIDAMED offers free screening tools and prevention and treatment research and information that physicians can share with their patients. It also offers curriculum resources to provide scientifically accurate information to medical students, residents and faculty about substance use, addiction and its consequences. ${ }^{276}$

> The Substance Abuse and Mental Health Services Administration's SBIRT ${ }^{*}$ Medical Residency Program, which seeks to develop and implement training programs for medical residents in evidence-based screening, brief intervention, brief treatment and referral to specialty treatment for risky substance use and addiction involving alcohol and other drugs, and to promote more broadly the adoption of these practices in the medical field. ${ }^{277}$

\section{The Health Resources and Services} Administration's Project MAINSTREAM, which provides resources for educating a broad range of health care professionals ${ }^{\dagger}$ in screening, brief intervention, referral to treatment,

\footnotetext{
* Screening, Brief Intervention, Referral and Treatment.

${ }^{\dagger}$ Including audiologists, dentists, dieticians, nurse midwives, nurse practitioners, nurses, occupational therapists, pharmacists, physical therapists, physician assistants, physicians, psychologists, public health professionals, social workers and speech pathologists.
}

identifying and assisting children of parents with addiction and helping communities implement effective prevention programs related to addiction involving all substances. ${ }^{278}$

- Smaller-scale efforts to provide brief training to health professionals ${ }^{\ddagger}$ in addiction science in order to improve the attitudes, proficiency and practices of all health professionals ${ }^{279}$--not just those who choose to specialize in addiction medicine or addiction psychiatry. Such brief trainings have proven effective in improving health professionals' knowledge, attitudes, confidence, motivation and practices with regard to providing smoking cessation services; ${ }^{280}$ conducting screening and brief interventions for risky alcohol use in emergency departments ${ }^{281}$ and for risky alcohol and other drug use in primary care facilities; ${ }^{282}$ and for prescribing pharmaceutical medications such as methadone and buprenorphine for patients with addiction involving opioids. ${ }^{283}$

- The emergence of Patient-Centered Medical Homes $^{284}$--in which a team of health professionals provides comprehensive and continuous medical care--is proving to be a strong model for addiction treatment to be integrated into the primary medical care system. ${ }^{285}$ Recent research within the Medicaid and Veterans Health Administration systems demonstrates the success of this approach ${ }^{286}$ and the potential to incorporate addiction care into this integrated system.

- Efforts by quality assurance organizations to encourage services that address risky substance use and addiction within mainstream health care. For example, in 2012, the Joint Commission announced new, voluntary measures for hospitals that choose to provide screening, brief intervention and referral to treatment for tobacco, alcohol and

\footnotetext{
${ }^{\ddagger}$ Including but not limited to physicians, physician assistants, nurses, nurse practitioners, dental professionals, pharmacists, social workers.
} 
other drug use. The performance measurement sets related to alcohol and other drugs include screening, brief interventions, treatment, discharge planning and follow up. ${ }^{287}$ The specifications for the tobacco cessation performance measures include screening all patients to identify tobacco users, providing or offering evidence-based counseling and medications for smokers during hospitalization and upon discharge, and assessing tobacco use 30 days post-discharge. ${ }^{288}$ Hospitals are required to choose four out of 14 possible core performance measurement sets, with no requirements about which sets must be chosen. ${ }^{289}$ For each core performance measurement set that the hospital chooses, the hospital is held accountable for collecting data and measuring performance related to the activities associated with the set. However, other sets of measures that hospitals may choose to be held accountable for include those that they already perform routinely, limiting the reach of this promising development which would require more effort and resources than most other measurement sets. ${ }^{290}$
Our perspective is that, although tactically impressive, the [Joint Commission's] measure set [regarding tobacco screening and cessation services] is strategically flawed because its adoption is optional. ${ }^{291}$

--Fiore, M.C., Goplerud, E., \& Schroeder, S. A. (2012)

If the Joint Commission would require hospitals to report their outcomes on SBIRT, it could do more to medicalize how we deal with risky substance use than all the urging and pleading we've undertaken for the past 25 to 30 years. $^{292}$

--Larry M. Gentilello, MD Trauma Services Kaiser Permanente Hospital Sacramento, CA 


\section{Chapter XI \\ Recommendations and Next Steps}

As this report has documented, addiction is a complex brain disease and the risky use of addictive substances is a significant public health problem. Together they result in untold human suffering and cost taxpayers billions of dollars each year. Effective, evidence-based interventions and treatment options exist that can and should be delivered through the health care system. A substantial body of research demonstrates that providing effective prevention, intervention, treatment and disease management services yields improvements in health and considerable reductions in costs to government and taxpayers; research also suggests that providing these services does not result in significant increases in insurance costs. In the face of these facts, it is unethical, inhumane and cost prohibitive to continue to deny effective care and treatment for the 40.3 million Americans with this disease or to fail to screen and intervene with the 80.4 million who engage in risky use of addictive substances.

No one group or sector alone can realize the changes required in health care practice, government regulation and spending, insurance coverage, and public understanding to bring addiction prevention and treatment and reductions in risky substance use in line with the standard of care for other public health and medical conditions. Concerted action is required on the part of physicians and other medical and health professionals, policy makers, insurers and the general public.

This is not an unprecedented challenge. There have been many examples where health care practice has lagged behind the science. Only recently, depression was considered a character flaw before the brain science was understood and HIV/AIDS was considered a moral scourge before it was seen as a virus that can be prevented, treated, managed and perhaps cured. Likewise, addiction has been seen for too long 
as a character flaw and a moral failing rather than a preventable and treatable disease.

It is past time for health care practice to catch up with the science. Efforts already underway to close this gap must be expanded and accelerated. Toward this end, CASA Columbia makes the following recommendations:

\section{Reform Health Care Practice}

\section{Incorporate Screening and Intervention for Risky Substance Use, and Diagnosis, Treatment and Disease Management for Addiction into Routine Medical Practice}

As essential components of routine medical care practice, all physicians and other medical professionals should provide their patients with:

- $\quad$ Routine screening for all forms of risky substance use--including tobacco, alcohol, illicit drugs and controlled prescription drugs--at initial visit to a primary care or specialty care physician and routinely thereafter, and upon entry into a hospital, emergency department, trauma center or clinic;

- Brief interventions as needed;

- Comprehensive assessment to determine disease stage and severity as well as the presence of co-occurring health conditions and special population needs;

- Patient stabilization (e.g., detoxification), when necessary, as a precursor to treatment;

- Development of a tailored treatment plan that includes:

Acute treatment via evidence-based psychosocial and/or pharmaceutical interventions;

Chronic disease management, as needed;
$>$ Connection to support and auxiliary services--including legal, educational, employment, housing and family supports, nutrition and exercise counseling, and mutual support programs; and

$>$ Referral to physicians trained in addiction medicine or addiction psychiatry for specialty care as needed.

Evidence-based screening can be conducted by a broad-range of licensed providers with general training in addiction and specific training in how to conduct such screens and what to do with patients who screen positive. Brief interventions can be provided by health professionals-licensed graduate-level medical or mental health clinicians--trained in addiction care.

Treatment and disease management services can be provided through a multi-disciplinary team of appropriately trained and credentialed health professionals managed by a physician:

- Comprehensive assessment, diagnosis, stabilization, acute treatment and disease management must be performed or managed by physicians in collaboration with a team of licensed graduate-level medical or mental health clinicians trained in addiction care;

- Case management can be provided by licensed physician assistants, nurses and nurse practitioners and mental health clinicians trained in addiction care;

- Auxiliary services can be provided by a range of professional and paraprofessional personnel working within the treatment and disease management plan;

- Peer support, often an important component of the larger treatment plan, can be provided by those who have learned to manage the disease. 


\section{All Medical Schools and Residency Training Programs Should Educate and Train Physicians to Address Risky Substance Use and Addiction}

All physicians should be educated and trained in the origins of risky substance use and addiction; prevention, intervention, treatment and management options; co-occurring conditions; and special population and specialty-care needs, including:

- What constitutes risky substance use, the harms of such use to health and safety and the importance of reducing risky use;

- How to screen for risky substance use and conduct brief interventions when indicated;

- The causes and correlates of addiction;

- How to diagnose addiction; evaluate disease stage, severity, co-occurring disorders and needs of special populations; and develop a treatment and disease management plan including appropriate support services;

- How to collaborate with and manage a multidisciplinary team of providers;

- How to provide or supervise psychosocial and pharmaceutical treatments for addiction and disease management;

- How to arrange for and connect patients with auxiliary support services; and

- How to determine the need for specialty care and connect patients with such care.

These core clinical competencies should be required components of all medical school curricula, medical residency training programs, medical licensing exams, board certification exams and continuing medical education (CME) requirements, including maintenance of certification programs.

\section{Require Non-Physician Health Professionals to be Educated and Trained to Address Risky Substance Use and Addiction}

Develop core clinical competencies in addressing risky substance use and preventing and treating addiction for each type of nonphysician health professional including physician assistants, nurses and nurse practitioners, dentists, pharmacists and graduatelevel clinical mental health professionals (psychologists, social workers, counselors). These competencies should include:

- What constitutes risky substance use, the harms of such use to health and safety and the importance of reducing risky use;

- How to screen for risky substance use and conduct brief interventions when indicated;

- The causes and correlates of addiction;

- Available psychosocial and pharmaceutical treatments for addiction and disease management;

- How to arrange for and connect patients with auxiliary support services; and

- How to determine the need for specialty care and connect patients with such care.

Assure that these core clinical competencies and specialized training are required components of all professional health care program curricula, graduate fellowship training programs, professional licensing exams and continuing education (CE) requirements.

For non-physician health professionals involved in the provision of addiction care, core competencies also should include how to work in concert with a patient's physician and other health care providers to screen for risky substance use; provide brief interventions; diagnose addiction; evaluate disease stage, severity and co-occurring disorders; and develop 
a treatment plan, including appropriate support services.

Require all non-physician health professionals providing psychosocial addiction treatment services to have graduate-level clinical training in delivering these services.

Require that all pharmaceutical treatments for addiction be provided only by a physician or in accordance with a treatment plan managed by a physician.

\section{Develop Improved Screening and Assessment Instruments}

Screening instruments should be adjusted or developed to coincide with appropriate definitions of risky substance use, and assessment instruments should be adjusted or developed to mirror diagnostic criteria for addiction. Both screening and assessment instruments should address all types of addictive substances.

\section{Establish National Accreditation Standards for All Addiction Treatment Facilities and Programs that Reflect Evidence-Based Care}

As a condition of accreditation, accrediting organizations should stipulate requirements for all facilities and programs providing addiction treatment with regard to professional staffing, intervention and treatment services and quality assurance:

- Professional Staffing. All facilities and programs providing addiction treatment should be required to have a full-time certified addiction physician specialist on staff to serve as medical director, oversee patient care and be responsible for all treatment services. All individual providers of patient care in these facilities and programs should be required to be licensed in their field of practice and demonstrate mastery of the core clinical competencies. Professionals who are in the process of becoming licensed must be supervised at all times by a licensed professional.

- Intervention and Treatment Services. All facilities and programs providing addiction treatment should be required to provide comprehensive assessment and evidencebased treatment for addiction involving all substances that is tailored to the stage and severity of the disease, co-occurring conditions and patient characteristics.

- Quality Assurance. All facilities and programs providing addiction treatment should be required to collect and report comprehensive quality assessment data, including process and outcome measurements related to screening, intervention, treatment and disease management, in accordance with established guidelines developed in collaboration with the American Board of Addiction Medicine.

\section{Standardize Language Used to Describe the Full Spectrum of Substance Use and Addiction}

Recognize addiction as a medical disease and standardize the language related to the spectrum of substance use severity in current and forthcoming diagnostic instruments. Develop a classification system based both on observable behavior and neurobiological measures that underlie different manifestations of addiction and related conditions which currently are classified and addressed as distinct conditions.

\footnotetext{
* Currently, the provision of such services frequently is optional. For example, the Joint Commission currently has voluntary performance measures for hospitals that choose to provide these addictionrelated services. However, hospitals are required to choose four out of 14 possible core performance measurements sets and may completely avoid those related to addiction care (see Chapter X).
} 


\section{Use the Leverage of Public Policy to Speed Reform in Health Care Practice}

\section{Condition Grants and Contracts for Addiction Services on the Provision of Quality Care}

Federal, state and local governments should require--as a condition of receipt of public funds--that grants, contracts and non-insurance reimbursement for addiction treatment services:

- Utilize evidence-based prevention and treatment approaches, including pharmaceutical therapies (provided or managed by a physician demonstrating the core competencies of addiction medicine or addiction psychiatry) and psychosocial therapies (provided by medical professionals or graduate-level clinical mental health professionals trained and licensed in the core competencies of addiction treatment), as indicated;

- Involve other health professionals, individuals providing auxiliary services and those providing peer support, working in a multidisciplinary team; and

- Generate positive and measurable long-term patient outcomes.

\section{Educate Non-Health Professionals about Risky Substance Use and Addiction}

Require that the topic of risky substance use and addiction be included in the education and training of government-funded professionals who do not provide direct addiction-related services but who come into contact with significant numbers of individuals who engage in risky substance use or who may have addiction. These include, but are not limited to law enforcement and other criminal justice personnel, legal staff, child welfare and other social service workers and educators. Substance use- and addiction-related content should include:
- What constitutes risky substance use, the harms of such use to health and safety and the importance of reducing risky use;

- The nature of the disease of addiction and the medical and other health services needed to address it; and

- How to identify signs and symptoms of risky use and addiction and connect those with such signs and symptoms to professional services.

\section{Identify Patients at Risk in Government Programs and Services where Costs of Risky Use and Addiction Are High}

Federal, state and local governments should require that routine screening and brief interventions be provided by trained professionals in all educational, mental health, developmental disabilities, child welfare, housing, juvenile justice and adult corrections services that receive public funding; and that patients who screen positive for risky use or a potential diagnosis of addiction be connected with a trained health professional for intervention, diagnosis, treatment and disease management.

\section{Develop Tools to Improve Service Quality}

Federal and state governments in collaboration with professional associations, accrediting organizations and other non-profit organizations focusing on health care quality--such as the Washington Circle, ${ }^{*}$ the National Committee for

\footnotetext{
* The Washington Circle is a group of national experts in addiction-related policy, research and performance management who seek to improve the quality and effectiveness of prevention and treatment services through the use of performance measurement systems.
} 
Quality Assurance (NCQA) ${ }^{*}$ and the National Quality Forum (NQF) ${ }^{\dagger}$--should develop and disseminate evidence-based tools, practice guidelines and performance measures oriented toward patient outcomes to improve the quality of addiction care (involving all substances) and require their implementation as a condition of continued licensure and/or accreditation.

\section{License Addiction Treatment Facilities as Health Care Providers}

Federal, state and local governments should subject all addiction treatment facilities and programs to the same mandatory licensing processes as other health care facilities.

\section{Require Adherence to National Accreditation Standards that Reflect Evidence-Based Care}

As a condition of licensure, federal, state and local governments should stipulate that all facilities and programs providing addiction treatment adhere to established national minimum standards for accreditation (see recommendation under Reform Health Care Practice above).

\section{Require that All Insurers Provide Coverage for Comprehensive Addiction Care}

- $\quad$ Require that all health insurers--public and private--provide coverage for all insured individuals for patient education, screening and intervention for risky substance use and treatment and management of addiction (involving all substances associated with

\footnotetext{
* NCQA focuses on improving health care quality and manages the Healthcare Effectiveness Data and Information Set (HEDIS), a standardized performance measurement tool used by the majority of health insurance plans in the United States to measure performance on important dimensions of care and service.

${ }^{\dagger} \mathrm{NQF}$ is a public-private partnership that establishes priorities for quality improvement and endorses standards for evidence-based addiction practice and measuring performance.
}

addiction) consistent with standards of medical practice, eliminating exemptions.

- As a condition of reimbursement, public payers and private insurance companies should be mandated to require that all addiction interventions and treatment be directly provided, supervised or managed by trained medical professionals.

- Public payers and private health insurance companies should use all available tools-including quality assurance measurements, pay-for-performance contracting and other incentives--to encourage participating providers and facilities to adopt evidencebased practices, institute qualityimprovement measures and assess patient outcomes.

- Eliminate the Uniform Accident and Sickness Policy Provision Law (UPPL) which bars insurance coverage for injuries sustained by a person who was under the influence of alcohol or other drugs at the time of the injury; this law impedes appropriate medical care.

\section{Expand the Addiction Medicine Workforce}

Accelerate the work begun by the American Board of Addiction Medicine Foundation to develop residency training programs in addiction medicine and secure residency accreditation from the Accreditation Council for Graduate Medical Education (ACGME). Pursue and gain recognition of addiction medicine by the American Board of Medical Specialties (ABMS). Support the efforts of ACGMEaccredited addiction psychiatry residencies to increase the number of enrolled residents. Through these actions, assure that addiction medicine training programs are available to physicians, that training opportunities within addiction psychiatry are expanded, and that such specialty care will be available in every hospital throughout the country and through every health care system, including the Federally Qualified 
Health Centers (FQHCs), ${ }^{*}$ the Department of Veterans Affairs (VA) and the Indian Health Service (IHS):

- Allocate a designated portion of the federally-funded (primarily through Medicare) medical residency training positions to residency training in the specialty of addiction medicine, including addiction psychiatry.

- Allocate residency training slots through the VA and the IHS to addiction medicine to help ensure the availability of specialty care for veterans and Native Americans.

- $\quad$ Provide additional resources as needed to immediately increase the training and availability of addiction medicine specialists to meet the need nationwide.

I think we have to be mindful that creating a subspecialty is not a substitute for physicians and other providers developing greater knowledge and skill in the addiction field. There are too many patients. We're not going to be able to train enough specialists to treat all those folks. The general medicine field needs to accept that these are legitimate medical conditions for which they should take responsibility. ${ }^{1}$

--Peter D. Friedmann, MD, MPH Professor of Medicine Professor of Health Services, Policy and Practice Brown University

We want addiction prevention, screening, intervention, and treatment to become routine aspects of medical care, available virtually any place health care is provided. ${ }^{2}$

--Kevin B. Kunz, MD Former President American Board of Addiction Medicine

(ABAM)

\footnotetext{
${ }^{*}$ FQHCs serve populations such as the homeless, those residing in public housing and migrant workers.
}

\section{Implement a National Public Health Campaign}

Implement a nationwide public health campaign through the Centers for Disease Control and Prevention (CDC), the Food and Drug Administration (FDA) and other federal agencies charged with protecting the public health to educate the public about all forms of risky substance use and addiction, specifically with regard to:

- What constitutes risky substance use and the nature of the disease of addiction;

- Risk factors for each as well as their health, social, safety and economic consequences;

- The importance of preventing all forms of substance use among adolescents to protect their immediate health and safety and because of the link between early use and later addiction;

- How to spot signs of risk in individuals of all ages; and

- When to seek help and where to turn for effective intervention and care.

\section{Invest in Research and Data Collection to Improve and Track Progress in Addiction Prevention, Treatment and Disease Management}

Invest in research designed to:

- Develop reliable and valid screening and assessment tools that address risky substance use and addiction involving all substances and addictive behaviors, and that can be tailored to special populations.

- Identify bio-markers associated with disease stages.

- Provide evidence from clinical trials to further the understanding of: 
The efficacy of stabilization, acute treatment (psychosocial and pharmaceutical interventions) and disease management for addiction involving all substances; and

The effectiveness of these approaches in various real-world settings, including among people of different ages, races/ethnicities, in the justice population and among other special populations.

- Determine best practices in addiction care and how best to move evidence-based interventions into practice.

- Evaluate the effectiveness of current and newly-developed interventions and treatments against models of best practice, as well as the effectiveness of various types of support services as adjuncts to clinical treatment.

- Better understand the shared genetic, biological and environmental mechanisms underlying different manifestations of addiction.

- Develop the technological infrastructure (including electronic health records) necessary to collect and disseminate performance and outcomes measures for research and evaluation.

- Develop practice support tools such as those for electronic screening and brief intervention (e.g., via the Internet, smart phones and other electronic devices), based on findings from implementation research, that facilitate integration of addiction care into medical practice.

- Provide incentives to the pharmaceutical industry to develop and market new and effective pharmaceutical interventions for addiction treatment.

- Collect data in areas that currently are devoid of evidence, such as:
The screening and intervention services gap--data collected on a unified sample regarding both the prevalence of risky substance use and the prevalence of receipt of specific screening and intervention services.

$>$ Treatment of addiction involving nicotine alone and in combination with other substances and behaviors-prevalence, types of treatments, expenditures and outcomes.

- $\quad$ Find a cure for the disease of addiction.

\section{Implement the National Institutes of Health's (NIH) Recommendation to Create a Single Institute Addressing Substance Use and Addiction}

Create a unified national institute focused on substance use and addiction, recognizing the overarching disease of addiction rather than continuing the focus on different manifestations of substance use and the disease--tobacco, alcohol, other drug use--and including the risky use of all addictive substances. Include in the research portfolio addiction involving behaviors other than substance use, and focus on the causes, correlates, consequences, interventions, policies and possible cures for all manifestations of the disease. The portfolio of the institute also should include health conditions resulting from risky use and addiction (such as birth defects, cardiac complications, Hepatitis C, substancerelated HIV-AIDS) and other conditions which increase the risk of developing addiction (such as post-traumatic stress disorder, traumatic brain injury and other mental health disorders). 


\section{Appendix A Methodology}

CASA Columbia performed the following activities to present a comprehensive analysis of addiction and its treatment in the United States:

- A thorough review of more than 7,000 scientific articles, reports, books and other reference materials related to the science of addiction, the consequences of risky use and addiction, the prevention of risky use and treatment of addiction and barriers to improved care;

- Secondary analyses of five national data sets;

- Interviews with and suggestions from 174 leading experts in a broad range of fields relevant to the report, including researchers, physicians and other health professionals, other treatment providers, policymakers and members of professional associations, advocacy organizations, health insurers, pharmaceutical companies and organizations of people with the disease of addiction. Whereas the majority of these experts provided their thoughts in the context of an open-ended interview guide designed by CASA Columbia to explore key themes related to this project (see Appendix B for a list of the questions asked and of the individuals who were interviewed), some provided advice, suggestions and feedback about specific content to be included in this report;

- Focus groups and a national general population survey assessing the attitudes and beliefs of 1,303 adults with regard to addiction and its treatment: the National Addiction Belief and Attitude Survey (NABAS) (see Appendix C for the survey instrument); 
- Two statewide surveys of addiction treatment providers in New York State: one of 83 program directors and one of 141 staff treatment providers (see Appendices D and E for survey instruments);

- A national panel of treatment providers and an online survey of 1,142 members of professional associations involved in addiction care (see Appendix F for a list of panel members and for the survey instrument);

- An online survey of 360 individuals with a history of addiction who are managing the disease (i.e., in "long-term recovery") (see Appendix $\mathrm{G}$ for the survey instrument);

- An in-depth analysis of state, federal and professional associations' licensing and certification requirements for individual treatment providers and addiction treatment facilities and programs, as well as accreditation requirements for facilities and programs; and

- A case study of addiction treatment in New York State and New York City that drew from the research described above and the findings of which are incorporated into the report where relevant.

\section{Literature Review}

CASA Columbia research staff identified and reviewed more than 7,000 scientific articles, reports, books and other reference materials.

\section{Analysis of National Data Sets}

CASA Columbia's Substance Abuse Data Analysis Center (SADAC ${ }^{\mathrm{SM}}$ ) conducted extensive analyses of the following five national data sets:

- National Survey on Drug Use and Health (NSDUH);

- $\quad$ Treatment Episode Data Set (TEDS);
- National Survey of Substance Abuse Treatment Services (N-SSATS);

- $\quad$ Drug Abuse Warning Network (DAWN); and

- Fatality Analysis Reporting System (FARS).

\section{National Survey on Drug Use and Health}

The National Survey on Drug Use and Health (NSDUH), administered by the U.S. Department of Health and Human Services' Substance Abuse and Mental Health Services Administration (SAMHSA), is a cross-sectional national survey of approximately 70,000 (per year) randomly selected non-institutionalized individuals ages 12 and older in the United States. Because of changes made in survey methodology, time series data are available only from 2002.

The NSDUH is known to underestimate considerably the rate of substance use, particularly among young people, because it is administered in the home where a parent or other adult is present, increasing the risk that respondents will under-report substance use and other high-risk or illegal activities. ${ }^{1}$ The NSDUH also does not include high-risk institutionalized populations, such as prison inmates, hospital patients, patients in residential addiction treatment and others who cannot be reached in a home (e.g., the homeless), who tend to engage in substance use at higher rates than non-institutionalized populations. ${ }^{2}$

For each type of addictive substance, the NSDUH provides data on lifetime use, current use, frequency patterns, past-month diagnosis of nicotine addiction and past-year diagnosis of alcohol or other drug addiction. Data related to determining whether a respondent met clinical criteria for a past-year addiction diagnosis involving alcohol, controlled prescription drugs or illicit drugs correspond to the diagnostic criteria for alcohol or other drug abuse or dependence presented in the Diagnostic and Statistical Manual of Mental Disorders, 4th edition (DSM-IV). ${ }^{3}$ Data related to determining 
whether a respondent met clinical criteria for a past-month addiction diagnosis involving nicotine are based on the Nicotine Dependence Syndrome Scale (NDSS). ${ }^{4}$ The NDSS measures five dimensions of nicotine dependence based on symptoms and characteristics outlined in the DSM-IV. ${ }^{5}$ The NDSS was designed for adult smokers; although an adolescent version of the NDSS has been developed, ${ }^{6}$ the NSDUH uses the adult version.

CASA Columbia analyzed NSDUH data on individuals ages 12 and older living in U.S. households to examine the risky use of tobacco, alcohol, illicit drugs and controlled prescription drugs; addiction involving these substances; the prevalence of co-occurring health conditions; and rates of risky use and addiction among those who had past-year involvement in the justice system.

\section{Treatment Episode Data Set}

The Treatment Episode Data Set (TEDS), sponsored by the U.S. Department of Health and Human Services' Substance Abuse and Mental Health Services Administration (SAMHSA), provides information on the demographic and substance use characteristics of the approximately 2.0 million annual admissions in 2009 to addiction treatment programs in facilities that report to individual state administrative data systems. TEDS does not include all treatment admissions. Rather, it includes admissions to facilities that are licensed or certified by the designated state substance abuse agency to provide treatment (or are administratively tracked by the agency for other reasons). Facilities reporting TEDS data generally are those that receive state alcohol and/or other drug agency funds (including Federal Block Grant funds) for the provision of addiction treatment services; states report on all admissions to programs receiving public funds and on admissions to private facilities for which they have data. TEDS is an admissions-based system and TEDS admissions do not represent individuals. Thus, an individual admitted to treatment twice within a calendar year would be counted as two admissions.
The scope of admissions included in TEDS is affected by differences in state reporting practices, varying definitions of treatment admission, availability of public funds and public funding constraints. For example, treatment programs based in the criminal justice system may or may not be administered through the state substance abuse agency.

Detoxification facilities, which can generate large numbers of admissions, are not uniformly considered treatment facilities and are not uniformly reported by all states.

Facilities operated by federal agencies (e.g., the Bureau of Prisons, the Department of Defense and the Department of Veterans Affairs) generally do not report TEDS data to the state substance abuse agency, although some facilities operated by the Indian Health Service are included. Hospital-based addiction treatment facilities frequently are not licensed through the state substance abuse agency and do not report TEDS data. Correctional facilities (state prisons and local jails) are monitored by the state substance abuse agency and report TEDS data in some states but not in others.

In addition to admissions data, TEDS has a separate data set for patient discharges from addiction treatment. The discharge variables include treatment completion, length of stay, substances of addiction, type of services offered, demographic information and other data about those ages 12 and older who were discharged from addiction treatment facilities in 2008.

CASA Columbia analyzed TEDS admissions data for the years 2002 through 2009 to characterize the sources of referral, types of treatment and primary substances involved in the addiction of individuals ages 11 and older, as well as TEDS discharge data from 2008 to assess the completion and dropout rates for treatment admissions. (Note that after 2009, TEDS only reported admissions for those ages 12 and older). 


\section{National Survey of Substance Abuse Treatment Services}

The National Survey of Substance Abuse Treatment Services (N-SSATS), sponsored by the U.S. Department of Health and Human Services' Substance Abuse and Mental Health Services Administration (SAMHSA), is designed to collect information from all treatment facilities in the United States, both public and private, that provide addiction treatment. N-SSATS data allows examination of the composition of the U.S. addiction treatment delivery system. CASA Columbia analyzed this data to investigate variations in care by providers, the patterns of accreditation and licensing of facilities by various characteristics and the availability of services to specialized populations (e.g., adolescents, women, patients with co-occurring disorders).

\section{Drug Abuse Warning Network}

The Drug Abuse Warning Network (DAWN) public health surveillance system, conducted by the U.S. Department of Health and Human Services' Substance Abuse and Mental Health Services Administration (SAMHSA), monitors substance-related emergency department (ED) visits from a national sample of general, nonfederal hospitals that operate 24-hour EDs, with oversampling of hospitals in selected metropolitan areas. In participating hospitals, ED medical records are reviewed retrospectively to identify visits related to recent substance use. Illicit drugs, prescription and over-the-counter pharmaceuticals, dietary supplements and nonpharmaceutical inhalants are included in the analysis. Alcohol, when present in combination with another drug, is included as well. When alcohol is the only substance implicated in a visit, it is included only for patients younger than age 21.

CASA Columbia analyzed DAWN data to examine the number of ED visits involving alcohol and other drugs (excluding nicotine).

\section{Fatality Analysis Reporting System}

The National Center for Statistics and Analysis (NCSA) of the National Highway Traffic Safety Administration (NHTSA) conducts the Fatality Analysis Reporting System (FARS), a nationwide census providing yearly data regarding fatal injuries from motor vehicle traffic crashes. FARS contains data derived from a census of fatal traffic crashes within the 50 States, the District of Columbia and Puerto Rico. The results of alcohol and other drug tests are recorded, as well as police officers' determination of alcohol and/or other drug involvement. The FARS database contains descriptions--in standardized formats--of each fatal crash reported.

CASA Columbia analyzed FARS data to examine the role of alcohol and other drug use in fatal motor vehicle crashes.

\section{Key Informants}

CASA Columbia staff reached out to leading experts in a broad range of fields relevant to the study. Responses from key informants ${ }^{*}$ were solicited and received between July 2007 and May 2012. Comments were received from 174 individuals (109 by phone, 62 via e-mail and three in person). Informants were identified through a literature review, past research, referrals from CASA Columbia's National Advisory Commission which was convened for this study, and through a snowball sample where respondents recommended other qualified interviewees. (See Appendix B for the interview guide and list of participants.)

Where informants were amenable, an interview guide was used. The methodology and interview protocol were approved by CASA Columbia’s Institutional Review Board (IRB).

\footnotetext{
* The term "key informant" is used in reference to the list of individuals specifically contacted and asked to answer questions identified in Appendix B or to provide comments and suggestions on the scope of the study, key issues to be addressed and suggestions for recommendations. The full list of Key Informants is included in Appendix B.
} 
In other cases, informants made specific comments related to the scope of the study, key issues to be addressed and suggestions for recommendations. The responses were analyzed by CASA Columbia staff to identify key themes, provide illustrative quotes and inform subsequent research efforts.

\section{The National Addiction Belief and Attitude Survey (NABAS)}

CASA Columbia designed and developed a focus group protocol and a survey to assess the addiction-related attitudes and beliefs of a nationally-representative sample of adults. CASA Columbia staff, in collaboration with Peter D. Hart Research Associates, a survey research firm, developed the focus group discussion guide and survey instruments. Peter D. Hart Research Associates arranged for and moderated the focus groups and fielded the survey.

The recruiting and screening materials, consent protocol, focus group discussion guides, methodology and survey instrument were approved by CASA Columbia's IRB.

\section{Focus Groups}

Focus groups were conducted at research facilities in Atlanta, GA and Philadelphia, PA. The first set of focus groups took place in Atlanta, GA on May 13, 2008. The second set of groups was held in Philadelphia, PA on May 20 , 2008. In each city, one group was composed of respondents without a college education and the other was made up of respondents who had completed college.

All participants were administered a screening questionnaire as part of the recruitment process. Because it was considered unlikely that we would be able to recruit a representative sample of those managing their disease to participate in the focus groups, and inclusion of just several such people in the group might bias the

\footnotetext{
${ }^{*}$ No qualitative differences were found in the responses of these two groups of participants.
}

discussion, individuals with addiction who were currently managing the disease were excluded from participation. Efforts also were made to split the groups as evenly as possible between participants who had immediate family members with a history of addiction and those who did not. Participants also had to be between the ages of 25 and 70. A relatively even balance of men and women was sought in each group.

Participants were paid $\$ 90$ for participating in the focus group session.

\section{The National Survey}

Using results of the focus groups, gaps identified in the literature and the goals of this study as a guide, a pretest survey was developed by CASA Columbia staff with input from Peter D. Hart Research Associates. This pretest survey was administered and then modified and the final questionnaire was administered by means of a telephone survey. (See Appendix $\mathrm{C}$ for the survey instrument and response frequencies.)

Peter D. Hart Research Associates staff completed interviews with 1,303 adults by telephone between October 21, 2008 and November 3, 2008.

Of the respondents, 196 reported personal experience with addiction, while 887 respondents reported having family members or close friends with a history of addiction.

The margins of error for the telephone survey were:

- $\quad$ +/- 2.7 percent among all respondents;

- $\quad$ +- 7.0 percent among those with personal experience with addiction; and

- $\quad$ +/- 3.3 percent among those with family members or close friends with a history of addiction.

Telephone numbers in the sample were called up to four times. A total of 3,663 households answered the phone and attempts were made to 
complete an interview with an adult member in each of those households:

- Refusal Rate Prior to Obtaining Informed Consent: Thirty-seven percent $(1,364$ respondents) broke off before the interviewer could obtain informed consent. The remaining 63 percent $(2,299$ respondents) stayed on the line and answered the informed consent question.

- Refusal Rate of Informed Consent: Sixtynine percent (1,595 respondents) agreed to the informed consent question. The remaining 31 percent (704 respondents) refused at the point of informed consent and terminated the survey.

- Breakoff Rate: Once the survey questions began, 82 percent (1,303 respondents) completed the interview. The remaining 18 percent (292 respondents) terminated the survey before it was completed.

Overall, 35.6 percent (1303/3663) of those we attempted to contact completed the survey.

\section{Surveys of Addiction Treatment Directors and Staff Providers in New York State}

CASA Columbia collaborated with the Survey Research Laboratory (SRL) of the University of Illinois at Chicago to obtain a representative sample of treatment facilities and programs (and their directors and staff) in New York State. The goal of the surveys was to explore the types of treatment services provided in addiction treatment facilities and programs in New York, how performance and outcomes are assessed and the attitudes and beliefs of treatment providers concerning addiction and its treatment. (See Appendix D for the program directors' survey instrument and response frequencies and Appendix E for the staff providers' survey instrument and response frequencies.) The target population included addiction treatment providers, including Department of Veterans Affairs (VA) facilities, located in New York State. Because the number of facilities that treated adolescents was too few for us to draw a representative sample across New York State given our limited resources, only adultonly treatment centers were eligible to participate in the survey. We received the initial sample frame from the New York State Office of Alcoholism and Substance Abuse Services (OASAS). The goal was to complete interviews with the director and two staff members at 75 treatment facilities, for a total of 225 interviews (75 directors plus 150 staff members). We estimated that we would need to begin with a sample of 500 treatment facilities in order to obtain the target number of completed interviews.

The methodology, recruiting and screening materials, consent protocols and survey instruments were approved by CASA Columbia’s IRB.

Between December 17, 2008 and February 27, 2009, 83 facilities agreed to participate, resulting in interviews with 83 facility directors and 141 staff treatment providers within the 83 facilities. The survey protocol utilized multiple data collection modalities including telephone, fax and the Internet. To construct the initial frame of 500 facilities, SRL staff telephoned facilities to ensure that they were still in business and still seeing patients/clients. Once a pool of more than 500 eligible facilities was created, we issued screening forms to the pool to assess which facilities would be willing to participate; 224 of the 549 eligible facilities completed the screening instrument, resulting in a 40.8 percent screening response rate. Since our goal was 75 completed facility surveys, we recruited in blocks of 20 from the 224 facilities that completed the screening instrument. We exceeded the goal, resulting in a completion rate of 15.1 percent $(83 / 549)$ of eligible facilities, or 37.1 percent (83/224) of responding facilities.

The simple margin of error (calculated without taking into account the complex sampling structure) was approximately $+/-10$ percent for the director survey and approximately $+/-8$ percent for the staff provider survey. 


\section{National Panel of Treatment Providers and National Online Survey of Members of Professional Associations Involved in Addiction Care}

CASA Columbia convened a small, national panel of treatment providers. Panel members were asked to provide their perspectives on issues and barriers they faced in providing quality services and their recommendations for improvement in the treatment system. Organizations that assisted in identifying panel members included: NAADAC, the Association for Addiction Professionals; ${ }^{*}$ the State Associations of Addiction Services (SAAS); the National Council for Community Behavioral Healthcare (National Council); the National Association of Addiction Treatment Providers (NAATP); and the American Society of Addiction Medicine (ASAM). Panel members are listed in Appendix F.

This panel also assisted CASA Columbia in developing a brief, online national convenience survey of members of professional associations involved in addiction care to help understand their views of addiction treatment including: the relative importance of various components of the treatment process, the goals of treatment, the barriers to the implementation of high-quality treatment and recommendations for improving access to treatment and quality care. (See Appendix F for the survey instrument and response frequencies.)

Eleven professional associations agreed to share the link to the survey either via a group e-mail, a posting on their Web site or in an association newsletter. The 11 associations were: The American Academy of Addiction Psychiatry (AAAP); the American Association for the Treatment of Opioid Dependence (AATOD); the American Psychological Association (APA); the American Society of Addiction Medicine (ASAM); the Association for the Treatment of

\footnotetext{
* Formerly named the National Association for Alcoholism and Drug Abuse Counselors (NAADAC).
}

Tobacco Use and Dependence (ATTUD); NAADAC, the Association for Addiction Professionals; the National Association of Addiction Treatment Providers (NAATP); the National Association of County Behavioral Health and Developmental Disability Directors (NACBHDD); the National Council for Community Behavioral Healthcare (National Council); the State Associations of Addiction Services (SAAS); and Treatment Communities of America (TCA). ${ }^{\dagger}$

The methodology, recruiting and screening materials, consent protocol and survey instrument were approved by CASA Columbia's IRB.

Between May 2007 and May 2008, 1,142 members of the treatment provider associations completed the survey. The survey was anonymous.

\section{Online Survey of Individuals Managing Their Addiction}

CASA Columbia developed an online survey using a convenience sample of individuals who have been managing their addiction (i.e., "in recovery") to explore factors that have helped them manage the disease and challenges they have faced over the years in doing so. (See Appendix $G$ for specific survey questions and response frequencies.)

To qualify for participation, respondents had to be age 18 or older.

The following agencies and organizations agreed either to send an e-mail blast with an embedded survey link to their members or to include the link in a newsletter or other material that would reach those in the recovery groups: Hazelden, Freedom Institute, Faces and Voices of Recovery, Betty Ford Center, National Council on Alcoholism and Drug Dependence, Inc. (NCADD), Treatment Communities of America (TCA), Alcoholism and Substance Abuse

\footnotetext{
${ }^{\dagger}$ Formerly named Therapeutic Communities of America (TCA).
} 
Providers of New York State, Inc. (ASAP) and an anonymous treatment program alumni group.

The survey was anonymous; therefore, there is no way to determine with certainty if the responses provided by those who chose to respond are representative of the membership as a whole or of the broader group of individuals managing the disease of addiction ("in recovery”).

The methodology, recruiting and screening materials, consent protocol and survey instrument were approved by CASA Columbia's IRB.

Respondents self-defined as being in "long-term recovery" (i.e., not currently undergoing detoxification or acute treatment); 360 respondents completed the survey. The average reported length of being "clean and sober" was 10 or more years. The survey links were open from July 2007 to July 2008.

\section{Review of Licensing, Certification and Accreditation Requirements for Treatment Providers}

Between 2009 and 2012, CASA Columbia conducted online ${ }^{*}$ reviews and analyses of publicly available documents to develop a summary overview of the regulatory landscape related to government, professional association and other accrediting agency requirements related to the provision of addiction treatment services. In some cases where key information could not be identified or where requirements were unclear, we called or e-mailed the relevant state agency or organization for clarification. Because licensing and certification requirements are found in a wide variety of laws and regulations and can change on a state-by-state basis, findings from this review cannot be guaranteed to be complete and current.

\footnotetext{
* Using the Lexis/Nexis database to supplement information related to state laws and regulations available on the Internet.
}

\section{Individual Provider Licensing and Certification Requirements}

CASA Columbia reviewed publicly-available federal and state laws and regulations (in all 50 states and the District of Columbia) and professional association Web sites, to determine the minimum education and training requirements to become licensed or certified in each of the following professions most likely to provide addiction treatment in the United States: physicians, physician assistants, nurses, psychologists, mental health counselors/ therapists, social workers, acupuncturists and addiction counselors. We collected data on state licensing requirements for each profession, including addiction-related requirements and voluntary certification requirements for addiction specialists in each profession.

\section{Addiction Facility/Program Licensing and Accreditation Requirements}

CASA Columbia reviewed publicly-available federal and state laws and regulations that govern addiction treatment facilities in all 50 states and the District of Columbia. We also reviewed accreditation standards for addiction facilities and programs set forth in the Commission on Accreditation of Rehabilitation Facilities' (CARF) Behavioral Health Standards Manual and the Joint Commission's Standards for Behavioral Health Care. CARF and the Joint Commission are the two largest accreditors of addiction treatment programs. ${ }^{\dagger}$ We collected data on requirements pertaining to staff composition and qualifications, provided services, quality assurance activities and the use of patient outcomes data.

\section{Case Study of Addiction Treatment in New York}

CASA Columbia conducted a case study of addiction treatment in New York State and New York City with support from the New York Community Trust. The goal of this work was to provide an in-depth look at one state/city parallel

\footnotetext{
${ }^{\dagger}$ See Chapter IX.
} 
with the objectives of the larger study. To conduct the case study, CASA Columbia staff drew from the research methods described above, to the extent that the findings pertained to New York, including, for example, a literature review focused on addiction treatment in New York; key informant interviews with experts from New York; the surveys of New York State addiction treatment directors and staff providers; the survey of individuals managing their addiction--80 respondents were from New York and accessed through two New York-based organizations: New York Association of Alcoholism and Substance Abuse Providers, Inc. (ASAP) and National Council on Alcoholism and Drug Dependence, Inc. (NCADD); and the credentialing requirements for treatment providers in New York. Relevant findings from these analyses and illustrative quotes from key informants are incorporated into the report. 
$-244-$ 


\section{Appendix B \\ Key Informant Interview Guide and List of Key Informants}

Name:

Title:

Organization:

Interviewer:

Interview Date:

Q1 How do you think the average person in the US today perceives (thinks about) substance abuse and addiction (e.g., physical health problem, mental health problem, behavioral problem, moral problem, social problem, criminal justice problem)?

Q2 Do you think this perception has changed in the last 20 years?

Q3 How do you perceive (think about) substance abuse and addiction (e.g., physical health problem, mental health problem, behavioral problem, moral problem, social problem, criminal justice problem)?

Q4 Do you think addiction is a family disease? If so, do you think it requires some type of intervention or treatment for members other than the addict? Please explain.

Q5 How easy is it, in your opinion, to get effective help for an addiction problem in America (or in NYC, for NYC Case Study key informants) today?

Q6 When people are looking for help for an addiction problem, who do they usually turn to or where do they go and why?

Q7 When someone gets help for an addiction problem, what type of help do they usually receive?

Q8 How would you define treatment for substance abuse or addiction?

Q9 What is your definition of effective treatment for substance abuse or addiction?

Q10 What should the goals of treatment be (e.g., complete abstinence, reduction in use, reduction in harmful consequences; remission of DSM symptoms)?

Q11 Under what conditions does effective treatment of addiction require treatment of co-morbid psychiatric conditions?

Q12 What do you think can be done to make treatment more science- or evidence-based? Is evidence from research findings accessible and understandable to providers, as well as to policymakers and advocacy groups?

Q13 What is your assessment of the capacity of treatment programs in the U.S. (or in NYC, for NYC Case Study key informants) to deliver effective and appropriate treatment to those with substance use disorders?

Q14 What do you think stands in the way of people getting quality, effective treatment and of providers offering quality, effective treatment? 
Q15 Do you think that most treatment providers are qualified to help individuals with substance abuse or addiction problems? Please explain.

Q16 Do you think there should be minimum standards of knowledge, skills and/or training for an individual to provide treatment? If so, what are some examples or suggestions for those minimum standards?

Q17 What do you think are three key things that would improve the quality of addiction treatment in America (or in NYC, for NYC Case Study key informants)?

Q18 Where do you think the substance use and addiction field will be in 20 years? Where would you like it to be?

Q19 Is there anything we have not asked you that you would like to discuss? 


\section{New York State-Specific Key Informants*}

Barry, Justin, Citywide Drug Court Coordinator, Criminal Court of the City of New York

Carpenter-Palumbo, Karen M., Commissioner, New York State Office of Alcoholism and Substance Abuse Services - OASAS

Conlon, Peggy, President and Chief Executive Officer, Advertising Council, Inc.

Coppola, John, Executive Director, New York Association of Alcoholism and Substance Abuse Providers, Inc. (ASAP)

Deitch, MD, David, Chief Clinical Officer, Phoenix House New York

Donowitz, Stephen C., Director, Adolescent Services, Phoenix House New York

Feinblatt, JD, John, Criminal Justice Coordinator, City of New York

Galanter, MD, Marc, Professor, Department of Psychiatry and Director, Division of Alcohol and Drug Abuse, New York University

Gebbie, PhD, RN, Kristine, Elizabeth Standish Gill Associate Professor of Nursing, Columbia University School of Nursing

Gitlow, MD, MPH, MBA, Stuart, Executive Director, Annenberg Physician Training Program

Gourevitch, MD, MPH, Marc N., Professor Medicine and Professor of Psychiatry, Director, Division of General Internal Medicine, Bellevue Primary Care Clinic

Hawkins, PhD, Barry, Director, Chemical Dependency Services, Orange County Department of Mental Health

Hogan, PhD, Michael F., Commissioner, New York State, Office of Mental Health

Hynes, Charles, District Attorney, Kings County

Johnson, MD, Brian, Director of Addiction Psychiatry and Associate Professor, State University of New York, Upstate Medical University

Josepher, Howard, Co-Founder and Executive Director, Exponents

Kellogg, PhD, Scott, Clinical Assistant Professor, Psychology, New York University, FAS Psychology Department

Kistenmacher, PhD, Barbara, Director, Addictions Treatment, Bronx Lebanon

Kleber, MD, Herbert D., Professor of Psychiatry and Director of the Division on Substance Abuse at the Columbia University College of Physicians and Surgeons and the New York State Psychiatric Institute, New York State Psychiatric Center

Leary, Robyn, Executive Director, Recovery Network Foundation

Levounis, MD, Petros, Director, Addiction Institute of New York

Lindsey, Robert, President, National Council on Alcoholism and Drug Dependence

Lopez, MD, Ralph I., Clinical Associate Professor of Pediatrics and Associate Attending Physician, Weill Cornell Medical Center

McDonald, George T., Founder and President, The Doe Fund, Inc.

Meitiner, Howard P., President and Chief Executive Officer, Phoenix House New York

Nadelmann, Ethan, Executive Director, Drug Policy Alliance

Newman, MD, MPH, Robert G., Director, The Baron Edmond de Rothschild Chemical Dependency Institute, Beth Israel Hospital Center

Pantin, MSW, Debra, Vice President, Outpatient and Centralized Services, Palladia, Inc.

Raine, Valerie, Director, Drug Court Programs, Center for Court Innovation

Randolph, MD, Chris, Medical Director, Phoenix House New York

Riddle, Kathleen A., President and Chief Executive Officer, Outreach Project

Rosen, Paul, Staff Director, Senate Caucus on International Narcotics Control, Office of U.S. Senator Biden

\footnotetext{
${ }^{*}$ Titles and affiliations represent those at the time of Key Informant participation.
} 
Rosenthal, MD, Richard N., Head, Public Policy Section, American Academy of Addiction Psychiatry, and Professor of Clinical Psychiatry and Chairman, Department of Psychiatry, St. Luke's Roosevelt Hospital Center

Ross, MD, Stephen, Director, Substance Abuse Services, Bellevue Hospital Center and South Manhattan Healthcare Network

Rotrosen, MD, John, Professor, Department of Psychiatry, New York University, School of Medicine

Samuels, JD, Paul N., Director and President, Legal Action Center

Savoy, LCSW, CASAC, Jeffrey R., Vice President and Director of Clinical Support Services, Odyssey House, Inc.

Schwartz, MD, Bruce J., Director of Clinical Services for Substance Abuse, Montefiore Medical Center

Sederer, MD, Lloyd I., Medical Director, New York State, Office of Mental Health

Swanson, PhD, Arthur J., Program Director, University Behavioral Associates, Montefiore Medical Center

Swern, Anne, District Attorney's Office, Kings County

Travis, JD, Jeremy, President, John Jay College of Criminal Justice

Wadalavage, Kevin, Vice President, Outreach Development Corporation

Weinstein, MPH, Naomi, Director, Phoenix House Center on Addiction and the Family (COAF)

Wilkens, PhD, Carrie, Clinical Director, Center for Motivation and Change

\section{General Study Key Informants ${ }^{\dagger}$}

Allem, Johnny W., President and Chief Executive Officer, Johnson Institute

Amico, MDiv, Joseph M., President, National Association of Lesbian and Gay Addiction Professionals

Babor, PhD, MPH, Thomas F., Professor, Physicians Health Services Chair in Community Medicine and Public Health, Department of Community Medicine and Health Care, University of Connecticut Health Center

Bart, MD, FACP, FASAM, Gavin, Director, Division of Addiction Medicine, Department of Medicine, Hennepin County Medical Center; Associate Professor of Medicine, University of Minnesota

Baxter, Sr., MD, FASM, Louis E., Executive Medical Director, Professional Assistance Program of New Jersey

Beauchemin, Patricia, Executive Director, Therapeutic Communities of America ${ }^{\ddagger}$

Berger, PhD, Thomas J., National Chair, PTSD \& Substance Abuse Committee

Breyer, Ellen L., President and Chief Executive Officer, Hazelden Foundation

Brown, PhD, Stephanie, Director, The Addictions Institute

Callahan, DPA, James F., Executive Vice President, The American Board of Addiction Medicine, and The ABAM Foundation

Carroll, PhD, Kathleen M., Professor of Psychiatry, Yale University, School of Medicine, Division of Substance Abuse

Cimaglio, Barbara, Deputy Commissioner for Alcohol and Drug Abuse Programs, Vermont Department of Health

Clark, MD, JD, MPH, H. Westley, Director, Center for Substance Abuse Treatment

Cohen, Michael, Executive Director, Florida Lawyers Assistance Program

Colston, MA, Stephenie W., Director, Substance Abuse Program, Florida Department of Children and Families

Compton, MD, MPE, Wilson M., Director, National Institutes of Health, Division of Epidemiology, Services and Prevention Research

Condon, PhD, Timothy P., Deputy Director, National Institute on Drug Abuse

\footnotetext{
${ }^{\dagger}$ Titles and affiliations represent those at the time of Key Informant participation.

${ }^{\ddagger}$ Currently named Treatment Communities of America (TCA).
} 
Covington, PhD, Stephanie, Director, Institute for Relational Development/The Center for Gender and Justice

Cropper, Cabell C., Executive Director, National Criminal Justice Association

Cundiff, MD, MPH, Dave, Secretary, American Association of Public Health Physicians

Curie, Charles G., President, The Curie Group, LLC

Dackis, MD, Charles A., Medical Director of Clinical Services, Department of Psychiatry, University of Pennsylvania Health System

Daley, PhD, Dennis C., Professor of Psychiatry, Chief, Addiction Medicine Services, Principal Investigator, Appalachian Tri-State Node of the NIDA Clinical Trails Network, Western Psychiatric Institute and Clinic, University of Pittsburgh Medical Center

D’Aunno, PhD, Thomas, Professor of Health Policy and Management, Mailman School of Public Health, Columbia University

Davis, MD, Ronald M., President, American Medical Association

Dawes, Jacqueline, Founder and Owner, Brookhaven Retreat

Dean, Gen. Arthur T., Chairman and Chief Executive Officer, Community Anti-Drug Coalitions of America

Delos Reyes, MD, Christina M., Assistant Professor of Psychiatry, Department of Psychiatry, School of Medicine, Case Western Reserve University; Director, Addiction Psychiatry Fellowship, University Hospitals Case Medical Center

Dentzer, Susan, Health Correspondent and Head, Health Policy Unit, The News Hours with Jim Lehrer, PBS

Duffy, MD, F. Daniel, Senior Advisor to the President, American Board of Internal Medicine

Dukakis, JD, Michael S., Chair, Blueprint for the States, Join Together Policy Panel Members

Durham, PhD, Thomas G., Executive Director, The Danya Institute

Dyak, Marie, Executive Vice President, Program Services \& Government Relations, Entertainment Industries Council

Erickson, PhD, Carlton K., Director, Addiction Science Research and Education Center and Distinguished Professor of Pharmacology/Toxicology, University of Texas at Austin, College of Pharmacy

Evans, Jr., PhD, Arthur C., Acting Commissioner, Department of Human Services, Pennsylvania Department of Behavioral Health/Mental Retardation Services

Farabee, PhD, David, Research Psychologist at the University of California, Los Angeles and Director of the Integrated Substance Abuse Programs (ISAP) Juvenile Justice Research, UCLA Integrated Substance Abuse Programs

Finney, John W., Director, HSR\&D Center For Health Care Evaluation, VA Palo Alto Health Care System

Flaherty, PhD, Michael T., Executive Director, IRETA -- Institute for Research, Education and Training in Addiction

French, PhD, Michael T., Professor of Health Economics and Director, Health Economics Research Group, University of Miami, Department of Sociology

Galea, MD, DrPH, MPH, Sandro, Associate Professor, Department of Epidemiology, University of Michigan, School of Public Health

Gallon, PhD, Steve, Director, Northwest Frontier ATTC, Department of Public Health and Preventive Medicine, Oregon Health \& Science University

Gertig, JD, June, Director, CSAT Recovery Community Services Program Technical Assistance Program, Health Systems Research, Inc.

Glantz, PhD, Meyer D., Associate Director for Science, National Institute on Drug Abuse

Goplerud, Eric, Senior Vice President, Substance Abuse, Mental Health and Criminal Justice Studies, NORC at the University of Chicago 
Greer, Patricia M., President, National Association of Alcoholism and Drug Abuse Counselors $(\text { NAADAC) })^{\S}$

Gustafson, PhD, David, Professor Emeritus, University of Wisconsin, Madison

Guthrie, PhD, RN, FAAN, Barbara J., Associate Dean of Academic Affairs, Associate Professor School of Nursing, Yale University, School of Nursing

Hajela, MD, MPH, Raju, President and Medical Director, Health Upwardly Mobile, Inc. (HUM); Region IX (International) Director, American Society of Addiction Medicine

Hamburg, MD, David A., President Emeritus, Carnegie Corporation of New York; former President, American Association for the Advancement of Science and the Institute of Medicine, DeWitt Wallace Distinguished Scholar, Weill Cornell Medical College

Hamilton, Nancy, Chief Executive Officer, Operation PAR

Harwood, Henrick, Director of Research and Program Applications, National Association of State Alcohol and Drug Abuse Directors, Inc.

Hatcher, EdD, Anne S., Director, Center for Addiction Studies, Metropolitan State College of Denver Higgins-Biddle, PhD, John C., Assistant Professor (Retired), University of Connecticut Health Center, Department of Community Medicine and Health Care

Hill, MD, J. Edward, Immediate Past President, American Medical Association

Hoffman, PhD, Norman G., President, Evince Clinical Assessments

Howell, MD, FASAM, DFAPA, Elizabeth F., Associate Professor (Clinical), Department of Psychiatry, University of Utah School of Medicine

Huddleston, III, C. West, Chief Executive Officer and Executive Director, National Association of Drug Court Professionals, National Drug Court Institute

Humphreys, PhD, Keith N., Professor (Research) of Psychiatry and Behavioral Sciences; CHP/PCOR Associate, Stanford School of Medicine, Department of Psychiatry

Hurley, MD, MBA, Brian, Chair, Physicians-In-Training Committee, American Society of Addiction Medicine, National Vice President, American Medical Student Association

Jackson, MSW, Ron, Executive Director, Evergreen Treatment Services

Janes, William H., Former Director, Florida Office of Drug Control, Office of the Governor, Florida

Karlin, PhD, Barry W., Chairman and Chief Executive Officer, CRC Health Group

Katz, MD, MS, Nathaniel, Founder, Analgesic Research

Kosten, MD, Thomas R., Professor, Department of Neuroscience, Baylor College of Medicine

Kosterman, PhD, Judi Marie, Senior Vice President, WestCare Foundation, Inc.

Kressler, MA, Harry, Executive Director and Superintendent, Pima Prevention Partnership

Kunz, MD, MPH, FASAM, Kevin, Director and Immediate Past President, American Board of Addiction Medicine and The ABAM Foundation

Leary, William R., Executive Director, Louisiana Lawyers' Assistance Program

Leshner, PhD, Alan I., Chief Executive Officer, American Association for the Advancement of Science

Lewis, MD, David C., Professor Emeritus of Medicine and Community Health, Brown University

Ling, MD, Walter, Professor of Psychiatry and Director, Integrated Substance Abuse Programs, Department of Psychiatry \& Behavioral Sciences, David Geffen School of Medicine at UCLA

Lundberg, MD, ScD, George D., Co-Chair, Board of Directors, PLNDP, PLNDP National Office, Center for Alcohol and Addiction Studies, Brown University

Maine, PhD, Margo D., Co-Founder, Maine \& Weinstein Specialty Group

Malliarakis, Kate, President, KAM Associates

Marlatt, PhD, G. Alan, Professor and Director, Addictive Behaviors Research Center, University of Washington, Department of Psychology

Martin, MD, Judith, Medical Director, BAART Turk Street Clinic

McAuliffe, PhD, William, Director, North Charles Research and Planning Group

McCaffree, MD, Robert, Chief of Staff, VA Medical Center, Oklahoma City, OK

${ }^{\S}$ Currently named NAADAC, the Association for Addiction Professionals. 
McCaffrey, Gen. Barry R., Founder, BR McCaffrey Associates LLC

McDonough, James, Secretary, Florida Department of Corrections

McLellan, PhD, A. Thomas, Chief Executive Officer, Treatment Research Institute

McMillen, LSAC, MPA, Shawn M., Executive Director, First Step House

McVay, Doug A., Director of Research and Editor, Common Sense for Drug Policy

Miller, MD, Michael M., Medical Director, Herrington Recovery Center, Rogers Memorial Hospital, Oconomowoc, WI; Past President, American Society of Addiction Medicine; Director, American Board of Addiction Medicine and The ABAM Foundation

Miller, PhD, William R., Distinguished Professor of Psychology and Psychiatry, Center on Alcoholism, Substance Abuse and Addictions

Mills, MBA, Penny S., Executive Vice President and Chief Executive Officer, American Society of Addiction Medicine

Minkoff, MD, Kenneth, Clinical Assistant Professor of Psychiatry, Harvard University

Molloy, JD, J. Paul, Chief Executive Officer, Oxford House

Moore, EdD, Dennis C., Director, Substance Abuse Resources and Disability Issues and Professor, Department of Community Health, Boonshoft School of Medicine, Wright State University Building

Moos, PhD, Rudolph, Professor, Department of Psychiatry and Behavioral Sciences, Stanford University School of Medicine

Moyers, William Cope, Vice President, External Affairs, Hazelden Foundation

Murphy, Steven W., Executive Director, The Right Step-San Antonio

Newhouse, Eric, Project Editor, Great Falls Tribune

Nicolaus, Martin, Chief Executive Officer, LifeRing Service Center

Norman, Cathy, Prosecutor, Vermont Attorney General's Office

O’Brien, MD, PhD, Charles P., Kenneth E. Appel Professor and Vice Chair of Psychiatry, University of Pennsylvania and Director of Research, MIRECC, Philadelphia Veterans Affairs Medical, Charles O'Brien Center for Addiction Treatment

Powell, Richard, Director of Addiction and Violence Services, Vermont Department of Corrections

Rawson, PhD, Richard, Associate Director, Professor-in-Residence, UCLA Integrated Substance Abuse Programs

Ries, MD, Richard K., Professor of Psychiatry and Director, Addictions Division, University of Washington Department of Psychiatry

Rivara, MD, Frederick P., Professor, Pediatrics and Adjunct Professor, Epidemiology

Robinson, Stephen L., Government Relations Director, Veterans for America

Rosen, Paul, Staff Director, Senate Caucus on International Narcotics Control, Senator Biden

Rosenbloom, PhD, David, Professor, Boston University, Join Together

Samet, MD, MA, MPH, Jeffrey H., Clinical Addiction Research and Education (CARE) Program, Professor of Medicine and Social and Behavioral Sciences, Boston University School of Medicine

Satel, MD, Sally, Resident Scholar, American Enterprise Institute for Public Policy Research

Schwarzlose, John T., President, Betty Ford Center

Sindelar, PhD, Jody L., Professor and Head, Division of Health Policy and Administration, Yale University, School of Public Health

Sloboda, PhD, Zili, Senior Research Associate, Institute for Health and Social Policy, University of Akron

Snow, PhD, Diane, Clinical Professor and Director, Psychiatric Mental Health NP Program and CoDirector, Center for Psychopharmacology Education and Research, University of Texas, Arlington School of Nursing

Starer, MD, FACOG, FASAM, Jacquelyn, Coordinator, CSAC Division of Opioid Dependent Pregnant Patients at Community Substance Abuse Centers

Stitzer, PhD, Maxine, Professor of Behavioral Biology, Johns Hopkins University, School of Medicine

Swift, MD, PhD, Robert M., Professor of Psychiatry and Human Behavior, Brown University, Department of Bio Med Psychiatry and Human Behavior 
Szapocznik, PhD, Jose, Professor, Psychiatry and Behavioral Sciences and Director, Center for Family Studies, University of Miami, School of Medicine

Taylor, Patricia A., Project Director, Faces and Voices of Recovery

Tieman, Doug, President and Chief Executive Officer, Caron Foundation

Trojan, Jodi, Program Evaluator, Cook Inlet Tribal Council

Valentine, Phillip, Executive Director, Connecticut Community for Addiction Recovery

Vocci, PhD, Francis, Director, Division of Pharmacotherapy and Medical Consequences, National Institute on Drug Abuse

Walker, MSW, LCSW, MDiv, Robert J., Assistant Professor, University of Kentucky, Center on Drug and Alcohol Research

Walsh, PhD, J. Michael, President, Walsh Group, PA

Wenger, Sis, President and Chief Executive Officer, National Association for Children of Alcoholics

Wetsman, MD, FASAM, Howard C., Chief Medical Officer, Townsend; Author, QAA: Questions and Answers on Addiction

White, MA, William, Senior Research Coordinator, Chestnut Health Systems

Willenbring, MD, Mark, Director, Addictive Disorders Section, Minneapolis VA Medical Center

Wilson, PhD, Julie Boatright, Director, Malcolm Wiener Center for Social Policy, John F. Kennedy School of Government, Harvard University

Woods, Mary R., Chief Executive Officer, WestBridge Community Services

Woody, MD, George E., Professor, Department of Psychiatry, University of Pennsylvania, Treatment Research Institute

Yoast, PhD, MA, Richard A., Director, Office of Alcohol and Other Drug Abuse Prevention, Division of Healthy Lifestyles, American Medical Association

Ziegler, MD, Penelope P., Medical Director Emeritus and Senior Consulting Psychiatrist, Williamsburg Place and The William J. Farley Center, and Associate Clinical Professor of Psychiatry, Virginia Commonwealth University 


\section{Appendix C National Addiction Belief and Attitude Survey (NABAS)}

Responses from 1,303 survey participants. The number corresponding to each response option represents the percent, among those responding to the question, that provided the particular response.

1. I am going to read you a list of health conditions and then please tell me which TWO or THREE do you think is most common in the United States. Would you like me to read this list again?

64.4 Obesity and diabetes

46.1 Cancer

45.5 Heart disease

29.7 Addiction to tobacco

22.2 Addiction to alcohol

16.8 Addiction to illegal drugs

14.5 Depression

11.0 Addiction to prescription drugs

9.0 Asthma

0.4 Not sure

2. Now I am going to read the same list again and this time please tell me which TWO or THREE do you believe cause the greatest harm in the United States.

56.4 Obesity and diabetes

52.2 Cancer

43.7 Heart disease

28.0 Addiction to tobacco

26.4 Addiction to illegal drugs

23.6 Addiction to alcohol

11.0 Addiction to prescription drugs

10.6 Depression

3.2 Asthma

0.2 Not sure

3. I am going to mention some issues that could be a problem in your community. For each one I mention, please tell me how much of a problem you think it is in your community--a very serious problem, somewhat serious problem, not too much of a problem, or not a problem at all.

Insufficient treatment programs and services for people addicted to illegal drugs

28.9 Very serious problem

27.2 Somewhat serious problem

16.8 Not too much of a problem

17.5 Not a problem at all

9.7 Not sure

Insufficient treatment programs and services for people with obesity and diabetes

26.5 Very serious problem

32.4 Somewhat serious problem

16.0 Not too much of a problem

17.8 Not a problem at all

7.3 Not sure 
Insufficient treatment programs and services for people addicted to tobacco

19.9 Very serious problem

28.0 Somewhat serious problem

21.4 Not too much of a problem

21.4 Not a problem at all

9.2 Not sure

Insufficient treatment programs and services for people with depression

18.7 Very serious problem

33.4 Somewhat serious problem

20.1 Not too much of a problem

16.9 Not a problem at all

10.9 Not sure

Insufficient treatment programs and services for people addicted to prescription drugs

18.2 Very serious problem

28.8 Somewhat serious problem

18.7 Not too much of a problem

19.3 Not a problem at all

15.0 Not sure

Insufficient treatment programs and services for people addicted to alcohol

17.1 Very serious problem

29.1 Somewhat serious problem

23.9 Not too much of a problem

22.0 Not a problem at all

7.9 Not sure

Insufficient treatment programs and services for people with cancer

17.6 Very serious problem

20.5 Somewhat serious problem

23.7 Not too much of a problem

31.1 Not a problem at all

7.0 Not sure

Discrimination against people in recovery from addiction to alcohol, tobacco, prescription or other drugs

15.8 Very serious problem

25.6 Somewhat serious problem

30.0 Not too much of a problem

20.5 Not a problem at all

8.2 Not sure

Insufficient treatment programs and services for people with heart disease

16.3 Very serious problem

23.1 Somewhat serious problem

23.9 Not too much of a problem

31.0 Not a problem at all

5.6 Not sure

Insufficient treatment programs and services for people with diabetes

13.4 Very serious problem

27.6 Somewhat serious problem

19.0 Not too much of a problem

27.7 Not a problem at all

12.3 Not sure 
Discrimination against people with mental illness

12.9 Very serious problem

22.7 Somewhat serious problem

28.1 Not too much of a problem

30.2 Not a problem at all

6.0 Not sure

Insufficient treatment programs and services for people with asthma

6.9 Very serious problem

18.1 Somewhat serious problem

26.7 Not too much of a problem

35.0 Not a problem at all

13.2 Not sure

4. If you were responsible for deciding who to hire at your place of work, and you learned that a qualified applicant (READ ITEM), would that make you more likely to hire that person, less likely, or would it not make a difference either way? (IF ANSWERED “MORE LIKELY,” ASK:)

Would you be much more likely or only somewhat more likely to hire someone who (READ ITEM)? (IF ANSWERED “LESS LIKELY,” ASK:) Would you be much less likely or only somewhat less likely to hire someone (READ ITEM)?

Is in recovery from an addiction to illegal drugs

3.7 Much more likely

3.7 Somewhat more likely

37.9 Would not make a difference

33.6 Somewhat less likely

20.0 Much less likely

1.2 Not sure

Is in recovery from an addiction to prescription medications

1.4 Much more likely

5.9 Somewhat more likely

48.7 Would not make a difference

28.8 Somewhat less likely

12.3 Much less likely

2.9 Not sure

Is in recovery from an addiction to alcohol

2.3 Much more likely

6.7 Somewhat more likely

60.5 Would not make a difference

18.7 Somewhat less likely

9.2 Much less likely

2.6 Not sure

Has been treated for addiction to alcohol

1.8 Much more likely

2.9 Somewhat more likely

65.8 Would not make a difference

19.0 Somewhat less likely

8.1 Much less likely

2.5 Not sure 
Has been treated for depression

2.3 Much more likely

4.7 Somewhat more likely

71.9 Would not make a difference

14.1 Somewhat less likely

3.9 Much less likely

3.0 Not sure

Has been treated for obesity

2.8 Much more likely

2.3 Somewhat more likely

84.6 Would not make a difference

7.2 Somewhat less likely

2.2 Much less likely

0.9 Not sure

Has been treated for heart disease

1.4 Much more likely

4.2 Somewhat more likely

83.5 Would not make a difference

6.5 Somewhat less likely

2.7 Much less likely

1.7 Not sure

Is a former smoker

1.4 Much more likely

2.1 Somewhat more likely

89.0 Would not make a difference

4.9 Somewhat less likely

1.7 Much less likely

1.0 Not sure

Is in treatment for diabetes

2.0 Much more likely

2.2 Somewhat more likely

88.8 Would not make a difference

4.2 Somewhat less likely

1.2 Much less likely

1.6 Not sure

Is in treatment for asthma

2.1 Much more likely

1.2 Somewhat more likely

90.7 Would not make a difference

3.8 Somewhat less likely

1.0 Much less likely

1.0 Not sure

Has been treated for cancer

4.0 Much more likely

4.3 Somewhat more likely

85.6 Would not make a difference

3.1 Somewhat less likely

1.6 Much less likely

1.4 Not sure 
5. Now I am going to mention various substances some people may consume and I would like you to tell me what level of use would, in your personal opinion, indicate that a person has a serious problem. To give you an example, some people might say that a person who eats fried foods once a week does not have a problem but if someone eats fried foods several times a day then they do have a serious problem and should seek help to change their diet.

When it comes to (READ ITEM), do you think any use at all indicates a person has a serious problem and should seek treatment, or is monthly use, weekly use, daily use, or use several times a day that would indicate a serious problem--or do you not think that any level of use of (READ ITEM) indicates a serious problem for which treatment is needed?

Heroin

84.0 Any use at all

3.0 Monthly use

2.5 Weekly use

5.5 Daily use

2.6 More than daily use

1.0 Does not indicate a problem at any level of use

1.6 Not sure

Cocaine

76.0 Any use at all

4.6 Monthly use

5.1 Weekly use

8.2 Daily use

3.6 More than daily use

1.1 Does not indicate a problem at any level of use

1.5 Not sure

Methamphetamine

73.9 Any use at all

4.0 Monthly use

3.9 Weekly use

9.4 Daily use

4.0 More than daily use

0.9 Does not indicate a problem at any level of use

3.9 Not sure

Non-medical use of prescription medications

42.5 Any use at all

8.2 Monthly use

9.9 Weekly use

19.7 Daily use

9.9 More than daily use

5.5 Does not indicate a problem at any level of use

4.3 Not sure

Marijuana

35.8 Any use at all

7.6 Monthly use

9.2 Weekly use

21.4 Daily use

14.5 More than daily use

7.0 Does not indicate a problem at any level of use

4.5 Not sure 
Cigarettes/Tobacco

24.3 Any use at all

1.9 Monthly use

6.6 Weekly use

22.0 Daily use

27.5 More than daily use

12.9 Does not indicate a problem at any level of use

4.8 Not sure

Alcohol

6.8 Any use at all

2.0 Monthly use

7.4 Weekly use

39.5 Daily use

38.5 More than daily use

2.8 Does not indicate a problem at any level of use

3.0 Not sure

6. Now let me read you a list of factors different people say may cause people to become addicted to (READ ITEM) and have you tell me which TWO or THREE you think are the primary factors causing addiction to (READ ITEM)?

Tobacco

43.5 Inability to resist peer pressure

38.7 Easy availability of tobacco among youth

37.7 Stress or anxiety about work, family or other problems

33.0 Lack of willpower or self control

25.4 A predisposition to addiction due to genetics or family history

20.0 Lack of knowledge about addiction

17.3 Emotional disorders or mental illness such as depression or anxiety

3.8 Absence of religious faith or spiritual grounding

3.0 Distorted moral values

2.0 Other

1.6 None

1.4 Not sure

Alcohol

47.6 A predisposition to addiction due to genetics or family history

44.9 Stress or anxiety about work, family or other problems

35.3 Emotional disorders or mental illness such as depression or anxiety

29.7 Lack of willpower or self control

29.2 Easy availability of alcohol among youth

28.2 Inability to resist peer pressure

11.1 Lack of knowledge about addiction

6.8 Absence of religious faith or spiritual grounding

5.9 Distorted moral values

1.1 Other

0.1 None

0.6 Not sure 
Prescription medications

40.8 Emotional disorders or mental illness such as depression or anxiety

36.7 Easy availability of prescription medications among youth

36.9 Stress or anxiety about work, family or other problems

27.5 A predisposition to addiction due to genetics or family history

26.9 Lack of willpower or self control

25.8 Lack of knowledge about addiction

17.8 Inability to resist peer pressure

5.6 Absence of religious faith or spiritual grounding

6.8 Distorted moral values

3.6 Other

0.8 None

1.8 Not sure

Illegal drugs

41.9 Inability to resist peer pressure

35.1 Easy availability of illegal drugs among youth

34.8 Emotional disorders or mental illness such as depression or anxiety

29.9 A predisposition to addiction due to genetics or family history

29.9 Stress or anxiety about work, family or other problems

28.7 Lack of willpower or self control

19.6 Lack of knowledge about addiction

10.2 Distorted moral values

9.4 Absence of religious faith or spiritual grounding

1.8 Other

1.0 None

1.2 Not sure

7. What should the main treatment goal for someone addicted to (READ ITEM)? Should it be complete abstinence, reduced use, fewer negative consequences from use or the goal should be set by the patient?

Illegal drugs

65.7 Complete abstinence

8.8 Reduced use

4..0 Fewer negative consequences from use

17.3 Goal should be set by the patient

4.2 Not sure

Alcohol

60.0 Complete abstinence

14.6 Reduced use

1.5 Fewer negative consequences from use

21.1 Goal should be set by the patient

2.8 Not sure

Tobacco

50.1 Complete abstinence

17.6 Reduced use

0.8 Fewer negative consequences from use

29.7 Goal should be set by the patient

1.9 Not sure 
Prescription medications

47.1 Complete abstinence

22.7 Reduced use

3.5 Fewer negative consequences from use

20.8 Goal should be set by the patient

6.0 Not sure

8a. Suppose someone close to you realized they had a major problem with addiction to alcohol, tobacco, prescription or other drugs, how confident would you be that you knew or could find out where to go or call or send them to get the help they would need: very confident, somewhat confident, not too confident or not at all confident?

50.9 Very confident

30.7 Somewhat confident

8.6 Not too confident

8.5 Not confident at all

1.3 Not sure

8b. If someone close to you needed help for an addiction, where would you turn for information or help? (PROBE) Is there anywhere else you think of where you could turn for help or information?

27.8 My doctor or primary care physician

18.8 Internet or Yellow Pages or "look it up" or do "research"

19.7 Other doctor, nurse, hospital, health clinic or health professional

12.0 Alcoholics Anonymous (AA)

10.7 Friend or family member

9.8 Church, clergy or religious or spiritual leader

11.0 Addiction treatment center

9.2 Psychologist, psychiatrist or other mental health counselor

7.2 Addiction "hotline" or "helpline"

6.0 Narcotics Anonymous (NA) or other similar 12-step programs

3.6 Place of work, office, Employee Assistance Program (EAP)

3.0 Other mutual support or self-help programs such as Smart Recovery

2.0 Student guidance counselor for a minor

0.6 My insurance company

1.2 Would not know where to turn for help

1.8 Not sure

24.2 Other*

* The number of responses in the "Other” option are quite varied and therefore, not specified here.

8c. (ASKED ONLY OF THOSE WHO SAY “FRIEND/FAMILY MEMBER” OR “SOMEONE ELSE” in Q 8b) And is that someone with special knowledge or training when it comes to dealing with addictions or just someone you count on for good advice in general?

56.8 Special knowledge or training

30.7 Good advice in general

12.4 Both 
9. In general, how effective do you think treatment is for addiction to (READ ITEM) if the person admits they have a problem and wants to get better? Would you say treatment for (READ ITEM) addiction is usually very effective, somewhat effective, not too effective, or not effective at all? (IF RESPONDENT SAYS “IT DEPENDS OR IS “NOT SURE, ASK:) Well, if you had to give an answer about addiction treatment for people in general would you say it is usually very effective, somewhat effective, not too effective, or not effective at all?

Prescription medications

31.7 Very effective

48.2 Somewhat effective

8.0 Not too effective

2.7 Not effective at all

3.6 It depends

5.8 Not sure

Alcohol

30.6 Very effective

51.9 Somewhat effective

7.1 Not too effective

3.1 Not effective at all

4.3 It depends

3.0 Not sure

Tobacco

25.3 Very effective

47.8 Somewhat effective

13.4 Not too effective

5.4 Not effective at all

4.3 It depends

3.8 Not sure

Illegal drugs

24.4 Very effective

49.2 Somewhat effective

13.8 Not too effective

3.7 Not effective at all

3.6 It depends

5.2 Not sure

10a. How much would you say you know about treatment for addiction? Would you say you are very confident that you know what treatment for addiction involves, somewhat confident, not too confident, or not at all confident that you know what is really involved when someone gets treatment for addiction?

18.8 Very confident

42.8 Somewhat confident

20.6 Not too confident

16.5 Not confident at all

1.4 Not sure 
10b. When you think about treatment for addiction, what kinds of treatments come to mind? What are different types of addiction treatment you know about? (PROBE) Anything else? Are there any other types of treatment available for addiction that you know of?

40.1 Narcotic Anonymous (NA) or other similar 12-step programs

39.7 Hospitals

20.1 Alcoholics Anonymous (AA)

12.1 Prescription medications for addiction--patches and pills, suboxone, naltrexone or methadone

11.4 Residential rehabilitation clinics such as Betty Ford or Hazelden

10.1 Outpatient clinic or day treatment programs

8.2 Psychological therapies in either an individual or group setting

6.0 Comprehensive addictions treatment combining prescribed medication, individual and group therapy, and mutual support groups

5.6 Office-based treatment by a therapist or counselor

5.3 Religious or spiritual recovery programs

4.5 Hospital inpatient detoxification

3.5 Other mutual support or self-help programs such as Smart Recovery

2.3 Detoxification in a jail cell or prison

1.8 Talking to priest or pastor

0.7 Hotlines/Help lines, Quit lines

0.5 Office-based treatment by a physician

4.8 Other* (specify)

* The number of responses in the "Other" option are quite varied and therefore, not specified here.

4.0 None

7.5 Not sure

11a. Now I would like to read two views about medicines to treat addictions and have you tell me which one comes closer to your personal point of view.

Statement A: It is good news that there are medicines to treat addictions.

81.0 Good news

Statement B: It is not good news that there are medicines to treat addictions.*

14.2 Not good news

4.8 Not sure

11b. Now I would like to read two views about medicines to treat addictions and have you tell me which one comes closest to your personal point of view.

Statement A: It is good news that there are medicines to treat addictions, because addictions are medical conditions that medicine can help.

54.6 Good news

Statement B: It is not good news that there are medicines to treat addictions, because this only replaces one addiction with another.*

38.3 Not good news

7.1 Not sure

\footnotetext{
${ }^{*}$ Asked of one-half of the respondents.
} 
12. People have suggested various reasons why some people with addiction do not get the help they need. Which TWO or THREE of the following do you personally think are the main reasons why people with an addiction to alcohol, prescription or other drugs do not get help? Please let me know if you would like me to read the list again. (IF “ALL,” ASK:) If you had to choose just two or three, which would you choose?

72.0 Denial--refusal to admit the problem or not wanting to quit

38.0 Fear of consequences like losing job, getting expelled from school or losing child custody

29.1 Fear of social embarrassment or shame

28.3 Insufficient available or affordable treatment programs

20.3 Don't believe treatment would help

19.1 Fear of physical or emotional pain of withdrawal

15.9 Insufficient information about how and where to get help

1.0 Other

3.4 All

0.0 None

0.5 Not sure

13. Which TWO or THREE of the following are your main source of information about treatments for addiction to alcohol, tobacco, prescription or other drugs?

37.7 A friend or family member's personal experience

32.5 Health care professionals

25.7 Internet

24.4 News media such as newspapers or television news

20.1 Your own personal experience

15.4 Advertisements for treatment programs

13.2 Employee Assistance Programs (EAP)

12.6 Entertainment media like movies and TV shows

11.4 Academic research and scholarly journals

10.1 School guidance or health offices

8.2 Magazines about celebrities and entertainment news

2.6 Not sure

14. Do you think public health insurance plans like Medicare or Medicaid that are paid for by the tax dollars of people like you should cover treatment for addiction to (READ ITEM) or do you not think so? (IF "YES,” ASK:) Do you think public health insurance plans should provide patients with as much treatment as they need for the addictions to (READ ITEM) without limits or do you think there should be limits on addictions treatment such as " $\mathrm{X}$ " number of physician visits or " $Y$ " number of days per year of hospital or other residential care?

Prescription medications

25.6 Yes, should cover treatment without limits

42.3 Yes, should cover treatment with limits on days

3.4 Not sure about limits

22.8 No, should not cover treatment

6.0 Not sure 
Alcohol

22.6 Yes, should cover treatment without limits

41.6 Yes, should cover treatment with limits on days

4.0 Not sure about limits

28.3 No, should not cover treatment

3.4 Not sure

Illegal drugs

25.1 Yes, should cover treatment without limits

38.1 Yes, should cover treatment with limits on days

4.4 Not sure about limits

27.8 No, should not cover treatment

4.6 Not sure

Tobacco

21.0 Yes, should cover treatment without limits

30.1 Yes, should cover treatment with limits on days

2.8 Not sure about limits

42.4 No, should not cover treatment

3.8 Not sure

15. Now I am going to mention some approaches society could take to address the problem of addiction to alcohol, tobacco, prescription and other drugs. For each approach, please tell me how important you think it is--very important, somewhat important, not too important, or not important at all?

Educate the public about the disease of addiction and the possibility of recovery

73.1 Very important

22.1 Somewhat important

2.0 Not too important

1.9 Not important at all

0.9 Not sure

Fund research to improve treatment and recovery options for addiction to alcohol, tobacco, prescription or other drugs

57.6 Very important

36.5 Somewhat important

2.6 Not too important

1.9 Not important at all

1.4 Not sure

Increase the number and availability of effective treatment and recovery programs

57.4 Very important

34.2 Somewhat important

3.7 Not too important

2.7 Not important at all

2.0 Not sure

End discrimination in the areas of employment, insurance and housing against people who have used drugs in the past but are now in recovery

48.6 Very important

35.2 Somewhat important

8.2 Not too important

5.4 Not important at all

2.6 Not sure 
Increase criminal penalties for people who use drugs illegally

38.6 Very important

27.4 Somewhat important

14.9 Not too important

16.7 Not important at all

2.3 Not sure

The following questions are personal in nature, but we can assure you that this is a confidential survey and your responses will be kept completely private.

16. To your knowledge, has anybody close to you, like a parent, child, sibling, close friend, etc., ever had a problem with addiction to alcohol, prescription drugs or other drugs?

68.3 Yes

30.3 No

0.6 Not sure

0.8 Refused

17. To your knowledge, has anybody close to you, like a parent, child, sibling, close friend, etc., ever had a problem with addiction to tobacco?

78.2 Yes

19.7 No

1.2 A smoker but not addicted

0.5 Not sure

0.4 Refused

18a. Are you, yourself, addicted to alcohol, or prescription or other drugs right now, or have you been addicted to them in the past? I know this is a sensitive topic, but let me reassure you that this is for research purposes only and that all your responses will be completely anonymous and confidential.

16.9 Yes

82.0 No

0.7 Not sure

0.3 Refused

18b. What are you now or have you been addicted to in the past?

40.0 Alcohol

12.5 Prescription drugs

50.9 Other drugs

5.1 Not sure

7.6 Refused 
18c. Are you, yourself, addicted to tobacco right now, or have you been addicted to it in the past? I know this is a sensitive topic, but let me reassure you that this is for research purposes only and that all your responses will be completely anonymous and confidential.

34.3 Yes

61.6 No

3.1 A smoker but not addicted

0.7 Not sure

0.2 Refused

19a. Do you currently have health insurance?
85.8 Yes
13.0 No
1.0 Not sure
0.3 Refused

19b. (ASKED ONLY OF THOSE WHO SAY “YES," THEY CURRENTLY HAVE HEALTH INSURANCE IN Q. 19a) Which of the following most closely describes the type of health care plan you have--a traditional fee-for-service plan, in which the patient or the insurance company pays for each doctor visit or hospitalization, and HMO or PPO, in which most doctor visits or hospitalizations are covered without charging separately, Medicare or Medicaid, or some other type of plan?

21.6 Yes, traditional fee-for-service plan

46.1 Yes, an HMO or PPO

24.3 Yes, Medicare or Medicaid

10.3 Yes, some other type of plan

2.2 Yes, not sure which type of plan

1.0 Not sure

0.4 Refused

FACTUALS: Now I am going to ask you a few questions for statistical purposes only.

F1. How old are you? (IF “REFUSED,” ASK:) Well, would you tell me which age group you belong to?
$9.6 \quad 18-24$
$9.5 \quad 25-29$
$11.9 \quad 30-34$
$5.4 \quad 35-39$
$10.3 \quad 40-44$
$12.2 \quad 45-49$
$8.3 \quad 50-54$
$7.8 \quad 55-59$
$7.9 \quad 60-64$
$5.3 \quad 65-69$
$4.4 \quad 70-74$
7.375 and over
0.2 Refused 
F2a. Are you currently employed? (IF “CURRENTLY EMPLOYED,” ASK:) What type of work do you do? (IF “NOT CURRENTLY EMPLOYED,” ASK:) Are you a student, homemaker, retired, or unemployed and looking for work?

Not currently employed

3.3 Student

5.5 Homemaker

20.6 Retired

5.4 Unemployed, looking for work

63.1 Other - Currently employed

2.0 Not sure

F2b. Do you work full-time or part-time?

47.9 Yes, employed full-time

14.4 Yes, employed part-time

35.4 No, not currently employed

2.3 Not sure

F3. What is the last grade that you completed in school?

1.2 Grade school

6.8 Some high school

30.5 High school graduate

19.4 Some college, no degree

12.1 Vocational training/2 year college

14.6 4 year college/bachelor's degree

2.3 Some postgraduate work, no degree

8.7 2-3 years postgraduate work/master's degree

2.4 Doctoral/law degree

2.0 Not sure/refused

F4. Regardless of how you may be registered, how would you describe your overall point of view in terms of the political parties? Would you say that you are?

30.4 Mostly Democratic

11.1 Leaning Democratic

21.2 Completely Independent

9.5 Leaning Republican

18.8 Mostly Republican

9.1 Not sure

F5. Thinking about your general approach to issues, do you consider yourself to be liberal, moderate or conservative?

21.7 Liberal

35.5 Moderate

32.5 Conservative

10.3 Not sure 
F6. For statistical purposes only, would you please tell me which one of the following categories represents your total household income? Just stop me when I get to the correct category.

18.2 Less than $\$ 25,000$

$24.5 \$ 25,000$ to $\$ 50,000$

$19.4 \$ 50,000$ to $\$ 75,000$

$11.7 \$ 75,000$ to $\$ 100,000$

$8.9 \$ 100,000$ to $\$ 150,000$

5.5 More than $\$ 150,000$

11.8 Not sure/refused

F7. Finally, are you from a Hispanic or Spanish-speaking background?

13.0 Yes, Hispanic

86.7 No, not Hispanic

0.3 Not sure/refused

F8. What is your race--white, black, Asian or something else?

76.0 White

11.7 Black

1.3 Asian

3.0 Other

7.0 Hispanic

1.0 Not sure/refused 


\section{Appendix D \\ Survey of New York State Addiction Treatment Directors}

Responses from 83 survey participants. The number corresponding to each response option represents the percent, among those responding to the question, that provided the particular response.

\section{ORGANIZATION AND PRACTICE}

1. Which of the following provides the funding to operate your facility? (CHECK ALL THAT APPLY)

10.8 A private for-profit organization

54.2 A private nonprofit organization

53.0 State/federal government

19.3 Other

2. What types of payment for addiction/substance abuse treatment services are accepted by your facility? (CHECK ALL THAT APPLY)

10.8 Payment is not necessary (free treatment) GO TO \#3

88.0 Cash or self-payment

30.1 Medicare

86.7 Medicaid

39.8 State financed health insurance plan other than Medicaid (e.g., Children's Health Insurance Plan)

28.9 Municipal, county, or state-administered grant funding (either derivative of the federal Substance Abuse Treatment Block Grants, or not)

16.9 Federal military insurance (TriCare, CHAMPUS, or CHAMPVA)

65.1 Private health insurance

20.5 Other

3. What are the primary types of service offered in your program? (CHECK ALL THAT APPLY)

8.4 Inpatient general hospital

12.0 Inpatient specialty (addiction or psychiatric) hospital

28.9 Residential non-hospital

68.7 Outpatient non-methadone

13.3 Outpatient methadone

22.9 Other* (PLEASE SPECIFY)

*The high number of responses in the "Other" option are quite varied and therefore, not specified here. Other responses include chemical dependency centers, case management, and counseling.

4. Which of the following describes the kind of services provided in your program? (CHECK ALL

THAT APPLY)

28.9 Medical care

47.0 Pharmacological treatments

48.2 Treatment for co-occurring mental and physical health issues

100.0 Counseling/therapy

59.0 Self-help/mutual aid/social support

26.5 Other 
5. Is your facility affiliated with a religious organization, or is it not?

3.6 Affiliated

96.4 Not affiliated

6. Is your facility currently smoke free, or is it not?

98.8 Smoke free

1.2 Not smoke free

7. Does your facility offer treatment for nicotine addiction, or does it not?

89.2 Offers

10.8 Does not offer

8. What is the name of the county in which the treatment facility of which you are the director is located?

3.6 Albany

9.6 Bronx

1.2 Broome

1.2 Chemung

1.2 Clinton

1.2 Columbia

1.2 Cortland

1.2 Dutchess

3.6 Erie

1.2 Genesee

1.2 Greene

10.8 Kings

3.6 Nassau

3.6 Monroe

1.2 Montgomery

9.6 New York

1.2 Oneida

3.6 Onondaga

1.2 Ontario

2.4 Orange

1.2 Putnam

3.6 Queens

1.2 Rensselaer

1.2 Richmond

2.4 Rockland

2.4 Schenectady

1.2 Seneca

2.4 St. Lawrence

8.4 Suffolk

1.2 Sullivan

1.2 Ulster

1.2 Wayne

4.8 Westchester

1.2 Yates 
9. For each type of clinical staff listed below, how many full-time equivalent (FTE) staff who provide treatment for addiction/substance abuse are currently employed at your facility? (Average FTE staff reported below).

1.2 Physicians (MD/DO) e.g., general practitioner, internist, family physician, psychiatrist, addiction medicine specialist

1.2 Other doctoral-level (PhD, PsyD, SciD, DrSW) e.g., psychology, social work, counseling

0.7 Physician Assistants/Nurse Practitioners

4.4 Master's e.g., psychology, social work, counseling

6.7 Certified/licensed addiction and substance abuse counselors e.g., CDCs, CASACs, LACs (even if counted in categories above)

5.4 Other* (PLEASE SPECIFY)

* The high number of responses in the "Other" option are quite varied and therefore, not specified here. Other responses include CASACs, registered nurses and BA-level counselors.

10. What is the total number of full-time and part-time clinical staff currently employed at your facility? Please include all staff members in your count. (Average number of clinical staff reported below).

25.0 Clinical staff employed.

11. Last month, about how many staff members in total resigned, were let go, retired or left your facility? Please include all staff members in your count.

64.2 None

21.01

12.32

2.53

12. On average, about how long do staff who are directly involved in providing client treatment stay employed with your facility?

0.01 month or less

0.0 More than 1 month to less than 3 months

0.03 months to less than 6 months

0.06 months to less than 9 months

1.39 months to less than 1 year

6.31 year to less than 1.5 years

3.81 .5 years to less than 2 years

20.32 years to less than 3 years

24.13 years to less than 5 years

44.3 More than 5 years 
13. Under which of the following conditions would a client/patient be dismissed by your center or asked to leave the program before completing the treatment course? (CHECK ALL THAT

APPLY)

31.3 Client/patient using drugs or alcohol

75.9 Client/patient bringing drugs or alcohol onto the facility premises

12.0 Client/patient being unable to pay for treatment (e.g., private sources of payment run out; insurance stops covering costs)

69.9 Other* (PLEASE SPECIFY)

* The high number of responses in the "Other" option are quite varied and therefore, not specified here. Other responses include aggressive and violent behaviors, non-compliance, smoking, and legal issues.

14. What are the top two sources from which clients/patients are referred to your facility for treatment? (CHECK UP TO TWO)

27.7 Individual/self-referrals

6.0 Family

1.2 Private physician

22.9 Alcohol or other drug counselors (e.g., CASACs)

2.4 Other health care professionals (e.g., physicians, nurses)

3.6 Schools/educational organizations

3.6 Employers/employee assistance programs

18.1 Other community organizations

42.2 Criminal justice/court/drug court (exclusive of DUI/OWI)

45.8 Criminal justice/court/drug court referrals(DUI/OWI)

22.9 Other sources

15. How would you describe the attitude of the surrounding community toward having a treatment facility in the neighborhood? (CHECK THE ONE THAT BEST APPLIES)

2.4 Generally hostile

43.4 Neither hostile nor supportive

48.2 Generally supportive

6.0 Not applicable

16. If your facility does any advertising or outreach to attract patients/clients, which two of the following methods does it use most often? (CHECK UP TO TWO)

12.0 This facility does not do any advertising or outreach

23.5 Local print/fliers

4.8 Television

27.7 Internet

10.8 Hospitals/doctors' offices

21.7 Community centers

48.2 Other 
17. Which of the following steps does this facility take to continually improve treatment quality? (CHECK ALL THAT APPLY)

0.0 There are no quality improvement efforts at this facility

85.5 Require continuing education for staff

91.6 Provide continuing education opportunities for staff

75.9 Make available relevant publications discussing advances in treatment practices

85.5 Provide mentorship/supervision for staff

63.9 Forge collaborations with other facilities/organizations to offer additional off-site services to patient/clients

28.9 Other* (PLEASE SPECIFY)

* The high number of responses in the "Other” option are quite varied and therefore, not specified here. Other responses include performance improvement committees and inservice training.

\section{ATTITUDES TOWARD ADDICTION}

18. For each of the following health conditions please indicate whether you think...

- It cannot be treated at all; once a person has it, he or she always will suffer from it and its symptoms;

- It can be managed so that the symptoms are kept in check even though the individual continues to have the underlying problem; or

- It can be treated successfully so that the individual no longer suffers from the problem.

$\begin{array}{llll} & \begin{array}{l}\text { Cannot be } \\ \text { treated at all }\end{array} & \begin{array}{l}\text { Can be } \\ \text { managed }\end{array} & \begin{array}{l}\text { Can be treated } \\ \text { successfully }\end{array} \\ \text { Depression } & 1.2 & 47.0 & 51.8 \\ \text { Addiction to alcohol } & 1.2 & 41.0 & 57.8 \\ \text { Diabetes } & 1.2 & 79.5 & 19.3 \\ \text { Asthma } & 2.4 & 79.5 & 18.1 \\ \text { Addiction to drugs other than nicotine or alcohol } & 1.2 & 42.2 & 56.6 \\ \text { Heart disease } & 2.4 & 73.5 & 24.1 \\ \text { Addiction to nicotine } & 1.2 & 33.7 & 65.1\end{array}$

19. Which of the following do you think are the main factors involved in developing...

(i) Addiction to tobacco? (Please select a maximum of two primary factors)

(ii) Addiction to alcohol? (Please select a maximum of two primary factors)

(iii) Addiction to prescription/illegal drugs? (Please select a maximum of two primary factors)

Addiction to

A physical health problem

A mental health problem

A genetic problem

A behavioral problem

A moral problem

A spiritual problem

A reliance on the substance as an emotional crutch in response to negative life events

A problem of willpower or self control
Addiction to Addiction

tobacco to alcohol

26.5

18.1

22.9

48.2

6.0

7.2

49.4

12.0
26.5

38.6

56.6

44.6

15.7

21.7

62.7

12.0 prescription

illegal/drugs

51.8

39.8

38.6

43.4

13.3

14.5

60.2

10.8 
20. What should be a treatment provider's main treatment goal for...*

(i) Someone addicted to tobacco?

(ii) Someone addicted to alcohol?

(iv) Someone addicted to prescription/illegal drugs?

*The survey allowed respondent to choose more than one option for this question.

Complete abstinence from the substance

Reduced use of the substance

Fewer negative consequences from use of the

substance

Goal should be set by the patient

Other

$\begin{array}{lll}\begin{array}{l}\text { Addicted to } \\ \text { tobacco }\end{array} & \begin{array}{l}\text { Addicted } \\ \text { to alcohol }\end{array} & \begin{array}{l}\text { Addicted to } \\ \text { prescription } \\ \text { illegal drugs }\end{array} \\ 10.4 & 61.4 & 66.3 \\ & 3.6 & 8.4 \\ 4.8 & & \\ 41.0 & 10.8 & 13.3 \\ 3.6 & 25.3 & 30.1 \\ & 2.4 & 3.6\end{array}$

21. In your opinion, where should the money come from to pay for treating substance abuse and addiction? (CHECK UP TO THREE SOURCES)

If a source that you think the money should come from is not on the list below, please check

"Other" and write in your answer.

30.1 General tax revenues (e.g., income tax)

60.2 Increased taxes on alcohol

54.2 Increased taxes on cigarettes

43.4 Fines for individuals driving under the influence of alcohol or other drugs (DUIs)

25.3 Fines for commercial establishments that sell alcohol or cigarettes to minors

20.5 Funds saved from cutting government spending in other areas

34.9 Private insurance

13.3 Self-pay

9.6 Other* (PLEASE SPECIFY)

* The high number of responses in the "Other" option are quite varied and therefore, not specified here. Other responses include health insurance and Medicaid.

\section{ATTITUDES TOWARD TREATMENT}

22. How important is it for a treatment facility to have each of the following comprehensive assessment services available to clients/patients?

Substance use behavior

Other mental health conditions

Other physical health conditions

Family history of substance use

Social/peer history of substance abuse

Family/social network support systems

Economic circumstances

Religious/spiritual involvement
Not at all

important

0.0

0.0

0.0

0.0

0.0

0.0

1.2

1.2

\section{Slightly} important

0.0

0.0

1.2

0.0

3.7

1.2

8.5

13.3
Moderately Very important important

0.0

2.4

16.9

12.0

19.5

12.2

35.4

41.0
100.0

97.6

81.9

88.0

76.8

86.6

54.9

44.6 
23. How important is it for a treatment facility to have each of the following interventions/therapies available to clients/patients?

Detoxification

Motivational interviewing

Cognitive behavioral therapy

Family therapy

Religious/spiritual interventions

Mental health management (offered

along with substance abuse treatment)

Medical treatment for conditions such

as Hepatitis C, HIV, TB, STDs, and

other chronic medical illnesses (offered

along with substance abuse treatment)

Pharmacology therapies

Methadone maintenance for addictive

disorders

Other pharmacological interventions

for addictive disorders (e.g.,

buprenorphine, disulfiram, naltrexone)

Pharmacological interventions for co-

occurring mental health conditions

Pharmacological interventions for pain

management

Complementary/alternative medicine

approaches, such as acupuncture,

meditation

Recreation therapy/leisure skills

training

$\begin{array}{llll}\begin{array}{l}\text { Not at all } \\ \text { important }\end{array} & \begin{array}{l}\text { Slightly } \\ \text { important }\end{array} & \begin{array}{l}\text { Moderately } \\ \text { important }\end{array} & \begin{array}{l}\text { Very } \\ \text { important }\end{array} \\ 8.8 & 12.5 & 21.3 & 57.5 \\ 0.0 & 3.6 & 24.1 & 72.3 \\ 0.0 & 3.6 & 24.1 & 72.3 \\ 0.0 & 4.9 & 45.1 & 50.0 \\ 7.4 & 29.6 & 40.7 & 22.2 \\ 0.0 & 3.6 & 16.9 & 79.5\end{array}$

$\begin{array}{llll}2.4 & 11.0 & 26.8 & 59.8\end{array}$

$\begin{array}{llll}4.9 & 17.1 & 34.1 & 43.9\end{array}$

$\begin{array}{llll}1.2 & 6.1 & 18.3 & 74.4\end{array}$

$\begin{array}{llll}7.5 & 18.8 & 35.0 & 38.8\end{array}$

$\begin{array}{llll}4.8 & 31.3 & 33.7 & 30.1\end{array}$

$\begin{array}{llll}0.0 & 9.6 & 38.6 & 51.8\end{array}$

24. How important is it for a treatment facility to offer each of the following?

\begin{tabular}{|c|c|c|c|c|}
\hline & $\begin{array}{l}\text { Not at all } \\
\text { important }\end{array}$ & $\begin{array}{l}\text { Slightly } \\
\text { important }\end{array}$ & $\begin{array}{l}\text { Moderately } \\
\text { important }\end{array}$ & $\begin{array}{l}\text { Very } \\
\text { important }\end{array}$ \\
\hline Transportation services & 4.8 & 21.7 & 26.5 & 47.0 \\
\hline Child care services & 7.2 & 21.7 & 27.7 & 43.4 \\
\hline Employment/vocational services & 2.4 & 7.3 & 31.7 & 58.5 \\
\hline Housing services & 3.7 & 14.6 & 32.9 & 48.8 \\
\hline $\begin{array}{l}\text { Referral to programs such as AA, } \\
\text { Narcotics Anonymous, SMART } \\
\text { Recovery }\end{array}$ & 1.2 & 2.4 & 21.7 & 74.7 \\
\hline $\begin{array}{l}\text { Recovery support programs (e.g., AA, } \\
\text { Narcotics Anonymous, SMART } \\
\text { Recovery) }\end{array}$ & 1.2 & 9.6 & 25.3 & 63.9 \\
\hline $\begin{array}{l}\text { Relapse prevention (e.g., long-term } \\
\text { case management) }\end{array}$ & 0.0 & 2.4 & 10.8 & 86.7 \\
\hline
\end{tabular}


25. Which one of the following types of professionals do you think is best qualified to provide addiction treatment services?

71.3 Substance abuse counselors

16.3 Social workers

1.3 Nurses

7.5 Psychologists

1.3 Physicians

2.5 Persons who are recovered addicts or recovering from addiction

26. Do you think that only graduate-level clinicians/staff should provide addiction treatment services while non-graduate-level substance abuse counselors provide recovery support services $\mathbf{O R}$ do you think that non-graduate-level clinicians/staff should provide both addiction treatment services and recovery support services?

Note:

Graduate-level clinicians/staff refers to those with degrees/qualifications such as the following: $\mathrm{MD}, \mathrm{PhD}, \mathrm{MA}, \mathrm{RN}, \mathrm{NP}$.

Addiction treatment services refers to services such as the following: cognitive/behavioral therapy, pharmacotherapy.

Recovery support services refers to services such as the following: connection to mutual support programs; legal, housing, other social and health services; providing social support.

25.6 Only graduate-level professionals should provide the actual treatment, while counselors provide recovery support services

74.4 Non-graduate-level clinicians/staff should provide actual treatment and recovery support services

27. How important do you think it is for addiction treatment clinicians/staff to have each of the following qualifications?

Personal experience with addiction

Not at all important

College degree (e.g., BA/BS)

38.6

6.1

10.8

Slightly important 28.9

13.4

19.3

Moderately Very

Master's degree (e.g.,

MA/MS/MPH/MSW)

Doctoral degree (e.g., $\mathrm{PhD} / \mathrm{DSW}) \quad 40.7$

Medical degree (e.g., MD/DO)

35.8

Specific training in addiction treatment

0.0

33.3

27.2

0.0 important important

25.3

53.7

45.8

26.8

24.1

22.2

3.7

27.2

9.9

0.0 100.0 
28. If training were offered, which one or two training topics would be most helpful to you personally? (CHECK UP TO TWO RESPONSES)

12.0 Diagnosis/assessment via interview

7.2 Using standardized screening instruments

25.3 Etiology and prevalence of co-occurring disorders

37.3 Preferred treatment practices

14.5 Motivational interviewing

22.9 Specialized group treatments

16.9 Pharmacological treatments

8.4 Assertive case management approaches

9.6 Specialized 12-step facilitation approaches

19.3 Individual/group psychotherapies

12.1 Other* (PLEASE SPECIFY)

* The high number of responses in the "Other" option are quite varied and therefore, not specified here. Other responses include Evidence-Based practice and Cognitive-behavioral therapy.

29. Which of the following describes your opinion on the best way to structure the delivery of substance-addiction treatment in the U.S.? (CHECK THE ONE THAT BEST APPLIES)

11.3 The system should be integrated into the medical system such that addiction is treated as a health condition by heath care professionals (GO TO \#31)

7.5 The system should revolve around a recovery support model, with self-help/mutual support programs as the main intervention (GO TO \#31)

66.3 The system should be two-tiered with health care professionals providing psychotherapeutic and pharmacological interventions and individuals in recovery providing recovery support services (GO TO \#31)

15.0 Nothing should change

30. Why did you say that nothing should change? $(n=12$, those who answered "Nothing should change" in Q29)

33.3 Because the system is structured in the best possible way

8.3 Because a change in the way that treatment for addiction is delivered would be too costly

8.3 Because change in the way that treatment for addiction is delivered would be too disruptive to individuals involved in treatment delivery

0.0 Because we do not know how to change the treatment system

50.0 Other reason 
31. To what extent does each of the following stand in the way of people looking for needed treatment for addiction/substance abuse?

\begin{tabular}{|c|c|c|c|}
\hline & Not at all & Somewhat & Very much \\
\hline Denial of an addiction problem & 0.0 & 14.5 & 85.5 \\
\hline Belief that treatment does not work & 3.6 & 63.9 & 32.5 \\
\hline $\begin{array}{l}\text { Fear that one will be discriminated against if } \\
\text { looking for treatment }\end{array}$ & 12.0 & 48.2 & 39.8 \\
\hline $\begin{array}{l}\text { Fear of losing child custody (to the state or to } \\
\text { another individual competing for custody) }\end{array}$ & 1.2 & 25.3 & 73.5 \\
\hline Fear of losing job & 3.6 & 34.9 & 61.4 \\
\hline Fear of being sent to prison or jail & 16.9 & 41.0 & 42.2 \\
\hline $\begin{array}{l}\text { Inability to pay/lack of or insufficient medical } \\
\text { insurance }\end{array}$ & 9.6 & 44.6 & 45.8 \\
\hline Waiting lists that are too long & 37.8 & 40.2 & 22.0 \\
\hline
\end{tabular}

32. To what extent does each of the following stand in the way of people receiving needed treatment for addiction/substance abuse?

Lack of a treatment facility that is conveniently Not at all Somewhat Very much located

Lack of a treatment facility providing services that are suited to the individual client's needs Lack of ability to pay for treatment (including lack of private sources of funds and/or insurance 62.2 17.1

15.9

45.1

39.0 coverage)

$\begin{array}{lll}14.6 & 42.7 & 42.7\end{array}$

33. To what extent does each of the following stand in the way of treatment providers in New York State's ability to provide effective services to people in need of addiction/substance abuse treatment?

Insufficient program funding Not at all Somewhat Very much Insufficient insurance coverage for clients/patients 2.4

3.6 35.4 62.2 Insufficient salaries to attract and retain high3.6 36.1 60.2 quality staff

Insufficient number of trained counselors

10.8

21.7

74.7

(CASACs)

Insufficient number of trained Master's-level

47.0

42.2

professionals

Insufficient number of trained PhD-level

54.3

52.4

28.0

professionals

Insufficient number of trained MD/DO-level

35.8

37.0

8.6

professionals

Insufficient number of appropriately trained staff

3.7

51.9

12.3

with the qualifications necessary to provide treatment for co-morbid physical or mental health conditions

Insufficient number of appropriately trained staff with the qualifications necessary to provide pharmacological therapies 
Insufficient federal- or state-level education and training requirements for individuals providing addiction treatment Insufficient professional standards related to knowledge and clinical skills for individuals providing addiction treatment Limited motivation among staff members to be knowledgeable about evidence-based best practices Insufficient access to information about best practices

High volume of paperwork (reporting requirements that take up too much time and resources)
Not at all Somewhat Very much

34.9

47.0

18.1

41.0

38.6

20.5

42.2

36.1

50.6

1.2

22.0

76.8

34. How important do you think it is that there be national standards for how addiction/substance abuse treatment services should be delivered to patients/clients?

11.0 Not at all important (GO TO \#36)

18.3 Slightly important

29.3 Moderately important

41.5 Very important

35. Which of the following would be in the best position to decide on such national standards for the delivery of addiction/substance abuse treatment services? (CHECK THE ONE THAT BEST

APPLIES) ( $\mathrm{n}=72$, those who did not answer "Not at all Important” in Q34)

11.1 The federal government

40.3 National professional organizations

36.1 State professional organizations

12.5 Other (PLEASE SPECIFY)

\section{EVALUATION}

36. What are the three main ways your program evaluates how well it is doing? (CHECK UP TO THREE RESPONSES)

0.0 Our program does not conduct evaluations (GO TO \#37)

8.4 Informal reports of staff perceptions

54.2 Random client feedback/testimonials

43.4 Drug test results

68.7 Program completion rates

2.4 Reduction in use based on self-report of clients only

24.1 Reduction in use based on self-report of clients AND information from other sources

7.2 Abstinence from use based on self-report of clients only

42.2 Abstinence from use based on self-report of clients AND information from other sources

2.4 Remission of symptoms based on self-report of clients only

22.9 Remission of symptoms based on self-report of clients AND information from other sources

1.2 Reduced recidivism rates for criminal offenders based on self-report of clients only

13.3 Reduced recidivism rates for criminal offenders based on self-report of clients AND other information from justice departments 
37. At what stage(s), if any, in the treatment of an individual patient, does your program assess how well treatment is working? (CHECK ALL THAT APPLY)

2.4 Our program does not assess how well treatment is working (GO TO \#38)

60.2 At treatment program exit

20.5 One month after treatment

37.32 to 6 months after treatment

24.17 to 12 months after treatment

8.4 More than one year but less than five years after treatment

2.4 Five years or more post-treatment

38. In your opinion, what are the three primary ways a program should assess its effectiveness, assuming that a program has sufficient resources for this? (CHECK UP TO THREE

RESPONSES)

0.0 Program effectiveness does not require evaluation

7.2 Informal reports of staff perceptions

45.8 Random client feedback/testimonials

32.5 Drug test results

57.8 Program completion rates

2.4 Reduction in use based on self-report of clients only

33.7 Reduction in use based on self-report of clients AND information from other sources

1.2 Abstinence from use based on self-report of clients only

45.8 Abstinence from use based on self-report of clients AND information from other sources

1.2 Remission of symptoms based on self-report of clients only

34.9 Remission of symptoms based on self-report of clients AND information from other sources

0.0 Reduced recidivism rates for criminal offenders based on self-report of clients only

26.5 Reduced recidivism rates for criminal offenders based on self-report of clients AND other information from justice departments

\section{RECOMMENDATIONS}

39. Given sufficient resources, what are three ways you would change your program to improve treatment quality at your facility?

26.8 Hire more staff with experience/higher education

19.1 More access to education/resources/training

10.0 Decrease caseload/paperwork

9.6 More sessions/groups/treatment options

6.8 Better case management (employment, housing, child care)

6.4 Better pay for staff

4.6 More space/change facility

3.6 Add mental health component in treatment

1.8 Use electronic records/administrative technology

11.4 Other 
40. Given sufficient resources, what are three ways you would suggest for improving the treatment system for addiction or substance abuse in New York?

15.1 Better funding for training/staff resources

13.7 Decrease paperwork/regulations

9.0 Multi-care approach that includes mental health/referrals

8.5 Better access to inpatient care/treatment

8.0 Better pay for staff

6.6 Enhance quality of services and programs

6.6 Hire more staff with experience/higher education

6.1 Fix insurance issues/billing

5.6 Better case management (employment, housing, child care)

2.4 Use electronic records/administrative technology

1.9 More sessions and groups available in treatment

1.4 Decrease caseload

16.5 Other

VI. INDIVIDUAL DEMOGRAPHICS OF PROGRAM DIRECTOR PARTICIPATING IN THIS SURVEY

41. In what year were you born?
$29.51938-1950$
$60.31951-1970$
$10.31971-1981$

42. Are you Hispanic/Latino/Latina or of Spanish origin?

7.4 Yes

92.6 No

43. With which racial or ethnic group do you identify?

75.6 White

15.9 African American

0.0 Asian American/Pacific Islander

0.0 Native American

8.5 Other

44. What is your gender?

47.6 Male

52.4 Female

45. What is the highest level of education you have completed?

0.0 Less than high school graduation (GO TO \#47)

1.2 High school graduate or GED (GO TO \#47)

14.5 Some college or associate degree (GO TO \#47)

16.9 Four-year college degree (GO TO \#47)

67.5 Graduate or post-graduate degree 
46. What is your professional title? (CHECK THE ONE BEST APPLIES) (n=54, of those who answered "Graduate or post-graduate degree" in Q45).

0.0 Physician

11.1 Psychologist

0.0 Physician Assistant

0.0 Nurse Practitioner

0.0 Registered Nurse

0.0 Licensed Practicing Nurse

40.7 Social worker

5.6 Addictions counselor

42.6 Other* (PLEASE SPECIFY)

* The high number of responses in the "Other" option are quite varied and therefore, not specified here. Other responses include Director, MBA, and Administrator

47. Do you have specific training in addiction treatment, or do you not?

88.0 Have specific training* (PLEASE SPECIFY)

* The high number of responses in the "Have Specific training" option are quite varied and therefore, not specified here. Other responses include CASAC training hours, addiction fellowships and classes.

12.0 Do not have specific training

48. How many years of experience do you have in the field of addiction treatment?

1.2 Less than 1 year

1.21 year to less than 2 years

0.02 years to less than 4 years

2.44 years to less than 6 years

7.26 years to less than 9 years

6.09 years to less than 10 years

81.910 years or more

49. Do you think that being a recovered addict or recovering from addiction should be a prerequisite for being a treatment director, or should it not?

30.1 No, it would not help the position and should not be a prerequisite

68.7 It might help but should not be a prerequisite for the position

1.2 Yes, it would help and it should be a prerequisite 


\section{REFERRALS}

50. Do you ever refer patients to see private physicians who practice addiction medicine outside of your facility, or do you never do that?

53.0 Refer

47.0 Never refer (END. THANK YOU)

51. If yes, about what proportion of patients do you refer per year?

48.8 Less than $5 \%$

$26.85 \%-10 \%$

$17.1 \quad 11 \%-25 \%$

$4.926 \%-50 \%$

2.4 Over 50\% 
$-284-$ 


\section{Appendix E \\ Survey of New York State Addiction Treatment Staff}

Responses from 141 survey participants. The number corresponding to each response option represents the percent, among those responding to the question, that provided the particular response.

\section{ATTITUDES TOWARD ADDICTION}

1. For each of the following health conditions please indicate whether you think...

- It cannot be treated at all; once a person has it, he or she always will suffer from it and its symptoms;

- It can be managed so that the symptoms are kept in check even though the individual continues to have the underlying problem; or

- It can be treated successfully so that the individual no longer suffers from the problem.

Depression

Addiction to alcohol

Diabetes

Asthma

Addiction to drugs other than nicotine or alcohol

Heart disease

Addiction to nicotine

$\begin{array}{ccc}\begin{array}{c}\text { Cannot be } \\ \text { treated at all }\end{array} & \begin{array}{c}\text { Can be } \\ \text { managed }\end{array} & \begin{array}{c}\text { Can be treated } \\ \text { successfully }\end{array} \\ 0.0 & 60.7 & 39.3 \\ 0.0 & 55.4 & 44.6 \\ 0.0 & 84.9 & 15.1 \\ 0.0 & 84.2 & 15.8 \\ 0.0 & 57.6 & 42.4 \\ 2.2 & 75.5 & 22.3 \\ 0.7 & 40.6 & 58.7\end{array}$

2. Which of the following do you think are the main factors involved in developing...

(i) Addiction to tobacco? (Please select a maximum of two primary factors)

(ii) Addiction to alcohol? (Please select a maximum of two primary factors)

(iii) Addiction to prescription/illegal drugs? (Please select a maximum of two primary factors)

A physical health problem

A mental health problem

A genetic problem

A behavioral problem

A moral problem

A spiritual problem

A reliance on the substance as an emotional crutch

A problem of willpower or self control

$\begin{array}{ccc}\begin{array}{c}\text { Addiction to } \\ \text { tobacco }\end{array} & \begin{array}{c}\text { Addiction } \\ \text { to alcohol }\end{array} & \begin{array}{c}\text { Addiction to } \\ \text { prescription } \\ \text { illegal/drugs }\end{array} \\ 36.2 & 40.4 & 45.4 \\ 19.1 & 46.8 & 45.4 \\ 18.4 & 64.5 & 38.3 \\ 47.5 & 50.4 & 48.9 \\ 10.6 & 30.5 & 24.1 \\ 12.1 & 41.8 & 32.6 \\ 53.9 & 81.6 & 66.7 \\ 29.8 & 26.2 & 19.1\end{array}$


3. What should be a treatment provider's main treatment goal for...*

(i) Someone addicted to tobacco?

(ii) Someone addicted to alcohol?

(iv) Someone addicted to prescription/illegal drugs?

*The survey allowed respondent to choose more than one option for this question.

\begin{tabular}{|c|c|c|c|}
\hline & $\begin{array}{l}\text { Addicted to } \\
\text { tobacco }\end{array}$ & $\begin{array}{l}\text { Addicted } \\
\text { to alcohol }\end{array}$ & $\begin{array}{l}\text { Addicted to } \\
\text { prescription } \\
\text { illegal/drugs }\end{array}$ \\
\hline Complete abstinence from the substance & 48.2 & 70.2 & 65.2 \\
\hline Reduced use of the substance & 21.3 & 9.2 & 9.2 \\
\hline $\begin{array}{l}\text { Fewer negative consequences from use of the } \\
\text { substance }\end{array}$ & 11.3 & 9.2 & 9.9 \\
\hline Goal should be set by the patient & 41.1 & 22.0 & 26.2 \\
\hline Other & 6.4 & 3.5 & 5.7 \\
\hline
\end{tabular}

4. In your opinion, where should the money come from to pay for treating substance abuse and addiction? (CHECK UP TO THREE SOURCES)

If a source that you think the money should come from is not on the list below, please check "Other" and write in your answer.

18.4 General tax revenues (e.g., income tax)

48.2 Increased taxes on alcohol

42.6 Increased taxes on cigarettes

54.6 Fines for individuals driving under the influence of alcohol or other drugs (DUIs)

33.3 Fines for commercial establishments that sell alcohol or cigarettes to minors

20.6 Funds saved from cutting government spending in other areas

39.7 Private insurance

28.4 Self-pay

9.9 Other (PLEASE SPECIFY)

\section{ATTITUDES TOWARD TREATMENT}

5. How important is it for a treatment facility to have each of the following comprehensive assessment services available to clients/patients?

Substance use behavior

Other mental health conditions

Other physical health conditions

Family history of substance use

Social/peer history of substance abuse

Family/social network support systems

Economic circumstances

Religious/spiritual involvement

$\begin{array}{cccc}\begin{array}{c}\text { Not at all } \\ \text { important }\end{array} & \begin{array}{c}\text { Slightly } \\ \text { important }\end{array} & \begin{array}{c}\text { Moderately } \\ \text { important }\end{array} & \begin{array}{c}\text { Very } \\ \text { important }\end{array} \\ 0.0 & 0.7 & 1.4 & 97.9 \\ 0.7 & 0.7 & 7.9 & 90.7 \\ 0.7 & 3.6 & 29.5 & 66.2 \\ 0.7 & 3.6 & 20.7 & 75.0 \\ 0.7 & 4.3 & 28.1 & 66.9 \\ 0.0 & 2.2 & 15.1 & 82.7 \\ 1.4 & 14.4 & 40.3 & 43.9 \\ 1.4 & 15.0 & 42.9 & 40.7\end{array}$


6. How important is it for a treatment facility to have each of the following interventions/therapies available to clients/patients?

Detoxification

Not at all

important

Motivational interviewing

Cognitive behavioral therapy

Family therapy

3.6

0.7

0.0

0.0

7.9

0.0

Slightly
important

Moderately

Very

Religious/spiritual interventions

Mental health management (offered

along with substance abuse treatment)

Medical treatment for conditions such

as Hepatitis C, HIV, TB, STDs, and

other chronic medical illnesses (offered

along with substance abuse treatment)

Pharmacology therapies

Methadone maintenance for

addictive disorders

Other pharmacological

interventions for addictive disorders

(e.g., buprenorphine, disulfiram, naltrexone)

Pharmacological interventions for

co-occurring mental health

conditions

Pharmacological interventions for pain management


2.2

$\begin{array}{llll}2.2 & 11.6 & 29.7 & 56.5\end{array}$
56.5

10.9

7.2

important

important

67.4

64.5

74.6

60.9

25.2

84.1

Complementary/alternative medicine approaches, such as acupuncture, meditation

Recreation therapy/leisure skills

7.9

3.6

23.2

35.5

43.2

14.5

training

$\begin{array}{llll}2.2 & 22.5 & 29.7 & 45.7\end{array}$

$\begin{array}{llll}0.7 & 5.8 & 20.9 & 72.7\end{array}$

$\begin{array}{llll}4.4 & 19.0 & 38.7 & 38.0\end{array}$

$\begin{array}{llll}2.9 & 24.5 & 37.4 & 35.3\end{array}$

$\begin{array}{llll}0.7 & 14.4 & 30.2 & 54.7\end{array}$

7. How important is it for a treatment facility to offer each of the following?

$\begin{array}{lcccc} & \begin{array}{c}\text { Not at all } \\ \text { important }\end{array} & \begin{array}{c}\text { Slightly } \\ \text { important }\end{array} & \begin{array}{c}\text { Moderately } \\ \text { important }\end{array} & \begin{array}{c}\text { Very } \\ \text { important }\end{array} \\ \text { Transportation services } & 2.2 & 23.0 & 32.4 & 42.4 \\ \text { Child care services } & 6.5 & 23.9 & 33.3 & 36.2 \\ \text { Employment/vocational services } & 2.2 & 7.2 & 27.3 & 63.3 \\ \begin{array}{l}\text { Housing services } \\ \text { Referral to programs such as AA, }\end{array} & 4.3 & 17.4 & 26.8 & 51.4 \\ \begin{array}{l}\text { Narcotics Anonymous, SMART } \\ \text { Recovery }\end{array} & 2.2 & 4.3 & 13.8 & 79.7 \\ \begin{array}{l}\text { Recovery support programs (e.g., AA, } \\ \text { Narcotics Anonymous, SMART }\end{array} & 1.4 & & & \\ \begin{array}{l}\text { Recovery) } \\ \text { Relapse prevention (e.g., long-term }\end{array} & & & & \\ \text { case management) } & 0.0 & & & \\ \end{array}$


8. Which one of the following types of professionals do you think is best qualified to provide addiction treatment services?

72.6 Substance abuse counselors

17.8 Social workers

0.7 Nurses

6.7 Psychologists

1.5 Physicians

0.7 Persons who are recovered addicts or recovering from addiction

9. Do you think that only graduate-level clinicians/staff should provide addiction treatment services while non-graduate-level substance abuse counselors provide recovery support services $\mathbf{O R}$ do you think that non-graduate-level clinicians/staff should provide both addiction treatment services and recovery support services?

Note:

Graduate level clinicians/staff refers to those with degrees/qualifications such as the following: $\mathrm{MD}, \mathrm{PhD}, \mathrm{MA}, \mathrm{RN}, \mathrm{NP}$.

Addiction treatment services refers to services such as the following: cognitive/behavioral therapy, pharmacotherapy.

Recovery support services refers to services such as the following: connection to mutual support programs; legal, housing, other social and health services; providing social support.

35.6 Only graduate-level professionals should provide the actual treatment, while counselors provide recovery support service

64.4 Non-graduate-level clinicians/staff should provide actual treatment and recovery support services

10. How important do you think it is for addiction treatment clinicians/staff to have each of the following qualifications?

Personal experience with addiction

Not at all important

College degree (e.g., BA/BS)

23.7

12.9

Master's degree (e.g.,

18.8

MA/MS/MPH/MSW)

Doctoral degree (e.g., $\mathrm{PhD} / \mathrm{DSW})$

Medical degree (e.g., MD/DO)

43.9

Specific training in addiction treatment

41.7

0.7

Slightly
important
36.0
19.4
23.2

Moderately important

21.6

40.3

31.9

Very
important
18.7
27.3
26.1

28.1

21.6

6.5

24.5

20.1

13.7

0.7

7.9

90.6 
11. If training were offered, which one or two training topics would be most helpful to you personally? (CHECK UP TO TWO RESPONSES)

17.0 Diagnosis/assessment via interview

7.8 Using standardized screening instruments

21.3 Etiology and prevalence of co-occurring disorders

17.0 Preferred treatment practices

25.5 Motivational interviewing

24.8 Specialized group treatments

12.8 Pharmacological treatments

19.9 Assertive case management approaches

7.1 Specialized 12-step facilitation approaches

35.5 Individual/group psychotherapies

3.6 Other (PLEASE SPECIFY)

12. Which of the following describes your opinion on the best way to structure the delivery of substance-addiction treatment in the U.S.? (CHECK THE ONE THAT BEST APPLIES)

8.1 The system should be integrated into the medical system such that addiction is treated as a health condition by heath care professionals (GO TO \#14)

21.3 The system should revolve around a recovery support model, with self-help/mutual support programs as the main intervention (GO TO \#14)

60.3 The system should be two-tiered with health care professionals providing psychotherapeutic and pharmacological interventions and individuals in recovery providing recovery support services (GO TO \#14)

10.3 Nothing should change

13. Why did you say that nothing should change? (Those who answered "Nothing should change" in Q12)

28.6 Because the system is structured in the best possible way

0.0 Because a change in the way that treatment for addiction is delivered would be too costly

7.1 Because change in the way that treatment for addiction is delivered would be too disruptive to individuals involved in treatment recovery

7.1 Because we do not know how to change the treatment system

57.1 Other reason

14. To what extent does each of the following stand in the way of people looking for needed treatment for addiction/substance abuse?

Denial of an addiction problem

Belief that treatment does not work

Fear that one will be discriminated against if

looking for treatment

Fear of losing child custody (to the state or to

another individual competing for custody)

Fear of losing job

Fear of being sent to prison or jail

Inability to pay/lack of or insufficient medical

insurance

Waiting lists that are too long

$\begin{array}{ccc}\text { Not at all } & \text { Somewhat } & \text { Very much } \\ 2.9 & 12.2 & 84.9 \\ 9.4 & 59.0 & 31.7 \\ 18.1 & 51.4 & 30.4\end{array}$

$\begin{array}{lll}4.3 & 37.0 & 58.7\end{array}$

$\begin{array}{lll}5.8 & 38.4 & 55.8\end{array}$

$\begin{array}{lll}20.3 & 42.8 & 37.0\end{array}$

$\begin{array}{lll}6.5 & 42.8 & 50.7\end{array}$

$\begin{array}{lll}29.7 & 49.3 & 21.0\end{array}$ 
15. To what extent does each of the following stand in the way of people receiving needed treatment for addiction/substance abuse?

Lack of a treatment facility that is conveniently
Not at all

21.6

18.0

7.9

$\begin{array}{cc}\text { Somewhat } & \text { Very much } \\ 56.8 & 21.6\end{array}$

56.1

25.9

are suited to the individual client's needs

Lack of ability to pay for treatment (including lack

of private sources of funds and/or insurance

coverage)

16. To what extent does each of the following stand in the way of treatment providers in New York State's ability to provide effective services to people in need of addiction/substance abuse treatment?

Insufficient program funding

Insufficient insurance coverage for clients/patients

Not at all

2.9

3.6

4.3

Insufficient salaries to attract and retain high-

quality staff

Insufficient number of trained counselors

(CASACs)

Insufficient number of trained Master's-level

professionals

Insufficient number of trained $\mathrm{PhD}$-level

professionals

Insufficient number of trained MD/DO-level

professionals

Insufficient number of appropriately trained staff

with the qualifications necessary to provide

treatment for co-morbid physical or mental health

conditions

Insufficient number of appropriately trained staff

with the qualifications necessary to provide

pharmacological therapies

Insufficient federal- or state-level education and

training requirements for individuals providing

addiction treatment

Insufficient professional standards related to

knowledge and clinical skills for individuals

providing addiction treatment

Limited motivation among staff members to be

knowledgeable about evidence-based best practices

Insufficient access to information about best

practices

High volume of paperwork (reporting requirements

that take up too much time and resources)
15.

35.3

43.9

34.1

42.8

54.0

8.6

19.0

61.3

30.4

46.4

35.5

42.8

25.5

54.7

34.8

49.3

2.9

24.5
37.4

19.7

23.2

21.7

19.7

15.9

63.3

61.6

69.8

33.8

20.9

7.2

13.0

72.7 
17. How important do you think it is that there be national standards for how addiction/substance abuse treatment services should be delivered to patients/clients?

4.3 Not at all important (GO TO \#19)

15.1 Slightly important

29.5 Moderately important

51.1 Very important

18. Which of the following would be in the best position to decide on such national standards for the delivery of addiction/substance abuse treatment services? (CHECK THE ONE THAT BEST APPLIES)

13.7 The federal government

33.6 National professional organizations

38.2 State professional organizations

14.5 Other (PLEASE SPECIFY)

\section{EVALUATION}

19. What are the three main ways your program evaluates how well it is doing? (CHECK UP TO THREE RESPONSES)

2.8 Our program does not conduct evaluations (GO TO \#20)

24.1 Informal reports of staff perceptions

48.9 Random client feedback/testimonials

31.2 Drug test results

59.6 Program completion rates

2.8 Reduction in use based on self-report of clients only

19.9 Reduction in use based on self-report of clients AND information from other sources

2.8 Abstinence from use based on self-report of clients only

34.8 Abstinence from use based on self-report of clients AND information from other sources

0.7 Remission of symptoms based on self-report of clients only

18.4 Remission of symptoms based on self-report of clients AND information from other sources

0.0 Reduced recidivism rates for criminal offenders based on self-report of clients only

22.0 Reduced recidivism rates for criminal offenders based on self-report of clients AND other information from justice departments

20. At what stage(s), if any, in the treatment of an individual patient, does your program assess how well treatment is working? (CHECK ALL THAT APPLY)

7.8 Our program does not assess how well treatment is working (GO TO \#21)

43.3 At treatment program exit

27.7 One month after treatment

41.12 to 6 months after treatment

14.97 to 12 months after treatment

12.8 More than one year but less than five years after treatment

2.1 Five years or more post-treatment 
21. In your opinion, what are the three primary ways a program should assess its effectiveness, assuming that a program has sufficient resources for this? (CHECK UP TO THREE

RESPONSES)

2.8 Program effectiveness does not require evaluation

16.3 Informal reports of staff perceptions

46.1 Random client feedback/testimonials

29.1 Drug test results

55.3 Program completion rates

1.4 Reduction in use based on self-report of clients only

21.3 Reduction in use based on self-report of clients AND information from other sources

2.1 Abstinence from use based on self-report of clients only

53.2 Abstinence from use based on self-report of clients AND information from other sources

0.0 Remission of symptoms based on self-report of clients only

32.6 Remission of symptoms based on self-report of clients AND information from other sources

0.0 Reduced recidivism rates for criminal offenders based on self-report of clients only

21.3 Reduced recidivism rates for criminal offenders based on self-report of clients AND other information from justice departments

\section{WORK ENVIRONMENT}

22. In a typical day, about how many hours do you work at the treatment facility?

2.11 to 4 hours

44.3 More than 4 hours to 8 hours

50.7 More than 8 hours to 12 hours

2.9 More than 12 hours

23. In a typical day, about how many total hours a day would you say you spend on each of the following tasks?

If you perform any other task in a typical day on which you spend one or more hours but that task is not on the list below, please specify the task and indicate how many hours you spend on it.

3.2 Seeing/counseling/treating clients face-to-face

1.1 Working with clients over the phone/via email

0.8 Tracking clients because of missed appointments

1.4 Finding and scheduling referral services for clients

3.1 Completing paperwork

2.7 Other (PLEASE SPECIFY)

24. From the list below, please select the top two things a client might do that would keep you from doing your job well. (CHECK UP TO TWO RESPONSES)

57.4 Miss appointments

33.3 Never return after initial visit

32.6 Fail to follow treatment plan

41.1 Lie about behavior

12.1 Spend time with you just chatting instead of working with you

7.1 Other (PLEASE SPECIFY) 
25. From the list below, please select the top two factors that mainly motivate you to keep you doing your job. (CHECK UP TO TWO RESPONSES)

73.0 The work is rewarding

29.8 The work is enjoyable

15.6 I like my colleagues

34.0 I like my clients

2.1 The pay is good

5.0 The hours are good

5.0 The benefits are good

2.8 No other jobs are available

15.6 I made it out of addiction and consider it my responsibility to help others

6.4 Other (PLEASE SPECIFY)

26. If you were to leave your job, what are the top two reasons you would do so? (CHECK UP TO TWO RESPONSES)

42.6 I do not plan to leave my job

17.7 The work is frustrating

5.7 I dislike my colleagues

0.0 I dislike my clients

47.5 The pay is too low

8.5 The hours are not good

10.6 The benefits are not good

0.7 I made it out of addiction and do not want to deal with this issue anymore

28.4 Other (PLEASE SPECIFY)

\section{RECOMMENDATIONS}

27. Given sufficient resources, what are three ways you would change your program to improve treatment quality at your facility?

9.6 Higher pay/incentives for staff

9.3 Improve facilities/more space and activities

9.0 Hire more qualified staff

8.7 Spend more time with clients/lower caseloads

8.3 Decrease paperwork/improve efficiency in process

8.3 Provide more training/education to staff/team

6.1 Provide more resources for client (child care, transportation, etc.)/case management

5.8 Insurance coverage/funding

5.8 Eliminate/add treatment, effective treatment

5.4 Better counselors, more specialized for cultural/language differences

2.9 Customized services for women/children/families

2.6 Better materials/services/procedures (quality assurance)

18.3 Other (PLEASE SPECIFY) 
28. Given sufficient resources, what are three ways you would suggest for improving the treatment system for addiction or substance abuse in New York?

11.4 Case management services (housing, job training, child care, etc.)

9.6 Uniform system/less paperwork

8.9 Better education/training system for providers

8.6 Effective/more proven treatments, customized/quality treatments

7.5 Incentives for workers/providers, higher pay

6.8 Affordable healthcare/insurance

6.4 Better funding/cut costs

5.7 Improve availability of programs, more time with clients

5.4 Women/family/children customized treatment

5.0 Hire more qualified staff

24.6 Other (PLEASE SPECIFY)

V. INDIVIDUAL DEMOGRAPHICS OF STAFF MEMBER PARTICIPATING IN THIS SURVEY

29. In what year were you born?
17.9 Before 1950
$32.1 \quad 1950-1959$
25.4 1960-1969
$16.4 \quad 1970-1979$
$6.0 \quad 1980-1989$
2.2 Other (PLEASE SPECIFY)

30. Are you Hispanic/Latino/Latina or of Spanish origin?
14.1 Yes
85.9 No

31. Which racial or ethnic group do you identify?

63.0 White

21.5 African American

0.7 Asian American/Pacific Islander

0.7 Native American

14.1 Other

32. What is your gender?

39.4 Male

60.6 Female

33. What is the highest level of education you have completed?

0.7 Less than high school graduation (Go to \#35)

6.6 High school graduate or GED (Go to \#35)

29.9 Some college or associate degree (Go to \#35)

27.7 Four-year college degree (Go to \#35)

35.0 Graduate or postgraduate degree 
34. What is your professional title? (Check the ONE that best applies)

0.0 Physician

4.2 Psychologist

0.0 Physician Assistant

0.0 Nurse Practitioner

0.0 Registered Nurse

0.0 Licensed Practicing Nurse

41.7 Social worker

12.5 Addictions counselor

41.7 Other* (PLEASE SPECIFY)

* A significant proportion of those who responded with the "Other" option specified that they are some form of addiction counselor or that they define themselves as both an addiction counselor and another professional title.

35. Do you have specific training in addiction treatment or do you not?

93.5 Have specific training (PLEASE SPECIFY)

6.5 Do not have specific training

36. How many years of experience do you have in the field of addiction treatment?

1.4 Less than 1 year

5.81 year to less than 2 years

14.42 years to less than 4 years

10.14 years to less than 6 years

12.96 years to less than 9 years

5.09 years to less than 10 years

50.410 years or more

37. On average, how many clients are on your treatment caseload at any point of time?

$5.11-5$

$15.9 \quad 6-10$

$13.8 \quad 11-15$

$9.4 \quad 16-20$

$22.5 \quad 21-30$

$9.4 \quad 31-40$

7.2 More than 40

16.7 I do not carry a caseload

38. Do you think that being a recovered addict or recovering from addiction should be a prerequisite for being a treatment provider, or should it not?

23.0 No, it would not help the position and should not be a prerequisite

71.9 It might help but should not be a prerequisite for the position

5.0 Yes, it would help and it should be a prerequisite

\section{THANK YOU!}


-296- 


\section{Appendix F \\ National Panel and National Online Survey of Members of Professional Associations Involved in Addiction Care*}

\section{National Panel of Treatment Providers}

Beauchemin, Patricia, Executive Director, Therapeutic Communities of America ${ }^{\dagger}$ (TCA)

Budney, MD, Alan J., Professor of Psychiatry and Research Scientist, Center for Addiction Research, University of Arkansas for Medical Sciences (American Psychological Association - APA)

Femino, MD, John, Medical Director, Meadows Edge Recovery Center (American Society of Addiction Medicine - ASAM)

Fleming, MPA, LSAC, Patrick J., Director, Salt Lake County Division of Substance Abuse Services (National Council for Community Behavioral Healthcare - National Council)

Grant, Linda, Executive Director, Evergreen Manor (State Associations of Addiction Services - SAAS)

Greer, Patricia M., President, National Association of Alcoholism and Drug Abuse Counselors NAADAC $^{\ddagger}$

Hurt, MD, Richard D., Director and Professor of Medicine, Nicotine Dependence Center, Mayo Clinic College of Medicine (Association for the Treatment of Tobacco Use and Dependence - ATTUD)

Marion, MA, Ira, AATOD First Vice President and NYS Board delegate and Executive Director, Albert Einstein College of Medicine (American Association for the Treatment of Opioid Dependence - AATOD)

Mumbauer, Daniel, President and Chief Executive Officer, Southeast Regional Network, Highpoint Treatment Center (National Council for Community Behavioral Healthcare - National Council)

Olsen, LCSW, CASAC, SAP, Edward, Director, EAC Outpatient Clinic (National Association of Alcoholism and Drug Abuse Counselors, NAADAC)

Roy, III, MD, A. Kenison, Fellow, American Society of Addiction Medicine; Distinguished Fellow, American Psychiatric Association; Assistant Clinical Professor of Psychiatry, Tulane Medical School; and Medical Director, Addiction Recovery Resources of New Orleans (American Society of Addiction Medicine - ASAM)

Schwarzlose, John T., President and Chief Executive Officer, Betty Ford Center (National Association of Addiction Treatment Providers - NAATP)

Scimeca, MD, Michael M., Area II Director (American Academy of Addiction Psychiatry - AAAP)

\footnotetext{
* Names and titles of individuals and organizations represent those at time of Panel.

${ }^{\dagger}$ This organization currently is named Treatment Communities of America (TCA).

₹ This organization currently is named NAADAC, the Association for Addiction Professionals.
} 
Washton, PhD, Arnold M., Founder and Executive Director, Recovery Options (American Psychological Association - APA)

Whitman, MS, Ellen G., Executive Director, National Association of County Behavioral Health and Developmental Disability Directors - NACBHDD

Wilkins, Christopher R., Senior Vice President, DePaul Addiction Services (State Associations of Addiction Services - SAAS) 


\section{National Online Survey of Members of Professional Associations Involved in Addiction Care}

Responses from 1,142 survey participants. The number corresponding to each response option represents the percent, among those responding to the question, that provided the particular response.

1. To what professional organization do you belong?

9.9 American Academy of Addiction Psychiatry (AAAP)

2.7 American Association for the Treatment of Opioid Dependence (AATOD)

8.9 American Psychological Association (APA)

26.0 American Society of Addiction Medicine (ASAM)

5.7 Association for the Treatment of Tobacco Use and Dependence (ATTUD)

17.1 National Association of Alcoholism and Drug Abuse Counselors (NAADAC)

7.2 National Association of Addiction Treatment Providers (NAATP)

2.5 National Association of County Behavioral Health and Developmental Disability Directors (NACBHDD)

1.7 National Council for Community Behavioral Healthcare (NCCBH)

2.2 State Association of Addiction Services (SAAS)

0.3 Therapeutic Communities of America (TCA)

15.8 Other

2. If you were designing a treatment program to meet the needs of individuals in your community, how important would it be to include each of the following? (Please rate each option on a scale from Not At All Important to Very Important as presented below).

Screenings and Brief Interventions

$0.21=$ Not at all Important

$3.12=$

$8.73=$

$18.74=$

69.3 5=Very Important

Comprehensive Assessments

$0.21=$ Not at all Important

$1.72=$

$7.0 \quad 3=$

$17.9 \quad 4=$

73.2 5=Very Important

Detoxification Services

$1.21=$ Not at all Important

$4.52=$

$10.73=$

20.8 4=

$62.85=$ =Very Important

Individual cognitive-behavioral therapy

$0.51=$ Not at all Important

$4.42=$

$15.83=$

$29.0 \quad 4=$

50.4 5=Very Important 
Family therapy

$0.71=$ Not at all Important

$4.72=$

$15.33=$

$32.74=$

46.6 5=Very Important

Complementary and alternative medicine (e.g., acupuncture, meditation)

$9.21=$ Not at all Important

$23.92=$

$29.6 \quad 3=$

$22.24=$

15.1 5=Very Important

Concurrent treatment/management of other physical and mental health conditions

$0.41=$ Not at all Important

$1.52=$

$9.83=$

$27.24=$

$61.25=$ =Very Important

Pharmacological options

$0.91=$ Not at all Important

$4.0 \quad 2=$

$11.53=$

$27.14=$

56.5 5=Very Important

Mutual recovery groups

$0.51=$ Not at all Important

$3.22=$

$13.93=$

$28.64=$

53.8 5=Very Important

Assistance with occupational/legal/housing issues

$2.81=$ Not at all Important

$6.32=$

$22.7 \quad 3=$

$32.94=$

$35.35=$ =Very Important

Assistance with child care or transportation

$2.71=$ Not at all Important

$8.92=$

$25.43=$

$32.14=$

30.9 5=Very Important

Comprehensive case management services

$1.41=$ Not at all Important

$4.42=$

$19.73=$

$30.94=$

43.6 5=Very Important 
Long-term disease management support

$1.71=$ Not at all Important

$5.72=$

$18.53=$

$32.34=$

41.7 5=Very Important

Are there other services that you think are Very Important to provide in order to appropriately treat substance use disorders? ( $\mathrm{n}=247$ out of 1,142 respondents)

10.9 Co-occurring/mental health diagnoses or services

7.3 Alternative therapies/treatment or more customized treatment options

6.1 Community/family education and support services

6.1 Mutual support groups

5.7 Long-term residential treatment

4.9 Aftercare, after-treatment services such as job placement, basic life skills

4.0 Spirituality counseling/services

3.6 Relapse management/prevention

2.8 Motivational enhancement therapy

2.8 Nutrition and fitness

42.1 Other (each individual/other response was provided by less than 2.4 percent of respondents responding to this question)

3. To what extent do you agree that each of the following is an important goal of treatment for substance use disorders? (Please rate each option on a scale from Strongly Disagree to Strongly Agree as presented below).

Complete Abstinence

$2.71=$ Strongly Disagree

$4.72=$

$14.0 \quad 3=$

$25.84=$

52.5 5=Strongly Agree

Reductions in use

$11.91=$ =Strongly Disagree

$8.02=$

$9.93=$

$26.34=$

43.8 5=Strongly Agree

Remission of DSM symptoms

$1.11=$ Strongly Disagree

$3.52=$

$13.53=$

$37.9 \quad 4=$

44.0 5=Strongly Agree

Improvements in functioning (e.g., employment, education, parenting, family stability, crime and recidivism, health, happiness, citizenship, etc.)

$\begin{array}{rl}0.4 & 1=\text { Strongly Disagree } \\ 0.5 & 2= \\ 2.3 & 3= \\ 18.4 & 4= \\ 78.5 & 5=\text { Strongly Agree }\end{array}$


Allowing the patient to set the goal that's right for him or her

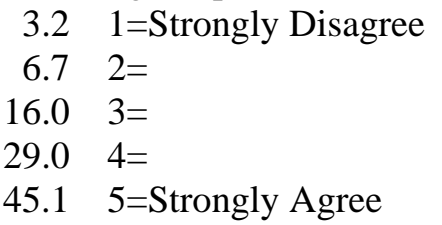

Are there other Very Important goals of treatment for substance use disorders? ( $n=169$ out of 1,142 respondents)

17.8 Self-care (self-esteem/confidence), understanding disease

13.6 Relapse prevention/harm reduction/pain management programs

12.4 Aftercare services

11.2 Mutual support groups/group therapy

5.3 Some level of abstinence

4.1 Mental health care

3.6 Spirituality

3.6 Family support/education

3.0 Accountability

2.4 Acceptance

23.1 Other (each individual/other response was provided by less than 1.2 percent of respondents responding to this question)

4. To what extent is each of the following a barrier to your ability to provide high quality treatment for your clients/patients with substance use disorders? (Please rate each option on a scale from Not At All a Barrier to A Very Big Barrier as present below).

Difficulty engaging the client (i.e., due to client denial, stigma, lack of family support, etc.)

$7.11=$ Not at all a barrier

$20.82=$

$29.03=$

$28.24=$

$15.05=$ A very big barrier

Insufficient evidenced-based treatment options

$26.21=$ Not at all a barrier

$31.92=$

$22.73=$

$12.34=$

$6.95=$ A very big barrier

Insufficient support to implement evidence-based treatments

$17.91=$ Not at all a barrier

$20.62=$

$24.13=$

$20.64=$

$16.85=$ A very big barrier

Difficulty tailoring treatment program to client needs

$17.11=$ Not at all a barrier

$24.22=$

$28.53=$

$22.0 \quad 4=$

$8.25=$ A very big barrier 
Difficulty recruiting and retaining qualified staff

$9.51=$ Not at all a barrier

$13.52=$

$22.53=$

$28.24=$

$26.45=$ A very big barrier

Insufficient staff or resources to address co-morbid conditions

$7.01=$ Not at all a barrier

$11.22=$

$21.93=$

$28.8 \quad 4=$

$31.15=$ A very big barrier

Insufficient staff or resources to provide pharmaceutical options

$15.01=$ Not at all a barrier

$17.22=$

$19.13=$

$27.34=$

$21.45=$ A very big barrier

Insufficient insurance coverage

$5.51=$ Not at all a barrier

$5.82=$

$11.33=$

$18.14=$

$59.35=$ A very big barrier

Insufficient time and resources to assess outcomes

$5.71=$ Not at all a barrier

$10.82=$

$20.73=$

$32.34=$

$30.45=$ A very big barrier

Community resistance to addiction treatment

$15.41=$ Not at all a barrier

$25.42=$

$23.8 \quad 3=$

$19.84=$

$15.65=$ A very big barrier

Are there other Very Big Barriers to your ability to provide high quality treatment for your clients/patients with substance use disorders? ( $\mathrm{n}=229$ out of 1,142 respondents)

22.3 Money/funding

12.7 Lack of resources and services (housing, transportation, facilities)

11.4 Insurance coverage/Medicaid/Medicare

9.2 Lack of appropriate or customized treatment

8.3 Public administration/government structure and restrictions

6.6 Lack of training or knowledge for providers

3.5 Inadequate communication/coordination with health providers

3.5 Community/medical provider resistance

3.5 Need more family support and education for addiction, lessen stigma

19.2 Other (each individual/other response was provided by less than 1.7 percent of respondents responding to this question) 
5. What are the top three recommendations you would make to improve access to and quality of treatment for substance use disorders in the U.S.?

21.6 Insurance coverage

17.7 Alternative/customized therapies (comprehensive care, co-occurring conditions)

12.0 Money/funding

8.2 More training/education for practitioners, service providers

4.9 Accessibility to services, treatment on demand

4.6 Adequate resources/education/awareness in community

3.5 Incentives for treatment staff, increase staffing, better pay

3.3 Provide services to those in treatment (child care, transportation, housing, etc.)

2.2 Higher education requirements for practitioners and service providers

1.8 Change stigma/opinions of public

1.6 Cross-medical coordination between specialists and providers

1.6 Government involvement, change laws and policies

1.6 Decriminalization, drug courts

1.4 Provide support/education for families and individuals/clients

1.2 Explain and treat addiction as public health, chronic issue

1.1 Early intervention/prevention

0.9 Provide aftercare services (job training, etc.)

0.9 More research/outcome studies

0.7 Reduce paperwork, reduce bureaucracy

0.6 Outreach to other populations (rural, poor, etc.)

0.6 Accountability, mutual support groups

0.5 Marketing/media on substance use disorders

7.3 Other 


\section{Appendix G \\ Survey of Participants in Recovery}

Responses from 360 survey participants. The number corresponding to each response option represents the percent, among those responding to the question, that provided the particular response.

1. How long have you been in recovery? Mean is 194.1 months (approximately 16.2 years).

2. Looking back over your recovery process, what are the three main factors to which you attribute your ability to maintain long term recovery?

29.3 Mutual support groups (12 Step/NA/AA)

12.8 Spirituality/religion

11.5 Peer support/fellowship/connection with others

6.8 Desire/commitment to stay clean and sober/setting goals

6.4 Family support system

5.0 Dedicated sponsor

4.7 Balance/change in lifestyle

3.4 Individual counseling

2.7 Acceptance/honesty/admitting problem

17.5 Other (each individual/other response was provided by less than 2.0 percent of respondents responding to this question)

3. To what extent is or was each of the following critical to your recovery? (Please check the box under the number that is right for you using a scale of 1 to 5 , with 1 being "Not at all critical" to 5 being "Extremely critical").

Medical detox

$39.61=$ Not at all critical

$7.72=$

$8.33=$

$4.84=$

$16.0 \quad 5=$ Extremely critical

$23.7 \quad$ N/A

In-patient treatment

$23.51=$ Not at all critical

$4.52=$

$4.53=$

$10.24=$

36.7 5=Extremely critical

20.6 N/A

Out-patient treatment

$26.41=$ Not at all critical

$8.32=$

$10.73=$

10.4 4=

20.5 5=Extremely critical

23.7 N/A 
Private therapy (primary care physician, psychiatrist, psychologist, social worker, counselor)

$18.91=$ Not at all critical

$7.72=$

$14.93=$

16.6 4=

26.0 5=Extremely critical

16.0 N/A

Pharmacological treatment

$43.91=$ Not at all critical

$4.52=$

$6.83=$

$6.84=$

9.0 5=Extremely critical

29.0 N/A

Mutual support programs such as AA or NA

$42.51=$ Not at all critical

$14.22=$

$2.33=$

$4.84=$

83.3 5=Extremely critical

4.0 N/A

Spiritual practice

$3.91=$ Not at all critical

$2.82=$

$9.93=$

$14.14=$

$66.85=$ Extremely critical

2.5 N/A

Family support

$8.81=$ Not at all critical

$9.72=$

$18.53=$

$20.54=$

40.5 5=Extremely critical

2.0 N/A

Employer support

$24.31=$ Not at all critical

$10.22=$

$16.13=$

$16.74=$

16.7 5=Extremely critical

16.1 N/A

Support from friends

$6.81=$ Not at all critical

$4.82=$

$13.33=$

$27.24=$

$45.65=$ 5xtremely critical

2.3 N/A 
Requirements of the Criminal Justice System for treatment

$33.11=$ Not at all critical

$6.82=$

$4.73=$

$3.84=$

6.8 5=Extremely critical

44.9 N/A

Legal assistance

$36.91=$ Not at all critical

$4.72=$

$5.13=$

$3.04=$

$3.45=$ Extremely critical

47.0 N/A

4. What are some of the major challenges or barriers you face or faced in maintaining long-term recovery?

Being separated from family

$39.61=$ Not a major challenge/barrier

$7.72=$

$8.33=$

$4.84=$

$16.05=$ Very major challenge/barrier

23.7 N/A

Lack of housing options

$41.21=$ Not a major challenge/barrier

$12.22=$

$9.73=$

$8.44=$

9.7 5= Very major challenge/barrier

18.9 N/A

Housing discrimination

$52.11=$ Not a major challenge/barrier

$3.82=$

$9.23=$

$6.34=$

$6.35=$ Very major challenge/barrier

26.1 N/A

Lack of employment options

$43.41=$ Not a major challenge/barrier

$9.82=$

$9.83=$

$4.74=$

$9.85=$ Very major challenge/barrier

22.6 N/A 
Employment discrimination

$47.21=$ Not a major challenge/barrier

$5.52=$

$8.93=$

$3.84=$

$6.45=$ Very major challenge/barrier

28.1 N/A

Lack of family support

$43.51=$ Not a major challenge/barrier

$10.12=$

$13.53=$

$7.64=$

$4.25=$ Very major challenge/barrier

21.1 N/A

Lack of peer support

$47.71=$ Not a major challenge/barrier

$14.92=$

$9.43=$

$4.74=$

$5.15=$ Very major challenge/barrier

17.5 N/A

Lack of other support systems

$44.41=$ Not a major challenge/barrier

$13.32=$

$12.43=$

$4.34=$

7.7 5= Very major challenge/barrier

18.0 N/A

Lack of medical attention

$50.91=$ Not a major challenge/barrier

$8.92=$

$6.43=$

$4.24=$

$6.85=$ Very major challenge/barrier

22.9 N/A

Lack of insurance coverage

$37.81=$ Not a major challenge/barrier

$7.62=$

$8.83=$

$7.6 \quad 4=$

19.3 5= Very major challenge/barrier

18.9 N/A 
5. If there is anything else you would like to add to help us better understand the recovery process, please feel free to comment on your thoughts and experiences.

\section{Main Themes from Participants' Responses:}

Inadequate training of health care providers: physicians and other health professionals have insufficient education and training on the subject of addiction

The need for more affordable and accessible treatment facilities for people of different demographic backgrounds

Addiction treatment should address co-occurring mental health disorders

Inadequate insurance coverage for addiction treatment and chronic disease management

Limited availability of auxiliary support services (e.g., housing, transportation) and continued medical management for those completing acute treatment

The need to reduce the stigma, misunderstandings and lack of knowledge surrounding addiction as a disease

Addiction should be addressed as a medical/public health problem rather than a legal/enforcement issue 
$-310-$ 


\section{Appendix $\mathrm{H}$}

\section{Screening and Assessment Instruments}

A screening instrument is meant to identify early signs of a disease or health condition and distinguish between individuals who require no intervention and those who may require a brief intervention or more intensive treatment. ${ }^{1}$ In contrast, an assessment instrument should be utilized once a patient has been screened for a condition--in this case, risky substance use--as a necessary precursor to the initiation of an intervention or treatment. ${ }^{2}$ The goals of the assessment are to help health care professionals determine the nature, stage and severity of a condition and whether the patient meets clinical criteria for an addiction diagnosis; establish whether co-occurring mental health or other medical problems exist; and allow for the development of an appropriate and specific treatment plan. ${ }^{3}$

Despite this theoretical distinction between screening and assessment, the term screening often is used to subsume the concept of assessment or interchangeably with the term in the clinical and research literatures. Instruments designed to screen for risky substance use and those designed to assess symptoms of addiction frequently do not fit neatly into these two categories. For example, many instruments that are described as screening tools use diagnostic criteria for addiction ${ }^{*}$ to evaluate their validity rather than measures of risky substance use. In addition, some instruments are designed to measure risky use or addiction across substances (typically not including nicotine), whereas others are more substance specific; none measures all substances that may be involved in risky use or addiction as a unified dimension.

With these caveats in mind, the following review attempts to lay out the characteristics of commonly-used instruments and specify, when

\footnotetext{
* Primarily based on the Diagnostic and Statistical Manual of Mental Disorders (DSM).
} 
possible, the extent to which they have clinical utility for screening versus assessment purposes.

\section{Determining the Clinical Utility of a Screening or Assessment Instrument}

The psychometric properties of instruments typically are established prior to their use in research or clinical settings. The main properties examined are validity and reliability. ${ }^{4}$

There are three primary measures of validity: construct, content and criterion validity. ${ }^{5}$ Construct validity determines the degree to which the instrument is related to the theoretical concept being measured; ${ }^{6}$ content validity is the extent to which items included in the instrument represent the area of interest that the instrument is designed to measure; ${ }^{7}$ and criterion validity determines whether the instrument is related to external criteria, ${ }^{8}$ such as diagnostic criteria for a disease.

Reliability determines whether the instrument produces consistent results that can be reproduced. ${ }^{9}$ Test-retest reliability refers to the stability of the instrument in terms of the consistency of a respondent's score when tested multiple times; ${ }^{10}$ inter-rater reliability determines whether the instrument produces stable results across different observers; and internal reliability (or consistency) determines whether the items in a multi-item instrument correlate with one another. ${ }^{11}$

Two other important properties of an instrument are its sensitivity--its ability to identify accurately those with a particular characteristic, in this case a certain level of substance involvement, as having the characteristic, and specificity--its ability to identify accurately those without a particular characteristic as not having the characteristic.

Although the statistics associated with these properties are beyond the scope of this report, these properties are discussed in a general way, where available and relevant, to help give a sense of the clinical utility of a particular instrument.

\section{General Substance Use Screening and Assessment Tools*}

\section{Alcohol, Smoking and Substance Involvement Screening Test (ASSIST)}

Developed by the World Health Organization (WHO) in 1997, the Alcohol, Smoking and Substance Involvement Screening Test is an interviewer-administered screening tool for primary care settings. ${ }^{12}$ The eight-question instrument measures the frequency of current and lifetime use of tobacco, alcohol and illicit drugs and the problems adult respondents have experienced due to their use. ${ }^{13}$ Each question is structured to identify tobacco, alcohol, cannabis, cocaine, amphetamine-type stimulant, inhalant, sedative, hallucinogen, opioid and other drug use and related problems resulting from use. ${ }^{14}$

Each question has a set of responses with corresponding numerical scores. For tobacco and illicit drugs, the WHO recommends that patients with specific substance involvement scores of three or lower receive no intervention aside from information about the substances they use; those with scores between four and 26 receive a brief intervention; and those with scores of 27 or higher receive an intensive intervention or treatment. For alcohol, this breakdown is 10 or lower, 11 to 26 and 27 or higher, respectively. ${ }^{\dagger 15}$

The ASSIST requires training to administer properly. ${ }^{16}$ The WHO recommends practitioners understand why screening is important; how to implement, administer and score the screening instrument; and how to conduct follow-up activities to help patients at different levels of risk. Trainings also should include role-play and supervised practice. ${ }^{17}$

Although it is copyrighted, the instrument along with the scoring manual are available free of charge through the WHO. ${ }^{18}$

\footnotetext{
* Select substance-specific screening tools are presented later in the appendix.

${ }^{\dagger}$ The total substance involvement score is calculated by adding scores based on a patient's responses to all eight questions across all 10 substance categories.
} 
Populations and Settings Served. The ASSIST was developed to serve patients in primary care settings and has been used successfully as the screening component of a screening and brief intervention program. ${ }^{19}$ The instrument also is appropriate for use in outpatient clinics, emergency departments, general hospital wards, psychiatric offices, antenatal and prenatal clinics, criminal justice settings and welfare facilities. ${ }^{20}$

Clinical Utility. According to a government review conducted by the Agency for Healthcare Research and Quality (AHRQ), research supports the ASSIST's reliability; in a subgroup of WHO study participants, " the ASSIST demonstrated both internal reliability and testretest reliability. ${ }^{21}$ When testing the validity of the ASSIST, WHO researchers studied its efficacy at identifying substance involvement across populations of seven countries. ${ }^{\dagger} 22$ The ASSIST was able to discriminate among levels of risky substance use and addiction for each substance individually and for total substance involvement. ${ }^{\ddagger 23}$ Scores on the ASSIST strongly correspond to scores on other related screening instruments. ${ }^{24}$ The ASSIST also has demonstrated acceptable sensitivity and specificity, particularly with regard to its ability to distinguish accurately between those who engage in alcohol and illicit drug ${ }^{\S}$ use versus abuse $^{25}$ and slightly lower sensitivity and specificity in distinguishing between abuse and dependence. ${ }^{26}$

\section{Simple Screening Instrument for Substance Abuse (SSI)}

The Simple Screening Instrument for Substance Abuse is a 16-item yes/no questionnaire that was

\footnotetext{
* 236 participants (60 percent from addiction treatment programs, 40 percent from a general medical facility).

${ }^{\dagger}$ Australia, Brazil, India, Thailand, the United Kingdom, the United States of America and Zimbabwe.

₹ The WHO study group $(n=1047)$ answered questions from other validated instruments and gave hair samples for purposes of comparison.

$\S$ Including marijuana, cocaine, amphetamine-type stimulants, sedatives and opioids.
}

developed in 1994 by the Center for Substance Abuse Treatment (CSAT) of the Substance Abuse and Mental Health Services Administration (SAMHSA) to screen for risky substance use in adult and adolescent populations. ${ }^{27}$ SSI measures an individual's alcohol and other drug consumption (excluding nicotine), preoccupation and loss of control, adverse consequences, problem recognition and tolerance and withdrawal levels. ${ }^{28}$ The screening can be conducted through an interview or self-administered. ${ }^{29}$

A cut-off score of four is recommended for identifying individuals at high to moderate risk who may be in need of further assessment. ${ }^{30}$

SSI is in the public domain and can be copied and administered free of charge. Minimal training is needed to administer and score it. ${ }^{31}$

Populations and Settings Served. SSI was developed for use in adult and adolescent populations regardless of gender, ethnicity, cultural background, literacy level or socioeconomic status. Originally it was designed to be used by outreach workers, health professionals and paraprofessionals in populations with infectious diseases ${ }^{32}$ and to encompass a broad spectrum of signs and symptoms of addiction; it may be particularly useful for screening patients in mental health settings for co-occurring substance-related problems. ${ }^{33}$

Clinical Utility. SSI has not been tested as rigorously as some other instruments. While one study found that it was valid and reliable in an inmate population, ${ }^{34}$ another study found that it had low validity in a college population: in a group of 201 college students, the SSI correctly classified only 58.9 percent of cases that met DSM criteria for substance abuse. ${ }^{35}$ The SSI also has lower sensitivity (it is more likely to produce false positives) than similar instruments. ${ }^{36}$ 


\section{The CAGE Questionnaire and the CAGE- Adapted to Include Drugs (AID)}

The CAGE Questionnaire, developed in 1984, is a four-item alcohol screening tool that gets its name from the questions that are asked:

1. Have you ever felt you should Cut down on your drinking?

2. Have people Annoyed you by criticizing your drinking?

3. Have you ever felt bad or Guilty about your drinking?

4. Have you ever had a drink first thing in the morning to steady your nerves or get rid of a hangover (Eye-opener)? ${ }^{37}$

Generally, if respondents answer affirmatively to at least two of these questions they are thought to be at risk for addiction involving alcohol and in need of a brief intervention or further diagnostic assessments. ${ }^{38}$ The CAGE can be administered through an interview with a professional or technician or it can be selfadministered. $^{39}$

The CAGE-Adapted to Include Drugs (AID) instrument, developed in 1991, is identical to the CAGE with the exception of the phrase "or drug use” (which appears to excludes smoking) tacked on to the end of each question. ${ }^{40}$

Neither of the questionnaires is copyrighted and both are widely available. ${ }^{41}$ The tools require no training to administer and the scoring process is straightforward.

Populations and Settings Served. The CAGE instrument can identify risky alcohol use in adolescent, adult and elderly populations. ${ }^{42}$ Due to its brevity and ease of administration and scoring, CAGE is well-suited to primary care settings. It has proven to be a practical tool for identifying risky alcohol use in emergency department settings as well. ${ }^{43}$ The CAGE-AID questionnaire generally is recommended for use with adult primary care patients. ${ }^{44}$
Clinical Utility. Multiple studies with different populations have found the CAGE and the CAGE-AID instruments to be valid and reliable. ${ }^{45}$ Across various primary care settings, the CAGE has demonstrated high sensitivity-identifying accurately up to 94 percent of patients with current or lifetime addiction involving alcohol, and high specificity-identifying accurately 97 percent of those without addiction involving alcohol. ${ }^{46}$ When used in an emergency department setting, CAGE results were correlated with biological indicators of addiction involving alcohol. ${ }^{47}$ When tested in a general population sample, individuals with positive scores were 37 times more likely than those with negative scores to have addiction involving alcohol, based on criteria for alcohol dependence in the DSM-IV and the International Classification of Diseases (ICD). ${ }^{48}$

The CAGE instrument is less effective at detecting risky substance use (vs. addiction involving alcohol); ${ }^{49}$ it does not appear to be effective at detecting low but risky alcohol use among college students--an important category of risky users; ${ }^{50}$ and its utility in screening pregnant populations has yet to be established. ${ }^{51}$

Like its counterpart, the CAGE-AID questionnaire is better at identifying symptoms of addiction than risky substance use. The instruments also do not distinguish between current and former risky substance use. ${ }^{52}$

\section{CRAFFT}

The CRAFFT is a six-item questionnaire for screening for lifetime risky alcohol and other drug use (excluding nicotine) among adolescents. $^{53}$ The items are: ${ }^{54}$

1. Have you ever ridden in a Car driven by someone (including yourself) who was high or had been using alcohol or drugs?

2. Do you ever use alcohol or drugs to Relax, feel better about yourself or fit in?

3. Do you ever use alcohol or drugs while you are by yourself (Alone)? 
4. Do you ever Forget things you did while using alcohol or drugs?

5. Do your Family or Friends ever tell you that you should cut down on your drinking or drug use?

6. Have you ever gotten into Trouble while you were using alcohol or drugs?

An affirmative answer to each question is worth one point. A cut-off score of two is recommended for identifying risky alcohol and other drug use or the risk of addiction. ${ }^{55} \mathrm{~A}$ positive test is a good indicator that respondents are in need of further assessment.

The CRAFFT is widely available free of charge. ${ }^{56}$

Populations and Settings Served. The CRAFFT has been validated among adolescent primary care clinic patients ${ }^{57}$ and there is some evidence that it is effective in identifying risky substance use among pregnant women as well. ${ }^{58}$

Clinical Utility. The CRAFFT has been found to be a valid indicator of substance involvement. A cut-off score of two accurately recognized 76 percent of adolescent risky users, 80 percent of adolescents with addiction ${ }^{*}$ and 92 percent of adolescents who met diagnostic criteria specifically for substance dependence. ${ }^{59}$ The CRAFFT has adequate internal and test-retest reliability as well. ${ }^{60}$ It has demonstrated a 92.3 percent sensitivity rate and an 82.1 percent specificity rate among adolescents with a history of alcohol and other drug use, ${ }^{61}$ and an 86 percent sensitivity rate and a 76 percent specificity rate in American Indian and Alaska Native adolescents with regard to alcohol and other drug use. $^{\dagger} 62$

\footnotetext{
${ }^{*}$ Including both substance abuse and substance dependence, as identified by the Adolescent Diagnostic Interview.

${ }^{\dagger}$ Including over-the-counter drugs, marijuana, cocaine, amphetamines, barbiturates/sedatives, inhalants, hallucinogens, opioids and other drugs (excluding nicotine).
}

\section{Substance Abuse Subtle Screening Inventory (SASSI)}

Developed in 1988, the Substance Abuse Subtle Screening Inventory can help practitioners identify individuals who are likely to have addiction involving alcohol or other drugs. ${ }^{63}$ The instrument is available in separate versions for adults (SASSI-3) and adolescents (SASSIA); only the adolescent version includes items related to smoking. ${ }^{64}$ To measure substance involvement, the SASSI also contains some indirect, "subtle" true-false items to help practitioners identify respondents who may have misrepresented the extent of their substance use. $^{\ddagger 65}$ The inclusion of these subtle items may decrease erroneous assessments by as much as 76 percent. $^{66}$

Both instruments can be self-, computer- or staff-administered and generally take 10 to 15 minutes to complete and 10 minutes for practitioners to score and interpret. ${ }^{67}$

Neither instrument requires extensive training to administer. ${ }^{68}$ A user's guide and manual are provided with the test. ${ }^{69}$ Both versions of the SASSI tools are copyrighted and available for purchase only. $^{70}$

Populations and Settings Served. SASSI may be useful in inpatient and outpatient mental health and medical settings, as well as criminal justice, employee assistance and education settings. ${ }^{71}$ It has been used with inmates, college students, pregnant women, brain injury patients and psychiatric inpatients. ${ }^{72}$

\footnotetext{
${ }^{\ddagger}$ Examples include: I have never done anything dangerous just for fun; There have been times when I have done things I didn't remember later; People who use drugs have more fun; In school I have often been in trouble for misbehaving; I can be depended on to do the things I'm supposed to.
} 
Clinical Utility. The SASSI-3 has been found to be valid and reliable among patients in a variety of clinical settings. ${ }^{*} 73$ It has been found to be 93 percent accurate at identifying individuals who are likely to have addiction involving alcohol or other drugs (excluding nicotine) ${ }^{74}$ and 97 percent accurate at classifying patients who met diagnostic criteria for substance dependence. The SASSI-3 demonstrates a 92 percent test-retest reliability rate and 93 percent internal reliability rate. ${ }^{75}$ One study found that it correctly identified 69.8 percent of cases with addiction (sensitivity) and 62.0 percent of cases without addiction (specificity). ${ }^{76}$

SASSI-A has not undergone extensive empirical examination. ${ }^{77}$ According to its manual, the screening tool can identify accurately up to 95 percent of 12- to 18-year olds with addiction (sensitivity) and 89 percent of those without addiction (specificity). ${ }^{78}$

Some research has questioned the effectiveness of the subtle items of the SASSI instruments in helping to detect addiction among adults and adolescents. $^{79}$

\section{Problem-Oriented Screening Instrument for Teenagers (POSIT)}

The Problem-Oriented Screening Instrument for Teenagers was developed by the National Institute on Drug Abuse (NIDA) in 1991 as a screening instrument. ${ }^{80}$ It can identify potential problems in adolescents between the ages of 12 and 19 and covers 10 areas, including alcohol and other drug use (excluding nicotine), physical health and social relations. ${ }^{\dagger 81}$ The POSIT

\footnotetext{
* Participants were drawn from clinical settings across the country including addiction treatment centers, general psychiatric hospitals, dual-diagnosis hospitals, vocational rehabilitation programs, sexoffender treatment programs and correctional centers; responses to the instrument were compared to DSMbased clinical criteria.

† The 10 domains relate to substance use, mental health status, physical health status, aggressive behavior/delinquency, social skills, family relations, educational status, vocational status, peer relations and leisure/recreation.
}

questionnaire used most often is made up of 139 yes/no items and takes 20 to 30 minutes to administer. $^{82}$ There are two versions of POSIT's substance use screening domain: a 17-question version and an 11-question version. ${ }^{83}$

Practitioners can score the instrument in a matter of minutes. The POSIT tool is not copyrighted and is available free of charge through NIDA. It does not require training to administer. ${ }^{84}$

Populations and Settings Served. POSIT can be used in school, juvenile justice, family court, medical, psychiatric and addiction treatment settings. ${ }^{85}$

Clinical Utility. Examinations of the POSIT among 12- to 19 year-olds demonstrate the instrument's validity and reliability in screening for symptoms of addiction. ${ }^{86}$ Using the 17-item version of the substance use scale, a cut-off score of two accurately classified 84 percent of cases of addiction ${ }^{\ddagger}$ in adolescents (95 percent sensitivity, 79 percent specificity). For the 11item scale, a cut-off score of two was found to be 85 percent accurate at identifying addiction (91 percent sensitivity; 82 percent specificity). ${ }^{87}$

\section{Drug Use Screening Inventory (DUSI)}

The Drug Use Screening Inventory was developed in 1990 to screen and/or assess adolescents in need of help for risky use of alcohol and other drugs (excluding nicotine) or addiction involving these substances. ${ }^{88}$ It has been revised to identify the severity of substance involvement in adult and adolescent populations. The instrument includes 159 items and takes 20 to 40 minutes to complete. ${ }^{89}$

DUSI measures problems across 10 domains: alcohol and other drug use (excluding nicotine), behavior problems, health status, psychiatric disorders, school adjustment, work adjustment, ${ }^{\S}$ family adjustment, peer relationships, social competence and leisure and recreation. ${ }^{90}$ DUSI

\footnotetext{
${ }^{\ddagger}$ Based on DSM criteria.

$\S$ The adolescent version of the DUSI does not measure work adjustment.
} 
can be administered via an interview or selfadministered. ${ }^{91}$

The instrument is scored on a scale from zero to 100. Practitioners tally relative scores across each domain to identify which areas of life have been affected most severely by a patient's substance use. ${ }^{92}$ Scoring the instrument takes up to 10 minutes. ${ }^{93}$

The revised version of the DUSI also includes a "lie scale," allowing practitioners to verify the truthfulness of patients' responses and identify inconsistencies. The instrument does not require training to administer. ${ }^{94}$

The DUSI is copyrighted. Paper and online questionnaires are available at a low cost and software licenses for online scoring and monitoring of progress over time can be purchased. $^{95}$

Populations and Settings Served. The instrument is recommended for use among known or suspected alcohol and other drug users and for identifying adolescents at risk. ${ }^{96}$

Clinical Utility. The DUSI substance use scale was found to be valid in an adolescent sample ${ }^{97}$ and scores on the DUSI in adolescence significantly correlated with the presence of addiction in young adulthood. ${ }^{98}$ The DUSI also has demonstrated good internal reliability as well as good sensitivity and specificity rates in an adult population. ${ }^{99}$

\section{The Addiction Severity Index (ASI)}

Developed in 1982, the Addiction Severity Index is an assessment instrument that not only measures self-reported lifetime and current alcohol and other drug use (excluding nicotine), but also related domains including medical status, employment and financial status, legal status, family history, social relationships and psychiatric status. ${ }^{100}$ This information can help practitioners determine the best course of treatment for patients. ${ }^{101}$

The instrument is a 200-item semi-structured interview that takes a trained practitioner about one hour to conduct and five minutes to score. It also can be self-administered on paper or a computer. $^{102}$

Practitioners can be trained to administer and score the index using manuals provided by the developers. On-site trainings run by experienced trainers also are available. The index and basic manuals are available for a minimal fee. ${ }^{103}$

Populations and Settings Served. The ASI primarily is used in treatment planning. It has been used with psychiatric, homeless, pregnant and prisoner populations. ${ }^{104}$ The ASI also is used by researchers to assess patient outcomes in clinical studies or by clinicians to assess the progress of a patient's disease during and after treatment. ${ }^{105}$ Thus far, the validity of the ASI has been studied only in adult populations; ${ }^{106}$ however, a version for adolescents, the Teen Addiction Severity Index (T-ASI), has been developed. ${ }^{107}$ The T-ASI is helpful for identifying adolescents with addiction and cooccurring mental health conditions. ${ }^{108}$

Clinical Utility. The ASI has demonstrated adequate test-retest reliability, ${ }^{109}$ but the validity of the ASI at identifying addiction varies among studies. ${ }^{110}$ The ASI measures aimed at identifying addiction involving alcohol and other drugs are significantly correlated with DSM-IV diagnostic criteria. The alcohol and other drug composite scores accurately identify 85 percent of individuals with addiction and 80 percent of individuals without addiction. ${ }^{111}$

However, the specificity of the instrument has been questioned in certain studies-misidentifying some patients as having addiction when they do not--particularly among certain psychiatric and international populations. ${ }^{112}$

The index frequently is used by researchers to track the progress of treatment patients over time. However, its length and complexity in administering and scoring make it impractical for use in primary care and emergency department settings. ${ }^{113}$ 
In clinical samples of adolescents, the T-ASI has been shown to be valid and reliable. ${ }^{114}$

\section{Structured Clinical Interview for the DSM (SCID)}

The Structured Clinical Interview for the DSMIV Axis I Disorders (SCID-I) is a semi-structured assessment instrument for diagnosing addiction involving alcohol and other drugs (excluding nicotine) based on the DSM-IV Axis 1 categories. $^{*} 115$ The SCID can be used for current and lifetime diagnoses among clinical and community samples, college students and the homeless. ${ }^{116}$ An adolescent version of the SCID has been developed. ${ }^{117}$ Clinicians or trained mental health professionals may administer the SCID, and the developer recommends that clinicians should spend at least 20 hours learning how to administer the instrument. ${ }^{118}$ The SCID is available for a fee. ${ }^{119}$ Administering the interview takes an average of 90 minutes. ${ }^{120}$

Clinical Utility. Addiction researchers and community-based addiction treatment providers in NIDA's Clinical Trials Network (CTN) rated the SCID as the preferred instrument for diagnosing addiction. ${ }^{\dagger 121}$ The SCID has demonstrated good reliability and validity in diagnosing addiction. ${ }^{122}$ Compared to other diagnostic instruments, the SCID is less structured, allowing interviewers to ask followup questions based on their judgment. ${ }^{123}$ Reliability of assessments may vary depending on the clinical judgment of the interviewer in modifying and asking follow-up questions. ${ }^{124}$ Among adolescents, the SCID showed high inter-rater reliability for DSM-IV diagnoses of

\footnotetext{
* Axis I includes major mental disorders, addiction and learning disorders, whereas Axis II includes personality disorders and intellectual disabilities. The other three axes include related medical, psychosocial and environmental factors, as well as assessments of functioning for children.

${ }^{\dagger}$ Compared to other diagnostic instruments including the addiction-related sections of the Composite International Diagnostic Interview, the Diagnostic Interview Schedule for DSM-IV Diagnosis, the Diagnostic Statistical Manual-IV Checklist and the Substance Dependence Severity Scale.
}

alcohol and other drug addiction. ${ }^{125}$ The SCID has been less accurate in identifying cooccurring disorders than in identifying addiction. ${ }^{126}$

\section{Composite International Diagnostic Interview (CIDI)}

The Composite International Diagnostic Interview was developed by the WHO in 1988 for assessing lifetime and past-year addiction in accordance with DSM-IV and ICD-10 classification systems. ${ }^{127}$ The CIDI is composed of 20 questions and 59 sub-questions. Another version, CIDI-SAM, focuses specifically on the substance use section of the CIDI in a more detailed and expansive manner than the original instrument. ${ }^{128}$ The substance-specific sections of the CIDI generally take 20 to 30 minutes to administer, ${ }^{129}$ and can be self-administered via paper or computer or by a trained interviewer. ${ }^{130}$ The information collected is useful for treatment planning. ${ }^{131}$ NIDA has adopted a version of the CIDI for use among clinicians and investigators in the CTN. ${ }^{132}$

Populations and Settings Served. The CIDI has been used with adults and adolescents and with individuals with co-occurring disorders. ${ }^{133}$

Clinical Utility. Reviews have found that the CIDI and CIDI-SAM are reliable and valid instruments for assessing mental health disorders and addiction. ${ }^{134}$ However, potential barriers to using the CIDI are the length of time required for administration and training costs: the instrument takes 75 minutes to administer and the scoring program is available only to those who have attended a WHO-authorized CIDI training session (a four-day training session that costs approximately \$1,100). ${ }^{135}$ 


\section{Tobacco-Specific Screening and Assessment Tools}

\section{Fagerstrom Test for Nicotine Dependence (FTND)}

The Fagerstrom Test for Nicotine Dependence, originally developed in 1991, is a six-item instrument that assesses addiction involving nicotine (dependence) based on quantity of cigarette consumption and compulsion to smoke. ${ }^{136}$ It is one of the most commonly used measures of addiction involving nicotine. ${ }^{137}$ Scored from one to $10,{ }^{138}$ the instrument requires no training to use and can be administered and scored in less than five minutes. ${ }^{139}$

Although the FTND is copyrighted it can be used and reproduced without the author's permission and free of charge. ${ }^{140}$

Populations and Settings Served. In addition to screening for addiction involving nicotine in cigarette smokers, the FTND has been adapted for screening for smokeless tobacco use. The FTND screening tool can be used alone or incorporated into comprehensive clinical and non-clinical health screenings. ${ }^{141}$ The instrument also is commonly used in research settings. ${ }^{142}$

Clinical Utility. The FTND is a valid instrument, ${ }^{143}$ with scores reflecting biological and psychological indicators of addiction involving nicotine. ${ }^{144}$ Among a group of inpatients with addiction, FTND scores were significantly correlated with patients' smokingrelated physical symptoms, frequency and quantity of cigarette use and with biological markers such as carbon monoxide and cotinine levels. FTND scores also are correlated with the number of puffs per cigarette patients took and the average amount of time they spent puffing on cigarettes. ${ }^{145}$ The instrument has marginal internal reliability ${ }^{146}$ but high test-retest reliability. ${ }^{147}$ At a score of four or more, the FTND showed a sensitivity of 94 percent and specificity of 88 percent among samples from the U.S. and Spain. ${ }^{148}$

\section{Nicotine Dependence Syndrome Scale (NDSS)}

The Nicotine Dependence Syndrome Scale is an assessment tool that measures five dimensions of nicotine dependence based on symptoms and characteristics outlined in the DSM, ${ }^{149}$ including craving and withdrawal (drive), preference for smoking over other activities (priority), decreased response to the effects of smoking (tolerance), patterns of tobacco use (stereotypy) and smoking at a regular rate (continuity). ${ }^{150}$ The NDSS was designed to assess addiction involving nicotine in a more comprehensive way than other scales, such as the FTND. ${ }^{151}$

Population and Settings Served. The NDSS was designed for adult smokers, but an adolescent version has been developed. ${ }^{152}$

Clinical Utility. The NDSS has been found to be a valid and reliable instrument. ${ }^{153}$ Several studies using samples of smokers of various ages provide support for the multidimensional nature of the scale and show that the scale identifies features of risky tobacco use that are below clinical thresholds of dependence. ${ }^{154}$

\section{Alcohol-Specific Screening and Assessment Tools}

\section{The Alcohol Use Disorders Identification Test (AUDIT)}

The Alcohol Use Disorders Identification Test is a screening tool that was created in 1992 to identify risky drinking ${ }^{*}$ in adult primary care settings. ${ }^{155}$ The 10-question interview takes only a few minutes to administer ${ }^{156}$ and score ${ }^{157}$ and covers consumption levels, drinking

\footnotetext{
* Defined by the developers as "hazardous" and "harmful” drinking involving an average daily alcohol intake exceeding $60 \mathrm{~g}$ per day for men and $40 \mathrm{~g}$ per day for women; recurrent intoxication; fulfilling at least one criterion of alcohol dependence on the drinking behavior scale on a monthly basis; experiencing at least one alcohol-related problem in the past year or having an alcohol-related disease or a perceived drinking problem.
} 
behavior, adverse reactions and alcohol-related problems. ${ }^{158}$

AUDIT was developed through a six-country ${ }^{*}$ WHO project to find a screening instrument suited for primary care settings. ${ }^{159}$

The questionnaire can be self-administered or administered through an interview with a trained practitioner. ${ }^{160}$ Respondents' answers to each question are scored from zero to four, with a maximum score of $40 .{ }^{161}$ Researchers have suggested the use of a cut-off score of eight as an indicator of risk. ${ }^{162}$

The AUDIT is copyrighted but available through the WHO. ${ }^{\dagger} 163$ It can be self-administered via computer. $^{164}$

Populations and Settings Served. The AUDIT was developed for use in primary care settings, but also has been deemed appropriate by the National Institute on Alcohol Abuse and Alcoholism (NIAAA) for use with hospital, emergency department and psychiatric patients as well as in criminal justice, armed forces, workplace and college settings. ${ }^{165}$

Clinical Utility. The AUDIT has demonstrated good validity and reliability across multiple populations with vastly differing cultural norms and behaviors. ${ }^{166}$ Using a cut-off score of eight results in high sensitivity and specificity: according to WHO data evaluating the AUDIT, 92 percent of risky drinkers had AUDIT scores of eight or higher and 94 percent of non-risky drinkers had scores lower than eight. ${ }^{\ddagger 167}$ A cutoff score of eight identified accurately 95 percent of drinkers who had experienced medical, trauma, domestic or social problems associated with their alcohol use and 97 percent of male drinkers who consumed five or more drinks per day or female drinkers who drank three or more drinks per day. ${ }^{168}$

\footnotetext{
* Australia, Bulgaria, Kenya, Mexico, Norway and the United States.

† The training module can be purchased through the Division of Mental Health and Prevention of Substance Abuse at the WHO.

${ }^{\ddagger}$ In the American study group, these figures were 90 percent and 92 percent, respectively.
}

The AUDIT's greatest strengths include its brevity, focus on current practices, ease of administration and cultural neutrality. ${ }^{169} \mathrm{~A}$ limitation of the AUDIT is that its threshold for identifying risky alcohol use is higher than that recommended by the U.S. Department of Agriculture's (USDA) Dietary Guidelines for safe alcohol use. ${ }^{\S 170}$ Some research suggests that a lower AUDIT cut-off score would be more effective in identifying risky alcohol use, particularly in female populations. ${ }^{171}$

\section{The T-ACE Questionnaire}

$T$-ACE is a four-question screening instrument for identifying risky alcohol use, including lifetime use and prenatal use in pregnant women. ${ }^{172}$ It was created in 1989 and is based on the CAGE:

1. How many drinks does it take to make you feel high (Tolerance)?

2. Have people Annoyed you by criticizing your drinking?

3. Have you ever felt you ought to Cut down on your drinking?

4. Have you ever had a drink first thing in the morning to steady your nerves or get rid of a hangover (Eye-opener)? ${ }^{173}$

Affirmative answers to questions two through four are scored as one point each, and a tolerance greater than two drinks is worth two points. Patients whose total score is at least two screen positive for risky alcohol use. ${ }^{174}$ The screening and scoring process takes only a few minutes. ${ }^{175}$

Although the T-ACE is copyrighted, it is widely available for use. It does not require training to administer. ${ }^{176}$

\footnotetext{
$\S$ Among the USDA Dietary Guidelines for safe alcohol use are the guidelines of no more than one drink a day for women and no more than two drinks a day for men--which are lower thresholds of risk than those included in the AUDIT.
} 
Populations and Settings Served. The T-ACE was created by obstetricians and gynecologists as a screening device for risky alcohol use among pregnant women. ${ }^{177}$

Clinical Utility. Research on the psychometric properties of the T-ACE is limited. The T-ACE can identify 88 percent of risky and non-risky drinkers. ${ }^{178}$ It has been found to be better at detecting risky alcohol use relative to some other alcohol screening instruments. ${ }^{* 179}$ In obstetric settings, the instrument accurately measures current prenatal consumption, pre-pregnancy risky drinking ${ }^{\dagger}$ and lifetime DSM-III diagnosis for addiction involving alcohol. ${ }^{180}$

\section{TWEAK}

Developed in 1994, the five-item screening instrument, TWEAK is an acronym for Tolerance, Worried, Eye-openers, Amnesia and Cut-down. ${ }^{181}$ These words describe the questions that measure respondents' tolerance level, drinking behavior and personal as well as family attitudes towards their drinking behavior. The "tolerance" and "worried" questions are worth up to two points and affirmative answers on the other three questions are one point each. A score of three or more indicates that a respondent may be a risky drinker. ${ }^{182}$

TWEAK is brief and easy to score. It is available at no cost and can be administered through interviews, via a computer or paper and pencil questionnaire. Training is not required or necessary. ${ }^{183}$

Populations and Settings Served. The instrument originally was intended for use in screening for risky drinking among pregnant women. ${ }^{\neq 184}$ However, it has been used in the general population and in outpatient, hospital and emergency room settings. ${ }^{185}$

\footnotetext{
* Such as the CAGE and MAST.

${ }^{\dagger}$ Defined as more than two drinks per drinking day.

₹ It should be noted that any level of drinking by pregnant women should be considered risky alcohol use.
}

Clinical Utility. Although research on the psychometric properties of the TWEAK is limited, ${ }^{186}$ the TWEAK appears to be better at identifying risky drinking in pregnant women than some other alcohol use screening instruments. ${ }^{\S 187}$ In one population of women in a prenatal setting, TWEAK identified accurately 71 percent of risky drinkers and 79 percent of non-risky drinkers. ${ }^{188}$

In a sample of adults from the general population, TWEAK identified accurately 79 percent of individuals with addiction involving alcohol and 89 percent of individuals without addiction. Respondents with a score of three or higher were 30 times likelier to have addiction involving alcohol than those with lower scores. In a general population sample, TWEAK was more accurate at identifying addiction involving alcohol among men (83 percent) than women (66 percent), ${ }^{189}$ a surprising finding given that women were the intended target population.

\section{Alcohol Dependence Scale (ADS)}

The Alcohol Dependence Scale is a 25-item assessment instrument developed in 1984 to identify addiction involving alcohol in adult populations. ${ }^{190}$ It also is useful for measuring disease severity. The instrument covers withdrawal and tolerance, compulsion to drink and salience of alcohol-seeking behavior. The ADS takes about 10 minutes to complete and score. It can be administered through an interview or self-administered using a computer or paper questionnaire. Generally, a cut-off score of nine is recommended to identify addiction. ${ }^{191}$

Some basic training is required to administer and score the instrument. The ADS is copyrighted and questionnaires and user guides are available for a fee. ${ }^{192}$

Populations and Settings Served. Although it was developed for adults, the ADS has been used in adolescent populations. It has been used in both research and clinical settings, including

\footnotetext{
$\S$ Such as the CAGE and MAST.
} 
in health care, education, workplace and criminal justice settings. ${ }^{193}$

Clinical Utility. The ADS has been found to be a reliable and valid instrument. ${ }^{194}$ In one study, the ADS identified accurately 88 percent of individuals based on DSM criteria. ${ }^{195}$ Using a cut-off score of eight, the ADS had a sensitivity rate of 78 percent and a specificity rate of 88 percent among a sample of homeless women in treatment. ${ }^{196}$ When examined in an inmate population, the ADS was found to have good test-retest reliability and identified accurately 79.6 percent of inmates with regard to their addiction status; however, the instrument's sensitivity in this population was found to be low (51.0 percent) relative to its specificity (95.7 percent). ${ }^{197}$

\section{The Michigan Alcoholism Screening Test (MAST)}

The Michigan Alcoholism Screening Test, originally developed in 1971, is one of the most well-known assessment tools for identifying lifetime symptoms of addiction involving alcohol. ${ }^{198}$ It consists of 25 yes/no questions concerning drinking behavior and alcoholrelated problems. ${ }^{199}$ The instrument can be selfadministered or administered in an interview format. The entire process, including scoring, takes less than 15 minutes and training is not required. ${ }^{200}$

There also is a 13-item version, referred to as SMAST, for Short Michigan Alcoholism Screening Test. ${ }^{201}$ The SMAST consists of yes/no items and affirmative answers are worth one point each. Generally a score of three indicates respondents are risky alcohol users and a score of four or higher indicates potential addiction involving alcohol. ${ }^{202}$

\footnotetext{
* Other variations of the instrument exist, including a 10-item version called the Brief MAST, a version that incorporates other drug diagnoses called MAST for Alcohol and Drugs (AD) and a version for geriatric patients (MAST-G). Since these variations are not used as commonly as MAST and SMAST, they are not elaborated upon here.
}

The MAST is not copyrighted and there is no fee for its use, however there is a small fee to obtain a copy of the instrument from the developers. ${ }^{203}$ There is no cost for using the SMAST. ${ }^{204}$

Populations and Settings Served. The MAST and SMAST can be used in a wide variety of adolescent and adult populations and in both clinical and research settings. ${ }^{205}$ SMAST has demonstrated strong reliability among Hispanic individuals ${ }^{206}$ and has been well validated in primary care settings. ${ }^{207}$ Since the MAST is better at detecting more severe rather than less advanced risky alcohol use or addiction, it may not be as useful among college students. ${ }^{208}$

Clinical Utility. Studies have found the MAST to be valid ${ }^{209}$ and reliable. ${ }^{210}$ Among adult outpatients, the SMAST identified 82 percent of individuals with lifetime addiction involving alcohol and 100 percent of those with current addiction. The corresponding specificity rates were 96 and 85 percent, respectively. ${ }^{211}$ However, another study using the same cut-off score found that the SMAST identified accurately only 48 percent of individuals with addiction involving alcohol and 95 percent of individuals without addiction. ${ }^{212}$ While the SMAST has demonstrated good test-retest reliability in inmate populations, its validity in this group is less strong; in one study, the SMAST identified accurately 100 percent of inmates with addiction involving alcohol, but 66.3 percent of inmates without addiction were misidentified as having addiction. ${ }^{213}$

\section{Drug-Specific Screening and Assessment Tools}

\section{The Drug Abuse Screening Test (DAST)}

The Drug Abuse Screening Test was developed in 1982 to screen for lifetime risky drug use (excluding nicotine and alcohol) and assess addiction in adult populations. ${ }^{214}$ There are four variations of the DAST: a 28-item, a 20-item, a 10 -item and a 27-item questionnaire--the last is intended for adolescents. ${ }^{215}$ 
When used to screen for risky drug use in adults, the measures take approximately 10 minutes to administer and score. ${ }^{216}$ Although it is copyrighted, the instrument is available for use by clinicians, educators and researchers. ${ }^{217}$ The DAST does not require training to administer. ${ }^{218}$

Populations and Settings Served. The DAST instruments have been used successfully among adolescents and adult treatment seekers, psychiatric patients, females involved in the justice system as well as in the workplace. ${ }^{219}$

Clinical Utility. The DAST has been found to be both valid and reliable. ${ }^{220}$ The DAST-28 can identify up to 96 percent of individuals with addiction involving drugs and has been found to misidentify as few as nine percent of those without addiction. ${ }^{221}$ Its overall accuracy in classifying patients according to DSM criteria for substance abuse is 89 percent. ${ }^{222}$ The 28item version was found to be better at assessing lifetime addiction involving alcohol and other drugs than current addiction among recently admitted psychiatric public hospital patients. ${ }^{223}$

The DAST-20 has been found to be a valid instrument in psychiatric outpatient ${ }^{224}$ and inmate $^{225}$ populations.

DAST-10 has demonstrated sensitivity rates ranging from 41 to 95 percent and specificity rates ranging from 68 to 99 percent, depending on the cut-off score used and the population group studied. $^{226}$ This shorter version is more accurate at identifying risky users than individuals with addiction. ${ }^{227}$

Among adolescents at inpatient evaluation and crisis intervention units, the adolescent version of DAST identified accurately 78.6 percent of those with addiction and 84.5 percent of those without addiction. ${ }^{228}$

\section{Additional Screening and Assessment Instruments}

Many instruments have been developed to screen for or assess risky tobacco, alcohol or other drug use and addiction. Researchers continue to combine, restructure and create questionnaires to better identify individuals at risk for addiction or those in need of treatment. The tools described above are some of the most commonly used, well-studied and recommended.

Other validated instruments exist, however, including: the Adolescent Diagnostic Interview; the Adolescent Drug Abuse Diagnosis questionnaire; the Alcohol Expectancy Questionnaire; the Alcohol Use Inventory; the Chemical Use, Abuse, and Dependence Scale; the Drinker Inventory of Consequences instrument; the Global Appraisal of Individual Needs (GAIN) assessment instrument; the MacAndrew Alcoholism Scale; the ObsessiveCompulsive Drinking Scale; the Personal Experience Inventory; the Rutgers Alcohol Problem Index; the Texas Christian University Drug Screen; and the Young Adult Alcohol Problems Screening. ${ }^{229}$ 
$-324-$ 


\section{Chapter I \\ Notes}

${ }^{1}$ American Society of Addiction Medicine. (2011b).

Hajela, R. \& Miller, M. M. (2012).

Leshner, A. I. (1997).

Leshner, A. I. (2001a).

Volkow, N. D., \& Li, T.-K. (2005).

${ }^{2}$ Brown, R. L., Leonard, T., Saunders, L. A., \& Papasouliotis, O. (1998).

Greene, J. M., Ennett, S. T., \& Ringwalt, C. L. (1997).

Substance Abuse and Mental Health Services Administration. (2006).

The National Center on Addiction and Substance Abuse (CASA) at Columbia University. (2010).

The National Center on Addiction and Substance Abuse at Columbia University (CASA Columbia). (2012n).

${ }^{3}$ Centers for Disease Control and Prevention. (2011h).

Centers for Disease Control and Prevention. (2012b).

${ }^{4}$ The National Center on Addiction and Substance Abuse at Columbia University (CASA Columbia). (2012n).

${ }^{5}$ American Cancer Society. (2009).

American Diabetes Association. (2009).

Centers for Disease Control and Prevention. (2008a).

Centers for Disease Control and Prevention. (2008b).

Centers for Disease Control and Prevention, Coordinating Center for Health Promotion. (2008).

Corso, P., Finkelstein, E., Miller, T., Fiebelkorn, I., \& Zaloshnja, E. (2006).

Insel, T. R. (2008).

Lloyd-Jones, D., Adams, R., Carnethon, M., De, S. G., Ferguson, T. B., Flegal, K., et al. (2009).

Mokdad, A. H., Marks, J. S., Stroup, D. F., \& Gerberding, J. L. (2004).

Robert Wood Johnson Foundation. (2001).

${ }^{6}$ Centers for Disease Control and Prevention. (2008b).

Kochanek, K., Xu, J. Q., Murphy, S. L., Minino, A. M., \& Kung, H.-C. (2011).

The National Center on Addiction and Substance Abuse at Columbia University (CASA Columbia). (2012a).

${ }^{7}$ Centers for Disease Control and Prevention. (2004b).

Centers for Disease Control and Prevention. (2008a).

Centers for Disease Control and Prevention. (2008b).

Merrill, J. \& Fox, K. (1998).

The National Center on Addiction and Substance Abuse (CASA) at Columbia University. (1993).

The National Center on Addiction and Substance Abuse (CASA) at Columbia University. (1994).

${ }^{8}$ American Academy of Pediatrics, Committee on Substance Abuse. (2001).

Centers for Disease Control and Prevention. (2011d).

Asbridge, M., Hayden, J. A., \& Cartwright, J. L. (2012).

Center for Substance Abuse Treatment. (2003).

French, M. T., Rachal, V., Harwood, H. J., \& Hubbard, R. (1990).

Naimi, T. S., Lipscomb, L. E., Brewer, R. D., \& Gilbert, B. C. (2003).

National Institute on Drug Abuse. (2005a).

Office of Juvenile Justice and Delinquency Prevention. (1998).

Office of National Drug Control Policy. (2004).

The National Center on Addiction and Substance Abuse (CASA) at Columbia University. (1999).

The National Center on Addiction and Substance Abuse (CASA) at Columbia University. (2010).

The National Center on Addiction and Substance Abuse (CASA) at Columbia University . (2011a).

U.S. Department of Health and Human Services. (2000).

Wechsler, H., Davenport, A., Dowdall, G., Moeykens, B., \& Castillo, S. (1994).

${ }^{9}$ The National Center on Addiction and Substance Abuse (CASA) at Columbia University. (2009d).

${ }^{10}$ The National Center on Addiction and Substance Abuse at Columbia University (CASA Columbia). (2012n).

${ }^{11}$ Roy, K., \& Miller, M. (2010).

Pating, D. R., Miller, M. M., Goplerud, E., Martin, J., \& Ziedonis, D. M. (2012).

Roy, A. K., \& Miller, M. M. (2012).

${ }^{12}$ Centers for Disease Control and Prevention. (2012b). 
${ }^{13}$ American Society of Addiction Medicine. (1997).

${ }^{14}$ Conn. Agencies Regs. § 17-134d-63 (2012).

Mass. Regs. Code 130, § 450.204 (2011).

N.Y. Soc Serv § 365-a (Consol. 2012).

Social Security Online. (2011).

${ }^{15}$ Appel, P. W., Ellison, A. A., Jansky, H. K., \& Oldak, R. (2004).

Appel, P. W., \& Oldak, R. (2007).

Hser, Y.-I., Maglione, M., Polinsky, M. L., \& Anglin, M. D. (1998).

Redko, C., Rapp, R. C., \& Carlson, R. G. (2006).

${ }^{16}$ Bishop, E. S. (1919).

${ }^{17}$ The National Center on Addiction and Substance Abuse at Columbia University (CASA Columbia). (2012i).

${ }^{18}$ The National Center on Addiction and Substance Abuse at Columbia University (CASA Columbia). (2012i).

${ }^{19}$ The National Center on Addiction and Substance Abuse at Columbia University (CASA Columbia). (2012n).

${ }^{20}$ U.S. Department of Agriculture and U.S. Department of Health and Human Services. (2010).

${ }^{21}$ U.S. Drug Enforcement Administration. (2010).

${ }^{22}$ The National Center on Addiction and Substance Abuse at Columbia University (CASA Columbia). (2012n).

${ }^{23}$ American Society of Addiction Medicine. (2011b).

Hajela, R. \& Miller, M. M. (2012).

Leshner, A. I. (1997).

Leshner, A. I. (2001a).

Volkow, N. D., \& Li, T.-K. (2005).

${ }^{24}$ Erickson, C. K. (2007).

Filbey, F. M., Schacht, J. P., Myers, U. S., Chavez, R. S., \& Hutchison, K. E. (2009).

Hyman, S. E. (2007).

Spanagel, R., \& Heilig, M. (2005).

Thombs, D. L. (2006).

Volkow, N. (2007).

${ }^{25}$ American Society of Addiction Medicine. (2011b).

Dackis, C., \& O'Brien, C. P. (2005).

Kalivas, P. W., \& Volkow, N. D. (2005).

Leshner, A. I. (1997).

Leshner, A. I. (2001a).

Robison, A. J., \& Nestler, E. J. (2011).

Volkow, N. D., \& Li, T.-K. (2005).

World Health Organization. (2004).

${ }^{26}$ American Society of Addiction Medicine. (2011b).

Hajela, R. \& Miller, M. M. (2012).

Kalivas, P. W., \& Volkow, N. D. (2005).

Leshner, A. I. (2001a).

${ }^{27}$ American Society of Addiction Medicine. (2011b).

Ersche, K. D., Jones, P. S., Williams, G. B., Turton, A. J., Robbins, T. W., \& Bullmore, E. T. (2012).

Gitlow, S. (2011).

Whelan, R., Conrod, P. J., Poline, J. B., Lourdusamy, A., Banaschewski, T., Barker, G. J., et al. (2012).

${ }^{28}$ Han, C., McGue, M. K., \& Iacono, W. G. (1999).

Lynskey, M. T., Agrawal, A., \& Heath, A. C. (2010).

Merikangas, K. R., \& McClair, V. L. (2012).

National Institute on Drug Abuse. (2003b).

Vink, J. M., Willemsen, G., \& Boomsma, D. I. (2005).

${ }^{29}$ Austin, G. A., \& Prendergast, M. (1991).

Lynskey, M. T., Agrawal, A., \& Heath, A. C. (2010).

Merikangas, K. R., \& McClair, V. L. (2012).

Merikangas, K. R., Stolar, M., Stevens, D. E., Goulet, J., Preisig, M. A., Fenton, B., et al. (1998).

Vink, J. M., Willemsen, G., \& Boomsma, D. I. (2005).

${ }^{30}$ The National Center on Addiction and Substance Abuse at Columbia University (CASA Columbia). (2012n).

${ }^{31}$ American Society of Addiction Medicine. (2011b). 
Andersen, S. L., \& Teicher, M. H. (2009).

Bava, S., Frank, L. R., McQueeny, T., Schweinsburg, B. C., Schweinsburg, A. D., \& Tapert, S. F. (2009).

Benowitz, N. L. (2010).

Chambers, R. A., Taylor, J. R., \& Potenza, M. N. (2003).

Crews, F. T., \& Boettiger, C. A. (2009).

Crews, F., He, J., \& Hodge, C. (2007).

Doremus-Fitzwater, T. L., Varlinskaya, E. I., \& Spear, L. P. (2010).

Ersche, K. D., Jones, P. S., Williams, G. B., Turton, A. J., Robbins, T. W., \& Bullmore, E. T. (2012).

Gitlow, S. (2011).

Guerri, C., \& Pascual, M. (2010).

Placzek, A. N., Zhang, T. A., \& Dani, J. A. (2009).

Riggs, N. R., \& Greenberg, M. T. (2009).

Schramm-Sapyta, N. L., Walker, Q. D., Caster, J. M., Levin, E. D., \& Kuhn, C. M. (2009).

The National Center on Addiction and Substance Abuse (CASA) at Columbia University. (2011a).

Whelan, R., Conrod, P. J., Poline, J. B., Lourdusamy, A., Banaschewski, T., Barker, G. J., et al. (2012).

${ }^{32}$ Centers for Disease Control and Prevention. (2004b).

Centers for Disease Control and Prevention. (2008a).

Centers for Disease Control and Prevention. (2008b).

Merrill, J. \& Fox, K. (1998).

The National Center on Addiction and Substance Abuse (CASA) at Columbia University. (1993).

The National Center on Addiction and Substance Abuse (CASA) at Columbia University. (1994).

${ }^{33}$ Armstrong, T. D., \& Costello, E. J. (2002).

Chan, Y. F., Dennis, M. L., \& Funk, R. R. (2008).

Drake, R. E., \& Mueser, K. T. (2002).

Epstein, J., Barker, P., Vorburger, M., \& Murtha, C. (2004).

Grella, C. E., Hser, Y.-I., Vandana, J., \& Rounds-Bryant, J. (2001).

National Institute of Mental Health. (2008).

Upadhyaya, H. P., Deas, D., Brady, K. T., \& Kruesi, M. (2002).

${ }^{34}$ Hasin, D. S., Stinson, F. S., Ogburn, E., \& Grant, B. F. (2007).

Willenbring, M. L. (2008a).

${ }^{35}$ Dawson, D. A., Grant, B. F., Stinson, F. S., Chou, P. S., Huang, B., \& Ruan, W. J. (2005).

Erickson, C. K. (2007).

Hudson, N. L., \& Mannino, D. M. (2010).

Lewis, D. C. (1991).

McLellan, A. T., Lewis, D. C., O'Brien, C. P., \& Kleber, H. D. (2000).

Roan, S. (2009).

The Partnership at DrugFree.org. (2012).

White, W. L. (2008).

${ }^{36}$ Chandler, R. K. (2012).

Miller, W. R., Sorensen, J. L., Selzer, J. A., \& Brigham, G. S. (2006).

National Quality Forum. (2005).

Pollack, H. A., \& D'Aunno, T. (2008).

Willenbring, M. L. (2008a).

${ }^{37}$ McLellan, A. T., Lewis, D. C., O'Brien, C. P., \& Kleber, H. D. (2000).

National Institute on Drug Abuse. (2009e).

Willenbring, M. L. (2008a).

${ }^{38}$ The National Center on Addiction and Substance Abuse at Columbia University (CASA Columbia). (2012n).

${ }^{39}$ Blum, K., Werner, T., Carnes, S., Carnes, P., Bowirrat, A., Giordano, J., et al. (2012).

Blume, S. B. (1994).

Çepik, A., Arikan, Z., Boratav, C., \& Isik, E. (1995).

Fortuna, J. L. (2012).

Grant, J. E., Brewer, J. A., \& Potenza, M. N. (2006).

Grant, J. E., Potenza, M. N., Weinstein, A., \& Gorelick, D. A. (2010).

Karim, R., \& Chaudhri, P. (2012).

Kenny, P. J. (2011). 
Shaffer, H. J., LaPlante, D. A., LaBrie, R. A., Kidman, R. C., Donato, A. N., \& Stanton, M. V. (2004).

Volkow, N. D., Wang, G. J., Fowler, J. S., \& Telang, F. (2008).

${ }^{40}$ Shaffer, H. J., LaPlante, D. A., LaBrie, R. A., Kidman, R. C., Donato, A. N., \& Stanton, M. V. (2004).

${ }^{41}$ The National Center on Addiction and Substance Abuse (CASA) at Columbia University. (2008a).

${ }^{42}$ American Society of Addiction Medicine. (2010a).

${ }^{43}$ Zanis, D. A., Melochick, J., \& Wagner, J. (2011).

${ }^{44}$ Aseltine, R. H. (2010).

Bertholet, N., Daeppen, J., Wietlisbach, V., Fleming, M., \& Burnand, B. (2005).

Gryczynski, J., Mitchell, S. G., Peterson, T. R., \& Gonzales, A. (2011).

National Association of State Alcohol and Drug Abuse Directors. (2006).

Schaus, J. F., Sole, M. L., McCoy, T. P., Mullett, N., \& O'Brien, M. C. (2009).

Spirito, A., Sindelar-Manning, H., Colby, S. M., Barnett, N. P., Lewander, W., Rohsenow, D. J., et al. (2011).

Sullivan, L. E., Tetrault, J. M., Braithwaite, R. S., Turner, B. J., \& Fiellin, D. A. (2011).

${ }^{45}$ Baker, A., Lee, N. K., Claire, M., Lewin, T. J., Grant, T., Pohlman, S., et al. (2005).

Bernstein, J., Bernstein, E., Tassiopoulos, K., Heeren, T., Levenson, S., \& Hingson, R. (2005).

Bernstein, E., Edwards, E., Dorfman, D., Heeren, T., Bliss, C., \& Bernstein, J. (2009).

D'Amico, E. J., Miles, J. N. V., Stern, S. A., \& Meredith, L. S. (2008).

Gryczynski, J., Mitchell, S. G., Peterson, T. R., \& Gonzales, A. (2011).

Madras, B. K., Compton, W. M., Avula, D., Stegbauer, T., Stein, J. B., \& Clark, H. W. (2009).

Magill, M., Barnett, N. P., Apodaca, T. R., Rohsenow, D. J., \& Monti, P. M. (2009).

Schonfeld, L., King-Kallimanis, B. L., Duchene, D. M., Etheridge, R. L., Herrera, J. R., Barry, K. L., et al. (2010).

Stein, M. D., Hagerty, C. E., Herman, D. S., Phipps, M. G., \& Anderson, B. J. (2011).

${ }^{46}$ Aseltine, R. H. (2010).

Bernstein, E., Edwards, E., Dorfman, D., Heeren, T., Bliss, C., \& Bernstein, J. (2009).

Bertholet, N., Daeppen, J., Wietlisbach, V., Fleming, M., \& Burnand, B. (2005).

D'Amico, E. J., Miles, J. N. V., Stern, S. A., \& Meredith, L. S. (2008).

Gryczynski, J., Mitchell, S. G., Peterson, T. R., \& Gonzales, A. (2011).

Madras, B. K., Compton, W. M., Avula, D., Stegbauer, T., Stein, J. B., \& Clark, H. W. (2009).

Magill, M., Barnett, N. P., Apodaca, T. R., Rohsenow, D. J., \& Monti, P. M. (2009).

Moore, A. A., Blow, F. C., Hoffing, M., Welgreen, S., Davis, J. W., Ramirez, K. D., et al. (2010).

Schaus, J. F., Sole, M. L., McCoy, T. P., Mullett, N., \& O'Brien, M. C. (2009).

Spirito, A., Sindelar-Manning, H., Colby, S. M., Barnett, N. P., Lewander, W., Rohsenow, D. J., et al. (2011).

Stein, M. D., Hagerty, C. E., Herman, D. S., Phipps, M. G., \& Anderson, B. J. (2011).

Sullivan, L. E., Tetrault, J. M., Braithwaite, R. S., Turner, B. J., \& Fiellin, D. A. (2011).

${ }^{47}$ National Institute on Drug Abuse. (2009e).

${ }^{48}$ American Society of Addiction Medicine. (2012).

National Institute on Drug Abuse. (2009e).

National Quality Forum. (2005).

${ }^{49}$ American Society of Addiction Medicine. (2010b).

National Quality Forum. (2005).

${ }^{50}$ National Institute on Drug Abuse. (2011h).

Richter, L., \& Richter, D. M. (2001).

U.S. Department of Health and Human Services, Substance Abuse and Mental Health Services Administration. (2009).

51 The National Center on Addiction and Substance Abuse (CASA) at Columbia University. (2011a).

${ }^{52}$ Center for Substance Abuse Treatment. (1998b).

Dowling, G. J., Weiss, S., \& Condon, T. P. (2008).

Institute of Alcohol Studies. (2011).

Rigler, S. K. (2000).

${ }^{53}$ Calhoun, P. S., Elter, J. R., Jones, E. R., Jr., Kudler, H., \& Straits-Troster, K. (2008).

Eisen, S., Schultz, M. R., Vogt, D., Glickman, M. E., Elwy, R., Drainoni, M.-L., et al. (2012).

Hassija, C. M., Jakupcak, M., Maguen, S., \& Shipherd, J. C. (2012).

Institute of Medicine. (2008).

Marshall, B. D. L., Prescotta, M. R., Liberzonb, I., Tamburrinod, M. B., Calabresee, J. R., \& Galeaa, S. (2012).

RTI International. (2009). 
Santiago, P. N., Wilk, J. E., Milliken, C. S., Castro, C. A., Engel, C. C., \& Hoge, C. W. (2010).

Shen, Y. C., Arkes, J., \& Williams, T. V. (2012).

Smith, B., Ryan, M. A., Wingard, D. L., Patterson, T. L., Slymen, D. J., \& Macera, C. A. (2008).

VHA Office of Public Health and Environmental Hazards. (2009).

Wilk, J. E., Bliese, P. D., Kim, P. Y., Thomas, J. L., McGurk, D., \& Hoge, C. W. (2010).

${ }^{54}$ Center for Substance Abuse Treatment. (2005b).

Chesher, N. J., Bousman, C. A., Gale, M., Norman, S. B., Twamley, E. W., Heaton, R. K., et al. (2011).

Epstein, J., Barker, P., Vorburger, M., \& Murtha, C. (2004).

Kessler, R. C., Anthony, J. C., Blazer, D. G., Bromet, E., Eaton, W. W., Kendler, K. S., et al. (1997).

McFall, M., \& Cook, J. (2006).

National Institute on Drug Abuse. (2010a).

The National Center on Addiction and Substance Abuse at Columbia University (CASA Columbia). (2012n).

Volkow, N. D. (2007a).

${ }^{55}$ The National Center on Addiction and Substance Abuse (CASA) at Columbia University . (2010).

The National Center on Addiction and Substance Abuse at Columbia University (CASA Columbia). (2012n).

${ }^{56}$ Liddle, H. A. (1999).

National Institute on Drug Abuse. (2009e).

Willenbring, M. L. (2008a).

Ziedonis, D., Kalman, D., Kolodziej, M., Johnson, C. W., \& Kim, S. (2011).

${ }^{57}$ National Institute on Drug Abuse. (2009e).

National Institute on Drug Abuse. (2011g).

${ }^{58}$ The National Center on Addiction and Substance Abuse (CASA) at Columbia University. (2008a).

${ }^{59}$ American Society of Addiction Medicine. (2006).

${ }^{60}$ Centers for Disease Control and Prevention. (2011h).

Ostchega, Y., Yoon, S. S., Huges, J., \& Louis, T. (2008).

The National Center on Addiction and Substance Abuse at Columbia University (CASA Columbia). (2012n).

${ }^{61}$ The National Center on Addiction and Substance Abuse at Columbia University (CASA Columbia). (2012n).

${ }^{62}$ The National Center on Addiction and Substance Abuse at Columbia University (CASA Columbia). (2012l).

The National Center on Addiction and Substance Abuse at Columbia University (CASA Columbia). (2012n).

${ }^{63}$ The National Center on Addiction and Substance Abuse (CASA) at Columbia University. (2008a).

${ }^{64}$ The National Center on Addiction and Substance Abuse at Columbia University (CASA Columbia). (2012r).

${ }^{65}$ The National Center on Addiction and Substance Abuse at Columbia University (CASA Columbia). (2012r).

${ }^{66}$ The National Center on Addiction and Substance Abuse at Columbia University (CASA Columbia). (2012p).

${ }^{67}$ The National Center on Addiction and Substance Abuse at Columbia University (CASA Columbia). (2012p).

${ }^{68}$ Substance Abuse and Mental Health Services Administration. (2010a).

The National Center on Addiction and Substance Abuse (CASA) at Columbia University. (2008a).

Xu, J., Wang, J., Rapp, R. C., \& Carlson, R. G. (2007).

${ }^{69}$ Center for Tobacco Research and Intervention, University of Wisconsin Medical School. (2005).

Gariti, P., Levin, S., Whittingham, T., Barou, D., Kampman, K. M., Lynch, K., et al. (2008).

Lazev, A. B., Vidrine, D. J., Arduino, R. C., \& Gritz, E. R. (2004).

${ }^{70}$ Agency for Healthcare Research and Quality. (2012d).

The National Center on Addiction and Substance Abuse at Columbia University (CASA Columbia). (2012h).

${ }^{71}$ Centers for Disease Control and Prevention. (2011h).

${ }^{72}$ Agency for Healthcare Research and Quality. (2012d).

The National Center on Addiction and Substance Abuse at Columbia University (CASA Columbia). (2012h).

${ }^{73}$ Centers for Disease Control and Prevention. (2012b).

${ }^{74}$ Agency for Healthcare Research and Quality. (2012d).

The Na (2010tional Center on Addiction and Substance Abuse at Columbia University (CASA Columbia). (2012h).

${ }^{75}$ Centers for Disease Control and Prevention. (2012b).

${ }^{76}$ The National Center on Addiction and Substance Abuse at Columbia University (CASA Columbia). (2012i).

${ }^{77}$ The National Center on Addiction and Substance Abuse (CASA) at Columbia University. (2009d).

${ }^{78}$ The National Center on Addiction and Substance Abuse at Columbia University (CASA Columbia). (2012t).

${ }^{79}$ The National Center on Addiction and Substance Abuse at Columbia University (CASA Columbia). (2012i).

${ }^{80}$ Bouchery, E. E., Harwood, H. J., Dilonardo, J., \& Vandivort-Warren, R. (2012).

${ }^{81}$ National Quality Forum. (2005). 
National Institute on Drug Abuse. (2009e).

Smith, M. J. W., Whitaker, T., \& Weismiller, T. (2006).

${ }^{82}$ Rieckmann, T. R., Kovas, A. E., Fussell, H. E., \& Stettler, N. M. (2009).

${ }^{83}$ Institute of Medicine, Committee on Crossing the Quality Chasm:Adaptation to Mental Health and Addictive Disorder. (2006).

McGlynn, E. A., Asch, S. M., Adams, J., Keesey, J., Hicks, J., DeCristofaro, A., et al. (2003). 


\section{Chapter II \\ Notes}

${ }^{1}$ American Society of Addiction Medicine. (2011b).

Hajela, R. \& Miller, M. M. (2012).

Leshner, A. I. (1997).

Leshner, A. I. (2001a).

Volkow, N., \& Li, T.-K. (2005).

${ }^{2}$ American Society of Addiction Medicine. (2011b).

Andersen, S. L., \& Teicher, M. H. (2009).

Bava, S., Frank, L. R., McQueeny, T., Schweinsburg, B. C., Schweinsburg, A. D., \& Tapert, S. F. (2009).

Benowitz, N. L. (2010).

Chambers, R. A., Taylor, J. R., \& Potenza, M. N. (2003).

Crews, F. T., \& Boettiger, C. A. (2009).

Crews, F., He, J., \& Hodge, C. (2007).

Doremus-Fitzwater, T. L., Varlinskaya, E. I., \& Spear, L. P. (2010).

Ersche, K. D., Jones, P. S., Williams, G. B., Turton, A. J., Robbins, T. W., \& Bullmore, E. T. (2012).

Gitlow, S. (2011).

Guerri, C., \& Pascual, M. (2010).

Merikangas, K. R., \& McClair, V. L. (2012).

Placzek, A. N., Zhang, T. A., \& Dani, J. A. (2009).

Riggs, N. R., \& Greenberg, M. T. (2009).

Schramm-Sapyta, N. L., Walker, Q. D., Caster, J. M., Levin, E. D., \& Kuhn, C. M. (2009).

The National Center on Addiction and Substance Abuse (CASA) at Columbia University. (2011a).

Whelan, R., Conrod, P. J., Poline, J. B., Lourdusamy, A., Banaschewski, T., Barker, G. J., et al. (2012).

${ }^{3}$ Erickson, C. K. (2007).

Hudson, N. L., \& Mannino, D. M. (2010).

Lewis, D. C. (1991).

McLellan, A. T., Lewis, D. C., O'Brien, C. P., \& Kleber, H. D. (2000).

White, W. L. (2008).

${ }^{4}$ American Society of Addiction Medicine. (2011b).

Leshner, A. I. (1997).

Leshner, A. I. (2001a).

Volkow, N., \& Li, T.-K. (2005).

${ }^{5}$ Heath, A. C., Bucholz, K. K., Madden, P. A., Dinwiddie, S. H., Slutske, W. S., Bierut, L. J., et al. (1997).

Kaufman, J., Yang, B.-Z., Douglas-Palumberi, H., Crouse-Artus, M., Lipschitz, D., Krystal, J. H., et al. (2007).

Kendler, K. S., Jacobson, K. C., Prescott, C. A., \& Neale, M. C. (2003).

Kreek, M. J., Nielsen, D. A., Butelman, E. R., \& LaForge, K. S. (2005).

Prescott, C. A., \& Kendler, K. S. (1999).

Tsuang, M. T., Lyons, M. J., Harley, R. M., Xian, H., Eisen, S., Goldberg, J., et al. (1999).

van den Bree, M. B., Johnson, E. O., Neale, M. C., \& Pickens, R. W. (1998).

Verweij, K. J. H., Zietsch, B. P., Lynskey, M. T., Medland, S. E., Neale, M. C., Martin, N. G., et al. (2010).

${ }^{6}$ Blum, K., Werner, T., Carnes, S., Carnes, P., Bowirrat, A., Giordano, J., et al. (2012).

Fortuna, J. L. (2012).

Karim, R., \& Chaudhri, P. (2012).

Grant, J. E., Brewer, J. A., \& Potenza, M. N. (2006).

Grant, J. E., Potenza, M. N., Weinstein, A., \& Gorelick, D. A. (2010).

Kenny, P. J. (2011).

Shaffer, H. J., LaPlante, D. A., LaBrie, R. A., Kidman, R. C., Donato, A. N., \& Stanton, M. V. (2004).

Volkow, N. D., Wang, G. J., Fowler, J. S., \& Telang, F. (2008).

${ }^{7}$ Hazelden Foundation. (1998).

Shaffer, H. J., LaPlante, D. A., LaBrie, R. A., Kidman, R. C., Donato, A. N., \& Stanton, M. V. (2004).

${ }^{8}$ American Society of Addiction Medicine. (2011b).

Dackis, C., \& O'Brien, C. P. (2005). 
Kalivas, P. W., \& Volkow, N. D. (2005).

Leshner, A. I. (1997).

Leshner, A. I. (2001a).

Robison, A. J., \& Nestler, E. J. (2011).

Volkow, N., \& Li, T.-K. (2005).

World Health Organization. (2004).

${ }^{9}$ Anthony, J. C. \& Helzer, J. E. (1991).

Anthony, J. C., Warner, L. A., \& Kessler, R. C. (1994).

Orson, F. M., Kinsey, B. M., Singh, R. A. K., Wu, Y., Gardner, T., \& Kosten, T. R. (2008).

Wagner, F. A., \& Anthony, J. C. (2002).

Whitten, L. (2010).

${ }^{10}$ American Psychiatric Association. (1994).

American Society of Addiction Medicine. (2011b).

Kalivas, P. W., \& Volkow, N. D. (2005).

Leshner, A. I. (2001a).

${ }^{11}$ American Society of Addiction Medicine. (2011b).

${ }^{12}$ American Society of Addiction Medicine. (2011b).

Ersche, K. D., Jones, P. S., Williams, G. B., Turton, A. J., Robbins, T. W., \& Bullmore, E. T. (2012).

Gitlow, S. (2011).

Whelan, R., Conrod, P. J., Poline, J. B., Lourdusamy, A., Banaschewski, T., Barker, G. J., et al. (2012).

${ }^{13}$ Hajela, R. \& Miller, M. M. (2012).

${ }^{14}$ American Society of Addiction Medicine. (2011a).

${ }^{15}$ Dackis, C., \& O'Brien, C. P. (2005).

Erickson, C. K. (2007).

Filbey, F. M., Schacht, J. P., Myers, U. S., Chavez, R. S., \& Hutchison, K. E. (2009).

Goldstein, R. Z., \& Volkow, N. D. (2011).

Gould, T. J. (2010).

Groenewegen, H. J. (2007).

Hyman, S. E. (2005).

Koob, G. F. (2008).

National Institute on Drug Abuse. (2001).

Volkow, N. D. (2009).

Volkow, N. D., Fowler, J. S., \& Wang, G.-J. (2003).

Volkow, N. D., Fowler, J. S., \& Wang, G.-J. (2004).

Volkow, N., \& Li, T.-K. (2005).

${ }^{16}$ Hyman, S. E. (2005).

Hyman, S. E. (2007).

Kelley, A. E., \& Berridge, K. C. (2002).

${ }^{17}$ Hyman, S. E. (2007).

${ }^{18}$ Volkow, N. D. (2007b).

${ }^{19}$ Erickson, C. K. (2007).

Filbey, F. M., Schacht, J. P., Myers, U. S., Chavez, R. S., \& Hutchison, K. E. (2009).

Hyman, S. E. (2007).

Volkow, N. D. (2007b).

National Institute on Drug Abuse. (2009a).

National Institute on Drug Abuse. (2010f).

Spanagel, R., \& Heilig, M. (2005).

Thombs, D. L. (2006).

${ }^{20}$ Hyman, S. E. (2005).

Hyman, S. E. (2007).

${ }^{21}$ American Society of Addiction Medicine. (2011b).

Hyman, S. E. (2007).

${ }^{22}$ Levine, A., Huang, Y. Y., Drisaldi, B., Griffin, E. A., Pollak, D. D., Xu, S., et al. (2011).

${ }^{23}$ Hyman, S. E. (2007).

McLellan, A. T., Lewis, D. C., O'Brien, C. P., \& Kleber, H. D. (2000). 
${ }^{24}$ American Society of Addiction Medicine. (2011b).

${ }^{25}$ Hyman, S. E. (2007).

${ }^{26}$ Denizet-Lewis, B. (2006).

National Institute on Drug Abuse. (2011i).

${ }^{27}$ Spence, R. T., DiNitto, D. M., \& Straussner, S. L. A.(Eds.). (2001).

${ }^{28}$ Volkow, N. D., Chang, L., Wang, G. J., Fowler, J. S., Ding, Y. S., Sedler, M., et al. (2001).

Volkow, N. D., Fowler, J. S., Wolf, A. P., Schlyer, D., Shiue, C. Y., Alpert, R., et al. (1990).

Volkow, N. D., Wang, G. J., Fowler, J. S., Logan, J., Hitzemann, R., Ding, Y. S., et al. (1996).

Volkow, N. D., Wang, G. J., Fowler, J. S., Logan, J., Gatley, S. J., Hitzemann, R., et al. (1997).

Wang, G. J., Volkow, N. D., Fowler, J. S., Logan, J., Abumrad, N. N., Hitzemann, R. J., et al. (1997).

${ }^{29}$ Kalivas, P. W., \& Vokow, N. D. (2005).

Leshner, A. I. (1997).

Leshner, A. I. (2001a).

${ }^{30}$ Dackis, C., \& O'Brien, C. P. (2005).

Hyman, S. E. (2007).

Institute of Medicine. (1997).

Leshner, A. I. (1997).

Leshner, A. I. (2001a).

${ }^{31}$ Gitlow, S. (2011).

Kreek, M. J., Nielsen, D. A., Butelman, E. R., \& LaForge, K. S. (2005).

Robison, A. J., \& Nestler, E. J. (2011).

${ }^{32}$ Gitlow, S. (2011).

Kreek, M. J., Nielsen, D. A., Butelman, E. R., \& LaForge, K. S. (2005).

Robison, A. J., \& Nestler, E. J. (2011).

Shaham, Y., \& Hope, B. T. (2005).

${ }^{33}$ Han, C., McGue, M. K., \& Iacono, W. G. (1999).

Lynskey, M. T., Agrawal, A., \& Heath, A. C. (2010).

Merikangas, K. R., \& McClair, V. L. (2012).

National Institute on Drug Abuse. (2003a).

Vink, J. M., Willemsen, G., \& Boomsma, D. I. (2005).

${ }^{34}$ Austin, G. A., \& Prendergast, M. (1991).

Lynskey, M. T., Agrawal, A., \& Heath, A. C. (2010).

Merikangas, K. R., \& McClair, V. L. (2012).

Merikangas, K. R., Stolar, M., Stevens, D. E., Goulet, J., Preisig, M. A., Fenton, B., et al. (1998).

Vink, J. M., Willemsen, G., \& Boomsma, D. I. (2005).

${ }^{35}$ The National Center on Addiction and Substance Abuse at Columbia University (CASA Columbia). (2012n).

${ }^{36}$ Agrawal, A., \& Lynskey, M. T. (2006).

Erickson, C. K. (2007).

Grant, J. D., Scherrer, J. F., Lynskey, M. T., Lyons, M. J., Eisen, S. A., Tsuang, M. T., et al. (2006).

Heath, A. C., Bucholz, K. K., Madden, P. A., Dinwiddie, S. H., Slutske, W. S., Bierut, L. J., et al. (1997).

Hopfer, C. J., Lessem, J. M., Hartman, C. A., Stallings, M. C., Cherny, S. S., Corley, R. P., et al. (2007).

Karkowski, L. M., Prescott, C. A., \& Kendler, K. S. (2000).

Kendler, K. S., Karkowski, L., \& Prescott, C. A. (1999).

Lynskey, M. T., Agrawal, A., \& Heath, A. C. (2010).

Kendler, K. S., Neale, M. C., Heath, A. C., Kessler, R. C., \& Eaves, L. J. (1994).

Kendler, K. S., Neale, M. C., Sullivan, P., Corey, L. A., Gardner, C. O., \& Prescott, C. A. (1999).

Maes, H. H., Neale, M. C., Chen, X., Chen, J., Prescott, C. A., \& Kendler, K. S. (2011).

Maes, H. H., Woodard, C. E., Murrelle, L., Meyer, J. M., Silberg, J. L., Hewitt, J. K., et al. (1999).

Merikangas, K. R., \& McClair, V. L. (2012).

Uhl, G. R., Drgon, T., Johnson, C., Fatusin, O. O., Liu, Q. R., Contoreggi, C., et al. (2008).

Han, C., McGue, M. K., \& Iacono, W. G. (1999).

Prescott, C. A., Aggen, S. H., \& Kendler, K. S. (1999).

van den Bree, M. B., Johnson, E. O., Neale, M. C., \& Pickens, R. W. (1998).

Segal, N. L. (1993).

Verweij, K. J. H., Zietsch, B. P., Lynskey, M. T., Medland, S. E., Neale, M. C., Martin, N. G., et al. (2010). 
Yuferov, V., Levran, O., Proudnikov, D., Nielsen, D. A., \& Kreek, M. J. (2010).

${ }^{37}$ Tsuang, M. T., Lyons, M. J., Harley, R. M., Xian, H., Eisen, S., Goldberg, J., et al. (1999).

Tsuang, M. T., Lyons, M. J., Meyer, J. M., Doyle, T., Eisen, S. A., Goldberg, J., et al. (1998).

Tsuang, M. T., Bar, J. L., Harley, R. M., \& Lyons, M. J. (2001).

${ }^{38}$ Karkowski, L. M., Prescott, C. A., \& Kendler, K. S. (2000).

${ }^{39}$ Bart, G., Kreek, M. J., Ott, J., LaForge, K. S., Proudnikov, D., Pollak, L., et al. (2005).

Mroziewicz, M., \& Tyndale, R. F. (2010).

Hartz, S. M., \& Bierut, L. J. (2010).

${ }^{40}$ Mroziewicz, M., \& Tyndale, R. F. (2010).

41 Treistman, S. N., \& Martin, G. E. (2009).

${ }^{42}$ Kurtz, D. L., Stewart, R. B., Zweifel, M., Li, T. K., \& Froehlich, J. C. (1996).

Treistman, S. N., \& Martin, G. E. (2009).

${ }^{43}$ Agrawal, A., \& Lynskey, M. T. (2006).

Heath, A. C., Bucholz, K. K., Madden, P. A., Dinwiddie, S. H., Slutske, W. S., Bierut, L. J., et al. (1997).

Kendler, K. S., Jacobson, K. C., Prescott, C. A., \& Neale, M. C. (2003).

Kendler, K. S., Neale, M. C., Sullivan, P., Corey, L. A., Gardner, C. O., \& Prescott, C. A. (1999).

Kreek, M. J., Nielsen, D. A., Butelman, E. R., \& LaForge, K. S. (2005).

Prescott, C. A., Aggen, S. H., \& Kendler, K. S. (1999).

Prescott, C. A., \& Kendler, K. S. (1999).

True, W. R., Heath, A. C., Bucholz, K., Slutske, W., Romeis, J. C., Scherrer, J. F., et al. (1996).

Tsuang, M. T., Lyons, M. J., Harley, R. M., Xian, H., Eisen, S., Goldberg, J., et al. (1999).

Tsuang, M. T., Lyons, M. J., Meyer, J. M., Doyle, T., Eisen, S. A., Goldberg, J., et al. (1998).

Tsuang, M. T., Bar, J. L., Harley, R. M., \& Lyons, M. J. (2001).

Vink, J. M., Willemsen, G., \& Boomsma, D. I. (2005).

Volkow, N. D. (2007b).

${ }^{44}$ Agrawal, A., Sartor, C. E., Lynskey, M. T., Grant, J. D., Pergadia, M. L., Grucza, R., et al. (2009).

${ }^{45}$ Audrain-McGovern, J., Al, K. N., Rodriguez, D., Wileyto, E. P., Shields, P. G., \& Tyndale, R. F. (2007).

${ }^{46}$ Bierut, L. J., Madden, P. A., Breslau, N., Johnson, E. O., Hatsukami, D., Pomerleau, O. F., et al. (2007).

Maes, H. H., Neale, M. C., Chen, X., Chen, J., Prescott, C. A., \& Kendler, K. S. (2011).

Saccone, S. F., Hinrichs, A. L., Saccone, N. L., Chase, G. A., Konvicka, K., Madden, P. A., et al. (2007).

${ }^{47}$ Schuckit, M. A., Tapert, S., Matthews, S. C., Paulus, M. P., Tolentino, N. J., Smith, T. L., et al. (2011).

Schuckit, M. A., Smith, T. L., Trim, R. S., Allen, R. C., Fukukura, T., Knight, E. E., et al. (2011).

${ }^{48}$ Belcher, H. M., \& Shinitzky, H. E. (1998).

49 Thomasson, H. R., Edenberg, H. J., Crabb, D. W., Mai, X.-L., Jerome, R. E., Li, T.-K., et al. (1991).

Neumark, Y. D., Friedlander, Y., Thomasson, H. R., \& Li, T. K. (1998).

${ }^{50}$ Roan, S. (2009).

${ }^{51}$ Agrawal, A., \& Lynskey, M. T. (2006).

${ }^{52}$ Ehlers, C. L., Gizer, I. R., Vieten, C., \& Wilhelmsen, K. C. (2010).

Ehlers, C. L., Gizer, I. R., Vieten, C., Gilder, D. A., Stouffer, G. M., Lau, P., et al. (2010).

${ }^{53}$ Kendler, K. S., Karkowski, L., \& Prescott, C. A. (1999).

${ }^{54}$ American Society of Addiction Medicine. (2011b).

Ersche, K. D., Jones, P. S., Williams, G. B., Turton, A. J., Robbins, T. W., \& Bullmore, E. T. (2012).

Gitlow, S. (2011).

Whelan, R., Conrod, P. J., Poline, J. B., Lourdusamy, A., Banaschewski, T., Barker, G. J., et al. (2012).

${ }^{55}$ George, O., \& Koob, G. F. (2010).

${ }^{56}$ Hommer, D. W., Bjork, J. M., \& Gilman, J. M. (2011).

Carter, J. C. \& Davis, C. (2011).

${ }^{57}$ Kennerly, S. W., Behrens, T. E. J., \& Wallis, J. D. (2011).

Lubman, D. I., Yucel, M., \& Pantelis, C. (2004).

${ }^{58}$ Brook, D. W., Brook, J. S., Zhang, C., \& Koppel, J. (2010).

Disney, E. R., Elkins, I. J., McGue, M., \& Iacono, W. G. (1999).

Elkins, I. J., McGue, M., \& Iacono, W. G. (2007).

Heath, A. C., Bucholz, K. K., Madden, P. A., Dinwiddie, S. H., Slutske, W. S., Bierut, L. J., et al. (1997).

Lewis, C. E., \& Bucholz, K. K. (1991).

Lopez, B., Schwartz, S. J., Prado, G., Huang, S., Rothe, E. M., Wang, W., et al. (2008). 
Moss, H. B., \& Lynch, K. G. (2001).

Windle, M., \& Windle, R. C. (2006).

${ }^{59}$ Audrain-McGovern, J., Rodriguez, D., \& Kassel, J. D. (2009).

Crum, R. M., Green, K. M., Storr, C. L., Chan, Y. F., Ialongo, N., Stuart, E. A., et al. (2008).

Crum, R. M., Storr, C. L., Ialongo, N., \& Anthony, J. C. (2008).

Escobedo, L. G., Reddy, M., \& Giovino, G. A. (1998).

Marmorstein, N. R. (2009).

Munafo, M. R., Hitsman, B., Rende, R., Metcalfe, C., \& Niaura, R. (2008).

Owens, T. J., \& Shippee, N. D. (2009).

The National Center on Addiction and Substance Abuse (CASA) at Columbia University. (2011a).

Upadhyaya, H. P., Deas, D., Brady, K. T., \& Kruesi, M. (2002).

Windle, M., \& Windle, R. C. (2001).

${ }^{60}$ Armstrong, T. D., \& Costello, E. J. (2002).

Audrain-McGovern, J., Rodriguez, D., \& Kassel, J. D. (2009).

Chan, Y. F., Dennis, M. L., \& Funk, R. R. (2008).

Center for Substance Abuse Treatment. (2005b).

Epstein, J., Barker, P., Vorburger, M., \& Murtha, C. (2004).

Grella, C. E., Hser, Y.-I., Vandana, J., \& Rounds-Bryant, J. (2001).

Heath, A. C., Bucholz, K. K., Madden, P. A., Dinwiddie, S. H., Slutske, W. S., Bierut, L. J., et al. (1997).

Khantzian, E. J. (1985).

Lopez-Quintero, C., de los Cobos, J. P., Hasin, D. S., Okuda, M., Wang, S., Grant, B. F., et al. (2011).

National Institute on Drug Abuse. (2010a).

O'Neil, K. A., Conner, B. T., \& Kendall, P. C. (2011).

Volkow, N. D. (2007a).

Wilens, T. E., Biederman, J., Adamson, J. J., Henin, A., Sgambati, S., Gignac, M., et al. (2008).

${ }^{61}$ Andersen, S. L., \& Teicher, M. H. (2009).

Kaufman, J., Yang, B.-Z., Douglas-Palumberi, H., Crouse-Artus, M., Lipschitz, D., Krystal, J. H., et al. (2007).

Meaney, M. J., Brake, W., \& Gratton, A. (2002).

${ }^{62}$ Kessler, R. C., Sonnega, A., Bromet, E., Hughes, M., \& Nelson, C. B. (1995).

Lasser, K., Boyd, J. W., Woolhandler, S., Himmelstein, D. U., McCormick, D., \& Bor, D. H. (2000).

Litz, B. T., \& Schlenger, W. E. (2009).

McFall, M., \& Cook, J. (2006).

Milliken, C. S., Auchterlonie, J. L., \& Hoge, C. W. (2007).

Tanielian, T., Jaycox, L. H., \& (eds). (2008).

U.S. Department of Veteran Affairs, National Center for PTSD. (2012).

U.S. Department of Veteran Affairs, National Center for PTSD. (2011).

${ }^{63}$ Adams, J. B., Heath, A. J., Young, S. E., Hewitt, J. K., Corley, R. P., \& Stallings, M. C. (2003).

Burt, R. D., Dinh, K. T., Peterson, A. V., Jr., \& Sarason, I. G. (2000).

Hill, K. G., Hawkins, J. D., Bailey, J. A., Catalano, R. F., Abbott, R. D., \& Shapiro, V. B. (2010).

Wills, T. A., Walker, C., Mendoza, D., \& Ainette, M. G. (2006).

${ }^{64}$ Donnelly, J., Young, M., Pearson, R., Penhollow, T. M., \& Hernandez, A. (2008).

Epstein, J. A., Griffin, K. W., \& Botvin, G. J. (2004).

Mouttapa, M., Weiss, J. W., \& Hermann, M. (2009).

Taylor, J., lloyd, D. A., \& Warheit, G. J. (2005).

${ }^{65}$ Hawkins, J. D., Catalano, R. F., \& Miller, J. Y. (1992).

${ }^{66}$ Dube, S. R., Felitti, V. J., Dong, M., Chapman, D. P., Giles, W. H., \& Anda, R. F. (2003).

Harrison, P. A., Hoffman, N. G., \& Edwall, G. E. (1989).

Kilpatrick, D. G., Acierno, R., Saunders, B., Resnick, H. S., Best, C. L., \& Schnurr, P. P. (2000).

Rogosch, F. A., Oshri, A., \& Cicchetti, D. (2010).

Schoen, C., Davis, K., Collins, K. S., Greenberg, L., Des Roches, C., \& Abrams, M. (1997).

${ }^{67}$ Epstein, J. A., Griffin, K. W., \& Botvin, G. J. (2008).

McCabe, S. E., Boyd, C. J., Cranford, J. A., \& Teter, C. J. (2009).

Song, A. V., Morrell, H. E., Cornell, J. L., Ramos, M. E., Biehl, M., Kropp, R. Y., et al. (2009).

The National Center on Addiction and Substance Abuse (CASA) at Columbia University. (2011a).

${ }^{68}$ Castro, F. G., Brook, J. S., Brook, D. W., \& Rubenstone, E. (2006). 
Hawkins, J. D., Catalano, R. F., \& Miller, J. Y. (1992).

Li, C., Pentz, M. A., \& Chou, C. P. (2002).

Tyc, V. L., Hadley, W., Allen, D., Varnell, S., Ey, S., Rai, S. N., et al. (2004).

Walden, B., Iacono, W. G., \& McGue, M. (2007).

Wills, T. A., Sandy, J. M., Yaeger, A., \& Shinar, O. (2001).

${ }^{69}$ Caughlin, J. P., \& Malis, R. S. (2004).

Miller, T. Q., \& Volk, R. J. (2002).

Skeer, M., McCormick, M. C., Normand, S. L., Buka, S. L., \& Gilman, S. E. (2009).

Wills, T. A., Sandy, J. M., Yaeger, A., \& Shinar, O. (2001).

${ }^{70}$ Bryant, A. L., \& Zimmerman, M. A. (2002).

Connell, C. M., Gilreath, T. D., Aklin, W. M., \& Brex, R. A. (2010).

Galea, S., Nandi, A., \& Vlahov, D. (2004).

Kennedy, D. P., Tucker, J. S., Pollard, M. S., Go, M. H., \& Green, H. D., Jr. (2011).

Lipperman-Kreda, S., Grube, J. W., \& Paschall, M. J. (2010).

Martins, S. S., Storr, C. L., Alexandre, P. K., \& Chilcoat, H. D. (2008).

Maxwell, K. (2002).

Prinstein, M. J., Boergers, J., \& Spirito, A. (2001).

Simons-Morton, B., \& Chen, R. S. (2006).

The National Center on Addiction and Substance Abuse (CASA) at Columbia University. (2011b).

${ }^{71}$ Rosack, J. (2004).

${ }^{72}$ Campbell, C. A., Hahn, R. A., Elder, R., Brewer, R., Chattopadhyay, S., Fielding, J., et al. (2009).

Chen, M. J., Grube, J. W., \& Gruenewald, P. J. (2009).

Novak, S. P., Reardon, S. F., Raudenbush, S. W., \& Buka, S. L. (2006).

Rasmussen, S. (2000).

Truong, K. D., \& Sturm, R. (2009).

${ }^{73}$ The National Center on Addiction and Substance Abuse (CASA) at Columbia University. (2011a).

The National Center on Addiction and Substance Abuse (CASA) at Columbia University. (2005).

${ }^{74}$ Campbell, C. A., Hahn, R. A., Elder, R., Brewer, R., Chattopadhyay, S., Fielding, J., et al. (2009).

Chen, M. J., Grube, J. W., \& Gruenewald, P. J. (2009).

Hawkins, J. D., Catalano, R. F., \& Miller, J. Y. (1992).

Henriksen, L., Schleicher, N. C., Feighery, E. C., \& Fortmann, S. P. (2010).

Novak, S. P., Reardon, S. F., Raudenbush, S. W., \& Buka, S. L. (2006).

Truong, K. D., \& Sturm, R. (2009).

${ }^{75}$ The National Center on Addiction and Substance Abuse (CASA) at Columbia University. (2005).

${ }^{76}$ Cerda, M., Wall, M., Keyes, K. M., Galea, S., \& Hasin, D. (2012).

Harper, S., Strumpf, E. C., \& Kaufman, J. S. (2012).

Wall, M. M., Poh, E., Cerda, M., Keyes, K. M., Galea, S., \& Hasin, D. S. (2011).

${ }^{77}$ The National Center on Addiction and Substance Abuse (CASA) at Columbia University. (2011a).

${ }^{78}$ Charlesworth, A., \& Glantz, S. A. (2005).

Dalton, M. A., Sargent, J. D., Beach, M. L., Titus-Ernstoff, L., Gibson, J. J., Ahrens, M. B., et al. (2003).

Hanewinkel, R., Isensee, B., Sargent, J. D., \& Morgenstern, M. (2011).

Sargent, J. D., Beach, M. L., Adachi-Mejia, A. M., Gibson, J. J., Titus-Ernstoff, L. T., Carusi, C. P., et al. (2005).

Biener, L., \& Siegel, M. (2000).

Pierce, J. P., Choi, W. S., Gilpin, E. A., Farkas, A. J., \& Berry, C. C. (1998).

Snyder, L. B., Milici, F. F., Slater, M., Sun, H., \& Strizhakova, Y. (2006).

79 Triggle, D. J. (2007).

${ }^{80}$ Andersen, S. L., \& Teicher, M. H. (2009).

Gordon, H. W. (2002).

Mroziewicz, M., \& Tyndale, R. F. (2010).

Paaver, M., Kurrikoff, T., Nordquist, N., Oreland, L., \& Harro, J. (2008).

${ }^{81}$ Merikangas, K. R., \& McClair, V. L. (2012).

The National Center on Addiction and Substance Abuse (CASA) at Columbia University. (2011a).

${ }^{82}$ The National Center on Addiction and Substance Abuse at Columbia University (CASA Columbia). (2012n).

${ }^{83}$ Dahl, R. E. (2004).

Giedd, J. N., Blumenthal, J., Jeffries, N. O., Castellanos, F. X., Liu, H., Zijdenbos, A., et al. (1999). 
Guerri, C., \& Pascual, M. (2010).

Riggs, N. R., \& Greenberg, M. T. (2009).

Steinberg, L. (2008).

The National Center on Addiction and Substance Abuse (CASA) at Columbia University. (2011a).

Wagner, F. A., \& Anthony, J. C. (2002).

${ }^{84}$ American Society of Addiction Medicine. (2011c).

Chambers, R. A., Taylor, J. R., \& Potenza, M. N. (2003).

Gould, T. J. (2010).

Volkow, N. D. (2007b).

Volkow, N. D. (2005).

${ }^{85}$ The National Center on Addiction and Substance Abuse (CASA) at Columbia University. (2011a).

Lopez-Quintero, C., de los Cobos, J. P., Hasin, D. S., Okuda, M., Wang, S., Grant, B. F., et al. (2011).

${ }^{86}$ Chan, Y. F., Dennis, M. L., \& Funk, R. R. (2008).

Grant, J. D., Scherrer, J. F., Lynskey, M. T., Lyons, M. J., Eisen, S. A., Tsuang, M. T., et al. (2006a).

Lynskey, M. T., Agrawal, A., \& Heath, A. C. (2010).

${ }^{87}$ Chan, Y. F., Dennis, M. L., \& Funk, R. R. (2008).

Epstein, J., Barker, P., Vorburger, M., \& Murtha, C. (2004).

${ }^{88}$ Centers for Disease Control and Prevention. (2004b).

${ }^{89}$ Centers for Disease Control and Prevention. (2008b).

${ }^{90}$ Robert Wood Johnson Foundation. (2001).

${ }^{91}$ Burge, S. K., \& Schneider, F. D. (1999).

${ }^{92}$ Rehm, J., Samokhvalov, A. V., Neuman, M. G., Room, R., Parry, C., Lonnroth, K., et al. (2009).

${ }^{93}$ National Institute on Drug Abuse. (2011a).

Centers for Disease Control and Prevention. (2002a).

${ }^{94}$ Thygesen, L. C., Mikkelsen, P., Andersen, T. V., Tonnesen, H., Juel, K., Becker, U., et al. (2009).

${ }^{95}$ Masoumi, M., Shahesmaeili, A., Mirzazadeh, A., Tavakoli, M., \& Ali, A. Z. (2010).

${ }^{96}$ Cook, R. L., Comer, D. M., Wiesenfeld, H. C., Chang, C. C., Tarter, R., Lave, J. R., et al. (2006).

${ }^{97}$ Quenqua, D. (2011, July 11).

${ }^{98}$ Armstrong, T. D., \& Costello, E. J. (2002).

Chan, Y. F., Dennis, M. L., \& Funk, R. R. (2008).

Epstein, J., Barker, P., Vorburger, M., \& Murtha, C. (2004).

Grella, C. E., Hser, Y.-I., Vandana, J., \& Rounds-Bryant, J. (2001).

King, S. M., Iacono, W. G., \& McGue, M. (2004).

The National Center on Addiction and Substance Abuse (CASA) at Columbia University. (2003a).

Upadhyaya, H. P., Deas, D., Brady, K. T., \& Kruesi, M. (2002).

${ }^{99}$ Johnson, J. G., Cohen, P., Pine, D. S., Klein, D. F., Kasen, S., \& Brook, J. S. (2000).

National Institute on Drug Abuse. (2010a).

Patton, G. C., Coffey, C., Carlin, J. B., Degenhardt, L., Lynskey, M., \& Hall, W. (2002).

Rohde, P., Lewinsohn, P. M., Kahler, C. W., Seeley, J. R., \& Brown, R. A. (2001).

Steuber, T. L., \& Danner, F. (2006).

${ }^{100}$ Biederman, J., Wilens, T. E., Mick, E., Faraone, S. V., \& Spencer, T. (1998).

National Institute on Drug Abuse. (2010a).

Swendsen, J., Conway, K. P., Degenhardt, L., Glantz, M., Jin, R., Merikangas, K. R., et al. (2010).

Wilens, T. E., Biederman, J., Adamson, J. J., Henin, A., Sgambati, S., Gignac, M., et al. (2008).

${ }^{101}$ Khantzian, E. J. (1985).

National Institute on Drug Abuse. (2010a).

The National Center on Addiction and Substance Abuse (CASA) at Columbia University. (2005).

${ }^{102}$ National Institute on Drug Abuse. (2010a).

${ }^{103}$ American Society of Addiction Medicine. (2011b).

Gitlow, S. (2011).

${ }^{104}$ Erickson, C. K. (2007).

Hudson, N. L., \& Mannino, D. M. (2010).

Lewis, D. C. (1991).

McLellan, A. T., Lewis, D. C., O'Brien, C. P., \& Kleber, H. D. (2000).

White, W. L. (2008). 
105 Hyman, S. E. (2007).

Hyman, S. E. (2005).

Shaham, Y., \& Hope, B. T. (2005).

${ }^{106}$ Leshner, A. I. (1997).

White, W. L. (2008).

${ }^{107}$ Willenbring, M. L. (2008a).

${ }^{108}$ National Quality Forum. (2005).

Pollack, H. A., \& D'Aunno, T. (2008).

Willenbring, M. L. (2008a).

${ }^{109}$ McLellan, A. T., Lewis, D. C., O'Brien, C. P., \& Kleber, H. D. (2000).

National Institute on Drug Abuse. (2009e).

Willenbring, M. L. (2008a).

${ }^{110}$ Lee, P. R., Lee, D. R., Lee, P., \& Arch, M. (2010).

Russell, C., Davies, J. B., \& Hunter, S. C. (2011).

${ }^{111}$ Leshner, A. I. (1997).

Leshner, A. I. (2001a).

Volkow, N., \& Li, T.-K. (2005).

112 Kumpfer, K. L., Trunnell, E. P., \& Whiteside, H. O. (1990).

Leshner, A. I. (1997).

Volkow, N. D. (2007b).

National Institute on Drug Abuse. (2005a).

113 Bishop, E. S. (1919).

${ }^{114}$ Lee, P. R., Lee, D. R., Lee, P., \& Arch, M. (2010).

${ }^{115}$ Centers for Disease Control and Prevention. (2002c).

Frank, D. (2011).

Leshner, A. I. (1997).

116 Satel, S. L. (1999).

117 Associated Press. (2011).

National Conference of State Legislatures. (2012).

Sulzberger, A. G. (2011).

${ }^{118}$ Bierut, L. J., Dinwiddie, S. H., Begleiter, H., Crowe, R. R., Hesselbrock, V., Nurnberger, J. I., Jr., et al. (1998).

Dackis, C., \& O'Brien, C. P. (2005).

Erickson, C. K. (2007).

Grant, J. D., Scherrer, J. F., Lynskey, M. T., Lyons, M. J., Eisen, S. A., Tsuang, M. T., et al. (2006).

Heath, A. C., Bucholz, K. K., Madden, P. A., Dinwiddie, S. H., Slutske, W. S., Bierut, L. J., et al. (1997).

Leshner, A. I. (1997).

Volkow, N. D. (2007b).

Lynskey, M. T., Agrawal, A., \& Heath, A. C. (2010).

Kendler, K. S., Neale, M. C., Heath, A. C., Kessler, R. C., \& Eaves, L. J. (1994).

Kendler, K. S., Neale, M. C., Sullivan, P., Corey, L. A., Gardner, C. O., \& Prescott, C. A. (1999).

Merikangas, K. R., Stolar, M., Stevens, D. E., Goulet, J., Preisig, M. A., Fenton, B., et al. (1998).

Mroziewicz, M., \& Tyndale, R. F. (2010).

Prescott, C. A., Aggen, S. H., \& Kendler, K. S. (1999).

van den Bree, M. B., Johnson, E. O., Neale, M. C., \& Pickens, R. W. (1998).

Zickler, P. (2000).

${ }^{119}$ Satel, S. L. (2007).

120 Peele, S. (2007).

Satel, S. L. (2007).

${ }^{121}$ Kluger, R. (1996).

${ }^{122}$ Gardner, M. N., \& Brandt, A. M. (2006).

Pierce, J. P., \& Gilpin, E. A. (1995).

The National Center on Addiction and Substance Abuse (CASA) at Columbia University. (2003b).

${ }^{123}$ U.S. Department of Health, Education and Welfare, Public Health Service. (1964).

${ }^{124}$ Narconon International. (2011).

National Conference of State Legislatures. (2011b). 
ProCon.org. (2012).

Spillane, J. F. (2000).

${ }^{125}$ Brecher, E. M. (1972).

${ }^{126}$ Public Broadcasting System. (2011).

${ }^{127}$ Gray, M. (2009).

${ }^{128}$ The National Center on Addiction and Substance Abuse (CASA) at Columbia University. (2005).

${ }^{129}$ Courtwright, D. T. (2010).

${ }^{130}$ American Medical Association. (2011d).

American Medical Association. (1956).

Block, M. A. (1956).

${ }^{131}$ Cameron, D. C. (1967).

${ }^{132}$ Comprehensive Alcohol Abuse and Alcoholism Prevention, Treatment, and Rehabilitation Act of $1970 . \S 1848$ (1970).

${ }^{133}$ American Medical Association. (1956).

${ }^{134}$ Bowen, O. R., \& Sammons, J. H. (1988).

American Medical Association, Council on Scientific Affairs. (1979).

${ }^{135}$ American Medical Association. (2011b).

${ }^{136}$ American Psychiatric Association. (1952).

${ }^{137}$ American Psychiatric Association. (1980).

${ }^{138}$ Leshner, A. I. (1997).

Leshner, A. I. (2001a).

${ }^{139}$ Volkow, N. D. (2007b).

${ }^{140}$ Leshner, A. I. (1997).

${ }^{141}$ Kluger, R. (1996).

${ }^{142}$ Gardner, M. N., \& Brandt, A. M. (2006).

${ }^{143}$ Wynder, E. L., \& Graham, E. A. (1950).

${ }^{144}$ Centers for Disease Control and Prevention. (1999).

${ }^{145}$ U.S. Department of Health, Education and Welfare, Public Health Service. (1964).

${ }^{146}$ Goldberg, R. (2009).

Williams, S. E. (1980).

${ }^{147}$ Eyewitness to History. (2007).

Ohio State University, Department of History. (2012).

PBS.org. (2012).

${ }^{148}$ PBS.org. (2012).

${ }^{149}$ Library of Congress. (2011).

National Center for Natural Products Research. (2009).

${ }^{150}$ National Conference of State Legislatures. (2011b).

Voth, E. A. (2003).

${ }^{151}$ Kandall, S. R., \& Petrillo, J. (1996).

${ }^{152}$ Brecher, E. M. (1972).

Courtwright, D. T. (2001).

${ }^{153}$ University at Buffalo, Addiction Research Unit. (2001).

${ }^{154}$ Brecher, E. M. (1972).

155 Brecher, E. M. (1972).

Kandall, S. R. (1998).

${ }^{156}$ The National Center on Addiction and Substance Abuse (CASA) at Columbia University. (2005).

The National Center on Addiction and Substance Abuse (CASA) at Columbia University. (2009c).

${ }^{157}$ The National Center on Addiction and Substance Abuse (CASA) at Columbia University. (2005).

${ }^{158}$ Gitlow, S. (2011).

${ }^{159}$ O'Brien, C. P., \& McLellan, A. T. (1996).

National Institute on Drug Abuse. (2008).

National Institute on Drug Abuse. (2009e).

${ }^{160}$ Chen, K., Kandel, D. B., \& Davies, M. (1997).

U.S. Department of Health and Human Services. (2010a).

Zakhari, S., \& Li, T. K. (2007). 
${ }^{161}$ Gold, M., MD, University of Florida, College of Medicine and McKnight Brain Institute, Departments of Psychiatry, Neuroscience, Anesthesiology, Community Health \& Family Medicine, Chairman, Department of Psychiatry (personal communication, March 19, 2012).

${ }^{162}$ U.S. Department of Agriculture and U.S. Department of Health and Human Services. (2010).

${ }^{163}$ Catholic University of America. (2010).

Center for Substance Abuse Treatment. (2006c).

U.S. Department of Agriculture and U.S. Department of Health and Human Services. (2010).

Catholic University of America. (2010).

${ }^{164}$ Gitlow, S., MD, MPH, MBA, Executive Director, Annenberg Physician Training Program in Addictive Disease, Associate Clinical Professor, Mount Sinai School of Medicine, Acting President \& AMA Delegate, Amercian Society of Addiction Medicine (personal communication, August 7, 2007).

${ }^{165}$ American Psychiatric Association. (1994).

${ }^{166}$ American Society of Addiction Medicine. (2011b).

Hajela, R. \& Miller, M. M. (2012).

${ }^{167}$ American Psychiatric Association. (1994).

${ }^{168}$ American Psychiatric Association. (1994).

${ }^{169}$ Babor, T. F., \& Hall, W. (2007).

${ }^{170}$ American Psychiatric Association. (1994).

${ }^{171}$ O'Brien, C. P. (1996).

${ }^{172}$ American Psychiatric Association. (1994).

${ }^{173}$ Erickson, C. K. (2007).

Leshner, A. I. (1997).

Leshner, A. I. (2001a).

Leshner, A. I. (2005).

${ }^{174}$ Savage, S. R. (2002).

Savage, S. R., Joranson, D. E., Covington, E. C., Schnoll, S. H., Heit, H. A., \& Gilson, A. M. (2003).

175 O'Brien, C. P., Volkow, N., \& Li, T. K. (2006).

${ }^{176}$ U.S. Drug Enforcement Administration. (2011).

Miller, N. S., \& Gold, M. S. (1990).

Savage, S. R., Joranson, D. E., Covington, E. C., Schnoll, S. H., Heit, H. A., \& Gilson, A. M. (2003).

177 Savage, S. R. (2002).

${ }^{178}$ Savage, S. R. (2002).

The National Center on Addiction and Substance Abuse (CASA) at Columbia University. (2005).

${ }^{179}$ O'Brien, C. P., Volkow, N., \& Li, T. K. (2006).

Dhalla, I. A. (2011).

${ }^{180}$ Baker, T. B., Breslau, N., Covey, L., \& Shiffman, S. (2012).

${ }^{181}$ American Psychiatric Association. (2011b).

American Psychiatric Association. (2010b).

${ }^{182}$ Clark, M. (2011).

American Psychiatric Association. (2011b).

${ }^{183}$ Courtwright, D. T. (2011).

${ }^{184}$ American Psychiatric Association. (2010a).

American Psychiatric Association. (2010c).

Van Ornum, W. (2011).

${ }^{185}$ Harford, T. C., Yi, H. Y., \& Grant, B. F. (2010).

McBride, O., \& Adamson, G. (2010).

${ }^{186}$ Knopf, A. (2010).

${ }^{187}$ World Health Organization. (2011d).

${ }^{188}$ World Health Organization. (2011c).

${ }^{189}$ World Health Organization. (2011a).

American Psychiatric Association. (2011a).

${ }^{190}$ World Health Organization. (2011b).

PsychiatryOnline. (2011).

${ }^{191}$ Centers for Disease Control and Prevention. (2011g).

Mezzich, J. E. (2002). 
${ }^{192}$ Baker, T. B., Breslau, N., Covey, L., \& Shiffman, S. (2012).

${ }^{193}$ Babor, T. F., \& Hall, W. (2007).

${ }^{194}$ American Society of Addiction Medicine. (2011b).

${ }^{195}$ National Institute of Mental Health. (2012).

${ }^{196}$ Leshner, A. I. (1997).

${ }^{197}$ Rivlin, A. (2005).

${ }^{198}$ Russell, C., Davies, J. B., \& Hunter, S. C. (2011).

${ }^{199}$ Substance Abuse and Mental Health Services Administration, Office of Applied Studies. (2010).

${ }^{200}$ Office of Communications. (2008).

${ }^{201}$ Molloy, J. P., JD, Chief Executive Officer, Oxford House (personal communication, November 27, 2007).

${ }^{202}$ Hazelden Foundation. (2009).

${ }^{203}$ Hazelden Foundation. (2009).

${ }^{204}$ Lake Research Partners. (2009).

${ }^{205}$ Corrigan, P. W., Kuwabara, S. A., \& O'Shaughnessy, J. (2009).

Volkow, N. D. (2007b).

${ }^{206}$ The National Center on Addiction and Substance Abuse (CASA) at Columbia University. (2008a).

${ }^{207}$ Vink, J. M., Willemsen, G., \& Boomsma, D. I. (2005).

${ }^{208}$ The National Center on Addiction and Substance Abuse (CASA) at Columbia University. (2008a).

${ }^{209}$ Prescott, C. A., Aggen, S. H., \& Kendler, K. S. (1999).

Heath, A. C., Bucholz, K. K., Madden, P. A., Dinwiddie, S. H., Slutske, W. S., Bierut, L. J., et al. (1997).

Lynskey, M. T., Agrawal, A., \& Heath, A. C. (2010).

Kendler, K. S., Neale, M. C., Heath, A. C., Kessler, R. C., \& Eaves, L. J. (1994).

Kreek, M. J., Nielsen, D. A., Butelman, E. R., \& LaForge, K. S. (2005).

Tsuang, M. T., Bar, J. L., Harley, R. M., \& Lyons, M. J. (2001).

Volkow, N. D. (2005).

Lynskey, M. T., Agrawal, A., \& Heath, A. C. (2010).

${ }^{210}$ The National Center on Addiction and Substance Abuse (CASA) at Columbia University. (2008a).

${ }^{211}$ Agrawal, A., \& Lynskey, M. T. (2008).

Kendler, K. S., Jacobson, K. C., Prescott, C. A., \& Neale, M. C. (2003).

Kreek, M. J., Nielsen, D. A., Butelman, E. R., \& LaForge, K. S. (2005).

Tsuang, M. T., Lyons, M. J., Harley, R. M., Xian, H., Eisen, S., Goldberg, J., et al. (1999).

${ }^{212}$ The National Center on Addiction and Substance Abuse (CASA) at Columbia University. (2008a).

${ }^{213}$ Kreek, M. J., Nielsen, D. A., Butelman, E. R., \& LaForge, K. S. (2005).

${ }^{214}$ The National Center on Addiction and Substance Abuse (CASA) at Columbia University. (2008a).

${ }^{215}$ The National Center on Addiction and Substance Abuse (CASA) at Columbia University. (2008a).

${ }^{216}$ The National Center on Addiction and Substance Abuse (CASA) at Columbia University. (2008). 


\section{Chapter III \\ Notes}

${ }^{1}$ Brown, R. L., Leonard, T., Saunders, L. A., \& Papasouliotis, O. (1998).

Greene, J. M., Ennett, S. T., \& Ringwalt, C. L. (1997).

Substance Abuse and Mental Health Services Administration. (2006).

The National Center on Addiction and Substance Abuse (CASA) at Columbia University. (2010).

The National Center on Addiction and Substance Abuse at Columbia University (CASA Columbia). (2012n).

${ }^{2}$ Centers for Disease Control and Prevention. (2011h).

Centers for Disease Control and Prevention. (2012b).

${ }^{3}$ The Partnership at DrugFree.org. (2012).

${ }^{4}$ The National Center on Addiction and Substance Abuse at Columbia University (CASA Columbia). (2012n).

${ }^{5}$ American Cancer Society. (2009).

American Diabetes Association. (2009).

Centers for Disease Control and Prevention. (2011b).

Centers for Disease Control and Prevention. (2008).

Centers for Disease Control and Prevention, Coordinating Center for Health Promotion. (2008).

Corso, P., Finkelstein, E., Miller, T., Fiebelkorn, I., \& Zaloshnja, E. (2006).

Insel, T. R. (2008).

Lloyd-Jones, D., Adams, R., Carnethon, M., De, S. G., Ferguson, T. B., Flegal, K., et al. (2009).

Mokdad, A. H., Marks, J. S., Stroup, D. F., \& Gerberding, J. L. (2004).

Robert Wood Johnson Foundation. (2001).

${ }^{6}$ Centers for Disease Control and Prevention. (2004b).

Centers for Disease Control and Prevention. (2008a).

Centers for Disease Control and Prevention. (2008b).

Merrill, J. \& Fox, K. (1998).

The National Center on Addiction and Substance Abuse (CASA) at Columbia University. (1993).

The National Center on Addiction and Substance Abuse (CASA) at Columbia University. (1994).

${ }^{7}$ The National Center on Addiction and Substance Abuse at Columbia University (CASA Columbia). (2012n).

${ }^{8}$ U.S. Department of Agriculture and U.S. Department of Health and Human Services. (2010).

${ }^{9}$ U.S. Drug Enforcement Administration. (2010).

${ }^{10}$ American Society of Addiction Medicine. (2011b).

Andersen, S. L., \& Teicher, M. H. (2009).

Bava, S., Frank, L. R., McQueeny, T., Schweinsburg, B. C., Schweinsburg, A. D., \& Tapert, S. F. (2009).

Benowitz, N. L. (2010).

Chambers, R. A., Taylor, J. R., \& Potenza, M. N. (2003).

Crews, F. T., \& Boettiger, C. A. (2009).

Crews, F., He, J., \& Hodge, C. (2007).

Doremus-Fitzwater, T. L., Varlinskaya, E. I., \& Spear, L. P. (2010a).

Ersche, K. D., Jones, P. S., Williams, G. B., Turton, A. J., Robbins, T. W., \& Bullmore, E. T. (2012).

Gitlow, S. (2011).

Guerri, C., \& Pascual, M. (2010).

Merikangas, K. R., \& McClair, V. L. (2012).

Placzek, A. N., Zhang, T. A., \& Dani, J. A. (2009).

Riggs, N. R., \& Greenberg, M. T. (2009).

Schramm-Sapyta, N. L., Walker, Q. D., Caster, J. M., Levin, E. D., \& Kuhn, C. M. (2009).

The National Center on Addiction and Substance Abuse (CASA) at Columbia University. (2011a).

Whelan, R., Conrod, P. J., Poline, J. B., Lourdusamy, A., Banaschewski, T., Barker, G. J., et al. (2012).

${ }^{11}$ Fendrich, M., \& Johnson, T. P. (2001).

Substance Abuse and Mental Health Services Administration. (2011b).

${ }^{12}$ The National Center on Addiction and Substance Abuse at Columbia University (CASA Columbia). (2012n).

${ }^{13}$ The National Center on Addiction and Substance Abuse at Columbia University (CASA Columbia). (2012n).

${ }^{14}$ The National Center on Addiction and Substance Abuse at Columbia University (CASA Columbia). (2012n).

${ }^{15}$ The National Center on Addiction and Substance Abuse at Columbia University (CASA Columbia). (2012n). 
${ }^{16}$ The National Center on Addiction and Substance Abuse at Columbia University (CASA Columbia). (2012n).

${ }^{17}$ The National Center on Addiction and Substance Abuse at Columbia University (CASA Columbia). (2012n).

${ }^{18}$ The National Center on Addiction and Substance Abuse at Columbia University (CASA Columbia). (2012n).

${ }^{19}$ The National Center on Addiction and Substance Abuse at Columbia University (CASA Columbia). (2012n).

${ }^{20}$ The National Center on Addiction and Substance Abuse at Columbia University (CASA Columbia). (2012n).

${ }^{21}$ The National Center on Addiction and Substance Abuse at Columbia University (CASA Columbia). (2012n).

${ }^{22}$ The National Center on Addiction and Substance Abuse at Columbia University (CASA Columbia). (2012n).

${ }^{23}$ The National Center on Addiction and Substance Abuse at Columbia University (CASA Columbia). (2012n).

${ }^{24}$ The National Center on Addiction and Substance Abuse at Columbia University (CASA Columbia). (2012n).

${ }^{25}$ The National Center on Addiction and Substance Abuse at Columbia University (CASA Columbia). (2012n).

${ }^{26}$ The National Center on Addiction and Substance Abuse at Columbia University (CASA Columbia). (2012n).

${ }^{27}$ The National Center on Addiction and Substance Abuse at Columbia University (CASA Columbia). (2012n).

${ }^{28}$ Centers for Disease Control and Prevention. (2012a).

The National Center on Addiction and Substance Abuse at Columbia University (CASA Columbia). (2012n).

${ }^{29}$ The National Center on Addiction and Substance Abuse at Columbia University (CASA Columbia). (2012n).

${ }^{30}$ The National Center on Addiction and Substance Abuse at Columbia University (CASA Columbia). (2012n).

${ }^{31}$ The National Center on Addiction and Substance Abuse at Columbia University (CASA Columbia). (2012n).

32 The National Center on Addiction and Substance Abuse at Columbia University (CASA Columbia). (2012n).

${ }^{33}$ The National Center on Addiction and Substance Abuse at Columbia University (CASA Columbia). (2012n).

${ }^{34}$ The National Center on Addiction and Substance Abuse at Columbia University (CASA Columbia). (2012n).

${ }^{35}$ The National Center on Addiction and Substance Abuse at Columbia University (CASA Columbia). (2012n).

${ }^{36}$ The National Center on Addiction and Substance Abuse at Columbia University (CASA Columbia). (2012n).

${ }^{37}$ The National Center on Addiction and Substance Abuse at Columbia University (CASA Columbia). (2012n).

${ }^{38}$ The National Center on Addiction and Substance Abuse at Columbia University (CASA Columbia). (2012n).

${ }^{39}$ The National Center on Addiction and Substance Abuse at Columbia University (CASA Columbia). (2012n).

${ }^{40}$ The National Center on Addiction and Substance Abuse at Columbia University (CASA Columbia). (2012n).

${ }^{41}$ The National Center on Addiction and Substance Abuse at Columbia University (CASA Columbia). (2012n).

${ }^{42}$ The National Center on Addiction and Substance Abuse at Columbia University (CASA Columbia). (2012n).

${ }^{43}$ The National Center on Addiction and Substance Abuse at Columbia University (CASA Columbia). (2012n).

${ }^{44}$ The National Center on Addiction and Substance Abuse at Columbia University (CASA Columbia). (2012n).

${ }^{45}$ The National Center on Addiction and Substance Abuse at Columbia University (CASA Columbia). (2012n).

${ }^{46}$ The National Center on Addiction and Substance Abuse at Columbia University (CASA Columbia). (2012n).

${ }^{47}$ The National Center on Addiction and Substance Abuse at Columbia University (CASA Columbia). (2012n).

${ }^{48}$ The National Center on Addiction and Substance Abuse at Columbia University (CASA Columbia). (2012n).

${ }^{49}$ The National Center on Addiction and Substance Abuse at Columbia University (CASA Columbia). (2012n).

${ }^{50}$ The National Center on Addiction and Substance Abuse at Columbia University (CASA Columbia). (2012n).

${ }^{51}$ The National Center on Addiction and Substance Abuse at Columbia University (CASA Columbia). (2012n).

${ }^{52}$ The National Center on Addiction and Substance Abuse at Columbia University (CASA Columbia). (2012n).

${ }^{53}$ The National Center on Addiction and Substance Abuse at Columbia University (CASA Columbia). (2012n).

${ }^{54}$ The National Center on Addiction and Substance Abuse at Columbia University (CASA Columbia). (2012n).

${ }^{55}$ The National Center on Addiction and Substance Abuse at Columbia University (CASA Columbia). (2012n).

${ }^{56}$ The National Center on Addiction and Substance Abuse at Columbia University (CASA Columbia). (2012n).

${ }^{57}$ The National Center on Addiction and Substance Abuse at Columbia University (CASA Columbia). (2012n).

${ }^{58}$ The National Center on Addiction and Substance Abuse at Columbia University (CASA Columbia). (2012n).

${ }^{59}$ National Institute on Drug Abuse. (2011h).

Richter, L., \& Richter, D. M. (2001).

U.S. Department of Health and Human Services, Substance Abuse and Mental Health Services Administration. (2009).

${ }^{60}$ The National Center on Addiction and Substance Abuse (CASA) at Columbia University. (2011a).

${ }^{61}$ The National Center on Addiction and Substance Abuse at Columbia University (CASA Columbia). (2012n).

${ }^{62}$ Center for Substance Abuse Treatment. (1998b).

Dowling, G. J., Weiss, S., \& Condon, T. P. (2008).

Gambert, S. R. \& Albrecht, A. E. (2005).

Institute of Alcohol Studies. (2011).

Rigler, S. K. (2000). 
${ }^{63}$ Culberson, J. W., \& Ziska, M. (2008).

National Institutes of Health. (2011b).

Leshner, A. I. (2001).

${ }^{64}$ Substance Abuse and Mental Health Services Administration. (2011a).

Wu, L. T., \& Blazer, D. G. (2011).

${ }^{65}$ The National Center on Addiction and Substance Abuse at Columbia University (CASA Columbia). (2012n).

${ }^{66}$ Center for Substance Abuse Treatment. (2005b).

Chesher, N. J., Bousman, C. A., Gale, M., Norman, S. B., Twamley, E. W., Heaton, R. K., et al. (2011).

Epstein, J., Barker, P., Vorburger, M., \& Murtha, C. (2004).

Kessler, R. C., Anthony, J. C., Blazer, D. G., Bromet, E., Eaton, W. W., Kendler, K. S., et al. (1997).

McFall, M., \& Cook, J. (2006).

National Institute on Drug Abuse. (2010a).

The National Center on Addiction and Substance Abuse at Columbia University (CASA Columbia). (2012n).

Volkow, N. D. (2007a).

${ }^{67}$ The National Center on Addiction and Substance Abuse at Columbia University (CASA Columbia). (2012n).

${ }^{68}$ The National Center on Addiction and Substance Abuse at Columbia University (CASA Columbia). (2012n).

${ }^{69}$ The National Center on Addiction and Substance Abuse (CASA) at Columbia University. (2010).

The National Center on Addiction and Substance Abuse at Columbia University (CASA Columbia). (2012n).

${ }^{70}$ Chesher, N. J., Bousman, C. A., Gale, M., Norman, S. B., Twamley, E. W., Heaton, R. K., et al. (2011).

${ }^{71}$ Chan, Y. F., Dennis, M. L., \& Funk, R. R. (2008).

${ }^{72}$ Chesher, N. J., Bousman, C. A., Gale, M., Norman, S. B., Twamley, E. W., Heaton, R. K., et al. (2011).

Lasser, K., Boyd, J. W., Woolhandler, S., Himmelstein, D. U., McCormick, D., \& Bor, D. H. (2000).

${ }^{73}$ The National Center on Addiction and Substance Abuse at Columbia University (CASA Columbia). (2012n).

${ }^{74}$ Hughes, J. R., Hatsukami, D. K., Mitchell, J. E., \& Dahlgren, L. A. (1986).

Kalman, D., Morissette, S. B., \& George, T. P. (2005).

${ }^{75}$ The National Center on Addiction and Substance Abuse at Columbia University (CASA Columbia). (2012n).

${ }^{76}$ Price, J. L. (2010).

Drug Policy Alliance. (2009).

${ }^{77}$ Petrakis, I. L., Rosenheck, R., \& Desai, R. (2011).

${ }^{78}$ Calhoun, P. S., Elter, J. R., Jones, E. R., Jr., Kudler, H., \& Straits-Troster, K. (2008).

Eisen, S., Schultz, M. R., Vogt, D., Glickman, M. E., Elwy, R., Drainoni, M.-L., et al. (2012).

Hassija, C. M., Jakupcak, M., Maguen, S., \& Shipherd, J. C. (2012).

Institute of Medicine. (2008).

Marshall, B. D. L., Prescotta, M. R., Liberzonb, I., Tamburrinod, M. B., Calabresee, J. R., \& Galeaa, S. (2012).

RTI International. (2009).

Santiago, P. N., Wilk, J. E., Milliken, C. S., Castro, C. A., Engel, C. C., \& Hoge, C. W. (2010).

Shen, Y. C., Arkes, J., \& Williams, T. V. (2012).

Smith, B., Ryan, M. A., Wingard, D. L., Patterson, T. L., Slymen, D. J., \& Macera, C. A. (2008).

VHA Office of Public Health and Environmental Hazards. (2009).

Wilk, J. E., Bliese, P. D., Kim, P. Y., Thomas, J. L., McGurk, D., \& Hoge, C. W. (2010).

${ }^{79}$ Smith, B., Ryan, M. A., Wingard, D. L., Patterson, T. L., Slymen, D. J., \& Macera, C. A. (2008).

${ }^{80}$ Calhoun, P. S., Elter, J. R., Jones, E. R., Jr., Kudler, H., \& Straits-Troster, K. (2008).

${ }^{81}$ Hoge, C. W., Castro, C. A., Messer, S. C., McGurk, D., Cotting, D. I., \& Koffman, R. L. (2004).

Brown, R. L., Leonard, T., Saunders, L. A., \& Papasouliotis, O. (2001).

${ }^{82}$ Wilk, J. E., Bliese, P. D., Kim, P. Y., Thomas, J. L., McGurk, D., \& Hoge, C. W. (2010).

${ }^{83}$ Jacobson, I. G., Ryan, M. A., Hooper, T. I., Smith, T. C., Amoroso, P. J., Boyko, E. J., et al. (2008).

${ }^{84}$ Kehle, S. M., Ferrier-Auerbach, A. G., Meis, L. A., Arbisi, P. A., Erbes, C. R., \& Polusny, M. A. (2012).

${ }^{85}$ RTI International. (2009).

${ }^{86}$ The National Center on Addiction and Substance Abuse at Columbia University (CASA Columbia). (2012n).

${ }^{87}$ Ommaya, A. K., Salazar, A. M., Dannenberg, A. L., Ommaya, A., Chervinsky, A. B., \& Schwab, K. (1996).

${ }^{88}$ Heltemes, K. J., Dougherty, A. L., MacGregor, A. J., \& Galarneau, M. R. (2011).

${ }^{89}$ Seal, K. H., Cohen, G., Waldrop, A., Cohen, B. E., Maguen, S., \& Ren, L. (2011).

${ }^{90}$ The National Center on Addiction and Substance Abuse at Columbia University (CASA Columbia). (2012n).

${ }^{91}$ The National Center on Addiction and Substance Abuse at Columbia University (CASA Columbia). (2012n).

${ }^{92}$ The National Center on Addiction and Substance Abuse (CASA) at Columbia University. (2004). 
${ }^{93}$ The National Center on Addiction and Substance Abuse (CASA) at Columbia University. (2010).

${ }^{94}$ The National Center on Addiction and Substance Abuse at Columbia University (CASA Columbia). (2012n).

${ }^{95}$ The National Center on Addiction and Substance Abuse (CASA) at Columbia University. (2010).

${ }^{96}$ Burge, S. K., \& Schneider, F. D. (1999).

Centers for Disease Control and Prevention. (2002a).

Centers for Disease Control and Prevention. (2004b).

Centers for Disease Control and Prevention. (2008b).

Cook, R. L., Comer, D. M., Wiesenfeld, H. C., Chang, C. C., Tarter, R., Lave, J. R., et al. (2006).

Masoumi, M., Shahesmaeili, A., Mirzazadeh, A., Tavakoli, M., \& Ali, A. Z. (2010).

National Institute on Drug Abuse. (2011a).

Rehm, J., Samokhvalov, A. V., Neuman, M. G., Room, R., Parry, C., Lonnroth, K., et al. (2009).

Robert Wood Johnson Foundation. (2001).

Thygesen, L. C., Mikkelsen, P., Andersen, T. V., Tonnesen, H., Juel, K., Becker, U., et al. (2009).

${ }^{97}$ American Cancer Society. (2009).

American Diabetes Association. (2009).

Centers for Disease Control and Prevention. (2011b).

Centers for Disease Control and Prevention. (2008).

Centers for Disease Control and Prevention, Coordinating Center for Health Promotion. (2008).

Corso, P., Finkelstein, E., Miller, T., Fiebelkorn, I., \& Zaloshnja, E. (2006).

Insel, T. R. (2008).

Lloyd-Jones, D., Adams, R., Carnethon, M., De, S. G., Ferguson, T. B., Flegal, K., et al. (2009).

Mokdad, A. H., Marks, J. S., Stroup, D. F., \& Gerberding, J. L. (2004).

Robert Wood Johnson Foundation. (2001).

${ }^{98}$ Kochanek, K., Xu, J. Q., Murphy, S. L., Minino, A. M., \& Kung, H.-C. (2011).

The National Center on Addiction and Substance Abuse at Columbia University (CASA Columbia). (2012a).

U.S. Department of Health and Human Services. (2010a).

${ }^{99}$ The National Center on Addiction and Substance Abuse at Columbia University (CASA Columbia). (2012o).

${ }^{100}$ Centers for Disease Control and Prevention. (2010d).

${ }^{101}$ Moisse, K. (2011).

102 Thygesen, L. C., Mikkelsen, P., Andersen, T. V., Tonnesen, H., Juel, K., Becker, U., et al. (2009).

${ }^{103}$ Masoumi, M., Shahesmaeili, A., Mirzazadeh, A., Tavakoli, M., \& Ali, A. Z. (2010).

${ }^{104}$ Cook, R. L., Comer, D. M., Wiesenfeld, H. C., Chang, C. C., Tarter, R., Lave, J. R., et al. (2006).

${ }^{105}$ Centers for Disease Control and Prevention. (2004b).

Centers for Disease Control and Prevention. (2008).

${ }^{106}$ Burge, S. K., \& Schneider, F. D. (1999).

Robert Wood Johnson Foundation. (2001).

${ }^{107}$ National Institute on Drug Abuse. (2011a).

Centers for Disease Control and Prevention. (2002a).

${ }^{108}$ Centers for Disease Control and Prevention. (2004b).

Centers for Disease Control and Prevention. (2008a).

Centers for Disease Control and Prevention. (2008b).

Merrill, J. \& Fox, K. (1998).

The National Center on Addiction and Substance Abuse (CASA) at Columbia University. (1993).

The National Center on Addiction and Substance Abuse (CASA) at Columbia University. (1994).

${ }^{109}$ Califano, J. A. (2007).

National Institute on Drug Abuse. (2005a).

${ }^{110}$ American Academy of Pediatrics, Committee on Substance Abuse. (2001).

American Academy of Pediatrics, Committee on Substance Abuse and Committee on Children With Disabilities. (2000).

Asbridge, M., Hayden, J. A., \& Cartwright, J. L. (2012).

Center for Substance Abuse Treatment. (2003).

French, M. T., Rachal, V., Harwood, H. J., \& Hubbard, R. (1990).

McLellan, A. T., Lewis, D. C., O'Brien, C. P., \& Kleber, H. D. (2000).

Naimi, T. S., Lipscomb, L. E., Brewer, R. D., \& Gilbert, B. C. (2003).

National Institute on Drug Abuse. (2005a). 
Office of Juvenile Justice and Delinquency Prevention. (1998).

Office of National Drug Control Policy. (2004).

Reisfield, G. M., Goldberger, B. A., Gold, M. S., \& Dupont, R. L. (2012).

The National Center on Addiction and Substance Abuse (CASA) at Columbia University. (1999).

The National Center on Addiction and Substance Abuse (CASA) at Columbia University. (2010).

The National Center on Addiction and Substance Abuse (CASA) at Columbia University. (2011a).

U.S. Department of Health and Human Services. (2000).

U.S. Department of Health and Human Services. (2006).

Volkow, N. D., \& Li, T.-K. (2005).

Wechsler, H., Davenport, A., Dowdall, G., Moeykens, B., \& Castillo, S. (1994).

111 Ray, G. T., Mertens, J. R., \& Weisner, C. (2007).

112 Andersen, S. L., \& Teicher, M. H. (2009).

Bava, S., Frank, L. R., McQueeny, T., Schweinsburg, B. C., Schweinsburg, A. D., \& Tapert, S. F. (2009).

Benowitz, N. L. (2010).

Chambers, R. A., Taylor, J. R., \& Potenza, M. N. (2003).

Crews, F., He, J., \& Hodge, C. (2007).

Crews, F. T., Mdzinarishvili, A., Kim, D., He, J., \& Nixon, K. (2006).

DeWit, D. J., Adlaf, E. M., Offord, D. R., \& Ogborne, A. C. (2000).

Doremus-Fitzwater, T. L., Varlinskaya, E. I., \& Spear, L. P. (2010).

Ehrlich, M. E., Sommer, J., Canas, E., \& Unterwald, E. M. (2002).

Grant, B. F., \& Dawson, D. A. (1997).

Grant, B. F., \& Dawson, D. A. (1998).

Jacobus, J., McQueeny, T., Bava, S., Schweinsburg, B. C., Frank, L. R., Yang, T. T., et al. (2009).

Lopez-Larson, M. P., Bogorodzki, P., Rogowska, J., McGlade, E., King, J. B., Terry, J., et al. (2011).

McQueeny, T., Schweinsburg, B. C., Schweinsburg, A. D., Jacobus, J., Bava, S., Frank, L. R., et al. (2009).

Medina, K. L., Schweinsburg, A. D., Cohen-Zion, M., Nagel, B. J., \& Tapert, S. F. (2007).

Monti, P. M., Miranda, R., Jr., Nixon, K., Sher, K. J., Swartzwelder, H. S., Tapert, S. F., et al. (2005).

Placzek, A. N., Zhang, T. A., \& Dani, J. A. (2009).

Rubinstein, M. L., Benowitz, N. L., Auerback, G. M., \& Moscicki, A. B. (2009).

Rubinstein, M. L., Luks, T. L., Moscicki, A. B., Dryden, W., Rait, M. A., \& Simpson, G. V. (2011).

Slotkin, T. A. (2002).

Squeglia, L. M., Jacobus, J., \& Tapert, S. F. (2009).

Squeglia, L. M., Spadoni, A. D., Infante, M. A., Myers, M. G., \& Tapert, S. F. (2009).

113 The National Center on Addiction and Substance Abuse (CASA) at Columbia University. (2001b).

${ }^{114}$ VanDeMark, N. R., Russel, L. A., O'Keefe, M., Finkelstein, N., Noether, C. D., \& Gampel, J. C. (2005).

${ }^{115}$ Ray, G. T., Mertens, J. R., \& Weisner, C. (2007).

${ }^{116}$ Centers for Disease Control and Prevention. (2008).

U.S. Department of Health and Human Services. (2010a).

${ }^{117}$ Centers for Disease Control and Prevention. (2011f).

${ }^{118}$ National Institute on Drug Abuse. (2005a).

Ockene, I. S., \& Miller, N. H. (1997).

${ }^{119}$ U.S. Department of Health and Human Services. (2010a).

Yacoub, R., Habib, H., Lahdo, A., Al, A. R., Varjabedian, L., Atalla, G., et al. (2010).

${ }^{120}$ Centers for Disease Control and Prevention. (2010c).

${ }^{121}$ Centers for Disease Control and Prevention. (1992).

Centers for Disease Control and Prevention. (2011).

${ }^{122}$ Centers for Disease Control and Prevention. (2004a).

Centers for Disease Control and Prevention. (2008b).

${ }^{123}$ National Institute on Drug Abuse. (2007b).

Centers for Disease Control and Prevention, Coordinating Center for Health Promotion. (2008).

${ }^{124}$ National Institute on Drug Abuse. (2007b).

U.S. Department of Health and Human Services. (2010a).

125 Behrman, R. E., \& Butler, A. S. (2007).

${ }^{126}$ Centers for Disease Control and Prevention. (2007).

${ }^{127}$ Moore, M. L., \& Zaccaro, D. J. (2000). 
Visscher, W. A., Feder, M., Burns, A. M., Brady, T. M., \& Bray, R. M. (2003).

${ }^{128}$ Datar, A., \& Jacknowitz, A. (2009).

Goldenberg, R. L., \& Culhane, J. F. (2007).

McCormick, M. C. (1985).

Schendel, D. E., Stockbauer, J. W., Hoffman, H. J., Herman, A. A., Berg, C. J., \& Schramm, W. F. (1997).

Singh, G. K., \& Yu, S. M. (1995).

Strang-Karlsson, S., Andersson, S., Paile-Hyvarinen, M., Darby, D., Hovi, P., Raikkonen, K., et al. (2009).

Visscher, W. A., Feder, M., Burns, A. M., Brady, T. M., \& Bray, R. M. (2003).

${ }^{129}$ Fergusson, D. M., Woodward, L. J., \& Horwood, J. (1998).

${ }^{130}$ Mick, E., Biederman, J., Faraone, S., Julie, S., \& Kleinman, S. (2002).

National Institute on Drug Abuse. (2005a).

Richter, L., \& Richter, D. M. (2001).

Toschke, A. M., Montgomery, M. A., Pfeiffer, U., \& von Kries, R. (2003).

Wakschlag, L. S., Pickett, K. E., Cook Jr, E., Benowitz, N., \& Leventhal, B. (2002).

Toschke, A. M., Montgomery, M. A., Pfeiffer, U., \& von Kries, R. (2003).

${ }^{131}$ Goodwin, R. D., Lewinsohn, P. M., \& Seeley, J. R. (2005).

Johnson, J. G., Cohen, P., Pine, D. S., Klein, D. F., Kasen, S., \& Brook, J. S. (2000).

The National Center on Addiction and Substance Abuse (CASA) at Columbia University. (2007a).

${ }^{132}$ Glantz, S. A., \& Parmley, W. W. (1991).

U.S. Department of Health and Human Services. (2010a).

133 California Environmental Protection Agency. (2005).

Ontario Camapign for Action on Tobacco. (2007).

${ }^{134}$ Larsson, M. L., Frisk, M., Hallstrom, J., Kiviloog, J., \& Lundback, B. (2001).

${ }^{135}$ Miller, M. D., Marty, M. A., Broadwin, R., Johnson, K. C., Salmon, A. G., Winder, B., et al. (2007).

Ontario Camapign for Action on Tobacco. (2007).

136 CBS News. (2011).

Rabin, R. C. (2009).

Rehan, V. K., Sakurai, R., \& Torday, J. S. (2011).

Sleiman, M., Gundel, L. A., Pankow, J. F., Jacob, P., III, Singer, B. C., \& Destaillats, H. (2010).

137 Sleiman, M., Gundel, L. A., Pankow, J. F., Jacob, P., III, Singer, B. C., \& Destaillats, H. (2010).

${ }^{138}$ Mokdad, A. H., Marks, J. S., Stroup, D. F., \& Gerberding, J. L. (2004).

139 The National Center on Addiction and Substance Abuse at Columbia University (CASA Columbia). (2012a).

140 The National Center on Addiction and Substance Abuse at Columbia University (CASA Columbia). (2012o).

${ }^{141}$ Bonnie, R. J., \& O'Connell, M. E. (2004).

${ }^{142}$ U.S. Department of Transportation, National Highway Traffic Safety Administration. (2011).

143 The National Center on Addiction and Substance Abuse at Columbia University (CASA Columbia). (2012f).

${ }^{144}$ Corrao, G., Bagnardi, V., Zambon, A., \& La, V. C. (2004).

Corrao, G., Rubbiati, L., Zambon, A., \& Arico, S. (2002).

King, M. B. (1986).

National Library of Medicine. (2011a).

${ }^{145}$ Krishnamoorthy, S., Lip, G. Y. J., \& Lane, D. A. (2009).

MacMahon, S. (1987).

Rehm, J., Gmel, G., Sempos, C. T., \& Trevisan, M. (2003).

${ }^{146}$ Castaneda, R., Sussman, N., Westreich, L., Levy, R., \& O'Malley, M. (1996).

Centers for Disease Control and Prevention. (2009a).

Flensborg-Madsen, T., Becker, U., Gronbaek, M., Knop, J., Sher, L., \& Mortensen, E. L. (2011).

${ }^{147}$ Heron, M. (2007).

Schiff, E. R. (1997).

National Library of Medicine. (2010h).

National Library of Medicine. (2011b).

${ }^{148}$ Kelly, J. P., Kaufman, D. W., Koff, R. S., Laszlo, A., Wiholm, B., \& Shapiro, S. (1995).

Lesher, S. D., \& Lee, Y. T. (1989).

${ }^{149}$ Baan, R., Straif, K., Grosse, Y., Secretan, B., El, G. F., Bouvard, V., et al. (2007).

${ }^{150}$ Forsyth, C. B., Tang, Y., Shaikh, M., Zhang, L., \& Keshavarzian, A. (2010).

${ }^{151}$ Kesmodel, U., Wisborg, K., Olsen, S. F., Henriksen, T. B., \& Secher, N. J. (2002a). 
Kesmodel, U., Wisborg, K., Olsen, S. F., Henriksen, T. B., \& Secher, N. J. (2002b).

Lui, S., Terplan, M., \& Smith, E. J. (2008).

Spohr, H. L., Willms, J., \& Steinhausen, H. C. (1993).

National Library of Medicine. (2011c).

${ }^{152}$ Stratton, K., Howe, C., Battaglia, F., Institute of Medicine, Division of Biobehavioral Sciences and Mental Disorders, \& Committee to Study Fetal Alcohol Syndrome (1996).

153 The National Center on Addiction and Substance Abuse at Columbia University (CASA Columbia). (2012c).

154 The National Center on Addiction and Substance Abuse at Columbia University (CASA Columbia). (2012o).

155 The National Center on Addiction and Substance Abuse at Columbia University (CASA Columbia). (2012f).

156 Substance Abuse and Mental Health Services Administration. (2010b).

${ }^{157}$ National Institute on Drug Abuse. (2006a).

${ }^{158}$ Robert Wood Johnson Foundation. (2001).

Volkow, N. D. (2007b).

${ }^{159}$ National Institute on Drug Abuse. (2010d).

National Institute on Drug Abuse. (2012b).

${ }^{160}$ Han, B., Gfroerer, J. C., \& Colliver, J. D. (2010).

${ }^{161}$ Fergusson, D. M., \& Horwood, L. J. (2004).

Moore, T. H. M., Zammit, S., Lingford-Hughes, A., Barnes, T. R. E., Jones, P. B., Burke, M., et

al. (2007).

National Institute on Drug Abuse. (2011f).

Rey, J. M. (2007).

Smit, F., Bolier, L., \& Cuijpers, P. (2004).

${ }^{162}$ Kuepper, R., van Os, J., Lieb, R., Wittchen, H.-U., Hofler, M., \& Henquet, C. (2011).

Pierre, J. M. (2011).

${ }^{163}$ Han, B., Gfroerer, J. C., \& Colliver, J. D. (2010).

164 National Institute on Drug Abuse. (2010c).

National Institute on Drug Abuse. (2010e).

National Institute on Drug Abuse. (2010d).

U.S. Department of Health and Human Services. (1989).

165 American Psychiatric Association. (1994).

${ }^{166}$ National Abandoned Infants Assistance Resource Center. (2008).

167 The National Center on Addiction and Substance Abuse (CASA) at Columbia University. (2006).

${ }^{168}$ Brady, J. P., Posner, M., Lang, C., \& Rosati, M. J. (1994).

${ }^{169}$ National Institute on Drug Abuse. (2009g).

${ }^{170}$ Volkow, N. D. (2007b).

Visscher, W. A., Feder, M., Burns, A. M., Brady, T. M., \& Bray, R. M. (2003).

${ }^{171}$ National Institute on Drug Abuse. (2009g).

Richter, L., \& Richter, D. M. (2001).

Volkow, N. D. (2007b).

${ }^{172}$ Centers for Disease Control and Prevention. (2011j).

173 Paulozzi, L. J., \& Xi, Y. (2008).

${ }^{174}$ Centers for Disease Control and Prevention, National Center for Injury Prevention and Control. (2011).

175 The National Center on Addiction and Substance Abuse at Columbia University (CASA Columbia). (2012o).

176 The National Center on Addiction and Substance Abuse at Columbia University (CASA Columbia). (2012f).

${ }^{177}$ National Institute on Drug Abuse. (2009b).

${ }^{178}$ White, A. G., Birnbaum, H. G., Mareva, M. N., Daher, M., Vallow, S., Schein, J., et al. (2005).

${ }^{179}$ National Institute on Drug Abuse. (2011d).

${ }^{180}$ U.S. Department of Health and Human Services, National Institutes of Health, National Institute on Drug Abuse. (2009).

${ }^{181}$ National Library of Medicine. (2010e).

National Library of Medicine. (2010i).

${ }^{182}$ Barker, M. J., Greenwood, K. M., Jackson, M., \& Crowe, S. F. (2004).

Busto, U., Sellers, E. M., Naranjo, C. A., Cappell, H., Sanchez-Craig, M., \& Sykora, K. (1986).

Golombok, S., Moodley, P., \& Lader, M. (1988).

Longo, L. P., \& Johnson, B. (2000). 
Tanaka, E. (2002).

${ }^{183}$ National Library of Medicine. (2010j).

Drug Information Online. (2011).

National Library of Medicine. (2010b).

${ }^{184}$ National Institute on Drug Abuse. (2009).

${ }^{185}$ National Library of Medicine. (2010a).

National Library of Medicine. (2010j). 


\section{Chapter IV \\ Notes}

${ }^{1}$ The National Center on Addiction and Substance Abuse at Columbia University (CASA Columbia). (2012n).

${ }^{2}$ Anderson, P., Aromaa, S., Rosenbloom, D., \& Enos, G. (2008).

Fleming, M. F. (2004).

${ }^{3}$ The National Center on Addiction and Substance Abuse at Columbia University (CASA Columbia). (2012n).

${ }^{4}$ Anderson, P., Aromaa, S., Rosenbloom, D., \& Enos, G. (2008).

${ }^{5}$ Bauermeister, J. A., Zimmerman, M. A., Gee, G. C., Caldwell, C., \& Xue, Y. (2009).

Bruce, K. D., \& Hanson, M. A. (2010).

Mertens, M. J. (2010).

National Research Council and Institute of Medicine (2009).

van Abeelen, A. F. M., Elias, S. G., Bossuyt, P. M. M., Grobbee, D. E., van der Schouw, Y. T., Roseboom, T. J., et al. (2011).

${ }^{6}$ The National Center on Addiction and Substance Abuse (CASA) at Columbia University. (2011a).

${ }^{7}$ Merikangas, K. R., \& McClair, V. L. (2012).

The National Center on Addiction and Substance Abuse (CASA) at Columbia University. (2003).

The National Center on Addiction and Substance Abuse (CASA) at Columbia University. (2011a).

${ }^{8}$ American Society of Addiction Medicine. (2011b).

Ersche, K. D., Jones, P. S., Williams, G. B., Turton, A. J., Robbins, T. W., \& Bullmore, E. T. (2012).

Gitlow, S. (2011).

Whelan, R., Conrod, P. J., Poline, J. B., Lourdusamy, A., Banaschewski, T., Barker, G. J., et al. (2012).

${ }^{9}$ Brook, J. S., Whiteman, M., Cohen, P., \& Tanaka, J. S. (1992).

Ensminger, M. E., Brown, C. H., \& Kellam, S. G. (1982).

Rose, R. J. (1998).

${ }^{10}$ American Academy of Child and Adolescent Psychiatry. (2009).

American Psychological Association. (1995).

Webster-Stratton, C., \& Taylor, T. (2001).

${ }^{11}$ Kim, M. J., Catalano, R. F., Haggerty, K. P., \& Abbott, R. D. (2011).

${ }^{12}$ Wong, M. M., Brower, K. J., \& Zucker, R. A. (2009).

${ }^{13}$ Ellason, J. W., \& Ross, C. A. (1996).

Shin, S. H., Hong, H. G., \& Hazen, A. L. (2010).

Topitzes, J., Mersky, J. P., \& Reynolds, A. J. (2010).

${ }^{14}$ Volkow, N. D. (2007b).

${ }^{15}$ Crews, F. T., \& Boettiger, C. A. (2009).

Volkow, N. D. (2007b).

Riggs, N. R., \& Greenberg, M. T. (2009).

${ }^{16}$ Steinberg, L. (2007).

Institute of Medicine and National Research Council. (2011).

Volkow, N. D. (2007b).

${ }^{17}$ The National Center on Addiction and Substance Abuse (CASA) at Columbia University. (2003b).

${ }^{18}$ Arnett, J. J. (2005).

${ }^{19}$ The National Center on Addiction and Substance Abuse (CASA) at Columbia University. (2007b).

${ }^{20}$ Arnett, J. J. (2005).

Gibb, S. J., Fergusson, D. M., \& Horwood, L. J. (2011).

Wiesner, M., Windle, M., \& Freeman, A. (2005).

${ }^{21}$ Arnett, J. J. (2005).

The National Center on Addiction and Substance Abuse (CASA) at Columbia University. (2003b).

Grant, B. F., \& Dawson, D. A. (1997).

${ }^{22}$ Volkow, N. D. (2007b).

${ }^{23}$ Frone, M. R., Barnes, G. M., \& Farrell, M. P. (1994).

Frone, M. R., Russell, M., \& Cooper, M. L. (1993).

Pelham, W. E., \& Lang A. R. (1999).

${ }^{24}$ Burton, L. M. (1992). 
Center for Substance Abuse Treatment. (1998b).

Evercare. (2006).

Glass, T. A., Prigerson, H., Kasl, S. V., \& Mendes de Leon, C. F. (1995).

Jennison, K. M. (1992).

Longoria, R. A. (2005).

${ }^{25}$ Brennan, P. L., \& Moos, R. H. (1996).

Finlayson, R. E., Hurt, R. D., Davis, L. J., \& Morse, R. M. (1988).

Klein, W. C., \& Jess, C. (2002).

Richardson, V. E., \& Kilty, K. M. (1995).

Shaw, B. A., Agahi, N., \& Krause, N. (2011).

Scheinholtz, M. K. (2011).

${ }^{26}$ Atkinson, R. M., Ganzini, L., \& Bernstein, M. J. (1992).

Jinks, M. J., \& Raschko, R. R. (1990).

${ }^{27}$ Center for Substance Abuse Treatment. (2001).

Dowling, G. J., Weiss, S., \& Condon, T. P. (2008).

Gambert, S. R. \& Albrecht, A. E. (2005).

Institute of Alcohol Studies. (2011).

${ }^{28}$ Atkinson, R. M., Ganzini, L., \& Bernstein, M. J. (1992).

Barry, P. P. (1986).

Braude, M. C. (1986).

Dufour, M. C., Archer, L., \& Gordis, E. (1992).

Gambert, S. R. \& Albrecht, A. E. (2005).

Greenblatt, D. J., Sellers, E. M., \& Shader, R. I. (1982).

Institute of Alcohol Studies. (2011).

Lamy, P. P. (1988).

Lowinson, J. H., Ruiz, P., Millman, R. B., \& Langrod, J. G. (eds.). (2005).

Montamat, S. C., Cusack, B. J., \& Vestal, R. E. (1989).

Vestal, R. E., McGuire, E. A., Tobin, J. D., Andres, R., Norris, A. H., \& Mezey, E. (1977).

Vogel-Sprott, M., \& Barrett, P. (1984).

${ }^{29}$ Han, B., Gfroerer, J. C., Colliver, J. D., \& Penne, M. A. (2009).

Patterson, T. L., \& Jeste, D. V. (1999).

${ }^{30}$ Culberson, J. W., \& Ziska, M. (2008).

National Institutes of Health. (2011b).

Leshner, A. I. (2001c).

National Institute on Alcohol Abuse and Alcoholism. (1988).

National Institute on Drug Abuse. (2011c).

${ }^{31}$ Armstrong, T. D., \& Costello, E. J. (2002).

Chan, Y. F., Dennis, M. L., \& Funk, R. R. (2008).

Epstein, J., Barker, P., Vorburger, M., \& Murtha, C. (2004).

Gould, T. J. (2010).

Grella, C. E., Hser, Y.-I., Vandana, J., \& Rounds-Bryant, J. (2001).

Rohde, P., Kahler, C. W., Lewinsohn, P. M., \& Brown, R. A. (2004).

Upadhyaya, H. P., Deas, D., Brady, K. T., \& Kruesi, M. (2002).

${ }^{32}$ Center for Substance Abuse Treatment. (2005b).

${ }^{33}$ Susser, M. (1985).

${ }^{34}$ National Institute on Alcohol Abuse and Alcoholism. (2005c).

${ }^{35}$ Babor, T. F., \& Kadden, R. M. (2005).

Babor, T. F., McRee, B. G., Kassebaum, P. A., Grimaldi, P. L., Ahmed, K., \& Bray, J. (2007).

${ }^{36}$ American Society of Addiction Medicine. (1997).

Centers for Disease Control and Prevention. (2010e).

Centers for Disease Control and Prevention. (2011k).

${ }^{37}$ Diemert, L. M., Bondy, S. J., Victor, J. C., Cohen, J. E., Brown, K. S., Ferrence, R., et al. (2008).

${ }^{38}$ Hungerford, D. (2009).

${ }^{39}$ National Institute on Alcohol Abuse and Alcoholism. (2005a).

${ }^{40}$ National Institute on Alcohol Abuse and Alcoholism. (2011a). 
${ }^{41}$ Smith, P. C., Schmidt, S. M., Allensworth-Davies, D., \& Saitz, R. (2010).

${ }^{42}$ Smith, P. C., Schmidt, S. M., Allensworth-Davies, D., \& Saitz, R. (2010).

${ }^{43}$ National Institute on Drug Abuse. (2012c).

${ }^{44}$ Higgins-Biddle, J. C., PhD, Assistant Professor (Retired), Community Medicine and Health Care, University of Connecticut Health Center (personal communication, February 28, 2012).

${ }^{45}$ Gorber, S. C., Schofield-Hurwitz, S., Hardt, J., Levasseur, G., \& Tremblay, M. (2009).

Parker, D. R., Lasater, T. M., Windsor, R., Wilkins, J., \& Upegui, D. I. H. J. (2002).

${ }^{46}$ Fiellin, D. A., Reid, M. C., \& O'Connor, P. G. (2000).

${ }^{47}$ Center for Substance Abuse Treatment. (1995a).

Dolan, K., Rouen, D., \& Kimber, J. (2004).

${ }^{48}$ Center for Substance Abuse Treatment. (1995a).

${ }^{49}$ National Institute on Alcohol Abuse and Alcoholism. (2002).

${ }^{50}$ National Institute on Alcohol Abuse and Alcoholism. (2002).

Saunders, J. B., Aasland, O. G., Babor, T. F., de la Fuente, J. R., \& Grant, M. (1993).

${ }^{51}$ National Institute on Alcohol Abuse and Alcoholism. (2002).

${ }^{52}$ Center for Substance Abuse Treatment. (1995a).

${ }^{53}$ Center for Substance Abuse Treatment. (1995a).

U.S. Department of Health and Human Services, Substance Abuse and Mental Health Services Administration, Center for Substance Abuse Treatment. (2006).

Lab Tests Online. (2011).

Wojcik, M. H., \& Hawthorne, J. S. (2007).

${ }^{54}$ American Civil Liberties Union. (1999).

American Civil Liberties Union, Drug Policy Alliance. (2006).

${ }^{55}$ American Civil Liberties Union, Drug Policy Alliance. (2006).

${ }^{56}$ DuPont, R. L., \& Baumgartner, W. A. (1995).

Reisfield, G. M., Salazar, E., \& Bertholf, R. L. (2007).

${ }^{57}$ Allen, J. P., \& Litten, R. Z. (2001).

National Institute on Alcohol Abuse and Alcoholism. (2002).

${ }^{58}$ Jacobs, W. S., Repetto, M., Vinson, S., Pomm, R., \& Gold, M. S. (2004).

Merlo, L. J., \& Gold, M. (2008).

Reisfield, G. M., Graham, N. A., \& Gold, M. S. (2011).

${ }^{59}$ National Institute on Drug Abuse. (2009e).

${ }^{60}$ Barnes, H. N., \& Samet, J. H. (1997).

${ }^{61}$ Babor, T. F., McRee, B. G., Kassebaum, P. A., Grimaldi, P. L., Ahmed, K., \& Bray, J. (2007).

Fleming, M. F. (2004).

${ }^{62}$ National Association of State Alcohol and Drug Abuse Directors. (2006).

${ }^{63}$ Babor, T. F., Higgins-Biddle, J. C., Dauser, D., Burleson, J. A., Zarkin, G. A., \& Bray, J. (2006).

Moyer, A., Finney, J. W., Swearingen, C. E., \& Vergun, P. (2002).

${ }^{64}$ National Institute on Drug Abuse. (2009e).

${ }^{65}$ Substance Abuse and Mental Health Services Administration, Center for Substance Abuse Treatment. (1999).

Clinical Practice Guideline Treating Tobacco Use and Dependence 2008 Update Panel, Liaisons, and Staff. (2008).

${ }^{66}$ Fiore,M., U.S. Department of Health and Human Services. (2008).

Clinical Practice Guideline Treating Tobacco Use and Dependence 2008 Update Panel, Liaisons, and Staff. (2008).

${ }^{67}$ Clinical Practice Guideline Treating Tobacco Use and Dependence 2008 Update Panel, Liaisons, and Staff. (2008).

${ }^{68}$ Clinical Practice Guideline Treating Tobacco Use and Dependence 2008 Update Panel, Liaisons, and Staff. (2008).

${ }^{69}$ Elliott, V. S. (2005).

${ }^{70}$ Aveyard, P., Begh, R., Parsons, A., \& West, R. (2012).

${ }^{71}$ Rohsenow, D. J., Monti, P. M., Colby, S. M., \& Martin, R. A. (2002).

${ }^{72}$ Substance Abuse and Mental Health Services Administration, Center for Substance Abuse Treatment. (1999).

${ }^{73}$ Nathan, P. E., \& Gorman, J. M. (2007).

${ }^{74}$ National Institute on Alcohol Abuse and Alcoholism. (1999).

${ }^{75}$ National Institute on Alcohol Abuse and Alcoholism. (1999).

${ }^{76}$ Bien, T. H., Miller, W. R., \& Tonigan, J. S. (1993). 
Henry-Edwards, S., Humeniuk, R., Ali, R., Monteiro, M., \& Poznyak, V. (2003).

Miller, W. R. \& Sanchez, V. C. (1993).

National Institute on Alcohol Abuse and Alcoholism. (1999).

${ }^{77}$ National Institute on Alcohol Abuse and Alcoholism. (1999).

${ }^{78}$ Bien, T. H., Miller, W. R., \& Tonigan, J. S. (1993).

National Institute on Alcohol Abuse and Alcoholism. (1999).

${ }^{79}$ Babor, T. F., McRee, B. G., Kassebaum, P. A., Grimaldi, P. L., Ahmed, K., \& Bray, J. (2007).

${ }^{80}$ Zanis, D. A., Melochick, J., \& Wagner, J. (2011).

${ }^{81}$ Aseltine, R. H. (2010).

Bertholet, N., Daeppen, J., Wietlisbach, V., Fleming, M., \& Burnand, B. (2005).

Gryczynski, J., Mitchell, S. G., Peterson, T. R., \& Gonzales, A. (2011).

National Association of State Alcohol and Drug Abuse Directors. (2006).

Schaus, J. F., Sole, M. L., McCoy, T. P., Mullett, N., \& O'Brien, M. C. (2009).

Spirito, A., Sindelar-Manning, H., Colby, S. M., Barnett, N. P., Lewander, W., Rohsenow, D. J., et al. (2011).

Sullivan, L. E., Tetrault, J. M., Braithwaite, R. S., Turner, B. J., \& Fiellin, D. A. (2011).

${ }^{82}$ Baker, A., Lee, N. K., Claire, M., Lewin, T. J., Grant, T., Pohlman, S., et al. (2005).

Bernstein, J., Bernstein, E., Tassiopoulos, K., Heeren, T., Levenson, S., \& Hingson, R. (2005).

Bernstein, E., Edwards, E., Dorfman, D., Heeren, T., Bliss, C., \& Bernstein, J. (2009).

D'Amico, E. J., Miles, J. N. V., Stern, S. A., \& Meredith, L. S. (2008).

Gryczynski, J., Mitchell, S. G., Peterson, T. R., \& Gonzales, A. (2011).

Madras, B. K., Compton, W. M., Avula, D., Stegbauer, T., Stein, J. B., \& Clark, H. W. (2009).

Magill, M., Barnett, N. P., Apodaca, T. R., Rohsenow, D. J., \& Monti, P. M. (2009).

Schonfeld, L., King-Kallimanis, B. L., Duchene, D. M., Etheridge, R. L., Herrera, J. R., Barry, K. L., et al. (2010).

Stein, M. D., Hagerty, C. E., Herman, D. S., Phipps, M. G., \& Anderson, B. J. (2011).

${ }^{83}$ Aseltine, R. H. (2010).

Bernstein, E., Edwards, E., Dorfman, D., Heeren, T., Bliss, C., \& Bernstein, J. (2009).

Bertholet, N., Daeppen, J., Wietlisbach, V., Fleming, M., \& Burnand, B. (2005).

D'Amico, E. J., Miles, J. N. V., Stern, S. A., \& Meredith, L. S. (2008).

Gryczynski, J., Mitchell, S. G., Peterson, T. R., \& Gonzales, A. (2011).

Madras, B. K., Compton, W. M., Avula, D., Stegbauer, T., Stein, J. B., \& Clark, H. W. (2009).

Magill, M., Barnett, N. P., Apodaca, T. R., Rohsenow, D. J., \& Monti, P. M. (2009).

Moore, A. A., Blow, F. C., Hoffing, M., Welgreen, S., Davis, J. W., Ramirez, K. D., et al. (2010).

Schaus, J. F., Sole, M. L., McCoy, T. P., Mullett, N., \& O'Brien, M. C. (2009).

Spirito, A., Sindelar-Manning, H., Colby, S. M., Barnett, N. P., Lewander, W., Rohsenow, D. J., et al. (2011).

Stein, M. D., Hagerty, C. E., Herman, D. S., Phipps, M. G., \& Anderson, B. J. (2011).

Sullivan, L. E., Tetrault, J. M., Braithwaite, R. S., Turner, B. J., \& Fiellin, D. A. (2011).

${ }^{84}$ Baker, A., Lee, N. K., Claire, M., Lewin, T. J., Grant, T., Pohlman, S., et al. (2005).

Fleming, M. F., Mundt, M. P., French, M. T., Manwell, L. B., Stauffacher, E. A., \& Barry, K. L. (2002).

Madras, B. K., Compton, W. M., Avula, D., Stegbauer, T., Stein, J. B., \& Clark, H. W. (2009).

${ }^{85}$ Fleming, M. F., Mundt, M. P., French, M. T., Manwell, L. B., Stauffacher, E. A., \& Barry, K. L. (2002).

${ }^{86}$ Fleming, M. F., Mundt, M. P., French, M. T., Manwell, L. B., Stauffacher, E. A., \& Barry, K. L. (2002).

${ }^{87}$ Madras, B. K., Compton, W. M., Avula, D., Stegbauer, T., Stein, J. B., \& Clark, H. W. (2009).

${ }^{88}$ Babor, T. F., McRee, B. G., Kassebaum, P. A., Grimaldi, P. L., Ahmed, K., \& Bray, J. (2007).

Krupski, A., Sears, J. M., Joesch, J. M., Estee, S., He, L., Dunn, C., et al. (2010).

${ }^{89}$ Babor, T. F., McRee, B. G., Kassebaum, P. A., Grimaldi, P. L., Ahmed, K., \& Bray, J. (2007).

${ }^{90}$ Clinical Practice Guideline Treating Tobacco Use and Dependence 2008 Update Panel, Liaisons, and Staff. (2008).

${ }^{91}$ Rice, V. H., \& Stead, L. F. (2008).

${ }^{92}$ Ong, M. K., Zhou, Q., \& Sung, H. Y. (2011).

${ }^{93}$ Aseltine, R. H. (2010).

Babor, T. F., Higgins-Biddle, J. C., Dauser, D., Burleson, J. A., Zarkin, G. A., \& Bray, J. (2006).

Bertholet, N., Daeppen, J., Wietlisbach, V., Fleming, M., \& Burnand, B. (2005).

Moore, A. A., Blow, F. C., Hoffing, M., Welgreen, S., Davis, J. W., Ramirez, K. D., et al. (2010).

Schaus, J. F., Sole, M. L., McCoy, T. P., Mullett, N., \& O'Brien, M. C. (2009).

Spirito, A., Sindelar-Manning, H., Colby, S. M., Barnett, N. P., Lewander, W., Rohsenow, D. J., et al. (2011). 
Sullivan, L. E., Tetrault, J. M., Braithwaite, R. S., Turner, B. J., \& Fiellin, D. A. (2011).

U.S. Preventive Services Task Force. (2004a).

${ }^{94}$ Fleming, M. F., Mundt, M. P., French, M. T., Manwell, L. B., Stauffacher, E. A., \& Barry, K. L. (2002).

${ }^{95}$ Schermer, C. R., Moyers, T. B., Miller, W. R., \& Bloomfield, L. A. (2006).

${ }^{96}$ D'Onofrio, G., \& Degutis, L. C. (2002).

${ }^{97}$ Kip, M. J., Neumann, T., Jugel, C., Kleinwaechter, R., Weiss-Gerlach, E., Guill, M. M., et al. (2008).

${ }^{98}$ Gentilello, L. M., Rivara, F. P., Donovan, D. M., Jurkovich, G. J., Daranciang, E., Dunn, C. W., et al. (1999).

${ }^{99}$ National Association of State Alcohol and Drug Abuse Directors. (2006).

Whitlock, E. P., Polen, M. R., Green, C. A., Orleans, T., \& Klein, J. (2004).

${ }^{100}$ Whitlock, E. P., Polen, M. R., Green, C. A., Orleans, T., \& Klein, J. (2004).

${ }^{101}$ U.S. Preventive Services Task Force. (2008).

U.S. Preventive Services Task Force. (2011).

${ }^{102}$ Bernstein, E., Edwards, E., Dorfman, D., Heeren, T., Bliss, C., \& Bernstein, J. (2009).

D'Amico, E. J., Miles, J. N. V., Stern, S. A., \& Meredith, L. S. (2008).

Madras, B. K., Compton, W. M., Avula, D., Stegbauer, T., Stein, J. B., \& Clark, H. W. (2009).

Magill, M., Barnett, N. P., Apodaca, T. R., Rohsenow, D. J., \& Monti, P. M. (2009).

Stein, M. D., Hagerty, C. E., Herman, D. S., Phipps, M. G., \& Anderson, B. J. (2011).

World Health Organization. (2008).

${ }^{103}$ Madras, B. K., Compton, W. M., Avula, D., Stegbauer, T., Stein, J. B., \& Clark, H. W. (2009).

${ }^{104}$ Madras, B. K., Compton, W. M., Avula, D., Stegbauer, T., Stein, J. B., \& Clark, H. W. (2009).

${ }^{105}$ Bernstein, J., Bernstein, E., Tassiopoulos, K., Heeren, T., Levenson, S., \& Hingson, R. (2005).

${ }^{106}$ Baker, A., Lee, N. K., Claire, M., Lewin, T. J., Grant, T., Pohlman, S., et al. (2005).

${ }^{107}$ Substance Abuse and Mental Health Services Administration. (2009a).

${ }^{108}$ Curie, C. G. (2006).

${ }^{109}$ Harris, S. K., Csemy, L., Sherritt, L., Starostova, O., Van Hook, S., Johnson, J., et al. (2012).

Vaca, F. E., Winn, D., Anderson, C. L., Kim, D., \& Arcila, M. (2011).

${ }^{110}$ Centers for Disease Control and Prevention. (2012b).

${ }^{111}$ American Society of Addiction Medicine. (1997).

${ }^{112}$ Bernstein, J., Bernstein, E., Tassiopoulos, K., Heeren, T., Levenson, S., \& Hingson, R. (2005).

Fleming, M. F., Mundt, M. P., French, M. T., Manwell, L. B., Stauffacher, E. A., \& Barry, K. L. (2002).

Clinical Practice Guideline Treating Tobacco Use and Dependence 2008 Update Panel, Liaisons, and Staff. (2008).

${ }_{113}^{113}$ Office of National Drug Control Policy. (2010).

${ }_{114}^{14}$ U.S. Preventive Services Task Force. (2011).

${ }_{116}^{115}$ American Society of Addiction Medicine. (1997).

${ }^{116}$ American Academy of Family Physicians. (2011b).

American Academy of Family Physicians. (2011a).

${ }^{117}$ American College of Obstetricians and Gynecologists. (2008).

${ }^{118}$ Galloway, T. (2010).

${ }^{119}$ U.S. Preventive Services Task Force. (2004).

${ }^{120}$ U.S. Preventive Services Task Force. (2003).

U.S. Preventive Services Task Force. (2009).

U.S. Preventive Services Task Force. (2008).

U.S. Preventive Services Task Force. (2011).

${ }^{121}$ National Quality Forum. (2007).

${ }^{122}$ Gold, M. S., Frost-Pineda, K., Goldberger, B. A., \& DuPont, R. L. (2006).

The National Center on Addiction and Substance Abuse (CASA) at Columbia University. (2011a).

${ }^{123}$ Clinical Practice Guideline Treating Tobacco Use and Dependence 2008 Update Panel, Liaisons, and Staff. (2008).

${ }^{124}$ Committee on Substance Abuse. (2011).

American Medical Association. (1997).

American Academy of Pediatrics. (2009).

${ }^{125}$ American Medical Association. (1997).

Kulig, J. W., \& Committee on Substance Abuse. (2005). 
${ }^{126}$ Knight, J. R., MD, Director, Center for Adolescent Substance Abuse Research, Associate Professor of Pediatrics, Harvard Medical School, Senior Associate in Medicine, Associate in Psychiatry, Children's Hospital Boston (personal communication, March 13, 2012).

${ }^{127}$ Kokotailo, P. K., \& Committee on Substance Abuse. (2010).

Committee on Substance Abuse. (2011).

Kulig, J. W., \& Committee on Substance Abuse. (2005).

American Academy of Pediatrics. (2009).

${ }^{128}$ D'Onofrio, G., \& Degutis, L. C. (2002).

Gentilello, L. M., Donato, A., Nolan, S., Mackin, R. E., Liebich, F., Hoyt, D. B., et al. (2005).

${ }^{129}$ Soderstrom, C. A., Smith, G. S., Dischinger, P. C., McDuff, D. R., Hebel, J. R., Gorelick, D. A., et al. (1997).

${ }^{130}$ Hungerford, D. W., \& Pollock, D. A. (2002).

McLellan, A. T., \& Meyers, K. (2004).

${ }^{131}$ Bernstein, E., Bernstein, J. A., Stein, J. B., \& Saitz, R. (2009).

Higgins-Biddle, J. C., Hungerford, D., \& Cates-Wessel, K. (2009).

${ }_{132}$ Physicians and Lawyers for National Drug Policy. (2008).

${ }^{133}$ Monti, P. M., Barnett, N. P., Colby, S. M., Gwaltney, C. J., Spirito, A., Rohsenow, D. J., et al. (2007).

${ }^{134}$ Gentilello, L. M. (2010).

${ }^{135}$ Centers for Disease Control and Prevention. (2011c).

Higgins-Biddle, J. C., Hungerford, D., \& Cates-Wessel, K. (2009).

${ }_{137}^{136}$ American College of Emergency Physicians. (2011).

${ }^{137}$ American College of Surgeons. (2007).

${ }^{138}$ Substance Abuse and Mental Health Services Administration. (2010b).

The National Center on Addiction and Substance Abuse at Columbia University (CASA Columbia). (2012d).

${ }^{139}$ Walton, M. A., Chermack, S. T., Shope, J. T., Bingham, C. R., Zimmerman, M. A., Blow, F. C., et al. (2010).

${ }^{140}$ Bernstein, E., Bernstein, J. A., Stein, J. B., \& Saitz, R. (2009).

Chafetz, M. E., Blane, H. T., Abram, H. S., Golner, J., Lacy, E., McCourt, W. F., et al. (1962).

${ }^{141}$ Center for Substance Abuse Treatment. (2009c).

${ }^{142}$ American College of Obstetricians and Gynecologists, Committee on Health Care for Underserved Women.

(2011).

${ }^{143}$ Ferreira-Borges, C. (2005).

${ }^{144}$ Pbert, L., Ockene, J. K., Zapka, J., Ma, Y., Goins, K. V., Oncken, C., et al. (2004).

${ }^{145}$ Chang, G. (2004).

Chang, G., McNamara, T. K., Orav, E. J., Koby, D., Lavigne, A., Ludman, B., et al. (2005).

Chang, G., Wilkins-Haug, L., Berman, S., \& Goetz, M. A. (1999a).

Handmaker, N. S., Miller, W. R., \& Manicke, M. (1999).

Handmaker, N. S., \& Wilbourne, P. (2001).

Nilsen, P. (2009).

Winhusen, T., Kropp, F., Babcock, D., Hague, D., Erickson, S. J., Renz, C., et al. (2008).

${ }^{146}$ Hankin, J. R. (2002).

${ }^{147}$ O'Connor, M. J., \& Whaley, S. E. (2007).

${ }^{148}$ Chang, G., McNamara, T. K., Orav, E. J., Koby, D., Lavigne, A., Ludman, B., et al. (2005).

${ }^{149}$ The National Center on Addiction and Substance Abuse at Columbia University (CASA Columbia). (2012n).

${ }^{150}$ Association of American Medical Colleges. (2007).

Deas-Nesmith, D., Campbell, S., \& Brady, K. T. (1998).

Friedmann, P. D., McCullough, D., Chin, M. H., \& Saitz, R. (2000).

Friedmann, P. D., McCullough, D., \& Saitz, R. (2001).

Lichtenstein, D. P., Spirito, A., \& Zimmermann, R. P. (2010).

Revell, C. C., \& Schroeder, S. A. (2005).

Schroeder, S. A. (2005).

Schroeder, S. A., \& Morris, C. D. (2010).

Tong, E. K., Strouse, R., Hall, J., Kovac, M., \& Schroeder, S. A. (2010).

${ }^{151}$ Perrone, J., De Roos, F., Jayaraman, S., \& Hollander, J. E. (2001).

Satre, D. D., Chi, F. W., Eisendrath, S., \& Weisner, C. (2011).

Satre, D., Wolfe, W., Eisendrath, S., \& Weisner, C. (2008).

Spear, S., Tillman, S., Moss, C., Gong-Guy, E., Ransom, L., \& Rawson, R. A. (2009). 
Teitelbaum, L. M., \& Carey, K. B. (2000).

${ }^{152}$ Martens, M. P., Cimini, M. D., Barr, A. R., Rivero, E. M., Vellis, P. A., Desemone, G. A., et al. (2007).

Milner, K. K., Barry, K. L., Blow, F. C., \& Welsh, D. (2010).

${ }^{153}$ Martens, M. P., Cimini, M. D., Barr, A. R., Rivero, E. M., Vellis, P. A., Desemone, G. A., et al. (2007).

${ }^{154}$ Denisco, R. C., Kenna, G. A., O'Neil, M. G., Kulich, R. J., Moore, P. A., Kane, W. T., et al. (2011).

Gordon, J. S., Albert, D. A., Crews, K. M., \& Fried, J. (2009).

${ }^{155}$ American Dental Association. (2007).

Shaw, J. (2011).

Crews, K. M., Cobb, G. W., Seago, D., \& Williams, N. (1999).

Gordon, J. S., Albert, D. A., Crews, K. M., \& Fried, J. (2009).

Johnson, G. K., \& Slach, N. A. (2001).

Johnson, N. (2001).

Robb, N. D., \& Smith, B. G. (1996).

Sciubba, J. J. (2001).

${ }^{156}$ Denisco, R. C., Kenna, G. A., O'Neil, M. G., Kulich, R. J., Moore, P. A., Kane, W. T., et al. (2011).

Hersh, E. V., Ochs, H., Quinn, P., MacAfee, K., Cooper, S. A., \& Barasch, A. (1993).

Leonard, R. H. (1991).

Murphy, D. C., \& Wilmers, S. (2002).

Robb, N. D., \& Smith, B. G. (1996).

${ }^{157}$ Gordon, J. S., Albert, D. A., Crews, K. M., \& Fried, J. (2009).

${ }^{158}$ Shaw, J. (2011).

${ }^{159}$ Patel, D. (2005).

${ }^{160}$ National Institute on Drug Abuse. (2011c).

${ }^{161}$ Substance Abuse and Mental Health Services Administration. (2008a).

${ }^{162}$ McLellan, A. T., \& Meyers, K. (2004).

${ }^{163}$ The National Center on Addiction and Substance Abuse (CASA) at Columbia University. (2011a).

${ }^{164}$ Substance Abuse and Mental Health Services Administration. (2008a).

${ }^{165}$ Substance Abuse and Mental Health Services Administration. (2003a

${ }^{166}$ Substance Abuse and Mental Health Services Administration. (2008a).

${ }^{167}$ Lee, S., Aos, S., Drake, E., Pennucci, A., Miller, M., \& Anderson, L. (2012).

Washington State Institute for Public Policy. (2012a).

${ }_{168}$ Baer, J. S., Kivlahan, D. R., Blume, A. W., McKnight, P., \& Marlatt, G. A. (2001).

${ }^{169}$ The National Center on Addiction and Substance Abuse (CASA) at Columbia University. (2007b).

The National Center on Addiction and Substance Abuse at Columbia University (CASA Columbia). (2012n).

${ }^{170}$ The National Center on Addiction and Substance Abuse (CASA) at Columbia University. (2007b).

${ }^{171}$ D'Onofrio, G., \& Degutis, L. C. (2002).

Martens, M. P., Cimini, M. D., Barr, A. R., Rivero, E. M., Vellis, P. A., Desemone, G. A., et al. (2007).

White, H. R., Morgan, T., Pugh, L., Celinska, K., Labouvie, E. W., \& Pandina, R. (2006).

${ }^{172}$ Brown, R. L. (2009).

${ }^{173}$ The National Center on Addiction and Substance Abuse (CASA) at Columbia University. (2004).

${ }^{174}$ Lapham, S. (2004).

Krisberg, B. (1998).

${ }^{175}$ Grisso, T., \& Underwood, L. (2004).

${ }^{176}$ Hockenberry, S., Sickmund, M., \& Sladky, A. (2009).

${ }^{177}$ The National Center on Addiction and Substance Abuse (CASA) at Columbia University. (2004).

${ }^{178}$ The National Center on Addiction and Substance Abuse (CASA) at Columbia University. (2010).

${ }^{179}$ Center for Substance Abuse Treatment. (2005c).

${ }^{180}$ Babor, T. F., Higgins-Biddle, J. C., Dauser, D., Burleson, J. A., Zarkin, G. A., \& Bray, J. (2006).

Ensuring Solutions to Alcohol Problems. (2008).

Fleming, M. F., Mundt, M. P., French, M. T., Manwell, L. B., Stauffacher, E. A., \& Barry, K. L. (2002).

Mundt, M. P. (2006).

National Association of State Alcohol and Drug Abuse Directors. (2006).

Solberg, L. I., Maciosek, M. V., \& Edwards, N. M. (2008).

Whitlock, E. P., Polen, M. R., Green, C. A., Orleans, T., \& Klein, J. (2004).

${ }^{181}$ McLearen, A. M., \& Ryba, N. L. (2003). 
${ }^{182}$ Alberti v. Sheriff of Harris County, 406 F. Supp. 649 (S.D. Tex. 1975).

McLearen, A. M., \& Ryba, N. L. (2003).

Palmigiano v. Garrahy, 443 F. Supp. 956 (D.R.I. 1977).

Peters, R. H. \& Matthews, C. O. (2003).

Ruiz v. Estelle, 503 F. Supp. 1265 (S.D. Tex. 1980).

${ }^{183}$ Lapham, S. (2004).

${ }^{184}$ The National Center on Addiction and Substance Abuse at Columbia University (CASA Columbia). (2012n).

${ }^{185}$ Substance Abuse and Mental Health Services Administration. (2008b).

Substance Abuse and Mental Health Services Administration. (2008c).

${ }^{186}$ Goplerud, E., \& McPherson, T. L. (2010b).

${ }^{187}$ U.S.Office of Personnel Management. (2011).

${ }^{188}$ Society for Human Resource Management. (2011).

${ }^{189}$ Goplerud, E., Sharar, D., \& McPherson, T. (2010b).

${ }^{190}$ Goplerud, E., \& McPherson, T. L. (2010b).

${ }^{191}$ Goplerud , E., \& McPherson, T. L. (2010a).

Greenwood, G. L., Goplerud, E., McPherson, T. L., Azocar, F., Baker, E. M., \& Dybdahl, S. (2010).

McPherson, T. L., Goplerud, E., Derr, D., Mickenberg, J., \& Courtemanche, S. (2010).

${ }_{192}$ McPherson, T., Lusby-Treber, K., Goplerud , E., \& Walsh, T. (2007).

${ }^{193}$ White, T. (2003).

${ }^{194}$ DeCew, J. W. (1994).

${ }^{195}$ American Civil Liberties Union. (1999).

${ }^{196}$ American Society of Addiction Medicine. (2003).

${ }_{197}^{197}$ Addiction Technology Transfer Center Network. (2010).

${ }^{198}$ Illinois Department of Human Services. (2011).

Madras, B. K., Compton, W. M., Avula, D., Stegbauer, T., Stein, J. B., \& Clark, H. W. (2009).

Stuart, G. L. (2005).

The National Center on Addiction and Substance Abuse (CASA) at Columbia University. (2009d).

U.S. Department of Justice, Office of Justice Programs. (2009).

U.S. Department of Justice, National Drug Intelligence Center. (2010).

${ }^{199}$ Associated Press. (2011).

Cohen, A. (2011).

Couwels, J. (2011).

National Conference of State Legislatures. (2012).

Sulzberger, A. G. (2011).

${ }^{200}$ Anderson, P., Aromaa, S., Rosenbloom, D., \& Enos, G. (2008).

Anthony, E. K., Taylor, S. A., \& Raffo, Z. (2011).

Babor, T. F., McRee, B. G., Kassebaum, P. A., Grimaldi, P. L., Ahmed, K., \& Bray, J. (2007).

McLellan, A. T., \& Meyers, K. (2004).

Forum on Integration. (2011).

Van Hook, S., Harris, S. K., Brooks, T., Carey, P., Kossack, R., Kulig, J., et al. (2007).

The National Center on Addiction and Substance Abuse (CASA) at Columbia University. (2011a).

The National Center on Addiction and Substance Abuse (CASA) at Columbia University. (2007b).

${ }^{201}$ The National Center on Addiction and Substance Abuse (CASA) at Columbia University. (2000).

Miller, N. S., Sheppard, L. M., Colenda, C. C., \& Magen, J. (2001).

${ }^{202}$ Miller, N. S., Sheppard, L. M., Colenda, C. C., \& Magen, J. (2001).

${ }^{203}$ Anderson, P., Aromaa, S., Rosenbloom, D., \& Enos, G. (2008).

${ }^{204}$ Anderson, P., Aromaa, S., Rosenbloom, D., \& Enos, G. (2008).

Knopf, A. (2011).

${ }^{205}$ The National Center on Addiction and Substance Abuse (CASA) at Columbia University. (2011a).

The National Center on Addiction and Substance Abuse (CASA) at Columbia University. (2001a).

${ }^{206}$ The National Center on Addiction and Substance Abuse (CASA) at Columbia University. (2011a).

${ }^{207}$ Burrow-Sanchez, J. J., \& Lopez, A. L. (2009).

Burrow-Sanchez, J., Call, M. E., Adolphson, S. L., \& Hawken, L. S. (2009).

${ }^{208}$ The National Center on Addiction and Substance Abuse (CASA) at Columbia University. (2011a).

${ }^{209}$ The National Center on Addiction and Substance Abuse (CASA) at Columbia University. (1999). 
${ }^{210}$ The National Center on Addiction and Substance Abuse (CASA) at Columbia University. (2010).

${ }^{211}$ Boyle, M. G., \& Murphy, T. P. (2005).

McLellan, A. T., \& Meyers, K. (2004).

Yoast, R. A., Wilford, B. B., \& Hayashi, S. W. (2008).

${ }^{212}$ Anderson, P., Aromaa, S., Rosenbloom, D., \& Enos, G. (2008).

Babor, T. F., McRee, B. G., Kassebaum, P. A., Grimaldi, P. L., Ahmed, K., \& Bray, J. (2007).

Rhode Island Hospital. (2011).

${ }^{213}$ The National Center on Addiction and Substance Abuse (CASA) at Columbia University. (2011a).

${ }^{214}$ The National Center on Addiction and Substance Abuse (CASA) at Columbia University. (2010).

${ }^{215}$ Anderson, P., Aromaa, S., Rosenbloom, D., \& Enos, G. (2008).

${ }^{216}$ Drabble, L. (2007).

Lapham, S. (2004).

The National Center on Addiction and Substance Abuse (CASA) at Columbia University. (2011a).

The National Center on Addiction and Substance Abuse (CASA) at Columbia University. (2001a).

The National Center on Addiction and Substance Abuse (CASA) at Columbia University. (2007b).

U.S. General Accounting Office. (1995).

${ }^{217}$ Allsop, S. J., \& Stevens, C. (2009).

Cryer, H. G. (2005).

Gentilello, L. M., Samuels, P. N., Henningfield, J. E., \& Santora, P. B. (2005).

Gentilello, L. M. (2005).

Anderson, P., Aromaa, S., Rosenbloom, D., \& Enos, G. (2008).

${ }^{218}$ U.S.Code Online via GPO Access. (2011).

U. S. Code of Federal Regulations. Title 52 Fed Reg 21796, 21797

${ }^{219}$ Anderson, P., Aromaa, S., Rosenbloom, D., \& Enos, G. (2008).

${ }^{220}$ Babor, T. F., McRee, B. G., Kassebaum, P. A., Grimaldi, P. L., Ahmed, K., \& Bray, J. (2007).

${ }^{221}$ Center for Substance Abuse Treatment. (1995a).

Lanier, D., \& Ko, S. (2008).

${ }^{222}$ U.S. Department of Agriculture and U.S. Department of Health and Human Services. (2010).

National Institute on Alcohol Abuse and Alcoholism. (2012b).

${ }^{223}$ University of Washington, Alcohol and Drug Abuse Institute. (2011).

${ }^{224}$ The National Center on Addiction and Substance Abuse (CASA) at Columbia University. (2011a).

${ }^{225}$ McLellan, A. T., \& Meyers, K. (2004).

Winters, K. C. (1999). 


\section{Chapter V \\ Notes}

${ }^{1}$ American Society of Addiction Medicine. (2010b).

National Quality Forum. (2005).

${ }^{2}$ Leshner, A. I. (2001b).

${ }^{3}$ Rotrosen, J., Professor, Department of Psychiatry, New York University School of Medicine, VA NY Harbor Healthcare System (personal communication, August 7, 2007).

${ }^{4}$ Clark, H. W. (2011).

${ }^{5}$ American Society of Addiction Medicine. (2011b).

Blum, K., Werner, T., Carnes, S., Carnes, P., Bowirrat, A., Giordano, J., et al. (2012).

Blume, S. B. (1994).

Çepik, A., Arikan, Z., Boratav, C., \& Isik, E. (1995).

Fortuna, J. L. (2012).

Grant, J. E., Brewer, J. A., \& Potenza, M. N. (2006).

Grant, J. E., Potenza, M. N., Weinstein, A., \& Gorelick, D. A. (2010).

Karim, R., \& Chaudhri, P. (2012).

Kenny, P. J. (2011).

Shaffer, H. J., LaPlante, D. A., LaBrie, R. A., Kidman, R. C., Donato, A. N., \& Stanton, M. V. (2004).

Volkow, N. D., Wang, G. J., Fowler, J. S., \& Telang, F. (2008).

${ }^{6}$ Kim, S. W., Grant, J. E., Adson, D. E., \& Shin, Y. C. (2001).

Shaffer, H. J., LaPlante, D. A., LaBrie, R. A., Kidman, R. C., Donato, A. N., \& Stanton, M. V. (2004).

${ }^{7}$ Shaffer, H. J., LaPlante, D. A., LaBrie, R. A., Kidman, R. C., Donato, A. N., \& Stanton, M. V. (2004).

${ }^{8}$ Koplewicz, H. (2011).

${ }^{9}$ United Nations, Office on Drugs and Crime. (2008).

${ }^{10}$ Center for Substance Abuse Treatment. (2005b).

University of Washington. (2011).

Center for Substance Abuse Treatment. (1999b).

Center for Substance Abuse Treatment. (2006b).

${ }^{11}$ Center for Substance Abuse Treatment. (1999b).

${ }^{12}$ National Institute on Alcohol Abuse and Alcoholism. (2005b).

United Nations, Office on Drugs and Crime. (2008).

${ }^{13}$ National Institute on Alcohol Abuse and Alcoholism. (2005b).

Winters, K. C., \& Zenilman, J. M. (1994).

${ }^{14}$ National Institute on Alcohol Abuse and Alcoholism. (2005b).

United Nations, Office on Drugs and Crime. (2008).

Winters, K. C., \& Zenilman, J. M. (1994).

${ }^{15}$ United Nations, Office on Drugs and Crime. (2008).

${ }^{16}$ National Institute on Alcohol Abuse and Alcoholism. (2005b).

${ }^{17}$ Grisso, T., \& Underwood, L. (2004).

${ }^{18}$ United Nations, Office on Drugs and Crime. (2008).

${ }^{19}$ Center for Substance Abuse Treatment. (1999b).

${ }^{20}$ Agency for Healthcare Research and Quality. (2010).

American Society of Addiction Medicine. (2010b).

U.S. Department of Health and Human Services. (2008).

${ }^{21}$ National Institute on Drug Abuse. (2006c).

O'Brien, C. P. \& McKay, J. (2007).

${ }^{22}$ National Institute on Drug Abuse. (2006c).

${ }^{23}$ Reus, V. I., \& Smith, B. J. (2008).

Stead, L. F., Perera, R., Bullen, C., Mant, D., \& Lancaster, T. (2008).

${ }^{24}$ Reus, V. I., \& Smith, B. J. (2008).

${ }^{25}$ Shiffman, S., Khayrallah, M., \& Nowak, R. (2000).

West, R., \& Shiffman, S. (2001).

${ }^{26}$ Reus, V. I., \& Smith, B. J. (2008). 
${ }^{27}$ Clinical Practice Guideline Treating Tobacco Use and Dependence 2008 Update Panel, Liaisons, and Staff. (2008).

Piper, M. E., Smith, S. S., Schlam, T. R., Fiore, M. C., Jorenby, D. E., Fraser, D., et al. (2009).

Smith, S. S., McCarthy, D. E., Japuntich, S. J., Christiansen, B., Piper, M. E., Jorenby, D. E., et al. (2009).

${ }^{28}$ Reus, V. I., \& Smith, B. J. (2008).

${ }^{29}$ United Nations, Office on Drugs and Crime. (2008).

${ }^{30}$ O'Brien, C. P. \& McKay, J. (2007).

${ }^{31}$ National Institute on Drug Abuse. (2005c).

U.S. Department of Health and Human Services. (2008).

${ }^{32}$ U.S. Department of Health and Human Services. (2008).

${ }^{33}$ Hazelden Foundation. (2012).

PsychCentral.org. (2010).

${ }^{34}$ Agency for Healthcare Research and Quality. (2010).

U.S. Department of Health and Human Services. (2008).

${ }^{35}$ Center for Substance Abuse Treatment. (2004).

Center for Substance Abuse Treatment. (2006b).

Finnegan, L. (2008).

Graham, A., Schultz, T., Mayo-Smith, M., Ries, R., \& Wilford, B. (2008).

Hudak, M. L, Tan, R. C., The Committee on Drugs, \& The Committee on Fetus and Newborn. (2012).

${ }^{36}$ U.S. Department of Health and Human Services. (2008).

${ }^{37}$ U.S. Department of Health and Human Services. (2008).

${ }^{38}$ U.S. Department of Health and Human Services. (2008).

${ }^{39}$ Allan, C., Smith, I., \& Mellin, M. (2000).

Foster, J. H., Marshall, E. J., \& Peters, T. J. (2000).

Holloway, H. C., Hales, R. E., \& Watanabe, H. K. (1984).

Kosten, T. R., \& O'Connor, P. G. (2003).

Myrick, H., \& Anton, R. F. (1998).

Parrott, S., Godfrey, C., Heather, N., Clark, J., \& Ryan, T. (2006).

${ }^{40}$ Myrick, H., \& Anton, R. F. (1998).

${ }^{41}$ Kosten, T. R., \& O'Connor, P. G. (2003).

Myrick, H., \& Anton, R. F. (1998).

${ }^{42}$ Myrick, H., \& Anton, R. F. (1998).

${ }^{43}$ Chang, P. H., \& Steinberg, M. B. (2001).

Myrick, H., \& Anton, R. F. (1998).

Tavel, M. E., Davidson, W., \& Batterton, T. D. (1961).

Victor, M., \& Adams, R. D. (1953).

Wright, T., Myrick, H., Henderson, S., Peters, H., \& Malcolm, R. (2006).

${ }^{44}$ Becker, H. C. (1998).

${ }^{45}$ Lejoyeux, M., Solomon, J., \& Ades, J. (1998).

${ }^{46}$ Amato, L., Minozzi, S., Vecchi, S., \& Davoli, M. (2010).

${ }^{47}$ National Library of Medicine. (2010f).

Ries, R. K., Fielin, D. A., Miller, S. C., \& Saitz, R. (2009

${ }^{48}$ National Library of Medicine. (2010c).

Ries, R. K., Fielin, D. A., Miller, S. C., \& Saitz, R. (2009).

${ }^{49}$ Bayard, M., McIntyre, J., Hill, K. R., \& Woodside, J., Jr. (2004).

Myrick, H., \& Anton, R. F. (1998).

${ }^{50}$ U.S.Drug Enforcement Administration. (2012).

Tanaka, E. (2002).

Innovations Report. (2005).

${ }^{51}$ French, D. D., Spehar, A. M., Campbell, R. R., Palacios, P., Coakley, R. W., Coblio, N., et al. (2005). Innovations Report. (2005).

${ }^{52}$ Longo, L. P., \& Johnson, B. (2000).

National Institute on Drug Abuse. (2005c).

${ }^{53}$ Bayard, M., McIntyre, J., Hill, K. R., \& Woodside, J., Jr. (2004).

National Library of Medicine. (2012). 
Malcolm, R., Ballenger, J. C., Sturgis, E. T., \& Anton, R. (1989).

${ }^{54}$ Bayard, M., McIntyre, J., Hill, K. R., \& Woodside, J., Jr. (2004).

Malcolm, R., Ballenger, J. C., Sturgis, E. T., \& Anton, R. (1989).

${ }^{55}$ Bayard, M., McIntyre, J., Hill, K. R., \& Woodside, J., Jr. (2004).

National Library of Medicine. (2012).

Saitz, R. (1998).

${ }^{56}$ Rounsaville, B. J., Kosten, T., \& Kleber, H. (1985).

U.S. Department of Health and Human Services. (2008).

${ }^{57}$ Amato, L., Davoli, M., Ferri, M., Gowing, L., \& Perucci, C. A. (2004).

${ }^{58}$ National Library of Medicine. (2008c).

${ }^{59}$ O'Connor, P. G., \& Fiellin, D. A. (2000).

${ }^{60}$ U.S. Department of Health and Human Services. (2008).

${ }^{61}$ Agency Medical Directors Group. (2012).

${ }^{62}$ Gold, M. S., Redmond, D. E., Jr., \& Kleber, H. D. (1978).

${ }^{63}$ Devenyi, P., Mitwalli, A., \& Graham, W. (1982).

Gold, M. S., Pottash, A. C., Sweeney, D. R., \& Kleber, H. D. (1980).

Kleber, H. D., Weiss, R. D., Anton, R. F., Rounsaville, B. J., George, T. P., Strain, E. C., et al. (2006).

Smithedajkul, P. Y., \& Cullen, M. W. (2009).

${ }^{64}$ Amato, L., Davoli, M., Ferri, M., Gowing, L., \& Perucci, C. A. (2004).

Center for Substance Abuse Treatment. (2006b).

Meader, N. (2010).

O'Connor, P. G., Carroll, K. M., Shi, Ju. M., Schottenfeld, R. S., Kosten, T. R., \& Rounsaville, B. J. (1997).

${ }^{65}$ U.S. Department of Health and Human Services. (2008).

U.S. Drug Enforcement Administration, Office of Diversion Control. (2011).

${ }^{66} 21$ C.F.R. § 1301.19-1301.32 (2010)

Center for Substance Abuse Treatment. (2004).

Center for Substance Abuse Treatment, Division of Pharmacologic Therapies. (2012).

${ }^{67}$ Amato, L., Davoli, M., Ferri, M., Gowing, L., \& Perucci, C. A. (2004).

Center for Substance Abuse Treatment. (2006b).

Center for Substance Abuse Treatment. (2004).

Centers for Disease Control and Prevention. (2002b).

Center for Substance Abuse Treatment. (2009d).

Agency Medical Directors Group. (2012).

Meader, N. (2010).

National Drug Intelligence Center. (2003).

O'Connor, P. G., Carroll, K. M., Shi, Ju. M., Schottenfeld, R. S., Kosten, T. R., \& Rounsaville, B. J. (1997).

O'Connor, P. G. (2005).

Sigmon, S. C., Dunn, K. E., Badger, G. J., Heil, S. H., \& Higgins, S. T. (2009).

Weiss, R. D., Potter, J. S., Fiellin, D. A., Byrne, M., Connery, H. S., Dickinson, W., et al. (2011).

${ }^{68}$ Center for Substance Abuse Treatment. (2004).

O'Brien, C. P. \& McKay, J. (2007).

${ }^{69}$ U.S. Department of Health and Human Services. (2008).

${ }^{70}$ Center for Substance Abuse Treatment. (2006b).

${ }^{71}$ Center for Substance Abuse Treatment. (2006b).

McGregor, C., Srisurapanont, M., Mitchell, A., Wickes, W., \& White, J. M. (2008).

Srisurapanont, M., Jarusuraisin, N., \& Jittiwutikan, J. (1999).

${ }^{72}$ Martell, B. A., Orson, F. M., Poling, J., Mitchell, E., Rossen, R. D., Gardner, T., et al. (2009).

Buddy, T. (2007).

Shen, X., Orson, F. M., \& Kosten, T. R. (2011).

${ }^{73}$ Buddy, T. (2007).

${ }^{74}$ Shoptaw, S. J., Kao, U., Heinzerling, K., \& Ling, W. (2009).

${ }^{75}$ Kampman, K. M. (2008).

${ }^{76}$ National Institute on Drug Abuse. (2011c).

${ }^{77}$ Center for Substance Abuse Treatment. (2006b).

${ }^{78}$ Center for Substance Abuse Treatment. (2006b). 
Tyrer, P., Rutherford, D., \& Huggert, T. (1981).

${ }^{79}$ Petursson, H. (1994).

${ }^{80}$ Ashton, H. (1984).

${ }^{81}$ Center for Substance Abuse Treatment. (2006b).

${ }^{82}$ Center for Substance Abuse Treatment. (2006b).

${ }^{83}$ Center for Substance Abuse Treatment. (2006b).

${ }^{84}$ Abbott, P. J., Quinn, D., \& Knox, L. (1995).

${ }^{85}$ Center for Substance Abuse Treatment. (2006b).

${ }^{86}$ Center for Substance Abuse Treatment. (2006b).

American Society of Addiction Medicine. (2007).

${ }^{87}$ Abbott, P. J., Quinn, D., \& Knox, L. (1995).

Center for Substance Abuse Treatment. (2006b).

${ }^{88}$ Amato, L., Minozzi, S., Davoli, M., Vecchi, S., Ferri, M. M., \& Mayet, S. (2009).

Arias, A. J., \& Kranzler, H. R. (2008).

Balldin, J., Berglund, M., Borg, S., Mansson, M., Bendtsen, P., Franck, J., et al. (2003).

Carroll, K. M., Fenton, L. R., Ball, S. A., Nich, C., Frankforter, T. L., Shi, J., et al. (2004).

Carroll, K. M., Nich, C., Ball, S. A., McCance, E., \& Rounsaville, B. J. (1998).

Carroll, K. M., Rounsaville, B. J., Nich, C., Gordon, L., \& Gawin, F. (1995).

Cepeda-Benito, A. (1993).

Fiore,M., U.S. Department of Health and Human Services. (2008).

Hughes, J. R. (1995).

Kosten, T., Oliveto, A., Feingold, A., Poling, J., Sevarino, K., Cance-Katz, E., et al. (2003).

National Institute on Drug Abuse. (2009c).

O'Brien, C. P., Woody, G. E., \& McLellan, A. T. (1995).

Poldrugo, F. (1997).

Poling, J., Oliveto, A., Petry, N., Sofuoglu, M., Gonsai, K., Gonzalez, G., et al. (2006).

Rigotti, N. A. (2009).

Rigotti, N. A., Munafo, M. R., \& Stead, L. F. (2008).

Silverman, K., Wong, C. J., Higgins, S. T., Brooner, R. K., Montoya, I. D., Contoreggi, C., et al. (1996).

Stitzer, M. L. (1999).

${ }^{89}$ Biery, J. R., Williford, J. H., Jr., \& McMullen, E. A. (1991).

Bock, B. C., Marcus, B. H., King, T. K., Borrelli, B., \& Roberts, M. R. (1999).

Forsander, O. A. (1998).

Grant, L. P., Haughton, B., \& Sachan, D. S. (2004).

Kochupillai, V., Kumar, P., Singh, D., Aggarwal, D., Bhardwaj, N., Bhutani, M., et al. (2005).

Kremer, D., Malkin, M. J., \& Benshoff, J. J. (1995).

Marcus, B. H., Albrecht, A. E., King, T. K., Parisi, A. F., Pinto, B. M., Roberts, M., et al. (1999).

Martin, J. E., Calfas, K. J., Patten, C. A., Polarek, M., Hofstetter, C. R., Noto, J., et al. (1997).

McClernon, F. J., Westman, E. C., \& Rose, J. E. (2004).

McIver, S., O'Halloran, P., \& McGartland, M. (2004).

Ussher, M. H., Taylor, A. H., West, R., \& McEwen, A. (2000).

Yung, L., Gordis, E., \& Holt, J. (1983).

${ }^{90}$ American Society of Addiction Medicine. (2012).

${ }^{91}$ Amato, L., Davoli, M., Perucci, C. A., Ferri, M., Faggiano, F., \& Mattick, R. P. (2005).

National Institute on Drug Abuse. (2009c).

${ }^{92}$ Center for Substance Abuse Treatment. (2004).

Center for Substance Abuse Treatment. (2009b).

Gerra, G., Fantoma, A., \& Zaimovic, A. (2006).

Mason, B. J., Goodman, A. M., Chabac, S., \& Lehert, P. (2006).

Orson, F. M., Kinsey, B. M., Singh, R. A. K., Wu, Y., Gardner, T., \& Kosten, T. R. (2008).

${ }^{93}$ Johns Hopkins Medicine. (2007).

SmokeFree.gov. (2012b).

SmokeFree.gov. (2012a).

U.S. Food and Drug Administration. (2009).

U.S. Food and Drug Administration. (2006a). 
U.S. Food and Drug Administration. (2007b).

U.S. Food and Drug Administration. (2011a).

U.S. Food and Drug Administration. (2012).

U.S. Food and Drug Administration. (2010a).

U.S. Food and Drug Administration. (2006b).

${ }^{94}$ Stafford, R. S. (2008).

${ }^{95}$ Quenqua, D. (2012).

${ }^{96}$ Heilig, M., Goldman, D., Berrettini, W., \& O'Brien, C. P. (2011).

${ }^{97}$ King, A. C., Volpicelli, J. R., Frazer, A., \& O'Brien, C. P. (1997).

Krishnan-Sarin, S., Krystal, J. H., Shi, J., Pittman, B., \& O'Malley, S. S. (2007).

Rubio, G., Ponce, G., Rodriguez-Jiminez, R., Jimenez-Arriero, A., Hoenicka, J., \& Palomo, T. (2005).

Wilcox, C. S., Noble, E. P., \& Oskooilar, N. (2011).

${ }^{98}$ Heilig, M., Goldman, D., Berrettini, W., \& O'Brien, C. P. (2011).

Wilcox, C. S., Noble, E. P., \& Oskooilar, N. (2011).

${ }^{99}$ Center for Substance Abuse Treatment. (2005a).

${ }^{100}$ Huebner, R. B., \& Kantor, L. W. (2011).

101 Johnson, B. A., \& Ait-Daoud, N. (1999).

National Library of Medicine. (2008c).

${ }^{102}$ Anton, R. F., O'Malley, S. S., Ciraulo, D. A., Cisler, R. A., Couper, D., Donovan, D. M., et al. (2006).

Center for Substance Abuse Treatment. (2009b).

Gueorguieva, R., Wu, R., Donovan, D., Rounsaville, B. J., Couper, D., Krystal, J. H., et al. (2011).

${ }^{103}$ Erickson, C. K. (2007).

Sass, H., Soyka, M., Mann, K., \& Zieglgansberger, W. (1996).

Whitworth, A. B., Fischer, F., Lesch, O. M., Nimmerrichter, A., Oberbauer, H., Platz, T., et al. (1996).

${ }^{104}$ Center for Substance Abuse Treatment. (2009b).

Johnson, B. A., O'Malley, S. S., Ciraulo, D. A., Roache, J. D., Chambers, R. A., Sarid-Segal, O., et al. (2003).

${ }^{105}$ Hughes, J. R., Stead, L. F., \& Lancaster, T. (2010).

${ }^{106}$ Fiore,M., U.S. Department of Health and Human Services. (2008).

${ }^{107}$ Durcan, M. J., Deener, G., White, J., Johnston, J. A., Gonzales, D., Niaura, R., et al. (2002).

Shiffman, S., Johnston, J. A., Khayrallah, M., Elash, C. A., Gwaltney, C. J., Paty, J. A., et al. (2000).

${ }^{108}$ Lerman, C., Roth, D., Kaufmann, V., Audrain, J., Hawk, L., Liu, A., et al. (2002).

${ }^{109}$ Fiore,M., U.S. Department of Health and Human Services. (2008).

Roddy, E. (2004).

${ }^{110}$ Erickson, C. K. (2007).

${ }^{111}$ Roddy, E. (2004).

${ }^{112}$ Fiore,M., U.S. Department of Health and Human Services. (2008).

Erickson, C. K. (2007).

${ }^{113}$ Fiore,M., U.S. Department of Health and Human Services. (2008).

${ }^{114}$ Hughes, J. R., Stead, L. F., \& Lancaster, T. (2007).

${ }^{115}$ U.S.Food and Drug Administration. (2007a).

${ }^{116}$ Fiore,M., U.S. Department of Health and Human Services. (2008).

${ }^{117}$ Hughes, J. R., Stead, L. F., \& Lancaster, T. (2007).

Jorenby, D. E., Leischow, S. J., Nides, M. A., Rennard, S. I., Johnston, J. A., Hughes, A. R., et al. (1999).

${ }^{118}$ Hughes, J. R., Stead, L. F., \& Lancaster, T. (2007).

${ }^{119}$ Newton, T. F., Roache, J. D., De La Garza, R., Fong, T., Wallace, C. L., Li, S. H., et al. (2006).

${ }^{120}$ Karila, L., Weinstein, A., Aubin, H. J., Benyamina, A., Reynaud, M., \& Batki, S. L. (2010).

Vocci, F., \& Ling, W. (2005).

${ }^{121}$ Hughes, J. R., Stead, L. F., \& Lancaster, T. (2007).

Hughes, J. R., Stead, L. F., \& Lancaster, T. (2005).

Roddy, E. (2004).

${ }^{122}$ Hughes, J. R., Stead, L. F., \& Lancaster, T. (2005).

${ }^{123}$ Fiore,M., U.S. Department of Health and Human Services. (2008).

${ }^{124}$ Gourlay, S. G., Stead, L. F., \& Benowitz, N. (2008).

Roddy, E. (2004). 
${ }^{125}$ Fiore,M., U.S. Department of Health and Human Services. (2008).

Gourlay, S. G., Stead, L. F., \& Benowitz, N. (2008).

Roddy, E. (2004).

${ }^{126}$ Addolorato, G., Caputo, F., Capristo, E., Domenicali, M., Bernardi, M., Janiri, L., et al. (2002).

Addolorato, G., Leggio, L., Ferrulli, A., Cardone, S., Bedogni, G., Caputo, F., et al. (2011).

Addolorato, G., Leggio, L., Ferrulli, A., Cardone, S., Vonghia, L., Mirijello, A., et al. (2007).

Flannery, B. A., Garbutt, J. C., Cody, M. W., Renn, W., Grace, K., Osborne, M., et al. (2004).

Liu, J., \& Wang, L. (2011).

${ }^{127}$ Addolorato, G., Caputo, F., Capristo, E., Domenicali, M., Bernardi, M., Janiri, L., et al. (2002).

${ }^{128}$ Addolorato, G., Leggio, L., Ferrulli, A., Cardone, S., Bedogni, G., Caputo, F., et al. (2011).

${ }^{129}$ National Library of Medicine. (2011g).

${ }^{130}$ Dawes, M. A., Johnson, B. A., Ait-Daoud, N., Ma, J. Z., \& Cornelius, J. R. (2005).

Dawes, M. A., Johnson, B. A., Ma, J. Z., Ait-Daoud, N., Thomas, S. E., \& Cornelius, J. R. (2005).

Kranzler, H. R., Pierucci-Lagha, A., Feinn, R., \& Hernandez-Avila, C. (2003).

${ }^{131}$ Kenna, G. A., Zywiak, W. H., McGeary, J. E., Leggio, L., McGeary, C., Wang, S., et al. (2009).

${ }^{132}$ Chu, L. F., Liang, D. Y., Li, X., Sahbaie, P., D'arcy, N., Liao, G., et al. (2009).

${ }^{133}$ Mason, B. J., Crean, R., Goodell, V., Light, J. M., Quello, S., Shadan, F., et al. (2012).

National Library of Medicine. (2011d).

${ }^{134}$ Anton, R. F., \& Swift, R. M. (2003).

Erickson, C. K. (2007).

Fett, D. L. \& Vukov, L. F. (1994).

Krampe, H., Stawicki, S., Wagner, T., Bartels, C., Aust, C., Ruther, E., et al. (2006).

Kitson, T. M. (1987).

Smith, J. A., Wolford, J. A., Weber, M., \& McLean, D. (1957).

135 Anton, R. F., \& Swift, R. M. (2003).

Erickson, C. K. (2007).

National Institute on Alcohol Abuse and Alcoholism. (2007).

${ }^{136}$ Anton, R. F., \& Swift, R. M. (2003).

Erickson, C. K. (2007).

${ }^{137}$ Anton, R. F., \& Swift, R. M. (2003).

${ }^{138}$ Suh, J. J., Pettinati, H. M., Kampman, K. M., \& O'Brien, C. P. (2006).

139 Johnson, B. A. (2008).

${ }^{140}$ Fuller, R. K., Branchey, L., Brightwell, D. R., Derman, R. M., Emrick, C. D., Iber, F. L., et al. (1986).

141 Erickson, C. K. (2007).

${ }^{142}$ Center for Substance Abuse Treatment. (2004).

National Institute on Alcohol Abuse and Alcoholism. (2008).

Tai, B., \& Blaine, J. (1997).

${ }^{143}$ Erickson, C. K. (2007).

Leavitt, S. B. (2002).

National Institute on Alcohol Abuse and Alcoholism. (2007).

National Institute on Drug Abuse. (2009e).

Sherman, C. (2007).

${ }^{144}$ National Institute on Alcohol Abuse and Alcoholism. (2007).

${ }^{145}$ Kjome, K. L., \& Moeller, F. G. (2011).

U.S.Food and Drug Administration. (2010b).

${ }^{146}$ Buddy, T. (2010).

Center for Substance Abuse Treatment. (2009e).

Haber, P. (2012).

National Institute on Alcohol Abuse and Alcoholism. (2008).

${ }^{147}$ Center for Substance Abuse Treatment. (2004).

Tai, B., \& Blaine, J. (1997).

Volpicelli, J. R., Rhines, K. C., Rhines, J. S., Volpicelli, L. A., Alterman, A. I., \& O'Brien, C. P. (1997).

148 Oncken, C., Van, K. J., \& Kranzler, H. R. (2001).

Kranzler, H. R., Modesto-Lowe, V., \& Van, K. J. (2000).

Rohsenow, D. J., Colby, S. M., Monti, P. M., Swift, R. M., Martin, R. A., Mueller, T. I., et al. (2000). 
${ }^{149}$ Chick, J., Anton, R., Checinski, K., Croop, R., Drummond, D. C., Farmer, R., et al. (2000).

Monti, P. M., Rohsenow, D. J., Swift, R. M., Gulliver, S. B., Colby, S. M., Mueller, T. I., et al. (2001).

Volpicelli, J. R., Rhines, K. C., Rhines, J. S., Volpicelli, L. A., Alterman, A. I., \& O'Brien, C. P. (1997).

${ }^{150}$ Comer, S. D., Sullivan, M. A., Yu, E., Rothenberg, J. L., Kleber, H. D., Kampman, K. M., et al. (2006).

${ }^{151}$ Garbutt, J. C., Kranzler, H. R., O'Malley, S. S., Gastfriend, D. R., Pettinati, H. M., Silverman, B. L., et al. (2005).

${ }^{152}$ Anton, R. F., Moak, D. H., Waid, L. R., Latham, P. K., Malcolm, R. J., \& Dias, J. K. (1999).

Morris, P. L. P., Hopwood, M., Whelan, G., Gardiner, J., \& Drummond, E. (2001).

${ }^{153}$ Anton, R. F., Moak, D. H., Waid, L. R., Latham, P. K., Malcolm, R. J., \& Dias, J. K. (1999).

O'Malley, S. S., Jaffe, A. J., Chang, G., Schottenfeld, R. S., Meyer, R. E., \& Rounsaville, B. (1992).

Volpicelli, J. R., Alterman, A. I., Hayashida, M., \& O'Brien, C. P. (1992).

${ }^{154}$ Tai, B., \& Blaine, J. (1997).

${ }^{155}$ Comer, S. D., Sullivan, M. A., Yu, E., Rothenberg, J. L., Kleber, H. D., Kampman, K. M., et al. (2006).

${ }^{156}$ Gerra, G., Fantoma, A., \& Zaimovic, A. (2006).

${ }^{157}$ Center for Substance Abuse Treatment. (2004).

Digiusto, E., Shakeshaft, A., Ritter, A., O'Brien, S., Mattick, R. P., \& NEPOD Research Group. (2004).

Kjome, K. L., \& Moeller, F. G. (2011).

${ }^{158}$ Aubin, H. J., Bobak, A., Britton, J. R., Oncken, C., Billing, C. B., Gong, J., et al. (2008).

Cahill, K., Stead, L. F., \& Lancaster, T. (2007).

Hays, J. T., Ebbert, J. O., \& Sood, A. (2008).

${ }^{159}$ Cahill, K., Stead, L. F., \& Lancaster, T. (2007).

Hays, J. T., Ebbert, J. O., \& Sood, A. (2008).

${ }^{160}$ Cahill, K., Stead, L. F., \& Lancaster, T. (2007).

${ }^{161}$ Cahill, K., Stead, L. F., \& Lancaster, T. (2007).

${ }^{162}$ U.S. Department of Health and Human Services, U.S. Food and Drug Administration, Center for Drug Evaluation and Research. (2008).

${ }^{163}$ U.S. Department of Health and Human Services, U.S. Food and Drug Administration, Center for Drug

Evaluation and Research. (2011).

${ }^{164}$ U.S.Food and Drug Administration. (2011b).

165 Arias, A. J., \& Kranzler, H. R. (2008).

Dackis, C. A., Lynch, K. G., Yu, E., Samaha, F. F., Kampman, K. M., Cornish, J. W., et al. (2003).

U.S. Department of Health and Human Services, U.S. Food and Drug Administration, Center for Drug Evaluation and Research. (2010).

Hart, C. L., Haney, M., Vosburg, S. K., Rubin, E., \& Foltin, R. W. (2008).

Malcolm, R., Swayngim, K., Donovan, J. L., DeVane, C. L., Elkashef, A., Chiang, N., et al. (2006).

${ }^{166}$ Arias, A. J., \& Kranzler, H. R. (2008).

Dackis, C. A., Lynch, K. G., Yu, E., Samaha, F. F., Kampman, K. M., Cornish, J. W., et al. (2003).

Hart, C. L., Haney, M., Vosburg, S. K., Rubin, E., \& Foltin, R. W. (2008).

${ }_{167}$ Dackis, C. A., Lynch, K. G., Yu, E., Samaha, F. F., Kampman, K. M., Cornish, J. W., et al. (2003).

168 Anderson, A. L., Reid, M. S., Li, S. H., Holmes, T., Shemanski, L., Slee, A., et al. (2009).

${ }^{169}$ Arias, A. J., \& Kranzler, H. R. (2008).

Johnson, B. A., Rosenthal, N., Capece, J. A., Wiegand, F., Mao, L., Beyers, K., et al. (2007).

Johnson, B. A., Rosenthal, N., Capece, J. A., Wiegand, F., Mao, L., Beyers, K., et al. (2008).

${ }^{170}$ Arias, A. J., \& Kranzler, H. R. (2008).

171 Johnson, B. A. (2004).

Ma, J. Z., Ait-Daoud, N., \& Johnson, B. A. (2006).

172 Johnson, B. A. (2005).

Kampman, K. M., Pettinati, H., Lynch, K. G., Dackis, C., Sparkman, T., Weigley, C., et al. (2004).

Karila, L., Reynaud, M., Aubin, H. J., Rolland, B., Guardia, D., Cottencin, O., et al. (2011).

Shinn, A. K., \& Greenfield, S. F. (2010).

${ }^{173}$ Arias, A. J., \& Kranzler, H. R. (2008).

Mueller, T. I., Stout, R. L., Rudden, S., Brown, R. A., Gordon, A., Solomon, D. A., et al. (1997).

Salloum, I. M., Cornelius, J. R., Daley, D. C., Kirisci, L., Himmelhoch, J. M., \& Thase, M. E. (2005).

${ }^{174}$ Kinsey, B. M., Jackson, D. C., \& Orson, F. M. (2009).

Orson, F. M., Kinsey, B. M., Singh, R. A. K., Wu, Y., Gardner, T., \& Kosten, T. R. (2008).

Shen, X., Orson, F. M., \& Kosten, T. R. (2011). 
Shorter, D., \& Kosten, T. R. (2011).

${ }^{175}$ Martell, B. A., Orson, F. M., Poling, J., Mitchell, E., Rossen, R. D., Gardner, T., et al. (2009).

Orson, F. M., Kinsey, B. M., Singh, R. A. K., Wu, Y., Gardner, T., \& Kosten, T. R. (2008).

Shorter, D., \& Kosten, T. R. (2011).

Whitten, L. (2010).

${ }^{176}$ Orson, F. M., Kinsey, B. M., Singh, R. A. K., Wu, Y., Gardner, T., \& Kosten, T. R. (2008).

${ }^{177}$ Chi, K. R. (2011).

Cornuz, J., Zwahlen, S., Jungi, W. F., Osterwalder, J., Klingler, K., van, M. G., et al. (2008).

LeSage, M. G., Keyler, D. E., \& Pentel, P. R. (2006).

Orson, F. M., Kinsey, B. M., Singh, R. A. K., Wu, Y., Gardner, T., \& Kosten, T. R. (2008).

Polosa, R., \& Benowitz, N. L. (2011).

${ }^{178}$ Pentel, P., \& Malin, D. (2002).

${ }^{179}$ Martell, B. A., Orson, F. M., Poling, J., Mitchell, E., Rossen, R. D., Gardner, T., et al. (2009).

${ }^{180}$ Byrnes-Blake, K. A., Laurenzana, E. M., Landes, R. D., Gentry, W. B., \& Owens, S. M. (2005).

Gentry, W. B., Laurenzana, E. M., Williams, D. K., West, J. R., Berg, R. J., Terlea, T., et al. (2006).

Moreno, A. Y., Mayorov, A. V., \& Janda, K. D. (2011).

Orson, F. M., Kinsey, B. M., Singh, R. A. K., Wu, Y., Gardner, T., \& Kosten, T. R. (2008).

Shen, X., Orson, F. M., \& Kosten, T. R. (2011).

${ }^{181}$ Hardin, J. S., Wessinger, W. D., Proksch, J. W., \& Owens, S. M. (1998).

Proksch, J. W., Gentry, W. B., \& Owens, S. M. (2000).

${ }^{182}$ Stead, L. F., Perera, R., Bullen, C., Mant, D., \& Lancaster, T. (2008).

${ }^{183}$ Clinical Practice Guideline Treating Tobacco Use and Dependence 2008 Update Panel, Liaisons, and Staff. (2008).

O'Brien, C. P. \& McKay, J. (2007).

Stead, L. F., Perera, R., Bullen, C., Mant, D., \& Lancaster, T. (2008).

${ }^{184}$ Nicorette. (2011).

helpwithsmoking.com. (2011).

Clinical Practice Guideline Treating Tobacco Use and Dependence 2008 Update Panel, Liaisons, and Staff. (2008).

${ }^{185}$ National Library of Medicine. (2011f).

${ }^{186}$ Stead, L. F., Perera, R., Bullen, C., Mant, D., \& Lancaster, T. (2008).

${ }^{187}$ Clinical Practice Guideline Treating Tobacco Use and Dependence 2008 Update Panel, Liaisons, and Staff. (2008).

${ }^{188}$ Clinical Practice Guideline Treating Tobacco Use and Dependence 2008 Update Panel, Liaisons, and Staff. (2008).

${ }^{189}$ Stead, L. F., Perera, R., Bullen, C., Mant, D., \& Lancaster, T. (2008).

${ }^{190}$ National Library of Medicine. (2008a).

National Library of Medicine. (2008b).

${ }^{191}$ Quitnet. (2009a).

${ }^{192}$ Clinical Practice Guideline Treating Tobacco Use and Dependence 2008 Update Panel, Liaisons, and Staff. (2008).

Stead, L. F., Perera, R., Bullen, C., Mant, D., \& Lancaster, T. (2008).

${ }^{193}$ Stead, L. F., Perera, R., Bullen, C., Mant, D., \& Lancaster, T. (2008).

${ }^{194}$ Quitnet. (2009b).

${ }^{195}$ Clinical Practice Guideline Treating Tobacco Use and Dependence 2008 Update Panel, Liaisons, and Staff. (2008).

Stead, L. F., Perera, R., Bullen, C., Mant, D., \& Lancaster, T. (2008).

${ }^{196}$ Stead, L. F., Perera, R., Bullen, C., Mant, D., \& Lancaster, T. (2008).

${ }^{197}$ Clinical Practice Guideline Treating Tobacco Use and Dependence 2008 Update Panel, Liaisons, and Staff. (2008).

${ }^{198}$ Clinical Practice Guideline Treating Tobacco Use and Dependence 2008 Update Panel, Liaisons, and Staff. (2008).

${ }^{199}$ Schnoll, R. A., Patterson, F., Wileyto, E. P., Heitjan, D. F., Shields, A. E., Asch, D. A., et al. (2010).

${ }^{200}$ Stead, L. F., Perera, R., Bullen, C., Mant, D., \& Lancaster, T. (2008).

${ }^{201}$ Clinical Practice Guideline Treating Tobacco Use and Dependence 2008 Update Panel, Liaisons, and Staff. (2008). 
${ }^{202}$ Clinical Practice Guideline Treating Tobacco Use and Dependence 2008 Update Panel, Liaisons, and Staff. (2008).

Stead, L. F., Perera, R., Bullen, C., Mant, D., \& Lancaster, T. (2008).

${ }^{203}$ Stead, L. F., Perera, R., Bullen, C., Mant, D., \& Lancaster, T. (2008).

${ }^{204}$ Fiore, M. C., Jorenby, D. E., Baker, T. B., \& Kenford, S. L. (1992).

Stead, L. F., Perera, R., Bullen, C., Mant, D., \& Lancaster, T. (2008).

${ }^{205}$ Alpert, H. R., Connolly, G. N., \& Biener, L. (2012).

${ }^{206}$ National Institute on Drug Abuse. (2009c).

${ }^{207}$ Centers for Disease Control and Prevention. (2002b).

${ }^{208}$ Centers for Disease Control and Prevention. (2002b).

Tinsley, J. A. (2011).

${ }^{209}$ McLellan, A. T. (2008).

Sadovsky, R. (2000).

${ }^{210}$ Centers for Disease Control and Prevention. (2002b).

McLellan, A. T. (2008).

${ }^{211}$ National Library of Medicine. (2009).

${ }^{212}$ Amato, L., Davoli, M., Perucci, C. A., Ferri, M., Faggiano, F., \& Mattick, R. P. (2005).

Gowing, L., Farrell, M., Bornemann, R., Sullivan, L., \& Ali, R. (2008).

Hubbard, R. L., Craddock, S. G., Flynn, P. M., Anderson, J., \& Etheridge, R. M. (1997).

Kleber, H. D. (2008).

Marsch, L. A. (1998).

Meandzija, B., O'Connor, P. G., Fitzgerald, B., Rounsaville, B. J., \& Kosten, T. R. (1994).

Executive Office of the President, Office of National Drug Control Policy. (2000).

${ }^{213}$ Drug Enforcement Administration. (2011).

${ }^{214}$ Fingerhut, L. A. (2008).

${ }^{215}$ National Highway Traffic Safety Administration. (2012).

${ }^{216}$ U.S. Department of Health and Human Services, Substance Abuse and Mental Health Services Administration, Center for Substance Abuse Treatment. (2009).

${ }^{217}$ Executive Office of the President, Office of National Drug Control Policy. (2000).

${ }^{218}$ Centers for Disease Control and Prevention. (2002b).

U.S. Drug Enforcement Administration, Office of Diversion Control. (2011).

${ }^{219}$ National Drug Intelligence Center. (2004).

${ }^{220}$ Center for Substance Abuse Treatment. (2011).

${ }^{221}$ Center for Substance Abuse Treatment. (2011).

${ }^{222}$ U.S. Food and Drug Administration, Center for Drug Evaluation and Research. (2002).

${ }^{223}$ Comer, S. D., Sullivan, M. A., Vosburg, S. K., Manubay, J., Amass, L., Cooper, Z. D., et al. (2010).

U.S. Food and Drug Administration, Center for Drug Evaluation and Research. (2002).

${ }^{224}$ Center for Substance Abuse Treatment. (2012).

${ }^{225}$ Center for Substance Abuse Treatment, Division of Pharmacologic Therapies. (2012).

Jones, H. E. (2004).

${ }^{226}$ Center for Substance Abuse Treatment, Division of Pharmacologic Therapies. (2012).

${ }^{227}$ New York Department of Health. (2011).

National Institute on Drug Abuse. (2012d).

Sobel, B.-F. X., Sigmon, S. C., Walsh, S. L., Johnson, R. E., Liebson, I. A., Nuwayser, E. S., et al. (2004).

${ }^{228}$ Amass, L., Kamien, J. B., \& Mikulich, S. K. (2000).

Johnson, R. E., Chutuape, M. A., Strain, E. C., Walsh, S. L., Stitzer, M. L., \& Bigelow, G. E. (2000).

Schottenfeld, R. S., Pakes, J., O'Connor, P., Chawarski, M., Oliveto, A., \& Kosten, T. R. (2000).

${ }^{229}$ Lanier, R. K., Umbricht, A., Harrison, J. A., Nuwayser, E. S., \& Bigelow, G. E. (2007).

Sobel, B.-F. X., Sigmon, S. C., Walsh, S. L., Johnson, R. E., Liebson, I. A., Nuwayser, E. S., et al. (2004).

${ }^{230}$ Center for Substance Abuse Treatment. (2012).

${ }^{231}$ Center for Substance Abuse Treatment. (2004).

Kraus, M. L., Alford, D. P., Kotz, M. M., Levounis, P., Mandell, T. W., Meyer, M., et al. (2011).

${ }^{232}$ Amato, L., Davoli, M., Perucci, C. A., Ferri, M., Faggiano, F., \& Mattick, R. P. (2005).

${ }^{233}$ Boothby, L. A., \& Doering, P. L. (2007).

Ling, W., Charuvastra, C., Collins, J. F., Batki, S., Brown, L. S., Jr., Kintaudi, P., et al. (1998). 
${ }^{234}$ Fudala, P. J., Bridge, T. P., Herbert, S., Williford, W. O., Chiang, C. N., Jones, K., et al. (2003).

${ }^{235}$ Pereira, F. C., Gough, B., Macedo, T. R., Ribeiro, C. F., Ali, S. F., \& Binienda, Z. K. (2011).

${ }^{236}$ Budney, A. J., Vandrey, R. G., Hughes, J. R., Moore, B. A., \& Bahrenburg, B. (2007).

Haney, M., Hart, C. L., Vosburg, S. K., Nasser, J., Bennett, A., Zubaran, C., et al. (2004).

${ }^{237}$ Vandrey, R., \& Haney, M. (2009).

${ }^{238}$ Carroll, K. M. (1996).

Center for Substance Abuse Treatment. (1999a).

National Institute on Drug Abuse. (2009c).

${ }^{239}$ Finney, J. W., Wilbourne, P. L., \& Moos, R. H. (2007).

${ }^{240}$ Arias, A. J., \& Kranzler, H. R. (2008).

Carroll, K. M. (1996).

Center for Substance Abuse Treatment. (1999a).

Cepeda-Benito, A. (1993).

National Institute on Drug Abuse. (2009c).

National Institute on Drug Abuse. (1995).

${ }^{241}$ National Institute on Drug Abuse. (2009e).

${ }^{242}$ Finney, J. W., Wilbourne, P. L., \& Moos, R. H. (2007).

National Institute on Drug Abuse. (2009c).

National Institute on Drug Abuse. (2009e).

${ }^{243}$ Center for Substance Abuse Treatment. (1999a).

${ }^{244}$ Center for Substance Abuse Treatment. (1999a).

${ }^{245}$ Center for Substance Abuse Treatment. (1999a).

Jones, P. (2009a).

${ }^{246}$ Center for Substance Abuse Treatment. (1999a).

Project MATCH Research Group. (1997).

Project MATCH Research Group. (1998).

${ }^{247}$ Burke, B. L., Arkowitz, H., \& Menchola, M. (2003).

Lee, S., Aos, S., Drake, E., Pennucci, A., Miller, M., \& Anderson, L. (2012).

Miller, W. R., Zweben, A., DiClemente, C. C., \& Rychtarik, R. G. (1994).

Project MATCH Research Group. (1998).

Sampl, S., Kadden, R., \& Center for Substance Abuse Treatment. (2001).

Stotts, A. L., Schmitz, J. M., Rhoades, H. M., \& Grabowski, J. (2001).

Tevyaw, T. O., \& Monti, P. M. (2004).

Vasilaki, E. I., Hosier, S. G., \& Cox, W. M. (2006).

Washington State Institute for Public Policy. (2012b).

Washington State Institute for Public Policy. (2012c).

Washington State Institute for Public Policy. (2012d).

Washington State Institute for Public Policy. (2012e).

${ }^{248}$ Carroll, K. M., Ball, S. A., Nich, C., Martino, S., Frankforter, T. L., Farentinos, C., et al. (2006).

Center for Substance Abuse Treatment. (1999a).

${ }^{249}$ Hser, Y.-I., Evans, E., Huang, D., \& Anglin, D. M. (2004).

Zhang, Z., Friedmann, P. D., \& Gerstein, D. R. (2003).

${ }^{250}$ Dornelas, E. A., Sampson, R. A., Gray, J. F., Waters, D., \& Thompson, P. D. (2000).

${ }^{251}$ Ball, S. A., Martino, S., Nich, C., Frankforter, T. L., Van, H. D., Crits-Christoph, P., et al. (2007).

${ }^{252}$ Center for Substance Abuse Treatment. (1999a).

Finney, J. W., Wilbourne, P. L., \& Moos, R. H. (2007).

${ }^{253}$ Finney, J. W., Wilbourne, P. L., \& Moos, R. H. (2007).

${ }^{254}$ National Institute on Drug Abuse. (2009e).

National Institute on Drug Abuse. (2007a).

${ }^{255}$ National Institute on Drug Abuse. (2007a).

${ }^{256}$ Baker, A., Lee, N. K., Claire, M., Lewin, T. J., Grant, T., Pohlman, S., et al. (2005).

Becker, S. J., \& Curry, J. F. (2008).

Carroll, K. M., Ball, S. A., Martino, S., Nich, C., \& Babuscio, T. (2009).

Carroll, K. M., Rounsaville, B. J., Nich, C., Lynn, G. T., Wirtz, P. W., \& Gawin, F. (1994).

Dennis, M., Godley, S. H., Diamond, G., Tims, F. M., Babor, T., Donaldson, J., et al. (2004). 
Waldron, H. B., \& Kaminer, Y. (2004).

Waldron, H. B., \& Turner, C. W. (2008).

${ }^{257}$ Hien, D. A., Cohen, L. R., Miele, G. M., Litt, L. C., \& Capstick, C. (2004).

Weiss, R. D., Griffin, M. L., Greenfield, S. F., Najavits, L. M., Wyner, D., Soto, J. A., et al. (2000).

Ziedonis, D. M., Smelson, D., Rosenthal, R. N., Batki, S. L., Green, A. I., Henry, R. J., et al. (2005).

${ }^{258}$ National Institute on Drug Abuse. (2009e).

${ }^{259}$ National Institute on Drug Abuse. (2009e).

Wolfe, B. L., \& Meyers, R. J. (1999).

${ }^{260}$ Finney, J. W., Wilbourne, P. L., \& Moos, R. H. (2007).

${ }^{261}$ Finney, J. W., Wilbourne, P. L., \& Moos, R. H. (2007).

Wolfe, B. L., \& Meyers, R. J. (1999).

${ }^{262}$ Finney, J. W., Wilbourne, P. L., \& Moos, R. H. (2007).

Roozen, H. G., Boulogne, J. J., van Tulder, M. W., van den Brink, W., De Jong, C. A. J., \& Kerkhof, A. J. F. M. (2004).

Smith, J. E., Meyers, R. J., \& Miller, W. R. (2001).

Wolfe, B. L., \& Meyers, R. J. (1999).

${ }^{263}$ Finney, J. W., Wilbourne, P. L., \& Moos, R. H. (2007).

Higgins, S. T., Budney, A. J., Bickel, W. K., Foerg, F. E., Donham, R., \& Badger, G. J. (1994).

Higgins, S. T., Wong, C. J., Badger, G. J., Huag Ogden, D. E., \& Dantona, R. L. (2000).

${ }^{264}$ Azrin, N. H. (1976).

Higgins, S. T., Sigmon, S. C., Wong, C. J., Heil, S. H., Badger, G. J., Donham, R., et al. (2003).

${ }^{265}$ Budney, A. J., Higgins, S. T., \& Sigmon, S. C. (2003).

Iguchi, M. Y., Stitzer, M. L., Bigelow, G. E., \& Liebson, I. A. (1988).

Kidorf, M., \& Stitzer, M. L. (1996).

${ }^{266}$ Finney, J. W., Wilbourne, P. L., \& Moos, R. H. (2007).

Iguchi, M. Y., Stitzer, M. L., Bigelow, G. E., \& Liebson, I. A. (1988).

Lussier, J. P., Heil, S. H., Mongeon, J. A., Badger, G. J., \& Higgins, S. T. (2006).

${ }^{267}$ Correia, C. J., \& Benson, T. A. (2006).

Higgins, S. T., Alessi, S. M., \& Dantona, R. L. (2002).

Higgins, S. T., \& Petry, N. M. (1999).

Jones, P. (2009b).

Krishnan-Sarin, S., Duhig, A. M., McKee, S. A., McMahon, T. J., Liss, T., McFetridge, A., et al. (2006).

Lussier, J. P., Heil, S. H., Mongeon, J. A., Badger, G. J., \& Higgins, S. T. (2006).

Petry, N. M., Peirce, J. M., Stitzer, M. L., Blaine, J., Roll, J. M., Cohen, A., et al. (2005).

Prendergast, M., Podus, D., Finney, J., Greenwell, L., \& Roll, J. (2006).

Roll, J. M. (2007).

Silverman, K., Wong, C. J., Higgins, S. T., Brooner, R. K., Montoya, I. D., Contoreggi, C., et al. (1996).

${ }^{268}$ Griffith, J. D., Rowan-Szal, G. A., Roark, R. R., \& Simpson, D. D. (2000).

Higgins, S. T., Alessi, S. M., \& Dantona, R. L. (2002).

Higgins, S. T., Heil, S. H., Dantona, R., Donham, R., Matthews, M., \& Badger, G. J. (2007).

Higgins, S. T., Sigmon, S. C., Wong, C. J., Heil, S. H., Badger, G. J., Donham, R., et al. (2003).

Lussier, J. P., Heil, S. H., Mongeon, J. A., Badger, G. J., \& Higgins, S. T. (2006).

${ }^{269}$ O'Farrell, T. J., \& Fals-Stewart, W. (2003).

Stanton, M. D., \& Shadish, W. R. (1997).

${ }^{270}$ Fals-Stewart, W., O'Farrell, T. J., Feehan, M., Birchler, G. R., Tiller, S., \& McFarlin, S. K. (2000).

Galanter, M., Dermatis, H., Glickman, L., Maslansky, R., Sellers, M. B., Neumann, E., et al. (2004).

Galanter, M., Dermatis, H., Keller, D., \& Trujillo, M. (2002).

Klostermann.K., Kelley, M. L., Mignone, T., Pusateri, L., \& Wills, K. (2011).

Liddle, H. A., Dakof, G. A., Parker, K., Diamond, G. S., Barrett, K., \& Tejeda, M. (2001).

Liddle, H. A., Dakof, G. A., Henderson, C. E., \& Greenbaum, P. E. (2009).

Liddle, H. A., Rowe, C. L., Dakof, G. A., Ungaro, R. A., \& Henderson, C. E. (2004).

O'Farrell, T. J., \& Fals-Stewart, W. (2003).

Stanton, M. D., \& Shadish, W. R. (1997).

${ }^{271}$ Copello, A. G., Templeton, L., \& Velleman, R. (2006).

Klostermann.K., Kelley, M. L., Mignone, T., Pusateri, L., \& Wills, K. (2011). 
Rowe, C. L., \& Liddle, H. A. (2003).

Stanton, M. D., \& Shadish, W. R. (1997).

${ }^{272}$ Fals-Stewart, W., Lam, W. K. K., \& Kelley, M. L. (2009).

Fals-Stewart, W., O'Farrell, T. J., Feehan, M., Birchler, G. R., Tiller, S., \& McFarlin, S. K. (2000).

Powers, M. B., Vedel, E., \& Emmelkamp, P. M. G. (2008).

Winters, J., Fals-Stewart, W., O'Farrell, T. J., Bircgler, G. R., \& Kelley, M. L. (2002).

${ }^{273}$ Amato, L., Minozzi, S., Davoli, M., Vecchi, S., Ferri, M. M., \& Mayet, S. (2009).

Anton, R. F., \& Swift, R. M. (2003).

Arias, A. J., \& Kranzler, H. R. (2008).

Balldin, J., Berglund, M., Borg, S., Mansson, M., Bendtsen, P., Franck, J., et al. (2003).

Carroll, K. M., Nich, C., Ball, S. A., McCance, E., \& Rounsaville, B. J. (1998).

Carroll, K. M., Rounsaville, B. J., Nich, C., Gordon, L., \& Gawin, F. (1995).

Foulds, J., Schmelzer, A. C., \& Steinberg, M. B. (2010).

Hughes, J. R. (1995).

Kosten, T., Oliveto, A., Feingold, A., Poling, J., Sevarino, K., Cance-Katz, E., et al. (2003).

National Institute on Drug Abuse. (2009c).

O'Brien, C. P., Woody, G. E., \& McLellan, A. T. (1995).

Poldrugo, F. (1997).

Poling, J., Oliveto, A., Petry, N., Sofuoglu, M., Gonsai, K., Gonzalez, G., et al. (2006).

Rigotti, N. A. (2009).

Rigotti, N. A., Munafo, M. R., \& Stead, L. F. (2008).

Roddy, E. (2004).

Silverman, K., Wong, C. J., Higgins, S. T., Brooner, R. K., Montoya, I. D., Contoreggi, C., et al. (1996).

Stitzer, M. L. (1999).

Stitzer, M. L., \& Walsh, S. L. (1997).

Tai, B., \& Blaine, J. (1997).

${ }^{274}$ Miller, N. S. (1997).

275 Schwarzlose, J., Chief Executive Officer, Betty Ford Center (personal communication, August 3, 2007).

${ }^{276}$ Hughes, J. R. (1995).

Stitzer, M. L. (1999).

${ }^{277}$ Hughes, J. R. (1995).

Stitzer, M. L. (1999).

${ }^{278}$ Amato, L., Minozzi, S., Davoli, M., Vecchi, S., Ferri, M. M., \& Mayet, S. (2009).

Carroll, K. M., Nich, C., Ball, S. A., McCance, E., \& Rounsaville, B. J. (1998).

Rigotti, N. A., Munafo, M. R., \& Stead, L. F. (2008).

Roddy, E. (2004).

${ }^{279}$ Hughes, J. R. (1995).

National Institute on Drug Abuse. (2009c).

Stitzer, M. L. (1999).

${ }^{280}$ Hughes, J. R. (1995).

${ }^{281}$ Cinciripini, P. M., Cinciripini, L. G., Wallfisch, A., Haque, W., \& Van, V. H. (1996).

Fiore, M. C., Kenford, S. L., Jorenby, D. E., Wetter, D. W., Smith, S. S., \& Baker, T. B. (1994).

Goldstein, M. G., Niaura, R., Follick, M. J., \& Abrams, D. B. (1989).

Hughes, J. R. (1995).

Stitzer, M. L. (1999).

${ }^{282}$ Hughes, J. R. (1995).

${ }^{283}$ Goldstein, M. G., Niaura, R., Follick, M. J., \& Abrams, D. B. (1989).

Hughes, J. R. (1995).

${ }^{284}$ Hurt, R. D., Sachs, D. P., Glover, E. D., Offord, K. P., Johnston, J. A., Dale, L. C., et al. (1997).

${ }^{285}$ Foulds, J., Schmelzer, A. C., \& Steinberg, M. B. (2010).

${ }^{286}$ Anton, R. F., Moak, D. H., Latham, P., Waid, L. R., Myrick, H., Voronin, K., et al. (2005).

${ }^{287}$ Feeney, G. F. X., Young, R. M., Connor, J. P., Tucker, J., \& McPherson, A. (2002).

${ }^{288}$ Azrin, N. H., Sisson, W., Myers, R., \& Godley, M. (1982).

${ }^{289}$ Fals-Stewart, W., \& O'Farrell, T. J. (2003).

${ }^{290}$ Griffith, J. D., Rowan-Szal, G. A., Roark, R. R., \& Simpson, D. D. (2000). 
${ }^{291}$ Carroll, K. M., Ball, S. A., Nich, C., O'Connor, P. G., Eagan, D. A., Frankforter, T. L., et al. (2001).

${ }^{292}$ Abbott, P. J., Weller, S. B., Delaney, H. D., \& Moore, B. A. (1998).

${ }^{293}$ Dackis, C. A., Kampman, K. M., Lynch, K. G., Pettinati, H. M., \& O'Brien, C. P. (2005).

${ }^{294}$ Poling, J., Oliveto, A., Petry, N., Sofuoglu, M., Gonsai, K., Gonzalez, G., et al. (2006).

${ }^{295}$ Kosten, T., Oliveto, A., Feingold, A., Poling, J., Sevarino, K., Cance-Katz, E., et al. (2003).

National Library of Medicine. (2010d).

${ }^{296}$ Kosten, T., Oliveto, A., Feingold, A., Poling, J., Sevarino, K., Cance-Katz, E., et al. (2003).

${ }^{297}$ Pettinati, H. M., Kampman, K. M., Lynch, K. G., Suh, J. J., Dackis, C. A., Oslin, D. W., et al. (2008).

${ }^{298}$ Biery, J. R., Williford, J. H., Jr., \& McMullen, E. A. (1991).

Bock, B. C., Marcus, B. H., King, T. K., Borrelli, B., \& Roberts, M. R. (1999).

Kochupillai, V., Kumar, P., Singh, D., Aggarwal, D., Bhardwaj, N., Bhutani, M., et al. (2005).

Marcus, B. H., Albrecht, A. E., King, T. K., Parisi, A. F., Pinto, B. M., Roberts, M., et al. (1999).

Martin, J. E., Calfas, K. J., Patten, C. A., Polarek, M., Hofstetter, C. R., Noto, J., et al. (1997).

McClernon, F. J., Westman, E. C., \& Rose, J. E. (2004).

Singh, V. N. (1992).

Ussher, M. H., Taylor, A. H., West, R., \& McEwen, A. (2000).

${ }^{299}$ Çepik, A., Arikan, Z., Boratav, C., \& Isik, E. (1995).

Edge, P. J., \& Gold, M. S. (2011).

Gold, J., \& Gold, M. S. (2011).

Kenny, P. J. (2011).

Shaffer, H. J., LaPlante, D. A., LaBrie, R. A., Kidman, R. C., Donato, A. N., \& Stanton, M. V. (2004).

Volkow, N. D., Wang, G. J., Fowler, J. S., \& Telang, F. (2008).

Volkow, N. D., Wang, G. J., Fowler, J. S., Tomasi, D., \& Baler, R. (2011).

${ }^{300}$ Benedict, J., Evans, W., \& Calder, J. C. (1999).

National Library of Medicine. (2010g).

U.S. Department of Health and Human Services. (1993).

Santolaria, F., Perez-Manzano, J. L., Milena, A., Gonzalez-Reimers, E., Gomez-Rodriguez, M. A., Martinez-Riera, A., et al. (2000).

${ }^{301}$ National Library of Medicine. (2010g).

Finnell, D. S. (2000).

${ }^{302}$ Forsander, O. A. (1998).

Yung, L., Gordis, E., \& Holt, J. (1983).

${ }^{303}$ The Economist. (2008, October 18).

${ }^{304}$ Harvard School of Public Health. (2012).

Wurtman, R. J., Hefti, F., \& Melamed, E. (1981).

${ }^{305}$ Foley, T. E., \& Fleshner, M. (2008).

${ }^{306}$ Bock, B. C., Marcus, B. H., King, T. K., Borrelli, B., \& Roberts, M. R. (1999).

Janse Van Rensburg, K., Taylor, A., Hodgson, T., \& Benattayallah, A. (2009).

${ }^{307}$ Bock, B. C., Marcus, B. H., King, T. K., Borrelli, B., \& Roberts, M. R. (1999).

Marcus, B. H., Albrecht, A. E., King, T. K., Parisi, A. F., Pinto, B. M., Roberts, M., et al. (1999).

Read, J. P., Brown, R. A., Marcus, B. H., Kahler, C. W., Ramsey, S. E., Dubreuil, M. E., et al. (2001).

Read, J. P., \& Brown, R. A. (2003).

${ }^{308}$ Bock, B. C., Marcus, B. H., King, T. K., Borrelli, B., \& Roberts, M. R. (1999).

Peterson, M., \& Johnstone, B. M. (1995).

Read, J. P., Brown, R. A., Marcus, B. H., Kahler, C. W., Ramsey, S. E., Dubreuil, M. E., et al. (2001).

${ }^{309}$ Palmer, J. A., Palmer, L. K., Michiels, K., \& Thigpen, B. (1995).

${ }^{310}$ Bock, B. C., Marcus, B. H., King, T. K., Borrelli, B., \& Roberts, M. R. (1999).

National Library of Medicine. (2011e).

${ }^{311}$ National Library of Medicine. (2011e).

O'Connor, P. J., \& Youngstedt, S. D. (1995).

${ }^{312}$ National Library of Medicine. (2011e).

Pronk, N. P., Crouse, S. F., \& Rohack, J. J. (1995).

${ }^{313}$ Marcus, B. H., Albrecht, A. E., King, T. K., Parisi, A. F., Pinto, B. M., Roberts, M., et al. (1999).

${ }^{314}$ Hasin, D. S., Stinson, F. S., Ogburn, E., \& Grant, B. F. (2007).

Willenbring, M. L. (2008a). 
${ }^{315}$ Dawson, D. A., Grant, B. F., Stinson, F. S., Chou, P. S., Huang, B., \& Ruan, W. J. (2005).

Erickson, C. K. (2007).

Hudson, N. L., \& Mannino, D. M. (2010).

Lewis, D. C. (1991).

McLellan, A. T., Lewis, D. C., O'Brien, C. P., \& Kleber, H. D. (2000).

Roan, S. (2009).

The Partnership at DrugFree.org. (2012).

White, W. L. (2008).

${ }^{316}$ Erickson, C. K. (2007).

McLellan, A. T., Lewis, D. C., O'Brien, C. P., \& Kleber, H. D. (2000).

White, W. L. (2008).

${ }^{317}$ Boyle, M. G., \& Murphy, T. P. (2005).

National Quality Forum. (2005).

${ }^{318}$ American Society of Addiction Medicine. (2011b).

O'Brien, C. P., \& McLellan, A. T. (1996).

${ }^{319}$ Festinger, D. S., Rubenstein, D. F., Marlowe, D. B., \& Platt, J. J. (2001).

${ }^{320}$ Rando, K., Hong, K. I., Bhagwagar, Z., Li.C.S.R., Bergquist, K., Guarnaccia, J., et al. (2011). Weiner, D. (2011).

${ }^{321}$ Willenbring, M. L. (2008a).

${ }^{322}$ Schaefer, J. A., Cronkite, R. C., \& Hu, K. U. (2011).

Watkins, K., Pincus, H. A., Tanielian, T. L., \& Lloyd, J. (2003).

${ }^{323}$ Center for Substance Abuse Treatment. (1999c).

${ }^{324}$ Friedmann, P. D., Saitz, R., \& Samet, J. H. (1998).

Forum on Integration. (2011).

Watkins, K., Pincus, H. A., Tanielian, T. L., \& Lloyd, J. (2003).

${ }^{325}$ American Society of Addiction Medicine. (2011b).

Friedmann, P. D., Saitz, R., \& Samet, J. H. (1998).

Caribbean Food \& Nutrition Institute for Caucus of Ministers of Health. (2002).

Watkins, K., Pincus, H. A., Tanielian, T. L., \& Lloyd, J. (2003).

${ }^{326}$ National Institute on Drug Abuse. (2009e).

Kosten, T. R. (1994).

Colker, A. C. (2004).

Weiss, R. D., Potter, J. S., Fiellin, D. A., Byrne, M., Connery, H. S., Dickinson, W., et al. (2011).

Woody, G. E., Poole, S. A., Subramaniam, G., Dugosh, K., Bogenschutz, M., Abbott, P., et al. (2008).

${ }^{327}$ Friedmann, P. D., Saitz, R., \& Samet, J. H. (1998).

${ }^{328}$ Verebey, K. G., Meenan, G., \& Buchan, B. J. (2005

${ }^{329}$ Butler, A. C., Chapman, J. E., Forman, E. M., \& Beck, A. T. (2006).

Carroll, K. M. (1996).

${ }^{330}$ Shore, J. H. (1987).

${ }^{331}$ Merlo, L. J., \& Gold, M. (2008).

Ross, S. (2003).

Federation of State Physician Health Programs. (2011).

${ }^{332}$ Merlo, L. J., \& Gold, M. (2008).

${ }^{333}$ Dupont, R. L., McLellan, A. T., Carr, G., Gendel, M., \& Skipper, G. E. (2009).

${ }^{334}$ Dupont, R. L., McLellan, A. T., Carr, G., Gendel, M., \& Skipper, G. E. (2009).

${ }^{335}$ Federaion of State Physician Health Program. (2005).

${ }^{336}$ Dupont, R. L., McLellan, A. T., Carr, G., Gendel, M., \& Skipper, G. E. (2009).

Merlo, L. J., \& Gold, M. (2008).

Ross, S. (2003).

${ }^{337}$ Merlo, L. J., \& Gold, M. (2008).

${ }^{338}$ Dupont, R. L., McLellan, A. T., Carr, G., Gendel, M., \& Skipper, G. E. (2009).

${ }^{339}$ Merlo, L. J., \& Gold, M. (2008).

${ }^{340}$ Merlo, L. J., \& Gold, M. (2008).

${ }^{341}$ Dupont, R. L., McLellan, A. T., Carr, G., Gendel, M., \& Skipper, G. E. (2009).

${ }^{342}$ Dupont, R. L., McLellan, A. T., Carr, G., Gendel, M., \& Skipper, G. E. (2009). 
Dupont, R. L., \& Skipper, G. E. (2012).

${ }^{343}$ Center for Substance Abuse Treatment. (1998a).

${ }^{344}$ Dennis, M. L., \& Scott, C. K. (2012).

345 Dennis, M., Scott, C. K., \& Funk, R. (2003).

Scott, C. K., \& Dennis, M. L. (2009).

${ }^{346}$ Scott, C. K., Dennis, M. L., \& Foss, M. A. (2005).

${ }^{347}$ Center for Substance Abuse Treatment. (1998a).

${ }^{348}$ Center for Substance Abuse Treatment. (1998a).

Watkins, K., Pincus, H. A., Tanielian, T. L., \& Lloyd, J. (2003).

${ }^{349}$ Scott, C. K., Dennis, M. L., \& Foss, M. A. (2005).

${ }^{350}$ Mejta, C. L., Bokos, P. J., Mickenberg, J., Maslar, M. E., \& Senary, E. (1997).

Ryan, J. P. (2006).

${ }^{351}$ Siegal, H. A., Rapp, R. C., Li, L., \& Saha, P. (2001).

352 Siegal, H. A., Fisher, J. H., Rapp, R. C., Kelliher, C. W., Wagner, J. H., O'Brien, W. F., et al. (1996).

${ }^{353}$ Hesse, M., Vanderplasschen, W., Rapp, R. C., Broekaert, E., \& Fridell, M. (2007).

${ }^{354}$ Morgenstern, J., Bux, D., Labouvie, E., Blanchard, K. A., \& Morgan, T. I. (2002).

Morgenstern, J., Blanchard, K. A., McCrady, B. S., McVeigh, K. H., Morgan, T. J., \& Pandina, R. J. (2006).

355 The National Center on Addiction and Substance Abuse (CASA) at Columbia University. (2009b).

${ }^{356}$ Biery, J. R., Williford, J. H., Jr., \& McMullen, E. A. (1991).

Bock, B. C., Marcus, B. H., King, T. K., Borrelli, B., \& Roberts, M. R. (1999).

Bodenheimer, T., Wagner, E. H., \& Grumbach, K. (2002).

Erickson, C. K. (2007).

Finney, J. W., Wilbourne, P. L., \& Moos, R. H. (2007).

Grant, L. P., Haughton, B., \& Sachan, D. S. (2004).

Institute of Medicine. (1990).

Kochupillai, V., Kumar, P., Singh, D., Aggarwal, D., Bhardwaj, N., Bhutani, M., et al. (2005).

Kremer, D., Malkin, M. J., \& Benshoff, J. J. (1995).

Mackay, P. W., \& Marlatt, G. A. (1991).

Marcus, B. H., Albrecht, A. E., King, T. K., Parisi, A. F., Pinto, B. M., Roberts, M., et al. (1999).

Master, R. J. (2011).

McClernon, F. J., Westman, E. C., \& Rose, J. E. (2004).

McIver, S., O'Halloran, P., \& McGartland, M. (2004).

Wagner, E. H., Austin, B. T., Davis, C., Hindmarsh, M., Schaefer, J., \& Bonomi, A. (2001).

White, W. L. (2008).

White, W. L. \& Kelly, J. F. (2011).

${ }^{357}$ Friedmann, P. D., Saitz, R., \& Samet, J. H. (1998).

Forum on Integration. (2011).

Watkins, K., Pincus, H. A., Tanielian, T. L., \& Lloyd, J. (2003).

${ }^{358}$ Chi, F. W., Campbell, C. I., Sterling, S., \& Weisner, C. (2011).

Workgroup on Substance Abuse Self-Help Organizations. (2003).

Kelly, J. F., Stout, R., Zywiak, W., \& Schneider, R. (2006).

Zemore, S. E., \& Kaskutas, L. A. (2008).

${ }^{359}$ Center for Substance Abuse Treatment. (2008).

${ }^{360}$ Humphreys, K. (2003).

McKellar, J., Stewart, E., \& Humphreys, K. (2003).

Tims, F. M., Leukefeld, C. G., \& Platt, J. J. (2001).

Workgroup on Substance Abuse Self-Help Organizations. (2003).

${ }^{361}$ Office of Applied Studies. (2008).

${ }^{362}$ Humphreys, K. (2004).

${ }^{363}$ Humphreys, K. (2004).

${ }^{364}$ Humphreys, K. (2004).

${ }^{365}$ Faces and Voices of Recovery. (2011).

Humphreys, K. (2004).

${ }^{366}$ Center for Substance Abuse Treatment. (2008).

Gamblers Anonymous. (2012). 
Sex Addicts Anonymous. (2012).

${ }^{367}$ Center for Substance Abuse Treatment. (2008).

${ }^{368}$ Friedmann, P. D., Saitz, R., \& Samet, J. H. (1998).

${ }^{369}$ Center for Substance Abuse Treatment. (2008).

${ }^{370}$ Center for Substance Abuse Treatment. (2008).

Friedmann, P. D., Saitz, R., \& Samet, J. H. (1998).

${ }^{371}$ Alcoholics Anonymous World Services. (2007).

${ }^{372}$ SMART Recovery. (2011).

${ }^{373}$ Center for Substance Abuse Treatment. (2008).

LifeRing. (2011).

${ }^{374}$ Center for Substance Abuse Treatment. (2008).

${ }^{375}$ Butler Center for Research. (2004).

Fiorentine, R. (1999).

Fiorentine, R., \& Hillhouse, M. P. (2000).

Kaskutas, L. A. (2009).

Kelly, J. F., Stout, R., Zywiak, W., \& Schneider, R. (2006).

McKellar, J., Stewart, E., \& Humphreys, K. (2003).

Timko, C., Moos, R. H., Finney, J. W., \& Lesar, M. D. (2000).

Workgroup on Substance Abuse Self-Help Organizations. (2003).

Zemore, S. E., Kaskutas, L. A., \& Ammon, L. N. (2004).

${ }^{376}$ Ferri, M. M. F., Amato, L., \& Davoli, M. (2006).

${ }^{377}$ Ferri, M. M. F., Amato, L., \& Davoli, M. (2006).

Tims, F. M., Leukefeld, C. G., \& Platt, J. J. (2001).

${ }^{378}$ Fiorentine, R. (1999).

McKellar, J., Stewart, E., \& Humphreys, K. (2003).

${ }^{379}$ Kaskutas, L. A. (2009).

Lile, B. (2003).

${ }^{380}$ Butler Center for Research. (2004).

Fiorentine, R., \& Hillhouse, M. P. (2000).

Kelly, J. F., Stout, R., Zywiak, W., \& Schneider, R. (2006).

Morgenstern, J., Labouvie, E., McCrady, B. S., Kahler, C. W., \& Frey, R. M. (1997).

${ }^{381}$ Kelly, J. F., Stout, R., Zywiak, W., \& Schneider, R. (2006).

${ }^{382}$ Kaskutas, L. A. (2009).

${ }^{383}$ Kelly, J. F., Magill, M., \& Stout, R. L. (2009).

${ }^{384}$ Humphreys, K. (2003).

McKellar, J., Stewart, E., \& Humphreys, K. (2003).

Tims, F. M., Leukefeld, C. G., \& Platt, J. J. (2001).

Workgroup on Substance Abuse Self-Help Organizations. (2003).

${ }^{385}$ Humphreys, K. (2003).

Kelly, J. F., \& Yeterian, J. D. (2011).

Nowinski, J. (2009).

Substance Abuse and Mental Health Services Administration. (2010c).

${ }^{386}$ Nowinski, J. (2009).

${ }^{387}$ Project MATCH Research Group. (1998).

${ }^{388}$ Drapkin, M. L., Tate, S. R., McQuaid, J. R., \& Brown, S. A. (2008).

Granholm, E., Tate, S. R., Link, P. C., Lydecker, K. P., Cummins, K. M., McQuaid, J., et al. (2011).

Worley, M. J., Trim, R. S., Tate, S. R., Hall, J. E., \& Brown, S. A. (2010).

${ }^{389}$ Finney, J. W., Wilbourne, P. L., \& Moos, R. H. (2007).

Humphreys, K. (2003).

Project MATCH Research Group. (1998).

Substance Abuse and Mental Health Services Administration. (2010c).

${ }^{390}$ Substance Abuse and Mental Health Services Administration. (2010c).

${ }^{391}$ National Institute on Drug Abuse. (2009e).

392 Jason, L. A., \& Ferrari, J. R. (2010).

Polcin, D. L. (2001). 
${ }^{393}$ Polcin, D. L. (2001).

Oxford House. (2000).

${ }^{394}$ Center for Substance Abuse Treatment. (2005c).

Inciardi, J. A., Martin, S. S., \& Butzin, C. A. (2004).

Treatment Communities of America. (2012).

Welsh, W. N. (2007).

${ }^{395}$ Finney, J. W., Wilbourne, P. L., \& Moos, R. H. (2007).

Institute of Medicine. (1997).

National Institute on Drug Abuse. (2009c).

${ }^{396}$ National Institute on Drug Abuse. (2002).

${ }^{397}$ National Institute on Drug Abuse. (2009c).

${ }^{398}$ National Institute on Drug Abuse. (2002).

${ }^{399}$ Simpson, D. D., Joe, G. W., \& Brown, B. S. (1997).

${ }^{400}$ Hubbard, R. L., Craddock, S. G., Flynn, P. M., Anderson, J., \& Etheridge, R. M. (1997).

${ }^{401}$ Center for Substance Abuse Treatment. (2005b).

${ }^{402}$ Polcin, D. L. (2001).

Polcin, D. L., \& Henderson, D. M. (2008).

${ }^{403}$ Oxford House. (2011).

Rees, T. (2011).

${ }^{404}$ Polcin, D. L., Korcha, R. A., Bond, J., \& Galloway, G. (2010).

${ }^{405}$ Jason, L. A., \& Ferrari, J. R. (2010).

Oxford House. (2000).

${ }^{406}$ Polcin, D. L., Korcha, R. A., Bond, J., \& Galloway, G. (2010).

${ }^{407}$ Oxford House. (2009).

Oxford House. (2007).

${ }^{408}$ DePaul University. (2011).

Oxford House. (2009).

Oxford House. (2011).

Oxford House. (2000).

${ }^{409}$ Oxford House. (2009).

Oxford House. (2000).

${ }^{410}$ Jason, L. A., Olson, B. D., Ferrari, J. R., \& Lo Sasso, A. T. (2006).

${ }^{411}$ Jason, L. A., Olson, B. D., Ferrari, J. R., Majer, J. M., Alvarez, J., \& Stout, J. (2007).

${ }^{412}$ Center for Substance Abuse Treatment. (1998a).

Laudet, A. B., \& White, W. (2010).

${ }^{413}$ United Nations Office on Drugs and Crime. (2002).

${ }^{414}$ The National Center on Addiction and Substance Abuse at Columbia University (CASA Columbia). (2012g).

415 The National Center on Addiction and Substance Abuse at Columbia University (CASA Columbia). (2012g).

${ }^{416}$ Hser, Y.-I., Polinsky, M. L., Maglione, M., \& Anglin, M. D. (1999).

${ }^{417}$ Huebner, R. B., \& Kantor, L. W. (2011).

${ }^{418}$ Dupont, R. L., \& Skipper, G. E. (2012).

Huebner, R. B., \& Kantor, L. W. (2011).

National Council for Community Behavioral Healthcare. (2012).

Substance Abuse and Mental Health Services Administration. (2005a).

${ }^{419}$ Copeland, J., \& Martin, G. (2004).

King, V. L., Stoller, K. B., Kidorf, M., Kindbom, K., Hursh, S., Brady, T., et al. (2009).

Powers, G., \& Hamilton, N. (2012).

${ }^{420}$ Copeland, J., \& Martin, G. (2004).

King, V. L., Stoller, K. B., Kidorf, M., Kindbom, K., Hursh, S., Brady, T., et al. (2009).

${ }^{421}$ Copeland, J., \& Martin, G. (2004).

Postel, M. G., de Haan, H. A., \& De Jong, C. A. (2008).

${ }^{422}$ Brendryen, H., \& Kraft, P. (2008).

${ }^{423}$ King, V. L., Stoller, K. B., Kidorf, M., Kindbom, K., Hursh, S., Brady, T., et al. (2009).

${ }^{424}$ Gustafson, D. H., Boyle, M. G., Shaw, B. R., Isham, A., McTavish, F., Richards, S., et al. (2011).

${ }^{425}$ Substance Abuse and Mental Health Services Administration. (2005a). 
${ }^{426}$ Institute of Medicine, Committee on Crossing the Quality Chasm:Adaptation to Mental Health and Addictive Disorder. (2006).

Substance Abuse and Mental Health Services Administration. (2005a).

${ }^{427}$ The National Center on Addiction and Substance Abuse (CASA) at Columbia University. (2008a).

${ }^{428}$ The National Center on Addiction and Substance Abuse (CASA) at Columbia University. (2008a).

${ }^{429}$ The National Center on Addiction and Substance Abuse (CASA) at Columbia University. (2008a).

${ }^{430}$ Frank, D. (2011).

${ }^{431}$ The National Center on Addiction and Substance Abuse (CASA) at Columbia University. (2008a).

${ }^{432}$ Office of Communications. (2008).

${ }^{433}$ Lake Research Partners. (2009). 


\section{Chapter VI \\ Notes}

${ }^{1}$ National Institute on Drug Abuse. (2009e).

National Institute on Drug Abuse. (2011g).

${ }^{2}$ Liddle, H. A. (1999).

National Institute on Drug Abuse. (2009e).

Willenbring, M. L. (2008a).

Ziedonis, D., Kalman, D., Kolodziej, M., Johnson, C. W., \& Kim, S. (2011).

${ }^{3}$ Skipper, G. E. (1997).

${ }^{4}$ Centers for Disease Control and Prevention. (2004b)

Centers for Disease Control and Prevention. (2008a)

Centers for Disease Control and Prevention. (2008b)

Merrill, J. \& Fox, K. (1998)

The National Center on Addiction and Substance Abuse (CASA) at Columbia University. (1993)

The National Center on Addiction and Substance Abuse (CASA) at Columbia University. (1994).

${ }^{5}$ Center for Substance Abuse Treatment. (2006b).

${ }^{6}$ Hays, L., Kirsh, K. L., \& Passik, S. D. (2003).

Ling, W., Wesson, D. R., \& Smith, D. E. (2003).

National Institute on Drug Abuse. (2009b).

${ }^{7}$ Center for Substance Abuse Treatment. (2006b).

${ }^{8}$ Center for Substance Abuse Treatment. (2006b).

${ }^{9}$ Center for Substance Abuse Treatment. (2005b).

${ }^{10}$ Drake, R. E., Mercer-McFadden, C., Mueser, K. T., McHugo, G. J., \& Bond, G. R. (1998).

Van Hoof-Haines, K. (2012).

National Institutes of Health. (1993).

${ }^{11}$ Bernhardt, A. (2009).

Hintz, T., \& Mann, K. (2006).

${ }^{12}$ Center for Substance Abuse Treatment. (2005b).

Substance Abuse and Mental Health Services Administration. (2002).

Legacy Foundation. (2011).

${ }^{13}$ Center for Substance Abuse Treatment. (2005b).

Drake, R. E., O'Neal, E. L., \& Wallach, M. A. (2008).

Legacy Foundation. (2011).

Substance Abuse and Mental Health Services Administration. (2002).

${ }^{14}$ Center for Substance Abuse Treatment. (2005b).

${ }^{15}$ Center for Substance Abuse Treatment. (2005b).

Kunz, K. B., President, \& Callahan, J. F., Executive Vice President, American Board of Addiction Medicine (personal communication, May 2, 2012).

${ }^{16}$ Center for Substance Abuse Treatment. (2005b).

Mangrum, L. F., Spence, R. T., \& Lopez, M. (2006).

Moggi, F., Ouimette, P. C., Moos, R. H., \& Finney, J. W. (1999).

${ }^{17}$ Center for Substance Abuse Treatment. (2006b).

${ }^{18}$ Hall, S. M., \& Prochaska, J. J. (2009).

${ }^{19}$ American Psychiatric Association. (2006).

Hall, S. M., \& Prochaska, J. J. (2009).

Fiore, M., U.S. Department of Health and Human Services. (2008).

National Institutes of Health. (2006).

${ }^{20}$ Ziedonis, D., Kalman, D., Kolodziej, M., Johnson, C. W., \& Kim, S. (2011).

${ }^{21}$ Schroeder, S. A. (2005).

${ }^{22}$ Fiore,M., U.S. Department of Health and Human Services. (2008).

Saxon, A. J., Baer, J. S., Davis, T. M., Sloan, K. L., Malte, C. A., Fitzgibbons, K., et al. (2003).

${ }^{23}$ Hall, S. M., \& Prochaska, J. J. (2009).

Heffner, J. L., \& Anthenelli, R. M. (2011). 
${ }^{24}$ Legacy Foundation. (2011).

${ }^{25}$ Morris, C., Waxmonsky, J., Giese A., Graves, M., \& Turnbull, J. (2009).

Ziedonis, D., Hitsman, B., Beckham, J. C., Zvolensky, M., Adler, L. E., Audrain-McGovern, J., et al. (2008).

Ziedonis, D., \& Williams, J. M. (2003).

${ }^{26}$ Bellack, A. S., Bennett, M. E., Gearon, J. S., Brown, C. H., \& Yang, Y. (2006).

${ }^{27}$ Barrowclough, C., Haddock, G., Tarrier, N., Lewis, S. W., Moring, J., O'Brien, R., et al. (2001).

Graeber, D. A., Moyers, T. B., Griffith, G., Guajardo, E., \& Tonigan, S. (2003).

${ }^{28}$ Jerrell, J. M., \& Ridgely, M. S. (1995).

${ }^{29}$ National Institute on Drug Abuse. (2010a).

Weiss, R. D., Griffin, M. L., Kolodziej, M. E., Greenfield, S. F., Najavits, L. M., Daley, D. C., et al. (2007).

${ }^{30}$ Rounsaville, B. J. (2004).

Nunes, E. V., \& Quitkin, F. M. (1997).

${ }^{31}$ Cornelius, J. R., Salloum, I. M., Haskett, R. F., Daley, D. C., Cornelius, M. D., Thase, M. E., et al. (2000).

Cornelius, J. R., Bukstein, O. G., Birmaher, B., Salloum, I. M., Lynch, K., Pollock, N. K., et al. (2001).

${ }^{32}$ Rounsaville, B. J. (2004).

${ }^{33}$ Mariani, J. J., \& Levin, F. R. (2008).

Salloum, I. M., Cornelius, J. R., Daley, D. C., Kirisci, L., Himmelhoch, J. M., \& Thase, M. E. (2005).

${ }^{34}$ Ford, J. D., \& Russo, E. (2006).

Finkelstein, N., VanDeMark, N. R., Fallot, R., Brown, V., Cadiz, S., \& Heckman, J. (2004).

Gulliver, S. B., \& Steffen, L. E. (2010).

${ }^{35}$ Center for Substance Abuse Treatment. (2005b).

${ }^{36}$ Drake, R. E., Essock, S. M., Shaner, A., Carey, K. B., Minkoff, K., Kola, L., et al. (2001).

Drake, R. E., McHugo, G. J., Clark, R. E., Teague, G. B., Xie, H., Miles, K., et al. (1998).

Essock, S. M., Mueser, K. T., Drake, R. E., Covell, N. H., McHugo, G. J., Frisman, L. K., et al. (2006).

Frisman, L. K., Mueser, K. T., Covell, N. H., Lin, H. J., Crocker, A., Drake, R. E., et al. (2009).

Substance Abuse and Mental Health Services Administration. (2009b).

${ }^{37}$ Center for Substance Abuse Treatment. (2005b).

Ozechowski, T. J., \& Waldron, H. B. (2010).

Ryan, P., \& Morgan, S. (2004).

${ }^{38}$ Center for Substance Abuse Treatment. (2005b).

${ }^{39}$ Center for Substance Abuse Treatment. (2005b).

Ozechowski, T. J., \& Waldron, H. B. (2010).

${ }^{40}$ Center for Substance Abuse Treatment. (2005b).

Drake, R. E., McHugo, G. J., Clark, R. E., Teague, G. B., Xie, H., Miles, K., et al. (1998).

Morse, G. A., Calsyn, R. J., Klinkenberg, W. D., Trusty, M. L., Gerber, F., Smith, R., et al. (1997).

Wingerson, D., \& Ries, R. K. (1999).

${ }^{41}$ The National Center on Addiction and Substance Abuse (CASA) at Columbia University. (2011a).

Addiction Technology Transfer Center Network. (2011).

${ }^{42}$ Andersen, S. L., \& Teicher, M. H. (2009).

Bava, S., Frank, L. R., McQueeny, T., Schweinsburg, B. C., Schweinsburg, A. D., \& Tapert, S. F. (2009).

Benowitz, N. L. (2010).

Chambers, R. A., Taylor, J. R., \& Potenza, M. N. (2003).

DeWit, D. J., Adlaf, E. M., Offord, D. R., \& Ogborne, A. C. (2000).

Grant, B. F., \& Dawson, D. A. (1997).

Grant, B. F., \& Dawson, D. A. (1998).

Placzek, A. N., Zhang, T. A., \& Dani, J. A. (2009).

Rubinstein, M. L., Benowitz, N. L., Auerback, G. M., \& Moscicki, A. B. (2009).

${ }^{43}$ The National Center on Addiction and Substance Abuse (CASA) at Columbia University. (2011a).

${ }^{44}$ Addiction Technology Transfer Center Network. (2011).

Center for Substance Abuse Treatment. (1999c).

Deas, D., \& Clark, A. (2009).

Hser, Y.-I., Grella, C. E., Hubbard, R. L., Hsieh, S.-C., Fletcher, B. W., Brown, B. S., et al. (2001).

The National Center on Addiction and Substance Abuse (CASA) at Columbia University. (2011a).

Wagner, E. F. (2009).

${ }^{45}$ Addiction Technology Transfer Center Network. (2011). 
${ }^{46}$ The National Center on Addiction and Substance Abuse (CASA) at Columbia University. (2011a).

Addiction Technology Transfer Center Network. (2011).

${ }^{47}$ Dishion, T. J., McCord, J., \& Poulin, F. (1999).

Dishion, T. J., Poulin, F., \& Burraston, B. (2001).

Addiction Technology Transfer Center Network. (2011).

Szalavitz, M. (2010).

${ }^{48}$ Addiction Technology Transfer Center Network. (2011).

${ }^{49}$ Moolchan, E. T., Robinson, M. L., Ernst, M., Cadet, J. L., Pickworth, W. B., Heishman, S. J., et al. (2005).

${ }^{50}$ Dino, G., Horn, K., Goldcamp, J., Fernandes, A., Kalsekar, I., \& Massey, C. (2001).

Horn, K., Dino, G., Kalsekar, I., \& Mody, R. (2005).

Joffe, A., McNeely, C., Colantuoni, E., An, M. W., Wang, W., \& Scharfstein, D. (2009).

American Lung Association. (2008).

American Lung Association. (2009).

Peterson, A. V., Jr., Kealey, K. A., Mann, S. L., Marek, P. M., Ludman, E. J., Liu, J., et al. (2009).

Pbert, L., Osganian, S. K., Gorak, D., Druker, S., Reed, G., O'Neill, K. M., et al. (2006).

Sussman, S., Sun, P., \& Dent, C. W. (2006).

Walsh, M. M., Langer, T. J., Kavanagh, N., Mansell, C., MacDougal, W., Kavanagh, C., et al. (2010).

${ }^{51}$ Sussman, S., Sun, P., \& Dent, C. W. (2006).

${ }^{52}$ Dowden, C., \& Latimer, J. (2006).

Vaughn, M. G., \& Howard, M. O. (2004).

Waldron, H. B., \& Turner, C. W. (2008).

${ }^{53}$ Addiction Technology Transfer Center Network. (2011).

Pumariega, A. J., Kilgus, M. D., \& Rodriguez, L. (2005).

${ }^{54}$ Latimer, W. W., Winters, K. C., D'Zurilla, T., \& Nichols, M. (2003).

Liddle, H. A., Dakof, G. A., Turner, R. M., Henderson, C. E., \& Greenbaum, P. E. (2008).

Waldron, H. B., \& Turner, C. W. (2008).

${ }^{55}$ Deas, D., \& Clark, A. (2009).

Lowinson, J. H., Ruiz, P., Millman, R. B., \& Langrod, J. G. (eds.). (2005).

Pumariega, A. J., Kilgus, M. D., \& Rodriguez, L. (2005).

${ }^{56}$ National Institute on Drug Abuse. (2009e).

SAMHSA's National Registry of Evidence-based Programs and Practices. (2008).

${ }^{57}$ Liddle, H. A., Dakof, G. A., Parker, K., Diamond, G. S., Barrett, K., \& Tejeda, M. (2001).

${ }^{58}$ Sexton, T. L., \& Alexander, J. F. (2000).

${ }^{59}$ Coppola, J., Executive Director, New York Association of Alcoholism and Substance Abuse Providers, Inc. (ASAP) (personal communication, July 27, 2007).

${ }^{60}$ Szapocznik, J., Professor and Chair, Department of Epidemiology and Public Health, Director, Center for Family Studies, Director, Miami Clinical Translational Science Institute (personal communication, August 6, 2007).

${ }^{61}$ National Institute on Drug Abuse. (2005b).

${ }^{62}$ Multisystemic Therapy Services. (2011).

National Institute on Drug Abuse. (2005b).

${ }^{63}$ National Institute on Drug Abuse. (2006b).

${ }^{64}$ Borduin, C. M., Mann, B. J., Cone, L. T., Henggeler, S. W., Fucci, B. R., Blaske, D. M., et al. (1995).

Henggeler, S. W., Clingempeel, W. G., Brondino, M. J., \& Pickrel, S. G. (2002).

National Institute on Drug Abuse. (2005b).

Schaeffer, C. M., \& Borduin, C. M. (2005).

Schoenwald, S. K., Ward, D. M., Henggeler, S. W., Pickrel, S. G., \& Patel, H. (1996).

University of Washington, Alcohol and Drug Abuse Institute. (2006).

${ }^{65}$ Hser, Y.-I., Grella, C. E., Hubbard, R. L., Hsieh, S.-C., Fletcher, B. W., Brown, B. S., et al. (2001).

${ }^{66}$ Godley, M. D. \& Godley, S. H. (2011).

${ }^{67}$ Godley, S. H., Godley, M. D., Karvinen, T., Slown, L. L., \& Wright, K. L. (2006).

${ }^{68}$ Godley, M. D., Godley, S. H., Dennis, M. L., Funk, R. R., \& Passetti, L. L. (2002).

Godley, M. D., Godley, S. H., Dennis, M. L., Funk, R. R., \& Passetti, L. L. (2007).

${ }^{69}$ Dawes, M. A., \& Johnson, B. A. (2004).

Niederhofer, H., \& Staffen, W. (2003).

Upadhyaya, H. \& Deas, D. (2008). 
Waxmonsky, J. G., \& Wilens, T. E. (2005).

Woody, G. E., Poole, S. A., Subramaniam, G., Dugosh, K., Bogenschutz, M., Abbott, P., et al. (2008).

${ }^{70}$ Clark, D. B., Bukstein, O., \& Cornelius, J. (2002).

Fournier, M. E., \& Levy, S. (2006).

${ }^{71}$ Greenfield, S. F., \& Grella, C. E. (2009).

Hser, Y.-I., Evans, E., Huang, D., \& Messina, N. P. (2011).

Prendergast, M. L., Messina, N. P., Hall, E. A., \& Warda, U. S. (2011).

72 The National Center on Addiction and Substance Abuse (CASA) at Columbia University. (2006).

${ }^{73}$ Ashley, O. S., Marsden, M. E., \& Brady, T. M. (2003).

Butler Center for Research. (1999).

The National Center on Addiction and Substance Abuse (CASA) at Columbia University. (2006).

${ }^{74}$ Center for Substance Abuse Treatment. (2002a).

Finkelstein, N., VanDeMark, N. R., Fallot, R., Brown, V., Cadiz, S., \& Heckman, J. (2004).

Ford, J. D., \& Russo, E. (2006).

Greenfield, S. F., \& Grella, C. E. (2009).

Blume, S. B. \& Zilberman, M. L. (2005).

${ }^{75}$ Brookhaven Retreat. (2011).

${ }^{76}$ Center for Substance Abuse Treatment. (2002a).

Center for Substance Abuse Treatment. (2002b).

Zilberman, M. L. \& Blume, S. B. (2005).

${ }^{77}$ Westermeyer, J., Wahmanholm, K., \& Thuras, P. (2001).

${ }^{78}$ Blom, T. J., \& Anthenelli, R. M. (2011).

Bollerud, K. (1990).

Center for Substance Abuse Treatment. (2002a).

Finkelstein, N., VanDeMark, N. R., Fallot, R., Brown, V., Cadiz, S., \& Heckman, J. (2004).

Sacks, J. Y., McKendrick, K., \& Banks, S. (2008).

Wadsworth, R., Spampneto, A. M., \& Halbrook, B. M. (1995).

${ }^{79}$ Wadsworth, R., Spampneto, A. M., \& Halbrook, B. M. (1995).

${ }^{80}$ Center for Substance Abuse Treatment. (1993).

Center for Substance Abuse Treatment. (2009c).

McCarthy, J. J. (2012).

National Institute on Drug Abuse. (2009e).

${ }^{81}$ Center for Substance Abuse Treatment. (2009c).

${ }^{82}$ Center for Substance Abuse Treatment. (2009c).

Jones, H. E., Kaltenbach, K., Heil, S. H., Stine, S. M., Coyle, M. G., Arria, A. M., et al. (2010).

${ }^{83}$ Center for Substance Abuse Treatment. (2009c).

${ }^{84}$ Center for Substance Abuse Treatment. (2009b).

Center for Substance Abuse Treatment. (2009c).

${ }^{85}$ Center for Substance Abuse Treatment. (2009c).

Kaltenbach, K., Berghella, V., \& Finnegan, L. (1998).

${ }^{86}$ Bastek-Karasow, C., \& Davis, R. (2007).

Burgdorf, K., \& Chen, X. (2004).

${ }^{87}$ Burgdorf, K., \& Chen, X. (2004).

Center for Substance Abuse Treatment. (1998a).

Finkelstein, N. (1993).

${ }^{88}$ Center for Substance Abuse Treatment. (2009c).

${ }^{89}$ Grella, C. E. (1997).

${ }^{90}$ U.S.Code Online via GPO Access. (2007).

${ }^{91}$ Center for Substance Abuse Treatment. (2009c).

${ }^{92}$ Clark, H. W. (2001).

93 Burgdorf, K., \& Chen, X. (2004).

${ }^{94}$ Center for Substance Abuse Treatment. (1998b).

Gambert, S. R. \& Albrecht, A. E. (2005).

Martin, L. M., Fleming, K. C., \& Evans, J. M. (1995).

The National Center on Addiction and Substance Abuse (CASA) at Columbia University. (1998). 
${ }^{95}$ Center for Substance Abuse Treatment. (1998b).

Grella, C. E. (2009).

${ }^{96}$ Center for Substance Abuse Treatment. (1998b).

SAMHSA's National Registry of Evidence-based Programs and Practices. (2007a).

${ }^{97}$ Center for Substance Abuse Treatment. (1998b).

${ }^{98}$ Center for Substance Abuse Treatment. (1998b).

${ }^{99}$ Caetano, R. (2003).

Castro, F. G., \& Garfinkle, J. (2003).

Hesselbrock, M. N., Hesselbrock, V. M., Segal, B., Schuckit, M. A., \& Bucholz, K. (2003).

Schmidt, L., Ye, Y., Greenfield, T., \& Bond, J. C. (2007).

${ }^{100}$ Burroughs, V. J., Maxey, R. W., \& Levy, R. A. (2002).

Ruiz, P., Varner, R. V., Small, D. R., \& Johnson, B. A. (1999).

${ }^{101}$ Finn, P. (1996).

${ }^{102}$ Campbell, C. I., \& Alexander, J. A. (2002).

Castro, F. G., \& Garfinkle, J. (2003).

Ruiz, P. \& Langrod, J. G. (2009).

${ }^{103}$ Campbell, C. I., \& Alexander, J. A. (2002).

${ }^{104}$ Cochran, S. D., Ackerman, D., Mays, V. M., \& Ross, M. W. (2004).

Drabble, L., Midanik.L.T., \& Trocki, K. (2005).

McCabe, S. E., Bostwick, W. B., Hughes, T. L., West, B. T., \& Boyd, C. J. (2010).

Meyer, I. H. (2003).

Pizacani, B. A., Rohde, K., Bushore, C., Stark, M. J., Maher, J. E., Dilley, J. A., et al. (2009).

${ }^{105}$ Center for Substance Abuse Treatment. (2009a).

Lipsky, S., Krupski, A., Roy-Byrne, P., Huber, A., Lucenko, B. A., \& Mancuso, D. (2012).

Meyer, I. H. (2003).

${ }^{106}$ Centers for Disease Control and Prevention. (2011econgress).

${ }^{107}$ Cochran, S. D., Sullivan, J. G., \& Mays, V. M. (2003).

Gilman, S. E., Cochran, S. D., Mays, V. M., Hughes, M., Ostrow, D., \& Kessler, R. C. (2001).

Lipsky, S., Krupski, A., Roy-Byrne, P., Huber, A., Lucenko, B. A., \& Mancuso, D. (2012).

Meyer, I. H. (2003).

${ }^{108}$ Rothman, E. F., Exner, D., \& Baughman, A. (2011).

${ }^{109}$ Center for Substance Abuse Treatment. (2009a).

110 Substance Abuse and Mental Health Services Administration, Office of Applied Studies. (2011a).

${ }^{111}$ Cochran, B. N., Peavy, K. M., \& Robohm, J. S. (2007).

112 Senreich, E. (2010).

113 Clinical Practice Guideline Treating Tobacco Use and Dependence 2008 Update Panel, Liaisons, and Staff. (2008).

U.S. Department of Defense, Department of Veteran Affairs. (2009).

U.S. Department of Defense, Department of Veteran Affairs. (2011).

${ }^{114}$ U.S. Department of Defense, Department of Veteran Affairs. (2009).

Clinical Practice Guideline Treating Tobacco Use and Dependence 2008 Update Panel, Liaisons, and Staff. (2008).

U.S. Department of Defense, Department of Veteran Affairs. (2011).

115 Seal, K. H., Cohen, G., Waldrop, A., Cohen, B. E., Maguen, S., \& Ren, L. (2011).

Watkins, K. E., Pincus, H. A., Paddock, S., Smith, B., Woodroffe, A., Farmer, C., et al. (2011).

${ }^{116}$ U.S. Department of Veteran Affairs, National Center for PTSD. (2010).

${ }^{117}$ Najavits, L. M., Kivlahan, D., \& Kosten, T. (2011).

Substance Abuse and Mental Health Services Administration. (2012a).

Boden, M. T., Kimerling, R., Jacobs-Lentz, J., Bowman, D., Weaver, C., Carney, D., et al. (2011).

Norman, S. B., Wilkins, K. C., Tapert, S. F., Lang, A. J., \& Najavits, L. M. (2010).

Triffleman, E., Carroll, K., \& Kellogg, S. (1999).

Donovan, B., Padin-Rivera, E., \& Kowaliw, S. (2001).

Desai, R. A., Harpaz-Rotem, I., Najavits, L. M., \& Rosenheck, R. A. (2008).

${ }^{118}$ Ouimette, P., Moos, R. H., \& Finney, J. W. (2003).

${ }^{119}$ Ouimette, P., Moos, R. H., \& Finney, J. W. (2003).

Ouimette, P. C., Finney, J. W., \& Moos, R. H. (1997). 
${ }^{120}$ National Institute on Drug Abuse. (2006b).

121 The National Center on Addiction and Substance Abuse(CASA) at Columbia University. (2004). The National Center on Addiction and Substance Abuse (CASA) at Columbia University. (2010).

122 The National Center on Addiction and Substance Abuse (CASA) at Columbia University. (2010).

${ }^{123}$ Center for Substance Abuse Treatment \& Denver Juvenile Justice Integrated Treatment Network. (2000). Lipsey, M. W. (1999).

Lipsey, M. W., \& Cothern, L. (2000).

${ }^{124}$ U.S. Department of Justice, Office of Justice Programs. (2004).

${ }^{125}$ National Institute on Drug Abuse. (2006b).

${ }^{126}$ National Institute on Drug Abuse. (2006b).

127 Aos, S., Miller, M., \& Drake, E. (2006).

National Institute on Drug Abuse. (2006b).

${ }^{128}$ National Institute on Drug Abuse. (2006b).

${ }^{129}$ Center for Substance Abuse Treatment. (2005c).

National Institute on Drug Abuse. (2006b).

${ }^{130}$ National Institute on Drug Abuse. (2006b).

${ }^{131}$ National Institute on Drug Abuse. (2006b).

The National Center on Addiction and Substance Abuse (CASA) at Columbia University. (2010). 


\section{Chapter VII Notes}

${ }^{1}$ The National Center on Addiction and Substance Abuse at Columbia University (CASA Columbia). (2012n).

${ }^{2}$ Centers for Disease Control and Prevention. (2011i).

${ }^{3}$ The National Center on Addiction and Substance Abuse at Columbia University (CASA Columbia). (2012i).

${ }^{4}$ United States Government Accountability Office. (2011).

${ }^{5}$ The National Center on Addiction and Substance Abuse at Columbia University (CASA Columbia). (2012q).

${ }^{6}$ Hoffman, K. A., Ford, J. H., Tillotson, C. J., Choi, D., \& McCarty, D. (2011).

${ }^{7}$ The National Center on Addiction and Substance Abuse at Columbia University (CASA Columbia). (2012p).

${ }^{8}$ The National Center on Addiction and Substance Abuse at Columbia University (CASA Columbia). (2012q).

${ }^{9}$ The National Center on Addiction and Substance Abuse at Columbia University (CASA Columbia). (2012n).

${ }^{10}$ The National Center on Addiction and Substance Abuse at Columbia University (CASA Columbia). (2012n).

${ }^{11}$ Centers for Disease Control and Prevention. (2011h).

Ostchega, Y., Yoon, S. S., Huges, J., \& Louis, T. (2008).

The National Center on Addiction and Substance Abuse at Columbia University (CASA Columbia). (2012n).

${ }^{12}$ Centers for Disease Control and Prevention. (2011i).

${ }^{13}$ The National Center on Addiction and Substance Abuse at Columbia University (CASA Columbia). (2012n).

${ }^{14}$ The National Center on Addiction and Substance Abuse at Columbia University (CASA Columbia). (2012n).

${ }^{15}$ Centers for Disease Control and Prevention. (2011i).

${ }^{16}$ Centers for Disease Control and Prevention. (2011i).

The National Center on Addiction and Substance Abuse at Columbia University (CASA Columbia). (2012n).

${ }^{17}$ The National Center on Addiction and Substance Abuse at Columbia University (CASA Columbia). (2012n).

${ }^{18}$ The National Center on Addiction and Substance Abuse at Columbia University (CASA Columbia). (2012n).

The National Center on Addiction and Substance Abuse (CASA) at Columbia University. (2011).

${ }^{19}$ The National Center on Addiction and Substance Abuse at Columbia University (CASA Columbia). (2012n).

${ }^{20}$ Centers for Disease Control and Prevention. (2011i).

${ }^{21}$ The National Center on Addiction and Substance Abuse at Columbia University (CASA Columbia). (2012n).

${ }^{22}$ The National Center on Addiction and Substance Abuse at Columbia University (CASA Columbia). (2012n).

${ }^{23}$ Centers for Disease Control and Prevention. (2011i).

${ }^{24}$ American College of Physicians. (2011).

Mead, H., Cartwright-Smith, L., Jones, K., Ramos, C., Siegel, B., \& Woods, K. (2008).

National Council of La Raza (NCLR). (2009b).

National Council of La Raza (NCLR). (2009a).

${ }^{25}$ The National Center on Addiction and Substance Abuse at Columbia University (CASA Columbia). (2012n).

${ }^{26}$ Cummings, J. R., Wen, H., \& Druss, B. G. (2011).

The National Center on Addiction and Substance Abuse at Columbia University (CASA Columbia). (2012n).

${ }^{27}$ The National Center on Addiction and Substance Abuse at Columbia University (CASA Columbia). (2012n).

${ }^{28}$ Centers for Disease Control and Prevention. (2011i).

${ }^{29}$ Fu, S. S., Kodl, M. M., Joseph, A. M., Hatsukami, D. K., Johnson, E. O., Breslau, N., et al. (2008).

Fu, S. S., Sherman, S. E., Yano, E. M., Van Ryn, M., Lanto, A. B., \& Joseph, A. M. (2005).

Ryan, K. K., Garrett-Mayer, E., Alberg, A. J., Cartmell, K. B., \& Carpenter, M. J. (2011).

Trinidad, D. R., Perez-Stable, E. J., White, M. M., Emery, S. L., \& Messer, K. (2011).

${ }^{30}$ Cummings, J. R., Wen, H., \& Druss, B. G. (2011).

Garland, A. F., Aarons, G. A., Brown, S. A., Wood, P. A., \& Hough, R. L. (2003).

Ilgen, M. A., Schulenberg, J., Kloska, D. D., Czyz, E., Johnston, L., \& O'Malley, P. (2011).

The National Center on Addiction and Substance Abuse at Columbia University (CASA Columbia). (2012n).

${ }^{31}$ The National Center on Addiction and Substance Abuse at Columbia University (CASA Columbia). (2012k).

${ }^{32}$ The National Center on Addiction and Substance Abuse at Columbia University (CASA Columbia). (2012n).

${ }^{33}$ Lasser, K., Boyd, J. W., Woolhandler, S., Himmelstein, D. U., McCormick, D., \& Bor, D. H. (2000).

The National Center on Addiction and Substance Abuse at Columbia University (CASA Columbia). (2012n).

${ }^{34}$ Prochaska, J. J., Rossi, J. S., Redding, C. A., Rosen, A. B., Tsoh, J. Y., Humfleet, G. L., et al. (2004).

Siru, R., Hulse, G. K., \& Tait, R. J. (2009).

${ }^{35}$ Association of American Medical Colleges. (2007). 
Himelhoch, S., \& Daumit, G. (2003).

Thorndike, A. N., Stafford, R. S., \& Rigotti, N. A. (2001).

${ }^{36}$ The National Center on Addiction and Substance Abuse at Columbia University (CASA Columbia). (2012n).

${ }^{37}$ The National Center on Addiction and Substance Abuse at Columbia University (CASA Columbia). (2012k).

${ }^{38}$ The National Center on Addiction and Substance Abuse (CASA) at Columbia University. (2010).

${ }^{39}$ The National Center on Addiction and Substance Abuse (CASA) at Columbia University. (2010).

The National Center on Addiction and Substance Abuse (CASA) at Columbia University. (2004).

${ }^{40}$ Porter, J. (2005).

The National Center on Addiction and Substance Abuse (CASA) at Columbia University. (2010).

${ }^{41}$ Porter, J. (2005).

${ }^{42}$ Calhoun, P. S., Elter, J. R., Jones, E. R., Jr., Kudler, H., \& Straits-Troster, K. (2008).

${ }^{43}$ Milliken, C. S., Auchterlonie, J. L., \& Hoge, C. W. (2007).

${ }^{44}$ U.S. Department of Veteran Affairs, National Center for PTSD. (2012).

${ }^{45}$ McAuliffe, W. E., \& Dunn, R. (2004).

Perron, B. E., Gillespie, D. F., Alexander-Eitzman, B., \& Delva, J. (2010).

${ }^{46}$ McAuliffe, W. E., \& Dunn, R. (2004).

${ }^{47}$ McAuliffe, W. E., \& Dunn, R. (2004).

${ }^{48}$ Substance Abuse and Mental Health Services Administration, Office of Applied Studies. (2001).

Weisner, C. (1993).

${ }^{49}$ McAuliffe, W. E., \& Dunn, R. (2004).

${ }^{50}$ The National Center on Addiction and Substance Abuse at Columbia University (CASA Columbia). (2012i).

${ }^{51}$ The National Center on Addiction and Substance Abuse at Columbia University (CASA Columbia). (2012i).

${ }^{52}$ Bouchery, E. E., Harwood, H. J., Dilonardo, J., \& Vandivort-Warren, R. (in press).

${ }^{53}$ The National Center on Addiction and Substance Abuse at Columbia University (CASA Columbia). (2012i).

${ }^{54}$ The National Center on Addiction and Substance Abuse at Columbia University (CASA Columbia). (2012i).

${ }^{55}$ The National Center on Addiction and Substance Abuse at Columbia University (CASA Columbia). (2012i).

${ }^{56}$ The National Center on Addiction and Substance Abuse (CASA) at Columbia University. (2008b).

${ }^{57}$ Mark, T. L., Levit, K. R., Vandivort-Warren, R., Coffey, R. M., \& Buck, J. A. (2007).

${ }^{58}$ The National Center on Addiction and Substance Abuse at Columbia University (CASA Columbia). (2012i).

${ }^{59}$ The National Center on Addiction and Substance Abuse at Columbia University (CASA Columbia). (2012i).

${ }^{60}$ The National Center on Addiction and Substance Abuse at Columbia University (CASA Columbia). (2012i).

${ }^{61}$ The National Center on Addiction and Substance Abuse at Columbia University (CASA Columbia). (2012q).

${ }^{62}$ The National Center on Addiction and Substance Abuse at Columbia University (CASA Columbia). (2012n).

${ }^{63}$ The National Center on Addiction and Substance Abuse at Columbia University (CASA Columbia). (2012q).

${ }^{64}$ The National Center on Addiction and Substance Abuse (CASA) at Columbia University. (2008a).

${ }^{65}$ Survey: People still unsure whether alcoholism is disease or moral weakness. (2005).

${ }^{66}$ The National Center on Addiction and Substance Abuse at Columbia University (CASA Columbia). (2012q).

${ }^{67}$ The National Center on Addiction and Substance Abuse at Columbia University (CASA Columbia). (2012q).

${ }^{68}$ The National Center on Addiction and Substance Abuse at Columbia University (CASA Columbia). (2012q).

${ }^{69}$ Substance Abuse and Mental Health Services Administration. (2010d).

${ }^{70}$ Substance Abuse and Mental Health Services Administration. (2009).

${ }^{71}$ The National Center on Addiction and Substance Abuse at Columbia University (CASA Columbia). (2012q).

${ }^{72}$ The National Center on Addiction and Substance Abuse at Columbia University (CASA Columbia). (2012q).

${ }^{73}$ The National Center on Addiction and Substance Abuse at Columbia University (CASA Columbia). (2012q).

${ }^{74}$ The National Center on Addiction and Substance Abuse at Columbia University (CASA Columbia). (2012q).

${ }^{75}$ The National Center on Addiction and Substance Abuse at Columbia University (CASA Columbia). (2012p).

${ }^{76}$ The National Center on Addiction and Substance Abuse at Columbia University (CASA Columbia). (2012p).

${ }^{77}$ The National Center on Addiction and Substance Abuse at Columbia University (CASA Columbia). (2012p).

${ }^{78}$ The National Center on Addiction and Substance Abuse at Columbia University (CASA Columbia). (2012p).

${ }^{79}$ The National Center on Addiction and Substance Abuse at Columbia University (CASA Columbia). (2012p).

${ }^{80}$ The National Center on Addiction and Substance Abuse at Columbia University (CASA Columbia). (2012p).

${ }^{81}$ The National Center on Addiction and Substance Abuse at Columbia University (CASA Columbia). (2012p).

Jacobson, J. O., Robinson, P. L., \& Bluthenthal, R. N. (2007).

${ }^{82}$ Bouchery, E. E., Harwood, H. J., Dilonardo, J., \& Vandivort-Warren, R. (2012). 
${ }^{83}$ U.S. Department of Health and Human Services, Substance Abuse and Mental Health Services Administration, Office of Applied Studies. (2011).

${ }^{84}$ U.S. Department of Health and Human Services, Substance Abuse and Mental Health Services Administration, Office of Applied Studies. (2011).

${ }^{85}$ Bouchery, E. E., Harwood, H. J., Dilonardo, J., \& Vandivort-Warren, R. (2012).

${ }^{86}$ Substance Abuse and Mental Health Services Administration. (2010e).

The National Center on Addiction and Substance Abuse (CASA) at Columbia University. (2008a).

Xu, J., Wang, J., Rapp, R. C., \& Carlson, R. G. (2007).

${ }^{87}$ Center for Tobacco Research and Intervention, University of Wisconsin Medical School. (2005).

Gariti, P., Levin, S., Whittingham, T., Barou, D., Kampman, K. M., Lynch, K., et al. (2008).

Lazev, A. B., Vidrine, D. J., Arduino, R. C., \& Gritz, E. R. (2004).

${ }^{88}$ Battjes, R. J., Onken, L. S., \& Delany, P. J. (1999).

Survey: People still unsure whether alcoholism is disease or moral weakness. (2005).

Xu, J., Wang, J., Rapp, R. C., \& Carlson, R. G. (2007).

${ }^{89}$ Croog, S. H., Shapiro, D. S., \& Levine, S. (1971).

Dusing, R. (2006).

Graziani, C., Rosenthal, M. P., \& Diamond, J. J. (1999).

Joslin Diabetes Center. (2012).

National Institutes of Health, National Institute of Nursing Research. (2006).

Ogedegbe, G. (2008).

Wolfer, S. (2007).

Zyczynski, T. M., \& Coyne, K. S. (2000).

${ }^{90}$ Grella, C. E., Karno, M. P., Warda, U. S., Moore, A. A., \& Niv, N. (2009).

Hedden, S. L., \& Gfroerer, J. C. (2011).

Tsogia, D., Copello, A., \& Orford, J. (2001).

${ }^{91}$ Hedden, S. L., \& Gfroerer, J. C. (2011).

92 Saunders, S. M., Zygowicz, K. M., \& D'Angelo, B. R. (2006).

Tucker, J. A. (1995).

${ }^{93}$ Goldstein, R. Z., Craig, A. D., Bechara, A., Garavan, H., Childress, A. R., Paulus, M. P., et al. (2009).

Moeller, S. J., Maloney, T., Parvaz, M. A., Alia-Klein, N., Woicik, P. A., Telang, F., et al. (2010).

Volkow, N. D., Baler, R. D., \& Goldstein, R. Z. (2011).

${ }^{94}$ American Society of Addiction Medicine. (2011b).

American Psychiatric Association. (1995).

${ }^{95}$ American Society of Addiction Medicine. (2011b).

${ }^{96}$ Survey: People still unsure whether alcoholism is disease or moral weakness. (2005).

97 The National Center on Addiction and Substance Abuse (CASA) at Columbia University. (2008a).

${ }^{98}$ Rapp, R. C., Xu, J., Carr, C. A., Lane, D. T., Wang, J., \& Carlson, R. (2006).

${ }^{99}$ Substance Abuse and Mental Health Services Administration. (2010).

${ }^{100}$ Center for Tobacco Research and Intervention, University of Wisconsin Medical School. (2005).

${ }^{101}$ Hedden, S. L., \& Gfroerer, J. C. (2011).

102 The National Center on Addiction and Substance Abuse (CASA) at Columbia University. (2009a).

103 Cunningham, J. A., Sobell, L. C., Sobell, M. B., Agrawal, S., \& Toneatto, T. (1993).

McLellan, A. T., \& Meyers, K. (2004).

Survey: People still unsure whether alcoholism is disease or moral weakness. (2005).

White, W. L. (2008).

Wu, L. T., Blazer, D. G., Li, T.-K., \& Woody, G. E. (2011).

${ }^{104}$ Luoma, J. B., Twohig, M. P., Waltz, T., Hayes, S. C., Roget, N., Padilla, M., et al. (2007).

Semple, S. J., Grant, I., \& Patterson, T. L. (2005).

Sosin, M. R., \& Durkin, E. (2007).

105 Allen, K. (1994).

Appel, P. W., Ellison, A. A., Jansky, H. K., \& Oldak, R. (2004).

Grant, B. F. (1997).

Krahn, G., Farrell, N., Gabriel, R. M., \& Deck, D. (2006).

Rapp, R. C., Xu, J., Carr, C. A., Lane, D. T., Wang, J., \& Carlson, R. (2006).

Saunders, S. M., Zygowicz, K. M., \& D'Angelo, B. R. (2006). 
Xu, J., Wang, J., Rapp, R. C., \& Carlson, R. G. (2007).

106 The National Center on Addiction and Substance Abuse (CASA) at Columbia University. (2008a).

${ }^{107}$ Hart, P. D. (2004).

${ }^{108}$ Rivlin, A. (2005).

${ }^{109}$ Stuber, J., Galea, S., \& Link, B. G. (2008).

110 Stuber, J., Galea, S., \& Link, B. G. (2008).

111 The National Center on Addiction and Substance Abuse (CASA) at Columbia University. (2008a).

112 The National Center on Addiction and Substance Abuse (CASA) at Columbia University. (2009a).

113 Baldwin, M. L., Marcus, S. C., \& DeSimone, J. (2010).

${ }^{114}$ Keyes, K. M., Hatzenbuehler, M. L., McLaughlin, K. A., Link, B., Olfson, M., Grant, B. F., et al. (2010).

115 The National Center on Addiction and Substance Abuse (CASA) at Columbia University. (2008a).

116 The National Center on Addiction and Substance Abuse (CASA) at Columbia University. (2008a).

117 TThe National Center on Addiction and Substance Abuse (CASA) at Columbia University. (2008a).

${ }^{118}$ Hart, P. D. (2004).

${ }^{119}$ Library of Congress. (2008).

United States Government Accountability Office. (2011).

${ }^{120}$ Library of Congress. (2008).

Roy, K., \& Miller, M. (2010).

111th Congress. (2010).

${ }^{121}$ Angermeyer, M. C., \& Dietrich, S. (2006).

Corrigan, P. W., Kuwabara, S. A., \& O'Shaughnessy, J. (2009).

${ }^{122}$ Pescosolido, B. A., Martin, J. K., Long, J. S., Medina, T. R., Phelan, J. C., \& Link, B. G. (2010).

123 Tucker, J. A., Vuchinich, R. E., \& Rippens, P. D. (2004).

${ }^{124}$ Battjes, R. J., Onken, L. S., \& Delany, P. J. (1999).

${ }^{125}$ Clay, R. A. (2007).

${ }^{126}$ Rapp, R. C., Xu, J., Carr, C. A., Lane, D. T., Wang, J., \& Carlson, R. (2006).

Xu, J., Wang, J., Rapp, R. C., \& Carlson, R. G. (2007).

${ }^{127}$ Rapp, R. C., Xu, J., Carr, C. A., Lane, D. T., Wang, J., \& Carlson, R. (2006).

128 Tucker, J. A. (1995).

${ }^{129}$ Allen, K. (1994).

Center for Tobacco Research and Intervention, University of Wisconsin Medical School. (2005).

Clay, R. A. (2007).

Farabee, D., Leukefeld, C. G., \& Hays, L. (1998).

Grant, B. F. (1997).

Hser, Y.-I., Maglione, M., Polinsky, M. L., \& Anglin, M. D. (1998).

Schober, R., \& Annis, H. M. (1996).

Sturm, R., \& Sherbourne, C. D. (2001).

Substance Abuse and Mental Health Services Administration. (2009).

Survey: People still unsure whether alcoholism is disease or moral weakness. (2005).

Tucker, J. A. (1995).

Tucker, J. A., Vuchinich, R. E., \& Rippens, P. D. (2004).

${ }^{130}$ U.S. Census Bureau. (2011).

${ }^{131}$ Schoen, C., Doty, M. M., Robertson, R. H., \& Collins, S. R. (2011).

132 Adelmann, P. K. (2003).

${ }^{133}$ Collins, S. R., Doty, M. M., Robertson, R., \& Garber, T. (2011).

Institute of Medicine. (2002).

Kaiser Commission on Medicaid and the Uninsured. (2003).

Wilper, A. P., Woolhandler, S., Lasser, K. E., McCormick, D., Bor, D. H., \& Himmelstein, D. U. (2009).

${ }^{134}$ Lake Research Partners. (2009).

135 Sturm, R., \& Sherbourne, C. D. (2001).

136 Substance Abuse and Mental Health Services Administration. (2010d).

${ }^{137}$ Hodgkin, D., Horgan, C. M., Garnick, D. W., \& Merrick, E. L. (2009).

${ }^{138}$ Allem, Johnny W., Founder and President, Aquila Recovery, Chartered, Former President and CEO, Johnson Institute (personal communication, July 31, 2007).

${ }^{139}$ Sturm, R., \& Sherbourne, C. D. (2001). 
${ }^{140}$ Bouchery, E. E., Harwood, H. J., Dilonardo, J., \& Vandivort-Warren, R. (in press).

${ }^{141}$ The National Center on Addiction and Substance Abuse (CASA) at Columbia University. (2008a).

${ }^{142}$ The National Center on Addiction and Substance Abuse (CASA) at Columbia University. (2009a).

${ }^{143}$ Maddux, J. F., Prihoda, T. J., \& Desmond, D. P. (1994).

${ }^{144}$ Perron, B. E., Mowbray, O. P., Glass, J. E., Delva, J., Vaughn, M. G., \& Howard, M. O. (2009).

The National Center on Addiction and Substance Abuse (CASA) at Columbia University. (2011a).

The National Center on Addiction and Substance Abuse (CASA) at Columbia University. (2008a).

145 The National Center on Addiction and Substance Abuse (CASA) at Columbia University. (2008a).

${ }^{146}$ Appel, P. W., Ellison, A. A., Jansky, H. K., \& Oldak, R. (2004).

Battjes, R. J., Onken, L. S., \& Delany, P. J. (1999).

Buck, J. A. (2011).

Galea, S., Factor, S. H., Palermo, A.-G., Aaaron, D., Canales, E., \& Vlahov, D. (2002).

Survey: People still unsure whether alcoholism is disease or moral weakness. (2005).

White, W. L. (2008).

${ }^{147}$ The National Center on Addiction and Substance Abuse (CASA) at Columbia University. (2008a).

${ }^{148}$ Open Society Foundations. (2009).

149 The National Center on Addiction and Substance Abuse (CASA) at Columbia University. (2009a).

${ }^{150}$ Appel, P. W., Ellison, A. A., Jansky, H. K., \& Oldak, R. (2004).

Appel, P. W., \& Oldak, R. (2007).

Battjes, R. J., Onken, L. S., \& Delany, P. J. (1999).

Hser, Y.-I., Maglione, M., Polinsky, M. L., \& Anglin, M. D. (1998).

Redko, C., Rapp, R. C., \& Carlson, R. G. (2006).

${ }^{151}$ Social Security Online. (2011).

${ }^{152}$ Carr, C. J. A., Xu, J., Redko, C., Lane, D. T., Rapp, R. C., Goris, J., et al. (2008).

Hoffman, K. A., Ford, J. H., Tillotson, C. J., Choi, D., \& McCarty, D. (2011).

Redko, C., Rapp, R. C., \& Carlson, R. G. (2006).

${ }^{153}$ Carr, C. J. A., Xu, J., Redko, C., Lane, D. T., Rapp, R. C., Goris, J., et al. (2008).

Farabee, D., Leukefeld, C. G., \& Hays, L. (1998).

Friedmann, P. D., Lemon, S. C., Stein, M. D., \& D'Aunno, T. A. (2003).

White, W. L. (2008).

${ }^{154}$ Battjes, R. J., Onken, L. S., \& Delany, P. J. (1999).

${ }^{155}$ Best, D., Noble, A., Ridge, G., Gossop, M., Farrell, M., \& Strang, J. (2002).

${ }^{156}$ Chawdhary, A., Sayre, S. L., Green, C., Schmitz, J. M., Grabowski, J., \& Mooney, M. E. (2007).

Hser, Y.-I., Maglione, M., Polinsky, M. L., \& Anglin, M. D. (1998).

Pollini, R. A., McCall, L., Mehta, S. H., Vlahov, D., \& Strathdee, S. A. (2006).

${ }^{157}$ Hoffman, K. A., Ford, J. H., Tillotson, C. J., Choi, D., \& McCarty, D. (2011).

158 The National Center on Addiction and Substance Abuse (CASA) at Columbia University. (2009a).

159 Appel, P. W., Ellison, A. A., Jansky, H. K., \& Oldak, R. (2004).

Rapp, R. C., Xu, J., Carr, C. A., Lane, D. T., Wang, J., \& Carlson, R. (2006).

${ }^{160}$ Goehl, L., Nunes, E., Quitkin, F., \& Hilton, I. (1993).

Walton, M. A., Blow, F. C., Bingham, C. R., \& Chermack, S. T. (2003).

${ }^{161}$ Allen, K. (1994).

Appel, P. W., Ellison, A. A., Jansky, H. K., \& Oldak, R. (2004).

McCollister, K. E., French, M. T., Pyne, J. M., Booth, B., Rapp, R., \& Carr, C. (2009).

Rapp, R. C., Xu, J., Carr, C. A., Lane, D. T., Wang, J., \& Carlson, R. (2006).

Sturm, R., \& Sherbourne, C. D. (2001).

Tucker, J. A. (1995).

Tucker, J. A., Vuchinich, R. E., \& Rippens, P. D. (2004).

Ussher, M., Etter, J.-F., \& West, R. (2006).

162 McCollister, K. E., French, M. T., Pyne, J. M., Booth, B., Rapp, R., \& Carr, C. (2009).

${ }^{163}$ Battjes, R. J., Onken, L. S., \& Delany, P. J. (1999).

George, A. A., \& Tucker, J. A. (1996).

Grant, B. F. (1997).

Krahn, G., Farrell, N., Gabriel, R. M., \& Deck, D. (2006).

Saunders, S. M., Zygowicz, K. M., \& D'Angelo, B. R. (2006). 
Tucker, J. A. (1995).

Ussher, M., Etter, J.-F., \& West, R. (2006).

Wu, L. T., Blazer, D. G., Li, T.-K., \& Woody, G. E. (2011).

${ }^{164}$ Grant, B. F. (1997).

Hser, Y.-I., Maglione, M., Polinsky, M. L., \& Anglin, M. D. (1998).

Rapp, R. C., Xu, J., Carr, C. A., Lane, D. T., Wang, J., \& Carlson, R. (2006).

Peter D.Hart Research Associates. (2001).

165 The National Center on Addiction and Substance Abuse (CASA) at Columbia University. (2008a).

166 The National Center on Addiction and Substance Abuse (CASA) at Columbia University. (2009a).

${ }^{167}$ Battjes, R. J., Onken, L. S., \& Delany, P. J. (1999).

Xu, J., Wang, J., Rapp, R. C., \& Carlson, R. G. (2007).

Appel, P. W., Ellison, A. A., Jansky, H. K., \& Oldak, R. (2004).

Krahn, G., Farrell, N., Gabriel, R. M., \& Deck, D. (2006).

Tucker, J. A. (1995).

${ }^{168}$ Allen, K. (1994).

Appel, P. W., Ellison, A. A., Jansky, H. K., \& Oldak, R. (2004).

Battjes, R. J., Onken, L. S., \& Delany, P. J. (1999).

Rapp, R. C., Xu, J., Carr, C. A., Lane, D. T., Wang, J., \& Carlson, R. (2006).

${ }^{169}$ Ryan, K. K., Garrett-Mayer, E., Alberg, A. J., Cartmell, K. B., \& Carpenter, M. J. (2011).

Shiffman, S., Ferguson, S. G., Rohay, J., \& Gitchell, J. G. (2008).

${ }^{170}$ Shiffman, S., Ferguson, S. G., Rohay, J., \& Gitchell, J. G. (2008).

${ }^{171}$ The National Center on Addiction and Substance Abuse (CASA) at Columbia University. (2009a).

${ }^{172}$ Substance Abuse and Mental Health Services Administration. (2012b).

Waldrop, J., \& Stern, S. M. (2003).

${ }^{173}$ Cherry, L. (1993).

Krahn, G., Farrell, N., Gabriel, R. M., \& Deck, D. (2006).

Krahn, G., Deck, D., Gabriel, R., \& Farrell, N. (2007).

The National Center on Addiction and Substance Abuse at Columbia University (CASA Columbia). (2012n).

West, S. L. (2007).

174 The National Center on Addiction and Substance Abuse at Columbia University (CASA Columbia). (2012n).

175 Cherry, L. (1993).

Krahn, G., Farrell, N., Gabriel, R. M., \& Deck, D. (2006).

Krahn, G., Deck, D., Gabriel, R., \& Farrell, N. (2007).

The National Center on Addiction and Substance Abuse at Columbia University (CASA Columbia). (2012n).

West, S. L. (2007).

${ }^{176}$ Center for Substance Abuse Treatment. (1998c).

${ }^{177}$ U.S. Department of Health and Human Services. (2012).

${ }^{178}$ Center for Substance Abuse Treatment. (1998c).

Substance Abuse and Mental Health Services Administration. (2011c).

${ }^{179}$ Krahn, G., Farrell, N., Gabriel, R. M., \& Deck, D. (2006).

${ }^{180}$ Voss, C. P., Ceasar, K. W., Tymus, T., \& Fielder, I. G. (2002).

West, S. L. (2007).

${ }^{181}$ Krahn, G., Farrell, N., Gabriel, R. M., \& Deck, D. (2006).

Lutz, P. (1994).

${ }^{182}$ The National Center on Addiction and Substance Abuse at Columbia University (CASA Columbia). (2012k).

${ }^{183}$ Lui, S., Terplan, M., \& Smith, E. J. (2008).

${ }^{184}$ The National Center on Addiction and Substance Abuse at Columbia University (CASA Columbia). (2012k).

185 Brown, J. D., Vartivarian, S., \& Alderks, C. E. (2011).

${ }^{186}$ Berg, C. J., Park, E. R., Chang, Y., \& Rigotti, N. A. (2008).

${ }^{187}$ DiClemente, C. C., Dolan-Mullen, P., \& Windsor, R. A. (2000).

Ershoff, D. H., Solomon, L. J., \& Dolan-Mullen, P. (2000).

Public Health Research Institute. (2005).

Arkansas Department of Health. (2011).

${ }^{188}$ Battjes, R. J., Onken, L. S., \& Delany, P. J. (1999).

Schober, R., \& Annis, H. M. (1996). 
${ }^{189}$ Ussher, M., Etter, J.-F., \& West, R. (2006).

${ }^{190}$ Appel, P. W., Ellison, A. A., Jansky, H. K., \& Oldak, R. (2004).

Battjes, R. J., Onken, L. S., \& Delany, P. J. (1999).

Flavin, J., \& Paltrow, L. M. (2010).

191 Allen, K. (1994).

Battjes, R. J., Onken, L. S., \& Delany, P. J. (1999).

Grella, C. E., \& Greenwell, L. (2004).

Clay, R. A. (2007).

Grant, B. F. (1997).

192 The National Center on Addiction and Substance Abuse (CASA) at Columbia University. (2009a).

193 Guttmacher Institute. (2011).

Dailard, C., \& Nash, E. (2000).

${ }^{194}$ Dailard, C., \& Nash, E. (2000).

Marshall, M. F., Menikoff, J., \& Paltrow, L. M. (2003).

195 Guttmacher Institute. (2011).

${ }^{196}$ Ilgen, M. A., Schulenberg, J., Kloska, D. D., Czyz, E., Johnston, L., \& O'Malley, P. (2011).

Monti, P. M., Barnett, N. P., Colby, S. M., Gwaltney, C. J., Spirito, A., Rohsenow, D. J., et al. (2007).

Wu, L. T., Blazer, D. G., Li, T.-K., \& Woody, G. E. (2011).

${ }^{197}$ Cummings, J. R., Wen, H., \& Druss, B. G. (2011).

198 The National Center on Addiction and Substance Abuse (CASA) at Columbia University. (2011a).

${ }^{199}$ Morreale, M. C., Kapphahn, C. J., Elster, A. B., Juszczak, L., \& Klein, J. D. (2004).

VanderWaal, C. J., McBride, D. C., Terry-McElrath, Y., \& Bishop, R. M. (2006).

${ }^{200}$ Weisner, C. \& Schmidt, L. (2001).

${ }^{201}$ Deas, D., \& Clark, A. (2009).

Pumariega, A. J., Kilgus, M. D., \& Rodriguez, L. (2005).

Center for Substance Abuse Treatment. (1999c).

${ }^{202}$ Dowden, C., \& Latimer, J. (2006).

Sexton, T. L., \& Alexander, J. F. (2000).

Sussman, S., Sun, P., \& Dent, C. W. (2006).

Waldron, H. B., \& Turner, C. W. (2008).

203 The National Center on Addiction and Substance Abuse at Columbia University (CASA Columbia). (2012k).

${ }^{204}$ Kealey, K. A., Ludman, E. J., Mann, S. L., Marek, P. M., Phares, M. M., Riggs, K. R., et al. (2007).

Massey, C. J., Dino, G. A., Horn, K. A., Lacey-McCracken, A., Goldcamp, J., \& Kalsekar, I. (2003).

${ }^{205}$ Massey, C. J., Dino, G. A., Horn, K. A., Lacey-McCracken, A., Goldcamp, J., \& Kalsekar, I. (2003).

Breland, A. B., Colby, S., Dino, G., Smith, G., \& Taylor, M. (2009).

${ }^{206}$ Wu, L. T., Blazer, D. G., Li, T.-K., \& Woody, G. E. (2011).

${ }^{207}$ Breland, A. B., Colby, S., Dino, G., Smith, G., \& Taylor, M. (2009).

Clark, D. B., Bukstein, O., \& Cornelius, J. (2002).

Dawes, M. A., \& Johnson, B. A. (2004).

Niederhofer, H., \& Staffen, W. (2003).

Upadhyaya, H. \& Deas, D. (2008).

Waxmonsky, J. G., \& Wilens, T. E. (2005).

Woody, G. E., Poole, S. A., Subramaniam, G., Dugosh, K., Bogenschutz, M., Abbott, P., et al. (2008).

${ }^{208}$ Center for Substance Abuse Treatment. (1998b).

Curtis, J. R., Geller, G., Stokes, E. J., Levine, D. M., \& Moore, R. D. (1989).

${ }^{209}$ Center for Substance Abuse Treatment. (1998b).

210 The National Center on Addiction and Substance Abuse at Columbia University (CASA Columbia). (2012k).

211 Jeremias, E., Chatkin, J. M., Chatkin, G., Seibert, J., Martins, M., \& Wagner, M. (2012).

Marinho, V., Laks, J., Coutinho, E. S. F., \& Blay, S. L. (2010).

Maryland's Tobacco Resource Center. (2012).

Orleans, C. T., Jepson, C., Resch, N., \& Rimer, B. K. (1994).

Maryland's Tobacco Resource Center. (2012).

212 Jeremias, E., Chatkin, J. M., Chatkin, G., Seibert, J., Martins, M., \& Wagner, M. (2012).

Kerr, S., Watson, H., Tolson, D., Lough, M., \& Brown, M. (2006).

Maryland's Tobacco Resource Center. (2012). 
213 Jeremias, E., Chatkin, J. M., Chatkin, G., Seibert, J., Martins, M., \& Wagner, M. (2012).

${ }^{214}$ Atkinson, R. M., Tolson, R. L., \& Turner, J. A. (1990).

Center for Substance Abuse Treatment. (1998b).

Gambert, S. R. \& Albrecht, A. E. (2005).

Trevisan, L. A., Boutros, N., Petrakis, I. L., \& Krystal, J. H. (1998).

215 The National Center on Addiction and Substance Abuse (CASA) at Columbia University. (1998).

${ }^{216}$ Paquette, K. (2010).

${ }^{217}$ Kraybill, K., Zerger, S., \& National Health Care for the Homeless Council. (2003).

McNiel, D. E., \& Binder, R. L. (2005).

National Alliance to End Homelessness. (2012).

Padgett, D., Struening, E. L., \& Andrews, H. (1990).

Pearson, D. A., Bruggman, A. R., \& Haukoos, J. S. (2007).

Wenzel, S. L., Burnam, A. M., Koegel, P., Morton, S. C., Miu, A., Jinnett, K. J., et al. (2001).

${ }^{218}$ Connor, S. E., Cook, R. L., Herbert, M. I., Neal, S. M., \& Williams, J. T. (2002).

Crosier, A. (2004).

Goldade, K., Whembolua, G. L., Thomas, J., Eischen, S., Guo, H., Connett, J., et al. (2011).

National Coalition for the Homeless. (2009).

National Coalition for the Homeless. (2009).

Okuyemi, K. S., Caldwell, A. R., Thomas, J. L., Born, W., Richter, K. P., Nollen, N., et al. (2006).

${ }^{219}$ Break Free Alliance. (2009).

Goldade, K., Whembolua, G. L., Thomas, J., Eischen, S., Guo, H., Connett, J., et al. (2011).

Connor, S. E., Cook, R. L., Herbert, M. I., Neal, S. M., \& Williams, J. T. (2002).

Okuyemi, K. S., Caldwell, A. R., Thomas, J. L., Born, W., Richter, K. P., Nollen, N., et al. (2006).

${ }^{220}$ National Coalition for the Homeless. (2009).

National Coalition for the Homeless. (2009).

Okuyemi, K. S., Caldwell, A. R., Thomas, J. L., Born, W., Richter, K. P., Nollen, N., et al. (2006).

${ }^{221}$ Break Free Alliance. (2009).

${ }^{222}$ Drake, R. E., Osher, F. C., \& Wallach, M. A. (1991).

National Coalition for the Homeless. (2009).

${ }^{223}$ Freund, P. D., \& Hawkins, D. (2004).

${ }^{224}$ Wenzel, S. L., Burnam, A. M., Koegel, P., Morton, S. C., Miu, A., Jinnett, K. J., et al. (2001).

${ }^{225}$ U.S. Department of Defense Task Force on Mental Health. (2007).

${ }^{226}$ Institute of Medicine. (2010).

U.S.Department of Defense. (1985).

${ }^{227}$ United States Army Center for Substance Abuse. (2012).

GI Rights Hotline. (2012).

Institute of Medicine. (2010).

${ }^{228}$ Institute of Medicine. (2010).

${ }^{229}$ United States Army Center for Substance Abuse. (2012).

GI Rights Hotline. (2012).

Zwerdling, D. (2007).

${ }^{230}$ Zwerdling, D. (2007).

${ }^{231}$ Daly, R. (2006).

Institute of Medicine. (2010).

${ }^{232}$ Bernhardt, A. (2009).

${ }^{233}$ Institute of Medicine. (2010).

${ }^{234}$ Borders, T. F., \& Booth, B. M. (2007).

Fortney, J., \& Booth, B. M. (2001).

Pringle, J. L., Emptage, N. P., \& Hubbard, R. L. (2006).

Stevens, S., Colwell, B., \& Hutchison, L. (2003).

${ }^{235}$ Fortney, J., \& Booth, B. M. (2001).

Stoops, W. W., Dallery, J., Fields, N. M., Nuzzo, P. A., Schoenberg, N. E., Martin, C. A., et al. (2009).

Stevens, S., Colwell, B., \& Hutchison, L. (2003).

${ }^{236}$ Rost, K., Fortney, J., Zhang, M., Smith, J., \& Smith, G. R., Jr. (1999).

Stevens, S., Colwell, B., \& Hutchison, L. (2003). 
${ }^{237}$ Lenardson, J. D., \& Gale, J. A. (2007).

${ }^{238}$ U.S. Department of Transportation, Research and Innovative Technology Administration. (2011).

${ }^{239}$ Stevens, S., Colwell, B., \& Hutchison, L. (2003).

${ }^{240}$ Fortney, J., \& Booth, B. M. (2001).

${ }^{241}$ Lenardson, J. D., \& Gale, J. A. (2007).

Pennsylvania Rural Health Association. (2010).

U.S. Department of Housing and Urban Development, Office of Community Planning and Development. (2009).

242 Schmidt, L. A., \& Mulia, N. (2009).

243 Caraballo, R. S., Yee, S. L., Gfroerer, J., \& Mirza, S. A. (2008).

American Lung Association. (2010a).

Grant, B. F., Dawson, D. A., Stinson, F. S., Chou, S. P., Dufour, M. C., \& Pickering, R. P. (2004).

Smith, S. M., Stinson, F. S., Dawson, D. A., Goldstein, R., Huang, B., \& Grant, B. F. (2006).

${ }^{244}$ Schmidt, L. A., \& Mulia, N. (2009).

Schmidt, L., Tam, T., \& Larson, M. J. (2007).

${ }^{245}$ Schmidt, L. A., \& Mulia, N. (2009). 


\section{Chapter VIII \\ Notes}

${ }^{1}$ Cassel, E. J. (1982).

Larson, J. S. (1999).

Nordgren, A., Hanson, M. J., \& Callahan, D. (1999).

Callahan, D. (1999).

Social Security Act, 42 U.S.C. § 1395y(a)(1) (2012).

${ }^{2}$ Social Security Act, 42 U.S.C. § 1395y(a)(1) (2012).

${ }^{3}$ Conn. Agencies Regs. § 17-134d-63 (2012).

N.Y. Soc Serv § 365-a (Consol. 2012).

Mass. Regs. Code 130, § 450.204 (2011).

${ }^{4}$ Garber, A. M. (2004).

Garber, A. M. (2011).

${ }^{5}$ Social Security Act, 42 U.S.C. § 1395y(a)(1) (2012).

Conn. Agencies Regs. § 17-134d-63 (2012).

N.Y. Soc Serv § 365-a (Consol. 2012).

Mass. Regs. Code 130, § 450.204 (2011).

${ }^{6}$ Mass. Regs. Code 130, § 450.204 (2011).

${ }^{7}$ American Medical Association. (1990).

${ }^{8}$ Beauchamp, T. L., \& Childress, J. F. (2001).

Daniels, N. (1985).

${ }^{9}$ Pollak, M., Paicheler, G., \& Pierret, J. (1992).

Sontag, S. (1990).

Steen, M. (1991).

U.S.Department of Health and Human Services. (2011b).

Willenbring, M. L. (2008a).

${ }^{10}$ Willenbring, M. L. (2008a).

${ }^{11}$ American Society of Addiction Medicine. (2011a).

Hajela, R. \& Miller, M. M. (2012).

Leshner, A. I. (1997).

Leshner, A. I. (2001a).

Volkow, N., \& Li, T.-K. (2005).

${ }^{12}$ American Society of Addiction Medicine. (2011a).

Leshner, A. I. (1997).

Leshner, A. I. (2001a).

${ }^{13}$ Hyman, S. E. (2007).

National Institute on Drug Abuse. (2010b).

${ }_{15}^{14}$ American Psychiatric Association. (1995).

${ }^{15}$ American Cancer Society. (2009).

American Diabetes Association. (2009).

Centers for Disease Control and Prevention. (2008a).

Centers for Disease Control and Prevention. (2008b).

Centers for Disease Control and Prevention, Coordinating Center for Health Promotion. (2008).

Corso, P., Finkelstein, E., Miller, T., Fiebelkorn, I., \& Zaloshnja, E. (2006).

Insel, T. R. (2008).

Lloyd-Jones, D., Adams, R., Carnethon, M., De, S. G., Ferguson, T. B., Flegal, K., et al. (2009).

Mokdad, A. H., Marks, J. S., Stroup, D. F., \& Gerberding, J. L. (2004).

Robert Wood Johnson Foundation. (2001).

The National Center on Addiction and Substance Abuse (CASA) at Columbia University. (1993).

U.S. Department of Health and Human Services. (2010a).

Volkow, N. D., \& Li, T.-K. (2005).

${ }^{16}$ Centers for Disease Control and Prevention. (2008b).

Centers for Disease Control and Prevention. (2010a). 
Ezzati, M., Lopez, A. D., Rodgers, A., Vander Hoorn, S., Murray, C. J. L., \& and the Comparative Risk Assessment Collaborating Group. (2002).

Kochanek, K., Xu, J. Q., Murphy, S. L., Minino, A. M., \& Kung, H.-C. (2011).

Mokdad, A. H., Marks, J. S., Stroup, D. F., \& Gerberding, J. L. (2004).

World Health Organization. (2009).

${ }^{17}$ The National Center on Addiction and Substance Abuse (CASA) at Columbia University. (2009d).

${ }^{18}$ The National Center on Addiction and Substance Abuse (CASA) at Columbia University. (2009d).

${ }^{19}$ The National Center on Addiction and Substance Abuse (CASA) at Columbia University. (1994).

Zook, C. J., \& Moore, F. D. (1980).

${ }^{20}$ Clark, R. E., Samnaliev, M., \& McGovern, M. P. (2009).

Curran, G. M., Sullivan, G., Williams, K., Han, X., Collins, K., Keys, J., et al. (2003).

Owens, P. L., Mutter, R., \& Stocks, C. (2010).

Rockett, I. R., Putnam, S. L., Jia, H., Chang, C. F., \& Smith, G. S. (2005).

Substance Abuse and Mental Health Services Administration. (2010f).

White, A. G., Birnbaum, H. G., Mareva, M. N., Daher, M., Vallow, S., Schein, J., et al. (2005).

${ }^{21}$ The National Center on Addiction and Substance Abuse at Columbia University (CASA Columbia). (2012t).

${ }^{22}$ Ray, G. T., Mertens, J. R., \& Weisner, C. (2007).

${ }^{23}$ The National Center on Addiction and Substance Abuse (CASA) at Columbia University. (1993).

${ }^{24}$ Santora, P. B., \& Hutton, H. E. (2008).

${ }^{25}$ The National Center on Addiction and Substance Abuse at Columbia University (CASA Columbia). (2012i).

${ }^{26}$ Agency for Healthcare Research and Quality. (2012d).

The National Center on Addiction and Substance Abuse at Columbia University (CASA Columbia). (2012h).

${ }^{27}$ Centers for Disease Control and Prevention. (2011h).

${ }^{28}$ Agency for Healthcare Research and Quality. (2012).

The National Center on Addiction and Substance Abuse at Columbia University (CASA Columbia). (2012h).

${ }^{29}$ Centers for Disease Control and Prevention. (2012b).

${ }^{30}$ Agency for Healthcare Research and Quality. (2012d).

The National Center on Addiction and Substance Abuse at Columbia University (CASA Columbia). (2012h).

${ }^{31}$ Centers for Disease Control and Prevention. (2012b).

${ }^{32}$ The National Center on Addiction and Substance Abuse at Columbia University (CASA Columbia). (2012i).

${ }^{33}$ The National Center on Addiction and Substance Abuse at Columbia University (CASA Columbia). (2012n).

The National Center on Addiction and Substance Abuse at Columbia University (CASA Columbia). (2012j).

${ }^{34}$ Office of National Drug Control Policy. (2009).

${ }^{35}$ Humphreys, K., Wagner, T. H., \& Gage, M. (2011).

McCollister, K. E., \& French, M. T. (2003).

Maciosek, M. V., Coffield, A. B., Edwards, N. M., Flottemesch, T. J., Goodman, M. J., \& Solberg, L. I. (2006).

Solberg, L. I., Maciosek, M. V., \& Edwards, N. M. (2008).

${ }^{36}$ Humphreys, K., Wagner, T. H., \& Gage, M. (2011).

${ }^{37}$ Allen, J. M. (2010).

Humphreys, K., Wagner, T. H., \& Gage, M. (2011).

${ }^{38}$ McCollister, K. E., \& French, M. T. (2003).

${ }^{39}$ Sturm, R., Zhang, W., \& Schoenbaum, M. (1999).

${ }^{40}$ Sturm, R., Zhang, W., \& Schoenbaum, M. (1999).

Sturm, R. (1999).

${ }^{41}$ Congressional Budget Office. (2012).

${ }^{42}$ Azzone, V., Frank, R. G., Normand, S. L., \& Burnam, M. A. (2011).

Goldman, H. H., Frank, R. G., Burnam, M. A., Huskamp, H. A., Ridgely, M. S., Normand, S.-L. T., et al. (2006).

${ }^{43}$ Adams, E. K., Miller, V. P., Ernst, C., Nishimura, B. K., Melvin, C., \& Merritt, R. (2002).

American Legacy Foundation. (2007).

Babor, T. F., McRee, B. G., Kassebaum, P. A., Grimaldi, P. L., Ahmed, K., \& Bray, J. (2007).

Fleming, M. F., Mundt, M. P., French, M. T., Manwell, L. B., Stauffacher, E. A., \& Barry, K. L. (2002a).

Klug, M. G., \& Burd, L. (2003).

Neighbors, C. J., Barnett, N. P., Rohsenow, D. J., Colby, S. M., \& Monti, P. M. (2010).

${ }^{44}$ Clark, R. E., Samnaliev, M., \& McGovern, M. P. (2009).

Holder, H. D. (1998). 
${ }^{45}$ Parkin, D. (2009).

Partnership for Prevention. (2012).

${ }^{46}$ Gold, M. R., Siegel, J. E., Russell, L. B., \& Weinstein, M. C. (1996).

Hillner, B. E., \& Smith, T. J. (2009).

Parkin, D. (2009).

Partnership for Prevention. (2012).

${ }^{47}$ Russell, L. B. (2007).

${ }^{48}$ Pignone, M., Earnshaw, S., Tice, J. A., \& Pletcher, M. J. (2006).

${ }^{49}$ Russell, L. B. (2007).

${ }^{50}$ Adams, E. K., Miller, V. P., Ernst, C., Nishimura, B. K., Melvin, C., \& Merritt, R. (2002).

${ }^{51}$ Lightwood, J. M., Phibbs, C. S., \& Glantz, S. A. (1999).

${ }^{52}$ American Legacy Foundation. (2007).

${ }^{53}$ Maciosek, M. V., Coffield, A. B., Edwards, N. M., Flottemesch, T. J., Goodman, M. J., \& Solberg, L. I. (2006).

Solberg, L. I., Maciosek, M. V., \& Edwards, N. M. (2008).

${ }^{54}$ Solberg, L. I., Maciosek, M. V., \& Edwards, N. M. (2008).

${ }^{55}$ Fleming, M. F., Mundt, M. P., French, M. T., Manwell, L. B., Stauffacher, E. A., \& Barry, K. L. (2002b).

${ }^{56}$ Fleming, M. F., Mundt, M. P., French, M. T., Manwell, L. B., Stauffacher, E. A., \& Barry, K. L. (2000).

${ }^{57}$ Gentilello, L. M., Ebel, B. E., Wickizer, T. M., Salkever, D. S., \& Rivara, F. P. (2005).

Neighbors, C. J., Barnett, N. P., Rohsenow, D. J., Colby, S. M., \& Monti, P. M. (2010).

${ }^{58}$ Gentilello, L. M., Ebel, B. E., Wickizer, T. M., Salkever, D. S., \& Rivara, F. P. (2005).

${ }^{59}$ Neighbors, C. J., Barnett, N. P., Rohsenow, D. J., Colby, S. M., \& Monti, P. M. (2010).

${ }^{60}$ Klug, M. G., \& Burd, L. (2003).

${ }^{61}$ Curran, G. M., Sullivan, G., Williams, K., Han, X., Collins, K., Keys, J., et al. (2003).

Rockett, I. R., Putnam, S. L., Jia, H., Chang, C. F., \& Smith, G. S. (2005).

The National Center on Addiction and Substance Abuse at Columbia University (CASA Columbia). (2012j).

Centers for Disease Control and Prevention. (2010a).

${ }^{62}$ Barnett, P. G. (1999).

Leonardson, G. R. (2005).

McCollister, K. E., \& French, M. T. (2003).

Parthasarathy, S., Weisner, C., Hu, T., \& Moore, C. (2001).

Polsky, D., Glick, H. A., Yang, J., Subramaniam, G. A., Poole, S. A., \& Woody, G. E. (2010).

Volkow, N. D. (2006).

Wickizer, T. M., Krupski, A., Stark, K. D., Mancuso, D., \& Campbell, K. (2006).

${ }^{63}$ Harwood, H. (2008).

${ }^{64}$ Harwood, H. (2008).

${ }^{65}$ Humphreys, K., Wagner, T. H., \& Gage, M. (2011).

${ }^{66}$ Walter, L. J., Ackerson, L., \& Allen, S. (2005).

${ }^{67}$ Parthasarathy, S., Weisner, C., Hu, T., \& Moore, C. (2001).

${ }^{68}$ Ettner, S. L., Huang, D., Evans, E., Ash, D. R., Hardy, M., Jourabchi, M., et al. (2006).

${ }^{69}$ Wickizer, T. M., Krupski, A., Stark, K. D., Mancuso, D., \& Campbell, K. (2006).

${ }^{70}$ Mancuso, D., \& Felver, B. (2010).

${ }^{71}$ Office of the State Auditor, State of Colorado. (2010).

${ }^{72}$ Bhati, A. S., Roman, J. K., \& Chalfin, A. (2008).

${ }^{73}$ Barnett, P. G., Wong, W., \& Hall, S. (2008).

${ }^{74}$ Land, T., Rigotti, N. A., Levy, D. E., Paskowsky, M., Warner, D., Kwass, J. A., et al. (2010).

${ }^{75}$ Zarkin, G. A., Bray, J. W., Aldridge, A., Mitra, D., Mills, M. J., Couper, D. J., et al. (2008).

${ }^{76}$ Kranzler, H. R., Montejano, L. B., Stephenson, J. J., Wang, S., \& Gastfriend, D. R. (2010).

${ }^{77}$ Svikis, D., Golden, A., Huggins, G., Pickens, R., McCaul, M. E., Velez, M. L., et al. (1997).

${ }^{78}$ Baser, O., Chalk, M., Fiellin, D. A., \& Gastfriend, D. R. (2011).

${ }^{79}$ Gold, M. R., Siegel, J. E., Russell, L. B., \& Weinstein, M. C. (1996).

Hillner, B. E., \& Smith, T. J. (2009).

Parkin, D. (2009).

Partnership for Prevention. (2012).

Polsky, D., Glick, H. A., Yang, J., Subramaniam, G. A., Poole, S. A., \& Woody, G. E. (2010).

Russell, L. B., Gold, M. R., Siegel, J. E., Daniels, N., \& Weinstein, M. C. (1996). 
Weinstein, M. C., Siegel, J. E., Gold, M. R., Kamlet, M. S., \& Russell, L. B. (1996).

${ }^{80}$ Barnett, P. G. (1999).

81 Zaric, G. S., Barnett, P. G., \& Brandeau, M. L. (2000).

${ }^{82}$ Samet, J., MD, Professor of Medicine and Social Behavior, Clinical Addiction Research and Education (CARE) Program, Boston University School of Medicine (personal communication, August 7, 2007).

${ }^{83}$ Centers for Medicare and Medicaid Services, Center for Consumer Information and Insurance Oversight. (2012). House Office of the Legislative Counsel. (2010).

${ }^{84}$ Library of Congress. (2012a).

National Conference of State Legislatures. (2011a).

${ }^{85}$ Dave, D., \& Mukerjee, S. (2011).

Roy, K., \& Miller, M. (2010).

${ }^{86}$ Library of Congress. (2012a).

Dave, D., \& Mukerjee, S. (2011).

Solis, H. L. (2012).

${ }^{87}$ Social Security Act, 42 U.S.C. $§ 1937$ (2012).

${ }^{88}$ Mann, C., \& Center for Medicaid and State Operations. (2009).

Sarata, A. K. (2011).

U.S. Department of Labor, Employee Benefits Security Administration. (2010).

${ }^{89}$ Advocates for Human Potential. (2010).

Library of Congress. (2012a).

U.S. Department of Labor, Employee Benefits Security Administration. (2010).

U.S.Government Printing Office. (2010).

${ }^{90}$ National Conference of State Legislatures. (2011a).

${ }^{91}$ United States Government Accountability Office. (2011).

92 Dave, D., \& Mukerjee, S. (2011).

${ }^{93}$ Dierker, L. C., Sledjeski, E. M., Marshall, S., \& Johnson, S. (2009).

${ }^{94}$ Commonwealth Fund. (2010).

HealthCare.gov. (2012c).

${ }^{95}$ U.S. Department of Health and Human Services, Office of the Assistant Secretary for Planning and Evaluation. (2011).

${ }^{96}$ Henry J.Kaiser Family Foundation. (2010).

Substance Abuse and Mental Health Services Administration. (2011d).

${ }^{97}$ Center for Consumer Information and Insurance Oversight. (2011).

98 Sarata, A. K. (2011).

${ }^{99}$ Collins, S. R., Doty, M. M., Robertson, R., \& Garber, T. (2011).

${ }^{100}$ House Office of the Legislative Counsel. (2010).

Collins, S. R., Doty, M. M., Robertson, R., \& Garber, T. (2011).

HealthCare.gov. (2011c).

HealthCare.gov. (2011b).

${ }^{101}$ U.S. Department of Health and Human Services. (2010b).

${ }^{102}$ Centers for Medicare and Medicaid Services. (2010a).

Buck, J. A. (2011).

${ }^{103}$ Crosson, F. J. (2011).

Centers for Medicare and Medicaid Services. (2011b).

Druss, B. G., \& Mauer, B. J. (2012).

${ }^{104}$ U.S.Department of Health and Human Services. (2011a).

${ }^{105}$ Buck, J. A. (2011).

Humphreys, K., \& McLellan, A. T. (2011).

${ }^{106}$ American Medical Association. (2009).

American Psychiatric Association. (2008).

Ensuring Solutions to Alcohol Problems. (2009).

Porter, S. (2008).

Substance Abuse and Mental Health Services Administration. (2010g).

${ }^{107}$ Madras, B. K. (2010).

Substance Abuse and Mental Health Services Administration. (2010g). 
${ }^{108}$ Substance Abuse and Mental Health Services Administration. (2011e).

${ }^{109}$ National Association of Community Health Centers. (2011).

Substance Abuse and Mental Health Services Administration. (2009e).

Substance Abuse and Mental Health Services Administration. (2010g).

Substance Abuse and Mental Health Services Administration. (2011e).

${ }^{110}$ Center for Tobacco Cessation. (2011).

Health Resources and Services Administration. (2011a).

Health Resources and Services Administration. (2011b).

${ }^{111}$ U.S. Department of Health and Human Services, Centers for Medicare \& Medicaid Services. (2008).

Substance Abuse and Mental Health Services Administration. (2011e).

${ }^{112}$ Centers for Medicare and Medicaid Services. (2012g).

${ }^{113}$ Centers for Medicare and Medicaid Services. (2012g).

U.S. Department of Health and Human Services, Centers for Medicare \& Medicaid Services. (2011).

${ }^{114}$ Centers for Medicare and Medicaid Services. (2012g).

U.S. Department of Health and Human Services, Centers for Medicare \& Medicaid Services. (2011).

${ }^{115}$ U.S. Department of Health and Human Services, Centers for Medicare \& Medicaid Services. (2012a).

${ }^{116}$ Centers for Medicare and Medicaid Services. (2010d).

${ }^{117}$ American Medical Association. (2009).

American Medical Association. (2009).

${ }^{118}$ American Medical Association. (2009).

American Medical Association. (2009).

Porter, S. (2008).

${ }^{119}$ Jacobus-Kantor, L., \& Goplerud, E. (2011).

Mercure, S., \& Greenberg, L. (2009).

${ }^{120}$ Jacobus-Kantor, L., \& Goplerud, E. (2011).

${ }^{121}$ Mercure, S., \& Greenberg, L. (2009).

${ }^{122}$ Henry J.Kaiser Family Foundation. (2010).

HealthCare.gov. (2011e).

${ }^{123}$ Bazelon Center for Mental Health Law. (2010).

${ }^{124}$ Johnson, N., Oliff, P., \& Williams, E. (2011).

${ }^{125}$ U.S. Department of Transportation, National Highway Traffic Safety Administration. (2008).

${ }^{126}$ Physicians and Lawyers for National Drug Policy. (2008).

Higgins-Biddle, J. C., Hungerford, D., \& Cates-Wessel, K. (2009).

${ }^{127}$ Gentilello, L. M., Samuels, P. N., Henningfield, J. E., \& Santora, P. B. (2005).

Anderson, P., Aromaa, S., Rosenbloom, D., \& Enos, G. (2008).

${ }^{128}$ American Medical Association. (2011).

American Medical Association. (2011).

Gentilello, L. M. (2005).

Grill, C. (2011).

Physicians and Lawyers for National Drug Policy. (2008).

U.S. Department of Transportation, National Highway Traffic Safety Administration. (2008).

${ }^{129}$ National Institute on Alcohol Abuse and Alcoholism. (2011b).

${ }^{130}$ Chezem, L. (2005).

${ }^{131}$ Kaiser Commission on Medicaid and the Uninsured. (2005).

${ }^{132}$ Bouchery, E. E., Harwood, H. J., Dilonardo, J., \& Vandivort-Warren, R. (2012).

Sarata, A. K. (2011).

${ }^{133}$ Robinson, G., Kaye, N., Bergman, D., Moreaux, M., \& Baxter, c. (2005).

Substance Abuse and Mental Health Services Administration. (2010f).

${ }^{134}$ Substance Abuse and Mental Health Services Administration. (2010f).

${ }^{135}$ Substance Abuse and Mental Health Services Administration. (2010f).

Rogowski, J. A. (1992).

Kaiser Commission on Medicaid and the Uninsured. (2011a).

${ }^{136}$ Rogowski, J. A. (1992).

${ }^{137}$ Substance Abuse and Mental Health Services Administration. (2010f).

Kaiser Commission on Medicaid and the Uninsured. (2011b). 
${ }^{138}$ Crowley, J. S., \& O'Malley, M. (2007).

Substance Abuse and Mental Health Services Administration. (2010f).

${ }^{139}$ Substance Abuse and Mental Health Services Administration. (2010f).

Rogowski, J. A. (1992).

${ }^{140}$ Substance Abuse and Mental Health Services Administration. (2010f).

${ }^{141}$ Maglione, M., \& Ridgely, M. S. (2006).

${ }^{142}$ Substance Abuse and Mental Health Services Administration. (2010f).

${ }^{143}$ Center for Medicaid and State Operations. (2011).

${ }^{144}$ Centers for Disease Control and Prevention. (2010b).

${ }^{145}$ American Lung Association. (2010).

${ }^{146}$ Centers for Medicare and Medicaid Services. (2011d).

${ }^{147}$ Centers for Medicare and Medicaid Services. (2012b).

Centers for Medicare and Medicaid Services. (2012c).

${ }^{148}$ Centers for Medicare and Medicaid Services. (2012b).

Centers for Medicare and Medicaid Services. (2012c).

${ }^{149}$ Centers for Medicare and Medicaid Services. (2012d).

Centers for Medicare and Medicaid Services. (2012b).

${ }^{150}$ Centers for Medicare and Medicaid Services. (2012e).

Centers for Medicare and Medicaid Services. (2012).

Centers for Medicare and Medicaid Services. (2011a).

${ }^{151}$ Centers for Medicare and Medicaid Services. (2010b).

U.S. Department of Health and Human Services, Centers for Medicare \& Medicaid Services. (2012b).

${ }^{152}$ Centers for Medicare and Medicaid Services. (2010c).

Medicare Interactive. (2012).

153 Ostrow, L., \& Manderscheid, R. (2010).

${ }^{154}$ Sundararaman, R., \& Redhead, S. (2007).

HealthCare.gov. (2012a).

HealthCare.gov. (2012b).

${ }^{155}$ Hodgkin, D., Horgan, C. M., Garnick, D. W., \& Merrick, E. L. (2009).

${ }^{156}$ Congressional Budget Office. (2007).

${ }^{157}$ Center for Mental Health Services, Substance Abuse and Mental Health Services Administration. (2000).

${ }^{158}$ Library of Congress. (2012b).

Congressional Budget Office. (2007).

${ }^{159}$ Center for Substance Abuse Treatment. (2000).

${ }^{160}$ Center for Mental Health Services, Substance Abuse and Mental Health Services Administration. (2000).

${ }^{161}$ Paul Wellstone and Pete Domenici Mental Health Parity and Addiction Equity Act of 2008 (H.R. 1424).

${ }^{162}$ U.S.Government Printing Office. (2012).

Paul Wellstone and Pete Domenici Mental Health Parity and Addiction Equity Act of 2008 (H.R. 1424).

${ }^{163}$ Cornell University Law School, Legal Information Institute. (2012).

House Office of the Legislative Counsel. (2010).

Mann, C., \& Center for Medicaid and State Operations. (2009).

${ }^{164}$ National Institute on Drug Abuse. (2009e).

White, W. L., \& McLellan, A. T. (2008).

${ }^{165}$ American Board of Addiction Medicine Foundation. (2009).

Frost-Pineda, K., VanSusteren, T., \& Gold, M. S. (2004).

Miller, N. S., Sheppard, L. M., Colenda, C. C., \& Magen, J. (2001).

Isaacson, J. H., Fleming, M., Kraus, M., Kahn, R., \& Mundt, M. (2000).

Weintraub, T. A., Saitz, R., \& Samet, J. H. (2003).

Advocates for Human Potential. (2009).

16629 U.S.C. § 18 (2011).

Dave, D., \& Mukerjee, S. (2011).

National Academy for State Health Policy. (2012).

${ }^{167}$ Buck, J. A. (2011).

${ }^{168}$ Capoccia, V., Grazier, K. L., Toal, C., Ford, J. H., \& Gustafson, D. H. (2012).

Kaiser Commission on Medicaid and the Uninsured. (2007). 
${ }^{169}$ Capoccia, V., Grazier, K. L., Toal, C., Ford, J. H., \& Gustafson, D. H. (2012).

${ }_{170}^{17}$ Azzone, V., Frank, R. G., Normand, S. L., \& Burnam, M. A. (2011).

${ }^{171}$ HealthCare.gov. (2011d).

${ }^{172}$ Center for Consumer Information and Insurance Oversight. (2011).

${ }^{173}$ Centers for Medicare and Medicaid Services. (2012a).

${ }^{174}$ Commonwealth Fund. (2010).

Center for Consumer Information and Insurance Oversight. (2011).

${ }^{175}$ Smith, V. K., Gifford, K., \& Ellis, E. (2011).

${ }^{176}$ Henry J.Kaiser Family Foundation. (2012). 


\section{Chapter IX \\ Notes}

${ }^{1}$ National Institute on Drug Abuse. (2009e).

National Quality Forum. (2005).

Smith, M. J. W., Whitaker, T., \& Weismiller, T. (2006).

${ }^{2}$ Rieckmann, T. R., Kovas, A. E., Fussell, H. E., \& Stettler, N. M. (2009).

${ }^{3}$ American Board of Addiction Medicine Foundation. (2009).

Addiction Technology Transfer Center Network. (2009b).

Institute of Medicine, Committee on Crossing the Quality Chasm:Adaptation to Mental Health and Addictive

Disorder. (2006).

Mulvey, K. P., Hubbard, S., \& Hayashi, S. (2003).

O'Connor, P. G., Nyquist, J. G., \& McLellan, T. (2011).

Partners for Recovery. (2003).

${ }^{4}$ Substance Abuse and Mental Health Services Administration. (2003b).

${ }^{5}$ Substance Abuse and Mental Health Services Administration. (2003b).

${ }^{6}$ McLellan, A. T., \& Meyers, K. (2004).

${ }^{7}$ Substance Abuse and Mental Health Services Administration. (2003b).

${ }^{8}$ Mulvey, K. P., Hubbard, S., \& Hayashi, S. (2003).

${ }^{9}$ McLellan, A. T., \& Meyers, K. (2004).

Substance Abuse and Mental Health Services Administration. (2003b).

${ }^{10}$ U.S. Department of Labor, Bureau of Labor Statistics. (2012a).

${ }^{11}$ Substance Abuse and Mental Health Services Administration. (2003b).

${ }^{12}$ American Board of Addiction Medicine Foundation. (2009).

${ }^{13}$ American Medical Association. (2012c).

${ }^{14}$ American Medical Association. (2012c).

${ }^{15}$ Kunz, K. B., President, \& Callahan, J. F., Executive Vice President, American Board of Addiction Medicine (personal communication, May 2, 2012).

Gitlow, S., MD, MPH, MBA, Executive Director, Annenberg Physician Training Program in Addictive Disease, Associate Clinical Professor, Mount Sinai School of Medicine, Acting President \& AMA Delegate, Amercian Society of Addiction Medicine (personal communication, May 23, 2012).

${ }^{16}$ Kunz, K. B., President, \& Callahan, J. F., Executive Vice President, American Board of Addiction Medicine (personal communication, May 2, 2012).

${ }^{17}$ American Board of Addiction Medicine Foundation. (2009).

Kunz, K. B., President, \& Callahan, J. F., Executive Vice President, American Board of Addiction Medicine (personal communication, May 2, 2012).

${ }^{18}$ American Board of Addiction Medicine Foundation. (2009).

Madras, B. K., Compton, W. M., Avula, D., Stegbauer, T., Stein, J. B., \& Clark, H. W. (2009).

The National Center on Addiction and Substance Abuse at Columbia University (CASA Columbia). (2012b).

${ }^{19}$ American Board of Medical Specialties. (2011).

Glick, S., Manager, Credentials Department, American Board of Psychiatry and Neurolog (personal communication, March 15, 2012).

${ }^{20}$ American Medical Association. (2012d).

${ }^{21}$ American Board of Medical Specialties. (2012a).

${ }^{22}$ American Counseling Association. (2012).

American Board of Professional Psychology. (2012).

${ }^{23}$ American Counseling Association. (2012).

${ }^{24}$ Institute of Medicine, Committee on Crossing the Quality Chasm:Adaptation to Mental Health and Addictive Disorder. (2006).

McLellan, A. T., Carise, D., \& Kleber, H. D. (2003).

Substance Abuse and Mental Health Services Administration. (2005b).

${ }^{25}$ U.S. Department of Labor, Bureau of Labor Statistics. (2012b).

Keller, D. S., \& Dermatis, H. (1999).

${ }^{26}$ Kerwin, M. E., Walker-Smith, K., \& Kirby, K. C. (2006).

${ }^{27}$ McCarty, D. (2002). 
${ }^{28}$ Institute of Medicine, Committee on Crossing the Quality Chasm:Adaptation to Mental Health and Addictive Disorder. (2006).

Isaacson, J. H., Fleming, M., Kraus, M., Kahn, R., \& Mundt, M. (2000).

Miller, N. S., Sheppard, L. M., Colenda, C. C., \& Magen, J. (2001).

Olmstead, T. A., Abraham, A. J., Martino, S., \& Roman, P. M. (2012).

Weintraub, T. A., Saitz, R., \& Samet, J. H. (2003).

${ }^{29}$ McLellan, A. T., \& Meyers, K. (2004).

Substance Abuse and Mental Health Services Administration. (2003b).

${ }^{30}$ Institute of Medicine, Committee on Crossing the Quality Chasm:Adaptation to Mental Health and Addictive Disorder. (2006).

Keller, D. S., \& Dermatis, H. (1999).

McLellan, A. T., Carise, D., \& Kleber, H. D. (2003).

${ }^{31}$ Institute of Medicine, Committee on Crossing the Quality Chasm:Adaptation to Mental Health and Addictive Disorder. (2006).

${ }^{32}$ Richards, E. P. (1999).

${ }^{33}$ McLean, T. R. (2002).

${ }^{34}$ Institute of Medicine, Committee on Crossing the Quality Chasm:Adaptation to Mental Health and Addictive Disorder. (2006).

${ }^{35}$ Accreditation Council for Graduate Medical Education. (2012c).

${ }^{36}$ American Board of Addiction Medicine Foundation. (2009).

Dilonardo, J. (2011).

Frost-Pineda, K., VanSusteren, T., \& Gold, M. S. (2004).

Isaacson, J. H., Fleming, M., Kraus, M., Kahn, R., \& Mundt, M. (2000).

Miller, N. S., Sheppard, L. M., Colenda, C. C., \& Magen, J. (2001).

Weintraub, T. A., Saitz, R., \& Samet, J. H. (2003).

${ }^{37}$ American Medical Association. (2012d).

Indiana University, Health Professions and Prelaw Center. (2007).

${ }^{38}$ USMLE.org. (2012).

Young, A., Chaudhry, H. J., Rhyne, J., \& Dugan, M. (2011).

${ }^{39}$ United States Medical Licensing Examination. (2008a).

United States Medical Licensing Examination. (2008b).

United States Medical Licensing Examination. (2008c).

${ }^{40}$ American Association of Colleges of Osteopathic Medicine. (2012).

${ }^{41}$ National Board of Osteopathic Medical Examiners. (2009).

${ }^{42}$ Accreditation Council for Graduate Medical Education. (2012a).

Liaison Committee on Medical Education. (2011

National Board of Medical Examiners. (2012).

United States Medical Licensing Examination. (2012).

National Board of Osteopathic Medical Examiners. (2011).

${ }^{43}$ American Board of Addiction Medicine Foundation. (2009).

Haack, M. R., \& Adger, H. (2002).

Institute of Medicine, Committee on Crossing the Quality Chasm:Adaptation to Mental Health and Addictive Disorder. (2006).

Sandlow, L. J., \& Dos Santos, S. R. (1997).

${ }^{44}$ Isaacson, J. H., Fleming, M., Kraus, M., Kahn, R., \& Mundt, M. (2000).

${ }^{45}$ Etzel, S., Research Associate, American Medical Association (personal communication, January 25, 2012).

${ }^{46}$ Miller, N. S., Sheppard, L. M., Colenda, C. C., \& Magen, J. (2001).

${ }^{47}$ American Board of Medical Specialties. (2012c).

${ }^{48}$ American Board of Medical Specialties. (2012c).

American Board of Medical Specialties. (2012b).

${ }^{49}$ American Board of Internal Medicine. (2009c).

${ }^{50}$ American Board of Internal Medicine. (2009a).

American Board of Family Medicine. (2012b).

American Board of Family Medicine. (2012a).

American Board of Family Medicine. (2012c). 
American Board of Emergency Medicine. (2006).

${ }^{51}$ American Board of Internal Medicine. (2009b).

${ }^{52}$ American Board of Pediatrics. (2009a).

${ }^{53}$ American Board of Pediatrics. (2010a).

${ }^{54}$ American Board of Pediatrics. (2011).

${ }^{55}$ American Board of Pediatrics. (2009b).

American Board of Pediatrics. (2010b).

American Board of Pediatrics. (2010c).

${ }^{56}$ American Board of Family Medicine. (2009).

${ }^{57}$ American Board of Family Medicine. (2007).

${ }^{58}$ American Board of Psychiatry and Neurology. (2010a).

${ }^{59}$ American Board of Psychiatry and Neurology. (2009a). American Board of Psychiatry and Neurology. (2009b).

${ }^{60}$ American Board of Psychiatry and Neurology. (2009a).

American Board of Psychiatry and Neurology. (2009b).

American Board of Psychiatry and Neurology. (2010c).

American Board of Psychiatry and Neurology. (2010b).

${ }^{61}$ American Board of Emergency Medicine. (2009).

American Board of Emergency Medicine. (2011).

${ }^{62}$ American Board of Obstetrics and Gynecology. (2008).

${ }^{63}$ American Board of Obstetrics and Gynecology. (2007).

${ }^{64}$ American Board of Addiction Medicine. (2012c).

${ }^{65}$ American Board of Addiction Medicine Foundation. (2009).

${ }^{66}$ American Board of Addiction Medicine. (2012a).

${ }^{67}$ American Board of Addiction Medicine. (2012a).

${ }^{68}$ American Board of Addiction Medicine. (2012a).

${ }^{69}$ American Board of Addiction Medicine. (2012a).

${ }^{70}$ American Board of Addiction Medicine. (2012a).

${ }^{71}$ American Board of Addiction Medicine. (2012b).

${ }^{72}$ American Board of Addiction Medicine. (2012c).

${ }^{73}$ American Academy of Addiction Psychiatry. (2012).

${ }^{74}$ American Board of Medical Specialties. (2009).

${ }^{75}$ American Board of Psychiatry and Neurology. (2012).

${ }^{76}$ American Academy of Physician Assistants. (2012b).

${ }^{77}$ U.S. Department of Labor, Bureau of Labor Statistics. (2009a).

${ }^{78}$ American Academy of Physician Assistants. (2012a).

National Commission on Certification of Physician Assistants. (2010).

${ }^{79}$ American Medical Association. (2012b).

${ }^{80}$ Accreditation Review Commission on Education for the Physician Assistant. (2008).

${ }^{81}$ National Commission on Certification of Physician Assistants. (2009).

${ }^{82}$ American Academy of Physician Assistants. (2010a).

${ }^{83}$ American Academy of Physician Assistants. (2010b).

${ }^{84}$ U.S. Department of Labor, Bureau of Labor Statistics. (2009b).

${ }^{85}$ National Council of State Boards of Nursing. (2012).

${ }^{86}$ Howard, M. O., Walker, D. R., Walker, P. S., \& Suchinsky, R. T. (1997).

${ }^{87}$ Psychiatric Mental Health Substance Abuse Essential Competencies Taskforce of the American Academy of Nursing Psychiatric Mental Health Substance Abuse Expert Panel. (2012).

${ }^{88}$ National League for Nursing Accrediting Commission. (2002).

National League for Nursing Accrediting Commission. (2008b).

National League for Nursing Accrediting Commission. (2008a).

National League for Nursing Accrediting Commission. (2008c).

American Association of Colleges of Nursing. (2012).

American Association of Colleges of Nursing. (2009).

American Association of Colleges of Nursing. (2008).

${ }^{89}$ Wendt, A., Kenny, L., \& Schultz, L. (2010). 
Wendt, A., Kenny, L., \& Schultz, L. (2011).

${ }^{90}$ Psychiatric Mental Health Substance Abuse Essential Competencies Taskforce of the American Academy of Nursing Psychiatric Mental Health Substance Abuse Expert Panel. (2012).

${ }^{91}$ U.S. Department of Labor, Bureau of Labor Statistics. (2009b).

${ }^{92}$ International Nurses Society on Addictions (IntNSA). (2012).

${ }^{93}$ Association of State and Provincial Psychology Boards. (2008).

${ }^{94}$ U.S. Department of Labor, Bureau of Labor Statistics. (2012b).

Association of State and Provincial Psychology Boards. (2012b).

${ }_{95}^{95}$ Miller, W. R. (2002).

${ }^{96}$ Association of State and Provincial Psychology Boards. (2012a).

${ }^{97}$ American Psychological Association. (2009).

${ }^{98}$ Ciuccio, J., Assistant Executive Director for Administration, American Psychological Association Practice Organization (personal communication, March 30, 2012).

${ }^{99}$ U.S. Department of Labor, Bureau of Labor Statistics. (2012b).

${ }^{100}$ National Board for Certified Counselors. (2012a).

National Board for Certified Counselors. (2012b).

National Board for Certified Counselors. (2012c).

${ }^{101}$ National Board for Certified Counselors. (2005).

${ }^{102}$ National Board for Certified Counselors. (2012d).

${ }^{103}$ National Board for Certified Counselors. (2012c).

${ }^{104}$ American Medical Association. (2012a).

105 American Counseling Association. (2006).

${ }^{106}$ Council for Accreditation of Counseling and Related Educational Programs. (2009).

${ }^{107}$ Salyers, K. M., Ritchie, M. H., Cochrane, W. S., \& Roseman, C. P. (2006).

${ }^{108}$ American Association for Marriage and Family Therapy. (2012).

${ }^{109}$ Association of Marital and Family Therapy Regulatory Boards, \& Professional Examination Service. (2012).

${ }^{110}$ U.S. Department of Labor, Bureau of Labor Statistics. (2012b).

${ }^{111}$ Bina, R., Yum, J., Hall, D., Sowbel, L., Molette, A., Jani, J., et al. (2008).

${ }^{112}$ Association of Social Work Boards. (2011).

${ }^{113}$ Alaska Division of Corporations, Business, and Professional Licensing, \& Board of Social Work Examiners. (2012).

California Board of Behavioral Sciences. (2012).

${ }^{114}$ National Association of Social Workers. (2012).

${ }^{115}$ Gates, S., Smith Lesley A., \& Foxcroft, D. (2006).

${ }^{116}$ Bullock, M. L., Kiresuk, T. J., Pheley, A. M., Culliton, P. D., \& Lenz, S. K. (1999). Bullock, M. L., Kiresuk, T. J., Sherman, R. E., Lenz, S. K., Culliton, P. D., Boucher, T. A., et al. (2002).

Gates, S., Smith Lesley A., \& Foxcroft, D. (2006).

Jordan, J. B. (2006).

Margolin, A., Avants, S. K., \& Kleber, H. D. (1998).

Margolin, A., Kleber, H. D., Avants, S. K., Konefal, J., Gawin, F., Stark, E., et al. (2002).

Tahiri, M., Mottillo, S., Joseph, L., Pilote, L., \& Eisenberg, M. J. (2012).

Trumpler, F., Oez, S., Stahli, P., Brenner, H. D., \& Juni, P. (2003).

White, A. R., Rampes, H., \& Campbell, J. L. (2006).

${ }^{117}$ Acufinder.com. (2012a).

Acufinder.com. (2012b).

Acufinder.com. (2012c).

Acufinder.com. (2012d).

Acufinder.com. (2012e).

${ }^{118}$ Cal. Codes Regs. 16. 13.7, § 1399.434 (2012)

${ }^{119}$ National Certification Commission for Acupuncture and Oriental Medicine. (2012).

National Certification Commission for Acupuncture and Oriental Medicine. (2011).

Accreditation Commission for Acupuncture and Oriental Medicine. (2009).

${ }^{120}$ National Acupuncture Detoxification Association. (2012).

${ }^{121}$ National Acupuncture Detoxification Association. (2010).

${ }^{122}$ International Certification and Reciprocity Consortium. (2012c). 
McLellan, A. T., \& Meyers, K. (2004).

Substance Abuse and Mental Health Services Administration. (2003b).

Substance Abuse and Mental Health Services Administration. (2005b).

${ }^{123}$ American Counseling Association. (2012).

${ }^{124}$ Dilonardo, J. (2011).

${ }^{125}$ Libretto, S. V., Weil, J., Nemes, S., Linder, N. C., \& Johansson, A. C. (2004).

Substance Abuse and Mental Health Services Administration. (2005b).

${ }^{126}$ Harwood, H. J. (2002).

${ }^{127}$ Addiction Technology Transfer Center Network. (2012).

${ }^{128}$ American Counseling Association. (2012).

${ }^{129}$ Education-Portal.com. (2012).

${ }^{130}$ Kuehn, D. (2007).

NAADAC - The Association for Addiction Professionals. (2009a).

International Certification and Reciprocity Consortium. (2012a).

International Certification and Reciprocity Consortium. (2012d).

NAADAC - The Association for Addiction Professionals. (2009c).

${ }^{131}$ International Certification and Reciprocity Consortium. (2012b).

NAADAC - The Association for Addiction Professionals. (2009b).

${ }^{132}$ International Certification and Reciprocity Consortium. (2012b).

${ }^{133}$ International Certification and Reciprocity Consortium. (2012b).

${ }^{134}$ NAADAC, The Association for Addiction Professionals. (2012).

${ }^{135}$ NAADAC,The Association for Addiction Professionals. (2012).

${ }^{136}$ Kerwin, M. E., Walker-Smith, K., \& Kirby, K. C. (2006).

${ }^{137}$ The National Center on Addiction and Substance Abuse (CASA) at Columbia University. (2009a).

${ }^{138}$ Center for Substance Abuse Treatment. (2006a).

${ }^{139}$ Ganju, V. (2006).

Hermann, R. C., Chan, J. A., Zazzali, J. L., \& Lerner, D. (2006).

${ }^{140}$ Substance Abuse and Mental Health Services Administration. (2010d).

Substance Abuse and Mental Health Services Administration, Office of Applied Studies. (2011b).

Substance Abuse and Mental Health Services Administration. (2005b).

${ }^{141}$ Pollio, D. E., McClendon, J., \& Reid, D. (2004).

${ }^{142}$ Substance Abuse and Mental Health Services Administration. (2010d).

${ }^{143}$ Center for Substance Abuse Treatment. (2005b).

Drake, R. E., O'Neal, E. L., \& Wallach, M. A. (2008).

Institute of Medicine, Committee on Crossing the Quality Chasm:Adaptation to Mental Health and Addictive

Disorder. (2006).

Legacy Foundation. (2011).

Substance Abuse and Mental Health Services Administration. (2002).

${ }^{144}$ National Association of State Alcohol and Drug Abuse Directors. (2002).

${ }^{145}$ National Association of State Alcohol and Drug Abuse Directors. (2002).

${ }^{146}$ National Association of State Alcohol and Drug Abuse Directors. (2002).

${ }^{147}$ National Association of State Alcohol and Drug Abuse Directors. (2002).

${ }^{148}$ National Association of State Alcohol and Drug Abuse Directors. (2002).

14942 C.F.R. § 8 (2002).

Chriqui, J. F., Terry-McElrath, Y., McBride, D. C., Eidson, S. S., \& VanderWaal, C. J. (2007).

${ }^{150}$ Centers for Medicare and Medicaid Services. (2011c).

42 C.F.R. § 482 (2012).

Social Security Act, 42 U.S.C. § 1861 (2012).

U.S. Social Security Administration. (2011).

38 C.F.R. § 17.81 (2010).

U.S.Code Online via GPO Access. (2012b).

${ }^{151}$ Social Security Act, 42 U.S.C. § 1814 (2012).

Social Security Act, 42 U.S.C. § 1861 (2012).

${ }^{152}$ Smith, A. D. (2005).

15342 C.F.R. § 485 (2012). 
U.S.Code Online via GPO Access. (2003).

Office of Management and Budget. (2007).

15442 C.F.R. $\S 8$ (2002).

U.S. Department of Health and Human Services, Substance Abuse and Mental Health Services Administration, Center for Substance Abuse Treatment, Division of Pharmacologic Therapies. (2012).

15542 C.F.R. § 8 (2002).

U.S. Department of Health and Human Services, Substance Abuse and Mental Health Services Administration,

Center for Substance Abuse Treatment, Division of Pharmacologic Therapies. (2012).

Center for Substance Abuse Treatment, Division of Pharmacologic Therapies. (2007).

${ }^{156}$ Accreditation Association for Ambulatory Health Care. (2012).

${ }^{157}$ Institute of Medicine, Committee on Crossing the Quality Chasm:Adaptation to Mental Health and Addictive Disorder. (2006).

${ }^{158}$ Chriqui, J. F., Terry-McElrath, Y., McBride, D. C., Eidson, S. S., \& VanderWaal, C. J. (2007).

Substance Abuse and Mental Health Services Administration. (2005b).

${ }^{159}$ Chriqui, J. F., Terry-McElrath, Y., McBride, D. C., Eidson, S. S., \& VanderWaal, C. J. (2007).

${ }^{160}$ CARF International. (2011).

${ }^{161}$ Wells, R., Lemark, C. H., Alexander, J. A., Nahra, T. A., Ye, Y., \& Campbell, C. (2007).

Pollack, H. A., \& D'Aunno, T. (2008).

${ }^{162}$ Institute of Medicine, Committee on Crossing the Quality Chasm:Adaptation to Mental Health and Addictive Disorder. (2006).

${ }^{163}$ Chriqui, J. F., Terry-McElrath, Y., McBride, D. C., Eidson, S. S., \& VanderWaal, C. J. (2007).

${ }^{164}$ Wells, R., Lemark, C. H., Alexander, J. A., Nahra, T. A., Ye, Y., \& Campbell, C. (2007).

165 Pollack, H. A., \& D'Aunno, T. (2008).

166 Thompson, Kristen, BH/CYS Customer Service Units Account Services Coordinator, CARF International (personal communication, August 21, 2009).

Commission on Accreditation of Rehabilitation Facilities (CARF). (2012).

${ }^{167}$ Substance Abuse and Mental Health Services Administration. (2005b).

168 Joint Commission. (2012a).

169 Substance Abuse and Mental Health Services Administration. (2010d).

${ }^{170}$ Substance Abuse and Mental Health Services Administration. (2005b).

${ }^{171}$ Council on Accreditation. (2008).

172 Substance Abuse and Mental Health Services Administration. (2005b).

173 The National Committee for Quality Assurance (NCQA). (2012).

${ }^{174}$ Substance Abuse and Mental Health Services Administration. (2005b).

${ }^{175}$ National Commission on Correctional Health Care. (2012).

${ }^{176}$ Substance Abuse and Mental Health Services Administration. (2010d).

177 Alaska Admin. Code 7, § 12.110 (January 2012).

Ariz. Admin. Code R9-10-207 (2010).

Georgia Department of Human Services. (2007).

New Jersey Department of Health and Senior Services. (2007).

New Jersey Department of Health and Senior Services. (2008).

${ }^{178}$ Kerwin, M. E., Walker-Smith, K., \& Kirby, K. C. (2006).

${ }^{179}$ Crowley, J. S., \& O'Malley, M. (2007).

${ }^{180}$ N.J. Admin. Code 10.161B, §3.12 (2012).

Alaska Legislature. (2006).

Okla. Stat. 450. 18, § 1-2 (2011).

N.Y. Comp. Codes R \& Regs.14 §381.4 (2012).

${ }^{181}$ N.J. Admin. Code 10.161B, §3.12 (2012).

Alaska Legislature. (2006).

Okla. Stat. 450. 18, § 1-2 (2011).

Mass. Regs. Code 105, § 164.124 (2011).

18242 C.F.R. $\S 8$ (2002).

${ }^{183}$ Mulvey, K. P., Hubbard, S., \& Hayashi, S. (2003).

184 The National Center on Addiction and Substance Abuse (CASA) at Columbia University. (2009a).

185 Ala. Code 580 § 2-9.03. (2012). 
Idaho Admin Code r. 16.06.03.050. (2011).

Ky. Rev. Stat. § 222.231. (2012).

Code Me. R. § 14-118-005. (2012).

N.J. Admin. Code 10.161B, \$1.4. (2012).

N.J. Admin. Code 10.161B, §1.8. (2012).

Or. Admin. R. 415-020-0075. (2012).

28 Pa. Code § 704.6. (2012).

Code R.I. Reg. 18030 003. (2012).

N.Y. Comp. Codes R \& Regs.14 §381.4. (2012).

Delaware General Assembly. (2010).

${ }^{186}$ Idaho Admin Code r. 16.06.03.050. (2011).

Neb. Admin. R. \& Regs. 175 § 18. (2004).

Connecticut Department of Public Health. (2009).

Ark. Code. $016 \S 04$ 004. (2012).

Fla. Stat. 397.471. (2012).

Fla. Stat. 397.311. (2012).

18742 C.F.R. § 440.10. (2012).

42 C.F.R. § 440.90. (2012).

${ }^{188} 42$ C.F.R. § 440.130. (2012).

Crowley, J. S., \& O'Malley, M. (2007).

Tritz, K. (2006).

Terry-McElrath, Y. M., Chriqui, J. F., \& McBride, D. C. (2011).

18942 C.F.R. § 485. (2012).

${ }^{190}$ U.S.Code Online via GPO Access. (2012a).

${ }^{191}$ CARF International. (2011).

${ }^{192}$ CARF International. (2011).

193 Joint Commission. (2012d).

${ }^{194}$ Richards, E. P. (1999).

${ }^{195}$ Kerwin, M. E., Walker-Smith, K., \& Kirby, K. C. (2006).

${ }^{196}$ Fla. Admin. Code 59A-3.065 (2012).

Fla. Admin. Code 59A-33.001 (2012).

Fla. Admin. Code 65D-30.003 (2012).

${ }^{197}$ D. C. Mun. Regs. 29, § 2365 (2012).

${ }^{198} 908$ Ky. Admin. Regs.1:370 (2011).

${ }^{199}$ American Society of Addiction Medicine. (2012).

${ }^{200}$ N.Y. Comp. Codes R \& Regs.14 §381.4 (2012).

N.C. Admin. Code 10A. 27G.4401 (January 2012).

Ark. Code. $016 \S 04004$ (2012).

908 Ky. Admin. Regs.1:370 (2011).

${ }^{201}$ Rieckmann, T. R., Kovas, A. E., Fussell, H. E., \& Stettler, N. M. (2009).

${ }^{202}$ Or. Rev. Stat. § 182.515 (2009).

20342 C.F.R. § 8.12 (2002).

${ }^{204}$ CARF International. (2011).

${ }^{205}$ CARF International. (2011).

${ }^{206}$ Joint Commission. (2012d).

${ }^{207}$ Joint Commission. (2012d).

${ }^{208}$ Cooperberg, M. R., Birkmeyer, J. D., \& Litwin, M. S. (2009).

Hermann, R. C., Chan, J. A., Zazzali, J. L., \& Lerner, D. (2006).

${ }^{209}$ Cooperberg, M. R., Birkmeyer, J. D., \& Litwin, M. S. (2009).

${ }^{210} 42$ C.F.R. § 485 (2012).

${ }^{211} 42$ C.F.R. § 8.12 (2002).

${ }^{212}$ Office of Management and Budget. (2007).

${ }^{213}$ Substance Abuse and Mental Health Services Administration. (2011g).

42 U.S.C.S. § 300x-22 (2012).

42 U.S.C.S. § 300x-28 (2012). 
${ }^{214}$ Cowell, A., McCarty, D., \& Woodward, A. (2003).

${ }^{215}$ Substance Abuse and Mental Health Services Administration. (2011f).

${ }^{216}$ CARF International. (2011).

${ }^{217}$ Joint Commission. (2012d). 


\section{Chapter X \\ Notes}

${ }^{1}$ Institute of Medicine, Committee on Crossing the Quality Chasm:Adaptation to Mental Health and Addictive Disorder. (2006).

McGlynn, E. A., Asch, S. M., Adams, J., Keesey, J., Hicks, J., DeCristofaro, A., et al. (2003).

2 Anderson, P., Aromaa, S., Rosenbloom, D., \& Enos, G. (2008).

Denisco, R. C., Kenna, G. A., O'Neil, M. G., Kulich, R. J., Moore, P. A., Kane, W. T., et al. (2011).

Fleming, M. F. (2004).

Friedmann, P. D., McCullough, D., \& Saitz, R. (2001).

Gordon, J. S., Albert, D. A., Crews, K. M., \& Fried, J. (2009).

Gunderson, E. W., Levin, F. R., \& Smith, L. (2005).

Patel, D. (2005

${ }^{3}$ Russell, M. (2004).

${ }^{4}$ Russell, M. (2004).

Whitby, L. G. (1974).

Wilson, J. M. G., \& Jungner, G. (1968).

${ }^{5}$ Centers for Disease Control and Prevention. (2009b).

${ }^{6}$ Moyer, A., \& Finney, J. W. (2004).

${ }^{7}$ Denisco, R. C., Kenna, G. A., O'Neil, M. G., Kulich, R. J., Moore, P. A., Kane, W. T., et al. (2011).

Gordon, J. S., Albert, D. A., Crews, K. M., \& Fried, J. (2009).

Moyer, A., \& Finney, J. W. (2004).

New York State Office of Alcoholism and Substance Abuse Services. (2010).

Patel, D. (2005).

${ }^{8}$ Anderson, P., Aromaa, S., Rosenbloom, D., \& Enos, G. (2008).

Fleming, M. F. (2004).

Maciosek, M. V., Coffield, A. B., Edwards, N. M., Flottemesch, T. J., Goodman, M. J., \& Solberg, L. I. (2006).

Millstein, S. G., \& Marcell, A. V. (2003).

${ }^{9}$ The National Center on Addiction and Substance Abuse (CASA) at Columbia University. (2000).

${ }^{10}$ Boyle, M. G., \& Murphy, T. P. (2005).

${ }^{11}$ Petrone, K., \& Katz, P. (2005).

Rochon, P. A., \& Gurwitz, J. H. (1997).

The National Center on Addiction and Substance Abuse (CASA) at Columbia University. (1998).

The National Center on Addiction and Substance Abuse (CASA) at Columbia University. (2000).

12 Millstein, S. G., \& Marcell, A. V. (2003).

Tait, R. J., \& Hulse, G. K. (2003).

The National Center on Addiction and Substance Abuse (CASA) at Columbia University. (2011a).

Winters, K. C., \& Leitten, W. (2007).

${ }^{13}$ Centers for Disease Control and Prevention. (2011i).

${ }^{14}$ Centers for Disease Control and Prevention. (2011a).

The National Center on Addiction and Substance Abuse at Columbia University (CASA Columbia). (2012s).

${ }^{15}$ Hum, A. M., Robinson, L. A., Jackson, A. A., \& Ali, K. S. (2011).

${ }^{16}$ Maciosek, M. V., Coffield, A. B., Edwards, N. M., Flottemesch, T. J., Goodman, M. J., \& Solberg, L. I. (2006).

17 Thorndike, A. N., Regan, S., \& Rigotti, N. A. (2007).

${ }^{18}$ Steinberg, M. B., Akincigil, A., Delnevo, C. D., Crystal, S., \& Carson, J. L. (2006).

${ }^{19}$ Tong, E. K., Strouse, R., Hall, J., Kovac, M., \& Schroeder, S. A. (2010).

${ }^{20}$ Association of American Medical Colleges. (2007).

${ }^{21}$ Steinberg, M. B., Alvarez, M. S., Delnevo, C. D., Kaufman, I., \& Cantor, J. C. (2006).

${ }^{22}$ Akpanudo, S. M., Price, J. H., Jordan, T., Khuder, S., \& Price, J. A. (2009).

23 Tong, E. K., Strouse, R., Hall, J., Kovac, M., \& Schroeder, S. A. (2010).

${ }^{24}$ Sarna, L., Bialous, S. A., Wells, M., Kotlerman, J., Wewers, M. E., \& Froelicher, E. S. (2009).

${ }^{25}$ Fiore, M. C., Bailey, W. C., Cohen, S. J., Dorfman, S. F., Goldstein, M. G., Gritz, E. R., et al. (2000).

${ }^{26}$ Tong, E. K., Strouse, R., Hall, J., Kovac, M., \& Schroeder, S. A. (2010).

Tobacco Use and Dependence Clinical Practice Guideline Panel, Staff, and Consortium Representatives. (2000).

${ }^{27}$ Johnson, N. W., \& Warnakulasuriya, K. A. A. S. (2003). 
${ }^{28}$ Garvey, A. J. (1997).

${ }^{29}$ Couchenour, R. L., Carson, D. S., \& Segal, A. R. (2002).

Hudmon, K. S., Hemberger, K. K., Corelli, R. L., Kroon, L. A., \& Prokhorov, A. V. (2003).

${ }^{30}$ Williams, D. M. (2009).

${ }^{31}$ Couchenour, R. L., Carson, D. S., \& Segal, A. R. (2002).

${ }^{32}$ Hudmon, K. S., Hemberger, K. K., Corelli, R. L., Kroon, L. A., \& Prokhorov, A. V. (2003).

${ }^{33}$ Kilfoy, B. A., Prokhorov, A. V., \& Hudmon, K. S. (2006).

${ }^{34}$ D'Amico, E. J., Paddock, S. M., Burnam, A., \& Kung, F. Y. (2005).

${ }^{35}$ Hassan, A., Harris, S. K., Sherritt, L., Van, H. S., Brooks, T., Carey, P., et al. (2009).

${ }^{36}$ Hingson, R. W., Heeren, T., Edwards, E. M., \& Saitz, R. (2011).

${ }^{37}$ Fortney, J. C., Tripathi, S. P., Walton, M. A., Cunningham, R. M., \& Booth, B. M. (2011).

Mader, T. J., Smithline, H. A., Nyquist, S., \& Letourneau, P. (2001).

${ }^{38}$ American College of Surgeons. (1993).

Mader, T. J., Smithline, H. A., Nyquist, S., \& Letourneau, P. (2001).

${ }^{39}$ Gentilello, L. M., Donato, A., Nolan, S., Mackin, R. E., Liebich, F., Hoyt, D. B., et al. (2005).

${ }^{40}$ Cunningham, R. M., Harrison, S. R., McCay, M. P., Mello, M. J., Sochor, M., Shandro, M., et al. (2010).

${ }^{41}$ Gentilello, L. M., Donato, A., Nolan, S., Mackin, R. E., Liebich, F., Hoyt, D. B., et al. (2005).

Physicians and Lawyers for National Drug Policy. (2008).

${ }^{42}$ Gentilello, L. M., Donato, A., Nolan, S., Mackin, R. E., Liebich, F., Hoyt, D. B., et al. (2005).

${ }^{43}$ Physicians and Lawyers for National Drug Policy. (2008).

Center for Substance Abuse Treatment. (1995a).

${ }^{44}$ Bernstein, E., Edwards, E., Dorfman, D., Heeren, T., Bliss, C., \& Bernstein, J. (2009).

Spirito, A., Monti, P. M., Barnett, N. P., Colby, S. M., Sindelar, H., Rohsenow, D. J., et al. (2004).

Walton, M. A., Chermack, S. T., Shope, J. T., Bingham, C. R., Zimmerman, M. A., Blow, F. C., et al. (2010).

Tait, R. J., Hulse, G. K., Robertson, S. I., \& Sprivulis, P. C. (2005).

${ }^{45}$ Mader, T. J., Smithline, H. A., Nyquist, S., \& Letourneau, P. (2001).

${ }^{46}$ Willenbring, M. (2009).

${ }^{47}$ Larimer, M. E. \& Kilmer, J. R. (2000).

Peter D.Hart Research Associates, \& Peter. (2001).

Sobell, L. C., Cunningham, J. A., \& Sobell, M. B. (1996).

Sobell, L. C., Sobell, M. B., Toneatto, T., \& Leo, G. I. (1993).

White, W., \& Kurtz, E. (2005).

${ }^{48}$ Fisher, G. L., \& Roget, N. A. (2009).

Knudsen, H. K., Abraham, A. J., \& Oser, C. B. (2011).

LaPaglia, D. (2011).

Stilen, P., Carise, D., Roget, N., \& Wendler, A. (2007).

The National Center on Addiction and Substance Abuse (CASA) at Columbia University. (2000).

${ }^{49}$ Tong, E. K., Strouse, R., Hall, J., Kovac, M., \& Schroeder, S. A. (2010).

${ }^{50}$ The National Center on Addiction and Substance Abuse (CASA) at Columbia University. (2000).

${ }^{51}$ The National Center on Addiction and Substance Abuse (CASA) at Columbia University. (2000).

${ }^{52}$ Hassan, A., Harris, S. K., Sherritt, L., Van, H. S., Brooks, T., Carey, P., et al. (2009).

${ }^{53}$ Deas-Nesmith, D., Campbell, S., \& Brady, K. T. (1998).

${ }^{54}$ Breton, A. R., Taira, D. A., Burns, E., O'Leary, J., \& Chung, R. S. (2007).

${ }^{55}$ Rockett, I. R., Putnam, S. L., Jia, H., Chang, C. F., \& Smith, G. S. (2005).

${ }^{56}$ Fortney, J. C., Tripathi, S. P., Walton, M. A., Cunningham, R. M., \& Booth, B. M. (2011).

${ }^{57}$ Center for Substance Abuse Treatment. (2006b).

Kosten, T. R., \& O'Connor, P. G. (2003).

Merlo, L. J., \& Gold, M. (2008).

Millery, M., Kleinman, B. P., Polissar, N. L., Millman, R. B., \& Scimeca, M. (2002).

National Institute on Drug Abuse. (2005c).

O'Connor, P. G. (2005).

O'Connor, P. G., \& Fiellin, D. A. (2000).

Stein, B. D., Kogan, J. N., \& Sorbero, M. (2009).

${ }^{58}$ The National Center on Addiction and Substance Abuse at Columbia University (CASA Columbia). (2012q).

${ }^{59}$ Center for Substance Abuse Treatment. (2006b). 
Stein, B. D., Kogan, J. N., \& Sorbero, M. (2009).

O'Brien, C. P. (1997).

${ }^{60}$ National Institute on Drug Abuse. (2009c).

Volkow, N. D., Wang, G. J., Fowler, J. S., Tomasi, D., \& Baler, R. (2011).

${ }^{61}$ Hunt, J. J., Cupertino, A. P., Garrett, S., Friedmann, P. D., \& Richter, K. P. (2012).

${ }^{62}$ Hunt, J. J., Cupertino, A. P., Garrett, S., Friedmann, P. D., \& Richter, K. P. (2012).

${ }^{63}$ Prochaska, J. J., Gill, P., \& Hall, S. M. (2004).

${ }^{64}$ Hurt, R. D., Eberman, K. M., Croghan, I. T., Offord, K. P., Davis, L. J., Jr., Morse, R. M., et al. (1994

Richter, K. P. (2006).

Richter, K. P., McCool, R. M., Okuyemi, K. S., Mayo, M. S., \& Ahluwalia, J. S. (2002).

${ }^{65}$ Hurt, R. D., Eberman, K. M., Croghan, I. T., Offord, K. P., Davis, L. J., Jr., Morse, R. M., et al. (1994).

Reid, M. S., Fallon, B., Sonne, S., Flammino, F., Nunes, E. V., Jiang, H., et al. (2008).

Shoptaw, S., Rotheram-Fuller, E., Yang, X., Frosch, D., Nahom, D., Jarvik, M. E., et al. (2002).

${ }^{66}$ Bobo, J. K., McIlvain, H. E., Lando, H. A., Walker, R. D., \& Leed-Kelly, A. (1998).

Friend, K. B., \& Pagano, M. E. (2007).

Shoptaw, S., Rotheram-Fuller, E., Yang, X., Frosch, D., Nahom, D., Jarvik, M. E., et al. (2002).

${ }^{67}$ State of Washington. (2009).

New York State Office of Alcoholism and Substance Abuse Services. (2007).

New Jersey. (2009).

${ }^{68}$ Knudsen, H. K., Studts, J. L., \& Boyd, S. (2009).

${ }^{69}$ Knudsen, H. K., Studts, J. L., \& Boyd, S. (2009).

${ }^{70}$ American Heart Association. (2011).

American Lung Association. (2008).

American Lung Association. (2009).

Chun, J. S., Guydish, J., \& Chan, Y. F. (2007).

Dino, G., Horn, K., Goldcamp, J., Fernandes, A., Kalsekar, I., \& Massey, C. (2001).

Horn, K., Dino, G., Kalsekar, I., \& Mody, R. (2005).

Joffe, A., McNeely, C., Colantuoni, E., An, M. W., Wang, W., \& Scharfstein, D. (2009).

Myers, M. G., \& Brown, S. A. (1994).

Peterson, A. V., Jr., Kealey, K. A., Mann, S. L., Marek, P. M., Ludman, E. J., Liu, J., et al. (2009).

Pbert, L., Osganian, S. K., Gorak, D., Druker, S., Reed, G., O'Neill, K. M., et al. (2006).

Sussman, S., Sun, P., \& Dent, C. W. (2006).

Walsh, M. M., Langer, T. J., Kavanagh, N., Mansell, C., MacDougal, W., Kavanagh, C., et al. (2010).

${ }^{71}$ Knudsen, H. K. (2009).

${ }^{72}$ Knudsen, H. K., Studts, J. L., \& Boyd, S. (2009).

${ }^{73}$ Centers for Disease Control and Prevention. (2011j).

Guydish, J., Passalacqua, E., Tajima, B., Chan, M., Chun, J. S., \& Bostrom, A. (2011).

Knudsen, H. K., Studts, J. L., \& Boyd, S. (2009).

Guydish, J., Passalacqua, E., Tajima, B., Chan, M., Chun, J. S., \& Bostrom, A. (2011).

${ }^{74}$ Roman, P. M., Abraham, A. J., \& Knudsen, H. K. (2011).

${ }^{75}$ Abraham, A. J., \& Roman, P. M. (2010).

Garner, B. R. (2009).

Petrakis, I. L., Leslie, D., \& Rosenheck, R. (2003).

Roman, P. M., Abraham, A. J., \& Knudsen, H. K. (2011).

${ }^{76}$ The National Center on Addiction and Substance Abuse (CASA) at Columbia University. (2009a).

${ }^{77}$ Knudsen, H. K., \& Studts, J. L. (2011).

${ }^{78}$ Abraham, A. J., Knudsen, H. K., \& Roman, P. M. (2011).

Knudsen, H. K., Abraham, A. J., \& Oser, C. B. (2011).

Knudsen, H. K., \& Studts, J. L. (2011).

${ }^{79}$ Knudsen, H. K., Abraham, A. J., \& Oser, C. B. (2011).

Roman, P. M., Abraham, A. J., \& Knudsen, H. K. (2011).

${ }^{80}$ Abraham, A. J., \& Roman, P. M. (2010).

Knudsen, H. K., Abraham, A. J., \& Oser, C. B. (2011).

Roman, P. M., Abraham, A. J., \& Knudsen, H. K. (2011).

${ }^{81}$ Roman, P. M., Abraham, A. J., \& Knudsen, H. K. (2011). 
${ }^{82}$ Forman, R. F., Bovasso, G., \& Woody, G. (2001).

Garner, B. R. (2009).

Thomas, S. E., \& Miller, P. M. (2007).

${ }^{83}$ Anton, R. F., \& Swift, R. M. (2003).

Knudsen, H. K., Abraham, A. J., \& Oser, C. B. (2011).

Pollack, H. A., \& D'Aunno, T. (2008).

${ }^{84}$ Abraham, A. J., Knudsen, H. K., \& Roman, P. M. (2011).

D'Aunno, T. (2006).

Oser, C. B., \& Roman, P. M. (2007).

Roman, P. M., Abraham, A. J., \& Knudsen, H. K. (2011).

${ }^{85}$ The National Center on Addiction and Substance Abuse (CASA) at Columbia University. (2009a).

${ }^{86}$ Anton, R. F., \& Swift, R. M. (2003).

Roman, P. M., Abraham, A. J., \& Knudsen, H. K. (2011).

${ }^{87}$ Knudsen, H. K., Abraham, A. J., \& Oser, C. B. (2011).

${ }^{88}$ Abraham, A. J., Knudsen, H. K., \& Roman, P. M. (2011).

Roman, P. M., Abraham, A. J., \& Knudsen, H. K. (2011).

${ }^{89}$ Roman, P. M., Abraham, A. J., \& Knudsen, H. K. (2011).

${ }^{90}$ Olsen, Y., Bass, E., McCaul, M. E., \& Steinwachs, D. (2004).

${ }^{91}$ Barnett, P. G., Zaric, G. S., \& Brandeau, M. L. (2001).

Center for Substance Abuse Treatment. (2004).

Clark, R. E., Samnaliev, M., Baxter, J. D., \& Leung, G. Y. (2011).

${ }^{92}$ Center for Substance Abuse Treatment. (2004).

Kraus, M. L., Alford, D. P., Kotz, M. M., Levounis, P., Mandell, T. W., Meyer, M., et al. (2011).

${ }^{93}$ Barnett, P. G., Zaric, G. S., \& Brandeau, M. L. (2001).

Clark, R. E., Samnaliev, M., Baxter, J. D., \& Leung, G. Y. (2011).

Polsky, D., Glick, H. A., Yang, J., Subramaniam, G. A., Poole, S. A., \& Woody, G. E. (2010).

94 The National Center on Addiction and Substance Abuse at Columbia University (CASA Columbia). (2012q).

Wallack, S. S., Thomas, C. P., Martin, T. C., Chilingerian, J., \& Reif, S. (2010).

${ }^{95}$ Knudsen, H. K., Abraham, A. J., Johnson, J. A., \& Roman, P. M. (2009).

Knudsen, H. K., Ducharme, L. J., Roman, P. M., \& Link, T. (2005).

${ }^{96}$ Costello, D. (2004).

${ }^{97}$ Center for a Healthy Maryland. (2007).

Join Together. (2003a).

Netherland, J., Botsko, M., Egan, J. E., Saxon, A. J., Cunningham, C. O., Finkelstein, R., et al. (2009).

${ }^{98}$ Beletsky, L., Ruthazer, R., Macalino, G. E., Rich, J. D., Tan, L., \& Burris, S. (2007).

Olsen, Y., Bass, E., McCaul, M. E., \& Steinwachs, D. (2004).

${ }^{99}$ Olsen, Y., Bass, E., McCaul, M. E., \& Steinwachs, D. (2004).

${ }^{100}$ Greenfield, S., Associate Professor of Psychiatry, Harvard Medical School, Associate Clinical Director, Alcohol and Drug Abuse Treatment Program, McLean Hospital (personal communication, January 18, 2008).

${ }^{101}$ Curley, B. (2002).

102 Thomas, C. P., Reif, S., Haq, S., Wallack, S. S., Hoyt, A., \& Ritter, G. A. (2008).

Walley, A. Y., Alperen, J. K., Cheng, D. M., Botticelli, M., Castro-Donlan, C., Samet, J. H., et al. (2008).

${ }^{103}$ Biery, J. R., Williford, J. H., Jr., \& McMullen, E. A. (1991).

Bock, B. C., Marcus, B. H., King, T. K., Borrelli, B., \& Roberts, M. R. (1999).

Forsander, O. A. (1998).

Grant, L. P., Haughton, B., \& Sachan, D. S. (2004).

Marcus, B. H., Albrecht, A. E., King, T. K., Parisi, A. F., Pinto, B. M., Roberts, M., et al. (1999).

McClernon, F. J., Westman, E. C., \& Rose, J. E. (2004).

Ussher, M. H., Taylor, A. H., West, R., \& McEwen, A. (2000).

${ }^{104}$ Kenny, P. J. (2011).

Volkow, N. D., Wang, G. J., Fowler, J. S., \& Telang, F. (2008).

${ }^{105}$ Grant, L. P., Haughton, B., \& Sachan, D. S. (2004).

${ }^{106}$ American Society of Addiction Medicine. (2006).

Peter D.Hart Research Associates, \& Peter. (2001).

Sobell, L. C., Cunningham, J. A., \& Sobell, M. B. (1996). 
Sobell, L. C., Sobell, M. B., Toneatto, T., \& Leo, G. I. (1993).

The National Center on Addiction and Substance Abuse (CASA) at Columbia University. (2008a).

${ }^{107}$ Alcoholics Anonymous World Services. (1972).

${ }^{108}$ American Diabetes Association. (2005).

American Diabetes Association. (2012).

Fromer, L. (2010).

Mayo Clinic. (2012).

National Heart,Lung,and Blood Institute. (2007).

Agency for Healthcare Research and Quality. (2012c).

${ }^{109}$ Center for Substance Abuse Treatment. (1995b).

Peters, R. H., Bartoi, M. G., \& Sherman, P. B. (2008).

U.S. Department of Defense, Department of Veteran Affairs. (2009).

${ }^{110}$ Casares-Lopez, M. J., Diaz-Mesa, E., Garcia-Portilla, P., Saiz, P., Bobes-Bascaran, M. T., Fonseca-Pedrero, E., et al. (2011).

French, M. T., Salome, H. J., Sindelar, J. L., \& McLellan, A. T. (2002).

Institute of Medicine, Committee on Opportunities in Drug Abuse Research. (1996).

McLellan, A. T., Cacciola, J. C., Alterman, A. I., Rikoon, S. H., \& Carise, D. (2006).

National Institute on Alcohol Abuse and Alcoholism. (2012a).

Spear, S. E., Brown, A. H., \& Rawson, R. A. (2005).

Stilen, P., Carise, D., Roget, N., \& Wendler, A. (2007).

${ }^{111}$ Fisher, G. L. \& Roget, N. A. (2009).

Stilen, P., Carise, D., Roget, N., \& Wendler, A. (2007).

${ }^{112}$ Liddle, H. A., \& Dakof, G. A. (1995).

Marlatt, G. A., Blume, A. W., \& Parks, G. A. (2001).

Ritchie, D., Schulz, S., \& Bryce, A. (2007).

Rowe, C. L., Liddle, H. A., Greenbaum, P. E., \& Henderson, C. E. (2004).

${ }^{113}$ The National Center on Addiction and Substance Abuse at Columbia University (CASA Columbia). (2012q).

${ }^{114}$ O'Brien, C. P., Childress, A. R., Ehrman, R., \& Robbins, S. J. (1998).

O'Brien, C. P., \& McLellan, A. T. (1996).

Roan, S. (2008).

Quenqua, D. (2011, July 11).

Willenbring, M. L. (2008b).

Wall Street Journal. (2012).

${ }^{115}$ Willenbring, M. (2009).

${ }^{116}$ American Diabetes Association. (2005).

American Diabetes Association. (2012).

Defense Centers of Excellence. (2011).

Fromer, L. (2010).

${ }^{117}$ Hoof-Haines, K. V. (2012).

Sterling, S., Chi, F., \& Hinman, A. (2011).

Substance Abuse and Mental Health Services Administration, Office of Applied Studies. (2010).

${ }^{118}$ The National Center on Addiction and Substance Abuse (CASA) at Columbia University. (2009a).

${ }^{119}$ Boden, M. T., \& Moos, R. (2009)

${ }^{120}$ Mendell, G., Father, Lost his son Brian, age 25, to addiction and suicide (personal communication, April 3, 2012).

${ }^{121}$ Lasser, K., Boyd, J. W., Woolhandler, S., Himmelstein, D. U., McCormick, D., \& Bor, D. H. (2000).

Association of American Medical Colleges. (2007).

Thorndike, A. N., Stafford, R. S., \& Rigotti, N. A. (2001).

Himelhoch, S., \& Daumit, G. (2003).

Zvolensky, M. J., Baker, K., Yartz, A. R., Gregor, K., Leen-Feldner, E. W., \& Feldner, M. T. (2005).

${ }_{122}$ Association of American Medical Colleges. (2007).

${ }^{123}$ Hall, S. M., \& Prochaska, J. J. (2009).

${ }^{124}$ Association of American Medical Colleges. (2007).

${ }^{125}$ Siru, R., Hulse, G. K., \& Tait, R. J. (2009).

${ }^{126}$ Prochaska, J. J., Rossi, J. S., Redding, C. A., Rosen, A. B., Tsoh, J. Y., Humfleet, G. L., et al. (2004). 
${ }^{127}$ Alexander, J. A., Nahra, T. A., Lemak, C. H., Pollack, H., \& Campbell, C. I. (2008).

Knudsen, H. K., \& Studts, J. L. (2011).

Knudsen, H. K., Studts, C. R., \& Studts, J. L. (2011).

Olmstead, T., \& Sindelar, J. L. (2004).

Pollack, H. A., \& D'Aunno, T. (2008).

Substance Abuse and Mental Health Services Administration, Office of Applied Studies. (2010).

${ }^{128}$ Anglin, M. D., Hser, Y. I., \& Grella, C. E. (1997).

Dennis, M. L., Scott, C. K., Funk, R., \& Foss, M. A. (2005).

Leshner, A. I. (2001a).

Larimer, M. E. \& Kilmer, J. R. (2000).

Peter D.Hart Research Associates, \& Peter. (2001).

Sobell, L. C., Sobell, M. B., Toneatto, T., \& Leo, G. I. (1993).

129 American Diabetes Association. (2011).

${ }^{130}$ Appel, L. J., Brands, M. W., Daniels, S. R., Karanja, N., Elmer, P. J., \& Sacks, F. M. (2006).

National Library of Medicine. (2007).

131 Asthma and Allergy Foundation of America. (2005).

National Heart,Lung,and Blood Institute. (2011a).

National Heart,Lung,and Blood Institute. (2011b).

132 Buck, J. A. (2011).

Pating, D. R., Miller, M. M., Goplerud, E., Martin, J., \& Ziedonis, D. M. (2012).

133 American Society of Addiction Medicine. (2012).

Pating, D. R., Miller, M. M., Goplerud, E., Martin, J., \& Ziedonis, D. M. (2012).

White, W. L. (2008).

White, W. L., \& McLellan, A. T. (2008).

${ }^{134}$ Mackay, P. W., \& Marlatt, G. A. (1991).

O'Brien, C. P., \& McLellan, A. T. (1996).

Tims, F. M., Leukefeld, C. G., \& Platt, J. J. (2001).

White, W. L., \& McLellan, A. T. (2008).

${ }^{135}$ Centers for Medicare and Medicaid Services. (2012c).

${ }^{136}$ Rush, B. (1808). An inquiry into the effects of ardent spirits upon the human body and mind: With an account of the means of preventing, and of the remedies for curing them. Philadelphia: Thomas Dobson (cited in Anton,R. 2010).

${ }^{137}$ Katcher, B. S. (1993).

University of Pennsylvania. (2012).

${ }^{138}$ Kandall, S. R., \& Petrillo, J. (1996).

${ }^{139}$ Kandall, S. R., \& Petrillo, J. (1996).

${ }^{140}$ Caron, J. (2002).

White, W. L. (1998).

${ }^{141}$ Bottlebooks.com. (2009).

142 TheAddictionCure.com. (2012).

143 Norcross, J. C., Koocher, G. P., Fala, N. C., \& Wexler, H. K. (2010).

${ }^{144}$ Alcoholics Anonymous World Services. (1972).

Anderson, D. J., McGovern, J. P., \& Dupont, R. L. (1999).

White, W. L., \& Kurtz, E. (2008).

Spicer, J. (1993).

${ }^{145}$ Alcoholics Anonymous World Services. (1972).

146 Alcoholics Anonymous World Services. (1972).

Anderson, D. J., McGovern, J. P., \& Dupont, R. L. (1999).

Spicer, J. (1993).

${ }^{147}$ Roy, K., \& Miller, M. (2010).

Roy, A. K., \& Miller, M. M. (2012).

148 Humphreys, K., Ph.D., Professor, Stanford Medical School (personal communication, August 16, 2007).

${ }^{149}$ Lamb, S., Greenlick, M. R., \& McCarty, D. (eds.). (1998).

Condon, T. P., Miner, L. L., Balmer, C. W., \& Pintello, D. (2008).

${ }^{150}$ McLellan, A. T., \& Meyers, K. (2004). 
Olmstead, T. A., Abraham, A. J., Martino, S., \& Roman, P. M. (2012).

Substance Abuse and Mental Health Services Administration. (2003b).

${ }^{151}$ Institute of Medicine, Committee on Crossing the Quality Chasm:Adaptation to Mental Health and Addictive Disorder. (2006).

${ }^{152}$ The National Center on Addiction and Substance Abuse (CASA) at Columbia University. (2009a).

${ }^{153}$ The National Center on Addiction and Substance Abuse (CASA) at Columbia University. (2009a).

${ }^{154}$ The National Center on Addiction and Substance Abuse (CASA) at Columbia University. (2009a).

${ }^{155}$ The National Center on Addiction and Substance Abuse (CASA) at Columbia University. (2009a).

${ }^{156}$ The National Center on Addiction and Substance Abuse (CASA) at Columbia University. (2009a).

${ }^{157}$ D'Aunno, T. (2006).

Eby, L. T., Burk, H., \& Maher, C. P. (2010).

Lamb, S., Greenlick, M. R., \& McCarty, D. (eds.). (1998).

McLellan, A. T., Carise, D., \& Kleber, H. D. (2003).

McLellan, A. T., \& Meyers, K. (2004).

McNulty, T. L., Oser, C. B., Johnson, J. A., Knudsen, H. K., \& Roman, P. M. (2007).

Northeast Addiction Technology Transfer Center. (2005).

Olmstead, T. A., Johnson, J. A., Roman, P. M., \& Sindelar, J. L. (2005).

Powell, D. J. (2006).

${ }^{158}$ D'Aunno, T. (2006).

${ }^{159}$ Forum on Integration, A Collaborative for States. (2010).

Institute of Medicine, Committee on Crossing the Quality Chasm:Adaptation to Mental Health and Addictive

Disorder. (2006).

Knight, D. K., Landrum, B., Becan, J. E., \& Flynn, P. M. (2012).

${ }^{160}$ The National Center on Addiction and Substance Abuse (CASA) at Columbia University. (2009a).

${ }^{161}$ Clay, R. A. (2004).

McLellan, A. T., Carise, D., \& Kleber, H. D. (2003).

Olmstead, T. A., Abraham, A. J., Martino, S., \& Roman, P. M. (2012).

${ }^{162}$ Dilonardo, J. (2011).

Kerwin, M. E., Walker-Smith, K., \& Kirby, K. C. (2006).

${ }^{163}$ Dilonardo, J. (2011).

Kerwin, M. E., Walker-Smith, K., \& Kirby, K. C. (2006).

U.S. Department of Justice, Office of Diversion Control. (2012).

Van Hoof-Haines, K. (2012).

${ }^{164}$ Kerwin, M. E., Walker-Smith, K., \& Kirby, K. C. (2006).

Marinelli-Casey, P., Domier, C. P., \& Rawson, R. A. (2002).

${ }^{165}$ Brown, B. S., \& Flynn, P. M. (2002).

Condon, T. P., Miner, L. L., Balmer, C. W., \& Pintello, D. (2008).

${ }^{166}$ Garner, B. R. (2009).

${ }^{167}$ Rieckmann, T., Fuller, B. E., Saedi, G. A., \& McCarty, D. (2010).

168 Janes, W. H., Former Director, Florida Office of Drug Control, Office of the Governor (personal communication, August 14, 2007).

169 Amico, J. M., M.Div, President, National Association of Lesbian and Gay Addiction Professionals (now Vice President, and association now called The Association of Lesbian, Gay, Bisexual, Transgender Addiction

Professionals and Their Allies) (personal communication, August 16, 2007).

${ }^{170}$ Covington, S., PhD, LCSW, Co-Director, Center for Gender and Justice, Institute for Relational Development, La Jolla, CA (personal communication, May 21, 2012).

${ }^{171}$ Burke, B. L., Arkowitz, H., \& Menchola, M. (2003).

McCarty, D., Fuller, B. E., Arfken, C., Miller, M., Nunes, E. V., Edmundson, E., et al. (2007).

National Institute on Drug Abuse. (2012a).

172 McCarty, D., Fuller, B. E., Arfken, C., Miller, M., Nunes, E. V., Edmundson, E., et al. (2007).

${ }^{173}$ Knudsen, H. K. (2006).

${ }^{174}$ Lundgren, L., Amodeo, M., Krull, I., Chassler, D., Weidenfeld, R., de Saxe Zerden, L., et al. (2011).

McCarty, D., Fuller, B. E., Arfken, C., Miller, M., Nunes, E. V., Edmundson, E., et al. (2007).

Knudsen, H. K. (2006).

${ }^{175}$ D'Aunno, T. (2006). 
Knudsen, H. K. (2006).

${ }^{176}$ Aalto, M., Pekuri, P., \& Seppa, K. (2003).

Anderson, P., Aromaa, S., Rosenbloom, D., \& Enos, G. (2008).

Erickson, C. K., Wilcox, R. E., Miller, G. W., Littlefield, J. H., \& Lawson, K. A. (2003).

Gordon, A. J., Ettaro, L., Rodriguez, K. L., Mocik, J., \& Clark, D. B. (2011).

Lafferty, L., Hunter, T. S., \& Marsh, W. A. (2006).

Leshner, A. I. (1999).

Miller, N. S., Sheppard, L. M., Colenda, C. C., \& Magen, J. (2001).

Office of National Drug Control Policy. (2011).

Quinton, A. (2004).

Russell, M. (2004).

${ }^{177}$ Rawson, R. A., McCann, M. J., Hasson, A. J., \& Ling, W. (2000).

${ }^{178}$ SAMHSA's National Registry of Evidence-based Programs and Practices. (2007b).

${ }^{179}$ American Medical Association. (1997).

American Psychiatric Association. (2006).

Kokotailo, P. K., \& Committee on Substance Abuse. (2010).

${ }^{180}$ National Quality Forum. (2007).

${ }^{181}$ Agency for Healthcare Research and Quality. (2003).

Agency for Healthcare Research and Quality. (2012b).

West, S. L., Garbutt, J. C., Carey, T. S., Lux, L. J., Jackman, A. M., Tolleson-Rinehart, S., et al. (1999).

Agency for Healthcare Research and Quality. (2012a).

${ }^{182}$ Cabana, M. D., Rand, C. S., Powe, N. R., Wu, A. W., Wilson, M. H., Abboud, P.-A., et al. (1999).

Center for Substance Abuse Treatment. (2010).

Center for Substance Abuse Treatment. (2005b).

Maciosek, M. V., Coffield, A. B., Edwards, N. M., Flottemesch, T. J., Goodman, M. J., \& Solberg, L. I. (2006).

U.S. Preventive Services Task Force. (2010).

${ }^{183}$ Kunz, K. B., President, \& Callahan, J. F., Executive Vice President, American Board of Addiction Medicine (personal communication, May 2, 2012).

${ }^{184}$ Cabana, M. D., Rand, C. S., Powe, N. R., Wu, A. W., Wilson, M. H., Abboud, P.-A., et al. (1999).

${ }^{185}$ Aalto, M., Pekuri, P., \& Seppa, K. (2003).

Allsop, S. J., \& Stevens, C. (2009).

Anderson, P., Aromaa, S., Rosenbloom, D., \& Enos, G. (2008).

Friedmann, P. D., McCullough, D., Chin, M. H., \& Saitz, R. (2000).

Maciosek, M. V., Coffield, A. B., Edwards, N. M., Flottemesch, T. J., Goodman, M. J., \& Solberg, L. I. (2006).

Miller, N. S., Sheppard, L. M., \& Magen, J. (2001).

Miller, N. S., Sheppard, L. M., Colenda, C. C., \& Magen, J. (2001).

Substance Abuse and Mental Health Services Administration, Office of Applied Studies. (2010).

The National Center on Addiction and Substance Abuse (CASA) at Columbia University. (2000).

${ }^{186}$ National Institute on Drug Abuse. (2011b).

${ }^{187}$ Hurley, B., Chair, Physicians-in-training Committee, American Society of Addiction Medicine (personal communication, August 13, 2007).

${ }^{188}$ The National Center on Addiction and Substance Abuse (CASA) at Columbia University. (2000).

${ }^{189}$ Gohdes, D., Amundson, H., Oser, C. S., Helgerson, S. D., \& Harwell, T. S. (2009).

Harwell, T. S., Oser, C. S., Helgerson, S. D., \& Gohdes, D. (2007).

190 Tong, E. K., Strouse, R., Hall, J., Kovac, M., \& Schroeder, S. A. (2010).

${ }^{191}$ Prochaska, J. J., Fromont, S. C., Louie, A. K., Jacobs, M. H., \& Hall, S. M. (2006).

${ }^{192}$ Prochaska, J. J., Fromont, S. C., \& Hall, S. M. (2005).

${ }^{193}$ Springer, C. M., Niang, C. M. T., Matte, T. D., Miller, N., Bassett, M. T., \& Frieden, T. R. (2008).

${ }^{194}$ Powers, C. A., Zapka, J., Phelan, S., Ozcan, T., Biello, K. B., O'Donnell, J., et al. (2011).

${ }^{195}$ Anderson, P., Aromaa, S., Rosenbloom, D., \& Enos, G. (2008).

Miller, N. S., Sheppard, L. M., Colenda, C. C., \& Magen, J. (2001).

${ }^{196}$ Anderson, P., Aromaa, S., Rosenbloom, D., \& Enos, G. (2008).

Miller, N. S., Sheppard, L. M., Colenda, C. C., \& Magen, J. (2001).

${ }^{197}$ Fleming, M., Barry, K., Davis, A., Kropp, S., Kahn, R., \& Rivo, M. (1994).

Accreditation Council for Graduate Medical Education. (2012b). 
${ }^{198}$ Fitzgerald, K., Fourth year medical student (personal communication, September 17, 2008).

199 American Board of Psychiatry and Neurology. (2012).

${ }^{200}$ Renner, J. A., Jr., Karam-Hage, M., Levinson, M., Craig, T., \& Eld, B. (2009).

${ }^{201}$ Cromwell, J., Bartosch, W. J., Fiore, M. C., Hasselblad, V., \& Baker, T. (1997).

Stead, L. F., Bergson, G., \& Lancaster, T. (2008).

${ }^{202}$ Ferry, L. H., Grissino, L. M., \& Runfola, P. S. (1999).

Spangler, J. G., George, G., Foley, K. L., \& Crandall, S. J. (2002).

203 The National Center on Addiction and Substance Abuse (CASA) at Columbia University. (2008b).

${ }^{204}$ Washton, A. M. (2001).

Carey, K. B., Bradizza, C. M., Stasiewicz, P. R., \& Maisto, S. A. (1999).

${ }^{205}$ Washton, A. M. (2001).

Akpanudo, S. M., Price, J. H., Jordan, T., Khuder, S., \& Price, J. A. (2009).

206 Akpanudo, S. M., Price, J. H., Jordan, T., Khuder, S., \& Price, J. A. (2009).

${ }^{207}$ Dolan, T. A., McGorray, S. P., Grinstead-Skigen, C. L., \& Mecklenburg, R. (1997).

Gordon, J. S., Albert, D. A., Crews, K. M., \& Fried, J. (2009).

Huggett, K. N., Westerman, G. H., Barone, E. J., \& Lofgreen, A. S. (2011).

Pendharkar, B., Levy, S. M., McQuistan, M. R., Qian, F., Squier, C. A., Slach, N. A., et al. (2010).

Simoyan, O., Badner, V. M., \& Freeman, K. D. (2002).

${ }^{208}$ Denisco, R. C., Kenna, G. A., O'Neil, M. G., Kulich, R. J., Moore, P. A., Kane, W. T., et al. (2011).

Gordon, J. S., Albert, D. A., Crews, K. M., \& Fried, J. (2009).

${ }^{209}$ Gordon, J. S., Albert, D. A., Crews, K. M., \& Fried, J. (2009).

${ }^{210}$ Barker, G. J., \& Williams, K. B. (1999).

${ }^{211}$ Harris, J. L., Patton, L. L., Wilder, R. S., Peterson, C. A., \& Curran, A. E. (2009).

Mecklenburg, R. E. (2001).

${ }^{212}$ Yip, J. K., Hay, J. L., Ostroff, J. S., Stewart, R. K., \& Cruz, G. D. (2000).

${ }^{213}$ U.S. Department of Health and Human Services, Health Resources and Services Administration. (2012).

Kreitzer, M. J., Kligler, B., \& Meeker, W. C. (2009).

${ }^{214}$ Desy, P. M., Howard, P. K., Perhats, C., \& Li, S. (2010).

${ }^{215}$ Sarna, L., Bialous, S. A., Rice, V. H., \& Wewers, M. E. (2009).

${ }^{216}$ Sheffer, C. E., Barone, C., \& Anders, M. E. (2010).

217 Sarna, L., Bialous, S. A., Rice, V. H., \& Wewers, M. E. (2009).

Wewers, M. E., Kidd, K., Armbruster, D., \& Sarna, L. (2004).

${ }^{218}$ Dent, L. A., Harris, K. J., \& Noonan, C. W. (2007).

219 The American Society of Health System Pharmacists. (2012).

Patel, D. (2005).

National Institute on Drug Abuse. (2011e).

${ }^{220}$ Hudmon, K. S., Prokhorov, A. V., \& Corelli, R. L. (2006).

Lafferty, L., Hunter, T. S., \& Marsh, W. A. (2006).

Williams, D. M. (2009).

${ }^{221}$ Hudmon, K. S., Prokhorov, A. V., \& Corelli, R. L. (2006).

${ }^{222}$ Lafferty, L., Hunter, T. S., \& Marsh, W. A. (2006).

${ }^{223}$ Cahill, K., Stead, L. F., \& Lancaster, T. (2007).

Fiore, M. C., Jorenby, D. E., Baker, T. B., \& Kenford, S. L. (1992).

Hughes, J. R., Stead, L. F., \& Lancaster, T. (2007).

Stead, L. F., Perera, R., Bullen, C., Mant, D., \& Lancaster, T. (2008).

U.S. Department of Health and Human Services, U.S. Food and Drug Administration, Center for Drug Evaluation and Research. (2008).

U.S.Food and Drug Administration. (2007a).

U.S.Food and Drug Administration. (2011a).

National Library of Medicine. (2008a).

National Library of Medicine. (2008b).

${ }^{224}$ D'Aunno, T. (2006).

Pollack, H. A., \& D'Aunno, T. (2008).

${ }^{225}$ Fareed, A., Casarella, J., Amar, R., Vayalapalli, S., \& Drexler, K. (2010).

Strain, E. C., Bigelow, G. E., Liebson, I. A., \& Stitzer, M. L. (1999). 
${ }^{226}$ Lowinson, J. H., Ruiz, P., Millman, R. B., \& Langrod, J. G. (eds.). (2005).

Pollack, H. A., \& D'Aunno, T. (2008).

${ }^{227}$ D'Aunno, T., \& Pollack, H. A. (2002).

${ }^{228}$ Rettig, R. (1995).

${ }^{229}$ Salsitz, E., Joseph, H., Blanche, F., Perez, J., Richman, B. L., Salomon, N., et al. (2000).

${ }^{230}$ New York City Department of Health and Mental Hygiene. (2009).

${ }^{231}$ Volkow, N. D., \& Skolnick, P. (2012).

${ }^{232}$ Frank, R. G., Conti, R. M., \& Goldman, H. H. (2005).

Willenbring, M. (2009).

${ }^{233}$ Miller, G. (2010).

Volkow, N. D., \& Skolnick, P. (2012).

${ }^{234}$ Miller, G. (2010).

${ }^{235}$ Volkow, N. D., \& Skolnick, P. (2012).

${ }^{236}$ Volkow, N. D., \& Skolnick, P. (2012).

${ }^{237}$ Volkow, N. D., \& Skolnick, P. (2012).

${ }^{238}$ Volkow, N. D., \& Skolnick, P. (2012).

${ }^{239}$ Chao, S. (2007).

Institute of Medicine, Committee on Crossing the Quality Chasm:Adaptation to Mental Health and Addictive

Disorder. (2006).

Join Together. (2003b).

${ }^{240}$ Kohn, L. T., Corrigan, J., \& Donaldson, M. S. (2000).

Insitute of Medicine, Committee on Quality of Health Care in America. (2001).

Institute of Medicine, Committee on Crossing the Quality Chasm:Adaptation to Mental Health and Addictive

Disorder. (2006).

${ }^{241}$ Institute of Medicine, Committee on Crossing the Quality Chasm:Adaptation to Mental Health and Addictive Disorder. (2006).

242 Office of National Drug Control Policy. (2007).

${ }^{243}$ Abt Associates. (2006).

Institute of Medicine, Committee on Crossing the Quality Chasm:Adaptation to Mental Health and Addictive Disorder. (2006).

McLellan, A. T., McKay, J. R., Forman, R., Cacciola, J., \& Kemp, J. (2005).

${ }^{244}$ McLellan, A. T., McKay, J. R., Forman, R., Cacciola, J., \& Kemp, J. (2005).

${ }^{245}$ Institute of Medicine, Committee on Crossing the Quality Chasm:Adaptation to Mental Health and Addictive Disorder. (2006).

${ }^{246}$ Cassel, E. J. (1982).

Larson, J. S. (1999).

Nordgren, A. (1999).

Social Security Online. (2011).

${ }^{247}$ Institute of Medicine. (1990).

Laudet, A. B., \& White, W. (2010).

O'Brien, C. P. \& McKay, J. (2007).

Schuckit, M. A. (2009).

248 The National Center on Addiction and Substance Abuse (CASA) at Columbia University. (2008c).

249 The National Center on Addiction and Substance Abuse (CASA) at Columbia University. (2009a).

${ }^{250}$ Carey, K. B. (2008, December 22).

${ }^{251}$ Carey, K. B. (2008, December 22).

D'Aunno, T. (2006).

Ganju, V. (2006).

Institute of Medicine, Committee on Crossing the Quality Chasm:Adaptation to Mental Health and Addictive Disorder. (2006).

McLellan, A. T., Carise, D., \& Kleber, H. D. (2003).

${ }^{252}$ Institute of Medicine, Committee on Crossing the Quality Chasm:Adaptation to Mental Health and Addictive Disorder. (2006).

253 The National Center on Addiction and Substance Abuse (CASA) at Columbia University. (2009a). 
${ }^{254}$ Miller, M. M., MD., Past President, American Society of Addiction Medicine (personal communication, March 16, 2012).

${ }^{255}$ Ganju, V. (2006).

Institute of Medicine, Committee on Crossing the Quality Chasm:Adaptation to Mental Health and Addictive Disorder. (2006).

${ }^{256}$ Centers for Medicare and Medicaid Services, Center for Consumer Information and Insurance Oversight. (2012). House Office of the Legislative Counsel. (2010).

257 The National Center on Addiction and Substance Abuse (CASA) at Columbia University. (2009d).

${ }^{258}$ National Cancer Institute. (2012).

${ }^{259}$ American Heart Association, American Stroke Association. (2012).

${ }^{260}$ National Institutes of Health. (2012).

261 Roan, S. (2011).

${ }^{262}$ Addiction Technology Transfer Center Network. (2009a).

Hermann, R. C., Chan, J. A., Zazzali, J. L., \& Lerner, D. (2006).

Rieckmann, T. R., Kovas, A. E., Cassidy, E. F., \& McCarty, D. (2011).

Timmermans, S., \& Mauck, A. (2005).

${ }^{263}$ Addiction Technology Transfer Center Network. (2009a).

Center for Substance Abuse Treatment. (2000a).

Substance Abuse and Mental Health Services Administration. (2003c).

Hoge, M. A., Morris, J. A., Daniels, A. S., Stuart, G. W., Huey, L. Y., \& Adams, N. (2007).

Tomas-Rossello, J., Rawson, R. A., Zarza, M. J., Bellows, A., Busse, A., Saenz, E., et al. (2010).

${ }^{264}$ American Psychiatric Association. (2008b).

Garnick, D. W., Lee, M. T., Chalk, M., Gastfriend, D., Horgan, C. M., McCorry, F., et al. (2002).

Joint Commission. (2012e).

McCorry, F., Garnick, D. W., Bartlett, J., Cotter, F., \& Chalk, M. (2000).

Robert Wood Johnson Foundation. (2005).

Thomas, C. P., Garnick, D. W., Horgan, C. M., McCorry, F., Gmyrek, A., Chalk, M., et al. (2011).

${ }^{265}$ Alliance for Health Reform. (2006).

Ganju, V. (2006).

Join Together. (2003b).

Pelletier, L. R., \& Hoffamn, J. A. (2002).

Stitzer, M. L., Owen, P. L., Hall, S. M., Rawson, R. A., \& Petry, N. M. (2003).

Teruya, C., Hardy, M., Hser, Y.-I., \& Evans, E. (2006).

${ }^{266}$ Buck, J. A. (2011).

Hillestad, R., Bigelow, J., Bower, A., Girosi, F., Meili, R., Scoville, R., et al. (2005).

Humphreys, K., \& McLellan, A. T. (2011).

Substance Abuse and Mental Health Services Administration. (2011).

${ }^{267}$ Brucker, D. L., \& Stewart, M. (2011).

Ganju, V. (2006).

Hermann, R. C., Chan, J. A., Zazzali, J. L., \& Lerner, D. (2006).

Humphreys, K., \& McLellan, A. T. (2011).

Institute of Medicine, Committee on Crossing the Quality Chasm:Adaptation to Mental Health and Addictive Disorder. (2006).

Merrick, E. L., Garnick, D. W., Horgan, C. M., \& Hodgkin, D. (2002).

Shen, Y. (2003).

Werner, R. M., Kolstad, J. T., Stuart, E. A., \& Polsky, D. (2011).

${ }^{268}$ Forum on Integration, A Collaborative for States. (2010).

${ }^{269}$ Finney, J. W., Ouimette, P. C., Humphreys, K., \& Moos, R. H. (2001).

Forum on Integration, A Collaborative for States. (2010).

Miller, W. R., Baca, C., Compton, W. M., Ernst, D., Manuel, J. K., Pringle, B., et al. (2006).

${ }^{270}$ Berenson, R. A., \& Burton, R. A. (2011).

Buck, J. A. (2011).

Humphreys, K., \& McLellan, A. T. (2010).

Roy, K., \& Miller, M. (2010).

HealthCare.gov. (2011a). 
Patient protection and affordable care act, $42 \S 3022$ (2010).

${ }^{271}$ American Society of Addiction Medicine. (2009).

${ }^{272}$ American Board of Addiction Medicine. (2010).

Callahan, J. F., DPA., Executive Vice President, The American Board of Addiction Medicine and the ABAM

Foundation (personal communication, January 25, 2012).

Quenqua, D. (2011, July 11).

${ }^{273}$ News-Medical.Net. (2012).

${ }^{274}$ American Society of Health-System Pharmacists. (2012a).

American Society of Health-System Pharmacists. (2012b).

${ }^{275}$ National Institutes of Health. (2009).

National Institutes of Health. (2011a).

National Institute on Drug Abuse. (2009d).

Substance Abuse and Mental Health Services Administration. (2008e).

Substance Abuse and Mental Health Services Administration. (2008f).

${ }^{276}$ National Institutes of Health. (2009).

National Institute on Drug Abuse. (2009d).

${ }^{277}$ Substance Abuse and Mental Health Services Administration. (2008d).

${ }^{278}$ Haack, M. R., \& Adger, H. (2002).

${ }^{279}$ The Academic ED SBIRT Research Collaborative. (2007).

McLellan, A. T., \& Meyers, K. (2004).

Sheffer, C. E., Barone, C. P., \& Anders, M. E. (2009).

Studts, J. L., Burrie, J. L., Kearns, D. K., Worth, C. T., \& Sorrell, C. L. (2009).

${ }^{280}$ Sheffer, C. E., Barone, C. P., \& Anders, M. E. (2009).

${ }^{281}$ Bernstein, E., Bernstein, J., Feldman, J., Fernandez, W., Hagan, M., Mitchell, P., et al. (2007).

D'Onofrio, G., Nadel, E. S., Degutis, L. C., Sullivan, L. M., Casper, K., Bernstein, E., et al. (2002).

${ }^{282}$ Haack, M. R., \& Adger, H. (2002).

Ockene, J. K., Wheeler, E. V., Adams, A., Hurley, T. G., \& Hebert, J. (1997).

Saitz, R., Sullivan, L. M., \& Samet, J. H. (2000).

${ }^{283}$ Substance Abuse and Mental Health Services Administration. (2005c).

${ }^{284}$ Donaldson, M. S., Yordy, K. D., Lohr, K. N., \& Vanselow, N. A. (1996).

${ }^{285}$ Grumbach, K., \& Grundy, P. (2010).

Rosenthal, T. C. (2008).

${ }^{286}$ Grumbach, K., \& Grundy, P. (2010).

${ }^{287}$ Joint Commission. (2012b).

${ }^{288}$ Joint Commission. (2012c).

${ }^{289}$ Fiore, M. C., Goplerud, E., \& Schroeder, S. A. (2012).

${ }^{290}$ Fiore, M. C., Goplerud, E., \& Schroeder, S. A. (2012).

Saitz, R. (2010).

Joint Commission. (2012b).

${ }^{291}$ Fiore, M. C., Goplerud, E., \& Schroeder, S. A. (2012).

${ }^{292}$ Gentilello,L. M., M.D., Trauma Services, Kaiser Permanente Hospital, Sacramento, CA. (personal communication, March 8, 2012). 


\section{Chapter XI}

Notes

${ }^{1}$ Miller, N. S. (2011).

${ }^{2}$ National Institute on Drug Abuse. (2009e). 


\section{Appendix A \\ Notes}

${ }^{1}$ Fendrich, M., \& Johnson, T. P. (2001).

${ }^{2}$ Brown, R. L., Leonard, T., Saunders, L. A., \& Papasouliotis, O. (1998).

Greene, J. M., Ennett, S. T., \& Ringwalt, C. L. (1997).

Substance Abuse and Mental Health Services Administration. (2006).

The National Center on Addiction and Substance Abuse (CASA) at Columbia University. (2010).

The National Center on Addiction and Substance Abuse at Columbia University (CASA Columbia). (2012n).

${ }^{3}$ American Psychiatric Association. (1994).

${ }^{4}$ Shiffman, S., Waters, A. J., \& Hickcox, M. (2004).

U.S. Department of Health and Human Services, Substance Abuse and Mental Health Services Administration, Office of Applied Studies. (2008).

${ }^{5}$ American Psychiatric Association. (1994).

Colby, S. M., Tiffany, S. T., Shiffman, S., \& Niaura, R. S. (2000).

U.S. Department of Health and Human Services. (2010a).

${ }^{6}$ Nichter, M., Nichter, M., Thompson, P. J., Shiffman, S., \& Moscicki, A.-B. (2002). 


\section{Appendix $\mathbf{H}$ \\ Notes}

${ }^{1}$ Babor, T. F., \& Kadden, R. M. (2005).

Babor, T. F., McRee, B. G., Kassebaum, P. A., Grimaldi, P. L., Ahmed, K., \& Bray, J. (2007).

${ }^{2}$ United Nations, Office on Drugs and Crime. (2008).

${ }^{3}$ Center for Substance Abuse Treatment. (2005b).

University of Washington. (2011).

Center for Substance Abuse Treatment. (1999b).

Center for Substance Abuse Treatment. (2006b).

${ }^{4}$ Cook, D. A., \& Beckman, T. J. (2006).

${ }^{5}$ Goldstein, J. M., Cherkerzian, S., \& Simpson, J. C. (2011).

Fayers, P. M. (2007).

${ }^{6}$ Buhringer, G. \& Sassen, M. (2010).

Fayers, P. M. (2007).

${ }^{7}$ Fayers, P. M. (2007).

Buhringer, G. \& Sassen, M. (2010).

${ }^{8}$ Fayers, P. M. (2007).

Buhringer, G. \& Sassen, M. (2010).

${ }^{9}$ Fayers, P. M. (2007).

${ }^{10}$ Fayers, P. M. (2007).

Buhringer, G. \& Sassen, M. (2010).

${ }^{11}$ Fayers, P. M. (2007).

${ }^{12}$ Humeniuk, R., Ali, R., Babor, T. F., Farrell, M., Formigoni, M. L., Jittiwutikarn, J., et al. (2008).

Humeniuk, R., \& Ali, R. (2006).

WHO ASSIST Working Group. (2002).

${ }^{13}$ Humeniuk, R., Ali, R., Babor, T. F., Farrell, M., Formigoni, M. L., Jittiwutikarn, J., et al. (2008).

${ }^{14}$ Henry-Edwards, S., Humeniuk, R., Ali, R., Poznyak, V., \& Monteiro, M. (2003).

${ }^{15}$ Henry-Edwards, S., Humeniuk, R., Ali, R., Poznyak, V., \& Monteiro, M. (2003).

${ }^{16}$ Henry-Edwards, S., Humeniuk, R., Ali, R., Poznyak, V., \& Monteiro, M. (2003).

University of Washington. (2011).

${ }^{17}$ Henry-Edwards, S., Humeniuk, R., Ali, R., Poznyak, V., \& Monteiro, M. (2003).

${ }^{18}$ University of Washington. (2011).

${ }^{19}$ University of Washington. (2011).

${ }^{20}$ Henry-Edwards, S., Humeniuk, R., Ali, R., Poznyak, V., \& Monteiro, M. (2003).

${ }^{21}$ Lanier, D., \& Ko, S. (2008).

WHO ASSIST Working Group. (2002).

${ }^{22}$ Humeniuk, R., Ali, R., Babor, T. F., Farrell, M., Formigoni, M. L., Jittiwutikarn, J., et al. (2008).

${ }^{23}$ Humeniuk, R., \& Ali, R. (2006).

Humeniuk, R., Ali, R., Babor, T. F., Farrell, M., Formigoni, M. L., Jittiwutikarn, J., et al. (2008).

${ }^{24}$ Humeniuk, R., Ali, R., Babor, T. F., Farrell, M., Formigoni, M. L., Jittiwutikarn, J., et al. (2008).

${ }^{25}$ Humeniuk, R., \& Ali, R. (2006).

North Carolina School of Science and Mathematics. (1999).

${ }^{26}$ Humeniuk, R., \& Ali, R. (2006).

${ }^{27}$ Lanier, D., \& Ko, S. (2008).

University of Washington. (2011).

${ }^{28}$ Kills Small, N. J., Simons, J. S., \& Stricherz, M. (2007).

${ }^{29}$ University of Washington. (2011).

${ }^{30}$ Kills Small, N. J., Simons, J. S., \& Stricherz, M. (2007).

${ }^{31}$ University of Washington. (2011).

${ }^{32}$ Winters, K. C., \& Zenilman, J. M. (1994).

${ }^{33}$ University of Washington. (2011).

${ }^{34}$ Peters, R. H., Greenbaum, P. E., Steinberg, M. L., Carter, C. R., Ortiz, M. M., Fry, B. C., et al. (2000).

${ }^{35}$ Kills Small, N. J., Simons, J. S., \& Stricherz, M. (2007). 
${ }^{36}$ Center for Substance Abuse Treatment. (2005b).

${ }^{37}$ Humeniuk, R., Ali, R., Babor, T. F., Farrell, M., Formigoni, M. L., Jittiwutikarn, J., et al. (2008). University of Washington. (2011).

${ }^{38}$ National Institute on Alcohol Abuse and Alcoholism. (2005c).

University of Washington. (2011).

${ }^{39}$ Allen, J. P., \& Wilson, V. B. (Eds.). (2003).

${ }^{40}$ Lanier, D., \& Ko, S. (2008).

University of Washington. (2011).

${ }^{41}$ Allen, J. P., \& Wilson, V. B. (Eds.). (2003).

University of Washington. (2011).

${ }^{42}$ Allen, J. P., \& Wilson, V. B. (Eds.). (2003).

${ }^{43}$ National Institute on Alcohol Abuse and Alcoholism. (2002).

${ }^{44}$ Center for Substance Abuse Treatment. (1997).

${ }^{45}$ Dyson, V., Appleby, L., Altman, E., Doot, M., Luchins, D. J., \& Delehant, M. (1998).

Hinkin, C. H., Castellon, S. A., Dickson-Fuhrman, E., Daum, G., Jaffe, J., \& Jarvik, L. (2001).

Lanier, D., \& Ko, S. (2008).

${ }^{46}$ Fiellin, D. A., Reid, M. C., \& O'Connor, P. G. (2000).

${ }^{47}$ Nilssen, O., Ries, R. K., Rivara, F. P., Gurney, J. G., \& Jurkovich, G. J. (1994).

${ }^{48}$ Cherpitel, C. J. (1999).

${ }^{49}$ Fiellin, D. A., Reid, M. C., \& O'Connor, P. G. (2000).

National Institute on Alcohol Abuse and Alcoholism. (2002).

${ }^{50}$ Larimer, M. E., \& Cronce, J. M. (2002).

${ }^{51}$ National Institute on Alcohol Abuse and Alcoholism. (2002).

${ }^{52}$ University of Washington. (2011).

${ }^{53}$ Lanier, D., \& Ko, S. (2008).

${ }^{54}$ University of Washington. (2011).

${ }^{55}$ University of Washington. (2011).

${ }^{56}$ University of Washington. (2011).

${ }^{57}$ University of Washington. (2011).

Knight, J. R., Shrier, L. A., Bravender, T. D., Farrell, M., Vander Bilt, J., \& Shaffer, H. J. (1999).

${ }^{58}$ Chang, G., Orav, E. J., Jones, J. A., Buynitsky, T., Gonzalez, S., \& Wilkins-Haug, L. (2011).

${ }^{59}$ Knight, J. R., Sherritt, L., Shrier, L. A., Harris, S. K., \& Chang, G. (2002).

${ }^{60}$ Cummins, L. H., Chan, K. K., Burns, K. M., Blume, A. W., Larimer, M., \& Marlatt, G. A. (2003).

Lanier, D., \& Ko, S. (2008).

Levy, S., Sherritt, L., Harris, S. K., Gates, E. C., Holder, D. W., Kulig, J. W., et al. (2004).

${ }^{61}$ Knight, J. R., Shrier, L. A., Bravender, T. D., Farrell, M., Vander Bilt, J., \& Shaffer, H. J. (1999).

${ }^{62}$ Cummins, L. H., Chan, K. K., Burns, K. M., Blume, A. W., Larimer, M., \& Marlatt, G. A. (2003).

${ }^{63}$ Allen, J. P., \& Wilson, V. B. (Eds.). (2003).

Sweet, R. I., \& Saules, K. K. (2003).

University of Washington. (2011).

${ }^{64}$ Allen, J. P., \& Wilson, V. B. (Eds.). (2003).

${ }^{65}$ Feldstein, S. W., \& Miller, W. R. (2007).

Lazowski, L. E., Miller, F. G., Boye, M. W., \& Miller, G. A. (1998).

Allen, J. P., \& Wilson, V. B. (Eds.). (2003).

University of Washington. (2011).

${ }^{66}$ Lazowski, L. E., Miller, F. G., Boye, M. W., \& Miller, G. A. (1998).

${ }^{67}$ Allen, J. P., \& Wilson, V. B. (Eds.). (2003).

University of Washington. (2011).

${ }^{68}$ Allen, J. P., \& Wilson, V. B. (Eds.). (2003).

University of Washington. (2011).

${ }^{69}$ Substance Abuse Subtle Screening Inventory Institute. (2012).

${ }^{70}$ Allen, J. P., \& Wilson, V. B. (Eds.). (2003).

University of Washington. (2011).

${ }^{71}$ Allen, J. P., \& Wilson, V. B. (Eds.). (2003).

University of Washington. (2011). 
${ }^{72}$ Feldstein, S. W., \& Miller, W. R. (2007).

${ }^{73}$ Lazowski, L. E., Miller, F. G., Boye, M. W., \& Miller, G. A. (1998).

${ }^{74}$ National Institute on Alcohol Abuse and Alcoholism. (2012c).

University of Washington. (2011).

${ }^{75}$ Lazowski, L. E., Miller, F. G., Boye, M. W., \& Miller, G. A. (1998).

${ }^{76}$ Feldstein, S. W., \& Miller, W. R. (2007).

${ }^{77}$ Sweet, R. I., \& Saules, K. K. (2003).

${ }^{78}$ Feldstein, S. W., \& Miller, W. R. (2007).

${ }^{79}$ Feldstein, S. W., \& Miller, W. R. (2007).

Clements, R. (2002).

Sweet, R. I., \& Saules, K. K. (2003).

${ }^{80}$ McLaney, M. A., \& Boca, F. D. (1994).

Rahdert, E. (1991).

${ }^{81}$ National Institute on Drug Abuse. (1991).

Winters, K. C. (2004).

${ }^{82}$ Center for Substance Abuse Treatment. (1999b).

${ }^{83}$ Latimer, W. W., Winters, K. C., \& Stinchfield, R. D. (1997).

${ }^{84}$ Center for Substance Abuse Treatment. (1999b).

${ }^{85}$ Center for Substance Abuse Treatment. (1999b).

${ }^{86}$ Latimer, W. W., Winters, K. C., \& Stinchfield, R. D. (1997).

McLaney, M. A., \& Boca, F. D. (1994).

${ }^{87}$ Latimer, W. W., Winters, K. C., \& Stinchfield, R. D. (1997).

${ }^{88}$ Tarter, R. E., \& Kirisci, L. (2001).

University of Washington. (2011).

${ }^{89}$ University of Washington. (2011).

${ }^{90}$ Tarter, R. E., \& Kirisci, L. (2001).

University of Washington. (2011).

${ }^{91}$ University of Washington. (2011).

${ }^{92}$ University of Washington. (2011).

${ }^{93}$ Allen, J. P., \& Wilson, V. B. (Eds.). (2003).

${ }^{94}$ University of Washington. (2011).

${ }^{95}$ University of Washington. (2011).

${ }^{96}$ Allen, J. P., \& Wilson, V. B. (Eds.). (2003).

${ }^{97}$ Tarter, R. W., Laird, S. B., Bukstein, O., \& Kaminer, Y. (1992).

${ }^{98}$ Tarter, R. E., \& Kirisci, L. (2001).

${ }^{99}$ Tarter, R. E., \& Kirisci, L. (1997).

${ }^{100}$ Makela, K. (2004).

${ }^{101}$ University of Washington. (2011).

${ }^{102}$ Allen, J. P., \& Wilson, V. B. (Eds.). (2003).

${ }^{103}$ Allen, J. P., \& Wilson, V. B. (Eds.). (2003).

${ }^{104}$ Allen, J. P., \& Wilson, V. B. (Eds.). (2003).

${ }^{105}$ Allen, J. P., \& Wilson, V. B. (Eds.). (2003).

University of Washington. (2011).

${ }^{106}$ Allen, J. P., \& Wilson, V. B. (Eds.). (2003).

${ }^{107}$ Kaminer, Y., Bukstein, O., \& Tarter, R. E. (1991).

University of Washington. (2011).

${ }^{108}$ University of Washington, Alcohol and Drug Abuse Institute. (1999).

${ }^{109}$ Peters, R. H., Greenbaum, P. E., Steinberg, M. L., Carter, C. R., Ortiz, M. M., Fry, B. C., et al. (2000).

${ }^{110}$ Makela, K. (2004).

${ }^{111}$ Rikoon, S. H., Cacciola, J. S., Carise, D., Alterman, A. I., \& McLellan, A. T. (2006).

${ }^{112}$ Makela, K. (2004).

${ }^{113}$ Humeniuk, R., Ali, R., Babor, T. F., Farrell, M., Formigoni, M. L., Jittiwutikarn, J., et al. (2008).

McPherson, T. L., \& Hersch, R. K. (2000).

${ }^{114}$ Brodey, B. B., McMullin, D., Kaminer, Y., Winters, K. C., Mosshart, E., Rosen, C. S., et al. (2008).

Brodey, B. B., Rosen, C. S., Winters, K. C., Brodey, I. S., Sheetz, B. M., Steinfeld, R. R., et al. (2005). 
Diaz, R., Castro-Fornieles, J., Serrano, L., Gonzalez, L., Calvo, R., Goti, J., et al. (2008).

115 Spitzer, R. L., Williams, J. B., Gibbon, M., \& First, M. B. (1992).

New York State Psychiatric Institute, Biometrics Research. (2007).

American Psychiatric Association. (1994).

${ }^{116}$ New York State Psychiatric Institute, Biometrics Research. (2007).

${ }^{117}$ University of Washington. (2011).

${ }^{118}$ New York State Psychiatric Institute, Biometrics Research. (2007).

${ }^{119}$ New York State Psychiatric Institute, Biometrics Research. (2012).

${ }^{120}$ Samet, S., Waxman, R., Hatzenbuehler, M., \& Hasin, D. S. (2007).

${ }^{121}$ Forman, R. F., Svikis, D., Montoya, I. D., \& Blaine, J. (2004).

${ }^{122}$ Ross, H. E., Swinson, R., Doumani, S., \& Larkin, E. J. (1995).

Kranzler, H. R., Kadden, R. M., Babor, T. F., Tennen, H., \& Rounsaville, B. J. (1996).

${ }^{123}$ Spitzer, R. L., Williams, J. B., Gibbon, M., \& First, M. B. (1992).

University of Washington. (2011).

${ }^{124}$ Spitzer, R. L., Williams, J. B., Gibbon, M., \& First, M. B. (1992).

${ }^{125}$ Martin, C. S., Pollock, N. K., Bukstein, O. G., \& Lynch, K. G. (2000).

${ }^{126}$ Ross, H. E., Swinson, R., Doumani, S., \& Larkin, E. J. (1995).

Kranzler, H. R., Kadden, R. M., Babor, T. F., Tennen, H., \& Rounsaville, B. J. (1996).

${ }^{127}$ Samet, S., Waxman, R., Hatzenbuehler, M., \& Hasin, D. S. (2007).

Forman, R. F., Svikis, D., Montoya, I. D., \& Blaine, J. (2004).

University of Washington. (2011).

${ }^{128}$ University of Washington. (2011).

${ }^{129}$ Forman, R. F., Svikis, D., Montoya, I. D., \& Blaine, J. (2004).

${ }^{130}$ Allen, J. P., \& Wilson, V. B. (Eds.). (2003).

${ }^{131}$ Samet, S., Waxman, R., Hatzenbuehler, M., \& Hasin, D. S. (2007).

${ }^{132}$ Forman, R. F., Svikis, D., Montoya, I. D., \& Blaine, J. (2004).

133 University of Washington. (2011).

134 Andrews, G., \& Peters, L. (1998).

Cottler, L. B., Robins, L. N., \& Helzer, J. E. (1989).

Haro, J. M., Arbabzadeh-Bouchez, S., Brugha, T. S., de, G. G., Guyer, M. E., Jin, R., et al. (2006).

Horton, J., Compton, W., \& Cottler, L. B. (2000).

Wittchen, H. U. (1994).

${ }^{135}$ University of Washington. (2011).

${ }^{136}$ University of Washington. (2011).

${ }^{137}$ Piper, M. E., McCarthy, D. E., \& Baker, T. B. (2006).

138 Burling, A. S., \& Burling, T. A. (2003).

${ }^{139}$ University of Washington. (2011).

${ }^{140}$ University of Washington. (2011).

${ }^{141}$ University of Washington. (2011).

${ }^{142}$ Moolchan, E. T., Radzius, A., Epstein, D. H., Uhl, G., Gorelick, D. A., Cadet, J. L., et al. (2002).

${ }^{143}$ Burling, A. S., \& Burling, T. A. (2003).

Courvoisier, D., \& Etter, J.-F. (2008).

${ }^{144}$ Etter, J.-F., Duc, T. V., \& Perneger, T. V. (1999).

145 Burling, A. S., \& Burling, T. A. (2003).

146 Piper, M. E., McCarthy, D. E., \& Baker, T. B. (2006).

Hale, J. (2011).

${ }^{147}$ Etter, J.-F., Duc, T. V., \& Perneger, T. V. (1999).

University of Nebraska-Lincoln. (2012).

Pomerleau, C. S., Carton, S. M., Lutzke, M. L., Flessland, K. A., \& Pomerleau, O. F. (1994).

${ }_{148}$ de Leon, J., Diaz, F. J., Becona, E., Gurpegui, M., Jurado, D., \& Gonzalez-Pinto, A. (2003).

149 Colby, S. M., Tiffany, S. T., Shiffman, S., \& Niaura, R. S. (2000).

${ }^{150}$ Colby, S. M., Tiffany, S. T., Shiffman, S., \& Niaura, R. S. (2000).

U.S. Department of Health and Human Services. (2010a).

${ }^{151}$ Colby, S. M., Tiffany, S. T., Shiffman, S., \& Niaura, R. S. (2000).

152 Nichter, M., Nichter, M., Thompson, P. J., Shiffman, S., \& Moscicki, A.-B. (2002). 
${ }^{153}$ Clark, D. B., Wood, D. S., Martin, C. S., Cornelius, J. R., Lynch, K. G., \& Shiffman, S. (2005).

Shiffman, S., \& Sayette, M. A. (2005).

Shiffman, S., Waters, A. J., \& Hickcox, M. (2004).

${ }^{154}$ Costello, D., Dierker, L. C., Sledjeski, E. M., Flaherty, B., Flay, B., Shiffman, S., et al. (2007).

Clark, D. B., Wood, D. S., Martin, C. S., Cornelius, J. R., Lynch, K. G., \& Shiffman, S. (2005).

Okuyemia, K. S., Pulversc, K. M., Sanderson Cox, L., Thomas, J. L., Kaur, H., Mayod, M. S., et al. (2007).

Rubinstein, M. L., Thompson, P. J., Benowitz, N. L., Shiffman, S., \& Moscicki, A.-B. (2007).

Shiffman, S., \& Sayette, M. A. (2005).

Shiffman, S., Waters, A. J., \& Hickcox, M. (2004).

Strong, D. R., Kahler, C. W., Colby, S. M., Griesler, P. M., \& Kandel, D. B. (2009).

${ }^{155}$ Saunders, J. B., Aasland, O. G., Babor, T. F., de la Fuente, J. R., \& Grant, M. (1993).

University of Washington. (2011).

${ }^{156}$ Center for Substance Abuse Treatment. (1995a).

${ }^{157}$ Allen, J. P., \& Wilson, V. B. (Eds.). (2003).

${ }^{158}$ Saunders, J. B., Aasland, O. G., Babor, T. F., de la Fuente, J. R., \& Grant, M. (1993).

159 Saunders, J. B., Aasland, O. G., Babor, T. F., de la Fuente, J. R., \& Grant, M. (1993).

${ }^{160}$ Center for Substance Abuse Treatment. (1995a).

University of Washington. (2011).

${ }^{161}$ Saunders, J. B., Aasland, O. G., Babor, T. F., de la Fuente, J. R., \& Grant, M. (1993).

${ }^{162}$ Saunders, J. B., Aasland, O. G., Babor, T. F., de la Fuente, J. R., \& Grant, M. (1993).

Center for Substance Abuse Treatment. (1995a).

${ }^{163}$ University of Washington. (2011).

${ }^{164}$ National Institute on Alcohol Abuse and Alcoholism. (2002).

165 Allen, J. P., \& Wilson, V. B. (Eds.). (2003).

${ }^{166}$ Daeppen, J. B., Yersin, B., Landry, U., Pecoud, A., \& Decrey, H. (2000).

Maisto, S. A., Conigliaro, J., McNeil, M., Kraemer, K., \& Kelley, M. E. (2000).

Reinert, D. F., \& Allen, J. P. (2002).

Saunders, J. B., Aasland, O. G., Babor, T. F., de la Fuente, J. R., \& Grant, M. (1993).

Volk, R. J., Steinbauer, J. R., Cantor, S. B., \& Holzer, C. E., III. (1997).

${ }^{167}$ Saunders, J. B., Aasland, O. G., Babor, T. F., de la Fuente, J. R., \& Grant, M. (1993).

${ }^{168}$ Fiellin, D. A., Reid, M. C., \& O'Connor, P. G. (2000).

Saunders, J. B., Aasland, O. G., Babor, T. F., de la Fuente, J. R., \& Grant, M. (1993).

${ }^{169}$ Reinert, D. F., \& Allen, J. P. (2002).

University of Washington. (2011).

${ }^{170}$ U.S. Department of Agriculture and U.S. Department of Health and Human Services. (2010).

${ }^{171}$ Reinert, D. F., \& Allen, J. P. (2002).

${ }^{172}$ National Institute on Alcohol Abuse and Alcoholism. (2005c).

${ }^{173}$ National Institute on Alcohol Abuse and Alcoholism. (2005c).

University of Washington. (2011).

${ }^{174}$ National Institute on Alcohol Abuse and Alcoholism. (2005c).

${ }^{175}$ University of Washington. (2011).

${ }^{176}$ University of Washington. (2011).

177 Center for Substance Abuse Treatment. (1995a).

University of Washington. (2011).

Sokol, R. J., Martier, S. S., \& Ager, J. W. (1989).

${ }^{178}$ University of Washington. (2011).

${ }^{179}$ Center for Substance Abuse Treatment. (1995a).

${ }^{180}$ National Institute on Alcohol Abuse and Alcoholism. (2005c).

${ }^{181}$ University of Washington. (2011).

182 Allen, J. P., \& Wilson, V. B. (Eds.). (2003).

183 Allen, J. P., \& Wilson, V. B. (Eds.). (2003).

${ }^{184}$ University of Washington. (2011).

U.S. Department of Agriculture and U.S. Department of Health and Human Services. (2010).

${ }^{185}$ University of Washington. (2011).

${ }^{186}$ Allen, J. P., \& Wilson, V. B. (Eds.). (2003). 
${ }^{187}$ Center for Substance Abuse Treatment. (1995a).

${ }^{188}$ Chang, G., Wilkins-Haug, L., Berman, S., \& Goetz, M. A. (1999b).

${ }^{189}$ Cherpitel, C. J. (1999).

190 Allen, J. P., \& Wilson, V. B. (Eds.). (2003).

University of Washington. (2011).

${ }^{191}$ Allen, J. P., \& Wilson, V. B. (Eds.). (2003).

192 Allen, J. P., \& Wilson, V. B. (Eds.). (2003).

193 Allen, J. P., \& Wilson, V. B. (Eds.). (2003).

194 Skinner, H. A. (1993).

${ }^{195}$ Ross, H. E., Gavin, D. R., \& Skinner, H. A. (1990).

${ }^{196}$ Chantarujikapong, S. I., Smith, E. M., \& Fox, L. W. (1997).

${ }^{197}$ Peters, R. H., Greenbaum, P. E., Steinberg, M. L., Carter, C. R., Ortiz, M. M., Fry, B. C., et al. (2000).

198 Saunders, J. B., Aasland, O. G., Babor, T. F., de la Fuente, J. R., \& Grant, M. (1993).

Allen, J. P., \& Wilson, V. B. (Eds.). (2003).

University of Washington. (2011).

199 Allen, J. P., \& Wilson, V. B. (Eds.). (2003).

Selzer, M. L. (1971).

${ }^{200}$ Allen, J. P., \& Wilson, V. B. (Eds.). (2003).

${ }^{201}$ University of Washington. (2011).

${ }^{202}$ Center for Substance Abuse Treatment. (1994).

203 Allen, J. P., \& Wilson, V. B. (Eds.). (2003).

${ }^{204}$ Center for Substance Abuse Treatment. (1994).

${ }^{205}$ Allen, J. P., \& Wilson, V. B. (Eds.). (2003).

${ }^{206}$ Center for Substance Abuse Treatment. (1994).

${ }^{207}$ University of Washington. (2011).

${ }^{208}$ National Institute on Alcohol Abuse and Alcoholism. (2005c).

Svanun, S., \& McGrew, J. (1995).

209 Teitelbaum, L., \& Mullen, B. (2000).

${ }^{210}$ Conley, T. B. (2001).

Hirata, E. S., Almeida, O. P., Funari, R. R., \& Klein, E. L. (2001).

${ }^{211}$ Cleary, P. D., Miller, M., Bush, B. T., Warburg, M. M., DelBanco, T. L., \& Aronson, M. D. (1988).

212 Rumpf, H. J., Hapke, U., Hill, A., \& John, U. (1997).

${ }^{213}$ Peters, R. H., Greenbaum, P. E., Steinberg, M. L., Carter, C. R., Ortiz, M. M., Fry, B. C., et al. (2000).

${ }^{214}$ Skinner, H. A. (1982).

University of Washington. (2011).

${ }^{215}$ Yudko, E., Lozhkina, O., \& Fouts, A. (2007).

University of Washington. (2011).

${ }^{216}$ University of Washington. (2011).

${ }^{217}$ Yudko, E., Lozhkina, O., \& Fouts, A. (2007).

University of Washington. (2011).

${ }^{218}$ University of Washington. (2011).

${ }^{219}$ Lanier, D., \& Ko, S. (2008).

Yudko, E., Lozhkina, O., \& Fouts, A. (2007).

Martino, S., Grilo, C. M., \& Fehon, D. C. (2000).

McCann, B. S., Simpson, T. L., Ries, R., \& Roy-Byrne, P. (2000).

Skinner, H. A. (1982).

Staley, D., \& El-Guebaly, N. (1990).

Cocco, K. M., \& Carey, K. B. (1998).

Carey, K. B., Carey, M. P., \& Chandra, P. S. (2003).

Saltstone, R., Halliwell, S., \& Hayslip, M. A. (1994).

El-Bassel, N., Schilling, R. F., Schinke, S., Orlando, M., \& Sun, W. (1997).

${ }^{220}$ Lanier, D., \& Ko, S. (2008).

Peters, R. H., Greenbaum, P. E., Steinberg, M. L., Carter, C. R., Ortiz, M. M., Fry, B. C., et al. (2000).

Yudko, E., Lozhkina, O., \& Fouts, A. (2007).

Staley, D., \& El-Guebaly, N. (1990). 
University of Washington. (2011).

${ }^{221}$ Yudko, E., Lozhkina, O., \& Fouts, A. (2007).

Lanier, D., \& Ko, S. (2008).

Staley, D., \& El-Guebaly, N. (1990).

${ }^{222}$ University of Washington. (2011).

${ }^{223}$ Dyson, V., Appleby, L., Altman, E., Doot, M., Luchins, D. J., \& Delehant, M. (1998).

${ }^{224}$ Cocco, K. M., \& Carey, K. B. (1998).

${ }^{225}$ Peters, R. H., Greenbaum, P. E., Steinberg, M. L., Carter, C. R., Ortiz, M. M., Fry, B. C., et al. (2000).

${ }^{226}$ Yudko, E., Lozhkina, O., \& Fouts, A. (2007).

${ }^{227}$ Yudko, E., Lozhkina, O., \& Fouts, A. (2007).

Maisto, S. A., Carey, M. P., Carey, K. B., Gordon, C. M., \& Gleason, J. R. (2000).

${ }^{228}$ Martino, S., Grilo, C. M., \& Fehon, D. C. (2000)..

${ }^{229}$ University of Washington. (2011). 
$-428-$ 


\section{Bibliography}

111th Congress. (2010). Compilation of patient protection and affordable care act. [Online]. Retrieved February 2, 2012 from http://housedocs.house.gov..

21 C.F.R. $§ 1301.19-1301.32$ (2010).

$28 \mathrm{~Pa}$. Code $\S 704.6$ (2012).

29 U.S.C. $\S 18$ (2011).

38 C.F.R. $\S 17.81$ (2010).

42 C.F.R. $§ 8$ (2002).

42 C.F.R. § 8.12 (2002).

42 C.F.R. $\S 440.10$ (2012).

42 C.F.R. $\S 440.130$ (2012).

42 C.F.R. $\S 440.90$ (2012).

42 C.F.R. § 482 (2012).

42 C.F.R. $\S 485$ (2012).

42 U.S.C.S. § 300x-22 (2012).

42 U.S.C.S. § 300x-28 (2012).

908 Ky. Admin. Regs.1:370 (2011).

Aalto, M., Pekuri, P., \& Seppa, K. (2003). Obstacles to carrying out brief intervention for heavy drinkers in primary health care: A focus group study. Drug \& Alcohol Review, 22(2), 169-173.

Abbott, P. J., Quinn, D., \& Knox, L. (1995). Ambulatory medical detoxification for alcohol. American Journal of Drug \& Alcohol Abuse, 21(4), 549-563.

Abbott, P. J., Weller, S. B., Delaney, H. D., \& Moore, B. A. (1998). Community reinforcement approach in the treatment of opiate addicts. American Journal of Drug \& Alcohol Abuse, 24(1), 17-30.

Abraham, A. J., \& Roman, P. M. (2010). Early adoption of injectable naltrexone for alcohol-use disorders: Findings in the private-treatment sector. Journal of Studies on Alcohol \& Drugs, 71(3), 460-466.

Abraham, A. J., Knudsen, H. K., \& Roman, P. M. (2011). A longitudinal examination of alcohol pharmacotherapy adoption in substance use disorder treatment programs: Patterns of sustainability and discontinuation. Journal of Studies on Alcohol \& Drugs, 72(4), 669-677.

Abt Associates. (2006). Strengthening professional identity. Challenges of the addictions treatment workforce. A framework for discussion. [Online]. Retrieved January 27, 2012 from http://www.atforum.com.

Accreditation Association for Ambulatory Health Care. (2012). What is accreditation? [Online]. Retrieved March 22, 2012 from http://www.aaahc.org.

Accreditation Commission for Acupuncture and Oriental Medicine. (2009). Accreditation manual. Structure, scope, process, eligibility requirements and standards. [Online]. Retrieved February 14, 2012 from http://www.acaom.org.

Accreditation Council for Graduate Medical Education. (2012a). ACGME home page. [Online]. Retrieved May 31, 2012 from http://www.acgme.org. 
Accreditation Council for Graduate Medical Education. (2012b). List of ACGME accredited programs and sponsoring institutions. [Online]. Retrieved March 14, 2012 from http://www.acgme.org.

Accreditation Council for Graduate Medical Education. (2012c). Number of accredited programs for the current academic year (2011-2012). [Online]. Retrieved March 13, 2012 from http://www.acgme.org.

Accreditation Review Commission on Education for the Physician Assistant. (2008). Accreditation standards for physician assistant education. [Online]. Retrieved July 15, 2010 from http://arc-pa.org.

Acufinder.com. (2012a). Alabama laws and details. [Online]. Retrieved February 13, 2012 from https://www.acufinder.com.

Acufinder.com. (2012b). Kansas laws and details. [Online]. Retrieved February 13, 2012 from https://www.acufinder.com.

Acufinder.com. (2012c). North Dakota laws and details. [Online]. Retrieved February 12, 2012 from https://www.acufinder.com.

Acufinder.com. (2012d). Oklahoma laws and details. [Online]. Retrieved February 13, 2012 from https://www.acufinder.com.

Acufinder.com. (2012e). Wyoming laws and details. [Online]. Retrieved February 13, 2012 from https://www.acufinder.com.

Adams, E. K., Miller, V. P., Ernst, C., Nishimura, B. K., Melvin, C., \& Merritt, R. (2002). Neonatal health care costs related to smoking during pregnancy. Health Economics, 11(3), 193-206.

Adams, J. B., Heath, A. J., Young, S. E., Hewitt, J. K., Corley, R. P., \& Stallings, M. C. (2003). Relationships between personality and preferred substance and motivations for use among adolescent substance abusers. American Journal of Drug \& Alcohol Abuse, 29(3), 691-712.

Addiction Technology Transfer Center Network. (2009a). ATTC website: Who we are. [Online]. Retrieved December 22, 2009 from http://www.attcnetwork.org.

Addiction Technology Transfer Center Network. (2009b). Understanding America's substance use disorders treatment workforce: A summary report. [Online]. Retrieved February 27, 2012 from http://www.attcnetwork.org.

Addiction Technology Transfer Center Network. (2010). SBIRT. Part 3. Taking it to the field. [Online]. Retrieved December 16, 2011 from http://www.nattc.org.

Addiction Technology Transfer Center Network. (2011). Screening and treatment for adolescents with substance use disorders: Part 2. [Online]. Retrieved December 20, 2011 from http://www.attcnetwork.org.

Addiction Technology Transfer Center Network. (2012). Certification information. [Online]. Retrieved March 22, 2012 from http://www.attcnetwork.org.

Addolorato, G., Caputo, F., Capristo, E., Domenicali, M., Bernardi, M., Janiri, L., et al. (2002). Baclofen efficacy in reducing alcohol craving and intake: A preliminary double-blind randomized controlled study. Alcohol \& Alcoholism, 37(5), 504-508.

Addolorato, G., Leggio, L., Ferrulli, A., Cardone, S., Bedogni, G., Caputo, F., et al. (2011). Doseresponse effect of baclofen in reducing daily alcohol intake in alcohol dependence: Secondary analysis of a randomized, double-blind, placebo-controlled trial. Alcohol \& Alcoholism, 46(3), 312317. 
Addolorato, G., Leggio, L., Ferrulli, A., Cardone, S., Vonghia, L., Mirijello, A., et al. (2007). Effectiveness and safety of baclofen for maintenance of alcohol abstinence in alcohol-dependent patients with liver cirrhosis: Randomized, double-blind controlled study. Lancet, 370(9603), 19151922.

Adelmann, P. K. (2003). Mental and substance use disorders among Medicaid recipients: Prevalence estimates from two national surveys. Administration \& Policy in Mental Health, 31(2), 111-129.

Advocates for Human Potential. (2009). An environmental scan of the impact of parity: Practical implications of the Paul Wellstone and Pete Domenici Mental Health Parity and Addiction Equity Act of 2008 (AHP Behavioral Parity series White Paper \#1). Sudbury, MA: Author.

Advocates for Human Potential. (2010). Special report: MHPAEA regulations: Operational analysis of the mental health parity and addiction equity act interim final rule. Sudbury, MA: Author.

Agency for Healthcare Research and Quality. (2003). Program note 6: Compendium of AHRQ research related to mental health (AHRQ Pub. No. 03-0001). [Online]. Retrieved April 18, 2012 from http://www.ahrq.gov.

Agency for Healthcare Research and Quality. (2010). Guideline summary NGC-4931: Detoxification and substance abuse treatment: An overview of the psychosocial and biomedical issues during detoxification. [Online]. Retrieved December 13, 2011 from http://www.ngc.gov.

Agency for Healthcare Research and Quality. (2012a). About national quality measures clearinghouse (NQMC). [Online]. Retrieved April 18, 2012 from http://www.qualitymeasures.ahrq.gov.

Agency for Healthcare Research and Quality. (2012b). Guideline index. [Online]. Retrieved April 18, 2012 from http://www.guideline.gov.

Agency for Healthcare Research and Quality. (2012c). Guidelines by topic. [Online]. Retrieved June 5, 2012 from http://guideline.gov.

Agency for Healthcare Research and Quality. (2012d). Medical expenditure panel survey. Table 3: Total expenses and percent distribution for selected conditions by type of service: United States, 2009. [Online]. Retrieved April 3, 2012 from http://meps.ahrq.gov.

Agency Medical Directors Group. (2012). Interagency guideline on opioid dosing for chronic non-cancer pain: An educational aid to improve care and safety with opioid therapy: 2010 update. [Online]. Retrieved May 31, 2012 from http://www.agencymeddirectors.wa.gov.

Agrawal, A., \& Lynskey, M. T. (2006). The genetic epidemiology of cannabis use, abuse and dependence. Addiction, 101(6), 801-812.

Agrawal, A., \& Lynskey, M. T. (2008). Are there genetic influences on addiction: Evidence from family, adoption and twin studies. Addiction, 103(7), 1069-1081.

Agrawal, A., Sartor, C. E., Lynskey, M. T., Grant, J. D., Pergadia, M. L., Grucza, R., et al. (2009). Evidence for an interaction between age at first drink and genetic influences on DSM-IV alcohol dependence symptoms. Alcoholism: Clinical \& Experimental Research, 33(12), 2047-2056.

Akpanudo, S. M., Price, J. H., Jordan, T., Khuder, S., \& Price, J. A. (2009). Clinical psychologists and smoking cessation: Treatment practices and perceptions. Journal of Community Health, 34(6), 461471.

Ala. Code 580 § 2-9.03 (2012).

Alaska Admin. Code 7, § 12.110 (January 2012). 
Alaska Division of Corporations, Business, and Professional Licensing, \& Board of Social Work Examiners. (2012). Continuing education courses. [Online]. Retrieved March 19, 2012 from http://www.dced.state.ak.us.

Alaska Legislature. (2006). Substance abuse hospitals: 7 AAC 12.220. [Online]. Retrieved April 2, 2012 from http://www.touchngo.com.

Alberti v. Sheriff of Harris County, 406 F. Supp. 649 (S.D. Tex. 1975).

Alcoholics Anonymous World Services. (1972). A brief guide to Alcoholics Anonymous. New York: Author.

Alcoholics Anonymous World Services. (2007). A.A. at a glance. [Online]. Retrieved January 30, 2009 from http://www.aa.org.

Alexander, J. A., Nahra, T. A., Lemak, C. H., Pollack, H., \& Campbell, C. I. (2008). Tailored treatment in the outpatient substance abuse treatment sector: 1995-2005. Journal of Substance Abuse Treatment, 34(3), 282-292.

Allan, C., Smith, I., \& Mellin, M. (2000). Detoxification from alcohol: A comparison of home detoxification and hospital-based day patient care. Alcohol \& Alcoholism, 35(1), 66-69.

Allen, J. M. (2010). Economic/societal burden of metastatic breast cancer: A US perspective. American Journal of Managed Care, 16(9), 697-704.

Allen, J. P., \& Litten, R. Z. (2001). The role of laboratory tests in alcoholism treatment. Journal of Substance Abuse Treatment, 20(1), 81-85.

Allen, J. P., \& Wilson, V. B. (Eds.). (2003). Assessing alcohol problems: A guide for clinicians and researchers (NIH Publication No. 03-3745) (2nd ed.). Bethesda, MD: U.S. Department of Health and Human Services, National Institutes of Health, National Institute on Alcohol Abuse and Alcoholism.

Allen, K. (1994). Development of an instrument to identify barriers to treatment for addicted women from their perspective. International Journal of Addictions, 29(4), 429-444.

Alliance for Health Reform. (2006). Pay-for-performance: A promising start. Washington, DC: Author.

Allsop, S. J., \& Stevens, C. (2009). Evidence-based practice or imperfect seduction? Developing capacity to respond effectively to drug-related problems. Drug \& Alcohol Review, 28(5), 541-549.

Alpert, H. R., Connolly, G. N., \& Biener, L. (2012). A prospective cohort study challenging the effectiveness of population-based medical intervention for smoking cessation. Tobacco Control, tobaccocontrol-2011-050129 [pii]; doi:10.1136/tobaccocontrol-2011-050129.

Amass, L., Kamien, J. B., \& Mikulich, S. K. (2000). Efficacy of daily and alternate-day dosing regimens with the combination buprenorphine-naloxone tablet. Drug \& Alcohol Dependence, 58(1-2), 143-152.

Amato, L., Davoli, M., Ferri, M., Gowing, L., \& Perucci, C. A. (2004). Effectiveness of interventions on opiate withdrawal treatment: An overview of systematic reviews. Drug \& Alcohol Dependence, 73(3), 219-226.

Amato, L., Davoli, M., Perucci, C. A., Ferri, M., Faggiano, F., \& Mattick, R. P. (2005). An overview of systematic reviews of the effectiveness of opiate maintenance therapies: Available evidence to inform clinical practice and research. Journal of Substance Abuse, 28(4), 321-329.

Amato, L., Minozzi, S., Davoli, M., Vecchi, S., Ferri, M. M., \& Mayet, S. (2009). Psychosocial combined with agonist maintenance treatments versus agonist maintenance treatments alone for treatment of opioid dependence. Cochrane Database of Systematic Reviews, (4), CD004147. 
Amato, L., Minozzi, S., Vecchi, S., \& Davoli, M. (2010). Benzodiazepines for alcohol withdrawal. Cochrane Database of Systematic Reviews, (3), CD005063.

American Academy of Addiction Psychiatry. (2012). What is the addiction psychiatry subspecialty? [Online]. Retrieved March 6, 2012 from http://www2.aaap.org.

American Academy of Child and Adolescent Psychiatry. (2009). Oppositional defiant disorder: A guide for families. [Online]. Retrieved December 5, 2011 from http://www.aacap.org.

American Academy of Family Physicians. (2011a). Brief alcohol screening and intervention in family medicine. Rationale for screening and brief intervention for alcohol problems in primary care. [Online]. Retrieved December 13, 2011 from http://www.aafp.org.

American Academy of Family Physicians. (2011b). Tobacco use. [Online]. Retrieved December 13, 2011 from http://www.aafp.org.

American Academy of Pediatrics, Committee on Substance Abuse. (2001). Alcohol use and abuse: A pediatric concern. Pediatrics, 108(1), 185-189.

American Academy of Pediatrics, Committee on Substance Abuse and Committee on Children With Disabilities. (2000). Fetal alcohol syndrome and alcohol-related neurodevelopmental disorders. Pediatrics, 106(2 Pt 1), 358-361.

American Academy of Pediatrics. (2009). Technical report: Tobacco as a substance of abuse. Pediatrics, 124(5), e1045-e1053.

American Academy of Physician Assistants. (2010a). Prescribing. [Online]. Retrieved February 2, 2010 from http://www.aapa.org.

American Academy of Physician Assistants. (2010b). Summary of state laws for physician assistants: Abridged version. [Online]. Retrieved July 15, 2010 from http://www.aapa.org.

American Academy of Physician Assistants. (2012a). Becoming a PA. [Online]. Retrieved March 21, 2012 from http://www.aapa.org.

American Academy of Physician Assistants. (2012b). The pa profession. [Online]. Retrieved March 21, 2012 from http://www.aapa.org.

American Association for Marriage and Family Therapy. (2012). Qualifications and FAQs. [Online]. Retrieved February 15, 2012 from http://www.aamft.org.

American Association of Colleges of Nursing. (2008). The essentials of baccalaureate education for professional nursing practice. [Online]. Retrieved May 31, 2012 from http://www.aacn.nche.edu.

American Association of Colleges of Nursing. (2009). Standards for accreditation of baccalaureate and graduate degree nursing programs. [Online]. Retrieved May 31, 2012 from http://www.aacn.nche.edu.

American Association of Colleges of Nursing. (2012). Commission on Collegiate Nursing Education (CCNE) accreditation. [Online]. Retrieved May 31, 2012 from http://www.aacn.nche.edu.

American Association of Colleges of Osteopathic Medicine. (2012). Board examinations and licensure. [Online]. Retrieved May 14, 2012 from http://www.aacom.org.

American Board of Addiction Medicine Foundation. (2009). The need for addiction medicine physicians and for addiction medicine residency training programs: A report of The American Board of Addiction Medicine Foundation. Chevy Chase, MD: Author.

American Board of Addiction Medicine. (2010). About American Board of Addiction Medicine. [Online]. Retrieved January 6, 2010 from http://www.abam.net. 
American Board of Addiction Medicine. (2012a). 2012 Certification examination application. [Online]. Retrieved March 7, 2012 from http://www.csam.org.

American Board of Addiction Medicine. (2012b). ABAM maintenance of certification. [Online]. Retrieved May 31, 2011 from http://www.abam.net.

American Board of Addiction Medicine. (2012c). About American Board of Addiction Medicine. [Online]. Retrieved March 6, 2012 from http://www.abam.net.

American Board of Emergency Medicine. (2006). Pediatric emergency medicine overview. [Online]. Retrieved March 23, 2012 from http://www.abem.org.

American Board of Emergency Medicine. (2009). 2009 model of the clinical practice of emergency medicine. [Online]. Retrieved June 16, 2009 from https://www.abem.org.

American Board of Emergency Medicine. (2011). Qualifying examination description and content specifications. [Online]. Retrieved March 23, 2012 from http://www.abem.org.

American Board of Family Medicine. (2007). Sports medicine examination content. [Online]. Retrieved June 10, 2009 from https://www.theabfm.org,

American Board of Family Medicine. (2009). Certification/recertification examination modules. [Online]. Retrieved June 10, 2009 from https://www.theabfm.org.

American Board of Family Medicine. (2012a). Adolescent medicine. [Online]. Retrieved March 20, 2012 from https://www.theabfm.org.

American Board of Family Medicine. (2012b). Geriatric medicine. [Online]. Retrieved March 20, 2012 from https://www.theabfm.org.

American Board of Family Medicine. (2012c). Sports medicine. [Online]. Retrieved March 20, 2012 from https://www.theabfm.org.

American Board of Internal Medicine. (2009a). Geriatric medicine certification examination blueprint. [Online]. Retrieved June 10, 2009 from http://www.abim.org.

American Board of Internal Medicine. (2009b). Infectious disease certification examination blueprint. [Online]. Retrieved June 11, 9 A.D. from http://www.abim.org.

American Board of Internal Medicine. (2009c). Internal medicine certification examination blueprint. [Online]. Retrieved June 10, 2009 from http://www.abim.org.

American Board of Medical Specialties. (2009). Specialties and subspecialties. [Online]. Retrieved June 10, 2009 from http://www.abms.org.

American Board of Medical Specialties. (2011). Certificate statistics. [Online]. Retrieved May 15, 2012 from https://shopping.abms.org.

American Board of Medical Specialties. (2012a). ABMS maintenance of certification. MOC is the path. Better care is the destination. [Online]. Retrieved January 17, 2012 from http://www.abms.org.

American Board of Medical Specialties. (2012b). MOC competencies and criteria. [Online]. Retrieved May 31, 2012 from http://www.abms.org.

American Board of Medical Specialties. (2012c). What board certification means. [Online]. Retrieved January 17, 2012 from http://www.abms.org.

American Board of Obstetrics and Gynecology. (2007). Guide to learning in maternal-fetal medicine. [Online]. Retrieved June 16, 2009 from http://www.abog.org. 
American Board of Obstetrics and Gynecology. (2008). Bulletin for 2009. [Online]. Retrieved June 16, 2009 from http://www.abog.org.

American Board of Pediatrics. (2009a). Content outline: General pediatrics certification examination. [Online]. Retrieved June 10, 2009 from https://www.abp.org.

American Board of Pediatrics. (2009b). Content outline: Pediatric emergency medicine subspecialty intraining, certification, and maintenance of certification examinations. [Online]. Retrieved June 12, 2009 from https://www.abp.org.

American Board of Pediatrics. (2010a). Content outline: Adolescent medicine subspecialty in-training, certification, and maintenance of certification examinations. [Online]. Retrieved March 21, 2012 from https://www.abp.org.

American Board of Pediatrics. (2010b). Content outline: Child abuse pediatrics subspecialty in-training, certification, and maintenance of certification examinations. [Online]. Retrieved March 21, 2012 from https://www.abp.org.

American Board of Pediatrics. (2010c). Content outline: Neonatal-perinatal medicine subspecialty intraining, certification, and maintenance of certification examinations. [Online]. Retrieved March 21, 2012 from https://www.abp.org.

American Board of Pediatrics. (2011). Content outline: Developmental-behavioral pediatrics subspecialty in-training, certification, and maintenance of certification examinations. [Online]. Retrieved March 21, 2012 from https://www.abp.org.

American Board of Professional Psychology. (2012). The importance of board certification. [Online]. Retrieved April 11, 2012 from http://www.abpp.org.

American Board of Psychiatry and Neurology. (2009a). Subspecialty certification examination in forensic psychiatry: 2009 content outline. [Online]. Retrieved June 15, 2009 from http://www.abpn.com.

American Board of Psychiatry and Neurology. (2009b). Subspecialty certification examination in psychosomatic medicine: 2009 content outline. [Online]. Retrieved June 15, 2009 from http://www.abpn.com.

American Board of Psychiatry and Neurology. (2010a). Part I examination in psychiatry: 2010 content outline. [Online]. Retrieved March 21, 2012 from http://www.abpn.com.

American Board of Psychiatry and Neurology. (2010b). Subspecialty certification examination in geriatric psychiatry: 2010 content outline. [Online]. Retrieved April 1, 2010 from http://www.abpn.com.

American Board of Psychiatry and Neurology. (2010c). Written certification examination in child and adolescent psychiatry (Part 1): 2010 content outline. [Online]. Retrieved April 1, 2010 from http://www.abpn.com.

American Board of Psychiatry and Neurology. (2012). Initial certification in addiction psychiatry. [Online]. Retrieved February 24, 2012 from http://www.abpn.com.

American Cancer Society. (2009). Cancer facts and figures 2009. Atlanta, GA: Author.

American Civil Liberties Union, Drug Policy Alliance. (2006). Making sense of student drug testing: Why educators are saying no. New York: Authors.

American Civil Liberties Union. (1999). Drug testing: A bad investment. New York: Author.

American College of Emergency Physicians. (2011). Alcohol screening in the emergency department.

[Online]. Retrieved November 11, 2011 from http://www.acep.org. 
American College of Obstetricians and Gynecologists, Committee on Health Care for Underserved Women. (2011). Committee opinion no. 496: At-risk drinking and alcohol dependence: Obstetric and gynecologic implications. Obstetrics \& Gynecology, $118(2$ Pt 1), 383-388.

American College of Obstetricians and Gynecologists. (2008). Committee opinion No. 422 [replaces No. 249, May 2004] At-risk drinking and illicit drug use: Ethical issues in obstetric and gynecologic practice. [Online]. Retrieved June 1, 2012 from http://www.acog.org.

American College of Physicians. (2011). Racial and ethnic disparities in health care, updated 2010: A position paper. Philadelphia, PA: Health and Public Policy Committee of the American College of Physicians.

American College of Surgeons. (1993). Resources for the optimal care of the injured patient: 1993. Chicago, IL: Author.

American College of Surgeons. (2007). Alcohol screening and brief intervention (SBI) for trauma patients. COT quick guide. [Online]. Retrieved December 15, 2011 from http://www.facs.org.

American Counseling Association. (2006). Who are licensed professional counselors. [Online]. Retrieved August 5, 2009 from http://www.counseling.org.

American Counseling Association. (2012). Licensure and certification. [Online]. Retrieved March 14, 2012 from http://www.counseling.org.

American Dental Association. (2007). ADA applauds legislators for "meth mouth" bills. [Online]. Retrieved June 28, 2011 from http://www.dentalcompare.com.

American Diabetes Association. (2005). Standards of medical care in diabetes. Diabetes Care, 28(S1), S4-S36.

American Diabetes Association. (2009). Diabetes cost calculator. [Online]. Retrieved May 25, 2011 from http://www.diabetesarchive.net.

American Diabetes Association. (2011). Tight diabetes control. [Online]. Retrieved October 26, 2011 from http://www.diabetes.org.

American Diabetes Association. (2012). Executive summary: Standards of medical care in diabetes 2012. Diabetes Care, 35(S1), S4-S10.

American Heart Association, American Stroke Association. (2012). Route 2020: What drives us: Your road to a healthy life: Annual report 2010-2011. [Online]. Retrieved April 25, 2012 from http://www.heart.org.

American Heart Association. (2011). Nicotine substitutes / nicotine replacement therapy. [Online]. Retrieved March 30, 2011 from http://www.americanheart.org.

American Legacy Foundation. (2007). Saving lives, saving money II: Tobacco-free states spend less on Medicaid. [Online]. Retrieved February 22, 2012 from http://www.legacyforhealth.org.

American Lung Association. (2008). About N-O-T. [Online]. Retrieved March 30, 2011 from http://www.notontobacco.com.

American Lung Association. (2009). N-O-T publications. [Online]. Retrieved March 30, 2011 from http://www.notontobacco.com.

American Lung Association. (2010a). About smoking: Facts and figures: American Indians/Alaska Natives. [Online]. Retrieved April 3, 2012 from http://www.lung.org.

American Lung Association. (2010b). State cessation coverage 2010: Helping smokers quit. Washington, DC: Author. 
American Medical Association, Council on Scientific Affairs. (1979). Guidelines for alcoholism diagnosis: Treatment and referral. Chicago: Author.

American Medical Association. (1956). Reports of officers. JAMA, 162(8), 748-819.

American Medical Association. (1990). Fundamental elements of the patient-physician relationship. [Online]. Retrieved March 28, 2012 from http://www.ama-assn.org.

American Medical Association. (1997). Guidelines for Adolescent Preventive Services (GAPS). Chicago: Author.

American Medical Association. (2009). AMA healthier life steps: Coding for routine adult lifestyle screening, early intervention, and motivational interviewing. [Online]. Retrieved May 14, 2012 from http://www.ama-assn.org.

American Medical Association. (2009). Current Procedural Terminology codes (CPT codes). [Online]. Retrieved May 10, 2012 from http://www.businessgrouphealth.org.

American Medical Association. (2011a). Directives of the AMA house of delegates. [Online]. Retrieved December 20, 2011 from http://www.ama-assn.org.

American Medical Association. (2011b). H-95.976 Drug abuse in the United States: The next generation. [Online]. Retrieved March 15, 2012 from https://ssl3.ama-assn.org.

American Medical Association. (2011c). Report to the Board of Trustees: Background on the organization "Physicians and Lawyers for National Policy": Resolution 425, A-06. [Online]. Retrieved December 20, 2011 from http://www.docstoc.com.

American Medical Association. (2011d). Timelines in AMA history. [Online]. Retrieved December 2, 2011 from http://www.ama-assn.org.

American Medical Association. (2012a). Counseling. [Online]. Retrieved March 20, 2012 from http://www.ama-assn.org.

American Medical Association. (2012b). Physician assistant. [Online]. Retrieved March 19, 2012 from http://www.ama-assn.org.

American Medical Association. (2012c). Physician characteristics and distribution in the U.S. Chicago, IL: Author.

American Medical Association. (2012d). Requirements for becoming a physician. [Online]. Retrieved January 17, 2012 from http://www.ama-assn.org.

American Psychiatric Association. (1952). Diagnostic and statistical manual of mental disorders (1st ed.). Washington, DC: Author.

American Psychiatric Association. (1980). Diagnostic and statistical manual of mental disorders (3rd ed.). Washington, DC: Author.

American Psychiatric Association. (1994). Diagnostic and statistical manual of psychiatric disorders $(D S M-I V)$ (4th ed.). Washington, DC: Author.

American Psychiatric Association. (1995). Diagnostic and statistical manual of mental disorders, fourth edition: Primary care version. Washington, DC: Author.

American Psychiatric Association. (2006).Practice guidelines for the treatment of patients with substance use disorders (2nd ed.). Washington, DC: Author.

American Psychiatric Association. (2008a). CPT codes for screening, intervention. [Online]. Retrieved December 18, 2009 from http://pn.psychiatryonline.org. 
American Psychiatric Association. (2008b). Substance use disorders: Physician Performance Measurement Set: PCPI approved. [Online]. Retrieved February 27, 2012 from http://www.amaassn.org.

American Psychiatric Association. (2010a). APA announces draft diagnostic criteria for DSM-5. New proposed changes posted for leading manual of mental disorders. [Online]. Retrieved December 2, 2011 from http://www.dsm5.org.

American Psychiatric Association. (2010b). DSM-5 development: Timeline. [Online]. Retrieved December 2, 2011 from http://www.dsm5.org.

American Psychiatric Association. (2010c). R00-10 Substance use disorder. [Online]. Retrieved June 8, 2011 from http://www.dsm5.org.

American Psychiatric Association. (2011a). Diagnostic and statistical manual. [Online]. Retrieved December 2, 2011 from http://www.psych.org.

American Psychiatric Association. (2011b). Substance-related disorders. [Online]. Retrieved August 16, 2011 from http://www.dsm5.org.

American Psychological Association. (2009). Certificate of proficiency in the treatment of alcohol and other psychoactive substance use disorders. [Online]. Retrieved July 23, 2009 from http://www.apapracticecentral.org.

American Society of Addiction Medicine. (1997). Public policy statement on screening for addiction in primary care settings. [Online]. Retrieved October 2, 2008 from http://www.asam.org.

American Society of Addiction Medicine. (2003). Public policy statement on drug testing in workplace settings. Journal of Addictive Diseases, 22(3), 119-122.

American Society of Addiction Medicine. (2006). Public policy statement on relationship between treatment and self help. [Online]. Retrieved October 1, 2007 from http://www.asam.org.

American Society of Addiction Medicine. (2007). ASAM patient placement criteria for the treatment of substance-related disorders (PPC-R2) (2nd ed.). Chevy Chase, MD: Author.

American Society of Addiction Medicine. (2009). ASAM mission and goals. [Online]. Retrieved February 5, 2009 from http://www.asam.org.

American Society of Addiction Medicine. (2010a). Public policy statement on how to identify a physician recognized for expertness in the diagnosis and treatment of addiction and substance-related health conditions. [Online]. Retrieved June 12, 2011 from http://www.asam.org.

American Society of Addiction Medicine. (2010b). Public policy statement on treatment for alcohol and other drug addiction. [Online]. Retrieved May 11, 2012 from http://www.asam.org.

American Society of Addiction Medicine. (2011a). Definition of addiction: Frequently asked questions. [Online]. Retrieved November 14, 2011 from http://www.asam.org.

American Society of Addiction Medicine. (2011b). Public policy statement: Definition of addiction (long version). [Online]. Retrieved November 14, 2011 from http://www.asam.org.

American Society of Addiction Medicine. (2011c). Public policy statement: Short definition of addiction. [Online]. Retrieved November 21, 2011 from http://www.asam.org.

American Society of Addiction Medicine. (2012). ASAM patient placement criteria for the treatment of substance-related disorders (ASAM PPC-2R) (2nd ed.). Chevy Chase: MD: Author.

American Society of Health-System Pharmacists. (2012a). ASHP. [Online]. Retrieved February 27, 2012 from http://www.ashp.org. 
American Society of Health-System Pharmacists. (2012b). Tobacco cessation resource center. [Online]. Retrieved February 27, 2012 from http://www.ashp.org.

Andersen, S. L., \& Teicher, M. H. (2009). Desperately driven and no brakes: Developmental stress exposure and subsequent risk for substance abuse. Neuroscience \& Biobehavioral Reviews, 33(4), 516-524.

Anderson, A. L., Reid, M. S., Li, S. H., Holmes, T., Shemanski, L., Slee, A., et al. (2009). Modafinil for the treatment of cocaine dependence. Drug \& Alcohol Dependence, 104(1-2), 133-139.

Anderson, D. J., McGovern, J. P., \& Dupont, R. L. (1999). The origins of the Minnesota model of addiction treatment: A first person account. Journal of Addictive Diseases, 18(1), 107-114.

Anderson, P., Aromaa, S., Rosenbloom, D., \& Enos, G. (2008). Screening \& brief intervention: Making a public health difference. Boston: Join Together.

Andrews, G., \& Peters, L. (1998). The psychometric properties of the Composite International Diagnostic Interview. Social Psychiatry \& Psychiatric Epidemiology, 33(2), 80-88.

Angermeyer, M. C., \& Dietrich, S. (2006). Public beliefs about and attitudes towards people with mental illness: A review of population studies. Acta Psychiatrica Scandinavica, 113(3), 163-179.

Anglin, M. D., Hser, Y. I., \& Grella, C. E. (1997). Drug addiction and treatment careers among clients in the Drug Abuse Treatment Outcome Study (DATOS). Psychology of Addictive Behaviors, 11(4), 308323.

Anthony, E. K., Taylor, S. A., \& Raffo, Z. (2011). Early intervention for substance abuse among youth and young adults with mental health conditions: An exploration of community mental health practices. Administration \& Policy in Mental Health, 38(3), 131-141.

Anthony, J. C., \& Helzer, J. E. (1991). Syndromes of drug abuse and dependence. In L. N. Robins \& D. A. Regier (Eds.), Psychiatric disorders in America: The Epidemiologic Catchment Area Study (pp. 116-154). New York: Free Press.

Anthony, J. C., Warner, L. A., \& Kessler, R. C. (1994). Comparative epidemiology of dependence on tobacco, alcohol, controlled substances, and inhalants: Basic findings from the National Comorbidity Survey. Experimental \& Clinical Psychopharmacology, 2(3), 244-268.

Anton, R. (2010). Substance Abuse is a disease of the brain: Focus on alcohol. Journal of Law, Medicine \& Ethics, 38(4), 745-744.

Anton, R. F., \& Swift, R. M. (2003). Current pharmacotherapies of alcoholism: A U.S. perspective. American Journal on Addictions, 12(1), S53-S68.

Anton, R. F., Moak, D. H., Latham, P., Waid, L. R., Myrick, H., Voronin, K., et al. (2005). Naltrexone combined with either cognitive behavioral or motivational enhancement therapy for alcohol dependence. Journal of Clinical Psychopharmacology, 25(4), 349-357.

Anton, R. F., Moak, D. H., Waid, L. R., Latham, P. K., Malcolm, R. J., \& Dias, J. K. (1999). Naltrexone and cognitive behavioral therapy for the treatment of outpatient alcoholics: Results of a placebocontrolled trial. American Journal of Psychiatry, 156(11), 1758-1764.

Anton, R. F., O'Malley, S. S., Ciraulo, D. A., Cisler, R. A., Couper, D., Donovan, D. M., et al. (2006). Combined pharmacotherapies and behavioral interventions for alcohol dependence: The COMBINE Study: A randomized controlled trial. JAMA, 295(17), 2003-2017.

Aos, S., Miller, M., \& Drake, E. (2006). Evidence-based adult corrections programs: What works and what does not. Olympia, WA: Washington State Institute for Public Policy. 
Appel, L. J., Brands, M. W., Daniels, S. R., Karanja, N., Elmer, P. J., \& Sacks, F. M. (2006). Dietary approaches to prevent and treat hypertension: A scientific statement from the American Heart Association. Hypertension, 47(2), 296-308.

Appel, P. W., \& Oldak, R. (2007). A preliminary comparison of major kinds of obstacles to enrolling in substance abuse treatment (AOD) reported by injecting street outreach clients and other stakeholders. American Journal of Drug \& Alcohol Abuse, 33(5), 699-705.

Appel, P. W., Ellison, A. A., Jansky, H. K., \& Oldak, R. (2004). Barriers to enrollment in drug abuse treatment and suggestions for reducing them: Opinions of drug injecting street outreach clients and other system stakeholders. American Journal of Drug \& Alcohol Abuse, 30(1), 129-153.

Arias, A. J., \& Kranzler, H. R. (2008). Treatment of co-occurring alcohol and other drug use disorders. Alcohol Research \& Health, 31(2), 155-167.

Ariz. Admin. Code R9-10-207 (2010).

Ark. Code. $016 \S 04004$ (2012).

Arkansas Department of Health. (2011). Pregnancy risk assessment monitoring system: Barriers to quitting smoking, 2009. [Online]. Retrieved February 3, 2012 from http://www.healthy.arkansas.gov.

Armstrong, T. D., \& Costello, E. J. (2002). Community studies on adolescent substance use, abuse, or dependence and psychiatric comorbidity. Journal of Consulting and Clinical Psychology, 70(6), 1224-1239.

Arnett, J. J. (2005). The developmental context of substance use in emerging adulthood. Journal of Drug Issues, 35(2), 235-254.

Asbridge, M., Hayden, J. A., \& Cartwright, J. L. (2012). Acute cannabis consumption and motor vehicle collision risk: Systematic review of observational studies and meta-analysis. British Medical Journal, 344; doi:10.1136/bmj.e536

Aseltine, R. H. (2010). The impact of screening, brief intervention and referral for treatment in emergency department patients' alcohol use: A 3-, 6- and 12-month follow-up. Alcohol \& Alcoholism, 45(6), 514519.

Ashley, O. S., Marsden, M. E., \& Brady, T. M. (2003). Effectiveness of substance abuse treatment programming for women: A review. American Journal of Drug \& Alcohol Abuse, 29(1), 19-53.

Ashton, H. (1984). Benzodiazepine withdrawal: An unfinished story. British Medical Journal (Clinical Research Ed), 288(6424), 1135-1140.

Associated Press. (2011). Florida: Welfare recipients face drug tests. [Online]. New York Times. Retrieved December 13, 2011 from http://www.nytimes.com.

Association of American Medical Colleges. (2007). Physician behavior and practice patterns related to smoking cessation. Full report. [Online]. Retrieved February 7, 2012 from https://www.aamc.org.

Association of Marital and Family Therapy Regulatory Boards, \& Professional Examination Service. (2012). Information for candidates: Examination in marital and family therapy. [Online]. Retrieved March 19, 2012 from http://www.amftrb.org.

Association of Social Work Boards. (2011). ASWB examination candidate handbook. [Online]. Retrieved March 19, 2012 from http://www.aswb.org.

Association of State and Provincial Psychology Boards. (2008). ASPPB's guide for students and faculty. Entry requirements for the professional practice of psychology. [Online]. Retrieved May 14, 2012 from http://www.asppb.net. 
Association of State and Provincial Psychology Boards. (2012a). ASPPB information for EPPP candidates: Examination for Professional Practice in Psychology (EPPP). [Online]. Retrieved March 19, 2012 from http://www.asppb.net.

Association of State and Provincial Psychology Boards. (2012b). Handbook of licensing and certification requirements. [Online]. Retrieved March 19, 2012 from http://www.asppb.org.

Asthma and Allergy Foundation of America. (2005). Asthma overview. [Online]. Retrieved January 7 , 2009 from http://www.aafa.org.

Atkinson, R. M., Ganzini, L., \& Bernstein, M. J. (1992). Alcohol and substance-use disorders in the elderly. In J. E. Birren, R. B. Sloane, \& G. D. Cohen (Eds.), Handbook of mental health and aging (pp. 516-555). New York: Academic Press.

Atkinson, R. M., Tolson, R. L., \& Turner, J. A. (1990). Late versus early onset problem drinking in older men. Alcoholism: Clinical \& Experimental Research, 14(4), 574-579.

Aubin, H. J., Bobak, A., Britton, J. R., Oncken, C., Billing, C. B., Gong, J., et al. (2008). Varenicline versus transdermal nicotine patch for smoking cessation: Results from a randomised open-label trial. Thorax, 63, 717-724.

Audrain-McGovern, J., Al, K. N., Rodriguez, D., Wileyto, E. P., Shields, P. G., \& Tyndale, R. F. (2007). The role of CYP2A6 in the emergence of nicotine dependence in adolescents. Pediatrics, 119(1), e264-e274.

Audrain-McGovern, J., Rodriguez, D., \& Kassel, J. D. (2009). Adolescent smoking and depression: Evidence for self-medication and peer smoking mediation. Addiction, 104(10), 1743-1756.

Austin, G. A., \& Prendergast, M. (1991). Prevention research update No. 8: Young children of substance abusers. Portland, OR: Northwest Regional Educational Laboratory.

Aveyard, P., Begh, R., Parsons, A., \& West, R. (2012). Brief opportunistic smoking cessation interventions: A systematic review and meta-analysis to compare advice to quit and offer of assistance. Addiction, 107(6), 1066-1073.

Azrin, N. H. (1976). Improvements in the community-reinforcement approach to alcoholism. Behaviour Research \& Therapy., 14(5), 339-348.

Azrin, N. H., Sisson, W., Myers, R., \& Godley, M. (1982). Alcoholism treatment by disulfiram and community reinforcement therapy. Journal of Behavior Therapy \& Experimental Psychiatry, 13(2), 105-112.

Azzone, V., Frank, R. G., Normand, S. L., \& Burnam, M. A. (2011). Effect of insurance parity on substance abuse treatment. Psychiatric Services, 62(2), 129-134.

Baan, R., Straif, K., Grosse, Y., Secretan, B., El, G. F., Bouvard, V., et al. (2007). Carcinogenicity of alcoholic beverages. Lancet Oncology, 8(4), 292-293.

Babor, T. F., \& Hall, W. (2007). Editorial: Standardizing terminology in addiction science: To achieve the impossible dream. Addiction, 102(7), 1015-1018.

Babor, T. F., \& Kadden, R. M. (2005). Screening and interventions for alcohol and drug problems in medical settings: What works? Journal of Trauma, 59(Suppl. 3), S80-S87.

Babor, T. F., Higgins-Biddle, J. C., Dauser, D., Burleson, J. A., Zarkin, G. A., \& Bray, J. (2006). Brief interventions for at-risk drinking: Patient outcomes and cost-effectiveness in managed care organizations. Alcohol \& Alcoholism, 41(6), 624-631. 
Babor, T. F., McRee, B. G., Kassebaum, P. A., Grimaldi, P. L., Ahmed, K., \& Bray, J. (2007). Screening, Brief Intervention, and Referral to Treatment (SBIRT): Toward a public health approach to the management of substance abuse. Substance Abuse, 28(3), 7-30.

Baer, J. S., Kivlahan, D. R., Blume, A. W., McKnight, P., \& Marlatt, G. A. (2001). Brief intervention for heavy-drinking college students: 4-year follow-up and natural history. American Journal of Public Health, 91(8), 1310-1316.

Baker, A., Lee, N. K., Claire, M., Lewin, T. J., Grant, T., Pohlman, S., et al. (2005). Brief cognitive behavioural interventions for regular amphetamine users: A step in the right direction. Addiction, 100(3), 367-378.

Baker, T. B., Breslau, N., Covey, L., \& Shiffman, S. (2012). DMS criteria for tobacco use disorder and tobacco withdrawal: A critique and proposed revisions for DSM-5. Addiction, 107(2), 263-275.

Baldwin, M. L., Marcus, S. C., \& DeSimone, J. (2010). Job loss discrimination and former substance use disorders. Drug \& Alcohol Dependence, 110(1-2), 1-7.

Ball, S. A., Martino, S., Nich, C., Frankforter, T. L., Van, H. D., Crits-Christoph, P., et al. (2007). Site matters: Multisite randomized trial of motivational enhancement therapy in community drug abuse clinics. Journal of Consulting \& Clinical Psychology, 75(4), 556-567.

Balldin, J., Berglund, M., Borg, S., Mansson, M., Bendtsen, P., Franck, J., et al. (2003). A 6-month controlled naltrexone study: Combined effect with cognitive behavioral therapy in outpatient treatment of alcohol dependence. Alcoholism: Clinical \& Experimental Research, 27(7), 1142-1149.

Barker, G. J., \& Williams, K. B. (1999). Tobacco use cessation activities in U.S. dental and dental hygiene student clinics. Journal of Dental Education, 63(11), 828-833.

Barker, M. J., Greenwood, K. M., Jackson, M., \& Crowe, S. F. (2004). Cognitive effects of long-term benzodiazepine use. A meta-analysis. CNS Drugs, 18(1), 37-48.

Barnes, H. N., \& Samet, J. H. (1997). Brief interventions with substance-abusing patients. Medical Clinics of North America, 81(4), 867-879.

Barnett, P. G. (1999). The cost-effectiveness of methadone maintenance as a health care intervention. Addiction, 94(4), 479-488.

Barnett, P. G., Wong, W., \& Hall, S. (2008). The cost-effectiveness of a smoking cessation program for out-patients in treatment for depression. Addiction, 103(5), 834-840.

Barnett, P. G., Zaric, G. S., \& Brandeau, M. L. (2001). The cost-effectiveness of buprenorphine maintenance therapy for opiate addiction in the United States. Addiction, 96(9), 1267-1278.

Barrowclough, C., Haddock, G., Tarrier, N., Lewis, S. W., Moring, J., O'Brien, R., et al. (2001). Randomized controlled trial of motivational interviewing, cognitive behavior therapy, and family intervention for patients with comorbid schizophrenia and substance use disorders. American Journal of Psychiatry, 158(10), 1706-1713.

Barry, P. P. (1986). Gender as a factor in treating the elderly. In B. A. Ray \& M. C. Braude (Eds.), Women and drugs: A new era for research. NIDA research monograph 65 (pp. 65-69). Rockville, MD: U.S. Department of Health and Human Services, Public Health Service, Alcohol, Drug Abuse, and Mental Health Administration, National Institute on Drug Abuse.

Bart, G., Kreek, M. J., Ott, J., LaForge, K. S., Proudnikov, D., Pollak, L., et al. (2005). Increased attributable risk related to a functional mu-opioid receptor gene polymorphism in association with alcohol dependence in central Sweden. Neuropsychopharmacology, 30(2), 417-422. 
Baser, O., Chalk, M., Fiellin, D. A., \& Gastfriend, D. R. (2011). Cost and utilization outcomes of opioiddependence treatments. American Journal of Managed Care, 17(Suppl. 8), S235-S248.

Bastek-Karasow, C., \& Davis, R. (2007). Gender-responsive treatment for women with substance use disorders. [Online]. Retrieved January 12, 2012 from http://bcwf.org.

Battjes, R. J., Onken, L. S., \& Delany, P. J. (1999). Drug abuse treatment entry and engagement: Report of a meeting on treatment readiness. Journal of Clinical Psychology, 55(5), 643-657.

Bauermeister, J. A., Zimmerman, M. A., Gee, G. C., Caldwell, C., \& Xue, Y. (2009). Work and sexual trajectories among African American youth. Journal of Sex Research, 46(4), 290-300.

Bava, S., Frank, L. R., McQueeny, T., Schweinsburg, B. C., Schweinsburg, A. D., \& Tapert, S. F. (2009). Altered white matter microstructure in adolescent substance users. Psychiatry Research, 173(3), 228237.

Bayard, M., McIntyre, J., Hill, K. R., \& Woodside, J., Jr. (2004). Alcohol withdrawal syndrome. American Family Physician, 69(6), 1443-1450.

Bazelon Center for Mental Health Law. (2010). Medicaid reforms in the Patient Protection and Affordable Care Act and the Health Care and Education Reconciliation Act. [Online]. Retrieved November 11, 2011 from http://www.bazelon.org.

Beauchamp, T. L., \& Childress, J. F. (2001). Principles of biomedical ethics (5th ed.). New York: Oxford University Press.

Becker, H. C. (1998). Kindling in alcohol withdrawal. Alcohol Health \& Research World, 22(1), 25-33.

Becker, S. J., \& Curry, J. F. (2008). Outpatient interventions for adolescent substance abuse: A quality of evidence review. Journal of Consulting \& Clinical Psychology, 76(4), 531-543.

Behrman, R. E., \& Butler, A. S. (Eds.). (2007). Preterm birth: Causes, consequences, and prevention. Washington, DC: National Academies Press.

Belcher, H. M., \& Shinitzky, H. E. (1998). Substance abuse in children: Prediction, protection, and prevention. Archives of Pediatrics \& Adolescent Medicine, 152(10), 952-960.

Beletsky, L., Ruthazer, R., Macalino, G. E., Rich, J. D., Tan, L., \& Burris, S. (2007). Physicians' knowledge of and willingness to prescribe naloxone to reverse accidental opiate overdose: Challenges and opportunities. Journal of Urban Health, 84(1), 126-136.

Bellack, A. S., Bennett, M. E., Gearon, J. S., Brown, C. H., \& Yang, Y. (2006). A randomized clinical trial of a new behavioral treatment for drug abuse in people with severe and persistent mental illness. Archives of General Psychiatry, 63(4), 426-432.

Benedict, J., Evans, W., \& Calder, J. C. (1999). An exploratory study of recreational drug use and nutrition-related behaviors and attitudes among adolescents. Journal of Drug Education, 29(2), 139155.

Benowitz, N. L. (2010). Nicotine addiction. New England Journal of Medicine, 362(24), 2295-2303.

Berenson, R. A., \& Burton, R. A. (2011). Accountable care organizations in Medicare and the private sector: A status update: Timely analysis of immediate health policy issues. Washington, DC: Urban Institute.

Berg, C. J., Park, E. R., Chang, Y., \& Rigotti, N. A. (2008). Is concern about post-cessation weight gain a barrier to smoking cessation among pregnant women? Nicotine \& Tobacco Research 10(7), 11591163. 
Bernhardt, A. (2009). Rising to the challenge of treating OEF/OIF veterans with co-occurring PTSD and substance abuse. Smith College Studies in Social Work, 79(3-4), 344-367.

Bernstein, E., Bernstein, J. A., Stein, J. B., \& Saitz, R. (2009). SBIRT in emergency care settings: Are we ready to take it to scale? Academic Emergency Medicine, 16(11), 1072-1077.

Bernstein, E., Bernstein, J., Feldman, J., Fernandez, W., Hagan, M., Mitchell, P., et al. (2007). An evidence based alcohol screening, brief intervention and referral to treatment (SBIRT) curriculum for emergency department (ED) providers improves skills and utilization. Substance Abuse, 28(4), 79-92.

Bernstein, E., Edwards, E., Dorfman, D., Heeren, T., Bliss, C., \& Bernstein, J. (2009). Screening and brief intervention to reduce marijuana use among youth and young adults in a pediatric emergency department. Academic Emergency Medicine, 16(11), 1174-1185.

Bernstein, J., Bernstein, E., Tassiopoulos, K., Heeren, T., Levenson, S., \& Hingson, R. (2005). Brief motivational intervention at a clinic visit reduces cocaine and heroin use. Drug \& Alcohol Dependence, 77(1), 49-59.

Bertholet, N., Daeppen, J., Wietlisbach, V., Fleming, M., \& Burnand, B. (2005). Reduction of alcohol consumption by brief alcohol intervention in primary care. Archives of Internal Medicine, 165(9), 986-995.

Best, D., Noble, A., Ridge, G., Gossop, M., Farrell, M., \& Strang, J. (2002). The relative impact of waiting time and treatment entry on drug and alcohol use. Addiction Biology, 7(1), 67-74.

Bhati, A. S., Roman, J. K., \& Chalfin, A. (2008). To treat or not to treat: Evidence on the prospects of expanding treatment to drug-involved offenders. Washington, DC: Urban Institute.

Biederman, J., Wilens, T. E., Mick, E., Faraone, S. V., \& Spencer, T. (1998). Does attention-deficit hyperactivity disorder impact the developmental course of drug and alcohol abuse and dependence? Biological Psychiatry, 44(4), 269-273.

Bien, T. H., Miller, W. R., \& Tonigan, J. S. (1993). Brief interventions for alcohol problems: A review. Addiction, 88(3), 315-335.

Biener, L., \& Siegel, M. (2000). Tobacco marketing and adolescent smoking: More support for a causal inference. American Journal of Public Health, 90(3), 407-411.

Bierut, L. J., Dinwiddie, S. H., Begleiter, H., Crowe, R. R., Hesselbrock, V., Nurnberger, J. I., Jr., et al. (1998). Familial transmission of substance dependence: Alcohol, marijuana, cocaine, and habitual smoking: A report from the Collaborative Study on the Genetics of Alcoholism. Archives of General Psychiatry, 55(11), 982-988.

Bierut, L. J., Madden, P. A., Breslau, N., Johnson, E. O., Hatsukami, D., Pomerleau, O. F., et al. (2007). Novel genes identified in a high-density genome wide association study for nicotine dependence. Human Molecular Genetics, 16(1), 24-35.

Biery, J. R., Williford, J. H., Jr., \& McMullen, E. A. (1991). Alcohol craving in rehabilitation: Assessment of nutrition therapy. Journal of the American Dietetic Association, 91(4), 463-466.

Bina, R., Yum, J., Hall, D., Sowbel, L., Molette, A., Jani, J., et al. (2008). Substance abuse training and perceived knowledge: Predictors of perceived preparedness to work in substance abuse. Journal of Social Work Education, 44(3), 7-20.

Bishop, E. S. (1919). Narcotic drug addiction: A public health problem. American Journal of Public Health, 9(7), 481-488.

Block, M. A. (1956). Medical treatment of alcoholism. JAMA, 162(18), 1610-1619. 
Blom, T. J., \& Anthenelli, R. M. (2011). Gender differences in trauma history and symptoms as predictors of relapse to alcohol and drug use. American Journal on Addictions, 20(4), 307-311.

Blum, K., Werner, T., Carnes, S., Carnes, P., Bowirrat, A., Giordano, J., et al. (2012). Sex, drugs, and rock 'n' roll: Hypothesizing common mesolimbic activation as a function of reward gene polymorphisms. Journal of Psychoactive Drugs, 44(1), 38-55.

Blume, S. B. \& Zilberman, M. L. (2005). Alcohol and women. In J. Lowinson, P. Ruiz, R. B. Millman, \& J. G. Langrod (Eds.), Substance abuse: A comprehensive textbook ( $4^{\text {th }}$ ed., pp. 1049-1064). Baltimore, MD: Williams and Wilkins.

Blume, S. B. (1994). Pathological gambling and switching addictions: Report of a case. Journal of Gambling Studies, 10(1), 87-96.

Bobo, J. K., McIlvain, H. E., Lando, H. A., Walker, R. D., \& Leed-Kelly, A. (1998). Effect of smoking cessation counseling on recovery from alcoholism: Findings from a randomized community intervention trial. Addiction, 93(6), 877-887.

Bock, B. C., Marcus, B. H., King, T. K., Borrelli, B., \& Roberts, M. R. (1999). Exercise effects on withdrawal and mood among women attempting smoking cessation. Addictive Behaviors, 24(3), 399410.

Boden, M. T., \& Moos, R. (2009). Dually diagnosed patients' responses to substance use disorder treatment. Journal of Substance Abuse Treatment, 37(4), 335-345.

Boden, M. T., Kimerling, R., Jacobs-Lentz, J., Bowman, D., Weaver, C., Carney, D., et al. (2011). Seeking safety treatment for male veterans with a substance use disorder and post-traumatic stress disorder symptomology. Addiction, 107(3), 578-586.

Bodenheimer, T., Wagner, E. H., \& Grumbach, K. (2002). Improving primary care for patients with chronic illness: The chronic care model, Part 2. JAMA, 288(15), 1909-1914.

Bollerud, K. (1990). A model for the treatment of trauma-related syndromes among chemically dependent inpatient women. Journal of Substance Abuse Treatment, 7(2), 83-87.

Bonnie, R. J., \& O'Connell, M. E. (2004). Reducing underage drinking: A collective responsibility. Washington, DC: National Academies Press.

Boothby, L. A., \& Doering, P. L. (2007). Buprenorphine for the treatment of opioid dependence. American Journal of Health-System Pharmacy, 64(3), 266-272.

Borders, T. F., \& Booth, B. M. (2007). Research on rural residence and access to drug abuse services: Where are we and where do we go? Journal of Rural Health, 23(Suppl. 1), 79-83.

Borduin, C. M., Mann, B. J., Cone, L. T., Henggeler, S. W., Fucci, B. R., Blaske, D. M., et al. (1995). Multisystemic treatment of serious juvenile offenders: Long-term prevention of criminality and violence. Journal of Consulting \& Clinical Psychology, 63(4), 569-578.

Bottlebooks.com. (2009). The Keeley cure. [Online]. Retrieved February 17, 2012 from http://bottlebooks.com.

Bouchery, E. E., Harwood, H. J., Dilonardo, J., \& Vandivort-Warren, R. (2012). Type of health insurance and the substance abuse treatment gap. Journal of Substance Abuse Treatment, 42(3), 289-300.

Bowen, O. R., \& Sammons, J. H. (1988). The alcohol-abusing patient: A challenge to the profession. JAMA, 260(15), 2267-2270.

Boyle, M. G., \& Murphy, T. P. (2005). Guidelines for linking addiction treatment with primary healthcare developed for the Behavioral Health Recovery Management Project. [Online]. Retrieved December 19, 2011 from http://www.bhrm.org. 
Brady, J. P., Posner, M., Lang, C., \& Rosati, M. J. (1994). Risk and reality: The implications of prenatal exposure to alcohol and other drugs. Rockville, MD: U.S. Department of Health and Human Services, and the U. S. Department of Education, Educational Development Center.

Braude, M. C. (1986). Drugs and drug interactions in elderly women. In B. A. Ray \& M. C. Braude (Eds.), Women and drugs: A new era for research: NIDA research monograph 65 (pp. 58-64). Rockville, MD: U.S. Department of Health and Human Services, Public Health Service, Alcohol, Drug Abuse, and Mental Health Administration, National Institute on Drug Abuse.

Break Free Alliance. (2009). Addressing tobacco use in homeless populations. Recommendations of the expert panel. [Online]. Retrieved January 31, 2012 from http://smokingcessationleadership.ucsf.edu.

Brecher, E. M. (1972). The Consumers Union report on licit and illicit drugs. Opiates for pain relief, for tranquilization, and for pleasure. [Online]. Retrieved November 30, 2011 from http://www.druglibrary.org.

Breland, A. B., Colby, S., Dino, G., Smith, G., \& Taylor, M. (2009). Youth smoking cessation interventions: Treatments, barriers, and recommendations for Virginia. Richmond, VA:Virginia Commonwealth University, Institute for Drug and Alcohol Studies.

Brendryen, H., \& Kraft, P. (2008). Happy ending: A randomized controlled trial of a digital multi-media smoking cessation intervention. Addiction, 103(3), 478-484.

Brennan, P. L., \& Moos, R. H. (1996). Late-life drinking behavior: The influence of personal characteristics, life context, and treatment. Alcohol Research \& Health, 20(3), 197-204.

Breton, A. R., Taira, D. A., Burns, E., O'Leary, J., \& Chung, R. S. (2007). Follow-up services after an emergency department visit for substance abuse. American Journal of Managed Care, 13(9), 497505.

Brodey, B. B., McMullin, D., Kaminer, Y., Winters, K. C., Mosshart, E., Rosen, C. S., et al. (2008). Psychometric characteristics of the Teen Addiction Severity Index-Two (T-ASI-2). Substance Abuse, 29(2), 19-32.

Brodey, B. B., Rosen, C. S., Winters, K. C., Brodey, I. S., Sheetz, B. M., Steinfeld, R. R., et al. (2005). Conversion and validation of the Teen-Addiction Severity Index (T-ASI) for Internet and automatedtelephone self-report administration. Psychology of Addictive Behaviors, 19(1), 54-61.

Brook, D. W., Brook, J. S., Zhang, C., \& Koppel, J. (2010). Association between attentiondeficit/hyperactivity disorder in adolescence and substance use disorders in adulthood. Archives of Pediatrics and Adolescent Medicine, 164(10), 930-934.

Brook, J. S., Whiteman, M., Cohen, P., \& Tanaka, J. S. (1992). Childhood precursors of adolescent drug use: A longitudinal analysis. Genetic, Social, \& General Psychology Monographs, 118(2), 195-213.

Brookhaven Retreat. (2011). 20 things you should know about Brookhaven Retreat. [Online]. Retrieved August 9, 2011 from http://www.brookhavenretreat.com.

Brown, B. S., \& Flynn, P. M. (2002). The federal role in drug abuse technology transfer: A history and perspective. Journal of Substance Abuse Treatment, 22(4), 245-257.

Brown, J. D., Vartivarian, S., \& Alderks, C. E. (2011). Child care in outpatient substance abuse treatment facilities for women: Findings from the 2008 National Survey of Substance Abuse Treatment Services. Journal of Behavioral Health Services \& Research, 38(4), 478-487.

Brown, R. L. (2009). Expanding SBIRT to other behavioral risk factors a key to improving health and reducing costs. [Online]. Wisconsin Initiative to Promote Healthy Lifestyles Retrieved June 14, 2012 from http://www.wiphl.org. 
Brown, R. L., Leonard, T., Saunders, L. A., \& Papasouliotis, O. (1998). The prevalence and detection of substance use disorders among inpatients ages 18 to 49: An opportunity for prevention. Preventive Medicine, 27(1), 101-110.

Brown, R. L., Leonard, T., Saunders, L. A., \& Papasouliotis, O. (2001). A two-item conjoint screen for alcohol and other drug problems. Journal of the American Board of Family Practice, 14(2), 95-106.

Bruce, K. D., \& Hanson, M. A. (2010). The developmental origins, mechanisms, and implications of metabolic syndrome. Journal of Nutrition, 140(3), 648-652.

Brucker, D. L., \& Stewart, M. (2011). Performance-based contracting within a state substance abuse treatment system: A preliminary exploration of differences in client access and client outcomes. Journal of Behavioral Health Services \& Research, 38(3), 383-397.

Bryant, A. L., \& Zimmerman, M. A. (2002). Examining the effects of academic beliefs and behaviors on changes in substance use among urban adolescents. Journal of Educational Psychology, 94(3), 621637.

Buck, J. A. (2011). The looming expansion and transformation of public substance abuse treatment under the affordable care act. Health Affairs, 30(8), 1402-1410.

Buddy, T. (2007). New vaccines help stop drug addiction? [Online]. Retrieved September 9, 2011 from http://alcoholism.about.com.

Buddy, T. (2010). Naltrexone - Treatment for alcoholism and addiction: Blocks effects of opioids, reduces alcohol craving. [Online]. Retrieved January 23, 2012 from http://alcoholism.about.com.

Budney, A. J., Higgins, S. T., \& Sigmon, S. C. (2003). Contingency management in the substance abuse treatment clinic. In F. Rotgers, J. Morgenstern, \& S. Walters (Eds.), Treating substance abuse: Theory and technique (pp. 248-253). New York: Guilford Press.

Budney, A. J., Vandrey, R. G., Hughes, J. R., Moore, B. A., \& Bahrenburg, B. (2007). Oral delta-9tetrahydrocannabinol suppresses cannabis withdrawal symptoms. Drug \& Alcohol Dependence, 86(1), 22-29.

Buhringer, G. \& Sassen, M. (2010). Reliability and validity. In P. G. Miller, J. Strang, \& P. M. Miller (Eds.), Addiction research methods (pp. 11-25). Oxford: Wiley-Blackwell.

Bullock, M. L., Kiresuk, T. J., Pheley, A. M., Culliton, P. D., \& Lenz, S. K. (1999). Auricular acupuncture in the treatment of cocaine abuse. A study of efficacy and dosing. Journal of Substance Abuse Treatment, 16(1), 31-38.

Bullock, M. L., Kiresuk, T. J., Sherman, R. E., Lenz, S. K., Culliton, P. D., Boucher, T. A., et al. (2002). A large randomized placebo controlled study of auricular acupuncture for alcohol dependence. Journal of Substance Abuse Treatment, 22(2), 71-77.

Burgdorf, K., \& Chen, X. (2004). Residential women's treatment: Cost-benefit and outcome findings from a CSAT cross-site evaluation. [Online]. Retrieved November 12, 2007 from http://womenandchildren.treatment.org.

Burge, S. K., \& Schneider, F. D. (1999). Alcohol-related problems: Recognition and intervention. American Family Physician, 59(2), 361-70, 372.

Burke, B. L., Arkowitz, H., \& Menchola, M. (2003). The efficacy of motivational interviewing: A metaanalysis of controlled clinical trials. Journal of Consulting \& Clinical Psychology, 71(5), 843-861.

Burling, A. S., \& Burling, T. A. (2003). A comparison of self-report measures of nicotine dependence among male drug/alcohol-dependent cigarette smokers. Nicotine \& Tobacco Research, 5(5), 625-633. 
Burroughs, V. J., Maxey, R. W., \& Levy, R. A. (2002). Racial and ethnic differences in response to medicines: Towards individualized pharmaceutical treatment. Journal of the National Medical Association, 94(10), 1-26.

Burrow-Sanchez, J. J., \& Lopez, A. L. (2009). Identifying substance abuse issues in high schools: A national survey of high school counselors. Journal of Counseling \& Development, 87(1), 72-79.

Burrow-Sanchez, J., Call, M. E., Adolphson, S. L., \& Hawken, L. S. (2009). School psychologists' perceived competence and training needs for student substance abuse. Journal of School Health, 76(6), 269-276.

Burt, R. D., Dinh, K. T., Peterson, A. V., Jr., \& Sarason, I. G. (2000). Predicting adolescent smoking: A prospective study of personality variables. Preventive Medicine, 30(2), 115-125.

Burton, L. M. (1992). Black grandparents rearing children of drug-addicted parents: Stressors, outcomes, and social service needs. Gerontologist, 32(6), 744-751.

Busto, U., Sellers, E. M., Naranjo, C. A., Cappell, H., Sanchez-Craig, M., \& Sykora, K. (1986). Withdrawal reaction after long-term therapeutic use of benzodiazepines. New England Journal of Medicine, 315(14), 854-859.

Butler Center for Research. (1999). Women and substance abuse. [Online]. Retrieved January 12, 2012 from https://www.hazelden.org.

Butler Center for Research. (2004). Alcoholics Anonymous. Center City, MN: Hazelden Foundation.

Butler, A. C., Chapman, J. E., Forman, E. M., \& Beck, A. T. (2006). The empirical status of cognitivebehavioral therapy: A review of meta-analyses. Clinical Psychology Review, 26(1), 17-31.

Byrnes-Blake, K. A., Laurenzana, E. M., Landes, R. D., Gentry, W. B., \& Owens, S. M. (2005). Monoclonal IgG affinity and treatment time alters antagonism of (+)-methamphetamine effects in rats. European Journal of Pharmacology, 521(1-3), 86-94.

Cabana, M. D., Rand, C. S., Powe, N. R., Wu, A. W., Wilson, M. H., Abboud, P.-A., et al. (1999). Why don't physicians follow clinical practice guidelines? A framework for improvement. JAMA, 282(15), $1458-1465$

Caetano, R. (2003). Alcohol-related health disparities and treatment-related epidemiological findings among whites, blacks, and Hispanics in the United States. Alcoholism: Clinical \& Experimental Research, 27(8), 1337-1339.

Cahill, K., Stead, L. F., \& Lancaster, T. (2007). Nicotine receptor partial agonists for smoking cessation. Cochrane Database of Systematic Reviews, (1), CD006103.

Cal. Codes Regs. 16. 13.7, § 1399.434 (2012).

Calhoun, P. S., Elter, J. R., Jones, E. R., Jr., Kudler, H., \& Straits-Troster, K. (2008). Hazardous alcohol use and receipt of risk-reduction counseling among U.S. veterans of the wars in Iraq and Afghanistan. Journal of Clinical Psychiatry, 69(11), 1686-1693.

Califano, J. A. (2007). High society: How substance abuse ravages America and what to do about it. New York: Public Affairs.

California Board of Behavioral Sciences. (2012). Continuing education (CE) provider application. [Online]. Retrieved March 22, 2012 from http://www.bbs.ca.gov.

California Environmental Protection Agency. (2005). Proposed identification of environmental tobacco smoke as a toxic air contaminant. Part B health effects. Sacramento, CA: California Environmental Protection Agency, Office of Environmental Health Hazard Assessment, Air Resources Board. 
Callahan, D. (1999). Remembering the goals of medicine. Journal of Evaluation in Clinical Practice, 5(2), 103-106.

Cameron, D. C. (1967). Manual on alcoholism. JAMA, 202(6), 539-540.

Campbell, C. A., Hahn, R. A., Elder, R., Brewer, R., Chattopadhyay, S., Fielding, J., et al. (2009). The effectiveness of limiting alcohol outlet density as a means of reducing excessive alcohol consumption and alcohol-related harms. American Journal of Preventive Medicine, 37(6), 556-569.

Campbell, C. I., \& Alexander, J. A. (2002). Culturally competent treatment practices and ancillary service use in outpatient substance abuse treatment. Journal of Substance Abuse Treatment, 22(3), 109-119.

Capoccia, V., Grazier, K. L., Toal, C., Ford, J. H., \& Gustafson, D. H. (2012). Massachusetts's experience suggests coverage alone is insufficient to increase addiction disorders treatment. Health Affairs, 31(5), 1000-1008.

Caraballo, R. S., Yee, S. L., Gfroerer, J., \& Mirza, S. A. (2008). Adult tobacco use among racial and ethnic groups living in the United States, 2002-2005. Preventing Chronic Disease, 5(3), 1-9.

Carey, K. B. (2008, December 22). Drug rehabilitation or revolving door? New York Times, p. 1D.

Carey, K. B., Bradizza, C. M., Stasiewicz, P. R., \& Maisto, S. A. (1999). The case for enhanced addictions training in graduate programs. Behavior Therapist, 22(2), 27-31.

Carey, K. B., Carey, M. P., \& Chandra, P. S. (2003). Psychometric evaluation of the alcohol use disorders identification test and short drug abuse screening test with psychiatric patients in India. Journal of Clinical Psychiatry, 64(7), 767-774.

CARF International. (2011). Behavioral health standards manual: July 1, 2011-June 30, 2012. Tucson, AZ: Author.

Caribbean Food \& Nutrition Institute for Caucus of Ministers of Health. (2002). Towards the Nassau Declaration: Nutrition in mental health, non-communicable diseases and HIV/AIDS: The evidence and the challenge. [Online]. Retrieved June 14, 2012 from http://www.paho.org.

Caron, J. (2002). Keeley Institute: Fargo, ND. [Online]. Retrieved March 24, 2004 from http://www.fargo-history.com.

Carr, C. J. A., Xu, J., Redko, C., Lane, D. T., Rapp, R. C., Goris, J., et al. (2008). Individual and system influences on waiting time for substance abuse treatment. Journal of Substance Abuse Treatment, 34(2), 192-201.

Carroll, K. M. (1996). Relapse prevention as a psychosocial treatment: A review of controlled clinical trials. Experimental \& Clinical Psychopharmacology, 4(1), 46-54.

Carroll, K. M., Ball, S. A., Martino, S., Nich, C., \& Babuscio, T. (2009). Enduring effects of a computerassisted training program for cognitive behavioral therapy: A six-month follow-up of CBT4CBT. Drug \& Alcohol Dependence, 100(1-2), 178-181.

Carroll, K. M., Ball, S. A., Nich, C., Martino, S., Frankforter, T. L., Farentinos, C., et al. (2006). Motivational interviewing to improve treatment engagement and outcome in individuals seeking treatment for substance abuse: A multisite effectiveness study. Drug \& Alcohol Dependence, 81(3), 301-312.

Carroll, K. M., Ball, S. A., Nich, C., O'Connor, P. G., Eagan, D. A., Frankforter, T. L., et al. (2001). Targeting behavioral therapies to enhance naltrexone treatment of opioid dependence: Efficacy of contingency management and significant other involvement. Archives of General Psychiatry, 58(8), 755-761. 
Carroll, K. M., Fenton, L. R., Ball, S. A., Nich, C., Frankforter, T. L., Shi, J., et al. (2004). Efficacy of disulfiram and cognitive behavior therapy in cocaine-dependent outpatients: A randomized placebocontrolled trial. Archives of General Psychiatry, 61(3), 264-272.

Carroll, K. M., Nich, C., Ball, S. A., McCance, E., \& Rounsaville, B. J. (1998). Treatment of cocaine and alcohol dependence with psychotherapy and disulfiram. Addiction, 93(5), 713-728.

Carroll, K. M., Rounsaville, B. J., Nich, C., Gordon, L., \& Gawin, F. (1995). Integrating psychotherapy and pharmacotherapy for cocaine dependence: Results from a randomized clinical trial. NIDA Research Monograph, 150, 19-35.

Carroll, K. M., Rounsaville, B. J., Nich, C., Lynn, G. T., Wirtz, P. W., \& Gawin, F. (1994). One-year follow-up of psychotherapy and pharmacotherapy for cocaine dependence. Archives of General Psychiatry, 51(12), 989-997.

Carter, J. C., \& Davis, C. (2011). An addiction model of binge eating disorder. In B. A. Johnson (Ed.), Addiction medicine: Science and practice (pp. 633-647). New York: Springer.

Casares-Lopez, M. J., Diaz-Mesa, E., Garcia-Portilla, P., Saiz, P., Bobes-Bascaran, M. T., FonsecaPedrero, E., et al. (2011). Sixth version of the Addiction Severity Index: Assessing sensitivity to therapeutic change and retention predictors. International Journal of Clinical \& Health Psychology, $11(3), 495-508$.

Cassel, E. J. (1982). The nature of suffering and the goals of medicine. New England Journal of Medicine, 306(11), 639-645.

Castaneda, R., Sussman, N., Westreich, L., Levy, R., \& O'Malley, M. (1996). A review of the effects of moderate alcohol intake on the treatment of anxiety and mood disorders. Journal of Clinical Psychiatry, 57(5), 207-212.

Castro, F. G., \& Garfinkle, J. (2003). Critical issues in the development of culturally relevant substance abuse treatments for specific minority groups. Alcoholism: Clinical \& Experimental Research, 27(8), $1381-1388$.

Castro, F. G., Brook, J. S., Brook, D. W., \& Rubenstone, E. (2006). Paternal, perceived maternal, and youth risk factors as predictors of youth stage of substance use a longitudinal study. Journal of Addictive Diseases, 25(2), 65-75.

Catholic University of America. (2010). Summary of Federal laws. The Controlled Substances Act of 1970 (Title II of The Comprehensive Drug Abuse Prevention and Control Act of 1970). [Online]. Retrieved December 1, 2011 from http://counsel.cua.edu.

Caughlin, J. P., \& Malis, R. S. (2004). Demand/withdraw communication between parents and adolescents: Connections with self-esteem and substance use. Journal of Social \& Personal Relationships, 21(1), 125-148.

CBS News. (2011). California researchers raise concerns about 'thirdhand smoke'. [Online]. Retrieved January 26, 2012 from http://sanfrancisco.cbslocal.com.

Center for a Healthy Maryland. (2007). Improving patient access to buprenorphine treatment through physician offices in Maryland: Summary of findings, recommendations, and action steps. Baltimore, MD: Author.

Center for Consumer Information and Insurance Oversight. (2011). Essential health benefits bulletin . [Online]. Retrieved March 27, 2012 from http://cciio.cms.gov.

Center for Medicaid and State Operations. (2011). Letter from Director Cindy Mann to state Medicaid director on new Medicaid tobacco cessation services: SDL \# 11-007 ACA \# 17. [Online]. Retrieved May 17, 2012 from http://downloads.cms.gov. 
Center for Mental Health Services, Substance Abuse and Mental Health Services Administration. (2000). Mental health and substance abuse services under the state children's health insurance program: Designing benefits and estimating costs (DHHS Pub No. [SMA] 01-3473). [Online]. Retrieved April 5, 2012 from http://store.samhsa.gov.

Center for Substance Abuse Treatment \& Denver Juvenile Justice Integrated Treatment Network. (2000). Strategies for integrating substance abuse treatment and the juvenile justice system: A practice guide (DHHS Pub. No. (SMA) 00-3369). Rockville, MD: U.S. Department of Health and Human Services, Substance Abuse and Mental Health Services Administration, Center for Substance Abuse Treatment.

Center for Substance Abuse Treatment, Division of Pharmacologic Therapies. (2012). Physician waiver qualifications. [Online]. Retrieved January 12, 2012 from http://buprenorphine.samhsa.gov.

Center for Substance Abuse Treatment, Division of Pharmacologic Therapies. (2007). Guidelines for the accreditation of opioid treatment programs. [Online]. Retrieved August 14, 2009 from http://www.dpt.samhsa.gov.

Center for Substance Abuse Treatment. (1993). Pregnant, substance-using women. Treatment improvement protocol (TIP) Series 2 (DHHS Pub. No. (SMA) 95-3056). Rockville, MD: U.S. Department of Health and Human Services, Substance Abuse and Mental Health Services Administration, Center for Substance Abuse Treatment.

Center for Substance Abuse Treatment. (1994). Screening and assessment for alcohol and other drug abuse among adults in the criminal justice system. Treatment improvement protocol (TIP) Series 7 (DHHS Pub. No. (SMA) 94-2076). Rockville, MD: U.S. Department of Health and Human Services, Substance Abuse and Mental Health Services Administration, Center for Substance Abuse Treatment.

Center for Substance Abuse Treatment. (1995a). Alcohol and other drug screening of hospitalized trauma patients. Treatment improvement protocol (TIP) Series 16 (DHHS Pub. No. (SMA) 95-3039).

Rockville, MD: U.S. Department of Health and Human Services, Substance Abuse and Mental Health Services Administration, Center for Substance Abuse Treatment.

Center for Substance Abuse Treatment. (1995b). The role and current status of patient placement criteria in the treatment of substance use disorders. Treatment improvement protocol (TIP) Series 13 (DHHS Pub. No. (SMA) 09-3021). Rockville, MD: U.S. Department of Health and Human Services, Substance Abuse and Mental Health Services Administration, Center for Substance Abuse Treatment.

Center for Substance Abuse Treatment. (1997). A guide to substance abuse services for primary care clinicians. Treatment improvement protocol (TIP) Series 24 (DHHS Pub. No. (SMA) 97-3139). Rockville, MD: U.S. Department of Health and Human Services, Substance Abuse and Mental Health Services Administration, Center for Substance Abuse Treatment.

Center for Substance Abuse Treatment. (1998a). Comprehensive case management for substance abuse treatment. Treatment improvement protocol (TIP) Series 27. Rockville, MD: U.S. Department of Health and Human Services, Substance Abuse and Mental Health Services Administration, Center for Substance Abuse Treatment.

Center for Substance Abuse Treatment. (1998b). Substance abuse among older adults. Treatment improvement protocol (TIP) Series 26 (DHHS Pub. No. (SMA) 98-3179). Rockville, MD: U.S. Department of Health and Human Services, Substance Abuse and Mental Health Services Administration, Center for Substance Abuse Treatment.

Center for Substance Abuse Treatment. (1998c). Substance use disorder treatment for people with physical and cognitive disabilities. Treatment improvement protocol (TIP) Series 29 (DHHS Pub. No. (SMA) 98-3249). Rockville, MD: U.S. Department of Health and Human Services, Substance Abuse and Mental Health Services Administration, Center for Substance Abuse Treatment. 
Center for Substance Abuse Treatment. (1999a). Enhancing motivation for change in substance abuse treatment. Treatment improvement protocol (TIP) Series 35 (DHHS Pub. No. (SMA) 99-3354). Rockville, MD: U.S. Department of Health and Human Services, Substance Abuse and Mental Health Services Administration, Center for Substance Abuse Treatment.

Center for Substance Abuse Treatment. (1999b). Screening and assessing adolescents for substance use disorders. Treatment improvement protocol (TIP) Series 31 (DHHS Pub. No. (SMA) 99-3282).

Rockville, MD: U.S. Department of Health and Human Services, Substance Abuse and Mental Health Services Administration, Center for Substance Abuse Treatment.

Center for Substance Abuse Treatment. (1999c). Treatment of adolescents with substance use disorders. Treatment improvement protocol (TIP) Series 32 (DHHS Pub. No. (SMA) 99-3283). Rockville, MD: U.S. Department of Health and Human Services, Substance Abuse and Mental Health Services Administration, Center for Substance Abuse Treatment.

Center for Substance Abuse Treatment. (2000a). Improving substance abuse treatment: The national treatment plan initiative: Changing the conversation (DHHS Pub No. (SMA) 00-3480, NCADI Pub No. -BKD 383). Rockville, MD: U.S. Department of Health and Human Services, Substance Abuse and Mental Health Services Administration, Center for Substance Abuse Treatment.

Center for Substance Abuse Treatment. (2000b). Integrating substance abuse treatment and vocational services: Treatment Improvement Protocol (TIP) Series 38. (DHHS Pub No. (SMA) 00-3470). [Online]. Retrieved January 31, 2012 from http://lib.adai.washington.edu.

Center for Substance Abuse Treatment. (2001). KAP keys based on TIP 26 substance abuse among older adults: For clinicians. (DHHS Pub. No. (SMA) 01-3586). Rockville, MD: U.S. Department of Health and Human Services, Substance Abuse and Mental Health Services Administration.

Center for Substance Abuse Treatment. (2002a). Substance abuse treatment for persons with child abuse and neglect issues. Treatment improvement protocol (TIP) Series 36 (DHHS Pub. No. (SMA) 023694). Rockville, MD: U.S. Department of Health and Human Services, Substance Abuse and Mental Health Services Administration, Center for Substance Abuse Treatment.

Center for Substance Abuse Treatment. (2002b). Substance abuse treatment and domestic violence. Treatment improvement protocol (TIP) Series 25. Rockville, MD: U.S. Department of Health and Human Services, Substance Abuse and Mental Health Services Administration, Center for Substance Abuse Treatment.

Center for Substance Abuse Treatment. (2003). What you should know about alcohol problems. Substance Abuse in Brief, 2(1), 1-5.

Center for Substance Abuse Treatment. (2004). Clinical Guidelines for the Use of Buprenorphine in the Treatment of Opioid Addiction. Treatment Improvement Protocol (TIP) Series 40 (DHHS Pub. No. (SMA) 04-3939). Rockville, MD: U.S. Department of Health and Human Services, Substance Abuse and Mental Health Services Administration, Center for Substance Abuse Treatment.

Center for Substance Abuse Treatment. (2005a). Acamprosate: A new medication for alcohol use disorders (DHHS Pub. No. (SMA) 05-4114). Substance Abuse Treatment Advisory, 4(1).

Center for Substance Abuse Treatment. (2005b). Substance abuse treatment for persons with cooccurring disorders. Treatment improvement protocol (TIP) Series 42 (DHHS Pub. No. (SMA) 053992). Rockville, MD: U.S. Department of Health and Human Services, Substance Abuse and Mental Health Services Administration, Center for Substance Abuse Treatment. 
Center for Substance Abuse Treatment. (2005c). Substance abuse treatment for adults in the criminal justice system. Treatment improvement protocol (TIP) Series 44 (DHHS Pub. No. (SMA) 05-4056). Rockville, MD: U.S. Department of Health and Human Services, Substance Abuse and Mental Health Services Administration, Center for Substance Abuse Treatment.

Center for Substance Abuse Treatment. (2006a). Addiction counseling competencies: The knowledge, skills, and attitudes of professional practice: Technical assistance publication (TIP) Series 21 (DHHS Pub. No. (SMA) 08-4171). Rockville, MD: U.S. Department of Health and Human Services, Substance Abuse and Mental Health Services Administration, Center for Substance Abuse Treatment.

Center for Substance Abuse Treatment. (2006b). Detoxification and substance abuse treatment: Cooccurring medical and psychiatric conditions. Treatment improvement protocol (TIP) Series 45 (DHHS Pub. No. (SMA) 06-4131). Rockville, MD: U.S. Department of Health and Human Services, Substance Abuse and Mental Health Services Administration, Center for Substance Abuse Treatment.

Center for Substance Abuse Treatment. (2006c). Prescription medications: Misuse, abuse, dependence, and addiction. Substance Abuse Treatment Advisory, 5(2), 1-4.

Center for Substance Abuse Treatment. (2008). An introduction to mutual support groups for alcohol and drug abuse. Substance Abuse in Brief Fact Sheet, 5(1).

Center for Substance Abuse Treatment. (2009a). A provider's introduction to substance abuse treatment for lesbian, gay, bisexual, and transgender individuals. (DHHS Pub No. (SMA) 09-4104). [Online]. Retrieved May 7, 2012 from http://kap.samhsa.gov.

Center for Substance Abuse Treatment. (2009b). Incorporating alcohol pharmacotherapies into medical practice. Treatment Improvement Protocol (TIP) Series 49. (DHHS Pub. No. (SMA) 09-4380).

Rockville, MD: U.S. Department of Health and Human Services, Substance Abuse and Mental Health Services Administration.

Center for Substance Abuse Treatment. (2009c). Substance abuse treatment: Addressing the specific needs of women. Treatment Improvement Protocol (TIP) Series 51. (DHHS Pub No (SMA) 09-4426). Rockville, MD: U.S. Department of Health and Human Services, Substance Abuse and Mental Health Services Administration.

Center for Substance Abuse Treatment. (2009d). The facts about buprenorphine for the treatment of opioid addiction. [Online]. Retrieved May 25, 2012 from http://www.kap.samhsa.gov.

Center for Substance Abuse Treatment. (2009e). The facts about naltrexone for the treatment of opioid addiction. [Online]. Retrieved December 21, 2011 from http://www.kap.samhsa.gov.

Center for Substance Abuse Treatment. (2010). Incorporating alcohol pharmacotherapies into medical practice: A review of the literature. Update. Reviews literature from November 1, 2009 through June 10, 2010. Treatment Improvement Protocol (TIP) Series 49. Rockville, MD: U.S. Department of Health and Human Services, Substance Abuse and Mental Health Services Administration, Center for Substance Abuse Treatment.

Center for Substance Abuse Treatment. (2011). About buprenorphine therapy. [Online]. Retrieved August 10, 2011 from http://buprenorphine.samhsa.gov.

Center for Substance Abuse Treatment. (2012). Buprenorphine information center. [Online]. Retrieved January 24, 2012 from http://buprenorphine.samhsa.gov.

Center for Tobacco Cessation. (2011). Current treatment programs: What are states doing now on cessation? [Online]. Retrieved November 14, 2011 from http://www.ttac.org.

Center for Tobacco Research and Intervention, University of Wisconsin Medical School. (2005). Insights: Smoking in Wisconsin. Barriers to quitting smoking . Madison,WI: Author. 
Centers for Disease Control and Prevention, Coordinating Center for Health Promotion. (2008). Targeting tobacco use: The nation's leading cause of preventable death 2008. [Online]. Retrieved March 9, 2011 from http://www.cdc.gov.

Centers for Disease Control and Prevention, National Center for Injury Prevention and Control. (2011). Prescription painkiller overdoses in the U.S. [Online]. Retrieved January 9, 2012 from http://www.cdc.gov.

Centers for Disease Control and Prevention. (1992). Cigarette smoking among adults - United States, 1990. [Online]. Retrieved January 26, 2012 from http://www.cdc.gov.

Centers for Disease Control and Prevention. (1999). Achievements in public health, 1900-1999: Tobacco use -- United States, 1900-1999. [Online]. Retrieved November 30, 2011 from http://www.cdc.gov.

Centers for Disease Control and Prevention. (2002a). Drug-associated HIV transmission continues in the United States. [Online]. Retrieved May 10, 2012 from http://www.cdc.gov.

Centers for Disease Control and Prevention. (2002b). Methadone maintenance treatment. Atlanta, GA: U.S. Department of Health and Human Services.

Centers for Disease Control and Prevention. (2002c). Substance abuse treatment for injection drug users: A strategy with many benefits. [Online]. Retrieved March 16, 2012 from http://www.cdc.gov.

Centers for Disease Control and Prevention. (2004a). Surgeon General's reports: Highlights: Four major conclusions of the 2004 report. [Online]. Retrieved January 26, 2012 from http://www.cdc.gov.

Centers for Disease Control and Prevention. (2004b). The health consequences of smoking: A report of the Surgeon General. Altanta, GA: U.S. Department of Health and Human Services, Centers for Disease Control and Prevention, National Center for Chronic Disease Prevention and Health Promotion, Office on Smoking and Health.

Centers for Disease Control and Prevention. (2007). Births: Final data for 2005. National Vital Statistics Reports, 56(6), 1-104.

Centers for Disease Control and Prevention. (2008a). Alcohol and public health: Alcohol-Related Disease Impact (ARDI). [Online]. Retrieved August 16, 2011 from http://apps.nccd.cdc.gov.

Centers for Disease Control and Prevention. (2008b). Smoking-attributable mortality, years of potential life lost, and productivity losses: United States: 2000-2004. Morbidity \& Mortality Weekly Report, 57(45), 1226-1228.

Centers for Disease Control and Prevention. (2009a). Alcohol and suicide among racial/ethnic populations--17 states, 2005-2006. [Online]. Retrieved January 26, 2012 from http://www.cdc.gov.

Centers for Disease Control and Prevention. (2009b). Ambulatory care use and physician visits. [Online]. Retrieved February 27, 2009 from http://www.cdc.gov.

Centers for Disease Control and Prevention. (2010a). Online tools: Alcohol-Related Disease Impact (ARDI) software. [Online]. Retrieved August 16, 2011 from http://www.cdc.gov.

Centers for Disease Control and Prevention. (2010b). State Medicaid coverage for tobacco-dependence treatments --- United States, 2009. Morbidity \& Mortality Weekly Report, 59(41), 1340-1343.

Centers for Disease Control and Prevention. (2010c). Tobacco use: Targeting the nation's leading killer. Atlanta, GA: Centers for Disease Control and Prevention, National Center for Chronic Disease Prevention and Health Promotion.

Centers for Disease Control and Prevention. (2010d). Unintentional drug poisoning in the United States. Atlanta, GA: Author. 
Centers for Disease Control and Prevention. (2010e). Vaginal and vulvar cancers screening. [Online]. Retrieved December 8, 2011 from http://www.cdc.gov.

Centers for Disease Control and Prevention. (2011a). 2009 National Youth Tobacco Survey codebook. [Online]. Retrieved February 9, 2012 from http://www.cdc.gov.

Centers for Disease Control and Prevention. (2011b). Alcohol and public health: Alcohol-Related Disease Impact (ARDI). [Online]. Retrieved August 16, 2011 from http://apps.nccd.cdc.gov.

Centers for Disease Control and Prevention. (2011c). Alcohol screening. [Online]. Retrieved November 11, 2011 from http://www.cdc.gov.

Centers for Disease Control and Prevention. (2011d). Alcohol use and health. [Online]. Retrieved August 16, 2011 from http://www.cdc.gov/alcohol/fact-sheets/alcohol-use.htm

Centers for Disease Control and Prevention. (2011e). CDC fact sheet. HIV and AIDS among gay and bisexual men. [Online]. Retrieved May 7, 2012 from http://www.cdc.gov.

Centers for Disease Control and Prevention. (2011f). Fast facts: Smoking and tobacco use: Tobacco related mortality. [Online]. Retrieved May 10, 2012 from http://www.cdc.gov.

Centers for Disease Control and Prevention. (2011g). International classification of diseases, tenth revision, clinical modification (ICD-10-CM). [Online]. Retrieved July 11, 2011 from http://www.cdc.gov.

Centers for Disease Control and Prevention. (2011h). National diabetes fact sheet: National estimates and general information on diabetes and prediabetes in the United States, 2011. [Online]. Retrieved March 28, 2012 from http://www.cdc.gov.

Centers for Disease Control and Prevention. (2011i). Quitting smoking among adults - United States, 2001-2010. Morbidity \& Mortality Weekly Report, 60(44),1513-1519.

Centers for Disease Control and Prevention. (2011j). Vital signs: Overdoses of prescription opioid pain relievers--United States, 1999-2008. Morbidity \& Mortality Weekly Report, 60(43),1487-1492.

Centers for Disease Control and Prevention. (2011k). Vital signs: Colorectal cancer screening, incidence, and mortality--United States, 2002-2010. [Online]. Retrieved December 8, 2011 from http://www.cdc.gov.

Centers for Disease Control and Prevention. (20111). Vital signs: Current cigarette smoking among adults aged $=18$ years --- United States, 2005--2010. [Online]. Retrieved February 28, 2012 from http://www.cdc.gov.

Centers for Disease Control and Prevention. (2012a). 2010 Behavioral risk factor surveillance system combined landline and cell phone developmental dataset, adults aged 18 and older, US. Morbidity \& Mortality Weekly Report, 61(1), 14-19.

Centers for Disease Control and Prevention. (2012b). Summary health statistics for U.S. adults: National Health Interview Survey, 2010: Data from the National Health Interview Survey. Vital \& Health Statistics, 10(252).

Centers for Medicare and Medicaid Services, Center for Consumer Information and Insurance Oversight. (2012). The Mental Health Parity and Addiction Equity Act. [Online]. Retrieved March 14, 2012 from http://cciio.cms.gov.

Centers for Medicare and Medicaid Services. (2010a). Health homes for enrollees with chronic conditions. [Online]. Retrieved March 12, 2012 from https://www.cms.gov.

Centers for Medicare and Medicaid Services. (2010b). HHS announces Medicare expands coverage of tobacco cessation counseling. [Online]. Retrieved July 7, 2011 from http://www.cms.gov. 
Centers for Medicare and Medicaid Services. (2010c). Medicare prescription drug benefit manual. Chapter 6 - Part D drugs and formulary requirements. [Online]. Retrieved April 5, 2012 from https://www.cms.gov.

Centers for Medicare and Medicaid Services. (2010d). National Coverage Determination (NCD) for counseling to prevent tobacco use (210.4.1). [Online]. Retrieved May 14, 2012 from http://www.cms.gov.

Centers for Medicare and Medicaid Services. (2011a). "Incident to" services. [Online]. Retrieved April 5, 2012 from https://www.cms.gov.

Centers for Medicare and Medicaid Services. (2011b). Accountable care organizations: What providers need to know. [Online]. Retrieved March 28, 2012 from https://www.cms.gov.

Centers for Medicare and Medicaid Services. (2011c). Conditions for coverage (CfCs) and conditions of participations (CoPs). Overview. [Online]. Retrieved February 15, 2012 from http://www.cms.gov.

Centers for Medicare and Medicaid Services. (2011d). Medicare and you 2012. [Online]. Retrieved April 5, 2012 from http://www.medicare.gov.

Centers for Medicare and Medicaid Services. (2012a). Frequently asked questions on essential health benefits bulletin. [Online]. Retrieved April 6, 2012 from http://cciio.cms.gov.

Centers for Medicare and Medicaid Services. (2012b). National Coverage Determination (NCD) for treatment of drug abuse (chemical dependency) (130.6). [Online]. Retrieved April 4, 2012 from https://www.cms.gov.

Centers for Medicare and Medicaid Services. (2012c). National Coverage Determination (NCD) for inpatient hospital stays for treatment of alcoholism (130.1). [Online]. Retrieved February 17, 2012 from http://www.cms.gov.

Centers for Medicare and Medicaid Services. (2012d). National Coverage Determination (NCD) for outpatient hospital services for treatment of alcoholism (130.2). [Online]. Retrieved April 4, 2012 from https://www.cms.gov.

Centers for Medicare and Medicaid Services. (2012e). National Coverage Determination (NCD) for treatment of alcoholism and drug abuse in a freestanding clinic (130.5). [Online]. Retrieved April 4, 2012 from https://www.cms.gov.

Centers for Medicare and Medicaid Services. (2012f). National Coverage Determination (NCD) for withdrawal treatments for narcotic addictions (130.7). [Online]. Retrieved April 4, 2012 from https://www.cms.gov.

Centers for Medicare and Medicaid Services. (2012g). Substance (other than tobacco) abuse structured assessment and brief intervention (SBIRT) services. Medicare Learning Network. (ICN 904084). [Online]. Retrieved May 11, 2012 from http://www.cms.gov.

Cepeda-Benito, A. (1993). Meta-analytical review of the efficacy of nicotine chewing gum in smoking treatment programs. Journal of Consulting \& Clinical Psychology, 61(5), 822-830.

Çepik, A., Arikan, Z., Boratav, C., \& Isik, E. (1995). Bulimia in a male alcoholic: A symptom substitution in alcoholism. International Journal of Eating Disorders, 17(2), 201-204.

Cerda, M., Wall, M., Keyes, K. M., Galea, S., \& Hasin, D. (2012). Medical marijuana laws in 50 states: Investing the relationship between state legalization of medical marijuana and marijuana use, abuse and dependence. Drug \& Alcohol Dependence, 120(1-3), 22-27. 
Chafetz, M. E., Blane, H. T., Abram, H. S., Golner, J., Lacy, E., McCourt, W. F., et al. (1962). Establishing treatment relations with alcoholics. Journal of Nervous \& Mental Disease, 134(5), 395409.

Chambers, R. A., Taylor, J. R., \& Potenza, M. N. (2003). Developmental neurocircuitry of motivation in adolescence: A critical period of addiction vulnerability. American Journal of Psychiatry, 160(6), 1041-1052.

Chan, Y. F., Dennis, M. L., \& Funk, R. R. (2008). Prevalence and comorbidity of major internalizing and externalizing problems among adolescents and adults presenting to substance abuse treatment. Journal of Substance Abuse Treatment, 34(1), 14-24.

Chandler, R. K. (2012). Addiction, the brain, and evidence based treatment transcript. [Online]. Retrieved June 1, 2012 from http://www.nij.gov.

Chang, G. (2004). Screening and brief intervention in prenatal care settings. Alcohol Research \& Health, 28(2), 81-84.

Chang, G., McNamara, T. K., Orav, E. J., Koby, D., Lavigne, A., Ludman, B., et al. (2005). Brief intervention for prenatal alcohol use: A randomized trial. Obstetrics \& Gynecology, 105(5), 991-998.

Chang, G., Orav, E. J., Jones, J. A., Buynitsky, T., Gonzalez, S., \& Wilkins-Haug, L. (2011). Selfreported alcohol and drug use in pregnant young women: A pilot study of associated factors and identification. Journal of Addiction Medicine, 5(3), 221-226.

Chang, G., Wilkins-Haug, L., Berman, S., \& Goetz, M. A. (1999a). Brief intervention for alcohol use in pregnancy: A randomized trial. Addiction, 94(10), 1499-1508.

Chang, G., Wilkins-Haug, L., Berman, S., \& Goetz, M. A. (1999b). The TWEAK: Application in a prenatal setting. Journal of Studies on Alcohol, 60(3), 306-309.

Chang, P. H., \& Steinberg, M. B. (2001). Alcohol withdrawal. Medical Clinics of North America, 85(5), 1191-1212.

Chantarujikapong, S. I., Smith, E. M., \& Fox, L. W. (1997). Comparison of the Alcohol Dependence Scale and diagnostic interview schedule in homeless women. Alcoholism: Clinical \& Experimental Research, 21(4), 586-595.

Chao, S. (2007). Advancing quality improvement research: Challenges and opportunities - Workshop summary. Washington, DC: National Academies Press.

Charlesworth, A., \& Glantz, S. A. (2005). Smoking in the movies increases adolescent smoking: A review. Pediatrics, 116(6), 1516-1528.

Chawdhary, A., Sayre, S. L., Green, C., Schmitz, J. M., Grabowski, J., \& Mooney, M. E. (2007). Moderators of delay tolerance in treatment-seeking cocaine users. Addictive Behaviors, 32(2), 370376.

Chen, K., Kandel, D. B., \& Davies, M. (1997). Relationships between frequency and quantity of marijuana use and last year proxy dependence among adolescents and adults in the United States. Drug \& Alcohol Dependence, 46(1-2), 53-67.

Chen, M. J., Grube, J. W., \& Gruenewald, P. J. (2009). Community alcohol outlet density and underage drinking. Addiction, 105(2), 270-278.

Cherpitel, C. J. (1999). Screening for alcohol problems in the U.S. general population: A comparison of the CAGE and TWEAK by gender, ethnicity, and services utilization. Journal of Studies on Alcohol, 60(5), 705-711. 
Cherry, L. (1993). Institute on alcohol, drugs, and disability: From grassroots activity to systems changes. In A. W. Heinemann (Ed.), Substance abuse and physical disability (pp. 191-215). New York: Haworth Press.

Chesher, N. J., Bousman, C. A., Gale, M., Norman, S. B., Twamley, E. W., Heaton, R. K., et al. (2011). Chronic illness histories of adults entering treatment for co-occurring substance abuse and other mental health disorders. American Journal on Addictions, 21(1), 1-4.

Chezem, L. (2005). Legal barriers to alcohol screening in emergency departments and trauma centers. Alcohol Research \& Health, 28(2), 73-77.

Chi, F. W., Campbell, C. I., Sterling, S., \& Weisner, C. (2011). Twelve-step attendance trajectories over 7 years among adolescents entering substance use treatment in an integrated health plan. Addiction, 107(5), 933-942.

Chi, K. R. (2011). Vaccines move forward against a range of addictions. Nature Medicine, 17(2), 146146.

Chick, J., Anton, R., Checinski, K., Croop, R., Drummond, D. C., Farmer, R., et al. (2000). A multicentre, randomized, double-blind, placebo-controlled trial of naltrexone in the treatment of alcohol dependence or abuse. Alcohol \& Alcoholism, 35(6), 587-593.

Chriqui, J. F., Terry-McElrath, Y., McBride, D. C., Eidson, S. S., \& VanderWaal, C. J. (2007). Does state certification or licensure influence outpatient substance abuse treatment program practices? Journal of Behavioral Health Services \& Research, 34(3), 309-328.

Chu, L. F., Liang, D. Y., Li, X., Sahbaie, P., D'arcy, N., Liao, G., et al. (2009). From mouse to man: The 5-HT3 receptor modulates physical dependence on opioid narcotics. Pharmacogenetics \& Genomics, 19(3), 193-205.

Chun, J. S., Guydish, J., \& Chan, Y. F. (2007). Smoking among adolescents in substance abuse treatment: A study of programs, policy, and prevalence. Journal of Psychoactive Drugs, 39(4), 443-449.

Cinciripini, P. M., Cinciripini, L. G., Wallfisch, A., Haque, W., \& Van, V. H. (1996). Behavior therapy and the transdermal nicotine patch: Effects on cessation outcome, affect, and coping. Journal of Consulting \& Clinical Psychology, 64(2), 314-323.

Clark, D. B., Bukstein, O., \& Cornelius, J. (2002). Alcohol use disorders in adolescents: Epidemiology, diagnosis, psychosocial interventions, and pharmacological treatment. Paediatric Drugs, 4(8), 493502.

Clark, D. B., Wood, D. S., Martin, C. S., Cornelius, J. R., Lynch, K. G., \& Shiffman, S. (2005). Multidimensional assessment of nicotine dependence in adolescents. Drug \& Alcohol Dependence, 77(3), 235-242.

Clark, H. W. (2001). Residential substance abuse treatment for pregnant and postpartum women and their children: Treatment and policy implications. Child Welfare, 80(2), 179-1.

Clark, H. W. (2011). A truly integrated treatment system must be both patient- and process-focused. Addiction, 106(12), 2068-2069.

Clark, M. (2011). Conceptualising addiction: How useful is the construct? International Journal of Humanities \& Social Science, 1(13), 55-64.

Clark, R. E., Samnaliev, M., \& McGovern, M. P. (2009). Impact of substance disorders on medical expenditures for Medicaid beneficiaries with behavioral health disorders. Psychiatric Services, 60(1), $35-42$. 
Clark, R. E., Samnaliev, M., Baxter, J. D., \& Leung, G. Y. (2011). The evidence doesn't justify steps by state Medicaid programs to restrict opioid addiction treatment with buprenorphine. Health Affairs, 30(8), 1425-1433.

Clay, R. A. (2004). The workforce crisis: SAMHSA's response. SAMHSA News, 12(6).

Clay, R. A. (2007). Rural substance abuse: Overcoming barriers to prevention and treatment. SAMHSA News, 15(4), 1-6.

Cleary, P. D., Miller, M., Bush, B. T., Warburg, M. M., DelBanco, T. L., \& Aronson, M. D. (1988). Prevalence and recognition of alcohol abuse in a primary care population. American Journal of Medicine, 85(4), 466-471.

Clements, R. (2002). Psychometric properties of the Substance Abuse Subtle Screening Inventory-3. Journal of Substance Abuse Treatment, 23(4), 419-423.

Clinical Practice Guideline Treating Tobacco Use and Dependence 2008 Update Panel, Liaisons, and Staff. (2008). A clinical practice guideline for treating tobacco use and dependence: 2008 update. A U.S. Public Health Service report. American Journal of Preventive Medicine, 35(2), 158-176.

Cocco, K. M., \& Carey, K. B. (1998). Psychometric properties of the Drug Abuse Screening Test in psychiatric outpatients. Psychological Assessment, 10(4), 408-414.

Cochran, B. N., Peavy, K. M., \& Robohm, J. S. (2007). Do specialized services exist for LGBT individuals seeking treatment for substance misuse? A study of available treatment programs. Substance Use \& Misuse, 42(1), 161-176.

Cochran, S. D., Ackerman, D., Mays, V. M., \& Ross, M. W. (2004). Prevalence of non-medical drug use and dependence among homosexually active men and women in the US population. Addiction, 99(8), 989-998.

Cochran, S. D., Sullivan, J. G., \& Mays, V. M. (2003). Prevalence of mental disorders, psychological distress, and mental health services use among lesbian, gay, and bisexual adults in the United States. Journal of Consulting \& Clinical Psychology, 71(1), 53-61.

Code Me. R. § 14-118-005 (2012).

Code R.I. Reg. 18030003 (2012).

Cohen, A. (2011). Drug testing the poor: Bad policy, even worse law. [Online]. Retrieved December 16, 2011 from http://ideas.time.com.

Colby, S. M., Tiffany, S. T., Shiffman, S., \& Niaura, R. S. (2000). Measuring nicotine dependence among youth: A review of available approaches and instruments. Drug \& Alcohol Dependence, 59(Suppl. 1), s23-s29.

Colker, A. C. (2004). Pharmacotherapy treatment of alcoholism and drug addiction: Overview and bibliography. [Online]. Retrieved June 1, 2012 from http://www.ncsl.org.

Collins, S. R., Doty, M. M., Robertson, R., \& Garber, T. (2011). Help on the horizon. How the recession has left millions of workers without health insurance, and how health reform will bring relief. Findings from The Commonwealth Fund Biennial Health Insurance Survey of 2010. [Online]. Retrieved February 1, 2012 from http://www.commonwealthfund.org.

Comer, S. D., Sullivan, M. A., Vosburg, S. K., Manubay, J., Amass, L., Cooper, Z. D., et al. (2010). Abuse liability of intravenous buprenorphine/naloxone and buprenorphine alone in buprenorphinemaintained intravenous heroin abusers. Addiction, 105(4), 709-718. 
Comer, S. D., Sullivan, M. A., Yu, E., Rothenberg, J. L., Kleber, H. D., Kampman, K. M., et al. (2006). Injectable, sustained-release naltrexone for the treatment of opioid dependence: A randomized, placebo-controlled trial. Archives of General Psychiatry, 63(2), 210-218.

Commission on Accreditation of Rehabilitation Facilities (CARF). (2012). Quick facts about CARF. [Online].from http://www.carf.org.

Committee on Substance Abuse. (2011). Substance use screening, brief intervention, and referral to treatment for pediatricians. Journal of the American Academy of Pediatrics. 128, e1330. doi: 10.1542/peds.2011-1754.

Commonwealth Fund. (2010). Major provisions of the Affordable Care Act. [Online]. Retrieved March 27, 2012 from http://www.commonwealthfund.org.

Comprehensive Alcohol Abuse and Alcoholism Prevention, Treatment, and Rehabilitation Act of 1970, $84 \S 1848$ (1970).

Condon, T. P., Miner, L. L., Balmer, C. W., \& Pintello, D. (2008). Blending addiction research and practice: Strategies for technology transfer. Journal of Substance Abuse Treatment, 35(2), 156-160.

Congressional Budget Office. (2007). The State Children's Health Insurance Program. Washington, DC: Author.

Congressional Budget Office. (2012). Cost estimate: S. 543 Mental Health Equitable Treatment Act of 2001. [Online]. Retrieved May 16, 2012 from http://www.cbo.gov.

Conley, T. B. (2001). Construct validity of the MAST and AUDIT with multiple offender drunk drivers. Journal of Substance Abuse Treatment, 20(4), 287-295.

Conn. Agencies Regs. § 17-134d-63 (2012).

Connecticut Department of Public Health. (2009). Connecticut public health code, 19a-495-570. [Online]. Retrieved November 11, 2009 from http://www.ct.gov.

Connell, C. M., Gilreath, T. D., Aklin, W. M., \& Brex, R. A. (2010). Social-ecological influences on patterns of substance use among non-metropolitan high school students. American Journal of Community Psychology, 45(1-2), 36-48.

Connor, S. E., Cook, R. L., Herbert, M. I., Neal, S. M., \& Williams, J. T. (2002). Smoking cessation in a homeless population. There is a will, but is there a way? Journal of General Internal Medicine, 17(5), 369-372.

Cook, D. A., \& Beckman, T. J. (2006). Current concepts in validity and reliability for psychometric instruments: Theory and application. American Journal of Medicine, 119(2), 166-16.

Cook, R. L., Comer, D. M., Wiesenfeld, H. C., Chang, C. C., Tarter, R., Lave, J. R., et al. (2006). Alcohol and drug use and related disorders: An underrecognized health issue among adolescents and young adults attending sexually transmitted disease clinics. Sexually Transmitted Diseases, 33(9), 565-570.

Cooperberg, M. R., Birkmeyer, J. D., \& Litwin, M. S. (2009). Defining high quality health care. Urologic Oncology, 27(4), 411-416.

Copeland, J., \& Martin, G. (2004). Web-based interventions for substance use disorders: A qualitative review. Journal of Substance Abuse Treatment, 26(2), 109-116.

Copello, A. G., Templeton, L., \& Velleman, R. (2006). Family interventions for drug and alcohol misuse: Is there a best practice? Current Opinion in Psychiatry, 19(3), 271-276. 
Cornelius, J. R., Bukstein, O. G., Birmaher, B., Salloum, I. M., Lynch, K., Pollock, N. K., et al. (2001). Fluoxetine in adolescents with major depression and an alcohol use disorder: An open-label trial. Addictive Behaviors, 26(5), 735-739.

Cornelius, J. R., Salloum, I. M., Haskett, R. F., Daley, D. C., Cornelius, M. D., Thase, M. E., et al. (2000). Fluoxetine versus placebo in depressed alcoholics: A 1-year follow-up study. Addictive Behaviors, 25(2), 307-310.

Cornell University Law School, Legal Information Institute. (2012). 29 USC § 1185A: Parity in Mental Health and Substance Abuse. [Online]. from http://www.law.cornell.edu.

Cornuz, J., Zwahlen, S., Jungi, W. F., Osterwalder, J., Klingler, K., van, M. G., et al. (2008). A vaccine against nicotine for smoking cessation: A randomized controlled trial. PLoS Medicine, 3(6), e2547.

Corrao, G., Bagnardi, V., Zambon, A., \& La, V. C. (2004). A meta-analysis of alcohol consumption and the risk of 15 diseases. Preventive Medicine, 38(5), 613-619.

Corrao, G., Rubbiati, L., Zambon, A., \& Arico, S. (2002). Alcohol-attributable and alcohol-preventable mortality in Italy. A balance in 1983 and 1996. European Journal of Public Health, 12(3), 214-223.

Correia, C. J., \& Benson, T. A. (2006). The use of contingency management to reduce cigarette smoking among college students. Experimental \& Clinical Psychopharmacology, 14(2), 171-179.

Corrigan, P. W., Kuwabara, S. A., \& O'Shaughnessy, J. (2009). The public stigma of mental illness and drug addiction: Findings from a stratified random sample. Journal of Social Work, 9(2), 139-147.

Corso, P., Finkelstein, E., Miller, T., Fiebelkorn, I., \& Zaloshnja, E. (2006). Incidence and lifetime costs of injuries in the United States. Injury Prevention, 12(4), 212-218.

Costello, D. (2004). Addiction medication has a slow start in the U.S.: Many insurers, including MediCal, aren't covering the opiate substitute's cost. [Online]. Los Angeles Times. Retrieved June 4, 2012 from http://articles.latimes.com.

Costello, D., Dierker, L. C., Sledjeski, E. M., Flaherty, B., Flay, B., Shiffman, S., et al. (2007). Confirmatory factor analysis of the Nicotine Dependence Syndrome Scale in an American college sample of light smokers. Nicotine \& Tobacco Research, 9(8), 811-819.

Cottler, L. B., Robins, L. N., \& Helzer, J. E. (1989). The reliability of the CIDI-SAM: A comprehensive substance abuse interview. British Journal of Addiction, 84(7), 801-814.

Couchenour, R. L., Carson, D. S., \& Segal, A. R. (2002). Patients' views of pharmacists as providers of smoking cessation services. Journal of the American Pharmaceutical Association, 42(3), 510-512.

Council for Accreditation of Counseling and Related Educational Programs. (2009). 2009 Standards. [Online]. Retrieved July 16, 2009 from http://www.cacrep.org.

Council on Accreditation. (2008). About COA. [Online]. Retrieved February 14, 2012 from http://coastandards.org.

Courtwright, D. T. (2001). Dark paradise: A history of opiate addiction in America. Cambridge, MA: Harvard University Press.

Courtwright, D. T. (2010). The NIDA brain disease paradigm: History, resistance and spinoffs. [Online]. Retrieved December 5, 2011 from http://digitalcommons.unf.edu.

Courtwright, D. T. (2011). Language-use disorder: Comment on DSM-V's proposed 'addiction and related disorders' and Charles O'Brien's 'Addiction and dependence in DSM-V'. Addiction, 106(5), 878-879. 
Courvoisier, D., \& Etter, J.-F. (2008). Using item response theory to study the convergent and discriminant validity of three questionnaires measuring cigarette dependence. Psychology of Addictive Behaviors, 22(3), 391-401.

Couwels, J. (2011). Federal judge temporarily bars Florida's welfare drug-test law. [Online]. Retrieved January 17, 2011 from http://www.cnn.com.

Cowell, A., McCarty, D., \& Woodward, A. (2003). Impact of federal substance abuse block grants on state substance abuse spending: Literature and data review. Journal of Mental Health Policy \& Economics, 6(4), 173-179.

Crews, F. T., \& Boettiger, C. A. (2009). Impulsivity, frontal lobes and risk for addiction. Pharmacology Biochemistry \& Behavior, 93(3), 237-247.

Crews, F. T., Mdzinarishvili, A., Kim, D., He, J., \& Nixon, K. (2006). Neurogenesis in adolescent brain is potently inhibited by ethanol. Neuroscience, 137(2), 437-445.

Crews, F., He, J., \& Hodge, C. (2007). Adolescent cortical development: A critical period of vulnerability for addiction. Pharmacology Biochemistry \& Behavior, 86(2), 189-199.

Crews, K. M., Cobb, G. W., Seago, D., \& Williams, N. (1999). Tobacco and dental implants. General Dentistry, 47(5), 484-488.

Cromwell, J., Bartosch, W. J., Fiore, M. C., Hasselblad, V., \& Baker, T. (1997). Cost-effectiveness of the clinical practice recommendations in the AHCPR guideline for smoking cessation. JAMA, 278(21), 1759-1766.

Croog, S. H., Shapiro, D. S., \& Levine, S. (1971). Denial among male heart patients. An empirical study. Psychosomatic Medicine, 33(5), 385-397.

Crosier, A. (2004). Homelessness, smoking and health. London, England: Health Development Agency.

Crosson, F. J. (2011). The accountable care organization: Whatever its growing pains, the concept is too vitally important to fail. Health Affairs, 30(7), 1250-1255.

Crowley, J. S., \& O'Malley, M. (2007). Medicaid and the uninsured: Medicaid's rehabilitation services option: Overview and current policy issues. [Online]. Retrieved April 6, 2012 from http://www.kff.org.

Crum, R. M., Green, K. M., Storr, C. L., Chan, Y. F., Ialongo, N., Stuart, E. A., et al. (2008). Depressed mood in childhood and subsequent alcohol use through adolescence and young adulthood. Archives of General Psychiatry, 65(6), 702-712.

Crum, R. M., Storr, C. L., Ialongo, N., \& Anthony, J. C. (2008). Is depressed mood in childhood associated with an increased risk for initiation of alcohol use during early adolescence? Addictive Behaviors, 33(1), 24-40.

Cryer, H. G. (2005). Barriers to interventions for alcohol problems in trauma centers. Journal of Trauma, 59(Suppl. 3), S104-S111.

Culberson, J. W., \& Ziska, M. (2008). Prescription drug misuse/abuse in the elderly. Geriatrics, 63(9), 22-31.

Cummings, J. R., Wen, H., \& Druss, B. G. (2011). Racial/ethnic differences in treatment for substance use disorders among U.S. adolescents. Journal of the American Academy of Child \& Adolescent Psychiatry, 50(12), 1265-1274.

Cummins, L. H., Chan, K. K., Burns, K. M., Blume, A. W., Larimer, M., \& Marlatt, G. A. (2003). Validity of the CRAFFT in American-Indian and Alaska-Native adolescents: Screening for drug and alcohol risk. Journal of Studies on Alcohol, 64(5), 727-732. 
Cunningham, J. A., Sobell, L. C., Sobell, M. B., Agrawal, S., \& Toneatto, T. (1993). Barriers to treatment: Why alcohol and drug abusers delay or never seek treatment. Addictive Behaviors, 18(3), 347-353.

Cunningham, R. M., Harrison, S. R., McCay, M. P., Mello, M. J., Sochor, M., Shandro, M., et al. (2010). National survey of emergency department alcohol screening and intervention practices. Annals of Emergency Medicine, 55(6), 556-562.

Curie, C. G. (2006). From the administrator: The value of screening and brief intervention. SAMHSA News, 14(1).

Curley, B. (2002). FDA approves two forms of buprenorphine for opiate treatment. [Online]. Retrieved March 27, 2009 from http://www.jointogether.org.

Curran, G. M., Sullivan, G., Williams, K., Han, X., Collins, K., Keys, J., et al. (2003). Emergency department use of persons with comorbid psychiatric and substance abuse disorders. Annals of Emergency Medicine, 41(5), 659-667.

Curtis, J. R., Geller, G., Stokes, E. J., Levine, D. M., \& Moore, R. D. (1989). Characteristics, diagnosis, and treatment of alcoholism in elderly patients. Journal of the American Geriatrics Society, 37(4), 310-316.

D. C. Mun. Regs. 29, § 2365 (2012).

Dackis, C. A., Kampman, K. M., Lynch, K. G., Pettinati, H. M., \& O'Brien, C. P. (2005). A double-blind, placebo-controlled trial of modafinil for cocaine dependence. Neuropsychopharmacology, 30(1), 205211.

Dackis, C. A., Lynch, K. G., Yu, E., Samaha, F. F., Kampman, K. M., Cornish, J. W., et al. (2003). Modafinil and cocaine: A double-blind, placebo-controlled drug interaction study. Drug \& Alcohol Dependence, 70(1), 29-37.

Dackis, C., \& O'Brien, C. P. (2005). Neurobiology of addiction: Treatment and public policy ramifications. Nature Neuroscience, 8(11), 1431-1436.

Daeppen, J. B., Yersin, B., Landry, U., Pecoud, A., \& Decrey, H. (2000). Reliability and validity of the Alcohol Use Disorders Identification Test (AUDIT) imbedded within a general health risk screening questionnaire: Results of a survey in 332 primary care patients. Alcoholism: Clinical and Experimental Research, 24(5), 659-665.

Dahl, R. E. (2004). Adolescent brain development: A period of vulnerabilities and opportunities. Keynote address. Annals of the New York Academy of Sciences, 1021, 1-22.

Dailard, C., \& Nash, E. (2000). State responses to substance abuse among pregnant women. The Guttmacher Report on Public Policy, 3(6), 1-4.

Dalton, M. A., Sargent, J. D., Beach, M. L., Titus-Ernstoff, L., Gibson, J. J., Ahrens, M. B., et al. (2003). Effect of viewing smoking in movies on adolescent smoking initiation: A cohort study. Lancet, 362(9380), 281-285.

Daly, R. (2006). New Freedom Commission members assess report's impact. [Online]. from http://pnhw.psychiatryonline.org.

D'Amico, E. J., Miles, J. N. V., Stern, S. A., \& Meredith, L. S. (2008). Brief motivational interviewing for teens at risk of substance use consequences: A randomized pilot study in a primary care clinic.

Journal of Substance Abuse Treatment, 35(1), 53-61. 
D'Amico, E. J., Paddock, S. M., Burnam, A., \& Kung, F. Y. (2005). Identification of and guidance for problem drinking by general medical providers: Results from a national survey. Medical Care, 43(3), 229-236.

Daniels, N. (1985). Just health care. New York: Cambridge University Press.

Datar, A., \& Jacknowitz, A. (2009). Birth weight effects on children's mental, motor, and physical development: Evidence from twins data. Maternal \& Child Health Journal, 13(6), 780-794.

D'Aunno, T. (2006). The role of organization and management in substance abuse treatment: Review and roadmap. Journal of Substance Abuse Treatment, 31(3), 221-233.

D'Aunno, T., \& Pollack, H. A. (2002). Changes in methadone treatment practices. Results from a national panel study, 1988-2000. JAMA, 288(7), 850-856.

Dave, D., \& Mukerjee, S. (2011). Mental health parity legislation, cost-sharing and substance-abuse treatment admissions. Health Economics, 20(2), 161-183.

Dawes, M. A., \& Johnson, B. A. (2004). Pharmacotherapeutic trials in adolescent alcohol use disorders: Opportunities and challenges. Alcohol \& Alcoholism, 39(3), 166-177.

Dawes, M. A., Johnson, B. A., Ait-Daoud, N., Ma, J. Z., \& Cornelius, J. R. (2005). A prospective, openlabel trial of ondansetron in adolescents with alcohol dependence. Addictive Behaviors, 30(6), 10771085 .

Dawes, M. A., Johnson, B. A., Ma, J. Z., Ait-Daoud, N., Thomas, S. E., \& Cornelius, J. R. (2005). Reductions in and relations between "craving" and drinking in a prospective, open-label trial of ondansetron in adolescents with alcohol dependence. Addictive Behaviors, 30(9), 1630-1637.

Dawson, D. A., Grant, B. F., Stinson, F. S., Chou, P. S., Huang, B., \& Ruan, W. J. (2005). Recovery from DSM-IV alcohol dependence: United States, 2001-2002. Addiction, 100(3), 281-292.

de Leon, J., Diaz, F. J., Becona, E., Gurpegui, M., Jurado, D., \& Gonzalez-Pinto, A. (2003). Exploring brief measures of nicotine dependence for epidemiological surveys. Addictive Behaviors, 28(8), 14811486.

Deas, D., \& Clark, A. (2009). Current state of treatment for alcohol and other drug use disorders in adolescents. Alcohol Research \& Health, 32(1), 76-82.

Deas-Nesmith, D., Campbell, S., \& Brady, K. T. (1998). Substance use disorders in an adolescent inpatient psychiatric population. Journal of the National Medical Association, 90(4), 233-238.

DeCew, J. W. (1994). Testing balancing privacy and public safety. Hastings Center Report, 24(2), 17-23.

Defense Centers of Excellence. (2011). Co-occurring conditions toolkit: Mild traumatic brain injury and psychological health. Concussion, post-traumatic stress, depression, chronic pain, headache, substance use disorder. [Online]. Retrieved February 2, 2012 from http://www.dcoe.health.mil.

Delaware General Assembly. (2010). Delaware regulations: Administrative code: Title 16: 6000 Division of substance abuse \& mental health. [Online]. Retrieved April 2, 2012 from http://regulations.delaware.gov.

Denisco, R. C., Kenna, G. A., O'Neil, M. G., Kulich, R. J., Moore, P. A., Kane, W. T., et al. (2011). Prevention of prescription opioid abuse: The role of the dentist. Journal of the American Dental Association, 142(7), 800-810.

Denizet-Lewis, B. (June 25, 2006). An anti-addiction pill? [Online]. Retrieved January 30, 2008 from http://www.nytimes.com. 
Dennis, M. L., \& Scott, C. K. (2012). Four-year outcomes from the Early Re-Intervention (ERI) experiment using Recovery Management Checkups (RMCs). Drug \& Alcohol Dependence, 121(1-2), $10-17$.

Dennis, M. L., Scott, C. K., Funk, R., \& Foss, M. A. (2005). The duration and correlates of addiction and treatment careers. Journal of Substance Abuse Treatment, 28(Suppl. 1), S51-S62.

Dennis, M., Godley, S. H., Diamond, G., Tims, F. M., Babor, T., Donaldson, J., et al. (2004). The Cannabis Youth Treatment (CYT) Study: Main findings from two randomized trials. Journal of Substance Abuse Treatment, 27(3), 197-213.

Dennis, M., Scott, C. K., \& Funk, R. (2003). An experimental evaluation of recovery management checkups (RMC) for people with chronic substance use disorders. Evaluation \& Program Planning, 26(3), 339-352.

Dent, L. A., Harris, K. J., \& Noonan, C. W. (2007). Tobacco interventions delivered by pharmacists: A summary and systematic review. Pharmacotherapy, 27(7), 1040-1051.

DePaul University. (2011). Oxford House listed on the national registry of evidence based programs and practices (NREPP) website. [Online]. Retrieved January 25, 2012 from http://csh.depaul.edu.

Desai, R. A., Harpaz-Rotem, I., Najavits, L. M., \& Rosenheck, R. A. (2008). Impact of the Seeking Safety Program on clinical outcomes among homeless female veterans with psychiatric disorders. Psychiatric Services, 59(9), 996-1003.

Desy, P. M., Howard, P. K., Perhats, C., \& Li, S. (2010). Alcohol screening, brief intervention, and referral to treatment conducted by emergency nurses: An impact evaluation. Journal of Emergency Nursing, 36(6), 538-545.

Devenyi, P., Mitwalli, A., \& Graham, W. (1982). Clonidine therapy for narcotic withdrawal. Canadian Medical Association Journal, 127(10), 1009-1011.

DeWit, D. J., Adlaf, E. M., Offord, D. R., \& Ogborne, A. C. (2000). Age at first alcohol use: A risk factor for the development of alcohol disorders. American Journal of Psychiatry, 157(5), 745-750.

Dhalla, I. A. (2011). Facing up to the prescription opioid crisis. BJM, 343(d5142). doi: 10.1136/6mj.d5142.

Diaz, R., Castro-Fornieles, J., Serrano, L., Gonzalez, L., Calvo, R., Goti, J., et al. (2008). Clinical and research utility of Spanish Teen-Addiction Severity Index (T-ASI). Addictive Behaviors, 33(1), 188195.

DiClemente, C. C., Dolan-Mullen, P., \& Windsor, R. A. (2000). The process of pregnancy smoking cessation: Implications for interventions. Tobacco Control, 9 (Suppl. 3), III16-III21. doi: 10.1136/6c.9suppl_3.iii16.

Diemert, L. M., Bondy, S. J., Victor, J. C., Cohen, J. E., Brown, K. S., Ferrence, R., et al. (2008). Efficient screening of current smoking status in recruitment of smokers for population-based research. Nicotine \& Tobacco Research, 10(11), 1663-1667.

Dierker, L. C., Sledjeski, E. M., Marshall, S., \& Johnson, S. (2009). Substance parity laws and the detection and treatment of substance use disorders among adolescents in mental health care. Journal of Dual Diagnosis, 5(1), 2-13.

Digiusto, E., Shakeshaft, A., Ritter, A., O'Brien, S., Mattick, R. P., \& NEPOD Research Group. (2004). Serious adverse events in the Australian National Evaluation of Pharmacotherapies for Opioid Dependence (NEPOD). Addiction, 99(4), 450-460. 
Dilonardo, J. (2011). Workforce issues related to: Physical and behavioral healthcare integration: Specifically substance use disorders and primary care: A framework. [Online]. Retrieved January 27, 2012 from http://www.cimh.org.

Dino, G., Horn, K., Goldcamp, J., Fernandes, A., Kalsekar, I., \& Massey, C. (2001). A 2-year efficacy study of Not On Tobacco in Florida: An overview of program successes in changing teen smoking behavior. Preventive Medicine, 33(6), 600-605.

Dishion, T. J., McCord, J., \& Poulin, F. (1999). When interventions harm. Peer groups and problem behavior. American Psychologist, 54(9), 755-764.

Dishion, T. J., Poulin, F., \& Burraston, B. (2001). Peer group dynamics associated with iatrogenic effects in group interventions with high-risk young adolescents. New Directions for Child \& Adolescent Development, (91), 79-92. doi: 10.1002/cd.6.

Disney, E. R., Elkins, I. J., McGue, M., \& Iacono, W. G. (1999). Effects of ADHD, conduct disorder, and gender on substance use and abuse in adolescence. American Journal of Psychiatry, 156(10), 15151521.

Dolan, K., Rouen, D., \& Kimber, J. (2004). An overview of the use of urine, hair, sweat and saliva to detect drug use. Drug \& Alcohol Review, 23(2), 213-217.

Dolan, T. A., McGorray, S. P., Grinstead-Skigen, C. L., \& Mecklenburg, R. (1997). Tobacco control activities in U.S. dental practices. Journal of the American Dental Association, 128(12), 1669-1679.

Donaldson, M. S., Yordy, K. D., Lohr, K. N., \& Vanselow, N. A. (Eds.). (1996). Primary care: America's health in a new era. Washington, DC: National Academy Press.

Donnelly, J., Young, M., Pearson, R., Penhollow, T. M., \& Hernandez, A. (2008). Area specific selfesteem, values, and adolescent substance use. Journal of Drug Education, 38(4), 389-403.

D'Onofrio, G., \& Degutis, L. C. (2002). Preventive care in the emergency department: Screening and brief intervention for alcohol problems in the emergency department: A systematic review. Academic Emergency Medicine, 9(6), 627-638.

D'Onofrio, G., Nadel, E. S., Degutis, L. C., Sullivan, L. M., Casper, K., Bernstein, E., et al. (2002). Improving emergency medicine residents' approach to patients with alcohol problems: A controlled educational trial. Annals of Emergency Medicine, 40(1), 50-62.

Donovan, B., Padin-Rivera, E., \& Kowaliw, S. (2001). "Transend": Initial outcomes from a posttraumatic stress disorder/substance abuse treatment program. Journal of Traumatic Stress, 14(4), 757-772.

Doremus-Fitzwater, T. L., Varlinskaya, E. I., \& Spear, L. P. (2010). Motivational systems in adolescence: Possible implications for age differences in substance abuse and other risk-taking behaviors. Brain \& Cognition, 72(1), 114-123.

Dornelas, E. A., Sampson, R. A., Gray, J. F., Waters, D., \& Thompson, P. D. (2000). A randomized controlled trial of smoking cessation counseling after myocardial infarction. Preventive Medicine, 30(4), 261-268.

Dowden, C., \& Latimer, J. (2006). Providing effective substance abuse treatment for young-offender populations: What works! Child \& Adolescent Psychiatric Clinics of North America, 15(2), 517-537.

Dowling, G. J., Weiss, S., \& Condon, T. P. (2008). Drugs of abuse and the aging brain. Neuropsychopharmacology, 33(2), 209-218.

Drabble, L. (2007). Pathways to collaboration: Exploring values and collaborative practice between child welfare and substance abuse treatment fields. Child Maltreatment, 12(1), 31-42. 
Drabble, L., Midanik.L.T., \& Trocki, K. (2005). Reports of alcohol consumption and alcohol-related problems among homosexual, bisexual and heterosexual respondents: Results from the 2000 National Alcohol Survey. Journal of Studies on Alcohol, 66(1), 111-120.

Drake, R. E., \& Mueser, K. T. (2002). Co-occurring alcohol use disorder and schizophrenia. Alcohol Research \& Health, 26(2), 99-102.

Drake, R. E., Essock, S. M., Shaner, A., Carey, K. B., Minkoff, K., Kola, L., et al. (2001). Implementing dual diagnosis services for clients with severe mental illness. Psychiatric Services, 52(4), 469-476.

Drake, R. E., McHugo, G. J., Clark, R. E., Teague, G. B., Xie, H., Miles, K., et al. (1998). Assertive community treatment for patients with co-occurring severe mental illness and substance use disorder: A clinical trial. American Journal of Orthopsychiatry, 68(2), 201-215.

Drake, R. E., Mercer-McFadden, C., Mueser, K. T., McHugo, G. J., \& Bond, G. R. (1998). Review of integrated mental health and substance abuse treatment for patients with dual disorders. Schizophrenia Bulletin, 24(4), 589-608.

Drake, R. E., O'Neal, E. L., \& Wallach, M. A. (2008). A systematic review of psychosocial research on psychosocial interventions for people with co-occurring severe mental and substance use disorders. Journal of Substance Abuse Treatment, 34(1), 123-138.

Drake, R. E., Osher, F. C., \& Wallach, M. A. (1991). Homelessness and dual diagnosis. American Psychologist, 46(11), 1149-1158.

Drapkin, M. L., Tate, S. R., McQuaid, J. R., \& Brown, S. A. (2008). Does initial treatment focus influence outcomes for depressed substance abusers? Journal of Substance Abuse Treatment, 35(3), 343-350.

Drug Enforcement Administration. (2011). Controlled substance schedules. [Online]. Retrieved January 24, 2012 from http://www.deadiversion.usdoj.gov.

Drug Information Online. (2011). Butalbital and acetaminophen. [Online]. Retrieved May 31, 2012 from http://www.drugs.com.

Drug Policy Alliance. (2009). Healing a broken system: Veterans battling addiction and incarceration. [Online]. Retrieved May 31, 2012 from http://www.phoenixhouse.org.

Druss, B. G., \& Mauer, B. J. (2012). Health care reform and care at the behavioral health-primary care interface. Psychiatric Services, 61(11), 1087-1092.

Dube, S. R., Felitti, V. J., Dong, M., Chapman, D. P., Giles, W. H., \& Anda, R. F. (2003). Childhood abuse, neglect, and household dysfunction and the risk of illicit drug use: The adverse childhood experiences study. Pediatrics, 111(3), 564-572.

Dufour, M. C., Archer, L., \& Gordis, E. (1992). Alcohol and the elderly. Clinics in Geriatric Medicine, 8(1), 127-141.

DuPont, R. L., \& Baumgartner, W. A. (1995). Drug testing by urine and hair analysis: Complementary features and scientific issues. Forensic Science International, 70(1-3), 63-76.

Dupont, R. L., \& Skipper, G. E. (2012). Six lessons from state Physician Health Programs to promote long-term recovery. Journal of Psychoactive Drugs, 44(1), 72-78.

Dupont, R. L., McLellan, A. T., Carr, G., Gendel, M., \& Skipper, G. E. (2009). How are addicted physicians treated? A national survey of physician health programs. Journal of Substance Abuse Treatment, 37(1), 1-7. 
Durcan, M. J., Deener, G., White, J., Johnston, J. A., Gonzales, D., Niaura, R., et al. (2002). The effect of bupropion sustained-release on cigarette craving after smoking cessation. Clinical Therapeutics, 24(4), 540-551.

Dusing, R. (2006). Overcoming barriers to effective blood pressure control in patients with hypertension. Current Medical Research \& Opinion, 22(8), 1545-1553.

Dyson, V., Appleby, L., Altman, E., Doot, M., Luchins, D. J., \& Delehant, M. (1998). Efficiency and validity of commonly used substance abuse screening instruments in public psychiatric patients. Journal of Addictive Diseases, 17(2), 57-76.

Eby, L. T., Burk, H., \& Maher, C. P. (2010). How serious of a problem is staff turnover in substance abuse treatment? A longitudinal study of actual turnover. Journal of Substance Abuse Treatment, 39(3), 264-271.

Edge, P. J., \& Gold, M. S. (2011). Drug withdrawal and hyperphagia: Lessons from tobacco and other drugs. Current Pharmaceutical Design, 17(12), 1173-1179.

Education-Portal.com. (2012). Substance abuse counselor job duties and education requirements. [Online]. Retrieved March 19, 2012 from http://education-portal.com.

Ehlers, C. L., Gizer, I. R., Vieten, C., \& Wilhelmsen, K. C. (2010). Linkage analyses of cannabis dependence, craving, and withdrawal in the San Francisco family study. American Journal of Medical Genetics. Part B, Neuropsychiatric Genetics, 153B(3), 802-811.

Ehlers, C. L., Gizer, I. R., Vieten, C., Gilder, D. A., Stouffer, G. M., Lau, P., et al. (2010). Cannabis dependence in the San Francisco Family Study: Age of onset of use, DSM-IV symptoms, withdrawal, and heritability. Addictive Behaviors, 35(2), 102-110.

Ehrlich, M. E., Sommer, J., Canas, E., \& Unterwald, E. M. (2002). Periadolescent mice show enhanced FosB upregulation in response to cocaine and amphetamine. Journal of Neuroscience, 22(21), 91559159.

Eisen, S., Schultz, M. R., Vogt, D., Glickman, M. E., Elwy, R., Drainoni, M.-L., et al. (2012). Mental and physical health status and alcohol and drug use following return from deployment to Iraq or Afghanistan. American Journal of Public Health, 102(S1), S66-S73.

El-Bassel, N., Schilling, R. F., Schinke, S., Orlando, M., \& Sun, W. (1997). Assessing the utility of the Drug Abuse Screening Test in the workplace. Research on Social Work Practice, 7(1), 99-114.

Elkins, I. J., McGue, M., \& Iacono, W. G. (2007). Prospective effects of attention-deficit/hyperactivity disorder, conduct disorder, and sex on adolescent substance use and abuse. Archives of General Psychiatry, 64(10), 1145-1152.

Ellason, J. W., \& Ross, C. A. (1996). Axis I and II comorbidity and childhood trauma history in chemical dependency. Bulletin of the Menninger Clinic, 60(1), 39-51.

Elliott, V. S. (2005). Time to enhance "5 A's" of smoking cessation. [Online]. Retrieved March 26, 2012 from http://www.ama-assn.org.

Ensminger, M. E., Brown, C. H., \& Kellam, S. G. (1982). Sex differences in antecedents of substance use among adolescents. Journal of Social Issues, 38(2), 25-42.

Ensuring Solutions to Alcohol Problems. (2008). Workplace screening and brief intervention: What employers can and should do about excessive alcohol use. Washington, DC: George Washington University Medical Center.

Ensuring Solutions to Alcohol Problems. (2009). The promise of the new reimbursement codes: 2008 is the year of SBI. [Online]. Retrieved December 21, 2009 from http://www.ensuringsolutions.org. 
Epstein, J. A., Griffin, K. W., \& Botvin, G. J. (2004). Efficacy, self-derogation, and alcohol use among inner-city adolescents: Gender matters. Journal of Youth \& Adolescence, 33(2), 159-166.

Epstein, J. A., Griffin, K. W., \& Botvin, G. J. (2008). A social influence model of alcohol use for innercity adolescents: Family drinking, perceived drinking norms, and perceived social benefits of drinking. Journal of Studies on Alcohol \& Drugs, 69(3), 397-405.

Epstein, J., Barker, P., Vorburger, M., \& Murtha, C. (2004). Serious mental illness and its co-occurrence with substance use disorders, 2002 (DHHS Publication No. SMA 04-3905, Analytic Series A-24). Rockville, MD: U.S. Department of Health and Human Services, Substance Abuse and Mental Health Services Administration, Office of Applied Studies.

Erickson, C. K. (2007). The science of addiction: From neurobiology to treatment. New York: W.W. Norton.

Erickson, C. K., Wilcox, R. E., Miller, G. W., Littlefield, J. H., \& Lawson, K. A. (2003). Effectiveness of addiction science presentations to treatment professionals, using a modified Solomon study design. Journal of Drug Education, 33(2), 197-216.

Ersche, K. D., Jones, P. S., Williams, G. B., Turton, A. J., Robbins, T. W., \& Bullmore, E. T. (2012). Abnormal brain structure implicated in stimulant drug addiction. Science, 335(6068), 601-604.

Ershoff, D. H., Solomon, L. J., \& Dolan-Mullen, P. (2000). Predictors of intentions to stop smoking early in prenatal care. Tobacco Control, 9 (Suppl. 3), III41-III45.

Escobedo, L. G., Reddy, M., \& Giovino, G. A. (1998). The relationship between depressive symptoms and cigarette smoking in US adolescents. Addiction, 93(3), 433-440.

Essock, S. M., Mueser, K. T., Drake, R. E., Covell, N. H., McHugo, G. J., Frisman, L. K., et al. (2006). Comparison of ACT and standard case management for delivering integrated treatment for cooccurring disorders. Psychiatric Services, 57(2), 185-196.

Etter, J.-F., Duc, T. V., \& Perneger, T. V. (1999). Validity of the Fagerstrom test for nicotine dependence and of the Heaviness of Smoking Index among relatively light smokers. Addiction, 94(2), 269-281.

Ettner, S. L., Huang, D., Evans, E., Ash, D. R., Hardy, M., Jourabchi, M., et al. (2006). Benefit-cost in the California treatment outcome project: Does substance abuse treatment "pay for itself"? Health Services Research, 41(1), 192-213.

Evercare. (2006). Evercare study of caregivers in decline. A close-up look at the health risks of caring for a loved one. Minnetonka, MN: Author.

Executive Office of the President, Office of National Drug Control Policy. (2000). Methadone fact sheet. Washington, DC: Drug Policy Information Clearinghouse.

Eyewitness to History. (2007). Prohibition, 1927. [Online]. Retrieved December 13, 2011 from http://www.eyewitnesstohistory.com.

Ezzati, M., Lopez, A. D., Rodgers, A., Vander Hoorn, S., Murray, C. J. L., \& and the Comparative Risk Assessment Collaborating Group. (2002). Selected major risk factors and global and regional burden of disease. Lancet, 360(9343), 1347-1360.

Faces and Voices of Recovery. (2011). Organizing the recovery community: About us. [Online]. Retrieved January 11, 2012 from http://www.facesandvoicesofrecovery.org.

Fals-Stewart, W., \& O'Farrell, T. J. (2003). Behavioral family counseling and naltrexone for male opioiddependent patients. Journal of Consulting \& Clinical Psychology, 71(3), 432-442.

Fals-Stewart, W., Lam, W. K. K., \& Kelley, M. L. (2009). Learning sobriety together: Behavioural couples therapy for alcoholism and drug abuse. Journal of Family Therapy, 31(2), 115-125. 
Fals-Stewart, W., O'Farrell, T. J., Feehan, M., Birchler, G. R., Tiller, S., \& McFarlin, S. K. (2000). Behavioral couples therapy versus individual-based treatment for male substance-abusing patients. An evaluation of significant individual change and comparison of improvement rates. Journal of Substance Abuse Treatment, 18(3), 249-254.

Farabee, D., Leukefeld, C. G., \& Hays, L. (1998). Accessing drug-abuse treatment: Perceptions of out-oftreatment injectors. Journal of Drug Issues, 28(2), 381-394.

Fareed, A., Casarella, J., Amar, R., Vayalapalli, S., \& Drexler, K. (2010). Methadone maintenance dosing guideline for opioid dependence, a literature review. Journal of Addictive Diseases, 29(1), 1-14.

Fayers, P. M. (2007). Scores and measurements: Validity, reliability, sensitivity. In P. M. Fayers \& D. Machin (Eds.), Quality of life: The assessment, analysis and interpretation of patient-reported outcomes (pp. 77-108). New York: John Wiley \& Sons.

Federation of State Physician Health Program. (2005). Physician health program guidelines. [Online]. Retrieved January 25, 2012 from http://www.fsphp.org.

Federation of State Physician Health Programs. (2011). State program listings. [Online]. Retrieved August 16, 2011 from http://www.fsphp.org.

Feeney, G. F. X., Young, R. M., Connor, J. P., Tucker, J., \& McPherson, A. (2002). Cognitive behavioural therapy combined with the relapse-prevention medication acamprosate: Are short-term treatment outcomes for alcohol dependence improved? Australian \& New Zealand Journal of Psychiatry, 36(5), 622-628.

Feldstein, S. W., \& Miller, W. R. (2007). Does subtle screening for substance abuse work? A review of the Substance Abuse Subtle Screening Inventory (SASSI). Addiction, 102(1), 41-50.

Fendrich, M., \& Johnson, T. P. (2001). Examining prevalence differences in three national surveys of youth: Impact of consent procedures, mode, and editing rules. Journal of Drug Issues, 31(3), 615642.

Fergusson, D. M., \& Horwood, L. J. (2004). Tests of casual linkages between cannabis use and psychotic symptoms. Addiction, 100(3), 354-366.

Fergusson, D. M., Woodward, L. J., \& Horwood, J. (1998). Maternal smoking during pregnancy and psychiatric adjustment in late adolescence. Archives of General Psychiatry, 55(8), 721-727.

Ferreira-Borges, C. (2005). Effectiveness of a brief counseling and behavioral intervention for smoking cessation in pregnant women. Preventive Medicine, 41(1), 295-302.

Ferri, M. M. F., Amato, L., \& Davoli, M. (2006). Alcoholics Anonymous and other 12-step programmes for alcohol dependence. Cochrane Database of Systematic Reviews, (3), CD005032.

Ferry, L. H., Grissino, L. M., \& Runfola, P. S. (1999). Tobacco dependence curricula in US undergraduate medical education. JAMA, 282(9), 825-829.

Festinger, D. S., Rubenstein, D. F., Marlowe, D. B., \& Platt, J. J. (2001). Relapse: Contributing factors, causative models, and empirical considerations. In F. M. Tims, C. G. Leukefeld, \& J. J. Platt (Eds.), Relapse and recovery in addictions (pp. 122-142). New Haven, CT: Yale University Press.

Fett, D. L. \& Vukov, L. F. (1994). An unusual case of severe griseofulvin-alcohol interaction. Annals of Emergency Medicine, 24(1), 95-97.

Fiellin, D. A., Reid, M. C., \& O'Connor, P. G. (2000). Screening for alcohol problems in primary care: A systematic review. Archives of Internal Medicine, 160(13), 1977-1989. 
Filbey, F. M., Schacht, J. P., Myers, U. S., Chavez, R. S., \& Hutchison, K. E. (2009). Marijuana craving in the brain. Proceedings of the National Academy of Sciences of the United States of America, 106(31), 13016-13021.

Fingerhut, L. A. (2008). Increases in poisoning and methadone-related deaths: United States, 1999-2005. [Online]. Retrieved November 28, 2011 from http://www.cdc.gov.

Finkelstein, N. (1993). Treatment programming for alcohol and drug-dependent pregnant women. International Journal of the Addictions, 28(13), 1275-1309.

Finkelstein, N., VanDeMark, N. R., Fallot, R., Brown, V., Cadiz, S., \& Heckman, J. (2004). Enhancing substance abuse recovery through integrated trauma treatment. Sarasota, FL: National Trauma Consortium.

Finlayson, R. E., Hurt, R. D., Davis, L. J., \& Morse, R. M. (1988). Alcoholism in elderly persons: A study of the psychiatric and psychosocial features of 216 inpatients. Mayo Clinic Proceedings, 63(8), 761768.

Finn, P. (1996). Cultural responsiveness of drug user treatment programs: Approaches to improvement. Substance Use \& Misuse, 31(4), 493-518.

Finnegan, L. (2008). Figure 40-2. Neonatal abstinence scoring sheet used for the assessment of infants undergoing neonatal abstinence. In M. Galanter \& H. D. Kleber (Eds.), Textbook of Substance abuse treatment (p. 565-580). Washington, DC: American Psychiatric Publishing.

Finnell, D. S. (2000). The case for teaching patients about the neurobiological basis of addictions. Journal of Addictions Nursing, 12(3/4), 149-158.

Finney, J. W., Ouimette, P. C., Humphreys, K., \& Moos, R. H. (2001). A comparative, processeffectiveness evaluation of VA substance abuse treatment. In M. Galanter (Series Ed.), Recent developments in alcoholism: Vol. 15. Services research in the era of managed care (pp. 373-391). New York: Kluwer Academic/Plenum Press.

Finney, J. W., Wilbourne, P. L., \& Moos, R. H. (2007). Psychosocial treatments for substance use disorders. In P. E. Nathan \& J. M. Gorman (Eds.), A guide to treatments that work (pp. 179-202). New York: Oxford University Press.

Fiore, M. C., Bailey, W. C., Cohen, S. J., Dorfman, S. F., Goldstein, M. G., Gritz, E. R., et al. (2000). Treating tobacco use and dependence: A U.S. public health services clinical practice guideline. [Online]. Retrieved February 10, 2012 from http://www.surgeongeneral.gov.

Fiore, M. C., Goplerud, E., \& Schroeder, S. A. (2012). The Joint Commission's new tobacco-use measures -- will hospitals do the right thing? New England Journal of Medicine, 366(13), 1172-1174.

Fiore, M. C., Jorenby, D. E., Baker, T. B., \& Kenford, S. L. (1992). Tobacco dependence and the nicotine patch: Clinical guidelines for effective use. JAMA, 268(19), 2687-2694.

Fiore, M. C., Kenford, S. L., Jorenby, D. E., Wetter, D. W., Smith, S. S., \& Baker, T. B. (1994). Two studies of the clinical effectiveness of the nicotine patch with different counseling treatments. Chest, 105(2), 524-533.

Fiore,M., U.S. Department of Health and Human Services. (2008). Treating tobacco use and dependence: 2008 update U.S. Public Health Service Clinical Practice Guideline executive summary. Respiratory Care, 53(9), 1217-1222.

Fiorentine, R. (1999). After drug treatment: Are 12-step programs effective in maintaining abstinence? American Journal of Drug \& Alcohol Abuse, 25(1), 93-116. 
Fiorentine, R., \& Hillhouse, M. P. (2000). Drug treatment and 12-step program participation: The additive effects of integrated recovery activities. Journal of Substance Abuse Treatment, 18(1), 65-74.

Fisher, G. L., \& Roget, N. A. (Eds.). (2009). Encyclopedia of substance abuse prevention, treatment, and recovery. Thousand Oaks, CA: SAGE Publications.

Fla. Admin. Code 59A-3.065 (2012).

Fla. Admin. Code 59A-33.001 (2012).

Fla. Admin. Code 65D-30.003 (2012).

Fla. Stat. 397.311 (2012).

Fla. Stat. 397.471 (2012).

Flannery, B. A., Garbutt, J. C., Cody, M. W., Renn, W., Grace, K., Osborne, M., et al. (2004). Baclofen for alcohol dependence: A preliminary open-label study. Alcoholism, Clinical \& Experimental Research, 28(10), 1517-1523.

Flavin, J., \& Paltrow, L. M. (2010). Punishing pregnant drug-using women: Defying law, medicine, and common sense. Journal of Addictive Diseases, 29(2), 231-244.

Fleming, M. F. (2004). Screening and brief intervention in primary care settings. Alcohol Research \& Health, 28(2), 57-62.

Fleming, M. F., Mundt, M. P., French, M. T., Manwell, L. B., Stauffacher, E. A., \& Barry, K. L. (2002). Brief physician advice for problem drinkers: Long-term efficacy and benefit-cost analysis. Alcoholism: Clinical \& Experimental Research, 26(1), 36-43.

Fleming, M. F., Mundt, M. P., French, M. T., Manwell, L. B., Stauffacher, E. A., \& Barry, K. L. (2000). Benefit-cost analysis of brief physician advice with problem drinkers in primary care settings. Medical Care, 38(1), 7-18.

Fleming, M., Barry, K., Davis, A., Kropp, S., Kahn, R., \& Rivo, M. (1994). Medical education about substance abuse: Changes in curriculum and faculty between 1976 and 1992. Academic Medicine, 69(5), 362-369.

Flensborg-Madsen, T., Becker, U., Gronbaek, M., Knop, J., Sher, L., \& Mortensen, E. L. (2011). Alcohol consumption and later risk of hospitalization with psychiatric disorders: Prospective cohort study. Psychiatry Research, 187(1-2), 214-219.

Foley, T. E., \& Fleshner, M. (2008). Neuroplasticity of dopamine circuits after exercise: Implications for central fatigue. Neuromolecular Medicine, 10(1), 67-80.

Ford, J. D., \& Russo, E. (2006). Trauma-focused, present-centered, emotional self-regulation approach to integrated treatment for posttraumatic stress and addiction: Trauma Adaptive Recovery Group Education and Therapy (TARGET). American Journal of Psychotherapy, 60(4), 335-355.

Forman, R. F., Bovasso, G., \& Woody, G. (2001). Staff beliefs about addiction treatment. Journal of Substance Abuse Treatment, 21(1), 1-9.

Forman, R. F., Svikis, D., Montoya, I. D., \& Blaine, J. (2004). Selection of a substance use disorder diagnostic instrument by the National Drug Abuse Treatment Clinical Trials Network. Journal of Substance Abuse Treatment, 27(1), 1-8.

Forsander, O. A. (1998). Dietary influences on alcohol intake: A review. Journal of Studies on Alcohol, 59(1), 26-31. 
Forsyth, C. B., Tang, Y., Shaikh, M., Zhang, L., \& Keshavarzian, A. (2010). Alcohol stimulates activation of snail, epidermal growth factor receptor signaling, and biomarkers of epithelialmesenchymal transition in colon and breast cancer cells. Alcoholism: Clinical \& Experimental Research, 34(1), 19-31.

Fortney, J. C., Tripathi, S. P., Walton, M. A., Cunningham, R. M., \& Booth, B. M. (2011). Patterns of substance abuse treatment seeking following cocaine-related emergency department visits. Journal of Behavioral Health Services and Research, 38(2), 221-233.

Fortney, J., \& Booth, B. M. (2001). Access to substance abuse services in rural areas. In M. Galanter (Series Ed.), Recent developments in alcoholism: Vol. 15. Services research in the era of managed care (pp. 177-197). New York: Kluwer Academic/Plenum Press.

Fortuna, J. L. (2012). The obesity epidemic and food addiction: Clinical similarities to drug dependence. Journal of Psychoactive Drugs, 44(1), 56-63.

Forum on Integration, A Collaborative for States. (2010). Integrating appropriate services for substance use conditions in health care settings: An issue brief on lessons learned and challenges ahead. Philadelphia, PA: Treatment Research Institute.

Forum on Integration. (2011). Purchasing integrated services for substance use conditions in health care settings: An issue brief on lessons learned and challenges ahead. [Online]. from http://www.tresearch.org.

Foster, J. H., Marshall, E. J., \& Peters, T. J. (2000). Outcome after in-patient detoxification for alcohol dependence: A naturalistic comparison of 7 versus 28 days stay. Alcohol \& Alcoholism, 35(6), 580586.

Foulds, J., Schmelzer, A. C., \& Steinberg, M. B. (2010). Treating tobacco dependence as a chronic illness and a key modifiable predictor of disease. International Journal of Clinical Practice, 64(2), 142-146.

Fournier, M. E., \& Levy, S. (2006). Recent trends in adolescent substance use, primary care screening, and updates in treatment options. Current Opinions in Pediatrics, 18(4), 352-358.

Frank, D. (2011). The trouble with morality: The effects of 12-step discourse on addicts' decision-making. Journal of Psychoactive Drugs, 43(3), 245-256.

Frank, R. G., Conti, R. M., \& Goldman, H. H. (2005). Mental health policy and psychotropic drugs. The Milbank Quarterly, 83(2), 271-298.

French, D. D., Spehar, A. M., Campbell, R. R., Palacios, P., Coakley, R. W., Coblio, N., et al. (2005). Outpatient benzodiazepine prescribing, adverse events, and costs. In K. Henricksen, J. B. Battles, \& E. S. Marks (Eds.), Advances in patient safety: From research to implementation (Volume 1: Research findings) (pp. 185-198). Rockville, MD: Agency for Healthcare Research and Quality.

French, M. T., Rachal, V., Harwood, H. J., \& Hubbard, R. (1990). Does drug abuse treatment affect employment and earnings of clients? Benefits Quarterly, 6(2), 58-67.

French, M. T., Salome, H. J., Sindelar, J. L., \& McLellan, A. T. (2002). Benefit-cost analysis of addiction treatment: Methodological guidelines and empirical application using the DATCAP and ASI. Health Services Research, 37(2), 433-455.

Freund, P. D., \& Hawkins, D. (2004). What street people reported about service access and drug treatment. Journal of Health \& Social Policy, 18(3), 87-93.

Friedmann, P. D., Lemon, S. C., Stein, M. D., \& D'Aunno, T. A. (2003). Accessibility of addiction treatment: Results from a national survey of outpatient substance abuse treatment organizations. Health Services Research, 38(3), 887-903. 
Friedmann, P. D., McCullough, D., \& Saitz, R. (2001). Screening and intervention for illicit drug abuse: A national survey of primary care physicians and psychiatrists. Archives of Internal Medicine, 161(2), 248-251.

Friedmann, P. D., McCullough, D., Chin, M. H., \& Saitz, R. (2000). Screening and intervention for alcohol problems: A national survey of primary care physicians and psychiatrists. Journal of General Internal Medicine, 15(2), 84-91.

Friedmann, P. D., Saitz, R., \& Samet, J. H. (1998). Management of adults recovering from alcohol or other drug problems: Relapse prevention in primary care. JAMA, 279(15), 1227-1231.

Friend, K. B., \& Pagano, M. E. (2007). Timevarying predictors of smoking cessation among individuals in treatment for alcohol abuse and dependence: Findings from Project MATCH. Alcohol \& Alcoholism, 42(3), 234-240.

Frisman, L. K., Mueser, K. T., Covell, N. H., Lin, H. J., Crocker, A., Drake, R. E., et al. (2009). Use of integrated dual disorder treatment via assertive community treatment versus clinical case management for persons with co-occurring disorders and antisocial personality disorder. Journal of Nervous \& Mental Disease, 197(11), 822-828.

Fromer, L. (2010). Managing asthma: An evidence-based approach to optimizing inhaled corticosteroid treatment. Southern Medical Journal, 103(10), 1038-1044.

Frone, M. R., Barnes, G. M., \& Farrell, M. P. (1994). Relationship of work-family conflict to substance use among employed mothers: The role of negative affect. Journal of Marriage \& the Family, 56(4), 1019-1030.

Frone, M. R., Russell, M., \& Cooper, M. L. (1993). Relationship of work-family conflict, gender, and alcohol expectancies to alcohol use/abuse. Journal of Organizational Behavior, 14(6), 545-558.

Frost-Pineda, K., VanSusteren, T., \& Gold, M. S. (2004). Are physicians and medical students prepared to educate patients about alcohol consumption? Journal of Addictive Diseases, 23(2), 1-13.

Fu, S. S., Kodl, M. M., Joseph, A. M., Hatsukami, D. K., Johnson, E. O., Breslau, N., et al. (2008). Racial/ethnic disparities in the use of nicotine replacement therapy and quit ratios in lifetime smokers ages 25 to 44 years. Cancer Epidemiology, Biomarkers \& Prevention, 17(7), 1640-1647.

Fu, S. S., Sherman, S. E., Yano, E. M., Van Ryn, M., Lanto, A. B., \& Joseph, A. M. (2005). Ethnic disparities in the use of nicotine replacement therapy for smoking cessation in an equal access health care system. American Journal of Health Promotion, 20(2), 108-116.

Fudala, P. J., Bridge, T. P., Herbert, S., Williford, W. O., Chiang, C. N., Jones, K., et al. (2003). Officebased treatment of opiate addiction with a sublingual-tablet formulation of buprenorphine and naloxone. New England Journal of Medicine, 349(10), 949-958.

Fuller, R. K., Branchey, L., Brightwell, D. R., Derman, R. M., Emrick, C. D., Iber, F. L., et al. (1986). Disulfiram treatment of alcoholism. A Veterans Administration cooperative study. JAMA, 256(11), 1449-1455.

Galanter, M., Dermatis, H., Glickman, L., Maslansky, R., Sellers, M. B., Neumann, E., et al. (2004). Network therapy: Decreased secondary opioid use during buprenorphine maintenance. Journal of Substance Abuse Treatment, 26, 313-318.

Galanter, M., Dermatis, H., Keller, D., \& Trujillo, M. (2002). Network therapy for cocaine abuse: Use of family and peer supports. American Journal on Addictions, 11, 161-162.

Galea, S., Factor, S. H., Palermo, A.-G., Aaaron, D., Canales, E., \& Vlahov, D. (2002). Access to resources for substance users in Harlem, New York City: Service provider and client perspectives. Health Education \& Behavior, 29(3), 296-311. 
Galea, S., Nandi, A., \& Vlahov, D. (2004). The social epidemiology of substance use. Epidemiologic Reviews, 26(1), 36-52.

Galloway, T. (2010). Meth mouth: A modern epidemic. Journal of the Oklahoma Dental Association, 101(8), 27-28.

Gambert, S. R. \& Albrecht, A. E. (2005). The elderly. In S. Gitlow (Ed.), Substance use disorders: A practical guide (pp. 1038-1048). Philadelphia, PA: Lippincott, Williams and Wilkins.

Gamblers Anonymous. (2012). Recovery program. [Online]. Retrieved January 11, 2012 Retrieved from http://www.gamblersanonymous.org.

Ganju, V. (2006). Mental health quality and accountability: The role of evidence-based practices and performance measures. Administration and Policy in Mental Health, 33(6), 659-665.

Garber, A. M. (2004). Cost-effectiveness and evidence evaluation as criteria for coverage policy. Health Affairs, Suppl. Web Exclusives, W4-284-96.

Garber, A. M. (2011). Medical necessity, coverage policy, and evidence based medicine. [Online]. Retrieved April 5, 2012 from http://www.iom.edu.

Garbutt, J. C., Kranzler, H. R., O'Malley, S. S., Gastfriend, D. R., Pettinati, H. M., Silverman, B. L., et al. (2005). Efficacy and tolerability of long-acting injectable naltrexone for alcohol dependence: A randomized controlled trial. JAMA, 293(13), 1617-1625.

Gardner, M. N., \& Brandt, A. M. (2006). "The doctors' choice is America's choice": The physician in US cigarette advertisements, 1930-1953. American Journal of Public Health, 96(2), 222-232.

Gariti, P., Levin, S., Whittingham, T., Barou, D., Kampman, K. M., Lynch, K., et al. (2008). Why do those who request smoking treatment fail to attend the first appointment? Journal of Substance Abuse Treatment, 35(1), 62-67.

Garland, A. F., Aarons, G. A., Brown, S. A., Wood, P. A., \& Hough, R. L. (2003). Diagnostic profiles associated with use of mental health and substance abuse services among high-risk youths. Psychiatric Services, 54(4), 562-564.

Garner, B. R. (2009). Research on the diffusion of evidence-based treatments within substance abuse treatment: A systematic review. Journal of Substance Abuse Treatment, 36(4), 376-399.

Garnick, D. W., Lee, M. T., Chalk, M., Gastfriend, D., Horgan, C. M., McCorry, F., et al. (2002). Establishing the feasibility of performance measures for alcohol and other drugs. Journal of Substance Abuse Treatment, 23(4), 375-385.

Garvey, A. J. (1997). Dental office interventions are essential for smoking cessation. Journal of Massachusetts Dental Society, 46(1), 16-19.

Gates, S., Smith Lesley A., \& Foxcroft, D. (2006). Auricular acupuncture for cocaine dependence. Cochrane Database of Systematic Reviews, (1), CD005192.

Gentilello, L. M. (2005). Confronting the obstacles to screening and interventions for alcohol problems in trauma centers. Journal of Trauma, 59(Suppl. 3), S137-S143.

Gentilello, L. M. (2010). More patients or more competition? A physician believes widespread substance use screening in primary care will boost specialty treatment. Addiction Professional, 8(4), 11-15.

Gentilello, L. M., Donato, A., Nolan, S., Mackin, R. E., Liebich, F., Hoyt, D. B., et al. (2005). Effect of the Uniform Accident and Sickness Policy Provision Law on alcohol screening and intervention in trauma centers. Journal of Trauma-Injury Infection \& Critical Care, 59(3), 624-631. 
Gentilello, L. M., Ebel, B. E., Wickizer, T. M., Salkever, D. S., \& Rivara, F. P. (2005). Alcohol interventions for trauma patients treated in emergency departments and hospitals: A cost benefit analysis. Annals of Surgery, 241(4), 541-550.

Gentilello, L. M., Rivara, F. P., Donovan, D. M., Jurkovich, G. J., Daranciang, E., Dunn, C. W., et al. (1999). Alcohol interventions in a trauma center as a means of reducing the risk of injury recurrence. Annals of Surgery, 230(4), 473-483.

Gentilello, L. M., Samuels, P. N., Henningfield, J. E., \& Santora, P. B. (2005). Alcohol screening and intervention in trauma centers: Confidentiality concerns and legal considerations. Journal of Trauma: Injury, Infection, \& Critical Care, 59(5), 1250-1255.

Gentry, W. B., Laurenzana, E. M., Williams, D. K., West, J. R., Berg, R. J., Terlea, T., et al. (2006). Safety and efficiency of an anti-(+)-methamphetamine monoclonal antibody in the protection against cardiovascular and central nervous system effects of (+)-methamphetamine in rats. International Immunopharmacology, 6(6), 968-977.

George, A. A., \& Tucker, J. A. (1996). Help-seeking for alcohol-related problems: Social contexts surrounding entry into alcoholism treatment or Alcoholics Anonymous. Journal of Studies on Alcohol, 57(4), 449-457.

George, O., \& Koob, G. F. (2010). Individual differences in prefrontal cortex function and the transition from drug use to drug dependence. Neuroscience \& Biobehavioral Reviews, 35(2), 232-247.

Georgia Department of Human Services. (2007). Governing body and hospital administration: 290-9-7.09. [Online]. Retrieved April 2, 2012 from http://rules.sos.state.ga.us.

Gerra, G., Fantoma, A., \& Zaimovic, A. (2006). Naltrexone and buprenorphine combination in the treatment of opioid dependence. Journal of Psychopharmacology, 20(6), 806-814.

GI Rights Hotline. (2012). Misconduct (including drug and alchohol abuse): Department of Defense. [Online]. Retrieved June 4, 2012 from http://girightshotline.org.

Gibb, S. J., Fergusson, D. M., \& Horwood, L. J. (2011). Working hours and alcohol problems in early adulthood. Addiction, 107(1), 81-88.

Giedd, J. N., Blumenthal, J., Jeffries, N. O., Castellanos, F. X., Liu, H., Zijdenbos, A., et al. (1999). Brain development during childhood and adolescence: A longitudinal MRI study. Nature Neuroscience, 2(10), 861-863.

Gilman, S. E., Cochran, S. D., Mays, V. M., Hughes, M., Ostrow, D., \& Kessler, R. C. (2001). Risk of psychiatric disorders among individuals reporting same-sex sexual partners in the National Comorbidity Survey. American Journal of Public Health, 91(6), 933-939.

Gitlow, S. (2011). Addiction: The new definition. [Online]. Retrieved November 21, 2011 from http://counselormagazine.com.

Glantz, S. A., \& Parmley, W. W. (1991). Passive smoking and heart disease. Epidemiology, physiology, and biochemistry. Circulation, 83(1), 1-12.

Glass, T. A., Prigerson, H., Kasl, S. V., \& Mendes de Leon, C. F. (1995). The effects of negative life events on alcohol consumption among older men and women. Journals of Gerontology: Series B: Psychological Science and Social Science, 50(4), S205-S216.

Godley, M. D. \& Godley, S. H. (2011). Assertive continuing care for adolescents. In J.F.Kelly \& W.L.White (Eds.), Addiction recovery management: Theory, research and practice (pp. 103-126). New York: Springer. 
Godley, M. D., Godley, S. H., Dennis, M. L., Funk, R. R., \& Passetti, L. L. (2002). Preliminary outcomes from the assertive continuing care experiment for adolescents discharged from residential treatment. Journal of Substance Abuse Treatment, 23(1), 21-32.

Godley, M. D., Godley, S. H., Dennis, M. L., Funk, R. R., \& Passetti, L. L. (2007). The effect of assertive continuing care on continuing care linkage, adherence and abstinence following residential treatment for adolescents with substance use disorders. Addiction, 102(1), 81-93.

Godley, S. H., Godley, M. D., Karvinen, T., Slown, L. L., \& Wright, K. L. (2006). The assertive continuing care protocol: A clinician's manual for working with adolescents after treatment of alcohol and other substance abuse disorders (2nd ed.). [Online]. Retrieved December 21, 2011. from http://www.chestnut.org.

Goehl, L., Nunes, E., Quitkin, F., \& Hilton, I. (1993). Social networks and methadone treatment outcome: The costs and benefits of social ties. American Journal of Drug \& Alcohol Abuse, 19(3), 251-262.

Gohdes, D., Amundson, H., Oser, C. S., Helgerson, S. D., \& Harwell, T. S. (2009). How are we diagnosing cardiometabolic risk in primary care settings? Primary Care Diabetes, 3(1), 29-35.

Gold, J., \& Gold, M. S. (2011). Exercise for the overweight and obese. Current Pharmaceutical Design, 17(12), 1193-1197.

Gold, M. R., Siegel, J. E., Russell, L. B., \& Weinstein, M. C. (1996). Cost-effectiveness in health and medicine. New York: Oxford University Press.

Gold, M. S., Frost-Pineda, K., Goldberger, B. A., \& DuPont, R. L. (2006). Physicians and drug screening. Journal of Adolescent Health, 39(2), 154-155.

Gold, M. S., Pottash, A. C., Sweeney, D. R., \& Kleber, H. D. (1980). Opiate withdrawal using clonidine: A safe, effective, and rapid nonopiate treatment. JAMA, 243(4), 343-346.

Gold, M. S., Redmond, D. E., Jr., \& Kleber, H. D. (1978). Clonidine blocks acute opiate-withdrawal symptoms. Lancet, 2(8090), 599-602.

Goldade, K., Whembolua, G. L., Thomas, J., Eischen, S., Guo, H., Connett, J., et al. (2011). Designing a smoking cessation intervention for the unique needs of homeless persons: A community-based randomized clinical trial. Clinical Trials, 8(6), 744-754.

Goldberg, R. (2009). Drugs Across the Spectrum (6th ed.). Belmont, CA: Wadsworth.

Goldenberg, R. L., \& Culhane, J. F. (2007). Low birth weight in the United States. American Journal of Clinical Nutrition, 85(2), 584S-590S.

Goldman, H. H., Frank, R. G., Burnam, M. A., Huskamp, H. A., Ridgely, M. S., Normand, S.-L. T., et al. (2006). Behavioral health insurance parity for federal employees. New England Journal of Medicine, 354(13), 1378-1386.

Goldstein, J. M., Cherkerzian, S., \& Simpson, J. C. (2011). Validity: Definitions and applications to psychiatric research. In M. T. Tsuang, M. Tohen, \& P. B. Jones (Eds.), Textbook in psychiatric epidemiology (pp. 99-116). New York: John Wiley \& Sons.

Goldstein, M. G., Niaura, R., Follick, M. J., \& Abrams, D. B. (1989). Effects of behavioral skills training and schedule of nicotine gum administration on smoking cessation. American Journal of Psychiatry, 146(1), 56-60.

Goldstein, R. Z., \& Volkow, N. D. (2011). Dysfunction of the prefrontal cortex in addiction: Neuroimaging findings and clinical implications. Nature Reviews: Neuroscience, 12(11), 652-669. doi: $10.1038 / \mathrm{nm} 3119$. 
Goldstein, R. Z., Craig, A. D., Bechara, A., Garavan, H., Childress, A. R., Paulus, M. P., et al. (2009). The neurocircuitry of impaired insight in drug addiction. Trends in Cognitive Sciences, 13(9), 372380 .

Golombok, S., Moodley, P., \& Lader, M. (1988). Cognitive impairment in long-term benzodiazepine users. Psychological Medicine, 18(2), 365-374.

Goodwin, R. D., Lewinsohn, P. M., \& Seeley, J. R. (2005). Cigarette smoking and panic attacks among young adults in the community: The role of parental smoking and anxiety disorders. Biological Psychiatry, 58(9), 686-693.

Goplerud, E., \& McPherson, T. L. (2010a). SBIRT at work: The "big" initiative. Journal of Employee Assistance, 40(4), 16-19.

Goplerud, E., \& McPherson, T. L. (2010b). Workplace alcohol screening, brief intervention, and EAPs: The BIG (Brief Intervention Group) initiative. Washington, DC: Center for Integrated Behavioral Health Policy and Ensuring Solutions to Alcohol Problems; George Washington University.

Goplerud, E., Sharar, D., \& McPherson, T. (2010). Workplace alcohol screening, brief intervention, and EAPs: What employers need to know to improve productivity and reduce costs. Health \& Productivity Management, 8(1/2), 28-32.

Gorber, S. C., Schofield-Hurwitz, S., Hardt, J., Levasseur, G., \& Tremblay, M. (2009). The accuracy of self-reported smoking: A systematic review of the relationship between self-reported and cotinineassessed smoking status. Nicotine \& Tobacco Research, 11(1), 12-24.

Gordon, A. J., Ettaro, L., Rodriguez, K. L., Mocik, J., \& Clark, D. B. (2011). Provider, patient, and family perspectives of adolescent alcohol use and treatment in rural settings. Journal of Rural Health, 27(1), $81-90$.

Gordon, H. W. (2002). Early environmental stress and biological vulnerability to drug abuse. Psychoneuroendocrinology, 27(1-2), 115-126.

Gordon, J. S., Albert, D. A., Crews, K. M., \& Fried, J. (2009). Tobacco education in dentistry and dental hygiene. Drug \& Alcohol Review, 28(5), 517-532.

Gould, T. J. (2010). Addiction and cognition. Addiction Science \& Clinical Practice, 4-14.

Gourlay, S. G., Stead, L. F., \& Benowitz, N. (2008). Clonidine for smoking cessation. Cochrane Database of Systematic Reviews, (3), CD000058.

Gowing, L., Farrell, M., Bornemann, R., Sullivan, L., \& Ali, R. (2008). Substitution treatment of injecting opioid users for prevention of HIV infection. Cochrane Database of Systematic Reviews, (2), CD004145.

Graeber, D. A., Moyers, T. B., Griffith, G., Guajardo, E., \& Tonigan, S. (2003). A pilot study comparing motivational interviewing and an educational intervention in patients with schizophrenia and alcohol use disorders. Community Mental Health Journal, 39(3), 189-202.

Graham, A., Schultz, T., Mayo-Smith, M., Ries, R., \& Wilford, B. (2008). Principles of addiction medicine. Chevy Chase, MD: American Society of Addiction Medicine.

Granholm, E., Tate, S. R., Link, P. C., Lydecker, K. P., Cummins, K. M., McQuaid, J., et al. (2011). Neuropsychological functioning and outcomes of treatment for co-occurring depression and substance use disorders. American Journal of Drug \& Alcohol Abuse, 37(4), 240-249.

Grant, B. F. (1997). Barriers to alcoholism treatment: Reasons for not seeking treatment in a general population sample. Journal of Studies on Alcohol, 58(4), 365-371. 
Grant, B. F., \& Dawson, D. A. (1997). Age at onset of alcohol use and its association with DSM-IV alcohol abuse and dependence: Results from the National Longitudinal Alcohol Epidemiologic Survey. Journal of Substance Abuse, 9, 103-110.

Grant, B. F., \& Dawson, D. A. (1998). Age of onset of drug use and its association with DSM-IV drug abuse and dependence: Results from the National Longitudinal Alcohol Epidemiologic Survey. Journal of Substance Abuse Treatment, 10(2), 163-173.

Grant, B. F., Dawson, D. A., Stinson, F. S., Chou, S. P., Dufour, M. C., \& Pickering, R. P. (2004). The 12-month prevalence and trends in DSM-IV alcohol abuse and dependence: United States, 1991-1992 and 2001-2002. Drug \& Alcohol Dependence, 74(3), 223-234.

Grant, J. D., Scherrer, J. F., Lynskey, M. T., Lyons, M. J., Eisen, S. A., Tsuang, M. T., et al. (2006). Adolescent alcohol use is a risk factor for adult alcohol and drug dependence: Evidence from a twin design. Psychological Medicine, 36(1), 109-118.

Grant, J. E., Brewer, J. A., \& Potenza, M. N. (2006). The neurobiology of substance and behavioral addictions. CNS Spectrums, 11(12), 924-930.

Grant, J. E., Potenza, M. N., Weinstein, A., \& Gorelick, D. A. (2010). Introduction to behavioral addictions. American Journal of Drug \& Alcohol Abuse, 36(5), 233-241.

Grant, L. P., Haughton, B., \& Sachan, D. S. (2004). Nutrition education is positively associated with substance abuse treatment program outcomes. Journal of the American Dietetic Association, 104(4), 604-610.

Gray, M. (2009). A brief history of New York's Rockefeller Drug Laws. [Online]. Retrieved November 30, 2011 from http://www.time.com.

Graziani, C., Rosenthal, M. P., \& Diamond, J. J. (1999). Diabetes education program use and patientperceived barriers to attendance. [Online]. Retrieved April 30, 2012 from http://www.stfm.org.

Greenblatt, D. J., Sellers, E. M., \& Shader, R. I. (1982). Drug disposition in old age. New England Journal of Medicine, 306(18), 1081-1088.

Greene, J. M., Ennett, S. T., \& Ringwalt, C. L. (1997). Substance use among runaway and homeless youth in three national samples. American Journal of Public Health, 87(2), 229-235.

Greenfield, S. F., \& Grella, C. E. (2009). What is "women-focused" treatment for substance use disorders? Psychiatric Services, 60(7), 880-882.

Greenwood, G. L., Goplerud, E., McPherson, T. L., Azocar, F., Baker, E. M., \& Dybdahl, S. (2010). Alcohol screening and brief intervention (SBI) in telephonic employee assistance programs. Journal of Workplace Behavioral Health, 25(4), 233-240.

Grella, C. E. (1997). Services for perinatal women with substance abuse and mental health disorders: The unmet need. Journal of Psychoactive Drugs, 29(1), 67-78.

Grella, C. E. (2009). Older adults and co-occurring disorders. [Online]. Retrieved January 12, 2012 from http://www.aodpolicy.org.

Grella, C. E., \& Greenwell, L. (2004). Substance abuse treatment for women: Changes in the settings where women received treatment and types of services provided, 1987-1998. Journal of Behavioral Health Services \& Research, 31(4), 367-383.

Grella, C. E., Hser, Y.-I., Vandana, J., \& Rounds-Bryant, J. (2001). Drug treatment outcomes for adolescents with comcorbid mental and substance use disorders. Journal of Nervous \& Mental Disease, 189(6), 384-392. 
Grella, C. E., Karno, M. P., Warda, U. S., Moore, A. A., \& Niv, N. (2009). Perceptions of need and help received for substance dependence in a national probability survey. Psychiatric Services, 60(8), 10681074.

Griffith, J. D., Rowan-Szal, G. A., Roark, R. R., \& Simpson, D. D. (2000). Contingency management in outpatient methadone treatment: A meta-analysis. Drug \& Alcohol Dependence, 58(1-2), 55-66.

Grill, C. (2011). State STATs: Repeal of the UPPL. Bulletin of the American College of Surgeons, 95(11), 38-39.

Grisso, T., \& Underwood, L. (2004). Screening and assessing mental health and substance use disorders among youth in the juvenile justice system: A resource guide for practitioners (NCJ 204956). Washington, DC: U.S. Department of Justice, Office of Justice Programs, Office of Juvenile Justice and Delinquency Prevention.

Groenewegen, H. J. (2007). The ventral striatum as an interface between the limbic and motor systems. [Online]. Retrieved July 17, 2008 from http://www.cnsspectrums.com

Grumbach, K., \& Grundy, P. (2010). Outcomes of implementing patient centered medical home interventions: A review of the evidence from prospective evaluation studies in the United States. [Online]. Patient-Centered Primary Care Collaborative. Retrieved June 5, 2012 from http://www.pcpcc.net.

Gryczynski, J., Mitchell, S. G., Peterson, T. R., \& Gonzales, A. (2011). The relationship between services delivered and substance use outcomes in New Mexico's Screening, Brief Intervention, Referral and Treatment (SBIRT) initiative. Drug \& Alcohol Dependence, 118(2-3), 152-157.

Gueorguieva, R., Wu, R., Donovan, D., Rounsaville, B. J., Couper, D., Krystal, J. H., et al. (2011). Baseline trajectories of drinking moderate acamprosate and naltrexone effects in the COMBINE study. Alcoholism: Clinical \& Experimental Research, 35(3), 523-531.

Guerri, C., \& Pascual, M. (2010). Mechanisms involved in the neurotoxic, cognitive, and neurobehavioral effects of alcohol consumption during adolescence. Alcohol, 44(1), 15-26.

Gulliver, S. B., \& Steffen, L. E. (2010). Towards integrated treatments for PTSD and substance use disorders. PTSD Research Quarterly, 21(2), 1-3.

Gunderson, E. W., Levin, F. R., \& Smith, L. (2005). Screening and intervention for alcohol and illicit drug abuse: A survey of internal medicine housestaff. Journal of Addictive Diseases, 24(2), 1-18.

Gustafson, D. H., Boyle, M. G., Shaw, B. R., Isham, A., McTavish, F., Richards, S., et al. (2011). An ehealth solution for people with alcohol problems. Alcohol Research \& Health, 33(4), 327.

Guttmacher Institute. (2011). State policies in brief: Substance abuse during pregnancy. New York: Author.

Guydish, J., Passalacqua, E., Tajima, B., Chan, M., Chun, J. S., \& Bostrom, A. (2011). Smoking prevalence in addiction treatment: A review. Nicotine \& Tobacco Research, 13(6), 401-411.

Haack, M. R., \& Adger, H. (2002). Strategic plan for interdisciplinary faculty development: Arming the nation's health professional workforce for a new approach to substance use disorders. Providence, RI: Association for Medical Education and Research in Substance Abuse (AMERSA).

Haber, P. (2012). Pharmacotherapies for alcohol dependence. In E. Proude, O. Loptko, N. Lintzeris, \& P. Haber (Eds.), Guidelines for the treatment of alcohol problems: A review of evidence (pp. 172-169). Sydney, Australia: University of Sydney.

Hajela, R. \& Miller, M. M. (2012). Addiction is addiction: The development of the new ASAM definition. Unpublished manuscript. 
Hale, J. (2011). Scientific measures: Reliability and validity. [Online]. Retrieved April 23, 2012 from http://psychcentral.com.

Hall, S. M., \& Prochaska, J. J. (2009). Treatment of smokers with co-occurring disorders: Emphasis on integration in mental health and addiction treatment settings. Annual Review of Clinical Psychology, $5,409-431$.

Han, B., Gfroerer, J. C., \& Colliver, J. D. (2010). Associations between duration of illicit drug use and health conditions: Results from the 2005-2007 National Surveys on Drug Use and Health. Annals of Epidemiology, 20(4), 289-297.

Han, B., Gfroerer, J. C., Colliver, J. D., \& Penne, M. A. (2009). Substance use disorder among older adults in the United States in 2020. Addiction, 104(1), 88-96.

Han, C., McGue, M. K., \& Iacono, W. G. (1999). Lifetime tobacco, alcohol and other substance use in adolescent Minnesota twins: Univariate and multivariate behavioral genetic analyses. Addiction, 94(7), 981-993.

Handmaker, N. S., \& Wilbourne, P. (2001). Motivational interventions in prenatal clinics. Alcohol Research \& Health, 25(3), 219-221, 227-229.

Handmaker, N. S., Miller, W. R., \& Manicke, M. (1999). Findings of a pilot study of motivational interviewing with pregnant drinkers. Journal of Studies on Alcohol, 60(2), 285-287.

Hanewinkel, R., Isensee, B., Sargent, J. D., \& Morgenstern, M. (2011). Cigarette advertising and teen smoking initiation. Pediatrics, 127(2), e271-e278.

Haney, M., Hart, C. L., Vosburg, S. K., Nasser, J., Bennett, A., Zubaran, C., et al. (2004). Marijuana withdrawal in humans: Effects of oral THC or divalproex. Neuropsychopharmacology, 29(1), 158170 .

Hankin, J. R. (2002). Fetal alcohol syndrome prevention research. Alcohol Research \& Health, 26(1), 5865.

Hardin, J. S., Wessinger, W. D., Proksch, J. W., \& Owens, S. M. (1998). Pharmacodynamics of a monoclonal antiphencyclidine Fab with broad selectivity for phencyclidine-like drugs. Journal of Pharmacology \& Experimental Therapeutics, 285(3), 1113-1122.

Harford, T. C., Yi, H. Y., \& Grant, B. F. (2010). The five-year diagnostic utility of "diagnostic orphans" for alcohol use disorders in a national sample of young adults. Journal of Studies on Alcohol \& Drugs, 71(3), 410-417.

Haro, J. M., Arbabzadeh-Bouchez, S., Brugha, T. S., de, G. G., Guyer, M. E., Jin, R., et al. (2006). Concordance of the Composite International Diagnostic Interview Version 3.0 (CIDI 3.0) with standardized clinical assessments in the WHO World Mental Health surveys. International Journal of Methods in Psychiatric Research, 15(4), 167-180.

Harper, S., Strumpf, E. C., \& Kaufman, J. S. (2012). Do medical marijuana laws increase marijuana use: Replication study and extension. Annals of Epidemiology, 22(3), 207-212.

Harris, J. L., Patton, L. L., Wilder, R. S., Peterson, C. A., \& Curran, A. E. (2009). North Carolina dental hygiene students' opinions about tobacco cessation education and practices in their programs. Journal of Dental Education, 73(5), 539-549.

Harris, S. K., Csemy, L., Sherritt, L., Starostova, O., Van Hook, S., Johnson, J., et al. (2012). Computerfacilitated substance use screening and brief advice for teens in primary care: An international trial. Pediatrics, 129(6), 1-11. 
Harrison, P. A., Hoffman, N. G., \& Edwall, G. E. (1989). Sexual abuse correlates: Similarities between male and female adolescents in chemical dependency treatment. Journal of Adolescent Research, 4(3), 385-399.

Hart, C. L., Haney, M., Vosburg, S. K., Rubin, E., \& Foltin, R. W. (2008). Smoked cocaine selfadministration is decreased by modafinil. Neuropsychopharmacology, 33(4), 761-768.

Hart, P. D. (2004). Faces and Voices of Recovery Public Survey. [Online]. Retrieved February 27, 2009 from http://www.facesandvoicesofrecovery.org.

Hartz, S. M., \& Bierut, L. J. (2010). Genetics of addictions. Clinics in Laboratory Medicine, 30(4), 847864.

Harvard School of Public Health. (2012). The nutrition source: Protein: Moving closer to center stage. [Online]. Retrieved January 13, 2012 from http://www.hsph.harvard.edu.

Harwell, T. S., Oser, C. S., Helgerson, S. D., \& Gohdes, D. (2007). Primary care provider screening for diabetes and assessment of cardiometabolic risk. [Online]. Retrieved February 23, 2012 from http://professional.diabetes.org.

Harwood, H. (2008). An inventory of cost offset studies for state substance abuse agencies. Washington, DC: National Association of State Alcohol/Drug Abuse Directors.

Harwood, H. J. (2002). Survey on behavioral health workplace. Frontlines: Linking Alcohol Services Research \& Practices, (November), pp. 3.

Hasin, D. S., Stinson, F. S., Ogburn, E., \& Grant, B. F. (2007). Prevalence, correlates, disability, and comorbidity of DSM-IV alcohol abuse and dependence in the United States: Results from the National Epidemiologic Survey on Alcohol and Related Conditions. Archives of General Psychiatry, 64(7), 830-842.

Hassan, A., Harris, S. K., Sherritt, L., Van, H. S., Brooks, T., Carey, P., et al. (2009). Primary care follow-up plans for adolescents with substance use problems. Pediatrics, 124(1), 144-150.

Hassija, C. M., Jakupcak, M., Maguen, S., \& Shipherd, J. C. (2012). The influence of combat and interpersonal trauma on PTSD, depression, and alcohol misuse in U.S. Gulf War and OEF/OIF women veterans. Journal of Traumatic Stress, 25(2), 216-219.

Hawkins, J. D., Catalano, R. F., \& Miller, J. Y. (1992). Risk and protective factors for alcohol and other drug problems in adolescence and early adulthood: Implications for substance abuse prevention. Psychological Bulletin, 112(1), 64-105.

Hays, J. T., Ebbert, J. O., \& Sood, A. (2008). Efficacy and safety of varenicline for smoking cessation. American Journal of Medicine, 121(4) (Suppl. 1) , S32-S42.

Hays, L., Kirsh, K. L., \& Passik, S. D. (2003). Seeking drug treatment for OxyContin abuse: A chart review of consecutive admissions to a substance abuse treatment facility in Kentucky. Journal of the National Comprehensive Cancer Network, 1(3), 423-428.

Hazelden Foundation. (1998). Addiction: A disease defined. [Online]. Retrieved March 15, 2012 from www.hazelden.org.

Hazelden Foundation. (2009). Americans want insurance to cover addiction. [Online]. Retrieved August 16, 2011 from http://www.hazelden.org.

Hazelden Foundation. (2012). Addiction treatment, detoxification, withdrawal. [Online]. Retrieved May 23, 2012 from http://www.hazelden.org.

Health Resources and Services Administration. (2011a). EPSDT and Title V collaboration to improve child health. [Online]. Retrieved December 13, 2011 from http://mchb.hrsa.gov. 
Health Resources and Services Administration. (2011b). Mental health and EPSDT. [Online]. Retrieved December 13, 2011 from http://mchb.hrsa.gov.

HealthCare.gov. (2011a). Affordable care organizations: Improving care coordination for people with Medicare. [Online]. Retrieved September 16, 2011 from http://www.healthcare.gov.

HealthCare.gov. (2011b). Affordable insurance exchanges: Seamless access to affordable coverage overview. [Online]. Retrieved September 1, 2011 from http://www.healthcare.gov.

HealthCare.gov. (2011c). Coming in 2014: Affordable insurance exchanges. [Online]. Retrieved July 18, 2011 from http://www.healthcare.gov.

HealthCare.gov. (2011d). Essential health benefits: A set of health care service categories that must be covered by certain plans, starting in 2014. [Online]. Retrieved October 28, 2011 from http://www.healthcare.gov.

HealthCare.gov. (2011e). Preventive services covered under the Affordable Care Act. [Online]. Retrieved May 8, 2012 from http://www.healthcare.gov.

HealthCare.gov. (2012a). Glossary: Annual limit. [Online]. Retrieved April 26, 2012 from http://www.healthcare.gov.

HealthCare.gov. (2012b). Glossary: Lifetime limit. [Online]. Retrieved April 26, 2012 from http://www.healthcare.gov.

HealthCare.gov. (2012c). Key features of the law: The Affordable Care Act. [Online]. Retrieved March 29, 2012 from http://www.healthcare.gov.

Heath, A. C., Bucholz, K. K., Madden, P. A., Dinwiddie, S. H., Slutske, W. S., Bierut, L. J., et al. (1997). Genetic and environmental contributions to alcohol dependence risk in a national twin sample: Consistency of findings in women and men. Psychological Medicine, 27(6), 1381-1396.

Hedden, S. L., \& Gfroerer, J. C. (2011). Correlates of perceiving a need for treatment among adults with substance use disorder: Results from a national survey. Addictive Behaviors, 36(12), 1213-1222.

Heffner, J. L., \& Anthenelli, R. M. (2011). Strategies to help patients break the chains of tobacco addiction: Evidence-based treatments can help patients quit despite psychiatric illness. Current Psychiatry, 10(8), 41-49.

Heilig, M., Goldman, D., Berrettini, W., \& O'Brien, C. P. (2011). Pharmacogenetic approaches to the treatment of alcohol addiction. Nature Reviews, 12(11), 670-684.

helpwithsmoking.com. (2011). Nicotine lozenge. [Online]. Retrieved June 15, 2011 Retrieved from http://www.helpwithsmoking.com.

Heltemes, K. J., Dougherty, A. L., MacGregor, A. J., \& Galarneau, M. R. (2011). Alcohol abuse disorders among U.S. service members with mild traumatic brain injury. Military Medicine, 176(2), 147-150.

Henggeler, S. W., Clingempeel, W. G., Brondino, M. J., \& Pickrel, S. G. (2002). Four-year follow-up of multisystemic therapy with substance-abusing and substance-dependent juvenile offenders. Journal of the American Academy of Child \& Adolescent Psychiatry, 41(7), 868-874.

Henriksen, L., Schleicher, N. C., Feighery, E. C., \& Fortmann, S. P. (2010). A longitudinal study of exposure to retail cigarette advertising and smoking initiation. Pediatrics, 126(2), 232-238.

Henry J. Kaiser Family Foundation. (2010). Summary of new health reform law. [Online]. Retrieved April 15, 2011 from http://www.kff.org.

Henry J. Kaiser Family Foundation. (2012). A guide to the Supreme Court's review of the 2010 Health Care Reform law. [Online]. Retrieved March 12, 2012 from http://www.kff.org. 
Henry-Edwards, S., Humeniuk, R., Ali, R., Monteiro, M., \& Poznyak, V. (2003). Brief intervention for substance use: A manual for use in primary care. (Draft version 1.1 for field testing). [Online]. World Health Organization. Retrieved December 19, 2011 from http://www.who.int.

Henry-Edwards, S., Humeniuk, R., Ali, R., Poznyak, V., \& Monteiro, M. (2003). The Alcohol, Smoking and Substance Involvement Screening Test (ASSIST): Guidelines for use in primary care: (Draft version 1.1 for field testing). Geneva: World Health Organization.

Hermann, R. C., Chan, J. A., Zazzali, J. L., \& Lerner, D. (2006). Aligning measurement-based quality improvement with implementation of evidence-based practices. Administration and Policy in Mental Health, 33(6), 636-645.

Heron, M. (2007). Deaths: Leading causes for 2004. National Vital Statistics Reports, 56(5), 1-95.

Hersh, E. V., Ochs, H., Quinn, P., MacAfee, K., Cooper, S. A., \& Barasch, A. (1993). Narcotic receptor blockade and its effect on the analgesic response to placebo and ibuprofen after oral surgery. Oral Surgery, Oral Medicine, and Oral Pathology, 75(5), 539-546.

Hesse, M., Vanderplasschen, W., Rapp, R. C., Broekaert, E., \& Fridell, M. (2007). Case management for persons with substance use disorders. Cochrane Database Systematic Reviews, (4), CD006265.

Hesselbrock, M. N., Hesselbrock, V. M., Segal, B., Schuckit, M. A., \& Bucholz, K. (2003). Ethnicity and psychiatric comorbidity among alcohol-dependent persons who receive inpatient treatment: African Americans, Alaska natives, Caucasians, and Hispanics. Alcoholism: Clinical \& Experimental Research, 27(8), 1368-1373.

Hien, D. A., Cohen, L. R., Miele, G. M., Litt, L. C., \& Capstick, C. (2004). Promising treatments for women with comorbid PTSD and substance use disorders. American Journal of Psychiatry, 161(8), 1426-1432.

Higgins, S. T., \& Petry, N. M. (1999). Contingency management: Incentives for sobriety. Alcohol Research \& Health, 23(2), 122-127.

Higgins, S. T., Alessi, S. M., \& Dantona, R. L. (2002). Voucher-based incentives: A substance abuse treatment innovation. Addictive Behaviors, 27(6), 887-910.

Higgins, S. T., Budney, A. J., Bickel, W. K., Foerg, F. E., Donham, R., \& Badger, G. J. (1994). Incentives improve outcome in outpatient behavioral treatment of cocaine dependence. Archives of General Psychiatry, 51(7), 568-576.

Higgins, S. T., Heil, S. H., Dantona, R., Donham, R., Matthews, M., \& Badger, G. J. (2007). Effects of varying the monetary value of voucher-based incentives on abstinence achieved during and following treatment among cocaine-dependent outpatients. Addiction, 102(2), 271-281.

Higgins, S. T., Sigmon, S. C., Wong, C. J., Heil, S. H., Badger, G. J., Donham, R., et al. (2003). Community reinforcement therapy for cocaine-dependent outpatients. Archives of General Psychiatry, 60(10), 1043-1052.

Higgins, S. T., Wong, C. J., Badger, G. J., Huag Ogden, D. E., \& Dantona, R. L. (2000). Contingent reinforcement increases cocaine abstinence during outpatient treatment and 1 year of follow-up. Journal of Consulting \& Clinical Psychology, 68(1), 64-72.

Higgins-Biddle, J. C., Hungerford, D., \& Cates-Wessel, K. (2009). Screening \& brief interventions (SBI) for unhealthy alcohol use: A step-by-step implementation guide for trauma centers. Atlanta, GA: Centers for Disease Control and Prevention.

Hill, K. G., Hawkins, J. D., Bailey, J. A., Catalano, R. F., Abbott, R. D., \& Shapiro, V. B. (2010). Personenvironment interaction in the prediction of alcohol abuse and alcohol dependence in adulthood. Drug \& Alcohol Dependence, 110(1-2), 62-69. 
Hillestad, R., Bigelow, J., Bower, A., Girosi, F., Meili, R., Scoville, R., et al. (2005). Can electronic medical record systems transform health care? Potential health benefits, savings, and costs. Health Affairs, 24(5), 1103-1117.

Hillner, B. E., \& Smith, T. J. (2009). Efficacy does not necessarily translate to cost effectiveness: A case study in the challenges associated with 21 st-century cancer drug pricing. Journal of Clinical Oncology, 27(13), 2111-2113.

Himelhoch, S., \& Daumit, G. (2003). To whom do psychiatrists offer smoking-cessation counseling? American Journal of Psychiatry, 160(12), 2228-2230.

Hingson, R. W., Heeren, T., Edwards, E. M., \& Saitz, R. (2011). Young adults at risk for excess alcohol consumption are often not asked or counseled about drinking alcohol. Journal of General Internal Medicine doi 10.1007/s11606-011-1851-1.

Hinkin, C. H., Castellon, S. A., Dickson-Fuhrman, E., Daum, G., Jaffe, J., \& Jarvik, L. (2001). Screening for drug and alcohol abuse among older adults using a modified version of the CAGE. American Journal on Addictions, 10(4), 319-326.

Hintz, T., \& Mann, K. (2006). Co-occurring disorders: Policy and practice in Germany. American Journal on Addictions, 15(4), 261-267.

Hirata, E. S., Almeida, O. P., Funari, R. R., \& Klein, E. L. (2001). Validity of the Michigan Alcoholism Screening Test (MAST) for the detection of alcohol-related problems among male geriatric outpatients. American Journal of Geriatric Psychiatry, 9(1), 30-34.

Hockenberry, S., Sickmund, M., \& Sladky, A. (2009). Juvenile residential facility census, 2006: Selected findings. Washington, DC: U.S. Department of Justice, Office of Justice Programs, Office of Juvenile Justice and Delinquency Prevention.

Hodgkin, D., Horgan, C. M., Garnick, D. W., \& Merrick, E. L. (2009). Benefit limits for behavioral health care in private health plans. Administration \& Policy in Mental Health, 36(1), 15-23.

Hoffman, K. A., Ford, J. H., Tillotson, C. J., Choi, D., \& McCarty, D. (2011). Days to treatment and early retention among patients in treatment for alcohol and drug disorders. Addictive Behaviors, 36(6), 643647.

Hoge, C. W., Castro, C. A., Messer, S. C., McGurk, D., Cotting, D. I., \& Koffman, R. L. (2004). Combat duty in Iraq and Afghanistan, mental health problems, and barriers to care. New England Journal of Medicine, 351(1), 13-22.

Hoge, M. A., Morris, J. A., Daniels, A. S., Stuart, G. W., Huey, L. Y., \& Adams, N. (2007). An action plan for behavioral health workforce development: A framework for discussion. Rockville, MD: U.S. Department of Health and Human Services, Annapolis Coalition on the Behavioral Health Workforce.

Holder, H. D. (1998). Cost benefits of substance abuse treatment: An overview of results from alcohol and drug abuse. Journal of Mental Health Policy \& Economics, 1(1), 23-29.

Holloway, H. C., Hales, R. E., \& Watanabe, H. K. (1984). Recognition and treatment of acute alcohol withdrawal syndromes. Psychiatric Clinics of North America, 7(4), 729-743.

Hommer, D. W., Bjork, J. M., \& Gilman, J. M. (2011). Imaging brain response to reward in addictive disorders. Annals of the New York Academy of Sciences, 1216, 50-61.

Hoof-Haines, K. V. (2012). Dual diagnosis: The status of treating co-occurring disorders in the U.S. [Online]. Retrieved January 24, 2012 from http://www.drugfree.org. 
Hopfer, C. J., Lessem, J. M., Hartman, C. A., Stallings, M. C., Cherny, S. S., Corley, R. P., et al. (2007). A genome-wide scan for loci influencing adolescent cannabis dependence symptoms: Evidence for linkage on chromosomes 3 and 9. Drug \& Alcohol Dependence, 89(1), 34-41.

Horn, K., Dino, G., Kalsekar, I., \& Mody, R. (2005). The impact of Not on Tobacco on teen smoking cessation: End-of-program evaluation results, 1998 to 2003. Journal of Adolescent Research, 20(6), 640-661.

Horton, J., Compton, W., \& Cottler, L. B. (2000). Reliability of substance use disorder diagnoses among African-Americans and Caucasians. Drug \& Alcohol Dependence, 57(3), 203-209.

House Office of the Legislative Counsel. (2010). Patient Protection and Affordable Care Act: Public Law 111-148. [Online]. Retrieved June 14, 2012 from http://housedocs.house.gov.

Howard, M. O., Walker, D. R., Walker, P. S., \& Suchinsky, R. T. (1997). Alcohol and drug education in schools of nursing. Journal of Alcohol \& Drug Addiction, 42(3), 54-80.

Hser, Y.-I., Evans, E., Huang, D., \& Anglin, D. M. (2004). Relationship between drug treatment services, retention, and outcomes. Psychiatric Services, 55(7), 767-774.

Hser, Y.-I., Evans, E., Huang, D., \& Messina, N. P. (2011).Long-term outcomes among drug-dependent mothers treated in women-only versus mixed-gender programs. Journal of Substance Abuse Treatment, 41(2), 115-123.

Hser, Y.-I., Grella, C. E., Hubbard, R. L., Hsieh, S.-C., Fletcher, B. W., Brown, B. S., et al. (2001). An evaluation of drug treatments for adolescents in 4 US cities. Archives of General Psychiatry, 58(7), 689-695.

Hser, Y.-I., Maglione, M., Polinsky, M. L., \& Anglin, M. D. (1998). Predicting drug treatment entry among treatment-seeking individuals. Journal of Substance Abuse Treatment, 15(3), 213-220.

Hser, Y.-I., Polinsky, M. L., Maglione, M., \& Anglin, M. D. (1999). Matching clients' needs with drug treatment services. Journal of Substance Abuse Treatment, 16(4), 299-305.

Hubbard, R. L., Craddock, S. G., Flynn, P. M., Anderson, J., \& Etheridge, R. M. (1997). Overview of 1year follow-up outcomes in the Drug Abuse Treatment Outcome Study (DATOS). Psychology of Addictive Behaviors, 11(4), 261-278.

Hudak, M. L, Tan, R. C., The Committee on Drugs, \& The Committee on Fetus and Newborn. (2012). Neonatal drug withdrawal. Pediatrics, 129(2), e540-e560.

Hudmon, K. S., Hemberger, K. K., Corelli, R. L., Kroon, L. A., \& Prokhorov, A. V. (2003). The pharmacist's role in smoking cessation counseling: Perceptions of users of nonprescription nicotine replacement therapy. Journal of the American Pharmaceutical Association, 43(5), 573-582.

Hudmon, K. S., Prokhorov, A. V., \& Corelli, R. L. (2006). Tobacco cessation counseling: Pharmacists' opinions and practices. Patient Education \& Counseling, 61(1), 152-160.

Hudson, N. L., \& Mannino, D. M. (2010). Tobacco use: A chronic illness? Journal of Community Health, $35(5), 549-553$.

Huebner, R. B., \& Kantor, L. W. (2011). Advances in alcoholism treatment. Alcohol Research \& Health, 33(4), 295-299.

Huggett, K. N., Westerman, G. H., Barone, E. J., \& Lofgreen, A. S. (2011). Substance use and dependence education in predoctoral dental curricula: Results of a survey of U.S. and Canadian dental schools. Journal of Dental Education, 75(8), 1003-1009.

Hughes, J. R. (1995). Combining behavioral therapy and pharmacotherapy for smoking cessation: An update. NIDA Research Monograph, 150, 92-109. 
Hughes, J. R., Hatsukami, D. K., Mitchell, J. E., \& Dahlgren, L. A. (1986). Prevalence of smoking among psychiatric outpatients. American Journal of Psychiatry, 143(8), 993-997.

Hughes, J. R., Stead, L. F., \& Lancaster, T. (2005). Nortriptyline for smoking cessation: A review. Nicotine \& Tobacco Research, 7(4), 491-499.

Hughes, J. R., Stead, L. F., \& Lancaster, T. (2007). Antidepressants for smoking cessation. Cochrane Database System Reviews, (1), CD000031.

Hum, A. M., Robinson, L. A., Jackson, A. A., \& Ali, K. S. (2011). Physician communication regarding smoking and adolescent tobacco use. Pediatrics, 127(6), e1368-e1374.

Humeniuk, R., \& Ali, R. (2006). Validation of the Alcohol, Smoking and Substance Involvement Screening Test (ASSIST) and pilot brief intervention: A technical report of phase II findings of the WHO ASSIST Project. Geneva, Switzerland: World Health Organization.

Humeniuk, R., Ali, R., Babor, T. F., Farrell, M., Formigoni, M. L., Jittiwutikarn, J., et al. (2008). Validation of the Alcohol, Smoking and Substance Involvement Screening Test (ASSIST). Addiction, 103(6), 1039-1047.

Humphreys, K. (2003). Alcoholics Anonymous and 12-step alcoholism treatment programs. In M. Galanter (Series Ed.), Recent developments in alcoholism: Vol. 16. Methodology, psychosocial treatment, selected treatment topics, research priorities (pp. 149-64). New York: Kluwer Academic/Plenum Press.

Humphreys, K. (2004). Circles of recovery. Cambridge, MA: Cambridge University Press.

Humphreys, K., \& McLellan, A. T. (2010). Brief intervention, treatment, and recovery support services for Americans who have substance abuse disorders: An overview of policy in the Obama administration. Psychological Services, 7(4), 275-284.

Humphreys, K., \& McLellan, A. T. (2011). A policy-oriented review of strategies for improving the outcomes of services for substance use disorder patients. Addiction, 106(12), 2058-2066.

Humphreys, K., Wagner, T. H., \& Gage, M. (2011). If substance use disorder treatment more than offsets its costs, why don't more medical centers want to provide it? A budget impact analysis in the Veterans Health Administration. Journal of Substance Abuse Treatment, 41(3), 243-251.

Hungerford, D. (2009). Alcohol screening and brief intervention: An introduction. Altanta, GA: Centers for Disease Control and Prevention, National Center for Injury Prevention and Control.

Hungerford, D. W., \& Pollock, D. A. (2002). Alcohol problems among emergency department patients: Proceedings of a research conference on identification and intervention. Atlanta, GA: Centers for Disease Control and Prevention, National Center for Injury Prevention and Control.

Hunt, J. J., Cupertino, A. P., Garrett, S., Friedmann, P. D., \& Richter, K. P. (2012). How is tobacco treatment provided during drug treatment? Journal of Substance Abuse Treatment, 42(1), 4-15.

Hurt, R. D., Eberman, K. M., Croghan, I. T., Offord, K. P., Davis, L. J., Jr., Morse, R. M., et al. (1994). Nicotine dependence treatment during inpatient treatment for other addictions: A prospective intervention trial. Alcoholism: Clinical \& Experimental Research, 18(4), 867-872.

Hurt, R. D., Sachs, D. P., Glover, E. D., Offord, K. P., Johnston, J. A., Dale, L. C., et al. (1997). A comparison of sustained-release bupropion and placebo for smoking cessation. New England Journal of Medicine, 337(17), 1195-1202.

Hyman, S. E. (2005). Addiction: A disease of learning and memory. American Journal of Psychiatry, 162(8), 1414-1422. 
Hyman, S. E. (2007). The neurobiology of addiction: Implications for voluntary control of behavior. American Journal of Bioethics, 7(1), 8-11.

Idaho Admin Code r. 16.06.03.050 (2011).

Iguchi, M. Y., Stitzer, M. L., Bigelow, G. E., \& Liebson, I. A. (1988). Contingency management in methadone maintenance: Effects of reinforcing and aversive consequences on illicit polydrug use. Drug \& Alcohol Dependence, 22(1), 1-7.

Ilgen, M. A., Schulenberg, J., Kloska, D. D., Czyz, E., Johnston, L., \& O'Malley, P. (2011). Prevalence and characteristics of substance abuse treatment utilization by U.S. adolescents: National data from 1987 to 2008. Addictive Behaviors, 36(12), 1349-1352.

Illinois Department of Human Services. (2011). Screening, brief intervention and referral to treatment (SBIRT). [Online]. Retrieved December 19, 2011 from http://www.dhs.state.il.us.

Inciardi, J. A., Martin, S. S., \& Butzin, C. A. (2004). Five-year outcomes of therapeutic community treatment of drug-involved offenders after release from prison. Crime \& Delinquency, 50(1), 88-107.

Indiana University, Health Professions and Prelaw Center. (2007). Two kinds of physicians: Allopathic and osteopathic. [Online]. Retrieved May 25, 2012 from http://hpplc.indiana.edu.

Innovations Report. (2005). Benzodiazepines effective against alcohol withdrawal seizures. [Online]. Retrieved August 16, 2011 from http://www.innovations-report.com.

Insel, T. R. (2008). Assessing the economic costs of serious mental illness. American Journal of Psychiatry, 165(6), 663-665.

Institute of Alcohol Studies. (2011). Alcohol and the elderly: IAS factsheet. [Online]. Retrieved December 19, 2011 from http://www.ias.org.uk.

Institute of Medicine and National Research Council. (2011). The science of adolescent risk-taking. Workshop report. Washington, DC: National Academies Press.

Institute of Medicine, Committee on Crossing the Quality Chasm: Adaptation to Mental Health and Addictive Disorder. (2006). Improving the quality of health care for mental and substance-use conditions: Quality chasm series. Washington, DC: National Academies Press.

Institute of Medicine, Committee on Opportunities in Drug Abuse Research. (1996). Pathways of addiction: Opportunities in drug abuse research. [Online]. Retrieved February 3, 2012 from http://www.nap.edu.

Institute of Medicine, Committee on Quality of Health Care in America. (2001). Crossing the quality chasm: A new health system for for the 21st century. Washington, DC: National Academy Press.

Institute of Medicine. (1990). Broadening the base of treatment for alcohol problems. Washington, DC: National Academy Press.

Institute of Medicine. (1997). Dispelling the myths about addiction: Strategies to increase understanding and strengthen research. Washington, DC: National Academy Press.

Institute of Medicine. (2002). Care without coverage: Too little, too late. Washington, DC: National Academies Press.

Institute of Medicine. (2008). Gulf war and health: Physiologic, psychologic, and psychosocial effects of deployment-related stress (Vols. 6). Washington, DC: National Academies Press.

Institute of Medicine. (2010). Returning home from Iraq and Afghanistan: Preliminary assessment of readjustment needs of veterans, service members, and their families. Washington, DC: National Academies Press. 
International Certification and Reciprocity Consortium. (2012a). About IC \& RC. [Online]. Retrieved May 29, 2012 from http://internationalcredentialing.org.

International Certification and Reciprocity Consortium. (2012b). Alcohol and drug counselor (ADC). [Online]. Retrieved February 2, 2012 from http://internationalcredentialing.org.

International Certification and Reciprocity Consortium. (2012c). Credentialing 101. [Online]. Retrieved February 2, 2012 from http://internationalcredentialing.org.

International Certification and Reciprocity Consortium. (2012d). Find a board. IC\&RC member jurisdictions. [Online]. Retrieved February 13, 2012 from http://internationalcredentialing.org.

International Nurses Society on Addictions (IntNSA). (2012). Examination information. [Online]. Retrieved March 21, 2012 from http://www.intnsa.org.

Isaacson, J. H., Fleming, M., Kraus, M., Kahn, R., \& Mundt, M. (2000). A national survey of training in substance use disorders in residency programs. Journal of Studies on Alcohol, 61(6), 912-915.

Jacobs, W. S., Repetto, M., Vinson, S., Pomm, R., \& Gold, M. S. (2004). Random urine testing as an intervention for drug addiction. Psychiatric Annals, 34(10), 781-784.

Jacobson, I. G., Ryan, M. A., Hooper, T. I., Smith, T. C., Amoroso, P. J., Boyko, E. J., et al. (2008). Alcohol use and alcohol-related problems before and after military combat deployment. JAMA, 300(6), 663-675.

Jacobson, J. O., Robinson, P. L., \& Bluthenthal, R. N. (2007). Racial disparities in completion rates from publicly funded alcohol treatment: Economic resources explain more than demographics and addiction severity. Health Services Research, 42(2), 773-794.

Jacobus, J., McQueeny, T., Bava, S., Schweinsburg, B. C., Frank, L. R., Yang, T. T., et al. (2009). White matter integrity in adolescents with histories of marijuana use and binge drinking. Neurotoxicology \& Teratology, 31(6), 349-355.

Jacobus-Kantor, L., \& Goplerud, E. (2011). The current state of commercial behavioral healthcare services. Results from the 2010 eValue8 Request for Information. [Online]. Retrieved May 15, 2012 from http://www.nbch.org.

Janse Van Rensburg, K., Taylor, A., Hodgson, T., \& Benattayallah, A. (2009). Acute exercise modulates cigarette cravings and brain activation in response to smoking-related images: An FMRI study. Psychopharmacology, 203(3), 589-598.

Jason, L. A., \& Ferrari, J. R. (2010). Oxford House recovery homes: Characteristics and effectiveness. Psychological Services, 7(2), 92-102.

Jason, L. A., Olson, B. D., Ferrari, J. R., \& Lo Sasso, A. T. (2006). Communal housing settings enhance substance abuse recovery. American Journal of Public Health, 96(10), 1727-1729.

Jason, L. A., Olson, B. D., Ferrari, J. R., Majer, J. M., Alvarez, J., \& Stout, J. (2007). An examination of main and interactive effects of substance abuse recovery housing on multiple indicators of adjustment. Addiction, 102(7), 1114-1121.

Jennison, K. M. (1992). The impact of stressful life events and social support on drinking among older adults: A general population survey. International Journal of Aging \& Human Development, 35(2), 99-123.

Jeremias, E., Chatkin, J. M., Chatkin, G., Seibert, J., Martins, M., \& Wagner, M. (2012). Smoking cessation in older adults. International Journal of Tuberculosis \& Lung Disease, 16(2), 273-278. 
Jerrell, J. M., \& Ridgely, M. S. (1995). Comparative effectiveness of three approaches to serving people with severe mental illness and substance abuse disorders. Journal of Nervous \& Mental Disease, 183(9), 566-576.

Jinks, M. J., \& Raschko, R. R. (1990). A profile of alcohol and prescription drug abuse in a high-risk community-based elderly population. DICP Annals of Pharmacotherapy, 24(10), 971-975.

Joffe, A., McNeely, C., Colantuoni, E., An, M. W., Wang, W., \& Scharfstein, D. (2009). Evaluation of school-based smoking-cessation interventions for self-described adolescent smokers. Pediatrics, 124(2), e187-e194.

Johns Hopkins Medicine. (2007). Baclofen. [Online]. Retrieved April 3, 2012 from http://www.hopkinsmedicine.org.

Johnson, B. A. (2004). Progress in the development of topiramate for treating alcohol dependence: From a hypothesis to a proof-of-concept study. Alcoholism, Clinical \& Experimental Research, 28(8), $1137-1144$.

Johnson, B. A. (2005). Recent advances in the development of treatments for alcohol and cocaine dependence: Focus on topiramate and other modulators of GABA or glutamate function. CNS Drugs, 19(10), 873-896.

Johnson, B. A. (2008). Update on neuropharmacological treatments for alcoholism: Scientific basis and clinical findings. Biochemical Pharmacology, 75(1), 34-56.

Johnson, B. A., \& Ait-Daoud, N. (1999). Medications to treat alcoholism. Alcohol Research \& Health, 23(2), 99-106.

Johnson, B. A., O'Malley, S. S., Ciraulo, D. A., Roache, J. D., Chambers, R. A., Sarid-Segal, O., et al. (2003). Dose-ranging kinetics and behavioral pharmacology of naltrexone and acamprosate, both alone and combined, in alcohol-dependent subjects. Journal of Clinical Psychopharmacology, 23(3), 281-293.

Johnson, B. A., Rosenthal, N., Capece, J. A., Wiegand, F., Mao, L., Beyers, K., et al. (2007). Topiramate for treating alcohol dependence: A randomized controlled trial. JAMA, 298(14), 1641-1651.

Johnson, B. A., Rosenthal, N., Capece, J. A., Wiegand, F., Mao, L., Beyers, K., et al. (2008). Improvement of physical health and quality of life of alcohol-dependent individuals with topiramate treatment: US multisite randomized controlled trial. Archives of Internal Medicine, 168(11), 11881199.

Johnson, G. K., \& Slach, N. A. (2001). Impact of tobacco use on periodontal status. Journal of Dental Education, 65(4), 313-321.

Johnson, J. G., Cohen, P., Pine, D. S., Klein, D. F., Kasen, S., \& Brook, J. S. (2000). Association between cigarette smoking and anxiety disorders during adolescence and early adulthood. JAMA, 284(18), 2348-2351.

Johnson, N. (2001). Tobacco use and oral cancer: A global perspective. Journal of Dental Education, 65(4), 328-339.

Johnson, N. W., \& Warnakulasuriya, K. A. A. S. (2003). Do patients listen when we tell them to stop smoking? British Dental Journal, 194(3), 150-150.

Johnson, N., Oliff, P., \& Williams, E. (2011). An update on state budget cuts: At least 46 states have imposed cuts that hurt vulnerable residents and cause job loss. [Online]. Retrieved November 14, 2011 from http://www.cbpp.org. 
Johnson, R. E., Chutuape, M. A., Strain, E. C., Walsh, S. L., Stitzer, M. L., \& Bigelow, G. E. (2000). A comparison of levomethadyl acetate, buprenorphine, and methadone for opioid dependence. New England Journal of Medicine, 343(18), 1290-1297.

Join Together. (2003a). National poll of physicians on barriers to widespread buprenorphine use. Boston, MA: Join Together.

Join Together. (2003b). Rewarding results: Improving the quality of treatment for people with alcohol and drug problems. Administration and Policy in Mental Health, 31(4), 283-312.

Joint Commission. (2012a). About The Joint Commission. [Online]. Retrieved March 22, 2012 from http://www.jointcommission.org.

Joint Commission. (2012b). Specifications Manual for National Hospital Inpatient Quality Measures Version 4.0: Substance use (SUB) measures. [Online]. Retrieved February 28, 2012 from http://www.jointcommission.org.

Joint Commission. (2012c). Specifications Manual for National Hospital Inpatient Quality Measures Version 4.0: Tobacco treatment (TOB) measures. [Online]. Retrieved February 28, 2012 from http://www.prevent.org.

Joint Commission. (2012d). Standards for behavioral health care: Standards elements of performance scoring accreditation policies. Oakbrook Terrace, IL: Author.

Joint Commission. (2012e). Tobacco treatment. [Online]. Retrieved February 27, 2012 from http://www.jointcommission.org.

Jones, H. E. (2004). Practical considerations for the clinical use of buprenorphine. Science \& Practice Perspectives, 2(2), 4-20.

Jones, H. E., Kaltenbach, K., Heil, S. H., Stine, S. M., Coyle, M. G., Arria, A. M., et al. (2010). Neonatal abstinence syndrome after methadone or buprenorphine exposure. New England Journal of Medicine, $363(24), 2320-2331$.

Jones, P. (2009a). Confirming the effectiveness of an evidence-based practice: Use of motivational interviewing in the real world. In Central East Addiction Technology, An overview of evidence-based practices: Implementing science-based interventions in practical settings (pp. 10-13). Silver Spring, MD: Central East Addiction Technology Transfer Center/Danya Institute.

Jones, P. (2009b). Motivational incentives: Useful tool in the improvement of treatment outcomes. Providing positive reinforcement with motivational incentives. In Central East Addiction Technology, An overview of evidence-based practices: Implementing science-based interventions in practical settings (pp. 15-18). Silver Spring, MD: Central East Addiction Technology Transfer Center/Danya Institute.

Jordan, J. B. (2006). Acupuncture treatment for opiate addiction: A systematic review. Journal of Substance Abuse Treatment, 30(4), 309-314.

Jorenby, D. E., Leischow, S. J., Nides, M. A., Rennard, S. I., Johnston, J. A., Hughes, A. R., et al. (1999). A controlled trial of sustained-release bupropion, a nicotine patch, or both for smoking cessation. New England Journal of Medicine, 340(9), 685-691.

Joslin Diabetes Center. (2012). Diabetes denial: Acknowledging the disease for better care. [Online]. Retrieved April 27, 2012 from http://www.joslin.org.

Kaiser Commission on Medicaid and the Uninsured. (2003). The cost of not covering the uninsured. Project highlights. [Online]. Retrieved February 1, 2012 from http://www.kff.org. 
Kaiser Commission on Medicaid and the Uninsured. (2005). The Medicaid program at a glance. [Online]. Retrieved April 5, 2012 from http://www.kff.org.

Kaiser Commission on Medicaid and the Uninsured. (2007). Massachusetts health care reform plan: An update. [Online]. Retrieved May 16, 2012 from http://www.kff.org.

Kaiser Commission on Medicaid and the Uninsured. (2011a). Benefits by service: Inpatient hospital services, other than in an institution for mental diseases (October 2010). [Online]. Retrieved April 5, 2012 from http://medicaidbenefits.kff.org.

Kaiser Commission on Medicaid and the Uninsured. (2011b). Benefits by service: Outpatient hospital services (October 2010). [Online]. Retrieved April 5, 2012 from http://medicaidbenefits.kff.org.

Kalivas, P. W., \& Vokow, N. D. (2005). The neural basis of addiction: A pathology of motivation and choice. American Journal of Psychiatry, 162(8), 1403-1413.

Kalman, D., Morissette, S. B., \& George, T. P. (2005). Co-morbidity of smoking in patients with psychiatric and substance use disorders. American Journal on Addictions, 14(2), 106-123.

Kaltenbach, K., Berghella, V., \& Finnegan, L. (1998). Opioid dependence during pregnancy. Effects and management. Obstetrics \& Gynecology Clinics of North America, 25(1), 139-151.

Kaminer, Y., Bukstein, O., \& Tarter, R. E. (1991). The teen-addiction severity index: Rationale and reliability. International Journal of Addictions, 26(2), 219-226.

Kampman, K. M. (2008). The search for medications to treat stimulant dependence. Addiction Science \& Clinical Practice, 4(2), 28-35.

Kampman, K. M., Pettinati, H., Lynch, K. G., Dackis, C., Sparkman, T., Weigley, C., et al. (2004). A pilot trial of topiramate for the treatment of cocaine dependence. Drug \& Alcohol Dependence, 75(3), 233-240.

Kandall, S. R. (1998). Women and addiction in the United States: 1850 to 1920. In C. L. Wetherington \& A. B. Roman (Eds.), Drug addiction research and the health of women (pp. 33-52). Rockville, MD: U.S. Department of Health and Human Services, National Institutes of Health, National Institute on Drug Abuse.

Kandall, S. R., \& Petrillo, J. (1996). Substance and shadow: Women and addiction in the United States. Cambridge, MA: Harvard University Press.

Karila, L., Reynaud, M., Aubin, H. J., Rolland, B., Guardia, D., Cottencin, O., et al. (2011). Pharmacological treatments for cocaine dependence: Is there something new? Current Pharmaceutical Designs, 17(14), 1359-1368.

Karila, L., Weinstein, A., Aubin, H. J., Benyamina, A., Reynaud, M., \& Batki, S. L. (2010). Pharmacological approaches to methamphetamine dependence: A focused review. British Journal of Clinical Pharmacology, 69(6), 578-592.

Karim, R., \& Chaudhri, P. (2012). Behavioral addictions: An overview. Journal of Psychoactive Drugs, 44(1), 5-17.

Karkowski, L. M., Prescott, C. A., \& Kendler, K. S. (2000). Multivariate assessment of factors influencing illicit substance use in twins from female-female pairs. American Journal of Medical Genetics, 96(5), 665-670.

Kaskutas, L. A. (2009). Alcoholics Anonymous effectiveness: Faith meets science. Journal of Addictive Disorders, 28(2), 145-157.

Katcher, B. S. (1993). Benjamin Rush's educational campaign against hard drinking. American Journal of Public Health, 83(2), 273-281. 
Kaufman, J., Yang, B.-Z., Douglas-Palumberi, H., Crouse-Artus, M., Lipschitz, D., Krystal, J. H., et al. (2007). Genetic and environmental predictors of early alcohol use. Biological Psychiatry, 61, 12281234.

Kealey, K. A., Ludman, E. J., Mann, S. L., Marek, P. M., Phares, M. M., Riggs, K. R., et al. (2007). Overcoming barriers to recruitment and retention in adolescent smoking cessation. Nicotine \& Tobacco Research, 9(2), 257-270.

Kehle, S. M., Ferrier-Auerbach, A. G., Meis, L. A., Arbisi, P. A., Erbes, C. R., \& Polusny, M. A. (2012). Predictors of postdeployment alcohol use disorders in National Guard soldiers deployed to Operation Iraqi Freedom. Journal of Addictive Behaviors, 26(1), 42-50.

Keller, D. S., \& Dermatis, H. (1999). Current status of professional training in the addictions. Substance Abuse, 20(3), 123-140.

Kelley, A. E., \& Berridge, K. C. (2002). The neuroscience of natural rewards: Relevance to addictive drugs. Journal of Neuroscience, 22(9), 3306-3311.

Kelly, J. F., \& Yeterian, J. D. (2011). The role of mutual-help groups in extending the framework of treatment. [Online]. Retrieved November 22, 2011 from http://pubs.niaaa.nih.gov.

Kelly, J. F., Magill, M., \& Stout, R. L. (2009). How do people recover from alcohol dependence? A systematic review of the research on mechanisms of behavior change in Alcoholics Anonymous. Addiction Research \& Theory, 17(3), 236-259.

Kelly, J. F., Stout, R., Zywiak, W., \& Schneider, R. (2006). A 3-year study of addiction mutual-help group participation following intensive outpatient treatment. Alcoholism: Clinical \& Experimental Research, 30(8), 1381-1392.

Kelly, J. P., Kaufman, D. W., Koff, R. S., Laszlo, A., Wiholm, B., \& Shapiro, S. (1995). Alcohol consumption and the risk of major upper gastrointestinal bleeding. American Journal of Gastroenterology, 90(7), 1058-1061.

Kendler, K. S., Jacobson, K. C., Prescott, C. A., \& Neale, M. C. (2003). Specificity of genetic and environmental risk factors for use and abuse/dependence of cannabis, cocaine, hallucinogens, sedatives, stimulants, and opiates in male twins. American Journal of Psychiatry, 160(4), 687-695.

Kendler, K. S., Karkowski, L., \& Prescott, C. A. (1999). Hallucinogen, opiate, sedative and stimulant use and abuse in a population-based sample of female twins. Acta Psychiatrica Scandanavia, 99(5), 368376.

Kendler, K. S., Neale, M. C., Heath, A. C., Kessler, R. C., \& Eaves, L. J. (1994). A twin-family study of alcoholism in women. American Journal of Psychiatry, 151(5), 707-715.

Kendler, K. S., Neale, M. C., Sullivan, P., Corey, L. A., Gardner, C. O., \& Prescott, C. A. (1999). A population-based twin study in women of smoking initiation and nicotine dependence. Psychological Medicine, 29(2), 299-308.

Kenna, G. A., Zywiak, W. H., McGeary, J. E., Leggio, L., McGeary, C., Wang, S., et al. (2009). A within-group design of nontreatment seeking 5-HTTLPR genotyped alcohol-dependent subjects receiving ondansetron and sertraline. Alcoholism: Clinical \& Experimental Research, 33(2), 315-323.

Kennedy, D. P., Tucker, J. S., Pollard, M. S., Go, M. H., \& Green, H. D., Jr. (2011). Adolescent romantic relationships and change in smoking status. Addictive Behaviors, 36(4), 320-326.

Kennerly, S. W., Behrens, T. E. J., \& Wallis, J. D. (2011). Double dissociation of value computations in orbitofrontal and anterior cingulate neurons. Nature Neuroscience, 14(12), 1581-1591. 
Kenny, P. J. (2011). Common cellular and molecular mechanisms in obesity and drug addiction. [Online]. Retrieved December 2, 2011 from http://www.nature.com.

Kerr, S., Watson, H., Tolson, D., Lough, M., \& Brown, M. (2006). Smoking after the age of 65 years: A qualitative exploration of older current and former smokers' views on smoking, stopping smoking, and smoking cessation resources and services. Health \& Social Care in the Community, 14(6), 572582.

Kerwin, M. E., Walker-Smith, K., \& Kirby, K. C. (2006). Comparative analysis of state requirements for the training of substance abuse and mental health counselors. Journal of Substance Abuse Treatment, $30(3), 173-181$.

Kesmodel, U., Wisborg, K., Olsen, S. F., Henriksen, T. B., \& Secher, N. J. (2002a). Moderate alcohol intake during pregnancy and the risk of stillbirth and death in the first year of life. American Journal of Epidemiology, 155(4), 305-312.

Kesmodel, U., Wisborg, K., Olsen, S. F., Henriksen, T. B., \& Secher, N. J. (2002b). Moderate alcohol intake in pregnancy and the risk of spontaneous abortion. Alcohol \& Alcoholism, 37(1), 87-92.

Kessler, R. C., Anthony, J. C., Blazer, D. G., Bromet, E., Eaton, W. W., Kendler, K. S., et al. (1997). The US National Comorbidity Survey: Overview and future directions. [Online]. Retrieved August 16, 2011 from http://www.hcp.med.harvard.edu.

Kessler, R. C., Sonnega, A., Bromet, E., Hughes, M., \& Nelson, C. B. (1995). Posttraumatic stress disorder in the National Comorbidity Survey. Archives of General Psychiatry, 52(12), 1048-1060.

Keyes, K. M., Hatzenbuehler, M. L., McLaughlin, K. A., Link, B., Olfson, M., Grant, B. F., et al. (2010). Stigma and treatment for alcohol disorders in the United States. American Journal of Epidemiology, 172(12), 1364-1372.

Khantzian, E. J. (1985). The self-medication hypothesis of addictive disorders: Focus on heroin and cocaine dependence. American Journal of Psychiatry, 142(11), 1259-1264.

Kidorf, M., \& Stitzer, M. L. (1996). Contingent use of take-homes and split-dosing to reduce illicit drug use of methadone patients. Behavior Therapy, 27(1), 41-51.

Kilfoy, B. A., Prokhorov, A. V., \& Hudmon, K. S. (2006). Pharmacy placement of nonprescription nicotine replacement therapy products and community pharmacists' counseling for product use. Journal of the American Pharmaceutical Association, 46(6), 723-728.

Kills Small, N. J., Simons, J. S., \& Stricherz, M. (2007). Assessing criterion validity of the Simple Screening Instrument for Alcohol and Other Drug Abuse (SSI-AOD) in a college population. Addictive Behaviors, 32(10), 2425-2431.

Kilpatrick, D. G., Acierno, R., Saunders, B., Resnick, H. S., Best, C. L., \& Schnurr, P. P. (2000). Risk factors for adolescent substance abuse and dependence: Data from a national sample. Journal of Consulting \& Clinical Psychology, 68(1), 19-30.

Kim, M. J., Catalano, R. F., Haggerty, K. P., \& Abbott, R. D. (2011). Bullying at elementary school and problem behaviour in young adulthood: A study of bullying, violence and substance use from age 11 to age 21. Criminal Behaviour \& Mental Health, 21(2), 136-144.

Kim, S. W., Grant, J. E., Adson, D. E., \& Shin, Y. C. (2001). Double-blind naltrexone and placebo comparison study in the treatment of pathological gambling. Biological Psychiatry, 49(11), 914-921.

King, A. C., Volpicelli, J. R., Frazer, A., \& O'Brien, C. P. (1997). Effect of naltrexone on subjective alcohol response in subjects at high and low risk for future alcohol dependence.

Psychopharmacology, 129(1), 15-22. 
King, M. B. (1986). Alcohol abuse and dementia. International Journal of Geriatric Psychiatry, 1(1), 31 36.

King, S. M., Iacono, W. G., \& McGue, M. (2004). Childhood externalizing and internalizing psychopathology in the prediction of early substance use. Addiction, 99(12), 1548-1559.

King, V. L., Stoller, K. B., Kidorf, M., Kindbom, K., Hursh, S., Brady, T., et al. (2009). Assessing the effectiveness of an Internet-based videoconferencing platform for delivering intensified substance abuse counseling. Journal of Substance Abuse Treatment, 36(3), 331-338.

Kinsey, B. M., Jackson, D. C., \& Orson, F. M. (2009). Anti-drug vaccines to treat substance abuse. Immunology \& Cell Biology, 87(4), 309-314.

Kip, M. J., Neumann, T., Jugel, C., Kleinwaechter, R., Weiss-Gerlach, E., Guill, M. M., et al. (2008). New strategies to detect alcohol use disorders in the preoperative assessment clinic of a German university hospital. Anesthesiology, 109(2), 171-179.

Kitson, T. M. (1987). The effect of cephalosporin antibiotics on alcohol metabolism: A review. Alcohol, $4(3), 143-148$.

Kjome, K. L., \& Moeller, F. G. (2011). Long-acting injectable naltrexone for the management of patients with opioid dependence. Substance Abuse: Research \& Treatment, 5, 1-9.

Kleber, H. D. (2008). Methadone maintenance 4 decades later: Thousands of lives saved but still controversial. JAMA, 300(19), 2303-2305.

Kleber, H. D., Weiss, R. D., Anton, R. F., Rounsaville, B. J., George, T. P., Strain, E. C., et al. (2006). Part A: Treatment of patients with substance use disorders, second edition. American Psychiatric Association. American Journal of Psychiatry, 163(Suppl. 8), 5-82.

Klein, W. C., \& Jess, C. (2002). One last pleasure? Alcohol use among elderly people in nursing homes. Health \& Social Work, 193(203), 27-3.

Klostermann.K., Kelley, M. L., Mignone, T., Pusateri, L., \& Wills, K. (2011). Behavioral couples therapy for substance abusers: Where do we go from here? Substance Use \& Misuse, 46(12), 1502-1509.

Klug, M. G., \& Burd, L. (2003). Fetal alcohol syndrome prevention: Annual and cumulative cost savings. Neurotoxicology \& Teratology, 25(6), 763-765.

Kluger, R. (1996). Ashes to ashes. New York: Knopf.

Knight, D. K., Landrum, B., Becan, J. E., \& Flynn, P. M. (2012). Program needs and change orientation: Implications for counselor turnover. Journal of Substance Abuse Treatment, 42(2), 159-168.

Knight, J. R., Sherritt, L., Shrier, L. A., Harris, S. K., \& Chang, G. (2002). Validity of the CRAFFT substance abuse screening test among adolescent clinic patients. Archives of Pediatrics \& Adolescent Medicine, 156(6), 607-614.

Knight, J. R., Shrier, L. A., Bravender, T. D., Farrell, M., Vander Bilt, J., \& Shaffer, H. J. (1999). A new brief screen for adolescent substance abuse. Archives of Pediatrics \& Adolescent Medicine, 153(6), 591-596.

Knopf, A. (2010). Diagnostic uncertainty: Changes in the proposed DSM-5 worry addiction counselors, research professionals. Addiction Professional, 8(5), 8-13.

Knopf, A. (2011). CMS proposes free SBI for Medicare patients, but barriers still exist for PCPs. [Online]. Retrieved December 19, 2011 from http://www.alcoholismdrugabuseweekly.com. 
Knudsen, H. K. (2006). Clinical trials network: Counselor-level data on evidence-based treatment practices (National Treatment Center Study Summary Report No. 11). Atlanta, GA: Institute for Behavioral Research, Center for Research on Behavioral Health and Human Services Delivery, University of Georgia.

Knudsen, H. K. (2009). Smoking cessation services in adolescent substance abuse treatment: Opportunities missed? Journal of Drug Issues, 39(2), 257-276.

Knudsen, H. K., \& Studts, J. L. (2011). Availability of nicotine replacement therapy in substance use disorder treatment: Longitudinal patterns of adoption, sustainability, and discontinuation. Drug \& Alcohol Dependence, 118(2-3), 244-250.

Knudsen, H. K., Abraham, A. J., \& Oser, C. B. (2011). Barriers to the implementation of medicationassisted treatment for substance use disorders: The importance of funding policies and medical infrastructure. Evaluation \& Program Planning, 34(4), 375-381.

Knudsen, H. K., Abraham, A. J., Johnson, J. A., \& Roman, P. M. (2009). Buprenorphine adoption in the National Drug Abuse Treatment Clinical Trials Network. Journal of Substance Abuse Treatment, 37(3), 307-312.

Knudsen, H. K., Ducharme, L. J., Roman, P. M., \& Link, T. (2005). Buprenorphine diffusion: The attitudes of substance abuse treatment counselors. Journal of Substance Abuse Treatment, 29(2), 95106.

Knudsen, H. K., Studts, C. R., \& Studts, J. L. (2011). The implementation of smoking cessation counseling in substance abuse treatment. Journal of Behavioral Health Services Research, 39(1), 2841.

Knudsen, H. K., Studts, J. L., \& Boyd, S. (2009). Smoking cessation practices in community treatment programs. Project report March 2009. [Online].from http://www.uga.edu.

Kochanek, K., Xu, J. Q., Murphy, S. L., Minino, A. M., \& Kung, H.-C. (2011). Deaths: Preliminary data for 2009. National vital statistics reports (DHHS Pub No. (PHS) 2011-1120, Vols. 59). Hyattsville, MD: National Center for Health Statistics.

Kochupillai, V., Kumar, P., Singh, D., Aggarwal, D., Bhardwaj, N., Bhutani, M., et al. (2005). Effect of rhythmic breathing (Sudarshan Kriya and Pranayam) on immune functions and tobacco addiction. Annals of the New York Academy of Sciences, 1056, 242-252.

Kohn, L. T., Corrigan, J., \& Donaldson, M. S. (2000). To err is human: Building a safer health system. Washington, DC: Institute of Medicine.

Kokotailo, P. K., \& Committee on Substance Abuse. (2010). Policy statement: Alcohol use by youth and adolescents: A pediatric concern. Pediatrics, 125(5), 1078-1087.

Koob, G. F. (2008). A role for brain stress systems in addiction. Neuron, 59(1), 11-34.

Koplewicz, H. (2011). Drug addiction: Stigma paints it as a choice, not a mental illness. [Online]. Retrieved December 8, 2011 from http://www.huffingtonpost.com.

Kosten, T. R. (1994). Treatment for alcohol and other drug abuse: Opportunities for coordination: Technical assistance publication (TAP) series 11: Chapter 8-Pharmacotherapies for alcohol and drug dependence (DHHS Publication No. (SMA) 94-2075). Rockville, MD: U.S. Department of Health and Human Services, Substance Abuse and Mental Health Services Administration, Center for Substance Abuse Treatment.

Kosten, T. R., \& O'Connor, P. G. (2003). Management of drug and alcohol withdrawal. New England Journal of Medicine, 348(18), 1786-1795. 
Kosten, T., Oliveto, A., Feingold, A., Poling, J., Sevarino, K., Cance-Katz, E., et al. (2003). Desipramine and contingency management for cocaine and opiate dependence in buprenorphine maintained patients. Drug \& Alcohol Dependence, 70(3), 315-325.

Krahn, G., Deck, D., Gabriel, R., \& Farrell, N. (2007). A population-based study on substance abuse treatment for adults with disabilities: Access, utilization, and treatment outcomes. American Journal of Drug \& Alcohol Abuse, 33(6), 791-798.

Krahn, G., Farrell, N., Gabriel, R. M., \& Deck, D. (2006). Access barriers to substance abuse treatment for persons with disabilities: An exploratory study. Journal of Substance Abuse Treatment, 31(4), 375-384.

Krampe, H., Stawicki, S., Wagner, T., Bartels, C., Aust, C., Ruther, E., et al. (2006). Follow-up of 180 alcoholic patients for up to 7 years after outpatient treatment: Impact of alcohol deterrents on outcome. Alcoholism: Clinical \& Experimental Research, 30(1), 86-95.

Kranzler, H. R., Kadden, R. M., Babor, T. F., Tennen, H., \& Rounsaville, B. J. (1996). Validity of the SCID in substance abuse patients. Addiction, 91(6), 859-868.

Kranzler, H. R., Modesto-Lowe, V., \& Van, K. J. (2000). Naltrexone vs. nefazodone for treatment of alcohol dependence: A placebo-controlled trial. Neuropsychopharmacology, 22(5), 493-503.

Kranzler, H. R., Montejano, L. B., Stephenson, J. J., Wang, S., \& Gastfriend, D. R. (2010). Effects of naltrexone treatment for alcohol-related disorders on healthcare costs in an insured population. Alcoholism: Clinical \& Experimental Research, 34(6), 1090-1097.

Kranzler, H. R., Pierucci-Lagha, A., Feinn, R., \& Hernandez-Avila, C. (2003). Effects of ondansetron in early- versus late-onset alcoholics: A prospective, open-label study. Alcoholism: Clinical \& Experimental Research, 27(7), 1150-1155.

Kraus, M. L., Alford, D. P., Kotz, M. M., Levounis, P., Mandell, T. W., Meyer, M., et al. (2011). Statement of the American Society of Addiction Medicine Consensus Panel on the use of buprenorphine in office-based treatment of opioid addiction. Journal of Addiction Medicine, 5(4), 254-263.

Kraybill, K., Zerger, S., \& National Health Care for the Homeless Council. (2003). Providing treatment for homeless people with substance use disorders: Case studies of six programs. [Online]. Retrieved February 6, 2012 from http://www.nhchc.org.

Kreek, M. J., Nielsen, D. A., Butelman, E. R., \& LaForge, K. S. (2005). Genetic influences on impulsivity, risk taking, stress responsivity and vulnerability to drug abuse and addiction. Nature Neuroscience, 8(11), 1450-1457.

Kreitzer, M. J., Kligler, B., \& Meeker, W. C. (2009). Health professions education and integrative health care. [Online]. Retrieved March 1, 2012 from http://www.iom.edu.

Kremer, D., Malkin, M. J., \& Benshoff, J. J. (1995). Physical activity programs offered in substance abuse treatment facilities. Journal of Substance Abuse Treatment, 12(5), 327-333.

Krisberg, B. (1998). Substance abuse and the juvenile justice system: A paper presented at the Juvenile Justice \& Substance Abuse national Planning meeting sponsored by the Robert Wood Johnson Foundation. Annapolis, MD: National Council on Crime and Delinquency.

Krishnamoorthy, S., Lip, G. Y. J., \& Lane, D. A. (2009). Alcohol and illicit drug use as precipitants of atrial fibrillation in young adults: A case series and literature review. American Journal of Medicine, 122(9), 851-856. 
Krishnan-Sarin, S., Duhig, A. M., McKee, S. A., McMahon, T. J., Liss, T., McFetridge, A., et al. (2006). Contingency management for smoking cessation in adolescent smokers. Experimental \& Clinical Psychopharmacology, 14(3), 306-310.

Krishnan-Sarin, S., Krystal, J. H., Shi, J., Pittman, B., \& O'Malley, S. S. (2007). Family history of alcoholism influences naltrexone-induced reduction in alcohol drinking. Biological Psychiatry, 62(6), 694-697.

Krupski, A., Sears, J. M., Joesch, J. M., Estee, S., He, L., Dunn, C., et al. (2010). Impact of brief interventions and brief treatment on admissions to chemical dependency treatment. Drug \& Alcohol Dependence, 110(1-2), 126-136.

Kuehn, D. (2007). Looking back on 35 years: NAADAC's history reflects the establishment of a professional identity for addiction counseling. [Online]. Retrieved May 21, 2012 from http://www.thefreelibrary.com.

Kuepper, R., van Os, J., Lieb, R., Wittchen, H.-U., Hofler, M., \& Henquet, C. (2011). Continued cannabis use and risk of incidence and persistence of psychotic symptoms: 10 year follow-up cohort study. British Medical Journal, 342, d738-d746.

Kulig, J. W., \& Committee on Substance Abuse. (2005). Tobacco, alcohol, and other drugs: The role of the pediatrician in prevention, identification, and management of substance abuse. Pediatrics, 115(3), 816-821.

Kumpfer, K. L., Trunnell, E. P., \& Whiteside, H. O. (1990). The biopsychosocial model: Application to the addictions field. In R. C. Engs (Ed.), Controversies in the addiction field. (pp. 55-67). Dubuque, Iowa: Kendall-Hunt.http://www.indiana.edu.

Kurtz, D. L., Stewart, R. B., Zweifel, M., Li, T. K., \& Froehlich, J. C. (1996). Genetic differences in tolerance and sensitization to the sedative/hypnotic effects of alcohol. Pharmacology, Biochemistry, \& Behavior, 53(3), 585-591. .

Ky. Rev. Stat. § 222.231.

Lab Tests Online. (2011). Nicotine/cotinine. [Online]. Retrieved November 10, 2011 from http://labtestsonline.org.

Lafferty, L., Hunter, T. S., \& Marsh, W. A. (2006). Knowledge, attitudes and practices of pharmacists concerning prescription drug abuse. Journal of Psychoactive Drugs, 38(3), 229-232.

Lake Research Partners. (2009). New poll shows broad bi-partisan support for improving access to alcohol and drug addiction treatment. [Online]. Retrieved August 16, 2011 from http://www.soros.org.

Lake Research Partners. (2009). New poll shows majority of Americans support efforts to make alcohol and drug addiction treatment more accessible, affordable. [Online]. Retrieved November 16, 2011 from http://www.soros.org.

Lamb, S., Greenlick, M. R., \& McCarty, D. (Eds.). (1998). Bridging the gap between practice and research: Forging partnerships with community-based drug and alcohol treatment. Washington, DC: National Academy Press.

Lamy, P. P. (1988). Actions of alcohol and drugs in older people. Generations, 12(4), 9-13.

Land, T., Rigotti, N. A., Levy, D. E., Paskowsky, M., Warner, D., Kwass, J. A., et al. (2010). A longitudinal study of medicaid coverage for tobacco dependence treatments in Massachusetts and associated decreases in hospitalizations for cardiovascular disease. PLoS Medicine, 7(12), e1000375. 
Lanier, D., \& Ko, S. (2008). Screening in primary care settings for illicit drug use: Assessment of screening instruments--A supplemental evidence update for the U.S. Preventive Services Task Force: Evidence Synthesis No. 58, Part 2 (AHRQ Publication No. 08-05108-EF-2). Rockville, MD: U.S. Department of Health and Human Services, Agency for Healthcare Research and Quality.

Lanier, R. K., Umbricht, A., Harrison, J. A., Nuwayser, E. S., \& Bigelow, G. E. (2007). Evaluation of a transdermal buprenorphine formulation in opioid detoxification. Addiction, 102(10), 1648-1656.

LaPaglia, D. (2011). Challenges and solutions of adding medications treatment to specialty addiction treatment programs: A review with suggestions. Alcohol Research \& Health, 33(4), 305-307.

Lapham, S. (2004). Screening and brief intervention in the criminal justice system. Alcohol Research \& Health, 28(2), 85-92.

Larimer, M. E., \& Cronce, J. M. (2002). Identification, prevention and treatment: A review of individualfocused strategies to reduce problematic alcohol consumption by college students. Journal of Studies on Alcohol, (Suppl. 14), 148-163.

Larimer, M. E., \& Kilmer, J. R. (2000). Natural history. In G. Zernig, A. Saria, M. Kurz, \& S. S. O'Malley (Eds.), Handbook of alcoholism. (pp. 13-28). Boca Raton, FL: CRC Press.

Larson, J. S. (1999). The conceptualization of health. Medical Care Research \& Review, 56(2), 123-136.

Larsson, M. L., Frisk, M., Hallstrom, J., Kiviloog, J., \& Lundback, B. (2001). Environmental tobacco smoke exposure during childhood is associated with increased prevalence of asthma in adults. Chest, $120,711-717$.

Lasser, K., Boyd, J. W., Woolhandler, S., Himmelstein, D. U., McCormick, D., \& Bor, D. H. (2000). Smoking and mental illness: A population-based prevalence study. JAMA, 284(20), 2606-2610.

Latimer, W. W., Winters, K. C., \& Stinchfield, R. D. (1997). Screening for drug abuse among adolescents in clinical and correctional settings using the Problem-Oriented Screening Instrument for Teenagers. American Journal of Drug \& Alcohol Abuse, 23(1), 79-98.

Latimer, W. W., Winters, K. C., D'Zurilla, T., \& Nichols, M. (2003). Integrated family and cognitivebehavioral therapy for adolescent substance abusers: Stage I efficacy study. Drug \& Alcohol Dependence, 71, 303-317.

Laudet, A. B., \& White, W. (2010). What are your priorities right now? Identifying service needs across recovery stages to inform service development. Journal of Substance Abuse Treatment, 38(1), 51-59.

Lazev, A. B., Vidrine, D. J., Arduino, R. C., \& Gritz, E. R. (2004). Increasing access to smoking cessation treatment in a low-income, HIV-positive population: The feasibility of using cellular telephones. Nicotine \& Tobacco Research, 6(2), 281-286.

Lazowski, L. E., Miller, F. G., Boye, M. W., \& Miller, G. A. (1998). Efficacy of the Substance Abuse Subtle Screening Inventory-3 (SASSI-3) in identifying substance dependence disorders in clinical settings. Journal of Personality Assessment, 71(1), 114-128.

Leavitt, S. B. (2002). Evidence for the efficacy of naltrexone in the treatment of alcohol dependence. [Online]. Retrieved January 25, 2012 from http://dpt.samhsa.gov.

Lee, P. R., Lee, D. R., Lee, P., \& Arch, M. (2010). 2010: U.S. drug and alcohol policy, looking back and moving forward. Journal of Psychoactive Drugs, 42(2), 99-114.

Lee, S., Aos, S., Drake, E., Pennucci, A., Miller, M., \& Anderson, L. (2012). Return on investment: Evidence-based options to improve statewide outcomes, April 2012 update. [Online]. Retrieved May 7, 2012 from http://www.wsipp.wa.gov. 
Legacy Foundation. (2011). A hidden epidemic: Tobacco use and mental illness. [Online]. from http://www.legacyforhealth.org.

Lejoyeux, M., Solomon, J., \& Ades, J. (1998). Benzodiazepine treatment for alcohol-dependent patients. Alcohol \& Alcoholism, 33(6), 563-575.

Lenardson, J. D., \& Gale, J. A. (2007). Distribution of substance abuse treatment facilities across the rural-urban continuum. Portland, ME: Muskie School of Public Service.

Leonard, R. H. (1991). Alcohol, alcoholism, and dental treatment. Compendium, 12(4), 274, 276-278, 280-283.

Leonardson, G. R. (2005). Substance abuse treatment produces savings in South Dakota. [Online]. Retrieved March 29, 2012 from http://dhs.sd.gov.

Lerman, C., Roth, D., Kaufmann, V., Audrain, J., Hawk, L., Liu, A., et al. (2002). Mediating mechanisms for the impact of bupropion in smoking cessation treatment. Drug \& Alcohol Dependence, 67(2), 219223.

LeSage, M. G., Keyler, D. E., \& Pentel, P. R. (2006). Current status of immunologic approaches to treating tobacco dependence: Vaccines and nicotine-specific antibodies. AAPS Journal, 8(1), E65E75.

Lesher, S. D., \& Lee, Y. T. (1989). Acute pancreatitis in a military hospital. Military Medicine, 154(11), 559-564.

Leshner, A. I. (1997). Addiction is a brain disease, and it matters. Science, 278(5335), 45-47.

Leshner, A. I. (1999). Science is revolutionizing our view of addiction -- and what to do about it. American Journal of Psychiatry, 156(1), 1-3.

Leshner, A. I. (2001a). Addiction is a brain disease. Issues in Science and Technology, 17(3), 75-80.

Leshner, A. I. (2001b). Hearing before the Senate Committee on the Judiciary: Treatment, education, and prevention: Adding to the arsenal in the war on drugs. [Online]. Retrieved April 27, 2012 from http://www.hhs.gov.

Leshner, A. I. (2001c). Understanding the risks of prescription drug abuse. [Online]. Retrieved December 7, 2011 from http://archives.drugabuse.gov.

Leshner, A. I. (2005). The essence of drug addiction. [Online]. Retrieved October 23, 2006 from http://www.drugabuse.gov.

Levine, A., Huang, Y. Y., Drisaldi, B., Griffin, E. A., Pollak, D. D., Xu, S., et al. (2011). Molecular mechanism for a gateway drug: Epigenetic changes initiated by nicotine prime gene expression by cocaine. Science Translational Medicine, 3(107), 1-10.

Levy, S., Sherritt, L., Harris, S. K., Gates, E. C., Holder, D. W., Kulig, J. W., et al. (2004). Test-retest reliability of adolescents' self-report of substance use. Alcoholism: Clinical \& Experimental Research, 28(8), 1236-1241.

Lewis, C. E., \& Bucholz, K. K. (1991). Alcoholism, antisocial behavior and family history. British Journal of Addiction, 86(2), 177-194.

Lewis, D. C. (1991). Comparison of alcoholism and other medical diseases: An internist's view. Psychiatric Annals, 21(5), 256-265.

Li, C., Pentz, M. A., \& Chou, C. P. (2002). Parental substance use as a modifier of adolescent substance use risk. Addiction, 97(12), 1537-1550. 
Liaison Committee on Medical Education. (2011). Overview: Accreditation and the LCME. [Online]. Retrieved May 31, 2012 from http://www.lcme.org.

Library of Congress. (2008). Emergency Economic Stabilization Act of 2008: HR. 1424. [Online]. Retrieved January 6, 2010 from http://www.gpo.gov.

Library of Congress. (2011). Bill summary and status 100th Congress (1987-1988). H.R. 5210. All information. [Online]. Retrieved December 5, 2011 from http://thomas.loc.gov.

Library of Congress. (2012a). Bill summary and status 110th Congress (2007 - 2008) H.R.1424: CRS Summary. [Online]. Retrieved April 3, 2012 from http://thomas.loc.gov.

Library of Congress. (2012b). Bill summary and status 110th Congress (2007 - 2009) H.R. 6331: Medicare Improvements for Patients and Providers Act of 2008. [Online]. Retrieved April 3, 2012 from http://www.govtrack.us.

Libretto, S. V., Weil, J., Nemes, S., Linder, N. C., \& Johansson, A. C. (2004). Snapshot of the substance abuse treatment workforce in 2002: A synthesis of current literature. Journal of Psychoactive Drugs, 36(4), 489-497.

Lichtenstein, D. P., Spirito, A., \& Zimmermann, R. P. (2010). Assessing and treating co-occurring disorders in adolescents: Examining typical practice of community-based mental health and substance use treatment providers. Community Mental Health Journal, 46(3), 252-257.

Liddle, H. A. (1999). Theory development in a family-based therapy for adolescent drug abuse. Journal of Clinical Child Psychology, 28(4), 521-532.

Liddle, H. A., \& Dakof, G. A. (1995). Family-based treatment for adolescent drug use: State of the science. NIDA Research Monograph, 156, 218-254.

Liddle, H. A., Dakof, G. A., Henderson, C. E., \& Greenbaum, P. E. (2009). Multidimensional family therapy for young adolescent substance abuse: Twelve-month outcomes for a randomized controlled trial. Journal of Consulting \& Clinical Psychology, 77(1), 12-25.

Liddle, H. A., Dakof, G. A., Parker, K., Diamond, G. S., Barrett, K., \& Tejeda, M. (2001). Multidimensional family therapy for adolescent drug abuse: Results of a randomized clinical trial. American Journal of Drug \& Alcohol Abuse, 27(4), 651-688.

Liddle, H. A., Dakof, G. A., Turner, R. M., Henderson, C. E., \& Greenbaum, P. E. (2008). Treating adolescent drug abuse: A randomized trial comparing multidimensional family therapy and cognitive behavior therapy. Addiction, 103(10), 1660-1670.

Liddle, H. A., Rowe, C. L., Dakof, G. A., Ungaro, R. A., \& Henderson, C. E. (2004). Early intervention for adolescent substance abuse: Pretreatment to posttreatment outcomes of a randomized clinical trial comparing multidimensional family therapy and peer group treatment. Journal of Psychoactive Drugs, 36(1), 49-63.

LifeRing. (2011). LifeRing FAQs. [Online]. Retrieved November 15, 2011 from http://lifering.org/faqs.

Lightwood, J. M., Phibbs, C. S., \& Glantz, S. A. (1999). Short-term health and economic benefits of smoking cessation: Low birth weight. Pediatrics, 104(6), 1312-1320.

Lile, B. (2003). Twelve step programs: An update. Addictive Disorders \& Their Treatment, 2(1), 19-24.

Ling, W., Charuvastra, C., Collins, J. F., Batki, S., Brown, L. S., Jr., Kintaudi, P., et al. (1998). Buprenorphine maintenance treatment of opiate dependence: A multicenter, randomized clinical trial. Addiction, 93(4), 475-486. 
Ling, W., Wesson, D. R., \& Smith, D. E. (2003). Abuse of prescription opioids. In A. W. Graham, T. K. Schultz, M. Mayo-Smith, R. K. Ries, \& B. B. Wilford (Eds.), Principles of addiction medicine. Chevy Chase, MD: American Society of Addiction Medicine.

Lipperman-Kreda, S., Grube, J. W., \& Paschall, M. J. (2010). Community norms, enforcement of minimum legal drinking age laws, personal beliefs and underage drinking: An explanatory model. Journal of Community Health, 35(3), 249-257.

Lipsey, M. W. (1999). Can intervention rehabilitate serious delinquents? Annals of the American Academy of Political \& Social Science, 564, 142-166.

Lipsey, M. W., \& Cothern, L. (2000). Effective intervention for serious juvenile offenders (NCJ Pub. No. 181201). Washington, DC: U.S. Department of Justice, Office of Justice Programs, Office of Juvenile Justice and Delinquency Offenders.

Lipsky, S., Krupski, A., Roy-Byrne, P., Huber, A., Lucenko, B. A., \& Mancuso, D. (2012). Impact of sexual orientation and co-occurring disorders on chemical dependency treatment outcomes. Journal of Studies on Alcohol \& Drugs, 73(3), 401-412.

Litz, B. T., \& Schlenger, W. E. (2009). PTSD in service members and new veterans of the Iraq and Afghanistan wars: A bibliography and critique. [Online]. Retrieved May 10, 2012 from http://www.ptsd.va.gov.

Liu, J., \& Wang, L. (2011). Baclofen for alcohol withdrawal. Cochrane Database Systematic Reviews, (1), CD008502.

Lloyd-Jones, D., Adams, R., Carnethon, M., De, S. G., Ferguson, T. B., Flegal, K., et al. (2009). Heart disease and stroke statistics--2009 update: A report from the American Heart Association Statistics Committee and Stroke Statistics Subcommittee. Circulation, 119(3), e21-181.

Longo, L. P., \& Johnson, B. (2000). Addiction: Part 1. Benzodiazepines: Side effects, abuse risk and alternatives. American Family Physician, 61(7), 2121-2128.

Longoria, R. A. (2005). Grandparents raising grandchildren: Population characteristics, perceived neighborhood risk, alcohol use, and drug misuse as predictors of emotional well being. [Online]. Retrieved December 19, 2011 from http://repositories.lib.utexas.edu.

Lopez, B., Schwartz, S. J., Prado, G., Huang, S., Rothe, E. M., Wang, W., et al. (2008). Correlates of early alcohol and drug use in Hispanic adolescents: Examining the role of ADHD with comorbid conduct disorder, family, school, and peers. Journal of Clinical Child \& Adolescent Psychology, $37(4), 820-832$.

Lopez-Larson, M. P., Bogorodzki, P., Rogowska, J., McGlade, E., King, J. B., Terry, J., et al. (2011). Altered prefrontal and insular cortical thickness in adolescent marijuana users. Behavioural Brain Research, 220(1), 164-172.

Lopez-Quintero, C., de los Cobos, J. P., Hasin, D. S., Okuda, M., Wang, S., Grant, B. F., et al. (2011). Probability and predictors of transition from first use to dependence on nicotine, alcohol, cannabis, and cocaine: Results of the National Epidemiologic Survey on Alcohol and Related Conditions (NESARC). Drug \& Alcohol Dependence, 115(1-2), 120-130.

Lowinson, J. H., Ruiz, P., Millman, R. B., \& Langrod, J. G. (eds.). (2005). Substance abuse: A comprehensive textbook (4th ed). Philadelphia: Lippincott Williams \& Wilkins.

Lubman, D. I., Yucel, M., \& Pantelis, C. (2004). Addiction, a condition of compulsive behavior? Neuroimaging and neuropsychological evidence of inhibitory dysregulation. Addiction, 99(12), 14911502 . 
Lui, S., Terplan, M., \& Smith, E. J. (2008). Psychosocial interventions for women enrolled in alcohol treatment during pregnancy. Cochrane Database of Systematic Reviews, (3) CD006753.

Lundgren, L., Amodeo, M., Krull, I., Chassler, D., Weidenfeld, R., de Saxe Zerden, L., et al. (2011). Addiction treatment provider attitudes on staff capacity and evidence-based clinical training: Results from a national study. American Journal on Addictions, 20(3), 271-284.

Luoma, J. B., Twohig, M. P., Waltz, T., Hayes, S. C., Roget, N., Padilla, M., et al. (2007). An investigation of stigma in individuals receiving treatment for substance abuse. Addictive Behaviors, 32(7), 1331-1346.

Lussier, J. P., Heil, S. H., Mongeon, J. A., Badger, G. J., \& Higgins, S. T. (2006). A meta-analysis of voucher-based reinforcement therapy for substance use disorders. Addiction, 101(2), 192-203.

Lutz, P. (1994). Addiction programs opening up for disabled. [Online]. Retrieved February 3, 2012 from http://www.nytimes.com.

Lynskey, M. T., Agrawal, A., \& Heath, A. C. (2010). Genetically informative research on adolescent substance use: Methods, findings, and challenges. Journal of the American Academy of Child \& Adolescent Psychiatry, 49(12), 1202-1214.

Ma, J. Z., Ait-Daoud, N., \& Johnson, B. A. (2006). Topiramate reduces the harm of excessive drinking: Implications for public health and primary care. Addiction, 101(11), 1561-1568.

Maciosek, M. V., Coffield, A. B., Edwards, N. M., Flottemesch, T. J., Goodman, M. J., \& Solberg, L. I. (2006). Priorities among effective clinical preventive services: Results of a systematic review and analysis. American Journal of Preventive Medicine, 31(1), 52-61.

Mackay, P. W., \& Marlatt, G. A. (1991). Maintaining sobriety: Stopping is starting. International Journal on Addictions, 25(9A-10A), 1257-1276.

MacMahon, S. (1987). Alcohol consumption and hypertension. Hypertension, 9(2), 111-121.

Maddux, J. F., Prihoda, T. J., \& Desmond, D. P. (1994). Treatment fees and retention on methadone maintenance. Journal of Drug Issues, 24(3), 429-443.

Mader, T. J., Smithline, H. A., Nyquist, S., \& Letourneau, P. (2001). Social services referral of adolescent trauma patients admitted following alcohol-related injury. Journal of Substance Abuse Treatment, 21(3), 167-172.

Madras, B. K. (2010). Office of National Drug Control Policy: A scientist in drug policy in Washington, DC. Annals of the New York Academy of Sciences, 1187(1), 370-402.

Madras, B. K., Compton, W. M., Avula, D., Stegbauer, T., Stein, J. B., \& Clark, H. W. (2009). Screening, brief interventions, referral to treatment (SBIRT) for illicit drug and alcohol use at multiple healthcare sites: Comparison at intake and 6 months later. Drug \& Alcohol Dependence, 99(1-3), 280-295.

Maes, H. H., Neale, M. C., Chen, X., Chen, J., Prescott, C. A., \& Kendler, K. S. (2011). A twin association study of nicotine dependence with markers in the CHRNA3 and CHRNA5 genes. Behavior Genetics, 41(5), 680-690.

Maes, H. H., Woodard, C. E., Murrelle, L., Meyer, J. M., Silberg, J. L., Hewitt, J. K., et al. (1999). Tobacco, alcohol and drug use in eight- to sixteen-year-old twins: The Virginia Twin Study of Adolescent Behavioral Development. Journal of Studies on Alcohol, 60(3), 293-305.

Magill, M., Barnett, N. P., Apodaca, T. R., Rohsenow, D. J., \& Monti, P. M. (2009). The role of marijuana use in brief motivational intervention with young adult drinkers treated in an emergency department. Journal of Studies on Alcohol \& Drugs, 70(3), 409-413. 
Maglione, M., \& Ridgely, M. S. (2006). Is conventional wisdom wrong? Coverage for substance abuse treatment under Medicaid managed care. Journal of Substance Abuse Treatment, 30(4), 285-290.

Maisto, S. A., Carey, M. P., Carey, K. B., Gordon, C. M., \& Gleason, J. R. (2000). Use of the AUDIT and the DAST-10 to identify alcohol and drug use disorders among adults with a severe and persistent mental illness. Psychological Assessment, 12(2), 186-192.

Maisto, S. A., Conigliaro, J., McNeil, M., Kraemer, K., \& Kelley, M. E. (2000). An empirical investigation of the factor structure of the AUDIT. Psychological Assessment, 12(3), 346-353.

Makela, K. (2004). Studies of the reliability and validity of the Addiction Severity Index. Addiction, 99(4), 398-410.

Malcolm, R., Ballenger, J. C., Sturgis, E. T., \& Anton, R. (1989). Double-blind controlled trial comparing carbamazepine to oxazepam treatment of alcohol withdrawal. American Journal of Psychiatry, 146(5), 617-621.

Malcolm, R., Swayngim, K., Donovan, J. L., DeVane, C. L., Elkashef, A., Chiang, N., et al. (2006). Modafinil and cocaine interactions. American Journal of Drug \& Alcohol Abuse, 32(4), 577-587.

Mancuso, D., \& Felver, B. (2010). Bending the health care cost curve by expanding alcohol/drug treatment. Olympia, WA: Washington State Department of Social and Health Services, Planning, Performance and Accountability, Research and Data Analysis Division.

Mangrum, L. F., Spence, R. T., \& Lopez, M. (2006). Integrated versus parallel treatment of co-occurring psychiatric and substance use disorders. Journal of Substance Abuse Treatment, 30(1), 79-84.

Mann, C., \& Center for Medicaid and State Operations. (2009). Letter (SHO 09-014 CHIPRA \#9) to state health officials to provide general guidance on implementation of section 502 of the Children's Health Insurance Program Reauthorization Act of 2009. [Online]. Retrieved March 15, 2012 from https://www.cms.gov.

Marcus, B. H., Albrecht, A. E., King, T. K., Parisi, A. F., Pinto, B. M., Roberts, M., et al. (1999). The efficacy of exercise as an aid for smoking cessation in women: A randomized controlled trial. Archives of Internal Medicine, 159(11), 1229-1234.

Margolin, A., Avants, S. K., \& Kleber, H. D. (1998). Rationale and design of the Cocaine Alternative Treatments Study (CATS): A randomized, controlled trial of acupuncture. Journal of Alternative \& Complementary Medicine, 4(4), 405-418.

Margolin, A., Kleber, H. D., Avants, S. K., Konefal, J., Gawin, F., Stark, E., et al. (2002). Acupuncture for the treatment of cocaine addiction: A randomized controlled trial. JAMA, 287(1), 55-63.

Mariani, J. J., \& Levin, F. R. (2008). Levetiracetam for the treatment of co-occurring alcohol dependence and anxiety: Case series and review. American Journal of Drug \& Alcohol Abuse, 34(6), 683-691.

Marinelli-Casey, P., Domier, C. P., \& Rawson, R. A. (2002). The gap between research and practice in substance abuse treatment. Psychiatric Services, 53(8), 984-987.

Marinho, V., Laks, J., Coutinho, E. S. F., \& Blay, S. L. (2010). Tobacco use among the elderly: A systematic reivew and meta-analysis. Cadernos de Saude Publica, 26(12), 2213-2233.

Mark, T. L., Levit, K. R., Vandivort-Warren, R., Coffey, R. M., \& Buck, J. A. (2007). Trends in spending for substance abuse treatment, 1986-2003. Health Affairs, 26(4), 1118-1122.

Marlatt, G. A., Blume, A. W., \& Parks, G. A. (2001). Integrating harm reduction therapy and traditional substance abuse treatment. Journal of Psychoactive Drugs, 33(1), 13-21. 
Marmorstein, N. R. (2009). Longitudinal associations between alcohol problems and depressive symptoms: Early adolescence through early adulthood. Alcoholism: Clinical \& Experimental Research, 33(1), 49-59.

Marsch, L. A. (1998). The efficacy of methadone maintenance interventions in reducing illicit opiate use, HIV risk behavior and criminality: A meta-analysis. Addiction, 93(4), 515-532.

Marshall, B. D. L., Prescotta, M. R., Liberzonb, I., Tamburrinod, M. B., Calabresee, J. R., \& Galea, S. (2012). Coincident posttraumatic stress disorder and depression predict alcohol abuse during and after deployment among Army National Guard soldiers. Drug \& Alcohol Dependence, doi: 10.1016drugalcdep.2011.12.027.

Marshall, M. F., Menikoff, J., \& Paltrow, L. M. (2003). Perinatal substance abuse and human subjects research: Are privacy protections adequate? Mental Retardation \& Developmental Disabilities Research Reviews, 9(1), 54-59.

Martell, B. A., Orson, F. M., Poling, J., Mitchell, E., Rossen, R. D., Gardner, T., et al. (2009). Cocaine vaccine for the treatment of cocaine dependence in methadone-maintained patients: A randomized, double-blind, placebo-controlled efficacy trial. Archives of General Psychiatry, 66(10), 1116-1123.

Martens, M. P., Cimini, M. D., Barr, A. R., Rivero, E. M., Vellis, P. A., Desemone, G. A., et al. (2007). Implementing a screening and brief intervention for high-risk drinking in university-based health and mental health care settings: Reductions in alcohol use and correlates of success. Addictive Behaviors, $32(11), 2563-2572$.

Martin, C. S., Pollock, N. K., Bukstein, O. G., \& Lynch, K. G. (2000). Inter-rater reliability of the SCID alcohol and substance use disorders section among adolescents. Drug \& Alcohol Dependence, 59(2), 173-176.

Martin, J. E., Calfas, K. J., Patten, C. A., Polarek, M., Hofstetter, C. R., Noto, J., et al. (1997). Prospective evaluation of three smoking interventions in 205 recovering alcoholics: One-year results of Project SCRAP-Tobacco. Journal of Consulting \& Clinical Psychology, 65(1), 190-194.

Martin, L. M., Fleming, K. C., \& Evans, J. M. (1995). Recognition and management of anxiety and depression in elderly patients. Mayo Clinic Proceedings, 70(10), 999-1006.

Martino, S., Grilo, C. M., \& Fehon, D. C. (2000). Development of the Drug Abuse Screening Test for Adolescents (DAST-A). Addictive Behaviors, 25(1), 57-70.

Martins, S. S., Storr, C. L., Alexandre, P. K., \& Chilcoat, H. D. (2008). Adolescent ecstasy and other drug use in the National Survey of Parents and Youth: The role of sensation-seeking, parental monitoring and peer's drug use. Addictive Behaviors, 33(7), 919-933.

Maryland's Tobacco Resource Center. (2012). Older adults. [Online]. Retrieved February 3, 2012 from http://mdquit.org.

Mason, B. J., Crean, R., Goodell, V., Light, J. M., Quello, S., Shadan, F., et al. (2012). A proof-ofconcept randomized controlled study of gabapentin: Effects on cannabis use, withdrawal and executive function deficits in cannabis-dependent adults. Neuropsychopharmacology, 37(7), 16891698.

Mason, B. J., Goodman, A. M., Chabac, S., \& Lehert, P. (2006). Effects of oral acamprosate on abstinence in patients with alcohol dependence in a double-blind, placebo-controlled trial: The role of patient motivation. Journal of Psychiatric Research, 40(5), 383-393.

Masoumi, M., Shahesmaeili, A., Mirzazadeh, A., Tavakoli, M., \& Ali, A. Z. (2010). Opium addiction and severity of coronary artery disease: A case-control study. Journal of Research in Medical Science, 15(1), 27-32. 
Mass. Regs. Code 105, § 164.124 (2011).

Mass. Regs. Code 130, § 450.204 (2011).

Massey, C. J., Dino, G. A., Horn, K. A., Lacey-McCracken, A., Goldcamp, J., \& Kalsekar, I. (2003). Recruitment barriers and successes of the American Lung Association's Not-On-Tobacco Program. Journal of School Health, 73(2), 58-63.

Master, R. J. (2011). Improving care and managing costs for dually eligible, elderly and disabled populations. [Online]. Retrieved November 10, 2011 from http://www.innovations.harvard.edu.

Maxwell, K. (2002). Friends: The role of peer influence across adolescent risk behaviors. Journal of Youth \& Adolescence, 31(4), 267-277.

Mayo Clinic. (2012). Coronary artery disease. [Online]. Retrieved January 31, 2012 from http://www.mayoclinic.org.

McAuliffe, W. E., \& Dunn, R. (2004). Substance abuse treatment needs and access in the USA: Interstate variations. Addiction, 99(8), 999-1014.

McBride, O., \& Adamson, G. (2010). Are subthreshold alcohol dependence symptoms a risk factor for developing DSM-IV alcohol use disorders? A three-year prospective study of 'diagnostic orphans' in a national sample. Addictive Behaviors, 35(6), 586-592.

McCabe, S. E., Bostwick, W. B., Hughes, T. L., West, B. T., \& Boyd, C. J. (2010). The relationship between discrimination and substance use disorders among lesbian, gay, and bisexual adults in the United States. American Journal of Public Health, 100(10), 1946-1952.

McCabe, S. E., Boyd, C. J., Cranford, J. A., \& Teter, C. J. (2009). Motives for nonmedical use of prescription opioids among high school seniors in the United States: Self-treatment and beyond. Archives of Pediatric Adolescent Medicine, 163(8), 739-744.

McCann, B. S., Simpson, T. L., Ries, R., \& Roy-Byrne, P. (2000). Reliability and validity of screening instruments for drug and alcohol abuse in adults seeking evaluation for attention-deficit/hyperactivity disorder. American Journal on Addictions, 9, 1-9.

McCarthy, J. J. (2012). Intrauterine abstinence syndrome (IAS) during buprenorphine inductions and methadone tapers: Can we assure the safety of the fetus? Journal of Maternal-Fetal \& Neonatal Medicine, 25(2), 109-112.

McCarty, D. (2002). The alcohol and drug abuse treatment workforce. Frontlines: Linking Alcohol Services Research And Practice, 1-2, Washington, DC: National Institute on Alcohol Abuse and Alcoholism.

McCarty, D., Fuller, B. E., Arfken, C., Miller, M., Nunes, E. V., Edmundson, E., et al. (2007). Direct care workers in the National Drug Abuse Treatment Clinical Trials Network: Characteristics, opinions, and beliefs. Psychiatric Services, 58(2), 181-190.

McClernon, F. J., Westman, E. C., \& Rose, J. E. (2004). The effects of controlled deep breathing on smoking withdrawal symptoms in dependent smokers. Addictive Behaviors, 29(4), 765-772.

McCollister, K. E., \& French, M. T. (2003). The relative contribution of outcome domains in the total economic benefit of addiction interventions: A review of first findings. Addiction, 98(12), 1647-1659.

McCollister, K. E., French, M. T., Pyne, J. M., Booth, B., Rapp, R., \& Carr, C. (2009). The cost of treating addiction from the client's perspective: Results from a multi-modality application of the Client DATCAP. Drug \& Alcohol Dependence, 104(3), 241-248.

McCormick, M. C. (1985). The contribution of low birth weight to infant mortality and childhood morbidity. New England Journal of Medicine, 312(2), 82-90. 
McCorry, F., Garnick, D. W., Bartlett, J., Cotter, F., \& Chalk, M. (2000). Developing performance measures for alcohol and other drug services in managed care plans. Washington Circle Group. Joint Commission Journal on Quality Improvement, 26(11), 633-643.

McFall, M., \& Cook, J. (2006). PTSD and health risk behavior. PTSD Research Quarterly, 17(4), 1-8.

McGlynn, E. A., Asch, S. M., Adams, J., Keesey, J., Hicks, J., DeCristofaro, A., et al. (2003). The quality of health care delivered to adults in the United States. New England Journal of Medicine, 348(26), 2635-2645.

McGregor, C., Srisurapanont, M., Mitchell, A., Wickes, W., \& White, J. M. (2008). Symptoms and sleep patterns during inpatient treatment of methamphetamine withdrawal: A comparison of mirtazapine and modafinil with treatment as usual. Journal of Substance Abuse Treatment, 35(3), 334-342.

McIver, S., O'Halloran, P., \& McGartland, M. (2004). The impact of Hatha yoga on smoking behavior. Alternative Therapies in Health \& Medicine, 10(2), 22-23.

McKellar, J., Stewart, E., \& Humphreys, K. (2003). Alcoholics Anonymous involvement and positive alcohol-related outcomes: Cause, consequence, or just a correlate? A prospective 2-year study of 2,319 alcohol-dependent men. Journal of Consulting \& Clinical Psychology, 71(2), 302-308.

McLaney, M. A., \& Boca, F. D. (1994). A validation study of the Problem-Oriented Screening Instrument for Teenagers (POSIT). Journal of Mental Health, 3(3), 363-376.

McLean, T. R. (2002). Crossing the quality chasm: Autonomous physician extenders will necessitate a shift to enterprise liability coverage for health care delivery. Health Matrix, 12(2), 239-295.

McLearen, A. M., \& Ryba, N. L. (2003). Identifying severely mentally ill inmates: Can small jails comply with detection standards? Journal of Offender Rehabilitation, 37(1), 25-40.

McLellan, A. T. (2008).Evolution in addiction treatment concepts and methods. In M. Galanter \& H. D. Kleber (Eds.), Substance abuse treatment (pp. 93-108). Washington, DC: American Psychiatric Publishing.

McLellan, A. T., \& Meyers, K. (2004). Contemporary addiction treatment: A review of systems problems for adults and adolescents. Biological Psychiatry, 56(10), 764-770.

McLellan, A. T., Cacciola, J. C., Alterman, A. I., Rikoon, S. H., \& Carise, D. (2006). The Addiction Severity Index at 25: Origins, contributions and transitions. American Journal on Addictions, 15(2), 113-124.

McLellan, A. T., Carise, D., \& Kleber, H. D. (2003). Can the national addiction treatment infrastructure support the public's demand for quality care? Journal of Substance Abuse Treatment, 25(2), 117-121.

McLellan, A. T., Lewis, D. C., O'Brien, C. P., \& Kleber, H. D. (2000). Drug dependence, a chronic medical illness: Implications for treatment, insurance, and outcomes evaluation. JAMA, 284(13), 1689-1695.

McLellan, A. T., McKay, J. R., Forman, R., Cacciola, J., \& Kemp, J. (2005). Reconsidering the evaluation of addiction treatment: From retrospective follow-up to concurrent recovery monitoring. Addiction, 100(4), 447-458.

McNiel, D. E., \& Binder, R. L. (2005). Psychiatric emergency service use and homelessness, mental disorder, and violence. Psychiatric Services, 56(6), 699-704.

McNulty, T. L., Oser, C. B., Johnson, J. A., Knudsen, H. K., \& Roman, P. M. (2007). Counselor turnover in substance abuse treatment centers: An organizational-level analysis. Sociological Inquiry, 77(2), 166-193. 
McPherson, T. L., \& Hersch, R. K. (2000). Brief substance use screening instruments for primary care settings: A review. Journal of Substance Abuse Treatment, 18(2) 193-202.

McPherson, T. L., Goplerud, E., Derr, D., Mickenberg, J., \& Courtemanche, S. (2010). Telephonic screening and brief intervention for alcohol misuse among workers contacting the employee assistance program: A feasibility study. Drug \& Alcohol Review, 29(6), 641-646.

McPherson, T., Lusby-Treber, K., Goplerud , E., \& Walsh, T. (2007). Alcohol screening and brief intervention in the workplace: Opportunities for early identification and intervention. Washington, DC: George Washington University Medical Center, Department of Health Policy, Ensuring Solutions to Alcohol Problems.

McQueeny, T., Schweinsburg, B. C., Schweinsburg, A. D., Jacobus, J., Bava, S., Frank, L. R., et al. (2009). Altered white matter integrity in adolescent binge drinkers. Alcoholism: Clinical \& Experimental Research, 33(7), 1278-1285.

Mead, H., Cartwright-Smith, L., Jones, K., Ramos, C., Siegel, B., \& Woods, K. (2008). Racial and ethnic disparities in U.S. health care: A chartbook. [Online]. Retrieved April 18, 2012 from http://www.commonwealthfund.org.

Meader, N. (2010). A comparison of methadone, buprenorphine and alpha(2) adrenergic agonists for opioid detoxification: A mixed treatment comparison meta-analysis. Drug \& Alcohol Dependence, 108(1-2), 110-114.

Meandzija, B., O'Connor, P. G., Fitzgerald, B., Rounsaville, B. J., \& Kosten, T. R. (1994). HIV infection and cocaine use in methadone maintained and untreated intravenous drug users. Drug \& Alcohol Dependence, 36(2), 109-113.

Meaney, M. J., Brake, W., \& Gratton, A. (2002). Environmental regulation of the development of mesolimbic dopamine systems: A neurobiological mechanism for vulnerability to drug abuse? Psychoneuroendocrinology, 27(1-2), 127-138.

Mecklenburg, R. E. (2001). Tobacco prevention and control in dental practice: The future. Journal of Dental Education, 65(4), 375-384.

Medicare Interactive. (2012). Medicare coverage of treatment for alcoholism and drug abuse. [Online]. Retrieved March 19, 2012 from http://www.medicareinteractive.org.

Medina, K. L., Schweinsburg, A. D., Cohen-Zion, M., Nagel, B. J., \& Tapert, S. F. (2007). Effects of alcohol and combined marijuana and alcohol use during adolescence on hippocampal volume and asymmetry. Neurotoxicology \& Teratology, 29(1), 141-152.

Mejta, C. L., Bokos, P. J., Mickenberg, J., Maslar, M. E., \& Senary, E. (1997). Improving substance abuse treatment access and retention using a case management approach. Journal of Drug Issues, $27(2), 329-340$.

Mercure, S., \& Greenberg, L. (2009). eValue8 employer report: Health plan tobacco cessation performance. [Online]. Retrieved May 15, 2012 from http://www.nbch.org.

Merikangas, K. R., \& McClair, V. L. (2012). Epidemiology of substance use disorders. Human Genetics, 131(6), 779-789.

Merikangas, K. R., Stolar, M., Stevens, D. E., Goulet, J., Preisig, M. A., Fenton, B., et al. (1998). Familial transmission of substance use disorders. Archives of General Psychiatry, 55(11), 973-979.

Merlo, L. J., \& Gold, M. (2008). Prescription opioid abuse and dependence among physicians: Hypotheses and treatment. Harvard Review of Psychiatry, 16(3), 181-194. 
Merrick, E. L., Garnick, D. W., Horgan, C. M., \& Hodgkin, D. (2002). Quality measurement and accountability for substance abuse and mental health services in managed care organizations. Medical Care, 40(12), 1238-1248.

Merrill, J., \& Fox, K. (1998). The impact of substance abuse on federal spending. In W. J. Bukoski \& R. I. Evans (Eds.), Cost-benefit/cost effectiveness research of drug abuse prevention: Implications for programming and policy. (NIDA Monograph 176, pp. 5-36). Rockville, MD: National Institute on Drug Abuse.

Mertens, M. J. (2010). Weight status continuity and change from adolescence to young adulthood: Examining disease and health risk conditions. Obesity, 18(7), 1423-1428.

Meyer, I. H. (2003). Prejudice, social stress, and mental health in lesbian, gay, and bisexual populations: Conceptual issues and research evidence. Psychological Bulletin, 129(5), 674-697.

Mezzich, J. E. (2002). International surveys on the use of ICD-10 and related diagnostic systems. Psychopathology, 35(2-3), 72-75.

Mick, E., Biederman, J., Faraone, S., Julie, S., \& Kleinman, S. (2002). Case-control study of attentiondeficit hyperactivity disorder and maternal smoking, alcohol use and drug use during pregnancy. Journal of the American Academy of Child \& Adolescent Psychiatry, 41(4), 378-385.

Miller, G. (2010). Is pharma running out of brainy ideas? Science, 329(5991), 502-504.

Miller, M. D., Marty, M. A., Broadwin, R., Johnson, K. C., Salmon, A. G., Winder, B., et al. (2007). The association between exposure to environmental tobacco smoke and breast cancer: A review by the California Environmental Protection Agency. Preventive Medicine, 44(2), 93-106.

Miller, N. S. (1997). The integration of pharmacological and nonpharmacological treatments in drug/alcohol addiction. Journal of Addictive Diseases, 16(4), 1-5.

Miller, N. S. (2011). Addiction medicine on the road to subspecialty status. [Online]. Retrieved February 28, 2012 from http://www.internalmedicinenews.com.

Miller, N. S., \& Gold, M. S. (1990). Benzodiazepines: Tolerance, dependence, abuse, and addiction. Journal of Psychoactive Drugs, 22(1), 23-33.

Miller, N. S., Sheppard, L. M., \& Magen, J. (2001). Barriers to improving education and training in addiction medicine. Psychiatric Annals, 31(11), 649-656.

Miller, N. S., Sheppard, L. M., Colenda, C. C., \& Magen, J. (2001). Why physicians are unprepared to treat patients who have alcohol- and drug-related disorders. Academic Medicine, 76(5), 410-418.

Miller, T. Q., \& Volk, R. J. (2002). Family relationships and adolescent cigarette smoking: Results from a national longitudinal survey. Journal of Drug Issues, 32(3) 946-972.

Miller, W. R. (2002). Educating psychologists about substance abuse. In M. R. Haack \& H. Adger (Eds.), Strategic plan for interdisciplinary faculty development: Arming the nation's health professional workforce for a new approach to substance use disorders. (pp. 289-303). Providence, RI: Association for Medical Education and Research in Substance Abuse (AMERSA).

Miller, W. R., \& Sanchez, V. C. (1993). Motivating young adults for treatment and lifestyle change. In G. Howard \& P. E. Nathan (Eds.), Issues in alcohol use and misuse by young adults. (pp. 55-82). Notre Dame, IN: University of Notre Dame Press.

Miller, W. R., Baca, C., Compton, W. M., Ernst, D., Manuel, J. K., Pringle, B., et al. (2006). Addressing substance abuse in health care settings. Alcoholism: Clinical \& Experimental Research, 30(2), 292302. 
Miller, W. R., Sorensen, J. L., Selzer, J. A., \& Brigham, G. S. (2006). Disseminating evidence-based practices in substance abuse treatment: A review with suggestions. Journal of Substance Abuse Treatment, 31(1), 25-39.

Miller, W. R., Zweben, A., DiClemente, C. C., \& Rychtarik, R. G. (1994). Motivational enhancement therapy manual: A clinical research guide for therapists treating individuals with alcohol abuse and dependence (Vols. 2). Rockville, MD: National Institute on Alcohol Abuse and Alcoholism.

Millery, M., Kleinman, B. P., Polissar, N. L., Millman, R. B., \& Scimeca, M. (2002). Detoxification as a gateway to long-term treatment: Assessing two interventions. Journal of Substance Abuse Treatment, 23(3), 183-190.

Milliken, C. S., Auchterlonie, J. L., \& Hoge, C. W. (2007). Longitudinal assessment of mental health problems among active and reserve component soldiers returning from the Iraq war. JAMA, 298(18), 2141-2148.

Millstein, S. G., \& Marcell, A. V. (2003). Screening and counseling for adolescent alcohol use among primary care physicians in the United States. Pediatrics, 111(1), 114-122.

Milner, K. K., Barry, K. L., Blow, F. C., \& Welsh, D. (2010). Brief interventions for patients presenting to the Psychiatric Emergency Service (PES) with major mental illnesses and at-risk drinking. Community Mental Health Journal, 46(2), 149-155.

Moeller, S. J., Maloney, T., Parvaz, M. A., Alia-Klein, N., Woicik, P. A., Telang, F., et al. (2010). Impaired insight in cocaine addiction: Laboratory evidence and effects on cocaine-seeking behaviour. Brain, 133(5), 1484-1493.

Moggi, F., Ouimette, P. C., Moos, R. H., \& Finney, J. W. (1999). Dual diagnosis patients in substance abuse treatment: Relationship of general coping and substance-specific coping to 1-year outcomes. Addiction, 94(12), 1805-1816.

Moisse, K. (2011). Drug deaths exceed traffic deaths. [Online]. Retrieved November 22, 2011 from http://abcnews.go.com.

Mokdad, A. H., Marks, J. S., Stroup, D. F., \& Gerberding, J. L. (2004). Actual causes of death in the United States, 2000. JAMA, 291(10), 1238-1245.

Montamat, S. C., Cusack, B. J., \& Vestal, R. E. (1989). Management of drug therapy in the elderly. New England Journal of Medicine, 321(5), 303.

Monti, P. M., Barnett, N. P., Colby, S. M., Gwaltney, C. J., Spirito, A., Rohsenow, D. J., et al. (2007). Motivational interviewing versus feedback only in emergency care for young adult problem drinking. Addiction, 102(8), 1234-1243.

Monti, P. M., Miranda, R., Jr., Nixon, K., Sher, K. J., Swartzwelder, H. S., Tapert, S. F., et al. (2005). Adolescence: Booze, brains, and behavior. Alcoholism: Clinical \& Experimental Research, 29(2), 207-220.

Monti, P. M., Rohsenow, D. J., Swift, R. M., Gulliver, S. B., Colby, S. M., Mueller, T. I., et al. (2001). Naltrexone and cue exposure with coping and communication skills training for alcoholics: Treatment process and 1-year outcomes. Alcoholism: Clinical \& Experimental Research, 25(11), 1634-1647.

Moolchan, E. T., Radzius, A., Epstein, D. H., Uhl, G., Gorelick, D. A., Cadet, J. L., et al. (2002). The Fagerstrom Test for Nicotine Dependence and the Diagnostic Interview Schedule. Do they diagnose the same smokers? Addictive Behaviors, 27(1), 101-113.

Moolchan, E. T., Robinson, M. L., Ernst, M., Cadet, J. L., Pickworth, W. B., Heishman, S. J., et al. (2005). Safety and efficacy of the nicotine patch and gum for the treatment of adolescent tobacco addiction. Pediatrics, 115(4), e407-e414. 
Moore, A. A., Blow, F. C., Hoffing, M., Welgreen, S., Davis, J. W., Ramirez, K. D., et al. (2010). Primary care-based intervention to reduce at-risk drinking in older adults: A randomized controlled trial. Addiction, 106(1), 111-120.

Moore, M. L., \& Zaccaro, D. J. (2000). Cigarette smoking, low birth weight, and preterm births in lowincome African American women. Journal of Perinatology, 20(3), 176-180.

Moore, T. H. M., Zammit, S., Lingford-Hughes, A., Barnes, T. R. E., Jones, P. B., Burke, M., et al. (2007). Cannabis use and risk of psychotic or affective mental health outcomes: A systematic review. Lancet, 370(9584), 319-328.

Moreno, A. Y., Mayorov, A. V., \& Janda, K. D. (2011). Impact of distinct chemical structures for the development of a methamphetamine vaccine. Journal of the American Chemical Society, 133(17), 6587-6595.

Morgenstern, J., Blanchard, K. A., McCrady, B. S., McVeigh, K. H., Morgan, T. J., \& Pandina, R. J. (2006). Effectiveness of intensive case management for substance-dependent women receiving temporary assistance for needy families. American Journal of Public Health, 96(11), 2016-2023.

Morgenstern, J., Bux, D., Labouvie, E., Blanchard, K. A., \& Morgan, T. I. (2002). Examining mechanisms of action in 12-step treatment: The role of 12-step cognitions. Journal of Studies on Alcohol, 63(6), 665-672.

Morgenstern, J., Labouvie, E., McCrady, B. S., Kahler, C. W., \& Frey, R. M. (1997). Affiliation with Alcoholics Anonymous after treatment: A study of its therapeutic effects and mechanisms of action. Journal of Consulting \& Clinical Psychology, 65(5), 768-777.

Morreale, M. C., Kapphahn, C. J., Elster, A. B., Juszczak, L., \& Klein, J. D. (2004). Access to health care for adolescents and young adults. Position paper of the Society for Adolescent Medicine. Journal of Adolescent Health, 35(Suppl. 6), 342-344.

Morris, C., Waxmonsky, J., Giese A., Graves, M., \& Turnbull, J. (2009). Smoking cessation for persons with mental illness: A toolkit for mental health providers. Denver, CO: University of Colorado at Denver.

Morris, P. L. P., Hopwood, M., Whelan, G., Gardiner, J., \& Drummond, E. (2001). Naltrexone for alcohol dependence: A randomized controlled trial. Addiction, 96(11), 1565-1573.

Morse, G. A., Calsyn, R. J., Klinkenberg, W. D., Trusty, M. L., Gerber, F., Smith, R., et al. (1997). An experimental comparison of three types of case management for homeless mentally ill persons. Psychiatric Services, 48(4), 497-503.

Moss, H. B., \& Lynch, K. G. (2001). Comorbid disruptive behavior disorder symptoms and their relationship to adolescent alcohol use disorders. Drug \& Alcohol Dependence, 64(1), 75-83.

Mouttapa, M., Weiss, J. W., \& Hermann, M. (2009). Is image everything? The role of self-image in the relationship between family functioning and substance use among Hispanic adolescents. Substance Use \& Misuse, 44(5), 702-721.

Moyer, A., \& Finney, J. W. (2004). Brief interventions for alcohol problems: Factors that facilitate implementation. Alcohol Research \& Health, 28(1), 44-50.

Moyer, A., Finney, J. W., Swearingen, C. E., \& Vergun, P. (2002). Brief interventions for alcohol problems: A meta-analytic review of controlled investigations in treatment-seeking and nontreatment-seeking populations. Addiction, 97(3), 279-292.

Mroziewicz, M., \& Tyndale, R. F. (2010). Pharmacogenetics: A tool for identifying genetic factors in drug dependence and response to treatment. Addiction Science \& Clinical Practice, 5(2), 17-29. 
Mueller, T. I., Stout, R. L., Rudden, S., Brown, R. A., Gordon, A., Solomon, D. A., et al. (1997). A double-blind, placebo-controlled pilot study of carbamazepine for the treatment of alcohol dependence. Alcoholism: Clinical \& Experimental Research, 21(1), 86-92.

Multisystemic Therapy Services. (2011). Multisystemic therapy: An overview. [Online]. Retrieved January 3, 2008 from http://www.mstservices.com.

Mulvey, K. P., Hubbard, S., \& Hayashi, S. (2003). A national study of the substance abuse treatment workforce. Journal of Substance Abuse Treatment, 24(1), 51-57.

Munafo, M. R., Hitsman, B., Rende, R., Metcalfe, C., \& Niaura, R. (2008). Effects of progression to cigarette smoking on depressed mood in adolescents: Evidence from the National Longitudinal Study of Adolescent Health. Addiction, 103(1), 162-171.

Mundt, M. P. (2006). Analyzing the costs and benefits of brief intervention. Alcohol Research \& Health, 29(1), 34-36.

Murphy, D. C., \& Wilmers, S. (2002). Patients who are substance abusers. New York State Dental Journal, 68(5), 24-27.

Myers, M. G., \& Brown, S. A. (1994). Smoking and health in substance-abusing adolescents: A two-year follow-up. Pediatrics, 93(4), 561-566.

Myrick, H., \& Anton, R. F. (1998). Treatment of alcohol withdrawal. Alcohol Health \& Research World, 22(1), 38-43.

N.C. Admin. Code 10A. 27G.4401 (January 2012).

N.J. Admin. Code 10.161B, §1.4 (2012).

N.J. Admin. Code 10.161B, $§ 1.8$ (2012).

N.J. Admin. Code 10.161B, §3.12 (2012).

N.Y. Comp. Codes R \& Regs.14 §381.4 (2012).

N.Y. Soc Serv § 365-a (Consol. 2012).

NAADAC - The Association for Addiction Professionals. (2009a). About NAADAC. [Online]. Retrieved August 4, 2009 from http://www.naadac.org.

NAADAC - The Association for Addiction Professionals. (2009b). National Certified Addiction Counselor, Level I (NCAC I). [Online]. Retrieved May 16, 2012 from http://www.naadac.org.

NAADAC - The Association for Addiction Professionals. (2009c). Use of the NAADAC test at the state level: List of states. [Online]. Retrieved July 7, 2009 from http://www.naadac.org.

NAADAC - The Association for Addiction Professionals. (2012). Frequently asked questions about NAADAC certification. [Online]. Retrieved May 21, 2012 from http://www.naadac.org.

Naimi, T. S., Lipscomb, L. E., Brewer, R. D., \& Gilbert, B. C. (2003). Binge drinking in the preconception period and the risk of unintended pregnancy: Implications for women and their children. Pediatrics, 111(5 Part 2), 1136-1141.

Najavits, L. M., Kivlahan, D., \& Kosten, T. (2011). A national survey of clinicians' views of evidencebased therapies for PTSD and substance abuse. Addiction Research \& Theory, 19(2), 138-147.

Narconon International. (2011). History of marijuana. [Online]. Retrieved November 30, 2011 from http://www.narconon.org.

Nathan, P. E., \& Gorman, J. M. (2007). A guide to treatments that work (3rd ed.). New York: Oxford University Press. 
National Abandoned Infants Assistance Resource Center. (2008). Fact sheet: Prenatal substance exposure. [Online]. Retrieved February 3, 2012 from http://aia.berkeley.edu.

National Academy for State Health Policy. (2012). ERISA preemption primer. [Online]. Retrieved April 4, 2012 from http://www.nashp.org.

National Acupuncture Detoxification Association. (2010). About the National Acupuncture Detoxification Association. [Online]. Retrieved February 14, 2012 from http://acudetox.com.

National Acupuncture Detoxification Association. (2012). What is the National Acupuncture Detoxification Association? [Online]. Retrieved March 19, 2012 from http://www.acudetox.com.

National Alliance to End Homelessness. (2012). Cost of homelessness. [Online]. Retrieved February 6, 2012 from http://www.endhomelessness.org.

National Association of Community Health Centers. (2011). State policy report \#34: Health center reimbursement for behavioral health services in Medicaid. [Online]. Retrieved December 19, 2011 from http://www.nachc.com.

National Association of Social Workers. (2012). Certified clinical alcohol, tobacco, and other drugs social worker (C-CATODSW). [Online]. Retrieved February 23, 2012 from http://www.socialworkers.org.

National Association of State Alcohol and Drug Abuse Directors. (2006). State issue brief: Current research on screening and brief intervention and implications for state alcohol and other drug (AOD) systems. Washington, DC: Author.

National Association of State Alcohol and Drug Abuse Directors. (2002). Identification and description of multiple alcohol and other drug treatment systems: Final report. Washington, D.C.: Author.

National Board for Certified Counselors. (2005). Candidate handbook for state credentialing for the National Counselor Examination (NCE). Greensboro, NC: Author.

National Board for Certified Counselors. (2012a). About us. [Online]. Retrieved March 19, 2012 from http://www.nbcc.org.

National Board for Certified Counselors. (2012b). Exams. [Online]. Retrieved March 19, 2012 from http://www.nbcc.org.

National Board for Certified Counselors. (2012c). Master addictions counselor credential. [Online]. Retrieved April 6, 2012 from http://www.nbcc.org.

National Board for Certified Counselors. (2012d). The examination for master addictions counselor. [Online]. Retrieved April 6, 2012 from http://www.nbcc.org.

National Board of Medical Examiners. (2012). About the NBME. [Online]. Retrieved May 31, 2012 from http://www.nbme.org.

National Board of Osteopathic Medical Examiners. (2009). Computer based COMLEX content outline: Dimension 1: Patient presentation. [Online]. Retrieved March 20, 2012 from http://www.nbome.org.

National Board of Osteopathic Medical Examiners. (2011). Comlex -USA bulletin of information 20112012 . [Online]. Retrieved May 31, 2012 from http://www.nbome.org.

National Cancer Institute. (2012). MCI mission statement. [Online]. Retrieved April 25, 2012 from http://www.cancer.gov. 
National Center for Natural Products Research. (2009). Quarterly report potency monitoring project: Report 104: December 16, 2008 thru March 15, 2009. University, MS: University of Mississippi, School of Pharmacy, Research Institute of Pharmaceutical Sciences, National Center for Natural Products Research.

National Certification Commission for Acupuncture and Oriental Medicine. (2011). NCCAOM examination study guide for the diplomate in acupuncture certification for the 2012 examination administration. [Online]. Retrieved March 20, 2012 from http://www.nccaom.org.

National Certification Commission for Acupuncture and Oriental Medicine. (2012). NCCAOM certification in acupuncture. [Online]. Retrieved February 14, 2012 from http://www.nccaom.org.

National Coalition for the Homeless. (2009a). Substance abuse and homelessness. [Online]. Retrieved January 31, 2012 from http://www.nationalhomeless.org.

National Coalition for the Homeless. (2009b). Tobacco use and homelessness. [Online]. Retrieved January 31, 2012 from http://www.nationalhomeless.org.

National Commission on Certification of Physician Assistants. (2009). Exams: Content blueprint: Organ systems. [Online]. Retrieved March 21, 2012 from http://www.nccpa.net.

National Commission on Certification of Physician Assistants. (2010). PANCE. [Online]. Retrieved July 15, 2010 from http://www.nccpa.net.

National Commission on Correctional Health Care. (2012). OTP accreditation: NCCHC accreditation paves the way for correctional opioid treatment programs. [Online]. Retrieved February 14, 2012 from http://ncchc.org.

National Conference of State Legislatures. (2011a). State laws mandating or regulating mental health benefits. [Online]. Retrieved March 7, 2012 from http://www.ncsl.org.

National Conference of State Legislatures. (2011b). State medical marijuana laws. [Online]. Retrieved November 7, 2011 from http://www.ncsl.org.

National Conference of State Legislatures. (2012). Drug testing and public assistance. [Online]. Retrieved June 4, 2012 from http://www.ncsl.org.

National Council for Community Behavioral Healthcare. (2012). Mobile Apps gain popularity in the substance abuse field. [Online]. Retrieved April 18, 2012 from http://echo4.bluehornet.com.

National Council of La Raza (NCLR). (2009a). Question 7: Which health care services do Latinos have trouble obtaining? [Online]. Retrieved April 18, 2012 from http://www.nclr.org.

National Council of La Raza (NCLR). (2009b). Question1: Which health conditions disparately impact Latinos? [Online]. Retrieved April 18, 2012 from http://www.nclr.org.

National Council of State Boards of Nursing. (2012). NCLEX examinations. [Online]. Retrieved March 21, 2012 from https://www.ncsbn.org.

National Drug Intelligence Center. (2003). Methadone fast facts. [Online]. Retrieved December 14, 2011 from http://www.justice.gov.

National Drug Intelligence Center. (2004). Intelligence bulletin. Buprenorphine: Potential for abuse. Doc ID: 2004-L0424-013. [Online]. Retrieved December 14, 2011 from http://www.justice.gov.

National Heart, Lung, and Blood Institute. (2007). Expert panel report 3: Guidelines for the diagnosis and management of asthma: Full report 2007 (NIH Pub. No. 07-4051). Bethesda, MD: U.S. Department of Health and Human Services, National Institutes of Health, National Heart, Lung, and Blood Institute. 
National Heart, Lung, and Blood Institute. (2011a). What is asthma? [Online]. Retrieved November 10, 2011 from http://www.nhlbi.nih.gov/health/health-topics/topics/asthma/

National Heart, Lung, and Blood Institute. (2011b). How is asthma treated and controlled? [Online]. Retrieved November 18, 2011 from http://www.nhlbi.nih.gov.

National Highway Traffic Safety Administration. (2012). Drugs and human performance fact sheets: Methadone. [Online]. Retrieved January 24, 2012 from http://www.nhtsa.gov.

National Institute of Mental Health. (2008). Bipolar disorder (NIH Pub. No. 08-3679). Bethesda, MD: U.S. Department of Human Services, National Institute of Mental Health.

National Institute of Mental Health. (2012). Research Domain Criteria (RDoC). [Online]. Retrieved March 15, 2012 from http://www.nimh.nih.gov.

National Institute on Alcohol Abuse and Alcoholism. (1988). Alcohol alert: Alcohol and aging. [Online]. Retrieved December 7, 2011 from http://pubs.niaaa.nih.gov.

National Institute on Alcohol Abuse and Alcoholism. (1999). Brief interventions for alcohol problems. [Online]. Retrieved December 9, 2011 from http://pubs.niaaa.nih.gov.

National Institute on Alcohol Abuse and Alcoholism. (2002). Screening for alcohol problems--An update. Alcohol Alert, 56.

National Institute on Alcohol Abuse and Alcoholism. (2005a). Helping patients who drink too much: A clinician's guide (NIH Publication No. 07-3769). Bethesda, MD: U.S. Department of Health and Human Services, National Institutes of Health, National Institute on Alcohol Abuse and Alcoholism.

National Institute on Alcohol Abuse and Alcoholism. (2005b). Module 5: Diagnosis and assessment of alcohol use disorders. [Online]. Retrieved September 26, 2011 from http://pubs.niaaa.nih.gov.

National Institute on Alcohol Abuse and Alcoholism. (2005c). Screening for alcohol use and alcohol related problems. Alcohol Alert, 65.

National Institute on Alcohol Abuse and Alcoholism. (2007). Prescribing medications. [Online]. Retrieved July 18, 2008 from http://pubs.niaaa.nih.gov.

National Institute on Alcohol Abuse and Alcoholism. (2008). Answers to frequently asked medication questions. [Online]. Retrieved August 1, 2008 from http://pubs.niaaa.nih.gov.

National Institute on Alcohol Abuse and Alcoholism. (2011a). Alcohol screening and brief intervention for youth. A practitioner's guide. [Online]. Retrieved March 22, 2012 from http://pubs.niaaa.nih.gov.

National Institute on Alcohol Abuse and Alcoholism. (2011b). Health care services and financing: Health insurance: Losses due to intoxication. [Online]. Retrieved December 19, 2011 from http://www.alcoholpolicy.niaaa.nih.gov.

National Institute on Alcohol Abuse and Alcoholism. (2012a). Addiction Severity Index (ASI). [Online]. In McLellan, A.T., Luborsky, L., O'Brien, C.P. \& Woody, G.E. (1980). An improved diagnostic instrument for substance abuse patients: The Addiction Severity Index. Journal of Nervous \& Mental Diseases, 168(1), 26-33.

National Institute on Alcohol Abuse and Alcoholism. (2012b). CAGE Questionnaire. [Online]. Retrieved May 24, 2012 from http://pubs.niaaa.nih.gov.

National Institute on Alcohol Abuse and Alcoholism. (2012c). Substance Abuse Subtle Screening Inventory (SASSI) . [Online]. Retrieved April 20, 2012 from http://pubs.niaaa.nih.gov.

National Institute on Drug Abuse. (1991). Problem Oriented Screening Instrument for Teenagers (POSIT). Rockville, MD: Author. 
National Institute on Drug Abuse. (1995). Integrating behavioral therapies with medication in the treatment of drug dependence. (NIH Pub. No. 95-3899). Rockville, MD: U.S. Department of Health and Human Services, National Institutes of Health, National Institute on Drug Abuse.

National Institute on Drug Abuse. (2001). Imaging studies expand understanding of how methamphetamine affects the human brain. [Online]. Retrieved July 17, 2008 from http://www.nida.nih.gov.

National Institute on Drug Abuse. (2002). NIDA research report series: Therapeutic community (NIH Publication No. 02-4877). Rockville, MD: U.S Department of Health and Human Services, National Institutes of Health, National Institute on Drug Abuse.

National Institute on Drug Abuse. (2003a). Preventing drug abuse among children and adolescents: Chapter 1: Risk factors and protective factors. [Online]. Retrieved June 7, 2012 from http://www.drugabuse.gov.

National Institute on Drug Abuse. (2003b). Preventing drug use among children and adolescents: A research-based guide for parents, educators, and community leaders (NIH Publication No. 044212(A)) (2nd ed.). Bethesda, MD: U.S. Department of Health and Human Services, National Institutes of Health, National Institute on Drug Abuse.

National Institute on Drug Abuse. (2005a). Drug abuse and addiction: One of America's most challenging public health problems. [Online]. Retrieved April 30, 2007 from http://www.nida.nih.gov.

National Institute on Drug Abuse. (2005b). Principles of drug addiction treatment: A research based guide. [Online]. Retrieved February 8, 2006 from http://www.nida.nih.gov.

National Institute on Drug Abuse. (2005c). Research report series: Prescription drugs: Abuse and addiction (NIH Pub. No. 05-4881). Rockville, MD: U.S. Department of Health and Human Services, National Institutes of Health, National Institute on Drug Abuse.

National Institute on Drug Abuse. (2006a). NIDA Info Facts: Drug abuse and the link to HIV/AIDS and other infectious diseases. [Online]. Retrieved November 28, 2006 from http://www.nida.nih.gov.

National Institute on Drug Abuse. (2006b). Principles of drug abuse treatment for criminal justice populations: A research-based guide (NIH Publication No. 06-5316). Bethesda, MD: U.S.

Department of Health and Human Services, National Institutes of Health, National Institute on Drug Abuse.

National Institute on Drug Abuse. (2006c). Tobacco addiction. NIDA research report series (NIH Publication No.06-4342). Rockville, MD: U.S. Department of Health and Human Services, National Institutes of Health, National Institute on Drug Abuse.

National Institute on Drug Abuse. (2007a). A cognitive-behavioral approach: Treating cocaine. [Online]. Retrieved January 6, 2012 Retrieved from the World Wide Web http://archives.drugabuse.gov.

National Institute on Drug Abuse. (2007b). NIDA: Info Facts: Cigarettes and other tobacco products [Updated 2010]. [Online]. Retrieved December 10, 2007 from http://www.drugabuse.gov.

National Institute on Drug Abuse. (2008). Info Facts: Treatment approaches for drug addiction. [Online]. Retrieved January 20, 2008 from http://www.nida.nih.gov.

National Institute on Drug Abuse. (2009a). NIDA Info Facts. Hallucinogens: LSD, peyote, psilocybin, and $P C P$. [Online]. Retrieved December 5, 2011 from http://drugabuse.gov.

National Institute on Drug Abuse. (2009b). NIDA Info Facts. Prescriptions and over the counter medications. [Online]. Retrieved December 5, 2011 from http://www.nida.nih.gov. 
National Institute on Drug Abuse. (2009c). NIDA Info Facts: Treatment approaches for drug addiction. [Online]. Retrieved November 28, 2006 from http://www.nida.nih.gov.

National Institute on Drug Abuse. (2009d). NIDA launches new substance abuse resources to help fill gaps in medical education: First curriculum offerings from NIDA centers of excellence for physician information. [Online]. Retrieved November 18, 2009 from http://www.drugabuse.gov.

National Institute on Drug Abuse. (2009e). Principles of drug addiction treatment: A research-based guide (NIH Publication No. 09-4180) (2nd ed.). Rockville, MD: U.S. Department of Health and Human Services, National Institutes of Health, National Institute on Drug Abuse.

National Institute on Drug Abuse. (2009e). Program aims to expand physician training to treat drug addiction. NIDA Notes, 22(6), 1.

National Institute on Drug Abuse. (2009g). Topics in brief: Prenatal exposure to drugs of abuse. [Online]. Retrieved February 15, 2011 from http://www.nida.nih.gov.

National Institute on Drug Abuse. (2010a). Comorbidity: Addiction and other mental illnesses (NIH Pub. No 10-5771). [Online]. Retrieved December 12, 2011 from http://drugabuse.gov.

National Institute on Drug Abuse. (2010b). Developmental neural mechanisms of cognitive control: Implications for drug abuse interventions. [Online]. Retrieved March 28, 2012 from http://www.drugabuse.gov.

National Institute on Drug Abuse. (2010c). NIDA Info Facts: Club drugs (GHB, ketamine, and rohypnol). [Online]. Retrieved May 7, 2012 from http://www.drugabuse.gov.

National Institute on Drug Abuse. (2010d). NIDA Info Facts: Cocaine. [Online]. Retrieved May 7, 2012 from http://www.drugabuse.gov.

National Institute on Drug Abuse. (2010e). NIDA Info Facts: Methamphetamine. [Online]. Retrieved May 7, 2012 from http://www.drugabuse.gov.

National Institute on Drug Abuse. (2010f). NIDA Info Facts. MDMA (ecstasy). [Online]. Retrieved December 5, 2011 from http://www.drugabuse.gov.

National Institute on Drug Abuse. (2011a). NIDA Info Facts: Drug abuse and the link to HIV/AIDS and other infectious diseases. [Online]. Retrieved May 5, 2012 from http://www.drugabuse.gov.

National Institute on Drug Abuse. (2011b). NIH and ASAM launch new screening resources. [Online]. Retrieved February 23, 2012 from http://www.drugabuse.gov.

National Institute on Drug Abuse. (2011c). Prescription drugs: Abuse and addiction. (NIH Pub. No 114881). [Online]. Retrieved December 12, 2011 from http://www.drugabuse.gov.

National Institute on Drug Abuse. (2011d). Prescription medications. [Online]. Retrieved March 16, 2011 from http://www.nida.nih.gov.

National Institute on Drug Abuse. (2011e). Preventing and recognizing prescription drug abuse. [Online]. Retrieved June 29, 2011 from http://www.nida.nih.gov.

National Institute on Drug Abuse. (2011f). Research report series: Marijuana abuse. [Online]. Retrieved March 1, 2011 from http://www.nida.nih.gov.

National Institute on Drug Abuse. (2011g). Seeking drug abuse treatment: Know what to ask (NIH Pub No. 12-7764). [Online]. Retrieved June 4, 2012 from http://www.drugabuse.gov.

National Institute on Drug Abuse. (2011h). Topics in brief: Prenatal exposure to drugs of abuse. [Online]. Retrieved February 3, 2012 from http://www.drugabuse.gov. 
National Institute on Drug Abuse. (2011i). Understanding drug abuse and addiction. [Online]. Retrieved December 13, 2011 from http://www.drugabuse.gov.

National Institute on Drug Abuse. (2012a). About the CTN. [Online]. Retrieved March 13, 2012 from http://www.drugabuse.gov.

National Institute on Drug Abuse. (2012b). Drug facts: HIV/AIDS and drug abuse: Intertwined epidemics. [Online]. Retrieved May 10, 2012 from http://www.drugabuse.gov.

National Institute on Drug Abuse. (2012c). NIDA quick screen: Clinician's screening tool for drug use in general medical settings. [Online]. Retrieved March 26, 2012 from http://www.drugabuse.gov.

National Institute on Drug Abuse. (2012d). Research report series: Heroin abuse and addiction: What are the treatments for heroin addiction? [Online]. Retrieved January 5, 2012 Retrieved from the World Wide Web http://www.drugabuse.gov.

National Institutes of Health, National Institute of Nursing Research. (2006). Subtle and dangerous: Symptoms of heart disease in women (NIH Pub. No. 06-6079). [Online]. Retrieved April 30, 2012 from http://www.ninr.nih.gov.

National Institutes of Health. (1993). SAMHSA/CSAT Treatment Improvement Protocols: Chapter 3 -Mental health and addiction treatment systems: Philosophical and treatment approach issues. [Online]. Retrieved December 22, 2011 from http://www.ncbi.nlm.nih.gov.

National Institutes of Health. (2006). NIH State-of-the-Science Conference on Tobacco Use: Prevention, Cessation and Control. Bethesda, MD: U.S. Department of Health and Human Services, National Institutes of Health.

National Institutes of Health. (2009). NIDAMED: Medical \& health professionals. [Online]. Retrieved August 16, 2011 from http://www.drugabuse.gov.

National Institutes of Health. (2011a). New warm line helps clinicians tackle patients' substance abuse: NIH and ASAM launch new screening resources. [Online]. Retrieved February 27, 2012 from http://www.nih.gov.

National Institutes of Health. (2011b). Older adults and medications. [Online]. Retrieved December 7 , 2011 from http://nihseniorhealth.gov.

National Institutes of Health. (2012). Request for Information (RFI): Input into the scientific strategic plan for the proposed National Institute of Substance Use and Addiction Disorders: Notice No: NOTOD-12-045. [Online]. Retrieved April 25, 2012 from http://grants.nih.gov.

National League for Nursing Accrediting Commission. (2002). NLNAC mission homepage. [Online]. Retrieved May 31, 2012 from http://www.nlnac.org.

National League for Nursing Accrediting Commission. (2008a). NLNAC standards and criteria: Associate. [Online]. Retrieved May 31, 2012 from http://www.nlnac.org.

National League for Nursing Accrediting Commission. (2008b). NLNAC standards and criteria: Baccalaureate. [Online]. Retrieved May 31, 2012 from http://www.nlnac.org.

National League for Nursing Accrediting Commission. (2008c). NLNAC standards and criteria: Master's and post-master's certificate. [Online]. Retrieved May 31, 2012 from http://www.nlnac.org.

National Library of Medicine. (2007). High blood pressure (hypertension). [Online]. Retrieved December 23, 2008 from http://www.nlm.nih.gov.

National Library of Medicine. (2008a). Nicotine gum. [Online]. Retrieved January 24, 2012 from http://www.nlm.nih.gov. 
National Library of Medicine. (2008b). Nicotine lozenges. [Online]. January 24, 2012 from http://www.nlm.nih.gov.

National Library of Medicine. (2008c). Opiate withdrawal. [Online]. January 24, 2012 from http://www.nlm.nih.gov.

National Library of Medicine. (2008d). Acamprosate. [Online]. January 24, 2012 from http://www.nlm.nih.gov.

National Library of Medicine. (2009). Methadone. [Online]. Retrieved January 18, 2012 from http://www.ncbi.nlm.nih.gov

National Library of Medicine. (2010a). Alprazolam. [Online]. Retrieved January 11, 2012 from http://www.ncbi.nlm.nih.gov.

National Library of Medicine. (2010b). Barbiturate intoxication and overdose. [Online]. Retrieved November 22, 2011 from http://www.nlm.nih.gov.

National Library of Medicine. (2010c). Chlordiazepoxide. [Online]. Retrieved May 2, 2012 from http://www.ncbi.nlm.nih.gov.

National Library of Medicine. (2010d). Desipramine. [Online]. Retrieved December 20, 2011 from http://www.ncbi.nlm.nih.gov.

National Library of Medicine. (2010e). Dextroamphetamine and Amphetamine. [Online]. Retrieved January 11, 2012 from http://www.ncbi.nlm.nih.gov.

National Library of Medicine. (2010f). Diazepam. [Online]. Retrieved May 2, 2012 from http://www.ncbi.nlm.nih.gov.

National Library of Medicine. (2010g). Diet and substance abuse recovery. [Online]. Retrieved November 18, 2011 from http://www.nlm.nih.gov.

National Library of Medicine. (2010h). Hepatitis. [Online]. Retrieved January 26, 2012 from http://www.ncbi.nlm.nih.gov.

National Library of Medicine. (2010i). Methylphenidate. [Online]. Retrieved January 11, 2012 from http://www.ncbi.nlm.nih.gov.

National Library of Medicine. (2010j). Phenobarbital. [Online]. Retrieved January 11, 2012 from http://www.ncbi.nlm.nih.gov.

National Library of Medicine. (2011a). Alcoholic neuropathy. [Online]. Retrieved January 26, 2012 from http://www.nlm.nih.gov.

National Library of Medicine. (2011b). Cirrhosis. [Online]. Retrieved January 26, 2012 from http://www.ncbi.nlm.nih.gov.

National Library of Medicine. (2011c). Fetal alcohol syndrome. [Online]. Retrieved January 27, 2012 from http://www.ncbi.nlm.nih.gov.

National Library of Medicine. (2011d). Gabapentin. [Online]. Retrieved May 31, 2012 from http://www.ncbi.nlm.nih.gov.

National Library of Medicine. (2011e). Nicotine addiction and withdrawal. [Online]. Retrieved December 21, 2011 from http://www.nlm.nih.gov.

National Library of Medicine. (2011f). Nicotine replacement therapy. [Online]. Retrieved January 24, 2012 from http://www.nlm.nih.gov. 
National Library of Medicine. (2011g). Ondansetron. [Online]. Retrieved January 24, 2012 from http://www.nlm.nih.gov.

National Library of Medicine. (2012). Carbamazepine. [Online]. Retrieved January 10, 2012 Retrieved from http://www.nlm.nih.gov.

National Quality Forum. (2005). Evidence-based treatment practices for substance use disorders. Workshop proceedings. [Online]. Retrieved February 27, 2012 from http://www.tresearch.org.

National Quality Forum. (2007). National voluntary consensus standards for the treatment of substance use conditions: Evidence- based treatment practices. Washington, DC: Author.

National Research Council and Institute of Medicine (2009). Adolescent health services: Missing opportunities. Washington, DC: The National Academies Press.

Neb. Admin. R. \& Regs. 175 § 18 (2004).

Neighbors, C. J., Barnett, N. P., Rohsenow, D. J., Colby, S. M., \& Monti, P. M. (2010). Costeffectiveness of a motivational intervention for alcohol-involved youth in a hospital emergency department. Journal of Studies on Alcohol \& Drugs, 71(3), 384-394.

Netherland, J., Botsko, M., Egan, J. E., Saxon, A. J., Cunningham, C. O., Finkelstein, R., et al. (2009). Factors affecting willingness to provide buprenorphine treatment. Journal of Substance Abuse Treatment, 36(3), 244-251.

Neumark, Y. D., Friedlander, Y., Thomasson, H. R., \& Li, T. K. (1998). Association of the ADH2*2 allele with reduced ethanol consumption in Jewish men in Israel: A pilot study. Journal of Studies on Alcohol, 59(2), 133-139.

New Jersey Department of Health and Senior Services. (2007). Standards for licensure of ambulatory care facilities. NJAC Title 8. Chapter 43A. [Online]. Retrieved February 16, 2012 from http://www.nj.gov.

New Jersey Department of Health and Senior Services. (2008). Hospital licensing standards. N. J. Admin Code Title 8. Chapter 43G: N.J.S.A. 26:2H-1 et seq. [Online]. Retrieved February 16, 2012 from http://www.nj.gov.

New Jersey. (2009). Licensure of substance abuse treatment facilities. Chapter 161B. Standards for licensure of outpatient substance abuse treatment facilities. (Supp. 6-1-09). [Online]. Retrieved February 16, 2012 from http://www.state.nj.us.

New York City Department of Health and Mental Hygiene. (2009). Local governmental plan: Alcohol and drug use services. [Online]. Retrieved March 5, 2012 from http://www.nyc.gov.

New York Department of Health. (2011). Buprenorphine. [Online]. Retrieved January 5, 2012 Retrieved from the World Wide Web http://www.health.ny.gov.

New York State Office of Alcoholism and Substance Abuse Services. (2007). Tobacco-free services: Title 14 NYCRR Part 856. [Online]. Retrieved December 19, 2006 from http://www.oasas.ny.gov.

New York State Office of Alcoholism and Substance Abuse Services. (2010). SBIRT-If you don't ask...Addiction medicine educational series workbook. [Online]. Retrieved November 14, 2011 from http://www.oasas.ny.gov.

New York State Psychiatric Institute, Biometrics Research. (2007). SCID: Frequently asked questions. [Online]. Retrieved April 17, 2012 from http://www.scid4.org.

New York State Psychiatric Institute, Biometrics Research. (2012). SCID - order form. [Online]. Retrieved April 20, 2012 from https://secure.cumc.columbia.edu. 
News-Medical.Net. (2012). NIDA awards \$1.4M grant to aid development of formal addiction medicine training programs. [Online]. Retrieved April 18, 2012 from http://www.news-medical.net.

Newton, T. F., Roache, J. D., De La Garza, R., Fong, T., Wallace, C. L., Li, S. H., et al. (2006). Bupropion reduces methamphetamine-induced subjective effects and cue-induced craving. Neuropsychopharmacology, 31(7), 1537-1544.

Nichter, M., Nichter, M., Thompson, P. J., Shiffman, S., \& Moscicki, A.-B. (2002). Using qualitative research to inform survey development on nicotine dependence among adolescents. Drug \& Alcohol Dependence, 68(Suppl. 1), s41-s56.

Nicorette. (2011). Stop smoking with NICORETTE® Inhalator. [Online]. Retrieved June 16, 2011 from http://www.nicorette.co.uk.

Niederhofer, H., \& Staffen, W. (2003). Acamprosate and its efficacy in treating alcohol dependent adolescents. European Child \& Adolescent Psychiatry, 12(3), 144-148.

Nilsen, P. (2009). Brief alcohol intervention to prevent drinking during pregnancy: An overview of research findings. Current Opinion in Obstetrics and Gynecology, 21(6), 496-500.

Nilssen, O., Ries, R. K., Rivara, F. P., Gurney, J. G., \& Jurkovich, G. J. (1994). The CAGE questionnaire and the Short Michigan Alcohol Screening Test in trauma patients: Comparison of their correlations with biological alcohol markers. Journal of Trauma, 36(6), 784-788.

Norcross, J. C., Koocher, G. P., Fala, N. C., \& Wexler, H. K. (2010). What does not work? Expert consensus on discredited treatments in the addictions. Journal of Addiction Medicine, 4(3), 174-180.

Nordgren, A. (1999). Theoretic health care reform. Hastings Center Report, 29(5), 41-41.

Nordgren, A., Hanson, M. J., \& Callahan, D. (1999). The goals of medicine: The forgotten issues in health care reform. Washington, DC: Georgetown University Press.

Norman, S. B., Wilkins, K. C., Tapert, S. F., Lang, A. J., \& Najavits, L. M. (2010). A pilot study of seeking safety therapy with OEF/OIF veterans. Journal of Psychoactive Drugs, 42(1), 83-87.

North Carolina School of Science and Mathematics. (1999). Sensitivity and specificity. [Online]. Retrieved April 26, 2012 from http://courses.ncssm.edu.

Northeast Addiction Technology Transfer Center. (2005, October). Taking action to build a stronger addictions workforce: Workforce development summit II. Philadelphia, PA: Author.

Novak, S. P., Reardon, S. F., Raudenbush, S. W., \& Buka, S. L. (2006). Retail tobacco outlet density and youth cigarette smoking: A propensity-modeling approach. American Journal of Public Health, 96(4), 670-676.

Nowinski, J. (2009). Twelve-step facilitation. [Online]. Retrieved January 16, 2009 from http://www.nida.nih.gov.

Nunes, E. V., \& Quitkin, F. M. (1997). Treatment of depression in drug-dependent patients: Effects on mood and drug use. NIDA Research Monograph, 172, 61-85.

O'Brien, C. P. \& McKay, J. (2007). Psychopharmacological treatments for substance use disorders. In P. E. Nathan \& J. M. Gorman (Eds.), A guide to treatments that work (3rd ed., pp. 145-177). New York: Oxford University Press.

O'Brien, C. P. (1996). Drug addiction and drug abuse. In J. G. Hardman, L. E. Limbird, L. S. Goodman, \& A. G. Gilman (Eds.), The pharmacological basis of therapeutics (pp. 557-577). New York: McGraw-Hill. 
O'Brien, C. P. (1997). A range of research-based pharmacotherapies for addiction. Science, 278(5335), 66-70.

O'Brien, C. P., \& McKay, J. (2007). Psychopharmacological treatments for substance use disorders. In P. E. Nathan \& J. M. Gorman (Eds.), A guide to treatments that work. (3rd ed., pp. 145-177). New York: Oxford University Press.

O'Brien, C. P., \& McLellan, A. T. (1996). Myths about the treatment of addiction. Lancet, 347(8996), 237-240.

O'Brien, C. P., Childress, A. R., Ehrman, R., \& Robbins, S. J. (1998). Conditioning factors in drug abuse: Can they explain compulsion? Journal of Psychopharmacology, 12(1), 15-22.

O'Brien, C. P., Volkow, N., \& Li, T. K. (2006). What's in a word? Addiction versus dependence in DSMV. American Journal of Psychiatry, 163(5), 764-765.

O'Brien, C. P., Woody, G. E., \& McLellan, A. T. (1995). Enhancing the effectiveness of methadone using psychotherapeutic interventions. NIDA Research Monograph, 150, 5-18.

Ockene, I. S., \& Miller, N. H. (1997). Cigarette smoking, cardiovascular disease, and stroke: A statement for healthcare professionals from the American Heart Association. American Heart Association Task Force on Risk Reduction. Circulation, 96(9), 3243-3247.

Ockene, J. K., Wheeler, E. V., Adams, A., Hurley, T. G., \& Hebert, J. (1997). Provider training for patient-centered alcohol counseling in a primary care setting. Archives of Internal Medicine, 157(20), 2334-2341.

O'Connor, M. J., \& Whaley, S. E. (2007). Brief intervention for alcohol use by pregnant women. American Journal of Public Health, 97(2), 252-258.

O'Connor, P. G. (2005). Methods of detoxification and their role in treating patients with opioid dependence. JAMA, 294(8), 961-963.

O'Connor, P. G., \& Fiellin, D. A. (2000). Pharmacologic treatment of heroin-dependent patients. Annals of Internal Medicine, 133(1), 40-54.

O'Connor, P. G., Carroll, K. M., Shi, Ju. M., Schottenfeld, R. S., Kosten, T. R., \& Rounsaville, B. J. (1997). Three methods of opioid detoxification in a primary care setting: A randomized trial. Annals of Internal Medicine, 127(7), 526-530.

O'Connor, P. G., Nyquist, J. G., \& McLellan, T. (2011). Integrating addiction medicine into graduate medical education in primary care: The time has come. Annals of Internal Medicine, 154(1), 56-59.

O'Connor, P. J., \& Youngstedt, S. D. (1995). Influence of exercise on human sleep. Exercise \& Sport Sciences Reviews, 23(1), 105-134.

O'Farrell, T. J., \& Fals-Stewart, W. (2003). Alcohol abuse. Journal of Marital \& Family Therapy, 29(1), 121-146.

Office of Applied Studies. (2008). The NSDUH report: Participation in self-help groups for alcohol and illicit drug use: 2006 and 2007. Rockville, MD: U.S. Department of Health and Human Services, Substance Abuse and Mental Health Services Administration, Office of Applied Studies.

Office of Communications. (2008). Summary report CARAVAN® survey for SAMHSA on addictions and recovery. Rockville, MD: U.S. Department of Health and Human Services, Substance Abuse and Mental Health Services Administration, Office of Communications.

Office of Juvenile Justice and Delinquency Prevention. (1998). Consequences of youth substance abuse. [Online]. Retrieved November 14, 2011 from http://www.ojjdp.gov. 
Office of Management and Budget. (2007). Government Performance Results Act of 1993. [Online]. Whitehouse.gov. Retrieved February 26, 2007 from http://www.whitehouse.gov.

Office of National Drug Control Policy. (2004). The economic costs of drug abuse in the United States, 1992-2002 (Publication No. 207303). Washington, DC: Executive Office of the President.

Office of National Drug Control Policy. (2007). National Leadership Conference on Medical Education in Substance Abuse [November 30 - December 1, 2006 (Draft 2/28/07, Updated 5/7/07)]. [Online]. Retrieved February 27, 2012 from https://www.ncjrs.gov.

Office of National Drug Control Policy. (2009). National drug control strategy: FY 2010 budget summary. [Online]. Retrieved March 29, 2012 from http://www.whitehouse.gov.

Office of National Drug Control Policy. (2011). National drug control strategy. [Online]. Retrieved March 1, 2012 from http://www.whitehouse.gov.

Office of National Drug Control Policy. (June 23, 2010). Treating addiction as a disease: The promise of medication assisted recovery: Written statement of Dr. A. Thomas McLellan, Deputy Director ONDCP. [Online]. House Committee on Oversight and Government Reform, Subcommittee on Domestic Policy. Retrieved December 19, 2011 from http://democrats.oversight.house.gov.

Office of the State Auditor, State of Colorado. (2010). Medicaid outpatient substance abuse treatment benefit. Department of Health Care Policy and Financing. Performance audit, November 2010. [Online]. Retrieved May 17, 2012 from http://www.leg.state.co.us.

Ogedegbe, G. (2008). Barriers to optimal hypertension control. Journal of Clinical Hypertension, 10(8), 644-646.

Ohio State University, Department of History. (2012). Clash of cultures in the 1910s and 1920s. Introduction. [Online]. Retrieved April 2, 2012 from http://ehistory.osu.edu.

Okla. Stat. 450. 18, § 1-2 (2011).

Okuyemi, K. S., Caldwell, A. R., Thomas, J. L., Born, W., Richter, K. P., Nollen, N., et al. (2006). Homelessness and smoking cessation: Insights from focus groups. Nicotine \& Tobacco Research, $8(2), 287-296$.

Okuyemia, K. S., Pulversc, K. M., Sanderson Cox, L., Thomas, J. L., Kaur, H., Mayod, M. S., et al. (2007). Nicotine dependence among African American light smokers: A comparison of three scales. Addictive Behaviors, 32(10), 1998-2002.

Olmstead, T. A., Abraham, A. J., Martino, S., \& Roman, P. M. (2012). Counselor training in several evidence-based psychosocial addiction treatments in private US substance abuse treatment centers. Drug \& Alcohol Dependence, 120(1-3), 149-154.

Olmstead, T. A., Johnson, J. A., Roman, P. M., \& Sindelar, J. L. (2005). What are the correlates of substance abuse treatment counselor salaries? Journal of Substance Abuse Treatment, 29(3), 181-189.

Olmstead, T., \& Sindelar, J. L. (2004). To what extent are key services offered in treatment programs for special populations? Journal of Substance Abuse Treatment, 27(1), 9-15.

Olsen, Y., Bass, E., McCaul, M. E., \& Steinwachs, D. (2004). Treating opioid addiction in office based settings: Findings from a physician survey. [Online]. Center for a Healthy Maryland. Retrieved February 16, 2012 from http://healthymaryland.org.

O'Malley, S. S., Jaffe, A. J., Chang, G., Schottenfeld, R. S., Meyer, R. E., \& Rounsaville, B. (1992). Naltrexone and coping skills therapy for alcohol dependence. A controlled study. Archives of General Psychiatry, 49(11), 881-887. 
Ommaya, A. K., Salazar, A. M., Dannenberg, A. L., Ommaya, A., Chervinsky, A. B., \& Schwab, K. (1996). Outcome after traumatic brain injury in the U.S. military medical system. Journal of Trauma, 41(6), 972-975.

Oncken, C., Van, K. J., \& Kranzler, H. R. (2001). Adverse effects of oral naltrexone: Analysis of data from two clinical trials. Psychopharmacology, 154(4), 397-402.

O'Neil, K. A., Conner, B. T., \& Kendall, P. C. (2011). Internalizing disorders and substance use disorders in youth: Comorbidity, risk, temporal order, and implications for intervention. Clinical Psychology Review, 31(1), 104-112.

Ong, M. K., Zhou, Q., \& Sung, H. Y. (2011). Primary care providers advising smokers to quit: Comparing effectiveness between those with and without alcohol, drug, or mental disorder. Nicotine \& Tobacco Research, 13(12), 1193-1201.

Ontario Campaign for Action on Tobacco. (2007). Health effects of second-hand smoke exposure. [Online]. Retrieved December 7, 2007 from http://www.ocat.org.

Open Society Foundations. (2009). Poll finds bipartisan agreement on one aspect of health care reform: Addiction treatment. [Online]. Retrieved August 16, 2011 from http://www.soros.org.

Or. Admin. R. 415-020-0075 (2012).

Or. Rev. Stat. § 182.515 (2009).

Orleans, C. T., Jepson, C., Resch, N., \& Rimer, B. K. (1994). Quitting motives and barriers among older smokers. The 1986 Adult Use of Tobacco Survey revisited. Cancer, 74(Suppl. 7), 2055-2061.

Orson, F. M., Kinsey, B. M., Singh, R. A. K., Wu, Y., Gardner, T., \& Kosten, T. R. (2008). Substance abuse vaccines. Annals of the New York Academy of Sciences, 1141, 257-269.

Oser, C. B., \& Roman, P. M. (2007). Organizational-level predictors of adoption across time: Naltrexone in private substance-use disorders treatment centers. Journal of Studies on Alcohol \& Drugs, 68(6), 852-861.

Ostchega, Y., Yoon, S. S., Huges, J., \& Louis, T. (2008). Hypertension awareness, treatment, and control -- continued disparities in adults: United States, 2005-2006 (NCHS data brief No. 3). Hyattsville, MD: National Center for Health Statistics.

Ostrow, L., \& Manderscheid, R. (2010). Medicare mental health parity: A high potential change that is long overdue. Journal of Behavioral Health Services \& Research, 37(3), 285-290.

Ouimette, P. C., Finney, J. W., \& Moos, R. H. (1997). Twelve-step and cognitive-behavioral treatment for substance abuse: A comparison of treatment effectiveness. Journal of Consulting \& Clinical Psychology, 65(2), 230-240.

Ouimette, P., Moos, R. H., \& Finney, J. W. (2003). PTSD treatment and 5-year remission among patients with substance use and posttraumatic stress disorders. Journal of Consulting \& Clinical Psychology, $71(2), 410-414$.

Owens, P. L., Mutter, R., \& Stocks, C. (2010). Mental health and substance abuse-related emergency department visits among adults, 2007. HCUP statistical brief\#92. Rockville, MD: Agency for Healthcare Research and Quality.

Owens, T. J., \& Shippee, N. D. (2009). Depressed mood and drinking occasions across high school: Comparing the reciprocal causal structures of a panel of boys and girls. Journal of Adolescence, 32(4), 763-780.

Oxford House. (2011). Suggested guidelines for expulsiosn and disruptive behavior: A compilation from several Oxford House sources. [Online]. Retrieved January 25, 2012 from http://oxfordhouseok.org. 
Oxford House. (2007). Profile of Oxford House residents in fifteen jurisdictions. [Online]. Retrieved from the World Wide Web http://www.oxfordhouse.org.

Oxford House. (2000). Oxford House Manual: An idea based on a sound system for recovering alcoholics and drug addicts to help themselves. Silver Spring, MD: Author.

Oxford House. (2009). Questions and answers. [Online]. Retrieved March 16, 2009 from http://www.oxfordhouse.org.

Ozechowski, T. J., \& Waldron, H. B. (2010). Assertive outreach strategies for narrowing the adolescent substance abuse treatment gap: Implications for research, practice, and policy. Journal of Behavioral Health Services \& Research, 37(1), 40-63.

Paaver, M., Kurrikoff, T., Nordquist, N., Oreland, L., \& Harro, J. (2008). The effect of 5-HTT gene promoter polymorphism on impulsivity depends on family relations in girls. Progress In NeuroPsychopharmacology \& Biological Psychiatry, 32(5), 1263-1268.

Padgett, D., Struening, E. L., \& Andrews, H. (1990). Factors affecting the use of medical, mental health, alcohol, and drug treatment services by homeless adults. Medical Care, 28(9), 805-821.

Palmer, J. A., Palmer, L. K., Michiels, K., \& Thigpen, B. (1995). Effects of type of exercise on depression in recovering substance abusers. Perceptual \& Motor Skills, 80(2), 523-530.

Palmigiano v. Garrahy, 443 F. Supp. 956 (D.R.I. 1977).

Paquette, K. (2010). Individuals experiencing chronic/long-term homelessness. [Online]. Retrieved March 9, 2012 from http://homeless.samhsa.gov.

Parker, D. R., Lasater, T. M., Windsor, R., Wilkins, J., \& Upegui, D. I. H. J. (2002). The accuracy of selfreported smoking status assessed by cotinine test strips. Nicotine \& Tobacco Research, 4(3), 305-309.

Parkin, D. (2009). Techniques of economic appraisal (including cost-effectiveness analysis and modelling, cost-utility analysis, option appraisal and cost-benefit analysis, the measurement of health benefits in terms of QALYs and related measures e.g. DALYs). Health economics: 5 - techniques of economic appraisal. [Online]. Retrieved April 2, 2012 from http://www.healthknowledge.org.uk.

Parrott, S., Godfrey, C., Heather, N., Clark, J., \& Ryan, T. (2006). Cost and outcome analysis of two alcohol detoxification services. Alcohol \& Alcoholism, 41(1), 84-91.

Parthasarathy, S., Weisner, C., Hu, T., \& Moore, C. (2001). Association of outpatient alcohol and drug treatment with health care utilization and cost: Revisiting the offset hypothesis. Journal of Studies on Alcohol, 62(1), 89-97.

Partners for Recovery. (2003). Substance abuse treatment workforce environmental scan. Rockville, MD: U.S. Department of Health and Human Services, Substance Abuse and Mental Health Services Administration, Center for Substance Abuse Treatment.

Partnership for Prevention. (2012). Prevention: A key indicator of quality. [Online]. Retrieved April 4, 2012 from http://www.prevent.org.

Patel, D. (2005). Pharmacists role in treating and preventing substance abuse. [Online]. Retrieved July 5, 2011 from http://rxethics.org.

Patient Protection and Affordable Care Act, 42 § 3022 (2010).

Pating, D. R., Miller, M. M., Goplerud, E., Martin, J., \& Ziedonis, D. M. (2012). New systems of care for substance use disorders: Treatment finance, and technology under health care reform. Psychiatric Clinics of North America, 35(2), 327-356. 
Patterson, T. L., \& Jeste, D. V. (1999). The potential impact of the baby-boom generation on substance abuse among elderly persons. Psychiatric Services, 50(9), 1184-1188.

Patton, G. C., Coffey, C., Carlin, J. B., Degenhardt, L., Lynskey, M., \& Hall, W. (2002). Cannabis use and mental health in young people: Cohort study. British Medical Journal, 325(1), 1195-1198.

Paul Wellstone and Pete Domenici Mental Health Parity and Addiction Equity Act of 2008 (H.R. 1424).

Paulozzi, L. J., \& Xi, Y. (2008). Recent changes in drug poisoning mortality in the United States by urban-rural status and by drug type. Pharmacoepidemiology \& Drug Safety, 17(10), 997-1005.

Pbert, L., Ockene, J. K., Zapka, J., Ma, Y., Goins, K. V., Oncken, C., et al. (2004). A community health center smoking-cessation intervention for pregnant and postpartum women. American Journal of Preventive Medicine, 26(5), 377-385.

Pbert, L., Osganian, S. K., Gorak, D., Druker, S., Reed, G., O'Neill, K. M., et al. (2006). A school nursedelivered adolescent smoking cessation intervention: A randomized controlled trial. Preventive Medicine, 43(4), 312-320.

PBS.org. (2012). Prohibition: Roots of prohibition. [Online]. Retrieved April 2, 2012 from http://www.pbs.org.

Pearson, D. A., Bruggman, A. R., \& Haukoos, J. S. (2007). Out-of-hospital and emergency department utilization by adult homeless patients. Annals of Emergency Medicine, 50(6), 646-652.

Peele, S. (2007). Addiction as disease: Policy, epidemiology, and treatment: Consequences of a bad idea. In J. E. Henningfield, P. B. Santora, \& W. K. Bickel (Eds.), Addiction treatment: Science and policy for the twenty first-century. (pp. 153-164). Baltimore, MD: John Hopkins University Press.

Pelham, W. E., \& Lang. A. R. (2011). Can your children drive you to drink? Stress and parenting in adults interacting with children with ADHD. Alcohol Research \& Health, 23(4), 292-298.

Pelletier, L. R., \& Hoffamn, J. A. (2002). A framework for selecting performance measures for opioid treatment programs. Journal of Health Care Quality, 24(3), 24-35.

Pendharkar, B., Levy, S. M., McQuistan, M. R., Qian, F., Squier, C. A., Slach, N. A., et al. (2010). Fourth-year dental students' perceived barriers to providing tobacco intervention services. Journal of Dental Education, 74(10), 1074-1084.

Pennsylvania Rural Health Association. (2010). Pennsylvania rural health care: Status check V. [Online]. Retrieved February 7, 2012 from http://www.paruralhealth.org.

Pentel, P., \& Malin, D. (2002). A vaccine for nicotine dependence: Targeting the drug rather than the brain. Respiration, 69(3), 193-197.

Pereira, F. C., Gough, B., Macedo, T. R., Ribeiro, C. F., Ali, S. F., \& Binienda, Z. K. (2011).

Buprenorphine modulates methamphetamine-induced dopamine dynamics in the rat caudate nucleus. Neurotoxicity Research, 19(1), 94-101.

Perron, B. E., Gillespie, D. F., Alexander-Eitzman, B., \& Delva, J. (2010). Availability of outpatient substance use disorder treatment programs in the United States. Substance Use \& Misuse, 45(7-8), 1097-111.

Perron, B. E., Mowbray, O. P., Glass, J. E., Delva, J., Vaughn, M. G., \& Howard, M. O. (2009). Differences in service utilization and barriers among blacks, Hispanics, and whites with drug use disorders. Substance Abuse Treatment, Prevention, \& Policy, 4(3), 1-10.

Perrone, J., De Roos, F., Jayaraman, S., \& Hollander, J. E. (2001). Drug screening versus history in detection of substance use in ED psychiatric patients. American Journal of Emergency Medicine, 19(1), 49-51. 
Pescosolido, B. A., Martin, J. K., Long, J. S., Medina, T. R., Phelan, J. C., \& Link, B. G. (2010). "A disease like any other"? A decade of change in public reactions to schizophrenia, depression, and alcohol dependence. American Journal of Psychiatry, 167(11), 1321-1330.

Peter D. Hart Research Associates. (2001). The face of recovery. Survey among the recovery community conducted August 2-22, 2001 for The Alliance Project. [Power Point Presenation].

Peter D.Hart Research Associates, \& Peter. (2001). The face of recovery. [Online]. Retrieved October 28, 2005 from http://www.facesandvoicesofrecovery.org.

Peters, R. H., \& Matthews, C. O. (2003). Substance abuse treatment programs in prisons and jails. In T. J. Fagan \& R. K. Ax (Eds.), Correctional mental health handbook (pp. 73-74). Thousand Oaks, CA: Sage.

Peters, R. H., Bartoi, M. G., \& Sherman, P. B. (2008). Screening and assessment of co-occurring disorders in the justice system. New York: CMHS National GAINS Center.

Peters, R. H., Greenbaum, P. E., Steinberg, M. L., Carter, C. R., Ortiz, M. M., Fry, B. C., et al. (2000). Effectiveness of screening instruments in detecting substance use disorders among prisoners. Journal of Substance Abuse Treatment, 18(4), 349-358.

Peterson, A. V., Jr., Kealey, K. A., Mann, S. L., Marek, P. M., Ludman, E. J., Liu, J., et al. (2009). Group-randomized trial of a proactive, personalized telephone counseling intervention for adolescent smoking cessation. Journal of the National Cancer Institute, 101(20), 1378-1392.

Peterson, M., \& Johnstone, B. M. (1995). The Atwood Hall Health Promotion Program, Federal Medical Center, Lexington, KY. Effects on drug-involved federal offenders. Journal of Substance Abuse Treatment, 12(1), 43-48.

Petrakis, I. L., Leslie, D., \& Rosenheck, R. (2003). Use of naltrexone in the treatment of alcoholism nationally in the Department of Veterans Affairs. Alcoholism: Clinical \& Experimental Research, 27(11), 1780-1784.

Petrakis, I. L., Rosenheck, R., \& Desai, R. (2011). Substance use comorbidity among veterans with posttraumatic stress disorder and other psychiatric illness. American Journal on Addictions, 20(3), 185-189.

Petrone, K., \& Katz, P. (2005). Approaches to appropriate drug prescribing for the older adult. Primary Care Clinical Office Practice, 32(3), 755-775.

Petry, N. M., Peirce, J. M., Stitzer, M. L., Blaine, J., Roll, J. M., Cohen, A., et al. (2005). Effect of prizebased incentives on outcomes in stimulant abusers in outpatient psychosocial treatment programs: A national drug abuse treatment clinical trials network study. Archives of General Psychiatry, 62(10), 1148-1156.

Pettinati, H. M., Kampman, K. M., Lynch, K. G., Suh, J. J., Dackis, C. A., Oslin, D. W., et al. (2008). Gender differences with high-dose naltrexone in patients with co-occurring cocaine and alcohol dependence. Journal of Substance Abuse Treatment, 34(4), 378-390.

Petursson, H. (1994). The benzodiazepine withdrawal syndrome. Addiction, 89(11), 1455-1459.

Physicians and Lawyers for National Drug Policy. (2008). Screening, brief intervention, referral, and treatment (SBIRT) of acute care patients for alcohol use disorders. [Online]. Retrieved October 8, 2008 from http://www.plndp.org.

Pierce, J. P., \& Gilpin, E. A. (1995). A historical analysis of tobacco marketing and the uptake of smoking by youth in the United States: 1890-1977. Health Psychology, 14(6), 500-508. 
Pierce, J. P., Choi, W. S., Gilpin, E. A., Farkas, A. J., \& Berry, C. C. (1998). Tobacco industry promotion of cigarettes and adolescent smoking. JAMA, 279(7), 511-515.

Pierre, J. M. (2011). Cannabis, synthetic cannabinoids, and psychosis risk: What the evidence says. Current Psychiatry, 10(9), 49-57.

Pignone, M., Earnshaw, S., Tice, J. A., \& Pletcher, M. J. (2006). Aspirin, statins, or both drugs for the primary prevention of coronary heart disease events in men: A cost-utility analysis. Annals of Internal Medicine, 144(5), 326-336.

Piper, M. E., McCarthy, D. E., \& Baker, T. B. (2006). Assessing tobacco dependence: A guide to measure evaluation and selection. Nicotine \& Tobacco Research, 8(3), 339-351.

Piper, M. E., Smith, S. S., Schlam, T. R., Fiore, M. C., Jorenby, D. E., Fraser, D., et al. (2009). A randomized placebo-controlled clinical trial of 5 smoking cessation pharmacotherapies. Archives of General Psychiatry, 66(11), 1253-1262.

Pizacani, B. A., Rohde, K., Bushore, C., Stark, M. J., Maher, J. E., Dilley, J. A., et al. (2009). Smokingrelated knowledge, attitudes and behaviors in the lesbian, gay and bisexual community: A populationbased study from the U.S. Pacific Northwest. Preventive Medicine, 48(6), 555-561.

Placzek, A. N., Zhang, T. A., \& Dani, J. A. (2009). Age dependent nicotinic influences over dopamine neuron synaptic plasticity. Biochemical Pharmacology, 78(7), 686-692.

Polcin, D. L. (2001). Sober living houses: Potential roles in substance abuse services and suggestions for research. Substance Use \& Misuse, 36(3), 301-311.

Polcin, D. L., \& Henderson, D. M. (2008). A clean and sober place to live: Philosophy, structure, and purported therapeutic factors in sober living houses. Journal of Psychoactive Drugs, 40(2), 153-159.

Polcin, D. L., Korcha, R. A., Bond, J., \& Galloway, G. (2010). Sober living houses for alcohol and drug dependence: 18-month outcomes. Journal of Substance Abuse Treatment, 38(4), 356-365.

Poldrugo, F. (1997). Integration of pharmacotherapies in the existing programs for the treatment of alcoholics: An international perspective. Journal of Addictive Diseases, 16(4), 65-82.

Poling, J., Oliveto, A., Petry, N., Sofuoglu, M., Gonsai, K., Gonzalez, G., et al. (2006). Six-month trial of bupropion with contingency management for cocaine dependence in a methadone-maintained population. Archives of General Psychiatry, 63(2), 219-228.

Pollack, H. A., \& D'Aunno, T. (2008). Dosage patterns in methadone treatment: Results from a national survey, 1988-2005. Health Services Research, 43(6), 2143-2163.

Pollak, M., Paicheler, G., \& Pierret, J. (1992). AIDS: A problem for sociological research. London: SAGE Publications.

Pollini, R. A., McCall, L., Mehta, S. H., Vlahov, D., \& Strathdee, S. A. (2006). Non-fatal overdose and subsequent drug treatment among injection drug users. Drug \& Alcohol Dependence, 83(2), 104-110.

Pollio, D. E., McClendon, J., \& Reid, D. (2004). Certification and program regulations for inpatient services to youth with addiction: A state-level analysis. Journal of Psychoactive Drugs, 36(4), 499509.

Polosa, R., \& Benowitz, N. L. (2011). Treatment of nicotine addiction: Present therapeutic options and pipeline developments. Trends in Pharmacological Sciences, 32(5), 281-289.

Polsky, D., Glick, H. A., Yang, J., Subramaniam, G. A., Poole, S. A., \& Woody, G. E. (2010). Costeffectiveness of extended buprenorphine-naloxone treatment for opioid-dependent youth: Data from a randomized trial. Addiction, 105(9), 1616-1624. 
Pomerleau, C. S., Carton, S. M., Lutzke, M. L., Flessland, K. A., \& Pomerleau, O. F. (1994). Reliability of the Fagerstrom tolerance questionnaire and the fagerstrom test for nicotine dependence. Addictive Behaviors, 19(1), 33-39.

Porter, J. (2005). Clearing the air on tobacco use in corrections. [Online]. Retrieved October 19, 2009 from the World Wide Web: http://www.ncchc.org.

Porter, S. (2008). New codes cover substance abuse screening, intervention: Check private payer coverage beforehand. [Online]. Retrieved October 27, 2011 from http://www.aafp.org.

Postel, M. G., de Haan, H. A., \& De Jong, C. A. (2008). E-therapy for mental health problems: A systematic review. Telemedicine Journal \& e-Health, 14(7), 707-714.

Powell, D. J. (2006). It's time for a national approach on staff development. Behavioral Healthcare, 26(3), 42-45.

Powers, C. A., Zapka, J., Phelan, S., Ozcan, T., Biello, K. B., O'Donnell, J., et al. (2011). Tobacco education and counseling in obstetrics and gynecology clerkships: A survey of medical school program directors. Maternal \& Child Health Journal, 15(8), 1153-1159.

Powers, G., \& Hamilton, N. (2012). eServices: Definition and standards. [Online]. Retrieved January 25, 2012 from http://www.fadaa.org.

Powers, M. B., Vedel, E., \& Emmelkamp, P. M. G. (2008). Behavioral couples therapy (BCT) for alcohol and drug use disorders: A meta-analysis. Clinical Psychology Review, 28(6), 952-962.

Prendergast, M. L., Messina, N. P., Hall, E. A., \& Warda, U. S. (2011). The relative effectiveness of women-only and mixed-gender treatment for substance-abusing women. Journal of Substance Abuse Treatment, 40(4), 336-348.

Prendergast, M., Podus, D., Finney, J., Greenwell, L., \& Roll, J. (2006). Contingency management for treatment of substance use disorders: A meta-analysis. Addiction, 101(11), 1546-1560.

Prescott, C. A., \& Kendler, K. S. (1999). Genetic and environmental contributions to alcohol abuse and dependence in a population-based sample of male twins. American Journal of Psychiatry, 156(1), 3440.

Prescott, C. A., Aggen, S. H., \& Kendler, K. S. (1999). Sex differences in the sources of genetic liability to alcohol abuse and dependence in a population-based sample of U.S. twins. Alcoholism: Clinical \& Experimental Research, 23(7), 1136-1144.

Price, J. L. (2010). Findings from the National Vietnam Veterans' Readjustment Study. [Online]. U.S. Department of Veterans Affairs. Retrieved May 31, 2012 from http://www.lb9.uscourts.gov.

Pringle, J. L., Emptage, N. P., \& Hubbard, R. L. (2006). Unmet needs for comprehensive services in outpatient addiction treatment. Journal of Substance Abuse Treatment, 30(3), 183-189.

Prinstein, M. J., Boergers, J., \& Spirito, A. (2001). Adolescents' and their friends' health-risk behavior: Factors that alter or add to peer influence. Journal of Pediatric Psychology, 26(5), 287-298.

Prochaska, J. J., Fromont, S. C., \& Hall, S. M. (2005). How prepared are psychiatry residents for treating nicotine dependence? Academic Psychiatry, 29(3), 256-261.

Prochaska, J. J., Fromont, S. C., Louie, A. K., Jacobs, M. H., \& Hall, S. M. (2006). Training in tobacco treatments in psychiatry: A national survey of psychiatry residency training directors. Academic Psychiatry, 30(5), 372-378.

Prochaska, J. J., Gill, P., \& Hall, S. M. (2004). Treatment of tobacco use in an inpatient psychiatric setting. Psychiatric Services, 55(11), 1265-1270. 
Prochaska, J. J., Rossi, J. S., Redding, C. A., Rosen, A. B., Tsoh, J. Y., Humfleet, G. L., et al. (2004). Depressed smokers and stage of change: Implications for treatment interventions. Drug \& Alcohol Dependence, 76(2), 143-151.

ProCon.org. (2012). Should marijuana be a medical option? [Online]. Retrieved March 15, 2012 from http://medicalmarijuana.procon.org.

Project MATCH Research Group. (1997). Matching alcoholism treatment to client heterogeneity: Project MATCH post treatment drinking outcomes. Journal of Studies on Alcohol, 58, 7-29.

Project MATCH Research Group. (1998). Matching alcoholism treatment to client heterogeneity: Project MATCH three-year drinking outcomes. Alcoholism: Clinical \& Experimental Research, 22(6), 13001311.

Proksch, J. W., Gentry, W. B., \& Owens, S. M. (2000). Anti-phencyclidine monoclonal antibodies provide long-term reductions in brain phencyclidine concentrations during chronic phencyclidine administration in rats. Journal of Pharmacology \& Experimental Therapeutics, 292(3), 831-837.

Pronk, N. P., Crouse, S. F., \& Rohack, J. J. (1995). Maximal exercise and acute mood response in women. Physiology \& Behavior, 57(1), 1-4.

PsychCentral.org. (2010). Substance abuse and dependence. Treatment. [Online]. Retrieved May 23, 2012 from http://psychcentral.com.

Psychiatric Mental Health Substance Abuse Essential Competencies Taskforce of the American Academy of Nursing Psychiatric Mental Health Substance Abuse Expert Panel. (2012). Essential psychiatric, mental health and substance use competencies for the registered nurse. Archives of Psychiatric Nursing, 26(2), 80-110.

PsychiatryOnline. (2011). DSM library. Chapter 4. Substance-related disorders. [Online]. Retrieved December 2, 2011 from http://dsm.psychiatryonline.org

Public Broadcasting System. (2011). Thirty years of America's drug war: A chronology. [Online]. Retrieved December 13, 2011 from http://www.pbs.org

Public Health Research Institute. (2005). Tobacco treatment for low-income pregnant women: Identifying patient barriers to smoking cessation and adherence to tobacco treatment alternatives. [Online]. Retrieved February 3, 2012 from http://www.une.edu.

Pumariega, A. J., Kilgus, M. D., \& Rodriguez, L. (2005). Adolescents. In J. H. Lowinson, P. Ruiz, R. B. Millman, \& J. G. Langrod (Eds.), Substance abuse: A comprehensive textbook (pp. 1021-1037). Baltimore, MD: Lippincott Williams and Wilkens.

Quenqua, D. (2011, July 11). Rethinking addiction's roots, and its treatment. New York Times, p. A11.

Quenqua, D. (2012). Drugs help tailor alcoholism treatment. [Online]. Retrieved May 22, 2012 from http://www.nytimes.com.

Quinton, A. (2004). Alcohol abuse: What doctors can do. [Online]. Retrieved August 29, 2008 from http://www.nhpr.org.

Quitnet. (2009a). Medication guide: Nicotine replacement therapy: The nicotine inhaler. [Online]. Retrieved January 4, 2012 Retrieved from http://quitnet.com.

Quitnet. (2009b). Medication guide: Nicotine replacement therapy: Nicotine nasal spray. [Online]. Retrieved January 4, 2012 Retrieved from http://www.quitnet.com.

Rabin, R. C. (2009). A new cigarette hazard: "Third-hand smoke". [Online]. Retrieved January 26, 2012 from http://www.nytimes.com. 
Rahdert, E. (1991). The adolescent assessment/referral system manual (DHHS Publication No. ADM911735). Rockville, MD: U.S. Department of Health and Human Services, National Institutes of Health, National Institute on Drug Abuse.

Rando, K., Hong, K. I., Bhagwagar, Z., Li. C. S. R., Bergquist, K., Guarnaccia, J., et al. (2011). Association of frontal and posterior cortical gray matter volume with time to alcohol relapse: A prospective study. American Journal of Psychiatry, 168(2), 183-192.

Rapp, R. C., Xu, J., Carr, C. A., Lane, D. T., Wang, J., \& Carlson, R. (2006). Treatment barriers identified by substance abusers assessed at a centralized intake unit. Journal of Substance Abuse Treatment, 30(3), 227-235.

Rasmussen, S. (2000). Addiction treatment: Theory and practice. Thousand Oaks, CA: Sage.

Rawson, R. A., McCann, M. J., Hasson, A. J., \& Ling, W. (2000). Addiction pharmacotherapy 2000: New options, new challenges. Journal of Psychoactive Drugs, 32(4), 371-378.

Ray, G. T., Mertens, J. R., \& Weisner, C. (2007). The excess medical cost and health problems of family members of persons diagnosed with alcohol or drug problems. Medical Care, 45(2), 116-122.

Read, J. P., \& Brown, R. A. (2003). The role of physical exercise in alcoholism treatment and recovery. Professional Psychology: Research \& Practice, 34(1), 49-56.

Read, J. P., Brown, R. A., Marcus, B. H., Kahler, C. W., Ramsey, S. E., Dubreuil, M. E., et al. (2001). Exercise attitudes and behaviors among persons in treatment for alcohol use disorders. Journal of Substance Abuse Treatment, 21(4), 199-206.

Redko, C., Rapp, R. C., \& Carlson, R. G. (2006). Waiting time as a barrier to treatment entry: Perceptions of substance users. Journal of Drug Issues, 36(4), 831-852.

Rees, T. (2011). Sober living code of conduct, house rules, and guest agreement template. [Online]. Retrieved January 12, 2012 Retrieved from the World Wide Web http://www.soberlivingsearch.com.

Rehan, V. K., Sakurai, R., \& Torday, J. S. (2011). Thirdhand smoke: A new dimension to the effects of cigarette smoke on the developing lung. American Journal of Physiology. Lung Cellular and Molecular Physiology, 301(1), L1-L8.

Rehm, J., Gmel, G., Sempos, C. T., \& Trevisan, M. (2003). Alcohol-related morbidity and mortality. Alcohol Research \& Health, 27(1), 39-51.

Rehm, J., Samokhvalov, A. V., Neuman, M. G., Room, R., Parry, C., Lonnroth, K., et al. (2009). The association between alcohol use, alcohol use disorders and tuberculosis (TB). A systematic review. BMC Public Health, 9, 450. doi:10.1186/147-2458-9-450.

Reid, M. S., Fallon, B., Sonne, S., Flammino, F., Nunes, E. V., Jiang, H., et al. (2008). Smoking cessation treatment in community-based substance abuse rehabilitation programs. Journal of Substance Abuse Treatment, 35(1), 68-77.

Reinert, D. F., \& Allen, J. P. (2002). The Alcohol Use Disorders Identification Test (AUDIT): A review of recent research. Alcoholism: Clinical \& Experimental Research, 26(2), 272-279.

Reisfield, G. M., Goldberger, B. A., Gold, M. S., \& Dupont, R. L. (2012). The mirage of impairing drug concentration thresholds: A rationale for zero tolerance per se driving under the influence of drug laws. Journal of Analytical Toxicology, 36(5), 353-356.

Reisfield, G. M., Graham, N. A., \& Gold, M. S. (2011). Letter to the editor. Re: "Estimates of nondisclosure of cigarette smoking among pregnant and nonpregnant women of reproductive age in the United States". American Journal of Epidemiology, 173(9), 1095-1096. 
Reisfield, G. M., Salazar, E., \& Bertholf, R. L. (2007). Review: Rational use and interpretation of urine drug testing in chronic opioid therapy. Annals of Clinical \& Laboratory Science, 37(4), 301-314.

Renner, J. A., Jr., Karam-Hage, M., Levinson, M., Craig, T., \& Eld, B. (2009). What do psychiatric residents think of addiction psychiatry as a career? Academic Psychiatry, 33(2), 139-142.

Rettig, R. (1995). Federal regulation of methadone: Table of contents and executive summary. [Online]. Retrieved September 26, 2006 from http://www.drugpolicy.org.

Reus, V. I., \& Smith, B. J. (2008). Multimodal techniques for smoking cessation: A review of their efficacy and utilization and clinical practice guidelines. International Journal of Clinical Practice, 62(11), 1753-1768.

Revell, C. C., \& Schroeder, S. A. (2005). Simplicity matters: Using system-level changes to encourage clinician intervention in helping tobacco users quit. Nicotine \& Tobacco Research, 7(Suppl. 1), S67S69.

Rey, J. M. (2007). Does marijuana contribute to psychotic illness? Cannabis abuse is not benign, especially in adolescents. Journal of Family Practice, 6(2), 158-162.

Rhode Island Hospital. (2011). Screening, Brief Intervention and Referral to Treatment (SBIRT). Addressing barriers to SBIRT implementation. [Online]. Retrieved December 16, 2011 from http://www.rhodeislandhospital.org.

Rice, V. H., \& Stead, L. F. (2008). Nursing interventions for smoking cessation. [Online]. Retrieved December 13, 2011 from http://www.thecochranelibrary.com.

Richards, E. P. (1999). The police power and the regulation of medical practice: A historical review and guide for medical licensing board regulation of physicians in ERISA-qualified managed care organizations. [Online]. Retrieved December 13, 2011 from http://biotech.law.lsu.edu.

Richardson, V. E., \& Kilty, K. M. (1995). Gender differences in mental health before and after retirement: A longitudinal analysis. Journal of Women \& Aging, 7(1/2), 19-35.

Richter, K. P. (2006). Good and bad times for treating cigarette smoking in drug treatment. Journal of Psychoactive Drugs, 38(3), 311-315.

Richter, K. P., McCool, R. M., Okuyemi, K. S., Mayo, M. S., \& Ahluwalia, J. S. (2002). Patients' views on smoking cessation and tobacco harm reduction during drug treatment. Nicotine \& Tobacco Research, 4(Suppl. 2), S175-S182.

Richter, L., \& Richter, D. M. (2001). Exposure to parental tobacco and alcohol use: Effects on children's health and development. American Journal of Orthopsychiatry, 71(2), 182-203.

Rieckmann, T. R., Kovas, A. E., Cassidy, E. F., \& McCarty, D. (2011). Employing policy and purchasing levers to increase the use of evidence-based practices in community-based substance abuse treatment settings: Reports from single state authorities. Evaluation \& Program Planning, 34(4), 366-374.

Rieckmann, T. R., Kovas, A. E., Fussell, H. E., \& Stettler, N. M. (2009). Implementation of evidencebased practices for treatment of alcohol and drug disorders: The role of the state authority. Journal of Behavioral Health Services \& Research, 36(4), 407-419.

Rieckmann, T., Fuller, B. E., Saedi, G. A., \& McCarty, D. (2010). Adoption of practice guidelines and assessment tools in substance abuse treatment. Substance Abuse Treatment, Prevention, \& Policy, 5(4), 1-9.

Ries, R. K., Fielin, D. A., Miller, S. C., \& Saitz, R. (2009). Principles of addiction medicine. Philadelphia, PA: Wolters Kluwer/Lippincott Williams \& Wilkins. 
Riggs, N. R., \& Greenberg, M. T. (2009). Neurocognition as a moderator and mediator in adolescent substance misuse prevention. American Journal on Drug \& Alcohol Abuse, 35(4), 209-213.

Rigler, S. K. (2000). Alcoholism in the elderly. [Online]. Retrieved December 7, 2011 from http://www.aafp.org.

Rigotti, N. A. (2009). The future of tobacco treatment in the health care system. Annals of Internal Medicine, 150(7), 496-497.

Rigotti, N. A., Munafo, M. R., \& Stead, L. F. (2008). Smoking cessation interventions for hospitalized smokers: A systematic review. Archives of Internal Medicine, 168(18), 1950-1960.

Rikoon, S. H., Cacciola, J. S., Carise, D., Alterman, A. I., \& McLellan, A. T. (2006). Predicting DSM-IV dependence diagnoses from Addiction Severity Index composite scores. Journal of Substance Abuse Treatment, 31(1), 17-24.

Ritchie, D., Schulz, S., \& Bryce, A. (2007). One size fits all? A process evaluation-the turn of the 'story' in smoking cessation. Public Health, 121(5), 341-348.

Rivlin, A. (2005). Views on alcoholism and treatment. [Online]. Retrieved December 12, 2011 from http://www.facesandvoicesofrecovery.org.

Roan, S. (2008). The 30-day myth. [Online]. Los Angeles Times. Retrieved March 5, 2012 from http://articles.latimes.com.

Roan, S. (2009). You can cut back on alcohol. [Online]. Los Angeles Times. Retrieved October 20, 2011 from http://www.latimes.com.

Roan, S. (2011). Federal substance-abuse agencies lumber toward a merger. [Online]. Los Angeles Times. Retrieved April 25, 2012 from http://articles.latimes.com.

Robb, N. D., \& Smith, B. G. (1996). Chronic alcoholism: An important condition in the dentist-patient relationship. Journal of Dentistry, 24(1-2), 17-24.

Robert Wood Johnson Foundation. (2001). Substance abuse: The nation's number one health problem: Key indicators for policy update. Princeton, NJ: Author.

Robert Wood Johnson Foundation. (2005). National drug treatment quality improvement program adds 13 new sites: Learning collaborative works to improve success rates in treating addictions. [Online]. Retrieved November 1, 2007 from http://www.rwjf.org.

Robinson, G., Kaye, N., Bergman, D., Moreaux, M., \& Baxter, C. (2005). State profiles of mental health and substance abuse services in Medicaid. Washington, DC: U.S. Department of Health and Human Services, Substance Abuse and Mental Health Services Administration, Center for Mental Health Services.

Robison, A. J., \& Nestler, E. J. (2011). Transcriptional and epigenetic mechanisms of addiction. Nature Reviews Neuroscience, 12(11), 623-637.

Rochon, P. A., \& Gurwitz, J. H. (1997). Optimising drug treatment for elderly people: The prescribing cascade. British Medical Journal, 315(7115), 1096-1099.

Rockett, I. R., Putnam, S. L., Jia, H., Chang, C. F., \& Smith, G. S. (2005). Unmet substance abuse treatment need, health services utilization, and cost: A population-based emergency department study. Annals of Emergency Medicine, 45(2), 118-127.

Roddy, E. (2004). Bupropion and other non-nicotine pharmacotherapies. British Medical Journal, $328(7438), 509-511$. 
Rogosch, F. A., Oshri, A., \& Cicchetti, D. (2010). From child maltreatment to adolescent cannabis abuse and dependence: A developmental cascade model. Developmental \& Psychopathology, 22(4), 883 897.

Rogowski, J. A. (1992). Insurance coverage for drug abuse. Health Affairs, 11(3), 137-148.

Rohde, P., Kahler, C. W., Lewinsohn, P. M., \& Brown, R. A. (2004). Psychiatric disorders, familial factors, and cigarette smoking: II. Associations with progression to daily smoking. Nicotine \& Tobacco Research, 6(1), 119-132.

Rohde, P., Lewinsohn, P. M., Kahler, C. W., Seeley, J. R., \& Brown, R. A. (2001). Natural course of alcohol use disorders from adolescence to young adulthood. Journal of the American Academy of Child \& Adolescent Psychiatry, 40(1), 83-90.

Rohsenow, D. J., Colby, S. M., Monti, P. M., Swift, R. M., Martin, R. A., Mueller, T. I., et al. (2000). Predictors of compliance with naltrexone among alcoholics. Alcoholism: Clinical \& Experimental Research, 24(10), 1542-1549.

Rohsenow, D. J., Monti, P. M., Colby, S. M., \& Martin, R. A. (2002). Brief interventions for smoking cessation in alcoholic smokers. Alcoholism: Clinical \& Experimental Research, 26(12), 1950-1951.

Roll, J. M. (2007). Contingency management: An evidence-based component of methamphetamine use disorder treatments. Addiction, 102(Suppl.\# 1), 114-120.

Roman, P. M., Abraham, A. J., \& Knudsen, H. K. (2011). Using medication-assisted treatment for substance use disorders: Evidence of barriers and facilitators of implementation. Addictive Behaviors, $36(6), 584-589$.

Roozen, H. G., Boulogne, J. J., van Tulder, M. W., van den Brink, W., De Jong, C. A. J., \& Kerkhof, A. J. F. M. (2004). A systematic review of the effectiveness of the community reinforcement approach in alcohol, cocaine and opioid addiction. Drug \& Alcohol Dependence, 74(1), 1-13.

Rosack, J. (2004). NIDA, APA collaborate on substance abuse series. [Online]. Retrieved December 2, 2011 from http://pnhw.psychiatryonline.org.

Rose, R. J. (1998). A developmental behavior-genetic perspective on alcoholism risk. Alcohol Health \& Research World, 22(2), 131-143.

Rosenthal, T. C. (2008). The medical home: Growing evidence to support a new approach to primary care. Journal of the American Board of Family Medicine, 21(5), 427-440.

Ross, H. E., Gavin, D. R., \& Skinner, H. A. (1990). Diagnostic validity of the MAST and the alcohol dependence scale in the assessment of DSM-III alcohol disorders. Journal of Studies on Alcohol, 51(6), 506-513.

Ross, H. E., Swinson, R., Doumani, S., \& Larkin, E. J. (1995). Diagnosing comorbidity in substance abusers: A comparison of the test-retest reliability of two interviews. American Journal on Drug \& Alcohol Abuse, 21(2), 167-185.

Ross, S. (2003). Identifying an impaired physician. [Online]. Retrieved September 8, 2008 from http://virtualmentor.ama-assn.org.

Rost, K., Fortney, J., Zhang, M., Smith, J., \& Smith, G. R., Jr. (1999). Treatment of depression in rural Arkansas: Policy implications for improving care. Journal of Rural Health, 15(3), 308-315.

Rothman, E. F., Exner, D., \& Baughman, A. (2011). The prevalence of sexual assault against people who identify as gay, lesbian or bisexual in the United States: A systematic review. Trauma, Violence \& Abuse, 12(2), 55-66. 
Rounsaville, B. J. (2004). Treatment of cocaine dependence and depression. Biological Psychiatry, 56(10), 803-809.

Rounsaville, B. J., Kosten, T., \& Kleber, H. (1985). Success and failure at outpatient opioid detoxification. Evaluating the process of clonidine- and methadone-assisted withdrawal. Journal of Nervous \& Mental Disease, 173(2), 103-110.

Rowe, C. L., \& Liddle, H. A. (2003). Substance abuse. Journal of Marital \& Family Therapy, 29(1), $97-$ 120.

Rowe, C. L., Liddle, H. A., Greenbaum, P. E., \& Henderson, C. E. (2004). Impact of psychiatric comorbidity on treatment of adolescent drug abusers. Journal of Substance Abuse Treatment, 26(2), 129-140.

Roy, A. K., \& Miller, M. M. (2012). The medicalization of addiction treatment professionals. Journal of Psychoactive Drugs, 44(2), 1-12.

Roy, K., \& Miller, M. (2010). Parity and the medicalization of addiction treatment. Journal of Psychoactive Drugs, 42(2), 115-121.

RTI International. (2009). 2008 Department of Defense survey of health related behaviors among active duty military personnel. A component of the Defense Lifestyle Assessment Program (DLAP). Ann Arbor, MI: Author.

Rubinstein, M. L., Benowitz, N. L., Auerback, G. M., \& Moscicki, A. B. (2009). Withdrawal in adolescent light smokers following 24-hour abstinence. Nicotine \& Tobacco Research, 11(2), 185189.

Rubinstein, M. L., Luks, T. L., Moscicki, A. B., Dryden, W., Rait, M. A., \& Simpson, G. V. (2011). Smoking-related cue-induced brain activation in adolescent light smokers. Journal of Adolescent Health, 48(1), 7-12.

Rubinstein, M. L., Thompson, P. J., Benowitz, N. L., Shiffman, S., \& Moscicki, A.-B. (2007). Cotinine levels in relation to smoking behavior and addiction in young adolescent smokers. Nicotine \& Tobacco Research, 9(1), 129-135.

Rubio, G., Ponce, G., Rodriguez-Jiminez, R., Jimenez-Arriero, A., Hoenicka, J., \& Palomo, T. (2005). Clinical predictors of response to naltrexone in alcoholic patients: Who benefits most from treatment with naltrexone? Alcohol \& Alcoholism, 40(3), 227-2333.

Ruiz v. Estelle, 503 F. Supp. 1265 (S.D. Tex. 1980).

Ruiz, P. \& Langrod, J. G. (2009). Hispanic Americans. In J. Lowinson, P. Ruiz, R. B. Millman, \& J. G. Langrod (Eds.), Substance abuse: A comprehensive textbook (pp. 1103-1112). Baltimore, MD: Williams and Wilkins.

Ruiz, P., Varner, R. V., Small, D. R., \& Johnson, B. A. (1999). Ethnic differences in the neuroleptic treatment of schizophrenia. Psychiatric Quarterly, 70(2), 163-172.

Rumpf, H. J., Hapke, U., Hill, A., \& John, U. (1997). Development of a screening questionnaire for the general hospital and general practices. Alcoholism: Clinical \& Experimental Research, 21(5), 894898.

Russell, C., Davies, J. B., \& Hunter, S. C. (2011). Predictors of addiction treatment providers' beliefs in the disease and choice models of addiction. Journal of Substance Abuse \& Treatment, 40(2), 150-164.

Russell, L. B. (2007). Prevention's potential for slowing the growth of medical spending. Washington, DC: Rutgers University, Institute of Health, National Coalition on Health Care. 
Russell, L. B., Gold, M. R., Siegel, J. E., Daniels, N., \& Weinstein, M. C. (1996). The role of costeffectiveness analysis in health and medicine. JAMA, 276(14), 1172-1177.

Russell, M. (2004). Screening in general health care. Alcohol Research \& Health, 28(1), 17-22.

Ryan, J. P. (2006). Illinois Alcohol and Other Drug Abuse (AODA) waiver demonstration: Final evaluation report. IL: The State of Illinois, Illinois Department of Children and Family Services.

Ryan, K. K., Garrett-Mayer, E., Alberg, A. J., Cartmell, K. B., \& Carpenter, M. J. (2011). Predictors of cessation pharmacotherapy use among Black and non-Hispanic White smokers. Nicotine \& Tobacco Research, 13(8), 646-652.

Ryan, P., \& Morgan, S. (2004). Assertive outreach: A strengths approach to policy and practice. New York: Churchill Livingstone.

Saccone, S. F., Hinrichs, A. L., Saccone, N. L., Chase, G. A., Konvicka, K., Madden, P. A., et al. (2007). Cholinergic nicotinic receptor genes implicated in a nicotine dependence association study targeting 348 candidate genes with 3713 SNPs. Human Molecular Genetics, 16(1), 36-49.

Sacks, J. Y., McKendrick, K., \& Banks, S. (2008). The impact of early trauma and abuse on residential substance abuse treatment outcomes for women. Journal of Substance Abuse Treatment, 34(1), 90100.

Sadovsky, R. (2000). Tips from other journals: Public health issue: Methadone maintenance therapy. [Online]. Retrieved January 4, 2012 Retrieved from the World Wide Web http://www.aafp.org.

Saitz, R. (1998). Introduction to alcohol withdrawal. Alcohol Health \& Research World, 22(1), 5-12.

Saitz, R. (2010). Candidate performance measures for screening for, assessing, and treating unhealthy substance use in hospitals: Advocacy or evidence-based practice? Annals of Internal Medicine, $153(1), 40-43$.

Saitz, R., Sullivan, L. M., \& Samet, J. H. (2000). Training community-based clinicians in screening and brief intervention for substance abuse problems: Translating evidence into practice. Substance Abuse, 21(1), 21-31.

Salloum, I. M., Cornelius, J. R., Daley, D. C., Kirisci, L., Himmelhoch, J. M., \& Thase, M. E. (2005). Efficacy of valproate maintenance in patients with bipolar disorder and alcoholism: A double-blind placebo-controlled study. Archives of General Psychiatry, 62(1), 37-45.

Salsitz, E., Joseph, H., Blanche, F., Perez, J., Richman, B. L., Salomon, N., et al. (2000). Methadone Medical Maintenance (MMM): Treating chronic opioid dependence in private medical practice: A summary report (1983-1998). Mount Sinai Journal of Medicine, 67(5 and 6), 388-451.

Saltstone, R., Halliwell, S., \& Hayslip, M. A. (1994). A multivariate evaluation of the Michigan Alcoholism Screening Test and the Drug Abuse Screening Test in a female offender population. Addictive Behaviors, 19(5), 455-462.

Salyers, K. M., Ritchie, M. H., Cochrane, W. S., \& Roseman, C. P. (2006). Inclusion of substance abuse training in CACREP-accredited programs. Journal of Addictions \& Offender Counseling, 27(1), 4758.

Samet, S., Waxman, R., Hatzenbuehler, M., \& Hasin, D. S. (2007). Assessing addiction: Concepts and instruments. Addiction Science \& Clinical Practice, 4(1), 19-31.

SAMHSA's National Registry of Evidence-based Programs and Practices. (2008). Multidimensional Family Therapy (MDFT). [Online]. Retrieved December 21, 2011 Retrieved from the World Wide Web http://nrepp.samhsa.gov. 
SAMHSA's National Registry of Evidence-based Programs and Practices. (2007a). Motivational Enhancement Therapy (MET). [Online]. Retrieved January 12, 2012 from http://nrepp.samhsa.gov.

SAMHSA's National Registry of Evidence-based Programs and Practices. (2007b). National Registry of Evidence-Based Programs and Practices (NREPP). [Online]. Review of this intervention was funded by the Center for Substance Abuse Treatment. Retrieved January 12, 2012 from http://nrepp.samhsa.gov.

Sampl, S., Kadden, R., \& Center for Substance Abuse Treatment. (2001). Motivational enhancement therapy and cognitive behavioral therapy for adolescent cannabis users: 5 sessions (DHHS (SMA) 01-3486). Rockville, MD: U.S. Department of Health and Human Services; Substance Abuse and Mental Health Services Administration; Center for Substance Abuse Treatment.

Sandlow, L. J., \& Dos Santos, S. R. (1997). Addiction medicine and continuing medical education. Journal of Psychoactive Drugs, 29(3), 275-284.

Santiago, P. N., Wilk, J. E., Milliken, C. S., Castro, C. A., Engel, C. C., \& Hoge, C. W. (2010). Screening for alcohol misuse and alcohol-related behaviors among combat veterans. Psychiatric Services, 61(6), $575-581$.

Santolaria, F., Perez-Manzano, J. L., Milena, A., Gonzalez-Reimers, E., Gomez-Rodriguez, M. A., Martinez-Riera, A., et al. (2000). Nutritional assessment in alcoholic patients. Its relationship with alcoholic intake, feeding habits, organic complications and social problems. Drug \& Alcohol Dependence, 59(3), 295-304.

Santora, P. B., \& Hutton, H. E. (2008). Longitudinal trends in hospital admissions with co-occurring alcohol/drug diagnoses, 1994-2002. Journal of Substance Abuse Treatment, 35(1), 1-12.

Sarata, A. K. (2011). Mental health parity and the Patient Protection and Affordable Care Act of 2010. [Online]. Retrieved March 12, 2012 from http://www.ncsl.org.

Sargent, J. D., Beach, M. L., Adachi-Mejia, A. M., Gibson, J. J., Titus-Ernstoff, L. T., Carusi, C. P., et al. (2005). Exposure to movie smoking: Its relation to smoking initiation among US adolescents. Pediatrics, 116(5), 1183-1191.

Sarna, L., Bialous, S. A., Rice, V. H., \& Wewers, M. E. (2009). Promoting tobacco dependence treatment in nursing education. Drug \& Alcohol Review, 28(5), 507-516.

Sarna, L., Bialous, S. A., Wells, M., Kotlerman, J., Wewers, M. E., \& Froelicher, E. S. (2009). Frequency of nurses' smoking cessation interventions: Report from a national survey. Journal of Clinical Nursing, 18(14), 2066-2077.

Sass, H., Soyka, M., Mann, K., \& Zieglgansberger, W. (1996). Relapse prevention by acamprosate: Results from a placebo-controlled study on alcohol dependence. Archives of General Psychiatry, 53(8), 673-680.

Satel, S. L. (1999). What should we expect from drug abusers? Psychiatric Services, 50(7), 861-861.

Satel, S. L. (2007). The human factor. [Online]. The American. Retrieved November 2, 2007 from http://www.american.com.

Satre, D. D., Chi, F. W., Eisendrath, S., \& Weisner, C. (2011). Subdiagnostic alcohol use by depressed men and women seeking outpatient psychiatric services: Consumption patterns and motivation to reduce drinking. Alcoholism: Clinical \& Experimental Research, 35(4), 695-702.

Satre, D., Wolfe, W., Eisendrath, S., \& Weisner, C. (2008). Computerized screening for alcohol and drug use among adults seeking outpatient psychiatric services. Psychiatric Services, 59(4), 441-444. 
Saunders, J. B., Aasland, O. G., Babor, T. F., de la Fuente, J. R., \& Grant, M. (1993). Development of the Alcohol Use Disorders Identification Test (AUDIT): WHO Collaborative Project on Early Detection of Persons with Harmful Alcohol Consumption--II. Addiction, 88(6), 791-804.

Saunders, S. M., Zygowicz, K. M., \& D'Angelo, B. R. (2006). Person-related and treatment-related barriers to alcohol treatment. Journal of Substance Abuse Treatment, 30(3), 261-270.

Savage, S. R. (2002). Assessment for addiction in pain-treatment settings. Clinical Journal of Pain, 18(Suppl. 4), S28-S38.

Savage, S. R., Joranson, D. E., Covington, E. C., Schnoll, S. H., Heit, H. A., \& Gilson, A. M. (2003). Definitions related to the medical use of opioids: Evolution towards universal agreement. Journal of Pain \& Symptom Management, 26(1), 655-667.

Saxon, A. J., Baer, J. S., Davis, T. M., Sloan, K. L., Malte, C. A., Fitzgibbons, K., et al. (2003). Smoking cessation treatment among dually diagnosed individuals: Preliminary evaluation of different pharmacotherapies. Nicotine \& Tobacco Research, 5(4), 589-596.

Schaefer, J. A., Cronkite, R. C., \& Hu, K. U. (2011). Differential relationships between continuity of care practices, engagement in continuing care, and abstinence among subgroups of patients with substance use and psychiatric disorders. Journal of Studies on Alcohol \& Drugs, 72(4), 611-621.

Schaeffer, C. M., \& Borduin, C. M. (2005). Long-term follow-up to a randomized clinical trial of multisystemic therapy with serious and violent juvenile offenders. Journal of Consulting \& Clinical Psychology, 73(3), 445-453.

Schaus, J. F., Sole, M. L., McCoy, T. P., Mullett, N., \& O'Brien, M. C. (2009). Alcohol screening and brief intervention in a college student health center: A randomized controlled trial. Journal of Studies on Alcohol \& Drugs, (Suppl. 16), 131-141.

Scheinholtz, M. K. (2011). Implementation of evidence-based practices: SAMHSA's older adults targeted capacity expansion grant program. Journal of the American Society on Aging, 34(1), 26-35.

Schendel, D. E., Stockbauer, J. W., Hoffman, H. J., Herman, A. A., Berg, C. J., \& Schramm, W. F. (1997). Relation between very low birth weight and developmental delay among preschool children without disabilities. American Journal of Epidemiology, 146(9), 740-749.

Schermer, C. R., Moyers, T. B., Miller, W. R., \& Bloomfield, L. A. (2006). Trauma center brief interventions for alcohol disorders decrease subsequent driving under the influence arrests. Journal of Trauma, 60(1), 29-34.

Schiff, E. R. (1997). Hepatitis C and alcohol. Hepatology, 26(3 Suppl. 1), 39S-42S.

Schmidt, L. A., \& Mulia, N. (2009). Racial/ethnic disparities in AOD treatment knowledge asset: Key results: Racial and ethnic disparities in substance abuse treatment. [Online]. Retrieved March 9, 2012 from http://saprp.org.

Schmidt, L., Tam, T., \& Larson, M. J. (2007). Understanding the substance abuse treatment gap. Washington, DC: U.S. Department of Health and Human Services, Substance Abuse and Mental Health Services Administration, Center for Substance Abuse Treatment.

Schmidt, L., Ye, Y., Greenfield, T., \& Bond, J. C. (2007). Ethnic disparities in clinical severity and services for alcohol problems: Results from the National Alcohol Survey. Alcoholism: Clinical \& Experimental Research, 31(1), 48-56.

Schnoll, R. A., Patterson, F., Wileyto, E. P., Heitjan, D. F., Shields, A. E., Asch, D. A., et al. (2010). Effectiveness of extended-duration transdermal nicotine therapy: A randomized trial. Annals of Internal Medicine, 152(3), 144-151. 
Schober, R., \& Annis, H. M. (1996). Barriers to help-seeking for change in drinking: A gender-focused review of the literature. Addictive Behaviors, 21(1), 81-92.

Schoen, C., Davis, K., Collins, K. S., Greenberg, L., Des Roches, C., \& Abrams, M. (1997). The Commonweatlth Fund Survey of the Health of Adolescent Girls Washington, DC: Commonwelath Fund's Commission on Women's Health.

Schoen, C., Doty, M. M., Robertson, R. H., \& Collins, S. R. (2011). Affordable Care Act reforms could reduce the number of underinsured US adults by 70 percent. Health Affairs, 30(9), 1762-1771.

Schoenwald, S. K., Ward, D. M., Henggeler, S. W., Pickrel, S. G., \& Patel, H. (1996). Multisystemic therapy treatment of substance abusing or dependent adolescent offenders: Costs of reducing incarceration, inpatient, and residential placement. Journal of Child \& Family Studies, 5(4), 431-444.

Schonfeld, L., King-Kallimanis, B. L., Duchene, D. M., Etheridge, R. L., Herrera, J. R., Barry, K. L., et al. (2010). Screening and brief intervention for substance misuse among older adults: The Florida BRITE project. American Journal of Public Health, 100(1), 108-114.

Schottenfeld, R. S., Pakes, J., O'Connor, P., Chawarski, M., Oliveto, A., \& Kosten, T. R. (2000). Thriceweekly versus daily buprenorphine maintenance. Biological Psychiatry, 47(12), 1072-1079.

Schramm-Sapyta, N. L., Walker, Q. D., Caster, J. M., Levin, E. D., \& Kuhn, C. M. (2009). Are adolescents more vulnerable to drug addiction than adults? Evidence from animal models. Psychopharmacology, 206(1), 1-21.

Schroeder, S. A. (2005). What to do with a patient who smokes. JAMA, 294(4), 482-487.

Schroeder, S. A., \& Morris, C. D. (2010). Confronting a neglected epidemic: Tobacco cessation for persons with mental illnesses and substance abuse problems. Annual Review of Public Health, 31, 297-314.

Schuckit, M. A. (2009). Alcohol-use disorders. Lancet, 373(9662), 492-501.

Schuckit, M. A., Smith, T. L., Trim, R. S., Allen, R. C., Fukukura, T., Knight, E. E., et al. (2011). A prospective evaluation of how a low level of response to alcohol predicts later heavy drinking and alcohol problems. American Journal of Drug \& Alcohol Abuse, 37(6), 479-486.

Schuckit, M. A., Tapert, S., Matthews, S. C., Paulus, M. P., Tolentino, N. J., Smith, T. L., et al. (2011). fMRI differences between subjects with low and high responses to alcohol during a stop signal task. Alcoholism: Clinical \& Experimental Research, 36(1), 130-140.

Sciubba, J. J. (2001). Oral cancer. The importance of early diagnosis and treatment. American Journal of Clinical Dermatology, 2(4), 239-251.

Scott, C. K., \& Dennis, M. L. (2009). Results from two randomized clinical trials evaluating the impact of quarterly recovery management checkups with adult chronic substance users. Addiction, 104(6), 959971.

Scott, C. K., Dennis, M. L., \& Foss, M. A. (2005). Utilizing recovery management checkups to shorten the cycle of relapse, treatment reentry, and recovery. Drug \& Alcohol Dependence, 78(3), 325-338.

Seal, K. H., Cohen, G., Waldrop, A., Cohen, B. E., Maguen, S., \& Ren, L. (2011). Substance use disorders in Iraq and Afghanistan veterans in VA healthcare, 2001-2010: Implications for screening, diagnosis and treatment. Drug \& Alcohol Dependence, 116(1-3), 93-101.

Segal, N. L. (1993). Twin, sibling and adoption methods. Tests of evolutionary hypotheses. American Psychologist, 48(9), 943-956.

Selzer, M. L. (1971). The Michigan Alcoholism Screening Test: The quest for a new diagnostic instrument. American Journal of Psychiatry, 127(12), 1653-1658. 
Semple, S. J., Grant, I., \& Patterson, T. L. (2005). Utilization of treatment programs by methamphetamine users: The role of social stigma. American Journal of Addictions, 14(4), 367-380.

Senreich, E. (2010). Are specialized LGBT program components helpful for gay and bisexual men in substance abuse treatment? Substance Use and Misuse, 45(7-8), 1077-1096.

Sex Addicts Anonymous. (2012). The twelve steps. [Online]. Retrieved January 11, 2012 Retrieved from the World Wide Web http://saa-recovery.org.

Sexton, T. L., \& Alexander, J. F. (2000). Juvenile justice bulletin: Functional family therapy (NCJ 184743). Washington, DC: U.S. Department of Justice, Office of Justice Programs, Office of Juvenile Justice and Delinquency Prevention.

Shaffer, H. J., LaPlante, D. A., LaBrie, R. A., Kidman, R. C., Donato, A. N., \& Stanton, M. V. (2004). Toward a syndrome of model addiction: Multiple expressions, common etiology. Harvard Review of Psychiatry, 12(6), 367-374.

Shaham, Y., \& Hope, B. T. (2005). The role of neuroadaptations in relapse to drug seeking. Nature Neuroscience, 8(11), 1437-1439.

Shaw, B. A., Agahi, N., \& Krause, N. (2011). Are changes in financial strain associated with changes in alcohol use and smoking among older adults? Journal of Studies on Alcohol \& Drugs, 72(6), 917 925.

Shaw, J. (2011). Alcohol addiction and dental treatment. [Online]. Retrieved June 30, 2011 from http://www.livestrong.com.

Sheffer, C. E., Barone, C. P., \& Anders, M. E. (2009). Training health care providers in the treatment of tobacco use and dependence: Pre- and post-training results. Journal of Evaluation in Clinical Practice, 15(4), 607-613.

Sheffer, C. E., Barone, C., \& Anders, M. E. (2010). Training nurses in the treatment of tobacco use and dependence: Pre- and post-training results. Journal of Advanced Nursing, 67(1), 176-183.

Shen, X., Orson, F. M., \& Kosten, T. R. (2011). Anti-addiction vaccines. F1000. Medicine Report, 3, 20. doi:10.3410-m3-20.

Shen, Y. (2003). Selection incentives in a performance-based contracting system. Health Services Research, 38(2), 535-552.

Shen, Y. C., Arkes, J., \& Williams, T. V. (2012). Effects of Iraq/Afghanistan deployments on major depression and substance use disorder: Analysis of active duty personnel in the U.S. military. American Journal of Public Health, 102(S1), S80-S87.

Sherman, C. (2007). Impacts of drugs on neurotransmission. NIDA Notes, 21(4).

Shiffman, S., \& Sayette, M. A. (2005). Validation of the nicotine dependence syndrome scale (NDSS): A criterion-group design contrasting chippers and regular smokers. Drug \& Alcohol Dependence, 79(1), 45-52.

Shiffman, S., Ferguson, S. G., Rohay, J., \& Gitchell, J. G. (2008). Perceived safety and efficacy of nicotine replacement therapies among US smokers and ex-smokers: Relationship with use and compliance. Addiction, 103(8), 1371-1378.

Shiffman, S., Johnston, J. A., Khayrallah, M., Elash, C. A., Gwaltney, C. J., Paty, J. A., et al. (2000). The effect of buproprion on nicotine craving and withdrawal. Psychopharmacology, 148(1), 33-40.

Shiffman, S., Khayrallah, M., \& Nowak, R. (2000). Efficacy of the nicotine patch for relief of craving and withdrawal 7-10 weeks after cessation. Nicotine \& Tobacco Research, 2(4), 371-378. 
Shin, S. H., Hong, H. G., \& Hazen, A. L. (2010). Childhood sexual abuse and adolescent substance use: A latent class analysis. Drug \& Alcohol Dependence, 109(1-3), 226-235.

Shinn, A. K., \& Greenfield, S. F. (2010). Topiramate in the treatment of substance-related disorders: A critical review of the literature. Journal of Clinical Psychiatry, 71(5), 634-648.

Shoptaw, S. J., Kao, U., Heinzerling, K., \& Ling, W. (2009). Treatment for amphetamine withdrawal. Cochrane Database of Systematic Reviews, (4), CD003021.

Shoptaw, S., Rotheram-Fuller, E., Yang, X., Frosch, D., Nahom, D., Jarvik, M. E., et al. (2002). Smoking cessation in methadone maintenance. Addiction, 97(10), 1317-1328.

Shore, J. H. (1987). The Oregon experience with impaired physicians on probation. An eight-year followup. JAMA, 257(21), 2931-2934.

Shorter, D., \& Kosten, T. R. (2011). Vaccines in the treatment of substance abuse. FOCUS: Journal of Lifelong Learning in Psychiatry, 9(1), 25-29.

Siegal, H. A., Fisher, J. H., Rapp, R. C., Kelliher, C. W., Wagner, J. H., O'Brien, W. F., et al. (1996). Enhancing substance abuse treatment with case management. Its impact on employment. Journal of Substance Abuse Treatment, 13(2), 93-98.

Siegal, H. A., Rapp, R. C., Li, L., \& Saha, P. (2001). Case management in substance abuse treatment: Perspectives, impact, and use. In F. M. Tims, C. G. Leukefeld, \& J. J. Platt (Eds.), Relapse and recovery in addictions (pp. 253-274). New Haven, CT: Yale University Press.

Sigmon, S. C., Dunn, K. E., Badger, G. J., Heil, S. H., \& Higgins, S. T. (2009). Brief buprenorphine detoxification for the treatment of prescription opioid dependence: A pilot study. Addictive Behaviors, 34(3), 304-311.

Silverman, K., Wong, C. J., Higgins, S. T., Brooner, R. K., Montoya, I. D., Contoreggi, C., et al. (1996). Increasing opiate abstinence through voucher-based reinforcement therapy. Drug \& Alcohol Dependence, 41(2), 157-165.

Simons-Morton, B., \& Chen, R. S. (2006). Over time relationships between early adolescent and peer substance use. Addictive Behaviors, 31(7), 1211-1223.

Simoyan, O., Badner, V. M., \& Freeman, K. D. (2002). Tobacco cessation services in dental offices. Are we doing all we can? New York State Dental Journal, 68(7), 34-40.

Simpson, D. D., Joe, G. W., \& Brown, B. S. (1997). Treatment retention and follow-up outcomes in the Drug Abuse Treatment Outcome Study (DATOS). Psychology of Addictive Behaviors, 11(4), 294307.

Singh, G. K., \& Yu, S. M. (1995). Infant mortality in the United States: Trends, differentials, and projections 1950 through 2010. American Journal of Public Health, 85(7), 957-964.

Singh, V. N. (1992). A current perspective on nutrition and exercise. Journal of Nutrition, 122, 760-765.

Siru, R., Hulse, G. K., \& Tait, R. J. (2009). Assessing motivation to quit smoking in people with mental illness: A review. Addiction, 104(5), 719-733.

Skeer, M., McCormick, M. C., Normand, S. L., Buka, S. L., \& Gilman, S. E. (2009). A prospective study of familial conflict, psychological stress, and the development of substance use disorders in adolescence. Drug \& Alcohol Dependence, 104(1-2), 65-72.

Skinner, H. A. (1982). The drug abuse screening test. Addictive Behaviors, 7(4), 363-371. 
Skinner, H. A. (1993). Detailed review of the Alcohol Dependence Scale (ADS). In Addiction Research Foundation (Ed.), A directory of client outcome measures for addictions treatment programs (pp. 7278). Toronto: Addiction Research Foundation.

Skipper, G. E. (1997). Treating the chemically dependent health professional. Journal of Addictive Diseases, 16(3), 67-73.

Sleiman, M., Gundel, L. A., Pankow, J. F., Jacob, P., III, Singer, B. C., \& Destaillats, H. (2010). Formation of carcinogens indoors by surface-mediated reactions of nicotine with nitrous acid, leading to potential thirdhand smoke hazards. Proceedings of the National Academy of Sciences of the United States of America, 107(15), 6576-6581.

Slotkin, T. A. (2002). Nicotine and the adolescent brain: Insights from an animal model. Neurotoxicology \& Teratology, 24(3), 369-384.

SMART Recovery. (2011). Frequently asked questions about SMART Recovery. [Online]. Retrieved November 15, 2011 from http://www.smartrecovery.org.

Smit, F., Bolier, L., \& Cuijpers, P. (2004). Cannabis use and the risk of later schizophrenia: A review. Addiction, 99(4), 425-430.

Smith, A. D. (2005). Medicaid EPSDT and AOD treatment services. [Online]. Retrieved March 7, 2012 from http://www.rwjf.org.

Smith, B., Ryan, M. A., Wingard, D. L., Patterson, T. L., Slymen, D. J., \& Macera, C. A. (2008). Cigarette smoking and military deployment: A prospective evaluation. American Journal of Preventive Medicine, 35(6), 539-546.

Smith, J. A., Wolford, J. A., Weber, M., \& McLean, D. (1957). Use of citrated calcium carbimide (temposil) in treatment of chronic alcoholism. Journal of American Medical Association, 165(17), 2181-2183.

Smith, J. E., Meyers, R. J., \& Miller, W. R. (2001). The community reinforcement approach to the treatment of substance use disorders. American Journal on Addictions, 10(Suppl.), 51-59.

Smith, M. J. W., Whitaker, T., \& Weismiller, T. (2006). Social workers in the substance abuse treatment field: A snapshot of service activities. Health \& Social Work, 31(2), 109-115.

Smith, P. C., Schmidt, S. M., Allensworth-Davies, D., \& Saitz, R. (2010). A single-question screening test for drug use in primary care. Archives of Internal Medicine, 170(13), 1155-1160.

Smith, S. M., Stinson, F. S., Dawson, D. A., Goldstein, R., Huang, B., \& Grant, B. F. (2006). Race/ethnic differences in the prevalence and co-occurrence of substance use disorders and independent mood and anxiety disorders: Results from the National Epidemiologic Survey on Alcohol and Related Conditions. Psychology Medicine, 36(7), 987-998.

Smith, S. S., McCarthy, D. E., Japuntich, S. J., Christiansen, B., Piper, M. E., Jorenby, D. E., et al. (2009). Comparative effectiveness of 5 smoking cessation pharmacotherapies in primary care clinics. Archives of Internal Medicine, 169(22), 2148-2155.

Smith, V. K., Gifford, K., \& Ellis, E. (2011). Moving ahead amid fiscal challenges: A look at Medicaid spending, coverage and policy trends: Results from a 50-state Medicaid budget survey for state fiscal years 2011 and 2012. [Online]. Retrieved March 12, 2012 from http://www.kff.org.

Smithedajkul, P. Y., \& Cullen, M. W. (2009). Managing acute opiate withdrawal in hospitalized patients. [Online]. Retrieved June 5, 2012 from http://www.acphospitalist.org.

SmokeFree.gov. (2012a). Clonidine fact sheet. [Online]. Retrieved April 3, 2012 from http://www.smokefree.gov. 
SmokeFree.gov. (2012b). Nortriptyline fact sheet. [Online]. Retrieved April 3, 2012 from http://www.smokefree.gov.

Snyder, L. B., Milici, F. F., Slater, M., Sun, H., \& Strizhakova, Y. (2006). Effects of alcohol advertising exposure on drinking among youth. Archives of Pediatrics \& Adolescent Medicine, 160(1), 18-24.

Sobel, B.-F. X., Sigmon, S. C., Walsh, S. L., Johnson, R. E., Liebson, I. A., Nuwayser, E. S., et al. (2004). Open-label trial of an injection depot formulation of buprenorphine in opioid detoxification. Drug \& Alcohol Dependence, 73(1), 11-22.

Sobell, L. C., Cunningham, J. A., \& Sobell, M. B. (1996). Recovery from alcohol problems with and without treatment: Prevalence in two population surveys. American Journal of Public Health, 86(7), 966-972.

Sobell, L. C., Sobell, M. B., Toneatto, T., \& Leo, G. I. (1993). What triggers the resolution of alcohol problems without treatment? Alcoholism: Clinical \& Experimental Research, 17(2), 217-224.

Social Security Act, 42 U.S.C. $§ 1814$ (2012).

Social Security Act, 42 U.S.C. § 1861 (2012).

Social Security Act, 42 U.S.C. § 1937 (2012).

Social Security Act, 42 U.S.C. $\$ 1395 y(a)(1)$ (2012).

Social Security Online. (2011). Compilation of the social security laws: Exclusions from coverage and Medicare as secondary payer. [Online]. Retrieved February 1, 2012 from http://www.ssa.gov.

Society for Human Resource Management. (2011). SHRM research spotlight: Wellness in the workplace. [Online]. Retrieved December 19, 2011 from http://www.shrm.org.

Soderstrom, C. A., Smith, G. S., Dischinger, P. C., McDuff, D. R., Hebel, J. R., Gorelick, D. A., et al. (1997). Psychoactive substance use disorders among seriously injured trauma center patients. JAMA, 277(22), 1769-1774.

Sokol, R. J., Martier, S. S., \& Ager, J. W. (1989). The T-ACE questions: Practical prenatal detection of risk-drinking. American Journal of Obstetrics \& Gynecology, 160(4), 863-868.

Solberg, L. I., Maciosek, M. V., \& Edwards, N. M. (2008). Primary care intervention to reduce alcohol misuse ranking its health impact and cost effectiveness. American Journal of Preventive Medicine, $34(2), 143-152$.

Solis, H. L. (2012). Compliance of group health plans (and health insurance coverage in connection with such plans) with the requirements of the Mental Health Parity and Addiction Equity Act of 2008. [Online]. Retrieved March 15, 2012 from http://www.dol.gov.

Song, A. V., Morrell, H. E., Cornell, J. L., Ramos, M. E., Biehl, M., Kropp, R. Y., et al. (2009). Perceptions of smoking-related risks and benefits as predictors of adolescent smoking initiation. American Journal of Public Health, 99(3), 487-492.

Sontag, S. (1990). Illness as metaphor and AIDS and its metaphors. New York: Picador: Farrar, Straus and Giroux.

Sosin, M. R., \& Durkin, E. (2007). Perceptions about services and dropout from a substance abuse case management program. Journal of Community Psychology, 35(5), 583-602.

Spanagel, R., \& Heilig, M. (2005). Addiction and its brain science. Addiction, 100(12), 1813-1822.

Spangler, J. G., George, G., Foley, K. L., \& Crandall, S. J. (2002). Tobacco intervention training: Current efforts and gaps in US medical schools. JAMA, 288(9), 1102-1109. 
Spear, S. E., Brown, A. H., \& Rawson, R. A. (2005). "Painting a picture of the client": Implementing the Addiction Severity Index in community treatment programs. Journal of Substance Abuse Treatment, 29(4), 277-282.

Spear, S., Tillman, S., Moss, C., Gong-Guy, E., Ransom, L., \& Rawson, R. A. (2009). Another way of talking about substance abuse: Substance abuse screening and brief intervention in a mental health clinic. Journal of Human Behavior in the Social Environment, 19(8), 959-977.

Spence, R. T., DiNitto, D. M., \& Straussner, S. L. A.(Eds.). (2001). Neurobiology of addictions: Implications for clinical practice. New York: NY: Haworth Press.

Spicer, J. (1993). The Minnesota Model: The evolution of the multidisciplinary approach to addiction recovery. Center City, MN: Hazelden.

Spillane, J. F. (2000). Cocaine: From medical marvel to modern menace in the United States, 1884-1920. Baltimore, MD: Johns Hopkins University Press.

Spirito, A., Monti, P. M., Barnett, N. P., Colby, S. M., Sindelar, H., Rohsenow, D. J., et al. (2004). A randomized clinical trial of a brief motivational intervention for alcohol-positive adolescents treated in an emergency department. Journal of Pediatrics, 145(3), 396-402.

Spirito, A., Sindelar-Manning, H., Colby, S. M., Barnett, N. P., Lewander, W., Rohsenow, D. J., et al. (2011). Individual and family motivational interventions for alcohol-positive adolescents treated in an emergency department: Results of a randomized clinical trial. Archives of Pediatrics \& Adolescent Medicine, 165(3), 269-274.

Spitzer, R. L., Williams, J. B., Gibbon, M., \& First, M. B. (1992). The Structured Clinical Interview for DSM-III-R (SCID). I: History, rationale, and description. Archives of General Psychiatry, 49(8), 624629.

Spohr, H. L., Willms, J., \& Steinhausen, H. C. (1993). Prenatal alcohol exposure and long-term developmental consequences. Lancet, 341(8850), 907-910.

Springer, C. M., Niang, C. M. T., Matte, T. D., Miller, N., Bassett, M. T., \& Frieden, T. R. (2008). Do medical students know enough about smoking to help their future patients? Assessment of New York City fourth-year medical students' knowledge of tobacco cessation and treatment for nicotine addiction. Academic Medicine, 83(10), 982-989.

Squeglia, L. M., Jacobus, J., \& Tapert, S. F. (2009). The influence of substance use on adolescent brain development. Clinical Electroencephalography \& Neuroscience, 40(1), 31-38.

Squeglia, L. M., Spadoni, A. D., Infante, M. A., Myers, M. G., \& Tapert, S. F. (2009). Initiating moderate to heavy alcohol use predicts changes in neuropsychological functioning for adolescent girls and boys. Psychology of Addictive Behaviors, 23(4), 715-722.

Srisurapanont, M., Jarusuraisin, N., \& Jittiwutikan, J. (1999). Amphetamine withdrawal: II. A placebocontrolled, randomised, double-blind study of amineptine treatment. Australian \& New Zealand Journal of Psychiatry, 33(1), 94-98.

Stafford, R. S. (2008). Regulating off-label drug use--rethinking the role of the FDA. New England Journal of Medicine, 358(14), 1427-1429.

Staley, D., \& El-Guebaly, N. (1990). Psychometric properties of the Drug Abuse Screening Test in a psychiatric patient population. Addictive Behaviors, 15(3), 257-264.

Stanton, M. D., \& Shadish, W. R. (1997). Outcome, attrition, and family-couples treatment for drug abuse: A meta-analysis and review of the controlled, comparative studies. Psychological Bulletin, 122(2), 170-191. 
State of Washington. (2009). Tobacco-free facility and grounds letter. [Online]. Retrieved February 16, 2012 from http://www.clallam.net.

Stead, L. F., Bergson, G., \& Lancaster, T. (2008). Physician advice for smoking cessation. Cochrane Database System Reviews, (2), CD000165.

Stead, L. F., Perera, R., Bullen, C., Mant, D., \& Lancaster, T. (2008). Nicotine replacement therapy for smoking cessation. Cochrane Database of Systematic Reviews, (1), CD000146.

Steen, M. (1991). Historical perspectives on women and mental illness and prevention of depression in women, using a feminist framework. Issues in Mental Health Nursing, 12(4), 359-374.

Stein, B. D., Kogan, J. N., \& Sorbero, M. (2009). Substance abuse detoxification and residential treatment among Medicaid-enrolled adults: Rates and duration of subsequent treatment. Drug \& Alcohol Dependence, 104(1-2), 100-106.

Stein, M. D., Hagerty, C. E., Herman, D. S., Phipps, M. G., \& Anderson, B. J. (2011). A brief marijuana intervention for non-treatment-seeking young adult women. Journal of Substance Abuse Treatment, 40(2), 189-198.

Steinberg, L. (2007). Risk taking in adolescence. New perspectives from brain and behavioral science. Current Directions in Psychological Science, 16(2), 55-59.

Steinberg, L. (2008). A social neuroscience perspective on adolescent risk-taking. Developmental Review, 28(1), 78-106.

Steinberg, M. B., Akincigil, A., Delnevo, C. D., Crystal, S., \& Carson, J. L. (2006). Gender and age disparities for smoking-cessation treatment. American Journal of Preventive Medicine, 30(5), 405412.

Steinberg, M. B., Alvarez, M. S., Delnevo, C. D., Kaufman, I., \& Cantor, J. C. (2006). Disparity of physicians' utilization of tobacco treatment services. American Journal of Health Behavior, 30(4), 375-386.

Sterling, S., Chi, F., \& Hinman, A. (2011). Integrating care for people with co-occurring alcohol and other drug, medical, and mental health conditions. Alcohol Research \& Health, 33(4), 338-349.

Steuber, T. L., \& Danner, F. (2006). Adolescent smoking and depression: Which comes first? Addictive Behaviors, 31(1), 133-136.

Stevens, S., Colwell, B., \& Hutchison, L. (2003). Tobacco use in rural areas: A literature review. Rural Healthy People 2010: A companion document to Healthy People 2010. Volume 2. College Station, TX: Texas A\&M University System Health Science Center, School of Rural Public Health, Southwest Rural Health Research Center.

Stilen, P., Carise, D., Roget, N., \& Wendler, A. (2007). Treatment planning M.A.T.R.S. utilizing the Addiction Severity Index (ASI) to make required data collection useful. Kansas City, MO: MidAmerica Addiction Technology Transfer Center in residence at the University of Missouri-Kansas City.

Stitzer, M. L. (1999). Combined behavioral and pharmacological treatments for smoking cessation. Nicotine \& Tobacco Research, 1(Suppl. 2), S181-S187.

Stitzer, M. L., \& Walsh, S. L. (1997). Psychostimulant abuse: The case for combined behavioral and pharmacological treatments. Pharmacology Biochemistry \& Behavior, 57(3), 457-470.

Stitzer, M. L., Owen, P. L., Hall, S. M., Rawson, R. A., \& Petry, N. M. (2003). CPDD policy statement: Standards for drug abuse treatment providers. Drug \& Alcohol Dependence, 70, 213-215. 
Stoops, W. W., Dallery, J., Fields, N. M., Nuzzo, P. A., Schoenberg, N. E., Martin, C. A., et al. (2009). An internet-based abstinence reinforcement smoking cessation intervention in rural smokers. Drug \& Alcohol Dependence, 105(1-2), 56-62.

Stotts, A. L., Schmitz, J. M., Rhoades, H. M., \& Grabowski, J. (2001). Motivational interviewing with cocaine-dependent patients: A pilot study. Journal of Consulting \& Clinical Psychology, 69(5), 858862.

Strain, E. C., Bigelow, G. E., Liebson, I. A., \& Stitzer, M. L. (1999). Moderate- vs high-dose methadone in the treatment of opioid dependence. JAMA, 281(11), 1000-1005.

Strang-Karlsson, S., Andersson, S., Paile-Hyvarinen, M., Darby, D., Hovi, P., Raikkonen, K., et al. (2009). Slower reaction times and impaired learning in young adults with birth weight $<1500 \mathrm{~g}$. Pediatrics, 125(1), e74-e82.

Stratton, K., Howe, C., Battaglia, F., Institute of Medicine, Division of Biobehavioral Sciences and Mental Disorders, \& Committee to Study Fetal Alcohol Syndrome (Eds.). (1996). Fetal alcohol syndrome. Diagnosis, epidemiology, prevention, and treatment. Washington, DC: National Academy Press.

Strong, D. R., Kahler, C. W., Colby, S. M., Griesler, P. M., \& Kandel, D. B. (2009). Linking measures of adolescent nicotine dependence to a common latent continuum. Drug \& Alcohol Dependency, 99(13), 296-308.

Stuart, G. L. (2005). Improving violence intervention outcomes by integrating alcohol treatment. Journal of Interpersonal Violence, 20(4), 388-393.

Stuber, J., Galea, S., \& Link, B. G. (2008). Smoking and the emergence of a stigmatized social status. Social Science \& Medicine, 67(3), 420-430.

Studts, J. L., Burrie, J. L., Kearns, D. K., Worth, C. T., \& Sorrell, C. L. (2009). "Providers practice prevention": Promoting dental hygenists' use of evidence-based treatment of tobacco use and dependence. Journal of Dental Education, 73(9), 1069-1082.

Sturm, R. (1999). Effects of substance abuse parity in private insurance plans under managed care. [Online]. Retrieved January 30, 2008 from http://rand.org.

Sturm, R., \& Sherbourne, C. D. (2001). Are barriers to mental health and substance abuse care still rising? Journal of Behavioral Health Services \& Research, 28(1), 81-88.

Sturm, R., Zhang, W., \& Schoenbaum, M. (1999). How expensive are unlimited substance abuse benefits under managed care? Journal of Behavioral Health Services \& Research, 26(2), 203-210.

Substance Abuse and Mental Health Services Administration, Center for Substance Abuse Treatment. (1999). Brief interventions and brief therapies for substance abuse. (TIP) Series 34. (DHHS Pub No. (SMA) 99-3353). [Online]. Retrieved December 9, 2011 from http://www.ncbi.nlm.nih.gov.

Substance Abuse and Mental Health Services Administration, Office of Applied Studies. (2001). The DASIS report: Growth of managed care in substance abuse treatment. [Online]. Retrieved January 30, 2012 from http://www.samhsa.gov.

Substance Abuse and Mental Health Services Administration, Office of Applied Studies. (2010a). National Survey of Substance Abuse Treatment Services (N-SSATS): 2009. Data on substance abuse treatment facilities (DASIS Series: S-54, HHS Pub No (SMA) 10-4579). Rockville, MD: U.S. Department of Health and Human Services, Substance Abuse and Mental Health Services Administration. 
Substance Abuse and Mental Health Services Administration, Office of Applied Studies. (2010b). National Survey of Substance Abuse Treatment Services (N-SSATS). 2010 state profile - United States. Rockville, MD: U.S. Department of Health and Social Services, Substance Abuse and Mental Health Services Administration, Office of Applied Studies.

Substance Abuse and Mental Health Services Administration, Office of Applied Studies. (2011a). The NSSATS report: Infectious disease screening. [Online]. Retrieved November 14, 2011 from http://oas.samhsa.gov.

Substance Abuse and Mental Health Services Administration, Office of Applied Studies. (2011b). National Survey of Substance Abuse Treatment Services (N-SSATS): 2010. Data on substance abuse treatment facilities. (DASIS Series: S-59, HHS Pub No. (SMA) 11-4665). Rockville, MD: U.S. Department of Health and Human Services, Substance Abuse and Mental Health Services Administration.

Substance Abuse and Mental Health Services Administration. (2002). Report to Congress on the prevention and treatment of co-occurring substance abuse disorders and mental disorders. Bethesda, MD: U.S. Department of Health and Human Services, Substance Abuse and Mental Health Services Administration.

Substance Abuse and Mental Health Services Administration. (2003a). BASICS: Brief alcohol screening and intervention of college students. [Online]. Retrieved October 6, 2008 from http://www.dontletminorsdrink.com.

Substance Abuse and Mental Health Services Administration. (2003b). Alcohol and Drug Services Study (ADSS): The national substance abuse treatment system: Facilities, clients, services, and staffing. Rockville, MD: U.S. Department of Health and Human Services, Substance Abuse and Mental Health Services Administration, Office of Applied Studies.

Substance Abuse and Mental Health Services Administration. (2003c). National treatment plan and national treatment network: Catalog of the status of national treatment plan activity within the states: Review of state activities. Rockville, MD: Prepared by the National Association of State Alcohol and Drug Abuse Directors and ABT Associates for the Substance Abuse and Mental Health Services Administration.

Substance Abuse and Mental Health Services Administration. (2005a). Conference explores high-tech treatment. SAMHSA News, 13(1).

Substance Abuse and Mental Health Services Administration. (2005b). A national review of state alcohol and drug treatment programs and certification standards for substance abuse counselors and prevention professionals (DHHS Pub No. 05-3994). Rockville, MD: U.S. Department of Health and Human Services, Substance Abuse and Mental Health Services Administration, Center for Substance Abuse Treatment.

Substance Abuse and Mental Health Services Administration. (2005c). Clinical support system available to assist physicians who treat patients dependent on opiates [Press release]. [Online]. Retrieved November 23, 2009 from http://www.samhsa.gov.

Substance Abuse and Mental Health Services Administration. (2006). National Survey on Drug Use and Health (NSDUH), 2002 (Codebook. ICPSR 3903). Ann Arbor, MI: U.S. Department of Health and Human Services, Substance Abuse and Mental Health Services Administration.

Substance Abuse and Mental Health Services Administration. (2008a). Brief alcohol screening and intervention for college students (BASICS). [Online]. Retrieved August 10, 2011 from http://nrepp.samhsa.gov. 
Substance Abuse and Mental Health Services Administration. (2008b). Issue brief \#4 for employers: What you need to know about mental and substance use disorders (SMA 08-4350 - 2008). [Online]. Retrieved December 19, 2011 from http://www.samhsa.gov.

Substance Abuse and Mental Health Services Administration. (2008c). Workplace substance use: Quick facts to inform managers. [Online]. Retrieved December 19, 2011 from http://workplace.samhsa.gov.

Substance Abuse and Mental Health Services Administration. (2008d). Request for Applications (RFA): Screening, brief intervention, referral and treatment (SBIRT): Medical residency program (initial announcement). [Online]. Retrieved February 27, 2012 from http://www.samhsa.gov.

Substance Abuse and Mental Health Services Administration. (2008e). SAMHSA awards $\$ 66$ million for programs teaching early intervention techniques to use with patients at risk for substance abuse. [Online]. Retrieved July 27, 2011 from http://www.samhsa.gov.

Substance Abuse and Mental Health Services Administration. (2008f). SAMHSA to provide \$3.75 million to train medical residents in SBIRT techniques. [Online]. Retrieved August 9, 2011 from http://www.samhsa.gov.

Substance Abuse and Mental Health Services Administration. (2009a). Screening, brief intervention, and referral to treatment: New populations, new effectiveness data. SAMHSA News, 19(6), 1-20.

Substance Abuse and Mental Health Services Administration. (2009b). Integrated treatment for cooccurring disorders: How to use the evidence-based practices KITs (DHHS Pub. No. SMA-08-4366). Rockville, MD: U.S. Department of Health and Human Services, Substance Abuse and Mental Health Services Administration.

Substance Abuse and Mental Health Services Administration. (2009c). Treatment Episode Data Set (TEDS): Highlights - 2007. National admissions to substance abuse treatment services: Table 4 (DASIS Series: S-45, DHHS Pub No. (SMA) 09-4360). Rockville, MD: U.S. Department of Health and Human Services, Substance Abuse and Mental Health Services Administration.

Substance Abuse and Mental Health Services Administration. (2009d). Results from the 2008 National Survey on Drug Use and Health: National findings (NSDUH Series H-36, HHS Pub. No. SMA 094434). Rockville, MD: U.S. Department of Health and Human Services, Substance Abuse and Mental Health Services Administration.

Substance Abuse and Mental Health Services Administration. (2009e). Overview of SBIRT coverage and payment in Medicaid and Medicare: SAMHSA SBIRT grantee conference. [Online]. Retrieved December 19, 2011 from http://sbirtgranteemeeting.com.

Substance Abuse and Mental Health Services Administration. (2010a). Results from the 2009 National Survey on Drug Use and Health: Volume I. Summary of national findings (NSDUH Series H-38A, HHS Publication No. SMA 10-4586). Rockville, MD: U.S. Department of Health and Human Services, Substance Abuse and Mental Health Services Administration, Office of Applied Studies.

Substance Abuse and Mental Health Services Administration. (2010b). National estimates of drug-related emergency department visits, 2004 - 2009 Analytic group: Drug misuse and abuse visits: Table: Age 12 - 17. [Online]. Retrieved April 20, 2011 from https://dawninfo.samhsa.gov

Substance Abuse and Mental Health Services Administration. (2010c). Twelve step facilitation therapy. [Online]. Retrieved August 16, 2011 from http://nrepp.samhsa.gov.

Substance Abuse and Mental Health Services Administration. (2010d). National Survey of Substance Abuse Treatment Services (N-SSATS). 2010 state profile - United States. [Online]. Retrieved April 2, 2012 from http://wwwdasis.samhsa.gov. 
Substance Abuse and Mental Health Services Administration. (2010e). Results from the 2009 National Survey on Drug Use and Health: Volume I. Summary of national findings (NSDUH Series H-38A, HHS Publication No. SMA 10-4586). Rockville, MD: U.S. Department of Health and Human Services, Substance Abuse and Mental Health Services Administration.

Substance Abuse and Mental Health Services Administration. (2010f). Mental health and substance abuse services in Medicaid, 2003: Charts and state tables (HHS Publication No. (SMA) 10-4608). Rockville, MD: U.S. Department of Health and Human Services, Substance Abuse and Mental Health Services Administration.

Substance Abuse and Mental Health Services Administration. (2010g). Examples of states' billing codes for mental health services, publicly funded. [Online]. Retrieved October 27, 2011 from http://www.samhsa.gov.

Substance Abuse and Mental Health Services Administration. (2011a). Illicit drug use among older adults. The NSDUH Report. [Online]. Retrieved January 26, 2012 from http://www.samhsa.gov.

Substance Abuse and Mental Health Services Administration. (2011b). Results from the 2010 National Survey on Drug Use and Health: Summary of national findings (NSDUH Series H-41, HHS Publication No. (SMA) 11-4658). [Online]. Retrieved November 21, 2011 from http://www.samhsa.gov.

Substance Abuse and Mental Health Services Administration. (2011c). Substance use disorders in people with physical and sensory disabilities (HHS Pub. No. (SMA) 11-4648). In Brief, 6(1), 1-8.

Substance Abuse and Mental Health Services Administration. (2011d). Leading change: A plan for SAMHSA's roles and actions 2011-2014. [Online]. Retrieved March 29, 2011 from http://store.samhsa.gov.

Substance Abuse and Mental Health Services Administration. (2011e). Coding for SBI reimbursement. [Online]. Retrieved November 14, 2011 from http://www.samhsa.gov.

Substance Abuse and Mental Health Services Administration. (2011f). FY 2012 online performance index. [Online]. Retrieved December 6, 2011 from http://www.samhsa.gov

Substance Abuse and Mental Health Services Administration. (2011g). SAMHSA block grants. [Online]. Retrieved February 24, 2012 from http://www.samhsa.gov

Substance Abuse and Mental Health Services Administration. (2011h). SAMHSA announces grants awards totaling up to $\$ 13.2$ million to build on health information technology investments. [Online]. Retrieved December 6, 2011 from http://www.samhsa.gov.

Substance Abuse and Mental Health Services Administration. (2012a). Co-occurring disorders in veterans and military service members. [Online]. Retrieved May 11, 2012 from http://www.samhsa.gov.

Substance Abuse and Mental Health Services Administration. (2012b). SAMHSA news release: National report finds one-in-five Americans experienced mental illness in the past year. [Online]. Retrieved February 2, 2012 from http://www.samhsa.gov.

Substance Abuse Subtle Screening Inventory Institute. (2012). Adolescent Substance Abuse Subtle Screening Inventory (SASSI-A2). [Online]. Retrieved April 20, 2012 from http://www.sassi.com.

Suh, J. J., Pettinati, H. M., Kampman, K. M., \& O'Brien, C. P. (2006). The status of disulfiram: A half of a century later. Journal of Clinical Psychopharmacology, 26(3), 290-302.

Sullivan, L. E., Tetrault, J. M., Braithwaite, R. S., Turner, B. J., \& Fiellin, D. A. (2011). A meta-analysis of the efficacy of nonphysician brief interventions for unhealthy alcohol use: Implications for the patient-centered medical home. American Journal on Addictions, 20(4), 343-356. 
Sulzberger, A. G. (2011). States adding drug test as hurdle for welfare. [Online]. New York Times. Retrieved November 17, 2011 from http://www.nytimes.com

Sundararaman, R., \& Redhead, S. (2007). CRS Report for Congress: The Mental Health Parity Act: A legislative history. [Online]. Retrieved January 30, 2008 from http://opencrs.com

Survey: People still unsure whether alcoholism is disease or moral weakness. (2005). Alcoholism \& Drug Abuse Weekly, 17(40), 1, 4-5.

Susser, M. (1985). Patient education: A public health point of view. Bulletin of the New York Academy of Medicine, 61(2), 189-202.

Sussman, S., Sun, P., \& Dent, C. W. (2006). A meta-analysis of teen cigarette smoking cessation. Health Psychology, 25(5), 549-557.

Svanun, S., \& McGrew, J. (1995). Prospective screening of substance dependence: The advantages of directness. Addictive Behaviors, 20(2), 205-213.

Svikis, D., Golden, A., Huggins, G., Pickens, R., McCaul, M. E., Velez, M. L., et al. (1997). Costeffectiveness of treatment for drug-abusing pregnant women. Drug \& Alcohol Dependence, 45, 105113.

Sweet, R. I., \& Saules, K. K. (2003). Validity of the substance abuse subtle screening inventoryadolescent version (SASSI-A). Journal of Substance Abuse Treatment, 24(4), 331-340.

Swendsen, J., Conway, K. P., Degenhardt, L., Glantz, M., Jin, R., Merikangas, K. R., et al. (2010). Mental disorders as risk factors for substance use, abuse and dependence: Results from the 10-year follow-up of the National Comorbidity Survey. Addiction, 105(6), 1117-1128.

Szalavitz, M. (2010). Does teen drug rehab cure addiction or create it? [Online]. Retrieved December 8, 2010 from http://www.time.com.

Tahiri, M., Mottillo, S., Joseph, L., Pilote, L., \& Eisenberg, M. J. (2012). Alternative smoking cessation aids: A meta-analysis of randomized controlled trials. American Journal of Medicine, 125(6), 523620.

Tai, B., \& Blaine, J. (1997). Naltrexone: An antagonist therapy for heroin addiction. [Online]. Retrieved January 30, 2008 from http://www.nida.nih.gov.

Tait, R. J., \& Hulse, G. K. (2003). A systematic review of the effectiveness of brief interventions with substance using adolescents by type of drug. Drug \& Alcohol Review, 22(3), 337-346.

Tait, R. J., Hulse, G. K., Robertson, S. I., \& Sprivulis, P. C. (2005). Emergency department-based intervention with adolescent substance users: 12-month outcomes. Drug \& Alcohol Dependence, 79(3), 359-363.

Tanaka, E. (2002). Toxicological interactions between alcohol and benzodiazepines. Journal of Toxicology: Clinical Toxicology, 40(1), 69-75.

Tanielian, T., Jaycox, L. H., \& (eds). (2008). Invisible wounds of war: Psychological and cognitive injuries, their consequences, and services to assist recovery. [Online]. Retrieved May 10, 2012 from http://www.rand.org.

Tarter, R. E., \& Kirisci, L. (1997). The Drug Use Screening Inventory for adults: Psychometric structure and discriminative sensitivity. American Journal of Drug \& Alcohol Abuse, 23(2), 207-219.

Tarter, R. E., \& Kirisci, L. (2001). Validity of the Drug Use Screening Inventory for predicting DSM-III$\mathrm{R}$ substance use disorder. Journal of Child \& Adolescent Substance Abuse, 10(4), 45-53. 
Tarter, R. W., Laird, S. B., Bukstein, O., \& Kaminer, Y. (1992). Validation of the adolescent drug use screening inventory: Preliminary findings. Psychology of Addictive Behaviors, 6(4), 233-236.

Tavel, M. E., Davidson, W., \& Batterton, T. D. (1961). A critical analysis of mortality associated with delirium tremens. Review of 39 fatalities in a 9-year period. American Journal of Medical Sciences, $242,18-29$.

Taylor, J., lloyd, D. A., \& Warheit, G. J. (2005). Self-derogation, peer factors, and drug dependence among a multiethnic sample of young adults. Journal of Child \& Adolescent Substance Abuse, 15(2), 39-51.

Teitelbaum, L. M., \& Carey, K. B. (2000). Temporal stability of alcohol screening measures in a psychiatric setting. Psychology of Addictive Behaviors, 14(4), 401-404.

Teitelbaum, L., \& Mullen, B. (2000). The validity of the MAST in psychiatric settings: A meta-analytic integration. Michigan Alcoholism Screening Test. Journal of Studies on Alcohol, 61(2), 254-261.

Terry-McElrath, Y. M., Chriqui, J. F., \& McBride, D. C. (2011). Factors related to Medicaid payment acceptance at outpatient substance abuse treatment programs. Health Services Research, 46(2), 632653.

Teruya, C., Hardy, M., Hser, Y.-I., \& Evans, E. (2006). Implementation of a statewide outcome monitoring system: Lessons learned from substance abuse treatment provider staff. Qualitative Health Research, 16(3), 337-352.

Tevyaw, T. O., \& Monti, P. M. (2004). Motivational enhancement and other brief interventions for adolescent substance abuse: Foundations, applications and evaluations. Addiction, 99(Suppl. 2), 6375.

The Academic ED SBIRT Research Collaborative. (2007). An evidence-based alcohol screening, brief intervention and referral to treatment (SBIRT) curriculum for emergency department (ED) providers improves skills and utilization. Substance Abuse, 28(4), 79-92.

The American Society of Health System Pharmacists. (2012). ASHP statement on the pharmacist's role in substance abuse prevention, education, and assistance. ASHP Reports, 60(19), 1995-1998.

The Economist. (2008, October 18). Drug addiction: Treatment on a plate. Economist, 91-92.

The National Center on Addiction and Substance Abuse (CASA) at Columbia University. (1993). The cost of substance abuse to America's health care system: Report 1, Medicaid hospital costs. New York: Author

The National Center on Addiction and Substance Abuse (CASA) at Columbia University. (1994). The cost of substance abuse to America's health care system: Report 2, Medicare hospital costs. New York: Author.

The National Center on Addiction and Substance Abuse (CASA) at Columbia University. (1998). Under the rug: Substance abuse and the mature woman. New York: Author.

The National Center on Addiction and Substance Abuse (CASA) at Columbia University. (1999). No safe haven: Children of substance-abusing parents. New York: Author.

The National Center on Addiction and Substance Abuse (CASA) at Columbia University. (2000). Missed opportunity: National survey of primary care physicians and patients on substance abuse. New York: Author.

The National Center on Addiction and Substance Abuse (CASA) at Columbia University. (2001a). Malignant neglect: Substance abuse and America's schools. New York: Author. 
The National Center on Addiction and Substance Abuse at Columbia University (CASA Columbia). (2001b). Shoveling Up: The impact of substance abuse on state budgets. New York: Author.

The National Center on Addiction and Substance Abuse (CASA) at Columbia University. (2003a). Food for thought: Substance abuse and eating disorders. New York: Author.

The National Center on Addiction and Substance Abuse (CASA) at Columbia University. (2003b). The formative years: pathways to substance abuse among girls and young women ages 8-22. New York: Author.

The National Center on Addiction and Substance Abuse (CASA) at Columbia University. (2004). Criminal neglect: Substance abuse, juvenile justice and the children left behind. New York: Author.

The National Center on Addiction and Substance Abuse (CASA) at Columbia University. (2005). Under the counter: The diversion and abuse of controlled prescription drugs in the United States. New York: Author.

The National Center on Addiction and Substance Abuse (CASA) at Columbia University. (2006). Women under the influence. Baltimore, MD: John Hopkins University Press.

The National Center on Addiction and Substance Abuse (CASA) at Columbia University. (2007a). Tobacco: The smoking gun. New York: Author.

The National Center on Addiction and Substance Abuse (CASA) at Columbia University. (2007b). Wasting the best and the brightest: Substance abuse at America's colleges and universities. New York: Author.

The National Center on Addiction and Substance Abuse (CASA) at Columbia University. (2008a). CASA Columbia National Addiction Belief and Attitude Survey (NABAS). Unpublished raw data. New York: Author.

The National Center on Addiction and Substance Abuse (CASA) at Columbia University. (2008b). CASA Columbia's survey of Individuals in Long-Term Recovery. Unpublished raw data. New York: Author.

The National Center on Addiction and Substance Abuse (CASA) at Columbia University. (2008c). CASA Columbia's National Online Survey of Members of Professional Associations Involved in Addiction Care. Unpublished raw data. New York: Author.

The National Center on Addiction and Substance Abuse (CASA) at Columbia University. (2009a). CASA Columbia's survey of New York State Addiction Treatment Providers. Unpublished raw data. New York: Author.

The National Center on Addiction and Substance Abuse (CASA) at Columbia University. (2009b). $C A S A S A R D^{s m}$ : Intensive case management for substance-dependent women receiving Temporary Assistance for Needy Families. New York: Author.

The National Center on Addiction and Substance Abuse (CASA) at Columbia University. (2009c). National survey of American attitudes on substance abuse XIV: Teens and parents. New York: Author.

The National Center on Addiction and Substance Abuse (CASA) at Columbia University. (2009d). Shoveling up II: The impact of substance abuse on federal, state and local budgets. New York: Author.

The National Center on Addiction and Substance Abuse (CASA) at Columbia University. (2010). Behind bars II: Substance abuse and America's prison population. New York: Author.

The National Center on Addiction and Substance Abuse (CASA) at Columbia University. (2011a). Adolescent substance use: America's \#1 public health problem. New York: Author. 
The National Center on Addiction and Substance Abuse (CASA) at Columbia University. (2011b). CASA's National Survey of High School Students, Parents of High School Students, and High School Personnel. Unpublished raw data. New York: Author.

The National Center on Addiction and Substance Abuse at Columbia University (CASA Columbia). (2012a). CASA Columbia analysis of the alcohol and public health: Alcohol-Related Disease Impact (ARDI). [Online]. Centers for Disease Control and Prevention. Retrieved January 11, 2012 from http://apps.nccd.cdc.gov.

The National Center on Addiction and Substance Abuse at Columbia University (CASA Columbia). (2012b). CASA Columbia analysis of American Board of Addiction Medicine Foundation. (2009). The need for addiction medicine physicians and for addiction medicine residency training programs. A report of The American Board of Addiction Medicine Foundation. Chevy Chase, MD: Author. And Kunz, K. B., President, \& Callahan, J. F., Executive Vice President, American Board of Addiction Medicine and Gentilello, L.M., MD, Trauma Services, Kaiser Permanente Hospital, Sacramento, CA (personal communication, March 12, 2012).

The National Center on Addiction and Substance Abuse at Columbia University (CASA Columbia). (2012c). CASA Columbia analysis of data from the Centers for Disease Control and Prevention. (2011). Vital Signs: Overdoses of prescription opioid pain relievers--United States, 1999-2008. Morbidity \& Mortality Weekly Report, 60(43),1487-1492.

The National Center on Addiction and Substance Abuse at Columbia University (CASA Columbia). (2012d). CASA Columbia analysis of the Drug Abuse Warning Network, 2009: Selected tables of national estimates of drug-related emergency department visits. Rockville, MD: Center for Behavioral Health Statistics and Quality, SAMHSA.

The National Center on Addiction and Substance Abuse at Columbia University (CASA Columbia). (2012e). CASA Columbia analysis of the Drug Abuse Warning Network (DAWN): National estimates of drug-related emergency department visits, 2004 - 2009: ED visits by drugs. Rockville, MD: U.S. Department of Health and Human Services, Substance Abuse and Mental Health Services Administration, Office of Applied Studies.

The National Center on Addiction and Substance Abuse at Columbia University (CASA Columbia). (2012f). CASA Columbia analysis of the Drug Abuse Warning Network (DAWN): National estimates of drug-related emergency department visits for 2004 - 2009: Frequency of drugs reported.

Rockville, MD: U.S. Department of Health and Human Services, Substance Abuse and Mental Health Services Administration, Office of Applied Studies.

The National Center on Addiction and Substance Abuse at Columbia University (CASA Columbia). (2012g). CASA Columbia analysis of Friedmann, P. D., et al. (2004). The effect of matching comprehensive services to patients' needs on drug use improvement in addiction treatment. Addiction, 99(8), 962-972.

The National Center on Addiction and Substance Abuse at Columbia University (CASA Columbia). (2012h). CASA Columbia analysis of the Medical expenditure panel survey. Table 3: Total expenses and percent distribution for selected conditions by type of service: United States, 2009. [Online]. Retrieved April 3, 2012 from http://meps.ahrq.gov. 
The National Center on Addiction and Substance Abuse at Columbia University (CASA Columbia). (2012i). CASA Columbia analysis of the National expenditures for mental health services and substance abuse treatment, 1986-2005. (Table A.1) SAMHSA spending estimates project, 2010; CMS Office of the Actuary, National Health Statistics Group (DHHS Publication No. (SMA) 10-4612). Rockville, MD: U.S. Department of Health \& Human Services, Substance Abuse and Mental Health Services Administration, Center for Mental Health Services and Center for Substance Abuse Treatment.

The National Center on Addiction and Substance Abuse at Columbia University (CASA Columbia). (2012j). CASA Columbia analysis of the National expenditures for mental health services and substance abuse treatment, 1986-2005 (DHHS Publication No. (SMA) 10-4612). Rockville, MD: U.S. Department of Health \& Human Services, Substance Abuse and Mental Health Services Administration, Center for Mental Health Services and Center for Substance Abuse Treatment.

The National Center on Addiction and Substance Abuse at Columbia University (CASA Columbia). (2012k). CASA Columbia analysis of the National Survey of Substance Abuse Treatment Services ( $N$ SSATS): 2010 ([Computer file]. United States Department of Health and Human Services. Substance Abuse and Mental Health Services Administration. Center for Behavioral Health Statistics and Quality. ICPSR32723-v2). Anne Arbor, MI: Inter-university Consortium for Political and Social Research.

The National Center on Addiction and Substance Abuse at Columbia University (CASA Columbia). (20121). CASA Columbia analysis of the National Survey on Drug Use and Health (NSDUH), 2002. Rockville, MD: U.S. Department of Health and Human Services, Substance Abuse and Mental Health Services Administration.

The National Center on Addiction and Substance Abuse at Columbia University (CASA Columbia). (2012m). CASA Columbia analysis of the National Survey on Drug Use and Health (NSDUH), 2009. Rockville, MD: U.S. Department of Health and Human Services, Substance Abuse and Mental Health Services Administration.

The National Center on Addiction and Substance Abuse at Columbia University (CASA Columbia). (2012n). CASA Columbia analysis of the National Survey on Drug Use and Health (NSDUH), 2010. Rockville, MD: U.S. Department of Health and Human Services, Substance Abuse and Mental Health Services Administration.

The National Center on Addiction and Substance Abuse at Columbia University (CASA Columbia). (2012o). CASA Columbia analysis of the NHTSA Fatality Analysis Reporting System (FARS) database. [Online]. National Highway Traffic Safety Administration. Retrieved January 11, 2012 from http://www.nhtsa.gov.

The National Center on Addiction and Substance Abuse at Columbia University (CASA Columbia). (2012p). CASA Columbia analysis of the Treatment Episode Data -- Discharges (TEDS-D), 2008 ([Computer file]. ICPSR29901-v1). Ann Arbor, MI: Inter-University Consortium for Political and Social Research [distributor], 2011-04-25.doi:10.3886/ICPSR29901.v1.

The National Center on Addiction and Substance Abuse at Columbia University (CASA Columbia). (2012q). CASA Columbia analysis of the Treatment Episode Data Set (TEDS): 1999-2009. National admissions to substance abuse treatment services (DASIS Series: S-56, HHS Pub No. (SMA) 114646). Rockville, MD: Substance Abuse and Mental Health Services Administration.

The National Center on Addiction and Substance Abuse at Columbia University (CASA Columbia). (2012r). CASA Columbia analysis of the Treatment Episode Data Set (TEDS): 2009. Rockville, MD: Substance Abuse and Mental Health Services Administration, Office of Applied Studies. 
The National Center on Addiction and Substance Abuse at Columbia University (CASA Columbia). (2012s). CASA Columbia analysis of the 2009 National Youth Tobacco Survey codebook. [Online]. Retrieved February 9, 2012 from http://www.cdc.gov.

The National Center on Addiction and Substance Abuse at Columbia University (CASA Columbia). (2012t). CASA Columbia analysis of data from CASA Columbia's report: Shoveling up II: The impact of substance abuse on federal, state and local budgets. New York: Author.

The National Committee for Quality Assurance (NCQA). (2012). About NCQA. [Online]. Retrieved March 20, 2012 from http://www.ncqa.org.

The Partnership at DrugFree.org. (2012). Survey: Ten percent of American adults report being in recovery from substance abuse or addiction. [Online]. Retrieved March 29, 2012 from http://www.drugfree.org.

TheAddictionCure.com. (2012). The addiction cure website. [Online]. Retrieved February 22, 2012 from http://www.theaddictioncure.com.

Thomas, C. P., Garnick, D. W., Horgan, C. M., McCorry, F., Gmyrek, A., Chalk, M., et al. (2011). Advancing performance measures for use of medications in substance abuse treatment. Journal of Substance Abuse Treatment, 40(1), 35-43.

Thomas, C. P., Reif, S., Haq, S., Wallack, S. S., Hoyt, A., \& Ritter, G. A. (2008). Use of buprenorphine for addiction treatment: Perspectives of addiction specialists and general psychiatrists. Psychiatric Services, 59(8), 909-916.

Thomas, S. E., \& Miller, P. M. (2007). Knowledge and attitudes about pharmacotherapy for alcoholism: A survey of counselors and administrators in community-based addiction treatment centers. Alcohol \& Alcoholism, 42(2), 113-118.

Thomasson, H. R., Edenberg, H. J., Crabb, D. W., Mai, X.-L., Jerome, R. E., Li, T.-K., et al. (1991). Alcohol and aldehyde dehydrogenase genotypes and alcoholism in Chinese men. American Journal of Human Genetics, 48, 677-681.

Thombs, D. L. (2006). Introduction to addictive behaviors. New York: Guilford Press.

Thorndike, A. N., Regan, S., \& Rigotti, N. A. (2007). The treatment of smoking by US physicians during ambulatory visits: 1994-2003. American Journal of Public Health, 97(10), 1878-1883.

Thorndike, A. N., Stafford, R. S., \& Rigotti, N. A. (2001). US physicians' treatment of smoking in outpatients with psychiatric diagnoses. Nicotine \& Tobacco Research, 3(1), 85-91.

Thygesen, L. C., Mikkelsen, P., Andersen, T. V., Tonnesen, H., Juel, K., Becker, U., et al. (2009). Cancer incidence among patients with alcohol use disorders: Long-term follow-up. Alcohol \& Alcoholism, 44(4), 387-391.

Timko, C., Moos, R. H., Finney, J. W., \& Lesar, M. D. (2000). Long-term outcomes of alcohol use disorders: Comparing untreated individuals with those in Alcoholics Anonymous and formal treatment. Journal of Studies on Alcohol, 61(4), 529-540.

Timmermans, S., \& Mauck, A. (2005). The promises and pitfalls of evidence-based medicine. Health Affairs, 24(1), 18-28.

Tims, F. M., Leukefeld, C. G., \& Platt, J. J. (2001). Relapse and recovery. In F. M. Tims, C. G. Leukefeld, \& J. J. Platt (Eds.), Relapse and recovery in addictions (pp. 3-17). New Haven, CT: Yale University Press.

Tinsley, J. A. (2011). Assessment and treatment of substance use disorders. FOCUS: Journal of Lifelong Learning in Psychiatry, 9(1), 3-14. 
Tobacco Use and Dependence Clinical Practice Guideline Panel, Staff, and Consortium Representatives. (2000). A clinical practice guideline for treating tobacco use and dependence: A U.S. Public Health Service report. JAMA, 283(24), 3244-3254.

Tomas-Rossello, J., Rawson, R. A., Zarza, M. J., Bellows, A., Busse, A., Saenz, E., et al. (2010). United Nations office on drugs and crime international network of drug dependence treatment and rehabilitation resource centers: Treatment. Substance Abuse, 31(4), 251-263.

Tong, E. K., Strouse, R., Hall, J., Kovac, M., \& Schroeder, S. A. (2010). National survey of U.S. health professionals' smoking prevalence, cessation practices, and beliefs. Nicotine \& Tobacco Research, $12(7), 724-733$.

Topitzes, J., Mersky, J. P., \& Reynolds, A. J. (2010). Child maltreatment and adult cigarette smoking: A long-term developmental model. Journal of Pediatric Psychology, 35(5), 484-498.

Toschke, A. M., Montgomery, M. A., Pfeiffer, U., \& von Kries, R. (2003). Early intrautrine exposure to tobacco-inhaled products and obesity. American Journal of Epidemiology, 158(11), 1068-1074.

Treatment Communities of America. (2012). Recovery through holistic client-based care. [Online]. Retrieved January 12, 2012 Retrieved from http://www.therapeuticcommunitiesofamerica.org.

Treistman, S. N., \& Martin, G. E. (2009). BK channels: Mediators and models for alcohol tolerance. Trends in Neurosciences, 32(12), 629-637.

Trevisan, L. A., Boutros, N., Petrakis, I. L., \& Krystal, J. H. (1998). Complications of alcohol withdrawal: Pathophysiological insights. Alcohol Health \& Research World, 22(1), 61-66.

Triffleman, E., Carroll, K., \& Kellogg, S. (1999). Substance dependence posttraumatic stress disorder therapy. Journal of Substance Abuse Treatment, 17(1-2), 3-14.

Triggle, D. J. (2007). Treating desires not diseases: A pill for every ill and an ill for every pill? Drug Discovery Today, 12(3/4), 161-166.

Trinidad, D. R., Perez-Stable, E. J., White, M. M., Emery, S. L., \& Messer, K. (2011). A nationwide analysis of US racial/ethnic disparities in smoking behaviors, smoking cessation, and cessationrelated factors. American Journal of Public Health, 101(4), 699-706.

Tritz, K. (2006). Integrating Medicare and Medicaid services through managed care. [Online]. Retrieved March 28, 2012 from http://ancor.org.

True, W. R., Heath, A. C., Bucholz, K., Slutske, W., Romeis, J. C., Scherrer, J. F., et al. (1996). Models of treatment seeking for alcoholism: The role of genes and environment. Alcoholism: Clinical \& Experimental Research, 20(9), 1577-1581.

Trumpler, F., Oez, S., Stahli, P., Brenner, H. D., \& Juni, P. (2003). Acupuncture for alcohol withdrawal: A randomized controlled trial. Alcohol \& Alcoholism, 38(4), 369-375.

Truong, K. D., \& Sturm, R. (2009). Alcohol environments and disparities in exposure associated with adolescent drinking in California. American Journal of Public Health, 99(2), 264-270.

Tsogia, D., Copello, A., \& Orford, J. (2001). Entering treatment for substance misuse: A review of the literature. Journal of Mental Health, 10(5), 481-499.

Tsuang, M. T., Bar, J. L., Harley, R. M., \& Lyons, M. J. (2001). The Harvard Twin Study of Substance Abuse: What we have learned. Harvard Review of Psychiatry, 9(6), 267-279.

Tsuang, M. T., Lyons, M. J., Harley, R. M., Xian, H., Eisen, S., Goldberg, J., et al. (1999). Genetic and environmental influences on transitions in drug use. Behavior Genetics, 29(6), 473-479. 
Tsuang, M. T., Lyons, M. J., Meyer, J. M., Doyle, T., Eisen, S. A., Goldberg, J., et al. (1998). Cooccurrence of abuse of different drugs in men. The role of drug-specific and share vulnerabilities. Archives of General Psychiatry, 55(11), 967-972.

Tucker, J. A. (1995). Predictors of help-seeking and the temporal relationship of help to recovery among treated and untreated recovered problem drinkers. Addiction, 90(6), 805-809.

Tucker, J. A., Vuchinich, R. E., \& Rippens, P. D. (2004). A factor analytic study of influences of patterns of help-seeking among treated and untreated alcohol dependent persons. Journal of Substance Abuse Treatment, 26(3), 237-242.

Tyc, V. L., Hadley, W., Allen, D., Varnell, S., Ey, S., Rai, S. N., et al. (2004). Predictors of smoking intentions and smoking status among nonsmoking and smoking adolescents. Addictive Behaviors, 29(6), 1143-1147.

Tyrer, P., Rutherford, D., \& Huggert, T. (1981). Benzodiazepine withdrawal symptoms and propranolol. Lancet, 1(8219), 520-522.

U.S. Census Bureau. (2011). Highlights 2010. [Online]. Retrieved February 1, 2012 from http://www.census.gov.

U.S. Code Online via GPO Access. (2003). 42 USC $\$ 300 x-22(a)(1):$ Public health and welfare: Certain allocations. [Online]. Retrieved February 16, 2007 from http://frwebgate.access.gpo.gov.

U.S. Code Online via GPO Access. (2007). 42 USC $\$ 300 x-27(a)$ : Public health and welfare: Treatment services for pregnant women. [Online]. Retrieved February 16, 2007 from http://frwebgate.access.gpo.gov.

U.S. Code Online via GPO Access. (2011). 42 USC Part 2: Confidentiality of alcohol and drug abuse patient records. [Online]. Retrieved September 16, 2011 from http://ecfr.gpoaccess.gov.

U.S. Code Online via GPO Access. (2012a). 42 CFR \$ 8.12: Public health: Certification of opioid treatment programs. Federal opioid standards. [Online]. Retrieved February 14, 2012 from http://ecfr.gpoaccess.gov.

U.S. Code Online via GPO Access. (2012b). Title 32: National defense. Part 199-Civilian Health and Medical Program of the Uniformed Services (CHAMPUS). \$199.6 TRICARE-authorized providers. [Online]. Retrieved February 13, 2012 from http://ecfr.gpoaccess.gov.

U. S. Code of Federal Regulations. Title 52 Fed Reg 21796, 21797.

U.S. Department of Agriculture and U.S. Department of Health and Human Services. (2010). Dietary guidelines for Americans, 2010. (7th Ed). Washington, DC: U.S. Government Printing Office.

U.S. Department of Defense Task Force on Mental Health. (2007). An achievable vision: Report of the Department of Defense Task Force on Mental Health. [Online]. Retrieved May 10, 2012 from http://www.health.mil.

U.S. Department of Defense, Department of Veteran Affairs. (2009). VA/DoD clinical practice guideline for management of Substance Use Disorders (SUD). [Online]. Retrieved May 10, 2012 from http://www.healthquality.va.gov.

U.S. Department of Defense, Department of Veteran Affairs. (2011). VA/DoD clinical practice guideline for management of tobacco use (MTU) 2008. [Online]. Retrieved May 10, 2012 from http://www.healthquality.va.gov.

U.S. Department of Defense. (1985). Rehabilitation and referral services for alcohol and drug abusers. [Online]. Retrieved May 10, 2012 from http://www.dtic.mil. 
U.S. Department of Health and Human Services, Centers for Medicare \& Medicaid Services. (2008). CMS manual system: Summary of policies in the 2008 Medicare physician fee schedule and the telehealth originating site facility fee payment amount (Pub 100-04 Medicare claims processing). [Online]. Retrieved November 15, 2011 from http://www.cms.gov.

U.S. Department of Health and Human Services, Centers for Medicare \& Medicaid Services. (2011). Decision memo for screening and behavioral counseling interventions in primary care to reduce alcohol misuse (CAG-00427N). [Online]. Retrieved November 11, 2011 from http://www.cms.gov.

U.S. Department of Health and Human Services, Centers for Medicare \& Medicaid Services. (2012a). Quick reference information: The ABCs of providing the initial preventive physical examination. [Online]. Retrieved May 11, 2012 from https://www.cms.gov.

U.S. Department of Health and Human Services, Centers for Medicare \& Medicaid Services. (2012b). Tobacco-use cessation counseling services. [Online]. Retrieved May 11, 2012 from https://www.cms.gov.

U.S. Department of Health and Human Services, Health Resources and Services Administration. (2012). The registered nurse population: Findings from the 2008 National Sample Survey of Registered Nurses. [Online]. Retrieved March 1, 2012 from http://bhpr.hrsa.gov.

U.S. Department of Health and Human Services, National Institutes of Health, National Institute on Drug Abuse. (2009). NIDA: Info Facts: Stimulant ADHD medications: Methylphenidate and amphetamines. [Online]. Retrieved December 10, 2007 from http://drugabuse.gov.

U.S. Department of Health and Human Services, Office of the Assistant Secretary for Planning and Evaluation. (2011). ASPE Issue Brief: Essential health benefits: Individual market coverage. [Online]. Retrieved February 28, 2012 from http://aspe.hhs.gov.

U.S. Department of Health and Human Services, Substance Abuse and Mental Health Services Administration. (2009). The NSDUH report: Substance use among women during pregnancy and following childbirth. [Online]. Retrieved February 6, 2012 from http://oas.samhsa.gov.

U.S. Department of Health and Human Services, Substance Abuse and Mental Health Services Administration, Center for Substance Abuse Treatment. (2006). The role of biomarkers in the treatment of alcohol use disorders. Substance Treatment Advisory, 5(4), 1-8.

U.S. Department of Health and Human Services, Substance Abuse and Mental Health Services Administration, Center for Substance Abuse Treatment. (2009). Emerging issues in the use of methadone. [Online]. Retrieved November 28, 2011 from http://kap.samhsa.gov.

U.S. Department of Health and Human Services, Substance Abuse and Mental Health Services Administration, Office of Applied Studies. (2011). Treatment Episode Data Set: Discharges (TEDSD), 2008 ([Computer file]. ICPSR29901-v1). Ann Arbor, MI: Inter-University Consortium for Political and Social Research.

U.S. Department of Health and Human Services, Substance Abuse and Mental Health Services Administration, Office of Applied Studies. (2008). The NSDUH report: Nicotine dependence: 2006. [Online]. Retrieved May 31, 2012 from http://www.samhsa.gov.

U.S. Department of Health and Human Services, Substance Abuse and Mental Health Services Administration, Center for Substance Abuse Treatment, Division of Pharmacologic Therapies. (2012). Opioid treatment program accreditation. [Online]. Retrieved March 20, 2012 from http://www.dpt.samhsa.gov.

U.S. Department of Health and Human Services, U.S. Food and Drug Administration, Center for Drug Evaluation and Research. (2008). Public health advisory: Important information on Chantix (varenicline). [Online]. Retrieved January 30, 2008 from http://www.fda.gov. 
U.S. Department of Health and Human Services, U.S. Food and Drug Administration, Center for Drug Evaluation and Research. (2011). Information for healthcare professionals: Varenicline (marketed as Chantix). [Online]. Retrieved January 23, 2012 from http://www.fda.gov.

U.S. Department of Health and Human Services, U.S. Food and Drug Administration, Center for Drug Evaluation and Research. (2010). Medication guide: Provigil C-IV tablets. [Online]. Retrieved January 23, 2012 from http://www.fda.gov.

U.S. Department of Health and Human Services. (1989). NIDA Research Monograph 94: Pharmacology and toxicology of amphetamine and related designer drugs. Rockville, MD: U.S. Department of Health and Human Services, National Institute on Drug Abuse.

U.S. Department of Health and Human Services. (1993). Alcohol alert: Alcohol and nutrition. [Online]. National Institute on Alcohol Abuse and Alcoholism. Retrieved November 11, 2011 from http://pubs.niaaa.nih.gov.

U.S. Department of Health and Human Services. (2000). 10th special report to the U.S. Congress on alcohol and health: Highlights on current research. [Online]. National Institute on Alcohol Abuse and Alcoholism. Retrieved January 15, 2008 from http://pubs.niaaa.nih.gov.

U.S. Department of Health and Human Services. (2006). Alcohol alert: National epidemiologic survey on alcohol and related conditions. [Online]. National Institute on Alcohol Abuse and Alcoholism. Retrieved December 10, 2007 from http://pubs.niaaa.nih.gov.

U.S. Department of Health and Human Services. (2008). Detoxification and substance abuse treatment: TIP 45. [Online]. Retrieved January 30, 2008 from http://www.ncbi.nlm.nih.gov.

U.S. Department of Health and Human Services. (2010a). How tobacco smoke causes disease: The biology and behavioral basis for smoking-attributable disease: A report of the Surgeon General. Atlanta, GA: U.S. Department of Health and Human Services, Centers for Disease Control and Prevention, National Center for Chronic Disease Prevention and Health Promotion, Office on Smoking and Health.

U.S. Department of Health and Human Services. (2010b). Young adult coverage until age 26. [Online]. Retrieved July 18, 2011 from http://www.healthcare.gov.

U.S. Department of Health and Human Services. (2011a). Affordable Care Act to support quality improvement and access to primary care for more Americans. [Online]. Retrieved March 9, 2012 from http://www.hhs.gov.

U.S. Department of Health and Human Services. (2011b). HIV/AIDS implementation science targeting drug using populations: A collaboration with PEPFAR (R01). [Online]. Retrieved January 31, 2012 from http://grants.nih.gov.

U.S. Department of Health and Human Services. (2012). Office on Disability - Substance abuse and disability: A companion to chapter 26 of healthy people 2010. [Online]. Retrieved February 9, 2012 from http://www.hhs.gov.

U.S. Department of Health, Education and Welfare, Public Health Service. (1964). Smoking and health. Report of the advisory committee to the Surgeon General of the Public Health Service. (Public Health Service Pub. No. 1103). Washington, DC: U.S. Department of Health, Education and Welfare.

U.S. Department of Housing and Urban Development, Office of Community Planning and Development. (2009). Rural continuums of care. HUD's homeless assistance programs. [Online]. Retrieved February 7, 12 A.D. from http://www.hudhre.info.

U.S. Department of Justice, National Drug Intelligence Center. (2010). Impact of drugs on society. [Online]. Retrieved December 19, 2011 Retrieved from http://www.justice.gov. 
U.S. Department of Justice, Office of Diversion Control. (2012). Practitioner's manual. Section V: Valid prescription requirements. [Online]. Retrieved March 1, 2012 from http://www.deadiversion.usdoj.gov.

U.S. Department of Justice, Office of Justice Programs. (2004). Screening and assessing mental health and substance use disorders among youth in the juvenile justice system: A resource guide for practitioners. [Online]. Retrieved September 27, 2011 from https://www.ncjrs.gov.

U.S. Department of Justice, Office of Justice Programs. (2009). Practical implications of current domestic violence research: For law enforcement, prosecutors and judges. [Online]. Retrieved December 19, 2011 from https://www.ncjrs.gov.

U.S. Department of Labor, Bureau of Labor Statistics. (2009a). Occupational outlook handbook (OOH), 2010-11 edition: Physician assistants. [Online]. Retrieved March 21, 2012 from http://www.bls.gov.

U.S. Department of Labor, Bureau of Labor Statistics. (2009b). Occupational outlook handbook (OOH), 2010-11 edition: Registered nurses. [Online]. Retrieved March 21, 2012 from http://www.bls.gov.

U.S. Department of Labor, Bureau of Labor Statistics. (2012a). Databases, tables and calculators. CPI inflation calculator. [Online]. Retrieved March 14, 2012 from http://www.bls.gov.

U.S. Department of Labor, Bureau of Labor Statistics. (2012b). Occupational employment statistics: Occupational employment and wages, May 2011: 21-1011 Substance abuse and behavioral disorder counselors. [Online]. Retrieved April 17, 2012 from http://www.bls.gov.

U.S. Department of Labor, Bureau of Labor Statistics. (2012c). Occupational outlook handbook (OOH), 2010-11 edition: Counselors. [Online]. Retrieved January 18, 2012 from http://www.bls.gov.

U.S. Department of Labor, Employee Benefits Security Administration. (2010). Fact sheet: The Mental Health Parity and Addiction Equity Act of 2008 (MHPAEA). [Online]. Retrieved April 4, 2012 from http://www.dol.gov.

U.S. Department of Transportation, National Highway Traffic Safety Administration. (2011). Time of day and demographic perspective of fatal alcohol-impaired-driving crashes. [Online]. Retrieved November 21, 2011 from http://www-nrd.nhtsa.dot.gov.

U.S. Department of Transportation, National Highway Traffic Safety Administration. (2008). Traffic safety facts: Laws: Alcohol exclusion laws. [Online]. Retrieved March 7, 2012 from http://www.nhtsa.gov.

U.S. Department of Transportation, Research and Innovative Technology Administration. (2011). The U.S. rural population and scheduled intercity transportation in 2010: A five-year decline in transportation access. [Online]. Retrieved February 3, 2012 from http://www.bts.gov.

U.S. Department of Veteran Affairs, National Center for PTSD. (2011). PTSD and problems with alcohol use. [Online]. Retrieved May 10, 2012 from http://www.ptsd.va.gov

U.S. Department of Veteran Affairs, National Center for PTSD. (2012). How common is PTSD? [Online]. Retrieved May 10, 2012 from http://www.ptsd.va.gov.

U.S. Department of Veteran Affairs, National Center for PTSD. (2010). Report of (VA) Consensus Conference: Practice recommendations for treatment of veterans with comorbid substance abuse and PTSD. [Online]. Retrieved May 14, 2012 from http://www.ptsd.va.gov.

U.S. Department of Veteran Affairs, National Center for PTSD. (2012). QUERI update: Improving access to opioid agonist therapy. [Online]. Retrieved May 14, 2012 from http://www.queri.research.va.gov.

U.S. Drug Enforcement Administration, Office of Diversion Control. (2011). General questions and answers. [Online]. from http://www.deadiversion.usdoj.gov. 
U.S. Drug Enforcement Administration. (2010). 21 USC: Controlled Substances Act. [Online]. Retrieved February 3, 2012 from http://www.dea.gov.

U.S. Drug Enforcement Administration. (2011). Drug classes. [Online]. Retrieved August 11, 2011 from http://www.justice.gov.

U.S. Drug Enforcement Administration. (2012). Benzodiazepines. [Online]. Retrieved May 23, 2012 from http://www.justice.gov.

U.S. Food and Drug Administration, Center for Drug Evaluation and Research. (2002). Subutex and Suboxone. Questions and answers. [Online]. Retrieved March 27, 2009 from http://www.fda.gov.

U.S. Food and Drug Administration. (2006a). FDA approves first generic ondansetron tablets, orally disintegrating tablets and oral solution. [Online]. Retrieved April 3, 2012 from http://www.fda.gov.

U.S. Food and Drug Administration. (2006b). Information for healthcare professionals Methadone hydrochloride text version. [Online]. Retrieved April 3, 2012 from http://www.fda.gov.

U.S. Food and Drug Administration. (2007a). Antidepressant use in children, adolescents, and adults. [Online]. Retrieved December 20, 2011 from http://www.fda.gov.

U.S. Food and Drug Administration. (2007b). PROVIGIL® (modafinil) tablets [C-IV]. [Online]. Retrieved April 3, 2012 from http://www.accessdata.fda.gov.

U.S. Food and Drug Administration. (2009). FDA approved smoking cessation products currently marketed. [Online]. Retrieved April 3, 2012 from http://www.fda.gov.

U.S. Food and Drug Administration. (2010a). FDA 101: Smoking cessation products. [Online]. Retrieved April 3, 2012 from http://www.fda.gov.

U.S. Food and Drug Administration. (2010b). FDA approves injectable drug to treat opioid-dependent patients. [Online]. Retrieved December 21, 2011 from http://www.fda.gov.

U.S. Food and Drug Administration. (2011a). FDA drug safety communication: Risk of oral clefts in children born to mothers taking Topamax (topiramate). [Online]. Retrieved April 3, 2012 from http://www.fda.gov.

U.S. Food and Drug Administration. (2011b). FDA drug safety communication: Chantix (varenicline) may increase the risk of certain cardiovascular adverse events in patients with cardiovascular disease. [Online]. Retrieved December 21, 2011 from http://www.fda.gov.

U.S. Food and Drug Administration. (2012). Complete list of vaccines licensed for immunization and distribution in the US. [Online]. Retrieved April 3, 2012 from http://www.fda.gov.

U.S. General Accounting Office. (1995). Child welfare: Complex needs strain capacity to provide services (GAO/HEHS-95-208). Washington, DC: U.S. General Accounting Office.

U.S. Government Printing Office. (2010). Interim final rules under the Paul Wellstone and Pete Domenici Mental Health Parity and Addiction Equity Act of 2008. Federal Register, 75(21), 5410-5451.

U.S. Government Printing Office. (2012). 26 C.F.R. \$ 54.9812-1T: Parity in mental health and substance use disorder benefits. [Online]. Retrieved March 27, 2012 from http://ecfr.gpoaccess.gov.

U.S. Office of Personnel Management. (2011). Confidentiality and the Employee Assistance Program: A question and answer guide for federal employees. [Online]. Retrieved September 9, 2011 from http://www.opm.gov.

U.S. Preventive Services Task Force. (2003). Counseling to prevent tobacco use and tobacco-caused disease. [Online]. Retrieved December 19, 2011 from http://www.uspreventiveservicestaskforce.org. 
U.S. Preventive Services Task Force. (2004). Screening and behavioral counseling interventions in primary care to reduce alcohol misuse: Recommendation statement. Annals of Internal Medicine, 140(7), 555-557.

U.S. Preventive Services Task Force. (2008). Screening for illicit drug use: Recommendation statement (AHRQ Publication No. No. 08-05108-EF-3). [Online]. Retrieved December 19, 2011 from http://www.uspreventiveservicestaskforce.org.

U.S. Preventive Services Task Force. (2009). Counseling and interventions to prevent tobacco use and tobacco-caused disease in adults and pregnant women: U.S. Preventive Services Task Force reaffirmation recommendation statement. Annals of Internal Medicine, 150(8), 551-555.

U.S. Preventive Services Task Force. (2010). Affordable Care Act: USPSTF A and B recommendations. [Online]. Retrieved September 1, 2011 from http://www.uspreventiveservicestaskforce.org

U.S. Preventive Services Task Force. (2011). First annual report to Congress: V. Current evidence gaps deserving of further research. [Online]. Retrieved December 19, 2011 from http://www.uspreventiveservicestaskforce.org.

U.S. Social Security Administration. (2011). Compilation of the social security laws: Sec. 1905. [Online]. Retrieved November 16, 2011 from http://www.ssa.gov.

Uhl, G. R., Drgon, T., Johnson, C., Fatusin, O. O., Liu, Q. R., Contoreggi, C., et al. (2008). "Higher Order" addiction molecular genetics: Covergent data from genome-wide association in humans and mice. Biochemical Pharmacology, 75(1), 98-111.

United Nations Office on Drugs and Crime. (2002). Contemporary drug abuse treatment: A review of the evidence base. Vienna: United Nations Office on Drugs and Crime.

United Nations, Office on Drugs and Crime. (2008). Principles of drug dependence treatment: Discussion paper. [Online]. Retrieved September 26, 2011 from http://www.unodc.org.

United States Army Center for Substance Abuse. (2012). Unit prevention leader (UPL) FAQ. [Online]. Retrieved June 4, 2012 from http://acsap.army.mil.

United States Government Accountability Office. (2011). Mental health and substance use. Employers' insurance coverage maintained or enhanced since Parity Act, but effect of coverage on enrollees varied. [Online]. Retrieved December 22, 2011 from http://www.gao.gov.

United States Medical Licensing Examination. (2008a). 2009 Step 1: Content description and general information. Philadelphia, PA: Federation of State Medical Boards of the United States; National Board of Medical Examiners.

United States Medical Licensing Examination. (2008b). 2009 Step 2 clinical knowledge (CK): Content description and general information. Philadelphia, PA: Federation of State Medical Boards of the United States; National Board of Medical Examiners.

United States Medical Licensing Examination. (2008c). 2009 Step 3: Content description and general information. Philadelphia, PA: Federation of State Medical Boards of the United States; National Board of Medical Examiners.

United States Medical Licensing Examination. (2012). USMLE bulletin: Eligibility. [Online]. Retrieved May 31, 2012 from http://www.usmle.org.

University at Buffalo, Addiction Research Unit. (2001). Before prohibition: Images from the preprohibition era when many psychotropic substances were legally available in America and Europe. [Online]. Retrieved December 2, 2011 from http://wings.buffalo.edu. 
University of Nebraska-Lincoln. (2012). BUROS Center for testing: Test-retest reliability. [Online]. Retrieved April 24, 2012 from http://www.unl.edu.

University of Pennsylvania. (2012). Dr. Benjamin Rush. [Online]. Retrieved March 5, 2012 from http://www.uphs.upenn.edu.

University of Washington, Alcohol and Drug Abuse Institute. (1999). Description of screening and assessment instruments: Teen Addiction Severity Index. [On-line]. Retrieved April 30, 2012 from http://bit.ly/T-ASI_inst.

University of Washington, Alcohol and Drug Abuse Institute. (2006). Evidence-based practices for treating substance use disorders: Matrix of interventions. [Online]. Retrieved September 18, 2008 from http://adai.washington.edu.

University of Washington, Alcohol and Drug Abuse Institute. (2011). Description of screening and assessment instruments: CRAFFT. [Online]. Retrieved March 15, 2011 from http://bit.ly/CRAFFT_inst.

University of Washington. (2011). Substance use screening \& assessment instruments database. [Online]. Retrieved October 26, 2011 from http://lib.adai.washington.edu.

Upadhyaya, H. \& Deas, D. (2008). Pharmacological interventions for adolescent substance use disorders. In Y. Kaminer \& O. G. Bukstein (Eds.), Adolescent substance abuse: Psychiatric comorbidity and high-risk behaviors (pp. 145-161). New York: Routledge.

Upadhyaya, H. P., Deas, D., Brady, K. T., \& Kruesi, M. (2002). Cigarette smoking and psychiatric comorbidity in children and adolescents. Journal of the American Academy of Child \& Adolescent Psychiatry, 41(11), 1294-1305.

USMLE.org. (2012). What is USMLE? [Online]. Retrieved May 24, 2012 from http://www.usmle.org/

Ussher, M. H., Taylor, A. H., West, R., \& McEwen, A. (2000). Does exercise aid smoking cessation? A systematic review. Addiction, 95(2), 199-208.

Ussher, M., Etter, J.-F., \& West, R. (2006). Perceived barriers to and benefits of attending a stop smoking course during pregnancy. Patient Education \& Counseling, 61(3), 467-472.

Vaca, F. E., Winn, D., Anderson, C. L., Kim, D., \& Arcila, M. (2011). Six-month follow-up of computerized alcohol screening, brief intervention, and referral to treatment in the emergency department. Substance Abuse, 32(3), 144-152.

van Abeelen, A. F. M., Elias, S. G., Bossuyt, P. M. M., Grobbee, D. E., van der Schouw, Y. T., Roseboom, T. J., et al. (2011). European Heart Journal, 33(4), 538-545.

van den Bree, M. B., Johnson, E. O., Neale, M. C., \& Pickens, R. W. (1998). Genetic and environmental influences on drug use and abuse/dependence in male and female twins. Drug \& Alcohol Dependency, 52(3), 231-241.

Van Hoof-Haines, K. (2012). Dual diagnosis: The status of treating co-occurring disorders in the U.S. [Online]. Retrieved January 24, 2012 from http://www.drugfree.org.

Van Hook, S., Harris, S. K., Brooks, T., Carey, P., Kossack, R., Kulig, J., et al. (2007). The "Six T's": Barriers to screening teens for substance abuse in primary care. Journal of Adolescent Health, 40(5), 456-461.

Van Ornum, W. (2011). DSM V changes to substance abuse disorders. [Online]. Retrieved June 8, 2011 from http://americanmentalhealthfoundation.org. 
VanDeMark, N. R., Russel, L. A., O'Keefe, M., Finkelstein, N., Noether, C. D., \& Gampel, J. C. (2005). Children of mothers with histories of substance abuse, mental illness, and trauma. Journal of Community Psychology, 33(4), 445-459.

VanderWaal, C. J., McBride, D. C., Terry-McElrath, Y., \& Bishop, R. M. (2006). The role of public health agencies in providing access to adolescent drug treatment services. Journal of Adolescent Health, 39(6), 916-924.

Vandrey, R., \& Haney, M. (2009). Pharmacotherapy for cannabis dependence. How close are we? CNS Drugs, 23(7), 543-553.

Vasilaki, E. I., Hosier, S. G., \& Cox, W. M. (2006). The efficacy of motivational interviewing as a brief intervention for excessive drinking: A meta-analytic review. Alcohol \& Alcoholism, 41(3), 328-335.

Vaughn, M. G., \& Howard, M. O. (2004). Adolescent substance abuse treatment: A synthesis of controlled evaluations. Research on Social Work Practice, 14(5), 325-335.

Verebey, K. G., Meenan, G., \& Buchan, B. J. (2005). Diagnostic laboratory: Screening for drug abuse. In J. H. Lowinson, P. Ruiz, R. B. Millman, \& M. Langrod (Eds.), Substance abuse: A comprehensive textbook (pp. 564-578). Philadelphia, PA: Lippincot Williams \& Wilkins.

Verweij, K. J. H., Zietsch, B. P., Lynskey, M. T., Medland, S. E., Neale, M. C., Martin, N. G., et al. (2010). Genetic and environmental influences on cannabis use initiation and problematic use: A metaanalysis of twin studies. Addiction, 105(3), 417-430.

Vestal, R. E., McGuire, E. A., Tobin, J. D., Andres, R., Norris, A. H., \& Mezey, E. (1977). Aging and ethanol metabolism. Clinical Pharmacology \& Therapeutics, 21(3), 343-354.

VHA Office of Public Health and Environmental Hazards. (2009). Analysis of VA health care utilization among U.S. global war on terrorism (GWOT) veterans: Operation Enduring Freedom and Operation Iraqi Freedom. [Online]. Retrieved May 10, 2012 from http://www.networkofcare.org.

Victor, M., \& Adams, R. D. (1953). The effect of alcohol on the nervous system. Research publications Association for Research in Nervous \& Mental Disease, 32, 526-573.

Vink, J. M., Willemsen, G., \& Boomsma, D. I. (2005). Heritability of smoking initiation and nicotine dependence. Behavior Genetics, 35(4), 397-406.

Visscher, W. A., Feder, M., Burns, A. M., Brady, T. M., \& Bray, R. M. (2003). The impact of smoking and other substance use by urban women on the birthweight of their infants. Substance Use \& Misuse, 38(8), 1063-1093.

Vocci, F., \& Ling, W. (2005). Medications development: Successes and challenges. Pharmacology \& Therapeutics, 108(1), 94-108.

Vogel-Sprott, M., \& Barrett, P. (1984). Age, drinking habits and the effects of alcohol. Journal of Studies on Alcohol, 45(6), 517-521.

Volk, R. J., Steinbauer, J. R., Cantor, S. B., \& Holzer, C. E., III. (1997). The Alcohol Use Disorders Identification Test (AUDIT) as a screen for at-risk drinking in primary care patients of different racial/ethnic backgrounds. Addiction, 92(2), 197-206.

Volkow, N. D. (2005). What do we know about drug addiction? American Journal of Psychiatry, 162(8), 1401-1402.

Volkow, N. D. (2006). Treat the addict, cut the crime rate. [Online]. Washington Post. Retrieved March 30, 2012 from http://www.washingtonpost.com.

Volkow, N. D. (2007a). Addiction and co-occurring mental disorders. NIDA Notes, 21(2). 
Volkow, N. D. (2007b). Drugs, brains, and behavior: The science of addiction [revised August 2010] (NIH Pub. No. 10-5605). Rockville, MD: U.S. Department of Health and Human Services, National Institute on Drug Abuse.

Volkow, N. D. (2009). Letter to the editor: Drug addiction as a brain disorder or disease. JAMA, 301(18), 1881-1882.

Volkow, N. D., \& Li, T.-K. (2005). Drugs and alcohol: Treating and preventing abuse, addiction and their medical consequences. Pharmacology \& Therapeutics, 108(1), 3-17.

Volkow, N. D., \& Li, T.-K. (2005). The neuroscience of addiction. Nature Neuroscience, 8(11), 14291430.

Volkow, N. D., \& Skolnick, P. (2012). New medications for substance use disorders: Challenges and opportunities. Neuropsychopharmacology, 37(1), 290-292.

Volkow, N. D., Baler, R. D., \& Goldstein, R. Z. (2011). Addiction: Pulling at the neural threads of social behaviors. Neuron, 69(4), 599-602.

Volkow, N. D., Chang, L., Wang, G. J., Fowler, J. S., Ding, Y. S., Sedler, M., et al. (2001). Low level of brain dopamine D2 receptors in methamphetamine abusers: Association with metabolism in the orbitofrontal cortex. American Journal of Psychiatry, 158(12), 2015-2021.

Volkow, N. D., Fowler, J. S., \& Wang, G.-J. (2003). The addicted human brain: Insights from imaging studies. Journal of Clinical Investigation, 111(10), 1444-1451.

Volkow, N. D., Fowler, J. S., \& Wang, G.-J. (2004). The addicted human brain viewed in the light of imaging studies: Brain circuits and treatment strategies. Neuropharmacology, 47(Suppl. 1), 3-13.

Volkow, N. D., Fowler, J. S., Wolf, A. P., Schlyer, D., Shiue, C. Y., Alpert, R., et al. (1990). Effects of chronic cocaine abuse on postsynaptic dopamine receptors. American Journal of Psychiatry, 147(6), 719-724.

Volkow, N. D., Wang, G. J., Fowler, J. S., \& Telang, F. (2008). Overlapping neuronal circuits in addiction and obesity: Evidence of systems pathology. Philosophical Transactions of the Royal Society of London. Series B, Biological Sciences, 363(1507), 3191-3200.

Volkow, N. D., Wang, G. J., Fowler, J. S., Logan, J., Gatley, S. J., Hitzemann, R., et al. (1997). Decreased striatal dopaminergic responsiveness in detoxified cocaine-dependent subjects. Nature, 386(6627), 830-833.

Volkow, N. D., Wang, G. J., Fowler, J. S., Logan, J., Hitzemann, R., Ding, Y. S., et al. (1996). Decreases in dopamine receptors but not in dopamine transporters in alcoholics. Alcoholism: Clinical \& Experimental Research, 20(9), 1594-1598.

Volkow, N. D., Wang, G. J., Fowler, J. S., Tomasi, D., \& Baler, R. (2011). Food and drug reward: Overlapping circuits in human obesity and addiction. Current Topics in Behavioral Neurosciences, $11,1-24$.

Volkow, N., \& Li, T.-K. (2005). The neuroscience of addiction. Nature Neuroscience, 8(11), 1429-1430.

Volpicelli, J. R., Alterman, A. I., Hayashida, M., \& O'Brien, C. P. (1992). Naltrexone in the treatment of alcohol dependence. Archives of General Psychiatry, 49(11), 876-880.

Volpicelli, J. R., Rhines, K. C., Rhines, J. S., Volpicelli, L. A., Alterman, A. I., \& O'Brien, C. P. (1997). Naltrexone and alcohol dependence. Role of subject compliance. Archives of General Psychiatry, 54(8), 737-742. 
Voss, C. P., Ceasar, K. W., Tymus, T., \& Fielder, I. G. (2002). Perceived versus actual physical accessibility of substance abuse treatment facilities. Topics in Spinal Cord Injury Rehabilitation, 7(3), 47-55.

Voth, E. A. (2003). A peek into Pandora's Box: The medical excuse marijuana controversy. Journal of Addictive Diseases, 22(4), 27-46.

Wadsworth, R., Spampneto, A. M., \& Halbrook, B. M. (1995). The role of sexual trauma in the treatment of chemically dependent women: Addressing the relapse issue. Journal of Counseling \& Development, 73(4), 401-406.

Wagner, E. F. (2009). Improving treatment through research: Directing attention to the role of development in adolescent treatment success. [Online]. Retrieved October 12, 2011 from http://pubs.niaaa.nih.gov.

Wagner, E. H., Austin, B. T., Davis, C., Hindmarsh, M., Schaefer, J., \& Bonomi, A. (2001). Improving chronic illness care: Translating evidence into action. Health Affairs, 20(6), 64-78.

Wagner, F. A., \& Anthony, J. C. (2002). From first drug use to drug dependence: Developmental periods of risk for dependence upon marijuana, cocaine, and alcohol. Neuropsychopharmacology, 26(4), 479488

Wakschlag, L. S., Pickett, K. E., Cook Jr, E., Benowitz, N., \& Leventhal, B. (2002). Maternal smoking during pregnancy and severe antisocial behavior in offspring: A review. American Journal of Public Health, 92(6), 966-974.

Walden, B., Iacono, W. G., \& McGue, M. (2007). Trajectories of change in adolescent substance use and symptomatology: Impact of paternal and maternal substance use disorders. Psychology of Addictive Behaviors, 21(1), 35-43.

Waldron, H. B., \& Kaminer, Y. (2004). On the learning curve: The emerging evidence supporting cognitive-behavioral therapies for adolescent substance abuse. Addiction, 99(Suppl. 2), 93-105.

Waldron, H. B., \& Turner, C. W. (2008). Evidence-based psychosocial treatments for adolescent substance abuse. Journal of Clinical Child \& Adolescent Psychology, 37(1), 238-261.

Waldrop, J., \& Stern, S. M. (2003). Disability status 2000: Census 2000 brief. Washington, DC: U.S. Department of Commerce, Economics and Statistics Administration, U.S. Census Bureau.

Wall Street Journal. (2012). A rewired brain: Many now see addiction as a chronic brain disease that requires new approaches to treatment. [Online]. Retrieved February 22, 2012 from http://online.wsj.com.

Wall, M. M., Poh, E., Cerda, M., Keyes, K. M., Galea, S., \& Hasin, D. S. (2011). Adolescent marijuana use from 2002 to 2008: Higher in states with medical marijuana laws, cause still unclear. Annals of Epidemiology, 21(9), 714-716.

Wallack, S. S., Thomas, C. P., Martin, T. C., Chilingerian, J., \& Reif, S. (2010). Substance abuse treatment organizations as mediators of social policy: Slowing the adoption of a congressionally approved medication. Journal of Behavioral Health Service Research, 37(1), 64-78.

Walley, A. Y., Alperen, J. K., Cheng, D. M., Botticelli, M., Castro-Donlan, C., Samet, J. H., et al. (2008). Office-based management of opioid dependence with buprenorphine: Clinical practices and barriers. Journal of General Internal Medicine, 23(9), 1393-1398.

Walsh, M. M., Langer, T. J., Kavanagh, N., Mansell, C., MacDougal, W., Kavanagh, C., et al. (2010). Smokeless tobacco cessation cluster randomized trial with rural high school males: Intervention interaction with baseline smoking. Nicotine \& Tobacco Research, 12(6), 543-550. 
Walter, L. J., Ackerson, L., \& Allen, S. (2005). Medicaid chemical dependency patients in a commercial health plan: Do high medical costs come down over time? Journal of Behavioral Health Services \& Research, 32(3), 253-263.

Walton, M. A., Blow, F. C., Bingham, C. R., \& Chermack, S. T. (2003). Individual and social/environmental predictors of alcohol and drug use 2 years following substance abuse treatment. Addictive Behaviors, 28(4), 627-642.

Walton, M. A., Chermack, S. T., Shope, J. T., Bingham, C. R., Zimmerman, M. A., Blow, F. C., et al. (2010). Effects of a brief intervention for reducing violence and alcohol misuse among adolescents: A randomized controlled trial. JAMA, 304(5), 527-535.

Wang, G. J., Volkow, N. D., Fowler, J. S., Logan, J., Abumrad, N. N., Hitzemann, R. J., et al. (1997). Dopamine D2 receptor availability in opiate-dependent subjects before and after naloxoneprecipitated withdrawal. Neuropsychopharmacology, 16(2), 174-182.

Washington State Institute for Public Policy. (2012a). Brief Alcohol Screening and Intervention for College Students (BASICS). [Online]. Retrieved May 11, 2012 from http://www.wsipp.wa.gov.

Washington State Institute for Public Policy. (2012b). Motivational interviewing / motivational enhancement therapy for cannabis abuse. [Online]. Retrieved May 11, 2012 from http://www.wsipp.wa.gov.

Washington State Institute for Public Policy. (2012c). Motivational interviewing / motivational enhancement therapy for illicit drug abuse. [Online]. Retrieved May 11, 2012 from http://www.wsipp.wa.gov..

Washington State Institute for Public Policy. (2012d). Motivational interviewing / motivational enhancement therapy for alcohol abuse. [Online]. Retrieved May 11, 2012 from http://www.wsipp.wa.gov.

Washington State Institute for Public Policy. (2012e). Motivational interviewing / motivational enhancement therapy for smoking. [Online]. Retrieved May 11, 2012 from http://www.wsipp.wa.gov.

Washton, A. M. (2001). Why psychologists should know how to treat substance use disorders. [Online]. Retrieved July 18, 2006 from http://www.apa.org.

Watkins, K. E., Pincus, H. A., Paddock, S., Smith, B., Woodroffe, A., Farmer, C., et al. (2011). Care for veterans with mental and substance use disorders: Good performance, but room to improve on many measures. Health Affairs, 30(11), 2194-2203.

Watkins, K., Pincus, H. A., Tanielian, T. L., \& Lloyd, J. (2003). Using the chronic care model to improve treatment of alcohol use disorders in primary care settings. Journal of Studies on Alcohol, 64(2), 209218.

Waxmonsky, J. G., \& Wilens, T. E. (2005). Pharmacotherapy of adolescent substance use disorders: A review of the literature. Journal of Child \& Adolescent Psychopharmacology, 15(5), 810-825.

Webster-Stratton, C., \& Taylor, T. (2001). Nipping early risk factors in the bud: Preventing substance abuse, delinquency, and violence in adolescence through interventions targeted at young children ( $0-8$ years). Prevention Science, 2(3), 165-192.

Wechsler, H., Davenport, A., Dowdall, G., Moeykens, B., \& Castillo, S. (1994). Health and behavioral consequences of binge drinking in college. A national survey of students at 140 campuses. JAMA, 272(21), 1672-1677.

Weiner, D. (2011). Brain scan predicts addiction. [Online]. Retrieved December 22, 2011 from http://www.yaledailynews.com. 
Weinstein, M. C., Siegel, J. E., Gold, M. R., Kamlet, M. S., \& Russell, L. B. (1996). Recommendations of the Panel on Cost-Effectiveness in Health and Medicine. JAMA, 276(15), 1253-1258.

Weintraub, T. A., Saitz, R., \& Samet, J. H. (2003). Education of preventive medicine residents. Alcohol, tobacco and other drug use. American Journal of Preventive Medicine, 24(1), 101-105.

Weisner, C. (1993). Toward an alcohol treatment entry model: A comparison of problem drinkers in the general population and in treatment. Alcohol: Clinical \& Experimental Research, 17(4), 746-752.

Weisner, C. \& Schmidt, L. (2001). Rethinking access to alcohol treatment. In M. Galanter (Series Ed.), Recent developments in alcoholism: Vol. 15. Services research in the era of managed care (pp. 107136). New York: Kluwer Academic/Plenum Press

Weiss, R. D., Griffin, M. L., Greenfield, S. F., Najavits, L. M., Wyner, D., Soto, J. A., et al. (2000). Group therapy for patients with bipolar disorder and substance dependence: Results of a pilot study. Journal of Clinical Psychiatry, 61(5), 361-367.

Weiss, R. D., Griffin, M. L., Kolodziej, M. E., Greenfield, S. F., Najavits, L. M., Daley, D. C., et al. (2007). A randomized trial of integrated group therapy versus group drug counseling for patients with bipolar disorder and substance dependence. American Journal of Psychiatry, 164(1), 100-107.

Weiss, R. D., Potter, J. S., Fiellin, D. A., Byrne, M., Connery, H. S., Dickinson, W., et al. (2011). Adjunctive counseling during brief and extended buprenorphine-naloxone treatment for prescription opioid dependence. A 2-phase randomized controlled trial. Archives of General Psychiatry, 68(12), 1238-1246.

Wells, R., Lemark, C. H., Alexander, J. A., Nahra, T. A., Ye, Y., \& Campbell, C. (2007). Do licensing and accreditation matter in outpatient substance abuse treatment programs? Journal of Substance Abuse Treatment, 33(1), 43-50.

Welsh, W. N. (2007). A multisite evaluation of prison-based therapeutic community drug treatment. Criminal Justice \& Behavior, 34(11), 1481-1498.

Wendt, A., Kenny, L., \& Schultz, L. (2010). 2010 NCLEX-RN® detailed test plan: Candidate version. Chicago, IL: National Council of State Boards of Nursing, NCLEX Examination Committee.

Wendt, A., Kenny, L., \& Schultz, L. (2011). 2011 NCLEX-RN® detailed test plan: Candidate version. Chicago, IL: National Council of State Boards of Nursing, NCLEX Examination Committee.

Wenzel, S. L., Burnam, A. M., Koegel, P., Morton, S. C., Miu, A., Jinnett, K. J., et al. (2001). Access to inpatient or residential substance abuse treatment among homeless adults with alcohol or other drug use disorders. Medical Care, 39(11), 1158-1169.

Werner, R. M., Kolstad, J. T., Stuart, E. A., \& Polsky, D. (2011). The effect of pay-for-performance in hospitals: Lessons for quality improvement. Health \& Social Care in the Community, 30(4), 690-698.

West, R., \& Shiffman, S. (2001). Effect of oral nicotine dosing forms on cigarette withdrawal symptoms and craving: A systematic review. Psychopharmacology, 155(2), 115-122.

West, S. L. (2007). The accessibility of substance abuse treatment facilities in the United States for persons with disabilities. Journal of Substance Abuse Treatment, 33(1), 1-5.

West, S. L., Garbutt, J. C., Carey, T. S., Lux, L. J., Jackman, A. M., Tolleson-Rinehart, S., et al. (1999). Evidence reports/Technology assessments No. 3: Pharmacotherapy for alcohol dependence. Rockville, MD: Agency for Health Care Policy and Research.

Westermeyer, J., Wahmanholm, K., \& Thuras, P. (2001). Effects of childhood physical abuse on course and severity of substance abuse. American Journal on Addictions, 10(2), 101-110. 
Wewers, M. E., Kidd, K., Armbruster, D., \& Sarna, L. (2004). Tobacco dependence curricula in U.S. baccalaureate and graduate nursing education. Nursing Outlook, 52(2), 95-101.

Whelan, R., Conrod, P. J., Poline, J. B., Lourdusamy, A., Banaschewski, T., Barker, G. J., et al. (2012). Adolescent impulsivity phenotypes characterized by distinct brain netowrks. Nature Neuroscience. 10.1038/nn.3092

Whitby, L. G. (1974). Definitions and criteria. Lancet, 304(7884), 819-821.

White, A. G., Birnbaum, H. G., Mareva, M. N., Daher, M., Vallow, S., Schein, J., et al. (2005). Direct costs of opioid abuse in an insured population in the United States. Journal of Managed Care Pharmacy, 11(6), 469-479.

White, A. R., Rampes, H., \& Campbell, J. L. (2006). Acupuncture and related interventions for smoking cessation. Cochrane Database of Systematic Reviews, (1), CD000009.

White, H. R., Morgan, T., Pugh, L., Celinska, K., Labouvie, E. W., \& Pandina, R. (2006). Evaluating two brief substance-use interventions for mandated college students. Journal of Studies on Alcohol, 67(2), 309-317.

White, T. (2003). Drug testing at work: Issues and perspectives. Substance Use and Misuse, 38(11-13), 1891-1902.

White, W. L. \& Kelly, J. F. (2011). Introduction: The theory, science, and practice of recovery management. In J. F.Kelly \& W. L.White (Eds.), Addiction recovery management: Theory, research and practice (pp. 1-6). New York: Springer.

White, W. L. (1998). Slaying the dragon: The history of addiction treatment and recovery in America. Bloomington, IL: Chestnut Health Systems, Lighthouse Institute.

White, W. L. (2008). Recovery management and recovery-oriented systems of care: Scientific rationale and promising practices. Philadelphia, PA: Northeast Addiction Technology Transfer Center, Great Lakes Addiction Technology Transfer Center, Philadelphia Department of Behavioral Health/Mental Retardation Services.

White, W. L., \& Kurtz, E. (2008). Twelve defining moments in the history of Alcoholics Anonymous. [Online]. Retrieved February 29, 2012 from http://www.williamwhitepapers.com

White, W. L., \& McLellan, A. T. (2008). Addiction as a chronic disorder: Key messages for clients, families and referral sources. Counselor, 9(3), 24-33.

White, W., \& Kurtz, E. (2005). The varieties of recovery experience: A primer for addiction treatment professionals and recovery advocates. Chicago, IL: Great Lakes Addiction Technology Transfer Center.

Whitlock, E. P., Polen, M. R., Green, C. A., Orleans, T., \& Klein, J. (2004). Behavioral counseling interventions in primary care to reduce risky/harmful alcohol use by adults: A summary of the evidence for the U.S. Preventive Services Task Force. Annals of Internal Medicine, 140(7), 557-568.

Whitten, L. (2010). Cocaine vaccine helps some reduce drug abuse. NIDA Notes, 23(3), 1, 6-7.

Whitworth, A. B., Fischer, F., Lesch, O. M., Nimmerrichter, A., Oberbauer, H., Platz, T., et al. (1996). Comparison of acamprosate and placebo in long-term treatment of alcohol dependence. Lancet, 347(9013), 1438-1442.

WHO ASSIST Working Group. (2002). The Alcohol, Smoking and Substance Involvement Screening Test (ASSIST): Development, reliability and feasibility. Addiction, 97(9), 1183-1194. 
Wickizer, T. M., Krupski, A., Stark, K. D., Mancuso, D., \& Campbell, K. (2006). The effect of substance abuse treatment on Medicaid expenditures among general assistance welfare clients in Washington state. Milbank Quarterly, 84(3), 555-576.

Wiesner, M., Windle, M., \& Freeman, A. (2005). Work stress, substance use, and depression among young adult workers: An examination of main and moderator effect model. Journal of Occupational Health \& Psychology, 10(2), 83-96.

Wilcox, C. S., Noble, E. P., \& Oskooilar, N. (2011). ANKK1/DRD2 locus variants are associated with rimonabant efficacy in aiding smoking cessation: Pilot data. Journal of Investigative Medicine, 59(8), $1280-1283$.

Wilens, T. E., Biederman, J., Adamson, J. J., Henin, A., Sgambati, S., Gignac, M., et al. (2008). Further evidence of an association between adolescent bipolar disorder with smoking and substance use disorders: A controlled study. Drug \& Alcohol Dependence, 95(3), 188-198.

Wilk, J. E., Bliese, P. D., Kim, P. Y., Thomas, J. L., McGurk, D., \& Hoge, C. W. (2010). Relationship of combat experiences to alcohol misuse among U.S. soldiers returning from the Iraq war. Drug \& Alcohol Dependence, 108(1-2), 115-121.

Willenbring, M. (2009). BHC Journal's interview with Mark Willenbring, M.D., About NESARC. [Online]. Retrieved February 4, 2009 from http://www.bhcjournal.com.

Willenbring, M. L. (2008a). Alcohol disorders: Does the treatment field have the courage to change? Addiction Professional, 6(5), 13-19.

Willenbring, M. L. (2008b). New research is redefining alcohol disorders: Does the treatment field have the courage to change? Addiction Professional, 6(5), 12-19.

Williams, D. M. (2009). Preparing pharmacy students and pharmacists to provide tobacco cessation counseling. Drug \& Alcohol Review, 28(5), 533-540.

Williams, S. E. (1980). The use of beverage alcohol as medicine, 1790-1860. Journal of Studies on Alcohol, 41(5), 543-566.

Wills, T. A., Sandy, J. M., Yaeger, A., \& Shinar, O. (2001). Family risk factors and adolescent substance use: Moderation effects for temperament dimensions. Developmental Psychology, 37(3), 283-297.

Wills, T. A., Walker, C., Mendoza, D., \& Ainette, M. G. (2006). Behavioral and emotional self-control: Relations to substance use in samples of middle and high school students. Psychology of Addictive Behaviors, 20(3), 265-278.

Wilper, A. P., Woolhandler, S., Lasser, K. E., McCormick, D., Bor, D. H., \& Himmelstein, D. U. (2009). Health insurance and mortality in US adults. American Journal of Public Health, 99(12), 2289-2295.

Wilson, J. M. G., \& Jungner, G. (1968). Principles and practice of screening for disease. [Online]. Retrieved February 9, 2012 from http://whqlibdoc.who.int.

Windle, M., \& Windle, R. C. (2001). Depressive symptoms and cigarette smoking among middle adolescents: Prospective associations and intrapersonal and interpersonal influences. Journal of Consulting \& Clinical Psychology, 69(2), 215-226.

Windle, M., \& Windle, R. C. (2006). Adolescent temperament and lifetime psychiatric and substance abuse disorders assessed in young adulthood. Personality \& Individual Differences, 41(1), 15-25.

Wingerson, D., \& Ries, R. K. (1999). Assertive community treatment for patients with chronic and severe mental illness who abuse drugs. Journal of Psychoactive Drugs, 31(1), 13-18. 
Winhusen, T., Kropp, F., Babcock, D., Hague, D., Erickson, S. J., Renz, C., et al. (2008). Motivational enhancement therapy to improve treatment utilization and outcome in pregnant substance users. Journal of Substance Abuse Treatment, 35(2), 161-173.

Winters, J., Fals-Stewart, W., O'Farrell, T. J., Bircgler, G. R., \& Kelley, M. L. (2002). Behavioral couples therapy for female substance-abusing patients: Effects on substance use and relationship adjustment. Journal of Consulting \& Clinical Psychology, 70(2), 344-355.

Winters, K. C. (1999). Treating adolescents with substance use disorders: An overview of practice issues and treatment outcome. Substance Abuse, 20(4), 203-225.

Winters, K. C. (2004). Assessment of alcohol and other drug use behaviors among adolescents. [Online]. Retrieved April 9, 2012 from http://pubs.niaaa.nih.gov.

Winters, K. C., \& Leitten, W. (2007). Brief intervention for drug-abusing adolescents in a school setting. Psychology of Addictive Behaviors, 21(2), 249-254.

Winters, K. C., \& Zenilman, J. M. (1994). Simple screening instruments for outreach for alcohol and other drug abuse and infectious diseases. Treatment improvement protocol (TIP) Series 11 (DHHS Pub. No. (SMA) 953058). Rockville, MD: U.S. Department of Health and Human Services, Substance Abuse and Mental Health Services Administration, Center for Substance Abuse Treatment.

Wittchen, H. U. (1994). Reliability and validity studies of the WHO--Composite International Diagnostic Interview (CIDI): A critical review. Journal of Psychiatric Research, 28(1), 57-84.

Wojcik, M. H., \& Hawthorne, J. S. (2007). Sensitivity of commercial ethyl glucuronide (ETG) testing in screening for alcohol abstinence. Alcohol \& Alcoholism, 42(4), 317-320.

Wolfe, B. L., \& Meyers, R. J. (1999). Cost-effective alcohol treatment: The community reinforcement approach. Cognitive \& Behavioral Practice, 6(2), 105-109.

Wolfer, S. (2007). Diabetes in denial. [Online]. Retrieved April 27, 2012 from http://www.psychologytoday.com.

Wong, M. M., Brower, K. J., \& Zucker, R. A. (2009). Childhood sleep problems, early onset of substance use and behavioral problems in adolescence. Sleep Medicine, 10(7), 787-796.

Woody, G. E., Poole, S. A., Subramaniam, G., Dugosh, K., Bogenschutz, M., Abbott, P., et al. (2008). Extended vs short-term buprenorphine-naloxone for treatment of opioid-addicted youth: A randomized trial. JAMA, 300(17), 2003-2011.

Workgroup on Substance Abuse Self-Help Organizations. (2003). Self-help organizations for alcohol and drug problems: Towards evidence-based practice and policy. Menlo Park, CA: U.S. Department of Veterans Affairs, Center for Health Care Evaluation.

World Health Organization. (2004). Neuroscience of psychoactive substance use and dependence. Geneva, Switzerland: World Health Organization.

World Health Organization. (2008). The effectiveness of a brief intervention for illicit drugs linked to the alcohol, smoking and substance involvement screening test (ASSIST) in primary health care settings: A technical report of phase III findings of the WHO ASSIST randomized controlled trial. Geneva, Switzerland: Prepared by Rachel Humeniuk, Victoria Dennington \& Robert Ali on behalf of the WHO ASSIST Phase III Study Group.

World Health Organization. (2009). Global health risks. Mortality and burden of disease attributable to selected major risks. [Online]. Retrieved March 13, 2012 from http://www.who.int/healthinfo.

World Health Organization. (2011a). Classifications. International classification of diseases (ICD). [Online]. Retrieved December 2, 2011 from http://www.who.int. 
World Health Organization. (2011b). International statistical classification of diseases and related health problems 10th revision (ICD-10_ version for 2010. Chapter V. Mental and behavioral disorders: F00F99). [Online]. Retrieved December 2, 2011 from http://apps. who.int.

World Health Organization. (2011c). Management of substance abuse. Lexicon of alcohol and drug terms published by the World Health Organization. [Online]. Retrieved December 2, 2011 from http://www.who.int.

World Health Organization. (2011d). The ICD-10 classification of mental and behavioral disorders: Diagnostic criteria for research. [Online]. Retrieved August 17, 2011 from http://www.who.int.

Worley, M. J., Trim, R. S., Tate, S. R., Hall, J. E., \& Brown, S. A. (2010). Service utilization during and after outpatient treatment for comorbid substance use disorder and depression. Journal of Substance Abuse Treatment, 39(2), 124-131.

Wright, T., Myrick, H., Henderson, S., Peters, H., \& Malcolm, R. (2006). Risk factors for delirium tremens: A retrospective chart review. American Journal on Addictions, 15(3), 213-219.

Wu, L. T., \& Blazer, D. G. (2011). Illicit and nonmedical drug use among older adults: A review. Journal of Aging \& Health, 23(3), 481-504.

Wu, L. T., Blazer, D. G., Li, T.-K., \& Woody, G. E. (2011). Treatment use and barriers among adolescents with prescription opioid use disorders. Addictive Behaviors, 36(12), 1233-1237.

Wurtman, R. J., Hefti, F., \& Melamed, E. (1981). Precursor control of neurotransmitter synthesis. Pharmacological Reviews, 32(4), 315-330.

Wynder, E. L., \& Graham, E. A. (1950). Tobacco smoking as a possible etiologic factor in bronchiogenic carcinoma. A study of six hundred and eighty-four proved cases. JAMA, 143(4), 329-336.

Xu, J., Wang, J., Rapp, R. C., \& Carlson, R. G. (2007). The multidimensional structure of internal barriers to substance abuse treatment and its invariance across gender, ethnicity, and age. Journal of Drug Issues, 37(2), 321-340.

Yacoub, R., Habib, H., Lahdo, A., Al, A. R., Varjabedian, L., Atalla, G., et al. (2010). Association between smoking and chronic kidney disease: A case control study. BMC Public Health, 10, 731. doi: 10.1186/1471-2458-10-731.

Yip, J. K., Hay, J. L., Ostroff, J. S., Stewart, R. K., \& Cruz, G. D. (2000). Dental students' attitudes toward smoking cessation guidelines. Journal of Dental Education, 64(9), 641-650.

Yoast, R. A., Wilford, B. B., \& Hayashi, S. W. (2008). Encouraging physicians to screen for and intervene in substance use disorders: Obstacles and strategies for change. Journal of Addictive Diseases, 27(3), 77-97.

Young, A., Chaudhry, H. J., Rhyne, J., \& Dugan, M. (2011). A census of actively licensed physicians in the United States, 2010. Journal of Medical Regulation, 96(4), 10-20.

Yudko, E., Lozhkina, O., \& Fouts, A. (2007). A comprehensive review of the psychometric properties of the Drug Abuse Screening Test. Journal of Substance Abuse Treatment, 32(2), 189-198.

Yuferov, V., Levran, O., Proudnikov, D., Nielsen, D. A., \& Kreek, M. J. (2010). Search for genetic markers and functional variants involved in the development of opiate and cocaine addiction and treatment. Annals of the New York Academy of Sciences, 1187, 184-207.

Yung, L., Gordis, E., \& Holt, J. (1983). Dietary choices and likelihood of abstinence among alcoholic patients in an outpatient clinic. Drug \& Alcohol Dependency, 12(4), 355-362.

Zakhari, S., \& Li, T. K. (2007). Determinants of alcohol use and abuse: Impact of quantity and frequency of patterns on liver disease. Hepatology, 46(6), 2032-2039. 
Zanis, D. A., Melochick, J., \& Wagner, J. (2011). Brief assessment oral health screening increase smokeless tobacco user enrollment. [Online]. Retrieved December 12, 2011 from http://www.smokelesstobaccosummit.com.

Zaric, G. S., Barnett, P. G., \& Brandeau, M. L. (2000). HIV transmission and the cost-effectiveness of methadone maintenance. American Journal of Public Health, 90(7), 1100-1111.

Zarkin, G. A., Bray, J. W., Aldridge, A., Mitra, D., Mills, M. J., Couper, D. J., et al. (2008). Cost and cost-effectiveness of the COMBINE Study in alcohol-dependent patients. Archives of General Psychiatry, 65(10), 1214-1221.

Zemore, S. E., \& Kaskutas, L. A. (2008). 12-step involvement and peer helping in day hospital and residential programs. Substance Use \& Misuse, 43(12-13), 1882-1903.

Zemore, S. E., Kaskutas, L. A., \& Ammon, L. N. (2004). In 12-step groups, helping helps the helper. Addiction, 99(8), 1015-1023.

Zhang, Z., Friedmann, P. D., \& Gerstein, D. R. (2003).Does retention matter? Treatment duration and improvement in drug use. Addiction, 98(5), 673-684.

Zickler, P. (2000). Evidence builds that genes influence cigarette smoking. NIDA Notes, 15(2), 1, 5.

Ziedonis, D. M., Smelson, D., Rosenthal, R. N., Batki, S. L., Green, A. I., Henry, R. J., et al. (2005). Improving the care of individuals with schizophrenia and substance use disorders: Consensus recommendations. Journal of Psychiatry Practice, 11(5), 315-339.

Ziedonis, D., \& Williams, J. M. (2003). Management of smoking in people with psychiatric disorders. Current Opinions in Psychiatry, 16(3), 305-315.

Ziedonis, D., Hitsman, B., Beckham, J. C., Zvolensky, M., Adler, L. E., Audrain-McGovern, J., et al. (2008). Tobacco use and cessation in psychiatric disorders: National Institute of Mental Health report. Nicotine \& Tobacco Research, 10(12), 1691-1715.

Ziedonis, D., Kalman, D., Kolodziej, M., Johnson, C. W., \& Kim, S. (2011). Tobacco addiction and psychological co-morbidities. In S. Pagoto (Ed.), Psychological co-morbidities of physical illness: A behavioral medicine perspective (pp. 207-232). New York, NY: Springer Science \& Business Media.

Zilberman, M. L. \& Blume, S. B. (2005). Drugs and women. In J. Lowinson, P. Ruiz, R. B. Millman, \& J. G. Langrod (Eds.), Substance abuse: A comprehensive textbook (p. 1064-1092). Baltimore, MD: Williams and Wilkins.

Zook, C. J., \& Moore, F. D. (1980). High-cost users of medical care. New England Journal of Medicine, 302(18), 996-1002.

Zvolensky, M. J., Baker, K., Yartz, A. R., Gregor, K., Leen-Feldner, E. W., \& Feldner, M. T. (2005). Mental health professionals with a specialty in anxiety disorders: Knowledge, training, and perceived competence in smoking cessation practices. Cognitive \& Behavioral Practice, 12(3), 312-318.

Zwerdling, D. (2007). Army dismissals for mental health, misconduct rise. [Online]. Retrieved May 9, 2012 from http://www.npr.org.

Zyczynski, T. M., \& Coyne, K. S. (2000). Hypertension and current issues in compliance and patient outcomes. Current Hypertension Reports, 2(6), 510-514. 